Prepared for the U.S. Department of Energy

under Contract DE-AC05-76RL01830

\title{
Recommended Parameter Values for GENII Modeling of Radionuclides in Routine Air and Water Releases
}
SF Snyder
BA Napier
Cl Arimescu
TR Hay

November 2013

Pacific Northwest

NATIONAL LABORATORY

Proudly Operated by Battelle Since 1965 


\title{
Recommended Parameter Values for GENII Modeling of Radionuclides in Routine Air and Water Releases
}

\author{
SF Snyder \\ BA Napier \\ CI Arimescu \\ TR Hay
}

November 2013

Pacific Northwest National Laboratory

Richland, Washington 99352

PR Reed, Project Manager

NRC Job Code V6237

U.S. Nuclear Regulatory Commission Office of Nuclear Regulatory Research

Washington, D.C. 20555-0001 


\section{Contents}

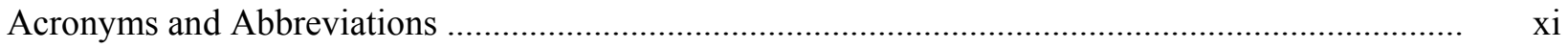

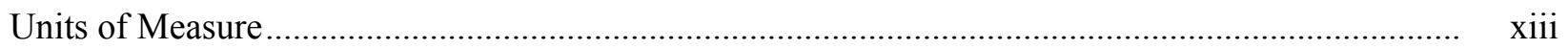

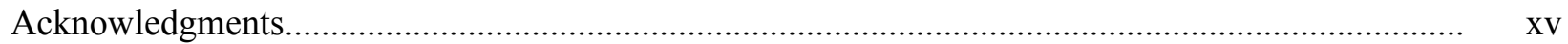

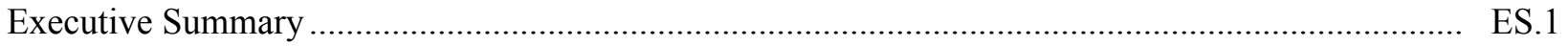

Executive Summary of Generic Parameters …............................................................................ ES.3

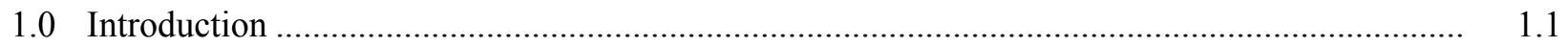

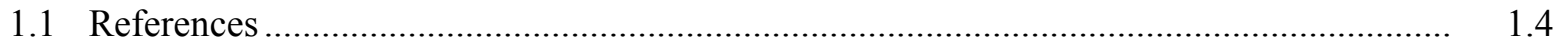

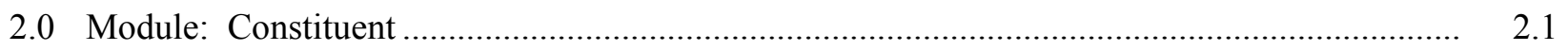

2.1 Tab: Edit Constituent Properties/Properties ................................................................... 2.1

2.1.1 CLKD Dry Soil-Water Partition Coefficient ..................................................... 2.1

2.1.2 CLANDF Inhalation Volatilization Factor ...................................................... 2.2

2.1.3 CLWPF Water Purification Factor................................................................... 2.2

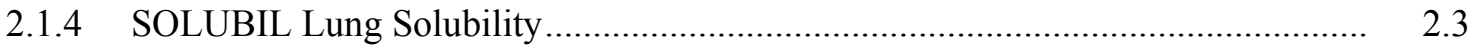

2.1.5 CLDFAx Inhalation Dose Factor ................................................................ 2.3

2.1.6 CLRDFGx Ingestion Dose Factor .................................................................... 2.4

2.1.7 CLDEX External Dose Factor, Air Immersion ................................................. 2.4

2.1.8 CLDIMR External Dose Factor, Water Immersion ............................................ 2.5

2.1.9 CLDSHx External Dose Factor, Ground Contaminated to $15 \mathrm{~cm}$.......................... 2.5

2.1.10 CLDIAM Inhalation Dose Factor, Particle Diameter ............................................ 2.6

2.1.11 CLBF $x$ and CLBM $x$ Bioaccumulation Factor for Aquatic Animals ..................... 2.7

2.1.12 CLBFP and CLBMP Bioaccumulation Factor in Aquatic Plants ........................ 2.17

2.1.13 CLBSAF Bioaccumulation in Aquatic Biota from Sediment ............................... 2.19

2.1.14 CLFMT Feed to Meat Transfer Factor.................................................................. 2.19

2.1.15 CLFMK Feed to Milk Transfer Factor............................................................... 2.22

2.1.16 CLFPL Feed to Poultry and CLFEG Egg Transfer Factor .................................... 2.25

2.1.17 CLBVx Bioconcentration to Edible Crop Portion from Soil ................................ 2.29

2.1.18 CLBVAx Bioconcentration in Animal Forage or Hay from Soil.......................... 2.38

2.1.19 CLBVAG Bioconcentration in Animal Feed Grain from Soil .............................. 2.38

2.1.20 CLBVOV Bioconcentration in Dry Other Vegetables from Soil.......................... 2.39

2.1.21 CLVD Atmospheric Deposition Velocity .......................................................... 2.39

2.1.22 CLSHALF Decay Half-Life in Soil ................................................................... 2.39

2.1.23 CLCLASS Atmospheric Deposition Class ....................................................... 2.39

2.2 References: Constituent Module .................................................................................. 2.40

3.0 Module: User-Defined Air....................................................................................... 3.1

3.1 References: User-Defined (AIR) Module ............................................................... 3.1

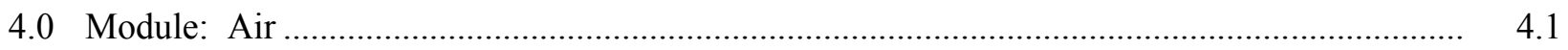


4.1 Tab: Model Information/Radial Grid Definition .........................................................

4.2 Tab: Model Information/Model Parameters .........................................................................

4.3 Tab: Model Information/Default Parameters ….............................................................

4.3.1 ARMINRISESPD Minimum Speed during Plume Rise ...................................... 4.1

4.3.2 ARMINSIGYSHIFT Sigma Shift to SI Cloud Shine........................................... 4.2

4.3.3 ARTRANSRESIST Transfer Resistance for Iodine and Particles ......................... 4.2

4.3.4 ARMINWIND Maximum Wind Speed for "Calm" ............................................. 4.2

4.4 References: Air Module ............................................................................................. 4.3

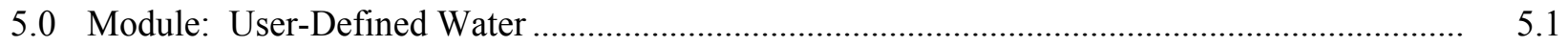

5.1 References: User-Defined (Water) Module ...................................................................... 5.1

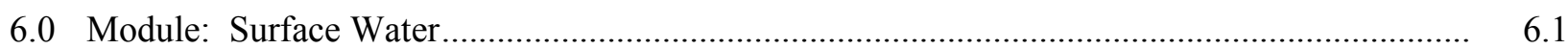

6.1 References: Surface Water Module..................................................................................

7.0 Module: Exposure Pathways - Chronic Exposure ..............................................................

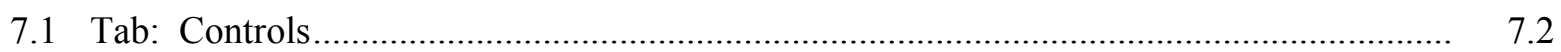

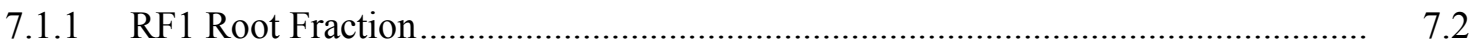

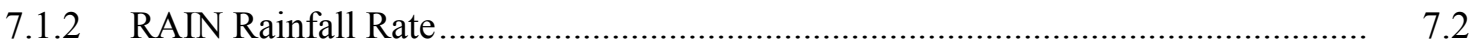

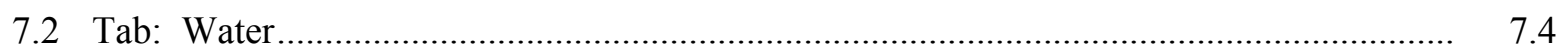

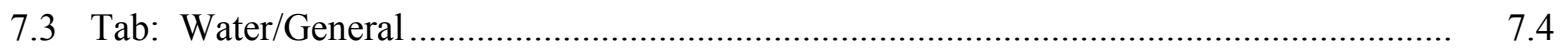

7.3.1 RIRRR Residential Land Irrigation, IRTIMR Irrigation Time for Residential

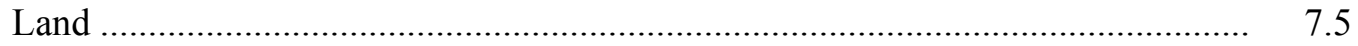

7.3.2 ANDKR Indoor Volatilization Factor for Radionuclides .................................... 7.6

7.3.3 HOLDDW Delay Time for Water Distribution ................................................... 7.7

7.3.4 SEDDN Shoreline Sediment Density .................................................................. 7.7

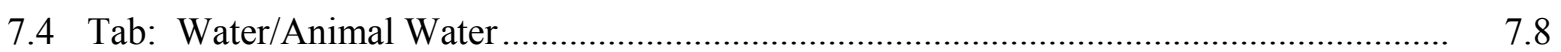

7.4.1 DWFACA and DWATER Contaminated Fraction and Animal Drinking Water Rate.

7.5 Tabs: Water/Irrigation Rates and Water/Irrigation Times............................................. 7.10

7.5.1 RIRR Crop Irrigation Rate, RIRRA Feed and Forage Irrigation Rate, IRTIMT Crop Irrigation Time, and IRTIMA Feed and Forage Irrigation Rate

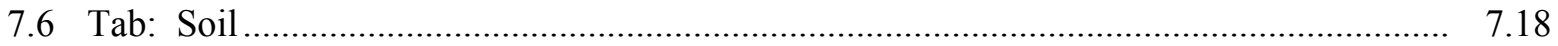

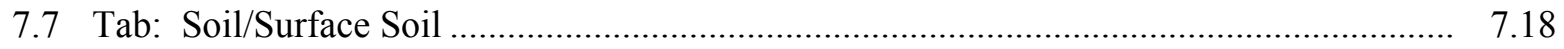

7.7.1 SLDN Surface Soil Areal Density and SURCM Surface Soil Thickness .............. 7.18

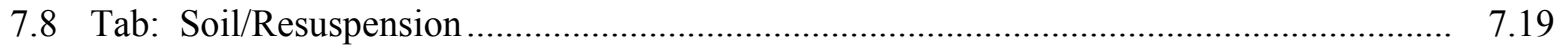

7.8.1 XMLF Mass Loading Factor for Resuspension .................................................. 7.20

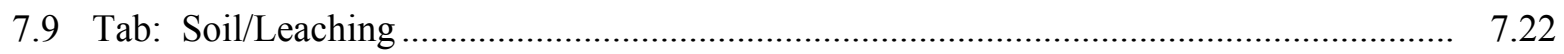

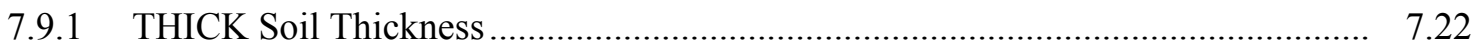

7.9.2 MOISTC Surface Soil Moisture Content …...................................................... 7.22

7.9.3 BULKD Surface Soil Bulk Density .................................................................... 7.22

7.9.4 VLEACH Total Infiltration Rate.......................................................................... 7.23 
7.9.5 SOILKD Parent and Progeny Soil Absorption Coefficient...................................... 7.23

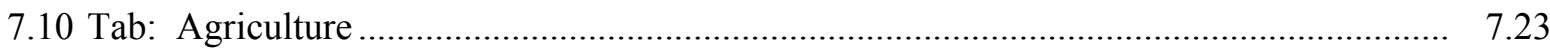

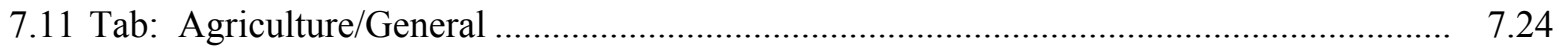

7.11.1 LEAFRS Soil-to-Plant Resuspension Factor ...................................................... 7.24

7.11.2 DPVRES Soil-to-Plant Deposition Velocity …………....................................... $\quad 7.25$

7.11.3 WTIM Plant Weathering Rate Constant ………………………….................... 7.25

7.12 Tab: Agriculture/Animal Feed ……………………............................................... 7.25

7.13 Tabs: Agriculture/Animal Feed/Biomass; /Growing Period; /Yield; and /Translocation

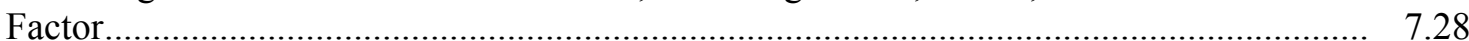

7.13.1 BIOMA2 Biomass; GRWPA Growing Period; YELDA Yield; and TRANSA Translocation Factor........................................................................................ 7.28

7.14 Tabs: Agriculture/Animal Feed/Consumption; /Storage Time; and /Diet Fraction ............ 7.34

7.14.1 CONSUM Consumption, STORTM Storage Time, and DIETFR Diet

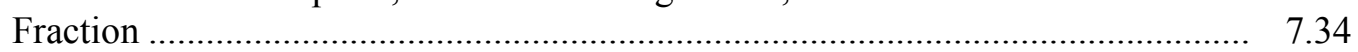

7.15 Tab: Agriculture/Animal Feed/Dry/Wet Ratio............................................................... 7.37

7.15.1 DRYFA2 Dry-to-Wet Ratio ........................................................................ 7.37

7.16 Tab: Agriculture/Animal Feed/Soil Intake.................................................................. 7.38

7.16.1 SLCONA Animal Soil Intake ……………………..................................... 7.38

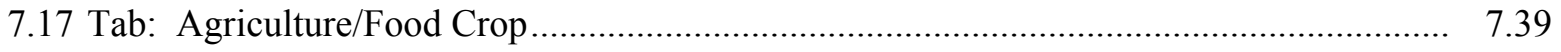

7.18 Tabs: Agriculture/Food Crop/Biomass; /Growing Period; /Yield; and /Translocation

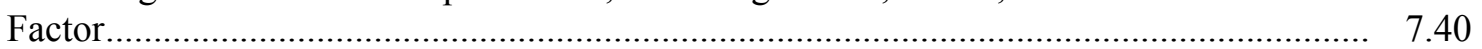

7.18.1 BIOMAS Biomass, GRWP Growing Period, YELD Yield, and TRANS

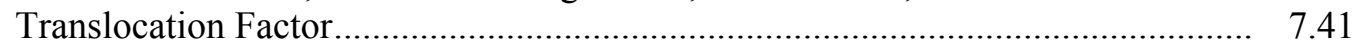

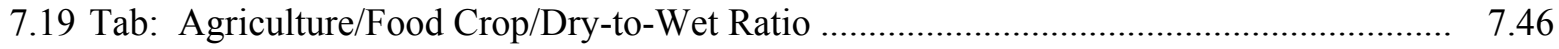

7.19.1 DRYFAC Dry-to-Wet Ratio ........................................................................... 7.46

7.20 Tab: Agriculture/Intake Delays .................................................................................... 7.47

7.20.1 HLDUP, HLDUPA, and HLDUP2 Intake Delays for Crops, Animal Products, and Aquatic Foods.................................................................................... 7.47

7.21 References: Chronic Exposure Module ...................................................................... 7.49

8.0 Module: Receptor Intake ………............................................................................. 8.1

8.1 Pathway Selection: External Exposure (multiple).......................................................... 8.2

8.1.1 External Exposure: Air and Ground ................................................................. 8.2

8.1.2 External Exposure: Surface Water from Swimming, Boating, and Shoreline ....... 8.6

8.2 Pathway Selection: Food Ingestion (multiple) ……………………………………....... 8.9

8.2.1 Ingestion Exposure: Food Crops ................................................................ 8.12

8.2.2 Ingestion Exposure: Terrestrial Animal Products ……………………………..... 8.14

8.2.3 Ingestion Exposure: Aquatic Foods ........................................................... 8.19

8.3 Pathway Selection: Water Ingestion (multiple)............................................................ 8.22

8.3.1 Water Ingestion Exposure: Drinking Water ...................................................... 8.22

8.3.2 Water Ingestion Exposure: Incidental Shower Water .......................................... 8.23 
8.3.3 Water Ingestion Exposure: Incidental Swimming Water ................................... 8.25

8.4 Pathway Selection: Inadvertent Soil Ingestion.......................................................... 8.25

8.4.1 USOIL Human Soil Ingestion Rate and TSOIL Soil Contact Days....................... 8.25

8.5 Pathway Selection: Inhalation (multiple) .................................................................. 8.27

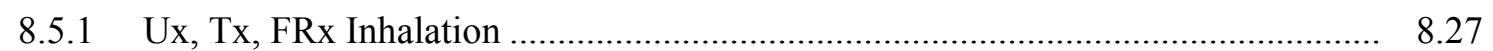

8.6 References: Receptor Intake Module .................................................................... 8.29

9.0 Module: Health Impacts ............................................................................................ 9.1

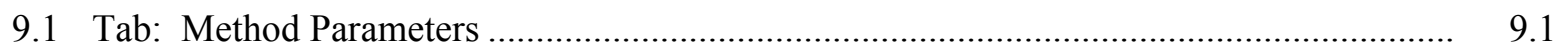

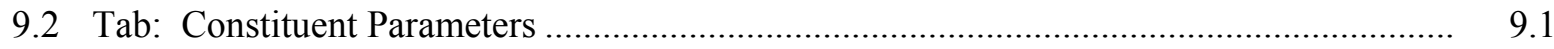

9.2.1 Additional Sources of Information.................................................................... 9.2

9.3 References: Health Impact Module ............................................................................ 9.2

10.0 Considerations for Tritium and Carbon-14.................................................................. 10.1

10.1 Tritium Model Considerations ........................................................................... 10.1

10.1.1 Module: Constituent............................................................................. 10.1

10.1.2 Module: User-Defined AIR and User-Defined WATER ................................... 10.2

10.1.3 Module: Surface Water ................................................................................ 10.2

10.1.4 Module: Chronic Exposure ........................................................................... 10.3

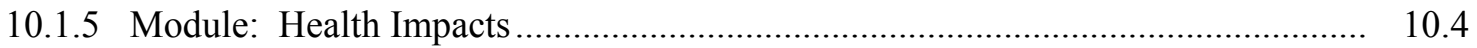

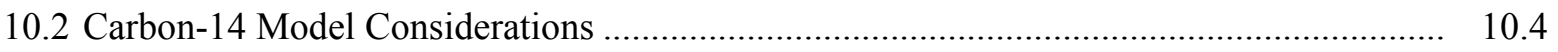

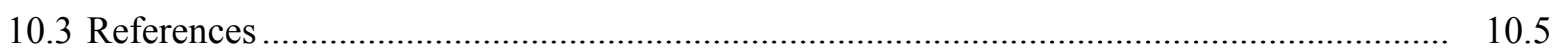

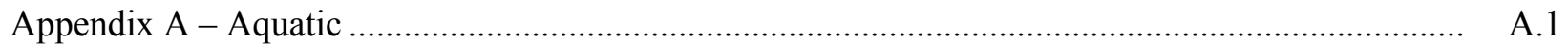

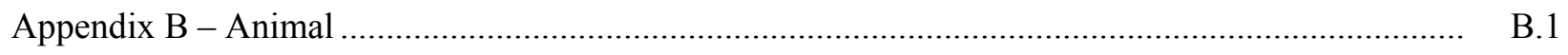

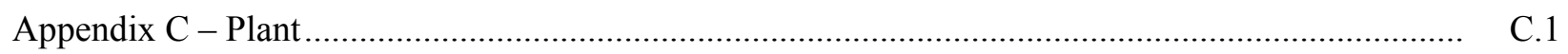

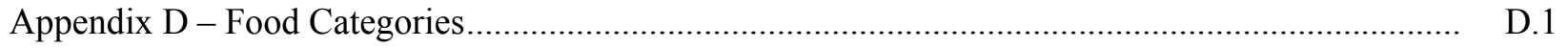

Appendix E - Rainfall Rate ….......................................................................................... E.

Appendix F - Growing Periods .................................................................................. F.

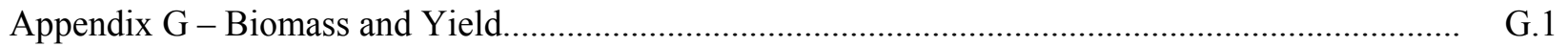

Appendix H - Dry-to-Wet Ratios ....................................................................................... H.

Appendix I - Hold-Up Times ..................................................................................... I. I

Appendix J - Distribution Assumptions ................................................................................... J. 


\section{Figures}

1.1 Example of GENIIv2 Chronic Air and Water Pathways Scenarios ....................................... 1.3

7.1 NCDC (1981) Frost-Free Days Map...............................................................................

7.2 USDA Census of Agriculture Reporting Regions …..................................................... 7.27

7.3 USDA Census of Agriculture Watershed Report Regions ................................................ 7.27

\section{Tables}

ES1.1 Constituent Module - Select Constituents of Concern ...................................................... ES.4

ES1.2 Constituent Module - Edit Constituent Properties …........................................................ ES.4

ES2.1 User-defined Module - Air .............................................................................. ES.6

ES2.2 User-defined Module - Water ................................................................................... ES.6

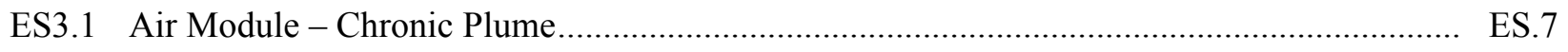

ES3.2 Surface Water Module ................................................................................................ ES.7

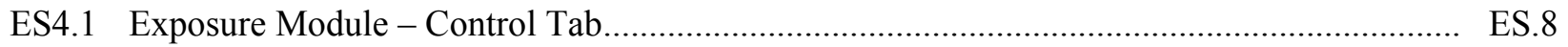

ES4.2 Exposure Module - Water Tab ….............................................................................. ES.9

ES4.3 Exposure Module - Soil Tab ........................................................................................ ES.12

ES4.4 Exposure Module - Agriculture Tab and Subtabs ….......................................................... ES.14

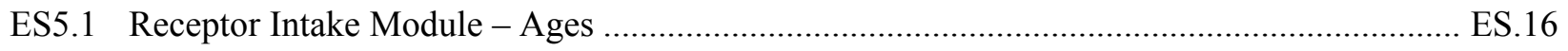

ES5.2 Receptor Intake Module - External Exposure Pathways...................................................... ES.17

ES5.3 Receptor Intake Module - Ingestion Pathways.................................................................. ES.18

ES5.4 Receptor Intake Module - Inhalation Pathways ….............................................................. ES.19

ES5.5 Receptor Intake Module - Summary of Age-dependent Generic Values .............................. ES.20

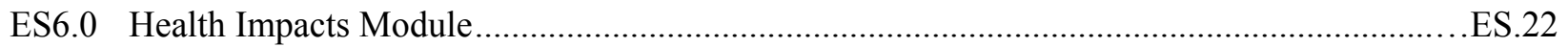

1.1 Applicable Models to Select for Modules of Chronic Release Scenarios.............................. 1.3

2.1 Generic Water Treatment Cleanup Factors for Alum Flocculation Filtration ....................... 2.2

2.2 CLBFF Bioaccumulation Factors - Freshwater Fish ...................................................... 2.8

2.3 CLBFM Bioaccumulation Factors - Freshwater Mollusk ............................................. 2.10

$2.4 \quad$ CLBFI Bioaccumulation Factor - Freshwater Crustacean .................................................. 2.11

2.5 CLBMF Bioaccumulation Factor - Marine Fish ......................................................... 2.12

2.6 CLBMM Bioaccumulation Factor - Marine Mollusk ..................................................... 2.14

$2.7 \quad$ CLBMI Bioaccumulation Factor - Marine Crustacean ....................................................... 2.15

$2.8 \quad$ CLBFP Bioaccumulation Factor - Freshwater Plants ................................................... 2.17

$2.9 \quad$ CLBMP Bioaccumulation Factor - Saltwater Plants....................................................... 2.18

2.10 CLFMT Feed to Meat Transfer Factor ........................................................................... 2.20

2.11 CLFMK Feed to Milk Transfer Factor ......................................................................... 2.23 
2.12 CLFPL Feed to Poultry Transfer Factor .......................................................................... 2.25

2.13 CLFEG Feed to Egg Transfer Factor......................................................................... 2.27

2.14 CLBVLV Concentration Ratio - Leafy Vegetable to Soil ..................................................... 2.31

2.15 CLBVRV Concentration Ratio - Root Vegetable to Soil....................................................... 2.33

2.16 CLBVFR Concentration Ratio - Fruit to Soil ..................................................................... 2.34

2.17 CLBVCL Concentration Ratio - Grain to Soil ............................................................... 2.36

4.1 Tabs and Sub-Tabs in the Air Module Chronic Plume Model.................................................. 4.1

7.1 Tabs and Sub-Tabs in the Chronic Exposure Module …………………………………..... 7.1

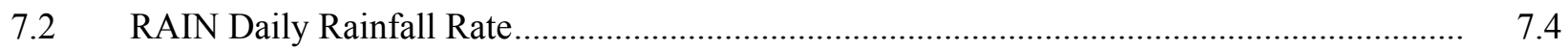

7.3 SEDDN Shoreline Sediment Density ........................................................................ 7.8

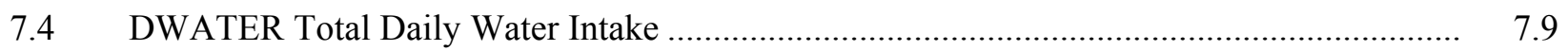

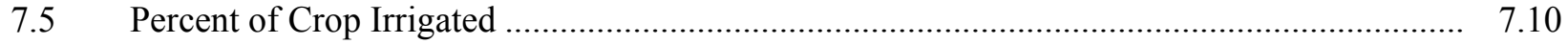

7.6 State- and Region-Specific Irrigation Rates for Human Food Crops ...................................... 7.13

7.7 State- and Region-Specific Irrigation Rates for Animal Feed Crops........................................ 7.16

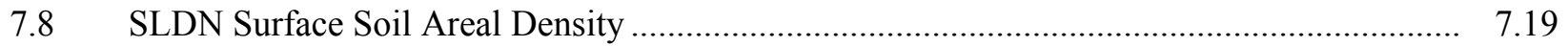

$7.9 \quad$ XMLF Mass Loading Factor ..................................................................................... 7.20

7.10 LEAFRS Soil-to-Plant Resuspension Factor ......................................................................... $\quad 7.24$

7.11 Feed and Forage Assumptions in GENII v2 ................................................................ 7.26

7.12 Gv2 Food Types and Feed and Forage Assumptions .......................................................... 7.26

7.13 BIOMA2 Standing Biomass - Animal Feed................................................................... 7.30

7.14 GRWPA Growing Period - Animal Feed.................................................................... 7.31

7.15 YELDA Crop Yield - Animal Feed ........................................................................... 7.32

7.16 TRANSA Translocation Factor - Animal Feed................................................................... $\quad 7.33$

7.17 Summary for BIOMA2, GRWPA, YELDA, and TRANSA …………………………......... 7.34

7.18 CONSUM Consumption Rate - Animal Feed ................................................................ 7.35

7.19 STORTM Storage Time - Animal Feed ....................................................................... 7.36

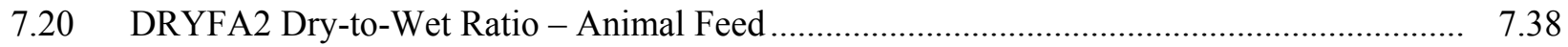

7.21 SLCONA Soil Intake - Animal Feed ....................................................................... 7.39

7.22 Suggested Categories for Food Crop Parameter Assignment ………..................................... 7.40

7.23 BIOMAS Standing Biomass - Human Food Crop Categories …………………………........... 7.44

7.24 GRWP Growing Period - Human Food Crop Categories ......................................................... 7.44

7.25 YELD Crop Yield - Human Food Crop Categories ............................................................... 7.45

7.26 TRANS Translocation - Human Food Crops ………………………………………........ 7.45

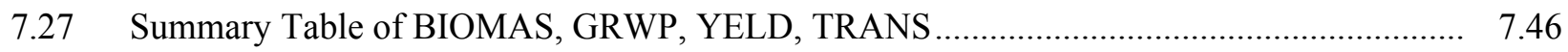

7.28 DRYFAC Dry-to-Wet Ratio - Human Food Categories ........................................................ 7.47

7.29 HLDUP, HLDUPA, HLDUP2 Hold-Up Time by Food Category .......................................... 7.49

8.1 Age Ranges of Six Age Categories............................................................................ 8.1

8.2 Receptor Intake Module External Exposure Parameters …………………………………..... 8.2

8.3 UEXAIR and TEXAIR Plume Exposure Time …………………………………………... 8.4 
PNNL-21950

8.4 FTIN and FTOUT Fraction of Time Indoors and Outdoors ….........................................

8.5 USWIM Incidental Ingestion Rate of Swimming Water .................................................... 8.8

8.6 Gv2 Animal Product Food Types and Examples ....................................................................

$8.7 \quad$ Gv2 Crop Food Types and Examples …......................................................................... 8.9

8.8 Gv2 Aquatic Foods Food Types and Examples.......................................................... 8.10

8.9 Distribution of Per Capita Food Consumption from 2000 to 2009.................................. 8.10

8.10 Age-Specific Body Weights. .................................................................................... 8.12

8.11 UCRP Ingestion Rate - Human Intake of Food Crop Categories........................................ 8.13

8.12 UANM Ingestion Rates - Human Intake of Animal Product Categories .............................. 8.16

8.13 UAQU Ingestion Rates - Human Intake of Aquatic Food Categories ................................. 8.20

8.14 UDW Drinking Water Ingestion Rate............................................................................ 8.23

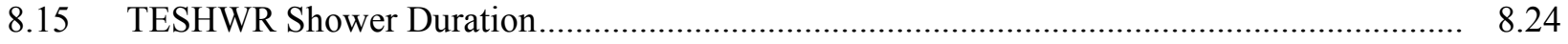

8.16 USHIN Incidental Ingestion of Shower Water ............................................................. 8.25

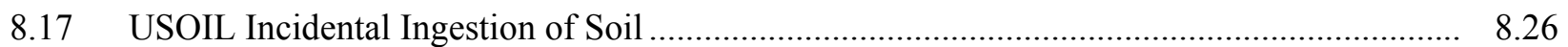

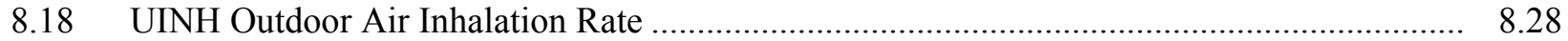

8.19 UINDRH Indoor Air Inhalation Rate............................................................................ 8.29

10.1 Tritium Nuclide Source Term Selection ...................................................................... 10.2

10.2 Relative Humidity by Watershed Region ................................................................... 10.3

10.3 Approximation of Absolute Humidity from RH ....................................................... 10.4 

PNNL-21950

\section{Acronyms and Abbreviations}

Note: This acronym list does not include the numerous GENII code parameter names (i.e., abbreviations) included in this document.

ATO

CFR

$\mathrm{CR}$

DOE

EPA

Gv2

IAEA

MEI

$\mathrm{NCDC}$

NRC

OBT

PNNL

$\mathrm{RH}$

SDD

SLDN

USCB

USDA atmospheric transport output

Code of Federal Regulations

concentration ratio

U.S. Department of Energy

U.S. Environmental Protection Agency

GENII version 2.10

International Atomic Energy Agency

maximally exposed individual

National Climatic Data Center

U.S. Nuclear Regulatory Commission

organically bound tritium

Pacific Northwest National Laboratory

relative humidity

Software Design Document

soil density values

U.S. Census Bureau

U.S. Department of Agriculture 

PNNL-21950

\section{Units of Measure}

\begin{tabular}{|c|c|}
\hline${ }^{\circ} \mathrm{C}$ & temperature in degrees Celsius $\left.\left[\mathrm{T}\left({ }^{\circ} \mathrm{C}\right)=\mathrm{T}(\mathrm{K})-273.15\right)\right]$ \\
\hline $\mathrm{ac}$ & acre(s) \\
\hline $\mathrm{Ci}$ & curie(s) \\
\hline $\mathrm{cm}$ & centimeter(s) \\
\hline d & $\operatorname{day}(\mathrm{s})$ \\
\hline $\mathrm{ft}$ & foot/feet \\
\hline g & $\operatorname{gram}(\mathrm{s})$ \\
\hline $\mathrm{hr}$ & hour(s) \\
\hline in. & $\operatorname{inch}(\mathrm{es})$ \\
\hline $\mathrm{L}$ & liter(s) \\
\hline $\mathrm{lb}$ & pound(s) \\
\hline $\mathrm{kg}$ & kilogram(s) \\
\hline $\mathrm{m}$ & meter(s) \\
\hline $\mathrm{mg}$ & milligram(s) \\
\hline $\mathrm{mL}$ & milliliter(s) \\
\hline $\mathrm{mm}$ & millimeter(s) \\
\hline mo & month(s) \\
\hline $\mathrm{yr}$ & year(s) \\
\hline$\mu$ & micro (prefix, $10^{-6}$ ) \\
\hline$\mu g$ & microgram(s) \\
\hline
\end{tabular}





\section{Acknowledgments}

Appendix $\mathrm{E}$ of this document contains daily rainfall rate values for numerous cities. While annual precipitation rates are readily available, the GENII version 2 code, RAIN parameter, requires daily rate values. The authors are grateful for JP Rishel for calculating the RAIN values tabulated in Appendix E. 



\section{Executive Summary}

The GENII v2 code is used to estimate dose to individuals or populations from the release of radioactive materials into air or water. Numerous parameter values are required for input into this code. User-defined parameters cover the spectrum from chemical data, meteorological data, agricultural data, and behavioral data. This document is a summary of parameter values that reflect conditions in the United States. Reasonable regional and age-dependent data is summarized. Data availability and quality varies. The set of parameters described address scenarios for chronic air emissions or chronic releases to public waterways. Considerations for the special tritium and carbon-14 models are briefly addressed. GENIIv2.10.1 is the current software version that this document supports. 



\section{Executive Summary of Generic Parameters}

Due to the volume of parameters the user may designate in the GENII v2 code, the initial task of designating appropriate values for the user's scenario at the location of interest can be somewhat daunting. Once this initial stage is passed, adjustments to a more limited number of parameters are generally required to establish scenario parameters. This summary of Generic Parameters was developed to serve as initial reference points for users to consider, then fine tune to their specific scenario of interest (e.g., fine tune for geography, climate, agriculture, exposure, and intake). The fine-tuning can be done upon consideration of details and references available in the cited document section or upon consideration of site-specific data.

Module input is presented in each table in the order in which it is requested. For age-specific parameters in the Receptor Intake section, a separate summary table of age-specific rates is provided.

ES1.0 Constituent Module

ES2.0 User-defined Modules - AFF, WFF - Initial Generic Parameters

ES3.0 Air Module, Surface Water Module - Initial Generic Parameters

ES4.0 Exposure Module

ES5.0 Receptor Intake Module - Initial Generic Parameters

ES6.0 Health Impacts Module 


\section{ES1.0 Constituent Module}

Refer to Section 2.0 for details.

! If a parameter is not listed, it is not implemented in current GENII models, nor of interest for record-keeping.

Table ES1.1. Constituent Module - Select Constituents of Concern

\section{SELECT CONSTITUENTS OF CONCERN}

$<$ user selection of nuclides of interest>

Table ES1.2. Constituent Module - Edit Constituent Properties

\begin{tabular}{|c|c|c|c|c|c|}
\hline \multicolumn{6}{|c|}{ EDIT CONSTITUENT PROPERTIES } \\
\hline Description Section & Symbol $^{(a)}$ & Parameter & Units & Suggested Value ${ }^{(\mathbf{b})}$ & Comment $^{(c)}$ \\
\hline Partition Coefficients & CLKD & Dry soil-water partition coefficient & $\mathrm{mL} / \mathrm{g}$ & $<$ record-keeping only $>$ & $\begin{array}{l}\text { Value used is assigned in Exposure module } \\
\text { SOILKD. }\end{array}$ \\
\hline Exposure Factors & CLANDF & Inhalation volatilization factor & $\mathrm{m}^{3} / \mathrm{L}$ & 0 & Value used is assigned in Exposure Module \\
\hline Exposure Factors & CLWPF & Water purification factor & - & $<$ see Table $2.1>$ & - \\
\hline $\begin{array}{l}\text { Radiation Dosimetry } \\
\text { Factors }\end{array}$ & SOLUBIL & Lung solubility & - & $<$ record-keeping only $>$ & $\begin{array}{l}\text { Value used is assigned in Health Impacts } \\
\text { Module, may want to enter here for record- } \\
\text { keeping. }\end{array}$ \\
\hline $\begin{array}{l}\text { Radiation Dosimetry } \\
\text { Factors }\end{array}$ & CLDFA $x$ & Inhalation dose factor & $\mathrm{rem} / \mathrm{pCi}$ & $<$ ICRP $66-72$ value $>$ & $\begin{array}{l}\text { <enter for only one class; record-keeping only } \\
\text { for Health Impacts module, ICRP } 60 \text { Method > }\end{array}$ \\
\hline $\begin{array}{l}\text { Radiation Dosimetry } \\
\text { Factors }\end{array}$ & CLRDFG $x$ & Ingestion dose factor & $\mathrm{rem} / \mathrm{pCi}$ & $<$ ICRP $66-72$ value $>$ & $\begin{array}{l}\text { <enter for only one solubility; record-keeping } \\
\text { only for Health Impacts module, ICRP } 60 \\
\text { Method }>\end{array}$ \\
\hline $\begin{array}{l}\text { Radiation Dosimetry } \\
\text { Factors }\end{array}$ & CLDEX & External dose factor, air immersion & $\begin{array}{c}\mathrm{mrem} / \mathrm{hr} \\
\text { per } \mathrm{pCi} / \mathrm{m}^{3}\end{array}$ & $<$ FGR-12 value $>$ & $<$ review supplied value for concurrence $>$ \\
\hline $\begin{array}{l}\text { Radiation Dosimetry } \\
\text { Factors }\end{array}$ & CLDIMR & $\begin{array}{l}\text { External dose factor, water } \\
\text { immersion }\end{array}$ & $\begin{array}{c}\mathrm{mrem} / \mathrm{hr} \\
\text { per } \mathrm{pCi} / \mathrm{m}^{3}\end{array}$ & $<$ FGR-12 value $>$ & $<$ review supplied value for concurrence $>$ \\
\hline $\begin{array}{l}\text { Radiation Dosimetry } \\
\text { Factors }\end{array}$ & CLDSH $x$ & External dose factor, ... to $15 \mathrm{~cm}$ & $\begin{array}{c}\mathrm{mrem} / \mathrm{hr} \\
\text { per } \mathrm{pCi} / \mathrm{m}^{3}\end{array}$ & $<$ FGR-12 value $>$ & $<$ review supplied value for concurrence $>$ \\
\hline $\begin{array}{l}\text { Radiation Dosimetry } \\
\text { Factors }\end{array}$ & CLDIAM & $\begin{array}{l}\text { Inhalation dose factor, particle } \\
\text { diameter }\end{array}$ & $\mu \mathrm{m}$ & $<$ record-keeping only $>$ & $\begin{array}{l}\text { Value used is assigned in User-defined AFF Air } \\
\text { Module. }\end{array}$ \\
\hline
\end{tabular}


Table ES1.2. (contd)

\begin{tabular}{|c|c|c|c|c|c|}
\hline \multicolumn{6}{|c|}{ EDIT CONSTITUENT PROPERTIES } \\
\hline Description Section & Symbol $^{(\mathbf{a})}$ & Parameter & Units & Suggested Value $^{(b)}$ & Comment $^{(c)}$ \\
\hline $\begin{array}{l}\text { Aquatic Food Transfer } \\
\text { Factors }\end{array}$ & CLBFx & $\begin{array}{l}\text { Bioaccumulation factors- } \\
\text { freshwater animals }\end{array}$ & $\mathrm{L} / \mathrm{kg}$ & $\begin{array}{c}<\text { see Table } 2.2 \text {, Table } 2.3, \\
\text { Table } 2.4>\end{array}$ & $\begin{array}{l}<\text { if applicable, review supplied value for } \\
\text { concurrence }>\end{array}$ \\
\hline $\begin{array}{l}\text { Aquatic Food Transfer } \\
\text { Factors }\end{array}$ & CLBFP & $\begin{array}{l}\text { Bioaccumulation factor-freshwater } \\
\text { plants }\end{array}$ & $\mathrm{L} / \mathrm{kg}$ & $<$ see Table $2.8>$ & $\begin{array}{l}<\text { if applicable, review supplied value for } \\
\text { concurrence }>\end{array}$ \\
\hline $\begin{array}{l}\text { Aquatic Food Transfer } \\
\text { Factors }\end{array}$ & CLBM $x$ & $\begin{array}{l}\text { Bioaccumulation factor-saltwater } \\
\text { animals }\end{array}$ & $\mathrm{L} / \mathrm{kg}$ & $\begin{array}{c}<\text { see Table } 2.5 \text {, Table } 2.6, \\
\text { Table } 2.7>\end{array}$ & $\begin{array}{l}<\text { if applicable, review supplied value for } \\
\text { concurrence }>\end{array}$ \\
\hline $\begin{array}{l}\text { Aquatic Food Transfer } \\
\text { Factors }\end{array}$ & CLBMP & $\begin{array}{l}\text { Bioaccumulation factors-saltwater } \\
\text { plants }\end{array}$ & $\mathrm{L} / \mathrm{kg}$ & $<$ see Table 2.9> & $\begin{array}{l}<\text { if applicable, review supplied value for } \\
\text { concurrence }>\end{array}$ \\
\hline Animal Transfer Factors & CLFMT & $\begin{array}{l}\text { Animal transfer factors-feed to } \\
\text { meat }\end{array}$ & $\mathrm{d} / \mathrm{kg}$ & $<$ see Table $2.10>$ & $<$ review supplied value for concurrence $>$ \\
\hline Animal Transfer Factors & CLFMK & Animal transfer factors-milk & $\mathrm{d} / \mathrm{L}$ & $<$ see Table 2.11> & $<$ review supplied value for concurrence $>$ \\
\hline Animal Transfer Factors & CLFPL & Animal transfer factors-poultry & $\mathrm{d} / \mathrm{kg}$ & $<$ see Table $2.12>$ & $<$ review supplied value for concurrence $>$ \\
\hline Animal Transfer Factors & CLFEG & Animal transfer factors-egg & $\mathrm{d} / \mathrm{kg}$ & $<$ see Table 2.13> & $<$ review supplied value for concurrence $>$ \\
\hline Plant Transfer Factors & CLBVx & $\begin{array}{l}\text { Bioconcentration to edible crop } \\
\text { from soil }\end{array}$ & $\mathrm{kg} / \mathrm{kg}$ & $\begin{array}{c}<\text { see Table } 2.14 \text {, Table } 2.15, \\
\text { Table } 2.16 \text {, Table } 2.17>\end{array}$ & $<$ review supplied value for concurrence $>$ \\
\hline Plant Transfer Factors & CLBVAx & $\begin{array}{l}\text { Bioconcentration to feed or forage } \\
\text { from soil }\end{array}$ & $\mathrm{kg} / \mathrm{kg}$ & Not used in current model. & - \\
\hline Plant Transfer Factors & CLBVAG & $\begin{array}{l}\text { Bioconcentration to animal feed } \\
\text { grain from soil }\end{array}$ & $\mathrm{kg} / \mathrm{kg}$ & Not used in current model. & - \\
\hline Plant Transfer Factors & CLBVOV & $\begin{array}{l}\text { Bioconcentration in dry other } \\
\text { vegetables from soil }\end{array}$ & $\mathrm{kg} / \mathrm{kg}$ & Not used in current model. & - \\
\hline Environmental Rates & CLVD & Atmospheric deposition velocity & $\mathrm{m} / \mathrm{sec}$ & $<$ record-keeping only $>$ & - \\
\hline Environmental Rates & CLCLASS & Atmospheric deposition class & - & $<$ record-keeping only $>$ & See help file for descriptions \\
\hline \multicolumn{6}{|c|}{$\begin{array}{l}\text { (a) For symbols ending in an italic } x \text {, parameter subsets are available (e.g., aquatic food types or soil horizons). See main document section cited for details. } \\
\text { (b) }<\text { record-keeping only }>\text { = Good practice to document value assumed, but the value entered in here is not applied by the code for a chronic air or surface water scenario. } \\
\text { (c) }<\text { review supplied value for concurrence }>=\text { When initial case is created, review the supplied default value or the parameter to determine acceptability. }\end{array}$} \\
\hline
\end{tabular}




\section{ES2.0 User-defined Modules - AFF, WFF - Initial Generic Parameters}

Refer to Section 3.0 (AFF) and Section 5.0 (WFF) for details.

Table ES2.1. User-defined Module - Air

\begin{tabular}{|c|c|c|c|c|}
\hline \multicolumn{5}{|l|}{ AFF - Air } \\
\hline Symbol & Parameter & Units & Suggested Value $^{(a)}$ & Comment \\
\hline media & Type of release & - & point & Typically a "point" source. \\
\hline one & Exit area of source & $\mathrm{m}^{2}$ & $<$ user input $>$ & - \\
\hline two & Exit height of source & $\mathrm{m}$ & $<$ user input $>$ & - \\
\hline three & Height of adjacent structure & $\mathrm{m}$ & $<$ user input $>$ & - \\
\hline four & Exit velocity of source & $\mathrm{m} / \mathrm{s}$ & $<$ user input $>$ & - \\
\hline five & Exit temperature of source & ${ }^{\circ} \mathrm{C}$ & $<$ user input $>$ & $\begin{array}{l}\text { Enter same value as ambient temperature if effective height of } \\
\text { source assigned as exit height. }\end{array}$ \\
\hline $\operatorname{six}$ & Ambient air temperature & ${ }^{\circ} \mathrm{C}$ & $<$ user input $>$ & - \\
\hline ctime & Release table- time & $\mathrm{yr}$ & 0 & first row entry \\
\hline ctime & Release table- time & yr & 1 & second row entry \\
\hline cval & Release table- Emission rate & $\mathrm{pCi} / \mathrm{yr}$ & $<$ user input $>$ & same value for each release time for a routine chronic release \\
\hline
\end{tabular}

Table ES2.2. User-defined Module - Water

\begin{tabular}{|c|c|c|c|c|}
\hline \multicolumn{5}{|c|}{ WFF - Surface Water } \\
\hline Symbol & Parameter & Units & Suggested Value $^{(a)}$ & Comment $^{(b)}$ \\
\hline one & Width of flux plane & $\mathrm{m}$ & $<$ user input $>$ & $<$ record-keeping only $>$ \\
\hline two & Height of flux plane & $\mathrm{m}$ & $<$ user input $>$ & $<$ record-keeping only $>$ \\
\hline ctime & Table - time & $\mathrm{yr}$ & 0 & first row entry \\
\hline ctime & Table - time & yr & 1 & second row entry \\
\hline cval & Constituent $=$ water & $\mathrm{m}^{3} / \mathrm{yr}$ & $<$ user input $>$ & $\begin{array}{l}\text { surface water source flow rate, same value for each time row } \\
\text { for routine chronic release }\end{array}$ \\
\hline cval & Constituent $=<$ nuclide $>$; Adsorbed flux & $\mathrm{pCi} / \mathrm{yr}$ & $<$ user input $>$ & - \\
\hline cval & Constituent $=<$ nuclide $>$; Dissolved flux & $\mathrm{pCi} / \mathrm{yr}$ & $<$ user input $>$ & Enter all activity here \\
\hline
\end{tabular}

(a) <user input $>$ indicates the value is very case-specific. No generic value is supplied.

(b) <record-keeping only > indicates that it is a good practice to document the value assumed here, but the entry is not applied by the code for a chronic air or surface water value. 


\section{ES3.0 Air Module, Surface Water Module - Initial Generic Parameters}

Refer to Section 4.0 (Air) and Section 6.0 (Surface Water) for details.

Table ES3.1. Air Module - Chronic Plume

\begin{tabular}{|c|c|c|c|c|c|}
\hline \multicolumn{6}{|l|}{ Air-Chronic Plume module } \\
\hline \multicolumn{6}{|l|}{ MODEL INFORMATION } \\
\hline Radial Grid Definition & - & Distances & $\mathrm{m}$ & $<$ user input $>$ & $\begin{array}{l}16 \text {-sector radial grid more typical } \\
\text { and will simplify detailed data } \\
\text { reviews }\end{array}$ \\
\hline Model Parameters & - & - & - & Briggs Open Country & - \\
\hline \multirow[t]{5}{*}{ Default Parameters } & ARMINRISESPD & Minimum wind speed & $\mathrm{m} / \mathrm{s}$ & 1.5 & - \\
\hline & ARMINSIGYSHIFT & Sigma to shift... & $\mathrm{m}$ & 400 & - \\
\hline & ARTRANSRESIST & Transfer resistance for iodine & $\mathrm{s} / \mathrm{m}$ & 10 & - \\
\hline & ARTRANSRESIST & Transfer resistance for particles & $\mathrm{s} / \mathrm{m}$ & 100 & - \\
\hline & ARMINWIND & Maximum windspeed for "calm" & $\mathrm{m} / \mathrm{s}$ & 0.8 & Minimum detected by anemometer. \\
\hline
\end{tabular}

Table ES3.2. Surface Water Module

\begin{tabular}{|c|c|c|c|c|c|}
\hline \multicolumn{6}{|c|}{ Surface Water - Surface Water module } \\
\hline Tab & Symbol & Parameter & Units & Suggested Value ${ }^{(a)}$ & Comment \\
\hline RIVER/LAKE & GNSWTREL & Duration of release to the surface water & $\mathrm{yr}$ & 1 & - \\
\hline & $<$ multiple $>$ & $<$ All other parameters on this tab $>$ & $<$ mult. $>$ & $<$ user input $>$ & scenario specific \\
\hline IMPOUNDMENT & - & $<$ All selections/parameters on this tab $>$ & $<$ mult. $>$ & $<$ user input $>$ & scenario specific \\
\hline
\end{tabular}




\section{ES4.0 Exposure Module}

Refer to Section 7.0 for most details. See last column of tables for specific sections.

Enter the Easting and Northing coordinates $(\mathrm{km})$ in the GENERAL INFO option of this Exposure Module to indicate location of receptor (or field) relative to emission point.

! NOTE: Environmental Conditions vary by location. Use suggested values at your own discretion.

Also, note that irrigation parameters are only necessary if irrigation with a contaminated water source is assumed in the scenario.

Table ES4.1. Exposure Module - Control Tab

\begin{tabular}{|c|c|c|c|c|c|c|}
\hline Tab: Top-level/2nd level & Symbol & Parameter & Units & Suggested Value & Comment & Details \\
\hline \multicolumn{7}{|l|}{ CONTROLS } \\
\hline & NTKEND & Duration of exposure period & $\mathrm{yr}$ & 1 & Required for chronic exposure & - \\
\hline & RELEND & End of release period & $\mathrm{yr}$ & 1 & - & - \\
\hline & BEFORE & Time from start to exposure & yr & 0 & - & - \\
\hline & ABSHUM & Absolute humidity & $\mathrm{kg} / \mathrm{m}^{3}$ & $<$ see Table $10.2>$ & $\begin{array}{l}\text { Usually, that during growing season. } \\
\text { Only used with tritium model. }\end{array}$ & Section 10.1.4 \\
\hline & $\mathrm{RF} 1$ & Fraction of roots in surface soil & - & 1 & - & Section 7.1.1 \\
\hline & RAIN & Average rain rate, when raining & $\mathrm{mm} / \mathrm{d}$ & $<$ see Appendix E $>$ & - & Section 7.1.2 \\
\hline & BEFAIR & Air deposition time prior to exposure & yr & 0 & - & \\
\hline
\end{tabular}


Table ES4.2. Exposure Module - Water Tab

\begin{tabular}{|c|c|c|c|c|c|c|}
\hline Tab: Top-level/2nd level & Symbol & Parameter $^{(\mathbf{a})}$ & Units & $\begin{array}{l}\text { Suggested } \\
\text { Value }\end{array}$ & Comment & Details \\
\hline \multicolumn{7}{|l|}{ WATER } \\
\hline General & RIRRR & Irrigation rate for residential land & in. $/ y r$ & 35 & Adjust by local climate. & Section 7.3.1 \\
\hline General & IRTIMR & Irrigation time for residential land & $\mathrm{mo} / \mathrm{yr}$ & 6 & $\begin{array}{l}\text { Adjust by local climate (see } \\
\text { Figure 7.1) or crop irrigation times, } \\
\text { below. }\end{array}$ & Section 7.3.1 \\
\hline General & BEFIRR & Irrigation water deposition time prior to exposure & yr & 0 & - & - \\
\hline General & ANDKRN & Indoor volatilization factor for radon & $\mathrm{L} / \mathrm{m}^{3}$ & 0.1 & - & Section 7.3.2 \\
\hline General & ANDKR & Indoor volatilization factor for radionuclides & $\mathrm{L} / \mathrm{m}^{3}$ & 0 & - & Section 7.3.2 \\
\hline General & HOLDDW & Delay time in water distribution system & $\mathrm{D}$ & 1 & - & Section 7.3.3 \\
\hline General & SEDDN & Shoreline sediment density-option 1 & $\mathrm{~kg} / \mathrm{m}^{2}$ & 200 & silty, sandy sediment & Section 7.3.4 \\
\hline General & SEDDN & Shoreline sediment density-option 2 & $\mathrm{~kg} / \mathrm{m}^{2}$ & 290 & rocky shoreline & Section 7.3 .4 \\
\hline Animal Water & DWFACA & contaminated fraction (all types of animal products) & - & 1 & - & Section 7.4.1 \\
\hline Animal Water & DWATER & Intake rate - meat animal & $\mathrm{L} / \mathrm{d}$ & 50 & assume beef cow, near butcher date & Section 7.4 .1 \\
\hline Animal Water & DWATER & Intake rate - poultry animal & $\mathrm{L} / \mathrm{d}$ & 0.26 & assume chicken & Section 7.4 .1 \\
\hline Animal Water & DWATER & Intake rate - milk animal & $\mathrm{L} / \mathrm{d}$ & 85 & assume cow milk & Section 7.4.1 \\
\hline Animal Water & DWATER & Intake rate - egg animal & $\mathrm{L} / \mathrm{d}$ & 0.30 & assume chicken & Section 7.4 .1 \\
\hline Irrigation sources & - & N/A & - & - & - & - \\
\hline Irrigation rates & RIRR & leafy vegetables, root vegetables-option 1 & in. $/ \mathrm{yr}$ & 0 & For Alaska & Section 7.5.1 \\
\hline Irrigation rates & RIRR & leafy vegetables, root vegetables-option 2 & in. $/ \mathrm{yr}$ & 4 & $\begin{array}{l}\text { For Census Reporting Regions of } \\
\text { Figure } 7.2: 1,2 \text { (North), } 5 \text { (W of } \\
\text { Cascade Mts) }\end{array}$ & Section 7.5.1 \\
\hline Irrigation rates & RIRR & leafy vegetables, root vegetables-option3 & in. $/ \mathrm{yr}$ & 10 & $\begin{array}{l}\text { For Census Reporting Regions of } \\
\text { Figure 7.2: } 4 \text { (North) }\end{array}$ & Section 7.5.1 \\
\hline Irrigation rates & RIRR & leafy vegetables, root vegetables-option 4 & in. $/ y r$ & 35 & $\begin{array}{l}\text { For Census Reporting Regions of } \\
\text { Figure 7.2: 2(South), 3, 4(South), } \\
\text { 5(E of the Cascade Mts), } 6\end{array}$ & Section 7.5.1 \\
\hline Irrigation rates & RIRR & leafy vegetables, root vegetables-option 5 & in. $/ y r$ & 44 & For Hawaii & Section 7.5.1 \\
\hline Irrigation rates & RIRR & fruits-option1 & in. $/ y r$ & 35 & For west of the Mississippi River & Section 7.5.1 \\
\hline
\end{tabular}


Table ES4.2. (contd)

\begin{tabular}{|c|c|c|c|c|c|c|}
\hline Tab: Top-level/2nd level & Symbol & Parameter $^{(\mathbf{a})}$ & Units & $\begin{array}{l}\text { Suggested } \\
\text { Value }\end{array}$ & Comment & Details \\
\hline Irrigation rates & RIRR & fruits-option2 & in./yr & 18 & For east of the Mississippi River & Section 7.5.1 \\
\hline Irrigation rates & RIRR & grains & in./yr & 0 & When dryland farming assumed & Section 7.5.1 \\
\hline Irrigation rates & RIRRA & meat animal feed-option 1 & in./yr & 6 & $\begin{array}{l}\text { For Census Reporting Regions of } \\
\text { Figure 7.2: } 1 \text { excluding Nebraska, } \\
2\end{array}$ & Section 7.5.1 \\
\hline Irrigation rates & RIRRA & meat animal feed-option 2 & in. $/ y r$ & 11 & $\begin{array}{l}\text { For Census Reporting Regions of } \\
\text { Figure 7.2: } 3,4 \text {, Nebraska }\end{array}$ & Section 7.5.1 \\
\hline Irrigation rates & RIRRA & meat animal feed-option 3 & in. $/ y r$ & 26 & $\begin{array}{l}\text { For Census Reporting Regions of } \\
\text { Figure 7.2: } 5\end{array}$ & Section 7.5.1 \\
\hline Irrigation rates & RIRRA & meat animal feed-option 4 & in./yr & 30 & $\begin{array}{l}\text { For Census Reporting Regions of } \\
\text { Figure 7.2: } 6\end{array}$ & Section 7.5.1 \\
\hline Irrigation rates & RIRRA & poultry\&egg animal feed & in./yr & 0 & $\begin{array}{l}\text { When dryland farming of grain } \\
\text { assumed }\end{array}$ & Section 7.5.1 \\
\hline Irrigation rates & RIRRA & milk animal feed-option 1 & in./yr & 7.5 & $\begin{array}{l}\text { For Census Reporting Regions of } \\
\text { Figure 7.2: } 1\end{array}$ & Section 7.5.1 \\
\hline Irrigation rates & RIRRA & milk animal feed-option 2 & in. $/ y r$ & 5 & $\begin{array}{l}\text { For Census Reporting Regions of } \\
\text { Figure } 7.2 \text { ): } 2\end{array}$ & Section 7.5.1 \\
\hline Irrigation rates & RIRRA & milk animal feed-option 3 & in. $/ y r$ & 14.5 & $\begin{array}{l}\text { For Census Reporting Regions of } \\
\text { Figure 7.2: } 3\end{array}$ & Section 7.5.1 \\
\hline Irrigation rates & RIRRA & milk animal feed-option 4 & in./yr & 18 & $\begin{array}{l}\text { For Census Reporting Regions of } \\
\text { Figure 7.2: } 4\end{array}$ & Section 7.5.1 \\
\hline Irrigation rates & RIRRA & milk animal feed-option5 & in./yr & 25 & $\begin{array}{l}\text { For Census Reporting Regions of } \\
\text { Figure 7.2: } 5\end{array}$ & Section 7.5.1 \\
\hline Irrigation rates & RIRRA & milk animal feed-option6 & in./yr & 48 & $\begin{array}{l}\text { For Census Reporting Regions of } \\
\text { Figure 7.2: } 6\end{array}$ & Section 7.5.1 \\
\hline Irrigation rates & RIRRA & meat animal forage & in. $/ y r$ & see above & see milk animal feed options & Section 7.5.1 \\
\hline Irrigation rates & RIRRA & milk animal forage-option 1 & in./yr & 7.5 & $\begin{array}{l}\text { For Census Reporting Regions of } \\
\text { Figure } 7.2: 1\end{array}$ & Section 7.5.1 \\
\hline Irrigation rates & RIRRA & milk animal forage-option 2 & in. $/ y r$ & 5 & $\begin{array}{l}\text { For Census Reporting Regions of } \\
\text { Figure 7.2: } 2\end{array}$ & Section 7.5.1 \\
\hline Irrigation rates & RIRRA & milk animal forage-option 3 & in./yr & 10 & $\begin{array}{l}\text { For Census Reporting Regions of } \\
\text { Figure 7.2: } 3\end{array}$ & Section 7.5.1 \\
\hline Irrigation rates & RIRRA & milk animal forage-option 4 & in./yr & 18 & $\begin{array}{l}\text { For Census Reporting Regions of } \\
\text { Figure 7.2: } 4\end{array}$ & Section 7.5.1 \\
\hline
\end{tabular}


Table ES4.2. (contd)

\begin{tabular}{|c|c|c|c|c|c|c|}
\hline Tab: Top-level/2nd level & Symbol & Parameter $^{(a)}$ & Units & $\begin{array}{l}\text { Suggested } \\
\text { Value }\end{array}$ & Comment & Details \\
\hline Irrigation rates & RIRRA & milk animal forage-option5 & in. $/ \mathrm{yr}$ & 22 & $\begin{array}{l}\text { For Census Reporting Regions of } \\
\text { Figure } 7.2: 5,7\end{array}$ & Section 7.5.1 \\
\hline Irrigation rates & RIRRA & milk animal forage-option 6 & in./yr & 30 & $\begin{array}{l}\text { For Census Reporting Regions of } \\
\text { Figure 7.2: } 6\end{array}$ & Section 7.5.1 \\
\hline Irrigation times $^{(\mathrm{b})}$ & IRTIMT $^{(b)}$ & any crop & $\mathrm{mo} / \mathrm{yr}$ & 1 & $\begin{array}{l}\text { For Watershed Reporting Regions } \\
\text { of Figure } 7.3: 1,2,4,9\end{array}$ & Section 7.5.1 \\
\hline Irrigation times $^{(\mathrm{b})}$ & or & any crop & $\mathrm{mo} / \mathrm{yr}$ & 2 & Section 7.5.1 & Section 7.5.1 \\
\hline Irrigation times $^{(\mathrm{b})}$ & IRTIMA $^{(\mathrm{b})}$ & any crop & $\mathrm{mo} / \mathrm{yr}$ & 3 & $\begin{array}{l}\text { Watershed Reporting Regions of } \\
\text { Figure } 7.3: 7,10,16\end{array}$ & Section 7.5.1 \\
\hline Irrigation times $^{(\mathrm{b})}$ & - & any crop & $\mathrm{mo} / \mathrm{yr}$ & 4 & $\begin{array}{l}\text { Watershed Reporting Regions of } \\
\text { Figure 7.3: } 17\end{array}$ & Section 7.5.1 \\
\hline Irrigation times $^{(\mathrm{b})}$ & - & any crop & $\mathrm{mo} / \mathrm{yr}$ & 5 & $\begin{array}{l}\text { Watershed Reporting Regions of } \\
\text { Figure } 7.3: 8,11,18\end{array}$ & Section 7.5.1 \\
\hline Irrigation times $^{(\mathrm{b})}$ & - & any crop & $\mathrm{mo} / \mathrm{yr}$ & 6 & $\begin{array}{l}\text { Watershed Reporting Regions of } \\
\text { Figure 7.3: } 3,12,13,15\end{array}$ & Section 7.5.1 \\
\hline Irrigation times ${ }^{(\mathrm{b})}$ & - & any crop & $\mathrm{mo} / \mathrm{yr}$ & 8 & $\begin{array}{l}\text { Watershed Reporting Regions of } \\
\text { Figure 7.3: } 20\end{array}$ & Section 7.5.1 \\
\hline $\begin{array}{l}\text { (a) Several options are pr } \\
\text { (b) Irrigation times have } t \\
\text { products ingested by }\end{array}$ & $\begin{array}{l}\text { ated for sor } \\
\text { symbol IRT } \\
\text { le. }\end{array}$ & $\begin{array}{l}\text { parameters, depending on } \\
\text { MT or IRTIMA. IRTIMT a }\end{array}$ & $\begin{array}{l}\text { terest a } \\
\text { eople. }\end{array}$ & cated in th & $\begin{array}{l}\text { omment. } \\
\text { crops ingested by animals that }\end{array}$ & animal \\
\hline
\end{tabular}


Table ES4.3. Exposure Module - Soil Tab

\begin{tabular}{|c|c|c|c|c|c|c|c|}
\hline Tab: & Top-level/2nd level & Symbol & Parameter $^{(a)}$ & Units & $\begin{array}{l}\text { Suggested } \\
\text { Value }\end{array}$ & Comment & Details \\
\hline \multicolumn{8}{|c|}{ SOIL } \\
\hline \multirow{13}{*}{ ati } & Surface soil & SLDN & surface soil areal density-option1 & $\mathrm{kg} / \mathrm{m}^{2}$ & 225 & sandy soil - for crop growth & Section 7.7.1 \\
\hline & Surface soil & SLDN & surface soil areal density-option2 & $\mathrm{kg} / \mathrm{m}^{2}$ & 195 & silty soil - for crop growth & Section 7.7.1 \\
\hline & Surface soil & SLDN & surface soil areal density-option3 & $\mathrm{kg} / \mathrm{m}^{2}$ & 155 & clay soil - for crop growth & Section 7.7.1 \\
\hline & Surface soil & SURCM & surface soil layer thickness used for density & $\mathrm{cm}$ & 0.15 & - & Section 7.7.1 \\
\hline & Resuspension & XMLF & Mass loading factor for resuspension-option1 & $\mathrm{g} / \mathrm{m}^{3}$ & $4.0 \mathrm{E}-05$ & $\begin{array}{l}\text { Selection: Use mass loading } \\
\text { model. Watershed Reporting } \\
\text { Regions (Figure } 7.3 \text { ): } 1\end{array}$ & Section 7.8.1 \\
\hline & Resuspension & XMLF & Mass loading factor for resuspension-option2 & $\mathrm{g} / \mathrm{m}^{3}$ & $5.0 \mathrm{E}-05$ & $\begin{array}{l}\text { Selection: Use mass loading } \\
\text { model. Watershed Reporting } \\
\text { Regions (Figure } 7.3 \text { ): } 2-6,8, \\
\text { 13,17west, } 19\end{array}$ & Section 7.8.1 \\
\hline & Resuspension & XMLF & Mass loading factor for resuspension-option3 & $\mathrm{g} / \mathrm{m}^{3}$ & $5.7 \mathrm{E}-05$ & $\begin{array}{l}\text { Selection: Use mass loading } \\
\text { model. Watershed Reporting } \\
\text { Regions (Figure } 7.3 \text { ): } 7 \text {, } \\
10-12\end{array}$ & Section 7.8.1 \\
\hline & Resuspension & XMLF & Mass loading factor for resuspension-option4 & $\mathrm{g} / \mathrm{m}^{3}$ & $6.7 \mathrm{E}-04$ & $\begin{array}{l}\text { Selection: Use mass loading } \\
\text { model. Watershed Reporting } \\
\text { Regions (Figure } 7.3 \text { ): } \\
\text { 14-16, 17east, } 18\end{array}$ & Section 7.8.1 \\
\hline & Leaching & LEACHOPTION & Selection: Leach rates calculated from user input & N/A & - & - & - \\
\hline & Leaching & THICK & Surface soil thickness & $\mathrm{cm}$ & 0.15 & $\begin{array}{l}\text { Same value as entered in } \\
\text { SOIL/Surface soil }\end{array}$ & Section 7.9.1 \\
\hline & Leaching & MOISTC & Surface soil moisture content & $\mathrm{mL} / \mathrm{cm}^{3}$ & 0.12 & Assumed loam soil value. & Section 7.9.2 \\
\hline & Leaching & BULKD & Surface soil bulk density-option1 & $\mathrm{g} / \mathrm{cm}^{3}$ & 1.5 & $\begin{array}{l}\text { Same value, but on a volume } \\
\text { basis, as entered in } \\
\text { SOIL/surface soil. Sandy soil }\end{array}$ & Section 7.9.3 \\
\hline & Leaching & BULKD & Surface soil bulk density-option2 & $\mathrm{g} / \mathrm{cm}^{3}$ & 1.3 & $\begin{array}{l}\text { Same value, but on a volume } \\
\text { basis, as entered in } \\
\text { SOIL/surface soil. Silty soil. }\end{array}$ & Section 7.9.3 \\
\hline
\end{tabular}


Table ES4.3. (contd)

\begin{tabular}{|c|c|c|c|c|c|c|c|}
\hline Tab: & :Top-level/2nd level & Symbol & Parameter $^{(a)}$ & Units & $\begin{array}{l}\text { Suggested } \\
\text { Value }\end{array}$ & Comment & Details \\
\hline & Leaching & BULKD & Surface soil bulk density-option3 & $\mathrm{g} / \mathrm{cm}^{3}$ & 1.03 & $\begin{array}{l}\text { Same value, but on a volume } \\
\text { basis, as entered in } \\
\text { SOIL/surface soil. Clay soil. }\end{array}$ & Section 7.9 .3 \\
\hline & Leaching & VLEACH & Total Infiltration rate & $\mathrm{cm} / \mathrm{yr}$ & 3.5 & $\begin{array}{l}\text { Approximated by } 10 \% \text { of } \\
\text { irrigation and precipitation } \\
\text { rates. Adjust by climate. }\end{array}$ & Section 7.9.4 \\
\hline & Leaching & SOILKD & Parent nuclide soil absorption coefficient & $\mathrm{mL} / \mathrm{g}$ & $<$ varies $>$ & - & Section 7.9.5 \\
\hline
\end{tabular}


Table ES4.4. Exposure Module - Agriculture Tab and Subtabs

\begin{tabular}{|c|c|c|c|c|c|c|}
\hline Tab: Top-level/2nd level/3rd Level & Symbol & Parameter & Units & $\begin{array}{l}\text { Suggested } \\
\text { Value }^{(a)}\end{array}$ & Comment $^{(\mathbf{b})}$ & Details \\
\hline \multicolumn{7}{|l|}{ AGRICULTURE } \\
\hline General & LEAFRS & Resuspension factor (soil-to-plant) & $1 / \mathrm{m}$ & $1.00 \mathrm{E}-10$ & - & Section 7.11 .1 \\
\hline General & DPVRES & Deposition velocity (soil-to-plant) & $\mathrm{m} / \mathrm{s}$ & 0.001 & - & Section 7.11 .2 \\
\hline General & WTIM & Weathering rate constant from plants & $\mathrm{d}$ & 14 & - & Section 7.11 .3 \\
\hline \multicolumn{7}{|l|}{ Animal Feed } \\
\hline Biomass & BIOMA2 & Biomass (wet) for animal feed and forage & $\mathrm{kg} / \mathrm{m}^{2}$ & $<$ see Table $7.17>$ & - & Section 7.13 .1 \\
\hline Consumption & CONSUM & Consumption rate for feed and forage & $\mathrm{kg} / \mathrm{d}$ & $<$ see Table $7.18>$ & - & Section 7.14 .1 \\
\hline Storage time & STORTM & Storage time for feed and forage & $\mathrm{d}$ & $<$ see Table 7.19> & - & Section 7.14 .1 \\
\hline Diet fraction & DIETFR & Diet fraction for feed and forage & - & $<$ user input $>$ & $\begin{array}{l}\text { Could also be } \\
\text { applied as fraction } \\
\text { of feed or forage } \\
\text { type from } \\
\text { contaminant region. }\end{array}$ & Section 7.14 .1 \\
\hline Growing period & GRWPA & Growing period for feed and forage & $\mathrm{d}$ & $<$ see Table $7.17>$ & - & Section 7.13 .1 \\
\hline Yield & YELDA & Yield for feed and forage & $\mathrm{kg} / \mathrm{m}^{2}$ & $<$ see Table $7.17>$ & - & Section 7.13 .1 \\
\hline Dry/Wet ratio & DRYFA2 & Dry/wet weight ratio for meat animal feed & - & 0.5 & $\begin{array}{l}\text { See Table } 7.20 \text {. Use } \\
\text { in conjunction with } \\
\text { Table } 7.16 \\
\text { comments of food } \\
\text { type. }\end{array}$ & Section 7.15.1 \\
\hline Dry/Wet ratio & DRYFA2 & Dry/wet weight ratio for poultry feed & - & 0.9 & - & Section 7.15 .1 \\
\hline Dry/Wet ratio & DRYFA2 & Dry/wet weight ratio for milk animal feed & - & 0.5 & - & Section 7.15 .1 \\
\hline Dry/Wet ratio & DRYFA2 & Dry/wet weight ratio for egg animal feed & - & 0.9 & - & Section 7.15 .1 \\
\hline Dry/Wet ratio & DRYFA2 & Dry/wet weight ratio for meat animal forage & - & 0.88 & $\begin{array}{l}\text { as fed value, } \\
\text { hay+grain }\end{array}$ & Section 7.15.1 \\
\hline Dry/Wet ratio & DRYFA2 & Dry/wet weight ratio for milk animal forage & - & 0.4 & - & Section 7.15 .1 \\
\hline Translocation factor & TRANSA & Translocation factor for feed and forage & - & $<$ see Table $7.17>$ & - & Section 7.13 .1 \\
\hline Soil intake & SLCONA & Soil intake for meat animal & $\mathrm{kg} / \mathrm{d}$ & 0.5 & - & Section 7.16 .1 \\
\hline Soil intake & SLCONA & Soil intake for poultry animal & $\mathrm{kg} / \mathrm{d}$ & 0.01 & assume chicken & Section 7.16.1 \\
\hline Soil intake & SLCONA & Soil intake for milk animal & $\mathrm{kg} / \mathrm{d}$ & 0.2 & assume cow & Section 7.16 .1 \\
\hline Soil intake & SLCONA & Soil intake for egg animal & $\mathrm{kg} / \mathrm{d}$ & 0.01 & assume chicken & Section 7.16 .1 \\
\hline
\end{tabular}


Table ES4.4. (contd)

\begin{tabular}{|c|c|c|c|c|c|c|c|}
\hline Tab & b: Top-level/2nd level/3rd Level & Symbol & Parameter & Units & $\begin{array}{l}\text { Suggested } \\
\text { Value }^{(a)}\end{array}$ & Comment $^{(\mathbf{b})}$ & Details \\
\hline \multicolumn{8}{|c|}{ Food Crop } \\
\hline & Biomass & BIOMAS & Biomass (wet) for human food crops & $\mathrm{kg} / \mathrm{m}^{2}$ & $<$ see Table 7.27> & - & Section 7.18.1 \\
\hline & Growing period & GRWP & Growing period for human food crops & d & $<$ see Table 7.27> & - & Section 7.18 .1 \\
\hline & Yield & YELD & Yield for human food crops & $\mathrm{kg} / \mathrm{m}^{2}$ & $<$ see Table 7.27> & - & Section 7.18 .1 \\
\hline & Dry/Wet ratio & DRYFAC & Dry/wet ratio for leafy vegetables & - & 0.08 & - & Section 7.19 .1 \\
\hline & Dry/Wet ratio & DRYFAC & Dry/wet ratio for other vegetables & - & 0.20 & - & Section 7.19 .1 \\
\hline & Dry/Wet ratio & DRYFAC & Dry/wet ratio for fruit & - & 0.25 & - & Section 7.19 .1 \\
\hline & Dry/Wet ratio & DRYFAC & Dry/wet ratio for grain & - & 0.22 & - & Section 7.19 .1 \\
\hline & Translocation factor & TRANS & Translocation factor for human food crops & - & $<$ see Table 7.27> & - & Section 7.18 .1 \\
\hline \multirow{12}{*}{\multicolumn{2}{|c|}{ Intake delays }} & HLDUP & Intake delay (harvest - consumption) leafy vegetable & $\mathrm{d}$ & 5 & $\mathrm{MEI}^{(\mathrm{b})}$ & Section 7.20 .1 \\
\hline & & HLDUP & Intake delay (harvest - consumption) other vegetable & $\mathrm{d}$ & 5 & MEI & Section 7.20 .1 \\
\hline & & HLDUP & Intake delay (harvest - consumption) fruit & $\mathrm{d}$ & 5 & MEI & Section 7.20 .1 \\
\hline & & HLDUP & Intake delay (harvest - consumption) grain & $\mathrm{d}$ & 180 & MEI & Section 7.20 .1 \\
\hline & & HLDUPA & Intake delay (harvest - consumption) meat & $\mathrm{d}$ & 15 & MEI & Section 7.20 .1 \\
\hline & & HLDUPA & Intake delay (harvest - consumption) poultry & $\mathrm{d}$ & 15 & MEI & Section 7.20 .1 \\
\hline & & HLDUPA & Intake delay (harvest - consumption) milk & $\mathrm{d}$ & 3 & MEI & Section 7.20 .1 \\
\hline & & HLDUPA & Intake delay (harvest - consumption) egg & $\mathrm{d}$ & 5 & MEI & Section 7.20 .1 \\
\hline & & HLDUP2 & Intake delay (harvest - consumption) fish & $\mathrm{d}$ & 2 & MEI & Section 7.20 .1 \\
\hline & & HLDUP2 & Intake delay (harvest - consumption) mollusca & $\mathrm{d}$ & 2 & MEI & Section 7.20 .1 \\
\hline & & HLDUP2 & Intake delay (harvest - consumption) crustacea & $\mathrm{d}$ & 2 & MEI & Section 7.20 .1 \\
\hline & & HLDUP2 & Intake delay (harvest - consumption) aquatic plants & $\mathrm{d}$ & 0 & MEI or population & Section 7.20 .1 \\
\hline $\begin{array}{l}\text { (a) } \\
\text { (b) }\end{array}$ & $\begin{array}{l}<\text { user input }>\text { indicates the valu } \\
\text { MEI = maximally exposed indi }\end{array}$ & $\begin{array}{l}\text { very case- } \\
\text { dal. }\end{array}$ & cific. No generic value is supplied. & & & & \\
\hline
\end{tabular}




\section{ES5.0 Receptor Intake Module - Initial Generic Parameters}

Six age categories are suggested for age-dependent parameters: under1 year old, 1-3 yrs, 3-8 yrs, 8-13 yrs, 13-18 yrs, and greater than 18 yrs. In GENII version2, these categories are symbolized as age1, age2, age3, age4, age5, and age6. Refer to Section 8.0 for details.

Many parameters in this module do not vary by age category. Intake rates and exposure times may. A summary table with age-appropriate suggested values for the six age categories follows the main tables.

! NOTE: Exposure time and intake rates can vary by location. Use suggested values at your own discretion. Suggested values are generally appropriate for the maximally-exposed individual.

Table ES5.1. Receptor Intake Module - Ages

\begin{tabular}{|c|c|c|c|c|c|}
\hline Pathway Selection & Symbol & Parameter & Units & Suggested Value & Comment \\
\hline \multirow[t]{2}{*}{$\mathrm{N} / \mathrm{A}$} & LOWAGE & Lower age & $\mathrm{yr}$ & $<$ see Table $8.1>$ & - \\
\hline & UPAGE & Upper age & $\mathrm{yr}$ & $<$ see Table $8.1>$ & - \\
\hline
\end{tabular}


Table ES5.2. Receptor Intake Module - External Exposure Pathways

\begin{tabular}{|c|c|c|c|c|c|}
\hline Pathway Selection & Symbol & Parameter & Units & Suggested Value & Comment \\
\hline \multirow[t]{2}{*}{ External exposure to air } & UEXAIR & Daily plume exposure time & $\mathrm{hr}$ & 24 & $\begin{array}{l}\text { All ages, maximizing; see Table } 8.3 \\
\text { for other options }\end{array}$ \\
\hline & TEXAIR & Yearly plume exposure time & $\mathrm{d}$ & 365 & $\begin{array}{l}\text { All ages, maximizing; see Table } 8.3 \\
\text { for other options }\end{array}$ \\
\hline \multirow[t]{6}{*}{ External ground exposure } & SHIN & Indoor shielding factor & - & 1 & - \\
\hline & SHOUT & Outdoor shielding factor & - & 1 & - \\
\hline & UEXGRD & Daily ground exposure time & $\mathrm{hr}$ & 24 & - \\
\hline & TEXGRD & Yearly ground exposure time & d & 365 & - \\
\hline & FTIN & Fraction of time spent indoors & - & 0 & - \\
\hline & FTOUT & Fraction of time spent outdoors & - & 1 & - \\
\hline \multirow[t]{4}{*}{ External exposure while swimming } & EVSWIM & Frequency of swimming event & event/d & 1 & - \\
\hline & TESWIM & Duration of swimming event & $\mathrm{hr}$ & 2 & - \\
\hline & TSWIM & Swimming days & $\mathrm{d}$ & 5 & - \\
\hline & USWIM & Ingestion of water while swimming & $\mathrm{L} / \mathrm{hr}$ & $<$ see Table $8.5>$ & - \\
\hline \multirow[t]{4}{*}{ External exposure while boating } & SFBOAT & Shielding factor & - & 1 & - \\
\hline & EVBOAT & Frequency of boating event & event/d & 1 & - \\
\hline & TEBOAT & Duration of boating event & $\mathrm{hr}$ & 1 & - \\
\hline & TBOAT & Boating days & $\mathrm{d}$ & 5 & - \\
\hline \multirow[t]{4}{*}{ External exposure while on shoreline } & EVSHOR & Frequency of shoreline use & event/d & 1 & - \\
\hline & TESHOR & Duration of shoreline use event & $\mathrm{hr}$ & 3.3 & - \\
\hline & TSHOR & Shoreline days & $\mathrm{d}$ & 5 & - \\
\hline & SWFAC & Shoreline width factor & $\mathrm{L} / \mathrm{hr}$ & 0.2 & - \\
\hline
\end{tabular}


Table ES5.3. Receptor Intake Module - Ingestion Pathways

\begin{tabular}{|c|c|c|c|c|c|}
\hline Pathway Selection & Symbol & Parameter & Units & Suggested Value & Comment \\
\hline \multirow[t]{2}{*}{ Food crop ingestion } & UCRP & All crop consumption rates & $\mathrm{kg} / \mathrm{d}$ & $<$ see Table $8.11>$ & - \\
\hline & TCRP & All consumption periods & $\mathrm{d} / \mathrm{yr}$ & 365 & - \\
\hline \multirow[t]{2}{*}{ Animal product ingestion } & UANM & All animal product consumption rates & $\mathrm{kg} / \mathrm{d}$ & $<$ see Table $8.12>$ & - \\
\hline & TANM & All consumption periods & $\mathrm{d} / \mathrm{yr}$ & 365 & - \\
\hline \multirow[t]{2}{*}{ Aquatic food ingestion } & UAQU & All aquatic consumption rates & $\mathrm{kg} / \mathrm{d}$ & $<$ see Table $8.13>$ & - \\
\hline & TAQU & All consumption periods & $\mathrm{d} / \mathrm{yr}$ & 365 & - \\
\hline \multirow[t]{2}{*}{ Drinking water ingestion } & UDW & Drinking water ingestion rate & $\mathrm{L} / \mathrm{d}$ & $<$ see Table $8.14>$ & - \\
\hline & TDW & Drinking water consumption period & $\mathrm{d} / \mathrm{yr}$ & 365 & - \\
\hline \multirow[t]{4}{*}{ Water ingestion while swimming } & EVSWIM & Frequency of swimming event & event/d & $<$ see Table ES5.2> & $\begin{array}{l}\text { User enters the same values as } \\
\text { External exposure while swimming. }\end{array}$ \\
\hline & TESWIM & Duration of swimming event & $\mathrm{hr}$ & $<$ see Table ES5.2> & - \\
\hline & TSWIM & Swimming days & $\mathrm{d}$ & $<$ see Table ES5.2> & - \\
\hline & USWIM & Ingestion of water while swimming & $\mathrm{L} / \mathrm{hr}$ & $<$ see Table ES5.2> & - \\
\hline \multirow[t]{4}{*}{ Water ingestion while showering } & EVSHWR & Frequency of showering event & event/d & 1 & - \\
\hline & TESHWR & Duration of showering event & $\mathrm{hr}$ & $<$ see Table $8.15>$ & - \\
\hline & TSHWR & Showering days & $\mathrm{d}$ & 365 & - \\
\hline & USHIN & Ingestion of water while showering & $\mathrm{L} / \mathrm{hr}$ & $<$ see Table $8.16>$ & - \\
\hline \multirow[t]{2}{*}{ Inadvertent soil ingestion } & TSOIL & Soil contact days & $\mathrm{d}$ & 365 & - \\
\hline & USOIL & Inadvertent soil ingestion rate & $\mathrm{mg} / \mathrm{d}$ & 200 & See Table 8.17 for other options. \\
\hline
\end{tabular}


Table ES5.4. Receptor Intake Module - Inhalation Pathways

\begin{tabular}{|c|c|c|c|c|c|}
\hline Pathway Selection & Symbol & Parameter & Units & Suggested Value & Comment \\
\hline \multirow[t]{3}{*}{ Air Inhalation [outdoor air] } & UINH & Air inhalation rate & $\mathrm{m} 3 / \mathrm{d}$ & $<$ see Table $8.18>$ & $\begin{array}{l}\text { If FRINH (see below) is not } 1 \text {, } \\
\text { UINH=FRINH*<Table } 8.18 \text { value }>\text {. }\end{array}$ \\
\hline & TINH & Air inhalation period & $\mathrm{d} / \mathrm{yr}$ & 365 & - \\
\hline & FRINH & Fraction of day for outdoor inhalation & - & 1 & $\begin{array}{l}\text { Maximizing. See Table } 8.4 \text { for } \\
\text { alternative fractions assumptions. }\end{array}$ \\
\hline \multirow[t]{3}{*}{ Resuspended soil inhalation } & UINHR & Resuspended soil inhalation rate & $\mathrm{m}^{3} / \mathrm{d}$ & $<$ see Table $8.18>$ & Enter same values as UINH \\
\hline & TINHR & Resuspended soil inhalation period & $\mathrm{d} / \mathrm{yr}$ & 365 & - \\
\hline & FRINHR & Fraction of day resuspended soil inhalation & - & $<1>$ & Enter same values as FRINH \\
\hline \multirow[t]{3}{*}{ Indoor inhalation } & UINDRH & Indoor inhalation rate & $\mathrm{m}^{3} / \mathrm{d}$ & 0 & $\begin{array}{l}\text { If FRINDR is not } 0 \text {, UINDRH } \\
\text { estimated by FRINDR*UINH. }\end{array}$ \\
\hline & TINDRH & Indoor inhalation period & $\mathrm{d} / \mathrm{yr}$ & 365 & - \\
\hline & FRINDR & Fraction of day for indoor inhalation & - & 0 & $\begin{array}{l}\text { If Outdoor inhalation rate is not } 0 \text {, } \\
\text { FRINDR=1-FRINH. }\end{array}$ \\
\hline
\end{tabular}




\section{ES5.1 Receptor Intake Module - Summary of Age-dependent Values}

Six age categories are suggested for age-dependent parameters, as indicated by the LOWAGE and UPAGE values of Table ES5.5. In GENII version2, these categories are symbolized as age 1, age2, age3, age4, age5, and age 6 .

Refer to Section 8.0 for details.

Table ES5.5. Summary of Age-dependent Generic Values

\begin{tabular}{|c|c|c|c|c|c|c|c|}
\hline Symbol (units) & Age1 & Age2 & Age3 & Age4 & Age5 & Age6 & Comment $^{(a)}$ \\
\hline \multirow{2}{*}{$\begin{array}{l}\text { LOWAGE (yr) } \\
\text { UPAGE (yr) }\end{array}$} & 0 & 1.5 & 2.5 & 7.5 & 12.5 & 17.5 & \multirow{2}{*}{$\begin{array}{l}\text { Age group lower bound } \\
\text { Age group upper bound }\end{array}$} \\
\hline & 1.5 & 2.5 & 7.5 & 12.5 & 17.5 & $17.5+$ & \\
\hline \multirow{2}{*}{$\begin{array}{l}\text { FTIN } \\
\text { FTOUT }\end{array}$} & 1 & 1 & 0.94 & 0.89 & 0.9 & 1 & \multirow{9}{*}{ Residential receptor MEI } \\
\hline & 0 & 0 & 0.06 & 0.11 & 0.1 & 0 & \\
\hline USWIM (L/hr) & 0 & 0.12 & 0.12 & 0.12 & 0.12 & 0.071 & \\
\hline \multirow{4}{*}{$\begin{array}{l}\text { UCRP, leafy }(\mathrm{kg} / \mathrm{d}) \\
\text { UCRP, root, other }(\mathrm{kg} / \mathrm{d}) \\
\text { UCRP, fruit }(\mathrm{kg} / \mathrm{d}) \\
\text { UCRP, grain }(\mathrm{kg} / \mathrm{d})\end{array}$} & 0.0121 & 0.0388 & 0.0474 & 0.0628 & 0.0918 & 0.1872 & \\
\hline & 0.1386 & 0.1765 & 0.2019 & 0.2679 & 0.2613 & 0.2928 & \\
\hline & 0.2100 & 0.2950 & 0.2809 & 0.2926 & 0.2440 & 0.3360 & \\
\hline & 0.1058 & 0.1711 & 0.2065 & 0.2608 & 0.3210 & 0.3240 & \\
\hline UANM, milk (kg/d) & 0.300 & 1.083 & 1.311 & 0.949 & 0.975 & 0.888 & \\
\hline UANM, egg (kg/d) & 0.022 & 0.022 & 0.024 & 0.031 & 0.034 & 0.036 & \\
\hline \multirow{4}{*}{$\begin{array}{l}\text { UAQU, fish }(\mathrm{kg} / \mathrm{d}) \\
\text { UAQU, mollusk }(\mathrm{kg} / \mathrm{d}) \\
\text { UAQU, crustacean }(\mathrm{kg} / \mathrm{d}) \\
\text { UAQU, aquatic plant }(\mathrm{kg} / \mathrm{d})\end{array}$} & 0.0068 & 0.0166 & 0.026 & 0.035 & 0.0449 & 0.088 & \multirow{4}{*}{$\begin{array}{l}\text { per capita } \\
\text { per capita }\end{array}$} \\
\hline & 0.0050 & 0.0119 & 0.0209 & 0.0241 & 0.0502 & 0.0730 & \\
\hline & \multicolumn{6}{|c|}{$<$ subtract from mollusk value, if applied $>$} & \\
\hline & $<$ apply si & ecific val & if relevar & & & & \\
\hline
\end{tabular}


Table ES5.5. (contd)

\begin{tabular}{|c|c|c|c|c|c|c|c|}
\hline Symbol (units) & Age1 & Age2 & Age3 & Age4 & Age5 & Age6 & Comment $^{(a)}$ \\
\hline UDW (L/d) & 0.97 & 0.865 & 0.928 & 1.36 & 1.841 & 2.73 & \\
\hline TESHWR (hr) & 0 & 0.73 & 0.57 & 0.68 & 0.71 & 0.75 & \\
\hline USHIN (L/hr) & 0 & 0.06 & 0.06 & 0.06 & 0.06 & 0.04 & \\
\hline $\operatorname{UINH}\left(\mathrm{m}^{3} / \mathrm{d}\right)$ & 6.8 & 9.2 & 13.8 & 16.6 & 23.3 & 19.4 & \\
\hline
\end{tabular}

N 


\section{ES6.0 Health Impacts Module}

Refer to Section 9.0 for details.

Table ES6.0. Health Impacts Module

\begin{tabular}{|c|c|c|c|c|c|}
\hline Tab & Symbol & Parameter & Units & Suggested Value & Comment \\
\hline METHOD SELECTION & FGR13 & N/A & - & “...ICRP-60; FGR 12/13” & \\
\hline \multirow[t]{6}{*}{ METHOD PARAMETERS } & SOILT & $\begin{array}{l}\text { Thickness of contaminated } \\
\text { soil/sediment }\end{array}$ & $\mathrm{m}$ & 0.15 & $\begin{array}{l}\text { Enter same value as SURCM of the } \\
\text { Exposure module. }\end{array}$ \\
\hline & SLDN & $\begin{array}{l}\text { Density of contaminated } \\
\text { soil/sediment }\end{array}$ & $\mathrm{kg} / \mathrm{m}^{3}$ & see options below & $\begin{array}{l}\text { Enter same value as (SLDN of the } \\
\text { Exposure module)* }(1 / 0.15) \text {. }\end{array}$ \\
\hline & SLDN & $\begin{array}{l}\text { Density of contaminated } \\
\text { soil/sediment-option } 1\end{array}$ & $\mathrm{~kg} / \mathrm{m}^{3}$ & 1466 & sandy soil - for crop growth \\
\hline & SLDN & $\begin{array}{l}\text { Density of contaminated } \\
\text { soil/sediment-option } 2\end{array}$ & $\mathrm{~kg} / \mathrm{m}^{3}$ & 1300 & silty soil - for crop growth \\
\hline & SLDN & $\begin{array}{l}\text { Density of contaminated } \\
\text { soil/sediment-option3 }\end{array}$ & $\mathrm{kg} / \mathrm{m}^{3}$ & 1033 & clay soil - for crop growth \\
\hline & SLDN & $\begin{array}{l}\text { Density of contaminated } \\
\text { soil/sediment-option } 4\end{array}$ & $\mathrm{~kg} / \mathrm{m}^{3}$ & 1933 & rocky shoreline \\
\hline
\end{tabular}




\subsection{Introduction}

Guidance in Regulatory Guide 1.109 (NRC 1977), "Calculation of Annual Doses to Man from Routine Releases in Reactor Effluents for the Purpose of Evaluating Compliance with 10 CFR Part 50, Appendix I," has been historically used as guidance to assess licensee compliance with public dose criteria. Public dose estimates calculated by nuclear power plant operators provide an extensive historical record of application of U.S. Nuclear Regulatory Commission (NRC) Regulatory Guide 1.109 ${ }^{1}$ information for the member of the public considered to be the maximally exposed individual (MEI). The NRC Office of New Reactors (NRO) has requested that a similar parameter document be developed for use in assessing compliance with both Title 10 Code of Federal Regulations (CFR) 50, Appendix I and 10 CFR Part 20 for use with the GENII version 2 (Gv2) computer code.

This document provides more detailed guidance for parameterization of the maximally exposed individual (MEI) and population doses for use with the GENII version 2 computer code (Gv2). Default parameter values are provided in the Users' Guide and Software Design Documentation. The Gv2 code requires a multitude of user-entered parameters, more than many environmental modeling codes. The numerous parameter entries are, in part, a result of the numerous linked modules that comprise a complete scenario definition from source to health impact. The numerous entries are also a consequence of the evolution of the code from a simpler, DOS-based code running on older computer systems to the advent of faster computer systems that allow more input to be processed in a practical amount of time.

The numerous user inputs make the code more flexible to user-specific conditions. Each user-defined parameter should have as strong a technical basis as possible. This document presents a compilation of U.S. generic and available regional parameter data and their technical bases. This document provides guidance for both MEI and population dose estimates. The user has the ultimate responsibility for ensuring the parameters entered are reasonable, technically strong, and coherent. Coherence means that the various parameters make sense; a simple example being that ingestion rates for youngsters would not result in a 150-lb six-year old. Although common for risk assessments to somewhat overestimate exposures or intakes to provide a reasonable upper bound, unreasonably overestimated impacts are unwarranted.

Typically, estimating dose to the MEI provides a prudently worst-case dose to a hypothetical individual. The MEI dose answers the question "What's the worst dose possible from annual radioactive emissions at my facility?" This individual is generally at an offsite location close to the emission source and harvests food, both animal products and crops, from this location. The public dose standards are based on an individual dose. The MEI intake and exposure assumptions are reasonable upper-bounds and would rarely be applicable to any actual person.

Population doses can be calculated by summing doses of more than one individual. Most of the U.S. population obtains the majority of their food from commercial sources from a wide variety of locations. Therefore, large numbers of individuals have smaller individual doses than the MEI. The population dose can be calculated for various population sets of interest and are generally limited to a greatest distance of 30-50 miles from the release point. The population dose has limited but informative

\footnotetext{
${ }^{1}$ See U.S. NRC Radioactive Effluent and Environmental Reports, last accessed at http://www.nrc.gov/reactors/operating/ops-experience/tritium/plant-info.html.
} 
applications, since there are no current population dose limits or guidelines. The population dose estimates and input parameters of the GENII code can be used to determine the several measures of interest.

- The dose to the average person can be calculated from average intake rates of regional food sources. ${ }^{1}$ The population dose within a user-defined region can also be calculated and tracked for trending purposes.

- The dose to the average person from a large commercial agricultural operation for a single crop or livestock operation in a region can be calculated. This can be used as a comparative baseline for accident impact estimates.

- A small number of additional calculations can be performed for regional commercial agricultural operations to determine the number of individuals exposed to the crop or livestock products of a given region. This will provide an indication of the extent of public impact as well as an indication of impact to the domestic market for that particular agricultural product.

"Generic" parameter data and model selections for chronic air and for liquid effluent releases to public bodies of water are presented. Considering schedule, data availability and format, and other factors, regionally-specific parameter data are also presented in varying levels of detail. Additional resources are also provided to prompt the user regarding additional information sources about a specific parameter. The user must be aware that these generic values and the indicated minimum and maximums are not extreme, bounding minimum and maximum values, but rather "generic," "typical" minimum and maximum values in the majority of cases. No attempt was made to quantify the percentile level that the minimum and maximum values represent. The user will need to evaluate the applicability of the parameter values they assign and how comprehensively they desire to represent the full range, likely range, or typical range of receptors that they are modeling.

Information specific to tritium and carbon-14 modeling is in Section 10.0. As is the standard, the environmental modeling of these two nuclides implements specific-activity equivalence models.

If the sensitivity module of the GENII code is implemented, output uncertainties can be estimated. Appendix $\mathrm{J}$ provides information regarding the distribution type suggested for routine release scenarios.

Parameter data are presented in the order of its occurrence in a Gv2 module. Air emissions are more common, therefore no water modules would be implemented. Modules are represented as icons in the Gv2 window (see Figure 1.1).

\footnotetext{
${ }^{1}$ This average individual dose can be compared to the regulatory dose limit, but has limited application because the MEI dose will always be larger.
} 


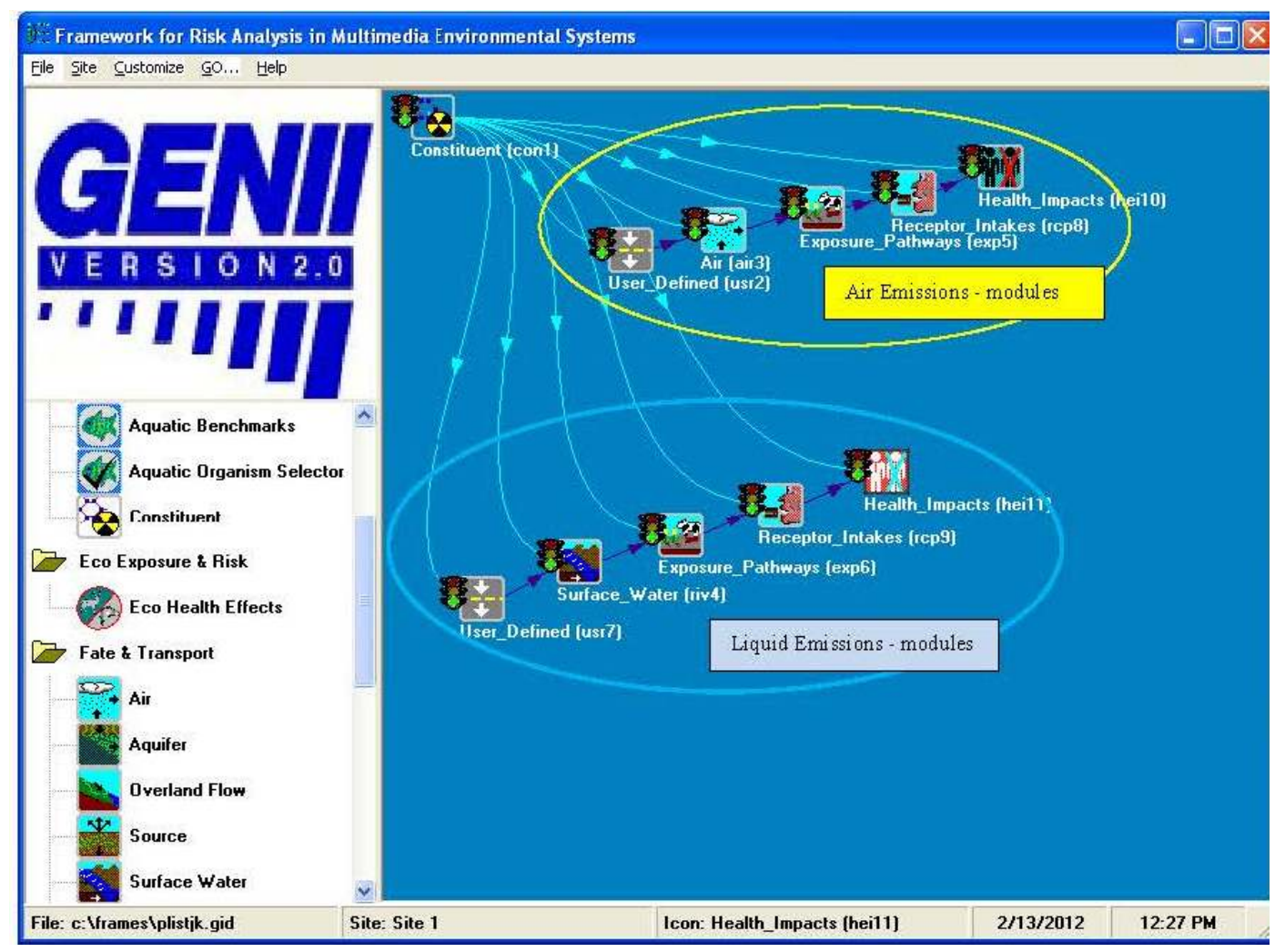

Figure 1.1. Example of GENIIv2 Chronic Air and Water Pathways Scenarios

One important concept to understand with use of the Gv2 code is the difference between a module and a model. Each icon represented in the blue field of Figure 1.1 represents a different module. Within each module, one or more models (i.e., algorithms) accept the user input and produce output. For example, the user can select a variety of models in the Air module (Acute $95^{\text {th }}$ Percentile, Acute Plume, Acute Puff, Chronic Plume, among others). The models selected for use with the parameters presented in this document are indicated in Table 1.1. The model is selected before parameter values are entered. Some, but not all, parameters values may differ if a model other than that indicated is selected.

Table 1.1. Applicable Models to Select for Modules of Chronic Release Scenarios

\begin{tabular}{lll}
\hline \multicolumn{1}{c}{ Pathway } & \multicolumn{1}{c}{ Module } & \multicolumn{1}{c}{ Applicable Model to Select } \\
\hline Air and Water & Constituent & GENII Radionuclide database selection \\
\hline Air & User-Defined & AFF Air Module \\
& Air & GENII V2 Air Module - Chronic Plume \\
& Exposure Pathways & GENII V2 Chronic Exposure Module \\
& Receptor Intakes & GENII V2 Receptor Intake Module \\
& Health Impacts & GENII V2 Health Impacts Module \\
\hline Water & User-Defined & WFF Surface Water Module \\
& Surface Water & GENII V2 Surface Water Module \\
& Exposure Pathways & GENII V2 Chronic Exposure Module \\
& Receptor Intakes & GENII V2 Receptor Intake Module \\
& Health Impacts & GENII V2 Health Impacts Module \\
\hline
\end{tabular}


GENIIv2 modules define the models that are linked to represent the scenario of interest. Module details are available in GENII v2 documentation (Napier 2012, Napier et al. 2012). Modules are summarized as follows for airborne and liquid releases:

- Chronic Airborne Release Modules

- Constituent: Chemical- or radionuclide-specific parameters entered

- User-Defined (AIR): Radionuclide release rates entered

- Air: Meteorological dispersion characterization

- Exposure Pathways: Environmental media defined and characterized

- Receptor Intakes: Intake rates and exposure times entered

- Health Impacts: Dose is calculated from intake and exposure estimate

- Chronic Liquid Release Modules

- Constituent: Chemical- or radionuclide-specific parameters entered

- User-Defined (WATER): Radionuclide release rates entered

- Surface Water: Mixing in surface water characterized

- Exposure Pathways: Environmental media defined and characterized

- Receptor Intakes: Intake rates and exposure times entered

- Health Impacts: Dose is calculated from intake and exposure estimate.

Default parameter values are provided in most modules. Parameters such as emission rates and release point characteristics are user-specific, so default values are not provided. This parameter document provides information to allow the user to modify the default parameters to better represent their release, local conditions, and regional receptor characteristics to model the release and the impact estimate that best describes their facility and region.

\subsection{References}

10 CFR 20. 2013. "Standards for Protection Against Radiation." Code of Federal Regulations, U.S. Department of Energy, Washington, D.C.

10 CFR 50, Appendix I. 2007. "Domestic Licensing of Production and Utilization Facilities." Appendix I, "Numerical Guides for Design Objectives and Limiting Conditions for Operation to Meet the Criterion "As Low as is Reasonably Achievable" for Radioactive Material in Light-Water-Cooled Nuclear Power Reactor Effluents." Code of Federal Regulations, U.S. Department of Energy, Washington, D.C.

Napier BA. 2012. GENII Version 2 Users' Guide. PNNL-14583, Rev. 4, Pacific Northwest National Laboratory, Richland, Washington. 
Napier BA, DL Strenge, JV Ramsdell, Jr., PW Eslinger, and C Fosmire. 2012. GENII Version 2

Software Design Document. PNNL-14584, Rev. 4, Pacific Northwest National Laboratory, Richland, Washington.

NRC. 1977. Calculation of Annual Doses to Man from Routine Releases of Reactor Effluents for the Purpose of Evaluating Compliance with 10 CFR Part 50, Appendix I. Regulatory Guide 1.109, Rev. 1, U.S. Nuclear Regulatory Commission, Washington, D.C. 



\subsection{Module: Constituent}

The Constituent module will be the first module for all cases. The module model selected is "GENII Radionuclide Database Selection." The Constituent Module is where the user indicates the radionuclides of interest and assigns most element- or nuclide-specific parameter values. Two Tabs exist in this module: Select Constituents of Concern and Edit Constituent Properties. No user-defined parameters are entered in the Select Constituents of Concern Tab. Therefore, only the numerous parameters of the Edit Constituent Properties Tab are detailed in this section. Sub-tabs include Properties and Degradation Chain, but only the Properties sub-tab allows user-defined values.

\subsection{Tab: Edit Constituent Properties/Properties}

Numerous parameters are listed, but not all require input. Those that do not require input are not noted in this section, unless a particular editorial comment was deemed note-worthy. There are some parameters that a user would expect to require input, but are actually handled in other modules or not required. This particular set of parameters includes:

CLANDF Inhalation Volatilization Factor for Indoors

SOLUBIL Lung Solubility

CLDFAx Inhalation Dose Factor, where $\mathrm{x}=\mathrm{D}, \mathrm{W}$, or $\mathrm{Y}$

CLDFGx Ingestion Dose Factor, where $\mathrm{x}=\mathrm{S}$ or I

CLBSAF Bioaccumulation in Aquatic Biota from Sediment

CLBVOV Bioconcentration in Other Feed Vegetables from Soil

CLVD Atmospheric Deposition Velocity

CLSCLASS Atmospheric Deposition Class

Inhalation and ingestion dose factors are not used from the entries in this module when the recommended Health Impact module selection [Method Selection of "Calculate Dose and/or Risk using ICRP-60 and EPA Risk Factors (Federal Guidance Reports 12/13)] is used (see Section 9.0), but are discussed briefly.

\subsubsection{CLKD Dry Soil-Water Partition Coefficient}

The default soil-water partition coefficients are listed here; for radionuclide modeling the value used by the code may also entered in a later module. If "Leach rates calculated from user input" is selected in the later Exposure Pathways-Chronic Exposure module (see Section 7.9), then the user may enter the values here that will be reflected as the defaults for SOILKD.

The units and range for this parameter in $\mathrm{Gv} 2$ are: $\mathrm{mL} / \mathrm{g}(0-10$ million).

See Chronic Exposure Module, Soil/Leaching tab (Section 7.9) for $\mathrm{K}_{\mathrm{d}}$ value used for radionuclide modeling. 


\subsubsection{CLANDF Inhalation Volatilization Factor}

CLANDF is intended to be used to calculate the indoor air contaminant concentrations that originate from shower water use, as the nuclide volatilizes from the heated, fine spray. The value applied for Gv2 modeling is assigned in the Chronic Exposure model, Water/General tab, parameter ANDKR (see Section 7.3.2).

The units and range for this parameter in $\mathrm{Gv} 2$ are: $\mathrm{m}^{3} / \mathrm{L}$ ( 0 to 100$)$.

It is suggested that all these values be set to zero, to remind the user that values are not applied at this point in water scenarios.

\subsubsection{CLWPF Water Purification Factor}

This value is used in modeling when the option for Treatment Plant Purification of Domestic Water is selected in the Chronic Exposure Module, Water/General tab (see Section 7.3). The parameter value indicates the ratio of the contaminant concentration after and before purification.

The units for this parameter in Gv2 are: fraction ( 0 to 1$)$.

A conservative value would be 1.0 .

Cleanup factors were developed for use in the original version of the GENII code used for U.S. Department of Energy (DOE) Hanford Site dose calculations (GENIIv1.485, Napier et al. 1988). The values indicated in the GENIIv1.485 BIOAC1.DAT file is shown in Table 2.1. These values reflect an alum-flocculation water treatment plant (Denham and Soldat 1974).

\subsubsection{Additional Sources of Information}

There are likely to be a number of additional sources of information available. None were identified at the time of publication.

\subsubsection{CLWPF Water Purification Factor Tables}

Table 2.1. Generic Water Treatment Cleanup Factors for Alum Flocculation Filtration

\begin{tabular}{cl}
\hline $\begin{array}{c}\text { ClWPF } \\
\text { (fraction) }\end{array}$ & \multicolumn{1}{c}{ Element } \\
\hline 1 & $\mathrm{C}, \mathrm{Cl}, \mathrm{H}$ \\
0.9 & $\mathrm{Bi}, \mathrm{Cs}, \mathrm{Mo}, \mathrm{Na}, \mathrm{Pb}, \mathrm{Rb}$ \\
0.8 & $\mathrm{~F}, \mathrm{I}, \mathrm{Po}, \mathrm{Sb}, \mathrm{Se}, \mathrm{Te}$ \\
0.7 & $\mathrm{Ac}, \mathrm{Ag}, \mathrm{Am}, \mathrm{As}, \mathrm{Cm}, \mathrm{Nb}, \mathrm{Np}, \mathrm{Pa}, \mathrm{Pu}, \mathrm{Ra}, \mathrm{Si}, \mathrm{Sn}, \mathrm{Tc}, \mathrm{Th}, \mathrm{U}, \mathrm{Zr}$ \\
0.6 & $\mathrm{Cd}$ \\
0.5 & $\mathrm{Mn}, \mathrm{Pd}, \mathrm{Rh}, \mathrm{Ru}$ \\
0.4 & $\mathrm{Ba}, \mathrm{P}, \mathrm{Zn}$ \\
0.3 & $\mathrm{Sc}$ \\
0.2 & $\mathrm{Be}, \mathrm{Ca}, \mathrm{Ce}, \mathrm{Co}, \mathrm{Er}, \mathrm{Eu}, \mathrm{Fe}, \mathrm{Ho}, \mathrm{La}, \mathrm{Nd}, \mathrm{Ni}, \mathrm{Pm}, \mathrm{Pr}, \mathrm{Sm}, \mathrm{Sr}, \mathrm{Tb}, \mathrm{Y}$ \\
0.1 & $<$ none $>$ \\
0 & gases \\
N/A & Bk, Br, Cf, Cr, Cu, Dy, Ga, Gd, Hf, Hg, In, Ir, S, Ta, Tl, Tm, W, Yb \\
\hline N/A $=$ No value indicated in BIOAC1.DAT file of Napier et al. (1988). \\
\hline
\end{tabular}




\subsubsection{SOLUBIL Lung Solubility}

SOLUBIL is not implemented from this entry when the ICRP-30 internal dose factors are used (the code takes the largest available). When the recommended internal dose factors (see next section) are implemented these define the default types. See CLDFAx, additional information (Section 2.1.5.1) for a reference that contains generic solubility types. See the Health Impacts module, Constituent Parameters tab for parameterization of lung solubility (see Section 9.2).

The units for this parameter in Gv2 are: N/A.

\subsubsection{CLDFAx Inhalation Dose Factor}

There are three lines of potential input for this parameter; however, these would only be used for calculations when the later Health Impacts module, the Method Selection was indicated to be use of “... ICRP 30/48..." The recommended Method Selection is "ICRP 60," which is more recent, and implements age-dependent internal dose factors consistent with ICRP 66-72. Both use the same non-agedependent external dose factors of Federal Guidance Report No. 12 (Eckerman and Ryman 1993).

If the user decides to use the ICRP 30/48 option in the later Health Impacts module, note the different data values for solubility class day (D) (CLDFAD), solubility class week (W) (CLDFAW), and solubility class year (Y) (CLDFAY). If more than one entry is provided, the code will use the greatest. If one particular class is desired for a nuclide, delete the others.

When the recommended later, Health Impacts module selection is made (Method Selection "...using ICRP $60 \ldots$.."), the dose factor implemented is implemented in code black-box processing and not defined explicitly by the user. Age-dependent inhalation dose factors are used, depending on the Receptor module age groups (see Section 8.0) and Health Impacts module inhalation class entered (see Section 9.2).

The units for this parameter in $\mathrm{Gv} 2$ are: $\mathrm{rem} / \mathrm{pCi}(0-1)$.

The inhalation dose factors implemented are those of Federal Guidance Report 13 (Eckerman et al. 1998), which is consistent with those in ICRP 66-72.

\subsubsection{Additional Sources of Information}

ICRP 66 (ICRP 1994). The inhalation dose factors of ICRP 71 and 72 (and ICRP 68 for workers) are based on this human respiratory tract model.

ICRP 71 and 72 (ICRP 1995b, 1995c). These publications provide inhalation dose factors that are based on the ICRP 66 respiratory tract model. These values update those of ICRP 56 (ICRP 1990), which use an older respiratory tract model. ICRP 72 does not include organ-specific data.

ICRP 119 (ICRP 2012). Supplement 1 to ICRP 119 is a Compendium of Dose Coefficients based on ICRP Publication 60. 
DOE Derived Concentration Technical Standard (DOE 2011). Table A-2 provides alternative effective dose coefficients from air inhalation $\left(\mathrm{Sv}_{\text {effective_dose }} / \mathrm{Bq}_{\text {inhaled }}\right)$. Table 4 indicates generic lung solubility type recommendations for each element in particulate form.

\subsubsection{CLDFAx Inhalation Dose Factor Tables}

Dose factors will not be tabulated here.

If the recommended "ICRP 60" method values of Federal Guidance Report 13 are implemented in the later Health Impacts module, the user can view the data implemented in the FRAMES/FGR13INH.HDB file, with different units indicated ( $\mathrm{Sv} / \mathrm{Bq})$. The format of the file is discussed in the GENII documentation (Napier et al. 2012) Appendix B, Section B.4. The Federal Guidance Report No. 13 can be accessed separately per information available at http://www.epa.gov/radiation/federal/techdocs.html.

Although discouraged, if the "ICRP 30" method values of Federal Guidance Report 11 are implemented in the later Health Impacts module, the dose factors can be access per information available at the same site: http://www.epa.gov/radiation/federal/techdocs.html, but choose Report No. 11.

\subsubsection{CLRDFGx Ingestion Dose Factor}

This parameter is implemented in a manner similar to the Inhalation Dose Factor, above. There are two lines of potential input for this parameter, however only one will be used for calculations when later, Health Impacts module, Method selection of “... ICRP38/40...” is chosen. This "ICRP 38/40" choice is not recommended. The recommended option in the later, Health Impacts module, Method selection is ICRP 60. There are different data entry points for soluble (CLRDFGS) and insoluble (CLRDFGI). If more than one entry is provided, the code will use the greatest.

The units for this parameter in Gv2 are: rem/pCi.

\subsubsection{Additional Sources of Information}

ICRP 67 and 69 (ICRP 1992, 1995a). Age-dependent ingestion dose factors.

\subsubsection{CLRDFGx Ingestion Dose Factor Tables}

Age-dependent ingestion dose factors will not be tabulated here.

If the recommended "ICPR 60" method values of Federal Guidance Report 13 are implemented in the later Health Impacts module, the user can view the data implemented in the FRAMES/FGR13ING.GDB file with data presented in different units $(\mathrm{Sv} / \mathrm{Bq})$. The format of the file is discussed in the GENII documentation (Napier et al. 2012) Appendix B, Section B.3.

\subsubsection{CLDEX External Dose Factor, Air Immersion}

The external dose factor for air immersion is the dose rate received by an individual standing in a large cloud or plume of contaminated air having a radionuclide concentration of $1 \mathrm{pCi} / \mathrm{m}^{3}$. 
The units and range for this parameter in Gv2 are: $\mathrm{mrem} / \mathrm{hr}$ per $\mathrm{pCi} / \mathrm{m}^{3}$ ( 0 to 1$)$.

The values implemented in GENII are taken from Federal Guidance Report 12 (Eckerman and Ryman 1993).

\subsubsection{Additional Sources of Information}

None provided.

\subsubsection{CLDEX External Dose Factor, Air Immersion Tables}

Not tabulated here. See the air submersion dose factors in Table III.1 of Eckerman and Ryman (1993).

\subsubsection{CLDIMR External Dose Factor, Water Immersion}

The water immersion, external dose factor is the dose rate received by an individual immersed in water (e.g., swimming) having a radionuclide concentration of $1 \mathrm{pCi}$ per L. For external exposure from boating, a 0.5 times the water immersion dose factor is applied for dose calculations.

The units and range for this parameter in Gv2 are: $\mathrm{mrem} / \mathrm{hr}$ per $\mathrm{pCi} / \mathrm{m}^{3}$ ( 0 to 1$)$.

Values applied are those in the U.S. Environmental Protection Agency (EPA) Federal Guidance Report No. 12 (Eckerman and Ryman 1993).

\subsubsection{Additional Sources of Information}

None provided.

\subsubsection{CLDIMR External Dose Factor, Water Immersion Tables}

Not tabulated here. See the water immersion dose factors in Table III.2 of Eckerman and Ryman (1993).

\subsubsection{CLDSHx External Dose Factor, Ground Contaminated to $15 \mathrm{~cm}$}

There are three lines of potential input for this parameter; however, only one will be used. The entire set of parameters includes dose factors for surface contamination (CLDSH1), or contamination to a depth of $5 \mathrm{~cm}$ (CLDSH5), or contamination to a depth of $15 \mathrm{~cm}$ (CLDSH15).

- The units and range for CLDSH1 in Gv2 are: $\mathrm{mrem} / \mathrm{hr}$ per $\mathrm{pCi} / \mathrm{m}^{2}$ ground surface $(0-1)$.

- The units and range for CLDSH5 in Gv2 are: $\mathrm{mrem} / \mathrm{hr}$ per $\mathrm{pCi} / \mathrm{m}^{3}$ for $5 \mathrm{~cm}$ depth $(0-1)$.

- The units and range for CLDSH15 in Gv2 are: $\mathrm{mrem} / \mathrm{hr}$ per $\mathrm{pCi} / \mathrm{m}^{3}$ for $15 \mathrm{~cm}$ depth $(0-1)$. 
Contamination to a depth of $15 \mathrm{~cm}$ is recommended. Values are applied from Federal Guidance Report No. 12 Table III.6 (Eckerman and Ryman 1993). No age-dependency is applied to these dose factors.

\subsubsection{Additional Sources of Information}

ICRP Report 74 (ICRP 1996) discusses age-dependency issues related to external dose coefficients in ICRP 74, Section 4.3.5.

\subsubsection{CLDSHX External Dose Factor, Ground Contaminated to $15 \mathrm{~cm}$ Tables}

Not tabulated here. See Table III.6 of Eckerman and Ryman (1993).

\subsubsection{CLDIAM Inhalation Dose Factor, Particle Diameter}

This parameter is not implemented in this module in GENII v2. The inhalation dose factors are defined for a specific particle size at the point of inhalation. For atmospheric transport modeling, the particle radius and density are parameterized in the next, User-Defined, AFF Air Module (select Flux Types button and enter values).

See User-Defined module, Section 3.0, for air pathway inputs 


\section{Bioconcentration and Transfer Factors}

\subsubsection{CLBFx and CLBMx Bioaccumulation Factor for Aquatic Animals (Freshwater and Saltwater)}

These parameters describe the bioaccumulation factor for wet fish, wet mollusks, and wet Crustacea in freshwater (CLBFF, CLBFM, and CLBFI, respectively) and saltwater (CLBMF, CLBMM, and CLBMI, respectively). These parameters are the ratio of the chemical concentration in a freshwater or saltwater organism to the chemical concentration in the water to which the organism is exposed. The bioaccumulation factor generally accounts for uptake by the organism of the chemical from water, sediments passing across the gills, and from consumption of various foods.

The units and range for these parameters in $\mathrm{Gv} 2$ are: $\mathrm{L} / \mathrm{kg}_{\text {wet }}$ which is a reduced from $\left(\mathrm{Ci} / \mathrm{kg}_{\mathrm{wet}}\right)$ per (Ci/L) (0 to 100,000 for all organisms).

To tabulate bioaccumulation factors for aquatic animals, sources considered to be the best available references were reviewed. Details on the references reviewed are available in Appendix A. The goal was to determine mean, median, geometric standard deviation, geometric mean, $5^{\text {th }}$ percentile, and $95^{\text {th }}$ percentile. Some of these values could be determined mathematically. In these cases, statistical calculations were performed, according to methods described in Strom and Stansbury (2000). (If, in assignment of statistical parameters, the assigned mean was greater than the $95^{\text {th }}$ percentile, it is not shown in the tables.) The full set of data reviewed to determine the values tabulated in this section are provided in Appendix A. Selection priority was given to recent International Atomic Energy Agency (IAEA) documents (IAEA 2004, 2010) with a preference for lognormality in the data sets.

\subsubsection{Additional Sources of Information}

The references that were reviewed contain additional information regarding data completeness, data limitations, and application of values to environmental models.

There are numerous chemicals for which no aquatic animal bioaccumulation factors are available. The user can refer to Staven et al. (2003) for an approach to approximate values for non-tabulated chemicals. The user is encouraged to review additional research on transfer factors in the literature, as well.

\subsubsection{CLBFx Tables - Freshwater Fish, Mollusks, and Crustaceans}

For CLBFF Bioconcentration factors for freshwater Fish, see Table 2.2.

For CLBFM Bioconcentration factors for freshwater Mollusk, see Table 2.3. Because relatively few data were available, this table has been enhanced by including additional results from the freshwater Crustacea where other data are lacking.

For CLBFI Bioconcentration factors for freshwater Crustacea, see Table 2.4. 


\subsubsection{CLBMx Tables - Saltwater Fish, Mollusks, and Crustaceans}

For CLBMF Bioconcentration factors for saltwater Fish, see Table 2.5

For CLBMM Bioconcentration factors for saltwater Mollusk, see Table 2.6

For CLBMI Bioconcentration factors for saltwater Crustacea, see Table 2.7

Table 2.2. CLBFF Bioaccumulation Factors - Freshwater Fish

\begin{tabular}{|c|c|c|c|c|c|c|c|}
\hline Element & Mean & STD & Median & GSD & GM & $5 \%$ & $95 \%$ \\
\hline $\mathrm{Ac}$ & $\mathrm{N} / \mathrm{A}$ & $\mathrm{N} / \mathrm{A}$ & $2.50 \mathrm{E}+01$ & $\mathrm{~N} / \mathrm{A}$ & N/A & N/A & $\mathrm{N} / \mathrm{A}$ \\
\hline $\mathrm{Ag}$ & $1.19 \mathrm{E}+02$ & $5.05 \mathrm{E}+01$ & N/A & $1.50 \mathrm{E}+00$ & $1.10 \mathrm{E}+02$ & $5.65 \mathrm{E}+01$ & $2.14 \mathrm{E}+02$ \\
\hline $\mathrm{Al}$ & $1.29 \mathrm{E}+02$ & $2.98 \mathrm{E}+02$ & $\mathrm{~N} / \mathrm{A}$ & $3.90 \mathrm{E}+00$ & $5.10 \mathrm{E}+01$ & $5.44 \mathrm{E}+00$ & $4.78 \mathrm{E}+02$ \\
\hline $\mathrm{Am}$ & $2.40 \mathrm{E}+02$ & N/A & N/A & N/A & N/A & N/A & N/A \\
\hline As & $4.35 \mathrm{E}+02$ & $3.72 \mathrm{E}+02$ & N/A & $2.10 \mathrm{E}+00$ & $3.30 \mathrm{E}+02$ & $9.74 \mathrm{E}+01$ & $1.12 \mathrm{E}+03$ \\
\hline $\mathrm{Au}$ & $3.16 \mathrm{E}+02$ & $2.71 \mathrm{E}+02$ & N/A & $2.10 \mathrm{E}+00$ & $2.40 \mathrm{E}+02$ & $7.08 \mathrm{E}+01$ & $8.13 \mathrm{E}+02$ \\
\hline $\mathrm{Ba}$ & $2.45 \mathrm{E}+00$ & $4.35 \mathrm{E}+00$ & N/A & $3.30 \mathrm{E}+00$ & $1.20 \mathrm{E}+00$ & $1.68 \mathrm{E}-01$ & $8.55 E+00$ \\
\hline $\mathrm{Be}$ & N/A & $\mathrm{N} / \mathrm{A}$ & $2.00 \mathrm{E}+00$ & N/A & N/A & N/A & N/A \\
\hline $\mathrm{Bi}$ & N/A & N/A & $1.50 \mathrm{E}+01$ & N/A & N/A & N/A & N/A \\
\hline $\mathrm{Br}$ & $1.29 \mathrm{E}+02$ & $1.29 \mathrm{E}+02$ & N/A & $2.30 \mathrm{E}+00$ & $9.10 \mathrm{E}+01$ & $2.31 \mathrm{E}+01$ & $3.58 \mathrm{E}+02$ \\
\hline $\mathrm{C}$ & $7.05 \mathrm{E}+05$ & $1.02 \mathrm{E}+06$ & $\mathrm{~N} / \mathrm{A}$ & $2.90 \mathrm{E}+00$ & $4.00 \mathrm{E}+05$ & $6.94 \mathrm{E}+04$ & $2.31 \mathrm{E}+06$ \\
\hline $\mathrm{Ca}$ & $1.83 \mathrm{E}+01$ & $2.09 \mathrm{E}+01$ & N/A & $2.50 \mathrm{E}+00$ & $1.20 \mathrm{E}+01$ & $2.66 \mathrm{E}+00$ & $5.42 \mathrm{E}+01$ \\
\hline $\mathrm{Cd}$ & $8.31 \mathrm{E}+02$ & $4.86 \mathrm{E}+03$ & $6.60 \mathrm{E}+00$ & $1.40 \mathrm{E}+02$ & N/A & $6.28 \mathrm{E}+00$ & $3.12 \mathrm{E}+03$ \\
\hline $\mathrm{Ce}$ & $3.15 \mathrm{E}+02$ & $3.96 \mathrm{E}+03$ & N/A & $9.50 \mathrm{E}+00$ & $2.50 \mathrm{E}+01$ & $6.16 \mathrm{E}-01$ & $1.01 \mathrm{E}+03$ \\
\hline $\mathrm{Cf}$ & N/A & N/A & $2.50 \mathrm{E}+01$ & N/A & N/A & N/A & N/A \\
\hline $\mathrm{Cl}$ & $5.46 \mathrm{E}+01$ & $5.07 \mathrm{E}+01$ & N/A & $2.20 \mathrm{E}+00$ & $4.00 \mathrm{E}+01$ & $1.09 \mathrm{E}+01$ & $1.46 \mathrm{E}+02$ \\
\hline $\mathrm{Cm}$ & N/A & N/A & $2.50 \mathrm{E}+02$ & N/A & N/A & N/A & N/A \\
\hline $\mathrm{Co}$ & $1.11 \mathrm{E}+02$ & $1.20 \mathrm{E}+02$ & N/A & $2.40 \mathrm{E}+00$ & $7.60 \mathrm{E}+01$ & $1.80 \mathrm{E}+01$ & $3.21 \mathrm{E}+02$ \\
\hline $\mathrm{Cr}$ & $5.09 \mathrm{E}+01$ & $3.99 \mathrm{E}+01$ & $\mathrm{~N} / \mathrm{A}$ & $2.00 \mathrm{E}+00$ & $4.00 \mathrm{E}+01$ & $1.28 \mathrm{E}+01$ & $1.25 \mathrm{E}+02$ \\
\hline Cs & $3.67 \mathrm{E}+03$ & $3.94 \mathrm{E}+03$ & N/A & $2.40 \mathrm{E}+00$ & $2.50 \mathrm{E}+03$ & $5.92 \mathrm{E}+02$ & $1.06 \mathrm{E}+04$ \\
\hline $\mathrm{Cu}$ & $2.65 \mathrm{E}+02$ & $1.51 \mathrm{E}+02$ & N/A & $1.70 \mathrm{E}+00$ & $2.30 \mathrm{E}+02$ & $9.61 \mathrm{E}+01$ & $5.51 \mathrm{E}+02$ \\
\hline Dy & $6.50 \mathrm{E}+02$ & N/A & $\mathrm{N} / \mathrm{A}$ & N/A & N/A & N/A & N/A \\
\hline $\mathrm{Eu}$ & $4.60 \mathrm{E}+02$ & $1.56 \mathrm{E}+03$ & N/A & $4.90 \mathrm{E}+00$ & $1.30 \mathrm{E}+02$ & $9.52 \mathrm{E}+00$ & $1.78 \mathrm{E}+03$ \\
\hline $\mathrm{F}$ & N/A & N/A & $1.00 \mathrm{E}+01$ & N/A & N/A & N/A & N/A \\
\hline $\mathrm{Fe}$ & $1.10 \mathrm{E}+03$ & $7.01 \mathrm{E}+03$ & N/A & $6.90 \mathrm{E}+00$ & $1.70 \mathrm{E}+02$ & $7.09 \mathrm{E}+00$ & $4.08 \mathrm{E}+03$ \\
\hline $\mathrm{Gd}$ & N/A & N/A & $2.50 \mathrm{E}+01$ & N/A & N/A & N/A & N/A \\
\hline $\mathrm{Ge}$ & $1.50 \mathrm{E}+00$ & N/A & N/A & N/A & N/A & N/A & N/A \\
\hline $\mathrm{Hf}$ & $1.35 \mathrm{E}+03$ & $9.65 \mathrm{E}+02$ & N/A & $1.90 \mathrm{E}+00$ & $1.10 \mathrm{E}+03$ & $3.83 \mathrm{E}+02$ & $3.16 \mathrm{E}+03$ \\
\hline $\mathrm{Hg}$ & $7.50 \mathrm{E}+03$ & $5.35 \mathrm{E}+03$ & N/A & $1.90 \mathrm{E}+00$ & $6.10 \mathrm{E}+03$ & $2.12 \mathrm{E}+03$ & $1.75 \mathrm{E}+04$ \\
\hline Ho & N/A & N/A & $2.50 \mathrm{E}+01$ & N/A & N/A & N/A & N/A \\
\hline I & $4.56 \mathrm{E}+01$ & $5.24 \mathrm{E}+01$ & N/A & $2.50 \mathrm{E}+00$ & $3.00 \mathrm{E}+01$ & $6.65 \mathrm{E}+00$ & $1.35 \mathrm{E}+02$ \\
\hline In & N/A & N/A & $1.00 \mathrm{E}+05$ & N/A & N/A & N/A & N/A \\
\hline $\mathrm{Ir}$ & N/A & N/A & $1.00 \mathrm{E}+01$ & N/A & N/A & N/A & N/A \\
\hline K & $3.57 \mathrm{E}+03$ & $1.78 \mathrm{E}+03$ & N/A & $1.60 \mathrm{E}+00$ & $3.20 \mathrm{E}+03$ & $1.48 \mathrm{E}+03$ & $6.93 \mathrm{E}+03$ \\
\hline $\mathrm{La}$ & $1.31 \mathrm{E}+02$ & $4.44 \mathrm{E}+02$ & $\mathrm{~N} / \mathrm{A}$ & $4.90 \mathrm{E}+00$ & $3.70 \mathrm{E}+01$ & $2.71 \mathrm{E}+00$ & $5.05 \mathrm{E}+02$ \\
\hline $\mathrm{Mg}$ & $5.05 \mathrm{E}+01$ & $4.69 \mathrm{E}+01$ & N/A & $2.20 \mathrm{E}+00$ & $3.70 \mathrm{E}+01$ & $1.01 \mathrm{E}+01$ & $1.35 \mathrm{E}+02$ \\
\hline $\mathrm{Mn}$ & $1.47 \mathrm{E}+03$ & $8.82 \mathrm{E}+03$ & N/A & $6.70 \mathrm{E}+00$ & $2.40 \mathrm{E}+02$ & $1.05 \mathrm{E}+01$ & $5.48 \mathrm{E}+03$ \\
\hline
\end{tabular}


Table 2.2. (contd)

\begin{tabular}{|c|c|c|c|c|c|c|c|}
\hline Element & Mean & STD & Median & GSD & GM & $5 \%$ & $95 \%$ \\
\hline Mo & $2.50 \mathrm{E}+00$ & $2.14 \mathrm{E}+00$ & $\mathrm{~N} / \mathrm{A}$ & $2.10 \mathrm{E}+00$ & $1.90 \mathrm{E}+00$ & $5.61 \mathrm{E}-01$ & $6.44 \mathrm{E}+00$ \\
\hline $\mathrm{N}$ & N/A & N/A & $1.50 \mathrm{E}+05$ & N/A & N/A & N/A & N/A \\
\hline $\mathrm{Na}$ & $1.39 \mathrm{E}+02$ & $2.13 \mathrm{E}+02$ & N/A & $3.00 \mathrm{E}+00$ & $7.60 \mathrm{E}+01$ & $1.25 \mathrm{E}+01$ & $4.63 \mathrm{E}+02$ \\
\hline $\mathrm{Nb}$ & $4.42 \mathrm{E}+01$ & $5.73 \mathrm{E}+01$ & $2.70 \mathrm{E}+00$ & $2.70 \mathrm{E}+01$ & N/A & $5.27 \mathrm{E}+00$ & $1.38 \mathrm{E}+02$ \\
\hline $\mathrm{Nd}$ & $4.30 \mathrm{E}+01$ & $3.91 \mathrm{E}+02$ & $8.20 \mathrm{E}+00$ & $4.70 \mathrm{E}+00$ & N/A & $1.48 \mathrm{E}-01$ & $1.50 \mathrm{E}+02$ \\
\hline $\mathrm{Ni}$ & $2.58 \mathrm{E}+01$ & $1.84 \mathrm{E}+01$ & N/A & $1.90 \mathrm{E}+00$ & $2.10 \mathrm{E}+01$ & $7.31 \mathrm{E}+00$ & $6.04 \mathrm{E}+01$ \\
\hline Os & $\mathrm{N} / \mathrm{A}$ & N/A & $1.00 \mathrm{E}+01$ & N/A & $\mathrm{N} / \mathrm{A}$ & N/A & $\mathrm{N} / \mathrm{A}$ \\
\hline $\mathrm{P}$ & $1.41 \mathrm{E}+05$ & $1.34 \mathrm{E}+04$ & N/A & $1.10 \mathrm{E}+00$ & $1.40 \mathrm{E}+05$ & $1.20 \mathrm{E}+05$ & $1.64 \mathrm{E}+05$ \\
\hline $\mathrm{Pa}$ & N/A & N/A & $1.10 \mathrm{E}+01$ & N/A & N/A & N/A & N/A \\
\hline $\mathrm{Pb}$ & $4.41 \mathrm{E}+01$ & $6.40 \mathrm{E}+01$ & N/A & $2.90 \mathrm{E}+00$ & $2.50 \mathrm{E}+01$ & $4.34 \mathrm{E}+00$ & $1.44 \mathrm{E}+02$ \\
\hline $\mathrm{Pd}$ & $7.13 \mathrm{E}+01$ & $1.09 \mathrm{E}+02$ & $3.00 \mathrm{E}+00$ & $3.90 \mathrm{E}+01$ & N/A & $6.40 \mathrm{E}+00$ & $2.38 \mathrm{E}+02$ \\
\hline $\mathrm{Pm}$ & N/A & N/A & $2.50 \mathrm{E}+01$ & N/A & N/A & N/A & N/A \\
\hline Po & $1.04 \mathrm{E}+02$ & $2.84 \mathrm{E}+02$ & N/A & $4.30 \mathrm{E}+00$ & $3.60 \mathrm{E}+01$ & $3.27 \mathrm{E}+00$ & $3.97 \mathrm{E}+02$ \\
\hline $\operatorname{Pr}$ & N/A & N/A & $2.50 \mathrm{E}+01$ & N/A & N/A & N/A & N/A \\
\hline $\mathrm{Pu}$ & $3.31 \mathrm{E}+04$ & $4.05 \mathrm{E}+04$ & N/A & $2.60 \mathrm{E}+00$ & $2.10 \mathrm{E}+04$ & $4.36 \mathrm{E}+03$ & $1.01 \mathrm{E}+05$ \\
\hline $\mathrm{Ra}$ & $2.58 \mathrm{E}+01$ & $1.65 \mathrm{E}+02$ & N/A & $6.90 \mathrm{E}+00$ & $4.00 \mathrm{E}+00$ & $1.67 \mathrm{E}-01$ & $9.59 \mathrm{E}+01$ \\
\hline $\mathrm{Rb}$ & $4.60 \mathrm{E}+03$ & $2.63 \mathrm{E}+03$ & $\mathrm{~N} / \mathrm{A}$ & $1.70 \mathrm{E}+00$ & $4.00 \mathrm{E}+03$ & $1.67 \mathrm{E}+03$ & $9.58 \mathrm{E}+03$ \\
\hline $\operatorname{Re}$ & N/A & N/A & $1.20 \mathrm{E}+02$ & N/A & N/A & N/A & N/A \\
\hline $\mathrm{Rh}$ & N/A & N/A & $1.00 \mathrm{E}+01$ & $\mathrm{~N} / \mathrm{A}$ & N/A & N/A & N/A \\
\hline $\mathrm{Ru}$ & N/A & N/A & $1.00 \mathrm{E}+02$ & N/A & $\mathrm{N} / \mathrm{A}$ & N/A & N/A \\
\hline $\mathrm{Ru}$ & $5.50 \mathrm{E}+01$ & N/A & $\mathrm{N} / \mathrm{A}$ & $\mathrm{N} / \mathrm{A}$ & N/A & N/A & N/A \\
\hline $\mathrm{S}$ & $3.50 \mathrm{E}+00$ & N/A & N/A & N/A & N/A & N/A & N/A \\
\hline $\mathrm{Sb}$ & $1.15 \mathrm{E}+02$ & $3.36 \mathrm{E}+02$ & N/A & $4.50 \mathrm{E}+00$ & $3.70 \mathrm{E}+01$ & $3.12 \mathrm{E}+00$ & $4.39 \mathrm{E}+02$ \\
\hline $\mathrm{Sc}$ & $2.50 \mathrm{E}+02$ & $2.14 \mathrm{E}+02$ & N/A & $2.10 \mathrm{E}+00$ & $1.90 \mathrm{E}+02$ & $5.61 \mathrm{E}+01$ & $6.44 \mathrm{E}+02$ \\
\hline $\mathrm{Se}$ & $6.21 \mathrm{E}+03$ & $1.66 \mathrm{E}+03$ & N/A & $1.30 \mathrm{E}+00$ & $6.00 \mathrm{E}+03$ & $3.90 \mathrm{E}+03$ & $9.24 \mathrm{E}+03$ \\
\hline $\mathrm{Sm}$ & N/A & N/A & $2.50 \mathrm{E}+01$ & N/A & N/A & N/A & $\mathrm{N} / \mathrm{A}$ \\
\hline $\mathrm{Sn}$ & N/A & N/A & $3.00 \mathrm{E}+03$ & N/A & N/A & N/A & N/A \\
\hline $\mathrm{Sr}$ & $7.32 \mathrm{E}+00$ & $1.70 \mathrm{E}+01$ & N/A & $3.90 \mathrm{E}+00$ & $2.90 \mathrm{E}+00$ & $3.09 \mathrm{E}-01$ & $2.72 \mathrm{E}+01$ \\
\hline $\mathrm{Tb}$ & $5.04 \mathrm{E}+02$ & $3.60 \mathrm{E}+02$ & N/A & $1.90 \mathrm{E}+00$ & $4.10 \mathrm{E}+02$ & $1.43 \mathrm{E}+02$ & $1.18 \mathrm{E}+03$ \\
\hline $\mathrm{Tc}$ & N/A & N/A & $1.50 \mathrm{E}+01$ & N/A & N/A & N/A & $\mathrm{N} / \mathrm{A}$ \\
\hline $\mathrm{Te}$ & $1.63 \mathrm{E}+02$ & $6.88 \mathrm{E}+01$ & N/A & $1.50 \mathrm{E}+00$ & $1.50 \mathrm{E}+02$ & $7.70 \mathrm{E}+01$ & $2.92 \mathrm{E}+02$ \\
\hline Th & N/A & N/A & N/A & N/A & $6.00 \mathrm{E}+00$ & N/A & $\mathrm{N} / \mathrm{A}$ \\
\hline $\mathrm{Ti}$ & $2.01 \mathrm{E}+02$ & $6.96 \mathrm{E}+01$ & N/A & $1.40 \mathrm{E}+00$ & $1.90 \mathrm{E}+02$ & $1.09 \mathrm{E}+02$ & $3.30 \mathrm{E}+02$ \\
\hline $\mathrm{Tl}$ & $1.42 \mathrm{E}+03$ & $1.74 \mathrm{E}+03$ & N/A & $2.60 \mathrm{E}+00$ & $9.00 \mathrm{E}+02$ & $1.87 \mathrm{E}+02$ & $4.33 \mathrm{E}+03$ \\
\hline $\mathrm{U}$ & $9.76 \mathrm{E}-01$ & $1.79 \mathrm{E}-01$ & N/A & $1.20 \mathrm{E}+00$ & $9.60 \mathrm{E}-01$ & $7.11 \mathrm{E}-01$ & $1.30 \mathrm{E}+00$ \\
\hline V & $1.19 \mathrm{E}+02$ & $8.51 \mathrm{E}+01$ & N/A & $1.90 \mathrm{E}+00$ & $9.70 \mathrm{E}+01$ & $3.37 \mathrm{E}+01$ & $2.79 \mathrm{E}+02$ \\
\hline W & N/A & N/A & $1.20 \mathrm{E}+03$ & N/A & N/A & N/A & $\mathrm{N} / \mathrm{A}$ \\
\hline $\mathrm{Y}$ & $6.09 \mathrm{E}+01$ & $6.98 \mathrm{E}+01$ & N/A & $2.50 \mathrm{E}+00$ & $4.00 \mathrm{E}+01$ & $8.86 \mathrm{E}+00$ & $1.81 \mathrm{E}+02$ \\
\hline $\mathrm{Zn}$ & $5.99 \mathrm{E}+03$ & $8.70 \mathrm{E}+03$ & N/A & $2.90 \mathrm{E}+00$ & $3.40 \mathrm{E}+03$ & $5.90 \mathrm{E}+02$ & $1.96 \mathrm{E}+04$ \\
\hline $\mathrm{Zr}$ & $3.23 \mathrm{E}+01$ & $3.46 \mathrm{E}+01$ & N/A & $2.40 \mathrm{E}+00$ & $2.20 \mathrm{E}+01$ & $5.21 \mathrm{E}+00$ & $9.29 \mathrm{E}+01$ \\
\hline $\begin{array}{l}\text { GM }= \\
\text { GSD }= \\
\text { N/A }= \\
\text { STD }=\end{array}$ & $\begin{array}{l}\text { Not available in } \\
\text { Standard Devia }\end{array}$ & reference & iewed. & & & & \\
\hline
\end{tabular}


Table 2.3. CLBFM Bioaccumulation Factors - Freshwater Mollusk

\begin{tabular}{|c|c|c|c|c|c|c|c|}
\hline Element & Mean & STD & Median & GSD & GM & $5 \%$ & $95 \%$ \\
\hline $\mathrm{Ag}$ & $2.30 \mathrm{E}+02$ & $1.40 \mathrm{E}+03$ & $3.73 \mathrm{E}+01$ & $6.74 \mathrm{E}+00$ & $3.73 \mathrm{E}+01$ & $1.62 \mathrm{E}+00$ & $8.60 \mathrm{E}+02$ \\
\hline $\mathrm{Al}$ & $3.40 \mathrm{E}+03$ & $4.00 \mathrm{E}+02$ & $3.38 \mathrm{E}+03$ & $1.12 \mathrm{E}+00$ & $3.38 \mathrm{E}+03$ & $2.78 \mathrm{E}+03$ & $4.10 \mathrm{E}+03$ \\
\hline $\mathrm{Am}$ & N/A & N/A & N/A & $7.00 \mathrm{E}+00$ & $2.40 \mathrm{E}+03$ & $9.77 \mathrm{E}+01$ & $5.89 \mathrm{E}+04$ \\
\hline As & $5.00 \mathrm{E}+01$ & N/A & N/A & N/A & N/A & N/A & N/A \\
\hline As & $1.50 \mathrm{E}+03$ & $7.10 \mathrm{E}+02$ & $1.36 \mathrm{E}+03$ & $1.57 \mathrm{E}+00$ & $1.36 \mathrm{E}+03$ & $6.47 \mathrm{E}+02$ & $2.84 \mathrm{E}+03$ \\
\hline $\mathrm{Au}$ & $1.40 \mathrm{E}+03$ & $3.50 \mathrm{E}+02$ & $1.36 \mathrm{E}+03$ & $1.28 \mathrm{E}+00$ & $1.36 \mathrm{E}+03$ & $9.06 \mathrm{E}+02$ & $2.04 \mathrm{E}+03$ \\
\hline $\mathrm{Ba}$ & $1.40 \mathrm{E}+02$ & $3.50 \mathrm{E}+00$ & $1.40 \mathrm{E}+02$ & $1.03 \mathrm{E}+00$ & $1.40 \mathrm{E}+02$ & $1.34 \mathrm{E}+02$ & $1.46 \mathrm{E}+02$ \\
\hline $\mathrm{Br}$ & $1.30 \mathrm{E}+03$ & $7.60 \mathrm{E}+02$ & $1.12 \mathrm{E}+03$ & $1.72 \mathrm{E}+00$ & $1.12 \mathrm{E}+03$ & $4.60 \mathrm{E}+02$ & $2.74 \mathrm{E}+03$ \\
\hline $\mathrm{C}$ & $5.17 \mathrm{E}+01$ & $5.93 \mathrm{E}+01$ & N/A & $2.50 \mathrm{E}+00$ & $3.40 \mathrm{E}+01$ & $7.53 \mathrm{E}+00$ & $1.53 \mathrm{E}+02$ \\
\hline $\mathrm{Ca}$ & N/A & N/A & $\mathrm{N} / \mathrm{A}$ & $3.90 \mathrm{E}+01$ & $1.00 \mathrm{E}+02$ & $2.41 \mathrm{E}-01$ & $4.14 \mathrm{E}+04$ \\
\hline $\mathrm{Cd}$ & N/A & N/A & N/A & $3.90 \mathrm{E}+01$ & $1.00 \mathrm{E}+02$ & $2.41 \mathrm{E}-01$ & $4.14 \mathrm{E}+04$ \\
\hline $\mathrm{Ce}$ & $2.30 \mathrm{E}+03$ & $6.00 \mathrm{E}+02$ & $2.23 \mathrm{E}+03$ & $1.29 \mathrm{E}+00$ & $2.23 \mathrm{E}+03$ & $1.46 \mathrm{E}+03$ & $3.39 \mathrm{E}+03$ \\
\hline $\mathrm{Cl}$ & $1.60 \mathrm{E}+02$ & $3.50 \mathrm{E}+01$ & $1.56 \mathrm{E}+02$ & $1.24 \mathrm{E}+00$ & $1.56 \mathrm{E}+02$ & $1.10 \mathrm{E}+02$ & $2.23 \mathrm{E}+02$ \\
\hline $\mathrm{Cm}$ & $9.50 \mathrm{E}+03$ & $7.10 \mathrm{E}+02$ & $9.47 \mathrm{E}+03$ & $1.08 \mathrm{E}+00$ & $9.47 \mathrm{E}+03$ & $8.38 \mathrm{E}+03$ & $1.07 \mathrm{E}+04$ \\
\hline Co & $2.50 \mathrm{E}+01$ & $6.00 \mathrm{E}+00$ & $2.43 \mathrm{E}+01$ & $1.27 \mathrm{E}+00$ & $2.43 \mathrm{E}+01$ & $1.65 \mathrm{E}+01$ & $3.59 \mathrm{E}+01$ \\
\hline $\mathrm{Cr}$ & $3.00 \mathrm{E}+01$ & $8.00 \mathrm{E}+00$ & $2.90 \mathrm{E}+01$ & $1.30 \mathrm{E}+00$ & $2.90 \mathrm{E}+01$ & $1.88 \mathrm{E}+01$ & $4.46 \mathrm{E}+01$ \\
\hline $\mathrm{Cr}$ & $3.00 \mathrm{E}+03$ & N/A & N/A & N/A & N/A & N/A & N/A \\
\hline Cs & $1.50 \mathrm{E}+01$ & $4.00 \mathrm{E}+00$ & $1.45 \mathrm{E}+01$ & $1.30 \mathrm{E}+00$ & $1.45 \mathrm{E}+01$ & $9.42 \mathrm{E}+00$ & $2.23 \mathrm{E}+01$ \\
\hline $\mathrm{Cu}$ & $6.00 \mathrm{E}+02$ & N/A & N/A & N/A & N/A & N/A & N/A \\
\hline $\mathrm{Eu}$ & $2.20 \mathrm{E}+02$ & $2.10 \mathrm{E}+01$ & $2.19 \mathrm{E}+02$ & $1.10 \mathrm{E}+00$ & $2.19 \mathrm{E}+02$ & $1.87 \mathrm{E}+02$ & $2.56 \mathrm{E}+02$ \\
\hline $\mathrm{Fe}$ & $7.30 \mathrm{E}+01$ & $1.80 \mathrm{E}+01$ & $7.09 \mathrm{E}+01$ & $1.27 \mathrm{E}+00$ & $7.09 \mathrm{E}+01$ & $4.75 \mathrm{E}+01$ & $1.06 \mathrm{E}+02$ \\
\hline $\mathrm{Hf}$ & $1.40 \mathrm{E}+03$ & $1.40 \mathrm{E}+02$ & $1.39 \mathrm{E}+03$ & $1.10 \mathrm{E}+00$ & $1.39 \mathrm{E}+03$ & $1.18 \mathrm{E}+03$ & $1.64 \mathrm{E}+03$ \\
\hline $\mathrm{Hg}$ & $1.23 \mathrm{E}+03$ & $1.59 \mathrm{E}+03$ & N/A & $2.70 \mathrm{E}+00$ & $7.50 \mathrm{E}+02$ & $1.46 \mathrm{E}+02$ & $3.84 \mathrm{E}+03$ \\
\hline I & $7.00 \mathrm{E}+00$ & $1.80 \mathrm{E}+00$ & $6.78 \mathrm{E}+00$ & $1.29 \mathrm{E}+00$ & $6.78 \mathrm{E}+00$ & $4.47 \mathrm{E}+00$ & $1.03 \mathrm{E}+01$ \\
\hline $\mathrm{K}$ & $5.80 \mathrm{E}+02$ & $5.00 \mathrm{E}+01$ & $5.78 \mathrm{E}+02$ & $1.09 \mathrm{E}+00$ & $5.78 \mathrm{E}+02$ & $5.02 \mathrm{E}+02$ & $6.66 \mathrm{E}+02$ \\
\hline $\mathrm{La}$ & $3.50 \mathrm{E}+02$ & $2.80 \mathrm{E}+01$ & $3.49 \mathrm{E}+02$ & $1.08 \mathrm{E}+00$ & $3.49 \mathrm{E}+02$ & $3.06 \mathrm{E}+02$ & $3.98 \mathrm{E}+02$ \\
\hline $\mathrm{Lu}$ & N/A & N/A & N/A & N/A & $1.10 \mathrm{E}+03$ & N/A & N/A \\
\hline $\mathrm{Mg}$ & $3.20 \mathrm{E}+01$ & $1.60 \mathrm{E}+00$ & $3.20 \mathrm{E}+01$ & $1.05 \mathrm{E}+00$ & $3.20 \mathrm{E}+01$ & $2.94 \mathrm{E}+01$ & $3.47 \mathrm{E}+01$ \\
\hline $\mathrm{Mn}$ & $1.30 \mathrm{E}+03$ & $3.00 \mathrm{E}+02$ & $1.27 \mathrm{E}+03$ & $1.26 \mathrm{E}+00$ & $1.27 \mathrm{E}+03$ & $8.71 \mathrm{E}+02$ & $1.84 \mathrm{E}+03$ \\
\hline Mo & $1.21 \mathrm{E}+01$ & $3.24 \mathrm{E}+02$ & N/A & $1.30 \mathrm{E}+01$ & 4.50E-01 & $6.62 \mathrm{E}-03$ & $3.06 \mathrm{E}+01$ \\
\hline $\mathrm{Na}$ & $2.00 \mathrm{E}+02$ & N/A & N/A & N/A & N/A & N/A & N/A \\
\hline $\mathrm{Np}$ & $9.50 \mathrm{E}+03$ & $1.10 \mathrm{E}+00$ & $9.50 \mathrm{E}+03$ & $1.00 \mathrm{E}+00$ & $9.50 \mathrm{E}+03$ & $9.50 \mathrm{E}+03$ & $9.50 \mathrm{E}+03$ \\
\hline $\mathrm{P}$ & $2.00 \mathrm{E}+04$ & N/A & N/A & N/A & N/A & N/A & N/A \\
\hline $\mathrm{Pb}$ & $1.96 \mathrm{E}+03$ & $1.74 \mathrm{E}+05$ & N/A & $2.00 \mathrm{E}+01$ & $2.20 \mathrm{E}+01$ & $1.59 \mathrm{E}-01$ & $3.04 \mathrm{E}+03$ \\
\hline Po & $7.20 \mathrm{E}+01$ & $1.80 \mathrm{E}+01$ & $6.99 \mathrm{E}+01$ & $1.28 \mathrm{E}+00$ & $6.99 \mathrm{E}+01$ & $4.66 \mathrm{E}+01$ & $1.05 \mathrm{E}+02$ \\
\hline $\mathrm{Pu}$ & N/A & N/A & N/A & $2.90 \mathrm{E}+01$ & $7.40 \mathrm{E}+03$ & $2.91 \mathrm{E}+01$ & $1.88 \mathrm{E}+06$ \\
\hline $\mathrm{Ra}$ & N/A & N/A & N/A & $3.00 \mathrm{E}+01$ & $1.00 \mathrm{E}+02$ & $3.72 \mathrm{E}-01$ & $2.69 \mathrm{E}+04$ \\
\hline $\mathrm{Rb}$ & $2.00 \mathrm{E}+03$ & $2.80 \mathrm{E}+02$ & $1.98 \mathrm{E}+03$ & $1.15 \mathrm{E}+00$ & $1.98 \mathrm{E}+03$ & $1.58 \mathrm{E}+03$ & $2.49 \mathrm{E}+03$ \\
\hline $\mathrm{Ru}$ & $1.20 \mathrm{E}+01$ & $3.00 \mathrm{E}+00$ & $1.16 \mathrm{E}+01$ & $1.28 \mathrm{E}+00$ & $1.16 \mathrm{E}+01$ & $7.76 \mathrm{E}+00$ & $1.75 \mathrm{E}+01$ \\
\hline $\mathrm{Sb}$ & $1.00 \mathrm{E}+01$ & $3.00 \mathrm{E}+00$ & $9.58 \mathrm{E}+00$ & $1.34 \mathrm{E}+00$ & $9.58 \mathrm{E}+00$ & $5.91 \mathrm{E}+00$ & $1.55 \mathrm{E}+01$ \\
\hline $\mathrm{Sc}$ & $3.50 \mathrm{E}+03$ & $2.80 \mathrm{E}+02$ & $3.49 \mathrm{E}+03$ & $1.08 \mathrm{E}+00$ & $3.49 \mathrm{E}+03$ & $3.06 \mathrm{E}+03$ & $3.98 \mathrm{E}+03$ \\
\hline $\mathrm{Se}$ & $2.23 \mathrm{E}+04$ & $8.72 \mathrm{E}+05$ & N/A & $1.50 \mathrm{E}+01$ & $5.70 \mathrm{E}+02$ & $6.63 \mathrm{E}+00$ & $4.90 \mathrm{E}+04$ \\
\hline $\mathrm{Sm}$ & $1.60 \mathrm{E}+03$ & $1.60 \mathrm{E}+03$ & $1.13 \mathrm{E}+03$ & $2.30 \mathrm{E}+00$ & $1.13 \mathrm{E}+03$ & $2.88 \mathrm{E}+02$ & $4.45 \mathrm{E}+03$ \\
\hline $\mathrm{Sr}$ & $2.40 \mathrm{E}+03$ & $6.00 \mathrm{E}+02$ & $2.33 \mathrm{E}+03$ & $1.28 \mathrm{E}+00$ & $2.33 \mathrm{E}+03$ & $1.55 \mathrm{E}+03$ & $3.49 \mathrm{E}+03$ \\
\hline
\end{tabular}


Table 2.3. (contd)

\begin{tabular}{cccccccc}
\hline Element & Mean & STD & Median & GSD & GM & $5 \%$ & $95 \%$ \\
\hline Tc & $3.60 \mathrm{E}+02$ & $4.97 \mathrm{E}+03$ & N/A & $9.90 \mathrm{E}+00$ & $2.60 \mathrm{E}+01$ & $5.99 \mathrm{E}-01$ & $1.13 \mathrm{E}+03$ \\
$\mathrm{Th}$ & $2.90 \mathrm{E}+03$ & $1.40 \mathrm{E}+01$ & $2.90 \mathrm{E}+03$ & $1.00 \mathrm{E}+00$ & $2.90 \mathrm{E}+03$ & $2.88 \mathrm{E}+03$ & $2.92 \mathrm{E}+03$ \\
$\mathrm{U}$ & $1.30 \mathrm{E}+04$ & $9.90 \mathrm{E}+05$ & N/A & $1.90 \mathrm{E}+01$ & $1.70 \mathrm{E}+02$ & $1.34 \mathrm{E}+00$ & $2.16 \mathrm{E}+04$ \\
$\mathrm{~V}$ & $3.80 \mathrm{E}+02$ & $2.80 \mathrm{E}+01$ & $3.79 \mathrm{E}+02$ & $1.08 \mathrm{E}+00$ & $3.79 \mathrm{E}+02$ & $3.36 \mathrm{E}+02$ & $4.28 \mathrm{E}+02$ \\
$\mathrm{Zn}$ & $2.00 \mathrm{E}+03$ & N/A & N/A & N/A & N/A & N/A & N/A \\
$\mathrm{Zr}$ & $1.50 \mathrm{E}+01$ & $4.00 \mathrm{E}+00$ & $1.45 \mathrm{E}+01$ & $1.30 \mathrm{E}+00$ & $1.45 \mathrm{E}+01$ & $9.42 \mathrm{E}+00$ & $2.23 \mathrm{E}+01$ \\
\hline GM $=$ Geometric mean. & & & & \\
GSD $=$ Geometric Standard Deviation. \\
N/A = Not available in the references reviewed. \\
STD = Standard Deviation.
\end{tabular}

Table 2.4. CLBFI Bioaccumulation Factor - Freshwater Crustacean

\begin{tabular}{|c|c|c|c|c|c|c|c|}
\hline Element & Mean & STD & Median & GSD & GM & $5 \%$ & $95 \%$ \\
\hline $\mathrm{Ag}$ & $2.30 \mathrm{E}+02$ & $1.40 \mathrm{E}+03$ & $3.73 \mathrm{E}+01$ & $6.74 \mathrm{E}+00$ & $3.73 \mathrm{E}+01$ & $1.62 \mathrm{E}+00$ & $8.60 \mathrm{E}+02$ \\
\hline $\mathrm{Al}$ & $3.40 \mathrm{E}+03$ & $4.00 \mathrm{E}+02$ & $3.38 \mathrm{E}+03$ & $1.12 \mathrm{E}+00$ & $3.38 \mathrm{E}+03$ & $2.78 \mathrm{E}+03$ & $4.10 \mathrm{E}+03$ \\
\hline $\mathrm{Am}$ & N/A & N/A & N/A & $7.00 \mathrm{E}+00$ & $2.40 \mathrm{E}+03$ & $9.77 \mathrm{E}+01$ & $5.89 \mathrm{E}+04$ \\
\hline As & $1.50 \mathrm{E}+03$ & $7.10 \mathrm{E}+02$ & $1.36 \mathrm{E}+03$ & $1.57 \mathrm{E}+00$ & $1.36 \mathrm{E}+03$ & $6.47 \mathrm{E}+02$ & $2.84 \mathrm{E}+03$ \\
\hline $\mathrm{Au}$ & $1.40 \mathrm{E}+03$ & $3.50 \mathrm{E}+02$ & $1.36 \mathrm{E}+03$ & $1.28 \mathrm{E}+00$ & $1.36 \mathrm{E}+03$ & $9.06 \mathrm{E}+02$ & $2.04 \mathrm{E}+03$ \\
\hline $\mathrm{Ba}$ & $1.40 \mathrm{E}+02$ & $3.50 \mathrm{E}+00$ & $1.40 \mathrm{E}+02$ & $1.03 \mathrm{E}+00$ & $1.40 \mathrm{E}+02$ & $1.34 \mathrm{E}+02$ & $1.46 \mathrm{E}+02$ \\
\hline $\mathrm{Br}$ & $1.30 \mathrm{E}+03$ & $7.60 \mathrm{E}+02$ & $1.12 \mathrm{E}+03$ & $1.72 \mathrm{E}+00$ & $1.12 \mathrm{E}+03$ & $4.60 \mathrm{E}+02$ & $2.74 \mathrm{E}+03$ \\
\hline $\mathrm{Ca}$ & $5.17 \mathrm{E}+01$ & $5.93 \mathrm{E}+01$ & N/A & $2.50 \mathrm{E}+00$ & $3.40 \mathrm{E}+01$ & $7.53 \mathrm{E}+00$ & $1.53 \mathrm{E}+02$ \\
\hline $\mathrm{Cd}$ & N/A & N/A & N/A & $3.90 \mathrm{E}+01$ & $1.00 \mathrm{E}+02$ & $2.41 \mathrm{E}-01$ & $4.14 \mathrm{E}+04$ \\
\hline $\mathrm{Ce}$ & $4.30 \mathrm{E}+02$ & $1.90 \mathrm{E}+02$ & $3.93 \mathrm{E}+02$ & $1.53 \mathrm{E}+00$ & $3.93 \mathrm{E}+02$ & $1.96 \mathrm{E}+02$ & $7.88 \mathrm{E}+02$ \\
\hline $\mathrm{Cl}$ & $1.60 \mathrm{E}+02$ & $3.50 \mathrm{E}+01$ & $1.56 \mathrm{E}+02$ & $1.24 \mathrm{E}+00$ & $1.56 \mathrm{E}+02$ & $1.10 \mathrm{E}+02$ & $2.23 \mathrm{E}+02$ \\
\hline $\mathrm{Cm}$ & $9.50 \mathrm{E}+03$ & $7.10 \mathrm{E}+02$ & $9.47 \mathrm{E}+03$ & $1.08 \mathrm{E}+00$ & $9.47 \mathrm{E}+03$ & $8.38 \mathrm{E}+03$ & $1.07 \mathrm{E}+04$ \\
\hline Co & N/A & N/A & N/A & $1.30 \mathrm{E}+02$ & $2.20 \mathrm{E}+01$ & 7.33E-03 & $6.60 \mathrm{E}+04$ \\
\hline $\mathrm{Cr}$ & $3.00 \mathrm{E}+02$ & $1.20 \mathrm{E}+02$ & $2.79 \mathrm{E}+02$ & $1.47 \mathrm{E}+00$ & $2.79 \mathrm{E}+02$ & $1.48 \mathrm{E}+02$ & $5.25 \mathrm{E}+02$ \\
\hline Cs & N/A & N/A & N/A & $7.50 \mathrm{E}+01$ & $2.30 \mathrm{E}+01$ & $1.89 \mathrm{E}-02$ & $2.79 \mathrm{E}+04$ \\
\hline $\mathrm{Cu}$ & $7.44 \mathrm{E}+02$ & $1.32 \mathrm{E}+04$ & N/A & $1.10 \mathrm{E}+01$ & $4.20 \mathrm{E}+01$ & $8.13 \mathrm{E}-01$ & $2.17 \mathrm{E}+03$ \\
\hline $\mathrm{Eu}$ & $2.20 \mathrm{E}+02$ & $2.10 \mathrm{E}+01$ & $2.19 \mathrm{E}+02$ & $1.10 \mathrm{E}+00$ & $2.19 \mathrm{E}+02$ & $1.87 \mathrm{E}+02$ & $2.56 \mathrm{E}+02$ \\
\hline $\mathrm{Fe}$ & $2.00 \mathrm{E}+03$ & $2.10 \mathrm{E}+02$ & $1.99 \mathrm{E}+03$ & $1.11 \mathrm{E}+00$ & $1.99 \mathrm{E}+03$ & $1.67 \mathrm{E}+03$ & $2.36 \mathrm{E}+03$ \\
\hline $\mathrm{Hf}$ & $1.40 \mathrm{E}+03$ & $1.40 \mathrm{E}+02$ & $1.39 \mathrm{E}+03$ & $1.10 \mathrm{E}+00$ & $1.39 \mathrm{E}+03$ & $1.18 \mathrm{E}+03$ & $1.64 \mathrm{E}+03$ \\
\hline $\mathrm{Hg}$ & $1.23 \mathrm{E}+03$ & $1.59 \mathrm{E}+03$ & N/A & $2.70 \mathrm{E}+00$ & $7.50 \mathrm{E}+02$ & $1.46 \mathrm{E}+02$ & $3.84 \mathrm{E}+03$ \\
\hline I & $3.01 \mathrm{E}+02$ & $5.33 \mathrm{E}+03$ & N/A & $1.10 \mathrm{E}+01$ & $1.70 \mathrm{E}+01$ & $3.29 \mathrm{E}-01$ & $8.78 \mathrm{E}+02$ \\
\hline $\mathrm{K}$ & $5.80 \mathrm{E}+02$ & $5.00 \mathrm{E}+01$ & $5.78 \mathrm{E}+02$ & $1.09 \mathrm{E}+00$ & $5.78 \mathrm{E}+02$ & $5.02 \mathrm{E}+02$ & $6.66 \mathrm{E}+02$ \\
\hline $\mathrm{La}$ & $3.50 \mathrm{E}+02$ & $2.80 \mathrm{E}+01$ & $3.49 \mathrm{E}+02$ & $1.08 \mathrm{E}+00$ & $3.49 \mathrm{E}+02$ & $3.06 \mathrm{E}+02$ & $3.98 \mathrm{E}+02$ \\
\hline $\mathrm{Lu}$ & N/A & N/A & N/A & N/A & $1.10 \mathrm{E}+03$ & N/A & N/A \\
\hline $\mathrm{Mg}$ & $3.20 \mathrm{E}+01$ & $1.60 \mathrm{E}+00$ & $3.20 \mathrm{E}+01$ & $1.05 \mathrm{E}+00$ & $3.20 \mathrm{E}+01$ & $2.94 \mathrm{E}+01$ & $3.47 \mathrm{E}+01$ \\
\hline $\mathrm{Mn}$ & N/A & N/A & N/A & $3.90 \mathrm{E}+02$ & $2.10 \mathrm{E}+01$ & $1.15 \mathrm{E}-03$ & $3.84 \mathrm{E}+05$ \\
\hline Mo & $1.21 \mathrm{E}+01$ & $3.24 \mathrm{E}+02$ & $\mathrm{~N} / \mathrm{A}$ & $1.30 \mathrm{E}+01$ & $4.50 \mathrm{E}-01$ & $6.62 \mathrm{E}-03$ & $3.06 \mathrm{E}+01$ \\
\hline $\mathrm{Na}$ & N/A & N/A & N/A & $3.60 \mathrm{E}+01$ & $3.40 \mathrm{E}+00$ & $9.36 \mathrm{E}-03$ & $1.23 \mathrm{E}+03$ \\
\hline $\mathrm{Np}$ & $9.50 \mathrm{E}+03$ & $1.10 \mathrm{E}+00$ & $9.50 \mathrm{E}+03$ & $1.00 \mathrm{E}+00$ & $9.50 \mathrm{E}+03$ & $9.50 \mathrm{E}+03$ & $9.50 \mathrm{E}+03$ \\
\hline $\mathrm{P}$ & $1.30 \mathrm{E}+04$ & N/A & N/A & N/A & N/A & N/A & N/A \\
\hline $\mathrm{Pb}$ & $1.96 \mathrm{E}+03$ & $1.74 \mathrm{E}+05$ & N/A & $2.00 \mathrm{E}+01$ & $2.20 \mathrm{E}+01$ & $1.59 \mathrm{E}-01$ & $3.04 \mathrm{E}+03$ \\
\hline Po & $9.90 \mathrm{E}+03$ & $1.40 \mathrm{E}+03$ & $9.80 \mathrm{E}+03$ & $1.15 \mathrm{E}+00$ & $9.80 \mathrm{E}+03$ & $7.78 \mathrm{E}+03$ & $1.24 \mathrm{E}+04$ \\
\hline
\end{tabular}


Table 2.4. (contd)

\begin{tabular}{cccccccc}
\hline Element & Mean & STD & Median & GSD & GM & $5 \%$ & $95 \%$ \\
\hline $\mathrm{Pu}$ & N/A & N/A & N/A & $2.90 \mathrm{E}+01$ & $7.40 \mathrm{E}+03$ & $2.91 \mathrm{E}+01$ & $1.88 \mathrm{E}+06$ \\
$\mathrm{Ra}$ & N/A & N/A & N/A & $3.00 \mathrm{E}+01$ & $1.00 \mathrm{E}+02$ & $3.72 \mathrm{E}-01$ & $2.69 \mathrm{E}+04$ \\
$\mathrm{Rb}$ & $2.00 \mathrm{E}+03$ & $2.80 \mathrm{E}+02$ & $1.98 \mathrm{E}+03$ & $1.15 \mathrm{E}+00$ & $1.98 \mathrm{E}+03$ & $1.58 \mathrm{E}+03$ & $2.49 \mathrm{E}+03$ \\
$\mathrm{Ru}$ & $4.02 \mathrm{E}+00$ & $4.14 \mathrm{E}+02$ & N/A & $2.10 \mathrm{E}+01$ & $3.90 \mathrm{E}-02$ & $2.61 \mathrm{E}-04$ & $5.84 \mathrm{E}+00$ \\
$\mathrm{~S}$ & $2.00 \mathrm{E}+01$ & N/A & N/A & N/A & N/A & N/A & N/A \\
$\mathrm{Sb}$ & $2.10 \mathrm{E}+02$ & $1.90 \mathrm{E}+02$ & $1.56 \mathrm{E}+02$ & $2.17 \mathrm{E}+00$ & $1.56 \mathrm{E}+02$ & $4.36 \mathrm{E}+01$ & $5.56 \mathrm{E}+02$ \\
$\mathrm{Sc}$ & $3.50 \mathrm{E}+03$ & $2.80 \mathrm{E}+02$ & $3.49 \mathrm{E}+03$ & $1.08 \mathrm{E}+00$ & $3.49 \mathrm{E}+03$ & $3.06 \mathrm{E}+03$ & $3.98 \mathrm{E}+03$ \\
$\mathrm{Se}$ & $2.23 \mathrm{E}+04$ & $8.72 \mathrm{E}+05$ & N/A & $1.50 \mathrm{E}+01$ & $5.70 \mathrm{E}+02$ & $6.63 \mathrm{E}+00$ & $4.90 \mathrm{E}+04$ \\
$\mathrm{Sm}$ & $1.60 \mathrm{E}+03$ & $1.60 \mathrm{E}+03$ & $1.13 \mathrm{E}+03$ & $2.30 \mathrm{E}+00$ & $1.13 \mathrm{E}+03$ & $2.88 \mathrm{E}+02$ & $4.45 \mathrm{E}+03$ \\
$\mathrm{Sr}$ & $5.31 \mathrm{E}+02$ & $8.99 \mathrm{E}+02$ & N/A & $3.20 \mathrm{E}+00$ & $2.70 \mathrm{E}+02$ & $3.98 \mathrm{E}+01$ & $1.83 \mathrm{E}+03$ \\
$\mathrm{Tc}$ & $3.60 \mathrm{E}+02$ & $4.97 \mathrm{E}+03$ & N/A & $9.90 \mathrm{E}+00$ & $2.60 \mathrm{E}+01$ & $5.99 \mathrm{E}-01$ & $1.13 \mathrm{E}+03$ \\
$\mathrm{Th}$ & $2.90 \mathrm{E}+03$ & $1.40 \mathrm{E}+01$ & $2.90 \mathrm{E}+03$ & $1.00 \mathrm{E}+00$ & $2.90 \mathrm{E}+03$ & $2.88 \mathrm{E}+03$ & $2.92 \mathrm{E}+03$ \\
$\mathrm{U}$ & $1.30 \mathrm{E}+04$ & $9.90 \mathrm{E}+05$ & N/A & $1.90 \mathrm{E}+01$ & $1.70 \mathrm{E}+02$ & $1.34 \mathrm{E}+00$ & $2.16 \mathrm{E}+04$ \\
$\mathrm{~V}$ & $3.80 \mathrm{E}+02$ & $2.80 \mathrm{E}+01$ & $3.79 \mathrm{E}+02$ & $1.08 \mathrm{E}+00$ & $3.79 \mathrm{E}+02$ & $3.36 \mathrm{E}+02$ & $4.28 \mathrm{E}+02$ \\
$\mathrm{Zn}$ & $2.67 \mathrm{E}+04$ & $7.73 \mathrm{E}+06$ & N/A & $2.90 \mathrm{E}+01$ & $9.20 \mathrm{E}+01$ & $3.62 \mathrm{E}-01$ & $2.34 \mathrm{E}+04$ \\
$\mathrm{Zr}$ & $2.60 \mathrm{E}+02$ & N/A & N/A & N/A & N/A & N/A & N/A \\
\hline GM $=$ Geometric mean. & & & & \\
$\mathrm{GSD}=$ Geometric Standard Deviation. & & & & & \\
$\mathrm{N} / \mathrm{A}=$ Not available in the references reviewed. & & & & \\
$\mathrm{STD}=$ Standard Deviation.
\end{tabular}

Table 2.5. CLBMF Bioaccumulation Factor - Marine Fish

\begin{tabular}{cccccccc}
\hline Element & Mean & SDT & Median & GSD & GM & $5 \%$ & $95 \%$ \\
\hline $\mathrm{Ac}$ & N/A & N/A & $5.00 \mathrm{E}+01$ & N/A & N/A & N/A & N/A \\
$\mathrm{Ag}$ & $4.20 \mathrm{E}+03$ & $4.10 \mathrm{E}+03$ & $3.01 \mathrm{E}+03$ & $2.27 \mathrm{E}+00$ & $3.01 \mathrm{E}+03$ & $7.82 \mathrm{E}+02$ & $1.15 \mathrm{E}+04$ \\
$\mathrm{Am}$ & $5.80 \mathrm{E}+01$ & $7.10 \mathrm{E}+01$ & $3.67 \mathrm{E}+01$ & $2.60 \mathrm{E}+00$ & $3.67 \mathrm{E}+01$ & $7.60 \mathrm{E}+00$ & $1.77 \mathrm{E}+02$ \\
$\mathrm{Am}$ & N/A & N/A & $1.00 \mathrm{E}+01$ & N/A & N/A & N/A & N/A \\
$\mathrm{Ba}$ & N/A & N/A & $1.00 \mathrm{E}+01$ & N/A & N/A & N/A & N/A \\
$\mathrm{Bk}$ & N/A & N/A & $1.00 \mathrm{E}+02$ & N/A & N/A & N/A & N/A \\
$\mathrm{Br}$ & N/A & N/A & $1.00 \mathrm{E}+01$ & N/A & N/A & N/A & N/A \\
$\mathrm{Ca}$ & N/A & N/A & $2.00 \mathrm{E}+00$ & N/A & N/A & N/A & N/A \\
$\mathrm{Cd}$ & $9.60 \mathrm{E}+03$ & $2.00 \mathrm{E}+04$ & $4.15 \mathrm{E}+03$ & $3.65 \mathrm{E}+00$ & $4.15 \mathrm{E}+03$ & $4.94 \mathrm{E}+02$ & $3.49 \mathrm{E}+04$ \\
$\mathrm{Ce}$ & $1.20 \mathrm{E}+02$ & $2.40 \mathrm{E}+02$ & $5.37 \mathrm{E}+01$ & $3.56 \mathrm{E}+00$ & $5.37 \mathrm{E}+01$ & $6.66 \mathrm{E}+00$ & $4.33 \mathrm{E}+02$ \\
$\mathrm{Cf}$ & N/A & N/A & $1.00 \mathrm{E}+02$ & N/A & N/A & N/A & N/A \\
$\mathrm{Cl}$ & N/A & N/A & $6.00 \mathrm{E}-02$ & N/A & N/A & N/A & N/A \\
$\mathrm{Cm}$ & N/A & N/A & $1.00 \mathrm{E}+02$ & N/A & N/A & N/A & N/A \\
$\mathrm{Co}$ & $5.30 \mathrm{E}+03$ & $1.50 \mathrm{E}+04$ & $1.77 \mathrm{E}+03$ & $4.40 \mathrm{E}+00$ & $1.77 \mathrm{E}+03$ & $1.54 \mathrm{E}+02$ & $2.02 \mathrm{E}+04$ \\
$\mathrm{Cs}$ & $8.70 \mathrm{E}+01$ & $1.20 \mathrm{E}+02$ & $5.11 \mathrm{E}+01$ & $2.81 \mathrm{E}+00$ & $5.11 \mathrm{E}+01$ & $9.35 \mathrm{E}+00$ & $2.79 \mathrm{E}+02$ \\
$\mathrm{Dy}$ & N/A & N/A & $3.00 \mathrm{E}+02$ & N/A & N/A & N/A & N/A \\
$\mathrm{Eu}$ & $4.40 \mathrm{E}+02$ & $3.00 \mathrm{E}+02$ & $3.64 \mathrm{E}+02$ & $1.85 \mathrm{E}+00$ & $3.64 \mathrm{E}+02$ & $1.32 \mathrm{E}+02$ & $1.00 \mathrm{E}+03$ \\
$\mathrm{Fe}$ & N/A & N/A & $3.00 \mathrm{E}+04$ & N/A & N/A & N/A & N/A \\
$\mathrm{Gd}$ & N/A & N/A & $3.00 \mathrm{E}+02$ & N/A & N/A & N/A & N/A \\
$\mathrm{Hf}$ & N/A & N/A & $5.00 \mathrm{E}+02$ & N/A & N/A & N/A & N/A \\
$\mathrm{Hg}$ & N/A & N/A & $3.00 \mathrm{E}+04$ & N/A & N/A & N/A & N/A \\
$\mathrm{I}$ & N/A & N/A & $9.00 \mathrm{E}+00$ & N/A & N/A & N/A & N/A \\
& & & & & & &
\end{tabular}


Table 2.5. (contd)

\begin{tabular}{|c|c|c|c|c|c|c|c|}
\hline Element & Mean & SDT & Median & GSD & GM & $5 \%$ & $95 \%$ \\
\hline In & $\mathrm{N} / \mathrm{A}$ & $\mathrm{N} / \mathrm{A}$ & $5.00 \mathrm{E}+02$ & $\mathrm{~N} / \mathrm{A}$ & $\mathrm{N} / \mathrm{A}$ & $\mathrm{N} / \mathrm{A}$ & $\mathrm{N} / \mathrm{A}$ \\
\hline $\mathrm{Ir}$ & N/A & N/A & $2.00 \mathrm{E}+01$ & N/A & N/A & N/A & N/A \\
\hline $\mathrm{Mn}$ & $2.40 \mathrm{E}+03$ & $1.50 \mathrm{E}+04$ & $3.79 \mathrm{E}+02$ & $6.83 \mathrm{E}+00$ & $3.79 \mathrm{E}+02$ & $1.61 \mathrm{E}+01$ & $8.94 \mathrm{E}+03$ \\
\hline $\mathrm{Na}$ & N/A & N/A & $1.00 \mathrm{E}+00$ & N/A & N/A & N/A & $\mathrm{N} / \mathrm{A}$ \\
\hline $\mathrm{Nb}$ & N/A & N/A & $3.00 \mathrm{E}+01$ & N/A & $\mathrm{N} / \mathrm{A}$ & $\mathrm{N} / \mathrm{A}$ & N/A \\
\hline $\mathrm{Ni}$ & $1.70 \mathrm{E}+02$ & $2.20 \mathrm{E}+02$ & $1.04 \mathrm{E}+02$ & $2.70 \mathrm{E}+00$ & $1.04 \mathrm{E}+02$ & $2.03 \mathrm{E}+01$ & $5.31 \mathrm{E}+02$ \\
\hline $\mathrm{Np}$ & N/A & N/A & $1.00 \mathrm{E}+00$ & N/A & N/A & N/A & $\mathrm{N} / \mathrm{A}$ \\
\hline $\mathrm{P}$ & $9.90 \mathrm{E}+04$ & $3.00 \mathrm{E}+04$ & $9.47 \mathrm{E}+04$ & $1.35 \mathrm{E}+00$ & $9.47 \mathrm{E}+04$ & $5.82 \mathrm{E}+04$ & $1.54 \mathrm{E}+05$ \\
\hline $\mathrm{Pa}$ & N/A & N/A & $5.00 \mathrm{E}+01$ & N/A & N/A & N/A & N/A \\
\hline $\mathrm{Pb}$ & $4.40 \mathrm{E}+03$ & $1.40 \mathrm{E}+04$ & $1.32 \mathrm{E}+03$ & $4.72 \mathrm{E}+00$ & $1.32 \mathrm{E}+03$ & $1.03 \mathrm{E}+02$ & $1.70 \mathrm{E}+04$ \\
\hline $\mathrm{Pd}$ & N/A & N/A & $3.00 \mathrm{E}+02$ & $\mathrm{~N} / \mathrm{A}$ & N/A & $\mathrm{N} / \mathrm{A}$ & N/A \\
\hline $\mathrm{Pm}$ & N/A & $\mathrm{N} / \mathrm{A}$ & $3.00 \mathrm{E}+02$ & N/A & $\mathrm{N} / \mathrm{A}$ & $\mathrm{N} / \mathrm{A}$ & N/A \\
\hline Po & $4.40 \mathrm{E}+04$ & $1.20 \mathrm{E}+05$ & $1.51 \mathrm{E}+04$ & $4.31 \mathrm{E}+00$ & $1.51 \mathrm{E}+04$ & $1.37 \mathrm{E}+03$ & $1.67 \mathrm{E}+05$ \\
\hline $\mathrm{Pu}$ & $1.60 \mathrm{E}+03$ & $6.40 \mathrm{E}+03$ & $3.88 \mathrm{E}+02$ & $5.38 \mathrm{E}+00$ & $3.88 \mathrm{E}+02$ & $2.43 \mathrm{E}+01$ & $6.19 \mathrm{E}+03$ \\
\hline $\mathrm{Ra}$ & $2.00 \mathrm{E}+02$ & $3.80 \mathrm{E}+02$ & $9.31 \mathrm{E}+01$ & $3.44 \mathrm{E}+00$ & $9.31 \mathrm{E}+01$ & $1.22 \mathrm{E}+01$ & $7.12 \mathrm{E}+02$ \\
\hline $\mathrm{Ru}$ & $3.00 \mathrm{E}+01$ & $4.30 \mathrm{E}+01$ & $1.72 \mathrm{E}+01$ & $2.88 \mathrm{E}+00$ & $1.72 \mathrm{E}+01$ & $3.02 \mathrm{E}+00$ & $9.76 \mathrm{E}+01$ \\
\hline $\mathrm{S}$ & N/A & N/A & $1.00 \mathrm{E}+00$ & N/A & N/A & N/A & N/A \\
\hline $\mathrm{Sb}$ & $2.20 \mathrm{E}+02$ & $7.60 \mathrm{E}+02$ & $6.12 \mathrm{E}+01$ & $4.95 \mathrm{E}+00$ & $6.12 \mathrm{E}+01$ & $4.40 \mathrm{E}+00$ & $8.50 \mathrm{E}+02$ \\
\hline $\mathrm{Sc}$ & N/A & N/A & $1.00 \mathrm{E}+03$ & N/A & N/A & N/A & N/A \\
\hline $\mathrm{Se}$ & N/A & N/A & $1.00 \mathrm{E}+04$ & N/A & $\mathrm{N} / \mathrm{A}$ & N/A & N/A \\
\hline $\mathrm{Sm}$ & N/A & N/A & $3.00 \mathrm{E}+02$ & N/A & N/A & N/A & N/A \\
\hline $\mathrm{Sn}$ & N/A & N/A & $5.00 \mathrm{E}+05$ & N/A & N/A & N/A & N/A \\
\hline $\mathrm{Sr}$ & $2.30 \mathrm{E}+01$ & $3.50 \mathrm{E}+01$ & $1.26 \mathrm{E}+01$ & $2.99 \mathrm{E}+00$ & $1.26 \mathrm{E}+01$ & $2.09 \mathrm{E}+00$ & $7.65 \mathrm{E}+01$ \\
\hline $\mathrm{Ta}$ & N/A & $\mathrm{N} / \mathrm{A}$ & $6.00 \mathrm{E}+01$ & $\mathrm{~N} / \mathrm{A}$ & $\mathrm{N} / \mathrm{A}$ & $\mathrm{N} / \mathrm{A}$ & N/A \\
\hline $\mathrm{Tb}$ & N/A & N/A & $6.00 \mathrm{E}+01$ & N/A & N/A & N/A & N/A \\
\hline $\mathrm{Tc}$ & $3.10 \mathrm{E}+01$ & $5.60 \mathrm{E}+01$ & $1.50 \mathrm{E}+01$ & $3.33 \mathrm{E}+00$ & $1.50 \mathrm{E}+01$ & $2.07 \mathrm{E}+00$ & $1.09 \mathrm{E}+02$ \\
\hline $\mathrm{Te}$ & N/A & N/A & $1.00 \mathrm{E}+03$ & N/A & N/A & N/A & N/A \\
\hline Th & $\mathrm{N} / \mathrm{A}$ & N/A & $6.00 \mathrm{E}+02$ & N/A & N/A & N/A & N/A \\
\hline $\mathrm{Tl}$ & N/A & $\mathrm{N} / \mathrm{A}$ & $5.00 \mathrm{E}+03$ & $\mathrm{~N} / \mathrm{A}$ & $\mathrm{N} / \mathrm{A}$ & N/A & N/A \\
\hline $\mathrm{Tm}$ & N/A & N/A & $3.00 \mathrm{E}+02$ & N/A & N/A & N/A & N/A \\
\hline $\mathrm{U}$ & $1.40 \mathrm{E}+01$ & $2.30 \mathrm{E}+01$ & $7.28 \mathrm{E}+00$ & $3.14 \mathrm{E}+00$ & $7.28 \mathrm{E}+00$ & $1.11 \mathrm{E}+00$ & $4.78 \mathrm{E}+01$ \\
\hline $\mathrm{W}$ & N/A & N/A & $9.00 \mathrm{E}+01$ & N/A & $\mathrm{N} / \mathrm{A}$ & N/A & N/A \\
\hline $\mathrm{Xe}$ & N/A & N/A & $1.00 \mathrm{E}+00$ & N/A & N/A & N/A & N/A \\
\hline $\mathrm{Y}$ & N/A & N/A & $2.00 \mathrm{E}+01$ & $\mathrm{~N} / \mathrm{A}$ & N/A & N/A & N/A \\
\hline $\mathrm{Yb}$ & N/A & N/A & $2.00 \mathrm{E}+02$ & N/A & N/A & N/A & N/A \\
\hline $\mathrm{Zn}$ & N/A & N/A & $1.00 \mathrm{E}+03$ & N/A & N/A & N/A & N/A \\
\hline $\mathrm{Zr}$ & $1.10 \mathrm{E}+02$ & $1.50 \mathrm{E}+02$ & $6.51 \mathrm{E}+01$ & $2.79 \mathrm{E}+00$ & $6.51 \mathrm{E}+01$ & $1.20 \mathrm{E}+01$ & $3.51 \mathrm{E}+02$ \\
\hline $\begin{array}{l}\text { GM }= \\
\text { GSD }= \\
\text { N/A }= \\
\text { STD }=\end{array}$ & $\begin{array}{l}\text { Geometric me } \\
\text { Geometric Sta } \\
\text { Not available } \\
\text { Standard Devi }\end{array}$ & $\begin{array}{l}\text { rd Deviati } \\
\text { he refereno } \\
\text { n. }\end{array}$ & viewed. & & & & \\
\hline
\end{tabular}


Table 2.6. CLBMM Bioaccumulation Factor - Marine Mollusk

\begin{tabular}{|c|c|c|c|c|c|c|c|}
\hline Element & Mean & STD & Median & GSD & GM & $5 \%$ & $95 \%$ \\
\hline $\mathrm{Ac}$ & $\mathrm{N} / \mathrm{A}$ & $\mathrm{N} / \mathrm{A}$ & $1.00 \mathrm{E}+03$ & N/A & N/A & N/A & $\mathrm{N} / \mathrm{A}$ \\
\hline $\mathrm{Ag}$ & N/A & N/A & $6.00 \mathrm{E}+04$ & $\mathrm{~N} / \mathrm{A}$ & N/A & N/A & N/A \\
\hline $\mathrm{Am}$ & $3.00 \mathrm{E}+01$ & $8.00 \mathrm{E}+00$ & N/A & $1.30 \mathrm{E}+00$ & $2.90 \mathrm{E}+01$ & $1.88 \mathrm{E}+01$ & $4.46 \mathrm{E}+01$ \\
\hline $\mathrm{Ba}$ & N/A & N/A & $1.00 \mathrm{E}+01$ & N/A & N/A & N/A & N/A \\
\hline $\mathrm{Bk}$ & N/A & N/A & $1.00 \mathrm{E}+03$ & N/A & N/A & N/A & N/A \\
\hline $\mathrm{Br}$ & N/A & N/A & $1.00 \mathrm{E}+01$ & N/A & N/A & N/A & N/A \\
\hline $\mathrm{Ca}$ & N/A & N/A & $3.00 \mathrm{E}+00$ & N/A & N/A & N/A & $\mathrm{N} / \mathrm{A}$ \\
\hline $\mathrm{Cd}$ & N/A & N/A & $8.00 \mathrm{E}+04$ & N/A & N/A & N/A & N/A \\
\hline $\mathrm{Ce}$ & N/A & N/A & $2.00 \mathrm{E}+03$ & N/A & N/A & N/A & N/A \\
\hline $\mathrm{Cf}$ & N/A & N/A & $1.00 \mathrm{E}+03$ & N/A & N/A & N/A & N/A \\
\hline $\mathrm{Cl}$ & N/A & N/A & $5.00 \mathrm{E}-02$ & N/A & N/A & N/A & N/A \\
\hline $\mathrm{Cm}$ & N/A & N/A & $1.00 \mathrm{E}+03$ & $\mathrm{~N} / \mathrm{A}$ & N/A & N/A & N/A \\
\hline $\mathrm{Co}$ & N/A & N/A & $2.00 \mathrm{E}+04$ & N/A & N/A & N/A & N/A \\
\hline Cs & N/A & N/A & $2.00 \mathrm{E}+01$ & $\mathrm{~N} / \mathrm{A}$ & N/A & N/A & N/A \\
\hline Cs & N/A & N/A & $6.00 \mathrm{E}+01$ & N/A & N/A & N/A & N/A \\
\hline Dy & N/A & N/A & $7.00 \mathrm{E}+03$ & N/A & N/A & N/A & $\mathrm{N} / \mathrm{A}$ \\
\hline $\mathrm{Eu}$ & N/A & N/A & $7.00 \mathrm{E}+03$ & N/A & N/A & N/A & N/A \\
\hline $\mathrm{Fe}$ & N/A & N/A & $5.00 \mathrm{E}+05$ & N/A & N/A & N/A & N/A \\
\hline $\mathrm{Gd}$ & N/A & N/A & $7.00 \mathrm{E}+03$ & N/A & N/A & N/A & $\mathrm{N} / \mathrm{A}$ \\
\hline $\mathrm{Hf}$ & N/A & N/A & $7.00 \mathrm{E}+03$ & N/A & N/A & N/A & N/A \\
\hline $\mathrm{Hg}$ & N/A & N/A & $2.00 \mathrm{E}+03$ & N/A & N/A & N/A & $\mathrm{N} / \mathrm{A}$ \\
\hline I & N/A & N/A & $1.00 \mathrm{E}+01$ & N/A & N/A & N/A & N/A \\
\hline In & N/A & N/A & $1.00 \mathrm{E}+04$ & $\mathrm{~N} / \mathrm{A}$ & N/A & N/A & N/A \\
\hline $\mathrm{Ir}$ & N/A & N/A & $1.00 \mathrm{E}+02$ & N/A & N/A & N/A & N/A \\
\hline $\mathrm{Kr}$ & N/A & N/A & $1.00 \mathrm{E}+00$ & N/A & N/A & N/A & N/A \\
\hline $\mathrm{Mn}$ & N/A & N/A & $5.00 \mathrm{E}+04$ & N/A & N/A & N/A & N/A \\
\hline $\mathrm{Na}$ & N/A & N/A & $3.00 \mathrm{E}-01$ & N/A & N/A & N/A & N/A \\
\hline $\mathrm{Nb}$ & N/A & N/A & $1.00 \mathrm{E}+03$ & N/A & N/A & N/A & N/A \\
\hline $\mathrm{Ni}$ & N/A & N/A & $2.00 \mathrm{E}+03$ & N/A & N/A & N/A & N/A \\
\hline $\mathrm{Np}$ & N/A & N/A & $4.00 \mathrm{E}+02$ & N/A & N/A & N/A & N/A \\
\hline $\mathrm{P}$ & $3.00 \mathrm{E}+04$ & N/A & N/A & N/A & N/A & N/A & N/A \\
\hline $\mathrm{Pa}$ & N/A & N/A & $5.00 \mathrm{E}+02$ & N/A & N/A & N/A & N/A \\
\hline $\mathrm{Pb}$ & N/A & N/A & $5.00 \mathrm{E}+04$ & N/A & N/A & N/A & N/A \\
\hline $\mathrm{Pd}$ & N/A & N/A & $3.00 \mathrm{E}+02$ & N/A & N/A & N/A & N/A \\
\hline $\mathrm{Pm}$ & N/A & N/A & $7.00 \mathrm{E}+03$ & N/A & N/A & N/A & N/A \\
\hline Po & N/A & N/A & $2.00 \mathrm{E}+04$ & N/A & N/A & N/A & N/A \\
\hline $\mathrm{Pu}$ & N/A & N/A & $3.00 \mathrm{E}+03$ & N/A & N/A & N/A & N/A \\
\hline $\mathrm{Ra}$ & N/A & N/A & $1.00 \mathrm{E}+02$ & N/A & N/A & N/A & N/A \\
\hline $\mathrm{Ru}$ & N/A & N/A & $5.00 \mathrm{E}+02$ & N/A & N/A & N/A & N/A \\
\hline $\mathrm{S}$ & N/A & N/A & $3.00 \mathrm{E}+00$ & N/A & N/A & N/A & N/A \\
\hline $\mathrm{Sb}$ & N/A & N/A & $3.00 \mathrm{E}+02$ & N/A & N/A & N/A & N/A \\
\hline $\mathrm{Sc}$ & N/A & N/A & $1.00 \mathrm{E}+05$ & N/A & N/A & N/A & N/A \\
\hline $\mathrm{Se}$ & N/A & N/A & $9.00 \mathrm{E}+03$ & N/A & N/A & N/A & N/A \\
\hline $\mathrm{Sm}$ & N/A & N/A & $7.00 \mathrm{E}+03$ & N/A & N/A & N/A & N/A \\
\hline $\mathrm{Sn}$ & N/A & N/A & $5.00 \mathrm{E}+05$ & N/A & N/A & N/A & N/A \\
\hline $\mathrm{Sr}$ & N/A & N/A & $1.00 \mathrm{E}+01$ & N/A & N/A & N/A & N/A \\
\hline
\end{tabular}


Table 2.6. (contd)

\begin{tabular}{cccccccc}
\hline Element & Mean & STD & Median & GSD & GM & $5 \%$ & $95 \%$ \\
\hline $\mathrm{Ta}$ & N/A & N/A & $7.00 \mathrm{E}+03$ & N/A & N/A & N/A & N/A \\
$\mathrm{Tb}$ & N/A & N/A & $3.00 \mathrm{E}+03$ & N/A & N/A & N/A & N/A \\
$\mathrm{Tc}$ & N/A & N/A & $5.00 \mathrm{E}+02$ & N/A & N/A & N/A & N/A \\
$\mathrm{Te}$ & N/A & N/A & $1.00 \mathrm{E}+03$ & N/A & N/A & N/A & N/A \\
$\mathrm{Th}$ & N/A & N/A & $1.00 \mathrm{E}+03$ & N/A & N/A & N/A & N/A \\
$\mathrm{Tl}$ & N/A & N/A & $6.00 \mathrm{E}+03$ & N/A & N/A & N/A & N/A \\
$\mathrm{Tm}$ & N/A & N/A & $7.00 \mathrm{E}+03$ & N/A & N/A & N/A & N/A \\
$\mathrm{U}$ & N/A & N/A & $3.00 \mathrm{E}+01$ & N/A & N/A & N/A & N/A \\
$\mathrm{W}$ & N/A & N/A & $6.00 \mathrm{E}+02$ & N/A & N/A & N/A & N/A \\
$\mathrm{Xe}$ & N/A & N/A & $1.00 \mathrm{E}+00$ & N/A & N/A & N/A & N/A \\
$\mathrm{Y}$ & N/A & N/A & $1.00 \mathrm{E}+03$ & N/A & N/A & N/A & N/A \\
$\mathrm{Yb}$ & N/A & N/A & $3.00 \mathrm{E}+03$ & N/A & N/A & N/A & N/A \\
$Z n$ & N/A & N/A & $8.00 \mathrm{E}+04$ & N/A & N/A & N/A & N/A \\
$Z r$ & N/A & N/A & & & & N/A \\
\hline GM $=$ Geometric mean. \\
GSD $=$ Geometric Standard Deviation. \\
N/A $=$ Not available in the references reviewed. \\
STD $=$ Standard Deviation. & & & & \\
\hline
\end{tabular}

Table 2.7. CLBMI Bioaccumulation Factor - Marine Crustacean

\begin{tabular}{cccccccc}
\hline Element & Mean & STD & Median & GSD & GM & $5 \%$ & $95 \%$ \\
\hline $\mathrm{Ac}$ & N/A & N/A & $1.00 \mathrm{E}+03$ & N/A & N/A & N/A & N/A \\
$\mathrm{Ag}$ & N/A & N/A & $2.00 \mathrm{E}+05$ & N/A & N/A & N/A & N/A \\
$\mathrm{Am}$ & $1.30 \mathrm{E}+03$ & $1.40 \mathrm{E}+03$ & $8.85 \mathrm{E}+02$ & $2.40 \mathrm{E}+00$ & $8.85 \mathrm{E}+02$ & $2.09 \mathrm{E}+02$ & $3.75 \mathrm{E}+03$ \\
$\mathrm{Ba}$ & N/A & N/A & $7.00 \mathrm{E}-01$ & N/A & N/A & N/A & N/A \\
$\mathrm{Bk}$ & N/A & N/A & $4.00 \mathrm{E}+02$ & N/A & N/A & N/A & N/A \\
$\mathrm{Br}$ & N/A & N/A & $1.00 \mathrm{E}+01$ & N/A & N/A & N/A & N/A \\
$\mathrm{Ca}$ & N/A & N/A & $5.00 \mathrm{E}+00$ & N/A & N/A & N/A & N/A \\
$\mathrm{Cd}$ & $2.60 \mathrm{E}+04$ & $4.90 \mathrm{E}+04$ & $1.22 \mathrm{E}+04$ & $3.42 \mathrm{E}+00$ & $1.22 \mathrm{E}+04$ & $1.61 \mathrm{E}+03$ & $9.23 \mathrm{E}+04$ \\
$\mathrm{Ce}$ & $3.40 \mathrm{E}+03$ & $5.70 \mathrm{E}+03$ & $1.74 \mathrm{E}+03$ & $3.18 \mathrm{E}+00$ & $1.74 \mathrm{E}+03$ & $2.60 \mathrm{E}+02$ & $1.17 \mathrm{E}+04$ \\
$\mathrm{Cf}$ & N/A & N/A & $4.00 \mathrm{E}+02$ & N/A & N/A & N/A & N/A \\
$\mathrm{Cl}$ & N/A & N/A & $6.00 \mathrm{E}-02$ & N/A & N/A & N/A & N/A \\
$\mathrm{Cm}$ & N/A & N/A & $4.00 \mathrm{E}+02$ & N/A & N/A & N/A & N/A \\
$\mathrm{Co}$ & $1.80 \mathrm{E}+03$ & $2.90 \mathrm{E}+03$ & $9.49 \mathrm{E}+02$ & $3.10 \mathrm{E}+00$ & $9.49 \mathrm{E}+02$ & $1.48 \mathrm{E}+02$ & $6.10 \mathrm{E}+03$ \\
$\mathrm{Cs}$ & $4.10 \mathrm{E}+01$ & $8.30 \mathrm{E}+01$ & $1.82 \mathrm{E}+01$ & $3.58 \mathrm{E}+00$ & $1.82 \mathrm{E}+01$ & $2.22 \mathrm{E}+00$ & $1.48 \mathrm{E}+02$ \\
$\mathrm{Dy}$ & N/A & N/A & $4.00 \mathrm{E}+03$ & N/A & N/A & N/A & N/A \\
$\mathrm{Eu}$ & N/A & N/A & $4.00 \mathrm{E}+03$ & N/A & N/A & N/A & N/A \\
$\mathrm{Fe}$ & N/A & N/A & $5.00 \mathrm{E}+05$ & N/A & N/A & N/A & N/A \\
$\mathrm{Gd}$ & N/A & N/A & $4.00 \mathrm{E}+03$ & N/A & N/A & N/A & N/A \\
$\mathrm{Hf}$ & N/A & N/A & $4.00 \mathrm{E}+03$ & N/A & N/A & N/A & N/A \\
$\mathrm{Hg}$ & N/A & N/A & $1.00 \mathrm{E}+04$ & N/A & N/A & N/A & N/A \\
$\mathrm{I}$ & N/A & N/A & $3.00 \mathrm{E}+00$ & N/A & N/A & N/A & N/A \\
$\mathrm{In}$ & N/A & N/A & $1.00 \mathrm{E}+04$ & N/A & N/A & N/A & N/A \\
$\mathrm{Ir}$ & N/A & N/A & $1.00 \mathrm{E}+02$ & N/A & N/A & N/A & N/A \\
$\mathrm{Kr}$ & N/A & N/A & $1.00 \mathrm{E}+00$ & N/A & N/A & N/A & N/A \\
& & & & & & &
\end{tabular}


Table 2.7. (contd)

\begin{tabular}{|c|c|c|c|c|c|c|c|}
\hline Element & Mean & STD & Median & GSD & GM & $5 \%$ & $95 \%$ \\
\hline $\mathrm{Mn}$ & $2.30 \mathrm{E}+04$ & $7.50 \mathrm{E}+04$ & $6.74 \mathrm{E}+03$ & $4.79 \mathrm{E}+00$ & $6.74 \mathrm{E}+03$ & $5.13 \mathrm{E}+02$ & $8.87 \mathrm{E}+04$ \\
\hline $\mathrm{Na}$ & N/A & N/A & $7.00 \mathrm{E}-02$ & N/A & N/A & N/A & N/A \\
\hline $\mathrm{Nb}$ & $1.00 \mathrm{E}+02$ & $1.20 \mathrm{E}+02$ & $6.40 \mathrm{E}+01$ & $2.57 \mathrm{E}+00$ & $6.40 \mathrm{E}+01$ & $1.35 \mathrm{E}+01$ & $3.03 \mathrm{E}+02$ \\
\hline $\mathrm{Ni}$ & $5.50 \mathrm{E}+02$ & $6.40 \mathrm{E}+02$ & $3.58 \mathrm{E}+02$ & $2.52 \mathrm{E}+00$ & $3.58 \mathrm{E}+02$ & $7.82 \mathrm{E}+01$ & $1.64 \mathrm{E}+03$ \\
\hline $\mathrm{Ni}$ & N/A & N/A & $1.00 \mathrm{E}+03$ & N/A & N/A & N/A & N/A \\
\hline $\mathrm{Np}$ & N/A & N/A & $1.00 \mathrm{E}+02$ & N/A & N/A & N/A & N/A \\
\hline $\mathrm{P}$ & $2.70 \mathrm{E}+04$ & N/A & N/A & N/A & $\mathrm{N} / \mathrm{A}$ & N/A & N/A \\
\hline $\mathrm{Pa}$ & N/A & N/A & $1.00 \mathrm{E}+01$ & N/A & N/A & N/A & N/A \\
\hline $\mathrm{Pb}$ & $7.50 \mathrm{E}+03$ & $2.10 \mathrm{E}+04$ & $2.52 \mathrm{E}+03$ & $4.38 \mathrm{E}+00$ & $2.52 \mathrm{E}+03$ & $2.22 \mathrm{E}+02$ & $2.86 \mathrm{E}+04$ \\
\hline $\mathrm{Pd}$ & N/A & N/A & $3.00 \mathrm{E}+02$ & N/A & N/A & N/A & N/A \\
\hline $\mathrm{Pm}$ & N/A & N/A & $4.00 \mathrm{E}+03$ & N/A & N/A & N/A & N/A \\
\hline Po & $5.60 \mathrm{E}+04$ & $6.60 \mathrm{E}+04$ & $3.62 \mathrm{E}+04$ & $2.54 \mathrm{E}+00$ & $3.62 \mathrm{E}+04$ & $7.81 \mathrm{E}+03$ & $1.68 \mathrm{E}+05$ \\
\hline $\mathrm{Pu}$ & $1.60 \mathrm{E}+02$ & $1.40 \mathrm{E}+02$ & $1.20 \mathrm{E}+02$ & $2.13 \mathrm{E}+00$ & $1.20 \mathrm{E}+02$ & $3.48 \mathrm{E}+01$ & $4.16 \mathrm{E}+02$ \\
\hline $\mathrm{Ra}$ & $1.10 \mathrm{E}+02$ & $8.10 \mathrm{E}+01$ & $8.86 \mathrm{E}+01$ & $1.93 \mathrm{E}+00$ & $8.86 \mathrm{E}+01$ & $3.00 \mathrm{E}+01$ & $2.62 \mathrm{E}+02$ \\
\hline $\mathrm{Ru}$ & $3.20 \mathrm{E}+02$ & $4.40 \mathrm{E}+02$ & $1.88 \mathrm{E}+02$ & $2.80 \mathrm{E}+00$ & $1.88 \mathrm{E}+02$ & $3.46 \mathrm{E}+01$ & $1.02 \mathrm{E}+03$ \\
\hline $\mathrm{Ru}$ & $1.00 \mathrm{E}+01$ & N/A & N/A & N/A & N/A & N/A & N/A \\
\hline $\mathrm{Ru}$ & N/A & N/A & $1.00 \mathrm{E}+02$ & N/A & $\mathrm{N} / \mathrm{A}$ & N/A & N/A \\
\hline $\mathrm{S}$ & N/A & N/A & $1.00 \mathrm{E}+00$ & N/A & N/A & N/A & N/A \\
\hline $\mathrm{Sb}$ & $1.40 \mathrm{E}+03$ & $2.60 \mathrm{E}+03$ & $6.64 \mathrm{E}+02$ & $3.39 \mathrm{E}+00$ & $6.64 \mathrm{E}+02$ & $8.90 \mathrm{E}+01$ & $4.95 \mathrm{E}+03$ \\
\hline $\mathrm{Sc}$ & N/A & N/A & $3.00 \mathrm{E}+02$ & N/A & N/A & N/A & N/A \\
\hline $\mathrm{Se}$ & $7.10 \mathrm{E}+03$ & $4.80 \mathrm{E}+03$ & $5.88 \mathrm{E}+03$ & $1.85 \mathrm{E}+00$ & $5.88 \mathrm{E}+03$ & $2.14 \mathrm{E}+03$ & $1.61 \mathrm{E}+04$ \\
\hline $\mathrm{Sm}$ & N/A & N/A & $4.00 \mathrm{E}+03$ & N/A & N/A & N/A & N/A \\
\hline $\mathrm{Sn}$ & N/A & N/A & $5.00 \mathrm{E}+05$ & N/A & N/A & N/A & N/A \\
\hline $\mathrm{Sr}$ & $1.20 \mathrm{E}+01$ & $1.20 \mathrm{E}+01$ & $8.49 \mathrm{E}+00$ & $2.30 \mathrm{E}+00$ & $8.49 \mathrm{E}+00$ & $2.16 \mathrm{E}+00$ & $3.34 \mathrm{E}+01$ \\
\hline $\mathrm{Ta}$ & N/A & N/A & $2.00 \mathrm{E}+03$ & N/A & N/A & N/A & N/A \\
\hline $\mathrm{Tb}$ & N/A & N/A & $4.00 \mathrm{E}+03$ & N/A & N/A & N/A & N/A \\
\hline Tc & $1.70 \mathrm{E}+04$ & $2.20 \mathrm{E}+04$ & $1.04 \mathrm{E}+04$ & $2.70 \mathrm{E}+00$ & $1.04 \mathrm{E}+04$ & $2.03 \mathrm{E}+03$ & $5.31 \mathrm{E}+04$ \\
\hline $\mathrm{Te}$ & N/A & N/A & $1.00 \mathrm{E}+03$ & N/A & N/A & N/A & N/A \\
\hline Th & N/A & N/A & $1.00 \mathrm{E}+03$ & N/A & N/A & N/A & N/A \\
\hline $\mathrm{Tl}$ & $\mathrm{N} / \mathrm{A}$ & N/A & $1.00 \mathrm{E}+03$ & N/A & $\mathrm{N} / \mathrm{A}$ & N/A & N/A \\
\hline $\mathrm{Tm}$ & N/A & N/A & $4.00 \mathrm{E}+03$ & N/A & N/A & N/A & N/A \\
\hline $\mathrm{U}$ & N/A & N/A & $1.00 \mathrm{E}+01$ & N/A & N/A & N/A & N/A \\
\hline W & N/A & N/A & $1.00 \mathrm{E}+01$ & N/A & N/A & N/A & N/A \\
\hline $\mathrm{Xe}$ & N/A & N/A & $1.00 \mathrm{E}+00$ & N/A & $\mathrm{N} / \mathrm{A}$ & N/A & N/A \\
\hline $\mathrm{Y}$ & N/A & N/A & $1.00 \mathrm{E}+03$ & N/A & N/A & N/A & N/A \\
\hline $\mathrm{Yb}$ & N/A & N/A & $4.00 \mathrm{E}+03$ & N/A & N/A & N/A & N/A \\
\hline $\mathrm{Zn}$ & N/A & N/A & $3.00 \mathrm{E}+05$ & N/A & N/A & N/A & N/A \\
\hline $\mathrm{Zr}$ & $2.20 \mathrm{E}+02$ & $4.00 \mathrm{E}+02$ & $1.06 \mathrm{E}+02$ & $3.35 \mathrm{E}+00$ & $1.06 \mathrm{E}+02$ & $1.45 \mathrm{E}+01$ & $7.74 \mathrm{E}+02$ \\
\hline $\begin{array}{l}\mathrm{GM}= \\
\mathrm{GSD}= \\
\mathrm{N} / \mathrm{A}= \\
\mathrm{STD}=\end{array}$ & $\begin{array}{l}\text { Geometric Sta } \\
\text { Not available } \\
\text { Standard Devi }\end{array}$ & n. & ewed. & & & & \\
\hline
\end{tabular}




\subsubsection{CLBFP and CLBMP Bioaccumulation Factor in Aquatic Plants (Freshwater and Saltwater)}

This parameter is the ratio of the chemical concentration in freshwater aquatic plants to the chemical concentration in the water to which the plants are exposed.

The units and range for this parameter in Gv2 are: $\mathrm{L} / \mathrm{kg}_{\text {wet }}$ which is a reduced from $\left(\mathrm{Ci} / \mathrm{kg}_{\text {wet }}\right)$ per $(\mathrm{Ci} / \mathrm{L})(0$ to 100,000$)$.

To tabulate bioaccumulation factors for aquatic plants, sources considered to be the best available references were reviewed. The references reviewed are listed in the corresponding appendices. The goal was to determine mean, median, geometric standard deviation, geometric mean, $5^{\text {th }}$ percentile, and $95^{\text {th }}$ percentile. Some of these values could be determined mathematically. In these cases, statistical calculations were performed, according to methods described in Strom and Stansbury (2000). The full set of data reviewed to determine the values tabulated in this section are provided in Appendix A.

\subsubsection{Additional Sources of Information}

The references that were reviewed contain additional information regarding data completeness, data limitations, and application of values to environmental models.

There are numerous chemicals for which no aquatic plant bioaccumulation factors are available. The user can refer to Staven et al. (2003) for an approach to approximate values for non-tabulated chemicals. The user is encouraged to review additional research on transfer factors in the literature, as well.

\subsubsection{CLBFP Bioaccumulation Factor in Aquatic Plants Tables}

Table 2.8. CLBFP Bioaccumulation Factor - Freshwater Plants

\begin{tabular}{cccccccc}
\hline Element & Mean & STD & Median & GSD & GM & $5 \%$ & $95 \%$ \\
\hline $\mathrm{Am}$ & $4.45 \mathrm{E}+04$ & $5.33 \mathrm{E}+05$ & N/A & $9.30 \mathrm{E}+00$ & $3.70 \mathrm{E}+03$ & $9.44 \mathrm{E}+01$ & $1.45 \mathrm{E}+05$ \\
$\mathrm{As}$ & $7.20 \mathrm{E}+03$ & $1.10 \mathrm{E}+03$ & $7.1 \mathrm{E}+03$ & $1.16 \mathrm{E}+00$ & $7.12 \mathrm{E}+03$ & $5.54 \mathrm{E}+03$ & $9.14 \mathrm{E}+03$ \\
$\mathrm{Cd}$ & $1.23 \mathrm{E}+05$ & $7.83 \mathrm{E}+05$ & N/A & $6.90 \mathrm{E}+00$ & $1.90 \mathrm{E}+04$ & $7.92 \mathrm{E}+02$ & $4.56 \mathrm{E}+05$ \\
$\mathrm{Ce}$ & $3.50 \mathrm{E}+03$ & N/A & N/A & N/A & N/A & N/A & N/A \\
$\mathrm{Cm}$ & $1.19 \mathrm{E}+05$ & $7.40 \mathrm{E}+05$ & N/A & $6.80 \mathrm{E}+00$ & $1.90 \mathrm{E}+04$ & $8.11 \mathrm{E}+02$ & $4.45 \mathrm{E}+05$ \\
$\mathrm{Co}$ & $2.68 \mathrm{E}+03$ & $9.73 \mathrm{E}+03$ & N/A & $5.10 \mathrm{E}+00$ & $7.10 \mathrm{E}+02$ & $4.87 \mathrm{E}+01$ & $1.04 \mathrm{E}+04$ \\
$\mathrm{Cs}$ & $4.53 \mathrm{E}+03$ & $2.11 \mathrm{E}+05$ & N/A & $1.60 \mathrm{E}+01$ & $9.70 \mathrm{E}+01$ & $1.01 \mathrm{E}+00$ & $9.28 \mathrm{E}+03$ \\
$\mathrm{Cu}$ & $5.04 \mathrm{E}+10$ & $8.47 \mathrm{E}+17$ & N/A & $3.20 \mathrm{E}+02$ & $3.00 \mathrm{E}+03$ & $2.27 \mathrm{E}-01$ & $3.96 \mathrm{E}+07$ \\
$\mathrm{Fe}$ & $1.12 \mathrm{E}+04$ & $7.98 \mathrm{E}+03$ & N/A & $1.90 \mathrm{E}+00$ & $9.10 \mathrm{E}+03$ & $3.17 \mathrm{E}+03$ & $2.62 \mathrm{E}+04$ \\
$\mathrm{I}$ & $3.06 \mathrm{E}+02$ & $6.52 \mathrm{E}+02$ & N/A & $3.70 \mathrm{E}+00$ & $1.30 \mathrm{E}+02$ & $1.51 \mathrm{E}+01$ & $1.12 \mathrm{E}+03$ \\
$\mathrm{Mn}$ & $3.01 \mathrm{E}+13$ & $7.56 \mathrm{E}+22$ & N/A & $7.20 \mathrm{E}+02$ & $1.20 \mathrm{E}+04$ & $2.39 \mathrm{E}-01$ & $6.02 \mathrm{E}+08$ \\
$\mathrm{Na}$ & $5.00 \mathrm{E}+02$ & N/A & N/A & N/A & N/A & N/A & N/A \\
$\mathrm{Ni}$ & $1.07 \mathrm{E}+08$ & $1.50 \mathrm{E}+13$ & N/A & $1.30 \mathrm{E}+02$ & $7.70 \mathrm{E}+02$ & $2.56 \mathrm{E}-01$ & $2.31 \mathrm{E}+06$ \\
$\mathrm{~Np}$ & $7.20 \mathrm{E}+03$ & N/A & N/A & N/A & N/A & N/A & N/A \\
$\mathrm{P}$ & $3.00 \mathrm{E}+02$ & N/A & N/A & N/A & N/A & N/A & N/A \\
$\mathrm{Pb}$ & N/A & N/A & N/A & $7.60 \mathrm{E}+01$ & $1.90 \mathrm{E}+03$ & $1.53 \mathrm{E}+00$ & $2.36 \mathrm{E}+06$ \\
$\mathrm{Pu}$ & $8.46 \mathrm{E}+05$ & $2.75 \mathrm{E}+07$ & N/A & $1.40 \mathrm{E}+01$ & $2.60 \mathrm{E}+04$ & $3.39 \mathrm{E}+02$ & $2.00 \mathrm{E}+06$
\end{tabular}


Table 2.8. (contd)

\begin{tabular}{cccccccc}
\hline Element & Mean & STD & Median & GSD & GM & $5 \%$ & $95 \%$ \\
\hline $\mathrm{Ra}$ & $7.85 \mathrm{E}+03$ & $1.97 \mathrm{E}+04$ & N/A & $4.10 \mathrm{E}+00$ & $2.90 \mathrm{E}+03$ & $2.85 \mathrm{E}+02$ & $2.95 \mathrm{E}+04$ \\
$\mathrm{Ru}$ & $3.69 \mathrm{E}+02$ & $2.90 \mathrm{E}+02$ & N/A & $2.00 \mathrm{E}+00$ & $2.90 \mathrm{E}+02$ & $9.27 \mathrm{E}+01$ & $9.07 \mathrm{E}+02$ \\
$\mathrm{Se}$ & $5.80 \mathrm{E}+03$ & $2.33 \mathrm{E}+04$ & N/A & $5.40 \mathrm{E}+00$ & $1.40 \mathrm{E}+03$ & $8.74 \mathrm{E}+01$ & $2.24 \mathrm{E}+04$ \\
$\mathrm{Sr}$ & $8.36 \mathrm{E}+02$ & $1.49 \mathrm{E}+03$ & N/A & $3.30 \mathrm{E}+00$ & $4.10 \mathrm{E}+02$ & $5.75 \mathrm{E}+01$ & $2.92 \mathrm{E}+03$ \\
$\mathrm{Tc}$ & $1.94 \mathrm{E}+01$ & $6.59 \mathrm{E}+01$ & N/A & $4.90 \mathrm{E}+00$ & $5.50 \mathrm{E}+00$ & $4.03 \mathrm{E}-01$ & $7.51 \mathrm{E}+01$ \\
$\mathrm{U}$ & $2.58 \mathrm{E}+02$ & $1.84 \mathrm{E}+02$ & N/A & $1.90 \mathrm{E}+00$ & $2.10 \mathrm{E}+02$ & $7.31 \mathrm{E}+01$ & $6.04 \mathrm{E}+02$ \\
$\mathrm{Zn}$ & $5.63 \mathrm{E}+05$ & $1.51 \mathrm{E}+07$ & N/A & $1.30 \mathrm{E}+01$ & $2.10 \mathrm{E}+04$ & $3.09 \mathrm{E}+02$ & $1.43 \mathrm{E}+06$ \\
\hline $\mathrm{GM}=$ Geometric mean. \\
$\mathrm{GSD}=$ Geometric Standard Deviation. \\
$\mathrm{N} / \mathrm{A}=$ Not available in the references reviewed. \\
$\mathrm{STD}=$ Standard Deviation.
\end{tabular}

Table 2.9. CLBMP Bioaccumulation Factor - Saltwater Plants

\begin{tabular}{ccccccc}
\hline Element & Mean & Median & GSD & GM & $5 \%$ & $95 \%$ \\
\hline $\mathrm{Ac}$ & N/A & $1.00 \mathrm{E}+03$ & N/A & N/A & N/A & N/A \\
$\mathrm{Ag}$ & N/A & $5.00 \mathrm{E}+03$ & N/A & N/A & N/A & N/A \\
$\mathrm{Am}$ & N/A & $8.00 \mathrm{E}+03$ & N/A & N/A & N/A & N/A \\
$\mathrm{Ba}$ & N/A & $7.00 \mathrm{E}+01$ & N/A & N/A & N/A & N/A \\
$\mathrm{Be}$ & N/A & $2.00 \mathrm{E}+00$ & N/A & N/A & N/A & N/A \\
$\mathrm{Bk}$ & N/A & $8.00 \mathrm{E}+03$ & N/A & N/A & N/A & N/A \\
$\mathrm{Br}$ & N/A & $1.00 \mathrm{E}+01$ & N/A & N/A & N/A & N/A \\
$\mathrm{Ca}$ & N/A & $6.00 \mathrm{E}+00$ & N/A & N/A & N/A & N/A \\
$\mathrm{Cd}$ & N/A & $2.00 \mathrm{E}+04$ & N/A & N/A & N/A & N/A \\
$\mathrm{Ce}$ & N/A & $5.00 \mathrm{E}+03$ & N/A & N/A & N/A & N/A \\
$\mathrm{Cf}$ & N/A & $8.00 \mathrm{E}+03$ & N/A & N/A & N/A & N/A \\
$\mathrm{Cl}$ & N/A & $5.00 \mathrm{E}-02$ & N/A & N/A & N/A & N/A \\
$\mathrm{Cm}$ & N/A & $5.00 \mathrm{E}+03$ & N/A & N/A & N/A & N/A \\
$\mathrm{Co}$ & N/A & $6.00 \mathrm{E}+03$ & N/A & N/A & N/A & N/A \\
$\mathrm{Cs}$ & N/A & $5.00 \mathrm{E}+01$ & N/A & N/A & N/A & N/A \\
$\mathrm{Dy}$ & N/A & $3.00 \mathrm{E}+03$ & N/A & N/A & N/A & N/A \\
$\mathrm{Eu}$ & N/A & $3.00 \mathrm{E}+03$ & N/A & N/A & N/A & N/A \\
$\mathrm{Fe}$ & 7.30E+02 & N/A & N/A & N/A & N/A & N/A \\
$\mathrm{Gd}$ & N/A & $3.00 \mathrm{E}+03$ & N/A & N/A & N/A & N/A \\
$\mathrm{Hf}$ & N/A & $3.00 \mathrm{E}+03$ & N/A & N/A & N/A & N/A \\
$\mathrm{Hg}$ & N/A & $2.00 \mathrm{E}+04$ & N/A & N/A & N/A & N/A \\
$\mathrm{I}$ & N/A & $1.00 \mathrm{E}+04$ & N/A & N/A & N/A & N/A \\
$\mathrm{In}$ & N/A & $5.00 \mathrm{E}+03$ & N/A & N/A & N/A & N/A \\
$\mathrm{Ir}$ & N/A & $1.00 \mathrm{E}+03$ & N/A & N/A & N/A & N/A \\
$\mathrm{Kr}$ & N/A & $1.00 \mathrm{E}+00$ & N/A & N/A & N/A & N/A \\
$\mathrm{Mn}$ & N/A & $6.00 \mathrm{E}+03$ & N/A & N/A & N/A & N/A \\
$\mathrm{Na}$ & N/A & $5.00 \mathrm{E}-01$ & N/A & N/A & N/A & N/A \\
$\mathrm{Nb}$ & N/A & $3.00 \mathrm{E}+03$ & N/A & N/A & N/A & N/A \\
$\mathrm{Ni}$ & N/A & $2.00 \mathrm{E}+03$ & N/A & N/A & N/A & N/A
\end{tabular}


Table 2.9. (contd)

\begin{tabular}{|c|c|c|c|c|c|c|}
\hline Element & Mean & Median & GSD & GM & $5 \%$ & $95 \%$ \\
\hline $\mathrm{Np}$ & $\mathrm{N} / \mathrm{A}$ & $5.00 \mathrm{E}+01$ & N/A & N/A & N/A & N/A \\
\hline $\mathrm{P}$ & $4.00 \mathrm{E}+03$ & N/A & N/A & N/A & N/A & N/A \\
\hline $\mathrm{Pa}$ & N/A & $1.00 \mathrm{E}+02$ & N/A & N/A & N/A & N/A \\
\hline $\mathrm{Pb}$ & $\mathrm{N} / \mathrm{A}$ & $1.00 \mathrm{E}+03$ & N/A & N/A & N/A & $\mathrm{N} / \mathrm{A}$ \\
\hline $\mathrm{Pd}$ & $\mathrm{N} / \mathrm{A}$ & $1.00 \mathrm{E}+03$ & N/A & N/A & N/A & N/A \\
\hline $\mathrm{Pm}$ & N/A & $3.00 \mathrm{E}+03$ & N/A & N/A & N/A & N/A \\
\hline Po & N/A & $1.00 \mathrm{E}+03$ & N/A & N/A & N/A & N/A \\
\hline $\mathrm{Pu}$ & N/A & $4.00 \mathrm{E}+03$ & N/A & N/A & N/A & N/A \\
\hline $\mathrm{Ra}$ & $\mathrm{N} / \mathrm{A}$ & $1.00 \mathrm{E}+02$ & N/A & N/A & N/A & N/A \\
\hline $\mathrm{Ru}$ & N/A & $2.00 \mathrm{E}+03$ & N/A & N/A & N/A & N/A \\
\hline $\mathrm{S}$ & N/A & $3.00 \mathrm{E}+00$ & N/A & N/A & N/A & N/A \\
\hline $\mathrm{Sb}$ & $\mathrm{N} / \mathrm{A}$ & $2.00 \mathrm{E}+01$ & N/A & N/A & N/A & N/A \\
\hline $\mathrm{Sc}$ & $\mathrm{N} / \mathrm{A}$ & $9.00 \mathrm{E}+04$ & N/A & N/A & N/A & N/A \\
\hline $\mathrm{Se}$ & N/A & $1.00 \mathrm{E}+03$ & N/A & N/A & N/A & N/A \\
\hline $\mathrm{Sm}$ & N/A & $3.00 \mathrm{E}+03$ & N/A & N/A & N/A & $\mathrm{N} / \mathrm{A}$ \\
\hline $\mathrm{Sr}$ & $\mathrm{N} / \mathrm{A}$ & $1.00 \mathrm{E}+01$ & N/A & N/A & N/A & N/A \\
\hline $\mathrm{Ta}$ & N/A & $3.00 \mathrm{E}+03$ & N/A & N/A & N/A & N/A \\
\hline $\mathrm{Tb}$ & N/A & $2.00 \mathrm{E}+03$ & N/A & N/A & N/A & $\mathrm{N} / \mathrm{A}$ \\
\hline Tc & $\mathrm{N} / \mathrm{A}$ & $3.00 \mathrm{E}+04$ & N/A & N/A & N/A & N/A \\
\hline $\mathrm{Te}$ & N/A & $1.00 \mathrm{E}+04$ & N/A & N/A & N/A & N/A \\
\hline Th & $\mathrm{N} / \mathrm{A}$ & $2.00 \mathrm{E}+02$ & N/A & N/A & N/A & N/A \\
\hline $\mathrm{Tl}$ & N/A & $1.00 \mathrm{E}+03$ & N/A & N/A & N/A & $\mathrm{N} / \mathrm{A}$ \\
\hline $\mathrm{Tm}$ & N/A & $3.00 \mathrm{E}+03$ & N/A & N/A & N/A & N/A \\
\hline $\mathrm{U}$ & N/A & $1.00 \mathrm{E}+02$ & N/A & N/A & N/A & $\mathrm{N} / \mathrm{A}$ \\
\hline $\mathrm{W}$ & N/A & $6.00 \mathrm{E}+02$ & N/A & N/A & N/A & N/A \\
\hline $\mathrm{Xe}$ & $\mathrm{N} / \mathrm{A}$ & $1.00 \mathrm{E}+00$ & N/A & N/A & N/A & N/A \\
\hline $\mathrm{Y}$ & N/A & $1.00 \mathrm{E}+03$ & N/A & N/A & N/A & N/A \\
\hline $\mathrm{Yb}$ & N/A & $8.00 \mathrm{E}+02$ & N/A & N/A & N/A & N/A \\
\hline $\mathrm{Zn}$ & N/A & $2.00 \mathrm{E}+03$ & N/A & N/A & N/A & $\mathrm{N} / \mathrm{A}$ \\
\hline $\mathrm{Zr}$ & $\mathrm{N} / \mathrm{A}$ & $3.00 \mathrm{E}+03$ & N/A & N/A & N/A & N/A \\
\hline $\begin{array}{l}\text { GM }= \\
\text { GSD }= \\
\text { N/A }=\end{array}$ & etric mean. & $\begin{array}{l}\text { on. } \\
\text { es reviewe }\end{array}$ & & & & \\
\hline
\end{tabular}

\subsubsection{CLBSAF Bioaccumulation in Aquatic Biota from Sediment}

This parameter is not implemented in human pathways for chronic water releases in Gv2.

The units and range for this parameter in Gv2 are: $\mathrm{kg}_{\text {wet_biota }} / \mathrm{kg}_{\text {wet_sediment }}(0$ to 100,000$)$.

\subsubsection{CLFMT Feed to Meat Transfer Factor}

The feed-to-meat transfer factor is the ratio of chemical concentration in fresh meat tissue to the daily intake of the radionuclide by the animal. This factor is generally based on beef as the meat animal. 
The units and range for this parameter in $\mathrm{Gv} 2$ are: $\mathrm{d} / \mathrm{kg}$ which is reduced from $\left(\mathrm{Ci} / \mathrm{kg}_{\mathrm{wet}}\right) \mathrm{per}(\mathrm{Ci} / \mathrm{d})$ (0 to 100,000$)$.

To tabulate bioaccumulation factors for feed-to-meat, sources considered to be the best available references were reviewed. The goal was to determine mean, median, geometric standard deviation, geometric mean, $5^{\text {th }}$ percentile, and $95^{\text {th }}$ percentile. Selection priority was given to IAEA (2010), Howard et al. (2009), and Staven et al. (2003), with a preference for lognormality in the datasets. Some of these values could be determined mathematically. In these cases, statistical calculations were performed, according to methods described in Strom and Stansbury (2000). The full set of data reviewed to determine the values tabulated in this section are provided in Appendix B.

\subsubsection{Additional Sources of Information}

There are numerous chemicals for which no meat transfer factors are available. The user can refer to Staven et al. (2003) for an approach to approximate values for non-tabulated chemicals. The user is encouraged to review additional research on transfer factors in the literature, as well.

\subsubsection{CLFMT Feed to Meat Transfer Factor Tables}

Table 2.10. CLFMT Feed to Meat Transfer Factor

\begin{tabular}{cccccccc}
\hline Element & Mean & STD & Median & GSD & GM & $5 \%$ & $95 \%$ \\
\hline $\mathrm{Ag}$ & $4.80 \mathrm{E}-04$ & N/A & $3.00 \mathrm{E}-03$ & N/A & N/A & N/A & N/A \\
$\mathrm{Am}$ & $5.00 \mathrm{E}-04$ & N/A & N/A & N/A & N/A & N/A & N/A \\
$\mathrm{As}$ & N/A & N/A & $2.00 \mathrm{E}-03$ & N/A & N/A & N/A & N/A \\
$\mathrm{Au}$ & $\mathrm{N} / \mathrm{A}$ & N/A & $5.00 \mathrm{E}-03$ & N/A & N/A & N/A & N/A \\
$\mathrm{Ba}$ & N/A & N/A & N/A & $5.00 \mathrm{E}-05$ & $1.40 \mathrm{E}-04$ & N/A & N/A \\
$\mathrm{Be}$ & N/A & N/A & $1.00 \mathrm{E}-03$ & N/A & N/A & N/A & N/A \\
$\mathrm{Bi}$ & N/A & N/A & $4.00 \mathrm{E}-04$ & N/A & N/A & N/A & N/A \\
$\mathrm{Br}$ & N/A & N/A & $2.50 \mathrm{E}-02$ & N/A & N/A & N/A & N/A \\
$\mathrm{Ca}$ & $2.00 \mathrm{E}-01$ & $3.50 \mathrm{E}-01$ & $9.92 \mathrm{E}-02$ & $3.27 \mathrm{E}+00$ & $9.92 \mathrm{E}-02$ & $1.42 \mathrm{E}-02$ & $6.96 \mathrm{E}-01$ \\
$\mathrm{Cd}$ & N/A & N/A & N/A & $7.80 \mathrm{E}+00$ & $5.80 \mathrm{E}-03$ & N/A & N/A \\
$\mathrm{Ce}$ & $2.50 \mathrm{E}-04$ & N/A & $2.00 \mathrm{E}-05$ & N/A & N/A & N/A & N/A \\
$\mathrm{Cf}$ & N/A & N/A & $4.00 \mathrm{E}-05$ & N/A & N/A & N/A & N/A \\
$\mathrm{Cl}$ & $1.70 \mathrm{E}-02$ & N/A & N/A & N/A & N/A & N/A & N/A \\
$\mathrm{Cm}$ & N/A & N/A & $4.00 \mathrm{E}-05$ & N/A & N/A & N/A & N/A \\
$\mathrm{Co}$ & N/A & N/A & N/A & $2.30 \mathrm{E}+00$ & $4.30 \mathrm{E}-04$ & N/A & N/A \\
$\mathrm{Cr}$ & N/A & N/A & $9.00 \mathrm{E}-03$ & N/A & N/A & N/A & N/A \\
$\mathrm{Cs}$ & $4.80 \mathrm{E}-01$ & $5.30 \mathrm{E}-01$ & $3.22 \mathrm{E}-01$ & $2.44 \mathrm{E}+00$ & $3.22 \mathrm{E}-01$ & $7.42 \mathrm{E}-02$ & 1.4 \\
$\mathrm{Cu}$ & N/A & N/A & $9.00 \mathrm{E}-03$ & N/A & N/A & N/A & N/A \\
$\mathrm{Dy}$ & N/A & N/A & $2.00 \mathrm{E}-05$ & N/A & N/A & N/A & N/A \\
$\mathrm{Er}$ & N/A & N/A & $2.00 \mathrm{E}-05$ & N/A & N/A & N/A & N/A \\
$\mathrm{Eu}$ & N/A & N/A & $2.00 \mathrm{E}-05$ & N/A & N/A & N/A & N/A \\
$\mathrm{F}$ & N/A & N/A & $1.50 \mathrm{E}-01$ & N/A & N/A & N/A & N/A \\
$\mathrm{Fe}$ & $1.50 \mathrm{E}-02$ & $6.70 \mathrm{E}-03$ & $1.37 \mathrm{E}-02$ & $1.53 \mathrm{E}+00$ & $1.37 \mathrm{E}-02$ & $6.79 \mathrm{E}-03$ & $2.76 \mathrm{E}-02$ \\
$\mathrm{Ga}$ & N/A & N/A & $5.00 \mathrm{E}-04$ & N/A & N/A & N/A & N/A \\
$\mathrm{Gd}$ & N/A & N/A & $2.00 \mathrm{E}-05$ & N/A & N/A & N/A & N/A \\
& & & & & & &
\end{tabular}


Table 2.10. (contd)

\begin{tabular}{|c|c|c|c|c|c|c|c|}
\hline Element & Mean & STD & Median & GSD & GM & $5 \%$ & $95 \%$ \\
\hline $\mathrm{Hf}$ & $\mathrm{N} / \mathrm{A}$ & $\mathrm{N} / \mathrm{A}$ & $1.00 \mathrm{E}-03$ & $\mathrm{~N} / \mathrm{A}$ & $\mathrm{N} / \mathrm{A}$ & N/A & $\mathrm{N} / \mathrm{A}$ \\
\hline $\mathrm{Hg}$ & N/A & N/A & $2.50 \mathrm{E}-01$ & N/A & N/A & N/A & N/A \\
\hline Ho & N/A & N/A & $2.00 \mathrm{E}-05$ & N/A & N/A & N/A & N/A \\
\hline I & N/A & $\mathrm{N} / \mathrm{A}$ & N/A & $3.20 \mathrm{E}+00$ & $6.70 \mathrm{E}-03$ & N/A & N/A \\
\hline In & N/A & N/A & 8.00E-03 & N/A & N/A & N/A & N/A \\
\hline $\mathrm{Ir}$ & $\mathrm{N} / \mathrm{A}$ & $\mathrm{N} / \mathrm{A}$ & $1.50 \mathrm{E}-03$ & N/A & $\mathrm{N} / \mathrm{A}$ & N/A & N/A \\
\hline $\mathrm{K}$ & N/A & N/A & $2.00 \mathrm{E}-02$ & N/A & N/A & N/A & N/A \\
\hline $\mathrm{La}$ & N/A & $2.00 \mathrm{E}-05$ & N/A & $1.20 \mathrm{E}+00$ & $1.30 \mathrm{E}-04$ & N/A & N/A \\
\hline $\mathrm{Mg}$ & N/A & N/A & $2.00 \mathrm{E}-02$ & N/A & N/A & N/A & N/A \\
\hline $\mathrm{Mn}$ & $6.00 \mathrm{E}-04$ & N/A & N/A & N/A & N/A & N/A & N/A \\
\hline Mo & $1.00 \mathrm{E}-03$ & N/A & N/A & $\mathrm{N} / \mathrm{A}$ & $\mathrm{N} / \mathrm{A}$ & N/A & N/A \\
\hline $\mathrm{N}$ & N/A & N/A & $7.50 \mathrm{E}-02$ & N/A & N/A & N/A & N/A \\
\hline $\mathrm{Na}$ & N/A & N/A & N/A & $1.00 \mathrm{E}-02$ & $1.50 \mathrm{E}-02$ & $\mathrm{~N} / \mathrm{A}$ & N/A \\
\hline $\mathrm{Nb}$ & $2.60 \mathrm{E}-07$ & N/A & N/A & N/A & N/A & N/A & N/A \\
\hline $\mathrm{Nd}$ & N/A & N/A & $3.00 \mathrm{E}-04$ & N/A & N/A & N/A & N/A \\
\hline $\mathrm{Ni}$ & N/A & $\mathrm{N} / \mathrm{A}$ & $6.00 \mathrm{E}-03$ & N/A & N/A & N/A & N/A \\
\hline $\mathrm{Np}$ & N/A & N/A & $1.00 \mathrm{E}-03$ & N/A & $\mathrm{N} / \mathrm{A}$ & N/A & N/A \\
\hline Os & N/A & N/A & $4.00 \mathrm{E}-01$ & N/A & N/A & N/A & N/A \\
\hline $\mathrm{P}$ & $5.50 \mathrm{E}-02$ & N/A & N/A & N/A & N/A & N/A & N/A \\
\hline $\mathrm{Pa}$ & N/A & $\mathrm{N} / \mathrm{A}$ & $4.00 \mathrm{E}-05$ & N/A & N/A & N/A & N/A \\
\hline $\mathrm{Pb}$ & N/A & N/A & N/A & $2.50 \mathrm{E}+00$ & $7.00 \mathrm{E}-04$ & N/A & N/A \\
\hline $\mathrm{Pd}$ & N/A & N/A & $4.00 \mathrm{E}-03$ & N/A & N/A & N/A & N/A \\
\hline $\mathrm{Pm}$ & N/A & N/A & $2.00 \mathrm{E}-05$ & N/A & N/A & N/A & N/A \\
\hline Po & N/A & N/A & $5.00 \mathrm{E}-03$ & N/A & N/A & N/A & N/A \\
\hline $\operatorname{Pr}$ & N/A & N/A & $3.00 \mathrm{E}-04$ & N/A & N/A & N/A & N/A \\
\hline $\mathrm{Pu}$ & N/A & $\mathrm{N} / \mathrm{A}$ & N/A & $2.48 \mathrm{E}+01$ & $1.10 \mathrm{E}-06$ & N/A & N/A \\
\hline $\mathrm{Ra}$ & $1.70 \mathrm{E}-03$ & $\mathrm{~N} / \mathrm{A}$ & N/A & N/A & N/A & N/A & N/A \\
\hline $\mathrm{Rb}$ & N/A & N/A & $1.00 \mathrm{E}-02$ & N/A & N/A & N/A & N/A \\
\hline $\operatorname{Re}$ & N/A & $\mathrm{N} / \mathrm{A}$ & $8.00 \mathrm{E}-03$ & N/A & $\mathrm{N} / \mathrm{A}$ & $\mathrm{N} / \mathrm{A}$ & N/A \\
\hline $\mathrm{Rh}$ & N/A & N/A & $2.00 \mathrm{E}-03$ & N/A & N/A & N/A & N/A \\
\hline $\mathrm{Ru}$ & N/A & N/A & N/A & $1.80 \mathrm{E}+00$ & $3.30 \mathrm{E}-03$ & N/A & N/A \\
\hline $\mathrm{S}$ & $1.70 \mathrm{E}+00$ & 4.70E-01 & $1.64 \mathrm{E}+00$ & $1.31 \mathrm{E}+00$ & $1.64 \mathrm{E}+00$ & $1.05 \mathrm{E}+00$ & 2.56 \\
\hline $\mathrm{Sb}$ & N/A & N/A & N/A & $1.10 \mathrm{E}-03$ & $1.20 \mathrm{E}-03$ & N/A & N/A \\
\hline $\mathrm{Sc}$ & N/A & N/A & $1.50 \mathrm{E}-02$ & N/A & N/A & N/A & N/A \\
\hline $\mathrm{Se}$ & N/A & N/A & $1.50 \mathrm{E}-02$ & N/A & N/A & N/A & N/A \\
\hline $\mathrm{Si}$ & N/A & $\mathrm{N} / \mathrm{A}$ & $4.00 \mathrm{E}-05$ & N/A & N/A & N/A & N/A \\
\hline $\mathrm{Sm}$ & N/A & N/A & $2.00 \mathrm{E}-05$ & N/A & $\mathrm{N} / \mathrm{A}$ & N/A & N/A \\
\hline $\mathrm{Sn}$ & N/A & N/A & $8.00 \mathrm{E}-02$ & N/A & N/A & N/A & N/A \\
\hline $\mathrm{Sr}$ & $3.00 \mathrm{E}-03$ & $5.10 \mathrm{E}-03$ & $1.52 \mathrm{E}-03$ & $3.21 \mathrm{E}+00$ & $1.52 \mathrm{E}-03$ & $2.24 \mathrm{E}-04$ & $1.03 \mathrm{E}-02$ \\
\hline $\mathrm{Ta}$ & N/A & N/A & $3.00 \mathrm{E}-07$ & N/A & N/A & N/A & N/A \\
\hline $\mathrm{Tb}$ & N/A & N/A & $2.00 \mathrm{E}-05$ & N/A & N/A & N/A & N/A \\
\hline $\mathrm{Tc}$ & N/A & N/A & $1.00 \mathrm{E}-04$ & N/A & N/A & N/A & N/A \\
\hline $\mathrm{Te}$ & $7.00 \mathrm{E}-03$ & $\mathrm{~N} / \mathrm{A}$ & N/A & N/A & N/A & N/A & N/A \\
\hline Th & N/A & N/A & N/A & $2.90 \mathrm{E}+00$ & $2.30 \mathrm{E}-04$ & N/A & N/A \\
\hline $\mathrm{Tl}$ & N/A & N/A & $4.00 \mathrm{E}-02$ & N/A & N/A & N/A & N/A \\
\hline $\mathrm{U}$ & N/A & N/A & N/A & $1.60 \mathrm{E}+00$ & $3.90 \mathrm{E}-04$ & N/A & N/A \\
\hline W & N/A & N/A & $4.00 \mathrm{E}-02$ & N/A & N/A & N/A & N/A \\
\hline
\end{tabular}


Table 2.10. (contd)

\begin{tabular}{cccccccc}
\hline Element & Mean & STD & Median & GSD & GM & $5 \%$ & $95 \%$ \\
\hline $\mathrm{Y}$ & N/A & N/A & $1.00 \mathrm{E}-03$ & N/A & N/A & N/A & N/A \\
$\mathrm{Zn}$ & N/A & N/A & N/A & $3.20 \mathrm{E}+00$ & $1.60 \mathrm{E}-01$ & N/A & N/A \\
$\mathrm{Zr}$ & $1.20 \mathrm{E}-06$ & N/A & N/A & N/A & N/A & N/A & N/A \\
\hline GM $=$ Geometric mean. & & & & \\
GSD $=$ Geometric Standard Deviation. \\
N/A $=$ Not available in the references reviewed. \\
STD $=$ Standard Deviation.
\end{tabular}

\subsubsection{CLFMK Feed to Milk Transfer Factor}

The feed-to-milk transfer factor is the ratio of chemical concentration in milk to the daily intake of the chemical by the milk animal.

Cow milk is the predominant milk consumed in the United States. The large majority of milk transfer factors are available for cow milk. If milk from another animal is assumed to be ingested, the user should perform a literature review to determine the applicability of cow milk transfer factor values.

The units and range for this parameter in Gv2 are: day/L (0 to 100,000).

To tabulate transfer factors for feed-to-milk, sources considered to be the best available references were reviewed. The goal was to determine mean, median, geometric standard deviation, geometric mean, $5^{\text {th }}$ percentile, and $95^{\text {th }}$ percentile. Selection priority was given to IAEA (2010), Howard et al. (2009), and Staven et al. (2003), with a preference for lognormality in the datasets. Some of these values could be determined mathematically. In these cases, statistical calculations were performed, according to methods described in Strom and Stansbury (2000). The full set of data reviewed to determine the values tabulated in this section are provided in Appendix B.

\subsubsection{Additional Sources of Information}

The references that were reviewed contain additional information regarding data completeness, data limitations, and application of values to environmental models.

There are numerous chemicals for which no milk transfer factors are available. The user can refer to Staven et al. (2003) for an approach to approximate values for non-tabulated chemicals. The user is encouraged to review additional research on transfer factors in the literature, as well. 
2.1.15.2 CLFMK Feed to Milk Transfer Factor Tables

Table 2.11. CLFMK Feed to Milk Transfer Factor

\begin{tabular}{|c|c|c|c|c|c|c|c|}
\hline Element & Mean & STD & Median & GSD & GM & $5 \%$ & $95 \%$ \\
\hline $\mathrm{Ac}$ & $\mathrm{N} / \mathrm{A}$ & N/A & $2.00 \mathrm{E}-05$ & $\mathrm{~N} / \mathrm{A}$ & N/A & N/A & N/A \\
\hline $\mathrm{Ag}$ & N/A & N/A & $5.00 \mathrm{E}-05$ & N/A & N/A & $\mathrm{N} / \mathrm{A}$ & N/A \\
\hline $\mathrm{Al}$ & N/A & N/A & $2.00 \mathrm{E}-04$ & N/A & N/A & N/A & N/A \\
\hline $\mathrm{Am}$ & $4.20 \mathrm{E}-07$ & N/A & N/A & N/A & N/A & N/A & N/A \\
\hline As & N/A & N/A & $6.00 \mathrm{E}-05$ & N/A & N/A & $\mathrm{N} / \mathrm{A}$ & N/A \\
\hline $\mathrm{Au}$ & N/A & N/A & $5.50 \mathrm{E}-06$ & N/A & N/A & $\mathrm{N} / \mathrm{A}$ & N/A \\
\hline $\mathrm{Ba}$ & $1.60 \mathrm{E}-04$ & N/A & N/A & $2.70 \mathrm{E}+00$ & N/A & N/A & N/A \\
\hline $\mathrm{Be}$ & 8.30E-07 & N/A & N/A & N/A & N/A & N/A & N/A \\
\hline $\mathrm{Bi}$ & N/A & N/A & $5.00 \mathrm{E}-04$ & N/A & N/A & N/A & N/A \\
\hline $\mathrm{Br}$ & N/A & N/A & $2.00 \mathrm{E}-02$ & N/A & N/A & N/A & N/A \\
\hline $\mathrm{Ca}$ & $1.00 \mathrm{E}-02$ & N/A & N/A & $1.70 \mathrm{E}+00$ & N/A & $\mathrm{N} / \mathrm{A}$ & N/A \\
\hline $\mathrm{Cd}$ & N/A & N/A & $1.00 \mathrm{E}-03$ & N/A & N/A & $\mathrm{N} / \mathrm{A}$ & N/A \\
\hline $\mathrm{Cd}$ & $1.90 \mathrm{E}-04$ & N/A & N/A & $1.50 \mathrm{E}+01$ & N/A & N/A & N/A \\
\hline $\mathrm{Ce}$ & $2.00 \mathrm{E}-05$ & N/A & N/A & $5.80 \mathrm{E}+00$ & $3.25 \mathrm{E}-05$ & 7.94E-06 & $1.33 \mathrm{E}-04$ \\
\hline $\mathrm{Cf}$ & N/A & N/A & $7.50 \mathrm{E}-07$ & N/A & $\mathrm{N} / \mathrm{A}$ & N/A & N/A \\
\hline $\mathrm{Cl}$ & N/A & N/A & $1.50 \mathrm{E}-02$ & N/A & N/A & N/A & N/A \\
\hline $\mathrm{Cm}$ & N/A & N/A & $9.60 \mathrm{E}-05$ & N/A & N/A & N/A & N/A \\
\hline $\mathrm{Co}$ & $1.30 \mathrm{E}-04$ & $1.10 \mathrm{E}-04$ & $9.92 \mathrm{E}-05$ & $2.09 \mathrm{E}+00$ & $9.92 \mathrm{E}-05$ & $2.96 \mathrm{E}-05$ & $3.32 \mathrm{E}-04$ \\
\hline $\mathrm{Cr}$ & N/A & N/A & N/A & $2.60 \mathrm{E}+01$ & $4.30 \mathrm{E}-04$ & N/A & N/A \\
\hline Cs & $4.48 \mathrm{E}-03$ & $3.05 \mathrm{E}-03$ & $3.70 \mathrm{E}-03$ & $1.85 \mathrm{E}+00$ & $3.70 \mathrm{E}-03$ & $1.34 \mathrm{E}-03$ & $1.02 \mathrm{E}-02$ \\
\hline $\mathrm{Cu}$ & N/A & N/A & $2.00 \mathrm{E}-03$ & N/A & N/A & N/A & N/A \\
\hline Dy & N/A & N/A & $2.00 \mathrm{E}-05$ & N/A & N/A & N/A & N/A \\
\hline Er & N/A & N/A & $2.00 \mathrm{E}-05$ & N/A & N/A & N/A & N/A \\
\hline $\mathrm{Eu}$ & N/A & N/A & $3.00 \mathrm{E}-05$ & N/A & N/A & N/A & N/A \\
\hline $\mathrm{F}$ & N/A & N/A & $1.00 \mathrm{E}-03$ & N/A & N/A & $\mathrm{N} / \mathrm{A}$ & N/A \\
\hline $\mathrm{Fe}$ & N/A & N/A & N/A & $2.00 \mathrm{E}+00$ & $3.50 \mathrm{E}-04$ & N/A & N/A \\
\hline $\mathrm{Ga}$ & N/A & N/A & $5.00 \mathrm{E}-05$ & N/A & $\mathrm{N} / \mathrm{A}$ & N/A & N/A \\
\hline $\mathrm{Gd}$ & N/A & N/A & $3.00 \mathrm{E}-05$ & N/A & N/A & N/A & N/A \\
\hline Hf & N/A & N/A & $5.50 \mathrm{E}-07$ & N/A & N/A & $\mathrm{N} / \mathrm{A}$ & N/A \\
\hline $\mathrm{Hg}$ & N/A & N/A & $4.50 \mathrm{E}-04$ & $\mathrm{~N} / \mathrm{A}$ & N/A & N/A & N/A \\
\hline Ho & N/A & N/A & $3.00 \mathrm{E}-05$ & N/A & N/A & N/A & N/A \\
\hline I & N/A & N/A & N/A & $2.40 \mathrm{E}+00$ & $5.40 \mathrm{E}-03$ & N/A & N/A \\
\hline In & N/A & N/A & $2.00 \mathrm{E}-04$ & N/A & N/A & $\mathrm{N} / \mathrm{A}$ & N/A \\
\hline $\mathrm{Ir}$ & N/A & N/A & $2.00 \mathrm{E}-06$ & N/A & N/A & N/A & N/A \\
\hline $\mathrm{K}$ & N/A & N/A & $7.20 \mathrm{E}-03$ & N/A & N/A & N/A & N/A \\
\hline $\mathrm{La}$ & N/A & N/A & $2.00 \mathrm{E}-05$ & N/A & N/A & N/A & N/A \\
\hline $\mathrm{Mg}$ & N/A & N/A & $3.90 \mathrm{E}-03$ & N/A & N/A & N/A & N/A \\
\hline $\mathrm{Mn}$ & N/A & N/A & N/A & $4.90 \mathrm{E}+00$ & $4.10 \mathrm{E}-05$ & N/A & N/A \\
\hline Mo & N/A & N/A & N/A & $2.30 \mathrm{E}+00$ & $1.10 \mathrm{E}-03$ & N/A & N/A \\
\hline $\mathrm{N}$ & N/A & N/A & $2.50 \mathrm{E}-02$ & N/A & N/A & N/A & N/A \\
\hline $\mathrm{Na}$ & $1.60 \mathrm{E}-02$ & $1.50 \mathrm{E}-02$ & $1.17 \mathrm{E}-02$ & $2.21 \mathrm{E}+00$ & $1.17 \mathrm{E}-02$ & $3.16 \mathrm{E}-03$ & $4.31 \mathrm{E}-02$ \\
\hline $\mathrm{Nb}$ & N/A & N/A & 4.10E-07 & N/A & N/A & N/A & N/A \\
\hline $\mathrm{Nd}$ & N/A & N/A & $3.00 \mathrm{E}-05$ & N/A & N/A & N/A & N/A \\
\hline
\end{tabular}


Table 2.11. (contd)

\begin{tabular}{|c|c|c|c|c|c|c|c|}
\hline Element & Mean & STD & Median & GSD & GM & $5 \%$ & $95 \%$ \\
\hline $\mathrm{Ni}$ & $\mathrm{N} / \mathrm{A}$ & $\mathrm{N} / \mathrm{A}$ & N/A & $6.50 \mathrm{E}-04$ & $9.50 \mathrm{E}-04$ & $\mathrm{~N} / \mathrm{A}$ & $\mathrm{N} / \mathrm{A}$ \\
\hline $\mathrm{Np}$ & N/A & N/A & $5.00 \mathrm{E}-06$ & N/A & N/A & $\mathrm{N} / \mathrm{A}$ & N/A \\
\hline Os & N/A & N/A & $5.00 \mathrm{E}-03$ & N/A & N/A & $\mathrm{N} / \mathrm{A}$ & N/A \\
\hline $\mathrm{P}$ & $2.00 \mathrm{E}-02$ & N/A & N/A & N/A & N/A & N/A & N/A \\
\hline $\mathrm{Pa}$ & N/A & N/A & $5.00 \mathrm{E}-06$ & N/A & N/A & N/A & N/A \\
\hline $\mathrm{Pb}$ & N/A & N/A & N/A & $1.00 \mathrm{E}+00$ & $1.90 \mathrm{E}-04$ & N/A & N/A \\
\hline$P d$ & $6.00 \mathrm{E}-03$ & N/A & N/A & N/A & N/A & N/A & N/A \\
\hline $\mathrm{Pm}$ & N/A & N/A & $3.00 \mathrm{E}-05$ & N/A & N/A & N/A & N/A \\
\hline Po & $2.30 \mathrm{E}-04$ & $9.70 \mathrm{E}-05$ & $2.12 \mathrm{E}-04$ & $1.50 \mathrm{E}+00$ & $2.12 \mathrm{E}-04$ & $1.09 \mathrm{E}-04$ & $4.12 \mathrm{E}-04$ \\
\hline $\operatorname{Pr}$ & N/A & N/A & $3.00 \mathrm{E}-05$ & N/A & N/A & N/A & N/A \\
\hline $\mathrm{Pu}$ & $1.00 \mathrm{E}-05$ & N/A & N/A & N/A & N/A & $\mathrm{N} / \mathrm{A}$ & N/A \\
\hline $\mathrm{Ra}$ & N/A & N/A & N/A & $2.30 \mathrm{E}+00$ & $3.80 \mathrm{E}-04$ & $\mathrm{~N} / \mathrm{A}$ & N/A \\
\hline $\mathrm{Rb}$ & N/A & N/A & $1.20 \mathrm{E}-02$ & N/A & N/A & N/A & N/A \\
\hline $\mathrm{Re}$ & N/A & N/A & $1.50 \mathrm{E}-03$ & N/A & N/A & $\mathrm{N} / \mathrm{A}$ & N/A \\
\hline $\mathrm{Rh}$ & N/A & N/A & $1.00 \mathrm{E}-02$ & N/A & N/A & N/A & N/A \\
\hline $\mathrm{Ru}$ & $3.60 \mathrm{E}-05$ & $5.30 \mathrm{E}-05$ & $2.02 \mathrm{E}-05$ & $2.93 \mathrm{E}+00$ & $2.02 \mathrm{E}-05$ & $3.46 \mathrm{E}-06$ & $1.18 \mathrm{E}-04$ \\
\hline $\mathrm{S}$ & 4.30E-02 & $1.90 \mathrm{E}-02$ & $3.93 \mathrm{E}-02$ & $1.53 \mathrm{E}+00$ & $3.93 \mathrm{E}-02$ & $1.96 \mathrm{E}-02$ & $7.88 \mathrm{E}-02$ \\
\hline $\mathrm{Sb}$ & N/A & N/A & N/A & $2.50 \mathrm{E}+00$ & $3.80 \mathrm{E}-05$ & N/A & N/A \\
\hline $\mathrm{Sc}$ & N/A & N/A & $5.00 \mathrm{E}-06$ & N/A & $\mathrm{N} / \mathrm{A}$ & N/A & N/A \\
\hline $\mathrm{Se}$ & N/A & N/A & N/A & $2.10 \mathrm{E}+00$ & $4.00 \mathrm{E}-03$ & N/A & N/A \\
\hline $\mathrm{Si}$ & N/A & N/A & $2.00 \mathrm{E}-05$ & N/A & N/A & $\mathrm{N} / \mathrm{A}$ & N/A \\
\hline $\mathrm{Sm}$ & N/A & N/A & $2.00 \mathrm{E}-05$ & N/A & N/A & $\mathrm{N} / \mathrm{A}$ & N/A \\
\hline $\mathrm{Sm}$ & N/A & N/A & $3.00 \mathrm{E}-05$ & N/A & N/A & $\mathrm{N} / \mathrm{A}$ & N/A \\
\hline $\mathrm{Sn}$ & N/A & N/A & $1.00 \mathrm{E}-03$ & N/A & N/A & $\mathrm{N} / \mathrm{A}$ & N/A \\
\hline $\mathrm{Sr}$ & $\mathrm{N} / \mathrm{A}$ & N/A & N/A & $1.70 \mathrm{E}+00$ & $1.30 \mathrm{E}-03$ & $\mathrm{~N} / \mathrm{A}$ & N/A \\
\hline $\mathrm{Ta}$ & N/A & N/A & 4.10E-07 & N/A & N/A & N/A & N/A \\
\hline $\mathrm{Tb}$ & $\mathrm{N} / \mathrm{A}$ & N/A & $3.00 \mathrm{E}-05$ & $\mathrm{~N} / \mathrm{A}$ & $\mathrm{N} / \mathrm{A}$ & $\mathrm{N} / \mathrm{A}$ & N/A \\
\hline $\mathrm{Tc}$ & N/A & N/A & $1.40 \mathrm{E}-04$ & N/A & $\mathrm{N} / \mathrm{A}$ & N/A & N/A \\
\hline $\mathrm{Te}$ & $3.40 \mathrm{E}-04$ & N/A & $4.50 \mathrm{E}-04$ & $2.40 \mathrm{E}+00$ & N/A & $\mathrm{N} / \mathrm{A}$ & N/A \\
\hline Th & N/A & N/A & $5.00 \mathrm{E}-06$ & N/A & $\mathrm{N} / \mathrm{A}$ & $\mathrm{N} / \mathrm{A}$ & N/A \\
\hline $\mathrm{Ti}$ & N/A & N/A & $1.00 \mathrm{E}-02$ & N/A & N/A & N/A & N/A \\
\hline $\mathrm{Tl}$ & N/A & N/A & $2.00 \mathrm{E}-03$ & N/A & $\mathrm{N} / \mathrm{A}$ & $\mathrm{N} / \mathrm{A}$ & N/A \\
\hline $\mathrm{U}$ & N/A & N/A & N/A & $3.50 \mathrm{E}+00$ & $1.80 \mathrm{E}-03$ & N/A & N/A \\
\hline $\mathrm{V}$ & N/A & N/A & $2.00 \mathrm{E}-05$ & N/A & N/A & N/A & N/A \\
\hline W & N/A & N/A & N/A & $3.10 \mathrm{E}+00$ & $1.90 \mathrm{E}-04$ & N/A & N/A \\
\hline $\mathrm{Y}$ & N/A & N/A & $2.00 \mathrm{E}-05$ & N/A & N/A & N/A & N/A \\
\hline $\mathrm{Zn}$ & N/A & N/A & N/A & $3.90 \mathrm{E}+00$ & $2.70 \mathrm{E}-03$ & N/A & N/A \\
\hline $\mathrm{Zr}$ & N/A & N/A & N/A & $4.30 \mathrm{E}+00$ & $3.60 \mathrm{E}-06$ & N/A & N/A \\
\hline \multicolumn{8}{|c|}{$\begin{array}{l}\text { GM }=\text { Geometric mean. } \\
\text { GSD }=\text { Geometric Standard Deviation. } \\
\text { N/A }=\text { Not available in the references reviewed. } \\
\text { STD }=\text { Standard Deviation. }\end{array}$} \\
\hline
\end{tabular}




\subsubsection{CLFPL Feed to Poultry and CLFEG Egg Transfer Factor}

These parameters characterize the ratio of constituent concentrations in poultry (CLFPL) and in eggs (CLFEG) to the concentration in the feed. The predominant type of egg consumed in the United States is that of a chicken. The majority of available transfer factor information for chicken poultry and eggs. If another type of poultry and egg is assumed to be ingested, the user should review the literature to determine if any transfer factors may need to be modified to reflect the different type of domesticated bird.

The units and range for these parameters in Gv2 are: day $/ \mathrm{kg}(0$ to 100,000$)$.

To tabulate transfer factors for feed-to-poultry and egg, sources considered to be the best available references were reviewed. The goal was to determine mean, median, geometric standard deviation, geometric mean, $5^{\text {th }}$ percentile, and $95^{\text {th }}$ percentile. Selection priority was given to IAEA (2010), Howard et al. (2009), and Napier et al. (2012), with a preference for lognormality in the datasets. Some of these values could be determined mathematically. In these cases, statistical calculations were performed, according to methods described in Strom and Stansbury (2000). The full set of data reviewed to determine the values tabulated in this section are provided in Appendix B.

\subsubsection{Additional Sources of Information}

There are numerous chemicals for which no poultry or egg transfer factors are available. The user can refer to Staven et al. (2003) for an approach to approximate values for non-tabulated chemicals. The user is encouraged to review additional research on transfer factors in the literature, as well.

\subsubsection{CLFPL Feed to Poultry and CLFEG Feed to Egg Transfer Factor Tables}

Table 2.12. CLFPL Feed to Poultry Transfer Factor

\begin{tabular}{cccccccc}
\hline Element & Mean & STD & Median & GSD & GM & $5 \%$ & $95 \%$ \\
\hline $\mathrm{Ac}$ & N/A & N/A & $6.00 \mathrm{E}-03$ & N/A & N/A & N/A & N/A \\
$\mathrm{Ag}$ & N/A & N/A & $2.00 \mathrm{E}+00$ & N/A & N/A & N/A & N/A \\
$\mathrm{Am}$ & N/A & N/A & $6.00 \mathrm{E}-03$ & N/A & N/A & N/A & N/A \\
$\mathrm{As}$ & N/A & N/A & $8.30 \mathrm{E}-01$ & N/A & N/A & N/A & N/A \\
$\mathrm{Au}$ & $\mathrm{N} / \mathrm{A}$ & $\mathrm{N} / \mathrm{A}$ & $\mathrm{N} / \mathrm{A}$ & $\mathrm{N} / \mathrm{A}$ & $1.00 \mathrm{E}+00$ & N/A & N/A \\
$\mathrm{Ba}$ & $1.90 \mathrm{E}-02$ & N/A & N/A & N/A & N/A & N/A & N/A \\
$\mathrm{Be}$ & N/A & N/A & $4.00 \mathrm{E}-01$ & N/A & N/A & N/A & N/A \\
$\mathrm{Bi}$ & N/A & N/A & N/A & N/A & $9.00 \mathrm{E}-02$ & N/A & N/A \\
$\mathrm{Br}$ & N/A & N/A & $4.00 \mathrm{E}-03$ & N/A & N/A & N/A & N/A \\
$\mathrm{Ca}$ & $4.40 \mathrm{E}-02$ & N/A & N/A & N/A & N/A & N/A & N/A \\
$\mathrm{Cd}$ & $1.70 \mathrm{E}+00$ & N/A & N/A & N/A & N/A & N/A & N/A \\
$\mathrm{Ce}$ & N/A & N/A & $2.00 \mathrm{E}-03$ & N/A & N/A & N/A & N/A \\
$\mathrm{Cf}$ & N/A & N/A & $4.00 \mathrm{E}-03$ & N/A & N/A & N/A & N/A \\
$\mathrm{Cl}$ & N/A & N/A & N/A & N/A & $3.00 \mathrm{E}-02$ & N/A & N/A \\
$\mathrm{Cm}$ & N/A & N/A & $4.00 \mathrm{E}-03$ & N/A & N/A & N/A & N/A \\
$\mathrm{Co}$ & $9.70 \mathrm{E}-01$ & N/A & N/A & N/A & N/A & N/A & N/A
\end{tabular}


Table 2.12. (contd)

\begin{tabular}{|c|c|c|c|c|c|c|c|}
\hline Element & Mean & STD & Median & GSD & GM & $5 \%$ & $95 \%$ \\
\hline $\mathrm{Cr}$ & $\mathrm{N} / \mathrm{A}$ & $\mathrm{N} / \mathrm{A}$ & $2.00 \mathrm{E}-01$ & $\mathrm{~N} / \mathrm{A}$ & $\mathrm{N} / \mathrm{A}$ & $\mathrm{N} / \mathrm{A}$ & $\mathrm{N} / \mathrm{A}$ \\
\hline Cs & $3.00 \mathrm{E}+00$ & $1.30 \mathrm{E}+00$ & $2.75 \mathrm{E}+00$ & $1.51 \mathrm{E}+00$ & $2.75 E+00$ & $1.39 \mathrm{E}+00$ & $5.45 \mathrm{E}+00$ \\
\hline $\mathrm{Cu}$ & $\mathrm{N} / \mathrm{A}$ & N/A & $5.00 \mathrm{E}-01$ & N/A & N/A & N/A & N/A \\
\hline Dy & N/A & N/A & $2.00 \mathrm{E}-03$ & N/A & $\mathrm{N} / \mathrm{A}$ & N/A & N/A \\
\hline Er & N/A & N/A & $2.00 \mathrm{E}-03$ & $\mathrm{~N} / \mathrm{A}$ & $\mathrm{N} / \mathrm{A}$ & N/A & N/A \\
\hline $\mathrm{Eu}$ & N/A & N/A & $2.00 \mathrm{E}-03$ & $\mathrm{~N} / \mathrm{A}$ & N/A & N/A & N/A \\
\hline $\mathrm{F}$ & N/A & N/A & N/A & N/A & $1.40 \mathrm{E}-02$ & N/A & N/A \\
\hline $\mathrm{Fe}$ & N/A & N/A & $1.00 \mathrm{E}+00$ & N/A & N/A & N/A & N/A \\
\hline $\mathrm{Ga}$ & N/A & N/A & $8.00 \mathrm{E}-01$ & $\mathrm{~N} / \mathrm{A}$ & $\mathrm{N} / \mathrm{A}$ & N/A & $\mathrm{N} / \mathrm{A}$ \\
\hline $\mathrm{Gd}$ & N/A & N/A & $2.00 \mathrm{E}-03$ & $\mathrm{~N} / \mathrm{A}$ & $\mathrm{N} / \mathrm{A}$ & N/A & N/A \\
\hline $\mathrm{Hf}$ & N/A & N/A & $6.00 \mathrm{E}-04$ & N/A & N/A & N/A & N/A \\
\hline $\mathrm{Hg}$ & N/A & N/A & $3.00 \mathrm{E}-02$ & $\mathrm{~N} / \mathrm{A}$ & $\mathrm{N} / \mathrm{A}$ & N/A & N/A \\
\hline Ho & N/A & N/A & $2.00 \mathrm{E}-03$ & N/A & N/A & N/A & N/A \\
\hline I & $1.00 \mathrm{E}-02$ & $5.60 \mathrm{E}-03$ & $8.73 \mathrm{E}-03$ & $1.69 \mathrm{E}+00$ & $8.73 \mathrm{E}-03$ & $3.70 \mathrm{E}-03$ & $2.06 \mathrm{E}-02$ \\
\hline In & N/A & $\mathrm{N} / \mathrm{A}$ & $8.00 \mathrm{E}-01$ & N/A & N/A & $\mathrm{N} / \mathrm{A}$ & N/A \\
\hline $\mathrm{Ir}$ & N/A & N/A & $2.00 \mathrm{E}+00$ & $\mathrm{~N} / \mathrm{A}$ & $\mathrm{N} / \mathrm{A}$ & N/A & N/A \\
\hline $\mathrm{K}$ & N/A & N/A & $4.00 \mathrm{E}-01$ & N/A & N/A & N/A & N/A \\
\hline $\mathrm{La}$ & N/A & N/A & $1.00 \mathrm{E}-01$ & N/A & N/A & N/A & N/A \\
\hline $\mathrm{Mg}$ & $\mathrm{N} / \mathrm{A}$ & N/A & $3.00 \mathrm{E}-02$ & N/A & $\mathrm{N} / \mathrm{A}$ & N/A & N/A \\
\hline $\mathrm{Mn}$ & $1.90 \mathrm{E}-03$ & N/A & $\mathrm{N} / \mathrm{A}$ & N/A & $\mathrm{N} / \mathrm{A}$ & N/A & $\mathrm{N} / \mathrm{A}$ \\
\hline Mo & N/A & N/A & $1.80 \mathrm{E}-02$ & $\mathrm{~N} / \mathrm{A}$ & N/A & N/A & $\mathrm{N} / \mathrm{A}$ \\
\hline $\mathrm{N}$ & N/A & N/A & N/A & N/A & $9.80 \mathrm{E}-02$ & N/A & N/A \\
\hline $\mathrm{Na}$ & $7.00 \mathrm{E}+00$ & N/A & N/A & N/A & N/A & N/A & N/A \\
\hline $\mathrm{Nb}$ & N/A & N/A & $3.10 \mathrm{E}-04$ & N/A & N/A & N/A & N/A \\
\hline $\mathrm{Nb}$ & N/A & N/A & $3.00 \mathrm{E}-04$ & $\mathrm{~N} / \mathrm{A}$ & $\mathrm{N} / \mathrm{A}$ & N/A & $\mathrm{N} / \mathrm{A}$ \\
\hline $\mathrm{Nb}$ & $3.00 \mathrm{E}-04$ & N/A & N/A & N/A & $\mathrm{N} / \mathrm{A}$ & N/A & N/A \\
\hline $\mathrm{Nd}$ & N/A & N/A & $2.00 \mathrm{E}-03$ & $\mathrm{~N} / \mathrm{A}$ & $\mathrm{N} / \mathrm{A}$ & N/A & N/A \\
\hline $\mathrm{Ni}$ & N/A & N/A & $1.00 \mathrm{E}-03$ & $\mathrm{~N} / \mathrm{A}$ & N/A & N/A & N/A \\
\hline $\mathrm{Np}$ & N/A & N/A & $6.00 \mathrm{E}-03$ & $\mathrm{~N} / \mathrm{A}$ & N/A & N/A & N/A \\
\hline Os & N/A & N/A & N/A & $\mathrm{N} / \mathrm{A}$ & $8.40 \mathrm{E}-02$ & N/A & N/A \\
\hline $\mathrm{P}$ & N/A & N/A & $1.90 \mathrm{E}-01$ & N/A & N/A & N/A & $\mathrm{N} / \mathrm{A}$ \\
\hline $\mathrm{Pa}$ & N/A & N/A & $6.00 \mathrm{E}-03$ & N/A & N/A & N/A & $\mathrm{N} / \mathrm{A}$ \\
\hline $\mathrm{Pb}$ & N/A & N/A & $8.00 \mathrm{E}-01$ & N/A & N/A & N/A & N/A \\
\hline $\mathrm{Pd}$ & N/A & N/A & $3.00 \mathrm{E}-04$ & N/A & N/A & N/A & $\mathrm{N} / \mathrm{A}$ \\
\hline $\mathrm{Pm}$ & N/A & N/A & $2.00 \mathrm{E}-03$ & $\mathrm{~N} / \mathrm{A}$ & $\mathrm{N} / \mathrm{A}$ & N/A & $\mathrm{N} / \mathrm{A}$ \\
\hline Po & $2.40 \mathrm{E}+00$ & N/A & N/A & N/A & N/A & N/A & $\mathrm{N} / \mathrm{A}$ \\
\hline $\operatorname{Pr}$ & N/A & N/A & $2.00 \mathrm{E}-03$ & $\mathrm{~N} / \mathrm{A}$ & $\mathrm{N} / \mathrm{A}$ & N/A & $\mathrm{N} / \mathrm{A}$ \\
\hline $\mathrm{Pu}$ & N/A & N/A & $3.00 \mathrm{E}-03$ & N/A & N/A & N/A & $\mathrm{N} / \mathrm{A}$ \\
\hline $\mathrm{Ra}$ & N/A & N/A & $3.00 \mathrm{E}-02$ & N/A & $\mathrm{N} / \mathrm{A}$ & N/A & N/A \\
\hline $\mathrm{Rb}$ & N/A & N/A & $2.00 \mathrm{E}+00$ & $\mathrm{~N} / \mathrm{A}$ & $\mathrm{N} / \mathrm{A}$ & N/A & $\mathrm{N} / \mathrm{A}$ \\
\hline $\operatorname{Re}$ & N/A & N/A & $4.00 \mathrm{E}-02$ & N/A & $\mathrm{N} / \mathrm{A}$ & N/A & $\mathrm{N} / \mathrm{A}$ \\
\hline $\mathrm{Rh}$ & N/A & N/A & $2.00 \mathrm{E}+00$ & $\mathrm{~N} / \mathrm{A}$ & N/A & N/A & $\mathrm{N} / \mathrm{A}$ \\
\hline $\mathrm{Ru}$ & $\mathrm{N} / \mathrm{A}$ & N/A & $7.00 \mathrm{E}-03$ & $\mathrm{~N} / \mathrm{A}$ & $\mathrm{N} / \mathrm{A}$ & N/A & N/A \\
\hline $\mathrm{S}$ & N/A & N/A & $\mathrm{N} / \mathrm{A}$ & N/A & $2.30 \mathrm{E}+00$ & N/A & $\mathrm{N} / \mathrm{A}$ \\
\hline $\mathrm{Sb}$ & N/A & N/A & $6.00 \mathrm{E}-03$ & N/A & N/A & N/A & $\mathrm{N} / \mathrm{A}$ \\
\hline $\mathrm{Sc}$ & $\mathrm{N} / \mathrm{A}$ & N/A & $4.00 \mathrm{E}-03$ & N/A & N/A & N/A & N/A \\
\hline $\mathrm{Se}$ & $1.26 \mathrm{E}+01$ & $1.06 \mathrm{E}+01$ & $9.64 \mathrm{E}+00$ & $2.08 \mathrm{E}+00$ & $9.64 \mathrm{E}+00$ & $2.89 \mathrm{E}+00$ & $3.21 \mathrm{E}+01$ \\
\hline
\end{tabular}


Table 2.12. (contd)

\begin{tabular}{|c|c|c|c|c|c|c|c|}
\hline Element & Mean & STD & Median & GSD & GM & $5 \%$ & $95 \%$ \\
\hline $\mathrm{Si}$ & $\mathrm{N} / \mathrm{A}$ & N/A & $8.00 \mathrm{E}-01$ & N/A & $\mathrm{N} / \mathrm{A}$ & $\mathrm{N} / \mathrm{A}$ & $\mathrm{N} / \mathrm{A}$ \\
\hline $\mathrm{Sm}$ & N/A & N/A & $2.00 \mathrm{E}-03$ & N/A & N/A & N/A & N/A \\
\hline $\mathrm{Sn}$ & N/A & N/A & $8.00 \mathrm{E}-01$ & N/A & N/A & N/A & N/A \\
\hline $\mathrm{Sr}$ & N/A & N/A & $8.00 \mathrm{E}-02$ & N/A & N/A & N/A & N/A \\
\hline $\mathrm{Sr}$ & $2.30 \mathrm{E}-02$ & $1.20 \mathrm{E}-02$ & 2.04E-02 & $1.63 \mathrm{E}+00$ & $2.04 \mathrm{E}-02$ & $9.10 \mathrm{E}-03$ & $4.57 \mathrm{E}-02$ \\
\hline $\mathrm{Ta}$ & N/A & N/A & $3.00 \mathrm{E}-04$ & N/A & N/A & N/A & N/A \\
\hline $\mathrm{Tb}$ & N/A & N/A & $2.00 \mathrm{E}-03$ & N/A & N/A & N/A & N/A \\
\hline $\mathrm{Tc}$ & N/A & N/A & $3.00 \mathrm{E}-02$ & N/A & N/A & N/A & $\mathrm{N} / \mathrm{A}$ \\
\hline $\mathrm{Te}$ & N/A & N/A & $8.50 \mathrm{E}-02$ & N/A & N/A & N/A & N/A \\
\hline Th & N/A & N/A & $6.00 \mathrm{E}-03$ & N/A & $\mathrm{N} / \mathrm{A}$ & N/A & N/A \\
\hline $\mathrm{Tl}$ & N/A & N/A & $8.00 \mathrm{E}-01$ & N/A & N/A & N/A & $\mathrm{N} / \mathrm{A}$ \\
\hline $\mathrm{U}$ & $7.50 \mathrm{E}-01$ & N/A & N/A & $3.00 \mathrm{E}-01$ & N/A & N/A & N/A \\
\hline $\mathrm{W}$ & N/A & $\mathrm{N} / \mathrm{A}$ & $2.00 \mathrm{E}-01$ & N/A & N/A & $\mathrm{N} / \mathrm{A}$ & N/A \\
\hline $\mathrm{Y}$ & N/A & N/A & $1.00 \mathrm{E}-02$ & N/A & N/A & N/A & N/A \\
\hline $\mathrm{Zn}$ & 4.70E-01 & $7.90 \mathrm{E}-02$ & 4.63E-01 & $1.18 \mathrm{E}+00$ & 4.63E-01 & $3.52 \mathrm{E}-01$ & $6.10 \mathrm{E}-01$ \\
\hline $\mathrm{Zr}$ & $6.00 \mathrm{E}-05$ & N/A & N/A & N/A & N/A & N/A & N/A \\
\hline \multicolumn{8}{|c|}{$\begin{array}{l}\text { GM }=\text { Geometric mean. } \\
\text { GSD }=\text { Geometric Standard Deviation. } \\
\text { N/A }=\text { Not available in the references reviewed. } \\
\text { STD }=\text { Standard Deviation. }\end{array}$} \\
\hline
\end{tabular}

Table 2.13. CLFEG Feed to Egg Transfer Factor

\begin{tabular}{|c|c|c|c|c|c|c|c|}
\hline Element & Mean & STD & Median & GSD & GM & $5 \%$ & $95 \%$ \\
\hline Ac & N/A & N/A & $2.00 \mathrm{E}-03$ & N/A & N/A & N/A & N/A \\
\hline $\mathrm{Ag}$ & N/A & N/A & $5.00 \mathrm{E}-01$ & N/A & N/A & N/A & N/A \\
\hline $\mathrm{Am}$ & $3.00 \mathrm{E}-03$ & N/A & N/A & N/A & N/A & N/A & N/A \\
\hline As & N/A & N/A & N/A & N/A & $2.60 \mathrm{E}-01$ & N/A & N/A \\
\hline $\mathrm{Au}$ & N/A & N/A & $5.00 \mathrm{E}-01$ & N/A & $\mathrm{N} / \mathrm{A}$ & N/A & N/A \\
\hline $\mathrm{Ba}$ & $8.70 \mathrm{E}-01$ & N/A & N/A & N/A & N/A & N/A & N/A \\
\hline $\mathrm{Be}$ & N/A & N/A & $2.00 \mathrm{E}-02$ & N/A & N/A & N/A & N/A \\
\hline $\mathrm{Bi}$ & N/A & N/A & N/A & N/A & $2.60 \mathrm{E}-01$ & N/A & N/A \\
\hline $\mathrm{Br}$ & N/A & N/A & $1.60 \mathrm{E}+00$ & N/A & N/A & N/A & N/A \\
\hline $\mathrm{Ca}$ & $4.40 \mathrm{E}-01$ & N/A & N/A & N/A & N/A & N/A & N/A \\
\hline $\mathrm{Cd}$ & N/A & N/A & $1.00 \mathrm{E}-01$ & N/A & N/A & N/A & N/A \\
\hline $\mathrm{Ce}$ & $3.10 \mathrm{E}-03$ & N/A & N/A & N/A & N/A & N/A & N/A \\
\hline $\mathrm{Cf}$ & N/A & N/A & $4.00 \mathrm{E}-03$ & N/A & N/A & N/A & N/A \\
\hline $\mathrm{Cl}$ & N/A & N/A & N/A & $\mathrm{N} / \mathrm{A}$ & $2.70 \mathrm{E}+00$ & N/A & N/A \\
\hline $\mathrm{Cm}$ & N/A & N/A & $4.00 \mathrm{E}-03$ & N/A & N/A & N/A & N/A \\
\hline Co & $3.30 \mathrm{E}-02$ & N/A & N/A & N/A & N/A & N/A & N/A \\
\hline $\mathrm{Cr}$ & N/A & N/A & $9.00 \mathrm{E}-01$ & N/A & N/A & N/A & N/A \\
\hline Cs & $4.30 \mathrm{E}-01$ & $1.60 \mathrm{E}-01$ & $4.03 \mathrm{E}-01$ & $1.43 \mathrm{E}+00$ & 4.03E-01 & $2.23 \mathrm{E}-01$ & $7.29 \mathrm{E}-01$ \\
\hline $\mathrm{Cu}$ & N/A & N/A & $5.00 \mathrm{E}-01$ & N/A & N/A & N/A & N/A \\
\hline Dy & N/A & N/A & $4.00 \mathrm{E}-05$ & N/A & N/A & N/A & N/A \\
\hline $\mathrm{Er}$ & N/A & N/A & $4.00 \mathrm{E}-05$ & N/A & N/A & N/A & N/A \\
\hline $\mathrm{Eu}$ & N/A & N/A & $4.00 \mathrm{E}-05$ & N/A & N/A & N/A & N/A \\
\hline
\end{tabular}


Table 2.13. (contd)

\begin{tabular}{|c|c|c|c|c|c|c|c|}
\hline Element & Mean & STD & Median & GSD & GM & $5 \%$ & $95 \%$ \\
\hline $\mathrm{Fe}$ & $1.80 \mathrm{E}+00$ & N/A & $\mathrm{N} / \mathrm{A}$ & $8.50 \mathrm{E}-01$ & $\mathrm{~N} / \mathrm{A}$ & $\mathrm{N} / \mathrm{A}$ & N/A \\
\hline $\mathrm{Ga}$ & N/A & N/A & $1.00 \mathrm{E}+00$ & N/A & N/A & N/A & N/A \\
\hline $\mathrm{Gd}$ & N/A & $\mathrm{N} / \mathrm{A}$ & $4.00 \mathrm{E}-05$ & N/A & N/A & N/A & N/A \\
\hline $\mathrm{Hf}$ & N/A & N/A & $2.00 \mathrm{E}-04$ & N/A & N/A & N/A & N/A \\
\hline $\mathrm{Hg}$ & N/A & N/A & $\mathrm{N} / \mathrm{A}$ & N/A & $5.00 \mathrm{E}-01$ & N/A & N/A \\
\hline Ho & $\mathrm{N} / \mathrm{A}$ & N/A & $4.00 \mathrm{E}-05$ & N/A & $\mathrm{N} / \mathrm{A}$ & $\mathrm{N} / \mathrm{A}$ & N/A \\
\hline I & $2.40 \mathrm{E}+00$ & $5.70 \mathrm{E}-01$ & $2.34 \mathrm{E}+00$ & $1.26 \mathrm{E}+00$ & $2.34 \mathrm{E}+00$ & $1.59 \mathrm{E}+00$ & $3.43 \mathrm{E}+00$ \\
\hline In & N/A & N/A & $1.00 \mathrm{E}+00$ & N/A & N/A & N/A & N/A \\
\hline $\mathrm{Ir}$ & N/A & N/A & $1.00 \mathrm{E}-01$ & N/A & N/A & $\mathrm{N} / \mathrm{A}$ & $\mathrm{N} / \mathrm{A}$ \\
\hline $\mathrm{K}$ & N/A & N/A & $1.00 \mathrm{E}+00$ & N/A & N/A & N/A & N/A \\
\hline $\mathrm{La}$ & N/A & N/A & $9.00 \mathrm{E}-03$ & N/A & N/A & N/A & N/A \\
\hline $\mathrm{Mg}$ & N/A & N/A & $2.00 \mathrm{E}+00$ & N/A & $\mathrm{N} / \mathrm{A}$ & N/A & N/A \\
\hline $\mathrm{Mn}$ & $4.40 \mathrm{E}-02$ & $1.60 \mathrm{E}-02$ & 4.14E-02 & $1.42 \mathrm{E}+00$ & $4.14 \mathrm{E}-02$ & 2.32E-02 & $7.38 \mathrm{E}-02$ \\
\hline Mo & $6.50 \mathrm{E}-01$ & $1.90 \mathrm{E}-01$ & $6.24 \mathrm{E}-01$ & $1.33 \mathrm{E}+00$ & $6.24 \mathrm{E}-01$ & $3.90 \mathrm{E}-01$ & $9.99 \mathrm{E}-01$ \\
\hline $\mathrm{N}$ & N/A & N/A & N/A & N/A & $2.60 \mathrm{E}-01$ & N/A & N/A \\
\hline $\mathrm{Na}$ & N/A & $\mathrm{N} / \mathrm{A}$ & N/A & $1.90 \mathrm{E}+00$ & $4.00 \mathrm{E}+00$ & $\mathrm{~N} / \mathrm{A}$ & $\mathrm{N} / \mathrm{A}$ \\
\hline $\mathrm{Nb}$ & $1.00 \mathrm{E}-03$ & N/A & N/A & N/A & N/A & N/A & N/A \\
\hline $\mathrm{Nd}$ & N/A & N/A & $4.00 \mathrm{E}-05$ & N/A & $\mathrm{N} / \mathrm{A}$ & N/A & N/A \\
\hline $\mathrm{Ni}$ & N/A & $\mathrm{N} / \mathrm{A}$ & $1.00 \mathrm{E}-01$ & $\mathrm{~N} / \mathrm{A}$ & N/A & N/A & $\mathrm{N} / \mathrm{A}$ \\
\hline $\mathrm{Np}$ & N/A & $\mathrm{N} / \mathrm{A}$ & $4.00 \mathrm{E}-03$ & N/A & N/A & $\mathrm{N} / \mathrm{A}$ & $\mathrm{N} / \mathrm{A}$ \\
\hline Os & N/A & $\mathrm{N} / \mathrm{A}$ & N/A & N/A & $7.10 \mathrm{E}-02$ & $\mathrm{~N} / \mathrm{A}$ & $\mathrm{N} / \mathrm{A}$ \\
\hline $\mathrm{P}$ & $6.40 \mathrm{E}-01$ & N/A & N/A & N/A & N/A & N/A & $\mathrm{N} / \mathrm{A}$ \\
\hline $\mathrm{Pa}$ & N/A & N/A & $4.00 \mathrm{E}-03$ & N/A & $\mathrm{N} / \mathrm{A}$ & N/A & $\mathrm{N} / \mathrm{A}$ \\
\hline $\mathrm{Pb}$ & N/A & N/A & $1.00 \mathrm{E}+00$ & N/A & N/A & N/A & $\mathrm{N} / \mathrm{A}$ \\
\hline $\mathrm{Pd}$ & N/A & $\mathrm{N} / \mathrm{A}$ & $4.00 \mathrm{E}-03$ & N/A & N/A & $\mathrm{N} / \mathrm{A}$ & $\mathrm{N} / \mathrm{A}$ \\
\hline $\mathrm{Pm}$ & N/A & N/A & $4.00 \mathrm{E}-05$ & N/A & N/A & N/A & N/A \\
\hline Po & $3.10 \mathrm{E}+00$ & $\mathrm{~N} / \mathrm{A}$ & N/A & N/A & $\mathrm{N} / \mathrm{A}$ & $\mathrm{N} / \mathrm{A}$ & N/A \\
\hline $\operatorname{Pr}$ & N/A & N/A & $4.00 \mathrm{E}-05$ & N/A & $\mathrm{N} / \mathrm{A}$ & N/A & N/A \\
\hline $\mathrm{Pu}$ & $1.20 \mathrm{E}-03$ & $\mathrm{~N} / \mathrm{A}$ & N/A & N/A & N/A & N/A & $\mathrm{N} / \mathrm{A}$ \\
\hline $\mathrm{Ra}$ & N/A & N/A & N/A & N/A & $3.10 \mathrm{E}-01$ & $\mathrm{~N} / \mathrm{A}$ & $\mathrm{N} / \mathrm{A}$ \\
\hline $\mathrm{Rb}$ & N/A & $\mathrm{N} / \mathrm{A}$ & $3.00 \mathrm{E}+00$ & N/A & N/A & $\mathrm{N} / \mathrm{A}$ & N/A \\
\hline $\operatorname{Re}$ & N/A & N/A & N/A & N/A & $4.20 \mathrm{E}-01$ & $\mathrm{~N} / \mathrm{A}$ & N/A \\
\hline $\mathrm{Rh}$ & N/A & N/A & $1.00 \mathrm{E}-01$ & N/A & N/A & N/A & N/A \\
\hline $\mathrm{Ru}$ & $4.00 \mathrm{E}-03$ & N/A & N/A & N/A & N/A & N/A & $\mathrm{N} / \mathrm{A}$ \\
\hline $\mathrm{S}$ & N/A & $\mathrm{N} / \mathrm{A}$ & N/A & $\mathrm{N} / \mathrm{A}$ & $7.00 \mathrm{E}+00$ & $\mathrm{~N} / \mathrm{A}$ & $\mathrm{N} / \mathrm{A}$ \\
\hline $\mathrm{Sb}$ & N/A & N/A & $7.00 \mathrm{E}-02$ & N/A & N/A & N/A & $\mathrm{N} / \mathrm{A}$ \\
\hline $\mathrm{Sc}$ & N/A & N/A & N/A & N/A & $4.20 \mathrm{E}-03$ & $\mathrm{~N} / \mathrm{A}$ & N/A \\
\hline $\mathrm{Se}$ & N/A & N/A & N/A & $1.90 \mathrm{E}+00$ & $1.60 \mathrm{E}+01$ & N/A & $\mathrm{N} / \mathrm{A}$ \\
\hline $\mathrm{Si}$ & N/A & N/A & $1.00 \mathrm{E}+00$ & N/A & N/A & N/A & $\mathrm{N} / \mathrm{A}$ \\
\hline $\mathrm{Sm}$ & N/A & N/A & $4.00 \mathrm{E}-05$ & N/A & N/A & N/A & N/A \\
\hline $\mathrm{Sn}$ & N/A & $\mathrm{N} / \mathrm{A}$ & $1.00 \mathrm{E}+00$ & N/A & N/A & $\mathrm{N} / \mathrm{A}$ & $\mathrm{N} / \mathrm{A}$ \\
\hline $\mathrm{Sr}$ & N/A & N/A & N/A & $1.40 \mathrm{E}+00$ & $3.50 \mathrm{E}-01$ & $\mathrm{~N} / \mathrm{A}$ & $\mathrm{N} / \mathrm{A}$ \\
\hline $\mathrm{Ta}$ & $\mathrm{N} / \mathrm{A}$ & $\mathrm{N} / \mathrm{A}$ & $1.00 \mathrm{E}-03$ & N/A & N/A & $\mathrm{N} / \mathrm{A}$ & $\mathrm{N} / \mathrm{A}$ \\
\hline $\mathrm{Tb}$ & N/A & N/A & $4.00 \mathrm{E}-05$ & N/A & $\mathrm{N} / \mathrm{A}$ & $\mathrm{N} / \mathrm{A}$ & $\mathrm{N} / \mathrm{A}$ \\
\hline Tc & N/A & N/A & $3.00 \mathrm{E}+00$ & N/A & N/A & N/A & $\mathrm{N} / \mathrm{A}$ \\
\hline $\mathrm{Te}$ & $5.10 \mathrm{E}+00$ & N/A & N/A & N/A & $\mathrm{N} / \mathrm{A}$ & N/A & $\mathrm{N} / \mathrm{A}$ \\
\hline Th & N/A & N/A & $4.00 \mathrm{E}-03$ & N/A & N/A & N/A & N/A \\
\hline
\end{tabular}


Table 2.13. (contd)

\begin{tabular}{cccccccc}
\hline Element & Mean & STD & Median & GSD & GM & $5 \%$ & $95 \%$ \\
\hline Tl & N/A & N/A & $1.00 \mathrm{E}+00$ & N/A & N/A & N/A & N/A \\
$\mathrm{U}$ & $1.10 \mathrm{E}+00$ & N/A & N/A & N/A & N/A & N/A & N/A \\
$\mathrm{W}$ & $9.00 \mathrm{E}-01$ & N/A & N/A & N/A & N/A & N/A & N/A \\
$\mathrm{Y}$ & $2.00 \mathrm{E}-03$ & N/A & N/A & N/A & N/A & N/A & N/A \\
$\mathrm{Zn}$ & $1.40 \mathrm{E}+00$ & $2.90 \mathrm{E}-01$ & $1.37 \mathrm{E}+00$ & $1.23 \mathrm{E}+00$ & $1.37 \mathrm{E}+00$ & $9.79 \mathrm{E}-01$ & $1.92 \mathrm{E}+00$ \\
$\mathrm{Zr}$ & $2.00 \mathrm{E}-04$ & N/A & N/A & N/A & N/A & N/A & N/A \\
\hline GM $=$ Geometric mean. & & & & & \\
GSD $=$ Geometric Standard Deviation. & & & & \\
N/A = Not available in the references reviewed. \\
STD = Standard Deviation.
\end{tabular}

\subsubsection{CLBVx Bioconcentration to Edible Crop Portion from Soil}

CLBVLV is the ratio of leafy vegetable plant concentration (dry weight) to the soil concentration (dry soil). The soil-to-plant concentration ratio (CR) accounts for the uptake from soil and transport through roots to the edible plant parts.

CLBVRV is the ratio of plant chemical concentration (dry weight) for root and other vegetables to the soil chemical concentration (dry soil). This parameter captures a wide range of food types, covering all categories beyond leafy vegetables, tree fruit, and grains. Although the range of crops encompassed by this bioconcentration factor covers a broad variety of plant and edible crop morphologies, it was determined to be the best approach to combine the paucity of data for crops beyond leafy vegetables, fruits, and grains. The soil-to-plant CR accounts for the uptake from soil and transport through roots to the edible plant parts. Especially with the CLBVRV category, if there is a particular crop that is predominantly consumed within this food crop type, the bioconcentration factors should reflect that particular food crop to the extent possible.

CLBVFR is the ratio of plant chemical concentration (dry weight) for the edible fruit portion to the soil chemical concentration (dry soil). The soil-to-plant CR accounts for the uptake from soil and transport through roots to the edible plant parts.

CLBVCL is the ratio of plant chemical concentration (dry weight) for cereal grain to the soil chemical concentration (dry soil). The soil-to-plant CR accounts for the uptake from soil and transport through roots to the edible plant parts. The Gv2 code currently applies the CLBVAG (see Section 2.1.19) value to grain. However, the user is encouraged to enter the same values in the CLBVCL and CLBVAG parameter entries. This action will capture any later changes to the Gv2 code if both parameters are applied separately for feed and food categories.

The units and range for CLBVx parameters in Gv2 are: $\mathrm{kg}_{\text {dry_plant }} / \mathrm{kg}_{\text {dry_soil }}$, which is reduced from $\left(\mathrm{Ci} / \mathrm{kg}_{\text {dry_soil }}\right)$ per ( $\left.\mathrm{Ci} / \mathrm{kg}_{\text {dry_plant }}\right)$ (0 to 5000$)$. 
For all food crop bioaccumulation factors, the goal was to determine mean, median, geometric standard deviation, geometric mean, $5^{\text {th }}$ percentile, and $95^{\text {th }}$ percentile. Some of these values could be determined mathematically. In these cases, statistical calculations were performed, according to methods described in Strom and Stansbury (2000).

CLBLV: To tabulate bioaccumulation factors for leafy vegetable, sources considered to be the best available references were reviewed. For leafy vegetable, the goal was to determine mean, median, geometric standard deviation, geometric mean, $5^{\text {th }}$ percentile, and $95^{\text {th }}$ percentile. Selection priority was given to IAEA (2010), Sheppard et al. (2010), and Staven et al. (2003), with a preference for lognormality in the datasets. Some of these values could be determined mathematically. In these cases, statistical calculations were performed, according to methods described in Strom and Stansbury (2000). The full set of data reviewed to determine the values tabulated in this section are provided in Appendix C.

CLBVRV: To tabulate bioaccumulation factors for root vegetables, sources considered to be the best available references were reviewed. For root vegetables, the goal was to determine mean, median, geometric standard deviation, geometric mean, $5^{\text {th }}$ percentile, and $95^{\text {th }}$ percentile. Selection priority was given to IAEA (2010), Sheppard et al. (2010), and Staven et al. (2003), with a preference for lognormality in the datasets. Some of these values could be determined mathematically. In these cases, statistical calculations were performed, according to methods described in Strom and Stansbury (2000). The full set of data reviewed to determine the values tabulated in this section are provided in Appendix C.

CLBVFR: To tabulate bioaccumulation factors for fruit, sources considered to be the best available references were reviewed. For fruit, the goal was to determine mean, median, geometric standard deviation, geometric mean, $5^{\text {th }}$ percentile, and $95^{\text {th }}$ percentile. Selection priority was given to IAEA (2010), Staven et al. (2003), and Napier et al. (2012) (when the distribution given there encompassed essentially all of the others), with a preference for lognormality in the datasets. Some of these values could be determined mathematically. In these cases, statistical calculations were performed, according to methods described in Strom and Stansbury (2000). The full set of data reviewed to determine the values tabulated in this section are provided in Appendix C.

CLBVCL: To tabulate bioaccumulation factors for cereal grain, sources considered to be the best available references were reviewed. For cereal grain, the goal was to determine mean, median, geometric standard deviation, geometric mean, $5^{\text {th }}$ percentile, and $95^{\text {th }}$ percentile. Selection priority was given to IAEA (2010), Sheppard et al. (2010), and Staven et al. (2003), with a preference for lognormality in the datasets. Some of these values could be determined mathematically. In these cases, statistical calculations were performed, according to methods described in Strom and Stansbury (2000). The full set of data reviewed to determine the values tabulated in this section are provided in Appendix C.

\subsubsection{Additional Sources of Information}

The references that were reviewed contain additional information regarding data completeness, data limitations, and application of values to environmental models.

There are numerous chemicals for which no food crop bioconcentration factors are available. The user can refer to Staven et al. (2003) for an approach to approximate values for non-tabulated chemicals. The user is encouraged to review additional research on bioconcentration factors in the literature, as well. 


\subsubsection{CLBVx Bioconcentration to Edible Crop Tables}

For CLBVLV values, see Table 2.14.

For CLBVRV values see Table 2.15.

For CLBVFR values, see Table 2.16.

For CLBVCL values see Table 2.17.

Table 2.14. CLBVLV Concentration Ratio - Leafy Vegetable to Soil

\begin{tabular}{|c|c|c|c|c|c|c|c|}
\hline Element & Mean & STD & Median & GSD & GM & $5 \%$ & $95 \%$ \\
\hline $\mathrm{Ac}$ & $\mathrm{N} / \mathrm{A}$ & $\mathrm{N} / \mathrm{A}$ & $4.70 \mathrm{E}-04$ & N/A & N/A & $\mathrm{N} / \mathrm{A}$ & $\mathrm{N} / \mathrm{A}$ \\
\hline $\mathrm{Ag}$ & N/A & N/A & N/A & $3.30 \mathrm{E}+00$ & $1.80 \mathrm{E}-04$ & N/A & N/A \\
\hline $\mathrm{Am}$ & N/A & N/A & N/A & $3.30 \mathrm{E}+00$ & $2.70 \mathrm{E}-04$ & N/A & N/A \\
\hline As & N/A & N/A & $4.00 \mathrm{E}-02$ & N/A & N/A & N/A & N/A \\
\hline $\mathrm{Au}$ & N/A & N/A & $1.00 \mathrm{E}-02$ & N/A & N/A & N/A & N/A \\
\hline $\mathrm{Ba}$ & $5.00 \mathrm{E}-03$ & N/A & N/A & N/A & N/A & N/A & N/A \\
\hline $\mathrm{Be}$ & $4.20 \mathrm{E}-01$ & N/A & N/A & N/A & N/A & N/A & N/A \\
\hline $\mathrm{Bi}$ & N/A & N/A & $5.00 \mathrm{E}-01$ & N/A & N/A & N/A & N/A \\
\hline $\mathrm{Br}$ & N/A & $\mathrm{N} / \mathrm{A}$ & $1.50 \mathrm{E}+00$ & N/A & N/A & N/A & N/A \\
\hline $\mathrm{Ca}$ & N/A & N/A & $3.50 \mathrm{E}+00$ & N/A & N/A & N/A & N/A \\
\hline $\mathrm{Cd}$ & N/A & N/A & $5.50 \mathrm{E}-01$ & N/A & N/A & N/A & N/A \\
\hline $\mathrm{Ce}$ & N/A & $\mathrm{N} / \mathrm{A}$ & N/A & N/A & $6.20 \mathrm{E}-03$ & N/A & N/A \\
\hline $\mathrm{Cf}$ & N/A & N/A & 4.70E-04 & N/A & N/A & N/A & N/A \\
\hline $\mathrm{Cl}$ & N/A & N/A & $7.00 \mathrm{E}+00$ & N/A & N/A & N/A & N/A \\
\hline $\mathrm{Cm}$ & N/A & $\mathrm{N} / \mathrm{A}$ & N/A & $4.50 \mathrm{E}+00$ & $1.40 \mathrm{E}-03$ & N/A & $\mathrm{N} / \mathrm{A}$ \\
\hline $\mathrm{Co}$ & N/A & N/A & N/A & $2.70 \mathrm{E}+00$ & $1.70 \mathrm{E}-01$ & N/A & N/A \\
\hline $\mathrm{Cr}$ & $1.00 \mathrm{E}-03$ & $\mathrm{~N} / \mathrm{A}$ & $\mathrm{N} / \mathrm{A}$ & N/A & N/A & N/A & $\mathrm{N} / \mathrm{A}$ \\
\hline Cs & N/A & N/A & N/A & $6.00 \mathrm{E}+00$ & $6.00 \mathrm{E}-02$ & N/A & N/A \\
\hline $\mathrm{Cu}$ & N/A & N/A & $4.00 \mathrm{E}-01$ & N/A & N/A & N/A & N/A \\
\hline Dy & N/A & N/A & $2.00 \mathrm{E}-02$ & N/A & N/A & N/A & N/A \\
\hline $\mathrm{Er}$ & N/A & N/A & $2.00 \mathrm{E}-02$ & N/A & N/A & N/A & N/A \\
\hline $\mathrm{Eu}$ & N/A & N/A & $2.00 \mathrm{E}-02$ & N/A & N/A & N/A & $\mathrm{N} / \mathrm{A}$ \\
\hline $\mathrm{F}$ & N/A & N/A & $6.00 \mathrm{E}-02$ & N/A & N/A & N/A & N/A \\
\hline $\mathrm{Fe}$ & $1.00 \mathrm{E}-03$ & N/A & N/A & N/A & N/A & N/A & $\mathrm{N} / \mathrm{A}$ \\
\hline $\mathrm{Ga}$ & $\mathrm{N} / \mathrm{A}$ & N/A & $4.00 \mathrm{E}-03$ & N/A & N/A & N/A & N/A \\
\hline $\mathrm{Gd}$ & N/A & N/A & $2.00 \mathrm{E}-02$ & N/A & N/A & N/A & N/A \\
\hline $\mathrm{Hf}$ & N/A & N/A & $1.00 \mathrm{E}-03$ & N/A & N/A & N/A & $\mathrm{N} / \mathrm{A}$ \\
\hline $\mathrm{Hg}$ & N/A & N/A & $8.50 \mathrm{E}-01$ & N/A & N/A & N/A & N/A \\
\hline Ho & N/A & N/A & $2.00 \mathrm{E}-02$ & N/A & N/A & N/A & N/A \\
\hline I & N/A & N/A & $4.00 \mathrm{E}-02$ & N/A & N/A & N/A & N/A \\
\hline In & N/A & $\mathrm{N} / \mathrm{A}$ & $4.00 \mathrm{E}-03$ & N/A & N/A & N/A & $\mathrm{N} / \mathrm{A}$ \\
\hline $\mathrm{Ir}$ & N/A & N/A & $5.50 \mathrm{E}-02$ & N/A & N/A & N/A & N/A \\
\hline $\mathrm{K}$ & $1.30 \mathrm{E}+00$ & $\mathrm{~N} / \mathrm{A}$ & N/A & N/A & N/A & N/A & N/A \\
\hline $\mathrm{La}$ & N/A & N/A & N/A & $2.70 \mathrm{E}+00$ & $5.70 \mathrm{E}-03$ & N/A & N/A \\
\hline $\mathrm{Mg}$ & N/A & N/A & $1.00 \mathrm{E}+00$ & N/A & N/A & N/A & N/A \\
\hline
\end{tabular}


Table 2.14. (contd)

\begin{tabular}{|c|c|c|c|c|c|c|c|}
\hline Element & Mean & STD & Median & GSD & GM & $5 \%$ & $95 \%$ \\
\hline $\mathrm{Mn}$ & $4.10 \mathrm{E}-01$ & N/A & N/A & $2.40 \mathrm{E}+00$ & $\mathrm{~N} / \mathrm{A}$ & N/A & N/A \\
\hline Mo & $5.10 \mathrm{E}-01$ & N/A & N/A & N/A & N/A & N/A & N/A \\
\hline $\mathrm{N}$ & N/A & N/A & $5.50 \mathrm{E}-02$ & N/A & N/A & N/A & N/A \\
\hline $\mathrm{Na}$ & $3.00 \mathrm{E}-02$ & N/A & N/A & N/A & N/A & N/A & N/A \\
\hline $\mathrm{Nb}$ & N/A & N/A & N/A & N/A & $1.70 \mathrm{E}-02$ & N/A & N/A \\
\hline $\mathrm{Nd}$ & N/A & N/A & $2.00 \mathrm{E}-02$ & N/A & N/A & N/A & N/A \\
\hline $\mathrm{Ni}$ & N/A & N/A & N/A & $2.60 \mathrm{E}+00$ & $1.70 \mathrm{E}-01$ & N/A & N/A \\
\hline $\mathrm{Np}$ & N/A & N/A & N/A & $3.00 \mathrm{E}+00$ & $2.70 \mathrm{E}-02$ & N/A & N/A \\
\hline Os & N/A & N/A & $1.50 \mathrm{E}-02$ & N/A & N/A & N/A & N/A \\
\hline $\mathrm{P}$ & $1.00 \mathrm{E}+00$ & N/A & N/A & N/A & N/A & N/A & N/A \\
\hline $\mathrm{Pa}$ & N/A & N/A & 4.70E-04 & N/A & N/A & N/A & N/A \\
\hline $\mathrm{Pb}$ & N/A & N/A & N/A & $1.30 \mathrm{E}+01$ & $8.00 \mathrm{E}-02$ & N/A & N/A \\
\hline $\mathrm{Pd}$ & N/A & N/A & $1.50 \mathrm{E}-01$ & N/A & N/A & N/A & N/A \\
\hline $\mathrm{Pm}$ & N/A & N/A & $2.00 \mathrm{E}-02$ & N/A & N/A & N/A & N/A \\
\hline Po & N/A & N/A & N/A & $6.90 \mathrm{E}+00$ & $7.40 \mathrm{E}-03$ & N/A & N/A \\
\hline $\operatorname{Pr}$ & $2.00 \mathrm{E}-02$ & N/A & N/A & N/A & N/A & N/A & N/A \\
\hline $\mathrm{Pu}$ & N/A & N/A & N/A & $2.70 \mathrm{E}+00$ & $8.30 \mathrm{E}-05$ & N/A & N/A \\
\hline $\mathrm{Rb}$ & N/A & N/A & N/A & N/A & $6.20 \mathrm{E}-01$ & N/A & N/A \\
\hline $\mathrm{Re}$ & N/A & N/A & $1.50 \mathrm{E}+00$ & N/A & N/A & N/A & N/A \\
\hline $\mathrm{Rh}$ & N/A & N/A & $1.50 \mathrm{E}-01$ & N/A & N/A & N/A & N/A \\
\hline $\mathrm{Ru}$ & N/A & N/A & N/A & $3.70 \mathrm{E}+00$ & $9.00 \mathrm{E}-02$ & N/A & N/A \\
\hline $\mathrm{S}$ & N/A & N/A & $1.50 \mathrm{E}+00$ & N/A & N/A & N/A & N/A \\
\hline $\mathrm{Sb}$ & N/A & N/A & N/A & $2.60 \mathrm{E}+00$ & $9.40 \mathrm{E}-05$ & N/A & N/A \\
\hline $\mathrm{Sc}$ & N/A & N/A & $6.00 \mathrm{E}-03$ & N/A & N/A & N/A & N/A \\
\hline $\mathrm{Se}$ & N/A & N/A & $2.50 \mathrm{E}-01$ & N/A & N/A & N/A & N/A \\
\hline $\mathrm{Se}$ & N/A & N/A & N/A & N/A & $1.00 \mathrm{E}+00$ & N/A & N/A \\
\hline $\mathrm{Si}$ & N/A & N/A & $3.50 \mathrm{E}-01$ & N/A & N/A & N/A & N/A \\
\hline $\mathrm{Sm}$ & N/A & N/A & $2.00 \mathrm{E}-02$ & N/A & N/A & N/A & N/A \\
\hline $\mathrm{Sn}$ & N/A & N/A & $3.00 \mathrm{E}-02$ & N/A & N/A & N/A & N/A \\
\hline $\mathrm{Sr}$ & N/A & N/A & N/A & $6.00 \mathrm{E}+00$ & $7.60 \mathrm{E}-01$ & N/A & N/A \\
\hline $\mathrm{Ta}$ & N/A & N/A & $2.50 \mathrm{E}-02$ & N/A & N/A & N/A & N/A \\
\hline $\mathrm{Tb}$ & N/A & N/A & $2.00 \mathrm{E}-02$ & N/A & N/A & N/A & N/A \\
\hline Tc & N/A & N/A & $2.10 \mathrm{E}+01$ & N/A & N/A & N/A & N/A \\
\hline $\mathrm{Te}$ & $3.00 \mathrm{E}-01$ & N/A & N/A & N/A & N/A & N/A & N/A \\
\hline Th & N/A & N/A & $6.60 \mathrm{E}-03$ & N/A & N/A & N/A & N/A \\
\hline $\mathrm{Tl}$ & N/A & N/A & $4.00 \mathrm{E}-03$ & N/A & N/A & N/A & N/A \\
\hline U & N/A & N/A & N/A & $7.30 \mathrm{E}+00$ & $2.00 \mathrm{E}-02$ & N/A & N/A \\
\hline $\mathrm{V}$ & N/A & N/A & N/A & N/A & 8.80E-03 & N/A & N/A \\
\hline W & N/A & N/A & $3.00 \mathrm{E}+00$ & N/A & N/A & N/A & N/A \\
\hline $\mathrm{Y}$ & $2.00 \mathrm{E}-03$ & N/A & N/A & N/A & N/A & N/A & N/A \\
\hline $\mathrm{Yb}$ & N/A & N/A & N/A & N/A & $8.00 \mathrm{E}-03$ & N/A & N/A \\
\hline $\mathrm{Zn}$ & N/A & N/A & N/A & $2.40 \mathrm{E}+00$ & $2.40 \mathrm{E}+00$ & N/A & N/A \\
\hline $\mathrm{Zr}$ & $4.00 \mathrm{E}-02$ & N/A & N/A & N/A & N/A & N/A & N/A \\
\hline \multicolumn{8}{|c|}{$\begin{array}{l}\text { GM }=\text { Geometric mean. } \\
\text { GSD }=\text { Geometric Standard Deviation. } \\
\text { N/A }=\text { Not available in the references reviewed. } \\
\text { STD }=\text { Standard Deviation. }\end{array}$} \\
\hline
\end{tabular}


Table 2.15. CLBVRV Concentration Ratio - Root (Other) Vegetable to Soil

\begin{tabular}{|c|c|c|c|c|c|c|c|}
\hline Element & Mean & STD & Median & GSD & GM & $5 \%$ & $95 \%$ \\
\hline $\mathrm{Ac}$ & $\mathrm{N} / \mathrm{A}$ & N/A & $3.50 \mathrm{E}-04$ & $\mathrm{~N} / \mathrm{A}$ & $\mathrm{N} / \mathrm{A}$ & N/A & N/A \\
\hline $\mathrm{Ag}$ & N/A & N/A & N/A & $2.00 \mathrm{E}+00$ & $1.30 \mathrm{E}-03$ & N/A & N/A \\
\hline $\mathrm{Am}$ & N/A & N/A & N/A & $2.40 \mathrm{E}+00$ & $6.70 \mathrm{E}-04$ & N/A & N/A \\
\hline As & N/A & N/A & $6.00 \mathrm{E}-03$ & N/A & N/A & N/A & N/A \\
\hline $\mathrm{Au}$ & N/A & $\mathrm{N} / \mathrm{A}$ & $1.80 \mathrm{E}-02$ & N/A & N/A & N/A & N/A \\
\hline $\mathrm{Ba}$ & $5.00 \mathrm{E}-03$ & N/A & N/A & N/A & N/A & N/A & N/A \\
\hline $\mathrm{Bi}$ & N/A & N/A & $1.00 \mathrm{E}-05$ & N/A & N/A & N/A & N/A \\
\hline $\mathrm{Br}$ & N/A & N/A & $1.50 \mathrm{E}+00$ & N/A & N/A & N/A & N/A \\
\hline $\mathrm{Ca}$ & N/A & N/A & N/A & N/A & N/A & N/A & N/A \\
\hline $\mathrm{Cd}$ & N/A & N/A & N/A & $2.70 \mathrm{E}-01$ & $2.70 \mathrm{E}-01$ & N/A & N/A \\
\hline $\mathrm{Ce}$ & $1.3 \mathrm{E}-02$ & N/A & N/A & N/A & N/A & N/A & N/A \\
\hline $\mathrm{Cf}$ & N/A & N/A & $3.50 \mathrm{E}-04$ & N/A & N/A & N/A & N/A \\
\hline $\mathrm{Cl}$ & N/A & N/A & $7.00 \mathrm{E}+00$ & N/A & N/A & N/A & N/A \\
\hline $\mathrm{Cm}$ & N/A & N/A & $\mathrm{N} / \mathrm{A}$ & $3.00 \mathrm{E}+00$ & $8.5 \mathrm{E}-04$ & N/A & N/A \\
\hline Co & N/A & N/A & N/A & $2.20 \mathrm{E}+00$ & $1.10 \mathrm{E}-01$ & N/A & N/A \\
\hline $\mathrm{Cr}$ & $1.00 \mathrm{E}-03$ & N/A & $\mathrm{N} / \mathrm{A}$ & $1.00 \mathrm{E}-03$ & N/A & N/A & N/A \\
\hline Cs & N/A & N/A & N/A & $3.00 \mathrm{E}+00$ & $4.20 \mathrm{E}-02$ & N/A & N/A \\
\hline $\mathrm{Cu}$ & N/A & N/A & $2.50 \mathrm{E}-01$ & N/A & N/A & N/A & N/A \\
\hline Dy & N/A & N/A & $2.00 \mathrm{E}-02$ & N/A & N/A & N/A & N/A \\
\hline $\mathrm{Er}$ & N/A & N/A & $2.00 \mathrm{E}-02$ & N/A & N/A & N/A & N/A \\
\hline $\mathrm{Eu}$ & N/A & N/A & $2.00 \mathrm{E}-02$ & N/A & N/A & N/A & N/A \\
\hline $\mathrm{F}$ & N/A & N/A & $6.00 \mathrm{E}-03$ & N/A & N/A & N/A & N/A \\
\hline $\mathrm{Fe}$ & $1.00 \mathrm{E}-03$ & N/A & N/A & N/A & N/A & N/A & N/A \\
\hline $\mathrm{Ga}$ & N/A & N/A & $4.00 \mathrm{E}-04$ & N/A & N/A & N/A & N/A \\
\hline $\mathrm{Gd}$ & N/A & N/A & $2.00 \mathrm{E}-02$ & N/A & N/A & N/A & N/A \\
\hline $\mathrm{Hf}$ & N/A & N/A & $1.00 \mathrm{E}-03$ & N/A & N/A & N/A & N/A \\
\hline $\mathrm{Hg}$ & N/A & N/A & $2.00 \mathrm{E}-01$ & N/A & N/A & N/A & N/A \\
\hline Ho & N/A & N/A & $2.00 \mathrm{E}-02$ & N/A & N/A & N/A & N/A \\
\hline I & N/A & N/A & $4.00 \mathrm{E}-02$ & N/A & $\mathrm{N} / \mathrm{A}$ & N/A & N/A \\
\hline In & N/A & N/A & $4.00 \mathrm{E}-04$ & N/A & N/A & N/A & N/A \\
\hline $\mathrm{Ir}$ & N/A & N/A & $1.50 \mathrm{E}-02$ & N/A & N/A & N/A & N/A \\
\hline $\mathrm{K}$ & N/A & N/A & $5.50 \mathrm{E}-01$ & N/A & N/A & N/A & N/A \\
\hline $\mathrm{La}$ & N/A & N/A & N/A & $2.70 \mathrm{E}+00$ & $1.60 \mathrm{E}-03$ & N/A & N/A \\
\hline $\mathrm{Mg}$ & N/A & N/A & $5.50 \mathrm{E}-01$ & N/A & N/A & N/A & N/A \\
\hline $\mathrm{Mn}$ & N/A & N/A & N/A & $4.10 \mathrm{E}+00$ & $3.10 \mathrm{E}-01$ & N/A & N/A \\
\hline Mo & $3.20 \mathrm{E}-01$ & N/A & N/A & N/A & N/A & N/A & N/A \\
\hline $\mathrm{N}$ & N/A & N/A & $4.20 \mathrm{E}-02$ & $\mathrm{~N} / \mathrm{A}$ & N/A & N/A & N/A \\
\hline $\mathrm{Na}$ & $3.00 \mathrm{E}-02$ & N/A & N/A & N/A & N/A & N/A & N/A \\
\hline $\mathrm{Nb}$ & 8.00E-03 & N/A & $\mathrm{N} / \mathrm{A}$ & N/A & N/A & N/A & N/A \\
\hline $\mathrm{Nd}$ & N/A & N/A & $2.00 \mathrm{E}-02$ & N/A & N/A & N/A & N/A \\
\hline $\mathrm{Ni}$ & N/A & N/A & N/A & $2.50 \mathrm{E}+00$ & $4.00 \mathrm{E}-01$ & N/A & N/A \\
\hline $\mathrm{Np}$ & N/A & N/A & N/A & $2.00 \mathrm{E}+00$ & $2.20 \mathrm{E}-02$ & N/A & N/A \\
\hline Os & N/A & N/A & $3.50 \mathrm{E}-03$ & N/A & N/A & N/A & N/A \\
\hline $\mathrm{P}$ & 1 & N/A & N/A & N/A & $\mathrm{N} / \mathrm{A}$ & N/A & N/A \\
\hline $\mathrm{Pa}$ & N/A & N/A & $3.50 \mathrm{E}-04$ & N/A & N/A & N/A & N/A \\
\hline $\mathrm{Pb}$ & N/A & N/A & $3.20 \mathrm{E}-03$ & $\mathrm{~N} / \mathrm{A}$ & $1.50 \mathrm{E}-02$ & N/A & N/A \\
\hline
\end{tabular}


Table 2.15. (contd)

\begin{tabular}{|c|c|c|c|c|c|c|c|}
\hline Element & Mean & STD & Median & GSD & GM & $5 \%$ & $95 \%$ \\
\hline $\mathrm{Pd}$ & $\mathrm{N} / \mathrm{A}$ & $\mathrm{N} / \mathrm{A}$ & $4.00 \mathrm{E}-02$ & $\mathrm{~N} / \mathrm{A}$ & N/A & N/A & N/A \\
\hline $\mathrm{Pm}$ & N/A & N/A & N/A & $1.20 \mathrm{E}+00$ & $4.20 \mathrm{E}-02$ & N/A & N/A \\
\hline Po & N/A & N/A & N/A & $4.30 \mathrm{E}+00$ & $5.80 \mathrm{E}-03$ & N/A & N/A \\
\hline $\operatorname{Pr}$ & $2.00 \mathrm{E}-02$ & N/A & $\mathrm{N} / \mathrm{A}$ & N/A & N/A & N/A & N/A \\
\hline $\mathrm{Pu}$ & N/A & N/A & N/A & $2.70 \mathrm{E}+00$ & $6.50 \mathrm{E}-05$ & N/A & N/A \\
\hline $\mathrm{Ra}$ & N/A & N/A & N/A & $8.40 \mathrm{E}+00$ & $1.70 \mathrm{E}-02$ & N/A & N/A \\
\hline $\mathrm{Rb}$ & $9.00 \mathrm{E}-01$ & N/A & $\mathrm{N} / \mathrm{A}$ & N/A & N/A & N/A & $\mathrm{N} / \mathrm{A}$ \\
\hline $\operatorname{Re}$ & N/A & N/A & $3.50 \mathrm{E}+00$ & N/A & N/A & N/A & N/A \\
\hline $\mathrm{Rh}$ & N/A & N/A & $4.00 \mathrm{E}-02$ & $\mathrm{~N} / \mathrm{A}$ & $\mathrm{N} / \mathrm{A}$ & N/A & N/A \\
\hline $\mathrm{Ru}$ & $2.00 \mathrm{E}-02$ & N/A & N/A & $\mathrm{N} / \mathrm{A}$ & $\mathrm{N} / \mathrm{A}$ & N/A & N/A \\
\hline $\mathrm{S}$ & N/A & N/A & $1.50 \mathrm{E}+00$ & N/A & N/A & N/A & N/A \\
\hline $\mathrm{Sb}$ & N/A & N/A & N/A & $6.70 \mathrm{E}+00$ & $1.30 \mathrm{E}-04$ & N/A & N/A \\
\hline $\mathrm{Sc}$ & N/A & N/A & $1.00 \mathrm{E}-03$ & N/A & N/A & N/A & N/A \\
\hline $\mathrm{Se}$ & N/A & N/A & $5.00 \mathrm{E}-02$ & N/A & N/A & N/A & N/A \\
\hline $\mathrm{Si}$ & N/A & N/A & 7.00E-02 & N/A & N/A & N/A & N/A \\
\hline $\mathrm{Sm}$ & N/A & N/A & $2.00 \mathrm{E}-02$ & N/A & $\mathrm{N} / \mathrm{A}$ & N/A & N/A \\
\hline Sn & N/A & N/A & $6.00 \mathrm{E}-03$ & N/A & N/A & N/A & N/A \\
\hline $\mathrm{Sr}$ & N/A & N/A & N/A & $4.10 \mathrm{E}+00$ & $7.20 \mathrm{E}-01$ & N/A & N/A \\
\hline $\mathrm{Ta}$ & N/A & N/A & $2.50 \mathrm{E}-02$ & N/A & N/A & N/A & N/A \\
\hline $\mathrm{Tb}$ & N/A & N/A & $2.00 \mathrm{E}-02$ & $\mathrm{~N} / \mathrm{A}$ & $\mathrm{N} / \mathrm{A}$ & N/A & N/A \\
\hline $\mathrm{Tc}$ & N/A & N/A & $2.40 \mathrm{E}-01$ & $\mathrm{~N} / \mathrm{A}$ & $\mathrm{N} / \mathrm{A}$ & N/A & N/A \\
\hline $\mathrm{Te}$ & $3.00 \mathrm{E}-01$ & N/A & N/A & N/A & N/A & N/A & N/A \\
\hline Th & $7.80 \mathrm{E}-04$ & & N/A & $6.80 \mathrm{E}+00$ & N/A & N/A & N/A \\
\hline $\mathrm{Tl}$ & N/A & N/A & $4.00 \mathrm{E}-04$ & N/A & N/A & N/A & N/A \\
\hline $\mathrm{U}$ & N/A & N/A & N/A & $4.20 \mathrm{E}+00$ & $1.50 \mathrm{E}-02$ & N/A & N/A \\
\hline $\mathrm{V}$ & N/A & N/A & N/A & N/A & $2.00 \mathrm{E}-03$ & N/A & N/A \\
\hline W & N/A & N/A & $3.00 \mathrm{E}+00$ & N/A & N/A & N/A & N/A \\
\hline $\mathrm{Y}$ & $2.00 \mathrm{E}-03$ & N/A & $\mathrm{N} / \mathrm{A}$ & N/A & N/A & N/A & N/A \\
\hline $\mathrm{Yb}$ & N/A & $\mathrm{N} / \mathrm{A}$ & N/A & N/A & $2.00 \mathrm{E}-03$ & N/A & N/A \\
\hline $\mathrm{Zn}$ & N/A & N/A & N/A & $1.80 \mathrm{E}+00$ & $3.00 \mathrm{E}-01$ & N/A & N/A \\
\hline $\mathrm{Zr}$ & $4.00 \mathrm{E}-03$ & N/A & N/A & N/A & N/A & N/A & N/A \\
\hline \multicolumn{8}{|c|}{$\begin{array}{l}\text { GM }=\text { Geometric mean. } \\
\text { GSD }=\text { Geometric Standard Deviation. } \\
\text { N/A }=\text { Not available in the references reviewed. } \\
\text { STD }=\text { Standard Deviation. }\end{array}$} \\
\hline
\end{tabular}

Table 2.16. CLBVFR Concentration Ratio - Fruit to Soil

\begin{tabular}{cccccccc}
\hline Element & Mean & STD & Median & GSD & GM & $5 \%$ & $95 \%$ \\
\hline $\mathrm{Ac}$ & N/A & N/A & $2.50 \mathrm{E}-04$ & N/A & N/A & N/A & N/A \\
$\mathrm{Ag}$ & N/A & N/A & $8.00 \mathrm{E}-04$ & N/A & N/A & N/A & N/A \\
$\mathrm{Al}$ & $1.00 \mathrm{E}-03$ & $2.00 \mathrm{E}-03$ & $2.81 \mathrm{E}-04$ & $3.56 \mathrm{E}+00$ & $4.47 \mathrm{E}-04$ & $5.55 \mathrm{E}-05$ & $3.60 \mathrm{E}-03$ \\
$\mathrm{Am}$ & N/A & N/A & $6.20 \mathrm{E}-06$ & 2.4 & N/A & N/A & N/A \\
$\mathrm{As}$ & $2.20 \mathrm{E}-01$ & $2.50 \mathrm{E}-02$ & $1.96 \mathrm{E}-01$ & $1.12 \mathrm{E}+00$ & $2.19 \mathrm{E}-01$ & $1.81 \mathrm{E}-01$ & $2.63 \mathrm{E}-01$ \\
$\mathrm{Au}$ & N/A & N/A & $1.40 \mathrm{E}-02$ & N/A & N/A & N/A & N/A \\
$\mathrm{Ba}$ & $3.00 \mathrm{E}-03$ & $3.00 \mathrm{E}-03$ & $1.30 \mathrm{E}-03$ & $2.30 \mathrm{E}+00$ & $2.12 \mathrm{E}-03$ & $5.39 \mathrm{E}-04$ & $8.34 \mathrm{E}-03$
\end{tabular}


Table 2.16. (contd)

\begin{tabular}{|c|c|c|c|c|c|c|c|}
\hline Element & Mean & STD & Median & GSD & GM & $5 \%$ & $95 \%$ \\
\hline $\mathrm{Be}$ & N/A & $\mathrm{N} / \mathrm{A}$ & $1.50 \mathrm{E}-03$ & $\mathrm{~N} / \mathrm{A}$ & N/A & N/A & N/A \\
\hline $\mathrm{Bi}$ & N/A & N/A & $5.00 \mathrm{E}-01$ & $\mathrm{~N} / \mathrm{A}$ & N/A & N/A & N/A \\
\hline Bk & N/A & N/A & $2.50 \mathrm{E}-04$ & N/A & N/A & $\mathrm{N} / \mathrm{A}$ & N/A \\
\hline $\mathrm{Br}$ & $1.81 \mathrm{E}-01$ & 1.69E-01 & $8.20 \mathrm{E}-02$ & $2.21 \mathrm{E}+00$ & $1.32 \mathrm{E}-01$ & $3.60 \mathrm{E}-02$ & $4.87 \mathrm{E}-01$ \\
\hline $\mathrm{Ca}$ & $1.80 \mathrm{E}-01$ & $1.34 \mathrm{E}-01$ & $9.27 \mathrm{E}-02$ & $1.94 \mathrm{E}+00$ & $1.44 \mathrm{E}-01$ & $4.84 \mathrm{E}-02$ & $4.30 \mathrm{E}-01$ \\
\hline $\mathrm{Ce}$ & $1.06 \mathrm{E}-04$ & N/A & N/A & N/A & N/A & N/A & N/A \\
\hline $\mathrm{Cm}$ & $1.00 \mathrm{E}-04$ & $2.60 \mathrm{E}-05$ & N/A & N/A & N/A & N/A & N/A \\
\hline Co & N/A & N/A & $7.00 \mathrm{E}-03$ & N/A & N/A & N/A & N/A \\
\hline Cs & $2.00 \mathrm{E}-03$ & $1.00 \mathrm{E}-03$ & $1.25 \mathrm{E}-03$ & $1.60 \mathrm{E}+00$ & $1.79 \mathrm{E}-03$ & $8.22 \mathrm{E}-04$ & $3.89 \mathrm{E}-03$ \\
\hline $\mathrm{Cu}$ & N/A & $\mathrm{N} / \mathrm{A}$ & $2.50 \mathrm{E}-01$ & N/A & N/A & N/A & N/A \\
\hline Dy & N/A & N/A & $2.00 \mathrm{E}-02$ & N/A & N/A & N/A & N/A \\
\hline $\mathrm{Er}$ & N/A & N/A & $2.00 \mathrm{E}-02$ & N/A & N/A & $\mathrm{N} / \mathrm{A}$ & N/A \\
\hline $\mathrm{Eu}$ & $1.00 \mathrm{E}-03$ & $1.00 \mathrm{E}-03$ & $4.35 \mathrm{E}-04$ & $2.30 \mathrm{E}+00$ & $7.07 \mathrm{E}-04$ & $1.80 \mathrm{E}-04$ & $2.78 \mathrm{E}-03$ \\
\hline $\mathrm{F}$ & N/A & N/A & $6.00 \mathrm{E}-03$ & N/A & N/A & N/A & N/A \\
\hline $\mathrm{Fe}$ & $2.00 \mathrm{E}-03$ & $5.00 \mathrm{E}-04$ & $1.56 \mathrm{E}-03$ & $1.28 \mathrm{E}+00$ & $1.94 \mathrm{E}-03$ & $1.29 \mathrm{E}-03$ & $2.91 \mathrm{E}-03$ \\
\hline $\mathrm{Ga}$ & N/A & N/A & 4.00E-04 & N/A & N/A & N/A & N/A \\
\hline $\mathrm{Gd}$ & $\mathrm{N} / \mathrm{A}$ & N/A & $2.00 \mathrm{E}-02$ & $\mathrm{~N} / \mathrm{A}$ & $\mathrm{N} / \mathrm{A}$ & $\mathrm{N} / \mathrm{A}$ & N/A \\
\hline Hf & $1.00 \mathrm{E}-03$ & $1.00 \mathrm{E}-03$ & 4.35E-04 & $2.30 \mathrm{E}+00$ & $7.07 \mathrm{E}-04$ & $1.80 \mathrm{E}-04$ & $2.78 \mathrm{E}-03$ \\
\hline $\mathrm{Hg}$ & N/A & N/A & $3.70 \mathrm{E}-01$ & N/A & N/A & N/A & N/A \\
\hline Ho & $\mathrm{N} / \mathrm{A}$ & $\mathrm{N} / \mathrm{A}$ & $2.00 \mathrm{E}-02$ & $\mathrm{~N} / \mathrm{A}$ & N/A & N/A & N/A \\
\hline I & N/A & N/A & N/A & 1.6 & $1.26 \mathrm{E}-03$ & N/A & N/A \\
\hline In & N/A & N/A & $4.00 \mathrm{E}-04$ & N/A & N/A & N/A & N/A \\
\hline $\mathrm{Ir}$ & N/A & N/A & $1.50 \mathrm{E}-02$ & N/A & N/A & N/A & $\mathrm{N} / \mathrm{A}$ \\
\hline $\mathrm{K}$ & $5.57 \mathrm{E}-01$ & 2.99E-01 & $3.37 \mathrm{E}-01$ & $1.65 \mathrm{E}+00$ & $4.91 \mathrm{E}-01$ & $2.14 \mathrm{E}-01$ & $1.12 \mathrm{E}+00$ \\
\hline $\mathrm{La}$ & $1.00 \mathrm{E}-03$ & $2.00 \mathrm{E}-04$ & $8.20 \mathrm{E}-04$ & $1.22 \mathrm{E}+00$ & $9.81 \mathrm{E}-04$ & $7.08 \mathrm{E}-04$ & $1.36 \mathrm{E}-03$ \\
\hline $\mathrm{La}$ & N/A & N/A & $4.00 \mathrm{E}-03$ & N/A & N/A & N/A & N/A \\
\hline $\mathrm{La}$ & $6.00 \mathrm{E}-03$ & N/A & N/A & $\mathrm{N} / \mathrm{A}$ & N/A & N/A & N/A \\
\hline $\mathrm{La}$ & $4.00 \mathrm{E}-03$ & $\mathrm{~N} / \mathrm{A}$ & N/A & $\mathrm{N} / \mathrm{A}$ & N/A & N/A & N/A \\
\hline $\mathrm{La}$ & N/A & N/A & N/A & N/A & $3.50 \mathrm{E}-04$ & N/A & N/A \\
\hline $\mathrm{Lu}$ & $2.00 \mathrm{E}-03$ & $2.00 \mathrm{E}-03$ & $8.70 \mathrm{E}-04$ & $2.30 \mathrm{E}+00$ & $1.41 \mathrm{E}-03$ & $3.60 \mathrm{E}-04$ & $5.56 \mathrm{E}-03$ \\
\hline $\mathrm{Mg}$ & $1.33 \mathrm{E}-01$ & $1.77 \mathrm{E}-01$ & $4.85 \mathrm{E}-02$ & $2.74 \mathrm{E}+00$ & 7.99E-02 & $1.52 \mathrm{E}-02$ & $4.21 \mathrm{E}-01$ \\
\hline $\mathrm{Mn}$ & $2.30 \mathrm{E}-02$ & $8.00 \mathrm{E}-03$ & $1.64 \mathrm{E}-02$ & $1.40 \mathrm{E}+00$ & $2.17 \mathrm{E}-02$ & $1.25 \mathrm{E}-02$ & 3.79E-02 \\
\hline Mo & N/A & $\mathrm{N} / \mathrm{A}$ & $6.00 \mathrm{E}-02$ & N/A & N/A & N/A & N/A \\
\hline $\mathrm{N}$ & N/A & N/A & $3.00 \mathrm{E}-02$ & N/A & N/A & N/A & N/A \\
\hline $\mathrm{Na}$ & $1.70 \mathrm{E}-02$ & $1.10 \mathrm{E}-02$ & $9.41 \mathrm{E}-03$ & $1.81 \mathrm{E}+00$ & $1.43 \mathrm{E}-02$ & $5.40 \mathrm{E}-03$ & $3.78 \mathrm{E}-02$ \\
\hline $\mathrm{Nb}$ & N/A & N/A & $2.50 \mathrm{E}-02$ & N/A & N/A & N/A & N/A \\
\hline $\mathrm{Nd}$ & $9.00 \mathrm{E}-03$ & $5.00 \mathrm{E}-03$ & $5.36 \mathrm{E}-03$ & $1.68 \mathrm{E}+00$ & $7.87 \mathrm{E}-03$ & $3.35 \mathrm{E}-03$ & $1.85 \mathrm{E}-02$ \\
\hline $\mathrm{Ni}$ & $2.63 \mathrm{E}-01$ & 8.90E-01 & $5.37 \mathrm{E}-02$ & $4.89 \mathrm{E}+00$ & $7.45 \mathrm{E}-02$ & $5.47 \mathrm{E}-03$ & $1.02 \mathrm{E}+00$ \\
\hline $\mathrm{Np}$ & N/A & N/A & $1.00 \mathrm{E}-02$ & N/A & N/A & N/A & N/A \\
\hline Os & $\mathrm{N} / \mathrm{A}$ & $\mathrm{N} / \mathrm{A}$ & $4.50 \mathrm{E}-02$ & $\mathrm{~N} / \mathrm{A}$ & $\mathrm{N} / \mathrm{A}$ & $\mathrm{N} / \mathrm{A}$ & N/A \\
\hline $\mathrm{P}$ & N/A & N/A & $3.50 \mathrm{E}+00$ & N/A & N/A & N/A & N/A \\
\hline $\mathrm{Pa}$ & $\mathrm{N} / \mathrm{A}$ & $\mathrm{N} / \mathrm{A}$ & $2.50 \mathrm{E}-04$ & $\mathrm{~N} / \mathrm{A}$ & $\mathrm{N} / \mathrm{A}$ & $\mathrm{N} / \mathrm{A}$ & N/A \\
\hline $\mathrm{Pb}$ & N/A & N/A & $1.00 \mathrm{E}-02$ & N/A & N/A & N/A & N/A \\
\hline $\mathrm{Pd}$ & $1.00 \mathrm{E}-02$ & $5.96 \mathrm{E}-03$ & $8.59 \mathrm{E}-03$ & $1.74 \mathrm{E}+00$ & $8.59 \mathrm{E}-03$ & $3.47 \mathrm{E}-03$ & $2.13 \mathrm{E}-02$ \\
\hline $\mathrm{Pm}$ & N/A & N/A & $2.00 \mathrm{E}-02$ & N/A & N/A & N/A & N/A \\
\hline Po & N/A & N/A & $1.20 \mathrm{E}-03$ & N/A & N/A & N/A & N/A \\
\hline $\operatorname{Pr}$ & N/A & N/A & $2.00 \mathrm{E}-02$ & N/A & N/A & N/A & N/A \\
\hline
\end{tabular}


Table 2.16. (contd)

\begin{tabular}{|c|c|c|c|c|c|c|c|}
\hline Element & Mean & STD & Median & GSD & GM & $5 \%$ & $95 \%$ \\
\hline $\mathrm{Pu}$ & N/A & $\mathrm{N} / \mathrm{A}$ & $4.50 \mathrm{E}-05$ & $\mathrm{~N} / \mathrm{A}$ & $\mathrm{N} / \mathrm{A}$ & N/A & N/A \\
\hline $\mathrm{Ra}$ & $2.69 \mathrm{E}-02$ & 4.61E-02 & $1.36 \mathrm{E}-02$ & $3.22 \mathrm{E}+00$ & $1.36 \mathrm{E}-02$ & $1.98 \mathrm{E}-03$ & $9.30 \mathrm{E}-02$ \\
\hline $\mathrm{Rb}$ & $1.45 \mathrm{E}-01$ & $5.30 \mathrm{E}-02$ & $1.02 \mathrm{E}-01$ & $1.42 \mathrm{E}+00$ & $1.36 \mathrm{E}-01$ & $7.61 \mathrm{E}-02$ & $2.44 \mathrm{E}-01$ \\
\hline $\operatorname{Re}$ & N/A & N/A & $3.50 \mathrm{E}-01$ & N/A & N/A & N/A & N/A \\
\hline $\mathrm{Rh}$ & N/A & N/A & $4.00 \mathrm{E}-02$ & N/A & $\mathrm{N} / \mathrm{A}$ & N/A & N/A \\
\hline $\mathrm{Ru}$ & N/A & N/A & $4.00 \mathrm{E}-02$ & N/A & N/A & N/A & N/A \\
\hline $\mathrm{S}$ & N/A & N/A & $1.50 \mathrm{E}+00$ & N/A & N/A & N/A & N/A \\
\hline $\mathrm{Sb}$ & $9.60 \mathrm{E}-02$ & $6.10 \mathrm{E}-02$ & $5.36 \mathrm{E}-02$ & $1.79 \mathrm{E}+00$ & $8.10 \mathrm{E}-02$ & $3.11 \mathrm{E}-02$ & $2.11 \mathrm{E}-01$ \\
\hline $\mathrm{Sc}$ & N/A & N/A & $3.00 \mathrm{E}-03$ & N/A & N/A & N/A & N/A \\
\hline $\mathrm{Se}$ & N/A & N/A & $5.00 \mathrm{E}-02$ & $\mathrm{~N} / \mathrm{A}$ & $\mathrm{N} / \mathrm{A}$ & N/A & N/A \\
\hline $\mathrm{Si}$ & N/A & N/A & $7.00 \mathrm{E}-02$ & N/A & N/A & N/A & N/A \\
\hline $\mathrm{Sm}$ & $1.00 \mathrm{E}-03$ & $1.00 \mathrm{E}-03$ & $4.35 \mathrm{E}-04$ & $2.30 \mathrm{E}+00$ & 7.07E-04 & $1.80 \mathrm{E}-04$ & $2.78 \mathrm{E}-03$ \\
\hline $\mathrm{Sn}$ & N/A & N/A & $6.00 \mathrm{E}-03$ & N/A & N/A & N/A & N/A \\
\hline $\mathrm{Sr}$ & $6.80 \mathrm{E}-02$ & $7.80 \mathrm{E}-02$ & 2.72E-02 & $2.50 \mathrm{E}+00$ & 4.47E-02 & $9.90 \mathrm{E}-03$ & $2.02 \mathrm{E}-01$ \\
\hline $\mathrm{Ta}$ & $1.00 \mathrm{E}-03$ & $1.00 \mathrm{E}-03$ & $4.35 \mathrm{E}-04$ & $2.30 \mathrm{E}+00$ & 7.07E-04 & $1.80 \mathrm{E}-04$ & $2.78 \mathrm{E}-03$ \\
\hline $\mathrm{Tb}$ & $2.00 \mathrm{E}-03$ & $2.00 \mathrm{E}-03$ & $8.70 \mathrm{E}-04$ & $2.30 \mathrm{E}+00$ & $1.41 \mathrm{E}-03$ & $3.60 \mathrm{E}-04$ & $5.56 \mathrm{E}-03$ \\
\hline $\mathrm{Tc}$ & N/A & N/A & $1.50 \mathrm{E}+00$ & N/A & N/A & N/A & N/A \\
\hline $\mathrm{Te}$ & N/A & N/A & $4.00 \mathrm{E}-03$ & N/A & N/A & N/A & N/A \\
\hline Th & $7.00 \mathrm{E}-04$ & $4.00 \mathrm{E}-04$ & 4.11E-04 & $1.70 \mathrm{E}+00$ & $6.08 \mathrm{E}-04$ & $2.53 \mathrm{E}-04$ & $1.46 \mathrm{E}-03$ \\
\hline $\mathrm{Ti}$ & $5.40 \mathrm{E}-05$ & N/A & N/A & N/A & N/A & N/A & N/A \\
\hline $\mathrm{Tl}$ & $\mathrm{N} / \mathrm{A}$ & N/A & $4.00 \mathrm{E}-04$ & N/A & N/A & N/A & N/A \\
\hline $\mathrm{U}$ & $5.72 \mathrm{E}-02$ & $1.15 \mathrm{E}-01$ & $2.55 \mathrm{E}-02$ & $3.57 \mathrm{E}+00$ & $2.55 \mathrm{E}-02$ & $3.14 \mathrm{E}-03$ & $2.06 \mathrm{E}-01$ \\
\hline $\mathrm{V}$ & $7.00 \mathrm{E}-03$ & $1.00 \mathrm{E}-02$ & $2.44 \mathrm{E}-03$ & $2.87 \mathrm{E}+00$ & 4.01E-03 & $7.08 \mathrm{E}-04$ & $2.28 \mathrm{E}-02$ \\
\hline $\mathrm{W}$ & N/A & N/A & $3.00 \mathrm{E}+00$ & N/A & N/A & N/A & N/A \\
\hline $\mathrm{Y}$ & N/A & N/A & $2.00 \mathrm{E}-02$ & N/A & N/A & N/A & N/A \\
\hline $\mathrm{Yb}$ & $5.00 \mathrm{E}-03$ & $5.00 \mathrm{E}-03$ & $2.17 \mathrm{E}-03$ & $2.30 \mathrm{E}+00$ & $3.54 \mathrm{E}-03$ & 8.99E-04 & $1.39 \mathrm{E}-02$ \\
\hline $\mathrm{Zn}$ & $1.37 \mathrm{E}-01$ & $6.20 \mathrm{E}-02$ & $8.90 \mathrm{E}-02$ & $1.54 \mathrm{E}+00$ & $1.25 \mathrm{E}-01$ & $6.14 \mathrm{E}-02$ & $2.54 \mathrm{E}-01$ \\
\hline $\mathrm{Zr}$ & $4.00 \mathrm{E}-03$ & $8.00 \mathrm{E}-03$ & $1.12 \mathrm{E}-03$ & $3.56 \mathrm{E}+00$ & $1.79 \mathrm{E}-03$ & $2.22 \mathrm{E}-04$ & $1.44 \mathrm{E}-02$ \\
\hline $\begin{array}{l}\mathrm{GM}=\mathrm{G} \\
\mathrm{GSD}=\mathrm{G} \\
\mathrm{N} / \mathrm{A}=\mathrm{N} \\
\mathrm{STD}=\mathrm{S}\end{array}$ & $\begin{array}{l}\text { vailable in } \\
\text { lard Deviat }\end{array}$ & forrate & ewed. & & & & \\
\hline
\end{tabular}

Table 2.17. CLBVCL Concentration Ratio - Grain to Soil

\begin{tabular}{cccccccc}
\hline Element & Mean & STD & Median & GSD & GM & $5 \%$ & $95 \%$ \\
\hline $\mathrm{Ac}$ & N/A & N/A & $2.20 \mathrm{E}-05$ & N/A & N/A & N/A & N/A \\
$\mathrm{Ag}$ & N/A & N/A & $2.50 \mathrm{E}-01$ & N/A & N/A & N/A & N/A \\
$\mathrm{Al}$ & $4.00 \mathrm{E}-03$ & $2.00 \mathrm{E}-03$ & $2.49 \mathrm{E}-03$ & $1.60 \mathrm{E}+00$ & $3.58 \mathrm{E}-03$ & $1.64 \mathrm{E}-03$ & $7.78 \mathrm{E}-03$ \\
$\mathrm{Am}$ & & N/A & N/A & $1.10 \mathrm{E}+01$ & $2.20 \mathrm{E}-05$ & N/A & N/A \\
$\mathrm{As}$ & $1.90 \mathrm{E}-02$ & $6.00 \mathrm{E}-03$ & $1.40 \mathrm{E}-02$ & $1.36 \mathrm{E}+00$ & $1.81 \mathrm{E}-02$ & $1.09 \mathrm{E}-02$ & $3.01 \mathrm{E}-02$ \\
$\mathrm{Au}$ & N/A & N/A & $2.50 \mathrm{E}-01$ & N/A & N/A & N/A & N/A \\
$\mathrm{Ba}$ & $5.00 \mathrm{E}-03$ & $2.00 \mathrm{E}-03$ & $3.40 \mathrm{E}-03$ & $1.47 \mathrm{E}+00$ & $4.64 \mathrm{E}-03$ & $2.46 \mathrm{E}-03$ & $8.75 \mathrm{E}-03$ \\
$\mathrm{Be}$ & N/A & N/A & $3.00 \mathrm{E}-03$ & N/A & N/A & N/A & N/A \\
$\mathrm{Bi}$ & N/A & N/A & $5.00 \mathrm{E}-01$ & N/A & N/A & N/A & N/A \\
$\mathrm{Br}$ & $1.06 \mathrm{E}+00$ & $3.01 \mathrm{E}-01$ & $8.00 \mathrm{E}-01$ & $1.32 \mathrm{E}+00$ & $1.02 \mathrm{E}+00$ & $6.43 \mathrm{E}-01$ & $1.61 \mathrm{E}+00$
\end{tabular}


Table 2.17. (contd)

\begin{tabular}{|c|c|c|c|c|c|c|c|}
\hline Element & Mean & STD & Median & GSD & GM & $5 \%$ & $95 \%$ \\
\hline $\mathrm{Ca}$ & $1.08 \mathrm{E}-01$ & $3.10 \mathrm{E}-02$ & $8.15 \mathrm{E}-02$ & $1.32 \mathrm{E}+00$ & $1.04 \mathrm{E}-01$ & $6.53 \mathrm{E}-02$ & $1.65 \mathrm{E}-01$ \\
\hline $\mathrm{Ca}$ & $\mathrm{N} / \mathrm{A}$ & N/A & $\mathrm{N} / \mathrm{A}$ & $3.70 \mathrm{E}+00$ & $8.70 \mathrm{E}+00$ & N/A & N/A \\
\hline $\mathrm{Cd}$ & N/A & N/A & N/A & $2.70 \mathrm{E}+00$ & $8.80 \mathrm{E}-01$ & N/A & N/A \\
\hline $\mathrm{Ce}$ & N/A & N/A & N/A & $3.70 \mathrm{E}+00$ & $3.10 \mathrm{E}-03$ & N/A & N/A \\
\hline $\mathrm{Cf}$ & $\mathrm{N} / \mathrm{A}$ & N/A & $2.20 \mathrm{E}-05$ & N/A & $\mathrm{N} / \mathrm{A}$ & N/A & N/A \\
\hline $\mathrm{Cl}$ & $1.16 \mathrm{E}+01$ & $2.61 \mathrm{E}+03$ & $4.30 \mathrm{E}-01$ & $2.69 \mathrm{E}+01$ & $5.12 \mathrm{E}-02$ & $2.28 \mathrm{E}-04$ & $1.15 \mathrm{E}+01$ \\
\hline $\mathrm{Cm}$ & N/A & N/A & N/A & $3.30 \mathrm{E}+00$ & $2.30 \mathrm{E}-05$ & N/A & N/A \\
\hline $\mathrm{Co}$ & N/A & N/A & N/A & $5.50 \mathrm{E}+00$ & $8.50 \mathrm{E}-03$ & N/A & N/A \\
\hline $\mathrm{Cr}$ & $2.00 \mathrm{E}-04$ & N/A & N/A & $\mathrm{N} / \mathrm{A}$ & $\mathrm{N} / \mathrm{A}$ & N/A & $\mathrm{N} / \mathrm{A}$ \\
\hline Cs & $\mathrm{N} / \mathrm{A}$ & N/A & N/A & $4.00 \mathrm{E}+00$ & $2.90 \mathrm{E}-02$ & N/A & $\mathrm{N} / \mathrm{A}$ \\
\hline $\mathrm{Cu}$ & N/A & N/A & $2.50 \mathrm{E}-01$ & $\mathrm{~N} / \mathrm{A}$ & $\mathrm{N} / \mathrm{A}$ & N/A & $\mathrm{N} / \mathrm{A}$ \\
\hline Dy & N/A & N/A & $2.00 \mathrm{E}-02$ & N/A & N/A & N/A & N/A \\
\hline $\mathrm{Er}$ & N/A & N/A & $2.00 \mathrm{E}-02$ & N/A & N/A & $\mathrm{N} / \mathrm{A}$ & N/A \\
\hline $\mathrm{Eu}$ & $2.00 \mathrm{E}-03$ & $1.00 \mathrm{E}-03$ & $1.25 \mathrm{E}-03$ & $1.60 \mathrm{E}+00$ & $1.79 \mathrm{E}-03$ & $8.22 \mathrm{E}-04$ & $3.89 \mathrm{E}-03$ \\
\hline $\mathrm{F}$ & $\mathrm{N} / \mathrm{A}$ & $\mathrm{N} / \mathrm{A}$ & $6.00 \mathrm{E}-03$ & N/A & N/A & N/A & $\mathrm{N} / \mathrm{A}$ \\
\hline $\mathrm{Fe}$ & $2.00 \mathrm{E}-04$ & N/A & N/A & N/A & N/A & N/A & $\mathrm{N} / \mathrm{A}$ \\
\hline $\mathrm{Ga}$ & $\mathrm{N} / \mathrm{A}$ & N/A & $4.00 \mathrm{E}-04$ & N/A & N/A & N/A & N/A \\
\hline $\mathrm{Gd}$ & N/A & N/A & $2.00 \mathrm{E}-02$ & N/A & N/A & N/A & N/A \\
\hline $\mathrm{Hf}$ & $2.00 \mathrm{E}-03$ & $1.00 \mathrm{E}-03$ & $1.25 \mathrm{E}-03$ & $1.60 \mathrm{E}+00$ & $1.79 \mathrm{E}-03$ & $8.22 \mathrm{E}-04$ & $3.89 \mathrm{E}-03$ \\
\hline $\mathrm{Hg}$ & $\mathrm{N} / \mathrm{A}$ & N/A & $4.90 \mathrm{E}-01$ & $\mathrm{~N} / \mathrm{A}$ & N/A & N/A & N/A \\
\hline Ho & N/A & N/A & $4.00 \mathrm{E}-03$ & $\mathrm{~N} / \mathrm{A}$ & N/A & N/A & $\mathrm{N} / \mathrm{A}$ \\
\hline I & N/A & N/A & $4.00 \mathrm{E}-02$ & N/A & $\mathrm{N} / \mathrm{A}$ & N/A & $\mathrm{N} / \mathrm{A}$ \\
\hline In & N/A & N/A & $4.00 \mathrm{E}-04$ & N/A & N/A & N/A & N/A \\
\hline $\mathrm{Ir}$ & N/A & N/A & $1.50 \mathrm{E}-02$ & N/A & N/A & $\mathrm{N} / \mathrm{A}$ & N/A \\
\hline $\mathrm{K}$ & $2.78 \mathrm{E}-01$ & $7.10 \mathrm{E}-02$ & $2.16 \mathrm{E}-01$ & $1.29 \mathrm{E}+00$ & $2.69 \mathrm{E}-01$ & $1.78 \mathrm{E}-01$ & $4.07 \mathrm{E}-01$ \\
\hline $\mathrm{La}$ & $2.00 \mathrm{E}-05$ & N/A & $\mathrm{N} / \mathrm{A}$ & N/A & N/A & N/A & N/A \\
\hline $\mathrm{Lu}$ & $4.00 \mathrm{E}-04$ & $1.00 \mathrm{E}-03$ & $9.79 \mathrm{E}-05$ & $4.09 \mathrm{E}+00$ & $1.49 \mathrm{E}-04$ & $1.47 \mathrm{E}-05$ & $1.50 \mathrm{E}-03$ \\
\hline $\mathrm{Mg}$ & $2.31 \mathrm{E}-01$ & $1.13 \mathrm{E}-01$ & $1.45 \mathrm{E}-01$ & $1.59 \mathrm{E}+00$ & $2.08 \mathrm{E}-01$ & $9.69 \mathrm{E}-02$ & $4.45 \mathrm{E}-01$ \\
\hline $\mathrm{Mn}$ & N/A & N/A & N/A & $3.30 \mathrm{E}+00$ & $2.80 \mathrm{E}-01$ & N/A & N/A \\
\hline Mo & $8.00 \mathrm{E}-01$ & N/A & N/A & N/A & N/A & N/A & $\mathrm{N} / \mathrm{A}$ \\
\hline $\mathrm{N}$ & N/A & N/A & $1.30 \mathrm{E}-01$ & N/A & N/A & N/A & N/A \\
\hline $\mathrm{Na}$ & $4.40 \mathrm{E}-02$ & $1.00 \mathrm{E}-02$ & $3.52 \mathrm{E}-02$ & $1.25 \mathrm{E}+00$ & $4.29 \mathrm{E}-02$ & $2.97 \mathrm{E}-02$ & $6.21 \mathrm{E}-02$ \\
\hline $\mathrm{Nb}$ & $1.40 \mathrm{E}-02$ & N/A & N/A & N/A & N/A & N/A & N/A \\
\hline $\mathrm{Nd}$ & $1.20 \mathrm{E}-02$ & $3.00 \mathrm{E}-03$ & $9.38 \mathrm{E}-03$ & $1.28 \mathrm{E}+00$ & $1.16 \mathrm{E}-02$ & $7.76 \mathrm{E}-03$ & $1.75 \mathrm{E}-02$ \\
\hline $\mathrm{Ni}$ & $3.09 \mathrm{E}-01$ & $5.02 \mathrm{E}-01$ & $9.92 \mathrm{E}-02$ & $3.12 \mathrm{E}+00$ & $1.62 \mathrm{E}-01$ & $2.50 \mathrm{E}-02$ & $1.05 \mathrm{E}+00$ \\
\hline $\mathrm{Np}$ & N/A & N/A & $\mathrm{N} / \mathrm{A}$ & $5.00 \mathrm{E}+00$ & $2.90 \mathrm{E}-03$ & N/A & $\mathrm{N} / \mathrm{A}$ \\
\hline $\mathrm{O}$ & N/A & N/A & $6.00 \mathrm{E}-03$ & N/A & N/A & N/A & N/A \\
\hline Os & N/A & N/A & $3.50 \mathrm{E}-03$ & N/A & $\mathrm{N} / \mathrm{A}$ & N/A & $\mathrm{N} / \mathrm{A}$ \\
\hline$P$ & N/A & N/A & $3.50 \mathrm{E}+00$ & N/A & $\mathrm{N} / \mathrm{A}$ & N/A & N/A \\
\hline $\mathrm{Pa}$ & N/A & N/A & $2.20 \mathrm{E}-05$ & N/A & N/A & N/A & N/A \\
\hline $\mathrm{Pb}$ & N/A & N/A & N/A & $3.60 \mathrm{E}+00$ & $1.10 \mathrm{E}-02$ & N/A & N/A \\
\hline $\mathrm{Pd}$ & N/A & N/A & $4.00 \mathrm{E}-02$ & N/A & N/A & N/A & $\mathrm{N} / \mathrm{A}$ \\
\hline $\mathrm{Pm}$ & N/A & N/A & N/A & $6.00 \mathrm{E}+00$ & $1.40 \mathrm{E}-02$ & N/A & N/A \\
\hline Po & $2.40 \mathrm{E}-04$ & N/A & N/A & N/A & N/A & N/A & N/A \\
\hline $\operatorname{Pr}$ & $2.00 \mathrm{E}-02$ & N/A & N/A & N/A & N/A & N/A & $\mathrm{N} / \mathrm{A}$ \\
\hline $\mathrm{Pu}$ & N/A & N/A & N/A & $6.70 \mathrm{E}+00$ & $9.50 \mathrm{E}-06$ & N/A & N/A \\
\hline $\mathrm{Ra}$ & N/A & N/A & N/A & $1.20 \mathrm{E}+01$ & $1.70 \mathrm{E}-02$ & N/A & N/A \\
\hline
\end{tabular}


Table 2.17. (contd)

\begin{tabular}{|c|c|c|c|c|c|c|c|}
\hline Element & Mean & STD & Median & GSD & GM & $5 \%$ & $95 \%$ \\
\hline $\mathrm{Rb}$ & $9.00 \mathrm{E}-01$ & $\mathrm{~N} / \mathrm{A}$ & $\mathrm{N} / \mathrm{A}$ & $\mathrm{N} / \mathrm{A}$ & N/A & $\mathrm{N} / \mathrm{A}$ & N/A \\
\hline $\mathrm{Re}$ & N/A & N/A & $3.50 \mathrm{E}-01$ & N/A & N/A & N/A & N/A \\
\hline $\mathrm{Rh}$ & $\mathrm{N} / \mathrm{A}$ & N/A & $4.00 \mathrm{E}-02$ & N/A & N/A & N/A & N/A \\
\hline $\mathrm{Ru}$ & N/A & $\mathrm{N} / \mathrm{A}$ & N/A & $2.60 \mathrm{E}+00$ & $3.00 \mathrm{E}-03$ & N/A & N/A \\
\hline $\mathrm{S}$ & N/A & N/A & $1.50 \mathrm{E}+00$ & N/A & N/A & N/A & N/A \\
\hline $\mathrm{Sb}$ & N/A & $\mathrm{N} / \mathrm{A}$ & N/A & $2.70 \mathrm{E}+00$ & $1.80 \mathrm{E}-03$ & N/A & N/A \\
\hline $\mathrm{Sc}$ & $1.00 \mathrm{E}-03$ & N/A & N/A & N/A & N/A & N/A & N/A \\
\hline $\mathrm{Se}$ & N/A & N/A & $2.50 \mathrm{E}-01$ & N/A & N/A & N/A & N/A \\
\hline $\mathrm{Si}$ & N/A & N/A & $7.00 \mathrm{E}-02$ & N/A & N/A & $\mathrm{N} / \mathrm{A}$ & N/A \\
\hline $\mathrm{Sm}$ & N/A & N/A & $2.00 \mathrm{E}-02$ & N/A & N/A & N/A & N/A \\
\hline $\mathrm{Sn}$ & N/A & N/A & $6.00 \mathrm{E}-03$ & N/A & N/A & N/A & N/A \\
\hline $\mathrm{Sr}$ & N/A & N/A & N/A & $2.70 \mathrm{E}+00$ & $1.10 \mathrm{E}-01$ & N/A & N/A \\
\hline $\mathrm{Ta}$ & $2.00 \mathrm{E}-03$ & $1.00 \mathrm{E}-03$ & $1.25 \mathrm{E}-03$ & $1.60 \mathrm{E}+00$ & $1.79 \mathrm{E}-03$ & 8.22E-04 & $3.89 \mathrm{E}-03$ \\
\hline $\mathrm{Tb}$ & $3.00 \mathrm{E}-03$ & $1.00 \mathrm{E}-03$ & $2.17 \mathrm{E}-03$ & $1.38 \mathrm{E}+00$ & $2.85 \mathrm{E}-03$ & $1.67 \mathrm{E}-03$ & $4.85 \mathrm{E}-03$ \\
\hline $\mathrm{Tc}$ & N/A & N/A & $7.30 \mathrm{E}-01$ & N/A & N/A & N/A & N/A \\
\hline $\mathrm{Te}$ & $1.00 \mathrm{E}+01$ & N/A & N/A & N/A & N/A & N/A & N/A \\
\hline Th & N/A & $\mathrm{N} / \mathrm{A}$ & N/A & $3.40 \mathrm{E}+00$ & $2.10 \mathrm{E}-03$ & $\mathrm{~N} / \mathrm{A}$ & N/A \\
\hline $\mathrm{Ti}$ & $5.40 \mathrm{E}-05$ & $\mathrm{~N} / \mathrm{A}$ & N/A & N/A & N/A & N/A & N/A \\
\hline $\mathrm{Tl}$ & N/A & N/A & 4.00E-04 & N/A & N/A & $\mathrm{N} / \mathrm{A}$ & N/A \\
\hline $\mathrm{U}$ & N/A & N/A & N/A & $7.70 \mathrm{E}+00$ & $6.20 \mathrm{E}-03$ & N/A & N/A \\
\hline $\mathrm{V}$ & $1.00 \mathrm{E}-02$ & $5.00 \mathrm{E}-03$ & $6.24 \mathrm{E}-03$ & $1.60 \mathrm{E}+00$ & 8.94E-03 & $4.11 \mathrm{E}-03$ & $1.95 \mathrm{E}-02$ \\
\hline $\mathrm{W}$ & N/A & N/A & $3.00 \mathrm{E}+00$ & N/A & N/A & N/A & N/A \\
\hline $\mathrm{Y}$ & $5.00 \mathrm{E}-04$ & N/A & N/A & N/A & N/A & N/A & N/A \\
\hline $\mathrm{Yb}$ & $1.10 \mathrm{E}-02$ & $4.00 \mathrm{E}-03$ & $7.73 \mathrm{E}-03$ & $1.42 \mathrm{E}+00$ & $1.03 \mathrm{E}-02$ & $5.79 \mathrm{E}-03$ & $1.85 \mathrm{E}-02$ \\
\hline $\mathrm{Zn}$ & N/A & N/A & N/A & $2.70 \mathrm{E}+00$ & $1.80 \mathrm{E}+00$ & N/A & N/A \\
\hline $\mathrm{Zr}$ & $5.00 \mathrm{E}-03$ & $4.00 \mathrm{E}-03$ & $2.47 \mathrm{E}-03$ & $2.02 \mathrm{E}+00$ & $3.90 \mathrm{E}-03$ & $1.23 \mathrm{E}-03$ & $1.24 \mathrm{E}-02$ \\
\hline \multicolumn{8}{|c|}{$\begin{array}{l}\text { GM }=\text { Geometric mean. } \\
\text { GSD }=\text { Geometric Standard Deviation. } \\
\text { N/A }=\text { Not available in the references reviewed. } \\
\text { STD }=\text { Standard Deviation. }\end{array}$} \\
\hline
\end{tabular}

\subsubsection{CLBVAx Bioconcentration in Animal Forage or Hay from Soil}

These parameters represent the bioconcentration of a constituent in dry forage (CLBVAF) and in dry hay (CLBVAH) when the constituent is in the soil. Due to data availability, identical values are assigned to both of these values. Examples of dry forage are alfalfa hay and sun-cured timothy hay. These values are not currently used in GENII (although the inputs are listed in the GENII database). Additional data have been reviewed and compiled and are available from the authors; they are not included here at this time because they are not directly used by the code.

\subsubsection{CLBVAG Bioconcentration in Animal Feed Grain from Soil}

This parameter value should be assigned a value identical to that of CLBVCL, the bioconcentration of a constituent in soil to the grain consumed by humans. These two parameters were established to 
differentiate between grain consumed by animals and by humans. However, these values are considered to be essentially identical. The user is responsible for entering the same values in CLBVAG and CLBVCL.

The units and range for CLBVAG in Gv2 are: $\mathrm{kg}_{\text {dry_plant }} / \mathrm{kg}_{\text {dry_soil }}(0$ to 5000$)$.

See CLBVCL in Section 2.1.17 for further information and parameter values.

\subsubsection{CLBVOV Bioconcentration in Dry Other Vegetables from Soil}

This is similar to the bioconcentration of a constituent in soil to the other vegetables consumed by humans, but is applied in this case to other vegetables consumed by meat and milk animals. This parameter is not implemented in the current models used for human ingestion of animal products.

The units and range for this parameter in Gv2 are: N/A.

\subsubsection{CLVD Atmospheric Deposition Velocity}

The deposition velocity is the rate of deposition of particulate matter from air to the ground. This parameter is not implemented via entries in this module, but is dealt with internally in the Air module (Section 4.0).

The units and range for this parameter in Gv2 are: $\mathrm{m} / \mathrm{sec}(0$ to 1$)$.

\subsubsection{CLSHALF Decay Half-Life in Soil}

CLSHALF describes the loss by radiological transformation or chemical reaction in soil. This parameter is typically applicable to irrigated soil leaching and will be implemented in the Exposure Module. The provided default values are not implemented for Chronic Air or Chronic Water emission scenarios.

The units and minimum for this parameter in Gv2 are: day (no range defined).

\subsubsection{CLCLASS Atmospheric Deposition Class}

CLCLASS describes characteristics of the constituent for evaluation of loses during atmospheric transport. This parameter is not implemented in the Gv2 modules for chronic air transport.

For information purposes only, the CLCLASS values are as follows:

- Class Description

- particles, radius $=7.5$ micron

- particles, radius $=3.0$ micron

- particles, radius $=0.3$ micron (default for inorganic chemicals, radionuclides)

- depositing gas, surface resistance of reactive gas 
- non-depositing gas (e.g., volatile organic chemicals)

- gas behaving as a particle (same deposition rate as class 3), absorbed on particulate material.

\subsection{References: Constituent Module}

Denham DH and JK Soldat. 1974. A Study of Selected Parameters Affecting the Radiation Dose from Drinking Water Downstream of Nuclear Facilities. BNWL-SA-4545, Pacific Northwest Laboratory, Richland, Washington.

DOE. 2011. Derived Concentration Technical Standard. DOE-STD-1196-2011, U.S. Department of Energy, Washington, D.C. Available at http://www.hss.doe.gov/nuclearsafety/techstds/standard.html\#1001.

Eckerman KF, AB Wolbarst, and ACB Richardson. 1988. Limiting Values of Radionuclide Intake and Air Concentration and Dose Conversion Factors for Inhalation, Submersion, and Ingestion. Federal Guidance Report No. 11, EPA-520/1-88-020, U.S. Environmental Protection Agency, Office of Radiation and Indoor Air, Washington, D.C. Available at http://www.epa.gov/radiation/federal/techdocs.html.

Eckerman KF and JC Ryman. 1993. External Exposure to Radionuclides in Air, Water, and Soil. Federal Guidance Report No. 12, EPA 402-R-93-081, U.S. Environmental Protection Agency, Office of Radiation and Indoor Air, Washington, D.C. Available at http://www.epa.gov/radiation/federal/techdocs.html.

Eckerman KF, RW Leggett, CB Nelson, JS Puskin, and ACB Richardson. 1998. Health Risks from Lowlevel Environmental Exposure to Radionuclides. Federal Guidance Report No. 13, Part I - Interim Version. Available at http://www.epa.gov/radiation/federal/techdocs.html.

IAEA. 2004. Sediment Distribution Coefficients and Concentration Factors for Biota in the Marine Environment. Technical Report No. 422, International Atomic Energy Agency, Vienna, Austria.

IAEA. 2010. Handbook of Parameter Value for the Prediction of Radionuclide Transfer in Terrestrial and Freshwater Environments. Technical Report No. 472, International Atomic Energy Agency, Vienna, Austria.

ICRP. 1990. Age-dependent Doses to Members of the Public from Intake of Radionuclides - Part 1. ICRP (International Commission on Radiological Protection) Publication 56, Ann. ICRP 20 (2).

ICRP. 1992. Age-dependent Doses to Members of the Public from Intake of Radionuclides - Part 2 Ingestion Dose Coefficients. ICRP (International Commission on Radiological Protection)

Publication 67, Ann. ICRP 22 (3-4).

ICRP. 1994. Human Respiratory Tract Model for Radiological Protection. ICRP (International Commission on Radiological Protection) Publication 66, Ann. ICRP 24 (1-3).

ICRP. 1995a. Age-dependent Doses to Members of the Public from Intake of Radionuclides - Part 3 Ingestion Dose Coefficients. ICRP (International Commission on Radiological Protection)

Publication 69, Ann. ICRP 25 (1). 
ICRP. 1995b. Age-dependent Doses to Members of the Public from Intake of Radionuclides - Part 4 Inhalation Dose Coefficients. ICRP (International Commission on Radiological Protection) Publication 71, Ann. ICRP 25 (3-4).

ICRP. 1995c. Age-dependent Doses to the Members of the Public from Intake of Radionuclides - Part 5 Compilation of Ingestion and Inhalation Coefficients. ICRP (International Commission on Radiological Protection) Publication 72, Ann. ICRP 26 (1).

ICRP. 1996. Conversion Coefficients for use in Radiological Protection against External Radiation. ICRP (International Commission on Radiological Protection) Publication 74, Ann. ICRP 26 (3-4).

ICRP. 2012. Compendium of Dose Coefficients based on ICRP Publication 60. ICRP (International Commission on Radiological Protection) Publication 119, Ann. ICRP 41(s). Available at: http://www.icrp.org/publication.asp?id=ICRP\%20Publication\%20119.

Napier BA, RA Peloquin, DL Strenge, and JV Ramsdell. 1988. Hanford Environmental Dosimetry Upgrade Project, GENII - The Hanford Environmental Radiation Dosimetry Software System, Vol. 1: Conceptual Representation. PNL-6584, Vol. 1, Pacific Northwest Laboratory, Richland, Washington.

Napier BA, DL Strenge, JV Ramsdell, Jr., PW Eslinger, and C Fosmire. 2012. GENII Version 2, Software Design Document. PNNL-14584, Rev. 4, Pacific Northwest National Laboratory, Richland, Washington.

Napier BA, RJ Fellows, and LD Minc. 2012. DRAFT. Transfer Factors for Contaminant Uptake by Tree Fruits. Pacific Northwest National Laboratory, Richland, Washington.

Sheppard SC, JM Long, and B Sanipelli. 2010a. "Measured Elemental Transfer Factors for Boreal Hunter/Gatherer Scenarios: Fish, Game and Berries." Journal of Environmental Radioactivity 101(11):902-909.

Staven LH, BA Napier, K Rhoads, and DL Strenge. 2003. A Compendium of Transfer Factors for Agricultural and Animal Products. PNNL-13421, Pacific Northwest National Laboratory, Richland, Washington.

Strom DJ and PS Stansbury. 2000. "Determining Parameters of Lognormal Distributions from Minimal Information.” American Industrial Hygiene Association Journal 61(6):977-880. 



\subsection{Module: User-Defined Air}

For scenarios with chronic atmospheric releases, the User-Defined module, AFF Air Module is selected. This module is downstream of the Constituent module and upstream of the Air Module. The user may wish to edit the User Label for this module to "Source term" or a more site-specific descriptive name for the facility creating the radioactive material release. The module model selected is "AFF Air Module." The user enters the annual activity released in this module.

The release information entered in this module is user-specific. The user enters the following facilityspecific information (default units listed):

- Type of release: point or area

- Exit area of source $\left(\mathrm{m}^{2}\right)$

- Exit height of source (point release only) (m)

- Height of adjacent structure (m)

- Exit velocity of source $(\mathrm{m} / \mathrm{s})$

- Exit temperature of source (degrees $\mathrm{C}$ )

- Ambient air temperature (degrees $\mathrm{C}$ ).

Consult the Gv2 Users Guide (Napier 2012) and Software Design Document (Napier et al. 2012, particularly, Section 5.3) for guidance on characterizing your facility specific emission point.

The radionuclide release information is entered at the bottom of the screen. The radionuclides and progeny selected in the Constituent module will self-populate. The user is then required to enter the annual release activity. Two rows of data should be entered. "Time" entries are 0 and 1. "Particle1" entries are identical and repeat the annual emission rate from the release point. For example if 10 microcuries of Cs-137 are released, enter $1 \mathrm{E} 7 \mathrm{pCi} / \mathrm{yr}$ in both the Time 0 row and Time 1 row. This will model a constant emission rate.

\subsection{References: User-Defined (AIR) Module}

Napier BA. 2012. GENII Version 2 Users' Guide. PNNL-14583, Rev. 4, Pacific Northwest National Laboratory, Richland, Washington.

Napier BA, DL Strenge, JV Ramsdell, Jr., PW Eslinger, and C Fosmire. 2012. GENII Version 2 Software Design Document. PNNL-14584, Rev. 4, Pacific Northwest National Laboratory, Richland, Washington. 



\subsection{Module: Air}

This module is downstream of the User-Defined AIR module and upstream of the Exposure Pathways Module. For scenarios with chronic atmospheric releases, the module model GENII V.2 Air module, Chronic Plume model," is selected. The user enters the characteristics of the atmosphere receiving the airborne effluent in this module.

Table 4.1. Tabs and Sub-Tabs in the Air Module Chronic Plume Model

\begin{tabular}{ll}
\hline \multicolumn{1}{c}{$\mathrm{Tab}$} & \multicolumn{1}{c}{ Second-Level Tab } \\
\hline Model Information & Radial Grid Definition \\
& Model Parameters \\
& Default Parameters \\
& Meteorological Files \\
Source Information & None \\
\hline
\end{tabular}

\subsection{Tab: Model Information/Radial Grid Definition}

The user sets the output grid that results from the atmospheric dispersion modeling. Sixteen-sector radial grids with user-entered distances are common; thirty-six sectors may be used if the available meteorological data are supplied in 10-degree increments. Distances are typically based on distance to the receptor(s) of interest and additional uniformly-spaced distances. A first distance of less than $100 \mathrm{~m}$ should not be used.

\subsection{Tab: Model Information/Model Parameters}

The user selects the model used with the meteorological input to estimate atmospheric dispersion via radio buttons in the Model Parameters subtab. "Brigg's Open Country" is recommended for generic use. Details on the various model assumptions and differences are discussed in Napier et al. (2012) and Napier et al. (2011).

\subsection{Tab: Model Information/Default Parameters}

The user assigns specific parameters values.

\subsubsection{ARMINRISESPD Minimum Speed during Plume Rise}

The minimum speed during plume rise is required for model calculations. When wind is calm a surrogate value is used to prevent numerical instability in the equations. A minimum stack height wind speed is specified because very low wind speeds cause unrealistic values. The user should not apply a value of less than $0.5 \mathrm{~m} / \mathrm{s}$.

\footnotetext{
${ }^{1}$ If the user believes the Chronic Puff model is more appropriate for their scenario, consult the Napier (2012a) and Napier et al. (2012). Only the Chronic Plume model inputs are described here.
} 
The units and range for this parameter in Gv2 are: $\mathrm{m} / \mathrm{s}(0-99.99)$.

A value of $1.5 \mathrm{~m} / \mathrm{s}$ is recommended.

\subsubsection{Additional Sources of Information}

No additional sources identified.

\subsubsection{ARMINSIGYSHIFT Sigma Shift to SI Cloud Shine}

When a semi-infinite cloud shine model is used, the width of the plume must be sufficient to model cloud-shine. A minimum Sigma shift of $400 \mathrm{~m}$ is required for appropriate modeling. This value, $400 \mathrm{~m}$, is assigned for generic applications.

The units and range for this parameter in Gv2 are: $\mathrm{m}(0-10,000)$.

\subsubsection{Additional Sources of Information}

No additional sources are provided.

\subsubsection{ARTRANSRESIST Transfer Resistance for lodine and Particles}

Dry deposition velocities consider aerodynamic resistance, surface resistance, and transfer resistance. Transfer resistance is generally a function of the depositing surface, for example, whether leaf stomatal openings are open or closed. Transfer resistance is used to establish an upper limit on the deposition velocity.

The units for this parameter in Gv2 are: $\mathrm{s} / \mathrm{m}(0-1,000,000)$.

The Gv2 Software Design Document (SDD) (Napier et al. 2012) indicates appropriate values to apply. A value of $10 \mathrm{~s} / \mathrm{m}$ is recommended for reactive gases (e.g., iodine) and $100 \mathrm{~s} / \mathrm{m}$ for fine particulates. Use of these values will result in dry deposition results that are consistent with reported values.

\subsubsection{Additional Sources of Information}

The information provided in the SDD references Ramsdell et al. (1994).

The impact of various transfer resistance values on deposition velocity results is available in Napier (2012b, p. 6).

\subsubsection{ARMINWIND Maximum Wind Speed for "Calm"}

This value represents the lowest speed reliably indicated by the measuring anemometer. This value is user-determined when user-provided meteorological data files are used and would be available from consultation with the person who created the meteorological data. 
The units for this parameter in Gv2 are: $\mathrm{m} / \mathrm{s}(0-2)$.

A generic value of $0.8 \mathrm{~m} / \mathrm{s}$ may be used.

\subsection{References: Air Module}

Napier BA. 2012a. GENII Version 2 Users' Guide. PNNL-14583, Rev. 4, Pacific Northwest National Laboratory, Richland, Washington.

Napier BA. 2012b. Dry Deposition Velocity Estimation for the Savannah River Site: Part 1 Parametric Analysis. PNNL-21144, Pacific Northwest National Laboratory, Richland, Washington.

Napier BA, JP Rishel, and NE Bixler. 2011. Final Review of Safety Assessment Issues at Savannah River Site, August 2011. PNNL-20990, (especially Appendix A), Pacific Northwest National Laboratory, Richland, Washington.

Napier BA, DL Strenge, JV Ramsdell, Jr., PW Eslinger, and C Fosmire. 2012. GENII Version 2 Software Design Document. PNNL-14584, Rev. 4, Pacific Northwest National Laboratory, Richland, Washington.

Ramsdell Jr, JV, CA Simonen, and KW Burk. 1994. Regional Atmospheric Transport Code for Hanford Emission Tracking (RATCHET). PNWD-2224 HEDR, Pacific Northwest National Laboratories, Richland, Washington. 



\subsection{Module: User-Defined Water}

For scenarios with chronic releases to surface waters, the User-Defined module with a module model "WFF Surface Water Module" selected. This module is downstream of the Constituent module and upstream of the Surface Water Module. The user enters the annual activity released in the liquid effluent in this module. The user may wish to edit the User Label for this module to "Source term" or a more sitespecific descriptive name for the facility creating the radioactive material release.

This module requires limited entries. The FRAMES system requires the facility-specific width and height of the liquid emission point; these are not used by the GENII models and unit vales may be entered. Consult the Gv2 Users Guide (Napier 2012) and Software Design Document (Napier et al. 2012) for guidance on characterizing your facility specific emission point.

The water volume and radionuclide release information is entered at the bottom of the screen. The "water" flux rate $\left(\mathrm{m}^{3} / \mathrm{s}\right)$ is used to determine its effective dilution in the surface water source defined in some available versions of the next module (Surface Water module); it is not used with the GENII models and a unit value may be entered. If the radionuclide release rate for diffuse sources was determined by downstream water sampling (i.e., radionuclide water concentration times water flow rate), then the user is responsible for entering the same water volume in both modules when Chronic Flow Dilution is selected in the Surface Water module. As indicated for the radionuclide information entries, next, two rows of data are entered for this chronic emission scenario, "Time" is 0 and 1; "Flux rate" is the same in both "Time" rows.

Select the nuclides one at a time in the dropdown box that first presents with the "water" entry. Both "Adsorbed Flux" and "Dissolved Flux" columns will present. The radionuclides and progeny selected in the Constituent module will self-populate. The user is required to enter the annual release activity. When using the GENII models, the "Adsorbed Flux" and "Dissolved Flux" are treated identically and summed; for other models enter the activity as "Dissolved Flux" as an over-estimating assumption. Two rows of data should be entered. "Time" entries are 0 and 1. Entries in a single flux column are identical and repeat the annual emission rate. For example if $10 \mu \mathrm{Ci}$ of Cs-137 are released, enter $1 \mathrm{E} 7 \mathrm{pCi} / \mathrm{yr}$ in both the Time 0 row and Time 1 row. This will model a constant emission rate.

\subsection{References: User-Defined (Water) Module}

Napier BA. 2012. GENII Version 2 Users' Guide. PNNL-14583, Rev. 4, Pacific Northwest National Laboratory, Richland, Washington.

Napier BA, DL Strenge, JV Ramsdell, Jr., PW Eslinger, and C Fosmire. 2012. GENII Version 2 Software Design Document. PNNL-14584, Rev. 4, Pacific Northwest National Laboratory, Richland, Washington. 



\subsection{Module: Surface Water}

This module is used for scenarios with chronic releases to surface waters. This module is downstream of the User-Defined (water) module and upstream of the Exposure Pathways Module. The user enters the characteristics of the surface water receiving the liquid effluent in this module.

Tabs in this module include River/Lake and Impoundment. The user would enter information in the Impoundment tab, only if liquid emissions are impounded in a non-publicly accessible location prior to release to the surface water body.

The user-specific information is entered to describe the receiving water body of the facility liquid effluent. Inputs information varies according to the "Type of release and body of water" defined. Consult the Gv2 Users Guide (Napier 2012) and Software Design Document (Napier et al. 2012) for guidance on characterizing your site-specific body of water.

\subsection{References: Surface Water Module}

Napier BA. 2012. GENII Version 2 Users' Guide. PNNL-14583, Rev. 4, Pacific Northwest National Laboratory, Richland, Washington.

Napier BA, DL Strenge, JV Ramsdell, Jr., PW Eslinger, and C Fosmire. 2012. GENII Version 2 Software Design Document. PNNL-14584, Rev. 4, Pacific Northwest National Laboratory, Richland, Washington. 



\subsection{Module: Exposure Pathways - Chronic Exposure}

This module is downstream of either the Surface Water module or Air (chronic plume) module. This module is upstream of the Receptor Intakes module. This module will receive the water or air radionuclide concentration data from the upstream module and use this information to estimate the concentration in various additional media.

The tabs and sub-tabs included in Gv2 Chronic Exposure Module are indicated in Table 7.1. This section is organized by tab and typical parameter input order. The parameters are presented with their code identification.

Table 7.1. Tabs and Sub-Tabs in the Chronic Exposure Module ${ }^{(a)}$

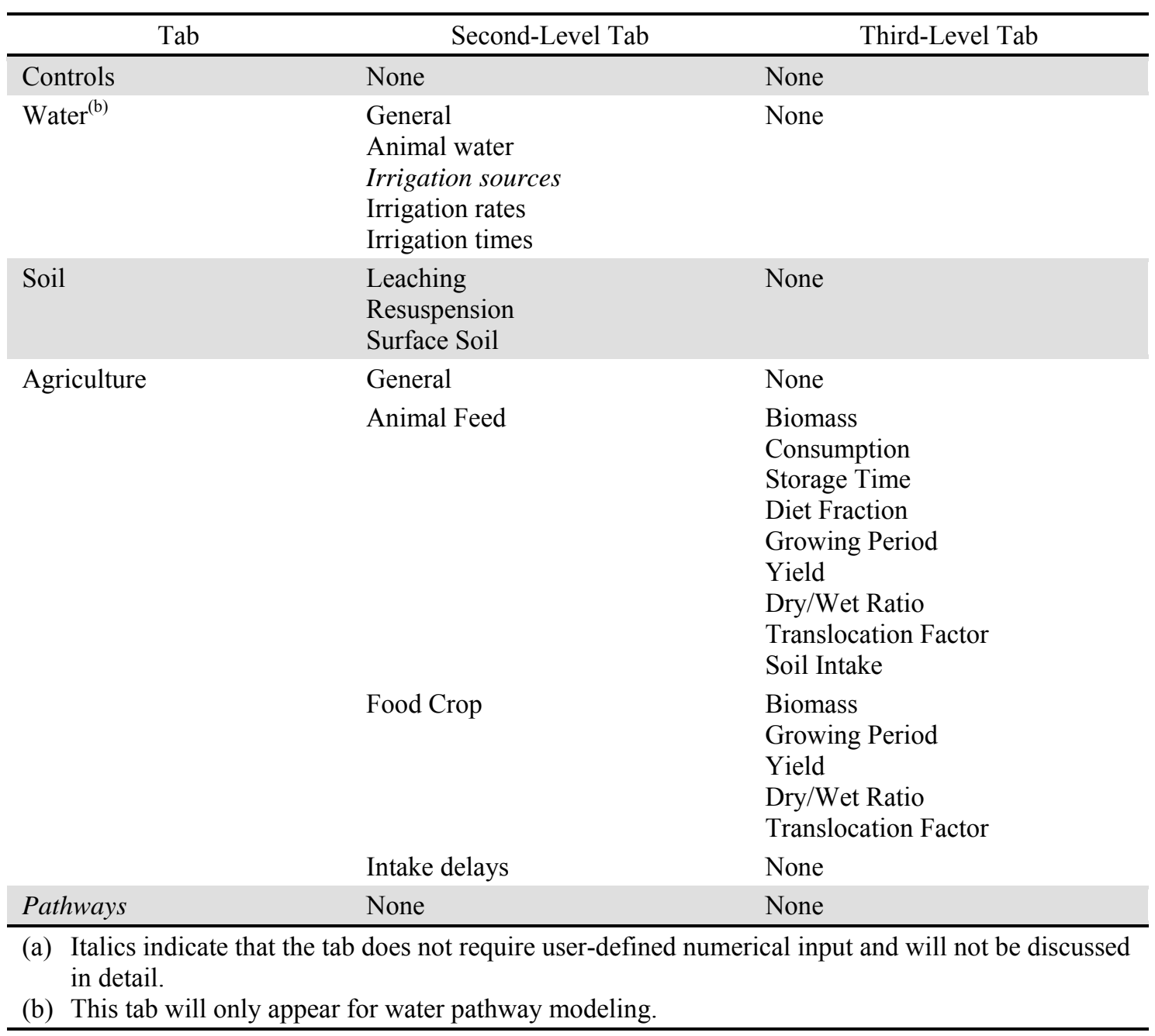

Those interested in an overview of concepts for environmental models for radioactive material releases are directed to IAEA (2009). An overview of pathways, definitions, standardized nomenclature, and other topics are provided for terrestrial and freshwater environments. The Gv2 models track those identified in IAEA (2009). 
A set of definitions provided in Table 1 of IAEA (2009) for CR, aggregate transfer factor, and feed transfer coefficient differs somewhat from that of Gv2. These terms describe the ratio of radioactive material concentrations in one compartment to that of another. The compartments considered include plant tissue-to-soil, aquatic food tissue-to-water, and animal tissue-to-feed. Gv2 uses the terms bioaccumulation factors for tissue-to-water ratios and tissue-to-sediment ratios (see Sections 2.1.11, 2.1.12, and 2.1.13), transfer factors for animal product-to-feed ratios (see Sections 2.1.14, 2.1.15, and 2.1.16) and bioconcentration factors for feed-to-soil ratios (see Sections 2.1.17, 2.1.18, 2.1.19, and 2.1.20).

\subsection{Tab: Controls}

There are limited inputs in the Control tab. Chronic scenarios (1 yr stable exposure) indicate a 1-yr Duration of Release Period (NTKEND), 1-yr End of Release Period (RELEND), and a 0-yr Time From Start to Exposure (BEFORE). The final parameter on this tab, Air Deposition Time Prior to Exposure (BEFAIR) has a variable range from 0 to the user-entered BEFORE value. When tritium is included in the source term, absolute humidity information is used; this parameter is discussed in Section 10.1.4. Other parameter value recommendations are indicated, below.

\subsubsection{RF1 Root Fraction}

The Fraction of plant roots in surface soil is used in the food crop and animal feed analyses and represents the fraction of plant roots that are in the contaminated soil layer. The uptake by plants is assumed to be proportional to this fraction.

The units for this parameter in Gv2 are: fraction (0 to 1).

This value is set to 1, as a conservative assumption, and is consistent with the assumptions used in the development of plant transfer factors. A value of 1 will be representative for most food crop applications.

\subsubsection{Additional sources of information}

Section 5.1 of IAEA (2010) indicates factors that affect radionuclide uptake from the soil. It is indicated that models sometimes consider variable root depth by crop type, with grass root depth at $10 \mathrm{~cm}$ and all other crop root depth at $20 \mathrm{~cm}$. These values may be considered with respect to the level of radionuclide mixing at various depths. Air deposition in dry climates would likely experience less radionuclide mixing than irrigation water depositions.

\subsubsection{RAIN Rainfall Rate}

Average daily rain rate is used to estimate the interception fraction from rain when wet deposition rates are provided in the atmospheric transport output file (ATO), and the user has selected the option to allow the code to calculate the wet deposition interception fraction. Note that the rainfall rates are not transferred with the ATO file, and a consistent value must be re-entered here. The value should reflect the rate "when it is raining," not the annual average.

The units for this parameter in Gv2 are: $\mathrm{mm} / \mathrm{d}(0.01$ to 500$)$. 
The user will enter the value determined from the meteorological data file used. Average the rainfall rate for the year for non-zero rainfall values. RAIN values for several cities are provided in Table 7.2, with a more comprehensive list in Appendix E. If precipitation data is not readily available the value can be approximated as follows.

The National Climatic Data Center (NCDC) provides access to climatological normals based on 30 years (1981-2010) of data for thousands of stations throughout the United States. Climatological values include normal annual precipitation amount and the normal number of days with precipitation greater than a trace (i.e., 0.01 inches or greater). The average daily rainfall rate can be estimated by dividing the normal precipitation amount by the normal number of precipitation days.

Estimation Procedure:

1. Go to http://www1.ncdc.noaa.gov/pub/data/normals/1981-2010/station-inventories/prep-inventory.txt and view the available stations. The first column in the file contains the unique station id that is needed to identify the station when viewing the other files. Columns two, three, and six provide the station latitude, longitude, and name, respectively. A complete description of the file format is available at http://www1.ncdc.noaa.gov/pub/data/normals/1981-2010/readme.txt.

2. Go to http://www1.ncdc.noaa.gov/pub/data/normals/1981-2010/products/precipitatioN/Ann-prcpnormal.txt and find the unique station id from Step 1. The second column provides the normal annual precipitation in hundredths of inches (note: the letter that immediately follows the numeric value is a processing flag; a description of the flag can be found at http://www1.ncdc.noaa.gov/pub/data/normals/1981-2010/readme.txt).

3. Go to http://www1.ncdc.noaa.gov/pub/data/normals/1981-2010/products/precipitatioN/Ann-prcpavgnds-ge001hi.txt and find the unique station id from Step 1. The second column provides the normal number of days with precipitation greater than a trace (i.e., 0.01 inches or greater) (note: the letter that immediately follows the numeric value is a processing flag; a description of the flag can be found at http://www1.ncdc.noaa.gov/pub/data/normals/1981-2010/readme.txt).

4. Convert the normal annual precipitation amount determined in Step 2 from inches (in.) to millimeters $(\mathrm{mm})$ by multiplying by 25.4 (i.e., $1 \mathrm{in} .=25.4 \mathrm{~mm}$ ). Divide the resulting value by the normal number of days with precipitation greater than a trace determined in Step 3-this is the average daily rainfall rate in $\mathrm{mm} / \mathrm{d}$.

In addition, a table of average daily rainfall rates for select cities is provided. It is recommended that the user acquire data using the above procedure, however, because precipitation is highly dependent on location and elevation.

\subsubsection{Additional Sources of Information}

The National Climatic Data Center allows queries of precipitation rates for a limited number of cities, all states, and an indication of whether a certain year's precipitation for a state was generally above, below, or about average. As an approximation, the annual rainfall can be divided by 365 to estimate the RAIN parameter. See the U.S. Climate at a Glance, web page, last accessed at http://www.ncdc.noaa.gov/oa/climate/research/cag3/cag3.html. 


\subsubsection{RAIN Rainfall Rate Table}

Table 7.2. RAIN Daily Rainfall Rate

\begin{tabular}{lcc}
\hline \multicolumn{1}{c}{ Station Name } & Elevation $(\mathrm{m})$ & $\begin{array}{c}\text { Average Rainfall } \\
\text { Rate }(\mathrm{mm} / \mathrm{d})\end{array}$ \\
\hline Boston, MA & 3.7 & 8.8 \\
Buffalo, NY & 214.9 & 6.2 \\
Pittsburgh, PA & 366.7 & 6.4 \\
Lexington, KY & 298.7 & 8.8 \\
Atlanta, GA & 307.8 & 11.1 \\
Tallahassee, FL & 16.8 & 13.5 \\
New Orleans, LA & 1.2 & 13.9 \\
Houston, TX & 13.4 & 13.4 \\
Oklahoma City, OK & 391.7 & 11.0 \\
Lincoln, NE & 362.7 & 7.7 \\
Bismarck, ND & 503.2 & 4.6 \\
Helena, MT & 1166.8 & 3.1 \\
Boulder, CO & 1671.5 & 5.2 \\
Albuquerque, NM & 1618.5 & 3.9 \\
Phoenix, AZ & 337.4 & 5.6 \\
Las Vegas, NV & 649.5 & 4.0 \\
Boise, ID & 857.7 & 3.4 \\
Seattle, WA & 112.8 & 6.3 \\
San Francisco, CA & 45.7 & 8.2 \\
San Diego, CA & 4.6 & 6.3 \\
Juneau, AK & 4.9 & 6.9 \\
Honolulu, HI & 2.1 & 4.9 \\
\hline
\end{tabular}

\subsection{Tab: Water}

The Water tab will be present only when a water pathway scenario is under evaluation. Inputs for this tab apply to

- ingestion of aquatic food types;

- animal water consumption rates and sources; and

- irrigation water rates, sources, and times.

\subsection{Tab: Water/General}

For Treatment Plant Purification Factors applied, see Constituent Module, CLWPF (see Section 2.1.3). For the first evaluation of an annual release, the Irrigation water deposition time prior to exposure (BEFIRR) is 0. Indoor volatilization factor for radon (ANDKRN) is implemented only in the radon model and is not discussed further since radons are not considered for release in the development of this publication. 


\subsubsection{RIRRR Residential Land Irrigation, IRTIMR Irrigation Time for Residential Land}

The IRTIMR parameter indicates the time period over which the annual irrigation is applied to a location where a person works or resides and is used for external exposure to ground pathways. Irrigation of foods for the ingestion pathway is discussed in Section 7.5.1.

The units and range for RIRRR in Gv2 are: in/yr (0-200).

The units and range for IRTIMR in Gv2 are: $\mathrm{mo} / \mathrm{yr}(0-12)$.

The U.S. Department of Agricultural conducts a Census of Agriculture every five years (USDA (2009). Part of the data collected is crop irrigation data. The data describing irrigation rates for pasture can be used to approximate irrigation rates for lawn irrigation. The irrigation rates in the Census are for a variety of crops and are reported by state or by region (see region map, Figure 7.3). See the pastureland irrigation rates indicate in Table 7.7 for rates that are most applicable to residential land irrigation. The rates indicated are those for the year 2007 or 2008. The user can go to the website to review data for other years. Data reported in units of annual ac-ft/ac are converted to the Gv2 units (in/yr) by multiplying ac-ft/ac by $12 \mathrm{in} / \mathrm{ft}$.

State-specific irrigation information for pastureland (see Table 7.5) may provide an indication of how likely irrigation of residential lawns occurs. Irrigation application rates for residential land are highly variable. Therefore for areas where contaminated irrigation water is applied, it is suggested that population irrigation rates are assumed to be about 75 percent of the MEI value. The user may wish to subtract annual rainfall amounts from the irrigation rate if a non-location-specific irrigation rate is applied.

For the IRTIMR parameter, the user can maximize the time of irrigation water application to residential land by assuming irrigation from last frost to first frost day. Find your location in the map shown in Figure 7.1. (See NCDC (1981) [last accessed at http://www.ncdc.noaa.gov/oa/climate/freezefrost/frostfreemaps.html] for a high resolution map.) The National Climatic Data Center mapped the number of frost-free days (days with temperature above 32 degrees F) for the U.S. based on data from 1951 through 1980. The user can estimate the number of frost-free days for their location, divide by $30 \mathrm{~d} / \mathrm{mo}$, and round down to estimate a maximum IRTIMR value for their location.

\subsubsection{Additional Sources of Information}

http://www.agcensus.usda.gov/Publications/2007/Online_Highlights/Farm and_Ranch_Irrigation_Su rvey/index.asp for Census of Agriculture 2007 results. There are links on this page to results for other Census years.

Annual rainfall can be obtained from numerous internet resources. A snapshot of United States, statelevel annual rainfall for a user-entered year can be obtained from the National Climatic Data Center, U.S. Climate at a Glance webpage, last accessed at http://www.ncdc.noaa.gov/oa/climate/research/cag3/cag3.html. The user enters the "Precipitation" 
element, year of interest, and "annual" period. The map will also indicate whether the annual precipitation is above, average, or below what is typical for the state.

\subsubsection{RIRRR and IRTIMR Irrigation Time Values}

See Section 7.5.1, Table 7.7 (pastureland) for RIRRR values.

Refer to Figure 7.1 for frost-free days to convert to mo/yr.

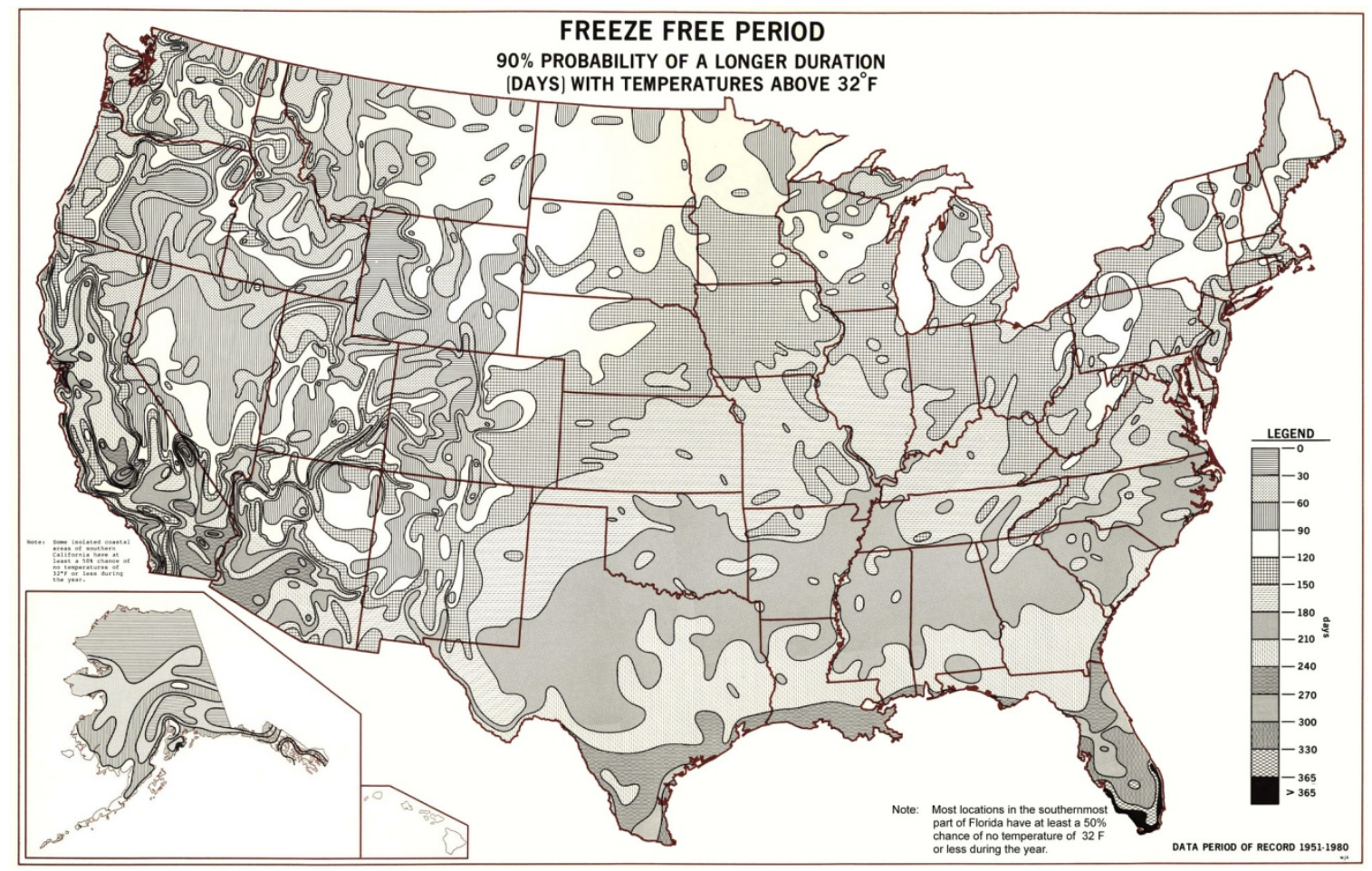

Figure 7.1. NCDC (1981) Frost-Free Days Map

\subsubsection{ANDKR Indoor Volatilization Factor for Radionuclides}

This value will be implemented if indoor air inhalation from water use is an exposure pathway of concern. The same value is applied to all radionuclide constituents, generally limited to noble gases and tritium. The value assigned is quite generic.

Note: The ANDKRN parameter (Radon indoor volatilization factor) is not discussed because radon modeling is not covered in this parameter document.

The units and range for this parameter in Gv2 are: $\mathrm{L} / \mathrm{m}^{3}(0-10)$.

Andelman (1990) proposes a rather simple model that tends to over-estimate this parameter value. A value of $0.5 \mathrm{~L} / \mathrm{m}^{3}$ is recommended; details on the value's derivation are found in EPA Risk Assessment Guidance (EPA 1991, p. 20). 


\subsubsection{Additional Sources of Information}

No additional sources identified.

\subsubsection{HOLDDW Delay Time for Water Distribution}

The time from water withdrawal to tap allows for radiological decay to occur.

The units for this parameter in Gv2 are: $\mathrm{d}(0-365)$.

A 1-day value is conservatively applied. The value would vary depending on the average time of withdrawal from the surface water source to the time the water appeared at the receptor tap.

\subsubsection{Additional Sources of Information}

Providers of public water supplies may have estimates of this information.

\subsubsection{SEDDN Shoreline Sediment Density}

If recreational shoreline use of a surface water source with radiological contaminants is considered, this parameter will be used. The parameter converts radionuclide transfer per unit area to sediment concentration per unit mass. The density reported should apply to a soil depth of $15 \mathrm{~cm}$ because the dose factor implemented is for this depth.

Other soil density values are explicitly entered for other pathways. Soil density values (SLDN) used for the outdoor air inhalation pathway and crop uptake calculations are entered in the Soil/Surface Soil tab (see Section 7.7) of this Chronic Exposure module. Soil density values (SLDN) for the external dose pathway from air pathway emissions or direct soil contamination are entered separately in the Health Impacts module (see Section 9.1). It is up to the user to define the two SLDN entries in the Chronic Exposure module and the Health Impacts module, identically. The SEDDN value for shoreline sediment density may differ.

The units for this parameter in Gv2 are: $\mathrm{kg} / \mathrm{m}^{2}(0-5000)$.

The USDA Soil Quality Indicators worksheet (USDA 2008) contains generic information on soil bulk density. GENII-appropriate values were derived from the generic information by applying a $0.15 \mathrm{~m}$ $(15 \mathrm{~cm})$ depth factor. Solid rock, as a rule of thumb has a density of $398 \mathrm{~kg} / \mathrm{m}^{2}$. A fraction of bulk pore space can be applied to the solid rock value approximate densities for cobbles. The data presented in Section 7.3.4.2 provide information for generic soil textures and limited vegetation growth. Sediment bulk density can be approximated from the values listed with a maximum value at the cited generic rock value.

\subsubsection{Additional Sources of Information}

No additional sources recommended. 


\subsubsection{SEDDN Shoreline Sediment Density Table}

Table 7.3. SEDDN Shoreline Sediment Density

\begin{tabular}{cccccccc}
\hline Gv2 ID & Gv2 Description & Reference & Comment & $\begin{array}{c}\text { Data } \\
\text { Reported }\end{array}$ & $\begin{array}{c}\text { Min } \\
\text { Value }\end{array}$ & $\begin{array}{c}\text { Max } \\
\text { Value }\end{array}$ & Units \\
\hline SEDDN & $\begin{array}{c}\text { Soil bulk density }- \\
\text { sediment }\end{array}$ & $\begin{array}{c}\text { USDA } \\
(2008)\end{array}$ & solid rock & datum & - & 398 & $\mathrm{~kg} / \mathrm{m}^{2}$ \\
SEDDN & $\begin{array}{c}\text { Soil bulk density }- \\
\text { sediment }\end{array}$ & $\begin{array}{c}\text { USDA } \\
(2008)\end{array}$ & $\begin{array}{c}\text { sandy soil texture, restricted } \\
\text { root growth }\end{array}$ & datum & 270 & - & $\mathrm{kg} / \mathrm{m}^{2}$ \\
SEDDN & $\begin{array}{c}\text { Soil bulk density }- \\
\text { sediment }\end{array}$ & $\begin{array}{c}\text { USDA } \\
(2008)\end{array}$ & $\begin{array}{c}\text { silty soil texture, restricted } \\
\text { root growth }\end{array}$ & datum & 248 & - & $\mathrm{kg} / \mathrm{m}^{2}$ \\
SEDDN & $\begin{array}{c}\text { Soil bulk density }- \\
\text { sediment }\end{array}$ & $\begin{array}{c}\text { USDA } \\
(2008)\end{array}$ & $\begin{array}{c}\text { clayey soil texture, restricted } \\
\text { root growth }\end{array}$ & datum & 221 & - & $\mathrm{kg} / \mathrm{m}^{2}$ \\
\hline
\end{tabular}

\subsection{Tab: Water/Animal Water}

The Water/Animal Water tab allows for input of the fraction of contaminated water consumed by the food-source animal and intake rates. For dairy cows, lactation can last just over 300 days with milk production peaking about 7 weeks post-calving.

\subsubsection{DWFACA and DWATER Contaminated Fraction and Animal Drinking Water Rate}

The fraction contaminated parameter (DWFACA) will fraction out the contaminated portion consumed by each animal. The contaminated fractions are scenario-specific, but are typically conservatively set to 1.0. Total daily water intake rate, whether from contaminated source or not, is input in DWATER. Except for locations with invariable climatic conditions, daily water intake will vary over the year. The drinking water rate entered is the average annual rate to determine the average animal product radionuclide levels.

The units for this parameter in Gv2 are: L/d (0.001-200).

Animal water requirements vary by several factors, including rate of weight gain, pregnancy, lactation, activity level, type of diet, feed intake, and ambient temperature (Lardy and Stoltenow 1999). The intake rate should reflect the average intake over the entire year. Beef cattle generally consume less water than dairy cattle. Dairy cattle consume about 4-4.5 L/d per L of milk produced (derived from Lardy and Stoltenow 1999). The USDA provides summary information on milk production in major milk producing states (e.g., see http://www.nass.usda.gov/Charts and_Maps/Milk_Production and Milk_Cows/mmlkpercow.asp). Other sources reviewed to determine animal water intake rates were Ensminger et al. (1990) and Ministry of the Environment (2001). 


\subsubsection{Additional Sources of Information}

Regional variations for water intake for goat and cattle can be estimated by assuming goats consume 1.4-1.7 L of water per kg of dry matter consumed and cattle consume $2.1 \mathrm{~L}$ of water per kg of dry matter consumed (Ensminger et al. 1990, p. 934).

Lardy and Stoltenow (1999) provide a summary of water intake information for beef cattle, sheep, dairy cows, and swine in a North Dakota State University publication, AS-954. Last accessed at http://www.ag.ndsu.edu/pubs/h2oqual/watanim/as954.html.

A Canadian resource with a range of water intake rates for a variety of domestic or wild animals (e.g., goats, sheep, rabbit, pigeon, numerous waterfowl, bear, mule deer, opossum, raccoon, and walrus) is available (Last accessed at http://www.env.gov.bc.ca/wat/wq/reference/foodandwater.html). There are also formulae to calculate water intake based on the animal weight for a non-listed mammal or bird.

The Agricultural Extension offices in a number of states have online information related to feeding and water consumption of animals.

\subsubsection{DWATER Animal Drinking Water Tables}

Table 7.4. DWATER Total Daily Water Intake

\begin{tabular}{|c|c|c|c|c|c|c|c|c|}
\hline Gv2_ID & $\begin{array}{c}\text { Gv2 } \\
\text { Description }\end{array}$ & $\begin{array}{l}\text { Food } \\
\text { Type }\end{array}$ & Reference & Comment & $\begin{array}{c}\text { Recom- } \\
\text { mendation }^{(a)}\end{array}$ & $\begin{array}{c}\text { Min } \\
\text { Value }\end{array}$ & $\begin{array}{c}\text { Max } \\
\text { Value }\end{array}$ & Units \\
\hline DWATER & $\begin{array}{l}\text { Intake rate of } \\
\text { water }\end{array}$ & 1.meat & $\begin{array}{l}\text { Lardy and Stoltenow } \\
\text { (1999) }\end{array}$ & $\begin{array}{l}\text { Beef-600 lb } \\
\text { finish wt }\end{array}$ & - & - & 33 & $\mathrm{~L} / \mathrm{d}$ \\
\hline DWATER & $\begin{array}{l}\text { Intake rate of } \\
\text { water }\end{array}$ & 1.meat & $\begin{array}{l}\text { Lardy and Stoltenow } \\
\text { (1999) }\end{array}$ & $\begin{array}{l}\text { Beef-800 lb } \\
\text { finish wt }\end{array}$ & - & - & 40 & $\mathrm{~L} / \mathrm{d}$ \\
\hline DWATER & $\begin{array}{l}\text { Intake rate of } \\
\text { water }\end{array}$ & 1.meat & $\begin{array}{l}\text { Lardy and Stoltenow } \\
\text { (1999); Ensminger et al. } \\
\text { (1990) }\end{array}$ & $\begin{array}{l}\text { Beef-1000 lb } \\
\text { finish wt }\end{array}$ & 50 & 25 & 47 & $\mathrm{~L} / \mathrm{d}$ \\
\hline DWATER & $\begin{array}{l}\text { Intake rate of } \\
\text { water }\end{array}$ & 1.meat & $\begin{array}{l}\text { Lardy and Stoltenow } \\
\text { (1999); Ensminger et al. } \\
\text { (1990) }\end{array}$ & $\begin{array}{l}\text { Beef-1200 lb } \\
\text { finish wt }\end{array}$ & - & 25 & 53 & $\mathrm{~L} / \mathrm{d}$ \\
\hline DWATER & $\begin{array}{l}\text { Intake rate of } \\
\text { water }\end{array}$ & 1.meat & $\begin{array}{l}\text { Lardy and Stoltenow } \\
\text { (1999); Ensminger et al. } \\
\text { (1990) }\end{array}$ & Pig-200 lb & 8.5 & 7.5 & 9.5 & $\mathrm{~L} / \mathrm{d}$ \\
\hline DWATER & $\begin{array}{l}\text { Intake rate of } \\
\text { water }\end{array}$ & 1.meat & $\begin{array}{l}\text { Lardy and Stoltenow } \\
\text { (1999); Ensminger et al. } \\
\text { (1990) }\end{array}$ & Sheep & 5 & 2 & 7 & $\mathrm{~L} / \mathrm{d}$ \\
\hline DWATER & $\begin{array}{l}\text { Intake rate of } \\
\text { water }\end{array}$ & 2.poultry & $\begin{array}{l}\text { Ensminger et al. (1990); } \\
\text { Ministry of the } \\
\text { Environment (2001) }\end{array}$ & Chicken & 0.26 & 0.2 & 0.32 & $\mathrm{~L} / \mathrm{d}$ \\
\hline DWATER & $\begin{array}{l}\text { Intake rate of } \\
\text { water }\end{array}$ & 2.poultry & $\begin{array}{l}\text { Ensminger et al. (1990); } \\
\text { Ministry of the } \\
\text { Environment (2001) }\end{array}$ & Turkey & 0.55 & 0.5 & 0.6 & $\mathrm{~L} / \mathrm{d}$ \\
\hline DWATER & $\begin{array}{l}\text { Intake rate of } \\
\text { water }\end{array}$ & 3.milk & $\begin{array}{l}\text { Lardy and Stoltenow } \\
\text { (1999); Ensminger et al. } \\
\text { (1990); Ministry of the } \\
\text { Environment (2011); Linn } \\
\text { et al. 2002. }\end{array}$ & $\begin{array}{c}\text { dairy cow } \\
(1200-1600 \mathrm{lb})\end{array}$ & 85 & 50 & 110 & $\mathrm{~L} / \mathrm{d}$ \\
\hline DWATER & $\begin{array}{l}\text { Intake rate of } \\
\text { water }\end{array}$ & 4.egg & $\begin{array}{l}\text { Ensminger et al. (1990); } \\
\text { Ministry of the } \\
\text { Environment (2001) }\end{array}$ & chicken, layers & 0.3 & 0.19 & 0.34 & $\mathrm{~L} / \mathrm{d}$ \\
\hline
\end{tabular}




\subsection{Tabs: Water/Irrigation Rates and Water/Irrigation Times}

Irrigation water rates and times are required for each human food crop (leafy vegetables, root vegetables, fruits, and grains) and each animal feed or forage type. Animal feed assumptions are: grain feed for meat, poultry, and egg; and hay for milk feed. Animal forage assumptions are hay for meat; and grass for milk. These values are only required if the contaminated water source is used for irrigation. No fractional fresh and contaminated water supplies are modeled, so the user enters only the rates and times when the contaminated water is used.

Table 7.5 provides information to indicate the prevalence of irrigation at a location. The prevalence of any farm to irrigate, irrigation prevalence for crop farms harvested in 2008, and irrigation prevalence for pastureland information is provided. This data provides the user with a general idea of how regionally common it is for farm, crop, and pasture irrigation.

Table 7.5. Percent of Crop Irrigated

\begin{tabular}{|c|c|c|c|c|c|c|c|}
\hline 2008 Data & $\begin{array}{c}\text { Farms }^{(a)} \\
\text { (number) }\end{array}$ & $\begin{array}{c}\text { Total Land in } \\
\text { Farms }^{(\mathrm{a})} \\
\text { (acres) }^{2}\end{array}$ & $\begin{array}{l}\text { Percent } \\
\text { Irrigated }^{(\mathrm{a})} \\
(\%)\end{array}$ & $\begin{array}{l}\text { Crop Land } \\
\text { Harvested }^{(\text {b) }} \\
\text { (acres) }^{\text {a }}\end{array}$ & $\begin{array}{c}\text { Percent } \\
\text { Irrigated }^{(\mathrm{b})} \\
(\%) \\
\end{array}$ & $\begin{array}{l}\text { Crop Land } \\
\text { Used Only for } \\
\text { Pasture or } \\
\text { Grazing }{ }^{(b)} \\
\text { (acres) } \\
\end{array}$ & $\begin{array}{l}\text { Percent } \\
\text { Irrigated }^{(b)} \\
(\%)\end{array}$ \\
\hline $\begin{array}{l}\text { United States } \\
\text { total }\end{array}$ & 206,834 & $198,160,896$ & $27.7 \%$ & $78,043,457$ & $65.3 \%$ & $21,826,263$ & $11.8 \%$ \\
\hline Alabama & 665 & 442,487 & $17 \%$ & 310,127 & $24 \%$ & 35,111 & $5 \%$ \\
\hline Alaska & 23 & 5,785 & $27 \%$ & 2,225 & N/A & N/A & N/A \\
\hline Arizona & 2,997 & $4,446,165$ & $19 \%$ & 803,563 & $100 \%$ & 112,483 & $29 \%$ \\
\hline Arkansas & 4,119 & $6,162,119$ & $73 \%$ & $5,454,373$ & $82 \%$ & 54,887 & $3 \%$ \\
\hline California & 45,136 & $15,866,424$ & $46 \%$ & $7,407,931$ & $94 \%$ & $4,484,832$ & $7 \%$ \\
\hline Colorado & 12,778 & $10,647,744$ & $27 \%$ & $3,132,687$ & $77 \%$ & $1,757,407$ & $18 \%$ \\
\hline Connecticut & 147 & 10,403 & $22 \%$ & 5,081 & $46 \%$ & 402 & 0 \\
\hline Delaware & 309 & 273,149 & $38 \%$ & 247,025 & $42 \%$ & 1,045 & N/A \\
\hline Florida & 5,250 & $2,535,664$ & $48 \%$ & $1,252,339$ & $91 \%$ & 352,681 & $14 \%$ \\
\hline Georgia & 3,584 & $2,999,922$ & $34 \%$ & $1,661,353$ & $59 \%$ & 179,213 & $11 \%$ \\
\hline Hawaii & 1,077 & 213,387 & $47 \%$ & 59,642 & $96 \%$ & 4,239 & $8 \%$ \\
\hline Idaho & 13,834 & $6,421,820$ & $52 \%$ & $3,161,743$ & $93 \%$ & 778,565 & $35 \%$ \\
\hline Illinois & 1,091 & $1,309,942$ & $35 \%$ & $1,206,759$ & $38 \%$ & 9,188 & N/A \\
\hline Indiana & 1,089 & $1,360,556$ & $30 \%$ & $1,261,582$ & $32 \%$ & 17,321 & N/A \\
\hline Iowa & 527 & 590,047 & $28 \%$ & 549,330 & $29 \%$ & 5,990 & N/A \\
\hline Kansas & 4,508 & $10,912,364$ & $24 \%$ & $6,887,476$ & $37 \%$ & $1,468,876$ & $2 \%$ \\
\hline Kentucky & 822 & 329,198 & $10 \%$ & 213,208 & $15 \%$ & 34,032 & $2 \%$ \\
\hline Louisiana & 1,692 & $2,074,497$ & $45 \%$ & $1,518,997$ & $61 \%$ & 85,057 & $0 \%$ \\
\hline Maine & 196 & 118,107 & $15 \%$ & 53,030 & N/A & 631 & N/A \\
\hline Maryland & 597 & 278,277 & $31 \%$ & 232,934 & $37 \%$ & 4,038 & $6 \%$ \\
\hline Massachusetts & 677 & 52,718 & $33 \%$ & 19,312 & N/A & 325 & N/A \\
\hline Michigan & 2,121 & $1,503,006$ & $35 \%$ & $1,292,404$ & $41 \%$ & 9,677 & $28 \%$ \\
\hline Minnesota & 1,546 & $1,397,018$ & $36 \%$ & $1,204,250$ & $42 \%$ & 24,885 & $2 \%$ \\
\hline Mississippi & 1,277 & $2,512,434$ & $58 \%$ & $2,265,515$ & $64 \%$ & 9,566 & 0 \\
\hline Missouri & 1,877 & $2,285,747$ & $54 \%$ & $2,063,897$ & $60 \%$ & 36,876 & N/A \\
\hline Montana & 8,507 & $19,637,380$ & $10 \%$ & $2,463,577$ & $63 \%$ & $2,577,266$ & $10 \%$ \\
\hline
\end{tabular}


Table 7.5. (contd)

\begin{tabular}{|c|c|c|c|c|c|c|c|}
\hline 2008 Data & $\begin{array}{l}\text { Farms }^{(a)} \\
\text { (number) }\end{array}$ & $\begin{array}{c}\text { Total Land in } \\
\text { Farms }^{(a)} \\
\text { (acres) }\end{array}$ & $\begin{array}{c}\text { Percent } \\
\text { Irrigated }^{(a)} \\
(\%) \\
\end{array}$ & $\begin{array}{l}\text { Crop Land } \\
\text { Harvested }^{(b)} \\
\text { (acres) }\end{array}$ & $\begin{array}{l}\text { Percent } \\
\text { Irrigated }^{(\mathbf{b})} \\
(\%) \\
\end{array}$ & $\begin{array}{l}\text { Crop Land } \\
\text { Used Only for } \\
\text { Pasture or } \\
\text { Grazing }^{(b)} \\
\text { (acres) }\end{array}$ & $\begin{array}{c}\text { Percent } \\
\text { Irrigated }^{(\mathrm{b})} \\
(\%) \\
\end{array}$ \\
\hline Nebraska & 14,812 & $21,853,534$ & $38 \%$ & $11,682,565$ & $71 \%$ & $2,007,591$ & $1 \%$ \\
\hline Nevada & 1,734 & $5,600,694$ & $12 \%$ & 538,941 & $98 \%$ & 585,529 & $19 \%$ \\
\hline New Hampshire & 86 & 9,043 & $8 \%$ & 3,366 & $21 \%$ & 81 & 0 \\
\hline New Jersey & 608 & 136,352 & $44 \%$ & 93,910 & $63 \%$ & 898 & $40 \%$ \\
\hline New Mexico & 8,878 & $12,816,722$ & $7 \%$ & 973,556 & $73 \%$ & 758,042 & $10 \%$ \\
\hline New York & 596 & 144,640 & $14 \%$ & 93,605 & $21 \%$ & 6,588 & 0 \\
\hline North Carolina & 1,975 & 990,106 & $15 \%$ & 607,620 & $23 \%$ & 37,303 & $30 \%$ \\
\hline North Dakota & 601 & $1,706,080$ & $15 \%$ & $1,103,232$ & $22 \%$ & 127,413 & $0 \%$ \\
\hline Ohio & 379 & 124,294 & $15 \%$ & 100,738 & $18 \%$ & 1,723 & $4 \%$ \\
\hline Oklahoma & 1,454 & $2,382,239$ & $19 \%$ & $1,119,486$ & $37 \%$ & 293,384 & $6 \%$ \\
\hline Oregon & 12,156 & $6,952,252$ & $25 \%$ & $1,767,412$ & $80 \%$ & $1,281,936$ & $18 \%$ \\
\hline Pennsylvania & 1,090 & 149,947 & $12 \%$ & 101,662 & $17 \%$ & 8,111 & N/A \\
\hline Rhode Island & 61 & 5,501 & $12 \%$ & 1,515 & $45 \%$ & 4 & $100 \%$ \\
\hline South Carolina & 712 & 443,293 & $23 \%$ & 282,162 & $36 \%$ & 24,054 & $11 \%$ \\
\hline South Dakota & 1,165 & $2,807,203$ & $13 \%$ & $1,379,726$ & $26 \%$ & 197,303 & $1 \%$ \\
\hline Tennessee & 582 & 424,980 & $17 \%$ & 347,419 & N/A & 21,852 & $2 \%$ \\
\hline Texas & 12,673 & $16,634,484$ & $32 \%$ & $7,912,212$ & $62 \%$ & $1,864,920$ & $14 \%$ \\
\hline Utah & 10,876 & $5,512,452$ & $19 \%$ & 899,493 & $90 \%$ & 651,964 & $28 \%$ \\
\hline Vermont & 66 & 8,186 & $6 \%$ & 1,637 & $30 \%$ & 205 & 0 \\
\hline Virginia & 657 & 323,778 & $14 \%$ & 191,990 & $23 \%$ & 15,960 & $2 \%$ \\
\hline Washington & 12,712 & $8,085,090$ & $21 \%$ & $1,925,767$ & $80 \%$ & 485,991 & $23 \%$ \\
\hline West Virginia & 90 & 13,493 & $7 \%$ & 6,301 & $14 \%$ & N/A & N/A \\
\hline Wisconsin & 1,261 & $1,065,575$ & $37 \%$ & 776,071 & $50 \%$ & 7,098 & $12 \%$ \\
\hline Wyoming & 5,164 & $15,584,598$ & $10 \%$ & $1,402,711$ & $77 \%$ & $1,401,854$ & $19 \%$ \\
\hline $\begin{array}{l}\text { (a) Information } \\
\text { http://www.a } \\
\text { Table } 2 . \\
\text { (b) Information } \\
\text { http://www.a } \\
\text { Table 3. }\end{array}$ & $\mathrm{m}$ & Publications & 207ine & (2) & and Ranch & Irrigation Survey & $\begin{array}{l}\text { /index.asp, } \\
\text { /index.asp, }\end{array}$ \\
\hline
\end{tabular}


Table 7.5. Percent of Crop Irrigated (contd)

\begin{tabular}{|c|c|c|c|c|c|c|c|}
\hline 2008 & $\begin{array}{l}\text { Farms }^{(a)} \\
\text { (number) }\end{array}$ & $\begin{array}{l}\text { Total Land } \\
\text { in Farms } \\
\text { (a) } \\
\text { (acres) }\end{array}$ & $\begin{array}{c}\text { Percent } \\
\text { Irrigated }^{(a)} \\
(\%)\end{array}$ & $\begin{array}{l}\text { Crop Land } \\
\text { Harvested }^{(b)} \\
\text { (acres) }\end{array}$ & $\begin{array}{c}\text { Percent } \\
\text { Irrigated }^{(a)} \\
(\%)\end{array}$ & $\begin{array}{l}\text { Crop Land } \\
\text { Used Only } \\
\text { for Pasture } \\
\text { or Grazing }{ }^{(b)} \\
\text { (acres) }\end{array}$ & $\begin{array}{c}\text { Percent } \\
\text { Irrigated }^{(\mathrm{a})} \\
(\%)\end{array}$ \\
\hline \multicolumn{8}{|c|}{ Water Resources Regions (see Figure 7.2) } \\
\hline Region 01 New England & 1,188 & 199,464 & $20 \%$ & 82,570 & N/A & 1,475 & $11 \%$ \\
\hline Region 02 Mid-Atlantic & 3,200 & $1,052,547$ & $29 \%$ & 816,004 & $38 \%$ & 25,300 & $4 \%$ \\
\hline $\begin{array}{l}\text { Region } 03 \text { South Atlantic- } \\
\text { Gulf }\end{array}$ & 12,428 & $7,495,555$ & $34 \%$ & $4,050,743$ & $60 \%$ & 629,405 & $14 \%$ \\
\hline Region 04 Great Lakes & 3,152 & $2,098,815$ & $34 \%$ & $1,810,691$ & $40 \%$ & 15,293 & $28 \%$ \\
\hline Region 05 Ohio & 2,056 & $1,461,874$ & $21 \%$ & $1,204,636$ & $25 \%$ & 57,505 & $2 \%$ \\
\hline Region 06 Tennessee & 527 & 283,315 & $15 \%$ & 213,451 & N/A & $\mathrm{N} / \mathrm{A}$ & N/A \\
\hline $\begin{array}{l}\text { Region } 07 \text { Upper } \\
\text { Mississippi }\end{array}$ & 3,863 & $3,827,858$ & $35 \%$ & $3,289,626$ & $40 \%$ & 42,095 & $2 \%$ \\
\hline $\begin{array}{l}\text { Region } 08 \text { Lower } \\
\text { Mississippi }\end{array}$ & 7,341 & $11,412,924$ & $64 \%$ & $10,291,786$ & $71 \%$ & 129,345 & $0 \%$ \\
\hline Region 09 Souris-Red-Rainy & 400 & 714,430 & $22 \%$ & 579,519 & $27 \%$ & 21,124 & $1 \%$ \\
\hline Region 10 Missouri & 32,727 & $66,817,228$ & $21 \%$ & $22,116,957$ & $59 \%$ & $6,484,836$ & $7 \%$ \\
\hline $\begin{array}{l}\text { Region } 11 \text { Arkansas-White- } \\
\text { Red }\end{array}$ & 10,336 & $24,462,413$ & $22 \%$ & $9,479,378$ & $53 \%$ & $3,207,945$ & $8 \%$ \\
\hline Region 12 Texas-Gulf & 10,224 & $10,145,409$ & $34 \%$ & $5,341,739$ & $60 \%$ & $1,022,165$ & $9 \%$ \\
\hline Region 13 Rio Grande & 8,031 & $8,037,090$ & $13 \%$ & $1,085,157$ & $85 \%$ & 401,947 & $24 \%$ \\
\hline Region 14 Upper Colorado & 10,771 & $5,822,461$ & $23 \%$ & 909,265 & $93 \%$ & $1,132,804$ & $32 \%$ \\
\hline Region 15 Lower Colorado & 3,551 & $4,727,545$ & $20 \%$ & 868,628 & $100 \%$ & 125,876 & $34 \%$ \\
\hline Region 16 Great Basin & 10,157 & $9,893,297$ & $16 \%$ & $1,448,602$ & $90 \%$ & $1,083,623$ & $20 \%$ \\
\hline Region 17 Pacific Northwest & 40,017 & $23,242,402$ & $29 \%$ & $6,920,803$ & $86 \%$ & $2,837,534$ & $23 \%$ \\
\hline Region 18 California & 45,765 & $16,247,097$ & $46 \%$ & $7,472,035$ & $94 \%$ & $4,577,809$ & $7 \%$ \\
\hline Region 19 Alaska & 23 & 5,785 & $27 \%$ & 2,225 & N/A & N/A & N/A \\
\hline Region 20 Hawaii & 1,077 & 213,387 & $47 \%$ & 59,642 & $96 \%$ & 4,239 & $8 \%$ \\
\hline
\end{tabular}

(a) Information from

http://www.agcensus.usda.gov/Publications/2007/Online_Highlights/Farm_and_Ranch_Irrigation_Survey/index.asp, Table 2.

(b) Information from

http://www.agcensus.usda.gov/Publications/2007/Online_Highlights/Farm_and_Ranch_Irrigation_Survey/index.asp, Table 3.

\subsubsection{RIRR Crop Irrigation Rate, RIRRA Feed and Forage Irrigation Rate, IRTIMT Crop Irrigation Time, and IRTIMA Feed and Forage Irrigation Rate}

The irrigation rates and times are used together for the code to determine the rate the contaminated water source is applied during the water application period. These rates and times are used for ingestion dose pathways. External exposure pathways use the residential land irrigation data (see Section 7.3.1).

The units and range for RIRR and RIRRA in Gv2 are: in/yr (0-200).

The units and range for IRTIMT and IRTIMA in Gv2 are: $\mathrm{mo} / \mathrm{yr}(0-12)$. 


\section{RIRR and RIRRA}

The U.S. Department of Agricultural conducts a Census of Agriculture every 5 years. Part of the data collected is crop irrigation data. The irrigation rates in the Census are for a variety of crops and are reported by state or by region (see region map, Figure 7.3). The rates indicated are those for the year 2007 or 2008 . The user can go to the website to review data for other years. Data reported in units of annual ac-ft/ac are converted to the Gv2 units (in/yr) by multiplying ac-ft/ac by $12 \mathrm{in} / \mathrm{ft}$.

\section{IRTIMT and IRTIMA}

The irrigation times are used to adjust the annual irrigation rate to the irrigation rate during the growing season. They are generally equal to the growing or fruiting period for the crop. However, the irrigation period for forage crops should be adjusted to account for continual browsing or multiple harvests, about 30 days. See Sections 7.13.1 and 7.18.1 for growing periods of animal feeds and food crops.

\subsubsection{Additional Sources of Information}

The USDA Census of Agriculture 2007 results are available. There are links on the indicated hyperlink to results for other Census years. Last accessed at http://www.agcensus.usda.gov/Publications/2007/Online_Highlights/Farm_and_Ranch_Irrigation_Survey lindex.asp.

\subsubsection{RIRR, RIRRA, IRTIMT, and IRTIMA Irrigation Rate Tables}

State-specific human food crops RIRR Table - see Table 7.6

State- and region-specific animal feed crops RIRRA Table - see Table 7.7

Table 7.6. State- and Region-Specific Irrigation Rates for Human Food Crops

\begin{tabular}{|c|c|c|c|c|c|c|c|c|c|}
\hline $\begin{array}{c}\text { GENII } \\
\text { Application } \\
\text { Comment }^{(a)}\end{array}$ & $\begin{array}{c}\text { Human } \\
\text { LV }\end{array}$ & $\begin{array}{c}\text { Human } \\
\text { RV }\end{array}$ & $\begin{array}{c}\text { Human } \\
\text { RV }\end{array}$ & $\begin{array}{c}\text { Human } \\
\text { F }\end{array}$ & $\underset{F^{(c)}}{\operatorname{Human}}$ & $\begin{array}{c}\text { Human } \\
\text { G }\end{array}$ & $\begin{array}{c}\text { Human } \\
\text { G }\end{array}$ & $\begin{array}{c}\text { Human } \\
\text { G }\end{array}$ & $\begin{array}{c}\text { Human } \\
\text { G }\end{array}$ \\
\hline 2008 & $\begin{array}{c}\text { Lettuce, } \\
\text { Romaine }^{(\mathrm{b})} \\
\text { RIRR } \\
\text { (in./yr) } \\
\end{array}$ & $\begin{array}{c}\text { Peanuts }^{(b)} \\
\text { RIRR } \\
\text { (in./yr) } \\
\end{array}$ & $\begin{array}{c}\text { Potatoes }^{(\mathrm{b})} \\
\text { RIRR } \\
\text { (in./yr) } \\
\end{array}$ & $\begin{array}{c}\text { All } \\
\text { Berries }^{(\mathrm{b})} \\
\text { RIRR } \\
\text { (in./yr) } \\
\end{array}$ & $\begin{array}{c}\text { Land in } \\
\text { Orchard, } \\
\text { Vineyard, } \\
\text { Nut Trees }{ }^{(b)} \\
\text { RIRR } \\
\text { (in./yr) }\end{array}$ & $\begin{array}{l}\begin{array}{c}\text { Soybean for } \\
\text { Beans }^{(b)}\end{array} \\
\text { RIRR (in./yr) }\end{array}$ & $\begin{array}{l}\text { Rice }^{(b)} \\
\text { RIRR } \\
\text { (in./yr) } \\
\end{array}$ & $\begin{array}{c}\text { Other } \\
\text { Small } \\
\text { Grains }^{(b)} \\
\text { RIRR } \\
\text { (in./yr) } \\
\end{array}$ & $\begin{array}{l}\text { Sweet } \\
\text { Corn }^{(b)} \\
\text { RIRR } \\
\text { (in./yr) }\end{array}$ \\
\hline United States & 34.8 & 12 & 21.6 & 26.4 & 28.8 & 8.4 & 27.6 & 16.8 & 18 \\
\hline Alabama & 4.8 & 4.8 & 12 & 28.8 & 9.6 & 7.2 & - & 10.8 & 8.4 \\
\hline Alaska & (D) & - & (D) & 3.6 & 3.6 & - & - & - & - \\
\hline Arizona & 34.8 & - & 33.6 & - & 70.8 & - & - & 44.4 & 48 \\
\hline Arkansas & - & - & - & - & 1.2 & 10.8 & 24 & 18 & - \\
\hline California & 36 & - & 33.6 & 44.4 & 31.2 & - & 49.2 & 18 & 40.8 \\
\hline Colorado & 16.8 & - & 21.6 & - & 9.6 & 14.4 & - & 14.4 & 36 \\
\hline Connecticut & 4.8 & - & 6 & 9.6 & 3.6 & - & - & - & 3.6 \\
\hline Delaware & - & - & 9.6 & 9.6 & - & 7.2 & - & (D) & 7.2 \\
\hline
\end{tabular}


Table 7.6. (contd)

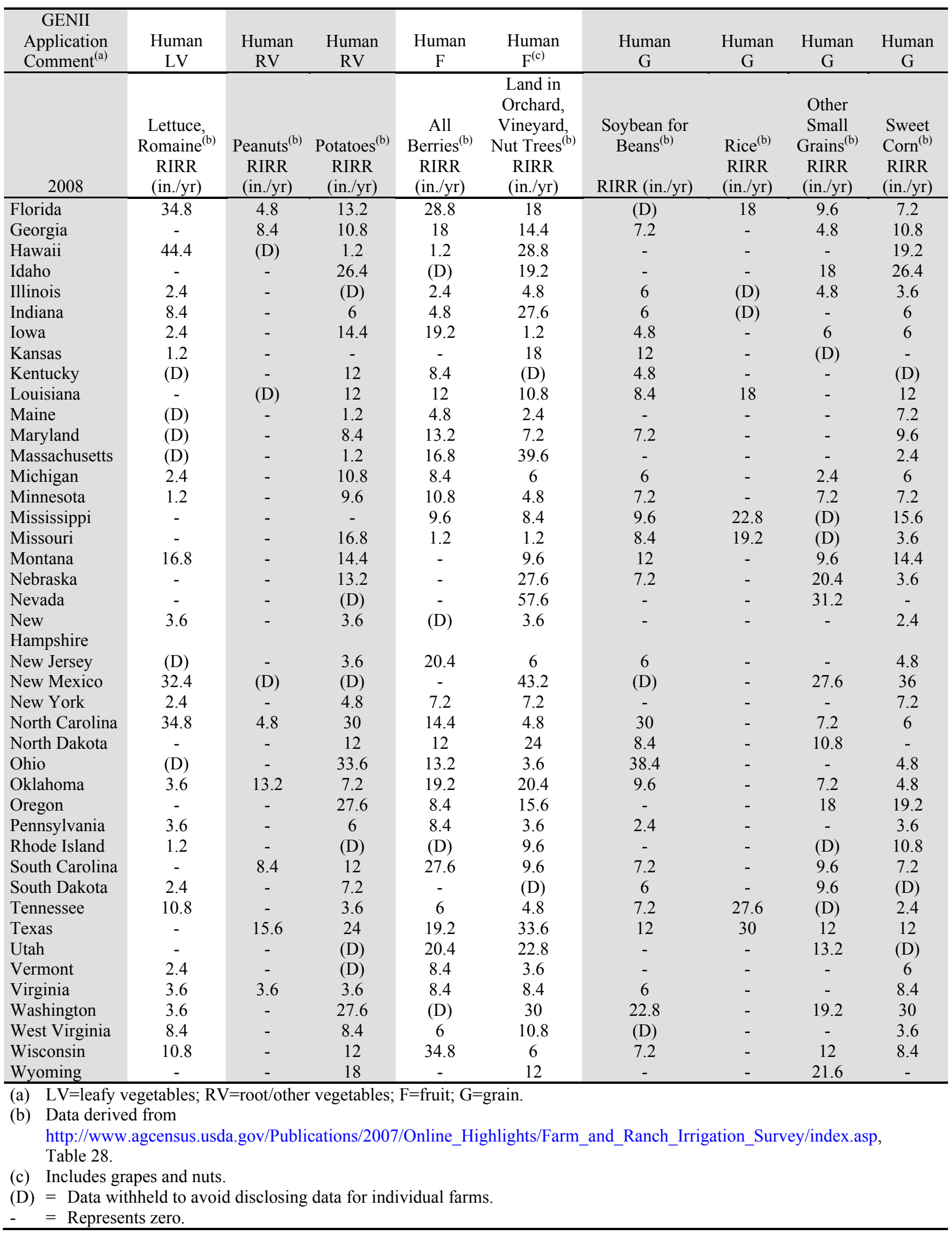


Table 7.6. (contd)

\begin{tabular}{|c|c|c|c|c|c|c|c|c|c|}
\hline $\begin{array}{l}\text { GENII Application } \\
\text { Comment }^{(\mathrm{a})}\end{array}$ & Human LV & $\begin{array}{c}\text { Human } \\
\text { RV }\end{array}$ & $\begin{array}{c}\text { Human } \\
\text { RV }\end{array}$ & $\begin{array}{c}\text { Human } \\
\mathrm{F}\end{array}$ & $\underset{F^{(c)}}{\text { Human }}$ & $\begin{array}{c}\text { Human } \\
\mathrm{G}\end{array}$ & $\begin{array}{c}\text { Human } \\
\mathrm{G}\end{array}$ & $\begin{array}{c}\text { Human } \\
\mathrm{G}\end{array}$ & $\begin{array}{c}\text { Human } \\
\text { G }\end{array}$ \\
\hline 2008 & $\begin{array}{l}\text { Lettuce, } \\
\text { Romaine }^{(\mathrm{b})} \\
\text { RIRR } \\
\text { (in./yr) } \\
\end{array}$ & $\begin{array}{l}\text { Peanuts }^{(\mathrm{b})} \\
\text { RIRR } \\
\text { (in./yr) } \\
\end{array}$ & $\begin{array}{l}\text { Potatoes }^{(\mathrm{b})} \\
\text { RIRR } \\
\text { (in./yr) } \\
\end{array}$ & $\begin{array}{l}\text { All } \\
\text { Berries }^{(b)} \\
\text { RIRR } \\
\text { (in./yr) } \\
\end{array}$ & $\begin{array}{c}\text { Land in } \\
\text { Orchard, } \\
\text { Vineyard, } \\
\text { Nut Trees }{ }^{(b)} \\
\text { RIRR } \\
\text { (in./yr) }\end{array}$ & $\begin{array}{c}\text { Soybeans } \\
\text { for } \\
\text { Beans }^{(b)} \\
\text { RIRR } \\
\text { (in./yr) }\end{array}$ & $\begin{array}{l}\text { Rice }^{(b)} \\
\text { RIRR } \\
\text { (in./yr) } \\
\end{array}$ & $\begin{array}{c}\text { Other } \\
\text { Small } \\
\text { Grains }{ }^{(b)} \\
\text { RIRR } \\
\text { (in./yr) } \\
\end{array}$ & $\begin{array}{l}\text { Sweet } \\
\text { Corn }{ }^{(b)} \\
\text { RIRR } \\
\text { (in./yr) }\end{array}$ \\
\hline \multicolumn{10}{|c|}{ See Figure 7.2 for reference map of regions. } \\
\hline Region 01 New England & 2.4 & - & 1.2 & 12 & 13.2 & - & - & (D) & 3.6 \\
\hline Region 02 Mid-Atlantic & 3.6 & - & 6 & 19.2 & 6 & 7.2 & - & (D) & 7.2 \\
\hline Region 03 South Atlantic-Gulf & 34.8 & 8.4 & 14.4 & 19.2 & 16.8 & 10.8 & 18 & 6 & 8.4 \\
\hline Region 04 Great Lakes & 3.6 & - & 10.8 & 8.4 & 4.8 & 6 & - & 8.4 & 6 \\
\hline Region 05 Ohio & 4.8 & - & 7.2 & 8.4 & 12 & 7.2 & (D) & (D) & 7.2 \\
\hline Region 06 Tennessee & 8.4 & - & 3.6 & 7.2 & 4.8 & 8.4 & - & (D) & 4.8 \\
\hline Region 07 Upper Mississippi & (D) & - & 10.8 & 33.6 & 7.2 & 6 & (D) & 8.4 & 8.4 \\
\hline Region 08 Lower Mississippi & (D) & (D) & 16.8 & 13.2 & 9.6 & 9.6 & 22.8 & 16.8 & 13.2 \\
\hline Region 09 Souris-Red-Rainy & - & - & 9.6 & - & 18 & 7.2 & - & 10.8 & - \\
\hline Region 10 Missouri & 4.8 & - & 13.2 & 12 & 22.8 & 7.2 & - & 15.6 & 6 \\
\hline $\begin{array}{l}\text { Region } 11 \text { Arkansas-White- } \\
\text { Red }\end{array}$ & 2.4 & (D) & (D) & 19.2 & 14.4 & 10.8 & 22.8 & 8.4 & (D) \\
\hline Region 12 Texas-Gulf & - & 15.6 & 21.6 & 19.2 & 25.2 & 12 & 30 & 19.2 & 12 \\
\hline Region 13 Rio Grande & 19.2 & (D) & 21.6 & - & 46.8 & - & - & 24 & 36 \\
\hline Region 14 Upper Colorado & - & - & (D) & - & 18 & 14.4 & - & 16.8 & 36 \\
\hline Region 15 Lower Colorado & 34.8 & - & 33.6 & - & 70.8 & - & - & 44.4 & 48 \\
\hline Region 16 Great Basin & - & - & 22.8 & 20.4 & 25.2 & - & - & 14.4 & (D) \\
\hline Region 17 Pacific Northwest & 3.6 & - & 27.6 & 8.4 & 28.8 & 22.8 & - & 18 & 27.6 \\
\hline Region 18 California & 36 & - & 33.6 & 44.4 & 31.2 & - & 49.2 & 18 & 40.8 \\
\hline Region 19 Alaska & (D) & - & (D) & 3.6 & 3.6 & - & - & - & - \\
\hline Region 20 Hawaii & 44.4 & (D) & 1.2 & 1.2 & 28.8 & - & - & - & 19.2 \\
\hline \multicolumn{10}{|c|}{ (a) $\mathrm{LV}=$ leafy vegetables; $\mathrm{RV}=$ root/other vegetables; $\mathrm{F}=$ fruit; $\mathrm{G}=$ grain. } \\
\hline $\begin{array}{l}\text { http://www.agcensus.usda } \\
\text { (c) Includes grapes and nuts. } \\
\text { (D) = Data withheld to avoid } \\
-\quad=\text { Represents zero. }\end{array}$ & $\begin{array}{l}\text { gov/Publicati } \\
\text { disclosing da }\end{array}$ & for indivi & dual farms. & tesorm & d Ponch & ration_Su & $y /$ index & sp, Table & \\
\hline
\end{tabular}


Table 7.7. State- and Region-Specific Irrigation Rates for Animal Feed Crops

\begin{tabular}{|c|c|c|c|c|c|c|c|}
\hline $\begin{array}{c}\text { GENII } \\
\text { Application } \\
\text { Comment }\end{array}$ & $\begin{array}{l}\text { FEED: } \\
\text { Meat, } \\
\text { Poultry, } \\
\text { Egg }\end{array}$ & $\begin{array}{l}\text { FEED: } \\
\text { Meat, } \\
\text { Poultry, } \\
\text { Egg }\end{array}$ & $\begin{array}{l}\text { FEED: } \\
\text { Meat, } \\
\text { Poultry, } \\
\text { Egg }\end{array}$ & $\begin{array}{c}\text { FEED:Milk; } \\
\text { FORAGE: } \\
\text { Meat }\end{array}$ & $\begin{array}{c}\text { FEED:Milk; } \\
\text { FORAGE: } \\
\text { Meat }\end{array}$ & $\begin{array}{c}\text { FEED:Milk; } \\
\text { FORAGE: } \\
\text { Meat }\end{array}$ & $\begin{array}{c}\text { FORAGE: } \\
\text { Milk }\end{array}$ \\
\hline 2008 & $\begin{array}{l}\text { Corn for } \\
\text { Grain or } \\
\text { Seed }^{(a)} \\
\text { RIRRA } \\
\text { (in./yr) } \\
\end{array}$ & $\begin{array}{l}\text { Sorghum } \\
\text { for Grain or } \\
\text { Seed }^{(a)} \\
\text { RIRRA } \\
\text { (in./yr) } \\
\end{array}$ & $\begin{array}{l}\text { Wheat for } \\
\text { Grain or } \\
\text { Seed }^{(a)} \\
\text { RIRRA } \\
\text { (in./yr) } \\
\end{array}$ & $\begin{array}{c}\text { Corn for } \\
\text { Silage or } \\
\text { Greenchop }^{(a)} \\
\text { RIRRA } \\
\text { (in./yr) }\end{array}$ & $\begin{array}{l}\text { Alfalfa and } \\
\text { Alfalfa } \\
\text { Mixtures }^{(a)} \\
\text { RIRRA } \\
\text { (in./yr) } \\
\end{array}$ & $\begin{array}{l}\text { All Other } \\
\text { Hay }^{\text {(a) }} \\
\text { RIRRA } \\
\text { (in./yr) } \\
\end{array}$ & $\begin{array}{c}\text { Pastureland, } \\
\text { All Types }{ }^{\text {(a) }} \\
\text { RIRRA } \\
\text { (in./yr) } \\
\end{array}$ \\
\hline United States & 12 & 10.8 & 16.8 & 25.2 & 28.8 & 21.6 & 19.2 \\
\hline Alabama & 8.4 & - & 4.8 & 7.2 & 6 & 4.8 & 4.8 \\
\hline Alaska & - & - & - & - & - & 3.6 & (D) \\
\hline Arizona & 33.6 & 40.8 & 43.2 & 55.2 & 74.4 & 46.8 & 30 \\
\hline Arkansas & 13.2 & 12 & 4.8 & (D) & - & 6 & 7.2 \\
\hline California & 24 & 21.6 & 26.4 & 34.8 & 48 & 30 & 30 \\
\hline Colorado & 19.2 & 7.2 & 16.8 & 18 & 20.4 & 18 & 18 \\
\hline Connecticut & - & - & - & - & - & - & - \\
\hline Delaware & 9.6 & - & 4.8 & 9.6 & 9.6 & 14.4 & 6 \\
\hline Florida & 8.4 & (D) & 6 & 10.8 & - & 19.2 & 12 \\
\hline Georgia & 13.2 & 14.4 & 4.8 & 15.6 & 7.2 & 10.8 & 8.4 \\
\hline Hawaii & (D) & (D) & - & - & - & - & 21.6 \\
\hline Idaho & 31.2 & - & 19.2 & 26.4 & 22.8 & 15.6 & 13.2 \\
\hline Illinois & 6 & 4.8 & 4.8 & 2.4 & 3.6 & 8.4 & (D) \\
\hline Indiana & 7.2 & (D) & 2.4 & 6 & 6 & 2.4 & 4.8 \\
\hline Iowa & 6 & - & (D) & 4.8 & 8.4 & (D) & (D) \\
\hline Kansas & 15.6 & 12 & 10.8 & 16.8 & 18 & 12 & 13.2 \\
\hline Kentucky & 6 & - & - & (D) & (D) & (D) & 6 \\
\hline Louisiana & 8.4 & 4.8 & 9.6 & 6 & 20.4 & 6 & 10.8 \\
\hline Maine & - & - & - & - & - & - & 1.2 \\
\hline Maryland & 9.6 & - & 4.8 & 4.8 & 4.8 & 3.6 & 3.6 \\
\hline Massachusetts & (D) & - & - & - & - & - & 1.2 \\
\hline Michigan & 7.2 & - & 3.6 & 4.8 & 4.8 & - & 3.6 \\
\hline Minnesota & 7.2 & - & 6 & 7.2 & 7.2 & 8.4 & 7.2 \\
\hline Mississippi & 10.8 & 10.8 & 2.4 & - & - & 1.2 & - \\
\hline Missouri & 9.6 & (D) & 4.8 & 8.4 & 3.6 & 2.4 & 4.8 \\
\hline Montana & 19.2 & - & 12 & 19.2 & 15.6 & 19.2 & 13.2 \\
\hline Nebraska & 9.6 & 6 & 9.6 & 9.6 & 10.8 & 26.4 & 28.8 \\
\hline Nevada & - & - & 33.6 & 38.4 & 37.2 & 20.4 & 31.2 \\
\hline New Hampshire & (D) & - & - & 25.2 & - & - & - \\
\hline New Jersey & 6 & - & 3.6 & 1.2 & 10.8 & 6 & 8.4 \\
\hline New Mexico & 24 & 19.2 & 20.4 & 25.2 & 32.4 & 20.4 & 14.4 \\
\hline New York & 4.8 & - & (D) & 25.2 & 1.2 & - & - \\
\hline North Carolina & 18 & (D) & 2.4 & 4.8 & 3.6 & 4.8 & 3.6 \\
\hline North Dakota & 9.6 & - & 10.8 & 13.2 & 13.2 & 6 & 9.6 \\
\hline Ohio & 18 & - & - & 8.4 & 24 & - & 9.6 \\
\hline Oklahoma & 16.8 & 9.6 & 10.8 & 15.6 & 15.6 & 12 & 8.4 \\
\hline Oregon & 36 & - & 20.4 & 21.6 & 25.2 & 19.2 & 22.8 \\
\hline Pennsylvania & 3.6 & - & - & 3.6 & 4.8 & 9.6 & 7.2 \\
\hline Rhode Island & - & - & - & - & - & - & 21.6 \\
\hline South Carolina & 9.6 & (D) & 6 & 7.2 & 9.6 & 4.8 & 12 \\
\hline South Dakota & 9.6 & 19.2 & 6 & 15.6 & 12 & 10.8 & 18 \\
\hline Tennessee & 7.2 & (D) & 6 & 2.4 & 1.2 & 3.6 & 20.4 \\
\hline Texas & 20.4 & 9.6 & 12 & 20.4 & 19.2 & 13.2 & 12 \\
\hline
\end{tabular}


Table 7.7. (contd)

\begin{tabular}{|c|c|c|c|c|c|c|c|}
\hline $\begin{array}{c}\text { GENII } \\
\text { Application } \\
\text { Comment }\end{array}$ & $\begin{array}{l}\text { FEED: } \\
\text { Meat, } \\
\text { Poultry, } \\
\text { Egg }\end{array}$ & $\begin{array}{l}\text { FEED: } \\
\text { Meat, } \\
\text { Poultry, } \\
\text { Egg }\end{array}$ & $\begin{array}{l}\text { FEED: } \\
\text { Meat, } \\
\text { Poultry, } \\
\text { Egg }\end{array}$ & $\begin{array}{c}\text { FEED:Milk; } \\
\text { FORAGE: } \\
\text { Meat }\end{array}$ & $\begin{array}{c}\text { FEED:Milk; } \\
\text { FORAGE: } \\
\text { Meat }\end{array}$ & $\begin{array}{c}\text { FEED:Milk; } \\
\text { FORAGE: } \\
\text { Meat }\end{array}$ & $\begin{array}{c}\text { FORAGE: } \\
\text { Milk }\end{array}$ \\
\hline & $\begin{array}{l}\text { Corn for } \\
\text { Grain or } \\
\text { Seed }^{(a)}\end{array}$ & $\begin{array}{l}\text { Sorghum for } \\
\text { Grain or } \\
\text { Seed }^{(a)}\end{array}$ & $\begin{array}{c}\text { Wheat for } \\
\text { Grain or } \\
\text { Seed }^{(a)}\end{array}$ & $\begin{array}{c}\text { Corn for } \\
\text { Silage or } \\
\text { Greenchop }^{(a)}\end{array}$ & $\begin{array}{c}\text { Alfalfa and } \\
\text { Alfalfa } \\
\text { Mixtures }^{(a)}\end{array}$ & $\begin{array}{l}\text { All Other } \\
\text { Hay }^{(a)}\end{array}$ & $\begin{array}{l}\text { Pastureland, } \\
\text { All Types }^{(a)}\end{array}$ \\
\hline 2008 & $\begin{array}{l}\text { RIRRA } \\
\text { (in./yr) }\end{array}$ & $\begin{array}{l}\text { RIRRA } \\
\text { (in./yr) }\end{array}$ & $\begin{array}{l}\text { RIRRA } \\
\text { (in./yr) }\end{array}$ & $\begin{array}{l}\text { RIRRA } \\
\text { (in./yr) }\end{array}$ & $\begin{array}{l}\text { RIRRA } \\
\text { (in./yr) }\end{array}$ & $\begin{array}{l}\text { RIRRA } \\
\text { (in./yr) }\end{array}$ & $\begin{array}{l}\text { RIRRA } \\
\text { (in./yr) }\end{array}$ \\
\hline Utah & 33.6 & 15.6 & 22.8 & 28.8 & 30 & 26.4 & 24 \\
\hline Vermont & - & - & - & - & - & - & - \\
\hline Virginia & 6 & - & 3.6 & 3.6 & 3.6 & 2.4 & 3.6 \\
\hline Washington & 28.8 & - & 22.8 & 30 & 28.8 & 28.8 & 24 \\
\hline West Virginia & (D) & - & - & (D) & (D) & - & 2.4 \\
\hline Wisconsin & 8.4 & - & 4.8 & 7.2 & 8.4 & 15.6 & 6 \\
\hline Wyoming & 20.4 & - & 12 & 24 & 25.2 & 20.4 & 20.4 \\
\hline
\end{tabular}

(a) Data derived from

http://www.agcensus.usda.gov/Publications/2007/Online_Highlights/Farm_and_Ranch_Irrigation_Survey/index.asp, Table 28.

(D) = Data withheld to avoid disclosing data for individual farms.

- $\quad=$ Represents zero.

Table 7.7. (contd)

\begin{tabular}{|c|c|c|c|c|c|c|c|}
\hline GENII Application Comment & $\begin{array}{c}\text { FEED: } \\
\text { Meat, } \\
\text { Poultry, Egg }\end{array}$ & $\begin{array}{l}\text { FEED:Meat, } \\
\text { Poultry, Egg }\end{array}$ & $\begin{array}{l}\text { FEED:Meat, } \\
\text { Poultry, Egg }\end{array}$ & $\begin{array}{c}\text { FEED:Milk; } \\
\text { FORAGE: } \\
\text { Meat }\end{array}$ & $\begin{array}{c}\text { FEED:Milk } \\
\text {; FORAGE: } \\
\text { Meat } \\
\end{array}$ & $\begin{array}{l}\text { FEED:Milk; } \\
\text { FORAGE: } \\
\text { Meat }\end{array}$ & $\begin{array}{l}\text { FORAGE: } \\
\text { Milk }\end{array}$ \\
\hline 2008 & $\begin{array}{l}\text { Corn for } \\
\text { Grain or } \\
\text { Seed }^{(a)} \\
\text { RIRRA } \\
\text { (in./yr) }\end{array}$ & $\begin{array}{l}\text { Sorghum for } \\
\text { Grain or } \\
\text { Seed }^{(a)} \\
\text { RIRRA } \\
\text { (in./yr) }\end{array}$ & $\begin{array}{l}\text { Wheat for } \\
\text { Grain or } \\
\text { Seed }^{(\text {a) }} \\
\text { RIRRA } \\
\text { (in./yr) }\end{array}$ & $\begin{array}{c}\text { Corn for } \\
\text { Silage or } \\
\text { Greenchop } \\
\text { (a) } \\
\text { RIRRA } \\
\text { (in./yr) }\end{array}$ & $\begin{array}{l}\text { Alfalfa and } \\
\text { Alfalfa } \\
\text { Mixtures }^{(a)} \\
\text { RIRRA } \\
\text { (in./yr) }\end{array}$ & $\begin{array}{l}\text { All Other } \\
\text { Hay }^{(a)} \\
\text { RIRRA } \\
\text { (in./yr) }\end{array}$ & $\begin{array}{l}\text { Pastureland, } \\
\text { All Types }^{(\mathrm{a})} \\
\text { RIRRA } \\
\text { (in./yr) }\end{array}$ \\
\hline \multicolumn{8}{|c|}{ See Figure 7.2 for reference map of regions. } \\
\hline Region 01 New England & 1.2 & - & - & 25.2 & - & - & 1.2 \\
\hline Region 02 Mid-Atlantic & 8.4 & - & 4.8 & 4.8 & 6 & 6 & (D) \\
\hline Region 03 South Atlantic-Gulf & 13.2 & (D) & 4.8 & 12 & 6 & 10.8 & 10.8 \\
\hline Region 04 Great Lakes & 7.2 & - & 3.6 & 6 & 6 & 14.4 & 4.8 \\
\hline Region 05 Ohio & 7.2 & - & 3.6 & 8.4 & 10.8 & 4.8 & 6 \\
\hline Region 06 Tennessee & 8.4 & - & 4.8 & 3.6 & 1.2 & 3.6 & 18 \\
\hline Region 07 Upper Mississippi & 7.2 & (D) & 4.8 & 6 & 7.2 & 9.6 & 7.2 \\
\hline Region 08 Lower Mississippi & 10.8 & 10.8 & 4.8 & 8.4 & 18 & 6 & 7.2 \\
\hline Region 09 Souris-Red-Rainy & 8.4 & - & 7.2 & 9.6 & 8.4 & 8.4 & 4.8 \\
\hline Region 10 Missouri & 10.8 & 7.2 & 10.8 & 15.6 & 18 & 19.2 & 18 \\
\hline $\begin{array}{l}\text { Region } 11 \text { Arkansas-White- } \\
\text { Red }\end{array}$ & 18 & 12 & 12 & 19.2 & 18 & 13.2 & 13.2 \\
\hline Region 12 Texas-Gulf & 19.2 & 9.6 & 12 & 20.4 & 24 & 12 & 9.6 \\
\hline Region 13 Rio Grande & (D) & 21.6 & 27.6 & 28.8 & 26.4 & 20.4 & 19.2 \\
\hline Region 14 Upper Colorado & 24 & - & 30 & 19.2 & 24 & 20.4 & 20.4 \\
\hline Region 15 Lower Colorado & 32.4 & 40.8 & 43.2 & 55.2 & 72 & 46.8 & 28.8 \\
\hline Region 16 Great Basin & 31.2 & 15.6 & 20.4 & 28.8 & 32.4 & 21.6 & 30 \\
\hline
\end{tabular}


Table 7.7. (contd)

\begin{tabular}{|c|c|c|c|c|c|c|c|}
\hline GENII Application Comment & $\begin{array}{l}\text { FEED: } \\
\text { Meat, } \\
\text { Poultry, } \\
\text { Egg } \\
\end{array}$ & $\begin{array}{l}\text { FEED:Meat, } \\
\text { Poultry, Egg } \\
\end{array}$ & $\begin{array}{l}\text { FEED:Meat, } \\
\text { Poultry, Egg } \\
\end{array}$ & $\begin{array}{c}\text { FEED:Milk; } \\
\text { FORAGE: } \\
\text { Meat }\end{array}$ & $\begin{array}{c}\text { FEED:Milk; } \\
\text { FORAGE: } \\
\text { Meat }\end{array}$ & $\begin{array}{c}\text { FEED:Milk; } \\
\text { FORAGE: } \\
\text { Meat }\end{array}$ & $\begin{array}{c}\text { FORAGE: } \\
\text { Milk }\end{array}$ \\
\hline 2008 & $\begin{array}{l}\text { Corn for } \\
\text { Grain or } \\
\text { Seed }^{(a)} \\
\text { RIRRA } \\
\text { (in./yr) }\end{array}$ & $\begin{array}{l}\text { Sorghum for } \\
\text { Grain or } \\
\text { Seed }^{(a)} \\
\text { RIRRA } \\
\text { (in./yr) }\end{array}$ & $\begin{array}{l}\text { Wheat for } \\
\text { Grain or } \\
\text { Seed } \\
\text { RIRRA } \\
\text { (in./yr) } \\
\end{array}$ & $\begin{array}{c}\text { Corn for } \\
\text { Silage or } \\
\begin{array}{c}\text { Greenchop } \\
\text { (a) }\end{array} \\
\text { RIRRA } \\
\text { (in./yr) } \\
\end{array}$ & $\begin{array}{l}\text { Alfalfa and } \\
\text { Alfalfa } \\
\text { Mixtures } \\
\text { RIRRA } \\
\text { (in./yr) } \\
\end{array}$ & $\begin{array}{l}\text { All Other } \\
\text { Hay }^{(a)} \\
\text { RIRRA } \\
\text { (in./yr) } \\
\end{array}$ & $\begin{array}{c}\text { Pastureland, } \\
\text { All Types }{ }^{(a)} \\
\text { RIRRA } \\
\text { (in./yr) }\end{array}$ \\
\hline Region 17 Pacific Northwest & 31.2 & - & 20.4 & 26.4 & 24 & 20.4 & 16.8 \\
\hline Region 18 California & 24 & 21.6 & 26.4 & 34.8 & 45.6 & 30 & 27.6 \\
\hline Region 19 Alaska & - & - & - & - & - & 3.6 & (D) \\
\hline Region 20 Hawaii & (D) & (D) & - & - & - & - & 21.6 \\
\hline \multicolumn{8}{|c|}{ 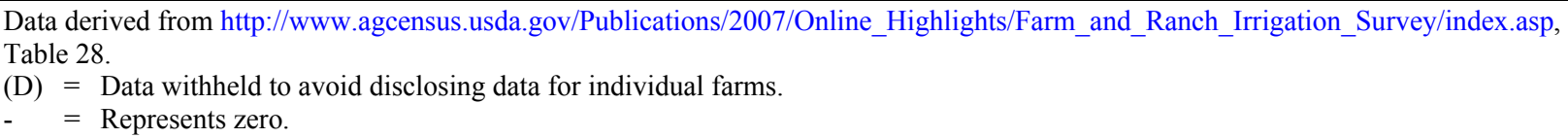 } \\
\hline
\end{tabular}

\subsection{Tab: Soil}

The soil tab contains three secondary tabs: Surface Soil tab (input used for food ingestion pathways), Resuspension tab (input used for inhalation of resuspended soil pathway), and Leaching tab (input used to model the movement of radionuclides in soil for the multi-year evaluations).

\subsection{Tab: Soil/Surface Soil}

The Surface soil density parameter (SSLDN) will always be gray-fonted on this tab; the equivalent information is derived from the SLDN and SURCM parameters.

\subsubsection{SLDN Surface Soil Areal Density and SURCM Surface Soil Thickness}

These parameters are used to determine crop uptake from soil and to determine soil resuspension (see Section 7.8). For other inputs of soil density, see Section 7.3.4 for river-shore surface soil density (SEDDN) and the Health Impacts module, Method Parameters tab (Section 9.1). It is up to the user to define the SLDN of the Chronic Exposure module, Soil/Soil Surface tab, and the SLDN of the Health Impacts module, Method Parameters tab, consistently. ${ }^{1}$

The units and range for SLDN in Gv2 are: $\mathrm{kg} / \mathrm{m}^{2}(0.00001-3000)$.

The units and range for SURCM in Gv2 are: $\mathrm{cm}(0.00001-500)$.

The USDA Soil Quality Indicators worksheet (USDA 2008) contains generic information on soil bulk density. Values were derived from the generic information by applying a $0.15 \mathrm{~m}(15 \mathrm{~cm})$ depth factor. As a comparative measure, solid rock, as a rule of thumb has a density of $398 \mathrm{~kg} / \mathrm{m}^{2}$ (equivalent to

\footnotetext{
${ }^{1}$ The (SLDN Health Impacts module) $\mathrm{kg} / \mathrm{m}^{3}=\left(\mathrm{SLDN}\right.$ Chronic Exposure module) $\mathrm{kg} / \mathrm{m}^{2} *(1 / 0.15 \mathrm{~m})$ because of units inconsistency issue in Gv2.10.
} 
$2.65 \mathrm{~g} / \mathrm{cm}^{3}$, as referenced in Burt 2011, p. 319). The data presented in Section 7.7.1.2 provide information for generic soil textures. The densities for restricted plant growth were about 15 percent greater than the densities reported for ideal plant growth. Therefore, the minimum values for ideal plant growth were generically calculated to be 15 percent less than the ideal value. The generic SURCM value is strongly recommended to be $15 \mathrm{~cm}$ for consistency with the soil-to-pant bioaccumulation and external dose rate conversion factors. If a different surface soil thickness is desired, the values can be adjusted. A generic value of $225 \mathrm{~kg} / \mathrm{m}^{3}$ is suggested.

\subsubsection{Additional Sources of Information}

Historic functions of the U.S. Soil Conservation Service have been delegated to the U.S. Department of Agriculture's Natural Resources Conservation Service (NRCS) (see www.nrcs.usda.gov). Detailed soil information can be obtained via the website's Web Soil Survey (WSS) electronic database (http://websoilsurvey.nrcs.usda.gov).

Acquiring the soil density data value is not technically difficult or expensive. The user may want to acquire their own local data values for soil areal density.

\subsubsection{SLDN Surface Soil Areal Density Table}

Table 7.8. SLDN Surface Soil Areal Density

\begin{tabular}{|c|c|c|c|c|c|c|c|}
\hline Gv2 ID & Gv2 Description & Reference & Comment & $\begin{array}{c}\text { Data } \\
\text { Reported }\end{array}$ & $\begin{array}{l}\text { Min } \\
\text { Value }\end{array}$ & $\begin{array}{l}\text { Max } \\
\text { Value }\end{array}$ & Units \\
\hline SLDN & $\begin{array}{l}\text { Soil bulk density - } \\
\text { sediment }\end{array}$ & $\begin{array}{l}\text { USDA } \\
(2008)\end{array}$ & solid rock & datum & - & 398 & $\mathrm{~kg} / \mathrm{m}^{2}$ \\
\hline SLDN & $\begin{array}{l}\text { Soil bulk density - } \\
\text { sediment }\end{array}$ & $\begin{array}{l}\text { USDA } \\
(2008)\end{array}$ & $\begin{array}{l}\text { sandy soil texture, ideal } \\
\text { for plant growth }\end{array}$ & range & 204 & 240 & $\mathrm{~kg} / \mathrm{m}^{2}$ \\
\hline SLDN & $\begin{array}{l}\text { Soil bulk density - } \\
\text { sediment }\end{array}$ & $\begin{array}{l}\text { USDA } \\
(2008)\end{array}$ & $\begin{array}{l}\text { silty soil texture, ideal } \\
\text { for plant growth }\end{array}$ & range & 179 & 210 & $\mathrm{~kg} / \mathrm{m}^{2}$ \\
\hline SLDN & $\begin{array}{l}\text { Soil bulk density - } \\
\text { sediment }\end{array}$ & $\begin{array}{l}\text { USDA } \\
(2008)\end{array}$ & $\begin{array}{l}\text { clayey soil texture, ideal } \\
\text { for plant growth }\end{array}$ & range & 140 & 165 & $\mathrm{~kg} / \mathrm{m}^{2}$ \\
\hline SLDN & $\begin{array}{l}\text { Soil bulk density - } \\
\text { sediment }\end{array}$ & $\begin{array}{l}\text { USDA } \\
(2008)\end{array}$ & $\begin{array}{l}\text { sandy soil texture, } \\
\text { restricted root growth }\end{array}$ & datum & 270 & - & $\mathrm{kg} / \mathrm{m}^{2}$ \\
\hline SLDN & $\begin{array}{l}\text { Soil bulk density - } \\
\text { sediment }\end{array}$ & $\begin{array}{l}\text { USDA } \\
(2008)\end{array}$ & $\begin{array}{l}\text { silty soil texture, } \\
\text { restricted root growth }\end{array}$ & datum & 248 & - & $\mathrm{kg} / \mathrm{m}^{2}$ \\
\hline SLDN & $\begin{array}{l}\text { Soil bulk density - } \\
\text { sediment }\end{array}$ & $\begin{array}{l}\text { USDA } \\
(2008)\end{array}$ & $\begin{array}{l}\text { clayey soil texture, } \\
\text { restricted root growth }\end{array}$ & datum & 221 & - & $\mathrm{kg} / \mathrm{m}^{2}$ \\
\hline
\end{tabular}

\subsection{Tab: Soil/Resuspension}

The recommended Type of Model to Run is: Use Mass Loading Model. If another model is desired, consult the Gv2 User's Guide (Napier 2012). This tab addresses resuspension of soil particulates into the outdoor air inhalation pathway and uses soil areal density information from the Soil/Surface Soil tab, see Section 7.7, above. 
Resuspension of soil onto food and feed crop surfaces is considered in tab Agriculture/General (see Section 7.11). Along with dry and wet depositions, it is used to calculate radionuclide translocation from the plant surface to the edible portion of the feed or food crop.

\subsubsection{XMLF Mass Loading Factor for Resuspension}

The mass loading factor for resuspension is the airborne particulate concentration of resuspended soil particles at the exposure location.

The units and range for this parameter in Gv2 are: $\mathrm{g} / \mathrm{m}^{3}(0-5)$.

Generic values for arid regions range from 1.4E-05 to 3.5E-04 g/m $\mathrm{m}^{3}$ (Snyder et al. 1994). A value of $2 \mathrm{E}-04 \mathrm{~g} / \mathrm{m}^{3}$ is cited in the RESRAD code, as appropriate conservative value that takes into account short periods of high mass loading and sustained periods of normal activity on a typical farm (Yu et al. 2001). As a reference point, the U.S. primary 24-hour air quality standard for larger particulates ( $\mathrm{PM}_{10}$ standard) in air is $1.5 \mathrm{E}-04 \mathrm{~g} / \mathrm{m}^{3}$. National trends in $\mathrm{PM}_{10}$ levels indicate a 2010 mean of $6.3 \mathrm{E}-05 \mathrm{~g} / \mathrm{m}^{3}$ with $10^{\text {th }}$ and $90^{\text {th }}$ percentile values of $3 \mathrm{E}-05$ and $9 \mathrm{E}-05 \mathrm{~g} / \mathrm{m}^{3}$, respectively (EPA 2012). A value of $2 \mathrm{E}-4$ is adequately generic and conservative for the region just above the soil surface.

\subsubsection{Additional Sources of Information}

Local particulate matter $\left(\mathrm{PM}_{10}\right)$ values can be found for numerous reporting locations at the EPA Air Trends website for particulate matter. A Google Earth file (KLM file) is available for the user to select their location of interest (last accessed at http://www.epa.gov/airtrends/pm.html). In addition, data trends for air quality constituents, including $\mathrm{PM}_{10}$ in units of microgram/cubic meter, are tabulated for U.S. counties or cities and can be found at the EPA Air Quality Monitoring Information website (last accessed at http://www.epa.gov/airtrends/factbook.html).

\subsubsection{XMLF Mass Loading Factor Table}

Table 7.9. XMLF Mass Loading Factor

\begin{tabular}{|c|c|c|c|c|c|c|c|c|}
\hline Gv2 ID & $\begin{array}{c}\text { Gv2 } \\
\text { Description }\end{array}$ & Reference & Comment & Data Reported & Average & $\begin{array}{l}\text { Mean } \\
\text { Min } \\
\text { Value }\end{array}$ & $\begin{array}{c}\text { Mean } \\
\text { Max } \\
\text { Value }\end{array}$ & Units \\
\hline XMLF & $\begin{array}{l}\text { Mass loading } \\
\text { factor for } \\
\text { resuspension }\end{array}$ & (a) & $\begin{array}{l}\text { Selection: Use mass loading } \\
\text { model. Watershed Reporting } \\
\text { Regions (Figure } 7.3 \text { ): } 1\end{array}$ & $\begin{array}{l}1 \text { city; annual 24-hr } \\
\mathrm{PM}_{10}, \text { 2nd maximum }\end{array}$ & $4.0 \mathrm{E}-05$ & $3.0 \mathrm{E}-05$ & $5.0 \mathrm{E}-05$ & $\mathrm{~g} / \mathrm{m}^{3}$ \\
\hline XMLF & $\begin{array}{l}\text { Mass loading } \\
\text { factor for } \\
\text { resuspension }\end{array}$ & (a) & $\begin{array}{l}\text { Selection: Use mass loading } \\
\text { model. Watershed Reporting } \\
\text { Regions (Figure } 7.3 \text { ): } 2\end{array}$ & $\begin{array}{l}4 \text { cities; annual 24-hr } \\
\mathrm{PM}_{10}, 2 \text { nd maximum }\end{array}$ & $6.0 \mathrm{E}-05$ & 4.3E-05 & 7.7E-05 & $\mathrm{g} / \mathrm{m}^{3}$ \\
\hline XMLF & $\begin{array}{l}\text { Mass loading } \\
\text { factor for } \\
\text { resuspension }\end{array}$ & (a) & $\begin{array}{l}\text { Selection: Use mass loading } \\
\text { model. Watershed Reporting } \\
\text { Regions (Figure } 7.3 \text { ): } 3\end{array}$ & $\begin{array}{l}7 \text { cities; annual 24-hr } \\
\mathrm{PM}_{10} \text {, 2nd maximum }\end{array}$ & $4.6 \mathrm{E}-05$ & $3.1 \mathrm{E}-05$ & $6.1 \mathrm{E}-05$ & $\mathrm{~g} / \mathrm{m}^{3}$ \\
\hline XMLF & $\begin{array}{l}\text { Mass loading } \\
\text { factor for } \\
\text { resuspension }\end{array}$ & (a) & $\begin{array}{l}\text { Selection: Use mass loading } \\
\text { model. Watershed Reporting } \\
\text { Regions (Figure } 7.3 \text { ): } 4\end{array}$ & $\begin{array}{l}1 \text { city; annual 24-hr } \\
\mathrm{PM}_{10}, \text { 2nd maximum }\end{array}$ & $4.2 \mathrm{E}-05$ & $2.8 \mathrm{E}-05$ & $5.5 \mathrm{E}-05$ & $\mathrm{~g} / \mathrm{m}^{3}$ \\
\hline
\end{tabular}


Table 7.9. (contd)

\begin{tabular}{|c|c|c|c|c|c|c|c|c|}
\hline Gv2 ID & $\begin{array}{c}\text { Gv2 } \\
\text { Description }\end{array}$ & Reference & Comment & Data Reported & Average & $\begin{array}{l}\text { Mean } \\
\text { Min } \\
\text { Value }\end{array}$ & $\begin{array}{l}\text { Mean } \\
\text { Max } \\
\text { Value }\end{array}$ & Units \\
\hline XMLF & $\begin{array}{l}\text { Mass loading } \\
\text { factor for } \\
\text { resuspension }\end{array}$ & (a) & $\begin{array}{l}\text { Selection: Use mass loading } \\
\text { model. Watershed Reporting } \\
\text { Regions (Figure 7.3): } 6\end{array}$ & $\begin{array}{l}1 \text { city; annual 24-hr } \\
\mathrm{PM}_{10}, 2 \text { nd maximum }\end{array}$ & $4.8 \mathrm{E}-05$ & $3.5 \mathrm{E}-05$ & $6.0 \mathrm{E}-05$ & $\mathrm{~g} / \mathrm{m}^{3}$ \\
\hline XMLF & $\begin{array}{l}\text { Mass loading } \\
\text { factor for } \\
\text { resuspension }\end{array}$ & (a) & $\begin{array}{l}\text { Selection: Use mass loading } \\
\text { model. Watershed Reporting } \\
\text { Regions (Figure } 7.3 \text { ): } 7\end{array}$ & $\begin{array}{l}3 \text { cities; annual 24-hr } \\
\mathrm{PM}_{10}, 2 \text { nd maximum }\end{array}$ & $5.6 \mathrm{E}-05$ & 3.7E-05 & $7.5 \mathrm{E}-05$ & $\mathrm{~g} / \mathrm{m}^{3}$ \\
\hline XMLF & $\begin{array}{l}\text { Mass loading } \\
\text { factor for } \\
\text { resuspension }\end{array}$ & (a) & $\begin{array}{l}\text { Selection: Use mass loading } \\
\text { model. Watershed Reporting } \\
\text { Regions (Figure } 7.3 \text { ): } 8\end{array}$ & $\begin{array}{l}2 \text { cities; annual 24-hr } \\
\mathrm{PM}_{10}, 2 \text { nd maximum }\end{array}$ & $5.9 \mathrm{E}-05$ & 4.3E-05 & $7.5 \mathrm{E}-05$ & $\mathrm{~g} / \mathrm{m}^{3}$ \\
\hline XMLF & $\begin{array}{l}\text { Mass loading } \\
\text { factor for } \\
\text { resuspension }\end{array}$ & (a) & $\begin{array}{l}\text { Selection: Use mass loading } \\
\text { model. Watershed Reporting } \\
\text { Regions (Figure 7.3): } 9\end{array}$ & $\begin{array}{l}1 \text { city; annual 24-hr } \\
\mathrm{PM}_{10}, 2 \text { nd maximum }\end{array}$ & $3.5 \mathrm{E}-05$ & $2.8 \mathrm{E}-05$ & 4.2E-05 & $\mathrm{g} / \mathrm{m}^{3}$ \\
\hline XMLF & $\begin{array}{l}\text { Mass loading } \\
\text { factor for } \\
\text { resuspension }\end{array}$ & (a) & $\begin{array}{l}\text { Selection: Use mass loading } \\
\text { model. Watershed Reporting } \\
\text { Regions (Figure } 7.3 \text { ): } 10\end{array}$ & $\begin{array}{l}4 \text { cities; annual 24-hr } \\
\mathrm{PM}_{10} \text {, 2nd maximum }\end{array}$ & $5.6 \mathrm{E}-05$ & $3.7 \mathrm{E}-05$ & $7.6 \mathrm{E}-05$ & $\mathrm{~g} / \mathrm{m}^{3}$ \\
\hline XMLF & $\begin{array}{l}\text { Mass loading } \\
\text { factor for } \\
\text { resuspension }\end{array}$ & (a) & $\begin{array}{l}\text { Selection: Use mass loading } \\
\text { model. Watershed Reporting } \\
\text { Regions (Figure } 7.3 \text { ): } 11\end{array}$ & $\begin{array}{l}2 \text { cities; annual 24-hr } \\
\mathrm{PM}_{10}, 2 \text { nd maximum }\end{array}$ & $5.3 \mathrm{E}-05$ & $4.0 \mathrm{E}-05$ & $6.5 \mathrm{E}-05$ & $\mathrm{~g} / \mathrm{m}^{3}$ \\
\hline XMLF & $\begin{array}{l}\text { Mass loading } \\
\text { factor for } \\
\text { resuspension }\end{array}$ & (a) & $\begin{array}{l}\text { Selection: Use mass loading } \\
\text { model. Watershed Reporting } \\
\text { Regions (Figure 7.3): } 12\end{array}$ & $\begin{array}{l}2 \text { cities; annual 24-hr } \\
\mathrm{PM}_{10}, 2 \text { nd maximum }\end{array}$ & $6.1 \mathrm{E}-05$ & 4.0E-05 & $8.3 \mathrm{E}-05$ & $\mathrm{~g} / \mathrm{m}^{3}$ \\
\hline XMLF & $\begin{array}{l}\text { Mass loading } \\
\text { factor for } \\
\text { resuspension }\end{array}$ & (a) & $\begin{array}{l}\text { Selection: Use mass loading } \\
\text { model. Watershed Reporting } \\
\text { Regions (Figure 7.3): } 13\end{array}$ & $\begin{array}{l}2 \text { cities; annual 24-hr } \\
\mathrm{PM}_{10}, 2 \text { nd maximum }\end{array}$ & $4.6 \mathrm{E}-05$ & 2.4E-05 & $6.9 \mathrm{E}-05$ & $\mathrm{~g} / \mathrm{m}^{3}$ \\
\hline XMLF & $\begin{array}{l}\text { Mass loading } \\
\text { factor for } \\
\text { resuspension }\end{array}$ & (a) & $\begin{array}{l}\text { Selection: Use mass loading } \\
\text { model. Watershed Reporting } \\
\text { Regions (Figure } 7.3 \text { ): } 14\end{array}$ & $\begin{array}{l}2 \text { cities; annual 24-hr } \\
\mathrm{PM}_{10}, 2 \text { nd maximum }\end{array}$ & $4.9 \mathrm{E}-05$ & $3.3 \mathrm{E}-05$ & $6.6 \mathrm{E}-05$ & $\mathrm{~g} / \mathrm{m}^{3}$ \\
\hline XMLF & $\begin{array}{l}\text { Mass loading } \\
\text { factor for } \\
\text { resuspension }\end{array}$ & (a) & $\begin{array}{l}\text { Selection: Use mass loading } \\
\text { model. Watershed Reporting } \\
\text { Regions (Figure } 7.3 \text { ): } 15\end{array}$ & $\begin{array}{l}2 \text { cities; annual 24-hr } \\
\mathrm{PM}_{10}, 2 \text { nd maximum }\end{array}$ & $6.7 \mathrm{E}-05$ & $4.9 \mathrm{E}-05$ & $8.6 \mathrm{E}-05$ & $\mathrm{~g} / \mathrm{m}^{3}$ \\
\hline XMLF & $\begin{array}{l}\text { Mass loading } \\
\text { factor for } \\
\text { resuspension }\end{array}$ & (a) & $\begin{array}{l}\text { Selection: Use mass loading } \\
\text { model. Watershed Reporting } \\
\text { Regions (Figure } 7.3 \text { ): } 16\end{array}$ & $\begin{array}{l}2 \text { cities; annual 24-hr } \\
\mathrm{PM}_{10}, 2 \text { nd maximum }\end{array}$ & $6.1 \mathrm{E}-05$ & $4.5 \mathrm{E}-05$ & $7.6 \mathrm{E}-05$ & $\mathrm{~g} / \mathrm{m}^{3}$ \\
\hline XMLF & $\begin{array}{l}\text { Mass loading } \\
\text { factor for } \\
\text { resuspension }\end{array}$ & (a) & $\begin{array}{l}\text { Selection: Use mass loading } \\
\text { model. Watershed Reporting } \\
\text { Regions (Figure } 7.3 \text { ): } 17 \text { east } \\
\text { of Cascade Mts. }\end{array}$ & $\begin{array}{l}3 \text { cities; annual } 24-\mathrm{hr} \\
\mathrm{PM}_{10}, 2 \text { nd maximum }\end{array}$ & $7.6 \mathrm{E}-05$ & $5.0 \mathrm{E}-05$ & $1.0 \mathrm{E}-04$ & $\mathrm{~g} / \mathrm{m}^{3}$ \\
\hline XMLF & $\begin{array}{l}\text { Mass loading } \\
\text { factor for } \\
\text { resuspension }\end{array}$ & (a) & $\begin{array}{l}\text { Selection: Use mass loading } \\
\text { model. Watershed Reporting } \\
\text { Regions (Figure } 7.3 \text { ): } 17 \text { west } \\
\text { of Cascade Mts. }\end{array}$ & $\begin{array}{l}2 \text { cities; annual 24-hr } \\
\mathrm{PM}_{10}, 2 \text { nd maximum }\end{array}$ & $5.4 \mathrm{E}-05$ & $3.9 \mathrm{E}-05$ & $6.9 \mathrm{E}-05$ & $\mathrm{~g} / \mathrm{m}^{3}$ \\
\hline XMLF & $\begin{array}{l}\text { Mass loading } \\
\text { factor for } \\
\text { resuspension }\end{array}$ & (a) & $\begin{array}{l}\text { Selection: Use mass loading } \\
\text { model. Watershed Reporting } \\
\text { Regions (Figure } 7.33 \text { ): } 18\end{array}$ & $\begin{array}{l}3 \text { cities; annual 24-hr } \\
\mathrm{PM}_{10}, 2 \text { nd maximum }\end{array}$ & $8.1 \mathrm{E}-05$ & $5.5 \mathrm{E}-05$ & $1.1 \mathrm{E}-04$ & $\mathrm{~g} / \mathrm{m}^{3}$ \\
\hline XMLF & $\begin{array}{l}\text { Mass loading } \\
\text { factor for } \\
\text { resuspension }\end{array}$ & (a) & $\begin{array}{l}\text { Selection: Use mass loading } \\
\text { model. Watershed Reporting } \\
\text { Regions (Figure 7.3): } 19\end{array}$ & $\begin{array}{l}1 \text { city; annual 24-hr } \\
\mathrm{PM}_{10}, 2 \text { nd maximum }\end{array}$ & $4.8 \mathrm{E}-05$ & $3.6 \mathrm{E}-05$ & $6.0 \mathrm{E}-05$ & $\mathrm{~g} / \mathrm{m}^{3}$ \\
\hline XMLF & $\begin{array}{l}\text { Mass loading } \\
\text { factor for } \\
\text { resuspension }\end{array}$ & (a) & $\begin{array}{l}\text { Selection: Use mass loading } \\
\text { model. Watershed Reporting } \\
\text { Regions (Figure 7.3): } 20\end{array}$ & $\begin{array}{l}1 \text { city; annual 24-hr } \\
\mathrm{PM}_{10}, 2 \text { nd maximum }\end{array}$ & 4.4E-05 & $2.8 \mathrm{E}-05$ & $6.0 \mathrm{E}-05$ & $\mathrm{~g} / \mathrm{m}^{3}$ \\
\hline (a) & ion from re & $\begin{array}{l}\text { entative } \\
\text { s/pm.htr }\end{array}$ & ies in indicated region from & (1) & T & $\mathrm{d}$ at: & & \\
\hline
\end{tabular}




\subsection{Tab: Soil/Leaching}

The recommended Type of Leach Rate Constant to select is: Leach rates calculated from user input. Do not select "GENII default leach rates" because this option is not functional (leach rates of zero are used). If the "User-provided leach rate constant" is selected, consult the Gv2 User's Guide (Napier 2012). For "Leach rates calculated from user input," four parameter entries plus radionuclide-specific soil absorption coefficients $\left(\mathrm{K}_{\mathrm{d}}\right)$ are required. Parameters applied here are used for the calculation of soil concentrations for several soil exposure pathways (e.g., soil inhalation; external exposure - excluding shoreline exposure; and food and feed crops) modeling.

\subsubsection{THICK Soil Thickness}

The user is responsible for entering the same soil thickness $(\mathrm{cm})$ value as entered in the Soil/Surface Soil tab (see Section 7.7.1). Coordinate with the value used for BULKD (see Section 7.9.3).

The units and range for THICK in Gv2 are: $\mathrm{cm}(0-10,000)$.

\subsubsection{MOISTC Surface Soil Moisture Content}

Surface soil moisture content is the volume fraction of the soil that is moisture.

The units and range for MOISTC in Gv2 are: $\mathrm{ml} / \mathrm{cm}^{3}(0-1.0)$.

For agricultural applications, this value is consistent with the field capacity of the soil, which is the water content of the soil after excess water has drained away. If values for available soil moisture are available, these may also be used. Available soil moisture is the amount of water usable by the plants. Chapter 2 of Brouwer et al. (1985) indicates generic values for available soil moisture: sand $\left(0.025-0.100 \mathrm{ml} / \mathrm{cm}^{3}\right)$, loam $\left(0.100-0.175 \mathrm{ml} / \mathrm{cm}^{3}\right)$, and clay $\left.0.175-0.250 \mathrm{ml} / \mathrm{cm}^{3}\right)$.

\subsubsection{Additional Sources of Information}

The USDA Natural Resources Conservation Service, formerly the Soil Conservation Service, has a number of web applications with national soil data details. The Web Soil Survey (WSS) (last accessed at: http://websoilsurvey.nrcs.usda.gov/app/HomePage.htm ) provides access the information. The level of detail available varies by location. Once in the WSS application and an area of interest is identified, the user can obtain information on the Soil Data Explorer tab, "Available water supply, 0 to $25 \mathrm{~cm}$ " to approximate the MOISTC value for their location of interest.

The National Weather Service reports soil moisture $(\mathrm{mm})$ under its Climate Prediction Center for drought surveillance. These data would be best applied to non-irrigated locations. Last accessed at: http://www.cpc.ncep.noaa.gov/cgi-bin/US_Soil-Moisture-Monthly.sh.

\subsubsection{BULKD Surface Soil Bulk Density}

The user is responsible for entering the same bulk density $\left(\mathrm{kg} / \mathrm{m}^{3}\right)$ value as entered in the Soil/Surface Soil tab (see Section 7.7.1). Coordinate with the value used for THICK (see Section 7.9.1). 
The units and range for BULKD in Gv2 are: $\mathrm{g} / \mathrm{cm}^{3}(0.5-3.0)$.

\subsubsection{VLEACH Total Infiltration Rate}

The total infiltration rate may be evaluated as the annual precipitation rate plus the irrigation rate minus the evapotranspiration rate. Unlike the rates applied in contaminated Surface Water scenarios, the irrigated water applications considered here may or may not be contaminated with radioactive materials. As a rough approximation for irrigated fields, assume 10 percent of the irrigation rate (see Section 7.3.1). Leaching rates are not crop-specific in Gv2, therefore, the user determines a representative rate to apply as VLEACH. Multiple modules could be used if variable leach rates are desired. Sandy soils generally have a greater infiltration rate, while consistently moist clay soils have lower rates.

The units and range for VLEACH in Gv2 are: $\mathrm{cm} / \mathrm{yr}(0-500)$.

\subsubsection{SOILKD Parent and Progeny Soil Absorption Coefficient}

Soil absorption coefficients are commonly known as $\mathrm{K}_{\mathrm{d}}$ values. The greater the number, the more tightly absorbed the nuclide is to the soil particle. The lower the number, the more likely the nuclide leaches out with the soil water. The user is responsible for entering the same value here and in the Constituent module (see CLKD, Section 2.1.1). If the values differ, the value entered in this Exposure module is the value applied for leaching calculations.

The units and range for SOILKD in Gv2 are: $\mathrm{ml} / \mathrm{g}(0-1,000,000)$.

Values recommended for a number of potential contaminants are those of Serne (2007). Serne (2007) also provides references for additional sources of $\mathrm{K}_{\mathrm{d}}$ values.

\subsubsection{Additional Sources of Information}

The EPA publication (EPA 1999) provides details regarding use and determinations of $\mathrm{K}_{\mathrm{d}}$ values for environmental modeling. Available at: http://www.epa.gov/radiation/cleanup/402-r-99-004.html\#vol1.

\subsection{Tab: Agriculture}

The agriculture tab has numerous sub-tabs to input data used for food ingestion pathways for human food crops and foods that livestock consume. U.S.-wide information is quite detailed for agricultural food commodities of interest, including but not limited to wheat, rice, corn, oats, barley, grain sorghums, mill feeds, butter, eggs, potatoes, peanuts, soybeans, soybean meal, livestock, livestock products, and frozen concentrated orange juice, but not onions.

Parameter documents similar to this one typically focus on a local view of agriculture practices as a consequence of the need for MEI impact determinations. This document attempts to provide a bigger view of agricultural production. This information provides an overview of the food production regions of commercially grown crops or animal products. Many distribution complexities (e.g., where a crop is processed, what parts of a crop are used in a final food product, what impact commercial processing 
practices have on the final radionuclide content, variable delay times between harvest and consumption) versus the location of the consumers are highly simplified and largely ignored.

Whether a crop is irrigated or not will have an impact on biomass and yield. In addition, the annual variation on these parameters is much less when crops are irrigated. Data in Table 7.5 provides some indication of prevalence of irrigation in each state.

\subsection{Tab: Agriculture/General}

For a 1-year emission calculation, which is a typical assumption for chronic release scenarios, no Radionuclide Removal Due to Harvesting is considered (leave box blank). Activation of this model (checking the box) would model soil radionuclide removal at the end of each year based on plant uptake and leaching. Also, do not check (leave box blank) the user-defined dry- and wet-deposition fraction to plant options.

\subsubsection{LEAFRS Soil-to-Plant Resuspension Factor}

The resuspension factor from soil to plant surfaces parameter is used for ingestion pathways to evaluate the air concentration above the plants from resuspension of soil particulates. See

Soil/Resuspension (Section 7.8) for resuspension factors used for the outdoor air inhalation pathway.

The units for this parameter in Gv2 are: $\mathrm{m}^{-1}(0-0.001)$.

A value of $1 \mathrm{E}-09 \mathrm{~m}^{-1}$ is used based on generic assumptions for atmospheric mass loading of $0.0002 \mathrm{~g} / \mathrm{m}^{3}$ soil in air and $225,000 \mathrm{~g} / \mathrm{m}^{2}$ areal soil density (to $15 \mathrm{~cm}$ ), dividing mass loading by areal density (Napier et al. 1988, p. 4.64). The user would modify this value based on their assumption for mass loading (see Section 7.8.1) and surface soil density (see Section 7.7.1). A range of values derived from the generic soil textures cited for the surface soil density section (Section 7.7.1) and the range of mass loading values for arid regions ( $\min 1.4 \mathrm{E}-05$; $\max 3.5 \mathrm{E}-04$; and central $2.0 \mathrm{E}-05 \mathrm{~g} / \mathrm{m}^{3}$ ) is provided, below.

\subsubsection{Additional Sources of Information}

See additional sources of information for mass loading (see Section 7.8.1) and surface soil density (see Section 7.7.1).

\subsubsection{LEAFRS Soil-to-Plant Resuspension Factor Table}

Table 7.10. LEAFRS Soil-to-Plant Resuspension Factor

\begin{tabular}{lcccc}
\hline \multicolumn{1}{c}{ Soil Texture } & & LEAFRS & LEAFRS & \\
& LEAFRS Min & Max & Central & Units \\
\hline sandy soil texture, ideal for plant growth & $7 \mathrm{E}-11$ & $1 \mathrm{E}-09$ & $1 \mathrm{E}-10$ & $\mathrm{~m}^{-1}$ \\
silty soil texture, ideal for plant growth & $8 \mathrm{E}-11$ & $2 \mathrm{E}-09$ & $1 \mathrm{E}-10$ & $\mathrm{~m}^{-1}$ \\
clayey soil texture, ideal for plant growth & $1 \mathrm{E}-10$ & $2 \mathrm{E}-09$ & $1 \mathrm{E}-10$ & $\mathrm{~m}^{-1}$ \\
\hline
\end{tabular}




\subsubsection{DPVRES Soil-to-Plant Deposition Velocity}

This parameter describes the amount of resuspended activity that deposits onto plants. One value is applied to all crops; therefore, this value is very generalized.

The units and range for this parameter in Gv2 are: $\mathrm{m} / \mathrm{s}(0-0.1)$.

Recommended value to assign is $0.001 \mathrm{~m} / \mathrm{s}$.

\subsubsection{Additional Sources of Information}

Although numerous publications have investigated deposition velocity, the single value is recommended for use in Gv2. This parameter in this application (contaminated soil resuspended at deposited on plant leaves) is not particularly sensitive within Gv2. Use of the default value is highly recommended.

\subsubsection{WTIM Plant Weathering Rate Constant}

This parameter indicates the weathering loss of a deposited radionuclide from a plant.

The units for this parameter in Gv2 are: $d(0.1-100)$.

A weathering rate constant of $14 \mathrm{~d}$ has been historically used in GENII (Napier et al. 1988). The RESRAD code (Yu et al. 2001) applies a $20 \mathrm{yr}^{-1}$ weathering removal constant, which converts to a 12.6-d removal rate for this lognormally distributed parameter. IAEA (2010) values relevant to Gv2 applications range from about 8-49 d. The professional opinion of the authors is that regional variability should not be considered for this parameter. The recommended value is $14 \mathrm{~d}$.

\subsubsection{Additional Sources of Information}

Weathering is reviewed in Section 3.2 of IAEA (2010). Weathering halftimes are reported from a limited number of samples, a limited number of nuclides, and a range of crops (cereal, grass, rice, and fruit). Be aware that Gv2 applications use one weathering rate for all crops.

\subsection{Tab: Agriculture/Animal Feed}

The Agriculture/Animal Feed tab contains numerous sub-tabs because several feed and forage crops (grain, hay, and grass) require separate parameterization. Also, incidental soil intake by livestock is addressed in this tab. The GENII model assumes the feed and forage types shown in Table 7.11. Gv2 code labels and specific examples of the food types that can be assigned are indicated in Table 7.12. The user should survey the region of interest to determine the mix and type of grains, silage, and forage commonly fed to the animal. Except for locations with large commercial operations, there will likely be a range of feed mixes to consider. The user can perform separate biomass and yield comparisons on the various mixtures to determine the range of possible values to assign. 
The creatures that provide animal products for human consumption which are modeled in Gv2 are assumed to be domesticated. Consider that wild or free-ranging counterparts will tend to consume more grass in their diet, which can increase intake rates of radionuclides.

Table 7.11. Feed and Forage Assumptions in GENII v2

\begin{tabular}{ccccc}
\hline \multirow{2}{*}{ Type } & & \multicolumn{3}{c}{ Feed or Forage Consumed $^{(\mathrm{a})}$} \\
\cline { 2 - 5 } FEED & Animal Product & Grain & Hay & Grass \\
& Meat & $\mathrm{X}$ & - & - \\
& Poultry & $\mathrm{X}$ & - & - \\
& Milk & - & $\mathrm{X}$ & - \\
& Egg & $\mathrm{X}$ & - & - \\
FORAGE & Meat & - & $\mathrm{X}$ & - \\
& Milk & - & - & $\mathrm{X}$ \\
\hline
\end{tabular}

(a) These are the default assumptions for feed and forage in the distribution version of Gv2. Parameter values recommended in this document reflect a change in feed composition (hay, silage, fodder mixture) for the meat and milk animal.

Table 7.12. Gv2 Food Types and Feed and Forage Assumptions

\begin{tabular}{cll}
\hline FdFrLabel & \multicolumn{1}{c}{$\begin{array}{c}\text { Feed for Animals that are Later } \\
\text { Consumed by People }\end{array}$} & \multicolumn{1}{c}{ Examples or Comment $^{(\mathrm{a})}$} \\
\hline 1 & meat animal feed & grain (e.g., primarily corn), silage, and hay \\
2 & poultry animal feed & grain (e.g., corn, wheat) \\
3 & milk animal feed & hay \\
4 & egg animal feed & grain (e.g., corn, wheat) \\
5 & meat animal forage & leafy vegetable (e.g., alfalfa, grass) \\
6 & milk animal forage & leafy vegetable (e.g., alfalfa, grass) \\
N/A & aquatic biota (all) & $\begin{array}{l}\text { Aquatic biota use only water as their } \\
\text { "contaminated food" source. }\end{array}$ \\
\hline
\end{tabular}

(a) These are the default assumptions for feed and forage in the distribution version of Gv2. Parameter values recommended in this document reflect a change in meat animal feed and milk animal feed composition (hay, silage, fodder mixture).

Meteorology, soil conditions, topography and other factors affect agricultural parameters. The U.S. Department of Agriculture conducts a census of agriculture every five years. The data is compiled by state, but Regions are also defined, as indicated in Figure 7.2 and Figure 7.3. State-specific data can be reviewed for a single census year or multiple census years, watershed specific data can be reviewed, and data from states with similar agriculture characteristics can be used to determine likely parameter ranges. For example, Figure 7.2 and Figure 7.3 indicate possible groupings of regions, which would permit regions from which ranges could be obtained. 


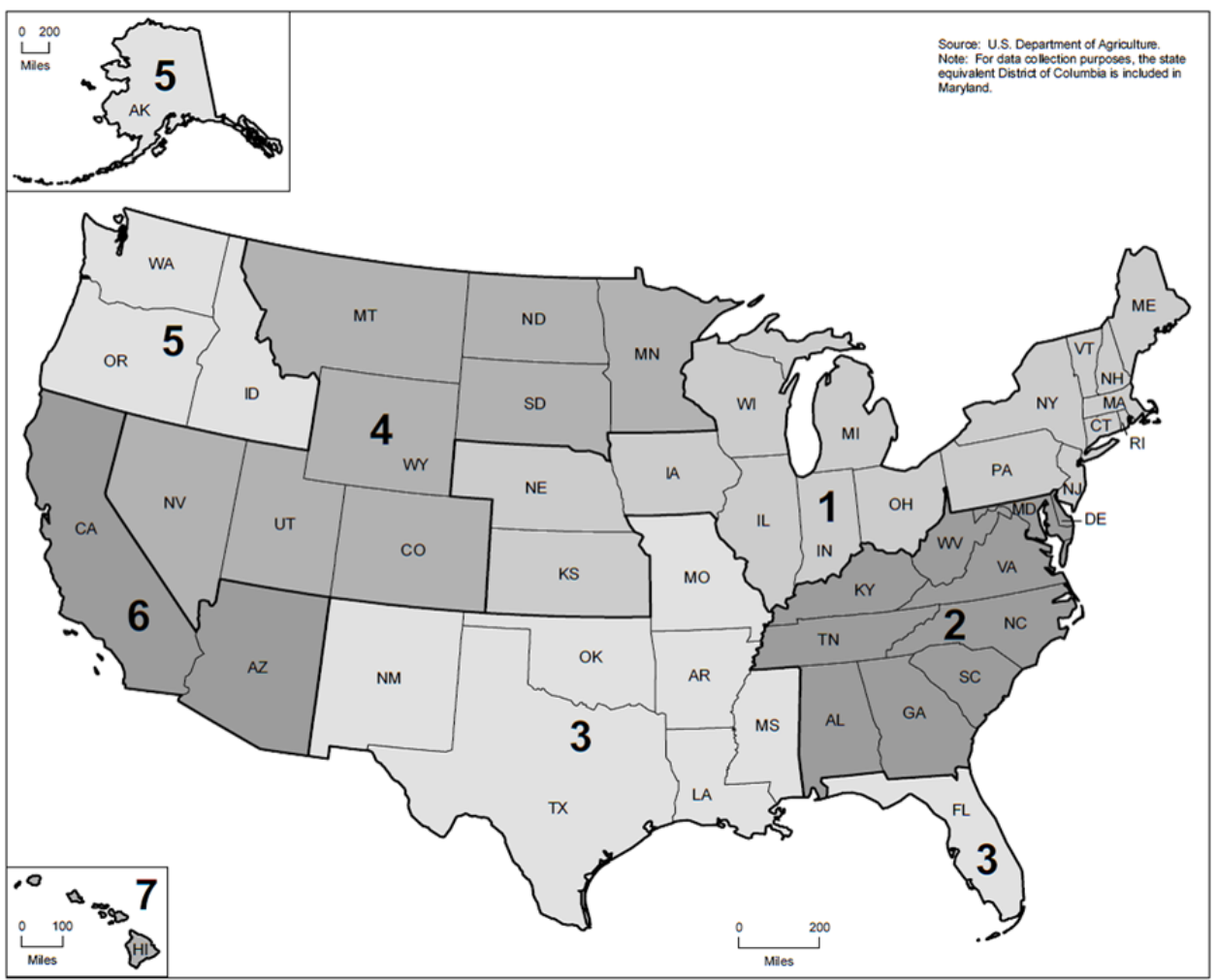

Figure 7.2. USDA Census of Agriculture Reporting Regions (USDA 2009)

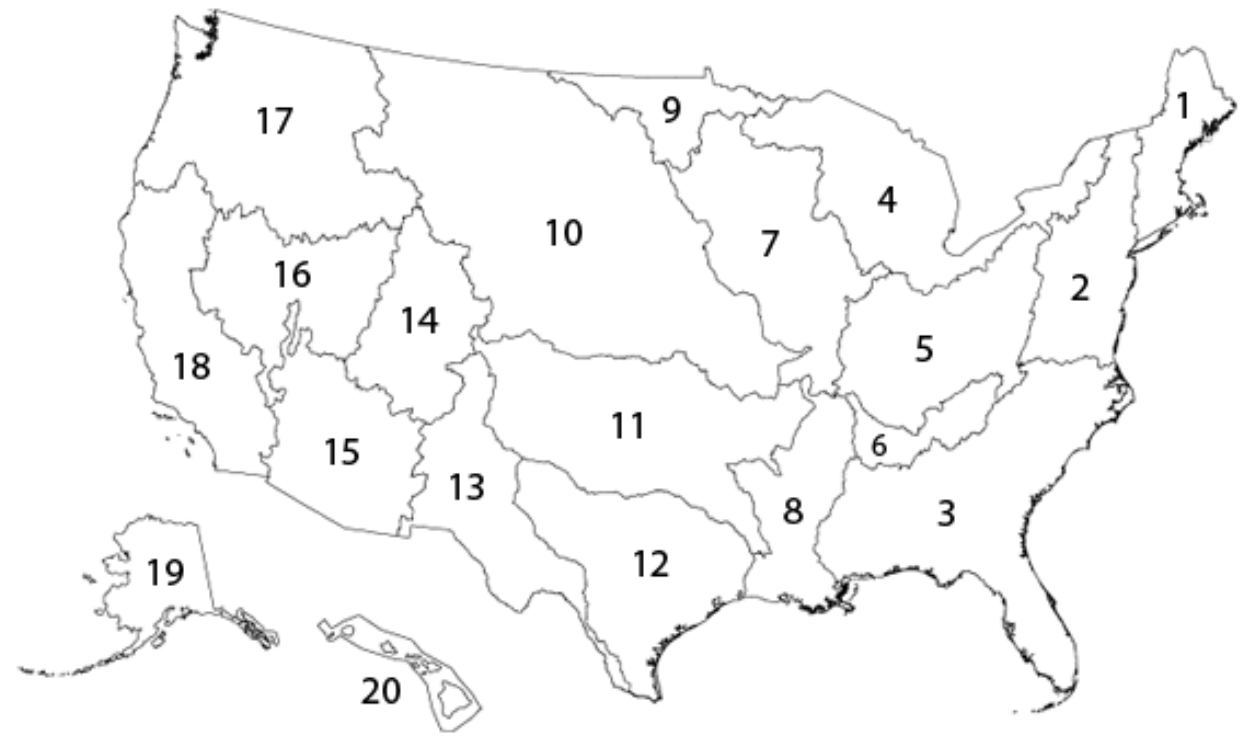

Figure 7.3. USDA Census of Agriculture Watershed Report Regions (USDA 2009) 


\subsection{Tabs: Agriculture/Animal Feed/Biomass; /Growing Period; IYield; and /Translocation Factor}

This section covers Animal Feed sub-tabs that pertain to the field characteristics of animal feed and forage. The input is entered in non-sequential tabs, but the data is presented together so that the field characteristic data can be compared among the various feed and forage crops. Crops considered are indicated in Table 7.11.

The biomass, growing period, yield and translocation factors for human food crops are covered in Section 7.18. The grain values for animal feed and human food should be comparable if not the same.

\subsubsection{BIOMA2 Biomass; GRWPA Growing Period; YELDA Yield; and TRANSA Translocation Factor}

The standing biomass for each animal feed type is the total above-ground plant mass (wet weight) used to estimate interception fractions for wet and dry deposition.

The growing period for each animal feed type defines the deposition period for irrigation and atmospheric deposition to plants. To the extent possible, the growing period should reflect the growing period of the edible portion of the crop. It may be approximated by 0.9 times the days between seed planting and harvesting. The entire plant-to-harvest period could also be used as a maximizing assumption.

The yield of each animal feed type gives the total annual production of edible feed crop mass (wet weight) per unit area of farmland. The yield is used to calculate the harvest removal losses from the soil. Radionuclide removal due to harvesting is modeled when opted on the Agriculture/General tab. This parameter only impacts scenarios that evaluate exposures which occur more than one-year after the release.

The translocation factor for each animal feed type is the fraction of activity reaching the plant surface that is transferred to the edible part of the plant. The significance of translocation depends on the radionuclide, kind of crop, time between harvest and consumption for fodder, and handling. This value differs from the animal feed or forage crop bioconcentration factors (CLBVAx and CLBVAG) assigned in the Constituent module (see Sections 2.1.17 through 2.1.19), which considers soil to plant transfer.

Similar parameters that are applicable to food crops ingested by people are found in Section 7.18.1.

The units and range for BIOMA2 in Gv2 are: $\mathrm{kg}_{\mathrm{wet}} / \mathrm{m}^{2}(0.1-10)$.

The units and range for GRWPA in Gv2 are: $d(0-365)$.

The units and range for YELDA in Gv2 are: $\mathrm{kg}_{\mathrm{wet}} / \mathrm{m}^{2}(0.1-10)$.

The units and range for TRANSA in Gv2 are: fraction (0-1). 


\section{Biomass}

Biomass values, in order of generally decreasing biomass are: fruit; root/other vegetables; leafy vegetables; human grain; feed for meat animal, poultry, and egg layer (grain); milk animal forage (grass); meat animal forage (hay); and milk animal feed (hay). A wide range of literature information is available to approximate biomass values. A limited set were reviewed and used for the evaluation (UC SAREP 2012; USDA 2009; Gallagher et al. 2003; Lalman and Sewell 1993).

\section{Growing Period}

USDA (2010) provided data on the planting and harvesting dates for a variety of crops and by state. Information on the area harvested was also provided, which provides an indication of the states that grow the most of a crop. Summary GRWPA data are provided in Table 7.14; state-specific details are provided in the Appendix F.

\section{Yield}

Yield information is collected in detail for commodity crops. Table 27 and Table 28 of the Census of Agriculture (USDA 2009) values were acquired and summarized for crops of interest. The values indicated are the wet-weight in the as-fed form of the crop (e.g., the wet weight of sun-cured hay is less than the wet-weight of the hay crop prior to sun-curing).

\section{Translocation}

Animal feed and forage translocation values (TRANSA) values are generally limited to 1.0 for leafy forages; a 0.9 "higher" value where 1.0 is not considered appropriate (e.g., corn silage or other non-grassy fodder); or a 0.1 "lower" value where a small fraction is appropriate to assign from translocation. The "lower" value would be appropriate to root feed crops (e.g., turnips) or grain feeds.

\subsubsection{Additional Sources of Information}

The USDA Agricultural Handbook Number 697 (USDA 1992) provides weights, measures, and conversion factors for agricultural commodities and their products. In particular, Table 6 provides commodity weights for a variety of crops and some crop products (e.g., bushels to $\mathrm{kg}$ ). It is noted that fruits and vegetables can have considerable variation in weight per unit volume because of differences in size, condition, packing, and other factors. These data can be used when evaluating mass, volume, or other data for farm product information in the open literature.

Dry-to-wet conversion factors can be useful when reviewing the literature. The USDA National Agricultural Library (USDA 2011) has compiled nutrient information on a long list of foods. Part of this data is the water content of various raw and processed crops. Access the database at http://www.nal.usda.gov/fnic/foodcomp/search/. Select "Start your search here", select the food group of interest on the left side of the screen (e.g., Vegetables and Vegetable Products), select the food of interest. The water content in $100 \mathrm{~g}$ of the food is listed. If you select "Statistics Report," the minimum, maximum, and standard deviation of the water content may be listed. 
Section 3.3 of IAEA (2010) reviews translocation factors. This reference also includes information regarding data availability and data quality.

The USDA Economics, Statistics, and Market Information System (e.g., http://usda.mannlib.cornell.edu/MannUsda/viewTaxonomy.do?taxonomyID=3) and the USDA Economic Research Service (e.g., http://www.ers.usda.gov/data-products/fruit-and-tree-nut-data.aspx) have summary information available on a variety of crops, including fruit, vegetables, nuts, berries, and field crops. In particular, biomass and yield information can be obtained from these resources. Some information is broken out by state, others are U.S. averages.

\subsubsection{BIOMA2, GRWPA, YELDA, TRANSA Animal Feed Biomass, Growing Period, Crop Yield, and Translocation Tables}

Table 7.13. BIOMA2 Standing Biomass - Animal Feed

\begin{tabular}{|c|c|c|c|c|c|c|c|}
\hline Gv2_ID & $\begin{array}{c}\text { Gv2 } \\
\text { Description }\end{array}$ & Food Type & Reference & Comment & $\begin{array}{l}\text { Low } \\
\text { Value }\end{array}$ & $\begin{array}{l}\text { High } \\
\text { Value }\end{array}$ & Units \\
\hline BIOMA2 & $\begin{array}{l}\text { Biomass for } \\
\text { FEED, } \\
\text { FORAGE }\end{array}$ & $\begin{array}{l}\text { 1.meat animal } \\
\text { feed (grain) }\end{array}$ & $\begin{array}{l}\text { UC SAREP (2012) and } \\
\text { Gallagher et al. (2003) }\end{array}$ & $\begin{array}{l}\text { barley, rapeseed, oats, } \\
\text { ryegrass. Dry:wet } \\
\text { assumption } 0.3\end{array}$ & 0.01 & 0.5 & $\mathrm{~kg}_{\text {wet }} / \mathrm{m}^{2}$ \\
\hline BIOMA2 & $\begin{array}{l}\text { Biomass for } \\
\text { FEED, } \\
\text { FORAGE }\end{array}$ & $\begin{array}{l}\text { 1.meat animal } \\
\text { feed (grain) }\end{array}$ & $\begin{array}{l}\text { Derived from Gallagher } \\
\text { et al. (2003) and USDA } \\
\text { (2011) }\end{array}$ & $\begin{array}{l}\text { winter and spring } \\
\text { wheat, grain and crop } \\
\text { residue }\end{array}$ & 0.64 & 1.41 & $\mathrm{~kg} \mathrm{wet}_{\text {wet }} / \mathrm{m}^{2}$ \\
\hline BIOMA2 & $\begin{array}{l}\text { Biomass for } \\
\text { FEED, } \\
\text { FORAGE }\end{array}$ & $\begin{array}{l}\text { 1.meat animal } \\
\text { feed (grain) }\end{array}$ & $\begin{array}{l}\text { Derived from Gallagher } \\
\text { et al. (2003) }\end{array}$ & sorghum & 1.5 & 1.8 & $\mathrm{~kg} \mathrm{wet}_{\text {wet }} / \mathrm{m}^{2}$ \\
\hline BIOMA2 & $\begin{array}{l}\text { Biomass for } \\
\text { FEED, } \\
\text { FORAGE }\end{array}$ & $\begin{array}{l}\text { 1.meat animal } \\
\text { feed (grain) }\end{array}$ & $\begin{array}{l}\text { Derived from Gallagher } \\
\text { et al. (2003) and USDA } \\
(2009 \text {, Tables } 27 \text { and } \\
28)\end{array}$ & corn & 2.2 & 6 & $\mathrm{~kg} g_{\text {wet }} / \mathrm{m}^{2}$ \\
\hline BIOMA2 & $\begin{array}{l}\text { Biomass for } \\
\text { FEED, } \\
\text { FORAGE }\end{array}$ & $\begin{array}{l}\text { 2.poultry feed } \\
\text { (grain) }\end{array}$ & $\begin{array}{l}\text { See meat animal feed, } \\
\text { above }\end{array}$ & $\begin{array}{l}\text { assumed } 60 \% \text { corn and } \\
40 \text { other (assumed } \\
\text { oats-equivalent) }\end{array}$ & 1.5 & 2.1 & $\mathrm{~kg} \mathrm{wet}_{\text {wet }} / \mathrm{m}^{2}$ \\
\hline BIOMA2 & $\begin{array}{l}\text { Biomass for } \\
\text { FEED, } \\
\text { FORAGE }\end{array}$ & $\begin{array}{l}\text { 3. milk feed } \\
\text { (corn silage) }\end{array}$ & $\begin{array}{l}\text { Derived from Gallagher } \\
\text { et al. (2003) and USDA } \\
(2009 \text {, Tables } 27 \text { and } \\
28)\end{array}$ & $\begin{array}{l}\text { corn silage. Dry:wet } \\
\text { assumption } 0.5 \text {. }\end{array}$ & 2.2 & 6 & $\mathrm{~kg}_{\text {wet }} / \mathrm{m}^{2}$ \\
\hline BIOMA2 & $\begin{array}{l}\text { Biomass for } \\
\text { FEED, } \\
\text { FORAGE }\end{array}$ & $\begin{array}{l}\text { 3. milk feed } \\
\text { (hay) }\end{array}$ & $\begin{array}{l}\text { Derived from Gallagher } \\
\text { et al. (2003), USDA } \\
\text { (2011), and USDA } \\
(2009 \text {, Tables } 27 \text { and } \\
28)\end{array}$ & $\begin{array}{l}\text { hay. Dry:wet } \\
\text { assumption } 0.5 \text {. }\end{array}$ & 0.2 & 1.3 & $\mathrm{~kg} \mathrm{wet} / \mathrm{m}^{2}$ \\
\hline BIOMA2 & $\begin{array}{l}\text { Biomass for } \\
\text { FEED, } \\
\text { FORAGE }\end{array}$ & $\begin{array}{l}\text { 4. egg feed } \\
\text { (grain) }\end{array}$ & See poultry feed, above & $\begin{array}{l}\text { assumed } 60 \% \text { corn and } \\
40 \text { other (assumed } \\
\text { oats-equivalent) }\end{array}$ & 1.5 & 2.1 & $\mathrm{~kg}{ }_{\text {wet }} / \mathrm{m}^{2}$ \\
\hline BIOMA2 & $\begin{array}{l}\text { Biomass for } \\
\text { FEED, } \\
\text { FORAGE }\end{array}$ & $\begin{array}{l}\text { 5.meat animal } \\
\text { forage (hay and } \\
\text { corn silage) }\end{array}$ & $\begin{array}{l}\text { See milk feed, hay and } \\
\text { corn, above }\end{array}$ & $\begin{array}{l}50 \% \text { corn silage, } 50 \% \\
\text { hay }\end{array}$ & 1.2 & 3.7 & $\mathrm{~kg}_{\text {wet }} / \mathrm{m}^{2}$ \\
\hline BIOMA2 & $\begin{array}{l}\text { Biomass for } \\
\text { FEED, } \\
\text { FORAGE }\end{array}$ & $\begin{array}{l}\text { 6.milk forage } \\
\text { (grass) }\end{array}$ & $\begin{array}{l}\text { Derived from Gallagher } \\
\text { et al. (2003), USDA } \\
(2011) \text {, and USDA } \\
(2009 \text {, Tables } 27 \text { and } \\
28)\end{array}$ & $\begin{array}{l}\text { hay. Dry:wet } \\
\text { assumption } 0.5 \text {. }\end{array}$ & 0.2 & 1.3 & $\mathrm{~kg}_{\text {wet }} / \mathrm{m}^{2}$ \\
\hline
\end{tabular}


Table 7.14. GRWPA Growing Period - Animal Feed

\begin{tabular}{|c|c|c|c|c|c|c|c|}
\hline Gv2_ID & $\begin{array}{c}\text { Gv2 } \\
\text { Description }\end{array}$ & Food Type & Reference & Comment & $\begin{array}{l}\text { Extremes } \\
\text { (d) }\end{array}$ & $\begin{array}{c}\text { Active } \\
\text { Period or } \\
\text { Average (d) }\end{array}$ & Units \\
\hline GRWPA & $\begin{array}{l}\text { Growing } \\
\text { period }\end{array}$ & $\begin{array}{l}\text { 1.meat animal } \\
\text { feed }\end{array}$ & $\begin{array}{l}\text { Calculated from } \\
\text { USDA (2010) }\end{array}$ & $\begin{array}{l}\text { Corn for grain, } \\
\text { planting to harvest }\end{array}$ & $123-199$ & $139-182$ & $\mathrm{~d}$ \\
\hline GRWPA & $\begin{array}{l}\text { Growing } \\
\text { period }\end{array}$ & $\begin{array}{l}\text { 1.meat animal } \\
\text { feed }\end{array}$ & $\begin{array}{l}\text { Calculated from } \\
\text { USDA (2010) }\end{array}$ & $\begin{array}{l}\text { Durum wheat, planting } \\
\text { to harvest }\end{array}$ & $105-237$ & $110-215$ & d \\
\hline GRWPA & $\begin{array}{l}\text { Growing } \\
\text { period }\end{array}$ & $\begin{array}{l}\text { 1.meat animal } \\
\text { feed }\end{array}$ & $\begin{array}{l}\text { Calculated from } \\
\text { USDA (2010) }\end{array}$ & $\begin{array}{l}\text { Spring wheat, planting } \\
\text { to harvest }\end{array}$ & $100-141$ & $105-131$ & d \\
\hline GRWPA & $\begin{array}{l}\text { Growing } \\
\text { period }\end{array}$ & $\begin{array}{l}\text { 1.meat animal } \\
\text { feed }\end{array}$ & $\begin{array}{l}\text { Calculated from } \\
\text { USDA (2010) }\end{array}$ & $\begin{array}{l}\text { Winter wheat, planting } \\
\text { to harvest }\end{array}$ & $151-350$ & $174-337$ & d \\
\hline GRWPA & $\begin{array}{l}\text { Growing } \\
\text { period }\end{array}$ & 2.poultry feed & $\begin{array}{l}\text { Calculated from } \\
\text { USDA (2010) }\end{array}$ & $\begin{array}{l}\text { Corn for grain, } \\
\text { planting to harvest }\end{array}$ & $123-199$ & $139-182$ & $d$ \\
\hline GRWPA & $\begin{array}{l}\text { Growing } \\
\text { period }\end{array}$ & 3. milk feed & $\begin{array}{l}\text { Calculated from } \\
\text { USDA (2010) }\end{array}$ & $\begin{array}{l}\text { Corn for silage, } \\
\text { planting to harvest }\end{array}$ & $61-183$ & $92-177$ & d \\
\hline GRWPA & $\begin{array}{l}\text { Growing } \\
\text { period }\end{array}$ & 3. milk feed & $\begin{array}{l}\text { Calculated from } \\
\text { USDA (2010) }\end{array}$ & $\begin{array}{l}\text { Alfalfa hay }{ }^{(\mathrm{a})} \text {, planting } \\
\text { to harvest }\end{array}$ & $6-74$ & $26-38$ & d \\
\hline GRWPA & $\begin{array}{l}\text { Growing } \\
\text { period }\end{array}$ & 3. milk feed & $\begin{array}{l}\text { Calculated from } \\
\text { USDA (2010) }\end{array}$ & $\begin{array}{l}\text { Other hay }{ }^{(\mathrm{a})} \text {, planting } \\
\text { to harvest }\end{array}$ & $6-80$ & $13-44$ & d \\
\hline GRWPA & $\begin{array}{l}\text { Growing } \\
\text { period }\end{array}$ & 4.egg layer feed & $\begin{array}{l}\text { Calculated from } \\
\text { USDA (2010) }\end{array}$ & $\begin{array}{l}\text { Corn for grain, } \\
\text { planting to harvest }\end{array}$ & 123-199 & $139-182$ & d \\
\hline GRWPA & $\begin{array}{l}\text { Growing } \\
\text { period }\end{array}$ & $\begin{array}{l}\text { 5.meat animal } \\
\text { forage }\end{array}$ & $\begin{array}{l}\text { Calculated from } \\
\text { USDA (2010) }\end{array}$ & $\begin{array}{l}\text { Corn for silage, } \\
\text { planting to harvest }\end{array}$ & $61-183$ & $92-177$ & d \\
\hline GRWPA & $\begin{array}{l}\text { Growing } \\
\text { period }\end{array}$ & $\begin{array}{l}\text { 5.meat animal } \\
\text { forage }\end{array}$ & $\begin{array}{l}\text { Calculated from } \\
\text { USDA (2010) }\end{array}$ & $\begin{array}{l}\text { Alfalfa hay }{ }^{(\mathrm{a})} \text {, planting } \\
\text { to harvest }\end{array}$ & $6-74$ & $26-38$ & d \\
\hline GRWPA & $\begin{array}{l}\text { Growing } \\
\text { period }\end{array}$ & $\begin{array}{l}\text { 5.meat animal } \\
\text { forage }\end{array}$ & $\begin{array}{l}\text { Calculated from } \\
\text { USDA (2010) }\end{array}$ & $\begin{array}{l}\text { Other hay }{ }^{(a)} \text {, planting } \\
\text { to harvest }\end{array}$ & $6-80$ & $13-44$ & d \\
\hline GRWPA & $\begin{array}{l}\text { Growing } \\
\text { period }\end{array}$ & 6. milk forage & $\begin{array}{l}\text { Calculated from } \\
\text { USDA (2010) }\end{array}$ & $\begin{array}{l}\text { Other hay }{ }^{(a)} \text {, planting } \\
\text { to harvest }\end{array}$ & $6-80$ & $13-44$ & d \\
\hline
\end{tabular}


Table 7.15. YELDA Crop Yield - Animal Feed

\begin{tabular}{|c|c|c|c|c|c|c|c|c|c|c|c|}
\hline GENII ID & YELDA, YELD & YELDA & YELDA & YELDA & YELDA, YELD & YELDA & YELDA & YELDA & YELDA & YELDA, YELD & YELD \\
\hline \multirow[t]{4}{*}{$\begin{array}{l}\text { GENII application } \\
\text { comment }\end{array}$} & \begin{tabular}{|c|} 
FEED: meat, \\
poultry, egg; \\
human grain \\
\end{tabular} & \begin{tabular}{|c|} 
FEED:milk; \\
FORAGE: meat
\end{tabular} & \begin{tabular}{|c} 
FEED:milk; \\
FORAGE: meat \\
\end{tabular} & \begin{tabular}{|c|} 
FEED:milk; \\
FORAGE: meat \\
\end{tabular} & \begin{tabular}{|c|} 
FEED: meat, \\
poultry, egg; \\
human grain
\end{tabular} & $\begin{array}{c}\text { FEED:milk; } \\
\text { FORAGE: meat } \\
\end{array}$ & $\begin{array}{c}\text { FEED:milk; } \\
\text { FORAGE: meat } \\
\end{array}$ & $\begin{array}{c}\text { FEED:milk; } \\
\text { FORAGE: } \\
\text { meat } \\
\end{array}$ & $\begin{array}{l}\text { FEED: meat, } \\
\text { poultry, egg }\end{array}$ & \begin{tabular}{|c|}
$\begin{array}{l}\text { FEED: meat, } \\
\text { poultry, egg; } \\
\text { human grain }\end{array}$ \\
\end{tabular} & $\begin{array}{c}\text { human } \\
\text { grain }\end{array}$ \\
\hline & $\begin{array}{c}\text { Corn for } \\
\text { grain } \\
\text { or seed }\end{array}$ & \begin{tabular}{|c|} 
silage \\
or \\
greenchop
\end{tabular} & $\begin{array}{l}\text { Alfalfa and } \\
\text { alfalfa } \\
\text { mixtures* }\end{array}$ & $\begin{array}{c}\text { All other } \\
\text { hay* }\end{array}$ & $\begin{array}{c}\text { Corn for } \\
\text { grain or seed }\end{array}$ & \begin{tabular}{|c|}
$\begin{array}{c}\text { Corn for } \\
\text { silage or } \\
\text { greenchop }\end{array}$ \\
\end{tabular} & $\begin{array}{l}\text { Alfalfa and } \\
\text { alfalfa } \\
\text { mixtures* }\end{array}$ & \begin{tabular}{|c} 
All other \\
hay*
\end{tabular} & $\begin{array}{c}\text { Sorghum } \\
\text { for grain } \\
\text { or seed }\end{array}$ & \begin{tabular}{|c|} 
Whe at for \\
grain or \\
seed
\end{tabular} & Rice \\
\hline & \begin{tabular}{|c|}
$\begin{array}{c}\text { Average yield } \\
\text { (irrigated) }\end{array}$ \\
\end{tabular} & $\begin{array}{c}\text { Average yield } \\
\text { (irrigated) }\end{array}$ & \begin{tabular}{|c|}
$\begin{array}{c}\text { Average yield } \\
\text { (irrigated) }\end{array}$ \\
\end{tabular} & \begin{tabular}{|c|}
$\begin{array}{c}\text { Average yield } \\
\text { (irrigated) }\end{array}$ \\
\end{tabular} & \begin{tabular}{|l|}
$\begin{array}{l}\text { Average yield } \\
\text { (nonIrrigated) }\end{array}$ \\
\end{tabular} & \begin{tabular}{|l|}
$\begin{array}{l}\text { Average yield } \\
\text { (nonlrrigated) }\end{array}$ \\
\end{tabular} & $\begin{array}{l}\begin{array}{l}\text { Average yield } \\
\text { (nonIrrigated) }\end{array} \\
\end{array}$ & $\begin{array}{c}\text { yield } \\
\text { (nonlrrigated }\end{array}$ & $\begin{array}{c}\text { yield } \\
\text { (irrigated) }\end{array}$ & $\begin{array}{c}\text { Average yield } \\
\text { (irrigated) }\end{array}$ & $\begin{array}{c}\text { yield } \\
\text { (irrigated) }\end{array}$ \\
\hline & kg_shelled $/ \mathrm{m} 2$ & $\mathrm{~kg}_{-}$wet $/ \mathrm{m} 2$ & kg_dry/m2 & kg_dry/m2 & kg_shelled $/ \mathrm{m} 2$ & kg_wet $/ \mathrm{m} 2$ & $\mathrm{~kg} \_\mathrm{dry} / \mathrm{m} 2$ & kg_dry/m2 & $\mathrm{kg} / \mathrm{m} 2$ & $\mathrm{~kg} / \mathrm{m} 2$ & $\mathrm{~kg} / \mathrm{m} 2$ \\
\hline United States average & 1.1 & 5.6 & 1.0 & 0.6 & 0.8 & 3.6 & 0.5 & 0.4 & 0.5 & 0.5 & 0.8 \\
\hline Alabama & 1.1 & 4.0 & 0.2 & 0.9 & 0.6 & 3.1 & (D) & 0.6 & - & 0.5 & - \\
\hline Alaska & - & - & - & 0.6 & - & - & - & (D) & - & - & - \\
\hline Arizona & 1.1 & 6.3 & 2.0 & 1.3 & - & - & (D) & - & 0.6 & 0.7 & - \\
\hline Arkansas & 1.0 & (D) & - & 0.8 & 0.8 & - & - & 0.5 & 0.6 & 0.4 & 0.8 \\
\hline California & 1.1 & 6.1 & 1.3 & 1.1 & 0.9 & 3.4 & 0.2 & 0.4 & 0.7 & 0.7 & 0.9 \\
\hline Colorado & 1.1 & 5.2 & 0.7 & 0.4 & 0.4 & 1.8 & 0.2 & 0.2 & 0.3 & 0.5 & - \\
\hline Connecticut & - & - & - & - & (D) & (D) & (D) & 1.3 & - & - & - \\
\hline Delaware & 1.2 & 4.7 & 0.8 & 0.7 & 0.7 & 2.9 & 0.4 & 0.5 & - & 0.6 & - \\
\hline Florida & 1.0 & 4.0 & - & 0.9 & 0.5 & (D) & 0.9 & 0.3 & (D) & 0.5 & 0.5 \\
\hline Georgia & 1.1 & 4.9 & 1.8 & 1.1 & 0.4 & 2.9 & 0.4 & 1.0 & 0.3 & 0.5 & - \\
\hline Hawaii & (D) & - & - & - & - & - & - & - & (D) & - & - \\
\hline Idaho & 1.1 & 6.1 & 1.0 & 0.4 & - & 3.8 & 0.2 & 0.2 & - & 0.7 & - \\
\hline Illinois & 1.2 & 3.1 & 0.5 & 0.7 & 1.1 & 3.1 & 0.5 & (D) & 0.7 & 0.4 & 0.7 \\
\hline Indiana & 1.1 & 4.7 & 0.7 & 0.2 & 1.0 & 4.5 & 0.5 & 0.2 & (D) & 0.4 & (D) \\
\hline Iowa & 1.1 & 5.4 & 1.2 & (D) & 1.0 & 2.9 & 0.8 & 0.4 & - & (D) & - \\
\hline Kansas & 1.2 & 4.9 & 1.4 & 1.0 & 0.5 & 4.0 & 0.6 & 0.5 & 0.6 & 0.4 & - \\
\hline Kentucky & 1.1 & 5.6 & (D) & 0.8 & 0.9 & 3.6 & 0.9 & 0.6 & - & - & - \\
\hline Louisiana & 1.0 & 2.2 & 0.1 & 1.1 & 0.9 & 3.4 & - & 0.6 & 0.5 & 0.4 & 0.7 \\
\hline Maine & - & - & - & - & (D) & (D) & - & (D) & - & - & - \\
\hline Maryland & 1.2 & 6.1 & 1.5 & 1.1 & 0.7 & 3.6 & 1.1 & 0.7 & - & 0.5 & - \\
\hline Massachusetts & (D) & - & - & - & - & - & 0.7 & 0.1 & - & - & - \\
\hline Michigan & 1.1 & 4.9 & 1.2 & - & 0.8 & 3.4 & 0.9 & 0.6 & - & 0.5 & - \\
\hline Minnesota & 1.1 & 4.5 & 1.0 & 0.5 & 0.9 & 3.8 & 0.6 & 0.3 & - & 0.4 & - \\
\hline Mississippi & 1.1 & - & - & 1.4 & 0.9 & (D) & - & 0.9 & 0.5 & 0.4 & 0.8 \\
\hline Missouri & 1.1 & 4.0 & 0.8 & 0.4 & 0.9 & 2.9 & 0.6 & 0.4 & 0.7 & 0.4 & 1.0 \\
\hline Montana & 0.9 & 4.9 & 0.7 & 0.4 & - & - & 0.2 & 0.2 & - & 0.5 & - \\
\hline Nebraska & 1.2 & 4.9 & 1.1 & 0.7 & 0.8 & 3.8 & 0.7 & 0.3 & 0.7 & 0.4 & - \\
\hline Nevada & - & 6.1 & 0.9 & 0.4 & - & - & 0.7 & 0.2 & - & 0.5 & - \\
\hline New Hampshire & (D) & 6.1 & - & - & - & (D) & 0.7 & (D) & - & - & - \\
\hline New Jersey & 1.1 & 5.4 & 1.4 & 0.2 & 0.7 & 3.4 & 0.2 & 0.6 & - & 0.5 & - \\
\hline New Mexico & 1.2 & 5.8 & 1.0 & 0.7 & 0.2 & (D) & 0.2 & 0.4 & 0.6 & 0.3 & - \\
\hline New York & 1.2 & 5.6 & 1.7 & - & 1.0 & 3.1 & 1.1 & 0.7 & - & (D) & - \\
\hline North Carolina & 0.8 & 6.1 & 0.2 & 0.7 & 0.4 & 3.6 & 0.8 & 0.6 & (D) & 0.3 & - \\
\hline North Dakota & 1.0 & 4.3 & 0.9 & 0.4 & 0.8 & 2.0 & 0.4 & 0.2 & - & 0.4 & - \\
\hline Ohio & 1.0 & 5.2 & 1.5 & - & 0.8 & 3.1 & 0.5 & 0.5 & - & - & - \\
\hline Oklahoma & 1.2 & 3.8 & 1.2 & 0.6 & 0.5 & - & 0.9 & 0.6 & 0.4 & 0.3 & - \\
\hline Oregon & 1.3 & 6.3 & 0.9 & 0.5 & - & 4.3 & 0.7 & 0.2 & - & 0.7 & - \\
\hline Pennsylvania & 0.9 & 5.4 & 0.5 & 0.6 & 0.9 & 4.3 & 0.5 & 0.4 & - & - & - \\
\hline Rhode Island & - & - & - & - & - & - & (D) & 0.7 & - & - & - \\
\hline South Carolina & 1.0 & 3.4 & 0.7 & 1.0 & 0.3 & 2.5 & - & 0.7 & (D) & 0.5 & - \\
\hline South Dakota & 1.1 & 4.0 & 0.8 & 0.4 & 0.8 & 3.1 & 0.4 & 0.4 & 0.6 & 0.4 & - \\
\hline Tennessee & 1.2 & 4.7 & 1.0 & (D) & 0.8 & 4.0 & 1.2 & 0.6 & (D) & 0.5 & (D) \\
\hline Texas & 1.2 & 5.6 & 1.0 & 0.7 & 0.3 & 2.9 & 0.9 & 0.4 & 0.4 & 0.3 & 0.8 \\
\hline Utah & 1.0 & 4.9 & 0.9 & 0.5 & - & 2.7 & 0.4 & 0.5 & 0.7 & 0.6 & - \\
\hline \begin{tabular}{|l} 
Vermont \\
\end{tabular} & - & - & - & - & - & - & - & 0.2 & - & - & - \\
\hline Virginia & 1.1 & 5.6 & 1.0 & 0.3 & 0.7 & 3.4 & 0.2 & 0.4 & - & 0.5 & - \\
\hline Washington & 1.2 & 6.5 & 1.1 & 1.0 & (D) & 5.8 & 0.5 & 0.2 & - & 0.7 & - \\
\hline West Virginia & (D) & (D) & (D) & - & 0.9 & 2.9 & 0.8 & 0.5 & - & - & - \\
\hline Wisconsin & 1.0 & 4.7 & 0.8 & 0.7 & 0.8 & 3.8 & 0.5 & 0.6 & - & 0.5 & - \\
\hline Wyoming & 0.9 & 5.4 & 0.8 & 0.4 & (D) & - & 0.3 & 0.2 & - & 0.4 & - \\
\hline
\end{tabular}


Table 7.15. (contd)

\begin{tabular}{|c|c|c|c|c|c|c|c|c|c|c|c|}
\hline GENII ID & YELDA, YELD & YELDA & YELDA & YELDA & KELDA, YELD & YELDA & YELDA & YELDA & YELDA & YELDA, YELD & YELD \\
\hline \multirow[t]{4}{*}{ GENII application comment } & $\begin{array}{c}\text { FEED: meat, } \\
\text { poultry, egg; } \\
\text { human grain }\end{array}$ & $\begin{array}{c}\text { FEED:milk; } \\
\text { FORAGE: meat }\end{array}$ & $\begin{array}{c}\text { FEED:milk; } \\
\text { FORAGE: meat }\end{array}$ & $\begin{array}{l}\text { FEED:milk; } \\
\text { FORAGE: meat }\end{array}$ & \begin{tabular}{|c|} 
FEED: meat, \\
poultry, egg; \\
human grain
\end{tabular} & $\begin{array}{c}\text { FEED:milk; } \\
\text { FORAGE: meat }\end{array}$ & $\begin{array}{c}\text { FEED:milk; } \\
\text { FORAGE: meat }\end{array}$ & $\begin{array}{c}\text { FEED:milk; } \\
\text { FORAGE: } \\
\text { meat }\end{array}$ & $\begin{array}{l}\text { FEED: meat, } \\
\text { poultry, egg }\end{array}$ & \begin{tabular}{|l|} 
FEED: meat, \\
poultry, egg; \\
human grain
\end{tabular} & $\begin{array}{c}\text { human } \\
\text { grain }\end{array}$ \\
\hline & $\begin{array}{c}\text { Corn for } \\
\text { grain } \\
\text { or seed }\end{array}$ & \begin{tabular}{|c|}
$\begin{array}{c}\text { Corn for } \\
\text { silage } \\
\text { or } \\
\text { greenchop }\end{array}$ \\
\end{tabular} & $\begin{array}{l}\text { Alfalfa and } \\
\text { alfalfa } \\
\text { mixtures* }\end{array}$ & $\begin{array}{l}\text { All other } \\
\text { hay* }\end{array}$ & $\begin{array}{c}\text { Corn for } \\
\text { grain or seed }\end{array}$ & \begin{tabular}{|c} 
Corn for \\
silage or \\
greenchop
\end{tabular} & $\begin{array}{l}\text { Alfalfa and } \\
\text { alfalfa } \\
\text { mixtures* }\end{array}$ & $\begin{array}{c}\text { All other } \\
\text { hay* }\end{array}$ & $\begin{array}{c}\text { Sorghum } \\
\text { for grain } \\
\text { or seed }\end{array}$ & $\begin{array}{l}\text { Wheat for } \\
\text { grain or } \\
\text { seed }\end{array}$ & Rice \\
\hline & $\begin{array}{c}\text { Average yield } \\
\text { (irrigated) }\end{array}$ & $\begin{array}{c}\text { Average yield } \\
\text { (irrigated) }\end{array}$ & $\begin{array}{l}\text { Average yield } \\
\text { (irrigated) }\end{array}$ & $\begin{array}{c}\text { Average yield } \\
\text { (irrigated) }\end{array}$ & $\begin{array}{l}\text { Average yield } \\
\text { (nonIrrigated) }\end{array}$ & $\begin{array}{l}\text { Average yield } \\
\text { (nonlrigated) }\end{array}$ & $\begin{array}{l}\text { Average yield } \\
\text { (nonIrrigated) }\end{array}$ & $\begin{array}{c}\text { Average } \\
\text { yield } \\
\text { (nonIrrigated } \\
\text { ) }\end{array}$ & $\begin{array}{c}\text { Average } \\
\text { yield } \\
\text { (irrigated) }\end{array}$ & $\begin{array}{c}\text { Average yield } \\
\text { (irrigated) }\end{array}$ & \begin{tabular}{|c} 
Average \\
yield \\
(irrigated)
\end{tabular} \\
\hline & $\mathrm{kg} \_$shelled $/ \mathrm{m} 2$ & kg_wet $/ \mathrm{m} 2$ & $\mathrm{~kg}$ dry $/ \mathrm{m} 2$ & $\mathrm{~kg}$ dry $/ \mathrm{m} 2$ & $\mathrm{~kg} \_$shelled $/ \mathrm{m} 2$ & kg_wet $/ \mathrm{m} 2$ & $\mathrm{~kg}$ dry $/ \mathrm{m} 2$ & $\mathrm{~kg}$ dry $/ \mathrm{m} 2$ & $\mathrm{~kg} / \mathrm{m} 2$ & $\mathrm{~kg} / \mathrm{m} 2$ & $\mathrm{~kg} / \mathrm{m} 2$ \\
\hline United States average & 1.1 & 5.6 & 1.0 & 0.6 & 0.8 & 3.6 & 0.5 & 0.4 & 0.5 & 0.5 & 0.8 \\
\hline \multicolumn{12}{|l|}{ Watershed Region } \\
\hline Region 01 New England & 0.7 & 6.1 & - & - & 0.7 & 4.3 & 0.7 & 1.0 & - & - & - \\
\hline Region 02 Mid-Atlantic & 1.2 & 5.6 & 1.1 & 0.8 & 0.7 & 3.8 & 0.5 & 0.5 & - & 0.5 & - \\
\hline Region 03 South Atlantic-Gulf & 1.0 & 4.5 & 1.1 & 0.9 & 0.4 & 2.9 & 0.5 & 0.7 & (D) & 0.4 & 0.5 \\
\hline Region 04 Great Lakes & 1.1 & 4.9 & 1.1 & 0.7 & 0.8 & 3.4 & 0.8 & 0.6 & - & 0.5 & - \\
\hline Region 05 Ohio & 1.1 & 4.7 & 0.8 & 0.8 & 1.0 & 4.3 & 0.7 & 0.6 & - & 0.4 & (D) \\
\hline Region 06 Tennessee & 1.2 & 4.7 & 0.9 & 0.5 & 0.7 & 4.0 & 1.3 & 0.6 & - & 0.6 & - \\
\hline Region 07 Upper Mississippi & 1.1 & 4.5 & 0.8 & 0.5 & 1.0 & 3.6 & 0.6 & 0.4 & (D) & 0.5 & (D) \\
\hline Region 08 Lower Mississippi & 1.1 & 4.0 & 0.2 & 1.1 & 0.9 & 3.1 & 0.8 & 0.6 & 0.6 & 0.4 & 0.8 \\
\hline Region 09 Souris-Red-Rainy & 1.0 & 4.5 & 1.3 & 0.5 & 0.8 & 4.0 & 0.6 & (D) & - & 0.4 & - \\
\hline Region 10 Missouri & 1.1 & 4.9 & 0.8 & 0.4 & 0.8 & 3.1 & 0.4 & 0.3 & 0.7 & 0.4 & - \\
\hline Region 11 Arkansas-White-Red & 1.2 & 5.4 & 1.1 & 0.6 & 0.5 & 4.3 & 0.6 & 0.5 & 0.5 & 0.3 & 0.8 \\
\hline \begin{tabular}{|l|l} 
Region 12 Texas-Gulf \\
\end{tabular} & 0.9 & 5.4 & 1.3 & 0.7 & (D) & 2.9 & (D) & 0.4 & 0.4 & 0.3 & 0.8 \\
\hline Region 13 Rio Grande & (D) & 6.1 & 0.9 & 0.6 & - & - & 0.2 & 0.3 & 0.6 & 0.6 & - \\
\hline Region 14 Upper Colorado & 1.2 & 5.2 & 0.6 & 0.4 & - & 2.7 & 0.2 & 0.3 & - & 0.6 & - \\
\hline Region 15 Lower Colorado & 1.1 & 6.3 & 1.9 & 1.2 & - & - & (D) & - & 0.6 & 0.7 & - \\
\hline Region 16 Great Basin & 0.9 & 5.2 & 0.9 & 0.4 & - & 3.1 & 0.4 & 0.4 & 0.7 & 0.6 & - \\
\hline Region 17 Pacific Northwest & 1.2 & 6.3 & 0.9 & 0.5 & (D) & 5.4 & 0.4 & 0.2 & - & 0.7 & - \\
\hline Region 18 California & 1.1 & 6.1 & 1.3 & 1.0 & 0.9 & 3.4 & 0.2 & 0.4 & 0.7 & 0.7 & 0.9 \\
\hline Region 19 Alaska & - & - & - & 0.6 & - & - & - & (D) & - & - & - \\
\hline Region 20 Hawaii & (D) & - & - & - & - & - & - & - & (D) & - & - \\
\hline Minimum yield & 0.7 & 2.2 & 0.1 & 0.2 & 0.2 & 1.8 & 0.2 & 0.1 & 0.3 & 0.3 & 0.5 \\
\hline Maximum yield & 1.3 & 6.5 & 2.0 & 1.4 & 1.1 & 5.8 & 1.3 & 1.3 & 0.7 & 0.7 & 1.0 \\
\hline \multicolumn{12}{|c|}{$\begin{array}{l}\text { See Figure } 7.2 \text { for Watershed Regions. } \\
\text { (D) = Witheld to avoid disclosing data for individual farms. } \\
\text { - = Represents zero. } \\
*=\text { Dry hay, green chop, and silage }\end{array}$} \\
\hline
\end{tabular}

Table 7.16. TRANSA Translocation Factor (Plant Surface to Edible Part) - Animal Feed

\begin{tabular}{|c|c|c|c|c|c|c|c|}
\hline Gv2_ID & Gv2_Description & Food Type & Reference & Comment & Min & Max & Units \\
\hline TRANSA & $\begin{array}{l}\text { Translocation Fraction } \\
\text { for animal feed }\end{array}$ & 1.meat_feed & $\begin{array}{l}\text { Assumption based on } \\
\text { feed/forage morphology }\end{array}$ & $\begin{array}{l}\text { hay, silage, } \\
\text { corn }\end{array}$ & 0.9 & 1 & fraction \\
\hline TRANSA & $\begin{array}{l}\text { Translocation Fraction } \\
\text { for animal feed }\end{array}$ & 2.poultry_feed & $\begin{array}{l}\text { Assumption based on } \\
\text { feed/forage morphology }\end{array}$ & grain & 0.1 & 0.1 & fraction \\
\hline TRANSA & $\begin{array}{l}\text { Translocation Fraction } \\
\text { for animal feed }\end{array}$ & 3.milk_feed & $\begin{array}{l}\text { Assumption based on } \\
\text { feed/forage morphology }\end{array}$ & $\begin{array}{l}\text { hay, silage, } \\
\text { fodder }\end{array}$ & 0.9 & 1 & fraction \\
\hline TRANSA & $\begin{array}{l}\text { Translocation Fraction } \\
\text { for animal feed }\end{array}$ & 4.egg_feed & $\begin{array}{l}\text { Assumption based on } \\
\text { feed/forage morphology }\end{array}$ & grain & 0.1 & 0.1 & fraction \\
\hline TRANSA & $\begin{array}{l}\text { Translocation Fraction } \\
\text { for animal forage }\end{array}$ & 5.meat_forage & $\begin{array}{l}\text { Assumption based on } \\
\text { feed/forage morphology }\end{array}$ & hay & 1 & 1 & fraction \\
\hline TRANSA & $\begin{array}{l}\text { Translocation Fraction } \\
\text { for animal forage }\end{array}$ & 6.milk_forage & $\begin{array}{l}\text { Assumption based on } \\
\text { feed/forage morphology }\end{array}$ & grass & 1 & 1 & fraction \\
\hline
\end{tabular}

To summarize generic values that may be used, the following table is provided. Varieties of feed and forage mixtures were considered in the development of this table. 
Table 7.17. Summary for BIOMA2, GRWPA, YELDA, and TRANSA

\begin{tabular}{lcccc}
\hline \multirow{2}{*}{$\begin{array}{c}\text { Animal Feed } \\
\text { and Forage }\end{array}$} & $\begin{array}{c}\text { Biomass } \\
\left(\mathrm{kg} / \mathrm{m}^{2}\right)\end{array}$ & $\begin{array}{c}\text { Growing } \\
\text { Period (d) }\end{array}$ & ${\text { Yield }\left(\mathrm{kg} / \mathrm{m}^{2}\right)}^{(\mathrm{c})}$ & $\begin{array}{c}\text { Translocation } \\
(\text { fraction })\end{array}$ \\
\cline { 2 - 5 } & $\mathrm{BIOMA2}^{(\mathrm{b})}$ & GRWPA $^{(\mathrm{c})}$ & YELDA $^{(\mathrm{d})}$ & TRANSA $^{(\mathrm{e})}$ \\
\hline 1.meat animal feed & 4.7 & 90 & 2.0 & 0.9 \\
2.poultry feed & 1.8 & 90 & 0.8 & 0.1 \\
3. milk feed & 2.0 & 90 & 1.1 & 0.9 \\
4.egg layer feed & 1.8 & 90 & 0.8 & 0.1 \\
5.meat animal forage & 2.0 & 30 & $3.3^{(\mathrm{a})}$ & 1 \\
6.milk forage & 1.3 & 30 & $1.5^{(\mathrm{a})}$ & 1 \\
\hline
\end{tabular}

(a) The yield for hay and grass forage reflects the replacement growth that occurs over the year. Values indicated reflect three harvests over the year. Southern states may want to increase this value by a factor of 1.3 to reflect an additional growth cycle.

(b) Details regarding BIOMA2 values can be found in Table 7.13.

(c) Details regarding GRWPA values can be found in Table 7.14.

(d) Details regarding YELDA values can be found in Table 7.15.

(e) Details regarding TRANSA values can be found in Table 7.16.

\subsection{Tabs: Agriculture/Animal Feed/Consumption; IStorage Time; and /Diet Fraction}

The Animal Feed sub-tabs Consumption, Storage Time, and Diet Fraction are grouped together as feed and forage parameters related animal intake of the crops.

The consumption parameter for human food crops is covered in the Receptor module (see Section 8.2.1). The storage time parameter for human foods is covered in the Agriculture/Intake delays tab (see Section 7.20).

\subsubsection{CONSUM Consumption, STORTM Storage Time, and DIETFR Diet Fraction}

The feed and forage consumption rates for each animal feed is entered. The value for each feed type represents the total intake of this type of feed. The types of animal feed assigned by GENII to each category are listed in parentheses and in Table 7.11, above. These consumption rate values are multiplied by their respective contaminated fraction (DIETFR) to determine the contaminated portion of the intake of each feed type.

The storage time (STORTM) between harvest and feeding to the animal is used to evaluate radioactive decay during the storage period. Maximum storage periods are 1 year in Gv2.

The contaminated fraction of feed for each animal type is the fraction of the feed type eaten by the animal that is contaminated. This fraction is multiplied by the feed consumption rate (CONSUM) to determine the total contaminated intake of each feed type. A 1.0 contaminated fraction is assumed. The user can adjust this value for their situation. Above-ideal temperature and humidity and feed intake rates for poultry are inversely impacted, where ideal levels are 60-75 degrees $F$ and 40-60 percent relative humidity (Ensminger et al. 1990). 
The units and range for CONSUM in Gv2 are: $\mathrm{kg} / \mathrm{d}(0.001-300)$.

The units and range for STORTM in Gv2 are: $\mathrm{d}(0-365)$.

The units and range for DIETFR in Gv2 are: fraction (0-1).

Consumption rates indicate consumption near the time of animal harvest for beef cattle and poultry. Milk cow consumption is related to the amount of milk they produce. Feed rates are indicated in as-fed weight. Beef cattle consume about 2-3 percent of their body weight in feed (dry weight) (Ensminger et al. 1990). Milk cows consume about 2.5-3.5 percent of their body weight (Ensminger et al. 1990). Beef cattle may be fed more grain than hay or silage near the time of animal harvest.

Storage time for animal feed and forages depend on the feed component. Storage minimum and maximums are indicated, with a single recommended value for each feed type. For specific cases, the harvest date of the animal relative to the harvest date of the feed crop may be given some consideration. The recommended value is an annual average.

\subsubsection{Additional Sources of Information}

Nutrient Requirements of Beef Cattle (NRC 1996). Available online from National Academy Press at http://www.nap.edu/catalog/9791.html. The year 2000 update may be available online.

Nutrient Requirements of Dairy Cattle (NRC 2001). National Research Council, National Academy Press, Washington, D.C. Last accessed at http://www.nap.edu/catalog.php?record id=9825.

Nutrient Requirements of Small Ruminants: Sheep, Goats, Cervids, and New World Camelids (NRC 2007). Not currently available online.

\subsubsection{CONSUM and STORTM Feed Consumption and Storage Time Tables}

Table 7.18. CONSUM Consumption Rate - Animal Feed

\begin{tabular}{|c|c|c|c|c|c|c|c|c|}
\hline Gv2_ID & $\begin{array}{c}\text { Gv2 } \\
\text { Description }\end{array}$ & $\begin{array}{c}\text { Animal Feed/ } \\
\text { Forage Type }\end{array}$ & Reference & Comment & $\begin{array}{l}\text { Recom- } \\
\text { mended }^{(a)}\end{array}$ & Min & Max & Units $^{(b)}$ \\
\hline CONSUM & $\begin{array}{l}\text { Animal feed } \\
\text { intake rate. }\end{array}$ & $\begin{array}{l}\text { 1.meat animal } \\
\text { feed }\end{array}$ & $\begin{array}{l}\text { Ensminger } \\
\text { et al. (1990) }\end{array}$ & $\begin{array}{l}\text { IF SOLELY ON FEED. } \\
\text { Derived from half grain, half } \\
\text { silage mixture. } 2-3 \% \text { (dry } \\
\text { basis) of animal weight } \\
(800-1200 \mathrm{lb}) .\end{array}$ & 18 & 11 & 29 & $\mathrm{~kg} / \mathrm{d}$ \\
\hline CONSUM & $\begin{array}{l}\text { Animal feed } \\
\text { intake rate. }\end{array}$ & 2.poultry & $\begin{array}{l}\text { Ensminger } \\
\text { et al. (1990) }\end{array}$ & grain. Chicken. & 0.12 & 0.07 & 0.14 & $\mathrm{~kg} / \mathrm{d}$ \\
\hline CONSUM & $\begin{array}{l}\text { Animal feed } \\
\text { intake rate. }\end{array}$ & 2.poultry & $\begin{array}{l}\text { Ensminger } \\
\text { et al. (1990) }\end{array}$ & grain. Turkey. & 0.26 & 0.20 & 0.30 & $\mathrm{~kg} / \mathrm{d}$ \\
\hline CONSUM & $\begin{array}{l}\text { Animal feed } \\
\text { intake rate. }\end{array}$ & 3.milk feed & $\begin{array}{l}\text { Ensminger } \\
\text { et al. (1990) }\end{array}$ & $\begin{array}{l}\text { IF SOLELY ON FEED. } \\
\text { Derived from half grain, half } \\
\text { silage mixture. } 2.5-3.5 \% \\
\text { (dry basis) of animal weight } \\
(900-1400 \mathrm{lb}) .\end{array}$ & 25 & 16 & 40 & $\mathrm{~kg} / \mathrm{d}$ \\
\hline
\end{tabular}


Table 7.18. (contd)

\begin{tabular}{|c|c|c|c|c|c|c|c|c|}
\hline Gv2_ID & $\begin{array}{c}\text { Gv2 } \\
\text { Description }\end{array}$ & $\begin{array}{l}\text { Animal Feed/ } \\
\text { Forage Type }\end{array}$ & Reference & Comment & $\begin{array}{l}\text { Recom- } \\
\text { mended }^{(\mathrm{a})}\end{array}$ & Min & Max & Units $^{(b)}$ \\
\hline CONSUM & $\begin{array}{l}\text { Animal feed } \\
\text { intake rate. }\end{array}$ & 4.egg layer feed & $\begin{array}{l}\text { Ensminger } \\
\text { et al. (1990) }\end{array}$ & grain & 0.12 & 0.07 & 0.14 & $\mathrm{~kg} / \mathrm{d}$ \\
\hline CONSUM & $\begin{array}{l}\text { Animal feed } \\
\text { intake rate. }\end{array}$ & $\begin{array}{l}\text { 5.meat animal } \\
\text { forage }\end{array}$ & $\begin{array}{l}\text { Ensminger } \\
\text { et al. (1990) }\end{array}$ & $\begin{array}{l}\text { IF SOLELY ON DRY } \\
\text { FORAGE. Derived from } \\
\text { hay-grain mixture }(90 / 10) \text {. }\end{array}$ & 13 & 8.4 & 19 & $\mathrm{~kg} / \mathrm{d}$ \\
\hline CONSUM & $\begin{array}{l}\text { Animal feed } \\
\text { intake rate. }\end{array}$ & $\begin{array}{l}\text { 5.meat animal } \\
\text { forage }\end{array}$ & $\begin{array}{l}\text { Ensminger } \\
\text { et al. (1990) }\end{array}$ & $\begin{array}{l}\text { IF SOLELY ON FRESH } \\
\text { FORAGE. Derived from } \\
\text { fresh pasture forage. }\end{array}$ & 50 & 18 & 60 & $\mathrm{~kg} / \mathrm{d}$ \\
\hline CONSUM & $\begin{array}{l}\text { Animal feed } \\
\text { intake rate. }\end{array}$ & 6.milk forage & $\begin{array}{l}\text { Ensminger } \\
\text { et al. (1990) }\end{array}$ & $\begin{array}{l}\text { IF SOLELY ON FORAGE. } \\
\text { Derived from fresh pasture } \\
\text { forage. }\end{array}$ & 40 & 25 & 65 & $\mathrm{~kg} / \mathrm{d}$ \\
\hline $\begin{array}{l}\text { (a) Recor } \\
\text { (b) As-fe }\end{array}$ & $\begin{array}{l}\text { nended single } \\
\text { nass. }\end{array}$ & alue in bold. & & & & & & \\
\hline
\end{tabular}

Table 7.19. STORTM Storage Time - Animal Feed

\begin{tabular}{|c|c|c|c|c|c|c|c|c|}
\hline Gv2_ID & Gv2_Description & Food Type & Reference & Comment & $\begin{array}{l}\text { Estimate } \\
\text { for a } \\
\text { Mixture }\end{array}$ & Min & Max & Units \\
\hline STORTM & $\begin{array}{l}\text { Storage Time for } \\
\text { animal feed }\end{array}$ & $\begin{array}{l}\text { 1.meat } \\
\text { animal } \\
\text { feed }\end{array}$ & $\begin{array}{l}\text { Estimate. Single annual } \\
\text { harvests. }\end{array}$ & corn grain & 140 & 45 & 365 & $\mathrm{~d}$ \\
\hline STORTM & $\begin{array}{l}\text { Storage Time for } \\
\text { animal feed }\end{array}$ & $\begin{array}{l}\text { 1.meat } \\
\text { animal } \\
\text { feed }\end{array}$ & $\begin{array}{l}\text { Estimate. Approximate } \\
\text { with end of spring wheat } \\
\text { harvest (about Sept) and } \\
\text { beginning of winter wheat } \\
\text { harvest (about June). }\end{array}$ & wheat & - & 45 & 280 & d \\
\hline STORTM & $\begin{array}{l}\text { Storage Time for } \\
\text { animal feed }\end{array}$ & $\begin{array}{l}\text { 1.meat } \\
\text { animal } \\
\text { feed }\end{array}$ & $\begin{array}{l}\text { Estimate. One month } \\
\text { minimum to cure. }\end{array}$ & corn silage & - & 30 & 180 & $d$ \\
\hline STORTM & $\begin{array}{l}\text { Storage Time for } \\
\text { animal feed }\end{array}$ & $\begin{array}{l}\text { 1.meat } \\
\text { animal } \\
\text { feed }\end{array}$ & Estimate. & stored hay & - & 30 & 180 & $\mathrm{~d}$ \\
\hline STORTM & $\begin{array}{l}\text { Storage Time for } \\
\text { animal feed }\end{array}$ & $\begin{array}{l}\text { 2.poultry } \\
\text { feed }\end{array}$ & $\begin{array}{l}\text { Estimate. See above, meat } \\
\text { animal feed, corn. }\end{array}$ & corn grain & 180 & 45 & 365 & $\mathrm{~d}$ \\
\hline STORTM & $\begin{array}{l}\text { Storage Time for } \\
\text { animal feed }\end{array}$ & $\begin{array}{l}\text { 3. milk } \\
\text { feed }\end{array}$ & $\begin{array}{l}\text { Estimate. See above, meat } \\
\text { animal feed, corn silage }\end{array}$ & corn silage & 140 & 30 & 180 & $\mathrm{~d}$ \\
\hline STORTM & $\begin{array}{l}\text { Storage Time for } \\
\text { animal feed }\end{array}$ & $\begin{array}{l}\text { 3. milk } \\
\text { feed }\end{array}$ & $\begin{array}{l}\text { Estimate. See above, meat } \\
\text { animal feed, stored hay. }\end{array}$ & stored hay & - & 30 & 180 & $\mathrm{~d}$ \\
\hline STORTM & $\begin{array}{l}\text { Storage Time for } \\
\text { animal feed }\end{array}$ & $\begin{array}{l}\text { 4.egg layer } \\
\text { feed }\end{array}$ & $\begin{array}{l}\text { Estimate. See above, meat } \\
\text { animal feed, corn. }\end{array}$ & corn grain & 180 & 45 & 365 & $\mathrm{~d}$ \\
\hline STORTM & $\begin{array}{l}\text { Storage Time for } \\
\text { animal feed }\end{array}$ & $\begin{array}{l}\text { 5.meat } \\
\text { animal } \\
\text { forage }\end{array}$ & $\begin{array}{l}\text { Estimate. Max value based } \\
\text { on a chronic, once-a-month } \\
\text { air emission. }\end{array}$ & pasture grass & 0 & 0 & 15 & d \\
\hline STORTM & $\begin{array}{l}\text { Storage Time for } \\
\text { animal feed }\end{array}$ & $\begin{array}{l}\text { 6.milk } \\
\text { forage }\end{array}$ & $\begin{array}{l}\text { Estimate. Max value based } \\
\text { on a chronic, once-a-month } \\
\text { air emission. }\end{array}$ & pasture grass & 0 & 0 & 15 & $\mathrm{~d}$ \\
\hline
\end{tabular}




\subsection{Tab: Agriculture/Animal Feed/Dry/Wet Ratio}

The dry/wet ratio for each animal feed type is used to convert between dry and wet weight bases. These data are also helpful when information found in open literature must be converted to the appropriate dry- or wet-bases for model input.

\subsubsection{DRYFA2 Dry-to-Wet Ratio}

The dry/wet ratio for each feed crop is used to convert between dry and wet weight bases. The most appropriate dry-to-wet ratio applied should be that of the edible portion; if edible portion data is not available, use data available (e.g., entire plant data). Oven-dry data, rather than ash weight data, are typically applied. One focus of animal nutrition is on the dry mass of the feed. Eliminating the water content, the dry matter contains all the other necessary nutrients required by the animals. As a result there is a large amount of information on the internet. Local animal producers, especially larger commercial operations, should also be able to provide regional information, as well.

Separate dry/wet ratio parameters for human food crops are covered in Section 7.19. The grain values for animal feed and human food should be comparable, if not the same.

The units and range for this parameter in Gv2 are: fraction (0.05-0.95).

Data has not changed over time for these data. However, consideration of feed mixtures may be required. Generalized values are provided in this section; minimum and maximum values are also generalized. Values for a number of specific feed and forage types are indicated in the Appendix H. Care should be exercised in the application of some values. For example, when considering hay, the user should determine whether the dry-to-wet ratio applies to the as-grown or as-fed dry-wet ratio.

\subsubsection{Additional Sources of Information}

IAEA (2010) Appendix I, Table 82 through 85, provides a list of dry matter content (percentage basis) for a variety of feed and crop types.

Ensminger et al. (1990), Section V, provides tables of dry-to-wet ratios for numerous energy feeds, protein feeds, dry forages, silages and haylages, and pasture and range plants.

Feed operations typically provide feed to livestock based on feed mass. One method of gauging sufficient animal nutrition is to determine the dry matter content of the feed. As a result, dry matter information is available at a variety of internet sites. 


\subsubsection{DRYFA2 Animal Feed Dry-to-wet Ratio Table}

Table 7.20. DRYFA2 Dry-to-Wet Ratio - Animal Feed

\begin{tabular}{|c|c|c|c|c|c|c|c|c|}
\hline Gv2_ID & Gv2_Description & Food Type & Reference & Comment & $\begin{array}{c}\text { Recom- }^{(\mathbf{a})} \\
\text { mendation }^{(2)}\end{array}$ & Min & Max & Units \\
\hline DRYFA2 & $\begin{array}{l}\text { Dry-to-wet ratio for } \\
\text { animal feed }\end{array}$ & $\begin{array}{l}\text { 1.meat animal } \\
\text { feed }\end{array}$ & $\begin{array}{l}\text { Ensminger } \\
\text { et al. (1990) }\end{array}$ & silage & 0.5 & 0.27 & 0.78 & $\mathrm{~kg}_{\text {dry }} / \mathrm{kg}_{\text {wet }}$ \\
\hline DRYFA2 & $\begin{array}{l}\text { Dry-to-wet ratio for } \\
\text { animal feed }\end{array}$ & $\begin{array}{l}\text { 1.meat animal } \\
\text { feed }\end{array}$ & $\begin{array}{l}\text { Ensminger } \\
\text { et al. (1990) }\end{array}$ & $\begin{array}{l}\text { grain plus } \\
\text { silage } \\
\text { mixture }\end{array}$ & 0.5 & - & - & $\mathrm{kg}_{\mathrm{dry}} / \mathrm{kg}_{\mathrm{wet}}$ \\
\hline DRYFA2 & $\begin{array}{l}\text { Dry-to-wet ratio for } \\
\text { animal feed }\end{array}$ & 2.poultry feed & $\begin{array}{l}\text { Ensminger } \\
\text { et al. (1990) }\end{array}$ & corn & 0.9 & 0.85 & 0.95 & $\mathrm{~kg}_{\text {dry }} / \mathrm{kg}_{\text {wet }}$ \\
\hline DRYFA2 & $\begin{array}{l}\text { Dry-to-wet ratio for } \\
\text { animal feed }\end{array}$ & 3. milk feed & $\begin{array}{l}\text { Ensminger } \\
\text { et al. (1990) }\end{array}$ & $\begin{array}{l}\text { grain plus } \\
\text { silage } \\
\text { mixture }\end{array}$ & 0.5 & - & - & $\mathrm{kg}_{\text {dry }} / \mathrm{kg}_{\text {wet }}$ \\
\hline DRYFA2 & $\begin{array}{l}\text { Dry-to-wet ratio for } \\
\text { animal feed }\end{array}$ & $\begin{array}{l}\text { 4.egg layer } \\
\text { feed }\end{array}$ & $\begin{array}{l}\text { Ensminger } \\
\text { et al. (1990) }\end{array}$ & corn & 0.9 & 0.85 & 0.95 & $\mathrm{~kg}_{\text {dry }} / \mathrm{kg}_{\text {wet }}$ \\
\hline DRYFA2 & $\begin{array}{l}\text { Dry-to-wet ratio for } \\
\text { animal feed }\end{array}$ & $\begin{array}{l}\text { 5.meat animal } \\
\text { forage }\end{array}$ & $\begin{array}{l}\text { Ensminger } \\
\text { et al. (1990) }\end{array}$ & grain & 0.9 & - & - & $\mathrm{kg}_{\mathrm{dry}} / \mathrm{kg}_{\mathrm{wet}}$ \\
\hline DRYFA2 & $\begin{array}{l}\text { Dry-to-wet ratio for } \\
\text { animal feed }\end{array}$ & $\begin{array}{l}\text { 5.meat animal } \\
\text { forage }\end{array}$ & $\begin{array}{l}\text { Ensminger } \\
\text { et al. (1990) }\end{array}$ & $\begin{array}{l}\text { sun-cured } \\
\text { hay }\end{array}$ & 0.85 & 0.82 & 0.93 & $\begin{array}{l}\mathrm{kg}_{\text {dry }} / \mathrm{kg}_{\text {wet }} \\
\text { (as fed) }\end{array}$ \\
\hline DRYFA2 & $\begin{array}{l}\text { Dry-to-wet ratio for } \\
\text { animal feed }\end{array}$ & $\begin{array}{l}\text { 5.meat animal } \\
\text { forage }\end{array}$ & $\begin{array}{l}\text { Ensminger } \\
\text { et al. (1990) }\end{array}$ & $\begin{array}{l}\text { hay+grain } \\
\text { mixture }\end{array}$ & 0.88 & - & - & $\begin{array}{c}\mathrm{kg}_{\text {dry }} / \mathrm{kg}_{\text {wet }} \\
\text { (as fed) }\end{array}$ \\
\hline DRYFA2 & $\begin{array}{l}\text { Dry-to-wet ratio for } \\
\text { animal feed }\end{array}$ & $\begin{array}{l}\text { 5.meat animal } \\
\text { forage }\end{array}$ & $\begin{array}{l}\text { Ensminger } \\
\text { et al. (1990) }\end{array}$ & $\begin{array}{l}\text { sun-cured } \\
\text { hay }\end{array}$ & 0.15 & 0.2 & 0.5 & $\begin{array}{l}\mathrm{kg}_{\text {dry }} / \mathrm{kg}_{\text {wet }} \\
\text { (as grown) }\end{array}$ \\
\hline DRYFA2 & $\begin{array}{l}\text { Dry-to-wet ratio for } \\
\text { animal feed }\end{array}$ & 6.milk forage & $\begin{array}{l}\text { Ensminger } \\
\text { et al. (1990) }\end{array}$ & $\begin{array}{l}\text { fresh pasture } \\
\text { grass }\end{array}$ & 0.4 & 0.2 & 0.5 & $\mathrm{~kg}_{\text {dry }} / \mathrm{kg}_{\text {wet }}$ \\
\hline
\end{tabular}

\subsection{Tab: Agriculture/Animal Feed/Soil Intake}

\subsubsection{SLCONA Animal Soil Intake}

The soil intake for each animal type is the mass of soil ingested daily by the animal. For incidental soil ingestion of humans, see Receptor module, Section 8.4.1.

The units and range for this parameter in Gv2 are: $\mathrm{kg} / \mathrm{d}(0-5)$.

Zach and Mayoh (1984) indicate that the soil intake rate, as a percent of dry-matter intake is greater for beef cattle (4-8 percent) than dairy cows (0.6-3.8 percent). Given that dry-matter intake rates are about 2-3 percent of the bovine mass (Ensminger et al. 1990), soil intake estimated for bovine categories: meat cattle, outdoor dairy cows, and housed dairy cows. The lower range values may be assigned for wet highly vegetated locations and higher values for dry or muddier locations. Free-range chickens were assigned a soil intake rate of 10 percent of their food intake rate (Schoeters and Hoogenboom 2006). Chickens cooped in commercial operations would not be assigned soil intakes. 


\subsubsection{Additional Sources of Information}

Zach and Mayoh (1984) provide information regarding animal incidental food ingestion. Animals included are domestic dairy and beef cattle (lactating and non-lactating), sheep, and mule deer. They also note how grazing land quality can impact soil ingestion. Ingestion rates are provided either by mass per day or by percent of food ingestion.

\subsubsection{SLCONA Animal Soil Intake Tables}

Table 7.21. SLCONA Soil Intake - Animal Feed

\begin{tabular}{|c|c|c|c|c|c|c|c|}
\hline Gv2_ID & $\begin{array}{c}\text { Gv2 } \\
\text { Description }\end{array}$ & $\begin{array}{l}\text { Food Type } \\
\text { (ANFlabel) }\end{array}$ & Reference & Comment & $\begin{array}{l}\text { Recom- } \\
\text { mendation }^{(a)}\end{array}$ & $\begin{array}{l}\text { Min/ } \\
\text { Max }\end{array}$ & Units \\
\hline SLCONA & $\begin{array}{l}\text { Soil intake } \\
\text { by animal }\end{array}$ & $\begin{array}{l}\text { 1.meat } \\
\text { animal }\end{array}$ & $\begin{array}{l}\text { Ensminger et al. } \\
\text { (1990); Zach and } \\
\text { Mayoh (1984) }\end{array}$ & $\begin{array}{l}\text { Cattle. Derived from animal } \\
\text { mass }(800-1200 \mathrm{lb}) \text {, dry } \\
\text { matter intake }(2-3 \% \text { of } \\
\text { animal mass }) \text {, soil intake } \\
\text { ( } 4-8 \% \text { of dry matter intake). }\end{array}$ & 0.5 & $0.3 / 0.9$ & $\mathrm{~kg} / \mathrm{d}$ \\
\hline SLCONA & $\begin{array}{l}\text { Soil intake } \\
\text { by animal }\end{array}$ & $\begin{array}{l}\text { 2.poultry } \\
\text { animal }\end{array}$ & $\begin{array}{l}\text { Schoeters and } \\
\text { Hoogenboom } \\
(2006)\end{array}$ & $\begin{array}{l}\text { Free range chicken. } 10 \% \text { of } \\
\text { dry matter intake. }\end{array}$ & 0.01 & $\begin{array}{l}0.007 / \\
0.015\end{array}$ & $\mathrm{~kg} / \mathrm{d}$ \\
\hline SLCONA & $\begin{array}{l}\text { Soil intake } \\
\text { by animal }\end{array}$ & $\begin{array}{l}\text { 3.milk } \\
\text { animal }\end{array}$ & $\begin{array}{l}\text { Ensminger et al. } \\
\text { (1990); Zach and } \\
\text { Mayoh (1984) }\end{array}$ & $\begin{array}{l}\text { Outdoor bovine. Derived } \\
\text { from animal mass } \\
(900-1400 \mathrm{lb}) \text {, dry matter } \\
\text { intake }(2-3 \% \text { of animal } \\
\text { mass), soil intake }(1.5-3.8 \% \\
\text { of dry matter intake). }\end{array}$ & 0.2 & $0.1 / 0.5$ & $\mathrm{~kg} / \mathrm{d}$ \\
\hline SLCONA & $\begin{array}{l}\text { Soil intake } \\
\text { by animal }\end{array}$ & $\begin{array}{l}\text { 3.milk } \\
\text { animal }\end{array}$ & $\begin{array}{l}\text { Ensminger et al. } \\
\text { (1990); Zach and } \\
\text { Mayoh (1984) }\end{array}$ & $\begin{array}{l}\text { Housed bovine. Derived } \\
\text { from animal mass } \\
(900-1400 \mathrm{lb}) \text {, dry matter } \\
\text { intake }(2-3 \% \text { of animal } \\
\text { mass }) \text {, soil intake }(0.6 \% \text { of } \\
\text { dry matter intake). }\end{array}$ & 0.08 & $\begin{array}{c}0.06 / \\
0.13\end{array}$ & $\mathrm{~kg} / \mathrm{d}$ \\
\hline SLCONA & $\begin{array}{l}\text { Soil intake } \\
\text { by animal }\end{array}$ & $\begin{array}{l}\text { 4.egg } \\
\text { animal }\end{array}$ & $\begin{array}{l}\text { Schoeters and } \\
\text { Hoogenboom } \\
(2006)\end{array}$ & $\begin{array}{l}\text { Free range chicken. } 10 \% \text { of } \\
\text { dry matter intake. }\end{array}$ & 0.01 & $\begin{array}{c}0.007 / \\
0.15\end{array}$ & $\mathrm{~kg} / \mathrm{d}$ \\
\hline
\end{tabular}

(a) Recommended discrete value in bold.

\subsection{Tab: Agriculture/Food Crop}

The human Food Crop tab parameters pertaining to field characteristics (biomass, growing period, yield, and translocation factor) are grouped in one section. The dry-to-wet ratio for food crops is covered in the next section. 


\subsection{Tabs: Agriculture/Food Crop/Biomass; /Growing Period; /Yield; and /Translocation Factor}

This section covers human food crop categories: leafy vegetables, root vegetables, ${ }^{1}$ fruit, and grain. The biomass, growing period, yield, and translocation factor parameters for animal feeds are covered in Section 7.13. The grain values should be comparable, if not the same, for both human food and animal feed. Grains are an important crop to consider as the per capita daily amount ingested (mass basis) is greatest for this category.

The most difficult food category to parameterize is the root vegetables because this category is essentially a catch-all for non-leafy and non-tree crops. As such it includes such wide-ranging crops as tomatoes, melons, strawberries, potatoes, carrots, and berries. The user should reasonably approximate the predominant human food crops grown in their region of interest, consider the volume of each food crop consumed, and then determine which crops are to be represented by the root vegetable category for their region.

Table 7.22 indicates a guideline for which crops fall into the four fixed Gv2 food categories. Some crops could be in two different categories, but the following guidance is suggested. A more detailed list of crops and Gv2 crop assignments is provided in Appendix D. Leafy vegetables are generally characterized as having a larger surface area to volume ratio. Difficult crops to model generically in Gv2 are tree nuts, fruit consumed as juice or wine, hops, and vegetable oils. If these foods are important to a scenario, they are best considered with a special Gv2 case supplemented with hand calculations.

Table 7.22. Suggested Categories for Food Crop Parameter Assignment

\begin{tabular}{cccc}
\hline Leafy Vegetables & Root/Other Vegetables & Fruit & Grain \\
\hline Head lettuce & Potatoes & Apples & Wheat \\
Romaine lettuce & Tomatoes & Citrus fruits & Corn \\
Leafy lettuce & Snap beans & Bananas & Lentils \\
Spinach & Green beans & Pears & \\
Cabbage & Carrots & Peaches & \\
Endive & Onions & Apricots & \\
Broccoli $^{(\text {a) }}$ & Cauliflower & Plums & \\
Brussels Sprouts $_{\text {Asparagus }}^{\text {(a) }}$ & Celery & Cherries & \\
& Cucumber & Grapes & \\
& Zucchini & & \\
& Melons & & \\
& Onions & & \\
& Peas & \\
& Peppers & \\
& Sweet potatoes & \\
& Sweet corn & & \\
& Pumpkin & & \\
& Beans & \\
& Strawberries &
\end{tabular}

\footnotetext{
${ }^{1}$ The term "root vegetables" is also indicated as "other vegetables" in Gv2. The terms are used interchangeably.
} 
Table 7.22. (contd)

\begin{tabular}{cccc}
\hline Leafy Vegetables & Root/Other Vegetables & Fruit & Grain \\
\hline Raspberries & & \\
& Blueberries & & \\
Mulberries & & \\
& Cranberries & & \\
& Peanuts & & \\
\hline
\end{tabular}

(a) Also could be considered root/other vegetable

(b) Also could be considered grain.

(c) Also could be considered fruit.

\subsubsection{BIOMAS Biomass, GRWP Growing Period, YELD Yield, and TRANS Translocation Factor}

The standing biomass for each human food crop type is the total above-ground plant mass (wet weight) used to estimate interception fractions for wet and dry deposition. Non-tree crop biomass values in the literature generally range from $0.1-3.0 \mathrm{~kg} / \mathrm{m} 2$. Gv2 biomass values may be greater or less than yield values. For example, fruit biomass values are generally greater than fruit yield values, but biomass values for other food types may be approximately the same or less than yield values.

The growing period for each human food crop type defines the deposition period for irrigation and atmospheric deposition to plants. Water availability, crop nutrient availability, soil conditions, weather conditions, and other factors impact this value. For a given location, the user should review available data sources relevant to their region. To the extent possible, the growing period should reflect the growing period of the edible portion of the crop. Values for each crop type may be determined from a number of available data sources (e.g., frost-free days, days to maturity, planting-to-harvest dates).

The yield of each human food crop type gives the total annual production of edible feed crop mass (wet weight) per unit area of farmland. The yield is used to calculate the harvest removal losses from the soil. Radionuclide removal due to harvesting is modeled when opted on the Agriculture/General tab. This parameter only impacts scenarios that evaluate exposures which occur more than one-year after the release. Parameter assignment should consider whether the vegetative, non-edible portion of the crop is harvested with the crop. Parameter estimation should also consider the relative consumption rates of the various crops within the four fixed food types (leafy vegetables, other vegetables, fruit, and grain).

The translocation factor for each human food crop type is the fraction of activity depositing on the plant surface that is translocated to the edible part of the plant. This value differs from the bioconcentration factor (CLBVx) assigned in the Constituent module (see Section 2.1.17), which considers soil to plant transfer.

The root vegetables BIOMAS, GRWP, and YELD values are the most challenging food category parameters to assign. The root vegetable category encompasses a number of crops. It is recommended that the user determine the predominantly consumed (mass basis) non-leafy, non-fruit crops grown in their region, then determine growing periods, biomass, and yields for those particular crops to determine the best user-specific value to apply. 
The units and range for BIOMAS in Gv2 are: $\mathrm{kg}_{\text {wet }} / \mathrm{m}^{2}(0.1-10)$.

The units and range for GRWP in Gv2 are: $d(0-365)$.

The units and range for YELD in Gv2 are: $\mathrm{kg}_{\mathrm{wet}} / \mathrm{m}^{2}(0.001-10)$.

The units and range for TRANS in Gv2 are: fraction (0-1).

The biomass was determined from USDA Census of Agriculture data in a manner similar to the animal feed (BIOMA2) data in Section 7.13.1. Specific crop and tree data (i.e., no crops grown; but native vegetation is modeled) are provided in Appendix G. Summary BIOMAS values are indicated in Table 7.27.

The Growing period reported in this document is based on planting and harvesting dates. USDA (2010) provided data on the planting and harvesting dates for a number of crops and by state. The data reflect 2008 or 2009 data. Grains are consumed by most people. Wheat is the predominant grain consumed per capita, and the types more commonly consumed are Spring and Winter wheat rather than durum wheat. Winter wheat has a long growing season, but is dormant for much of that time. GRWP data are summarized in Table 7.24; state-specific details are provided in the Appendix F. In reviewing the Appendix information, be cognizant of whether the crop is harvested multiple times over the growing season (e.g., melons, tomatoes, and berries) or harvested at the end of its growth cycle (e.g., wheat, potatoes, corn). For those harvested multiple times, a best estimate growing period closer to the minimum Appendix value would be more appropriate, whereas end-of-season crops would be approximated by average values.

The YELD values were determined from USDA Census of Agriculture data. Values for corn, wheat, and rice are presented with animal feed data in Table 7.15. Typically, total crop yields were divided by harvested acreage values. User-calculated values need to consider whether the vegetative, non-consumed, portion of the crop is harvested/removed annually; then consider whether the mass of the non-consumed plant mass is significant compared to the uncertainty in the crop yield value. If the non-consumed mass is harvested, approximate and document the additional fraction of the edible yield mass of the nonconsumed mass, so that nuclide harvest removal can be better accounted. Summary YELD values are indicated in Table 7.27; crop-specific details are provided in the Appendix G. State-specific online data from the USDA should be pursued for scenarios. YELD and BIOMAS values should be coherent. The YELD and BIOMAS values are likely biased high since the values represent commercial agriculture and would not be harvested if harvest costs were not recoverable in the market.

Translocation values (TRANS) are generally assigned as 1 for leafy plants; a 0.9 "higher" value where 1.0 is not considered appropriate; or a 0.1 "lower" value where a small fraction is appropriate to assign from translocation. The "lower" value would be applied to root crops (e.g., potatoes), crops where the edible portion is covered by a surface that is removed prior to consumption (e.g., orange, watermelon), or the edible portion is sizable in comparison to the surface area available for deposition (e.g., medium to large tomatoes).

A determination was made for the BIOMAS and YELD values for the root/other vegetables category. Assumptions are indicated in the footnotes of Table 7.27. Strictly root and tuber vegetable values are somewhat distinct from non-underground crops in this category. Therefore, generic values for 
underground and above-ground crops for the root/other vegetable category are provided and the user can determine which crops are more representative for their scenario, or alternatively to average the values.

\subsubsection{Additional Sources of Information}

The USDA Economics, Statistics, and Market Information System (e.g., http://usda.mannlib.cornell.edu/MannUsda/viewTaxonomy.do?taxonomyID=3) and the USDA Economic Research Service (e.g., http://www.ers.usda.gov/data-products/fruit-and-tree-nut-data.aspx) have summary information available on a variety of crops, including fruit, vegetables, nuts, berries, and field crops. In particular, biomass and yield information can be obtained from these resources. Some information is broken out by state, others are U.S. averages. The USDA Economics, Statistics, and Market Information System also provides summary information on U.S. per capita use of various crops, which can be used to estimate the mix of broad vegetative categories, such as "other vegetables."

USDA Agricultural Handbook 628 (USDA 2010). Provides planting and harvesting dates for a number of grain, feed, forage, and other crops. Agriculture Handbooks can be accessed at http://www.nal.usda.gov/ref/USDApubs/aghandbk.htm.

USDA Agricultural Handbook 507 (USDA 2007). Provides planting and harvesting dates for Vegetable crops. Data cover years 2004-2006. It can be accessed from the National Agricultural Library Digital Collections, last accessed at http://usda01.library.cornell.edu/usda/current/UsuPlant/UsuPlant-0531-2007.pdf.

Growing Degree Days (GDD) can provide growing season information. GDD is a measure of the amount of time a crop is exposed to temperatures amenable to plant growth. More data is available for certain crop and plant species and for specific locations than others. Growing degree days calculators are available at several websites (e.g., http://www.weather.com/outdoors/agriculture/forecast/99352:4).

Another growing period source is information on days to maturity for various crops which can be found from various sources on the internet. Some sources provide regional information. No one source is recommended in particular. 


\subsubsection{BIOMAS, GRWP, YELD, TRANS Biomass, Growing Period, Yield, and Translocation Tables}

Table 7.23. BIOMAS Standing Biomass - Human Food Crop Categories

\begin{tabular}{|c|c|c|c|c|c|c|c|}
\hline Gv2_ID & Description & Food Type & Comment & Reported & $\begin{array}{l}\text { Recom- } \\
\text { mended }^{(a)}\end{array}$ & $\begin{array}{l}\operatorname{Min} / \\
\operatorname{Max}\end{array}$ & Units \\
\hline BIOMAS & $\begin{array}{l}\text { Biomass- } \\
\text { food crop }\end{array}$ & $\begin{array}{l}\text { 1. Leafy } \\
\text { vegetables }\end{array}$ & $\begin{array}{l}\text { Assume biomass equals } \\
\text { yield }\end{array}$ & $\begin{array}{l}\text { Summarized data } \\
\text { from Appendix }\end{array}$ & 2.6 & $1.3 / 4.2$ & kg_wet $/ \mathrm{m}^{2}$ \\
\hline BIOMAS & $\begin{array}{l}\text { Biomass- } \\
\text { food crop }\end{array}$ & $\begin{array}{l}\text { 2. Other/Root } \\
\text { vegetables }\end{array}$ & $\begin{array}{l}\text { Above-ground crops. } \\
\text { BIOMAS assumed to be } \\
0.5 \text { YELD for melon, } \\
\text { tomatoes, peppers; equal } \\
\text { to YELD for beans. }\end{array}$ & $\begin{array}{l}\text { Assumed mix: } 30 \% \\
\text { melon, } 25 \% \\
\text { tomatoes, } 25 \% \\
\text { beans, } 20 \% \text { peppers }\end{array}$ & 1.3 & $1.2 / 1.5$ & kg_wet $/ \mathrm{m}^{2}$ \\
\hline BIOMAS & $\begin{array}{l}\text { Biomass- } \\
\text { food crop }\end{array}$ & $\begin{array}{l}\text { 2. Other/Root } \\
\text { vegetables }\end{array}$ & $\begin{array}{l}\text { Below-ground crops. } \\
\text { BIOMAS assumed to be } \\
0.5 \text { YELD. }\end{array}$ & $\begin{array}{l}\text { Assumed mix: } 55 \% \\
\text { potatoes, } 30 \% \\
\text { onions, } 15 \% \text { carrots }\end{array}$ & 2.2 & $1.9 / 2.4$ & kg_wet $/ \mathrm{m}^{2}$ \\
\hline BIOMAS & $\begin{array}{l}\text { Biomass- } \\
\text { food crop }\end{array}$ & 3. Fruit & $\begin{array}{l}\text { Tree fruit. Assumed to } \\
\text { be } 0.25 \text { YELD with } \\
\text { foliage added (average } \\
32 \mathrm{ft} \text { tree footprint) }{ }^{(\mathrm{b})} \text {. }\end{array}$ & $\begin{array}{l}\text { Assumed mix: } 80 \% \\
\text { apples, } 20 \% \text { peaches }\end{array}$ & 3.7 & $2.6 / 4.5$ & kg_wet $/ \mathrm{m}^{2}$ \\
\hline BIOMAS & $\begin{array}{l}\text { Biomass- } \\
\text { food crop }\end{array}$ & 4. Grain & $\begin{array}{l}\text { Assumed silage yield for } \\
\text { corn BIOMAS. }\end{array}$ & $\begin{array}{l}\text { Assumed mix: wheat } \\
80 \% \text {, silage } 20 \% \text {. }\end{array}$ & 1.1 & $0.6 / 1.7$ & kg_wet $/ \mathrm{m}^{2}$ \\
\hline
\end{tabular}

(a) Bold values are recommended for discrete input

(b) Jenkins et al. (2004).

Table 7.24. GRWP Growing Period - Human Food Crop Categories

\begin{tabular}{|c|c|c|c|c|c|c|c|}
\hline Gv2 ID & $\begin{array}{c}\text { Gv2 } \\
\text { Description }\end{array}$ & Food Type & Reference & Comment & $\begin{array}{l}\text { Extremes } \\
\text { (d) }\end{array}$ & $\begin{array}{l}\text { Active Period } \\
\text { or Average (d) }\end{array}$ & Units \\
\hline GRWP & $\begin{array}{l}\text { Growing } \\
\text { period }\end{array}$ & 1.leafy & $\begin{array}{l}\text { Calculated from } \\
\text { USDA (2010) }\end{array}$ & - & $56-137$ & $75-103$ & $\mathrm{~d}$ \\
\hline GRWP & $\begin{array}{l}\text { Growing } \\
\text { period }\end{array}$ & 2. root & $\begin{array}{l}\text { Calculated from } \\
\text { USDA (2010) }\end{array}$ & $\begin{array}{l}\text { full range of crops } \\
\text { included in category }\end{array}$ & $36-365$ & $110-152$ & $\mathrm{~d}$ \\
\hline GRWP & $\begin{array}{l}\text { Growing } \\
\text { period }\end{array}$ & 2. root & $\begin{array}{l}\text { Calculated from } \\
\text { USDA (2010) }\end{array}$ & potatoes & $98-139$ & 127 & $\mathrm{~d}$ \\
\hline GRWP & $\begin{array}{l}\text { Growing } \\
\text { period }\end{array}$ & 2. root & $\begin{array}{l}\text { Calculated from } \\
\text { USDA (2010) }\end{array}$ & tomatoes & $50-152$ & 97 & $\mathrm{~d}$ \\
\hline GRWP & $\begin{array}{l}\text { Growing } \\
\text { period }\end{array}$ & 2. root & $\begin{array}{l}\text { Calculated from } \\
\text { USDA (2010) }\end{array}$ & $\begin{array}{l}\text { generic } \\
\text { potato/tomato }\end{array}$ & $75-145$ & 112 & d \\
\hline GRWP & $\begin{array}{l}\text { Growing } \\
\text { period }\end{array}$ & 3.fruit & internet review & $\begin{array}{l}\text { fruit set to harvest } \\
\text { estimate; primarily } \\
\text { apples }\end{array}$ & & $55-90$ & $d$ \\
\hline GRWP & $\begin{array}{l}\text { Growing } \\
\text { period }\end{array}$ & 4.grain & $\begin{array}{l}\text { Calculated from } \\
\text { USDA (2010) }\end{array}$ & Spring wheat & $100-141$ & $105-131$ & $d$ \\
\hline GRWP & $\begin{array}{l}\text { Growing } \\
\text { period }\end{array}$ & 4.grain & $\begin{array}{l}\text { Calculated from } \\
\text { USDA (2010) }\end{array}$ & Winter wheat & $151-350$ & $174-337$ & d \\
\hline GRWP & $\begin{array}{l}\text { Growing } \\
\text { period }\end{array}$ & 4.grain & $\begin{array}{l}\text { Calculated from } \\
\text { USDA (2010) }\end{array}$ & $\begin{array}{l}\text { generic wheat, } \\
\text { average of spring } \\
\text { and winter wheat }\end{array}$ & $125-245$ & $140-234$ & $\mathrm{~d}$ \\
\hline
\end{tabular}


Table 7.25. YELD Crop Yield - Human Food Crop Categories

\begin{tabular}{|c|c|c|c|c|c|c|c|}
\hline $\begin{array}{r}\text { Gv2 } \\
\text { ID }\end{array}$ & Description & Food Type & Comment & Reported & $\begin{array}{l}\text { Recom- } \\
\text { mended }^{(a)}\end{array}$ & Min/Max & Units \\
\hline YELD & $\begin{array}{l}\text { Yield- food } \\
\text { crop }\end{array}$ & $\begin{array}{l}\text { 1. Leafy } \\
\text { vegetables }\end{array}$ & - & $\begin{array}{l}\text { "All lettuce", see } \\
\text { Appendix data. }\end{array}$ & 2.6 & $1.3 / 4.2$ & kg_wet $/ \mathrm{m}^{2}$ \\
\hline YELD & $\begin{array}{l}\text { Yield- food } \\
\text { crop }\end{array}$ & $\begin{array}{l}\text { 2. Other/ } \\
\text { Root } \\
\text { vegetables }\end{array}$ & $\begin{array}{l}\text { Above- } \\
\text { ground } \\
\text { crops }\end{array}$ & $\begin{array}{l}\text { Assumed mix: } 30 \% \\
\text { melon, } 25 \% \\
\text { tomatoes, } 25 \% \text { beans, } \\
20 \% \text { peppers }\end{array}$ & 2.5 & $2.1 / 2.7$ & kg_wet $/ \mathrm{m}^{2}$ \\
\hline YELD & $\begin{array}{l}\text { Yield- food } \\
\text { crop }\end{array}$ & $\begin{array}{l}\text { 2. Other/ } \\
\text { Root } \\
\text { vegetables }\end{array}$ & $\begin{array}{l}\text { Below- } \\
\text { ground } \\
\text { crops }\end{array}$ & $\begin{array}{l}\text { Assumed mix: } 55 \% \\
\text { potatoes, } 30 \% \\
\text { onions, } 15 \% \text { carrots }\end{array}$ & 4.3 & $3.9 / 4.7$ & kg_wet $/ \mathrm{m}^{2}$ \\
\hline YELD & $\begin{array}{l}\text { Yield- food } \\
\text { crop }\end{array}$ & 3. Fruit & Tree fruit & $\begin{array}{l}\text { Assumed mix: } 80 \% \\
\text { apples, } 20 \% \text { peaches. } \\
\text { Foliage assumed to } \\
\text { remain in orchard. }\end{array}$ & 2.6 & $2.2 / 2.9$ & kg_wet $/ \mathrm{m}^{2}$ \\
\hline YELD & $\begin{array}{l}\text { Yield- food } \\
\text { crop }\end{array}$ & 4. Grain & - & $\begin{array}{l}\text { Assumed mix: wheat } \\
80 \% \text {, sweet corn } \\
20 \% \text {. }\end{array}$ & 0.92 & $0.72 / 1.1$ & kg_wet $/ \mathrm{m}^{2}$ \\
\hline
\end{tabular}

(a) Bold values are recommended for discrete input.

Table 7.26. TRANS Translocation (Surface to Edible Portion) - Human Food Crops

\begin{tabular}{|c|c|c|c|c|c|c|c|}
\hline Gv2 ID & Gv2_Description & $\begin{array}{l}\text { Food } \\
\text { Type }\end{array}$ & Reference & Comment & Min & Max & Units \\
\hline TRANS & $\begin{array}{l}\text { Translocation } \\
\text { fraction for human } \\
\text { food crop }\end{array}$ & 1.leafy & $\begin{array}{l}\text { Assumption based } \\
\text { on crop } \\
\text { morphology }\end{array}$ & $\begin{array}{l}\text { Surface area to volume } \\
\text { ratio is "high" }\end{array}$ & 1 & 1 & fraction \\
\hline TRANS & $\begin{array}{l}\text { Translocation } \\
\text { fraction for human } \\
\text { food crop }\end{array}$ & 2. root & $\begin{array}{l}\text { Assumption based } \\
\text { on crop } \\
\text { morphology }\end{array}$ & $\begin{array}{l}\text { Wide variation in of non- } \\
\text { leafy and non-fruit crops. } \\
\text { Edible portion below- } \\
\text { ground or protected; or } \\
\text { surface area to volume } \\
\text { ratio of edible portion is } \\
\text { "low" }\end{array}$ & 0.1 & 0.25 & fraction \\
\hline TRANS & $\begin{array}{l}\text { Translocation } \\
\text { fraction for human } \\
\text { food crop }\end{array}$ & 3. fruit & $\begin{array}{l}\text { Assumption based } \\
\text { on crop } \\
\text { morphology }\end{array}$ & $\begin{array}{l}\text { Edible portion protected; } \\
\text { surface area to volume } \\
\text { ratio of edible portion is } \\
\text { "low"; or leafy tree } \\
\text { growing the edible crop }\end{array}$ & 0.1 & 0.1 & fraction \\
\hline TRANS & $\begin{array}{l}\text { Translocation } \\
\text { fraction for human } \\
\text { food crop }\end{array}$ & 4.grain & $\begin{array}{l}\text { Assumption based } \\
\text { on crop } \\
\text { morphology }\end{array}$ & grain & 0.1 & 0.1 & fraction \\
\hline
\end{tabular}


Table 7.27. Summary Table of BIOMAS, GRWP, YELD, TRANS

\begin{tabular}{lcccc}
\hline & $\begin{array}{c}\text { Biomass } \\
\left(\mathrm{kg} / \mathrm{m}^{2}\right)\end{array}$ & $\begin{array}{c}\text { Growing } \\
\text { Period (day) }\end{array}$ & Yield (kg/m $)^{2}$ & $\begin{array}{c}\text { Translocation } \\
\text { (fraction) }\end{array}$ \\
\cline { 2 - 5 } Human Food Crop & BIOMAS & GRWP & YELD & TRANS \\
\hline 1.leafy & $2.6^{(\mathrm{a}, \mathrm{b})}$ & 56 & $2.6^{(\mathrm{a})}$ & 1.0 \\
2.other (non-root) & $1.3^{(\mathrm{b})}$ & 75 & $2.5^{(\mathrm{c})}$ & 0.8 \\
2. other (root only) & $2.2^{(\mathrm{b})}$ & 110 & $4.3^{(\mathrm{d})}$ & 0.1 \\
3.fruit & $3.7^{(\mathrm{e})}$ & 60 & $2.6^{(\mathrm{f})}$ & 0.1 \\
4.grain & $1.1^{(\mathrm{b}, \mathrm{g})}$ & 120 & $0.92^{(\mathrm{g})}$ & 0.1 \\
\hline
\end{tabular}

(a) “All lettuce" (lettuce, cabbage, broccoli, spinach).

(b) Assumed to be half the YELD value for melon, tomato, peppers, potatoes, onions, and carrots; and same as YELD for leafy, beans, wheat, and silage.

(c) Mass fractions assumed: 30\% melon; 25\% tomato; $25 \%$ snap beans; $20 \%$ peppers.

(d) Mass fractions assumed: $55 \%$ potatoes, $30 \%$ onions, $15 \%$ carrots.

(e) Tree foliage of $32 \mathrm{ft}$ footprint tree plus $25 \%$ of YELD value.

(f) Mass fractions assumed: $80 \%$ apples; $20 \%$ peaches.

(g) Mass fractions assumed: $80 \%$ wheat and 20\% silage for BIOMAS; $80 \%$ wheat and 20\% sweet corn for YELD.

\subsection{Tab: Agriculture/Food Crop/Dry-to-Wet Ratio}

This section covers human food crop categories: leafy vegetables, root vegetables, fruit, and grain. Section 7.15 covers the dry-to-wet ratios for animal feed types. The grain values should be comparable, if not the same, for both animal feed and human food.

\subsubsection{DRYFAC Dry-to-Wet Ratio}

The dry-to-wet ratio for each food crop is used to convert between dry and wet weight bases. These data are also helpful when information found in open literature must be converted to the appropriate dryor wet-bases for model input. Aquatic plant concentrations are modeled solely from bioconcentration from water with no sediment-to-plant uptake, as with terrestrial crop models. Therefore, no dry-to-wet ratios for aquatic foods are provided.

The units and range for this parameter in Gv2 are: fraction (i.e., $\left.\mathrm{kg}_{\text {dry }} / \mathrm{kg}_{\text {wet }}\right)(0.05-0.95)$.

Values were derived from two large data sets, USDA (2011) and Gebhardt and Thomas (2002). Extracts of some data are provided in Appendix H.

\subsubsection{Additional Sources of Information}

The USDA Agricultural Research Service publishes the National Nutrient Database for Standard Reference information providing nutrition information for numerous foods. The current Standard Reference is Release 24 (September 2011). Reports by single nutrient, one of which is water, are located at the link provided. Examples of relevant values derived at this link are 0.05 for head lettuce to 0.98 for dry nuts. Data was accessed Jan 2012 at http://www.ars.usda.gov/Services/docs.htm?docid=22114 Another more comprehensive link for the data is http://ndb.nal.usda.gov/; select "Start your search here", 
and then choose a food group or scroll through the list. Once the food is selected, the variability in the data can be acquired by selecting "Statistics Report" near the top of the screen.

IAEA (2010) Appendix I, Table 82 through 85, provides a list of dry matter content (percentage basis) for a variety of feed and crop types.

\subsubsection{DRYFAC Food Crop/Dry-to-Wet Ratio Tables}

Table 7.28. DRYFAC Dry-to-Wet Ratio - Human Food Categories

\begin{tabular}{|c|c|c|c|c|c|c|c|c|}
\hline Gv2_ID & Description & Food Type & Reference & Comment & $\begin{array}{l}\text { Recom- } \\
\text { mendation }^{(a)}\end{array}$ & $\begin{array}{c}\text { Min } \\
\text { Value }\end{array}$ & $\begin{array}{c}\text { Max } \\
\text { Value }\end{array}$ & Units \\
\hline DRYFAC & $\begin{array}{l}\text { Dry/Wet } \\
\text { ratio }\end{array}$ & $\begin{array}{l}\text { 1. Leafy } \\
\text { vegetable }\end{array}$ & $\begin{array}{l}\text { USDA (2011); } \\
\text { Gebhardt and } \\
\text { Thomas (2002) }\end{array}$ & $\begin{array}{l}\text { lettuces, edible } \\
\text { greens, spinach, } \\
\text { cabbage, broccoli }\end{array}$ & 0.08 & 0.05 & 0.12 & dry/wet \\
\hline DRYFAC & $\begin{array}{l}\text { Dry/Wet } \\
\text { ratio }\end{array}$ & $\begin{array}{l}\text { 2. Other } \\
\text { vegetables }\end{array}$ & $\begin{array}{l}\text { USDA (2011); } \\
\text { Gebhardt and } \\
\text { Thomas (2002) }\end{array}$ & Root vegetables & 0.20 & 0.09 & 0.33 & dry/wet \\
\hline DRYFAC & $\begin{array}{l}\text { Dry/Wet } \\
\text { ratio }\end{array}$ & $\begin{array}{l}\text { 2. Other } \\
\text { vegetables }\end{array}$ & $\begin{array}{l}\text { USDA (2011); } \\
\text { Gebhardt and } \\
\text { Thomas (2002) }\end{array}$ & $\begin{array}{l}\text { Non-root } \\
\text { vegetables, ground- } \\
\text { growing fruits, } \\
\text { sweet corn }\end{array}$ & 0.25 & 0.06 & 0.31 & dry/wet \\
\hline DRYFAC & $\begin{array}{l}\text { Dry/Wet } \\
\text { ratio }\end{array}$ & 3. Fruit & $\begin{array}{l}\text { USDA (2011); } \\
\text { Gebhardt and } \\
\text { Thomas (2002) }\end{array}$ & $\begin{array}{l}\text { Tree fruit, Tree } \\
\text { nuts, fruit on } \\
\text { supported vines }\end{array}$ & 0.25 & 0.11 & 0.95 & dry/wet \\
\hline DRYFAC & $\begin{array}{l}\text { Dry/Wet } \\
\text { ratio }\end{array}$ & 4. Grain & $\begin{array}{l}\text { USDA (2011); } \\
\text { Gebhardt and } \\
\text { Thomas (2002) }\end{array}$ & $\begin{array}{l}\text { predominantly } \\
\text { wheat, some corn } \\
\text { prior to drying }\end{array}$ & 0.22 & 0.18 & 0.30 & $\begin{array}{l}\text { dry/wet_as } \\
\text { grown }\end{array}$ \\
\hline $\begin{array}{ll}\text { (a) Recor } \\
\text { estim } \\
\text { (b) Apprc } \\
\text { NOT }\end{array}$ & $\begin{array}{l}\text { nended generi } \\
\text { riate values to } \\
\text { applied in } \mathrm{G}\end{array}$ & $\begin{array}{l}\text { c value in bold. } \\
\text { use for dry/wet } \\
2 \text {, but could be }\end{array}$ & $\begin{array}{l}\text { as eaten would b } \\
\text { sed for conversic }\end{array}$ & $\begin{array}{l}\text { recommended }(\mathrm{min} / \mathrm{n} \\
\text { from literature value }\end{array}$ & $\begin{array}{l}\text { e food categor } \\
\text { x) of } 0.90(0.8\end{array}$ & consid & These & $\begin{array}{l}\text { ommended } \\
\text { lues would }\end{array}$ \\
\hline
\end{tabular}

\subsection{Tab: Agriculture/Intake Delays}

The intake delay for the ingestion pathway for human foods is assigned in the Agriculture/Intake Delays tab. Intake delays for food crops, terrestrial animal products, and aquatic foods are covered in this section. See Section 7.14 for delay times between harvest and animal consumption for animal feed crops (STORTM).

\subsubsection{HLDUP, HLDUPA, and HLDUP2 Intake Delays for Crops, Animal Products, and Aquatic Foods}

The HLDUP parameter assigns intake delays for food crops (leafy vegetables, root vegetables, fruit, and grain). The HLDUPA parameter assigns intake delays for animal products consumed by people (meat, poultry, milk, and eggs). The HLDUP2 parameter assigns intake delays for aquatic foods (fish, mollusca, crustacea, and aquatic plants), when surface water scenarios are evaluated. 
Although a number of feeds and dried, frozen, or processed foods can be stored longer than a year, the maximum storage time is limited in the Gv2 code to $365 \mathrm{~d}$. The user can run a separate evaluation to determine emissions in year 1 and consumption in year 2, if this scenario detail is important.

The units for HLDUP, HLDUPA, and HLDUP2 in Gv2 are: $d(0-365)$.

Assignment of intake delay values depends on the type of scenario being evaluated - whether MEI or population average. MEI scenarios assume more local food consumption and therefore, shorter intake delays. Population scenarios assume more consumption of commercially-purchased food and longer hold-up times are more likely. The shelf-life of various foods will provide a measure of upper-bound values for hold-up times. Storage times for a variety of food categories are provided in Appendix I. The appendix tables are more applicable to retail food purchases, rather than backyard sources. To support the determinations of population scenario intake delay times, Table 8.9 indicates per capita food consumption and provides an indication of the fractions and types of crops consumed fresh, frozen, and canned.

The U.S. Department of Agriculture, Food Safety Inspection Service, provides information regarding food storage. It is worth noting that foods frozen at 0 degrees $F$ or lower remain safe indefinitely, but food quality will degrade after a certain amount of time (USDA 2012). However, code limits provide an upper bound assumption of $365 \mathrm{~d}$.

\subsubsection{Additional Sources of Information}

There are numerous internet resources regarding food shelf-life.

The United Nations Food and Agriculture Organization and World Health Organization publish the Codex Alimentarius which are international food standards, guidelines, and codes of practice (website: http://www.codexalimentarius.org/codex-home/en/ ). The Codex Alimentarius ("food code") is a global reference point for consumers, food producers and processors, and national food control agencies. Official food standards were created for a number of foods and are available on the internet (last accessed at http://www.codexalimentarius.org/standards/list-of-standards/ ). Recommended practices for General Principals of Food Hygiene are also available at the same location. This hygiene information is generalized, but provides an overview of issues related to maintaining edible foods. FAO information is not specific to the hold-up parameter, but provides comprehensive information for various foods. 


\subsubsection{HLDUP, HLDUPA, and HLDUP2 Human Consumption Intake Delays for Crops, Animal Products, and Aquatic Foods Tables}

Table 7.29. HLDUP, HLDUPA, HLDUP2 Hold-Up Time by Food Category

\begin{tabular}{|c|c|c|c|c|c|c|}
\hline Gv2 ID & Gv2_Description & Food Type & Comment & $\begin{array}{l}\text { MEI Scenario } \\
\text { Recommended }\end{array}$ & $\begin{array}{l}\text { POP Scenario } \\
\text { Recommended }\end{array}$ & Units \\
\hline HLDUP & $\begin{array}{l}\text { Intake delay between } \\
\text { harvest and consumption. }\end{array}$ & $\begin{array}{l}\text { 1.leafy } \\
\text { vegetable }\end{array}$ & $\begin{array}{l}\text { POP Assumed } \\
\text { predominantly fresh, } \\
\text { but some canned or } \\
\text { frozen. }\end{array}$ & 5 & 14 & $\mathrm{~d}$ \\
\hline HLDUP & $\begin{array}{l}\text { Intake delay between } \\
\text { harvest and consumption. }\end{array}$ & $\begin{array}{l}\text { 2.other } \\
\text { vegetable }\end{array}$ & $\begin{array}{l}\text { POP Assumed } 50 \% \\
\text { fresh } / 25 \% \\
\text { canned } / 25 \% \text { frozen. }\end{array}$ & 5 & 80 & $d$ \\
\hline HLDUP & $\begin{array}{l}\text { Intake delay between } \\
\text { harvest and consumption. }\end{array}$ & 3.fruit & $\begin{array}{l}\text { POP Assumed half } \\
\text { fresh and half juice. }\end{array}$ & 5 & 80 & $d$ \\
\hline HLDUP & $\begin{array}{l}\text { Intake delay between } \\
\text { harvest and consumption. }\end{array}$ & 4.grain & $\begin{array}{l}\text { Predominantly } \\
\text { wheat. }\end{array}$ & 180 & 270 & $d$ \\
\hline HLDUPA & $\begin{array}{l}\text { Intake delay between } \\
\text { harvest and consumption. }\end{array}$ & 1.meat & $\begin{array}{l}\text { Predominantly beef } \\
\text { and pork. }\end{array}$ & 15 & 30 & $\mathrm{~d}$ \\
\hline HLDUPA & $\begin{array}{l}\text { Intake delay between } \\
\text { harvest and consumption. }\end{array}$ & 2.poultry & $\begin{array}{l}\text { Assumed all } \\
\text { chicken. }\end{array}$ & 15 & 30 & d \\
\hline HLDUPA & $\begin{array}{l}\text { Intake delay between } \\
\text { harvest and consumption. }\end{array}$ & 3.dairy & $\begin{array}{l}\text { POP Assumed } 75 \% \\
\text { milk, } 25 \% \text { other } \\
\text { dairy. }\end{array}$ & 3 & 10 & d \\
\hline HLDUPA & $\begin{array}{l}\text { Intake delay between } \\
\text { harvest and consumption. }\end{array}$ & 4.egg & Chicken egg. & 5 & 15 & $\mathrm{~d}$ \\
\hline HLDUP2 & $\begin{array}{l}\text { Intake delay between } \\
\text { harvest and consumption. }\end{array}$ & 1.fish & & 2 & 15 & $\mathrm{~d}$ \\
\hline HLDUP2 & $\begin{array}{l}\text { Intake delay between } \\
\text { harvest and consumption. }\end{array}$ & 2.mollusk & & 2 & 15 & $\mathrm{~d}$ \\
\hline HLDUP2 & $\begin{array}{l}\text { Intake delay between } \\
\text { harvest and consumption. }\end{array}$ & 3.crustacean & & 2 & 15 & d \\
\hline HLDUP2 & $\begin{array}{l}\text { Intake delay between } \\
\text { harvest and consumption. }\end{array}$ & $\begin{array}{l}\text { 4.aquatic } \\
\text { plant }\end{array}$ & $\begin{array}{l}\text { No intake assumed. } \\
\text { Special case } \\
\text { application. }\end{array}$ & 0 & 0 & $\mathrm{~d}$ \\
\hline
\end{tabular}

\subsection{References: Chronic Exposure Module}

Andelman JB. 1990. “Total Exposure to Volatile Organic Chemicals in Potable Water." In Significance and Treatment of Volatile Organic Compounds in Water, NM Ram, RF Christman, and KG Cantor (eds.). Lewis Publishers, Boca Raton, Florida.

Brouwer C, A Goffeau, and M Heibloem. 1985. Irrigation Water Management: Training Manual No. 1 - Introduction to Irrigation. ISSN: 1020-4261. Natural Resources Management and Environment Department, Food and Agriculture Organization of the United Nations, Rome, Italy. Last accessed at: http://www.fao.org/docrep/R4082E/r4082e00.htm\#Contents. 
Burt R (ed.). 2011. Soil Survey Laboratory Information Manual. Soil Survey Investigations Report No. 45, Version 2.0, U.S. Department of Agriculture, Natural Resources Conservation Service, Lincoln, Nebraska. Last accessed (2012) at ftp://ftp-fc.sc.egov.usda.gov/NSSC/Lab_Info_Manual/SSIR_45.pdf.

Ensminger ME, JE Oldfield, and WW Heinemann. 1990. Feeds and Nutrition, second edition. Ensminger Publishing Company, Clovis, California.

EPA. 1991. Risk Assessment Guidance for Superfund, Volume I: Human Health Evaluation Manual (Part B, Development of Risk-Based Preliminary Remediation Goals). EPA/540/R-92/003,

U.S. Environmental Protection Agency, Office of Emergency and Remedial Response, Washington, D.C. Last accessed at: http://rais.ornl.gov/documents/HHEMB.pdf.

EPA. 1999. Understanding Variation in Partition Coefficient Values. EPA 402-R-99-004A.

U.S. Environmental Protection Agency, Office of Air and Radiation, Washington D.C. Last accessed at: http://www.epa.gov/radiation/cleanup/402-r-99-004.html\#vol1.

EPA. 2012. Our Nations Air, Status and Trends Through 2010. EPA-454/R-12-001, U.S. Environmental Protection Agency, Office of Air Quality Planning and Standards, Research Triangle Park, North Carolina.

ERS. 2012. Loss-Adjusted Food Availability Data: Documentation. U.S. Department of Agriculture, Economic Research Service, Online data set. Last accessed (2012) at:

http://www.ers.usda.gov/Data/foodconsumption/FoodGuideSpreadsheets.htm, Spreadsheets; Vegetables; Total Vegetables - Fresh and Processed.

Gallagher P, M Dikeman, J Fritz, E Wailes, W Gauther, and H Shapouri. 2003. Biomass from Crop Residues: Cost and Supply Estimates. Agricultural Economic Report No. 819, U.S. Department of Agriculture, Office of the Chief Economist, Office of Energy Policy and New Uses.. Last accessed (2012) at: http://www.usda.gov/oce/reports/energy/AER819.pdf.

Gebhardt SE and RG Thomas. 2002. Nutritive Value of Foods. Home and Garden Bulletin Number 72 , U.S. Department of Agriculture, Agricultural Research Service, Nutrient Data Laboratory, Beltsville, Maryland.

IAEA. 2009. Quantification of Radionuclide Transfer in Terrestrial and Freshwater Environments for Radiological Assessments. IAEA-TECDOC-1616, International Atomic Energy Agency, Vienna.

IAEA. 2010. Handbook of Parameter Values for the Prediction of Radionuclide Transfer in Terrestrial and Freshwater Environments. Technical Report Series No. 472, International Atomic Energy Agency, Vienna.

Jenkins JC, DC Chojnacky, LS Heath, and RA Birdsey. 2004. Comprehensive Database of Diameterbased Biomass Regressions for North American Tree Species. General Technical Report NE-319, Newtown Square, Pennsylvania.

Lalman DL and HB Sewell. 1993. Rations for Growing and Finishing Beef Cattle. Publication G2066. University of Missouri, Extension. Last accessed (2012) at: http://extension.missouri.edu/p/G2066. 
Lardy G and C Stoltenow. 1999. Livestock and Water. AS-954, North Dakota State University, Fargo, North Dakota.

Linn JG, MF Hutjens, R Shaver, DE Otterby, WT Howard, and LH Kilmer. 2002. Feeding the Dairy Herd. North Central Regional Extension Publication 346, reviewed 2008, University of Minnesota, Extension, St. Paul, Minnesota. Last accessed (2012) at: http://www.extension.umn.edu/distribution/livestocksystems/DI0469.html\#tc.

Ministry of the Environment. 2001. Animal Weights and their Food and Water Requirements. Ministry of the Environment, Lands and Parks, Government of British Columbia, TBD, British Columbia. Last accessed (2012) at: http://www.env.gov.bc.ca/wat/wq/reference/foodandwater.html.

Napier BA. 2012. GENII Version 2 Users' Guide. PNNL-14583, Rev. 4, Pacific Northwest National Laboratory, Richland, Washington.

Napier BA, RA Peloquin, DL Strenge, and JV Ramsdell. 1988. GENII- The Hanford Environmental Radiation Dosimetry Software System, Vol. 1, Conceptual Representation. PNNL-6584, Vol. 1, Pacific Northwest Laboratory, Richland, Washington.

NCDC. 1981. Freeze Free Period, 90\% Probability of a (day) with Temperatures Above 32 F. Map, National Climatic Data Center, Asheville, North Carolina. Last accessed (2012) at: http://www.ncdc.noaa.gov/oa/climate/freezefrost/frostfreemaps.html.

NRC. 1996. Nutrient Requirements of Beef Cattle. National Research Council, National Academy Press, Washington, D.C. Last accessed (2012) at: http://www.nap.edu/catalog/9791.html.

NRC. 2001. Nutrient Requirements of Dairy Cattle, $7^{\text {th }}$ edition. National Research Council, National Academy Press, Washington, D.C. Last accessed (2012) at: http://www.nap.edu/catalog.php?record id=9825.

NRC. 2007. Nutrient Requirements of Small Ruminants: Sheep, Goats, Cervids, and New World Camelids. National Research Council, National Academy Press, Washington, D.C.

Schoeters G and R Hoogenboom. 2006. "Contamination of Free-range Chicken Eggs with Dioxins and Dioxin-like Polychlorinated Biphenyls.” Molecular Nutrition \& Food Research 50(10):908-914.

Serne RJ. 2007. Kd Values for Agricultural and Surface Soils for Use in Hanford Site Farm, Residential, and River Shoreline Scenarios. PNNL-16531, Pacific Northwest National Laboratory, Richland, Washington.

Snyder SF, WT Farris, BA Napier, TA Ikenberry, and RO Gilbert. 1994. Parameters Used in the Environmental and Radiological Dose Modules (DESCARTES, CIDER, and CRD Codes) of the Hanford Environmental Dose Reconstruction Integrated Codes (HEDRIC). PNWD-2023 HEDR, Rev. 1, Pacific Northwest Laboratory, Richland, Washington.

UC SAREP. 2012. UC SAREP (University of California Sustainable Agriculture Research and Education Program) Online, Cover Crop Database. Last accessed (2012) at: http://www.sarep.ucdavis.edu/cgi-bin/ccrop.EXE. 
USDA. 1992. Weights, Measures, and Conversion Factors for Agricultural Commodities and Their Products. USDA Handbook No. 697, U.S. Department of Agriculture, Washington, D.C.

USDA. 2007. Vegetables, Usual Planting and Harvesting Dates. USDA Agriculture Handbook Number 507, U.S. Department of Agriculture, National Agricultural Statistics Service, Washington, D.C. Last accessed at http://usda01.library.cornell.edu/usda/current/UsuPlant/UsuPlant-05-31-2007.pdf.

USDA. 2008. Soil Quality Indicators. USDA, Natural Resources Conservation Service, <city, state unknown>. Last accessed (2012) at:

http://soils.usda.gov/sqi/assessment/files/bulk density sq physical indicator sheet.pdf.

USDA. 2009. 2007 Census of Agriculture, United States Summary and State Data. Vol. 1, Geographic Area Series, Part 51, AC-07-A-51, USDA, National Agricultural Statistics Service, Washington, D.C. Last accessed (2012) at:

http://www.agcensus.usda.gov/Publications/2007/Online Highlights/Farm and Ranch_Irrigation_Survey lindex.asp.

USDA. 2010. Field Crops, Usual Planting and Harvesting Dates. Handbook Number 628, USDA National Agricultural Statistics Service, Washington, D.C. Last accessed (2012) at: http://usda.mannlib.cornell.edu/MannUsda/viewDocumentInfo.do?documentID=1251.

USDA. 2011. Composition of Foods, Raw, Processed, Prepared, USDA National Nutrient Database for Standard Reference, Release 24. USDA, Agricultural Research Service, Beltsville, Maryland. Last accessed (2012) at: http://www.nal.usda.gov/fnic/foodcomp/search/.

USDA. 2012. Food Safety and Inspection Service [online] Fact Sheets (multiple). USDA Food Safety and Inspection Service, Washington, D.C. Last accessed (2012) at: http://www.fsis.usda.gov/Fact_Sheets/.

Yu C, AJ Zielen, J-J Cheng, DJ LePoire, E Gnanapragasam, S Kamboj, J Arnish, A Wallo III, WA Williams, and H Peterson. 2001. User's Manual for RESRAD Version 6. ANL/EAD-4, Argonne National Laboratory, Argonne, Illinois.

Zach R and KR Mayoh. 1984. "Soil Ingestion by Cattle: A Neglected Pathway." Notes, Health Physics 46(2):426-431. 


\subsection{Module: Receptor Intake}

The Receptor Intake module is downstream of the Exposure Pathways module and upstream of the Health Impacts module. Receptor intakes describe behavioral factors for the receptor of interest. This receptor may be the maximally exposed individual (MEI) or the average member of the population. The user will need to determine the receptor required to address the purpose of the assessment. MEI parameters are prudent overestimates of exposure and intake rates, and address the question "what's the reasonably worst case impact?" Population estimates use average exposure and intake rate and indicate the average impact to the average person in the evaluated location or region.

The impact measure of importance to radioactive material exposure is radiation dose. Dose can come from internal radionuclide exposures from inhalation, ingestion, or less frequently from dermal uptake. Additional dose can result from external exposure to radioactive material external to the body. Internal dose varies by age at which the inhalation or ingestion intake occurs. Age-dependent inhalation and ingestion dose factors were published in ICRP Reports 68, 69, 71, and 72 (ICRP 1994b, 1995a, 1995b, 1995c). To apply the internal dose factor data most effectively, the intake data will be presented in the age categories considered for the age-dependent dose factors (Table 8.1). External dose factors have not been calculated on an age-dependent basis. Therefore, any air, ground, or water external exposure routes will apply the same pathway-appropriate dose factor to all ages.

Table 8.1. Age Ranges of Six Age Categories

\begin{tabular}{ccc}
\hline Age Category & $\begin{array}{c}\text { Minimum } \\
(\mathrm{yr})\end{array}$ & $\begin{array}{c}\text { Maximum } \\
(\mathrm{yr})\end{array}$ \\
\hline 1 & 0 & 1.49 \\
2 & 1.5 & 2.49 \\
3 & 2.5 & 7.49 \\
4 & 7.5 & 12.49 \\
5 & 12.5 & 17.49 \\
6 & 17.5 & 100 \\
\hline Note: If age category reported as 0, assume the value is \\
applicable to all age categories.
\end{tabular}

MEI characteristics for sites that routinely calculate dose estimates have stronger technical roots than population dose characteristics. MEI intake and exposure rates reported from historical sources can be used as a comparison set when establishing MEI values for a new site or considering comparative values for average or representative individual values or population values.

EPA published an updated Exposure Factors Handbook in 2011 (EPA 2011). The Exposure Factors Handbook is a resource used to provide guidance for risk assessment data applications. The data compilation and summaries is considered the best available for current model input. It also includes input data that allows the user to adjust the input value to their specific assessment purpose. Values for GENIIv2 input were derived from this reference. Recommended values are presented by age category and receptor type (i.e., MEI and population), with some indication of variability when possible. The user can refer to EPA (2011) for details on variability, data quality, and other information. 
The screen tab format is not used in this module. As in the Receptor Intake module screen, parameters listed below are grouped by exposure/intake pathway. If a source pathway (e.g., surface water) is not relevant the pathway parameter values need not be zeroed out. They simply will not be used in any calculation.

\subsection{Pathway Selection: External Exposure (multiple)}

Several external exposure pathways require parameterization to define the exposure scenario. Table 8.2 indicates the sources of contamination for external exposure pathways.

Table 8.2. Receptor Intake Module External Exposure Parameters

\begin{tabular}{lll}
\hline \multicolumn{1}{c}{$\begin{array}{c}\text { Source of } \\
\text { Contamination }\end{array}$} & \multicolumn{1}{c}{ Pathway Selection } & Media Causing Exposure \\
\hline Air & External exposure to air & Plume \\
Air and Water & External exposure to ground & $\begin{array}{l}\text { Ground - from plume } \\
\text { deposition, soil } \\
\text { contamination, and irrigation } \\
\text { application }\end{array}$ \\
& & $\begin{array}{l}\text { Surface water } \\
\text { Water }\end{array}$ \\
& External exposure while swimming & Surface water \\
& External exposure while boating & Shoreline - from surface \\
& External Exposure to shoreline & water \\
\hline
\end{tabular}

\subsubsection{External Exposure: Air and Ground}

Plume exposure from radioactive material in the air is estimated with exposure parameters for $\mathrm{hr} / \mathrm{d}$ (UEXAIR) and d/yr (TEXAIR). Ground exposure, from air and irrigation deposition, is calculated in a similar manner with UEXGRD and TEXGRD parameters, but allows for separate definition of indoor vs. outdoor shielding factors (SHIN and SHOUT) and specification of time spent indoors and out (FTIN and FTOUT).

Shielding factors are characteristic of the material between the surface deposition and the individual and are those appropriate for shielding of gamma radiation. They typically represent building materials. Applying appropriate shielding factors are generally more important for accident scenarios or chronic scenarios involving soil contamination exposure, rather than scenarios of low levels of chronic airborne emissions. A shielding factor of 1.0 is equivalent to no shielding.

When considering the fraction of time spent indoors and out, it is unnecessary for the indoor and outdoor fractions to add up to one if the individual is assumed to be "off location" for a fraction of the time. The user is responsible for making sure that the indoor and outdoor fractions add to the total fraction that the individual remains at a single grid location and for making sure that FTIN and FTOUT add to 1 . 
Ground exposure to shoreline soil from surface water contamination is addressed separately in the next section (Section 8.1.2).

- Air (plume) Exposure

- The units and range for UEXAIR in Gv2 are: hr (0-24)

- The units and range for TEXAIR in Gv2 are: $d(0-365)$

- Ground Exposure

- The units and range for UEXGRD in Gv2 are: hr (0-24)

- The units and range for TEXGRD in Gv2 are: $d(0-365)$

- The units and range for FTIN and FTOUT in Gv2 are: fraction (0-1)

- The units and range for SHIN and SHOUT in Gv2 are: fraction (0-1).

\section{Air Exposure}

Daily and annual exposure time to the plume should reflect occupancy at the location of interest. Constant occupancy is indicative of conservative residential exposure scenarios. Worksite occupancy for stationary jobs would be indicated by $9 \mathrm{hr} / \mathrm{d}, 250 \mathrm{~d} / \mathrm{yr}$ for a typical $8 \mathrm{hr} / \mathrm{d}$ work schedule. School exposure times could assume $7 \mathrm{hr} / \mathrm{d}, 180 \mathrm{~d} / \mathrm{yr}$. Assignment of school occupancy would depend on whether the school is located in the area of interest or outside the area of interest.

\section{Ground Exposure}

Shielding factors can be conservatively set to 1.0. Smaller shielding factors should be applied when ground contamination is a significant dose contributor to external dose. Jacob and Meckback (1987) indicate issues important to shielding factor determinations: more wet deposition and less vegetation results in less source strength; tree depositions will result in higher source strengths than lawn depositions; indoor deposition in openly ventilated homes is comparable to dry lawn depositions. They also looked at age-dependent doses and proposed factors of up to an 80 percent dose increase to youth since all their tissues are in closer proximity to the contaminated modeled plane. Shielding factors generally range from 0.2 to 0.8 . Structures with dense, thick materials have shielding factors in the lower end of this range, or even lower if gamma energies of $500 \mathrm{keV}$ or less are considered (LeGrand et al. 1987).

The user is responsible for aligning the fraction of time spent indoors and outdoors (FTIN and FTOUT) for external exposures with the indoor and outdoor fractions for inhalation exposure (see Section 8.5.1). The FTIN and FTOUT values tabulated in Section 8.1.1.3 apply to the residential MEI and population individuals. The values reflect the mostly indoor lifestyles of most of the United States. See the MEI table footnote for important information. Maximizing FTOUT values would be 1.0. Occupational times would apply to age group 6, only.

\subsubsection{Additional Sources of Information}

See Chapter 16 of EPA (2011) for extensive details regarding activity factors. 
The U.S. Census conducts an American Housing survey that includes information regarding the primary exterior wall materials used for single-family homes, by year and region. The year of construction of the houses in a region is also available. This information can be helpful when determining regionally specific material to assume for generic residential shielding factors. See http://www.census.gov/housing/ahs/data/national.html.

An NRC poster presentation (Barr et al. 2010) provides an excellent overview of shielding factors and summarizes SHIN options. Available at: http://pbadupws.nrc.gov/docs/ML1006/ML100610262.pdf.

Due to the high inherent uncertainty of shielding factors, radionuclide-specific values are rarely considered. However, Leung (1992) provides a glimpse of how shielding factors vary by nuclide.

\subsubsection{UEXAIR, TEXAIR Air and Ground External Exposure Time Tables}

Table 8.3. UEXAIR and TEXAIR Plume Exposure Time

\begin{tabular}{|c|c|c|c|c|c|c|}
\hline Gv2_Description & $\operatorname{Age}^{(a)}$ & Qualifier & Reference & Comment & $\begin{array}{l}\text { UEXAIR } \\
(\mathrm{hr} / \mathrm{d})\end{array}$ & $\begin{array}{l}\text { TEXAIR } \\
(\mathrm{d} / \mathrm{yr})\end{array}$ \\
\hline $\begin{array}{l}\text { External exposure, } \\
\text { Plume Air }\end{array}$ & 0 & MEI & $\begin{array}{c}\text { generic } \\
\text { assumption }\end{array}$ & residential & 24 & 365 \\
\hline $\begin{array}{l}\text { External exposure, } \\
\text { Plume Air }\end{array}$ & $1-4$ & MEI & $\begin{array}{c}\text { generic } \\
\text { assumption }\end{array}$ & $\begin{array}{l}\text { location of interest is } \\
\text { workplace }\end{array}$ & 0 & 0 \\
\hline $\begin{array}{l}\text { External exposure, } \\
\text { Plume Air }\end{array}$ & 5 & MEI & $\begin{array}{c}\text { generic } \\
\text { assumption }\end{array}$ & $\begin{array}{l}\text { location of interest is } \\
\text { workplace }\end{array}$ & 4 & 130 \\
\hline $\begin{array}{l}\text { External exposure, } \\
\text { Plume Air }\end{array}$ & 6 & MEI & $\begin{array}{c}\text { generic } \\
\text { assumption }\end{array}$ & $\begin{array}{c}\text { location of interest is } \\
\text { workplace }\end{array}$ & 9 & 250 \\
\hline $\begin{array}{l}\text { External exposure, } \\
\text { Plume Air }\end{array}$ & 1 & MEI & $\begin{array}{c}\text { generic } \\
\text { assumption }\end{array}$ & $\begin{array}{c}\text { location of interest is } \\
\text { school/daycare }\end{array}$ & 8 & 125 \\
\hline $\begin{array}{l}\text { External exposure, } \\
\text { Plume Air }\end{array}$ & 2 & MEI & $\begin{array}{c}\text { generic } \\
\text { assumption }\end{array}$ & $\begin{array}{c}\text { location of interest is } \\
\text { school/daycare }\end{array}$ & 8 & 125 \\
\hline $\begin{array}{l}\text { External exposure, } \\
\text { Plume Air }\end{array}$ & 3 & MEI & $\begin{array}{c}\text { generic } \\
\text { assumption }\end{array}$ & $\begin{array}{c}\text { location of interest is } \\
\text { school/daycare }\end{array}$ & 7 & 180 \\
\hline $\begin{array}{l}\text { External exposure, } \\
\text { Plume Air }\end{array}$ & 4 & MEI & $\begin{array}{c}\text { generic } \\
\text { assumption }\end{array}$ & $\begin{array}{c}\text { location of interest is } \\
\text { school/daycare }\end{array}$ & 7 & 180 \\
\hline $\begin{array}{l}\text { External exposure, } \\
\text { Plume Air }\end{array}$ & 5 & MEI & $\begin{array}{c}\text { generic } \\
\text { assumption }\end{array}$ & $\begin{array}{c}\text { location of interest is } \\
\text { school/daycare }\end{array}$ & 8 & 200 \\
\hline $\begin{array}{l}\text { External exposure, } \\
\text { Plume Air }\end{array}$ & 6 & MEI & $\begin{array}{c}\text { generic } \\
\text { assumption }\end{array}$ & $\begin{array}{c}\text { location of interest is } \\
\text { school/daycare }\end{array}$ & 0 & 0 \\
\hline \multicolumn{7}{|c|}{ (a) See Table 8.1 for age groups. } \\
\hline Gv2_Description & $\operatorname{Age}^{(\mathrm{a})}$ & Qualifier & Reference & Comment & $\begin{array}{c}\text { UEXAIR } \\
(\mathrm{hr} / \mathrm{d})\end{array}$ & $\begin{array}{c}\text { TEXAIR } \\
(\mathrm{d} / \mathrm{yr})\end{array}$ \\
\hline $\begin{array}{l}\text { External exposure, } \\
\text { Plume Air }\end{array}$ & 0 & POP & $\begin{array}{c}\text { generic } \\
\text { assumption }\end{array}$ & residential & 22 & 360 \\
\hline $\begin{array}{l}\text { External exposure, } \\
\text { Plume Air }\end{array}$ & $1-4$ & POP & $\begin{array}{c}\text { generic } \\
\text { assumption }\end{array}$ & $\begin{array}{l}\text { location of interest is } \\
\text { workplace }\end{array}$ & 0 & 0 \\
\hline $\begin{array}{l}\text { External exposure, } \\
\text { Plume Air }\end{array}$ & 5 & POP & $\begin{array}{c}\text { generic } \\
\text { assumption }\end{array}$ & $\begin{array}{l}\text { location of interest is } \\
\text { workplace }\end{array}$ & 3 & 80 \\
\hline $\begin{array}{l}\text { External exposure, } \\
\text { Plume Air }\end{array}$ & 6 & POP & $\begin{array}{c}\text { generic } \\
\text { assumption }\end{array}$ & $\begin{array}{l}\text { location of interest is } \\
\text { workplace, some off- } \\
\text { location travel assumed }\end{array}$ & 9 & 200 \\
\hline
\end{tabular}


Table 8.3. (contd)

\begin{tabular}{|c|c|c|c|c|c|c|}
\hline Gv2_Description & $\operatorname{Age}^{(a)}$ & Qualifier & Reference & Comment & $\begin{array}{l}\text { UEXAIR } \\
(\mathrm{hr} / \mathrm{d})\end{array}$ & $\begin{array}{c}\text { TEXAIR } \\
(\mathrm{d} / \mathrm{yr})\end{array}$ \\
\hline $\begin{array}{l}\text { External exposure, } \\
\text { Plume Air }\end{array}$ & 1 & POP & $\begin{array}{c}\text { generic } \\
\text { assumption }\end{array}$ & $\begin{array}{c}\text { location of interest is } \\
\text { school/childcare }\end{array}$ & 1 & 365 \\
\hline $\begin{array}{l}\text { External exposure, } \\
\text { Plume Air }\end{array}$ & 2 & POP & $\begin{array}{c}\text { generic } \\
\text { assumption }\end{array}$ & $\begin{array}{l}\text { location of interest is } \\
\text { school/childcare }\end{array}$ & 1 & 365 \\
\hline $\begin{array}{l}\text { External exposure, } \\
\text { Plume Air }\end{array}$ & 3 & POP & $\begin{array}{l}\text { generic } \\
\text { assumption }\end{array}$ & $\begin{array}{c}\text { location of interest is } \\
\text { school/daycare }\end{array}$ & 4 & 180 \\
\hline $\begin{array}{l}\text { External exposure, } \\
\text { Plume Air }\end{array}$ & 4 & POP & $\begin{array}{l}\text { generic } \\
\text { assumption }\end{array}$ & $\begin{array}{l}\text { location of interest is } \\
\text { school/daycare }\end{array}$ & 7 & 180 \\
\hline $\begin{array}{l}\text { External exposure, } \\
\text { Plume Air }\end{array}$ & 5 & POP & $\begin{array}{l}\text { generic } \\
\text { assumption }\end{array}$ & $\begin{array}{l}\text { location of interest is } \\
\text { school/daycare }\end{array}$ & 7 & 180 \\
\hline $\begin{array}{l}\text { External exposure, } \\
\text { Plume Air }\end{array}$ & 6 & POP & $\begin{array}{l}\text { generic } \\
\text { assumption }\end{array}$ & $\begin{array}{l}\text { location of interest is } \\
\text { school/daycare }\end{array}$ & 0 & 0 \\
\hline
\end{tabular}

\subsubsection{FTIN, FTOUT Air and Ground Exposure Fraction Tables}

Table 8.4. FTIN and FTOUT Fraction of Time Indoors and Outdoors

\begin{tabular}{llccccccc}
\hline \multicolumn{1}{c}{ Gv2_ID } & Gv2_Description & Age $^{(\mathrm{a})}$ & Qualifier & Reference & Comment & FTIN & FTOUT $^{(\mathrm{b})}$ & Units \\
\hline $\begin{array}{l}\text { FTIN, } \\
\text { FTOUT }\end{array}$ & $\begin{array}{l}\text { Fraction of time } \\
\text { indoors, outdoors }\end{array}$ & 1 & MEI & EPA (2011) & $\begin{array}{c}\text { FTOUT based } \\
\text { on (1-FTIN) }\end{array}$ & 1 & fraction \\
$\begin{array}{l}\text { FTIN, } \\
\text { FTOUT }\end{array}$ & $\begin{array}{l}\text { Fraction of time } \\
\text { indoors, outdoors }\end{array}$ & 2 & MEI & EPA (2011) & $\begin{array}{c}\text { FTOUT based } \\
\text { on (1-FTIN) }\end{array}$ & 1 & 0 & fraction \\
$\begin{array}{l}\text { FTIN, } \\
\text { FTOUT }\end{array}$ & $\begin{array}{l}\text { Fraction of time } \\
\text { indoors, outdoors }\end{array}$ & 3 & MEI & EPA (2011) & $\begin{array}{c}\text { FTOUT based } \\
\text { on (1-FTIN) }\end{array}$ & 0.94 & 0.06 & fraction \\
$\begin{array}{l}\text { FTIN, } \\
\text { FTOUT }\end{array}$ & $\begin{array}{l}\text { Fraction of time } \\
\text { indoors, outdoors }\end{array}$ & 4 & MEI & EPA (2011) & $\begin{array}{c}\text { FTOUT based } \\
\text { on (1-FTIN) }\end{array}$ & 0.89 & 0.11 & fraction \\
$\begin{array}{l}\text { FTIN, } \\
\text { FTOUT }\end{array}$ & $\begin{array}{l}\text { Fraction of time } \\
\text { indoors, outdoors }\end{array}$ & 5 & MEI & EPA (2011) & $\begin{array}{c}\text { FTOUT based } \\
\text { on (1-FTIN) }\end{array}$ & 0.9 & 0.1 & fraction \\
$\begin{array}{l}\text { FTIN, } \\
\text { FTOUT }\end{array}$ & $\begin{array}{l}\text { Fraction of time } \\
\text { indoors, outdoors }\end{array}$ & 6 & MEI & EPA (2011) & $\begin{array}{c}\text { FTOUT based } \\
\text { on (1-FTIN) }\end{array}$ & 1 & 0 & fraction \\
\hline
\end{tabular}

(a) See Table 8.1 for age groups.

(b) Maximizing the MEI would assign FTIN and FTOUT values of 0 and 1.0, respectively. Reported values indicate fraction of time spent indoors for scenarios where indoor contamination was of primary concern. If shielding fractions are set to 1.0, then apply values as reported. If shielding values are not zero, assign indicated FTIN to the FTOUT parameter entry and indicate FTOUT to the FTIN entry for conservatism.

\begin{tabular}{|c|c|c|c|c|c|c|c|c|c|}
\hline Gv2 ID & Gv2_Description & $\operatorname{Age}^{(a)}$ & $\begin{array}{l}\text { Qual- } \\
\text { ifier }\end{array}$ & Reference & Comment & $\begin{array}{l}\text { FTIN } \\
\text { Range }\end{array}$ & FTIN & FTOUT & Units \\
\hline $\begin{array}{l}\text { FTIN, } \\
\text { FTOUT }\end{array}$ & $\begin{array}{l}\text { Fraction of time } \\
\text { indoors, outdoors }\end{array}$ & 1 & POP & $\begin{array}{c}\text { EPA } \\
(2011)\end{array}$ & $\begin{array}{l}\text { FTOUT based } \\
\text { on }(1-\text { FTIN) }\end{array}$ & $0.77-1$ & 1 & 0 & fraction \\
\hline $\begin{array}{l}\text { FTIN, } \\
\text { FTOUT }\end{array}$ & $\begin{array}{l}\text { Fraction of time } \\
\text { indoors, outdoors }\end{array}$ & 2 & POP & $\begin{array}{c}\text { EPA } \\
(2011)\end{array}$ & $\begin{array}{l}\text { FTOUT based } \\
\text { on (1-FTIN) }\end{array}$ & $0.68-0.94$ & $0.81^{(b)}$ & 0.19 & fraction \\
\hline $\begin{array}{l}\text { FTIN, } \\
\text { FTOUT }\end{array}$ & $\begin{array}{l}\text { Fraction of time } \\
\text { indoors, outdoors }\end{array}$ & 3 & POP & $\begin{array}{c}\text { EPA } \\
(2011)\end{array}$ & $\begin{array}{l}\text { FTOUT based } \\
\text { on (1-FTIN) }\end{array}$ & $0.66-0.89$ & $0.77^{(\mathrm{b})}$ & 0.23 & fraction \\
\hline $\begin{array}{l}\text { FTIN, } \\
\text { FTOUT }\end{array}$ & $\begin{array}{l}\text { Fraction of time } \\
\text { indoors, outdoors }\end{array}$ & 4 & POP & $\begin{array}{l}\text { EPA } \\
(2011)\end{array}$ & $\begin{array}{l}\text { FTOUT based } \\
\text { on (1-FTIN) }\end{array}$ & $0.62-0.86$ & $0.74^{(\mathrm{b})}$ & 0.26 & fraction \\
\hline $\begin{array}{l}\text { FTIN, } \\
\text { FTOUT }\end{array}$ & $\begin{array}{l}\text { Fraction of time } \\
\text { indoors, outdoors }\end{array}$ & 5 & POP & $\begin{array}{c}\text { EPA } \\
(2011)\end{array}$ & $\begin{array}{l}\text { FTOUT based } \\
\text { on (1-FTIN) }\end{array}$ & $0.58-0.88$ & $0.73^{(b)}$ & 0.27 & fraction \\
\hline $\begin{array}{l}\text { FTIN, } \\
\text { FTOUT }\end{array}$ & $\begin{array}{l}\text { Fraction of time } \\
\text { indoors, outdoors }\end{array}$ & 6 & POP & $\begin{array}{l}\text { EPA } \\
(2011)\end{array}$ & $\begin{array}{l}\text { FTOUT based } \\
\text { on (1-FTIN) }\end{array}$ & $0.66-0.82$ & $0.74^{(\mathrm{b})}$ & 0.26 & fraction \\
\hline
\end{tabular}

(a) See Table 8.1 for age groups.

(b) FTIN datum values were calculated as simple averages of the reported range. 


\subsubsection{External Exposure: Surface Water from Swimming, Boating, and Shoreline}

The surface water pathway has three routes of external exposure: water immersion during swimming; water while in a boat; and shoreline contamination from water exposure. These parameters reflect exposure to the contaminated water source. Any water activity at non-contaminated sources is not included in the parameterization. The ground exposure from air deposition and irrigation water application is addressed in Section 8.1.1.

Swimming exposure uses three parameters to calculate external exposure from swimming: number of swimming events per day (EVSWIM), duration of a "swimming event" (TESWIM), number of days that swimming occurs in a year (TSWIM). The product of these three parameters indicates the hours of swimming time in a year. The "External exposure while swimming" pathway selection also prompts for the hourly water ingestion rate while swimming. The user is responsible for entering the same values in the Pathway Selections: External Exposure While Swimming and Water Ingestion while Swimming (see Section 8.3.3) parameters.

Boating external exposure uses four parameters to characterize: shielding factor (SFBOAT), frequency of boating events (EVBOAT), duration of a "boating event" (TEBOAT), and the number of days that boating is done in a year (TBOAT). The product of EVBOAT, TEBOAT, and TBOAT indicate the total boating hours in a year. Assuming most scenarios will not assume an individual is boating on highly contaminated waterways, a shielding factor of 1 should be assigned in virtually all routine emissions calculations. Other shielding factors, for building materials, are discussed in Section 8.1.1.

Shoreline exposure uses four parameters for characterization: daily frequency of shoreline events (EVSHOR), duration of a "shoreline event" (TESHOR), number of days that an individual partakes in shoreline activities in a year (TSHOR), and a shoreline width factor (SWFAC). The product of EVSHOR, TESHOR, and TSHOR indicate the hours of shoreline activity in a year. The shoreline width factor corrects for the fact that a person does not remain at, roughly, the average high water mark the entire time of their shoreline event.

- Swimming

- The units and range for EVSWIM in Gv2 are: events/d (0-10)

- The units and range for TESWIM in Gv2 are: $\mathrm{hr}(0-24)$

- The units and range for TSWIM in Gv2 are: $d(0-365)$

- The units and range for USWIM in Gv2 are: L/hr (0-10)

- Boating

- The units and range for SFBOAT in Gv2 are: fraction (0-1)

- The units and range for EVBOAT in Gv2 are: events/d (0-10)

- The units and range for TEBOAT in Gv2 are: $\mathrm{hr}(0-24)$

- The units and range for TBOAT in Gv2 are: $d(0-365)$ 
- Shoreline

- The units and range for EVSHOR in Gv2 are: events/d (0-10)

- The units and range for TESHOR in Gv2 are: $\mathrm{hr}(0-24)$

- The units and range for TSHOR in Gv2 are: $d(0-365)$

- The units and range for SWFAC in Gv2 are: fraction (0-1).

\section{Swimming}

The number of daily swim events, time per event, and swimming days per year is arbitrarily assigned as 1 event/d, $2 \mathrm{hr} /$ event, and $5 \mathrm{~d} / \mathrm{yr}$ for all age groups. The user must determine if these values are appropriate to their location. The incidental swimming ingestion rates (USWIM) are derived from EPA (2011) with the adult rate assigned to age group 6 and the children rate assigned to all other age groups. No incidental swimming ingestion is assigned for age category 1 (i.e., 1 year-old). The MEI is assumed to ingest the $97^{\text {th }}$ or $95^{\text {th }}$ percentile value reported, while the population is assigned the average value.

\section{Boating}

A shielding factor of 1.0 is recommended as a conservative assumption, meaning no shielding. Shielding value determinations would consider the height of the individual above the water surface and the construction material of the boat. Other boating parameter recommendations are somewhat arbitrary. The number of daily boating events, duration of the boating event, and boating days per year is assigned as 1 event/d, $1 \mathrm{hr} /$ event, and $5 \mathrm{~d} / \mathrm{yr}$ for all age groups. The user must determine if these values are appropriate to their location.

\section{Shoreline}

As with the other water recreation assumptions, the participation rate in shoreline activities is assigned somewhat arbitrarily. The number of daily shoreline events, duration of event, and number of annual shoreline days is assigned as 1 event/d, $3.3 \mathrm{hr} /$ event, and 5 shoreline $\mathrm{d} / \mathrm{yr}$ for all age groups. The user must determine if these values are appropriate to their location. No strong age-dependent information for water recreation times was identified. The shoreline width factors of NRC (1977, aka, Reg Guide 1.109), Table A-2, are still valid. The shoreline width factor can be approximated by the ratio of the (horizontal distance from the average high water mark to the average water level location) to the (total horizontal shore distance, i.e., total shore depth). A default shoreline width factor of 0.2 is assumed, considering a typical mixed fission source term is involved.

\subsubsection{Additional Sources of Information}

See Chapter 16, Table 16-1, of EPA (2011) for recommendations for mean and $95^{\text {th }}$ percentile minutes per month for swimming, by age.

See Chapter 16, Table 16-77 in the Moderately Active Activities row, of EPA (2011) for estimates of minutes per 2-d-period spent, in part, boating; values listed for children, by sex and age. 
See Chapter 3, Table 3-93, of EPA (2011) for details regarding swimming water ingestion during boating, canoeing, fishing, kayaking, rowing, and wading.

\subsubsection{Swimming Tables (EVSWIM, TESWIM, TSWIM, USWIM)}

For EVSWIM, TESWIM, and TSWIM, see text above.

Table 8.5. USWIM Incidental Ingestion Rate of Swimming Water

\begin{tabular}{|c|c|c|c|c|c|c|c|c|}
\hline Gv2 ID & Gv2 Description & $\operatorname{Age}^{(a)}$ & Qualifier & Reference & Comment & $\begin{array}{c}\text { Data } \\
\text { Reported }\end{array}$ & Value & Units \\
\hline$\overline{\text { USWIM }}$ & $\begin{array}{l}\text { Ingestion rate of } \\
\text { water while } \\
\text { swimming }\end{array}$ & 2 & MEI & $\begin{array}{c}\text { EPA } \\
(2011)\end{array}$ & $\begin{array}{l}\text { Chap 3, } \\
\text { 97th } \\
\text { percentile }\end{array}$ & $\begin{array}{l}97 \text { th } \\
\text { percentile } \\
\text { child }\end{array}$ & 0.12 & $\mathrm{~L} / \mathrm{hr}$ \\
\hline USWIM & $\begin{array}{l}\text { Ingestion rate of } \\
\text { water while } \\
\text { swimming }\end{array}$ & 3 & MEI & $\begin{array}{c}\text { EPA } \\
(2011)\end{array}$ & $\begin{array}{l}\text { Chap 3, } \\
\text { 97th } \\
\text { percentile }\end{array}$ & $\begin{array}{l}97 \text { th } \\
\text { percentile } \\
\text { child }\end{array}$ & 0.12 & $\mathrm{~L} / \mathrm{hr}$ \\
\hline USWIM & $\begin{array}{l}\text { Ingestion rate of } \\
\text { water while } \\
\text { swimming }\end{array}$ & 4 & MEI & $\begin{array}{c}\text { EPA } \\
(2011)\end{array}$ & $\begin{array}{l}\text { Chap 3, } \\
\text { 97th } \\
\text { percentile }\end{array}$ & $\begin{array}{l}97 \text { th } \\
\text { percentile } \\
\text { child }\end{array}$ & 0.12 & $\mathrm{~L} / \mathrm{hr}$ \\
\hline USWIM & $\begin{array}{l}\text { Ingestion rate of } \\
\text { water while } \\
\text { swimming }\end{array}$ & 5 & MEI & $\begin{array}{c}\text { EPA } \\
(2011)\end{array}$ & $\begin{array}{l}\text { Chap 3, } \\
\text { 97th } \\
\text { percentile }\end{array}$ & $\begin{array}{l}97 \text { th } \\
\text { percentile } \\
\text { child }\end{array}$ & 0.12 & $\mathrm{~L} / \mathrm{hr}$ \\
\hline USWIM & $\begin{array}{l}\text { Ingestion rate of } \\
\text { water while } \\
\text { swimming }\end{array}$ & 6 & MEI & $\begin{array}{c}\text { EPA } \\
(2011)\end{array}$ & $\begin{array}{l}\text { Chap 3, } \\
\text { 95th } \\
\text { percentile }\end{array}$ & $\begin{array}{l}95 \text { th } \\
\text { percentile } \\
\text { adult }\end{array}$ & 0.071 & $\mathrm{~L} / \mathrm{hr}$ \\
\hline
\end{tabular}

(a) See Table 8.1 for age groups.

\begin{tabular}{|c|c|c|c|c|c|c|c|c|}
\hline Gv2 ID & Gv2 Description & $\operatorname{Age}^{(a)}$ & Qualifier & Reference & Comment & $\begin{array}{c}\text { Data } \\
\text { Reported }\end{array}$ & Value & Units \\
\hline USWIM & $\begin{array}{l}\text { Ingestion rate of } \\
\text { water while } \\
\text { swimming }\end{array}$ & 2 & POP & $\begin{array}{c}\text { EPA } \\
(2011)\end{array}$ & $\begin{array}{l}\text { Chap 3, } \\
\text { average }\end{array}$ & $\begin{array}{l}\text { average } \\
\text { child }\end{array}$ & 0.049 & $\mathrm{~L} / \mathrm{hr}$ \\
\hline USWIM & $\begin{array}{l}\text { Ingestion rate of } \\
\text { water while } \\
\text { swimming }\end{array}$ & 3 & POP & $\begin{array}{c}\text { EPA } \\
(2011)\end{array}$ & $\begin{array}{l}\text { Chap } 3 \text {, } \\
\text { average }\end{array}$ & $\begin{array}{l}\text { average } \\
\text { child }\end{array}$ & 0.049 & $\mathrm{~L} / \mathrm{hr}$ \\
\hline USWIM & $\begin{array}{l}\text { Ingestion rate of } \\
\text { water while } \\
\text { swimming }\end{array}$ & 4 & POP & $\begin{array}{c}\text { EPA } \\
(2011)\end{array}$ & $\begin{array}{l}\text { Chap } 3 \text {, } \\
\text { average }\end{array}$ & $\begin{array}{l}\text { average } \\
\text { child }\end{array}$ & 0.049 & $\mathrm{~L} / \mathrm{hr}$ \\
\hline USWIM & $\begin{array}{l}\text { Ingestion rate of } \\
\text { water while } \\
\text { swimming }\end{array}$ & 5 & POP & $\begin{array}{c}\text { EPA } \\
(2011)\end{array}$ & $\begin{array}{l}\text { Chap } 3 \text {, } \\
\text { average }\end{array}$ & $\begin{array}{l}\text { average } \\
\text { child }\end{array}$ & 0.049 & $\mathrm{~L} / \mathrm{hr}$ \\
\hline USWIM & $\begin{array}{l}\text { Ingestion rate of } \\
\text { water while } \\
\text { swimming }\end{array}$ & 6 & POP & $\begin{array}{c}\text { EPA } \\
(2011)\end{array}$ & $\begin{array}{l}\text { Chap } 3 \text {, } \\
\text { average }\end{array}$ & $\begin{array}{l}\text { average } \\
\text { adult }\end{array}$ & 0.021 & $\mathrm{~L} / \mathrm{hr}$ \\
\hline
\end{tabular}

(a) See Table 8.1 for age groups. 


\subsubsection{Boating Tables (SFBOAT, EVBOAT, TEBOAT, TBOAT)}

See text, above. No tabulation necessary.

\subsubsection{Shoreline Sediments Tables (EVSHOR, TESHOR, TSHOR, SWFAC)}

See text above. No tabulation necessary.

\subsection{Pathway Selection: Food Ingestion (multiple)}

Food ingestion pathways require assignment of intake rates and consumption periods. The Pathway Selections are broken out by food crops, animal products, and aquatic food. All consumption periods for food crops (TCRP), animal products (TANM), and aquatic foods (TAQU) should be assigned as $365 \mathrm{~d} / \mathrm{yr}$. Table 8.6, Table 8.7, and Table 8.8 indicate the food types consumed by humans and examples of each. For use in reading Gv2 GID files, the ANFLabel, TFDLabel, and AQDLabel coding is indicated. Parameters related to the animal feed and forage crops of Table 8.6 were completed in the Exposure module, above. They are listed here as an indication of how crops are modeled to travel through the food chain to humans.

Note: There is no modeling of loses during food preparation in Gv2. If the assumption of nuclide loss during food preparation is desired, as indicated in IAEA (2010), external calculations could be performed on the scenario RIF or HIF files.

Table 8.6. Gv2 Animal Product Food Types and Examples

\begin{tabular}{cllcc}
\hline & Human Exposure and Receptor & & & \\
ANFLabel & Modules - Food Types & Examples or Comment & Feed & Forage \\
\hline 1 & meat animal & beef, pork & Silage+ Grain & Hay+ grain \\
2 & poultry animal & chicken, turkey & Grain & N/A \\
3 & milk animal & milk from cow or goat & Silage+ Grain & Grass \\
4 & egg animal & egg from chicken & Grain & N/A \\
\hline
\end{tabular}

Table 8.7. Gv2 Crop Food Types and Examples

\begin{tabular}{cll}
\hline & \multicolumn{1}{c}{$\begin{array}{c}\text { Human Exposure and Receptor } \\
\text { Modules - Food Types }\end{array}$} & \multicolumn{1}{c}{ Examples or Comment } \\
\hline 1 & leafy vegetables & lettuce, spinach \\
2 & root vegetables & carrot, potato \\
3 & fruits & apple, plum \\
4 & grains & Primarily wheat. Others, barley, oats \\
\hline
\end{tabular}


Table 8.8. Gv2 Aquatic Foods Food Types and Examples

\begin{tabular}{|c|c|c|}
\hline AQFLabel & $\begin{array}{c}\text { Human Exposure and Receptor } \\
\text { Modules - Food Types }\end{array}$ & Examples or Comment \\
\hline 1 & fish (fw) & pelagic fish - bass, trout \\
\hline 2 & mollusks (fw) & freshwater mussels, snails \\
\hline 3 & crustacea (fw) & crayfish, freshwater shrimp \\
\hline 4 & aquatic plants (fw) & watercress, water chestnut \\
\hline 1 & fish (marine) & pelagic fish - tuna, salmon \\
\hline 2 & mollusks (marine) & squid, oyster, clam, scallop \\
\hline 3 & crustacea (marine) & crab, lobster, shrimp \\
\hline 4 & aquatic plants (marine) & seaweed \\
\hline
\end{tabular}

Table 8.9 indicates the per capita consumption of major food commodities (mass per year) by retail mass. More important than its consumption rate information which would require correction for preparation losses is the information it provides regarding the fresh and processed fractions of food types consumed. When the relevant detailed information is available, this data can be used to apply appropriate bioconcentration factors in the Constituents module and intake delay times in the exposure module. It can also be used to appropriately distribute relative consumption rates of various food types (e.g., chicken intakes are about 65 percent of red meat intakes).

Table 8.9. Distribution of Per Capita Food Consumption (lb/yr) from 2000 to 2009

\begin{tabular}{|c|c|c|c|c|c|}
\hline & $\begin{array}{l}2000-2009 \\
\text { Average }^{(a)}\end{array}$ & $\begin{array}{c}2000-2009 \\
\operatorname{Min}^{(a)}\end{array}$ & $\begin{array}{c}2000-2009 \\
\operatorname{Max}^{(a)}\end{array}$ & $2009^{(a)}$ & $\begin{array}{c}2009 \text { Percent } \\
\text { of Category } \\
\text { Total }\end{array}$ \\
\hline Red meats total $^{(b)}$ & 110.6 & 105.7 & 114.1 & 105.7 & - \\
\hline Beef & 62.2 & 58.1 & 64.5 & 58.1 & $55 \%$ \\
\hline Veal & 0.4 & 0.3 & 0.5 & 0.3 & $0.3 \%$ \\
\hline Lamb and mutton & 0.8 & 0.7 & 0.9 & 0.7 & $0.7 \%$ \\
\hline Pork & 47.2 & 45.9 & 48.5 & 46.6 & $44 \%$ \\
\hline Poultry total $^{(b)}$ & 71.4 & 67.8 & 74.2 & 69.4 & - \\
\hline Chicken & 57.8 & 54.0 & 60.9 & 56.0 & $81 \%$ \\
\hline Turkey & 13.6 & 13.2 & 14.0 & 13.3 & $19 \%$ \\
\hline Fish and shellfish $^{(b)}$ & 15.9 & 14.7 & 16.5 & 15.8 & - \\
\hline Eggs (number) & 253 & 246 & 258 & 246 & - \\
\hline Listed Dairy Products total & 596.5 & 585.2 & 607.1 & 607.1 & - \\
\hline Cheese total (excluding cottage cheese) & 31.5 & 29.4 & 33.7 & 32.8 & - \\
\hline American & 12.9 & 12.5 & 13.4 & 13.4 & $41 \%$ \\
\hline Italian & 13.2 & 12.1 & 14.3 & 13.9 & $42 \%$ \\
\hline Other & 5.4 & 4.8 & 6.0 & 5.5 & $17 \%$ \\
\hline Cottage cheese & 2.6 & 2.3 & 2.7 & 2.4 & - \\
\hline Beverage milks (gallons) & 21.3 & 20.6 & 22.5 & 20.6 & - \\
\hline Fluid cream products $(1 / 2 \text { pints })^{(\mathrm{c})}$ & 22.4 & 18.3 & 24.7 & 23.6 & - \\
\hline Yogurt (excluding frozen) (1/2 pints) & 17.7 & 12.0 & 23.1 & 23.1 & - \\
\hline Ice cream & 15.1 & 13.4 & 16.7 & 13.4 & - \\
\hline
\end{tabular}


Table 8.9. (contd)

\begin{tabular}{|c|c|c|c|c|c|}
\hline & $\begin{array}{l}2000-2009 \\
\text { Average }^{(a)}\end{array}$ & $\begin{array}{c}2000-2009 \\
\operatorname{Min}^{(a)}\end{array}$ & $\begin{array}{c}2000-2009 \\
\operatorname{Max}^{(a)}\end{array}$ & $2009^{(a)}$ & $\begin{array}{c}2009 \text { Percent } \\
\text { of Category } \\
\text { Total }\end{array}$ \\
\hline Low-fat ice cream & 7.0 & 6.5 & 7.5 & 6.8 & - \\
\hline Sherbet & 1.2 & 1.1 & 1.3 & 1.1 & - \\
\hline Frozen yogurt & 1.4 & 1.1 & 2.0 & 1.1 & - \\
\hline Fats and oils ${ }^{(\mathrm{d})}$ & 84.3 & 78.6 & 87.3 & 78.6 & - \\
\hline Butter (product weight) & 4.6 & 4.4 & 5.0 & 4.9 & $7 \%$ \\
\hline Margarine (product weight) & 5.3 & 3.7 & 8.2 & 3.7 & $5 \%$ \\
\hline Lard (direct use) & 1.3 & 0.8 & 1.7 & 1.5 & $2 \%$ \\
\hline Edible beef tallow (direct use) & 3.2 & 0.7 & 4.0 & 0.7 & $1 \%$ \\
\hline Shortening & 27.0 & 15.9 & 32.8 & 15.9 & $21 \%$ \\
\hline Salad and cooking oils & 43.3 & 33.7 & 54.2 & 51.9 & $69 \%$ \\
\hline Other edible fats and oils & 1.6 & 1.3 & 2.1 & 1.7 & $2 \%$ \\
\hline Flour and cereal products ${ }^{(e)}$ & 194.3 & 191.3 & 199.2 & 194.5 & - \\
\hline Wheat flour & 137.5 & 134.3 & 146.3 & 134.6 & $69 \%$ \\
\hline Rice, milled & 20.1 & 18.9 & 21.2 & 21.2 & $11 \%$ \\
\hline Corn products & 31.0 & 28.4 & 33.0 & 33.0 & $17 \%$ \\
\hline 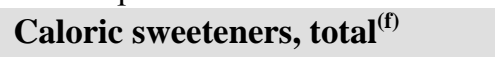 & 140.9 & 130.7 & 148.9 & 130.7 & - \\
\hline Sugar, refined cane and beet & 63.2 & 60.9 & 65.5 & 63.5 & $49 \%$ \\
\hline Corn sweeteners ${ }^{(\mathrm{g})}$ & 76.3 & 65.7 & 81.8 & 65.7 & $50 \%$ \\
\hline \multicolumn{6}{|l|}{ Fruits and vegetables ${ }^{(h)}$} \\
\hline Fruit total & 271.2 & 256.6 & 286.0 & 257.0 & - \\
\hline Fresh fruits & 126.7 & 123.5 & 128.5 & 127.5 & $50 \%$ \\
\hline Canned fruit & 16.5 & 15.4 & 17.6 & 15.5 & $6 \%$ \\
\hline Dried fruit & 9.9 & 9.2 & 10.5 & 9.2 & $4 \%$ \\
\hline Frozen fruit & 4.8 & 4.1 & 5.3 & 4.9 & $2 \%$ \\
\hline Selected fruit juices & 112.8 & 99.0 & 124.6 & 99.2 & $39 \%$ \\
\hline Vegetables total & 410.8 & 390.9 & 424.6 & 390.9 & - \\
\hline Fresh & 196.0 & 184.8 & 204.5 & 184.8 & $47 \%$ \\
\hline Canning & 99.4 & 94.4 & 104.8 & 100.4 & $26 \%$ \\
\hline Freezing & 76.4 & 71.3 & 79.4 & 71.3 & $18 \%$ \\
\hline Dehydrated and chips & 31.7 & 27.4 & 34.5 & 27.4 & $7 \%$ \\
\hline Legumes & 7.3 & 6.7 & 8.5 & 6.9 & $2 \%$ \\
\hline \multicolumn{6}{|l|}{ Other/Miscellaneous } \\
\hline Cocoa beans & 5.8 & 4.8 & 6.5 & 5.5 & - \\
\hline Coffee (green beans) & 9.5 & 9.1 & 10.3 & 9.1 & - \\
\hline Peanuts (shelled) & 6.3 & 5.8 & 6.6 & 6.5 & - \\
\hline Tree nuts (shelled) & 3.2 & 2.6 & 3.7 & 3.7 & - \\
\hline
\end{tabular}

Table derived from Food Supply and Use; Table 39--Per Capita Consumption of Major Food Commodities.

Found at http://www.ers.usda.gov/Publications/AgOutlook/AOTables/.
(a) Pounds retail weight unless otherwise indicated.
(b) Boneless trimmed weight.
(c) Includes heavy cream, light cream, half-and-half, and eggnog.
(d) Fat content mass, only.
(e) Includes rye, corn, oats, and barley products.
(f) Dry weight. Includes edible syrups (e.g., maple, molasses, honey)
(g) Includes high fructose corn syrup, glucose, and dextrose.
(h) Farm weight. 


\subsubsection{Ingestion Exposure: Food Crops}

Four food crop groups considered: leafy vegetables, root vegetables, fruit, and grain.

Set all consumption periods (TCRP) for all food groups and age groups to 365 .

The units and range for UCRP in Gv2 are: $\mathrm{kg} / \mathrm{d}(0-10)$.

The units and range for TCRP in Gv2 are: $\mathrm{d} / \mathrm{yr}(0-365)$.

Information at

www.census.gov/compendia/statab/cats/health nutrition/food_consumption and nutrition.html provides United States per capita consumption rates (mass based) for a variety of food categories over time.

Values are derived from EPA (2011), which are presented in units of $\mathrm{kg} / \mathrm{kg}$-d based on the body weight of the consumer. Assumed age-specific body weights used for units conversions are indicated in Table 8.10, from EPA (2011, Chapter 8). Then, intakes are averaged to determine Gv2 age-specific values, as indicate in the table.

Table 8.10. Age-Specific Body Weights.

\begin{tabular}{llc}
\hline \multicolumn{1}{c}{ Age Group } & $\mathrm{kg}$ & Gv2 Age Category \\
\hline Birth to 1 year & 6.825 & 1 \\
1 to $<2$ years & 11.4 & 1 \\
2 to $<3$ years & 13.8 & 2 \\
3 to $<6$ years & 18.6 & 3 \\
6 to $<11$ years & 31.8 & 4 \\
11 to $<16$ years & 56.8 & 5 \\
16 to $<21$ years & 71.6 & 5 \\
21 to $<50$ years & 80 & 6 \\
$\geq 50$ years & 80 & 6 \\
\hline
\end{tabular}

(a) See Table 8.1 for age ranges in each age category.

\subsubsection{Additional Sources of Information}

http://www.ars.usda.gov/Services/docs.htm?docid=22112. The USDA Agricultural Research Service publishes the National Nutrient Database for Standard Reference information providing nutrition information for numerous foods. Among the groups of foods reported are dairy and egg, finfish and shellfish, beef and other specific meat groups, fruits and juices, legumes, and vegetables. Useful information includes water content, mineral content, percent of refuse for specific foods, and mass of cooked meat from raw meat. The data were derived from Gebhardt and Thomas (2002), which also provides a table of particular foods high in evaluated minerals (Gebhardt and Thomas 2002, Table 5).

The USDA Agricultural Handbook Number 697 (USDA 1992) provides weights, measures, and conversion factors for agricultural commodities and their products. Conversions from farm weights are provided. Table 27 indicates corn fractions for various foods containing corn. Wheat, soybeans, and oils are covered in other tables. Table 38 indicates the mass of canned product from average masses of 
farm-weight fresh product. Table 40 indicates farm-weight per final volume of various juices. Tables 41 and 42 indicate farm-weight to processed weight conversions for several fruits. Tables 45 and 46 indicate farm-weight to canned weight for vegetables. Nut information is found in Table 51. Honey and maple syrup volume-to-mass conversions are found in Table 57.

\subsubsection{UCRP Ingestion Rate of Food Crops Tables}

Table 8.11. UCRP Ingestion Rate - Human Intake of Food Crop Categories

\begin{tabular}{|c|c|c|c|c|c|c|c|c|}
\hline Gv2 ID & Age & $\begin{array}{l}\text { Food } \\
\text { Type }\end{array}$ & Qualifier & Reference & Comment & Data Reported & Value & Units \\
\hline UCRP & 1 & 1.leafy & MEI & $\overline{\text { EPA (2011) }}$ & $\begin{array}{l}\text { Ch 9, consumers only } \\
\text { Veg }\end{array}$ & $\begin{array}{l}\text { derivation, consumers } 95 \text { th, } \\
\text { Root/Leafy breakout }\end{array}$ & 0.0121 & $\overline{\mathrm{kg} / \text { day }}$ \\
\hline UCRP & 1 & 2.root & MEI & EPA (2011) & Ch 9, consumers only & derivation, consumers 95 th, & 0.1386 & $\mathrm{~kg} /$ day \\
\hline UCRP & 1 & 3. fruit & MEI & EPA (2011) & $\begin{array}{l}\text { Ch } 9 \text {, consumers only } \\
\text { Fruit }\end{array}$ & $\begin{array}{l}\text { derivation, consumers } \\
\text { 95th percentile }\end{array}$ & 0.21 & $\mathrm{~kg} /$ day \\
\hline UCRP & 1 & 4.grain & MEI & EPA (2011) & $\begin{array}{l}\text { Table } 12-1 \text {, consumers } \\
\text { only } 95 \text { th, grain }\end{array}$ & $\begin{array}{l}\text { derivation, consumers only } \\
\text { 95th, grain }\end{array}$ & 0.1058 & $\mathrm{~kg} /$ day \\
\hline UCRP & 2 & 1.leafy & MEI & EPA (2011) & $\begin{array}{l}\text { Ch 9, consumers only } \\
\text { Veg }\end{array}$ & $\begin{array}{l}\text { derivation, consumers } 95 \text { th, } \\
\text { Root/Leafy breakout }\end{array}$ & 0.0388 & $\mathrm{~kg} /$ day \\
\hline UCRP & 2 & 2.root & MEI & EPA (2011) & $\begin{array}{l}\text { Ch } 9 \text {, consumers only } \\
\text { Veg }\end{array}$ & $\begin{array}{l}\text { derivation, consumers } 95 \text { th, } \\
\text { Root/Leafy breakout }\end{array}$ & 0.1765 & $\mathrm{~kg} /$ day \\
\hline UCRP & 2 & 3.fruit & MEI & EPA (2011) & $\begin{array}{l}\text { Ch } 9 \text {, consumers only } \\
\text { Fruit }\end{array}$ & $\begin{array}{l}\text { derivation, consumers } \\
\text { 95th percentile }\end{array}$ & 0.295 & $\mathrm{~kg} /$ day \\
\hline UCRP & 2 & 4.grain & MEI & EPA (2011) & $\begin{array}{l}\text { Table } 12-1 \text {, consumers } \\
\text { only } 95 \text { th, grain }\end{array}$ & $\begin{array}{l}\text { derivation, consumers only } \\
\text { 95th, grain }\end{array}$ & 0.1711 & $\mathrm{~kg} /$ day \\
\hline UCRP & 3 & 1.leafy & MEI & EPA (2011) & $\begin{array}{l}\text { Ch 9, consumers only } \\
\text { Veg }\end{array}$ & $\begin{array}{l}\text { derivation, consumers } 95 \text { th, } \\
\text { Root/Leafy breakout }\end{array}$ & 0.0474 & $\mathrm{~kg} /$ day \\
\hline UCRP & 3 & 2.root & MEI & EPA (2011) & $\begin{array}{l}\text { Ch } 9 \text {, consumers only } \\
\text { Veg }\end{array}$ & $\begin{array}{l}\text { derivation, consumers } 95 \text { th, } \\
\text { Root/Leafy breakout }\end{array}$ & 0.2019 & $\mathrm{~kg} /$ day \\
\hline UCRP & 3 & 3. fruit & MEI & EPA (2011) & $\begin{array}{l}\text { Ch } 9 \text {, consumers only } \\
\text { Fruit }\end{array}$ & $\begin{array}{l}\text { derivation, consumers } \\
\text { 95th percentile }\end{array}$ & 0.2809 & $\mathrm{~kg} /$ day \\
\hline UCRP & 3 & 4.grain & MEI & EPA (2011) & $\begin{array}{l}\text { Table } 12-1 \text {, consumers } \\
\text { only } 95 \text { th, grain }\end{array}$ & $\begin{array}{l}\text { derivation, consumers only } \\
\text { 95th, grain }\end{array}$ & 0.2065 & $\mathrm{~kg} /$ day \\
\hline UCRP & 4 & 1.leafy & MEI & EPA (2011) & $\begin{array}{l}\text { Ch } 9 \text {, consumers only } \\
\text { Veg }\end{array}$ & $\begin{array}{l}\text { derivation, consumers } 95 \text { th, } \\
\text { Root/Leafy breakout }\end{array}$ & 0.0628 & $\mathrm{~kg} /$ day \\
\hline UCRP & 4 & 2.root & MEI & EPA (2011) & $\begin{array}{l}\text { Ch } 9 \text {, consumers only } \\
\text { Veg }\end{array}$ & $\begin{array}{l}\text { derivation, consumers } 95 \text { th, } \\
\text { Root/Leafy breakout }\end{array}$ & 0.2679 & $\mathrm{~kg} /$ day \\
\hline UCRP & 4 & 3. fruit & MEI & EPA (2011) & $\begin{array}{l}\text { Ch } 9 \text {, consumers only } \\
\text { Fruit }\end{array}$ & $\begin{array}{l}\text { derivation, consumers } \\
\text { 95th percentile }\end{array}$ & 0.2926 & $\mathrm{~kg} /$ day \\
\hline UCRP & 4 & 4.grain & MEI & EPA (2011) & $\begin{array}{l}\text { Table } 12-1 \text {, consumers } \\
\text { only } 95 \text { th, grain }\end{array}$ & $\begin{array}{l}\text { derivation, consumers only } \\
\text { 95th, grain }\end{array}$ & 0.2608 & $\mathrm{~kg} /$ day \\
\hline UCRP & 5 & 1.leafy & MEI & EPA (2011) & $\begin{array}{l}\text { Ch } 9 \text {, consumers only } \\
\text { Veg }\end{array}$ & $\begin{array}{l}\text { derivation, consumers } 95 \text { th, } \\
\text { Root/Leafy breakout }\end{array}$ & 0.0918 & $\mathrm{~kg} /$ day \\
\hline UCRP & 5 & 2.root & MEI & EPA (2011) & $\begin{array}{l}\text { Ch } 9 \text {, consumers only } \\
\text { Veg }\end{array}$ & $\begin{array}{l}\text { derivation, consumers } 95 \text { th, } \\
\text { Root/Leafy breakout }\end{array}$ & 0.2613 & $\mathrm{~kg} /$ day \\
\hline UCRP & 5 & 3.fruit & MEI & EPA (2011) & $\begin{array}{l}\text { Ch } 9 \text {, consumers only } \\
\text { Fruit }\end{array}$ & $\begin{array}{l}\text { derivation, consumers } \\
\text { 95th percentile }\end{array}$ & 0.244 & $\mathrm{~kg} /$ day \\
\hline UCRP & 5 & 4. grain & MEI & EPA (2011) & $\begin{array}{l}\text { Table } 12-1 \text {, consumers } \\
\text { only } 95 \text { th, grain }\end{array}$ & $\begin{array}{l}\text { derivation, consumers only } \\
\text { 95th, grain }\end{array}$ & 0.3210 & $\mathrm{~kg}$ /day \\
\hline UCRP & 6 & 1.leafy & MEI & EPA (2011) & $\begin{array}{l}\text { Ch } 9 \text {, consumers only } \\
\text { Veg }\end{array}$ & $\begin{array}{l}\text { derivation, consumers } 95 \text { th, } \\
\text { Root/Leafy breakout }\end{array}$ & 0.1872 & $\mathrm{~kg} /$ day \\
\hline UCRP & 6 & 2.root & MEI & EPA (2011) & $\begin{array}{l}\text { Ch } 9 \text {, consumers only } \\
\text { Veg }\end{array}$ & $\begin{array}{l}\text { derivation, consumers } 95 \text { th, } \\
\text { Root/Leafy breakout }\end{array}$ & 0.2928 & $\mathrm{~kg} /$ day \\
\hline UCRP & 6 & 3.fruit & MEI & EPA (2011) & $\begin{array}{l}\text { Ch 9, consumers only } \\
\text { Fruit }\end{array}$ & $\begin{array}{l}\text { derivation, consumers } \\
\text { 95th percentile }\end{array}$ & 0.336 & $\mathrm{~kg} /$ day \\
\hline UCRP & 6 & 4.grain & MEI & EPA (2011) & $\begin{array}{l}\text { Table } 12-1 \text {, consumers } \\
\text { only } 95 \text { th, grain }\end{array}$ & $\begin{array}{l}\text { derivation, consumers only } \\
\text { 95th, grain }\end{array}$ & 0.3240 & $\mathrm{~kg} /$ day \\
\hline
\end{tabular}


Table 8.11. (contd)

\begin{tabular}{|c|c|c|c|c|c|c|c|c|}
\hline Gv2 ID & Age & $\begin{array}{l}\text { Food } \\
\text { Type }\end{array}$ & Qualifier & Reference & Comment & Data Reported & Value & Units \\
\hline UCRP & 1 & 1.leafy & POP & EPA (2011) & $\begin{array}{l}\text { Ch 9, consumers only } \\
\text { Veg }\end{array}$ & $\begin{array}{l}\text { derivation, consumers } \\
\text { mean, Root/Leafy breakout }\end{array}$ & 0.0049 & $\mathrm{~kg} /$ day \\
\hline UCRP & 1 & 2.root & POP & EPA (2011) & $\begin{array}{l}\text { Ch } 9 \text {, consumers only } \\
\text { Veg }\end{array}$ & $\begin{array}{l}\text { derivation, consumers } \\
\text { mean, Root/Leafy breakout }\end{array}$ & 0.0565 & $\mathrm{~kg}$ /day \\
\hline UCRP & 1 & 3. fruit & POP & EPA (2011) & $\begin{array}{l}\text { Ch 9, consumers only } \\
\text { Fruit }\end{array}$ & $\begin{array}{l}\text { derivation, consumers } \\
\text { mean }\end{array}$ & 0.0806 & $\mathrm{~kg}$ /day \\
\hline UCRP & 1 & 4.grain & POP & EPA (2011) & $\begin{array}{l}\text { Table } 12-1 \text {, consumers } \\
\text { only mean, grain }\end{array}$ & $\begin{array}{l}\text { derivation, consumers only } \\
\text { mean, grain }\end{array}$ & 0.0505 & $\mathrm{~kg}$ /day \\
\hline UCRP & 2 & 1.leafy & POP & EPA (2011) & $\begin{array}{l}\text { Ch } 9 \text {, consumers only } \\
\text { Veg }\end{array}$ & $\begin{array}{l}\text { derivation, consumers } \\
\text { mean, Root/Leafy breakout }\end{array}$ & 0.0166 & $\mathrm{~kg} /$ day \\
\hline UCRP & 2 & 2.root & POP & EPA (2011) & $\begin{array}{l}\text { Ch } 9 \text {, consumers only } \\
\text { Veg }\end{array}$ & $\begin{array}{l}\text { derivation, consumers } \\
\text { mean, Root/Leafy breakout }\end{array}$ & 0.0758 & $\mathrm{~kg}$ /day \\
\hline UCRP & 2 & 3. fruit & POP & EPA (2011) & $\begin{array}{l}\text { Ch } 9 \text {, consumers only } \\
\text { Fruit }\end{array}$ & $\begin{array}{l}\text { derivation, consumers } \\
\text { mean }\end{array}$ & 0.1118 & $\mathrm{~kg} /$ day \\
\hline UCRP & 2 & 4.grain & POP & EPA (2011) & $\begin{array}{l}\text { Table } 12-1 \text {, consumers } \\
\text { only mean, grain }\end{array}$ & $\begin{array}{l}\text { derivation, consumers only } \\
\text { mean, grain }\end{array}$ & 0.0883 & $\mathrm{~kg}$ /day \\
\hline UCRP & 3 & 1.leafy & POP & EPA (2011) & $\begin{array}{l}\text { Ch } 9 \text {, consumers only } \\
\text { Veg }\end{array}$ & $\begin{array}{l}\text { derivation, consumers } \\
\text { mean, Root/Leafy breakout }\end{array}$ & 0.0191 & $\mathrm{~kg}$ /day \\
\hline UCRP & 3 & 2.root & POP & EPA (2011) & $\begin{array}{l}\text { Ch } 9 \text {, consumers only } \\
\text { Veg }\end{array}$ & $\begin{array}{l}\text { derivation, consumers } \\
\text { mean, Root/Leafy breakout }\end{array}$ & 0.0814 & $\mathrm{~kg}$ /day \\
\hline UCRP & 3 & 3. fruit & POP & EPA (2011) & $\begin{array}{l}\text { Ch } 9 \text {, consumers only } \\
\text { Fruit }\end{array}$ & $\begin{array}{l}\text { derivation, consumers } \\
\text { mean }\end{array}$ & 0.0874 & $\mathrm{~kg}$ /day \\
\hline UCRP & 3 & 4.grain & POP & EPA (2011) & $\begin{array}{l}\text { Table } 12-1 \text {, consumers } \\
\text { only mean, grain }\end{array}$ & $\begin{array}{l}\text { derivation, consumers only } \\
\text { mean, grain }\end{array}$ & 0.1153 & $\mathrm{~kg}$ /day \\
\hline UCRP & 4 & 1.leafy & POP & EPA (2011) & $\begin{array}{l}\text { Ch 9, consumers only } \\
\text { Veg }\end{array}$ & $\begin{array}{l}\text { derivation, consumers } \\
\text { mean, Root/Leafy breakout }\end{array}$ & 0.0224 & $\mathrm{~kg}$ /day \\
\hline UCRP & 4 & 2.root & POP & EPA (2011) & $\begin{array}{l}\text { Ch } 9 \text {, consumers only } \\
\text { Veg }\end{array}$ & $\begin{array}{l}\text { derivation, consumers } \\
\text { mean, Root/Leafy breakout }\end{array}$ & 0.0953 & $\mathrm{~kg}$ /day \\
\hline UCRP & 4 & 3. fruit & POP & EPA (2011) & $\begin{array}{l}\text { Ch } 9 \text {, consumers only } \\
\text { Fruit }\end{array}$ & $\begin{array}{l}\text { derivation, consumers } \\
\text { mean }\end{array}$ & 0.0795 & $\mathrm{~kg}$ /day \\
\hline UCRP & 4 & 4.grain & POP & EPA (2011) & $\begin{array}{l}\text { Table } 12-1 \text {, consumers } \\
\text { only mean, grain }\end{array}$ & $\begin{array}{l}\text { derivation, consumers only } \\
\text { mean, grain }\end{array}$ & 0.1399 & $\mathrm{~kg} /$ day \\
\hline UCRP & 5 & 1.leafy & POP & EPA (2011) & $\begin{array}{l}\text { Ch } 9 \text {, consumers only } \\
\text { Veg }\end{array}$ & $\begin{array}{l}\text { derivation, consumers } \\
\text { mean, Root/Leafy breakout }\end{array}$ & 0.0384 & $\mathrm{~kg} /$ day \\
\hline UCRP & 5 & 2.root & POP & EPA (2011) & $\begin{array}{l}\text { Ch } 9 \text {, consumers only } \\
\text { Veg }\end{array}$ & $\begin{array}{l}\text { derivation, consumers } \\
\text { mean, Root/Leafy breakout }\end{array}$ & 0.1093 & $\mathrm{~kg}$ /day \\
\hline UCRP & 5 & 3. fruit & POP & EPA (2011) & $\begin{array}{l}\text { Ch 9, consumers only } \\
\text { Fruit }\end{array}$ & $\begin{array}{l}\text { derivation, consumers } \\
\text { mean }\end{array}$ & 0.0706 & $\mathrm{~kg} /$ day \\
\hline UCRP & 5 & 4.grain & POP & EPA (2011) & $\begin{array}{l}\text { Table } 12-1 \text {, consumers } \\
\text { only mean, grain }\end{array}$ & $\begin{array}{l}\text { derivation, consumers only } \\
\text { mean, grain }\end{array}$ & 0.1541 & $\mathrm{~kg}$ /day \\
\hline UCRP & 6 & 1.leafy & POP & EPA (2011) & $\begin{array}{l}\text { Ch } 9 \text {, consumers only } \\
\text { Veg }\end{array}$ & $\begin{array}{l}\text { derivation, consumers } \\
\text { mean, Root/Leafy breakout }\end{array}$ & 0.0796 & $\mathrm{~kg} /$ day \\
\hline UCRP & 6 & 2.root & POP & EPA (2011) & $\begin{array}{l}\text { Ch } 9 \text {, consumers only } \\
\text { Veg }\end{array}$ & $\begin{array}{l}\text { derivation, consumers } \\
\text { mean, Root/Leafy breakout }\end{array}$ & 0.1244 & $\mathrm{~kg}$ /day \\
\hline UCRP & 6 & 3.fruit & POP & EPA (2011) & $\begin{array}{l}\text { Ch } 9 \text {, consumers only } \\
\text { Fruit }\end{array}$ & $\begin{array}{l}\text { derivation, consumers } \\
\text { mean }\end{array}$ & 0.104 & $\mathrm{~kg}$ /day \\
\hline UCRP & 6 & 4.grain & POP & EPA (2011) & $\begin{array}{l}\text { Table } 12-1 \text {, consumers } \\
\text { only mean, grain }\end{array}$ & $\begin{array}{l}\text { derivation, consumers only } \\
\text { mean, grain }\end{array}$ & 0.1560 & $\mathrm{~kg}$ /day \\
\hline
\end{tabular}

\subsubsection{Ingestion Exposure: Terrestrial Animal Products}

Four land-based animal product groups are considered: meat, poultry, milk, and eggs. Assessed individuals are not assumed to be vegetarians. Consumption of the full range of animal products should be assumed in most cases. Set all consumption periods (TANM) for all food groups and age groups to 365. 
The units and range for UANM in Gv2 are: $\mathrm{kg} / \mathrm{d}(0-10)$.

The units and range for TANM in Gv2 are: $\mathrm{d} / \mathrm{yr}(0-365)$.

The MEI intake rates were approximated from U.S. Census Bureau (USCB) summaries (USCB 2012, Table 217) of U.S. per capita meat consumption ${ }^{1}$ data, which indicates that 2009 meat consumption $(0.131 \mathrm{~kg} / \mathrm{d})$ was predominantly beef (55 percent) and pork (44 percent). Poultry consumption, $0.087 \mathrm{~kg} / \mathrm{d}$ in 2009 , was predominantly chicken ( 81 percent) with turkey (19 percent) accounting for the remainder. Per capita beverage milk consumption was $0.213 \mathrm{~L} / \mathrm{d}$. Per capita egg consumption was $173 \mathrm{eggs} / \mathrm{yr}(0.474 \mathrm{~kg} / \mathrm{d})$ with an additional $73 \mathrm{eggs} / \mathrm{yr}(0.201 \mathrm{~kg} / \mathrm{d})$ consumed in various processed foods. These values were assumed to be adult intake rates. Age-dependency was assigned from the population data, described next. The rates for the MEI of each age were the product of the adult intake rate and the ratio of the age group $\mathrm{X}$ to age group 6 rate of the population values. The age ratios indicated that meat and egg consumption increased with age; poultry consumption increased with age with a slightly higher intake for age group 5 than for age group 6; and milk consumption decreased with age. Sebastian et al. (2010) noted a statistically significant decrease in milk consumption between the late 1970s and the early 2000 s in the United States. The largest decrease was in the 12-19 year old age group. In addition, the percentage reporting any milk consumption decreased in every age group.

Age-dependent population data were derived from EPA (2011), Table 11-5 for meat and poultry, Table 11-4 for total dairy products which are interpreted for modeling as fluid milk consumption, and Table 11-12 for eggs. The body masses used to convert units from $\mathrm{kg} / \mathrm{kg}-\mathrm{d}$ to $\mathrm{kg} / \mathrm{d}$ are found in Table 8.10, above. The indicated infant milk consumption rates are likely very conservative, since nursing or formula-fed infants would warrant adjusted values. Siega-Riz et al (2010) indicates that direct cow milk consumption (i.e., the not highly processed cow milk found in infant formulas) only becomes a significant part of infant diets after they are one year old.

\subsubsection{Additional Sources of Information}

The USDA Agricultural Handbook Number 697 (USDA 1992) provides weights, measures, and conversion factors for agricultural commodities and their products. In particular, Tables 7 and 11 provide factors to use in converting carcass weight to boneless, raw meat weights for beef, pork, veal, and lamb. Table 8 provides live weights and carcass weights for cattle, sheep, calves, and hogs. Milk and egg information is also provided in the reference.

The U.S. Census Bureau provides per capita consumption information for a variety of animal products and food commodities online. Last accessed at: www.census.gov/compendia/statab/cats/health_nutrition/food_consumption_and_nutrition.html.

\footnotetext{
${ }^{1}$ Information last accessed at: www.census.gov/compendia/statab/cats/health_nutrition/food_consumption_and_nutrition.html.
} 


\subsubsection{UANM Ingestion Rate of Meat, Poultry, Milk, and Eggs Tables}

Table 8.12. UANM Ingestion Rates - Human Intake of Animal Product Categories

\begin{tabular}{|c|c|c|c|c|c|c|c|c|}
\hline Gv2 ID & $\begin{array}{c}\text { Gv2 } \\
\text { Description }\end{array}$ & $\operatorname{Age}^{(a)}$ & $\begin{array}{l}\text { Food } \\
\text { Type }\end{array}$ & Qualifier & Reference & Comment & Value & Units \\
\hline UANM & $\begin{array}{l}\text { Animal } \\
\text { product } \\
\text { consumption }\end{array}$ & age. 1 & 1.meat & MEI & $\begin{array}{l}\text { USCB (2012) } \\
\text { with age- } \\
\text { dependency } \\
\text { from EPA } \\
(2011)\end{array}$ & $\begin{array}{l}\text { Intake is relatively } \\
\text { unsustainable. Assume all } \\
\text { protein is from this one } \\
\text { category and consider no } \\
\text { other animal product } \\
\text { categories. }\end{array}$ & 0.023 & $\mathrm{~kg} / \mathrm{d}$ \\
\hline UANM & $\begin{array}{l}\text { Animal } \\
\text { product } \\
\text { consumption }\end{array}$ & age. 2 & 1.meat & MEI & $\begin{array}{l}\text { USCB (2012) } \\
\text { with age- } \\
\text { dependency } \\
\text { from EPA } \\
(2011)\end{array}$ & $\begin{array}{l}\text { Intake is relatively } \\
\text { unsustainable. Assume all } \\
\text { protein is from this one } \\
\text { category and consider no } \\
\text { other animal product } \\
\text { categories. }\end{array}$ & 0.048 & $\mathrm{~kg} / \mathrm{d}$ \\
\hline UANM & $\begin{array}{l}\text { Animal } \\
\text { product } \\
\text { consumption }\end{array}$ & age. 3 & 1.meat & MEI & $\begin{array}{l}\text { USCB (2012) } \\
\text { with age- } \\
\text { dependency } \\
\text { from EPA } \\
(2011)\end{array}$ & $\begin{array}{l}\text { Intake is relatively } \\
\text { unsustainable. Assume all } \\
\text { protein is from this one } \\
\text { category and consider no } \\
\text { other animal product } \\
\text { categories. }\end{array}$ & 0.067 & $\mathrm{~kg} / \mathrm{d}$ \\
\hline UANM & $\begin{array}{l}\text { Animal } \\
\text { product } \\
\text { consumption }\end{array}$ & age. 4 & 1.meat & MEI & $\begin{array}{l}\text { USCB (2012) } \\
\text { with age- } \\
\text { dependency } \\
\text { from EPA } \\
(2011)\end{array}$ & $\begin{array}{l}\text { Intake is relatively } \\
\text { unsustainable. Assume all } \\
\text { protein is from this one } \\
\text { category and consider no } \\
\text { other animal product } \\
\text { categories. }\end{array}$ & 0.085 & $\mathrm{~kg} / \mathrm{d}$ \\
\hline UANM & $\begin{array}{l}\text { Animal } \\
\text { product } \\
\text { consumption }\end{array}$ & age. 5 & 1.meat & MEI & $\begin{array}{l}\text { USCB (2012) } \\
\text { with age- } \\
\text { dependency } \\
\text { from EPA } \\
(2011)\end{array}$ & $\begin{array}{l}\text { Intake is relatively } \\
\text { unsustainable. Assume all } \\
\text { protein is from this one } \\
\text { category and consider no } \\
\text { other animal product } \\
\text { categories. }\end{array}$ & 0.125 & $\mathrm{~kg} / \mathrm{d}$ \\
\hline UANM & $\begin{array}{l}\text { Animal } \\
\text { product } \\
\text { consumption }\end{array}$ & age. 6 & 1.meat & MEI & USCB (2012) & $\begin{array}{l}\text { Reference indicates beef } \\
(55 \%) \text {, veal, lamb }(1 \%) \\
\text { pork }(44 \%)\end{array}$ & 0.131 & $\mathrm{~kg} / \mathrm{d}$ \\
\hline UANM & $\begin{array}{l}\text { Animal } \\
\text { product } \\
\text { consumption }\end{array}$ & age. 1 & 2.poultry & MEI & $\begin{array}{l}\text { USCB (2012), } \\
\text { EPA (2011) }\end{array}$ & $\begin{array}{l}\text { Age-dependency derived } \\
\text { from EPA (2011) }\end{array}$ & 0.023 & $\mathrm{~kg} / \mathrm{d}$ \\
\hline UANM & $\begin{array}{l}\text { Animal } \\
\text { product } \\
\text { consumption }\end{array}$ & age. 2 & 2.poultry & MEI & $\begin{array}{l}\text { USCB (2012), } \\
\text { EPA (2011) }\end{array}$ & $\begin{array}{l}\text { Age-dependency derived } \\
\text { from EPA (2011) }\end{array}$ & 0.046 & $\mathrm{~kg} / \mathrm{d}$ \\
\hline UANM & $\begin{array}{l}\text { Animal } \\
\text { product } \\
\text { consumption }\end{array}$ & age. 3 & 2.poultry & MEI & $\begin{array}{l}\text { USCB (2012), } \\
\text { EPA (2011) }\end{array}$ & $\begin{array}{l}\text { Age-dependency derived } \\
\text { from EPA (2011) }\end{array}$ & 0.055 & $\mathrm{~kg} / \mathrm{d}$ \\
\hline UANM & $\begin{array}{l}\text { Animal } \\
\text { product } \\
\text { consumption }\end{array}$ & age. 4 & 2.poultry & MEI & $\begin{array}{l}\text { USCB (2012), } \\
\text { EPA (2011) }\end{array}$ & $\begin{array}{l}\text { Age-dependency derived } \\
\text { from EPA (2011) }\end{array}$ & 0.067 & $\mathrm{~kg} / \mathrm{d}$ \\
\hline UANM & $\begin{array}{l}\text { Animal } \\
\text { product } \\
\text { consumption }\end{array}$ & age. 5 & 2.poultry & MEI & $\begin{array}{l}\text { USCB (2012), } \\
\text { EPA (2011) }\end{array}$ & $\begin{array}{l}\text { Age-dependency derived } \\
\text { from EPA (2011) }\end{array}$ & 0.092 & $\mathrm{~kg} / \mathrm{d}$ \\
\hline UANM & $\begin{array}{l}\text { Animal } \\
\text { product } \\
\text { consumption }\end{array}$ & age. 6 & 2.poultry & MEI & USCB (2012) & $\begin{array}{c}\text { Reference indicates } \\
\text { chicken }(81 \%) \text {, turkey } \\
(19 \%)\end{array}$ & 0.086 & $\mathrm{~kg} / \mathrm{d}$ \\
\hline
\end{tabular}


Table 8.12. (contd)

\begin{tabular}{|c|c|c|c|c|c|c|c|c|}
\hline Gv2 ID & $\begin{array}{c}\text { Gv2 } \\
\text { Description }\end{array}$ & $\operatorname{Age}^{(a)}$ & $\begin{array}{l}\text { Food } \\
\text { Type }\end{array}$ & Qualifier & Reference & Comment & Value & Units \\
\hline UANM & $\begin{array}{l}\text { Animal } \\
\text { product } \\
\text { consumption }\end{array}$ & age.1 & 3.milk & MEI & $\begin{array}{l}\text { USCB (2012), } \\
\text { EPA (2011) }\end{array}$ & $\begin{array}{l}\text { Age-dependency derived } \\
\text { EPA (2011), Table 11-4, } \\
95^{\text {th }} \text { percentile }\end{array}$ & 0.300 & $\mathrm{~kg} / \mathrm{d}$ \\
\hline UANM & $\begin{array}{l}\text { Animal } \\
\text { product } \\
\text { consumption }\end{array}$ & age. 2 & 3.milk & MEI & $\begin{array}{l}\text { USCB (2012), } \\
\text { EPA (2011) }\end{array}$ & $\begin{array}{l}\text { Age-dependency derived } \\
\text { EPA }(2011) \text {, Table } 11-4, \\
95^{\text {th }} \text { percentile }\end{array}$ & 1.083 & $\mathrm{~kg} / \mathrm{d}$ \\
\hline UANM & $\begin{array}{l}\text { Animal } \\
\text { product } \\
\text { consumption }\end{array}$ & age. 3 & 3.milk & MEI & $\begin{array}{l}\text { USCB (2012), } \\
\text { EPA (2011) }\end{array}$ & $\begin{array}{l}\text { Age-dependency derived } \\
\text { EPA }(2011) \text {, Table } 11-4, \\
95^{\text {th }} \text { percentile }\end{array}$ & 1.311 & $\mathrm{~kg} / \mathrm{d}$ \\
\hline UANM & $\begin{array}{l}\text { Animal } \\
\text { product } \\
\text { consumption }\end{array}$ & age. 4 & 3.milk & MEI & $\begin{array}{l}\text { USCB (2012), } \\
\text { EPA (2011) }\end{array}$ & $\begin{array}{l}\text { Age-dependency derived } \\
\text { EPA }(2011) \text {, Table } 11-4, \\
95^{\text {th }} \text { percentile }\end{array}$ & 0.949 & $\mathrm{~kg} / \mathrm{d}$ \\
\hline UANM & $\begin{array}{l}\text { Animal } \\
\text { product } \\
\text { consumption }\end{array}$ & age. 5 & 3.milk & MEI & $\begin{array}{l}\text { USCB (2012), } \\
\text { EPA (2011) }\end{array}$ & $\begin{array}{l}\text { Age-dependency derived } \\
\text { EPA }(2011) \text {,Table } 11-4, \\
95^{\text {th }} \text { percentile }\end{array}$ & 0.975 & $\mathrm{~kg} / \mathrm{d}$ \\
\hline UANM & $\begin{array}{l}\text { Animal } \\
\text { product } \\
\text { consumption }\end{array}$ & age. 6 & 3.milk & MEI & $\begin{array}{l}\text { USCB (2012), } \\
\operatorname{EPA}(2011)\end{array}$ & $\begin{array}{l}\text { Age-dependency derived } \\
\text { EPA }(2011) \text {,Table } 11-4 \text {, } \\
95^{\text {th }} \text { percentile }\end{array}$ & 0.888 & $\mathrm{~kg} / \mathrm{d}$ \\
\hline UANM & $\begin{array}{l}\text { Animal } \\
\text { product } \\
\text { consumption }\end{array}$ & age. 1 & 4.egg & MEI & $\begin{array}{l}\text { USCB (2012), } \\
\text { EPA (2011) }\end{array}$ & $\begin{array}{l}\text { Age-dependency derived } \\
\text { from EPA (2011) }\end{array}$ & 0.022 & $\mathrm{~kg} / \mathrm{d}$ \\
\hline UANM & $\begin{array}{l}\text { Animal } \\
\text { product } \\
\text { consumption }\end{array}$ & age. 2 & 4.egg & MEI & $\begin{array}{l}\text { USCB (2012), } \\
\text { EPA (2011) }\end{array}$ & $\begin{array}{l}\text { Age-dependency derived } \\
\text { from EPA (2011) }\end{array}$ & 0.022 & $\mathrm{~kg} / \mathrm{d}$ \\
\hline UANM & $\begin{array}{l}\text { Animal } \\
\text { product } \\
\text { consumption }\end{array}$ & age. 3 & 4.egg & MEI & $\begin{array}{l}\text { USCB (2012), } \\
\text { EPA (2011) }\end{array}$ & $\begin{array}{l}\text { Age-dependency derived } \\
\text { from EPA (2011) }\end{array}$ & 0.024 & $\mathrm{~kg} / \mathrm{d}$ \\
\hline UANM & $\begin{array}{l}\text { Animal } \\
\text { product } \\
\text { consumption }\end{array}$ & age. 4 & 4.egg & MEI & $\begin{array}{l}\text { USCB (2012), } \\
\text { EPA (2011) }\end{array}$ & $\begin{array}{l}\text { Age-dependency derived } \\
\text { from EPA (2011) }\end{array}$ & 0.031 & $\mathrm{~kg} / \mathrm{d}$ \\
\hline UANM & $\begin{array}{l}\text { Animal } \\
\text { product } \\
\text { consumption }\end{array}$ & age. 5 & 4.egg & MEI & $\begin{array}{l}\text { USCB (2012), } \\
\text { EPA (2011) }\end{array}$ & $\begin{array}{l}\text { Age-dependency derived } \\
\text { from EPA (2011) }\end{array}$ & 0.034 & $\mathrm{~kg} / \mathrm{d}$ \\
\hline UANM & $\begin{array}{l}\text { Animal } \\
\text { product } \\
\text { consumption }\end{array}$ & age. 6 & 4.egg & MEI & USCB (2012) & $\begin{array}{l}53 \mathrm{~g} / \mathrm{egg} \text { ( } 21 \mathrm{~g} \text { yolk, } 32 \mathrm{~g} \\
\text { white) }\end{array}$ & 0.036 & $\mathrm{~kg} / \mathrm{d}$ \\
\hline
\end{tabular}

(a) See Table 8.1 for the ages of each age category.

\begin{tabular}{|c|c|c|c|c|c|c|c|c|}
\hline Gv2_ID & Gv2_Description & $\operatorname{Age}^{(a)}$ & $\begin{array}{l}\text { Food } \\
\text { Type }\end{array}$ & Qualifier & Reference & Comment & Value & Units \\
\hline UANM & $\begin{array}{l}\text { Animal product } \\
\text { consumption }\end{array}$ & age. 1 & 1.meat & POP & EPA (2011) & $\begin{array}{l}\text { Derived from } \\
\text { Table } 11-5 \text {, beef }+ \text { pork }\end{array}$ & 0.014 & $\mathrm{~kg} / \mathrm{d}$ \\
\hline UANM & $\begin{array}{l}\text { Animal product } \\
\text { consumption }\end{array}$ & age. 2 & 1.meat & POP & EPA (2011) & $\begin{array}{l}\text { Derived from } \\
\text { Table } 11-5 \text {, beef }+ \text { pork }\end{array}$ & 0.029 & $\mathrm{~kg} / \mathrm{d}$ \\
\hline UANM & $\begin{array}{l}\text { Animal product } \\
\text { consumption }\end{array}$ & age. 3 & 1.meat & POP & EPA (2011) & $\begin{array}{l}\text { Derived from } \\
\text { Table } 11-5 \text {, beef }+ \text { pork }\end{array}$ & 0.041 & $\mathrm{~kg} / \mathrm{d}$ \\
\hline UANM & $\begin{array}{l}\text { Animal product } \\
\text { consumption }\end{array}$ & age. 4 & 1.meat & POP & EPA (2011) & $\begin{array}{l}\text { Derived from } \\
\text { Table } 11-5 \text {, beef }+ \text { pork }\end{array}$ & 0.052 & $\mathrm{~kg} / \mathrm{d}$ \\
\hline UANM & $\begin{array}{l}\text { Animal product } \\
\text { consumption }\end{array}$ & age. 5 & 1.meat & POP & EPA (2011) & $\begin{array}{l}\text { Derived from } \\
\text { Table } 11-5 \text {, beef }+ \text { pork }\end{array}$ & 0.076 & $\mathrm{~kg} / \mathrm{d}$ \\
\hline UANM & $\begin{array}{l}\text { Animal product } \\
\text { consumption }\end{array}$ & age. 6 & 1.meat & POP & EPA (2011) & $\begin{array}{l}\text { Derived from } \\
\text { Table } 11-5 \text {, beef }+ \text { pork }\end{array}$ & 0.080 & $\mathrm{~kg} / \mathrm{d}$ \\
\hline
\end{tabular}


Table 8.12. (contd)

\begin{tabular}{|c|c|c|c|c|c|c|c|c|}
\hline Gv2_ID & Gv2_Description & $\operatorname{Age}^{(\mathrm{a})}$ & $\begin{array}{l}\text { Food } \\
\text { Type }\end{array}$ & Qualifier & Reference & Comment & Value & Units \\
\hline UANM & $\begin{array}{l}\text { Animal product } \\
\text { consumption }\end{array}$ & age.1 & 2.poultry & POP & EPA (2011) & $\begin{array}{l}\text { Derived from } \\
\text { Table } 11-5, \text { poultry }\end{array}$ & 0.013 & $\mathrm{~kg} / \mathrm{d}$ \\
\hline UANM & $\begin{array}{l}\text { Animal product } \\
\text { consumption }\end{array}$ & age. 2 & 2.poultry & POP & EPA (2011) & $\begin{array}{l}\text { Derived from } \\
\text { Table } 11-5 \text {, poultry }\end{array}$ & 0.026 & $\mathrm{~kg} / \mathrm{d}$ \\
\hline UANM & $\begin{array}{l}\text { Animal product } \\
\text { consumption }\end{array}$ & age. 3 & 2.poultry & POP & EPA (2011) & $\begin{array}{l}\text { Derived from } \\
\text { Table } 11-5 \text {, poultry }\end{array}$ & 0.031 & $\mathrm{~kg} / \mathrm{d}$ \\
\hline UANM & $\begin{array}{l}\text { Animal product } \\
\text { consumption }\end{array}$ & age. 4 & 2.poultry & POP & EPA (2011) & $\begin{array}{l}\text { Derived from } \\
\text { Table } 11-5 \text {, poultry }\end{array}$ & 0.038 & $\mathrm{~kg} / \mathrm{d}$ \\
\hline UANM & $\begin{array}{l}\text { Animal product } \\
\text { consumption }\end{array}$ & age. 5 & 2.poultry & POP & EPA (2011) & $\begin{array}{l}\text { Derived from } \\
\text { Table } 11-5 \text {, poultry }\end{array}$ & 0.051 & $\mathrm{~kg} / \mathrm{d}$ \\
\hline UANM & $\begin{array}{l}\text { Animal product } \\
\text { consumption }\end{array}$ & age. 6 & 2.poultry & POP & EPA (2011) & $\begin{array}{l}\text { Derived from } \\
\text { Table } 11-5 \text {, poultry }\end{array}$ & 0.048 & $\mathrm{~kg} / \mathrm{d}$ \\
\hline UANM & $\begin{array}{l}\text { Animal product } \\
\text { consumption }\end{array}$ & age. 1 & 3.milk & POP & EPA (2011) & $\begin{array}{l}\text { Derived from EPA } \\
(2011) \text {, Table 11-4, } \\
\text { mean }\end{array}$ & 0.080 & $\mathrm{~kg} / \mathrm{d}$ \\
\hline UANM & $\begin{array}{l}\text { Animal product } \\
\text { consumption }\end{array}$ & age. 2 & 3.milk & POP & EPA (2011) & $\begin{array}{l}\text { Derived from EPA } \\
(2011) \text {, Table 11-4, } \\
\text { mean }\end{array}$ & 0.493 & $\mathrm{~kg} / \mathrm{d}$ \\
\hline UANM & $\begin{array}{l}\text { Animal product } \\
\text { consumption }\end{array}$ & age. 3 & 3.milk & POP & EPA (2011) & $\begin{array}{l}\text { Derived from EPA } \\
(2011) \text {, Table 11-4, } \\
\text { mean }\end{array}$ & 0.596 & $\mathrm{~kg} / \mathrm{d}$ \\
\hline UANM & $\begin{array}{l}\text { Animal product } \\
\text { consumption }\end{array}$ & age. 4 & 3.milk & POP & EPA (2011) & $\begin{array}{l}\text { Derived from EPA } \\
(2011) \text {, Table 11-4, } \\
\text { mean }\end{array}$ & 0.446 & $\mathrm{~kg} / \mathrm{d}$ \\
\hline UANM & $\begin{array}{l}\text { Animal product } \\
\text { consumption }\end{array}$ & age. 5 & 3.milk & POP & EPA (2011) & $\begin{array}{l}\text { Derived from EPA } \\
(2011) \text {, Table 11-4, } \\
\text { mean }\end{array}$ & 0.361 & $\mathrm{~kg} / \mathrm{d}$ \\
\hline UANM & $\begin{array}{l}\text { Animal product } \\
\text { consumption }\end{array}$ & age. 6 & 3.milk & POP & EPA (2011) & $\begin{array}{l}\text { Derived from EPA } \\
(2011) \text {, Table 11-4, } \\
\text { mean }\end{array}$ & 0.301 & $\mathrm{~kg} / \mathrm{d}$ \\
\hline UANM & $\begin{array}{l}\text { Animal product } \\
\text { consumption }\end{array}$ & age. 1 & 4.egg & POP & EPA (2011) & $\begin{array}{l}\text { Derived from } \\
\text { Table 11-12, per } \\
\text { capita }\end{array}$ & 0.013 & $\mathrm{~kg} / \mathrm{d}$ \\
\hline UANM & $\begin{array}{l}\text { Animal product } \\
\text { consumption }\end{array}$ & age. 2 & 4.egg & POP & EPA (2011) & $\begin{array}{l}\text { Derived from } \\
\text { Table } 11-12 \text {, per } \\
\text { capita }\end{array}$ & 0.013 & $\mathrm{~kg} / \mathrm{d}$ \\
\hline UANM & $\begin{array}{l}\text { Animal product } \\
\text { consumption }\end{array}$ & age. 3 & 4.egg & POP & EPA (2011) & $\begin{array}{l}\text { Derived from } \\
\text { Table } 11-12 \text {, per } \\
\text { capita }\end{array}$ & 0.014 & $\mathrm{~kg} / \mathrm{d}$ \\
\hline UANM & $\begin{array}{l}\text { Animal product } \\
\text { consumption }\end{array}$ & age. 4 & 4.egg & POP & EPA (2011) & $\begin{array}{l}\text { Derived from } \\
\text { Table } 11-12 \text {, per } \\
\text { capita }\end{array}$ & 0.018 & $\mathrm{~kg} / \mathrm{d}$ \\
\hline UANM & $\begin{array}{l}\text { Animal product } \\
\text { consumption }\end{array}$ & age. 5 & 4.egg & POP & EPA (2011) & $\begin{array}{l}\text { Derived from } \\
\text { Table } 11-12 \text {, per } \\
\text { capita }\end{array}$ & 0.020 & $\mathrm{~kg} / \mathrm{d}$ \\
\hline UANM & $\begin{array}{l}\text { Animal product } \\
\text { consumption }\end{array}$ & age. 6 & 4.egg & POP & EPA (2011) & $\begin{array}{l}\text { Derived from } \\
\text { Table } 11-12 \text {, per } \\
\text { capita }\end{array}$ & 0.021 & $\mathrm{~kg} / \mathrm{d}$ \\
\hline
\end{tabular}




\subsubsection{Ingestion Exposure: Aquatic Foods}

Aquatic food ingestion somewhat differs from crop and animal product ingestion in that more people do not consume this category of food. Application of intake rates should be done with care. Few resources indicate freshwater vs. saltwater fish consumption. MEI rates are presented for consumersonly.

The daily intake rate (UAQU) and number of intake days per year (TAQU) require definition for each aquatic food type (fish, mollusk, crustacean, and aquatic plant). Set the consumption period for each aquatic food type to $365 \mathrm{~d} / \mathrm{yr}$.

The units and range for UAQU in Gv2 are: $\mathrm{kg} / \mathrm{d}(0-10)$.

The units and range for TAQU in Gv2 are: $\mathrm{d} / \mathrm{yr}(0-365)$.

Values are derived from EPA 2011, which are presented in units of $\mathrm{g} / \mathrm{kg}$-d base on the body weight of the consumer. Assumed body weights are indicated in Table 8.10 and then age-specific intake rates are averaged according to the Gv2 age categories shown in the same table. Population UAQU values tabulated below indicates both the per capita mean and a more conservative per capita $95^{\text {th }}$ percentile rates. MEI UAQU values tabulated below indicate both the consumers-only mean and the consumersonly $95^{\text {th }}$ percentile rates. It is suggested that the user assume the MEI $95^{\text {th }}$ percentile value if their state/region has a large aquaculture industry. It is suggested that the user assume the POP mean per capita rates for population estimates.

Aquatic plant consumption is not a major food source, currently, and should be set to 0. U.S. states with larger contributions to mollusk (abalone, clam, mussel, oyster, snail) aquaculture industry in 2007 are Virginia, Massachusetts, Florida, Maine, New York, Washington, and Connecticut (USDA 2009, Table 23 Aquaculture Sold). U.S. states with larger contributions to crustacean (crawfish, lobster, prawn, shrimp, softshell crabs) aquaculture industry in 2007 are Texas and Florida. Fish aquaculture is dominant in Mississippi (catfish) and Pennsylvania and Colorado (trout). Pennsylvania, Washington, and Alaska are dominant sportfish/gamefish states. Higher aquatic food consumption rates may be applied to these states.

\subsubsection{Additional Sources of Information}

The USDA Agricultural Handbook Number 697 (USDA 1992) provides weights, measures, and conversion factors for agricultural commodities and their products. In particular, Table 24 provides conversion factors for whole fish and shellfish masses, to the edible mass. Table 26 indicates the mass of various aquatic foods in a standard case of canned product.

The U.S. 2007 Census of Agriculture (USDA 2009) reports information on aquaculture. It can be accessed at http://www.agcensus.usda.gov/Publications/2007/Full_Report/index.asp. 


\subsubsection{UAQU Ingestion Rate of Aquatic Foods Tables}

Table 8.13. UAQU Ingestion Rates - Human Intake of Aquatic Food Categories

\begin{tabular}{|c|c|c|c|c|c|c|c|c|}
\hline Gv2_ID & $\operatorname{Age}^{(a)}$ & Food Type & Qualifier & Reference & Comment & Data Reported & Value & Units \\
\hline UAQU & 1 & 1.fish & MEI & EPA (2011) & $\begin{array}{l}\text { Ch } 10 \text {, consumers only } \\
\text { mean, fw or sw }\end{array}$ & $\begin{array}{l}\text { derivation, } \\
\text { consumers only } \\
\text { mean, Finfish }\end{array}$ & 0.0136 & $\mathrm{~kg} /$ day \\
\hline UAQU & 1 & 1.fish & MEI & EPA (2011) & $\begin{array}{l}\text { Ch } 10 \text {, consumers only } \\
95 \text { th, fw or sw }\end{array}$ & $\begin{array}{l}\text { derivation, } \\
\text { consumers only } \\
\text { 95th, Finfish }\end{array}$ & 0.0378 & $\mathrm{~kg}$ /day \\
\hline UAQU & 1 & 2.mollusk & MEI & EPA (2011) & $\begin{array}{l}\text { Ch } 10 \text {, consumers only } \\
95 \text { th, mollusk or } \\
\text { crustacea }\end{array}$ & $\begin{array}{l}\text { derivation, } \\
\text { consumers only } \\
\text { 95th, Shellfish }\end{array}$ & 0.0278 & $\mathrm{~kg} /$ day \\
\hline UAQU & 2 & 1.fish & MEI & EPA (2011) & $\begin{array}{l}\text { Ch } 10 \text {, consumers only } \\
\text { mean, fw or sw }\end{array}$ & $\begin{array}{l}\text { derivation, } \\
\text { consumers only } \\
\text { mean, Finfish }\end{array}$ & 0.0221 & $\mathrm{~kg} /$ day \\
\hline UAQU & 2 & 1.fish & MEI & EPA (2011) & $\begin{array}{l}\text { Ch } 10 \text {, consumers only } \\
95 \text { th, fw or sw }\end{array}$ & $\begin{array}{l}\text { derivation, } \\
\text { consumers only } \\
\text { 95th, Finfish }\end{array}$ & 0.0676 & $\mathrm{~kg} /$ day \\
\hline UAQU & 2 & 2.mollusk & MEI & EPA (2011) & $\begin{array}{l}\text { Ch } 10 \text {, consumers only } \\
95 \text { th, mollusk or } \\
\text { crustacea }\end{array}$ & $\begin{array}{l}\text { derivation, } \\
\text { consumers only } \\
\text { 95th, Shellfish }\end{array}$ & 0.0483 & $\mathrm{~kg} /$ day \\
\hline UAQU & 3 & 1.fish & MEI & EPA (2011) & $\begin{array}{l}\text { Ch } 10 \text {, consumers only } \\
\text { mean, fw or sw }\end{array}$ & $\begin{array}{l}\text { derivation, } \\
\text { consumers only } \\
\text { mean, Finfish }\end{array}$ & 0.0242 & $\mathrm{~kg} /$ day \\
\hline UAQU & 3 & 1.fish & MEI & EPA (2011) & $\begin{array}{l}\text { Ch } 10 \text {, consumers only } \\
95 \text { th, fw or sw }\end{array}$ & $\begin{array}{l}\text { derivation, } \\
\text { consumers only } \\
\text { 95th, Finfish }\end{array}$ & 0.067 & $\mathrm{~kg}$ /day \\
\hline UAQU & 3 & 2.mollusk & MEI & EPA (2011) & $\begin{array}{l}\text { Ch } 10 \text {, consumers only } \\
95 \text { th, mollusk or } \\
\text { crustacea }\end{array}$ & $\begin{array}{l}\text { derivation, } \\
\text { consumers only } \\
\text { 95th, Shellfish }\end{array}$ & 0.0539 & $\mathrm{~kg} /$ day \\
\hline UAQU & 4 & 1.fish & MEI & EPA (2011) & $\begin{array}{l}\text { Ch } 10 \text {, consumers only } \\
\text { mean, fw or sw }\end{array}$ & $\begin{array}{l}\text { derivation, } \\
\text { consumers only } \\
\text { mean, Finfish }\end{array}$ & 0.035 & $\mathrm{~kg} /$ day \\
\hline UAQU & 4 & 1.fish & MEI & EPA (2011) & $\begin{array}{l}\text { Ch } 10 \text {, consumers only } \\
95 \text { th, fw or sw }\end{array}$ & $\begin{array}{l}\text { derivation, } \\
\text { consumers only } \\
\text { 95th, Finfish }\end{array}$ & 0.0922 & $\mathrm{~kg} /$ day \\
\hline UAQU & 4 & 2.mollusk & MEI & EPA (2011) & $\begin{array}{l}\text { Ch } 10 \text {, consumers only } \\
95 \text { th, mollusk or } \\
\text { crustacea }\end{array}$ & $\begin{array}{l}\text { derivation, } \\
\text { consumers only } \\
\text { 95th, Shellfish }\end{array}$ & 0.0636 & $\mathrm{~kg} /$ day \\
\hline UAQU & 5 & 1.fish & MEI & EPA (2011) & $\begin{array}{l}\text { Ch } 10 \text {, consumers only } \\
\text { mean, fw or sw }\end{array}$ & $\begin{array}{l}\text { derivation, } \\
\text { consumers only } \\
\text { mean, Finfish }\end{array}$ & 0.0424 & $\mathrm{~kg} /$ day \\
\hline UAQU & 5 & 1.fish & MEI & EPA (2011) & $\begin{array}{l}\text { Ch } 10 \text {, consumers only } \\
95 \text { th, fw or sw }\end{array}$ & $\begin{array}{l}\text { derivation, } \\
\text { consumers only } \\
\text { 95th, Finfish }\end{array}$ & 0.1091 & $\mathrm{~kg} /$ day \\
\hline UAQU & 5 & 2.mollusk & MEI & EPA (2011) & $\begin{array}{l}\text { Ch } 10 \text {, consumers only } \\
95 \text { th, mollusk or } \\
\text { crustacea }\end{array}$ & $\begin{array}{l}\text { derivation, } \\
\text { consumers only } \\
\text { 95th, Shellfish }\end{array}$ & 0.122 & $\mathrm{~kg} /$ day \\
\hline UAQU & 6 & 1.fish & MEI & EPA (2011) & $\begin{array}{l}\text { Ch } 10 \text {, consumers only } \\
\text { mean, fw or sw }\end{array}$ & $\begin{array}{l}\text { derivation, } \\
\text { consumers only } \\
\text { mean, Finfish }\end{array}$ & 0.0532 & $\mathrm{~kg} /$ day \\
\hline UAQU & 6 & 1.fish & MEI & EPA (2011) & $\begin{array}{l}\text { Ch } 10 \text {, consumers only } \\
95 \text { th, fw or sw }\end{array}$ & $\begin{array}{l}\text { derivation, } \\
\text { consumers only } \\
\text { 95th, Finfish }\end{array}$ & 0.164 & $\mathrm{~kg} /$ day \\
\hline UAQU & 6 & 2.mollusk & MEI & EPA (2011) & $\begin{array}{l}\text { Ch } 10 \text {, consumers only } \\
95 \text { th, mollusk or } \\
\text { crustacea }\end{array}$ & $\begin{array}{l}\text { derivation, } \\
\text { consumers only } \\
\text { 95th, Shellfish }\end{array}$ & 0.136 & $\mathrm{~kg}$ /day \\
\hline
\end{tabular}


Table 8.13. (contd)

\begin{tabular}{|c|c|c|c|c|c|c|c|c|}
\hline Gv2 ID & $\operatorname{Age}^{(\mathrm{a})}$ & Food Type & Qualifier & Reference & Comment & Data Reported & Value & Units \\
\hline UAQU & 1 & 1.fish & POP & EPA (2011) & $\begin{array}{l}\text { Ch } 10 \text {, per capita mean } \\
\text { fw or sw }\end{array}$ & $\begin{array}{l}\text { derivation, per } \\
\text { capita mean, } \\
\text { Finfish }\end{array}$ & 0.0014 & $\mathrm{~kg} / \mathrm{day}$ \\
\hline UAQU & 1 & 1.fish & POP & EPA (2011) & $\begin{array}{l}\text { Ch } 10 \text {, per capita } 95 \text { th fw } \\
\text { or sw }\end{array}$ & $\begin{array}{l}\text { derivation, per } \\
\text { capita 95th, } \\
\text { Finfish }\end{array}$ & 0.0068 & $\mathrm{~kg}$ /day \\
\hline UAQU & 1 & 2.mollusk & POP & EPA (2011) & $\begin{array}{l}\text { Ch } 10 \text {, consumers only } \\
\text { mean, mollusk or } \\
\text { crustacea }\end{array}$ & $\begin{array}{l}\text { derivation, } \\
\text { consumers only } \\
\text { mean, Shellfish }\end{array}$ & 0.0068 & $\mathrm{~kg}$ /day \\
\hline UAQU & 2 & 1.fish & POP & EPA (2011) & $\begin{array}{l}\text { Ch } 10 \text {, per capita mean } \\
\text { fw or sw }\end{array}$ & $\begin{array}{l}\text { derivation, per } \\
\text { capita mean, } \\
\text { Finfish }\end{array}$ & 0.003 & $\mathrm{~kg} /$ day \\
\hline UAQU & 2 & 1.fish & POP & EPA (2011) & $\begin{array}{l}\text { Ch } 10 \text {, per capita } 95 \text { th fw } \\
\text { or sw }\end{array}$ & $\begin{array}{l}\text { derivation, per } \\
\text { capita 95th, } \\
\text { Finfish }\end{array}$ & 0.0166 & $\mathrm{~kg}$ /day \\
\hline UAQU & 2 & 2.mollusk & POP & EPA (2011) & $\begin{array}{l}\text { Ch } 10 \text {, consumers only } \\
\text { mean, mollusk or } \\
\text { crustacea }\end{array}$ & $\begin{array}{l}\text { derivation, } \\
\text { consumers only } \\
\text { mean, Shellfish }\end{array}$ & 0.013 & $\mathrm{~kg}$ /day \\
\hline UAQU & 3 & 1.fish & POP & EPA (2011) & $\begin{array}{l}\text { Ch } 10 \text {, per capita mean } \\
\text { fw or sw }\end{array}$ & $\begin{array}{l}\text { derivation, per } \\
\text { capita mean, } \\
\text { Finfish }\end{array}$ & 0.0035 & $\mathrm{~kg} /$ day \\
\hline UAQU & 3 & 1.fish & POP & EPA (2011) & $\begin{array}{l}\text { Ch } 10 \text {, per capita } 95 \text { th fw } \\
\text { or sw }\end{array}$ & $\begin{array}{l}\text { derivation, per } \\
\text { capita 95th, } \\
\text { Finfish }\end{array}$ & 0.026 & $\mathrm{~kg} /$ day \\
\hline UAQU & 3 & 2.mollusk & POP & EPA (2011) & $\begin{array}{l}\text { Ch } 10 \text {, consumers only } \\
\text { mean, mollusk or } \\
\text { crustacea }\end{array}$ & $\begin{array}{l}\text { derivation, } \\
\text { consumers only } \\
\text { mean, Shellfish }\end{array}$ & 0.0186 & $\mathrm{~kg}$ /day \\
\hline UAQU & 4 & 1.fish & POP & EPA (2011) & $\begin{array}{l}\text { Ch } 10 \text {, per capita mean } \\
\text { fw or sw }\end{array}$ & $\begin{array}{l}\text { derivation, per } \\
\text { capita mean, } \\
\text { Finfish }\end{array}$ & 0.0051 & $\mathrm{~kg}$ /day \\
\hline UAQU & 4 & 1.fish & POP & EPA (2011) & $\begin{array}{l}\text { Ch } 10 \text {, per capita } 95 \text { th fw } \\
\text { or sw }\end{array}$ & $\begin{array}{l}\text { derivation, per } \\
\text { capita 95th, } \\
\text { Finfish }\end{array}$ & 0.035 & $\mathrm{~kg}$ /day \\
\hline UAQU & 4 & 2.mollusk & POP & EPA (2011) & $\begin{array}{l}\text { Ch } 10 \text {, consumers only } \\
\text { mean, mollusk or } \\
\text { crustacea }\end{array}$ & $\begin{array}{l}\text { derivation, } \\
\text { consumers only } \\
\text { mean, Shellfish }\end{array}$ & 0.0229 & $\mathrm{~kg}$ /day \\
\hline UAQU & 5 & 1.fish & POP & EPA (2011) & $\begin{array}{l}\text { Ch } 10 \text {, per capita mean } \\
\text { fw or sw }\end{array}$ & $\begin{array}{l}\text { derivation, per } \\
\text { capita mean, } \\
\text { Finfish }\end{array}$ & 0.0064 & $\mathrm{~kg}$ /day \\
\hline UAQU & 5 & 1.fish & POP & EPA (2011) & $\begin{array}{l}\text { Ch } 10 \text {, per capita } 95 \text { th } \mathrm{fw} \\
\text { or sw }\end{array}$ & $\begin{array}{l}\text { derivation, per } \\
\text { capita 95th, } \\
\text { Finfish }\end{array}$ & 0.0449 & $\mathrm{~kg} /$ day \\
\hline UAQU & 5 & 2.mollusk & POP & EPA (2011) & $\begin{array}{l}\text { Ch } 10 \text {, consumers only } \\
\text { mean, mollusk or } \\
\text { crustacea }\end{array}$ & $\begin{array}{l}\text { derivation, } \\
\text { consumers only } \\
\text { mean, Shellfish }\end{array}$ & 0.0392 & $\mathrm{~kg}$ /day \\
\hline UAQU & 6 & 1.fish & POP & EPA (2011) & $\begin{array}{l}\text { Ch } 10 \text {, per capita mean } \\
\text { fw or sw }\end{array}$ & $\begin{array}{l}\text { derivation, per } \\
\text { capita mean, } \\
\text { Finfish }\end{array}$ & 0.014 & $\mathrm{~kg}$ /day \\
\hline UAQU & 6 & 1.fish & POP & EPA (2011) & $\begin{array}{l}\text { Ch } 10 \text {, per capita } 95 \text { th fw } \\
\text { or sw }\end{array}$ & $\begin{array}{l}\text { derivation, per } \\
\text { capita 95th, } \\
\text { Finfish }\end{array}$ & 0.088 & $\mathrm{~kg}$ /day \\
\hline UAQU & 6 & 2.mollusk & POP & EPA (2011) & $\begin{array}{l}\text { Ch } 10 \text {, consumers only } \\
\text { mean, mollusk or } \\
\text { crustacea }\end{array}$ & $\begin{array}{l}\text { derivation, } \\
\text { consumers only } \\
\text { mean, Shellfish }\end{array}$ & 0.0416 & $\mathrm{~kg} /$ day \\
\hline
\end{tabular}




\subsection{Pathway Selection: Water Ingestion (multiple)}

Water ingestion pathways include intakes from direct ingestion, incidental swimming-water ingestion, and incidental shower-water ingestion. These routes consider only ingestion exposure to the contaminated water. If a non-contaminated source is assumed for either drinking water, shower water, or swimming water, zero out the intake for that route.

Drinking water ingestion requires assignment of intake rate and consumption period. The swimming and showering exposures determine intakes based on hourly exposure rather than daily exposure, therefore more parameterization is required.

\subsubsection{Water Ingestion Exposure: Drinking Water}

Annual drinking water ingestion is described by the daily intake rate (UDW) and the number of days drinking water is acquired from the contaminated source (TDW).

The units for UDW in Gv2 are: $\mathrm{L} / \mathrm{d}(0-10)$.

The units for TDW in Gv2 are: d/yr (0-365).

The values provided assume that only water from the contaminated source is consumed. Therefore, TDW is set to $365 \mathrm{~d} / \mathrm{yr}$. EPA (2011) age-specific ranges of drinking water rates are assigned. The average of the range can be assigned as a discrete value. Pregnant and lactating female intake rates are also provided in the tables presented here.

Note: Compliance-specific calculations sometimes require the use of a $2 \mathrm{~L} / \mathrm{d}$ water ingestion rate. The values tabulated, below, indicate actual ingestion rates of water in the United States and should be applied when a certain water ingestion rate is not prescribed.

\subsubsection{Additional Sources of Information}

EPA (2004) provides additional information on U.S. water ingestion rates. Last accessed at: http://water.epa.gov/actioN/Advisories/drinking/percapita index.cfm. 


\subsubsection{UDW Drinking Water Ingestion Rate Table}

Table 8.14. UDW Drinking Water Ingestion Rate

\begin{tabular}{|c|c|c|c|c|c|c|c|c|c|}
\hline $\begin{array}{l}\text { Gv2 } \\
\text { ID }\end{array}$ & Gv2 Description & $\operatorname{Age}^{(a)}$ & Qualifier & Reference & Comment & Data Reported & Min & Max & Units \\
\hline UDW & $\begin{array}{l}\text { Drinking water } \\
\text { ingestion rate }\end{array}$ & 1 & MEI & EPA (2011) & $\begin{array}{l}\text { Chap 3, 95th } \\
\text { percentile }\end{array}$ & $\begin{array}{l}\text { 95th percentile } \\
\text { range }\end{array}$ & 0.839 & 1.101 & L/day \\
\hline UDW & $\begin{array}{l}\text { Drinking water } \\
\text { ingestion rate }\end{array}$ & 2 & MEI & EPA (2011) & $\begin{array}{l}\text { Chap 3, 95th } \\
\text { percentile }\end{array}$ & $\begin{array}{l}\text { 95th percentile } \\
\text { range }\end{array}$ & 0.837 & 0.893 & L/day \\
\hline UDW & $\begin{array}{l}\text { Drinking water } \\
\text { ingestion rate }\end{array}$ & 3 & MEI & EPA (2011) & $\begin{array}{l}\text { Chap 3, 95th } \\
\text { percentile }\end{array}$ & $\begin{array}{l}\text { 95th percentile } \\
\text { range }\end{array}$ & 0.877 & 0.979 & L/day \\
\hline UDW & $\begin{array}{l}\text { Drinking water } \\
\text { ingestion rate }\end{array}$ & 4 & MEI & EPA (2011) & $\begin{array}{l}\text { Chap 3, 95th } \\
\text { percentile }\end{array}$ & $\begin{array}{l}\text { 95th percentile } \\
\text { range }\end{array}$ & 1.316 & 1.404 & L/day \\
\hline UDW & $\begin{array}{l}\text { Drinking water } \\
\text { ingestion rate }\end{array}$ & 5 & MEI & EPA (2011) & $\begin{array}{l}\text { Chap 3, 95th } \\
\text { percentile }\end{array}$ & $\begin{array}{l}\text { 95th percentile } \\
\text { range }\end{array}$ & 1.783 & 1.899 & L/day \\
\hline UDW & $\begin{array}{l}\text { Drinking water } \\
\text { ingestion rate }\end{array}$ & 6 & MEI & EPA (2011) & $\begin{array}{l}\text { Chap 3, 95th } \\
\text { percentile }\end{array}$ & $\begin{array}{l}\text { 95th percentile } \\
\text { range }\end{array}$ & 2.368 & 3.092 & L/day \\
\hline UDW & $\begin{array}{l}\text { Drinking water } \\
\text { ingestion rate }\end{array}$ & 6 & $\begin{array}{l}\text { Pregnant } \\
\text { MEI }\end{array}$ & EPA (2011) & $\begin{array}{l}\text { Chap 3, 95th } \\
\text { percentile }\end{array}$ & $\begin{array}{l}\text { 95th percentile } \\
\text { range }\end{array}$ & 2.503 & 2.589 & L/day \\
\hline UDW & $\begin{array}{l}\text { Drinking water } \\
\text { ingestion rate }\end{array}$ & 6 & $\begin{array}{l}\text { Lactating } \\
\text { MEI }\end{array}$ & EPA (2011) & $\begin{array}{l}\text { Chap 3, 95th } \\
\text { percentile }\end{array}$ & $\begin{array}{l}95 \text { th percentile } \\
\text { range }\end{array}$ & 3.434 & 3.588 & L/day \\
\hline
\end{tabular}

(a) See Table 8.1 for the ages of each age category.

\begin{tabular}{|c|c|c|c|c|c|c|c|c|c|}
\hline $\begin{array}{c}\text { Gv2 } \\
\text { ID }\end{array}$ & Gv2 Description & $\operatorname{Age}^{(\mathrm{a})}$ & Qualifier & Reference & Comment & Data Reported & Min & Max & Units \\
\hline UDW & $\begin{array}{l}\text { Drinking water } \\
\text { ingestion rate }\end{array}$ & 1 & POP & EPA (2011) & $\begin{array}{l}\text { Chap 3, } \\
\text { average }\end{array}$ & average range & 0.184 & 0.467 & L/day \\
\hline UDW & $\begin{array}{l}\text { Drinking water } \\
\text { ingestion rate }\end{array}$ & 2 & POP & EPA (2011) & $\begin{array}{l}\text { Chap 3, } \\
\text { average }\end{array}$ & average range & 0.271 & 0.308 & L/day \\
\hline UDW & $\begin{array}{l}\text { Drinking water } \\
\text { ingestion rate }\end{array}$ & 3 & POP & EPA (2011) & $\begin{array}{l}\text { Chap 3, } \\
\text { average }\end{array}$ & average range & 0.317 & 0.382 & L/day \\
\hline UDW & $\begin{array}{l}\text { Drinking water } \\
\text { ingestion rate }\end{array}$ & 4 & POP & EPA (2011) & $\begin{array}{l}\text { Chap 3, } \\
\text { average }\end{array}$ & average range & 0.414 & 0.511 & L/day \\
\hline UDW & $\begin{array}{l}\text { Drinking water } \\
\text { ingestion rate }\end{array}$ & 5 & POP & EPA (2011) & $\begin{array}{l}\text { Chap 3, } \\
\text { average }\end{array}$ & average range & 0.52 & 0.702 & L/day \\
\hline UDW & $\begin{array}{l}\text { Drinking water } \\
\text { ingestion rate }\end{array}$ & 6 & POP & EPA (2011) & $\begin{array}{l}\text { Chap 3, } \\
\text { average }\end{array}$ & average range & 0.681 & 1.288 & L/day \\
\hline UDW & $\begin{array}{l}\text { Drinking water } \\
\text { ingestion rate }\end{array}$ & 6 & $\begin{array}{l}\text { Pregnant } \\
\text { POP }\end{array}$ & EPA (2011) & $\begin{array}{l}\text { Chap 3, } \\
\text { average }\end{array}$ & average range & 0.819 & 0.872 & L/day \\
\hline UDW & $\begin{array}{l}\text { Drinking water } \\
\text { ingestion rate }\end{array}$ & 6 & $\begin{array}{l}\text { Lactating } \\
\text { POP }\end{array}$ & EPA (2011) & $\begin{array}{l}\text { Chap 3, } \\
\text { average }\end{array}$ & average range & 1.379 & 1.665 & L/day \\
\hline
\end{tabular}

(a) See Table 8.1 for the ages of each age category.

\subsubsection{Water Ingestion Exposure: Incidental Shower Water}

Incidental shower water ingestion requires the assignment of four parameters: the number of showers per day (EVSHWR), the duration of the shower event (TESHWR), the number of days the individual showers (TSHWR), and the ingestion rate of water while showering (USHIN). 
The units and range for EVSHWR in Gv2 are: number (0-10).

The units and range for TESHWR in Gv2 are: $\mathrm{hr}(0-24)$.

The units and range for TSHWR in Gv2 are: $d(0-365)$.

The units and range for USHIN in Gv2 are: $\mathrm{L} / \mathrm{hr}(0-10)$.

Assume that both the MEI and population take 1 shower/d and that they take a shower every day. Chapter 16 of EPA (2011) indicates the shower minutes per day by age, for those who shower. Mean values are assigned to the population and $95^{\text {th }}$ percentile values assigned to the MEI, as adjusted for the age groups of interest. For the shower water ingestion rate, a reasonable assumption would be to assume half the swimming water ingestion rates presented in Section 8.1.2.2.

\subsubsection{Additional Sources of Information}

No additional sources that provide greater detail than EPA (2011) were identified.

\subsubsection{TESHWR, USHIN Incidental Shower Water Intake Tables}

Table 8.15. TESHWR Shower Duration

\begin{tabular}{|c|c|c|c|c|c|c|c|}
\hline Gv2 ID & Gv2 Description & $\operatorname{Age}^{(a)}$ & Qualifier & Reference & Comment & Value & Units \\
\hline TESHWR & Shower duration & 1 & MEI & EPA (2011) & $\begin{array}{l}\text { Table 16-1, } \\
\text { 95th percentile }\end{array}$ & 0 & $\mathrm{hr}$ \\
\hline TESHWR & Shower duration & 2 & MEI & EPA (2011) & $\begin{array}{l}\text { Table } 16-1 \text {, } \\
\text { 95th percentile }\end{array}$ & 0.73 & $\mathrm{hr}$ \\
\hline TESHWR & Shower duration & 3 & MEI & EPA (2011) & $\begin{array}{l}\text { Table } 16-1 \text {, } \\
\text { 95th percentile }\end{array}$ & 0.57 & $\mathrm{hr}$ \\
\hline TESHWR & Shower duration & 4 & MEI & EPA (2011) & $\begin{array}{l}\text { Table } 16-1 \text {, } \\
\text { 95th percentile }\end{array}$ & 0.68 & $\mathrm{hr}$ \\
\hline TESHWR & Shower duration & 5 & MEI & EPA (2011) & $\begin{array}{l}\text { Table 16-1, } \\
\text { 95th percentile }\end{array}$ & 0.71 & $\mathrm{hr}$ \\
\hline TESHWR & Shower duration & 6 & MEI & EPA (2011) & $\begin{array}{l}\text { Table } 16-1 \text {, } \\
\text { 95th percentile }\end{array}$ & 0.75 & $\mathrm{hr}$ \\
\hline
\end{tabular}

(a) See Table 8.1 for age groups.

\begin{tabular}{rlcclccc}
\hline Gv2 ID & Gv2 Description & Age $^{(\mathrm{a})}$ & Qualifier & Reference & Comment & Value & Units \\
\hline TESHWR & Shower duration & 1 & POP & EPA (2011) & Table 16-1 mean & 0.29 & $\mathrm{hr}$ \\
TESHWR & Shower duration & 2 & POP & EPA (2011) & Table 16-1 mean & 0.37 & $\mathrm{hr}$ \\
TESHWR & Shower duration & 3 & POP & EPA (2011) & Table 16-1 mean & 0.28 & $\mathrm{hr}$ \\
TESHWR & Shower duration & 4 & POP & EPA (2011) & Table 16-1 mean & 0.30 & $\mathrm{hr}$ \\
TESHWR & Shower duration & 5 & POP & EPA (2011) & Table 16-1 mean & 0.32 & $\mathrm{hr}$ \\
TESHWR & Shower duration & 6 & POP & EPA (2011) & Table 16-1 mean & 0.33 & $\mathrm{hr}$ \\
\hline
\end{tabular}

(a) See Table 8.1 for age groups. 
Table 8.16. USHIN Incidental Ingestion of Shower Water

\begin{tabular}{clccccc}
\hline Gv2 ID & \multicolumn{1}{c}{ Gv2_Description } & Age $^{(\mathrm{a})}$ & Qualifier & Reference & Value & Units \\
\hline USHIN & $\begin{array}{l}\text { Ingestion rate of water while } \\
\text { showering }\end{array}$ & 2 & MEI & Half of USWIM & 0.06 & L/hr \\
USHIN & $\begin{array}{l}\text { Ingestion rate of water while } \\
\text { showering }\end{array}$ & 3 & MEI & Half of USWIM & 0.06 & $\mathrm{~L} / \mathrm{hr}$ \\
USHIN & $\begin{array}{l}\text { Ingestion rate of water while } \\
\text { showering }\end{array}$ & 4 & MEI & Half of USWIM & 0.06 & $\mathrm{~L} / \mathrm{hr}$ \\
USHIN & $\begin{array}{l}\text { Ingestion rate of water while } \\
\text { showering }\end{array}$ & 5 & MEI & Half of USWIM & 0.06 & $\mathrm{~L} / \mathrm{hr}$ \\
USHIN & $\begin{array}{l}\text { Ingestion rate of water while } \\
\text { showering }\end{array}$ & 6 & MEI & Half of USWIM & 0.04 & $\mathrm{~L} / \mathrm{hr}$ \\
\hline
\end{tabular}

(a) See Table 8.1 for age groups.

\begin{tabular}{rlccccc}
\hline Gv2_ID & \multicolumn{1}{c}{ Gv2_Description } & Age $^{(\mathrm{a})}$ & qualifier & Reference & Value & Units \\
\hline USHIN & $\begin{array}{l}\text { Ingestion rate of water while } \\
\text { showering }\end{array}$ & 1 & POP & Half of USWIM, age 2 & 0.02 & L/hr \\
USHIN & $\begin{array}{l}\text { Ingestion rate of water while } \\
\text { showering }\end{array}$ & 2 & POP & Half of USWIM & 0.02 & L/hr \\
USHIN & $\begin{array}{l}\text { Ingestion rate of water while } \\
\text { showering }\end{array}$ & 3 & POP & Half of USWIM & 0.02 & $\mathrm{~L} / \mathrm{hr}$ \\
USHIN & $\begin{array}{l}\text { Ingestion rate of water while } \\
\text { showering }\end{array}$ & 4 & POP & Half of USWIM & 0.02 & $\mathrm{~L} / \mathrm{hr}$ \\
USHIN & $\begin{array}{l}\text { Ingestion rate of water while } \\
\text { showering }\end{array}$ & 5 & POP & Half of USWIM & 0.02 & $\mathrm{~L} / \mathrm{hr}$ \\
USHIN & $\begin{array}{l}\text { Ingestion rate of water while } \\
\text { showering }\end{array}$ & 6 & POP & Half of USWIM & 0.01 & $\mathrm{~L} / \mathrm{hr}$ \\
\hline
\end{tabular}

(a) See Table 8.1 for age groups.

\subsubsection{Water Ingestion Exposure: Incidental Swimming Water}

The user is responsible for entering the same values in the Pathway Selections: Water Ingestion while Swimming and External Exposure while Swimming (see Section 8.1.2) parameters.

The units and ranges for swimming water ingestion parameters are found in Section 8.1.2.

Additional information sources and tables of values are found in Section 8.1.2.2

\subsection{Pathway Selection: Inadvertent Soil Ingestion}

\subsubsection{USOIL Human Soil Ingestion Rate and TSOIL Soil Contact Days}

Annual incidental soil ingestion is determined by multiplying the USOIL and TSOIL parameters. Soil may be considered either outdoor soil or indoor dust accumulation. If the radiological characteristics of the outdoor and indoor soils are understood to be sufficiently different, the user may want to evaluate each in separate cases. Set the soil consumption period (TSOIL) for each age group to $365 \mathrm{~d}$.

Incidental ingestion of soil by animals is discussed in Section 7.16. 
The USOIL units and range in Gv2 are: $\mathrm{mg} / \mathrm{d}(0-15,000)$.

The TSOIL units and range in Gv2 are: $\mathrm{d}(0-365)$.

Values were obtained from Chapter 5 of EPA (2011). The range of MEI intakes are the central or upper values, at the low end, to the pica values at the high end. The MEI age 1 and MEI age 6 values can use the closest age category value listed. The range of Population values are the central to upper incidental soil and dust intakes. Single value assignments should use the minimum values listed for the population and the MEI. The TSOIL value assigned assumes fulltime occupancy at the location of interest and therefore, would be $365 \mathrm{~d} / \mathrm{yr}$.

\subsubsection{Additional Sources of Information}

No additional sources that provide greater detail than EPA (2011) were identified.

\subsubsection{USOIL Human Incidental Soil Ingestion Rate Table}

Table 8.17. USOIL Incidental Ingestion of Soil

\begin{tabular}{clccccccc}
\hline Gv2 ID & Gv2 Description & Age $^{(\mathrm{a})}$ & Qualifier & Reference & Comment & Min & Max & Units \\
\hline USOIL & $\begin{array}{l}\text { Inadvertent soil } \\
\text { ingestion rate }\end{array}$ & 2 & MEI & EPA (2011) & $\begin{array}{c}\text { Table 5-1, soil+dust } \\
\text { upper to pica }\end{array}$ & $\mathbf{2 0 0}$ & 1000 & $\mathrm{mg} / \mathrm{day}$ \\
USOIL & $\begin{array}{l}\text { Inadvertent soil } \\
\text { ingestion rate }\end{array}$ & 3 & MEI & EPA (2011) & $\begin{array}{c}\text { Table 5-1, soil+dust } \\
\text { upper to pica }\end{array}$ & $\mathbf{2 0 0}$ & 1000 & $\mathrm{mg} / \mathrm{day}$ \\
USOIL & $\begin{array}{l}\text { Inadvertent soil } \\
\text { ingestion rate }\end{array}$ & 4 & MEI & EPA (2011) & $\begin{array}{c}\text { Table 5-1, soil+dust } \\
\text { central to pica }\end{array}$ & $\mathbf{2 0 0}$ & 1000 & $\mathrm{mg} / \mathrm{day}$ \\
USOIL & $\begin{array}{l}\text { Inadvertent soil } \\
\text { ingestion rate }\end{array}$ & 5 & MEI & EPA (2011) & $\begin{array}{c}\text { Table 5-1, soil+dust } \\
\text { central to pica }\end{array}$ & $\mathbf{2 0 0}$ & 1000 & $\mathrm{mg} / \mathrm{day}$ \\
\hline
\end{tabular}

(a) See Table 8.1 for age groups.

Values in bold are recommended for discrete values.

\begin{tabular}{|c|c|c|c|c|c|c|c|c|}
\hline Gv2 ID & Gv2 Description & $\operatorname{Age}^{(a)}$ & Qualifier & Reference & Comment & Min & Max & Units \\
\hline USOIL & $\begin{array}{l}\text { Inadvertent soil } \\
\text { ingestion rate }\end{array}$ & 1 & POP & $\begin{array}{l}\text { EPA } \\
(2011)\end{array}$ & $\begin{array}{l}\text { Table 5-1, soil+dust; } \\
\text { central to upper }\end{array}$ & 60 & 200 & $\mathrm{mg} /$ day \\
\hline USOIL & $\begin{array}{l}\text { Inadvertent soil } \\
\text { ingestion rate }\end{array}$ & 2 & POP & $\begin{array}{c}\text { EPA } \\
(2011)\end{array}$ & $\begin{array}{l}\text { Table 5-1, soil+dust; } \\
\text { central to upper }\end{array}$ & 100 & 200 & $\mathrm{mg} /$ day \\
\hline USOIL & $\begin{array}{l}\text { Inadvertent soil } \\
\text { ingestion rate }\end{array}$ & 3 & POP & $\begin{array}{c}\text { EPA } \\
(2011)\end{array}$ & $\begin{array}{l}\text { Table 5-1, soil+dust; } \\
\text { central to upper }\end{array}$ & 100 & 200 & $\mathrm{mg} /$ day \\
\hline USOIL & $\begin{array}{l}\text { Inadvertent soil } \\
\text { ingestion rate }\end{array}$ & 4 & POP & $\begin{array}{c}\text { EPA } \\
(2011)\end{array}$ & $\begin{array}{l}\text { Table 5-1, soil+dust; } \\
\text { central to upper }\end{array}$ & 100 & 200 & $\mathrm{mg} /$ day \\
\hline USOIL & $\begin{array}{l}\text { Inadvertent soil } \\
\text { ingestion rate }\end{array}$ & 5 & POP & $\begin{array}{c}\text { EPA } \\
(2011)\end{array}$ & $\begin{array}{l}\text { Table 5-1, soil+dust; } \\
\text { central to upper }\end{array}$ & 100 & 200 & $\mathrm{mg} /$ day \\
\hline USOIL & $\begin{array}{l}\text { Inadvertent soil } \\
\text { ingestion rate }\end{array}$ & 6 & POP & $\begin{array}{c}\text { EPA } \\
(2011)\end{array}$ & $\begin{array}{l}\text { Table 5-1, soil+dust; } \\
\text { central to upper }\end{array}$ & 50 & 200 & $\mathrm{mg} /$ day \\
\hline
\end{tabular}

(a) See Table 8.1 for age groups.

Values in bold are recommended for discrete values. 


\subsection{Pathway Selection: Inhalation (multiple)}

Three inhalation pathways require parameterization. The boxes checked on the Pathways tab of the Exposure module will indicate which three inhalation pathways are calculated. The pathway selection "Air inhalation" will describe inhalation of plume contaminants (i.e., outdoor air). The pathway selection "Resuspended soil inhalation" describes suspended or resuspended soil particulate inhalation. The pathway selection "Indoor inhalation", of course, describes inhalation of indoor air. Indoor air inhalation will add in shower water contributions to the outdoor air levels. The user is responsible for making sure the fraction of the day that indoor and outdoor inhalation occurs is 1 or less.

\subsubsection{Ux, Tx, FRx Inhalation}

Each inhalation pathway selection requires three parameters: inhalation rate $(\mathrm{Ux})$, inhalation period $(\mathrm{T} x)$, and fraction of the day that inhalation of the indicated air type occurs (FRx). The product of these three within each air type will indicate the volume of each air type inhaled. The time periods (TINH, TINHR, TINDHR) for each age group should be assigned as $356 \mathrm{~d}$. The user is responsible for making the FRINH and FRINDR parameters add to one.

The user is responsible for making judgments on the reasonableness of the external ground exposure FTOUT value (see Section 8.1.1) and the FRINH and FRINHR values of outdoor and resuspended soil inhalation pathways parameters. One option for inhalation assignments is for the user to assign the same inhalation rate to all categories (i.e., outdoor air, resuspended soil air, and indoor air) and only vary the fractional exposure time to each route.

- Air inhalation (outdoor air)

- The units and range for UINH in Gv2 are: $\mathrm{m}^{3} / \mathrm{d}(0-50)$

- The units and range for TINH in Gv2 are: $\mathrm{d} / \mathrm{yr}(0-365)$

- The units and range for FRINH in Gv2 are: fraction (0-1)

- Resuspended soil inhalation

- The units and range for UINHR in Gv2 are: $\mathrm{m}^{3} / \mathrm{d}(0-50)$

- The units and range for TINHR in Gv2 are: $\mathrm{d} / \mathrm{yr}(0-365)$

- The units and range for FRINHR in Gv2 are: fraction (0-1)

- Indoor Air inhalation

- The units and range for UINDRH in Gv2 are: $\mathrm{m}^{3} / \mathrm{d}(0-50)$

- The units and range for TINDRH in Gv2 are: $\mathrm{d} / \mathrm{yr}(0-365)$

- The units and range for FRINDR in Gv2 are: fraction (0-1).

EPA 2011 provides detailed intake rates for a variety of ages and activities (Chapter 6) and fractions of time spent indoors and outdoors (Chapter 16). UINH values (outdoor air inhalation) rates are shown in Table 8.18 and assume the individual is outside 100 percent of the time. The UINDRH (indoor air inhalation) values use the recommended outdoor rate (UINH) value and multiply it by the age-dependent fraction of time that an individual remains indoors, according to the data in Table 16-1 of EPA (2011). If 
the individual is not assumed to be outdoors full time, the difference between the fulltime outdoors UINH value of Table 8.18 and the UINDRH value of Table 8.19 would provide an adjusted UINH value.

People generally remain indoors. See Section 8.1.1, FTIN and FTOUT values and associated notes. The values assigned here for FRINHR and FRINDR should be consistent. Some scenarios may consider that the individual is always outdoors.

\subsubsection{Additional Sources of Information}

ICRP Publications 66 and 71 (ICRP 1994a, 1995b). The ICRP publications indicate age-specific inhalation rates. ICRP information includes inhalation rates $(\mathrm{m} 3 / \mathrm{hr})$ for various activity levels. Hours of activity levels can then be assigned to determine daily inhalation volumes.

Table 3 of DOE (2011) provides age-dependent inhalation volumes for males and females.

\subsubsection{UINH, UINHR, UINDRH Inhalation Rate Tables}

Table 8.18. UINH Outdoor Air Inhalation Rate

\begin{tabular}{|c|c|c|c|c|c|c|c|c|c|}
\hline Gv2 ID & $\begin{array}{c}\text { Gv2 } \\
\text { Description }\end{array}$ & $\operatorname{Age}^{(a)}$ & Qualifier & Reference & Comment & $\begin{array}{l}\text { Recom- } \\
\text { mended }\end{array}$ & Min & Max & Units \\
\hline UINH & $\begin{array}{l}\text { Air inhalation } \\
\text { rate }\end{array}$ & 1 & MEI & $\begin{array}{c}\text { EPA } \\
(2011)\end{array}$ & $\begin{array}{l}\text { Ch } 6 \text {, assumes } \\
100 \% \text { outdoor }\end{array}$ & 6.8 & 6.8 & 6.8 & $\mathrm{~m}^{3} /$ day \\
\hline UINH & $\begin{array}{l}\text { Air inhalation } \\
\text { rate }\end{array}$ & 2 & MEI & $\begin{array}{c}\text { EPA } \\
(2011)\end{array}$ & $\begin{array}{l}\text { Ch } 6 \text {, assumes } \\
100 \% \text { outdoor }\end{array}$ & 9.2 & 9.2 & 9.2 & $\mathrm{~m}^{3} /$ day \\
\hline UINH & $\begin{array}{l}\text { Air inhalation } \\
\text { rate }\end{array}$ & 3 & MEI & $\begin{array}{c}\text { EPA } \\
(2011)\end{array}$ & $\begin{array}{l}\text { Ch } 6 \text {, assumes } \\
100 \% \text { outdoor }\end{array}$ & 13.8 & 13.8 & 13.8 & $\mathrm{~m}^{3} /$ day \\
\hline UINH & $\begin{array}{l}\text { Air inhalation } \\
\text { rate }\end{array}$ & 4 & MEI & $\begin{array}{c}\text { EPA } \\
(2011)\end{array}$ & $\begin{array}{l}\text { Ch } 6 \text {, assumes } \\
100 \% \text { outdoor }\end{array}$ & 16.6 & 16.6 & 16.6 & $\mathrm{~m}^{3} /$ day \\
\hline UINH & $\begin{array}{l}\text { Air inhalation } \\
\text { rate }\end{array}$ & 5 & MEI & $\begin{array}{c}\text { EPA } \\
(2011)\end{array}$ & $\begin{array}{l}\text { Ch } 6 \text {, assumes } \\
100 \% \text { outdoor }\end{array}$ & 23.3 & 23.3 & 23.3 & $\mathrm{~m}^{3} /$ day \\
\hline UINH & $\begin{array}{l}\text { Air inhalation } \\
\text { rate }\end{array}$ & 6 & MEI & $\begin{array}{l}\text { EPA } \\
(2011)\end{array}$ & $\begin{array}{l}\text { Ch } 6 \text {, assumes } \\
100 \% \text { outdoor }\end{array}$ & 19.4 & 19.4 & 19.4 & $\mathrm{~m}^{3} /$ day \\
\hline UINH & $\begin{array}{l}\text { Air inhalation } \\
\text { rate }\end{array}$ & 1 & POP & $\begin{array}{l}\text { EPA } \\
(2011)\end{array}$ & $\begin{array}{l}\text { Ch } 6 \text {, assumes } \\
100 \% \text { outdoor }\end{array}$ & 4.5 & 3.6 & 5.4 & $\mathrm{~m}^{3} /$ day \\
\hline UINH & $\begin{array}{l}\text { Air inhalation } \\
\text { rate }\end{array}$ & 2 & POP & $\begin{array}{c}\text { EPA } \\
(2011)\end{array}$ & $\begin{array}{l}\text { Ch } 6 \text {, assumes } \\
100 \% \text { outdoor }\end{array}$ & 7.2 & 5.4 & 8.9 & $\mathrm{~m}^{3} /$ day \\
\hline UINH & $\begin{array}{l}\text { Air inhalation } \\
\text { rate }\end{array}$ & 3 & POP & $\begin{array}{c}\text { EPA } \\
(2011)\end{array}$ & $\begin{array}{l}\text { Ch } 6 \text {, assumes } \\
100 \% \text { outdoor }\end{array}$ & 10.1 & 10.1 & 10.1 & $\mathrm{~m}^{3} /$ day \\
\hline UINH & $\begin{array}{l}\text { Air inhalation } \\
\text { rate }\end{array}$ & 4 & POP & $\begin{array}{l}\text { EPA } \\
(2011)\end{array}$ & $\begin{array}{l}\text { Ch } 6 \text {, assumes } \\
100 \% \text { outdoor }\end{array}$ & 12 & 12 & 12 & $\mathrm{~m}^{3} /$ day \\
\hline UINH & $\begin{array}{l}\text { Air inhalation } \\
\text { rate }\end{array}$ & 5 & POP & $\begin{array}{c}\text { EPA } \\
(2011)\end{array}$ & $\begin{array}{l}\text { Ch } 6 \text {, assumes } \\
100 \% \text { outdoor }\end{array}$ & 15.8 & 15.2 & 16.3 & $\mathrm{~m}^{3} /$ day \\
\hline UINH & $\begin{array}{l}\text { Air inhalation } \\
\text { rate }\end{array}$ & 6 & POP & $\begin{array}{l}\text { EPA } \\
(2011)\end{array}$ & $\begin{array}{l}\text { Ch } 6 \text {, assumes } \\
100 \% \text { outdoor }\end{array}$ & 14.1 & 12.2 & 16 & $\mathrm{~m}^{3} /$ day \\
\hline
\end{tabular}

(a) See Table 8.1 for the ages of each age category. 
Table 8.19. UINDRH Indoor Air Inhalation Rate

\begin{tabular}{|c|c|c|c|c|c|c|c|c|c|}
\hline Gv2 ID & $\begin{array}{c}\text { Gv2 } \\
\text { Description }\end{array}$ & $\operatorname{Age}^{(a)}$ & Qualifier & Reference & $\begin{array}{c}\text { Comment/Age- } \\
\text { Dependent Assumptions }\end{array}$ & $\begin{array}{l}\text { Recom- } \\
\text { mended }\end{array}$ & Min & Max & Units \\
\hline UINDRH & $\begin{array}{l}\text { Indoor air } \\
\text { inhalation rate }\end{array}$ & 1 & MEI & $\begin{array}{c}\text { EPA } \\
(2011)\end{array}$ & $\begin{array}{l}\text { UINH*Ch16frac. } \\
\text { indoors_age (1.0) }\end{array}$ & 6.8 & N/A & N/A & $\mathrm{m}^{3} / \mathrm{d}$ \\
\hline UINDRH & $\begin{array}{l}\text { Indoor air } \\
\text { inhalation rate }\end{array}$ & 2 & MEI & $\begin{array}{c}\text { EPA } \\
(2011)\end{array}$ & $\begin{array}{l}\text { UNIH*Ch16frac. } \\
\text { indoors (1.0) }\end{array}$ & 9.2 & N/A & N/A & $\mathrm{m}^{3} / \mathrm{d}$ \\
\hline UINDRH & $\begin{array}{l}\text { Indoor air } \\
\text { inhalation rate }\end{array}$ & 3 & MEI & $\begin{array}{c}\text { EPA } \\
(2011)\end{array}$ & $\begin{array}{l}\text { UINH*Ch16frac. } \\
\text { indoors }(0.94)\end{array}$ & 13 & N/A & N/A & $\mathrm{m}^{3} / \mathrm{d}$ \\
\hline UINDRH & $\begin{array}{l}\text { Indoor air } \\
\text { inhalation rate }\end{array}$ & 4 & MEI & $\begin{array}{l}\text { EPA } \\
(2011)\end{array}$ & $\begin{array}{l}\text { UINH*Ch16frac. } \\
\text { indoors }(0.89)\end{array}$ & 14.7 & N/A & N/A & $\mathrm{m}^{3} / \mathrm{d}$ \\
\hline UINDRH & $\begin{array}{l}\text { Indoor air } \\
\text { inhalation rate }\end{array}$ & 5 & MEI & $\begin{array}{l}\mathrm{EPA} \\
(2011)\end{array}$ & $\begin{array}{l}\text { UINH* Ch16frac. } \\
\text { indoors }(0.90)\end{array}$ & 21.0 & N/A & N/A & $\mathrm{m}^{3} / \mathrm{d}$ \\
\hline UINDRH & $\begin{array}{l}\text { Indoor air } \\
\text { inhalation rate }\end{array}$ & 6 & MEI & $\begin{array}{c}\text { EPA } \\
(2011)\end{array}$ & $\begin{array}{l}\text { UINH*Ch16frac. } \\
\text { indoors }(1.0)\end{array}$ & 19.4 & N/A & N/A & $\mathrm{m}^{3} / \mathrm{d}$ \\
\hline UINDRH & $\begin{array}{l}\text { Indoor air } \\
\text { inhalation rate }\end{array}$ & 1 & POP & $\begin{array}{c}\text { EPA } \\
(2011)\end{array}$ & $\begin{array}{l}\text { UINH_age*Ch16frac. } \\
\text { indoors_age }(0.77-1.0)\end{array}$ & 4.1 & 2.8 & 5.4 & $\mathrm{~m}^{3} / \mathrm{d}$ \\
\hline UINDRH & $\begin{array}{l}\text { Indoor air } \\
\text { inhalation rate }\end{array}$ & 2 & POP & $\begin{array}{c}\text { EPA } \\
(2011)\end{array}$ & $\begin{array}{l}\text { UINH*Ch16frac. } \\
\text { indoors }(0.68-0.94)\end{array}$ & 6.05 & 3.7 & 8.4 & $\mathrm{~m}^{3} / \mathrm{d}$ \\
\hline UINDRH & $\begin{array}{l}\text { Indoor air } \\
\text { inhalation rate }\end{array}$ & 3 & POP & $\begin{array}{c}\text { EPA } \\
(2011)\end{array}$ & $\begin{array}{l}\text { UINH*Ch16frac. } \\
\text { indoors }(0.66-0.89)\end{array}$ & 7.85 & 6.7 & 9 & $\mathrm{~m}^{3} / \mathrm{d}$ \\
\hline UINDRH & $\begin{array}{l}\text { Indoor air } \\
\text { inhalation rate }\end{array}$ & 4 & POP & $\begin{array}{c}\text { EPA } \\
(2011)\end{array}$ & $\begin{array}{l}\text { UINH*Ch16frac. } \\
\text { indoors }(0.62-0.86)\end{array}$ & 8.9 & 7.4 & 10.4 & $\mathrm{~m}^{3} / \mathrm{d}$ \\
\hline UINDRH & $\begin{array}{l}\text { Indoor air } \\
\text { inhalation rate }\end{array}$ & 5 & POP & $\begin{array}{c}\text { EPA } \\
(2011)\end{array}$ & $\begin{array}{l}\text { UINH*Ch16frac. } \\
\text { indoors }(0.58-0.88)\end{array}$ & 11.55 & 8.8 & 14.3 & $\mathrm{~m}^{3} / \mathrm{d}$ \\
\hline UINDRH & $\begin{array}{l}\text { Indoor air } \\
\text { inhalation rate }\end{array}$ & 6 & POP & $\begin{array}{c}\text { EPA } \\
(2011)\end{array}$ & $\begin{array}{l}\text { UINH*Ch16frac. } \\
\text { indoors }(0.66-0.82)\end{array}$ & 10.55 & 8 & 13.1 & $\mathrm{~m}^{3} / \mathrm{d}$ \\
\hline
\end{tabular}

(a) See Table 8.1 for the ages of each age category.

\subsection{References: Receptor Intake Module}

Barr C, D Schmidt, S Sherbini. 2010. Development of Site-specific Shielding Factors for Use in Radiological Risk Assessments. U.S. Nuclear Regulatory Commission, Washington, D.C. Available at: http://pbadupws.nrc.gov/docs/ML1006/ML100610262.pdf.

DOE. 2011. Derived Concentration Technical Standard. DOE-STD-1196-2011, U.S. Department of Energy, Washington, D.C. Available at:

http://www.hss.doe.gov/nuclearsafety/techstds/standard.html\#1001.

EPA. 2004. Estimated per Capita Water Ingestion and Body Weight in the United States-An Update. EPA-822-R-00-001, U.S. Environmental Protection Agency, Washington, D.C. Available online at http://www.epa.gov/waterscience/criteria/drinking/percapita/2004.pdf.

EPA. 2011. Exposure Factors Handbook: 2011 Edition. EPA/600/R-090/052F, U.S. Environmental Protection Agency, Office of Research and Development, Washington, D.C.

Gebhardt SE and RG Thomas. 2002. Nutritive Value of Foods. Home and Garden Bulletin 72, U.S. Department of Agriculture, Agricultural Research Service, Washington, D.C. 
IAEA. 2010. Handbook of Parameter Values for the Prediction of Radionuclide Transfer in Terrestrial and Freshwater Environments. Technical Report Series No. 472, International Atomic Energy Agency, Vienna.

ICRP. 1994a. Human Respiratory Tract Model for Radiological Protection. ICRP (International Commission on Radiological Protection) Publication 66, Ann. ICRP 24 (1-3).

ICRP. 1994b. Dose Coefficients for Intakes of Radionuclides by Workers. ICRP (International Commission on Radiological Protection) Publication 68, Ann. ICRP 24 (4).

ICRP. 1995a. Age-dependent Doses to Members of the Public from Intake of Radionuclides - Part 3 Ingestion Dose Coefficients. ICRP (International Commission on Radiological Protection) Publication 69, Ann. ICRP 25 (1).

ICRP. 1995b. Age-dependent Doses to Members of the Public from Intake of Radionuclides - Part 4 Inhalation Dose Coefficients. ICRP (International Commission on Radiological Protection) Publication 71, Ann. ICRP 25 (3-4).

ICRP. 1995c. Age-dependent Doses to the Members of the Public from Intake of Radionuclides - Part 5 Compilation of Ingestion and Inhalation Coefficients. ICRP (International Commission on Radiological Protection) Publication 72, Ann. ICRP 26 (1).

Jacob P and R Meckback. 1987. “Shielding Factors and External Dose Evaluation.” Radiation Protection Dosimetry 21(1-3):79-85.

LeGrand J, JC Croize, T deDorlodot, and Y Roux. 1987. "Statistical Survey of the Housing Characteristics and Evaluation of Shielding Factors in the Surroundings of French Nuclear Sites." Radiation Protection Dosimetry 21(1/3):87-95.

Leung JKC. 1992. "Application of Shielding Factors for Protection against Gamma Radiations during a Nuclear Accident." IEEE Transactions on Nuclear Science 39(5):1512-1518.

NRC. 1977. Calculation of Annual Doses to Man from Routine Releases of Reactor Effluents for the Purpose of Evaluating Compliance with 10 CFR Part 50, Appendix I. Regulatory Guide 1.109, Rev. 1, U.S. Nuclear Regulatory Commission, Washington, D.C.

Sebastian RS, JD Goldman, CW Enns, and RP LaComb. 2010. "Fluid Milk Consumption in the United States." Food Surveys Research Group, Dietary Data Brief No. 3. U.S. Department of Agriculture, Agriculture Research Service. Last accessed at:

www.ars.usda.gov/.../12355000/pdf/DBrief/3 milk_consumption_0506.pdf.

Siega-Riz AM, DM Deming, KC Reidy, MK Fox, E Condon, and RR Briefel. 2010. "Food Consumption Patterns of Infants and Toddlers: Where Are We Now?" Journal of the American Dietetic Association 110(12 Suppl):S38-S51.

USCB. 2012. U.S. Census Bureau, Statistical Abstract of the United States: 2012, The National Data Book. U.S. Census Bureau, Washington, D.C. Last accessed at: http://www.census.gov/compendia/statab/2012/tables/12s0217.pdf. 
USDA. 1992. Weights, Measures, and Conversion Factors for Agricultural Commodities and Their Products. USDA Handbook No. 697, U.S. Department of Agriculture, Washington, D.C.

USDA. 2009. 2007 Census of Agriculture, United States Summary and State Data. Vol. 1, Geographic Area Series, Part 51, AC-07-A-51, U.S. Department of Agriculture, National Agricultural Statistics Service, Washington, D.C. Last accessed at:

http://www.agcensus.usda.gov/Publications/2007/Full_Report/index.asp. 



\subsection{Module: Health Impacts}

This module is downstream of the Receptor Intake module and may be upstream of a Report Generator module, if the user selects the module. The Health Impacts module establishes the radiation dose factors applied. The intake estimates of the Receptor Intake module are translated into radiation doses in this module. Three tabs are in this module: Method Selection, Method Parameters, and Constituent Parameters. The user will select the "Calculate Dose and/or risk using ICRP-60 and EPA risk factors (Federal Guidance Reports 12/13)" in the Method Selection tab. The remaining tabs are discussed, below.

\subsection{Tab: Method Parameters}

The user will opt for "Calculate radiation effective dose equivalent (CEDE)" The soil thickness (SOILT) and soil density (SLDN) are to be entered as indicated in the Chronic Exposure module, see Section 7.7, assigning the SOILT parameter the value indicated for SURCM, with units conversion to meters.

The units and range for SOILT in Gv2 are: $\mathrm{m}(0-5)$.

The units and range for SLDN in Gv2 are: $\mathrm{kg} / \mathrm{m}^{3}(500-3000)$.

There is currently (Gv2.10) an inconsistency in the SLDN units assignment in the Health Impacts module and the Chronic Exposure module. This becomes evident only when the user reviews the GID file. Although the parameters will have the same ID (i.e., SLDN) enter the value appropriate to the units indicated in the user entry screen. SLDN of the Chronic Exposure module has the units $\mathrm{kg} / \mathrm{m}^{2}$ and the SLDN of the health impacts module has the units $\mathrm{kg} / \mathrm{m}^{3}$. The SLDN values indicated for each module will differ by a factor of $1 / 0.15$.

\subsection{Tab: Constituent Parameters}

The constituent parameters tab is important when inhalation pathways are considered. The user will scroll through the constituents and assign the appropriate lung transfer inhalation class solubility: gas, vapor, fast, medium, and slow. See ICRP Publications 68, 69, 71, and 72 (ICRP 1994, 1995a, 1995b, 1995c) for details regarding solubility class assumptions for inhalation dose factor calculations. Table 2 of ICRP Publication 72 (1995c) summarizes the solubility classes available for each chemical and provides reference to the ICRP publication that contains biokinetic model details.

Generic guidance for inhalation class designation is provided in the Help file. Help file values were based on two primary factors: the predominant solubility typically encountered and, when such information is unavailable or variable, the solubility class that would maximize the dose. Although the Help file lists suggestions by radionuclide, the user could reasonably assign inhalation classes by chemical rather than specific nuclide. 


\subsubsection{Additional Sources of Information}

DOE Derived Concentration Technical Standard (DOE 2011). Table 4 indicates generic lung solubility type recommendations for each element in particulate form.

\subsection{References: Health Impact Module}

ICRP. 1994. Dose Coefficients for Intakes of Radionuclides by Workers. ICRP (International Commission on Radiological Protection) Publication 68, Ann. ICRP 24 (4).

ICRP. 1995a. Age-dependent Doses to Members of the Public from Intake of Radionuclides - Part 3 Ingestion Dose Coefficients. ICRP (International Commission on Radiological Protection) Publication 69, Ann. ICRP 25 (1).

ICRP. 1995b. Age-dependent Doses to Members of the Public from Intake of Radionuclides - Part 4 Inhalation Dose Coefficients. ICRP (International Commission on Radiological Protection) Publication 71, Ann. ICRP 25 (3-4).

ICRP. 1995c. Age-dependent Doses to the Members of the Public from Intake of Radionuclides - Part 5 Compilation of Ingestion and Inhalation Coefficients. ICRP (International Commission on Radiological Protection) Publication 72, Ann. ICRP 26 (1).

DOE. 2011. Derived Concentration Technical Standard. DOE-STD-1196-2011, U.S. Department of Energy, Washington, D.C. Available at:

http://www.hss.doe.gov/nuclearsafety/techstds/standard.html\#1001. 


\subsection{Considerations for Tritium and Carbon-14}

Gv2 includes special models for tritium $\left({ }^{3} \mathrm{H}\right.$ or $\left.\mathrm{T}\right)$ and carbon-14 $\left({ }^{14} \mathrm{C}\right)$. The special considerations required for these two ubiquitous nuclides for chronic air or water emission cases are reviewed in this section. The model assumptions generally assume instantaneous equilibrium for the chronic emission cases, which is more reasonable for food and feed modeling but may overestimate inhalation, external exposure, and water ingestion pathway exposures. The magnitudes of the over-estimates are typically not excessive in most cases.

\subsection{Tritium Model Considerations}

Tritium can be released as a gas $\left(\mathrm{H}^{3} \mathrm{H}\right.$ or $\left.{ }^{3} \mathrm{H}^{3} \mathrm{H}\right)$, vapor $\left(\mathrm{H}^{3} \mathrm{HO}\right.$ or $\left.{ }^{3} \mathrm{H}^{3} \mathrm{HO}\right)$, where $\mathrm{H}$ indicates stable, non-tritium hydrogen. Organically bound tritium (OBT) is automatically included in the source term when tritium gas or tritiated water is selected. Tritium gas conversion into vapor; and biological conversion of gas and vapor into OBT is modeled. OBT describes tritium that is incorporated into food or feed. Any direct emission of OBT from a facility would be better handled by hand calculations or sampling. Chronic models for tritium are described in Section 9.6 of the Gv2 SDD (Napier et al. 2012).

The tritium model generally assumes specific activity equilibrium with some differences in modeling depending on the chemical form. As an example, a constant $\mathrm{Bq} \mathrm{H}^{3} \mathrm{HO} / \mathrm{m}^{3}$ emitted in air effluent is divided by the absolute humidity entered in the Chronic Exposure Module to determine the $\mathrm{Bq} / \mathrm{L}$ in air moisture.

To model food or feed crop tritiated water concentrations, the calculated equilibrium level of tritiated water $(\mathrm{Bq} / \mathrm{L})$ in air moisture is used with a 0.9 factor applied for leafy vegetable and pasture categories and a 0.8 factor applied for other crop categories (fruit, root, other vegetables, grain).

To model food or feed crop OBT concentrations, the calculated $\mathrm{Bq} / \mathrm{L}$ in air moisture is used with a 0.9 factor for all food and feed crops, as well as a 0.9 form discrimination factor, and factors to account for the relative moisture content of various crops (see Section 9.6.1 of Napier et al. 2012 for details). There is no defined root uptake pathway; all pathways related to root uptake (food or feed crop) are set equal to zero.

Modules itemized below require commentary for tritium modeling. Modules not itemized do not require additional considerations, if tritium is a contaminant of concern.

\subsubsection{Module: Constituent}

Constituent of concern choices are indicated in Table 10.1. When tritiated water or tritium gas is selected as a constituent of concern, OBT (organically-bound tritium) is automatically added. If OBT is deleted as a constituent of concern, the $\mathrm{H} 3$ or H3EL will be deleted as well. However, if $\mathrm{H} 3$ or H3EL is deleted, the OBT will remain. The OBT is indicated only for subsequent use by the code. The Gv2.10 tritium models only reflect environmental modeling of tritium gas and tritiated water. 
Table 10.1. Tritium Nuclide Source Term Selection

\begin{tabular}{|c|c|c|}
\hline Effluent Path & $\begin{array}{l}\text { Constituent Module - } \\
\text { Constituent of Concern }\end{array}$ & Comment \\
\hline \multicolumn{3}{|l|}{ Air Effluent } \\
\hline Tritiated Water & H3 or HTO & - \\
\hline Tritium gas & H3EL or HT & $\begin{array}{l}\text { Although soil conversion of tritium gas to tritiated } \\
\text { water is modeled, tritiated soil water is assumed to be } \\
\text { an insignificant dose contributor and is ignored for } \\
\text { soil exposure impacts. }\end{array}$ \\
\hline $\begin{array}{l}\text { Organically Bound } \\
\text { Tritium }\end{array}$ & $\mathrm{OBT}^{(\mathrm{a})}$ & $\begin{array}{l}\text { Environmental modeling of facility OBT emissions in } \\
\text { air is best performed by hand calculations or } \\
\text { environmental sampling. }\end{array}$ \\
\hline \multicolumn{3}{|l|}{ Liquid Effluent } \\
\hline Tritiated Water & H3 or HTO & $\begin{array}{l}\text { Enter release as dissolved flux in User-Defined Water } \\
\text { Module. }\end{array}$ \\
\hline Tritium gas & H3EL or HT & $\begin{array}{l}\text { Tritium gas in water not modeled in Gv2.10. Enter } \\
\text { release rate as zero. }\end{array}$ \\
\hline $\begin{array}{l}\text { Organically Bound } \\
\text { Tritium }\end{array}$ & $\mathrm{OBT}^{(\mathrm{a})}$ & $\begin{array}{l}\text { Environmental modeling of facility OBT emissions in } \\
\text { liquid is best performed by hand calculations or } \\
\text { environmental sampling. }\end{array}$ \\
\hline
\end{tabular}

(a) Automatically added when any other tritium constituent of concern is selected. Concentrations calculated by the code, downstream, in the Chronic Exposure Module.

Water purification-, aquatic food, animal product, and food crop transfer factors all default to 1 for H3, H3EL, and OBT. H3EL deposition will be zero because gases are not modeled to deposit on the ground or plant surfaces.

\subsubsection{Module: User-Defined AIR and User-Defined WATER}

For the User-Defined AIR module, the user is requested to enter release rates for the selected tritium form and OBT, as with other nuclides. The user is responsible for entering a zero for OBT because the Gv2 algorithms reflect only tritium gas and water vapor models. Any special entries of particle size, using the "Flux Types" button, will have no impact for H3 or OBT.

The required zero Ci OBT releases in the source term results in OBT media concentrations of zero in the output AFF file of the User-Defined module and in the ATO file of the AIR module; but concentrations are non-zero in the EPF file of the Exposure and subsequent modules for food and soil pathways.

\subsubsection{Module: Surface Water}

Water concentrations are modeled identically for tritiated water and OBT. As indicated earlier, the user is discouraged from entering an OBT source term because models reflect tritiated water environmental movement. 


\subsubsection{Module: Chronic Exposure}

The chronic exposure module requires the absolute humidity to be entered. Humidity values that reflect that of the growing season are preferred.

\section{Controls Tab: ABSHUM Absolute Humidity}

Relative humidity $(\mathrm{RH})$ information can be found at the NCDC website http://wf.ncdc.noaa.gov/oa/climate/online/ccd/avgrh.html (NCDC 2008). Data were summarized into the USDA watershed regions (see Figure 7.3) in Table 10.2 for the months of May through September, which are common growing season months in the US. Gv2 requires absolute, rather than relative, humidity values. The RH information can be converted into an approximate absolute humidity with the information provided in Table 10.3; the temperature assumed should at least correspond with that entered in the User-Defined module for ambient temperature (see Section 3.0) but is best assumed to be the average temperature during the growing season. Data in Table 10.3 were derived from TIS-GRD (2012); more precise calculations can be used to convert from relative to absolute humidity from calculations found in other resources. Specific humidity values could be used for absolute humidity values.

Chronic exposure module calculations for environmental media concentrations are modeled identically for tritiated water and OBT source terms in the EPF for aquatic foods, direct exposure to water pathways (i.e., boating, swimming, shower, and water ingestion), and shoreline exposure. Liquid effluent tritiated water source terms impact food and feed concentrations when irrigation is assumed.

Table 10.2. Relative Humidity (RH) by Watershed Region

\begin{tabular}{cccc}
\hline Watershed Region $^{(\text {a) }}$ & Average of Morning RH & Average of Afternoon RH & Average RH \\
\hline 1 & 80.2 & 59.7 & 69.9 \\
2 & 80.6 & 55.9 & 68.3 \\
3 & 87.4 & 59.9 & 73.7 \\
4 & 84.7 & 58.7 & 71.7 \\
5 & 85.2 & 58.8 & 72.0 \\
6 & 88.2 & 58.5 & 73.4 \\
7 & 83.7 & 60.2 & 72.0 \\
8 & 90.3 & 63.1 & 76.7 \\
9 & 81.3 & 54.8 & 68.0 \\
10 & 75.5 & 42.4 & 58.9 \\
11 & 82.3 & 56.9 & 69.6 \\
12 & 82.5 & 57.4 & 69.9 \\
13 & 65.5 & 31.7 & 48.6 \\
14 & 66.1 & 31.3 & 48.7 \\
15 & 51.8 & 23.4 & 37.6 \\
16 & 52.8 & 20.6 & 36.7 \\
17 & 77.3 & 42.6 & 60.0 \\
18 & 72.6 & 44.6 & 58.6 \\
19 & 78.9 & 67.2 & 73.1 \\
20 & 81.2 & 71.2 & 76.2 \\
\hline
\end{tabular}

(a) USDA (2009); see also Figure 7.3.

$\mathrm{RH}=$ Relative humidity. 
Table 10.3. Approximation of Absolute Humidity from RH

\begin{tabular}{ccccccccccccc}
\hline \multicolumn{2}{c}{ Relative Humidity: } & $10 \%$ & $20 \%$ & $30 \%$ & $40 \%$ & $50 \%$ & $60 \%$ & $70 \%$ & $80 \%$ & $90 \%$ & $100 \%$ \\
\hline $\begin{array}{c}\text { Temp } \\
\left({ }^{\circ} \mathrm{C}\right)\end{array}$ & $\begin{array}{c}\text { Temp } \\
\left({ }^{\circ} \mathrm{F}\right)\end{array}$ & \multicolumn{10}{c}{ Approximate Absolute Humidity $\left(\mathrm{g} / \mathrm{m}^{3}\right)$} & \\
\hline 50 & 122 & 8.3 & 16.6 & 24.9 & 33.2 & 41.5 & 49.8 & 58.1 & 66.4 & 74.7 & 83.0 \\
45 & 113 & 6.5 & 13.1 & 19.6 & 26.2 & 32.7 & 39.3 & 45.8 & 52.4 & 58.9 & 65.4 \\
40 & 104 & 5.1 & 10.2 & 15.3 & 20.5 & 25.6 & 30.7 & 35.8 & 40.9 & 46.0 & 51.1 \\
35 & 95 & 4.0 & 7.9 & 11.9 & 15.8 & 19.8 & 23.8 & 27.7 & 31.7 & 35.6 & 39.6 \\
30 & 86 & 3.0 & 6.1 & 9.1 & 12.1 & 15.2 & 18.2 & 21.3 & 24.3 & 27.3 & 30.4 \\
25 & 77 & 2.3 & 4.6 & 6.9 & 9.2 & 11.5 & 13.8 & 16.1 & 18.4 & 20.7 & 23.0 \\
20 & 68 & 1.7 & 3.5 & 5.2 & 6.9 & 8.7 & 10.4 & 12.1 & 13.8 & 15.6 & 17.3 \\
15 & 59 & 1.3 & 2.6 & 3.9 & 5.1 & 6.4 & 7.7 & 9.0 & 10.3 & 11.5 & 12.8 \\
10 & 50 & 0.9 & 1.9 & 2.8 & 3.8 & 4.7 & 5.6 & 6.6 & 7.5 & 8.5 & 9.4 \\
5 & 41 & 0.7 & 1.4 & 2.0 & 2.7 & 3.4 & 4.1 & 4.8 & 5.4 & 6.1 & 6.8 \\
0 & 32 & 0.5 & 1.0 & 1.5 & 1.9 & 2.4 & 2.9 & 3.4 & 3.9 & 4.4 & 4.8 \\
-5 & 23 & 0.3 & 0.7 & 1.0 & 1.4 & 1.7 & 2.1 & 2.4 & 2.7 & 3.1 & 3.4 \\
\hline
\end{tabular}

The shaded area reflects most absolute humidity values for typical U.S. growing season (May-September).

\subsubsection{Module: Health Impacts}

The sub-tab Constituent Parameters requires entry of Lung transfer inhalation class (SOLUBIL) assignments. The assignments will impact results. Be sure $\mathrm{H}^{3}$ and HTO are assigned as "Vapor;" H3EL and HT are assigned as "Gas;" and OBT is assigned "Vapor."

\subsection{Carbon-14 Model Considerations}

Carbon options in Gv2 are $\mathrm{C} 14$ and $\mathrm{C} 11$. Both are modeled using special models, however, the 20-minute half-life of ${ }^{11} \mathrm{C}$ results in essentially zero concentrations in media other than air. The topic of ${ }^{14} \mathrm{C}$ dose estimation has been brought to the forefront in recent years. ${ }^{1}$ Carbon-14 has become a more significant radioactive nuclide emission at nuclear power plants due to improved controls on other radioactive emissions and has been more intensely studied as a part of greenhouse gas emission research. The increase in $\mathrm{CO}_{2}$ levels in the atmosphere is largely the result of fossil fuel combustion; fossil fuel contains a lower ${ }^{14} \mathrm{C}$ to ${ }^{13} \mathrm{C}$ ratio, resulting in a net decrease in background ${ }^{14} \mathrm{CO}_{2}$ concentrations over time. As a result of the recent evolution of ${ }^{14} \mathrm{C}$ work, the ${ }^{14} \mathrm{C}$ models for air emissions as currently implemented in Gv2 are currently under review; estimates of dose from the current models should be considered to be minor overestimates.

Liquid effluent releases of ${ }^{14} \mathrm{C}$ are generally much less important than airborne releases. Liquid effluent releases of ${ }^{14} \mathrm{C}$ and subsequent irrigation water deposition are described in Section 9.6.2 of Napier

\footnotetext{
${ }^{1}$ For example, see the Electric Power Research Institute presentation at http://hps.ne.uiuc.edu/ rets-remp/PastWorkshops/2011/presentations/ 4B-C-14\%20Dose\%20Calculation\%20Methods\%20at\%20Nuclear.pdf (last accessed June 2012).
} 
et al. (2012). However, current coding of this model is simplistic and probably results in significant overestimates of dose. The short half-life of carbon-11 results in essentially zero concentrations in media other than surface water.

\subsection{References}

Napier BA, DL Strenge, JV Ramsdell, Jr., PW Eslinger, and C Fosmire. 2012. GENII Version 2 Software Design Document. PNNL-14584, Rev. 4, Pacific Northwest National Laboratory, Richland, Washington.

NCDC. 2008. Comparative Climatic Data: Average Relative Humidity (\%). National Climatic Data Center, online at http://lwf.ncdc.noaa.gov/oa/climate/online/ccd/avgrh.html. Last accessed July 3, 2012, NCDC, Ashville, North Carolina.

TIS-GDV. 2012. Climate/Humidity Table. Transportation Information Service-GDV, Berlin, Germany. Accessed July 3, 2012 at http://www.tis-gdv.de/tis e/misc/klima.htm.

USDA. 2009. 2007 Census of Agriculture, United States Summary and State Data. Vol. 1, Geographic Area Series, Part 51, AC-07-A-51, USDA, National Agricultural Statistics Service, Washington, D.C. Last accessed at:

http://www.agcensus.usda.gov/Publications/2007/Online_Highlights/Farm and Ranch_Irrigation_Survey lindex.asp. 

PNNL-21950, Appendix A

\section{Appendix A}

\section{Aquatic Biota}




\section{Appendix A}

\section{Aquatic Biota}

\section{A.1 Introduction}

This appendix contains tables of bioaccumulation factors for aquatic animals and plants, including both freshwater and saltwater values. References are indicated for each value.

\section{FRESHWATER}

Table A.1. Bioaccumulation in Wet Fish from Freshwater, L/kgwet (CLBFF)

Table A.2. Bioaccumulation in Wet Mollusk from Freshwater, L/kgwet (CLBFM)

Table A.3. Bioaccumulation in Wet Crustacea from Freshwater, L/kgwet (CLBFI)

Table A.4. Bioaccumulation in Wet Plants from Freshwater, L/kgwet (CLBFP)

\section{SALTWATER}

Table A.5. Bioaccumulation in Wet Fish from Saltwater, L/kgwet (CLBMF)

Table A.6. Bioaccumulation in Wet Mollusk from Saltwater, L/kgwet (CLBMM)

Table A.7. Bioaccumulation in Wet Crustacea from Saltwater, L/kgwet (CLBMI)

Table A.8. Bioaccumulation in Wet Plants from Saltwater, L/kgwet (CLBMP)

In all tables if an element is listed once and there is no data for that element, no bioaccumulation factor information was currently identified, preferred, or available. 

Table A.1. Bioaccumulation in Wet Fish from Freshwater, L/ $/ \mathrm{kg}_{\text {wet }}$ (CLBFF)

\begin{tabular}{|c|c|c|c|c|c|c|c|c|c|c|c|c|c|c|c|c|}
\hline Element & Atomic\# & $\mathrm{N}$ & Min & Max & AVG & Mean & Wmean & STD & GSD & GM & WGM & MED & 95\%ile & Document & Reference\# & Reference \\
\hline $\mathrm{Ac}$ & 89 & & & & & & & & & & & $2.50 \mathrm{E}+01$ & & $\begin{array}{l}\text { NUREG/CR-5512 } \\
\end{array}$ & & Strenge et al. (1986) \\
\hline $\mathrm{Ag}$ & 47 & & & & & & & & & & & $2.30 \mathrm{E}+00$ & & NUREG/CR-5512 & & Strenge et al. (1986) \\
\hline $\mathrm{Ag}$ & 47 & 23 & $5.70 \mathrm{E}+01$ & $1.80 \mathrm{E}+02$ & & & & & $1.30 \mathrm{E}+00$ & $1.10 \mathrm{E}+02$ & & & & TRS_364 & 4 & \\
\hline $\mathrm{Ag}$ & 47 & 27 & $4.00 \mathrm{E}+01$ & $2.10 \mathrm{E}+02$ & & & & & $1.50 \mathrm{E}+00$ & $1.10 \mathrm{E}+02$ & & & & TRS_472 & & \\
\hline $\mathrm{Al}$ & 13 & 93 & $4.50 \mathrm{E}+00$ & $5.20 \mathrm{E}+03$ & & & & & $7.10 \mathrm{E}+00$ & $6.60 \mathrm{E}+01$ & & & & TRS_364 & 3 & \\
\hline $\mathrm{Al}$ & 13 & 31 & $5.90 \mathrm{E}+00$ & $3.00 \mathrm{E}+02$ & & & & & $3.90 \mathrm{E}+00$ & $5.10 \mathrm{E}+01$ & & & & TRS_472 & & \\
\hline $\mathrm{Am}$ & 95 & & & & & & & & & & & $2.50 \mathrm{E}+02$ & & NUREG/CR-5512 & & Poston and Klopfer (1988) \\
\hline $\mathrm{Am}$ & 95 & & & & & $1.30 \mathrm{E}+03$ & & & & & & & & & & Fesenko et al. (2011) \\
\hline $\mathrm{Am}$ & 95 & 2 & $7.20 \mathrm{E}+01$ & $4.00 \mathrm{E}+02$ & & $2.40 \mathrm{E}+02$ & & & & & & & & TRS_472 & & \\
\hline $\mathrm{Ar}$ & 18 & & & & & & & & & & & & & & & \\
\hline As & 33 & & & & & & & & & & & $1.00 \mathrm{E}+02$ & & NUREG/CR-5512 & & Strenge et al. (1986) \\
\hline As & 33 & & & & $9.10 \mathrm{E}+01$ & & & & & & & & & Thompson et al. (1972) & 2723 & Davis et al. (1958) \\
\hline As & 33 & 33 & $8.10 \mathrm{E}+01$ & $1.00 \mathrm{E}+03$ & & & & & $2.30 \mathrm{E}+00$ & $3.80 \mathrm{E}+02$ & & & & TRS_364 & 4 & \\
\hline As & 33 & & & & & & & & $5.20 \mathrm{E}+00$ & $1.40 \mathrm{E}+01$ & & & & & & Sheppard et al. (2010a) \\
\hline As & 33 & 15 & $5.00 \mathrm{E}+01$ & $9.50 \mathrm{E}+02$ & & & & & $2.10 \mathrm{E}+00$ & $3.30 \mathrm{E}+02$ & & & & TRS_472 & & \\
\hline At & 85 & & & & & & & & & & & & & & & \\
\hline $\mathrm{Au}$ & 79 & & & & & & & & & & & $3.30 \mathrm{E}+01$ & & NUREG/CR-5512 & & Strenge et al. (1986) \\
\hline $\mathrm{Au}$ & 79 & 13 & $5.00 \mathrm{E}+01$ & $1.00 \mathrm{E}+03$ & & & & & $2.30 \mathrm{E}+00$ & $2.90 \mathrm{E}+02$ & & & & TRS_364 & 4 & \\
\hline $\mathrm{Au}$ & 79 & 17 & $5.00 \mathrm{E}+01$ & $9.00 \mathrm{E}+02$ & & & & & $2.10 \mathrm{E}+00$ & $2.40 \mathrm{E}+02$ & & & & TRS_472 & & \\
\hline $\mathrm{Ba}$ & 56 & & & & & & & & & & & $2.00 \mathrm{E}+02$ & & NUREG/CR-5512 & & Poston and Klopfer (1988) \\
\hline $\mathrm{Ba}$ & 56 & 92 & $5.00 \mathrm{E}+00$ & $2.20 \mathrm{E}+02$ & & & & & $1.70 \mathrm{E}+00$ & $4.70 \mathrm{E}+01$ & & & & TRS_364 & 4 & \\
\hline $\mathrm{Ba}$ & 56 & 111 & $5.30 \mathrm{E}-02$ & $3.20 \mathrm{E}+01$ & & & & & $3.30 \mathrm{E}+00$ & $1.20 \mathrm{E}+00$ & & & & TRS_472 & & \\
\hline $\mathrm{Be}$ & 4 & & & & & & & & & & & $2.00 \mathrm{E}+00$ & & NUREG/CR-5512 & & Strenge et al. (1986) \\
\hline $\mathrm{Bi}$ & 83 & & & & & & & & & & & $1.50 \mathrm{E}+01$ & & NUREG/CR-5512 & & Strenge et al. (1986) \\
\hline $\mathrm{Bk}$ & 97 & & & & & & & & & & & & & & & \\
\hline $\mathrm{Br}$ & 35 & & & & & & & & & & & $4.20 \mathrm{E}+02$ & & NUREG/CR-5512 & & Strenge et al. (1986) \\
\hline $\mathrm{Br}$ & 35 & 37 & $1.50 \mathrm{E}+01$ & $7.90 \mathrm{E}+02$ & & & & & $2.30 \mathrm{E}+00$ & $1.60 \mathrm{E}+03$ & & & & TRS_364 & 4 & \\
\hline $\mathrm{Br}$ & 35 & 15 & $1.90 \mathrm{E}+01$ & $3.00 \mathrm{E}+02$ & & & & & $2.30 \mathrm{E}+00$ & $9.10 \mathrm{E}+01$ & & & & TRS_472 & & \\
\hline C & 6 & & & & & & & & & & & $4.60 \mathrm{E}+03$ & & NUREG/CR-5512 & & Strenge et al. (1986) \\
\hline C & 6 & & & & & $6.30 \mathrm{E}+03$ & & $4.40 \mathrm{E}+03$ & & & & & & & & Fesenko et al. (2011) \\
\hline C & 6 & 6 & $1.90 \mathrm{E}+05$ & $3.20 \mathrm{E}+06$ & & & & & $2.90 \mathrm{E}+00$ & $4.00 \mathrm{E}+05$ & & & & TRS_472 & & \\
\hline $\mathrm{Ca}$ & 20 & & & & & & & & & & & $4.00 \mathrm{E}+01$ & & NUREG/CR-5512 & & Strenge et al. (1986) \\
\hline $\mathrm{Ca}$ & 20 & 118 & $8.40 \mathrm{E}+01$ & $5.60 \mathrm{E}+03$ & & & & & $3.40 \mathrm{E}+00$ & $1.00 \mathrm{E}+03$ & & & & TRS_364 & 5 & \\
\hline $\mathrm{Ca}$ & 20 & & & & & $8.80 \mathrm{E}+00$ & & & & & & & & & & Fesenko et al. (2011) \\
\hline $\mathrm{Ca}$ & 20 & & & & & & & & $4.30 \mathrm{E}+00$ & $9.00 \mathrm{E}+01$ & & & & & & Sheppard et al. (2010a) \\
\hline $\mathrm{Ca}$ & 20 & 104 & $2.00 \mathrm{E}+00$ & $9.70 \mathrm{E}+01$ & & & & & $2.50 \mathrm{E}+00$ & $1.20 \mathrm{E}+01$ & & & & TRS_472 & & \\
\hline $\mathrm{Cd}$ & 48 & & & & & & & & & & & $2.00 \mathrm{E}+02$ & & NUREG/CR-5512 & & Strenge et al. (1986) \\
\hline $\mathrm{Cd}$ & 48 & & & & & & & & $6.60 \mathrm{E}+00$ & $1.40 \mathrm{E}+02$ & & & & & & Sheppard et al. (2010a) \\
\hline $\mathrm{Ce}$ & 58 & & & & & & & & & & & $5.00 \mathrm{E}+02$ & & NUREG/CR-5512 & & Strenge et al. (1986) \\
\hline $\mathrm{Ce}$ & 58 & & & & $1.00 \mathrm{E}+00$ & & & & & & & & & Thompson et al. (1972) & 6012 & Polikarpov (1966) \\
\hline $\mathrm{Ce}$ & 58 & 90 & $3.00 \mathrm{E}+00$ & $1.10 \mathrm{E}+02$ & & & & & $2.70 \mathrm{E}+00$ & $1.20 \mathrm{E}+01$ & & & & TRS_364 & 5 & \\
\hline $\mathrm{Ce}$ & 58 & & & & & $3.50 \mathrm{E}+02$ & & & & & & & & & & Fesenko et al. (2011) \\
\hline $\mathrm{Ce}$ & 58 & & & & & & & & $9.00 \mathrm{E}+00$ & $4.80 \mathrm{E}+00$ & & & & & & Sheppard et al. (2010a) \\
\hline $\mathrm{Ce}$ & 58 & & & & & $2.20 \mathrm{E}+02$ & & & & & & & & & & Fesenko et al. (2011) \\
\hline $\mathrm{Ce}$ & 58 & 71 & $9.00 \mathrm{E}+01$ & $1.20 \mathrm{E}+03$ & & & & & $9.50 \mathrm{E}+00$ & $2.50 \mathrm{E}+01$ & & & & TRS_472 & & \\
\hline $\mathrm{Cf}$ & 98 & & & & & & & & & & & $2.50 \mathrm{E}+01$ & & NUREG/CR-5512 & & Strenge et al. (1986) \\
\hline $\mathrm{Cl}$ & 17 & & & & & & & & & & & $5.00 \mathrm{E}+01$ & & NUREG/CR-5512 & & Strenge et al. (1986) \\
\hline $\mathrm{Cl}$ & 17 & 37 & $2.50 \mathrm{E}+01$ & $2.30 \mathrm{E}+02$ & & & & & $1.60 \mathrm{E}+00$ & $8.50 \mathrm{E}+01$ & & & & TRS_364 & 4 & \\
\hline $\mathrm{Cl}$ & 17 & & & & & & & & $1.30 \mathrm{E}+00$ & $1.10 \mathrm{E}+02$ & & & & & & Sheppard et al. (2010a) \\
\hline $\mathrm{Cl}$ & 17 & 16 & $9.90 \mathrm{E}+00$ & $1.20 \mathrm{E}+02$ & & & & & $2.20 \mathrm{E}+00$ & $4.00 \mathrm{E}+01$ & & & & TRS_472 & & \\
\hline
\end{tabular}


Table A.1. (contd)

\begin{tabular}{|c|c|c|c|c|c|c|c|c|c|c|c|c|c|c|c|c|}
\hline Element & Atomic\# & $\mathrm{N}$ & Min & Max & AVG & Mean & Wmean & STD & GSD & GM & WGM & MED & 95\%ile & Document & Reference\# & $\begin{array}{l}\text { Reference } \\
\end{array}$ \\
\hline $\mathrm{Cm}$ & 96 & & & & & & & & & & & $2.50 \mathrm{E}+02$ & & NUREG/CR-5512 & & Poston and Klopfer (1988) \\
\hline Сo & 27 & & & & & & & & & & & $3.30 \mathrm{E}+02$ & & NUREG/CR-5512 & & Poston and Klopfer (1988) \\
\hline Co & 27 & & $3.00 \mathrm{E}+00$ & $4.50 \mathrm{E}+01$ & & & & & & & & & & Thompson et al. (1972) & 5731 & Perkins et al. (1967) \\
\hline Сo & 27 & 118 & $2.30 \mathrm{E}+02$ & $2.40 \mathrm{E}+03$ & & & & & $1.60 \mathrm{E}+00$ & $4.00 \mathrm{E}+02$ & & & & TRS_364 & 14 & \\
\hline Co & 27 & & & & & $2.10 \mathrm{E}+02$ & & & & & & & & & & Fesenko et al. (2011) \\
\hline Сo & 27 & & & & & $1.50 \mathrm{E}+01$ & & & & & & & & & & Fesenko et al. (2011) \\
\hline Co & 27 & & & & & $7.60 \mathrm{E}+01$ & & $2.20 \mathrm{E}+01$ & & & & & & & & Fesenko et al. (2011) \\
\hline Сo & 27 & & & & & $6.70 \mathrm{E}+01$ & & $3.10 \mathrm{E}+01$ & & & & & & & & Fesenko et al. (2011) \\
\hline Co & 27 & & & & & $5.30 \mathrm{E}+01$ & & $7.00 \mathrm{E}+00$ & & & & & & & & Fesenko et al. (2011) \\
\hline Сo & 27 & & & & & & & & $6.10 \mathrm{E}+00$ & $9.60 \mathrm{E}+01$ & & & & & & Sheppard et al. (2010a) \\
\hline Co & 27 & 65 & $9.00 \mathrm{E}+00$ & $5.60 \mathrm{E}+02$ & & & & & $2.40 \mathrm{E}+00$ & $7.60 \mathrm{E}+01$ & & & & TRS_472 & & \\
\hline $\mathrm{Cr}$ & 24 & & & & & & & & & & & $2.00 \mathrm{E}+02$ & & NUREG/CR-5512 & & Strenge et al. (1986) \\
\hline $\mathrm{Cr}$ & 24 & & & & $4.00 \mathrm{E}+03$ & & & & & & & & & Thompson et al. (1972) & 2723 & Davis et al. (1958) \\
\hline $\mathrm{Cr}$ & 24 & 51 & $3.50 \mathrm{E}+01$ & $7.60 \mathrm{E}+02$ & & & & & $2.00 \mathrm{E}+00$ & $2.10 \mathrm{E}+02$ & & & & TRS_364 & 5 & \\
\hline $\mathrm{Cr}$ & 24 & & & & & & & & $7.90 \mathrm{E}+00$ & 4.00E-01 & & & & & & Sheppard et al. (2010a) \\
\hline $\mathrm{Cr}$ & 24 & 57 & $1.30 \mathrm{E}+01$ & $1.20 \mathrm{E}+02$ & & & & & $2.00 \mathrm{E}+00$ & $4.00 \mathrm{E}+01$ & & & & TRS_472 & & \\
\hline Cs & 55 & & & & & & & & & & & $2.00 \mathrm{E}+03$ & & NUREG/CR-5512 & & Strenge et al. (1986) \\
\hline Cs & 55 & & & & $2.40 \mathrm{E}+03$ & & & & & & & & & Thompson et al. (1972) & 5731 & Perkins et al. (1967) \\
\hline Cs & 55 & & & & $2.40 \mathrm{E}+03$ & & & & & & & & & Thompson et al. (1972) & 5731 & Perkins et al. (1967) \\
\hline Cs & 55 & & $3.30 \mathrm{E}+03$ & $4.50 \mathrm{E}+03$ & $3.90 \mathrm{E}+03$ & & & & & & & & & Thompson et al. (1972) & 6335 & Preston et.al. (1967) \\
\hline Cs & 55 & & $4.60 \mathrm{E}+02$ & $1.10 \mathrm{E}+03$ & & & & & & & & & & Thompson et al. (1972) & 8625 & Kolehmainen et al. (1969) \\
\hline Cs & 55 & & $1.60 \mathrm{E}+03$ & $1.70 \mathrm{E}+03$ & & & & & & & & & & Thompson et al. (1972) & 9957 & Seelye $(1970)$ \\
\hline Cs & 55 & & $3.90 \mathrm{E}+02$ & $4.70 \mathrm{E}+03$ & & & & & & & & & & Thompson et al. (1972) & 10052 & Bigliocca et al. (1969) \\
\hline Cs & 55 & 145 & $7.50 \mathrm{E}+01$ & $2.40 \mathrm{E}+04$ & & & & & $2.60 \mathrm{E}+00$ & $3.00 \mathrm{E}+03$ & & & & TRS_364 & 52 & \\
\hline Cs & 55 & & & & & $8.50 \mathrm{E}+01$ & & $1.20 \mathrm{E}+02$ & & & & & & & & Fesenko et al. (2011) \\
\hline Cs & 55 & & & & & $1.00 \mathrm{E}+01$ & & & & & & & & & & Fesenko et al. (2011) \\
\hline Cs & 55 & & & & & $6.00 \mathrm{E}+02$ & & $9.50 \mathrm{E}+02$ & & & & & & & & Fesenko et al. (2011) \\
\hline Cs & 55 & & & & & $5.80 \mathrm{E}+02$ & & $9.50 \mathrm{E}+02$ & & & & & & & & Fesenko et al. (2011) \\
\hline Cs & 55 & & & & & & & & $6.00 \mathrm{E}+00$ & $6.90 \mathrm{E}+03$ & & & & & & Sheppard et al. (2010a) \\
\hline Cs & 55 & 106 & $1.40 \mathrm{E}+02$ & $1.50 \mathrm{E}+04$ & & & & & $2.40 \mathrm{E}+00$ & $2.50 \mathrm{E}+03$ & & & & TRS_472 & & \\
\hline $\mathrm{Cu}$ & 29 & & & & & & & & & & & $5.00 \mathrm{E}+01$ & & NUREG/CR-5512 & & Strenge et al. (1986) \\
\hline $\mathrm{Cu}$ & 29 & & & & $5.00 \mathrm{E}+01$ & & & & & & & & & Thompson et al. (1972) & 1679 & Krumholz et al. (1957) \\
\hline $\mathrm{Cu}$ & 29 & & $3.00 \mathrm{E}+00$ & $1.00 \mathrm{E}+01$ & & & & & & & & & & Thompson et al. (1972) & 2723 & Davis et al. (1958) \\
\hline $\mathrm{Cu}$ & 29 & 102 & $8.60 \mathrm{E}+01$ & $1.20 \mathrm{E}+03$ & & & & & $1.50 \mathrm{E}+00$ & $2.70 \mathrm{E}+02$ & & & & TRS_364 & 5 & \\
\hline $\mathrm{Cu}$ & 29 & & & & & $1.40 \mathrm{E}+03$ & & $3.00 \mathrm{E}+02$ & & & & & & & & Fesenko et al. (2011) \\
\hline $\mathrm{Cu}$ & 29 & & & & & & & & $2.50 \mathrm{E}+00$ & $1.24 \mathrm{E}+02$ & & & & & & Sheppard et al. (2010a) \\
\hline $\mathrm{Cu}$ & 29 & 96 & $9.90 \mathrm{E}+01$ & $7.20 \mathrm{E}+02$ & & & & & $1.70 \mathrm{E}+00$ & $2.30 \mathrm{E}+02$ & & & & TRS_472 & & \\
\hline Dy & 66 & 1 & & & & & & & & $3.00 \mathrm{E}+02$ & & & & TRS_364 & 3 & \\
\hline Dy & 66 & 2 & $2.20 \mathrm{E}+02$ & $1.10 \mathrm{E}+03$ & & $6.50 \mathrm{E}+02$ & & & & & & & & TRS_472 & & \\
\hline Er & 68 & & & & & & & & & & & & & & & \\
\hline Es & 99 & & & & & & & & & & & & & & & \\
\hline Eu & 63 & & & & & & & & & & & $2.50 \mathrm{E}+01$ & & NUREG/CR-5512 & & Strenge et al. (1986) \\
\hline $\mathrm{Eu}$ & 63 & 53 & $7.60 \mathrm{E}+00$ & $2.20 \mathrm{E}+03$ & & & & & $3.20 \mathrm{E}+00$ & $1.50 \mathrm{E}+02$ & & & & TRS_364 & 4 & \\
\hline $\mathrm{Eu}$ & 63 & 24 & $1.10 \mathrm{E}+01$ & $7.20 \mathrm{E}+02$ & & & & & $4.90 \mathrm{E}+00$ & $1.30 \mathrm{E}+02$ & & & & TRS_472 & & \\
\hline $\mathrm{F}$ & 9 & & & & & & & & & & & $1.00 \mathrm{E}+01$ & & NUREG/CR-5512 & & Strenge et al. (1986) \\
\hline $\mathrm{Fe}$ & 26 & & & & & & & & & & & $2.00 \mathrm{E}+03$ & & NUREG/CR-5512 & & Poston and Klopfer (1988) \\
\hline $\mathrm{Fe}$ & 26 & 114 & $1.60 \mathrm{E}+01$ & $5.30 \mathrm{E}+03$ & & & & & $5.70 \mathrm{E}+00$ & $1.40 \mathrm{E}+02$ & & & & TRS_364 & 6 & \\
\hline $\mathrm{Fe}$ & 26 & & & & & $1.00 \mathrm{E}+04$ & & & & & & & & & & Fesenko et al. (2011) \\
\hline $\mathrm{Fe}$ & 26 & & & & & & & & $6.80 \mathrm{E}+00$ & $3.60 \mathrm{E}+01$ & & & & & & Sheppard et al. (2010a) \\
\hline $\mathrm{Fe}$ & 26 & 96 & $6.60 \mathrm{E}+00$ & $2.00 \mathrm{E}+03$ & & & & & $6.90 \mathrm{E}+00$ & $1.70 \mathrm{E}+02$ & & & & TRS_472 & & \\
\hline $\mathrm{Fm}$ & 100 & & & & & & & & & & & & & & & \\
\hline $\mathrm{Fr}$ & 87 & & & & & & & & & & & & & & & \\
\hline
\end{tabular}


Table A.1. (contd)

\begin{tabular}{|c|c|c|c|c|c|c|c|c|c|c|c|c|c|c|c|c|}
\hline Element & Atomic\# & $\mathrm{N}$ & Min & Max & AVG & Mean & Wmean & STD & GSD & GM & WGM & MED & 95\%ile & Document & Reference\# & Reference \\
\hline $\mathrm{Ga}$ & 31 & & & & & & & & & & & & & & & \\
\hline Gd & 64 & & & & & & & & & & & $2.50 \mathrm{E}+01$ & & NUREG/CR-5512 & & Strenge et al. (1986) \\
\hline $\mathrm{Ge}$ & 32 & & & & & & & & & & & & & & & \\
\hline Ge & 32 & & & & & $1.50 \mathrm{E}+00$ & & & & & & & & & & Fesenko et al. (2011) \\
\hline $\mathrm{H}$ & 1 & & & & & & & & & & & $1.00 \mathrm{E}+00$ & & NUREG/CR-5512 & & Poston and Klopfer (1988) \\
\hline $\mathrm{Hf}$ & 72 & 20 & $3.00 \mathrm{E}+02$ & $2.80 \mathrm{E}+04$ & & & & & $3.20 \mathrm{E}+00$ & $2.10 \mathrm{E}+03$ & & & & TRS_364 & 3 & \\
\hline $\mathrm{Hf}$ & 72 & 10 & $3.30 \mathrm{E}+02$ & $2.00 \mathrm{E}+03$ & & & & & $1.90 \mathrm{E}+00$ & $1.10 \mathrm{E}+03$ & & & & TRS_472 & & \\
\hline $\mathrm{Hg}$ & 80 & & & & & & & & & & & $1.00 \mathrm{E}+03$ & & NUREG/CR-5512 & & Strenge et al. (1986) \\
\hline $\mathrm{Hg}$ & 80 & 20 & $1.10 \mathrm{E}+03$ & $2.20 \mathrm{E}+04$ & & & & & $2.20 \mathrm{E}+00$ & $4.50 \mathrm{E}+03$ & & & & TRS_364 & 3 & \\
\hline $\mathrm{Hg}$ & 80 & 14 & $1.90 \mathrm{E}+03$ & $1.70 \mathrm{E}+04$ & & & & & $1.90 \mathrm{E}+00$ & $6.10 \mathrm{E}+03$ & & & & TRS_472 & & \\
\hline Но & 67 & & & & & & & & & & & $2.50 \mathrm{E}+01$ & & NUREG/CR-5512 & & Strenge et al. (1986) \\
\hline I & 53 & & & & & & & & & & & $5.00 \mathrm{E}+02$ & & NUREG/CR-5512 & & Poston and Klopfer (1988) \\
\hline I & 53 & 84 & $1.00 \mathrm{E}+02$ & $4.50 \mathrm{E}+04$ & & & & & $2.10 \mathrm{E}+00$ & $6.50 \mathrm{E}+02$ & & & & TRS_364 & 8 & \\
\hline I & 53 & & & & & $1.70 \mathrm{E}+01$ & & $1.50 \mathrm{E}+01$ & & & & & & & & Fesenko et al. (2011) \\
\hline I & 53 & & & & & $2.50 \mathrm{E}+00$ & & & & & & & & & & Fesenko et al. (2011) \\
\hline I & 53 & & & & & & & & $2.70 \mathrm{E}+00$ & $3.40 \mathrm{E}+01$ & & & & & & Sheppard et al. (2010a) \\
\hline I & 53 & 50 & $1.10 \mathrm{E}+01$ & $4.00 \mathrm{E}+02$ & & & & & $2.50 \mathrm{E}+00$ & $3.00 \mathrm{E}+01$ & & & & TRS 472 & & \\
\hline In & 49 & & & & & & & & & & & $1.00 \mathrm{E}+05$ & & NUREG/CR-5512 & & Strenge et al. (1986) \\
\hline Ir & 77 & & & & & & & & & & & $1.00 \mathrm{E}+01$ & & NUREG/CR-5512 & & Strenge et al. (1986) \\
\hline $\mathrm{K}$ & 19 & & & & & & & & & & & $1.00 \mathrm{E}+03$ & & NUREG/CR-5512 & & Strenge et al. (1986) \\
\hline $\mathrm{K}$ & 19 & 120 & $5.70 \mathrm{E}+02$ & $1.50 \mathrm{E}+04$ & & & & & $2.00 \mathrm{E}+00$ & $4.00 \mathrm{E}+03$ & & & & TRS_364 & 5 & \\
\hline K & 19 & & & & & & & & $2.10 \mathrm{E}+00$ & $1.40 \mathrm{E}+04$ & & & & & & Sheppard et al. (2010a) \\
\hline $\mathrm{K}$ & 19 & 97 & $1.20 \mathrm{E}+03$ & $9.00 \mathrm{E}+03$ & & & & & $1.60 \mathrm{E}+00$ & $3.20 \mathrm{E}+03$ & & & & TRS_472 & & \\
\hline $\mathrm{Kr}$ & 36 & & & & & & & & & & & & & & & \\
\hline La & 57 & & & & & & & & & & & $2.50 \mathrm{E}+01$ & & NUREG/CR-5512 & & Strenge et al. (1986) \\
\hline La & 57 & 102 & $3.60 \mathrm{E}+00$ & $3.40 \mathrm{E}+02$ & & & & & $3.20 \mathrm{E}+00$ & $1.60 \mathrm{E}+01$ & & & & TRS_364 & 4 & \\
\hline La & 57 & & & & & & & & $7.60 \mathrm{E}+00$ & $5.40 \mathrm{E}+00$ & & & & & & Sheppard et al. (2010a) \\
\hline La & 57 & 74 & $1.10 \mathrm{E}+00$ & $6.60 \mathrm{E}+02$ & & & & & $4.90 \mathrm{E}+00$ & $3.70 \mathrm{E}+01$ & & & & TRS 472 & & \\
\hline $\mathrm{Lu}$ & 71 & & & & & & & & & & & & & & & \\
\hline Md & 101 & & & & & & & & & & & & & & & \\
\hline $\mathrm{Mg}$ & 12 & 111 & $1.40 \mathrm{E}+01$ & $4.30 \mathrm{E}+02$ & & & & & $3.00 \mathrm{E}+00$ & $1.10 \mathrm{E}+02$ & & & & TRS_364 & 4 & \\
\hline $\mathrm{Mg}$ & 12 & & & & & & & & $2.20 \mathrm{E}+00$ & $1.40 \mathrm{E}+02$ & & & & & & Sheppard et al. (2010a) \\
\hline $\mathrm{Mg}$ & 12 & 96 & $7.90 \mathrm{E}+00$ & $1.90 \mathrm{E}+02$ & & & & & $2.20 \mathrm{E}+00$ & $3.70 \mathrm{E}+01$ & & & & TRS 472 & & \\
\hline $\mathrm{Mn}$ & 25 & & & & & & & & & & & $4.00 \mathrm{E}+02$ & & NUREG/CR-5512 & & Poston and Klopfer (1988) \\
\hline $\mathrm{Mn}$ & 25 & & $6.00 \mathrm{E}-01$ & $2.00 \mathrm{E}+00$ & & & & & & & & & & Thompson et al. (1972) & 5731 & Perkins et al. (1967) \\
\hline $\mathrm{Mn}$ & 25 & & $1.50 \mathrm{E}+02$ & $6.60 \mathrm{E}+02$ & & & & & & & & & & Thompson et al. (1972) & 8687 & Bortoli et al. (1969) \\
\hline $\mathrm{Mn}$ & 25 & & & & $2.60 \mathrm{E}+01$ & & & & & & & & & Thompson et al. (1972) & 2723 & Davis et al. (1958) \\
\hline $\mathrm{Mn}$ & 25 & 110 & $4.80 \mathrm{E}+01$ & $7.00 \mathrm{E}+03$ & & & & & $4.00 \mathrm{E}+00$ & $4.50 \mathrm{E}+02$ & & & & TRS_364 & 6 & \\
\hline $\mathrm{Mn}$ & 25 & & & & & $6.30 \mathrm{E}+01$ & & $2.10 \mathrm{E}+01$ & & & & & & & & Fesenko et al. (2011) \\
\hline $\mathrm{Mn}$ & 25 & & & & & $2.20 \mathrm{E}+02$ & & & & & & & & & & Fesenko et al. (2011) \\
\hline $\mathrm{Mn}$ & 25 & & & & & & & & $8.10 \mathrm{E}+00$ & $1.60 \mathrm{E}+02$ & & & & & & Sheppard et al. (2010a) \\
\hline $\mathrm{Mn}$ & 25 & 97 & $1.30 \mathrm{E}+01$ & $1.40 \mathrm{E}+05$ & & & & & $6.70 \mathrm{E}+00$ & $2.40 \mathrm{E}+02$ & & & & TRS_472 & & \\
\hline Mo & 42 & & & & & & & & & & & $1.00 \mathrm{E}+01$ & & NUREG/CR-5512 & & Poston and Klopfer (1988) \\
\hline Mo & 42 & 81 & $2.10 \mathrm{E}+00$ & $1.90 \mathrm{E}+02$ & & & & & $1.90 \mathrm{E}+00$ & $2.70 \mathrm{E}+01$ & & & & TRS_364 & 5 & \\
\hline Mo & 42 & & & & & & & & $3.80 \mathrm{E}+00$ & $9.00 \mathrm{E}+00$ & & & & & & Sheppard et al. (2010a) \\
\hline Mo & 42 & 64 & $4.00 \mathrm{E}-03$ & $2.00 \mathrm{E}+01$ & & & & & $2.10 \mathrm{E}+00$ & $1.90 \mathrm{E}+00$ & & & & TRS_472 & & \\
\hline $\mathrm{N}$ & 7 & & & & & & & & & & & $1.50 \mathrm{E}+05$ & & NUREG/CR-5512 & & Strenge et al. (1986) \\
\hline $\mathrm{Na}$ & 11 & & & & & & & & & & & $1.00 \mathrm{E}+02$ & & NUREG/CR-5512 & & Poston and Klopfer (1988) \\
\hline $\mathrm{Na}$ & 11 & & $3.00 \mathrm{E}+01$ & $1.30 \mathrm{E}+02$ & & & & & & & & & & Thompson et al. (1972) & 2723 & Davis et al. (1958) \\
\hline $\mathrm{Na}$ & 11 & & $4.20 \mathrm{E}+01$ & $4.20 \mathrm{E}+02$ & & & & & & & & & & Thompson et al. (1972) & 5731 & Perkins et al. (1967) \\
\hline $\mathrm{Na}$ & 11 & 42 & $3.40 \mathrm{E}+01$ & $6.00 \mathrm{E}+02$ & & & & & $2.10 \mathrm{E}+00$ & $1.40 \mathrm{E}+02$ & & & & TRS_364 & 5 & \\
\hline $\mathrm{Na}$ & 11 & & & & & & & & $2.30 \mathrm{E}+00$ & $6.20 \mathrm{E}+01$ & & & & & & Sheppard et al. (2010a) \\
\hline
\end{tabular}


Table A.1. (contd)

\begin{tabular}{|c|c|c|c|c|c|c|c|c|c|c|c|c|c|c|c|c|}
\hline Element & Atomic\# & $\mathrm{N}$ & Min & Max & AVG & Mean & Wmean & STD & GSD & GM & WGM & MED & $95 \%$ ile & Document & Reference\# & Reference \\
\hline $\mathrm{Na}$ & 11 & 97 & $1.70 \mathrm{E}+01$ & $6.10 \mathrm{E}+02$ & & & & & $3.00 \mathrm{E}+00$ & $7.60 \mathrm{E}+01$ & & & & TRS_472 & & \\
\hline $\mathrm{Nb}$ & 41 & & & & & & & & & & & $2.00 \mathrm{E}+02$ & & NUREG/CR-5512 & & Poston and Klopfer (1988) \\
\hline $\mathrm{Nb}$ & 41 & & & & & & & & $2.70 \mathrm{E}+00$ & $2.70 \mathrm{E}+01$ & & & & & & Sheppard et al. (2010a) \\
\hline $\mathrm{Nd}$ & 60 & & & & & & & & & & & $2.50 \mathrm{E}+01$ & & NUREG/CR-5512 & & Strenge et al. (1986) \\
\hline $\mathrm{Nd}$ & 60 & & & & & & & & $8.20 \mathrm{E}+00$ & $4.70 \mathrm{E}+00$ & & & & & & Sheppard et al. (2010a) \\
\hline $\mathrm{Ne}$ & 10 & & & & & & & & & & & & & & & \\
\hline $\mathrm{Ni}$ & 28 & & & & & & & & & & & $1.00 \mathrm{E}+02$ & & NUREG/CR-5512 & & Poston and Klopfer (1988) \\
\hline $\mathrm{Ni}$ & 28 & 24 & $1.90 \mathrm{E}+01$ & $6.60 \mathrm{E}+02$ & & & & & $2.10 \mathrm{E}+00$ & $7.10 \mathrm{E}+01$ & & & & TRS_364 & 1 & \\
\hline $\mathrm{Ni}$ & 28 & & & & & & & & $3.70 \mathrm{E}+00$ & $9.60 \mathrm{E}+01$ & & & & & & Sheppard et al. (2010a) \\
\hline $\mathrm{Ni}$ & 28 & 5 & $1.10 \mathrm{E}+01$ & $4.40 \mathrm{E}+01$ & & & & & $1.90 \mathrm{E}+00$ & $2.10 \mathrm{E}+01$ & & & & TRS_472 & & \\
\hline $\mathrm{Np}$ & 93 & & & & & & & & & & & & & & & \\
\hline $\mathrm{O}$ & 8 & & & & & & & & & & & & & & & \\
\hline \multicolumn{17}{|l|}{ OBT } \\
\hline Os & 76 & & & & & & & & & & & $1.00 \mathrm{E}+01$ & & NUREG/CR-5512 & & Strenge et al. (1986) \\
\hline $\mathrm{P}$ & 15 & . & & & & & & & & & & $7.00 \mathrm{E}+04$ & & NUREG/CR-5512 & & Poston and Klopfer (1988) \\
\hline $\mathrm{P}$ & 15 & & $3.00 \mathrm{E}+04$ & $1.00 \mathrm{E}+05$ & & & & & & & & & & Thompson et al. (1972) & 1679 & Krumholz et al. (1957) \\
\hline $\mathrm{P}$ & 15 & & $1.20 \mathrm{E}+04$ & $1.00 \mathrm{E}+05$ & & & & & & & & & & Thompson et al. (1972) & 2723 & Davis et al. (1958) \\
\hline $\mathrm{P}$ & 15 & & & & $1.50 \mathrm{E}+05$ & & & & & & & & & Thompson et al. (1972) & 4081 & Ewool et al. (1963) \\
\hline $\mathrm{P}$ & 15 & & $5.00 \mathrm{E}+02$ & $5.50 \mathrm{E}+04$ & & & & & & & & & & Thompson et al. (1972) & 9576 & $<$ unknown> \\
\hline $\mathrm{P}$ & 15 & & & & & & & & & & & & & & & Smith et al. (2011) \\
\hline $\mathrm{P}$ & 15 & 39 & $1.20 \mathrm{E}+05$ & $1.70 \mathrm{E}+05$ & & & & & $1.10 \mathrm{E}+00$ & $1.40 \mathrm{E}+05$ & & & & TRS_472 & & \\
\hline $\mathrm{Pa}$ & 91 & & & & & & & & & & & $1.10 \mathrm{E}+01$ & & NUREG/CR-5512 & & Strenge et al. (1986) \\
\hline $\mathrm{Pb}$ & 82 & & & & & & & & & & & $1.00 \mathrm{E}+02$ & & NUREG/CR-5512 & & Strenge et al. (1986) \\
\hline $\mathrm{Pb}$ & 82 & & $5.00 \mathrm{E}-01$ & $1.50 \mathrm{E}+02$ & & & & & & & & & & Thompson et al. (1972) & 8956 & Wong_etal_1970 \\
\hline $\mathrm{Pb}$ & 82 & 82 & $5.80 \mathrm{E}+01$ & $5.70 \mathrm{E}+03$ & & & & & $3.00 \mathrm{E}+00$ & $3.70 \mathrm{E}+02$ & & & & TRS_364 & 1 & \\
\hline $\mathrm{Pb}$ & 82 & & & & & $2.30 \mathrm{E}+01$ & & $8.00 \mathrm{E}+00$ & & & & & & & & Fesenko et al. (2011) \\
\hline $\mathrm{Pb}$ & 82 & & & & & $1.90 \mathrm{E}+01$ & & $6.00 \mathrm{E}+00$ & & & & & & & & Fesenko et al. (2011) \\
\hline $\mathrm{Pb}$ & 82 & 39 & $1.00 \mathrm{E}-01$ & $2.70 \mathrm{E}+02$ & & & & & $2.90 \mathrm{E}+00$ & $2.50 \mathrm{E}+01$ & & & & TRS_472 & & \\
\hline $\mathrm{Pd}$ & 46 & & & & & & & & & & & $1.00 \mathrm{E}+01$ & & NUREG/CR-5512 & & Strenge et al. (1986) \\
\hline $\mathrm{Pd}$ & 46 & & & & & & & & $3.00 \mathrm{E}+00$ & $3.90 \mathrm{E}+01$ & & & & & & Sheppard et al. (2010a) \\
\hline $\mathrm{Pm}$ & 61 & & & & & & & & & & & $2.50 \mathrm{E}+01$ & & NUREG/CR-5512 & & Strenge et al. (1986) \\
\hline Po & 84 & & & & & & & & & & & $5.00 \mathrm{E}+02$ & & NUREG/CR-5512 & & Strenge et al. (1986) \\
\hline Po & 84 & & $6.70 \mathrm{E}+01$ & $4.50 \mathrm{E}+03$ & $3.00 \mathrm{E}+02$ & & & & & & & & & Thompson et al. (1972) & & Wong et al. (1970) \\
\hline Po & 84 & 5 & $6.00 \mathrm{E}+00$ & $1.70 \mathrm{E}+02$ & & & & & $4.30 \mathrm{E}+00$ & $3.60 \mathrm{E}+01$ & & & & TRS_472 & & \\
\hline Pr & 59 & & & & & & & & & & & $2.50 \mathrm{E}+01$ & & NUREG/CR-5512 & & Strenge et al. (1986) \\
\hline Pt & 78 & & & & & & & & & & & & & & & \\
\hline $\mathrm{Pu}$ & 94 & & & & & & & & & & & $2.50 \mathrm{E}+02$ & & NUREG/CR-5512 & & Poston and Klopfer (1988) \\
\hline $\mathrm{Pu}$ & 94 & & & & & $7.60 \mathrm{E}+01$ & & & & & & & & & & Fesenko et al. (2011) \\
\hline $\mathrm{Pu}$ & 94 & & & & & $3.10 \mathrm{E}+01$ & & & & & & & & & & Fesenko et al. (2011) \\
\hline $\mathrm{Pu}$ & 94 & & & & & $3.00 \mathrm{E}+00$ & & & & & & & & & & Fesenko et al. (2011) \\
\hline $\mathrm{Pu}$ & 94 & 3 & $7.70 \mathrm{E}+03$ & $5.00 \mathrm{E}+04$ & & & & & $2.60 \mathrm{E}+00$ & $2.10 \mathrm{E}+04$ & & & & TRS_472 & & \\
\hline $\mathrm{Ra}$ & 88 & & & & & & & & & & & $7.00 \mathrm{E}+01$ & & NUREG/CR-5512 & & Poston and Klopfer (1988) \\
\hline $\mathrm{Ra}$ & 88 & 2 & $1.60 \mathrm{E}+02$ & $2.50 \mathrm{E}+02$ & & $2.10 \mathrm{E}+02$ & & $6.00 \mathrm{E}+01$ & & & & & & TRS_364 & 3 & \\
\hline $\mathrm{Ra}$ & 88 & & & & & $1.60 \mathrm{E}+00$ & & $6.30 \mathrm{E}+00$ & & & & & & & & Fesenko et al. (2011) \\
\hline $\mathrm{Ra}$ & 88 & & & & & $4.00 \mathrm{E}+01$ & & & & & & & & & & Pyle and Clulow (1998) \\
\hline $\mathrm{Ra}$ & 88 & 21 & $6.00 \mathrm{E}-02$ & $1.50 \mathrm{E}+02$ & & & & & $6.90 \mathrm{E}+00$ & $4.00 \mathrm{E}+00$ & & & & TRS_472 & & \\
\hline $\mathrm{Rb}$ & 37 & & & & & & & & & & & $2.00 \mathrm{E}+03$ & & NUREG/CR-5512 & & Strenge et al. (1986) \\
\hline $\mathrm{Rb}$ & 37 & 113 & $1.20 \mathrm{E}+03$ & $1.60 \mathrm{E}+03$ & & & & & $1.60 \mathrm{E}+00$ & $6.10 \mathrm{E}+03$ & & & & TRS_364 & 4 & \\
\hline $\mathrm{Rb}$ & 37 & & & & & $3.40 \mathrm{E}+01$ & & & & & & & & & & Fesenko et al. (2011) \\
\hline $\mathrm{Rb}$ & 37 & & & & & & & & $3.90 \mathrm{E}+00$ & $5.10 \mathrm{E}+04$ & & & & & & Sheppard et al. (2010a) \\
\hline $\mathrm{Rb}$ & 37 & 92 & $1.00 \mathrm{E}+03$ & $1.40 \mathrm{E}+04$ & & & & & $1.70 \mathrm{E}+00$ & $4.00 \mathrm{E}+03$ & & & & TRS_472 & & \\
\hline $\mathrm{Re}$ & 75 & & & & & & & & & & & $1.20 \mathrm{E}+02$ & & NUREG/CR-5512 & & Strenge et al. (1986) \\
\hline
\end{tabular}


Table A.1. (contd)

\begin{tabular}{|c|c|c|c|c|c|c|c|c|c|c|c|c|c|c|c|c|}
\hline Element & Atomic\# & $\mathrm{N}$ & Min & Max & AVG & Mean & Wmean & STD & GSD & GM & WGM & MED & 95\%ile & Document & Reference\# & Reference \\
\hline $\mathrm{Rh}$ & 45 & & & & & & & & & & & $1.00 \mathrm{E}+01$ & & NUREG/CR-5512 & & Strenge et al. (1986) \\
\hline $\mathrm{Rn}$ & 86 & & & & & & & & & & & & & & & \\
\hline Ru & 44 & & & & & & & & & & & $1.00 \mathrm{E}+02$ & & NUREG/CR-5512 & & Poston and Klopfer (1988) \\
\hline $\mathrm{Ru}$ & 44 & & & & & $9.00 \mathrm{E}+01$ & & & & & & & & & & Fesenko et al. (2011) \\
\hline $\mathrm{Ru}$ & 44 & & & & & $2.00 \mathrm{E}+01$ & & & & & & & & & & Fesenko et al. (2011) \\
\hline $\mathrm{Ru}$ & 44 & 2 & $1.00 \mathrm{E}+01$ & $1.00 \mathrm{E}+02$ & & $5.50 \mathrm{E}+01$ & & & & & & & & TRS_472 & & \\
\hline $\mathrm{s}$ & 16 & & & & & & & & & & & $7.50 \mathrm{E}+02$ & & NUREG/CR-5512 & & Strenge et al. (1986) \\
\hline $\mathrm{s}$ & 16 & & & & & $3.50 \mathrm{E}+00$ & & & & & & & & & & Fesenko et al. (2011) \\
\hline Sb & 51 & & & & & & & & & & & $2.00 \mathrm{E}+02$ & & NUREG/CR-5512 & & Poston and Klopfer (1988) \\
\hline $\mathrm{Sb}$ & 51 & 37 & $4.70 \mathrm{E}+00$ & $9.30 \mathrm{E}+06$ & & & & & $8.80 \mathrm{E}+00$ & $7.10 \mathrm{E}+01$ & & & & TRS_364 & 4 & \\
\hline $\mathrm{Sb}$ & 51 & & & & & & & & $3.70 \mathrm{E}+00$ & $9.10 \mathrm{E}+00$ & & & & & & Sheppard et al. (2010a) \\
\hline $\mathrm{Sb}$ & 51 & 14 & $1.90 \mathrm{E}+00$ & $3.60 \mathrm{E}+02$ & & & & & $4.50 \mathrm{E}+00$ & $3.70 \mathrm{E}+01$ & & & & TRS_472 & & \\
\hline Sc & 21 & & & & & & & & & & & $1.00 \mathrm{E}+02$ & & NUREG/CR-5512 & & Poston and Klopfer (1988) \\
\hline Sc & 21 & & 6.00E-01 & $3.30 \mathrm{E}+00$ & & & & & & & & & & Thompson et al. (1972) & 5731 & Perkins et al. (1967) \\
\hline Sc & 21 & 30 & $6.70 \mathrm{E}+01$ & $3.70 \mathrm{E}+04$ & & & & & $3.60 \mathrm{E}+00$ & $9.30 \mathrm{E}+02$ & & & & TRS_364 & 3 & \\
\hline Sc & 21 & 20 & $3.30 \mathrm{E}+01$ & $7.30 \mathrm{E}+02$ & & & & & $2.10 \mathrm{E}+00$ & $1.90 \mathrm{E}+02$ & & & & TRS_472 & & \\
\hline Se & 34 & & & & & & & & & & & $1.70 \mathrm{E}+02$ & & NUREG/CR-5512 & & Strenge et al. (1986) \\
\hline Se & 34 & 28 & $3.60 \mathrm{E}+03$ & $1.20 \mathrm{E}+04$ & & & & & $1.30 \mathrm{E}+00$ & $6.80 \mathrm{E}+03$ & & & & TRS_364 & 6 & \\
\hline Se & 34 & & & & & & & & $3.70 \mathrm{E}+00$ & $1.10 \mathrm{E}+01$ & & & & & & \\
\hline Se & 34 & 14 & $3.50 \mathrm{E}+03$ & $9.40 \mathrm{E}+03$ & & & & & $1.30 \mathrm{E}+00$ & $6.00 \mathrm{E}+03$ & & & & TRS_472 & & \\
\hline $\mathrm{Si}$ & 14 & & & & & & & & & & & & & & & \\
\hline Sm & 62 & & & & & & & & & & & $2.50 \mathrm{E}+01$ & & NUREG/CR-5512 & & Strenge et al. (1986) \\
\hline $\mathrm{Sn}$ & 50 & & & & & & & & & & & $3.00 \mathrm{E}+03$ & & NUREG/CR-5512 & & Strenge et al. (1986) \\
\hline $\mathrm{Sr}$ & 38 & & & & & & & & & & & $5.00 \mathrm{E}+01$ & & NUREG/CR-5512 & & Poston and Klopfer (1988) \\
\hline $\mathrm{Sr}$ & 38 & & & & $3.00 \mathrm{E}+00$ & & & & & & & & & Thompson et al. (1972) & 9533 & Feldt (1971) \\
\hline $\mathrm{Sr}$ & 38 & & $5.80 \mathrm{E}+01$ & $1.70 \mathrm{E}+02$ & $1.60 \mathrm{E}+01$ & & & & & & & & & Thompson et al. (1972) & 10067 & Friend et al. (1965) \\
\hline $\mathrm{Sr}$ & 38 & 116 & $2.20 \mathrm{E}+01$ & $7.10 \mathrm{E}+02$ & & & & & $2.20 \mathrm{E}+00$ & $1.90 \mathrm{E}+02$ & & & & TRS_364 & 26 & \\
\hline $\mathrm{Sr}$ & 38 & & & & & $3.80 \mathrm{E}+02$ & & $7.50 \mathrm{E}+02$ & & & & & & & & Fesenko et al. (2011) \\
\hline $\mathrm{Sr}$ & 38 & & & & & $8.30 \mathrm{E}+01$ & & $1.50 \mathrm{E}+02$ & & & & & & & & Fesenko et al. (2011) \\
\hline $\mathrm{Sr}$ & 38 & & & & & $1.10 \mathrm{E}+02$ & & $2.50 \mathrm{E}+02$ & & & & & & & & Fesenko et al. (2011) \\
\hline $\mathrm{Sr}$ & 38 & & & & & $1.00 \mathrm{E}+03$ & & $2.20 \mathrm{E}+03$ & & & & & & & & Fesenko et al. (2011) \\
\hline $\mathrm{Sr}$ & 38 & & & & & $5.00 \mathrm{E}+01$ & & $1.20 \mathrm{E}+02$ & & & & & & & & Fesenko et al. (2011) \\
\hline $\mathrm{Sr}$ & 38 & & & & & $2.20 \mathrm{E}+02$ & & $9.80 \mathrm{E}+02$ & & & & & & & & Fesenko et al. (2011) \\
\hline $\mathrm{Sr}$ & 38 & & & & & $2.70 \mathrm{E}+01$ & & $8.00 \mathrm{E}+00$ & & & & & & & & Fesenko et al. (2011) \\
\hline $\mathrm{Sr}$ & 38 & & & & & & & & $5.40 \mathrm{E}+00$ & $1.20 \mathrm{E}+01$ & & & & & & Sheppard et al. (2010a) \\
\hline $\mathrm{Sr}$ & 38 & & & & & $1.16 \mathrm{E}+03$ & & $6.70 \mathrm{E}+02$ & & & & & & & & Outola et al. (2009) \\
\hline $\mathrm{Sr}$ & 38 & & & & & $1.60 \mathrm{E}+01$ & & $7.00 \mathrm{E}+00$ & & & & & & & & Outola et al. (2009) \\
\hline $\mathrm{Sr}$ & 38 & & & & & $3.50 \mathrm{E}+02$ & & $1.00 \mathrm{E}+02$ & & & & & & & & Outola et al. (2009) \\
\hline $\mathrm{Sr}$ & 38 & 99 & $1.40 \mathrm{E}-01$ & $6.90 \mathrm{E}+01$ & & & & & $3.90 \mathrm{E}+00$ & $2.90 \mathrm{E}+00$ & & & & TRS_472 & & \\
\hline $\mathrm{Ta}$ & 73 & & & & & & & & & & & & & & & \\
\hline $\mathrm{Tb}$ & 65 & & & & & & & & & & & $2.50 \mathrm{E}+01$ & & NUREG/CR-5512 & & Strenge et al. (1986) \\
\hline $\mathrm{Tb}$ & 65 & 18 & $8.00 \mathrm{E}+01$ & $2.40 \mathrm{E}+03$ & & & & & $2.60 \mathrm{E}+00$ & $7.50 \mathrm{E}+02$ & & & & TRS_364 & 3 & \\
\hline $\mathrm{Tb}$ & 65 & 11 & $2.00 \mathrm{E}+02$ & $1.70 \mathrm{E}+03$ & & & & & $1.90 \mathrm{E}+00$ & $4.10 \mathrm{E}+02$ & & & & TRS_472 & & \\
\hline Tc & 43 & & & & & & & & & & & $1.50 \mathrm{E}+01$ & & NUREG/CR-5512 & & Poston and Klopfer (1988) \\
\hline $\mathrm{Te}$ & 52 & & & & & & & & & & & $4.00 \mathrm{E}+02$ & & NUREG/CR-5512 & & Strenge et al. (1986) \\
\hline $\mathrm{Te}$ & 52 & 9 & $2.20 \mathrm{E}+02$ & $8.90 \mathrm{E}+02$ & & & & & $1.50 \mathrm{E}+00$ & $4.20 \mathrm{E}+02$ & & & & TRS_364 & 1 & \\
\hline $\mathrm{Te}$ & 52 & 3 & $9.60 \mathrm{E}+01$ & $2.10 \mathrm{E}+02$ & & & & & $1.50 \mathrm{E}+00$ & $1.50 \mathrm{E}+02$ & & & & TRS_472 & & \\
\hline Th & 90 & & & & & & & & & & & $1.00 \mathrm{E}+02$ & & NUREG/CR-5512 & & Poston and Klopfer (1988) \\
\hline Th & 90 & 2 & $3.80 \mathrm{E}+01$ & $3.80 \mathrm{E}+03$ & & $1.90 \mathrm{E}+02$ & & $2.60 \mathrm{E}+02$ & & & & & & TRS_364 & 5 & \\
\hline Th & 90 & & & & & & & & $3.50 \mathrm{E}+00$ & $1.30 \mathrm{E}+02$ & & & & & & Sheppard et al. (2010a) \\
\hline Th & 90 & 3 & $6.00 \mathrm{E}+00$ & $6.00 \mathrm{E}+00$ & & & & & & $6.00 \mathrm{E}+00$ & & & & TRS_472 & & \\
\hline $\mathrm{Ti}$ & 22 & 30 & $1.20 \mathrm{E}+02$ & $1.30 \mathrm{E}+03$ & & & & & $1.90 \mathrm{E}+00$ & $3.70 \mathrm{E}+02$ & & & & TRS_364 & 3 & \\
\hline
\end{tabular}


Table A.1. (contd)

\begin{tabular}{|c|c|c|c|c|c|c|c|c|c|c|c|c|c|c|c|c|}
\hline Element & Atomic\# & $\mathrm{N}$ & Min & Max & AVG & Mean & Wmean & STD & GSD & GM & WGM & MED & 95\%ile & Document & Reference\# & Reference \\
\hline $\mathrm{Ti}$ & 22 & 13 & $1.10 \mathrm{E}+02$ & $3.50 \mathrm{E}+02$ & & & & & $1.40 \mathrm{E}+00$ & $1.90 \mathrm{E}+02$ & & & & TRS_472 & & \\
\hline $\mathrm{Tl}$ & 81 & 81 & $6.40 \mathrm{E}+01$ & $3.10 \mathrm{E}+03$ & & & & & $1.90 \mathrm{E}+00$ & $5.80 \mathrm{E}+02$ & & & & TRS_364 & 3 & \\
\hline $\mathrm{Tl}$ & 81 & & & & & & & & $1.90 \mathrm{E}+00$ & $2.90 \mathrm{E}+03$ & & & & & & Sheppard et al. (2010a) \\
\hline $\mathrm{Tl}$ & 81 & 59 & $6.60 \mathrm{E}+01$ & $1.00 \mathrm{E}+04$ & & & & & $2.60 \mathrm{E}+00$ & $9.00 \mathrm{E}+02$ & & & & TRS_472 & & \\
\hline $\mathrm{Tm}$ & 69 & & & & & & & & & & & & & & & \\
\hline $\mathrm{U}$ & 92 & & & & & & & & & & & $5.00 \mathrm{E}+01$ & & NUREG/CR-5512 & & Poston and Klopfer (1988) \\
\hline $\mathrm{U}$ & 92 & & & & $2.00 \mathrm{E}+00$ & & & & & & & & & Thompson et al. (1972) & 10058 & Ouchi et al. (1970) \\
\hline $\mathrm{U}$ & 92 & 2 & $1.50 \mathrm{E}+00$ & $3.30 \mathrm{E}+00$ & & $1.30 \mathrm{E}+00$ & & $2.40 \mathrm{E}+00$ & & & & & & TRS_364 & 3 & \\
\hline $\mathrm{U}$ & 92 & & & & & & & & $2.10 \mathrm{E}+00$ & $2.30 \mathrm{E}+01$ & & & & & & Sheppard et al. (2010a) \\
\hline $\mathrm{U}$ & 92 & 9 & 2.00E-02 & $2.00 \mathrm{E}+01$ & & & & & $1.20 \mathrm{E}+00$ & $9.60 \mathrm{E}-01$ & & & & TRS_472 & & \\
\hline $\mathrm{V}$ & 23 & 103 & $3.00 \mathrm{E}+01$ & $1.10 \mathrm{E}+03$ & & & & & $2.00 \mathrm{E}+00$ & $2.80 \mathrm{E}+02$ & & & & TRS_364 & 4 & \\
\hline $\mathrm{V}$ & 23 & & & & & & & & $7.10 \mathrm{E}+00$ & $2.50 \mathrm{E}+00$ & & & & & & Sheppard et al. (2010a) \\
\hline $\mathrm{V}$ & 23 & 91 & $1.00 \mathrm{E}+01$ & $2.40 \mathrm{E}+02$ & & & & & $1.90 \mathrm{E}+00$ & $9.70 \mathrm{E}+01$ & & & & TRS_472 & & \\
\hline $\mathrm{W}$ & 74 & & & & & & & & & & & $1.20 \mathrm{E}+03$ & & NUREG/CR-5512 & & Strenge et al. (1986) \\
\hline Xe & 54 & & & & & & & & & & & & & & & \\
\hline $\mathrm{Y}$ & 39 & & & & & & & & & & & $2.50 \mathrm{E}+01$ & & NUREG/CR-5512 & & Strenge et al. (1986) \\
\hline $\mathrm{Y}$ & 39 & 12 & $1.10 \mathrm{E}+01$ & $6.20 \mathrm{E}+01$ & & & & & $1.60 \mathrm{E}+00$ & $3.10 \mathrm{E}+00$ & & & & TRS_364 & 1 & \\
\hline $\mathrm{Y}$ & 39 & 19 & $4.50 \mathrm{E}+00$ & $1.20 \mathrm{E}+02$ & & & & & $2.50 \mathrm{E}+00$ & $4.00 \mathrm{E}+01$ & & & & TRS_472 & & \\
\hline $\mathrm{Yb}$ & 70 & & & & & & & & & & & & & & & \\
\hline $\mathrm{Zn}$ & 30 & & & & & & & & & & & $2.50 \mathrm{E}+03$ & & NUREG/CR-5512 & & Poston and Klopfer (1988) \\
\hline $\mathrm{Zn}$ & 30 & & $3.20 \mathrm{E}+02$ & $8.50 \mathrm{E}+03$ & $1.70 \mathrm{E}+03$ & & & & & & & & & Thompson et al. (1972) & 2723 & Davis et al. (1958) \\
\hline $\mathrm{Zn}$ & 30 & 114 & $1.20 \mathrm{E}+03$ & $1.80 \mathrm{E}+04$ & & & & & $1.80 \mathrm{E}+00$ & $4.70 \mathrm{E}+03$ & & & & TRS_364 & 5 & \\
\hline $\mathrm{Zn}$ & 30 & & & & & $1.80 \mathrm{E}+01$ & & $8.00 \mathrm{E}+00$ & & & & & & & & Fesenko et al. (2011) \\
\hline $\mathrm{Zn}$ & 30 & & & & & $1.00 \mathrm{E}+03$ & & & & & & & & & & Fesenko et al. (2011) \\
\hline $\mathrm{Zn}$ & 30 & & & & & $1.10 \mathrm{E}+03$ & & & & & & & & & & Fesenko et al. (2011) \\
\hline $\mathrm{Zn}$ & 30 & & & & & & & & $3.40 \mathrm{E}+00$ & $2.50 \mathrm{E}+02$ & & & & & & Sheppard et al. (2010a) \\
\hline $\mathrm{Zn}$ & 30 & 96 & $3.30 \mathrm{E}+02$ & $1.60 \mathrm{E}+04$ & & & & & $2.90 \mathrm{E}+00$ & $3.40 \mathrm{E}+03$ & & & & TRS_472 & & \\
\hline $\mathrm{Zr}$ & 40 & & & & & & & & & & & $2.00 \mathrm{E}+02$ & & NUREG/CR-5512 & & Poston and Klopfer (1988) \\
\hline $\mathrm{Zr}$ & 40 & & & & & $1.00 \mathrm{E}+02$ & & & & & & & & & & Fesenko et al. (2011) \\
\hline $\mathrm{Zr}$ & 40 & & & & & $1.50 \mathrm{E}+01$ & & & & & & & & & & Fesenko et al. (2011) \\
\hline $\mathrm{Zr}$ & 40 & 10 & $9.20 \mathrm{E}+00$ & $1.20 \mathrm{E}+02$ & & & & & $2.40 \mathrm{E}+00$ & $2.20 \mathrm{E}+01$ & & & & TRS 472 & & \\
\hline
\end{tabular}


Table A.2. Bioaccumulation in Wet Mollusk from Freshwater, $\mathrm{L} / \mathrm{kg}_{\text {wet }}$ (CLBFM)

\begin{tabular}{|c|c|c|c|c|c|c|c|c|c|c|c|c|c|c|c|c|}
\hline Element & Atomic\# & $\mathrm{N}$ & Min & Max & AVG & Mean & Wmean & STD & GSD & GM & WGM & MED & 95\%ile & Document & Reference\# & Reference \\
\hline $\mathrm{Ac}$ & 89 & & & & & & & & & & & & & & & \\
\hline $\mathrm{Ag}$ & 47 & 2 & $1.30 \mathrm{E}+02$ & $3.30 \mathrm{E}+02$ & & $2.30 \mathrm{E}+02$ & & & & & & & & TRS_472 & & \\
\hline $\mathrm{Al}$ & 13 & 2 & $3.10 \mathrm{E}+03$ & $3.70 \mathrm{E}+03$ & & $3.40 \mathrm{E}+03$ & & & & & & & & TRS_472 & & \\
\hline Am & 95 & 17 & $5.90 \mathrm{E}+01$ & $9.00 \mathrm{E}+04$ & & & & & $7.00 \mathrm{E}+00$ & $2.40 \mathrm{E}+03$ & & & & TRS_472 & & \\
\hline $\mathrm{Ar}$ & 18 & & & & & & & & & & & & & & & \\
\hline As & 33 & & & & $5.00 \mathrm{E}+01$ & & & & & & & & & Thompson et al. (1972) & 2723 & Davis et al. (1958) \\
\hline As & 33 & 2 & $1.00 \mathrm{E}+03$ & $2.00 \mathrm{E}+03$ & & $1.50 \mathrm{E}+03$ & & & & & & & & TRS_472 & & \\
\hline At & 85 & & & & & & & & & & & & & & & \\
\hline $\mathrm{Au}$ & 79 & 2 & $1.00 \mathrm{E}+03$ & $1.50 \mathrm{E}+03$ & & $1.40 \mathrm{E}+03$ & & & & & & & & TRS_472 & & \\
\hline B & 5 & & & & & & & & & & & & & & & \\
\hline $\mathrm{Ba}$ & 56 & 2 & $1.10 \mathrm{E}+02$ & $1.60 \mathrm{E}+02$ & & $1.40 \mathrm{E}+02$ & & & & & & & & TRS_472 & & \\
\hline Be & 4 & & & & & & & & & & & & & & & \\
\hline $\mathrm{Bi}$ & 83 & & & & & & & & & & & & & & & \\
\hline $\mathrm{Bk}$ & 97 & & & & & & & & & & & & & & & \\
\hline $\mathrm{Br}$ & 35 & 2 & $7.20 \mathrm{E}+02$ & $1.90 \mathrm{E}+03$ & & $1.30 \mathrm{E}+03$ & & & & & & & & TRS_472 & & \\
\hline $\mathrm{C}$ & 6 & 24 & $1.30 \mathrm{E}+04$ & $5.70 \mathrm{E}+05$ & & & & & $2.60 \mathrm{E}+00$ & $6.50 \mathrm{E}+04$ & & & & TRS_472 & & \\
\hline $\mathrm{Ca}$ & 20 & 3 & $1.20 \mathrm{E}+01$ & $6.60 \mathrm{E}+01$ & & & & & $2.50 \mathrm{E}+00$ & $3.40 \mathrm{E}+01$ & & & & TRS_472 & & \\
\hline Cd & 48 & 149 & 1.40E-02 & $3.10 \mathrm{E}+04$ & & & & & $3.90 \mathrm{E}+01$ & $1.00 \mathrm{E}+02$ & & & & TRS_472 & & \\
\hline $\mathrm{Ce}$ & 58 & & & & & $2.30 \mathrm{E}+03$ & & $6.00 \mathrm{E}+02$ & & & & & & & & Fesenko et al. (2011) \\
\hline $\mathrm{Ce}$ & 58 & 2 & $2.90 \mathrm{E}+02$ & $5.60 \mathrm{E}+02$ & & $4.30 \mathrm{E}+02$ & & & & & & & & TRS_472 & & \\
\hline $\mathrm{Cf}$ & 98 & & & & & & & & & & & & & & & \\
\hline $\mathrm{Cl}$ & 17 & 2 & $1.30 \mathrm{E}+02$ & $1.90 \mathrm{E}+02$ & & $1.60 \mathrm{E}+02$ & & & & & & & & TRS_472 & & \\
\hline $\mathrm{Cm}$ & 96 & 29 & $9.00 \mathrm{E}+03$ & $1.00 \mathrm{E}+04$ & & $9.50 \mathrm{E}+03$ & & & & & & & & TRS_472 & & \\
\hline Co & 27 & & & & & $2.50 \mathrm{E}+01$ & & $6.00 \mathrm{E}+00$ & & & & & & & & Fesenko et al. (2011) \\
\hline Co & 27 & 2 & $1.90 \mathrm{E}-02$ & $4.10 \mathrm{E}+04$ & & & & & $1.30 \mathrm{E}+02$ & $2.20 \mathrm{E}+01$ & & & & TRS_472 & & \\
\hline $\mathrm{Cr}$ & 24 & & & & $3.00 \mathrm{E}+03$ & & & & & & & & & Thompson et al. (1972) & 2723 & Davis et al. (1958) \\
\hline $\mathrm{Cr}$ & 24 & & & & & $3.00 \mathrm{E}+01$ & & $8.00 \mathrm{E}+00$ & & & & & & & & Fesenko et al. (2011) \\
\hline $\mathrm{Cr}$ & 24 & 29 & $2.10 \mathrm{E}+02$ & $3.90 \mathrm{E}+02$ & & $3.00 \mathrm{E}+02$ & & & & & & & & TRS_472 & & \\
\hline Cs & 55 & & & & & $1.50 \mathrm{E}+01$ & & $4.00 \mathrm{E}+00$ & & & & & & & & Fesenko et al. (2011) \\
\hline Cs & 55 & 92 & $5.40 \mathrm{E}-03$ & $6.10 \mathrm{E}+03$ & & & & & $7.50 \mathrm{E}+01$ & $2.30 \mathrm{E}+01$ & & & & TRS_472 & & \\
\hline $\mathrm{Cu}$ & 29 & & & & $6.00 \mathrm{E}+02$ & & & & & & & & & Thompson et al. (1972) & 2723 & Davis et al. (1958) \\
\hline $\mathrm{Cu}$ & 29 & 2 & $5.60 \mathrm{E}+01$ & $1.40 \mathrm{E}+03$ & & & & & $1.10 \mathrm{E}+01$ & $4.20 \mathrm{E}+01$ & & & & TRS_472 & & \\
\hline Dy & 66 & & & & & & & & & & & & & & & \\
\hline Er & 68 & & & & & & & & & & & & & & & \\
\hline Es & 99 & & & & & & & & & & & & & & & \\
\hline Eu & 63 & 2 & $2.00 \mathrm{E}+02$ & $2.30 \mathrm{E}+02$ & & $2.20 \mathrm{E}+02$ & & & & & & & & TRS_472 & & \\
\hline $\mathrm{F}$ & 9 & & & & & & & & & & & & & & & \\
\hline $\mathrm{Fe}$ & 26 & & & & & $7.30 \mathrm{E}+01$ & & $1.80 \mathrm{E}+01$ & & & & & & & & Fesenko et al. (2011) \\
\hline $\mathrm{Fe}$ & 26 & 2 & $1.90 \mathrm{E}+03$ & $2.10 \mathrm{E}+03$ & & $2.00 \mathrm{E}+03$ & & & & & & & & TRS_472 & & \\
\hline Fm & 100 & & & & & & & & & & & & & & & \\
\hline $\mathrm{Fr}$ & 87 & & & & & & & & & & & & & & & \\
\hline $\mathrm{Ga}$ & 31 & & & & & & & & & & & & & & & \\
\hline $\mathrm{Gd}$ & 64 & & & & & & & & & & & & & & & \\
\hline $\mathrm{Ge}$ & 32 & 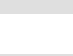 & & & & & & & & & & & & & & \\
\hline $\mathrm{H}$ & 1 & & & & & & & & & & & & & & & \\
\hline $\mathrm{Hf}$ & 72 & 2 & $1.30 \mathrm{E}+03$ & $1.50 \mathrm{E}+03$ & & $1.40 \mathrm{E}+03$ & & & & & & & & TRS_472 & & \\
\hline $\mathrm{Hg}$ & 80 & 31 & $2.00 \mathrm{E}+02$ & $5.20 \mathrm{E}+03$ & & & & & $2.70 \mathrm{E}+00$ & $7.50 \mathrm{E}+02$ & & & & TRS_472 & & \\
\hline Ho & 67 & & & & & & & & & & & & & & & \\
\hline I & 53 & & & & & $7.00 \mathrm{E}+00$ & & $1.80 \mathrm{E}+00$ & & & & & & & & Fesenko et al. (2011) \\
\hline I & 53 & 99 & $4.00 \mathrm{E}-01$ & $1.30 \mathrm{E}+03$ & & & & & $1.10 \mathrm{E}+01$ & $1.70 \mathrm{E}+01$ & & & & TRS_472 & & \\
\hline
\end{tabular}


Table A.2. (contd)

\begin{tabular}{|c|c|c|c|c|c|c|c|c|c|c|c|c|c|c|c|c|}
\hline Element & Atomic\# & $\mathrm{N}$ & Min & Max & AVG & Mean & Wmean & STD & GSD & GM & WGM & MED & 95\%ile & Document & Reference\# & Reference \\
\hline In & 49 & & & & & & & & & & & & & & & \\
\hline Ir & 77 & & & & & & & & & & & & & & & \\
\hline $\mathrm{K}$ & 19 & 2 & $5.40 \mathrm{E}+02$ & $6.10 \mathrm{E}+02$ & & $5.90 \mathrm{E}+02$ & & & & & & & & TRS_472 & & \\
\hline $\mathrm{Kr}$ & 36 & & & & & & & & & & & & & & & \\
\hline $\mathrm{La}$ & 57 & 2 & $3.30 \mathrm{E}+02$ & $3.70 \mathrm{E}+02$ & & $3.50 \mathrm{E}+02$ & & & & & & & & TRS_472 & & \\
\hline $\mathrm{Li}$ & 3 & & & & & & & & & & & & & & & \\
\hline $\mathrm{Lu}$ & 71 & 1 & & & & & & & & $1.10 \mathrm{E}+03$ & & & & TRS_472 & & \\
\hline Md & 101 & & & & & & & & & & & & & & & \\
\hline $\mathrm{Mg}$ & 12 & 2 & $2.10 \mathrm{E}+01$ & $4.30 \mathrm{E}+01$ & & $3.20 \mathrm{E}+01$ & & & & & & & & TRS_472 & & \\
\hline $\mathrm{Mn}$ & 25 & & & & $9.30 \mathrm{E}+03$ & & & & & & & & & Thompson et al. (1972) & & Gaglione et al. (1964) \\
\hline $\mathrm{Mn}$ & 25 & & & & & $1.30 \mathrm{E}+03$ & & $3.00 \mathrm{E}+02$ & & & & & & & & Fesenko et al. (2011) \\
\hline $\mathrm{Mn}$ & 25 & 4 & $1.10 \mathrm{E}-01$ & $3.70 \mathrm{E}+03$ & & & & & $3.90 \mathrm{E}+02$ & $2.10 \mathrm{E}+01$ & & & & TRS_472 & & \\
\hline Mo & 42 & 33 & $2.90 \mathrm{E}-02$ & $3.00 \mathrm{E}+03$ & & & & & $1.30 \mathrm{E}+01$ & $4.50 \mathrm{E}-01$ & & & & TRS_472 & & \\
\hline $\mathrm{N}$ & 7 & & & & & & & & & & & & & & & \\
\hline $\mathrm{Na}$ & 11 & & & & $2.00 \mathrm{E}+02$ & & & & & & & & & Thompson et al. (1972) & 2723 & Davis et al. (1958) \\
\hline $\mathrm{Na}$ & 11 & 4 & $1.40 \mathrm{E}-01$ & $1.10 \mathrm{E}+02$ & & & & & $3.60 \mathrm{E}+01$ & $3.40 \mathrm{E}+00$ & & & & TRS_472 & & \\
\hline $\mathrm{Nb}$ & 41 & & & & & & & & & & & & & & & \\
\hline $\mathrm{Nd}$ & 60 & & & & & & & & & & & & & & & \\
\hline $\mathrm{Ne}$ & 10 & & & & & & & & & & & & & & & \\
\hline $\mathrm{Ni}$ & 28 & & & & & & & & & & & & & & & \\
\hline $\mathrm{Np}$ & 93 & 2 & $9.00 \mathrm{E}+03$ & $1.00 \mathrm{E}+04$ & & $9.50 \mathrm{E}+03$ & & & & & & & & TRS_472 & & \\
\hline $\mathrm{O}$ & 8 & & & & & & & & & & & & & MNo-712 & & \\
\hline Os & 76 & & & & & & & & & & & & & & & \\
\hline $\mathrm{P}$ & 15 & & & & $2.00 \mathrm{E}+04$ & & & & & & & & & Thompson et al. (1972) & 2723 & Davis et al. (1958) \\
\hline Pa & 91 & & & & & & & & & & & & & & & \\
\hline $\mathrm{Pb}$ & 82 & 79 & $4.50 \mathrm{E}-02$ & $7.00 \mathrm{E}+02$ & & & & & $2.00 \mathrm{E}+01$ & $2.20 \mathrm{E}+01$ & & & & TRS_472 & & \\
\hline $\mathrm{Pd}$ & 46 & & & & & & & & & & & & & & & \\
\hline $\mathrm{Pm}$ & 61 & & & & & & & & & & & & & & & \\
\hline Po & 84 & & & & & $7.20 \mathrm{E}+01$ & & $1.80 \mathrm{E}+01$ & & & & & & & & Fesenko et al. (2011) \\
\hline Pr & 59 & & & & & & & & & & & & & & & \\
\hline Pt & 78 & & & & & & & & & & & & & & & \\
\hline Pu & 94 & 100 & $3.60 \mathrm{E}-01$ & $5.50 \mathrm{E}+06$ & & & & & $2.90 \mathrm{E}+01$ & $7.40 \mathrm{E}+03$ & & & & TRS_472 & & \\
\hline $\mathrm{Ra}$ & 88 & 5 & $1.90 \mathrm{E}+00$ & $1.90 \mathrm{E}+03$ & & & & & $3.00 \mathrm{E}+01$ & $1.00 \mathrm{E}+02$ & & & & TRS_472 & & \\
\hline $\mathrm{Rb}$ & 37 & 2 & $1.90 \mathrm{E}+03$ & $2.20 \mathrm{E}+03$ & & $2.00 \mathrm{E}+03$ & & & & & & & & TRS_472 & & \\
\hline $\mathrm{Re}$ & 75 & & & & & & & & & & & & & & & \\
\hline $\mathrm{Rh}$ & 45 & & & & & & & & & & & & & & & \\
\hline $\mathrm{Rn}$ & 86 & & & & & & & & & & & & & & & \\
\hline $\mathrm{Ru}$ & 44 & & & & & $1.20 \mathrm{E}+01$ & & $3.00 \mathrm{E}+00$ & & & & & & & & Fesenko et al. (2011) \\
\hline $\mathrm{Ru}$ & 44 & 9 & $1.90 \mathrm{E}-03$ & $9.30 \mathrm{E}+01$ & & & & & $2.10 \mathrm{E}+01$ & 3.90E-02 & & & & TRS_472 & & \\
\hline $\mathrm{s}$ & 16 & & & & & & & & & & & & & & & \\
\hline $\mathrm{Sb}$ & 51 & & & & & $1.00 \mathrm{E}+01$ & & $3.00 \mathrm{E}+00$ & & & & & & & & Fesenko et al. (2011) \\
\hline $\mathrm{Sb}$ & 51 & 2 & $7.40 \mathrm{E}+01$ & $3.50 \mathrm{E}+02$ & & $2.10 \mathrm{E}+02$ & & & & & & & & TRS_472 & & \\
\hline Sc & 21 & 2 & $3.30 \mathrm{E}+03$ & $3.70 \mathrm{E}+03$ & & $3.50 \mathrm{E}+03$ & & & & & & & & TRS_472 & & \\
\hline Se & 34 & 16 & $1.20 \mathrm{E}+01$ & $6.90 \mathrm{E}+04$ & & & & & $1.50 \mathrm{E}+01$ & $5.70 \mathrm{E}+02$ & & & & TRS_472 & & \\
\hline $\mathrm{Si}$ & 14 & & & & & & & & & & & & & & & \\
\hline $\mathrm{Sm}$ & 62 & 2 & $5.00 \mathrm{E}+02$ & $2.70 \mathrm{E}+03$ & & $1.60 \mathrm{E}+03$ & & & & & & & & TRS_472 & & \\
\hline Sn & 50 & & & & & & & & & & & & & & & \\
\hline $\mathrm{Sr}$ & 38 & & & & & $2.40 \mathrm{E}+03$ & & $6.00 \mathrm{E}+02$ & & & & & & & & Fesenko et al. (2011) \\
\hline $\mathrm{Sr}$ & 38 & 5 & $7.70 \mathrm{E}+01$ & $1.30 \mathrm{E}+03$ & & & & & $3.20 \mathrm{E}+00$ & $2.70 \mathrm{E}+02$ & & & & TRS_472 & & \\
\hline Та & 73 & & & & & & & & & & & & & & & \\
\hline $\mathrm{Tb}$ & 65 & & & & & & & & & & & & & & & \\
\hline Tc & 43 & 10 & $1.90 \mathrm{E}+00$ & $4.00 \mathrm{E}+02$ & & & & & $9.90 \mathrm{E}+00$ & $2.60 \mathrm{E}+01$ & & & & TRS_472 & & \\
\hline
\end{tabular}


Table A.2. (contd)

\begin{tabular}{|c|c|c|c|c|c|c|c|c|c|c|c|c|c|c|c|c|}
\hline Element & Atomic\# & $\mathrm{N}$ & Min & Max & AVG & Mean & Wmean & STD & GSD & GM & WGM & MED & 95\%ile & Document & Reference\# & Reference \\
\hline $\mathrm{Te}$ & 52 & & & & & & & & & & & & & & & \\
\hline Th & 90 & 2 & $2.90 \mathrm{E}+03$ & $2.90 \mathrm{E}+03$ & & $2.90 \mathrm{E}+03$ & & & & & & & & TRS_472 & & \\
\hline $\mathrm{Ti}$ & 22 & & & & & & & & & & & & & & & \\
\hline $\mathrm{Tl}$ & 81 & & & & & & & & & & & & & & & \\
\hline $\mathrm{Tm}$ & 69 & & & & & & & & & & & & & & & \\
\hline $\mathrm{U}$ & 92 & 9 & $3.60 \mathrm{E}+00$ & $6.00 \mathrm{E}+04$ & & & & & $1.90 \mathrm{E}+01$ & $1.70 \mathrm{E}+02$ & & & & TRS_472 & & \\
\hline $\mathrm{V}$ & 23 & 2 & $3.60 \mathrm{E}+02$ & $4.00 \mathrm{E}+02$ & & $3.90 \mathrm{E}+02$ & & & & & & & & TRS 472 & & \\
\hline W & 74 & & & & & & & & & & & & & & & \\
\hline Хе & 54 & & & & & & & & & & & & & & & \\
\hline $\begin{array}{l}\text { Ae } \\
\text { Y }\end{array}$ & 39 & & & & & & & & & & & & & & & \\
\hline $\mathrm{Yb}$ & 70 & & & & & & & & & & & & & & & \\
\hline $\mathrm{Zn}$ & 30 & & & & $2.00 \mathrm{E}+03$ & & & & & & & & & Thompson et al. (1972) & 2723 & Davis et al. (1958) \\
\hline $\mathrm{Zn}$ & 30 & 92 & $6.30 \mathrm{E}-02$ & $1.50 \mathrm{E}+03$ & & & & & $2.90 \mathrm{E}+01$ & $9.20 \mathrm{E}+01$ & & & & TRS_472 & & \\
\hline $\mathrm{Zr}$ & 40 & & & & & $1.50 \mathrm{E}+01$ & & $4.00 \mathrm{E}+00$ & & & & & & & & Fesenko et al. (2011) \\
\hline
\end{tabular}



Table A.3. Bioaccumulation in Wet Crustacea from Freshwater, $\mathrm{L} / \mathrm{kg}_{\text {wet }}$ (CLBFI)

\begin{tabular}{|c|c|c|c|c|c|c|c|c|c|c|c|c|c|c|c|c|}
\hline Element & Atomic\# & $\mathrm{N}$ & Min & Max & AVG & Mean & Wmean & STD & GSD & GM & WGM & MED & 95\%ile & Document & Reference\# & Reference \\
\hline $\mathrm{Ac}$ & 89 & & & & & & & & & & & & & & & \\
\hline $\mathrm{Ag}$ & 47 & 2 & $1.30 \mathrm{E}+02$ & $3.30 \mathrm{E}+02$ & & $2.30 \mathrm{E}+02$ & & $1.40 \mathrm{E}+03$ & & & & & & TRS_364 & 2 & \\
\hline $\mathrm{Ag}$ & 47 & 2 & $1.30 \mathrm{E}+02$ & $3.30 \mathrm{E}+02$ & & $2.30 \mathrm{E}+02$ & & & & & & & & TRS_472 & & \\
\hline $\mathrm{Al}$ & 13 & 2 & $3.10 \mathrm{E}+03$ & $3.70 \mathrm{E}+03$ & & $3.40 \mathrm{E}+03$ & & $4.00 \mathrm{E}+02$ & & & & & & TRS_364 & 2 & \\
\hline $\mathrm{Al}$ & 13 & 2 & $3.10 \mathrm{E}+03$ & $3.70 \mathrm{E}+03$ & & $3.40 \mathrm{E}+03$ & & & & & & & & TRS_472 & & \\
\hline $\mathrm{Am}$ & 95 & 17 & $5.90 \mathrm{E}+01$ & $8.00 \mathrm{E}+04$ & & & & & $7.00 \mathrm{E}+00$ & $2.40 \mathrm{E}+03$ & & & & TRS_364 & 7 & \\
\hline $\mathrm{Am}$ & 95 & 17 & $5.90 \mathrm{E}+01$ & $9.00 \mathrm{E}+04$ & & & & & $7.00 \mathrm{E}+00$ & $2.40 \mathrm{E}+03$ & & & & TRS_472 & & \\
\hline $\mathrm{Am}$ & 95 & & & & & $9.70 \mathrm{E}+01$ & & $1.50 \mathrm{E}+01$ & & & & & & & & Hosseini et al. (2008) \\
\hline $\mathrm{Ar}$ & 18 & & & & & & & & & & & & & & & \\
\hline As & 33 & & & & $3.00 \mathrm{E}+01$ & & & & & & & & & Thompson et al. (1972) & 2723 & Davis et al. (1958) \\
\hline As & 33 & 2 & $1.00 \mathrm{E}+03$ & $2.00 \mathrm{E}+03$ & & $1.50 \mathrm{E}+03$ & & $7.10 \mathrm{E}+02$ & & & & & & TRS_364 & 2 & \\
\hline As & 33 & 2 & $1.00 \mathrm{E}+03$ & $2.00 \mathrm{E}+03$ & & $1.50 \mathrm{E}+03$ & & & & & & & & TRS_472 & & \\
\hline At & 85 & & & & & & & & & & & & & & & \\
\hline $\mathrm{Au}$ & 79 & 2 & $1.00 \mathrm{E}+03$ & $1.50 \mathrm{E}+03$ & & $1.40 \mathrm{E}+03$ & & $3.50 \mathrm{E}+02$ & & & & & & TRS_364 & 2 & \\
\hline $\mathrm{Au}$ & 79 & 2 & $1.00 \mathrm{E}+03$ & $1.50 \mathrm{E}+03$ & & $1.40 \mathrm{E}+03$ & & & & & & & & TRS_472 & & \\
\hline Ва & 56 & 2 & $1.10 \mathrm{E}+02$ & $1.60 \mathrm{E}+02$ & & $1.40 \mathrm{E}+02$ & & $3.50 \mathrm{E}+00$ & & & & & & TRS_364 & 2 & \\
\hline $\mathrm{Ba}$ & 56 & 2 & $1.10 \mathrm{E}+02$ & $1.60 \mathrm{E}+02$ & & $1.40 \mathrm{E}+02$ & & & & & & & & TRS_472 & & \\
\hline $\mathrm{Be}$ & 4 & & & & & & & & & & & & & & & \\
\hline $\mathrm{Bi}$ & 83 & & & & & & & & & & & & & & & \\
\hline $\mathrm{Bk}$ & 97 & & & & & & & & & & & & & & & \\
\hline $\mathrm{Br}$ & 35 & 2 & $7.20 \mathrm{E}+02$ & $1.80 \mathrm{E}+03$ & & $1.30 \mathrm{E}+03$ & & $7.60 \mathrm{E}+02$ & & & & & & TRS_364 & 2 & \\
\hline $\mathrm{Br}$ & 35 & 2 & $7.20 \mathrm{E}+02$ & $1.90 \mathrm{E}+03$ & & $1.30 \mathrm{E}+03$ & & & & & & & & TRS_472 & & \\
\hline $\mathrm{C}$ & 6 & 24 & $1.30 \mathrm{E}+03$ & $5.70 \mathrm{E}+05$ & & & & & $2.60 \mathrm{E}+00$ & $6.50 \mathrm{E}+04$ & & & & TRS_364 & 3 & \\
\hline $\mathrm{C}$ & 6 & 24 & $1.30 \mathrm{E}+04$ & $5.70 \mathrm{E}+05$ & & & & & $2.60 \mathrm{E}+00$ & $6.50 \mathrm{E}+04$ & & & & TRS_472 & & \\
\hline $\mathrm{Ca}$ & 20 & 3 & $1.20 \mathrm{E}+01$ & $6.60 \mathrm{E}+01$ & & & & & $2.50 \mathrm{E}+00$ & $3.40 \mathrm{E}+01$ & & & & TRS_364 & 2 & \\
\hline Са & 20 & 3 & $1.20 \mathrm{E}+01$ & $6.60 \mathrm{E}+01$ & & & & & $2.50 \mathrm{E}+00$ & $3.40 \mathrm{E}+01$ & & & & TRS_472 & & \\
\hline $\mathrm{Cd}$ & 48 & 149 & $1.40 \mathrm{E}-02$ & $3.10 \mathrm{E}+04$ & & & & & $3.90 \mathrm{E}+01$ & $1.00 \mathrm{E}+02$ & & & & TRS_364 & 8 & \\
\hline $\mathrm{Cd}$ & 48 & 149 & $1.40 \mathrm{E}-02$ & $3.10 \mathrm{E}+04$ & & & & & $3.90 \mathrm{E}+01$ & $1.00 \mathrm{E}+02$ & & & & TRS_472 & & \\
\hline $\mathrm{Cd}$ & 48 & & & & & $5.00 \mathrm{E}+03$ & & & & & & & & & & Hosseini et al. (2008) \\
\hline $\mathrm{Ce}$ & 58 & 2 & $2.90 \mathrm{E}+02$ & $5.60 \mathrm{E}+02$ & & $4.30 \mathrm{E}+02$ & & $1.90 \mathrm{E}+02$ & & & & & & TRS_364 & 2 & \\
\hline $\mathrm{Ce}$ & 58 & 2 & $2.90 \mathrm{E}+02$ & $5.60 \mathrm{E}+02$ & & $4.30 \mathrm{E}+02$ & & & & & & & & TRS_472 & & \\
\hline $\mathrm{Ce}$ & 58 & & & & & $1.00 \mathrm{E}+03$ & & & & & & & & & & Hosseini et al. (2008) \\
\hline $\mathrm{Cf}$ & 98 & & & & & & & & & & & & & & & \\
\hline $\mathrm{Cl}$ & 17 & 2 & $1.30 \mathrm{E}+02$ & $1.80 \mathrm{E}+02$ & & $1.60 \mathrm{E}+02$ & & $3.50 \mathrm{E}+01$ & & & & & & TRS_364 & 2 & \\
\hline $\mathrm{Cl}$ & 17 & 2 & $1.30 \mathrm{E}+02$ & $1.90 \mathrm{E}+02$ & & $1.60 \mathrm{E}+02$ & & & & & & & & TRS_472 & & \\
\hline $\mathrm{Cl}$ & 17 & & & & & $5.00 \mathrm{E}+01$ & & & & & & & & & & Hosseini et al. (2008) \\
\hline $\mathrm{Cm}$ & 96 & 2 & $9.00 \mathrm{E}+03$ & $1.00 \mathrm{E}+04$ & & $9.50 \mathrm{E}+03$ & & $7.10 \mathrm{E}+02$ & & & & & & TRS_364 & 1 & \\
\hline $\mathrm{Cm}$ & 96 & 29 & $9.00 \mathrm{E}+03$ & $1.00 \mathrm{E}+04$ & & $9.50 \mathrm{E}+03$ & & & & & & & & TRS_472 & & \\
\hline Co & 27 & 28 & $1.80 \mathrm{E}-03$ & $4.10 \mathrm{E}+04$ & & & & & $1.30 \mathrm{E}+02$ & $2.20 \mathrm{E}+01$ & & & & TRS_364 & 9 & \\
\hline Сo & 27 & 2 & $1.90 \mathrm{E}-02$ & $4.10 \mathrm{E}+04$ & & & & & $1.30 \mathrm{E}+02$ & $2.20 \mathrm{E}+01$ & & & & TRS_472 & & \\
\hline Сo & 27 & & & & & $1.50 \mathrm{E}+03$ & & $5.00 \mathrm{E}+02$ & & & & & & & & Hosseini et al. (2008) \\
\hline $\mathrm{Cr}$ & 24 & & & & $2.00 \mathrm{E}+02$ & & & & & & & & & Thompson et al. (1972) & 2723 & Davis et al. (1958) \\
\hline $\mathrm{Cr}$ & 24 & 2 & $2.10 \mathrm{E}+02$ & $3.80 \mathrm{E}+02$ & & $3.00 \mathrm{E}+02$ & & $1.20 \mathrm{E}+02$ & & & & & & TRS_364 & 2 & \\
\hline $\mathrm{Cr}$ & 24 & 29 & $2.10 \mathrm{E}+02$ & $3.90 \mathrm{E}+02$ & & $3.00 \mathrm{E}+02$ & & & & & & & & TRS_472 & & \\
\hline Cs & 55 & 29 & $5.40 \mathrm{E}-03$ & $6.10 \mathrm{E}+03$ & & & & & $7.50 \mathrm{E}+01$ & $2.30 \mathrm{E}+01$ & & & & TRS_364 & 11 & \\
\hline Cs & 55 & 92 & $5.40 \mathrm{E}-03$ & $6.10 \mathrm{E}+03$ & & & & & $7.50 \mathrm{E}+01$ & $2.30 \mathrm{E}+01$ & & & & TRS_472 & & \\
\hline Cs & 55 & & & & & $7.60 \mathrm{E}+03$ & & $8.90 \mathrm{E}+03$ & & & & & & & & Hosseini et al. (2008) \\
\hline $\mathrm{Cu}$ & 29 & & & & $3.00 \mathrm{E}+01$ & & & & & & & & & Thompson et al. (1972) & 2723 & Davis et al. (1958) \\
\hline $\mathrm{Cu}$ & 29 & 82 & $5.60 \mathrm{E}+01$ & $1.40 \mathrm{E}+03$ & & & & & $1.10 \mathrm{E}+01$ & $4.20 \mathrm{E}+01$ & & & & TRS_364 & 4 & 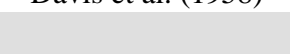 \\
\hline $\mathrm{Cu}$ & 29 & 2 & $5.60 \mathrm{E}+01$ & $1.40 \mathrm{E}+03$ & & & & & $1.10 \mathrm{E}+01$ & $4.20 \mathrm{E}+01$ & & & & TRS_472 & & \\
\hline
\end{tabular}


Table A.3. (contd)

\begin{tabular}{|c|c|c|c|c|c|c|c|c|c|c|c|c|c|c|c|c|}
\hline Element & Atomic\# & $\mathrm{N}$ & Min & Max & AVG & Mean & Wmean & STD & GSD & GM & WGM & MED & 95\%ile & Document & Reference\# & Reference \\
\hline Dy & 66 & & & & & & & & & & & & & & & \\
\hline Er & 68 & & & & & & & & & & & & & & & \\
\hline Es & 99 & & & & & & & & & & & & & & & \\
\hline $\mathrm{Eu}$ & 63 & 2 & $2.00 \mathrm{E}+02$ & $2.30 \mathrm{E}+02$ & & $2.20 \mathrm{E}+02$ & & $2.10 \mathrm{E}+01$ & & & & & & TRS_364 & 2 & \\
\hline $\mathrm{Eu}$ & 63 & 2 & $2.00 \mathrm{E}+02$ & $2.30 \mathrm{E}+02$ & & $2.20 \mathrm{E}+02$ & & & & & & & & TRS_472 & & \\
\hline $\mathrm{F}$ & 9 & & & & & & & & & & & & & & & \\
\hline $\mathrm{Fe}$ & 26 & 2 & $1.80 \mathrm{E}+03$ & $2.10 \mathrm{E}+03$ & & $2.00 \mathrm{E}+03$ & & $2.10 \mathrm{E}+02$ & & & & & & TRS_364 & 2 & \\
\hline $\mathrm{Fe}$ & 26 & 2 & $1.90 \mathrm{E}+03$ & $2.10 \mathrm{E}+03$ & & $2.00 \mathrm{E}+03$ & & & & & & & & TRS_472 & & \\
\hline Fm & 100 & & & & & & & & & & & & & & & \\
\hline $\mathrm{Fr}$ & 87 & & & & & & & & & & & & & & & \\
\hline $\mathrm{Ga}$ & 31 & & & & & & & & & & & & & & & \\
\hline $\mathrm{Gd}$ & 64 & & & & & & & & & & & & & & & \\
\hline $\mathrm{Ge}$ & 32 & & & & & & & & & & & & & & & \\
\hline $\mathrm{H}$ & 1 & & & & & & & & & & & & & & & \\
\hline $\mathrm{Hf}$ & 72 & 2 & $1.30 \mathrm{E}+03$ & $1.50 \mathrm{E}+03$ & & $1.40 \mathrm{E}+03$ & & $1.40 \mathrm{E}+02$ & & & & & & TRS_364 & 2 & \\
\hline $\mathrm{Hf}$ & 72 & 2 & $1.30 \mathrm{E}+03$ & $1.50 \mathrm{E}+03$ & & $1.40 \mathrm{E}+03$ & & & & & & & & TRS_472 & & \\
\hline $\mathrm{Hg}$ & 80 & 31 & $2.00 \mathrm{E}+02$ & $5.20 \mathrm{E}+03$ & & & & 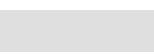 & $2.70 \mathrm{E}+00$ & $7.50 \mathrm{E}+02$ & & & & TRS_364 & 3 & \\
\hline $\mathrm{Hg}$ & 80 & 31 & $2.00 \mathrm{E}+02$ & $5.20 \mathrm{E}+03$ & & & & & $2.70 \mathrm{E}+00$ & $7.50 \mathrm{E}+02$ & & & & TRS_472 & & \\
\hline Ho & 67 & & & & & & & & & & & & & & & \\
\hline I & 53 & 99 & $4.00 \mathrm{E}-01$ & $1.30 \mathrm{E}+03$ & & & & & $1.10 \mathrm{E}+01$ & $1.70 \mathrm{E}+01$ & & & & TRS_364 & 5 & \\
\hline I & 53 & 99 & $4.00 \mathrm{E}-01$ & $1.30 \mathrm{E}+03$ & & & & & $1.10 \mathrm{E}+01$ & $1.70 \mathrm{E}+01$ & & & & TRS_472 & & \\
\hline I & 53 & & & & & $4.00 \mathrm{E}+02$ & & $2.30 \mathrm{E}+02$ & & & & & & & & Hosseini et al. (2008) \\
\hline In & 49 & 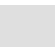 & & & & & & & & & & & & & & \\
\hline Ir & 77 & & & & & & & & & & & & & & & \\
\hline $\mathrm{K}$ & 19 & 2 & $5.40 \mathrm{E}+02$ & $6.10 \mathrm{E}+02$ & & $5.80 \mathrm{E}+02$ & & $5.00 \mathrm{E}+01$ & & & & & & TRS_364 & 2 & \\
\hline $\mathrm{K}$ & 19 & 2 & $5.40 \mathrm{E}+02$ & $6.10 \mathrm{E}+02$ & & $5.90 \mathrm{E}+02$ & & & & & & & & TRS_472 & & \\
\hline $\mathrm{Kr}$ & 36 & & & & & & & & & & & & & & & \\
\hline La & 57 & 2 & $3.30 \mathrm{E}+02$ & $3.70 \mathrm{E}+02$ & & $3.50 \mathrm{E}+02$ & & $2.80 \mathrm{E}+01$ & & & & & & TRS_364 & 2 & \\
\hline $\mathrm{La}$ & 57 & 2 & $3.30 \mathrm{E}+02$ & $3.70 \mathrm{E}+02$ & & $3.50 \mathrm{E}+02$ & & & & & & & & TRS_472 & & \\
\hline $\mathrm{Lu}$ & 71 & 1 & & & & & & & & $1.10 \mathrm{E}+03$ & & & & TRS_364 & 2 & \\
\hline $\mathrm{Lu}$ & 71 & 1 & & & & & & & & $1.10 \mathrm{E}+03$ & & & & TRS_472 & & \\
\hline Md & 101 & & & & & & & & & & & & & & & \\
\hline $\mathrm{Mg}$ & 12 & 2 & $2.10 \mathrm{E}+01$ & $4.30 \mathrm{E}+01$ & & $3.20 \mathrm{E}+01$ & & $1.60 \mathrm{E}+00$ & & & & & & TRS_364 & 2 & \\
\hline $\mathrm{Mg}$ & 12 & 2 & $2.10 \mathrm{E}+01$ & $4.30 \mathrm{E}+01$ & & $3.20 \mathrm{E}+01$ & & & & & & & & TRS_472 & & \\
\hline $\mathrm{Mn}$ & 25 & 4 & $1.10 \mathrm{E}-01$ & $3.70 \mathrm{E}+03$ & & & & & $3.90 \mathrm{E}+02$ & $2.10 \mathrm{E}+01$ & & & & TRS_364 & 3 & \\
\hline $\mathrm{Mn}$ & 25 & 4 & $1.10 \mathrm{E}-01$ & $3.70 \mathrm{E}+03$ & & & & & $3.90 \mathrm{E}+02$ & $2.10 \mathrm{E}+01$ & & & & TRS_472 & & \\
\hline $\mathrm{Mn}$ & 25 & & & & & $1.00 \mathrm{E}+04$ & & & & & & & & & & Hosseini et al. (2008) \\
\hline Mo & 42 & 33 & $2.90 \mathrm{E}-02$ & $3.00 \mathrm{E}+03$ & & & & & $1.30 \mathrm{E}+01$ & $4.50 \mathrm{E}-01$ & & & & TRS_364 & 3 & \\
\hline Mo & 42 & 33 & $2.90 \mathrm{E}-02$ & $3.00 \mathrm{E}+03$ & & & & & $1.30 \mathrm{E}+01$ & 4.50E-01 & & & & TRS_472 & & \\
\hline $\mathrm{N}$ & 7 & & & & & & & & & & & & & & & \\
\hline $\mathrm{Na}$ & 11 & & & & $2.00 \mathrm{E}+02$ & & & & & & & & & Thompson et al. (1972) & 2723 & Davis et al. (1958) \\
\hline $\mathrm{Na}$ & 11 & 4 & $1.40 \mathrm{E}-01$ & $1.10 \mathrm{E}+02$ & & & & & $3.60 \mathrm{E}+01$ & $3.40 \mathrm{E}+00$ & & & & TRS_364 & 3 & \\
\hline $\mathrm{Na}$ & 11 & 4 & $1.40 \mathrm{E}-01$ & $1.10 \mathrm{E}+02$ & & & & & $3.60 \mathrm{E}+01$ & $3.40 \mathrm{E}+00$ & & & & TRS_472 & & \\
\hline $\mathrm{Nb}$ & 41 & & & & & & & & & & & & & & & \\
\hline $\mathrm{Nd}$ & 60 & & & & & & & & & & & & & & & \\
\hline $\mathrm{Ne}$ & 10 & & & & & & & & & & & & & & & \\
\hline $\mathrm{Ni}$ & 28 & & & & & & & & & & & & & & & \\
\hline $\mathrm{Np}$ & 93 & 2 & $9.00 \mathrm{E}+03$ & $1.00 \mathrm{E}+04$ & & $9.50 \mathrm{E}+03$ & & $1.10 \mathrm{E}+00$ & & & & & & TRS_364 & 1 & \\
\hline $\mathrm{Np}$ & 93 & 2 & $9.00 \mathrm{E}+03$ & $1.00 \mathrm{E}+04$ & & $9.50 \mathrm{E}+03$ & & & & & & & & TRS_472 & 1 & \\
\hline $\mathrm{O}$ & 8 & & & & & & & & & & & & & & & \\
\hline
\end{tabular}


Table A.3. (contd)

\begin{tabular}{|c|c|c|c|c|c|c|c|c|c|c|c|c|c|c|c|c|}
\hline Element & Atomic\# & $\mathrm{N}$ & Min & Max & AVG & Mean & Wmean & STD & GSD & GM & WGM & MED & 95\%ile & Document & Reference\# & Reference \\
\hline $\mathrm{P}$ & 15 & & & & $1.00 \mathrm{E}+04$ & & & & & & & & & Thompson et al. (1972) & 2723 & Davis et al. (1958) \\
\hline $\mathrm{P}$ & 15 & & & & & $1.30 \mathrm{E}+04$ & & & & & & & & & & Hosseini et al. (2008) \\
\hline $\mathrm{Pa}$ & 91 & & & & & & & & & & & & & & & \\
\hline $\mathrm{Pb}$ & 82 & 79 & $4.50 \mathrm{E}-02$ & $7.00 \mathrm{E}+02$ & & & & & $2.00 \mathrm{E}+01$ & $2.20 \mathrm{E}+01$ & & & & TRS_364 & 3 & \\
\hline $\mathrm{Pb}$ & 82 & 79 & $4.50 \mathrm{E}-02$ & $7.00 \mathrm{E}+02$ & & & & & $2.00 \mathrm{E}+01$ & $2.20 \mathrm{E}+01$ & & & & TRS_472 & & \\
\hline $\mathrm{Pd}$ & 46 & & & & & & & & & & & & & & & \\
\hline $\mathrm{Pm}$ & 61 & & & & & & & & & & & & & & & \\
\hline Po & 84 & & & & & $9.90 \mathrm{E}+03$ & & $1.40 \mathrm{E}+03$ & & & & & & & & Hosseini et al. (2008) \\
\hline $\operatorname{Pr}$ & 59 & & & & & & & & & & & & & & & \\
\hline Pt & 78 & & & & & & & & & & & & & & & \\
\hline $\mathrm{Pu}$ & 94 & 100 & $3.60 \mathrm{E}-01$ & $5.50 \mathrm{E}+06$ & & & & & $2.90 \mathrm{E}+01$ & $7.40 \mathrm{E}+03$ & & & & TRS_364 & 13 & \\
\hline $\mathrm{Pu}$ & 94 & 100 & $3.60 \mathrm{E}-01$ & $5.50 \mathrm{E}+06$ & & & & & $2.90 \mathrm{E}+01$ & $7.40 \mathrm{E}+03$ & & & & TRS_472 & & \\
\hline $\mathrm{Pu}$ & 94 & & & & & $1.10 \mathrm{E}+03$ & & $4.30 \mathrm{E}+02$ & & & & & & & & Hosseini et al. (2008) \\
\hline Ra & 88 & 5 & $1.80 \mathrm{E}+00$ & $1.80 \mathrm{E}+03$ & & & & & $3.00 \mathrm{E}+01$ & $1.00 \mathrm{E}+02$ & & & & TRS_364 & 1 & \\
\hline $\mathrm{Ra}$ & 88 & 5 & $1.90 \mathrm{E}+00$ & $1.90 \mathrm{E}+03$ & & & & & $3.00 \mathrm{E}+01$ & $1.00 \mathrm{E}+02$ & & & & TRS_472 & & \\
\hline $\mathrm{Ra}$ & 88 & & & & & $1.50 \mathrm{E}+03$ & & $1.30 \mathrm{E}+03$ & & & & & & & & Hosseini et al. (2008) \\
\hline $\mathrm{Rb}$ & 37 & 2 & $1.80 \mathrm{E}+03$ & $2.20 \mathrm{E}+03$ & & $2.00 \mathrm{E}+03$ & & $2.80 \mathrm{E}+02$ & & & & & & TRS_364 & 2 & \\
\hline $\mathrm{Rb}$ & 37 & 2 & $1.90 \mathrm{E}+03$ & $2.20 \mathrm{E}+03$ & & $2.00 \mathrm{E}+03$ & & & & & & & & TRS_472 & & \\
\hline Re & 75 & & & & & & & & & & & & & & & \\
\hline $\mathrm{Rh}$ & 45 & & & & & & & & & & & & & & & \\
\hline $\mathrm{Rn}$ & 86 & & & & & & & & & & & & & & & \\
\hline $\mathrm{Ru}$ & 44 & 9 & $1.80 \mathrm{E}-03$ & $8.30 \mathrm{E}+01$ & & & & & $2.10 \mathrm{E}+01$ & $3.80 \mathrm{E}-02$ & & & & TRS_364 & 2 & \\
\hline $\mathrm{Ru}$ & 44 & 9 & $1.90 \mathrm{E}-03$ & $9.30 \mathrm{E}+01$ & & & & & $2.10 \mathrm{E}+01$ & $3.90 \mathrm{E}-02$ & & & & TRS_472 & & \\
\hline $\mathrm{S}$ & 16 & & & & & $2.00 \mathrm{E}+01$ & & & & & & & & & & Hosseini et al. (2008) \\
\hline $\mathrm{Sb}$ & 51 & 2 & $7.40 \mathrm{E}+01$ & $3.50 \mathrm{E}+02$ & & $2.10 \mathrm{E}+02$ & & $1.90 \mathrm{E}+02$ & & & & & & TRS_364 & 2 & \\
\hline $\mathrm{Sb}$ & 51 & 2 & $7.40 \mathrm{E}+01$ & $3.50 \mathrm{E}+02$ & & $2.10 \mathrm{E}+02$ & & & & & & & & TRS_472 & & \\
\hline Sc & 21 & 2 & $3.30 \mathrm{E}+03$ & $3.70 \mathrm{E}+03$ & & $3.50 \mathrm{E}+03$ & & $2.80 \mathrm{E}+02$ & & & & & & TRS_364 & 2 & \\
\hline Sc & 21 & 2 & $3.30 \mathrm{E}+03$ & $3.70 \mathrm{E}+03$ & & $3.50 \mathrm{E}+03$ & & & & & & & & TRS_472 & & \\
\hline Se & 34 & 16 & $1.20 \mathrm{E}+01$ & $6.90 \mathrm{E}+04$ & & & & & $1.50 \mathrm{E}+01$ & $5.70 \mathrm{E}+02$ & & & & TRS 364 & 5 & \\
\hline Se & 34 & 16 & $1.20 \mathrm{E}+01$ & $6.90 \mathrm{E}+04$ & & & & & $1.50 \mathrm{E}+01$ & $5.70 \mathrm{E}+02$ & & & & TRS_472 & & \\
\hline $\mathrm{Si}$ & 14 & & & & & & & & & & & & & & & \\
\hline Sm & 62 & 2 & $5.00 \mathrm{E}+02$ & $2.70 \mathrm{E}+03$ & & $1.60 \mathrm{E}+03$ & & $1.60 \mathrm{E}+03$ & & & & & & TRS_364 & 2 & \\
\hline $\mathrm{Sm}$ & 62 & 2 & $5.00 \mathrm{E}+02$ & $2.70 \mathrm{E}+03$ & & $1.60 \mathrm{E}+03$ & & & & & & & & TRS_472 & & \\
\hline Sn & 50 & & & & & & & & & & & & & & & \\
\hline $\mathrm{Sr}$ & 38 & 5 & $7.70 \mathrm{E}+01$ & $1.30 \mathrm{E}+03$ & & & & & $3.20 \mathrm{E}+00$ & $2.70 \mathrm{E}+03$ & & & & TRS_364 & 5 & \\
\hline $\mathrm{Sr}$ & 38 & 5 & $7.70 \mathrm{E}+01$ & $1.30 \mathrm{E}+03$ & & & & & $3.20 \mathrm{E}+00$ & $2.70 \mathrm{E}+02$ & & & & TRS_472 & & \\
\hline $\mathrm{Sr}$ & 38 & & & & & $2.00 \mathrm{E}+02$ & & $1.80 \mathrm{E}+02$ & & & & & & & & Hosseini et al. (2008) \\
\hline $\mathrm{Ta}$ & 73 & & & & & & & & & & & & & & & \\
\hline $\mathrm{Tb}$ & 65 & & & & & & & & & & & & & & & \\
\hline Tc & 43 & 10 & $1.80 \mathrm{E}+00$ & $4.00 \mathrm{E}+02$ & & & & & $9.80 \mathrm{E}+00$ & $2.60 \mathrm{E}+01$ & & & & TRS_364 & 1 & \\
\hline Tc & 43 & 10 & $1.90 \mathrm{E}+00$ & $4.00 \mathrm{E}+02$ & & & & & $9.90 \mathrm{E}+00$ & $2.60 \mathrm{E}+01$ & & & & TRS_472 & & \\
\hline $\mathrm{Te}$ & 52 & & & & & & & & & & & & & & & \\
\hline Th & 90 & 2 & $2.90 \mathrm{E}+03$ & $2.92 \mathrm{E}+03$ & & $2.90 \mathrm{E}+03$ & & $1.40 \mathrm{E}+01$ & & & & & & TRS_364 & 2 & \\
\hline Th & 90 & 2 & $2.90 \mathrm{E}+03$ & $2.90 \mathrm{E}+03$ & & $2.90 \mathrm{E}+03$ & & & & & & & & TRS_472 & & \\
\hline $\mathrm{Ti}$ & 22 & & & & & & & & & & & & & & & \\
\hline $\mathrm{Tl}$ & 81 & & & & & & & & & & & & & & & \\
\hline $\mathrm{Tm}$ & 69 & & & & & & & & & & & & & & & \\
\hline $\mathrm{U}$ & 92 & & & & $6.00 \mathrm{E}+01$ & & & & & & & & & Thompson et al. (1972) & 10058 & Ouchi et al. (1970) \\
\hline $\mathrm{U}$ & 92 & 8 & $3.60 \mathrm{E}+00$ & $6.00 \mathrm{E}+04$ & & & & & $1.90 \mathrm{E}+01$ & $1.70 \mathrm{E}+02$ & & & & TRS_364 & 1 & \\
\hline $\mathrm{U}$ & 92 & 9 & $3.60 \mathrm{E}+00$ & $6.00 \mathrm{E}+04$ & & & & & $1.90 \mathrm{E}+01$ & $1.70 \mathrm{E}+02$ & & & & TRS_472 & & \\
\hline $\mathrm{U}$ & 92 & & & & & $5.00 \mathrm{E}+02$ & & $7.00 \mathrm{E}+02$ & & & & & & & & Hosseini et al. (2008) \\
\hline $\mathrm{V}$ & 23 & 2 & $3.60 \mathrm{E}+02$ & $4.00 \mathrm{E}+02$ & & $3.80 \mathrm{E}+02$ & & $2.80 \mathrm{E}+01$ & & & & & & TRS_364 & 2 & \\
\hline
\end{tabular}


Table A.3. (contd)

\begin{tabular}{|c|c|c|c|c|c|c|c|c|c|c|c|c|c|c|c|c|}
\hline Element & Atomic\# & $\mathrm{N}$ & Min & Max & AVG & Mean & Wmean & STD & GSD & GM & WGM & MED & 95\%ile & Document & Reference\# & Reference \\
\hline $\mathrm{V}$ & 23 & 2 & $3.60 \mathrm{E}+02$ & $4.00 \mathrm{E}+02$ & & $3.90 \mathrm{E}+02$ & & & & & & & & TRS_472 & & \\
\hline $\mathrm{W}$ & 74 & & & & & & & & & & & & & & & \\
\hline Xe & 54 & & & & & & & & & & & & & & & \\
\hline $\mathrm{Y}$ & 39 & & & & & & & & & & & & & & & \\
\hline $\mathrm{Yb}$ & 70 & & & & & & & & & & & & & & & \\
\hline $\mathrm{Zn}$ & 30 & & & & $4.00 \mathrm{E}+03$ & & & & & & & & & Thompson et al. (1972) & 2723 & Davis et al. (1958) \\
\hline $\mathrm{Zn}$ & 30 & 82 & $6.30 \mathrm{E}-02$ & $1.50 \mathrm{E}+03$ & & & & & $2.90 \mathrm{E}+01$ & $9.20 \mathrm{E}+01$ & & & & TRS_364 & 4 & \\
\hline $\mathrm{Zn}$ & 30 & 92 & 6.30E-02 & $1.50 \mathrm{E}+03$ & & & & & $2.90 \mathrm{E}+01$ & $9.20 \mathrm{E}+01$ & & & & TRS_472 & & \\
\hline $\mathrm{Zr}$ & 40 & & & & & $2.60 \mathrm{E}+02$ & & & & & & & & & & Hosseini et al. (2008) \\
\hline
\end{tabular}


Table A.4. Bioaccumulation in Wet Plants from Freshwater, $\mathrm{L} / \mathrm{kg}_{\text {wet }}$ (CLBFP)

\begin{tabular}{|c|c|c|c|c|c|c|c|c|c|c|c|c|c|c|c|}
\hline Element & Atomic\# & $\mathrm{N}$ & Min & Max & AVG & Mean & Wmean & STD & GSD & GM & WGM & MED & Document & Reference\# & Reference \\
\hline Ac & 89 & & & & & & & & & & & & & & \\
\hline $\mathrm{Ag}$ & 47 & & & & & & & & & & & & & & \\
\hline $\mathrm{Al}$ & 13 & & & & & & & & & & & & & & \\
\hline $\mathrm{Am}$ & 95 & 16 & $7.50 \mathrm{E}+00$ & $3.80 \mathrm{E}+04$ & & & & & $8.30 \mathrm{E}+00$ & $3.70 \mathrm{E}+00$ & & & TRS_364 & 6 & \\
\hline $\mathrm{Am}$ & 95 & 16 & $7.50 \mathrm{E}+00$ & $3.90 \mathrm{E}+04$ & & & & & $9.30 \mathrm{E}+00$ & $3.70 \mathrm{E}+03$ & & & TRS_472 & & \\
\hline $\mathrm{Ar}$ & 18 & & & & & & & & & & & & & & \\
\hline As & 33 & & & & $3.50 \mathrm{E}+03$ & & & & & & & & Thompson et al. (1972) & 2723 & Davis et al. (1958) \\
\hline As & 33 & & & & & & & & & & & & & & \\
\hline At & 85 & & & & & & & & & & & & & & \\
\hline $\mathrm{Au}$ & 79 & & & & & & & & & & & & & & \\
\hline B & 5 & & & & & & & & & & & & & & \\
\hline $\mathrm{Ba}$ & 56 & & & & & & & & & & & & & & \\
\hline $\mathrm{Be}$ & 4 & & & & & & & & & & & & & & \\
\hline $\mathrm{Bi}$ & 83 & & & & & & & & & & & & & & \\
\hline $\mathrm{Bk}$ & 97 & . & & & & & & & & & & & & & \\
\hline $\mathrm{Br}$ & 35 & & & & & & & & & & & & & & \\
\hline $\mathrm{C}$ & 6 & 10 & $4.40 \mathrm{E}+01$ & $8.90 \mathrm{E}+04$ & & & & & $1.50 \mathrm{E}+01$ & $1.60 \mathrm{E}+04$ & & & TRS_364 & 1 & \\
\hline C & 6 & 10 & $4.40 \mathrm{E}+01$ & $9.90 \mathrm{E}+04$ & & & & & $1.50 \mathrm{E}+01$ & $1.60 \mathrm{E}+04$ & & & TRS_472 & & \\
\hline $\mathrm{Ca}$ & 20 & & & & & & & & & & & & & & \\
\hline $\mathrm{Cd}$ & 48 & 5 & $1.10 \mathrm{E}+04$ & $2.30 \mathrm{E}+04$ & & & & & $6.80 \mathrm{E}+00$ & $1.90 \mathrm{E}+04$ & & & TRS_364 & 2 & \\
\hline $\mathrm{Cd}$ & 48 & 5 & $1.10 \mathrm{E}+04$ & $2.30 \mathrm{E}+04$ & & & & & $6.90 \mathrm{E}+00$ & $1.90 \mathrm{E}+04$ & & & TRS_472 & & \\
\hline $\mathrm{Ce}$ & 58 & & $2.00 \mathrm{E}+03$ & $1.00 \mathrm{E}+04$ & & & & & & & & & Thompson et al. (1972) & 5335 & Bryan et al. (1966) \\
\hline $\mathrm{Cf}$ & 98 & & & & & & & & & & & & & & \\
\hline $\mathrm{Cl}$ & 17 & & & & & & & & & & & & & & \\
\hline $\mathrm{Cm}$ & 96 & 1 & & & & & & & & $8.00 \mathrm{E}+03$ & & & TRS_364 & 1 & \\
\hline $\mathrm{Cm}$ & 96 & 1 & & & & $9.00 \mathrm{E}+03$ & & & & & & & TRS_472 & & \\
\hline Co & 27 & 18 & $5.00 \mathrm{E}+01$ & $2.00 \mathrm{E}+04$ & & & & & $5.10 \mathrm{E}+00$ & $7.10 \mathrm{E}+02$ & & & TRS_364 & 11 & \\
\hline Co & 27 & 19 & $5.00 \mathrm{E}+01$ & $2.00 \mathrm{E}+04$ & & & & & $5.10 \mathrm{E}+00$ & $7.10 \mathrm{E}+02$ & & & TRS_472 & & \\
\hline $\mathrm{Cr}$ & 24 & & & & $4.00 \mathrm{E}+03$ & & & & & & & & Thompson et al. (1972) & 2723 & Davis et al. (1958) \\
\hline Cs & 55 & & $7.00 \mathrm{E}+01$ & $1.20 \mathrm{E}+03$ & & & & & & & & & Thompson et al. (1972) & 2722 & Pendleton et al. (1958) \\
\hline Cs & 55 & & $4.70 \mathrm{E}+02$ & $8.70 \mathrm{E}+02$ & & & & & & & & & Thompson et al. (1972) & 5811 & Ravera (1966) \\
\hline Cs & 55 & 26 & $1.80 \mathrm{E}+00$ & $3.30 \mathrm{E}+03$ & & & & & $1.60 \mathrm{E}+01$ & $8.70 \mathrm{E}+01$ & & & TRS_364 & 2 & \\
\hline Cs & 55 & 26 & $1.90 \mathrm{E}+00$ & $3.30 \mathrm{E}+03$ & & & & & $1.60 \mathrm{E}+01$ & $9.70 \mathrm{E}+01$ & & & TRS_472 & & \\
\hline $\mathrm{Cu}$ & 29 & & $5.00 \mathrm{E}+02$ & $2.00 \mathrm{E}+03$ & & & & & & & & & Thompson et al. (1972) & 1679 & Krumholz et al. (1957) \\
\hline $\mathrm{Cu}$ & 29 & & & & $6.00 \mathrm{E}+03$ & & & & & & & & Thompson et al. (1972) & 2723 & Davis et al. (1958) \\
\hline $\mathrm{Cu}$ & 29 & 5 & $2.40 \mathrm{E}+03$ & $3.60 \mathrm{E}+03$ & & & & & $3.19 \mathrm{E}+02$ & $3.00 \mathrm{E}+03$ & & & TRS_364 & 3 & \\
\hline $\mathrm{Cu}$ & 29 & 5 & $2.00 \mathrm{E}+03$ & $3.60 \mathrm{E}+03$ & & & & & $3.20 \mathrm{E}+02$ & $3.00 \mathrm{E}+03$ & & & TRS_472 & & \\
\hline Dy & 66 & & & & & & & & & & & & & & \\
\hline Er & 68 & & & & & & & & & & & & & & \\
\hline Es & 99 & & & & & & & & & & & & & & \\
\hline Eu & 63 & & & & & & & & & & & & & & \\
\hline $\mathrm{F}$ & 9 & & & & & & & & & & & & & & \\
\hline $\mathrm{Fe}$ & 26 & 5 & $5.20 \mathrm{E}+03$ & $1.50 \mathrm{E}+04$ & & & & & $1.80 \mathrm{E}+00$ & $9.10 \mathrm{E}+03$ & & & TRS_364 & 1 & \\
\hline $\mathrm{Fe}$ & 26 & 5 & $5.20 \mathrm{E}+03$ & $1.50 \mathrm{E}+04$ & & & & & $1.90 \mathrm{E}+00$ & $9.10 \mathrm{E}+03$ & & & TRS_472 & & \\
\hline $\mathrm{Fm}$ & 100 & & & & & & & & & & & & & & \\
\hline $\mathrm{Fr}$ & 87 & & & & & & & & & & & & & & \\
\hline $\mathrm{Ga}$ & 31 & & & & & & & & & & & & & & \\
\hline $\mathrm{Gd}$ & 64 & & & & & & & & & & & & & & \\
\hline Ge & 32 & & & & & & & & & & & & & & \\
\hline $\mathrm{H}$ & 1 & & & & & & & & & & & & & & \\
\hline
\end{tabular}


Table A.4. (contd)

\begin{tabular}{|c|c|c|c|c|c|c|c|c|c|c|c|c|c|c|c|}
\hline Element & Atomic\# & $\mathrm{N}$ & Min & Max & AVG & Mean & Wmean & STD & GSD & GM & WGM & MED & Document & Reference\# & Reference \\
\hline $\mathrm{He}$ & 2 & & & & & & & & & & & & & & \\
\hline $\mathrm{Hf}$ & 72 & & & & & & & & & & & & & & \\
\hline $\mathrm{Hg}$ & 80 & & & & & & & & & & & & & & \\
\hline Ho & 67 & & & & & & & & & & & & & & \\
\hline I & 53 & 3 & $7.80 \mathrm{E}+01$ & $2.70 \mathrm{E}+02$ & & & & & $3.70 \mathrm{E}+00$ & $1.30 \mathrm{E}+02$ & & & TRS_364 & 1 & \\
\hline I & 53 & 3 & $7.90 \mathrm{E}+01$ & $2.70 \mathrm{E}+02$ & & & & & $3.70 \mathrm{E}+00$ & $1.30 \mathrm{E}+02$ & & & TRS_472 & & \\
\hline In & 49 & & & & & & & & & & & & & & \\
\hline Ir & 77 & & & & & & & & & & & & & & \\
\hline $\mathrm{K}$ & 19 & & & & & & & & & & & & & & \\
\hline $\mathrm{Kr}$ & 36 & & & & & & & & & & & & & & \\
\hline La & 57 & . & & & & & & & & & & & & & \\
\hline $\mathrm{Li}$ & 3 & & & & & & & & & & & & & & \\
\hline $\mathrm{Lu}$ & 71 & & & & & & & & & & & & & & \\
\hline Md & 101 & & & & & & & & & & & & & & \\
\hline $\mathrm{Mg}$ & 12 & . & & & & & & & & & & & & & \\
\hline $\mathrm{Mn}$ & 25 & & & & $1.20 \mathrm{E}+04$ & & & & & & & & Thompson et al. (1972) & 5811 & Ravera (1966) \\
\hline $\mathrm{Mn}$ & 25 & 6 & $3.10 \mathrm{E}-01$ & $1.50 \mathrm{E}+05$ & & & & & $7.22 \mathrm{E}+02$ & $1.20 \mathrm{E}+04$ & & & TRS_364 & 4 & \\
\hline $\mathrm{Mn}$ & 25 & 6 & 3.10E-01 & $1.50 \mathrm{E}+05$ & & & & & $7.20 \mathrm{E}+02$ & $1.20 \mathrm{E}+04$ & & & TRS_472 & & \\
\hline Mo & 42 & & & & & & & & & & & & & & \\
\hline $\mathrm{N}$ & 7 & & & & & & & & & & & & & & \\
\hline $\mathrm{Na}$ & 11 & & & & $5.00 \mathrm{E}+02$ & & & & & & & & Thompson et al. (1972) & 1307 & Fontaine (1962) \\
\hline $\mathrm{Na}$ & 11 & 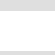 & & & $5.00 \mathrm{E}+02$ & & & & & & & & Thompson et al. (1972) & 1679 & Krumholz et al. (1957 \\
\hline $\mathrm{Na}$ & 11 & & & & $6.60 \mathrm{E}+02$ & & & & & & & & Thompson et al. (1972) & 2723 & Davis et al. (1958) \\
\hline $\mathrm{Nb}$ & 41 & & & & & & & & & & & & & & \\
\hline $\mathrm{Nd}$ & 60 & & & & & & & & & & & & & & \\
\hline $\mathrm{Ne}$ & 10 & & & & & & & & & & & & & & \\
\hline $\mathrm{Ni}$ & 28 & 5 & $2.50 \mathrm{E}+02$ & $1.10 \mathrm{E}+03$ & & & & & $1.29 \mathrm{E}+02$ & $7.70 \mathrm{E}+02$ & & & TRS_364 & 3 & \\
\hline $\mathrm{Ni}$ & 28 & 5 & $2.50 \mathrm{E}+02$ & $1.10 \mathrm{E}+03$ & & & & & $1.30 \mathrm{E}+02$ & $7.70 \mathrm{E}+02$ & & & TRS_472 & & \\
\hline $\mathrm{Np}$ & 93 & & & & $3.00 \mathrm{E}+02$ & & & & & & & & Thompson et al. (1972) & 2723 & Davis et al. (1958) \\
\hline $\mathrm{Np}$ & 93 & 2 & $6.50 \mathrm{E}+03$ & $8.00 \mathrm{E}+03$ & & $7.20 \mathrm{E}+03$ & & $1.10 \mathrm{E}+03$ & & & & & TRS_364 & 3 & \\
\hline $\mathrm{Np}$ & 93 & 2 & $6.50 \mathrm{E}+03$ & $9.00 \mathrm{E}+03$ & & $7.20 \mathrm{E}+03$ & & & & & & & TRS_472 & & \\
\hline $\mathrm{O}$ & 8 & & & & & & & & & & & & & & \\
\hline Os & 76 & & & & & & & & & & & & & & \\
\hline $\mathrm{P}$ & 15 & & $1.00 \mathrm{E}+05$ & $8.50 \mathrm{E}+05$ & & & & & & & & & Thompson et al. (1972) & 1679 & Krumholz et al. (1957 \\
\hline $\mathrm{P}$ & 15 & & & & $2.70 \mathrm{E}+05$ & & & & & & & & Thompson et al. (1972) & 2723 & Davis et al. (1958) \\
\hline Рa & 91 & & & & & & & & & & & & & & \\
\hline $\mathrm{Pb}$ & 82 & 5 & $1.30 \mathrm{E}+03$ & $2.20 \mathrm{E}+03$ & & & & & $7.60 \mathrm{E}+01$ & $1.90 \mathrm{E}+03$ & & & TRS_364 & 1 & \\
\hline $\mathrm{Pb}$ & 82 & 5 & $1.30 \mathrm{E}+03$ & $2.20 \mathrm{E}+03$ & & & & & $7.60 \mathrm{E}+01$ & $1.90 \mathrm{E}+03$ & & & TRS_472 & & \\
\hline Pd & 46 & & & & & & & & & & & & & & \\
\hline $\mathrm{Pm}$ & 61 & & & & & & & & & & & & & & \\
\hline Po & 84 & & & & & & & & & & & & & & \\
\hline $\operatorname{Pr}$ & 59 & & & & & & & & & & & & & & \\
\hline $\mathrm{Pt}$ & 78 & & & & & & & & & & & & & & \\
\hline Pu & 94 & 40 & $1.20 \mathrm{E}+02$ & $4.90 \mathrm{E}+07$ & & & & & $1.40 \mathrm{E}+01$ & $2.60 \mathrm{E}+04$ & & & TRS_364 & 4 & \\
\hline Pu & 94 & 40 & $1.20 \mathrm{E}+02$ & $4.90 \mathrm{E}+07$ & & & & & $1.40 \mathrm{E}+01$ & $2.60 \mathrm{E}+04$ & & & TRS_472 & & \\
\hline Ra & 88 & 8 & $6.40 \mathrm{E}+02$ & $1.10 \mathrm{E}+04$ & & & & & $4.10 \mathrm{E}+00$ & $2.80 \mathrm{E}+03$ & & & TRS_364 & 2 & \\
\hline Ra & 88 & & $1.10 \mathrm{E}+01$ & $4.40 \mathrm{E}+02$ & & & & & & & & & & & Jha et al. (2010) \\
\hline Ra & 88 & 9 & $6.40 \mathrm{E}+02$ & $1.10 \mathrm{E}+04$ & & & & & $4.10 \mathrm{E}+00$ & $2.90 \mathrm{E}+03$ & & & TRS_472 & & \\
\hline $\mathrm{Rb}$ & 37 & & & & & & & & & & & & & & \\
\hline Re & 75 & & & & & & & & & & & & & & \\
\hline Rh & 45 & & & & & & & & & & & & & & \\
\hline $\mathrm{Rn}$ & 86 & & & & & & & & & & & & & & \\
\hline
\end{tabular}


Table A.4. (contd)

\begin{tabular}{|c|c|c|c|c|c|c|c|c|c|c|c|c|c|c|c|}
\hline Element & Atomic\# & $\mathrm{N}$ & Min & Max & AVG & Mean & Wmean & STD & GSD & GM & WGM & MED & Document & Reference\# & Reference \\
\hline $\mathrm{Ru}$ & 44 & & $2.50 \mathrm{E}+02$ & $2.50 \mathrm{E}+03$ & & & & & & & & & Thompson et al. (1972) & 5335 & Bryan et al. (1966) \\
\hline Ru & 44 & & $1.80 \mathrm{E}+03$ & $1.20 \mathrm{E}+04$ & & & & & & & & & Thompson et al. (1972) & 5811 & Ravera (1966) \\
\hline $\mathrm{Ru}$ & 44 & 8 & $7.40 \mathrm{E}+01$ & $6.70 \mathrm{E}+02$ & & & & & $2.00 \mathrm{E}+00$ & $2.90 \mathrm{E}+02$ & & & TRS_364 & 1 & \\
\hline $\mathrm{Ru}$ & 44 & 9 & $7.40 \mathrm{E}+01$ & $6.70 \mathrm{E}+02$ & & & & & $2.00 \mathrm{E}+00$ & $2.90 \mathrm{E}+02$ & & & TRS_472 & & \\
\hline $\mathrm{s}$ & 16 & & & & & & & & & & & & & & \\
\hline $\mathrm{Sb}$ & 51 & & & & & & & & & & & & & & \\
\hline Sc & 21 & & & & & & & & & & & & & & \\
\hline Se & 34 & 31 & $9.00 \mathrm{E}+00$ & $8.20 \mathrm{E}+03$ & & & & & $5.40 \mathrm{E}+00$ & $1.40 \mathrm{E}+03$ & & & TRS_364 & 1 & \\
\hline Se & 34 & 31 & $9.40 \mathrm{E}+00$ & $9.20 \mathrm{E}+03$ & & & & & $5.40 \mathrm{E}+00$ & $1.40 \mathrm{E}+03$ & & & TRS_472 & & \\
\hline $\mathrm{Si}$ & 14 & & & & & & & & & & & & & & \\
\hline $\mathrm{Sm}$ & 62 & & & & & & & & & & & & & & \\
\hline Sn & 50 & & & & & & & & & & & & & & \\
\hline $\mathrm{Sr}$ & 38 & & & & $6.00 \mathrm{E}+02$ & & & & & & & & Thompson et al. (1972) & 1784 & Harvey (1964) \\
\hline $\mathrm{Sr}$ & 38 & & $3.30 \mathrm{E}+02$ & $6.90 \mathrm{E}+02$ & & & & & & & & & Thompson et al. (1972) & 3133 & Berg et al. (1961) \\
\hline $\mathrm{Sr}$ & 38 & & $2.40 \mathrm{E}+01$ & $2.80 \mathrm{E}+02$ & & & & & & & & & Thompson et al. (1972) & 10050 & Kalnina et al. (1969) \\
\hline $\mathrm{Sr}$ & 38 & 17 & $3.90 \mathrm{E}+01$ & $1.90 \mathrm{E}+03$ & & & & & $3.30 \mathrm{E}+00$ & $4.10 \mathrm{E}+02$ & & & TRS_364 & 16 & \\
\hline $\mathrm{Sr}$ & 38 & 17 & $3.90 \mathrm{E}+01$ & $1.90 \mathrm{E}+03$ & & & & & $3.30 \mathrm{E}+00$ & $4.10 \mathrm{E}+02$ & & & TRS_472 & & \\
\hline $\mathrm{Ta}$ & 73 & & & & & & & & & & & & & & \\
\hline $\mathrm{Tb}$ & 65 & & & & & & & & & & & & & & \\
\hline Tc & 43 & 8 & $2.80 \mathrm{E}-01$ & $8.80 \mathrm{E}+01$ & & & & & $4.90 \mathrm{E}+00$ & $5.50 \mathrm{E}+00$ & & & TRS_364 & 1 & \\
\hline Tc & 43 & 9 & $2.90 \mathrm{E}-01$ & $9.90 \mathrm{E}+01$ & & & & & $4.00 \mathrm{E}+00$ & $5.50 \mathrm{E}+00$ & & & TRS_472 & & \\
\hline $\mathrm{Te}$ & 52 & & & & & & & & & & & & & & \\
\hline Th & 90 & & & & & & & & & & & & & & \\
\hline $\mathrm{Ti}$ & 22 & & & & & & & & & & & & & & \\
\hline $\mathrm{Tl}$ & 81 & & & & & & & & & & & & & & \\
\hline $\mathrm{Tm}$ & 69 & & & & & & & & & & & & & & \\
\hline $\mathrm{U}$ & 92 & & $4.00 \mathrm{E}-01$ & $7.00 \mathrm{E}-01$ & $5.50 \mathrm{E}-01$ & & & & & & & & Thompson et al. (1972) & 10058 & Ouchi et al. (1970) \\
\hline $\mathrm{U}$ & 92 & 4 & $8.10 \mathrm{E}+01$ & $5.20 \mathrm{E}+02$ & & & & & $1.90 \mathrm{E}+00$ & $2.10 \mathrm{E}+02$ & & & TRS_364 & 1 & \\
\hline U & 92 & 4 & $9.10 \mathrm{E}+01$ & $5.20 \mathrm{E}+02$ & & & & & $1.90 \mathrm{E}+00$ & $2.10 \mathrm{E}+02$ & & & TRS_472 & & \\
\hline $\mathrm{V}$ & 23 & & & & & & & & & & & & & & \\
\hline $\mathrm{w}$ & 74 & & & & & & & & & & & & & & \\
\hline Хе & 54 & & & & & & & & & & & & & & \\
\hline $\mathrm{Y}$ & 39 & & & & & & & & & & & & & & \\
\hline $\mathrm{Yb}$ & 70 & & & & & & & & & & & & & & \\
\hline $\mathrm{Zn}$ & 30 & & & & $6.00 \mathrm{E}+03$ & & & & & & & & Thompson et al. (1972) & 1784 & Harvey (1964) \\
\hline $\mathrm{Zn}$ & 30 & & & & $1.30 \mathrm{E}+05$ & & & & & & & & Thompson et al. (1972) & 2723 & Davis et al. (1958) \\
\hline $\mathrm{Zn}$ & 30 & 5 & $1.40 \mathrm{E}+04$ & $2.70 \mathrm{E}+04$ & & & & & $1.30 \mathrm{E}+01$ & $2.10 \mathrm{E}+04$ & & & TRS_364 & 1 & \\
\hline Zn & 30 & 5 & $1.40 \mathrm{E}+04$ & $2.70 \mathrm{E}+04$ & & & & & $1.30 \mathrm{E}+01$ & $2.10 \mathrm{E}+04$ & & & TRS_472 & & \\
\hline $\mathrm{Zr}$ & 40 & & & & & & & & & & & & & & \\
\hline
\end{tabular}



Table A.5. Bioaccumulation in Wet Fish from Saltwater, $\mathrm{L} / \mathrm{kg}_{\text {wet }}$ (CLBMF)

\begin{tabular}{|c|c|c|c|c|c|c|c|c|c|c|c|c|c|c|c|c|}
\hline Element & Atomic\# & $\mathrm{N}$ & Min & Max & AVG & Mean & Wmean & STD & GSD & GM & WGM & MED & 95\%ile & Document & Reference\# & Reference \\
\hline $\mathrm{Ac}$ & 89 & & & & & & & & & & & $5.0 \mathrm{E}+01$ & & TRS 422 & & \\
\hline $\mathrm{Ag}$ & 47 & & & & & $4.20 \mathrm{E}+03$ & & $4.10 \mathrm{E}+03$ & & & & & & & & Hosseini et al. (2008) \\
\hline $\mathrm{Ag}$ & 47 & & & & & & & & & & & $1.0 \mathrm{E}+04$ & & TRS 422 & & \\
\hline $\mathrm{Al}$ & 13 & & & & & & & & & & & & & & & \\
\hline $\mathrm{Am}$ & 95 & & $7.00 \mathrm{E}+00$ & $2.30 \mathrm{E}+04$ & & & & & & & & $1.6 \mathrm{E}+02$ & & & & \\
\hline Am & 95 & & & & & $5.80 \mathrm{E}+01$ & & $7.10 \mathrm{E}+01$ & & & & & & & & Hosseini et al. (2008) \\
\hline $\mathrm{Am}$ & 95 & & & & & & & & & & & $1.0 \mathrm{E}+02$ & & TRS 422 & & \\
\hline $\mathrm{Ar}$ & 18 & & & & & & & & & & & & & & & \\
\hline As & 33 & & & & & & & & & & & & & & & \\
\hline At & 85 & & & & & & & & & & & & & & & \\
\hline $\mathrm{Au}$ & 79 & & & & & & & & & & & & & & & \\
\hline $\mathrm{Ba}$ & 56 & & & & & & & & & & & & & & & \\
\hline $\mathrm{Ba}$ & 56 & & & & & & & & & & & $1.0 \mathrm{E}+01$ & & TRS 422 & & \\
\hline $\mathrm{Be}$ & 4 & & & & & & & & & & & & & & & \\
\hline $\mathrm{Bi}$ & 83 & & & & & & & & & & & & & & & \\
\hline $\mathrm{Bk}$ & 97 & & & & & & & & & & & & & & & \\
\hline $\mathrm{Bk}$ & 97 & & & & & & & & & & & $1.0 \mathrm{E}+02$ & & TRS 422 & & \\
\hline $\mathrm{Br}$ & 35 & & & & & & & & & & & $1.0 \mathrm{E}+01$ & & Coughtrey et al. (1985) & 54.2 & \\
\hline $\mathrm{Br}$ & 35 & & & & & & & & & & & & & & & \\
\hline $\mathrm{C}$ & 6 & & & & & & & & & & & $2.0 \mathrm{E}+04$ & & TRS 422 & & \\
\hline $\mathrm{Ca}$ & 20 & & & & & $1.20 \mathrm{E}+04$ & & & & & & & & & & Hosseini et al. (2008) \\
\hline $\mathrm{Ca}$ & 20 & & & & & & & & & & & $2.0 \mathrm{E}+00$ & & TRS 422 & & \\
\hline $\mathrm{Cd}$ & 48 & & 1.00E-01 & $1.00 \mathrm{E}+02$ & & & & & & & & $2.00 \mathrm{E}+03$ & & Coughtrey et al. (1985) & S4.2.4, T4.13 & \\
\hline $\mathrm{Cd}$ & 48 & & & & & $9.60 \mathrm{E}+03$ & & $2.00 \mathrm{E}+04$ & & & & & & & & Hosseini et al. (2008) \\
\hline $\mathrm{Cd}$ & 48 & & & & & & & & & & & $5.0 \mathrm{E}+03$ & & TRS 422 & & \\
\hline $\mathrm{Ce}$ & 58 & & & & & & & & & & & $5.00 \mathrm{E}+01$ & & Coughtrey et al. (1985) & S4.2.3, T4.5 & \\
\hline $\mathrm{Ce}$ & 58 & & & & $1.00 \mathrm{E}+00$ & & & & & & & & & Thompson et al. (1972) & 1682 & Mauchline et al. (1964) \\
\hline $\mathrm{Ce}$ & 58 & & & & & $1.20 \mathrm{E}+02$ & & $2.40 \mathrm{E}+02$ & & & & & & & & Hosseini et al. (2008) \\
\hline $\mathrm{Ce}$ & 58 & & & & & & & & & & & $5.0 \mathrm{E}+01$ & & TRS 422 & & \\
\hline $\mathrm{Cf}$ & 98 & & & & & & & & & & & $1.0 \mathrm{E}+02$ & & TRS 422 & & \\
\hline $\mathrm{Cl}$ & 17 & & & & & $5.60 \mathrm{E}+02$ & & & & & & & & & & Hosseini et al. (2008) \\
\hline $\mathrm{Cl}$ & 17 & & & & & & & & & & & $6.0 \mathrm{E}-03$ & & TRS 422 & & \\
\hline $\mathrm{Cm}$ & 96 & & & & & & & & & & & $1.0 \mathrm{E}+02$ & & TRS 422 & & \\
\hline Co & 27 & & & & & $5.30 \mathrm{E}+03$ & & $1.50 \mathrm{E}+04$ & & & & & & & & Hosseini et al. (2008) \\
\hline Co & 27 & & & & & & & & & & & $7.0 \mathrm{E}+02$ & & TRS 422 & & \\
\hline $\mathrm{Cr}$ & 24 & & & & & & & & & & & $2.0 \mathrm{E}+02$ & & TRS 422 & & \\
\hline Cs & & & 5.00E-01 & $1.00 \mathrm{E}+04$ & & & & & & & & $7.00 \mathrm{E}+01$ & & Coughtrey et al. (1985) & S4.2.4 & \\
\hline Cs & 55 & & $3.00 \mathrm{E}+01$ & $4.50 \mathrm{E}+02$ & & & & & & & & & & Thompson et al. (1972) & 1799 & Agnedal (1965) \\
\hline Cs & 55 & & & & $1.00 \mathrm{E}+02$ & & & & & & & & & Thompson et al. (1972) & 8845 & Folsom et al. (1965) \\
\hline Cs & 55 & & $2.00 \mathrm{E}+00$ & $1.30 \mathrm{E}+02$ & & & & & & & & & & Thompson et al. (1972) & 9532 & Kamath et al. (1971) \\
\hline Cs & 55 & & & & $4.00 \mathrm{E}+00$ & & & & & & & & & Thompson et al. (1972) & 9533 & Feldt (1971) \\
\hline Cs & & & & & & $8.70 \mathrm{E}+01$ & & $1.20 \mathrm{E}+02$ & & & & & & & & Hosseini et al. (2008) \\
\hline Cs & 55 & & & & & & & & & & & $1.0 \mathrm{E}+02$ & & TRS 422 & & \\
\hline $\mathrm{Cu}$ & 29 & & & & & & & & & & & & & & & \\
\hline Dy & 66 & & & & & & & & & & & $3.0 \mathrm{E}+02$ & & TRS 422 & & \\
\hline Er & 68 & & & & & & & & & & & & & & & \\
\hline Es & 99 & & & & & & & & & & & & & & & \\
\hline $\mathrm{Eu}$ & 63 & & & & & $4.40 \mathrm{E}+02$ & & $3.00 \mathrm{E}+02$ & & & & & & & & Hosseini et al. (2008) \\
\hline $\mathrm{Eu}$ & 63 & & & & & & & & & & & $3.0 \mathrm{E}+02$ & & TRS 422 & & \\
\hline $\mathrm{F}$ & 9 & & & & & & & & & & & & & & & \\
\hline $\mathrm{Fe}$ & 26 & & & & & & & & & & & $3.0 \mathrm{E}+04$ & & TRS 422 & & \\
\hline
\end{tabular}


Table A.5. (contd)

\begin{tabular}{|c|c|c|c|c|c|c|c|c|c|c|c|c|c|c|c|c|}
\hline Element & Atomic\# & $\mathrm{N}$ & Min & Max & AVG & Mean & Wmean & STD & GSD & GM & WGM & MED & 95\%ile & Document & Reference\# & Reference \\
\hline $\mathrm{Fm}$ & 100 & & & & & & & & & & & & & & & \\
\hline $\mathrm{Fr}$ & 87 & & & & & & & & & & & & & & & \\
\hline Ga & 31 & & & & & & & & & & & & & & & \\
\hline Gd & 64 & & & & & & & & & & & $3.0 \mathrm{E}+02$ & & TRS 422 & & \\
\hline $\mathrm{Ge}$ & 32 & & & & & & & & & & & & & & & \\
\hline $\mathrm{H}$ & 1 & & & & & & & & & & & 1.0 & & TRS 422 & & \\
\hline $\mathrm{Hf}$ & 72 & & & & & & & & & & & $5.0 \mathrm{E}+02$ & & TRS 422 & & \\
\hline $\mathrm{Hg}$ & 80 & & & & & & & & & & & $3.0 \mathrm{E}+04$ & & TRS 422 & & \\
\hline Но & 67 & & & & & & & & & & & & & & & \\
\hline I & 53 & & & & & $3.50 \mathrm{E}+00$ & & & & & & & & & & Hosseini et al. (2008) \\
\hline I & 53 & & & & & & & & & & & $9.0 \mathrm{E}+00$ & & TRS 422 & & \\
\hline In & 49 & & & & & & & & & & & $5.0 \mathrm{E}+02$ & & TRS 422 & & \\
\hline Ir & 77 & & & & & & & & & & & $2.0 \mathrm{E}+01$ & & TRS 422 & & \\
\hline $\mathrm{K}$ & 19 & & & & & & & & & & & & & & & \\
\hline $\mathrm{Kr}$ & 36 & & & & & & & & & & & 1.0 & & TRS 422 & & \\
\hline $\mathrm{La}$ & 57 & & & & & & & & & & & & & & & \\
\hline $\mathrm{Lu}$ & 71 & & & & & & & & & & & & & & & \\
\hline $\mathrm{Md}$ & 101 & & & & & & & & & & & & & & & \\
\hline $\mathrm{Mg}$ & 12 & & & & & & & & & & & & & & & \\
\hline $\mathrm{Mn}$ & 25 & & & & $5.50 \mathrm{E}+02$ & & & & & & & & & Thompson et al. (1972) & 1405 & Folsom et al. (1963) \\
\hline Mn & 25 & & & & & $2.40 \mathrm{E}+03$ & & $1.50 \mathrm{E}+04$ & & & & & & 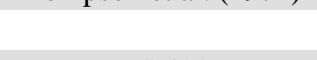 & & Hosseini et al. (2008) \\
\hline $\mathrm{Mn}$ & 25 & & & & & & & & & & & $1.0 \mathrm{E}+03$ & & TRS 422 & & \\
\hline Mo & 42 & & & & & & & & & & & & & & & \\
\hline $\mathrm{N}$ & 7 & & & & & & & & & & & & & & & \\
\hline $\mathrm{Na}$ & 11 & & & & & & & & & & & $1.0 \mathrm{E}+00$ & & TRS 422 & & \\
\hline $\mathrm{Nb}$ & 41 & & & & & & & & & & & $3.0 \mathrm{E}+02$ & & TRS 422 & & \\
\hline $\mathrm{Nd}$ & 60 & & & & & & & & & & & & & & & \\
\hline $\mathrm{Ne}$ & 10 & & & & & & & & & & & & & & & \\
\hline $\mathrm{Ni}$ & 28 & & & & & $1.70 \mathrm{E}+02$ & & $2.20 \mathrm{E}+02$ & & & & & & & & Hosseini et al. (2008) \\
\hline $\mathrm{Ni}$ & 28 & & & & & & & & & & & $1.0 \mathrm{E}+03$ & & TRS 422 & & \\
\hline $\mathrm{Np}$ & 93 & & & & & & & & & & & $1.0 \mathrm{E}+00$ & & TRS 422 & & \\
\hline \multirow{2}{*}{\multicolumn{17}{|c|}{ OBT }} \\
\hline & & & & & & & & & & & & & & & & \\
\hline Os & 76 & & & & & & & & & & & & & & & \\
\hline $\mathrm{P}$ & 15 & & & & & $9.90 \mathrm{E}+04$ & & $3.00 \mathrm{E}+04$ & & & & & & & & Hosseini et al. (2008) \\
\hline $\mathrm{Pa}$ & 91 & & & & & & & & & & & $5.0 \mathrm{E}+01$ & & TRS 422 & & \\
\hline $\mathrm{Pb}$ & 82 & & & & & $4.40 \mathrm{E}+03$ & & $1.40 \mathrm{E}+04$ & & & & & & & & Hosseini et al. (2008) \\
\hline $\mathrm{Pb}$ & 82 & & & & & & & & & & & $2.0 \mathrm{E}+02$ & & TRS 422 & & \\
\hline$P d$ & 46 & & & & & & & & & & & $3.0 \mathrm{E}+02$ & & TRS 422 & & \\
\hline Pm & 61 & & & & & & & & & & & $3.0 \mathrm{E}+02$ & & TRS 422 & & \\
\hline Po & 84 & & $3.00 \mathrm{E}+02$ & $4.50 \mathrm{E}+03$ & & & & & & & & & & Thompson et al. (1972) & 5832 & Folsom (1966) \\
\hline Po & 84 & & & & & $4.40 \mathrm{E}+04$ & & $1.20 \mathrm{E}+05$ & & & & & & & & Hosseini et al. (2008) \\
\hline Po & 84 & & & & & & & & & & & $2.0 \mathrm{E}+03$ & & TRS 422 & & \\
\hline Pr & 59 & & & & & & & & & & & & & & & \\
\hline $\mathrm{Pt}$ & 78 & & & & & & & & & & & & & & & \\
\hline $\mathrm{Pu}$ & 94 & & & & $3.00 \mathrm{E}+00$ & & & & & & & & & Thompson et al. (1972) & 1299 & Pillai et al. (1964) \\
\hline $\mathrm{Pu}$ & 94 & & & & $5.00 \mathrm{E}+00$ & & & & & & & & & Thompson et al. (1972) & 8956 & Wong et al. (1970) \\
\hline $\mathrm{Pu}$ & 94 & & $1.00 \mathrm{E}+00$ & $5.00 \mathrm{E}+00$ & & & & & & & & & & Thompson et al. (1972) & 9995 & Wong et al. (1971) \\
\hline $\mathrm{Pu}$ & 94 & & & & $1.30 \mathrm{E}+01$ & & & & & & & & & Thompson et al. (1972) & 10010 & Noshkin (1971) \\
\hline $\mathrm{Pu}$ & 94 & & & & & $1.60 \mathrm{E}+03$ & & $6.40 \mathrm{E}+03$ & & & & & & & & Hosseini et al. (2008) \\
\hline $\mathrm{Pu}$ & 94 & & & & & & & & & & & & & & & \\
\hline $\mathrm{Pu}$ & 94 & & & & & & & & & & & $1.0 \mathrm{E}+02$ & & TRS 422 & & \\
\hline
\end{tabular}


Table A.5. (contd)

\begin{tabular}{|c|c|c|c|c|c|c|c|c|c|c|c|c|c|c|c|c|}
\hline Element & Atomic\# & $\mathrm{N}$ & Min & Max & AVG & Mean & Wmean & STD & GSD & GM & WGM & MED & 95\%ile & Document & Reference\# & Reference \\
\hline $\mathrm{Ra}$ & 88 & & & & & $2.00 \mathrm{E}+02$ & & $3.80 \mathrm{E}+02$ & & & & & & & & Hosseini et al. (2008) \\
\hline $\mathrm{Ra}$ & 88 & & & & & & & & & & & $1.0 \mathrm{E}+02$ & & TRS 422 & & \\
\hline $\mathrm{Rb}$ & 37 & & & & & & & & & & & & & & & \\
\hline $\mathrm{Re}$ & 75 & & & & & & & & & & & & & & & \\
\hline $\mathrm{Rh}$ & 45 & & & & & & & & & & & & & & & \\
\hline $\mathrm{Rn}$ & 86 & & & & & & & & & & & & & & & \\
\hline $\mathrm{Ru}$ & 44 & & & & $1.00 \mathrm{E}+00$ & & & & & & & & & Thompson et al. (1972) & 1682 & Mauchline et al. (1964) \\
\hline Ru & 44 & & $1.00 \mathrm{E}+00$ & $1.00 \mathrm{E}+01$ & & & & & & & & & & Thompson et al. (1972) & 8915 & Preston et al. (1965) \\
\hline $\mathrm{Ru}$ & 44 & & & & & $3.00 \mathrm{E}+01$ & & $4.30 \mathrm{E}+01$ & & & & & & & & Hosseini et al. (2008) \\
\hline $\mathrm{Ru}$ & 44 & & & & & & & & & & & $2.0 \mathrm{E}+00$ & & TRS 422 & & \\
\hline $\mathrm{s}$ & 16 & & & & & $9.40 \mathrm{E}+01$ & & & & & & & & & & Hosseini et al. (2008) \\
\hline $\mathrm{s}$ & 16 & & & & & & & & & & & $1.0 \mathrm{E}+00$ & & TRS 422 & & \\
\hline $\mathrm{Sb}$ & 51 & & $1.50 \mathrm{E}-01$ & $1.00 \mathrm{E}+03$ & & & & & & & & $1-100$ & & Coughtrey et al. (1985) & S4.2.4,T4.11 & \\
\hline $\mathrm{sb}$ & 51 & & & & & $2.20 \mathrm{E}+02$ & & $7.60 \mathrm{E}+02$ & & & & & & & & Hosseini et al. (2008) \\
\hline $\mathrm{Sb}$ & 51 & & & & & & & & & & & $6.0 \mathrm{E}+02$ & & TRS 422 & & \\
\hline Sc & 21 & & & & & & & & & & & $1.0 \mathrm{E}+03$ & & TRS 422 & & \\
\hline Se & 34 & & & & & $9.30 \mathrm{E}+03$ & & $4.60 \mathrm{E}+03$ & & & & & & & & Hosseini et al. (2008) \\
\hline Se & 34 & & & & & & & & & & & $1.0 \mathrm{E}+04$ & & TRS 422 & & \\
\hline $\mathrm{Si}$ & 14 & & & & & & & & & & & & & & & \\
\hline $\mathrm{Sm}$ & 62 & & & & & & & & & & & $3.0 \mathrm{E}+02$ & & TRS 422 & & \\
\hline Sn & 50 & & & & & & & & & & & $5.0 \mathrm{E}+05$ & & TRS 422 & & \\
\hline $\mathrm{Sr}$ & 38 & & & & $6.00 \mathrm{E}-01$ & & & & & & & & & Thompson et al. (1972) & 1682 & Mauchline et al. (1964) \\
\hline $\mathrm{Sr}$ & 38 & & & & $3.00 \mathrm{E}-01$ & & & & & & & & & Thompson et al. (1972) & 5335 & Bryan et al. (1966) \\
\hline $\mathrm{Sr}$ & 38 & & $3.00 \mathrm{E}+00$ & $4.00 \mathrm{E}+00$ & & & & & & & & & & Thompson et al. (1972) & 9533 & Feldt (1971) \\
\hline $\mathrm{Sr}$ & 38 & & & & $3.00 \mathrm{E}+00$ & & & & & & & & & Thompson et al. (1972) & 10059 & Agnedal et al. (1958) \\
\hline $\mathrm{Sr}$ & 38 & & & & & $2.30 \mathrm{E}+01$ & & $3.50 \mathrm{E}+01$ & & & & & & & & Hosseini et al. (2008) \\
\hline Sr & 38 & & & & & & & & & & & $3.0 \mathrm{E}+00$ & & TRS 422 & & \\
\hline Та & 73 & & & & & & & & & & & $6.0 \mathrm{E}+01$ & & TRS 422 & & \\
\hline $\mathrm{Tb}$ & 65 & & & & & & & & & & & $6.0 \mathrm{E}+01$ & & TRS 422 & & \\
\hline Tc & 43 & & & & & 3..1e1 & & $5.60 \mathrm{E}+01$ & & & & & & & & Hosseini et al. (2008) \\
\hline Tc & 43 & & & & & & & & & & & $8.0 \mathrm{E}+01$ & & TRS 422 & & \\
\hline Te & 52 & & & & & & & & & & & $1.0 \mathrm{E}+03$ & & TRS 422 & & \\
\hline Th & 90 & & & & & $1.30 \mathrm{E}+03$ & & & & & & & & & & Hosseini et al. (2008) \\
\hline Th & 90 & & & & & & & & & & & $6.0 \mathrm{E}+02$ & & TRS 422 & & \\
\hline Ti & 22 & & & & & & & & & & & & & & & \\
\hline $\mathrm{Tl}$ & 81 & & & & & & & & & & & $5.0 \mathrm{E}+03$ & & TRS 422 & & \\
\hline $\mathrm{Tm}$ & 69 & & & & & & & & & & & $3.0 \mathrm{E}+02$ & & TRS 422 & & \\
\hline $\mathrm{U}$ & 92 & & & & & $1.40 \mathrm{E}+01$ & & $2.30 \mathrm{E}+01$ & & & & & & & & Hosseini et al. (2008) \\
\hline $\mathrm{U}$ & 92 & & & & & & & & & & & $1.0 \mathrm{E}+00$ & & TRS 422 & & \\
\hline $\mathrm{V}$ & 23 & & & & & & & & & & & & & & & \\
\hline $\mathrm{W}$ & 74 & & & & & & & & & & & $9.0 \mathrm{E}+01$ & & TRS 422 & & \\
\hline Хе & 54 & & & & & & & & & & & $1.0 \mathrm{E}+00$ & & TRS 422 & & \\
\hline $\mathrm{Y}$ & 39 & & & & & & & & & & & $2.0 \mathrm{E}+01$ & & TRS 422 & & \\
\hline $\mathrm{Yb}$ & 70 & & & & & & & & & & & $2.0 \mathrm{E}+02$ & & TRS 422 & & \\
\hline $\mathrm{Zn}$ & 30 & & & & & & & & & & & $1.0 \mathrm{E}+03$ & & TRS 422 & & \\
\hline
\end{tabular}



Table A.6. Bioaccumulation in Wet Mollusk from Saltwater, L/kg wet $_{\text {(CLBMM) }}$

\begin{tabular}{|c|c|c|c|c|c|c|c|c|c|c|c|c|c|c|c|c|}
\hline Element & Atomic\# & $\mathrm{N}$ & Min & Max & AVG & Mean & Wmean & STD & GSD & GM & WGM & $\begin{array}{l}\text { MED } \\
\end{array}$ & 95\%ile & Document & Reference\# & Reference \\
\hline $\mathrm{Ac}$ & 89 & & & & & & & & & & & $1.00 \mathrm{E}+3$ & & TRS 422 & & \\
\hline $\mathrm{Ag}$ & 47 & & & & & & & & & & & $6.00 \mathrm{E}+04$ & & TRS 422 & & \\
\hline $\mathrm{Al}$ & 13 & & & & & & & & & & & & & & & \\
\hline Am & 95 & & $2.00 \mathrm{E}+00$ & $4.00 \mathrm{E}+04$ & & & & & & & & $2.90 \mathrm{E}+02$ & & Coughtrey et al. (1985) & & \\
\hline $\mathrm{Am}$ & 95 & & & & & $3.00 \mathrm{E}+01$ & & $8.00 \mathrm{E}+00$ & & & & & & & & Metian et al. (2011) \\
\hline Am & 95 & & & & & & & & & & & $1.00 \mathrm{E}+03$ & & TRS 422 & & \\
\hline $\mathrm{Ar}$ & 18 & & & & & & & & & & & & & & & \\
\hline As & 33 & & & & & & & & & & & & & & & \\
\hline At & 85 & & & & & & & & & & & & & & & \\
\hline $\mathrm{Au}$ & 79 & & & & & & & & & & & & & & & \\
\hline $\mathrm{Ba}$ & 56 & & & & & & & & & & & $1.00 \mathrm{E}+01$ & & TRS 422 & & \\
\hline $\mathrm{Be}$ & 4 & & & & & & & & & & & & & & & \\
\hline $\mathrm{Bi}$ & 83 & & & & & & & & & & & & & & & \\
\hline $\mathrm{Bk}$ & 97 & & & & & & & & & & & $1.00 \mathrm{E}+03$ & & TRS 422 & & \\
\hline $\mathrm{Br}$ & 35 & & & & & & & & & & & $1.00 \mathrm{E}+01$ & & Coughtrey et al. (1985) & S4.2 & \\
\hline C & 6 & & & & & & & & & & & $2.00 \mathrm{E}+04$ & & TRS 422 & & \\
\hline $\mathrm{Ca}$ & 20 & & & & & & & & & & & $3.00 \mathrm{E}+00$ & & TRS 422 & & \\
\hline $\mathrm{Cd}$ & 48 & & $2.00 \mathrm{E}+00$ & $1.00 \mathrm{E}+04$ & & & & & & & & $1.00 \mathrm{E}+04$ & & Coughtrey et al. (1985) & S4.2.3, T4.10 & \\
\hline $\mathrm{Cd}$ & 48 & & & & & & & & & & & $8.00 \mathrm{E}+04$ & & TRS 422 & & \\
\hline $\mathrm{Ce}$ & 58 & & & & & & & & & & & $5.00 \mathrm{E}+02$ & & Coughtrey et al. (1985) & S4.2.2, T4.5 & \\
\hline $\mathrm{Ce}$ & 58 & & & & $2.00 \mathrm{E}+03$ & & & & & & & & & Thompson et al. (1972) & 506 & Mauchline (1963) \\
\hline $\mathrm{Ce}$ & 58 & & $2.00 \mathrm{E}+02$ & $2.00 \mathrm{E}+03$ & & & & & & & & & & Thompson et al. (1972) & 5335 & Bryan et al. (1966) \\
\hline $\mathrm{Ce}$ & 58 & & & & & & & & & & & $2.00 \mathrm{E}+03$ & & TRS 422 & & \\
\hline $\mathrm{Cf}$ & 98 & & & & & & & & & & & $1.00 \mathrm{E}+03$ & & TRS 422 & & \\
\hline $\mathrm{Cl}$ & 17 & & & & & & & & & & & $5.00 \mathrm{E}-02$ & & TRS 422 & & \\
\hline $\mathrm{Cm}$ & 96 & & & & & & & & & & & $1.00 \mathrm{E}+03$ & & TRS 422 & & \\
\hline Co & 27 & & & & & & & & & & & $2.00 \mathrm{E}+04$ & & TRS 422 & & \\
\hline $\mathrm{Cr}$ & 24 & & & & & & & & & & & $2.00 \mathrm{E}+03$ & & TRS 422 & & \\
\hline Cs & 55 & & $1.00 \mathrm{E}-01$ & $1.00 \mathrm{E}+03$ & & & & & & & & $2.00 \mathrm{E}+01$ & & Coughtrey et al. (1985) & S4.2.3 & \\
\hline Cs & 55 & & $6.00 \mathrm{E}+01$ & $1.50 \mathrm{E}+02$ & & & & & & & & & & Thompson et al. (1972) & 1799 & Agnedal (1965) \\
\hline Cs & 55 & & $9.00 \mathrm{E}+00$ & $7.20 \mathrm{E}+01$ & $3.70 \mathrm{E}+01$ & & & & & & & & & Thompson et al. (1972) & 5335 & Bryan et al. (1966) \\
\hline Cs & 55 & & $2.00 \mathrm{E}+01$ & $6.00 \mathrm{E}+01$ & & & & & & & & & & Thompson et al. (1972) & 9532 & Kamath et al. (1971) \\
\hline Cs & 55 & & & & $1.20 \mathrm{E}+01$ & & & & & & & & & Thompson et al. (1972) & 9533 & Feldt (1971) \\
\hline Cs & 55 & & & & & $3.00 \mathrm{E}+00$ & & $1.00 \mathrm{E}+00$ & & & & & & & & Metian et al. (2011) \\
\hline Cs & 55 & & & & & & & & & & & $6.00 \mathrm{E}+01$ & & TRS 422 & & \\
\hline $\mathrm{Cu}$ & 29 & & & & & & & & & & & & & & & \\
\hline Dy & 66 & & & & & & & & & & & $7.00 \mathrm{E}+03$ & & TRS 422 & & \\
\hline $\mathrm{Er}$ & 68 & & & & & & & & & & & & & & & \\
\hline Es & 99 & & & & & & & & & & & & & & & \\
\hline $\mathrm{Eu}$ & 63 & & & & & & & & & & & $7.00 \mathrm{E}+03$ & & TRS 422 & & \\
\hline $\mathrm{F}$ & 9 & & & & & & & & & & & & & & & \\
\hline $\mathrm{Fe}$ & 26 & & & & & & & & & & & $5.00+\mathrm{E} 05$ & & TRS 422 & & \\
\hline $\mathrm{Fm}$ & 100 & & & & & & & & & & & & & & & \\
\hline $\mathrm{Fr}$ & 87 & & & & & & & & & & & & & & & \\
\hline $\mathrm{Ga}$ & 31 & & & & & & & & & & & & & & & \\
\hline $\mathrm{Gd}$ & 64 & & & & & & & & & & & $7.00 \mathrm{E}+03$ & & TRS 422 & & \\
\hline Ge & 32 & & & & & & & & & & & & & & & \\
\hline $\mathrm{H}$ & 1 & & & & & & & & & & & $1.00 \mathrm{E}+00$ & & TRS 422 & & \\
\hline
\end{tabular}


Table A.6. (contd)

\begin{tabular}{|c|c|c|c|c|c|c|c|c|c|c|c|c|c|c|c|c|}
\hline Element & Atomic\# & $\mathrm{N}$ & Min & Max & AVG & Mean & Wmean & STD & GSD & GM & WGM & MED & 95\%ile & Document & Reference\# & Reference \\
\hline $\mathrm{Hf}$ & 72 & & & & & & & & & & & $7.00 \mathrm{E}+03$ & & TRS 422 & & \\
\hline $\mathrm{Hg}$ & 80 & & & & & & & & & & & $2.00 \mathrm{E}+03$ & & TRS 422 & & \\
\hline Ho & 67 & & & & & & & & & & & & & & & \\
\hline I & 53 & & & & & & & & & & & $1.00 \mathrm{E}+01$ & & TRS 422 & & \\
\hline In & 49 & & & & & & & & & & & $1.00 \mathrm{E}+04$ & & TRS 422 & & \\
\hline Ir & 77 & & & & & & & & & & & $1.00 \mathrm{E}+02$ & & TRS 422 & & \\
\hline $\mathrm{K}$ & 19 & & & & & & & & & & & & & & & \\
\hline $\mathrm{Kr}$ & 36 & & & & & & & & & & & $1.00 \mathrm{E}+00$ & & TRS 422 & & \\
\hline $\mathrm{La}$ & 57 & & & & & & & & & & & & & & & \\
\hline $\mathrm{Lu}$ & 71 & & & & & & & & & & & & & & & \\
\hline Md & 101 & & & & & & & & & & & & & & & \\
\hline $\mathrm{Mg}$ & 12 & & & & & & & & & & & & & & & \\
\hline $\mathrm{Mn}$ & 25 & & $8.00 \mathrm{E}+02$ & $8.30 \mathrm{E}+02$ & & & & & & & & & & Thompson et al. (1972) & 1405 & Folsom et al. (1963) \\
\hline $\mathrm{Mn}$ & 25 & & & & $8.30 \mathrm{E}+02$ & & & & & & & & & Thompson et al. (1972) & 5583 & Polikarpov et al. (1957) \\
\hline $\mathrm{Mn}$ & 25 & & & & & & & & & & & $5.00 \mathrm{E}+04$ & & TRS 422 & & \\
\hline Mo & 42 & & & & & & & & & & & & & & & \\
\hline $\mathrm{N}$ & 7 & & & & & & & & & & & & & & & \\
\hline $\mathrm{Na}$ & 11 & & & & & & & & & & & 3.00E-01 & & TRS 422 & & \\
\hline $\mathrm{Nb}$ & 41 & & & & & & & & & & & $1.00 \mathrm{E}+03$ & & TRS 422 & & \\
\hline $\mathrm{Nd}$ & 60 & & & & & & & & & & & & & & & \\
\hline $\mathrm{Ne}$ & 10 & & & & & & & & & & & & & & & \\
\hline $\mathrm{Ni}$ & 28 & & & & & & & & & & & $2.00 \mathrm{E}+03$ & & TRS 422 & & \\
\hline $\mathrm{Np}$ & 93 & & & & & & & & & & & $4.00 \mathrm{E}+02$ & & TRS 422 & & \\
\hline $\mathrm{O}$ & 8 & & & & & & & & & & & & & & & \\
\hline \multicolumn{17}{|l|}{$\mathrm{OBT}$} \\
\hline Os & 76 & & & & & & & & & & & & & & & \\
\hline $\mathrm{P}$ & 15 & & $2.40 \mathrm{E}+03$ & $4.10 \mathrm{E}+04$ & $3.00 \mathrm{E}+04$ & & & & & & & & & Thompson et al. (1972) & 8761 & Seymour (1969) \\
\hline $\mathrm{P}$ & 15 & & & & & & & & & & & & & & & \\
\hline $\mathrm{Pa}$ & 91 & & & & & & & & & & & $5.00 \mathrm{E}+02$ & & TRS 422 & & \\
\hline $\mathrm{Pb}$ & 82 & & & & & & & & & & & $5.00 \mathrm{E}+04$ & & TRS 422 & & \\
\hline $\mathrm{Pd}$ & 46 & & & & & & & & & & & $3.00 \mathrm{E}+02$ & & TRS 422 & & \\
\hline $\mathrm{Pm}$ & 61 & & & & & & & & & & & $7.00 \mathrm{E}+03$ & & TRS 422 & & \\
\hline Po & 84 & & & & $5.00 \mathrm{E}+03$ & & & & & & & & & Thompson et al. (1972) & 5832 & Folsom (1966) \\
\hline Po & 84 & & & & & & & & & & & $2.00 \mathrm{E}+04$ & & TRS 422 & & \\
\hline $\operatorname{Pr}$ & 59 & & & & & & & & & & & & & & & \\
\hline $\mathrm{Pt}$ & 78 & & & & & & & & & & & & & & & \\
\hline $\mathrm{Pu}$ & 94 & & $2.30 \mathrm{E}+02$ & $2.90 \mathrm{E}+02$ & $2.60 \mathrm{E}+02$ & & & & & & & & & Thompson et al. (1972) & 1299 & Pillai et al. (1964) \\
\hline $\mathrm{Pu}$ & 94 & & & & $2.90 \mathrm{E}+02$ & & & & & & & & & Thompson et al. (1972) & 8956 & Wong et al. (1970) \\
\hline $\mathrm{Pu}$ & 94 & & $1.60 \mathrm{E}+02$ & $3.80 \mathrm{E}+02$ & & & & & & & & & & Thompson et al. (1972) & 9995 & Wong et al. (1971) \\
\hline $\mathrm{Pu}$ & 94 & & $1.00 \mathrm{E}+02$ & $3.50 \mathrm{E}+02$ & & & & & & & & & & Thompson et al. (1972) & 10010 & Noshkin (1971) \\
\hline $\mathrm{Pu}$ & 94 & & & & & & & & & & & $3.00 \mathrm{E}+03$ & & TRS 422 & & \\
\hline $\mathrm{Ra}$ & 88 & & & & & & & & & & & $1.00 \mathrm{E}+02$ & & TRS 422 & & \\
\hline $\mathrm{Rb}$ & 37 & & & & & & & & & & & & & & & \\
\hline $\mathrm{Re}$ & 75 & & & & & & & & & & & & & & & \\
\hline $\mathrm{Rh}$ & 45 & & & & & & & & & & & & & & & \\
\hline $\mathrm{Rn}$ & 86 & & & & & & & & & & & & & & & \\
\hline $\mathrm{Ru}$ & 44 & & & & $2.90 \mathrm{E}+02$ & & & & & & & & & Thompson et al. (1972) & 6048 & Iwashima et al. (1966) \\
\hline $\mathrm{Ru}$ & 44 & & & & $3.20 \mathrm{E}+03$ & & & & & & & & & Thompson et al. (1972) & 6048 & Iwashima et al. (1966) \\
\hline $\mathrm{Ru}$ & 44 & & $1.20 \mathrm{E}+03$ & $2.00 \mathrm{E}+03$ & & & & & & & & & & Thompson et al. (1972) & 8915 & Preston et al. (1965) \\
\hline $\mathrm{Ru}$ & 44 & & & & & & & & & & & $5.00 \mathrm{E}+02$ & & TRS 422 & & \\
\hline
\end{tabular}


Table A.6. (contd)

\begin{tabular}{|c|c|c|c|c|c|c|c|c|c|c|c|c|c|c|c|c|}
\hline Element & Atomic\# & $\mathrm{N}$ & Min & Max & AVG & Mean & Wmean & STD & GSD & GM & WGM & MED & 95\%ile & Document & Reference\# & Reference \\
\hline $\mathrm{s}$ & 16 & & & & & & & & & & & $3.00 \mathrm{E}+00$ & & TRS 422 & & \\
\hline $\mathrm{Sb}$ & 51 & & $1.00 \mathrm{E}+00$ & $1.00 \mathrm{E}+01$ & & & & & & & & $5-100$ & & Coughtrey et al. (1985) & S4.2.3,T4.9 & \\
\hline $\mathrm{Sb}$ & 51 & & & & & & & & & & & $3.00 \mathrm{E}+02$ & & TRS 422 & & \\
\hline Sc & 21 & & & & & & & & & & & $1.00 \mathrm{E}+05$ & & TRS 422 & & \\
\hline Se & 34 & & & & & & & & & & & $9.00 \mathrm{E}+03$ & & TRS 422 & & \\
\hline $\mathrm{Si}$ & 14 & & & & & & & & & & & & & & & \\
\hline $\mathrm{Sm}$ & 62 & & & & & & & & & & & $7.00 \mathrm{E}+03$ & & TRS 422 & & \\
\hline $\mathrm{Sn}$ & 50 & & & & & & & & & & & $5.00 \mathrm{E}+05$ & & TRS 422 & & \\
\hline $\mathrm{Sr}$ & 38 & & $5.00 \mathrm{E}+00$ & $1.70 \mathrm{E}+01$ & & & & & & & & & & Thompson et al. (1972) & 5335 & Bryan et al. (1966) \\
\hline $\mathrm{Sr}$ & 38 & & & & $3.00 \mathrm{E}+00$ & & & & & & & & & Thompson et al. (1972) & 9533 & Feldt (1971) \\
\hline $\mathrm{Sr}$ & 38 & & & & & & & & & & & & & & & \\
\hline $\mathrm{Sr}$ & 38 & & & & & & & & & & & & & & & \\
\hline $\mathrm{Sr}$ & 38 & & & & & & & & & & & $1.00 \mathrm{E}+01$ & & TRS 422 & & \\
\hline $\mathrm{Ta}$ & 73 & & & & & & & & & & & $7.00 \mathrm{E}+03$ & & TRS 422 & & \\
\hline $\mathrm{Tb}$ & 65 & & & & & & & & & & & $3.00 \mathrm{E}+03$ & & TRS 422 & & \\
\hline Tc & 43 & & & & & & & & & & & $5.00 \mathrm{E}+02$ & & TRS 422 & & \\
\hline $\mathrm{Te}$ & 52 & & & & & & & & & & & $1.00 \mathrm{E}+03$ & & TRS 422 & & \\
\hline Th & 90 & & & & & & & & & & & $1.00 \mathrm{E}+03$ & & TRS 422 & & \\
\hline $\mathrm{Ti}$ & 22 & & & & & & & & & & & & & & & \\
\hline $\mathrm{Tl}$ & 81 & & & & & & & & & & & $6.0 \mathrm{E}+03$ & & TRS 422 & & \\
\hline $\mathrm{Tm}$ & 69 & & & & & & & & & & & $7.00 \mathrm{E}+03$ & & TRS 422 & & \\
\hline $\mathrm{U}$ & 92 & & & & & & & & & & & $3.00 \mathrm{E}+01$ & & TRS 422 & & \\
\hline $\mathrm{V}$ & 23 & & & & & & & & & & & & & & & \\
\hline $\mathrm{W}$ & 74 & & & & & & & & & & & $6.00 \mathrm{E}+02$ & & TRS 422 & & \\
\hline Xe & 54 & & & & & & & & & & & $1.00 \mathrm{E}+00$ & & TRS 422 & & \\
\hline Y & 39 & & & & & & & & & & & $1.00 \mathrm{E}+03$ & & TRS 422 & & \\
\hline $\mathrm{Yb}$ & 70 & & & & & & & & & & & $3.00 \mathrm{E}+03$ & & TRS 422 & & \\
\hline $\mathrm{Zn}$ & 30 & & $8.40 \mathrm{E}+03$ & $1.80 \mathrm{E}+04$ & & & & & & & & & & Thompson et al. (1972) & 2971 & Seymour et al. (1964) \\
\hline $\mathrm{Zn}$ & 30 & & & & $1.00 \mathrm{E}+05$ & & & & & & & & & Thompson et al. (1972) & 9903 & Preston (1968) \\
\hline $\mathrm{Zn}$ & 30 & & & & & & & & & & & $8.00 \mathrm{E}+04$ & & TRS 422 & & \\
\hline $\mathrm{Zr}$ & 40 & & $2.00 \mathrm{E}+01$ & $2.70 \mathrm{E}+02$ & & & & & & & & & & Thompson et al. (1972) & 140 & Cigna et al. (1963) \\
\hline $\mathrm{Zr}$ & 40 & & & & & & & & & & & $5.00 \mathrm{E}+03$ & & TRS 422 & & \\
\hline
\end{tabular}



Table A.7. Bioaccumulation in Wet Crustacea from Saltwater, $\mathrm{L} / \mathrm{kg}_{\text {wet }}$ (CLBMI)

\begin{tabular}{|c|c|c|c|c|c|c|c|c|c|c|c|c|c|c|c|c|}
\hline Element & Atomic\# & $\mathrm{N}$ & Min & Max & AVG & Mean & Wmean & STD & GSD & GM & WGM & MED & 95\%ile & Document & Reference\# & Reference \\
\hline Ac & 89 & & & & & & & & & & & $1.00 \mathrm{E}+03$ & & TRS 422 & & \\
\hline $\mathrm{Ag}$ & 47 & & & & & $1.60 \mathrm{E}+04$ & & & & & & & & & & Hosseini et al. (2008) \\
\hline $\mathrm{Ag}$ & 47 & & & & & & & & & & & $2.00 \mathrm{E}+05$ & & TRS 422 & & \\
\hline $\mathrm{Al}$ & 13 & & & & & & & & & & & & & & & \\
\hline Am & 95 & & $8.40 \mathrm{E}+01$ & $1.30 \mathrm{E}+03$ & & & & & & & & $3.60 \mathrm{E}+02$ & & Coughtrey et al. (1985) & & \\
\hline $\mathrm{Am}$ & 95 & & & & & $1.30 \mathrm{E}+03$ & & $1.40 \mathrm{E}+03$ & & & & & & & & Hosseini et al. (2008) \\
\hline $\mathrm{Am}$ & 95 & & & & & & & & & & & $4.00 \mathrm{E}+02$ & & TRS 422 & & \\
\hline $\mathrm{Ar}$ & 18 & & & & & & & & & & & & & & & \\
\hline As & 33 & & & & & & & & & & & & & & & \\
\hline At & 85 & & & & & & & & & & & & & & & \\
\hline $\mathrm{Au}$ & 79 & & & & & & & & & & & & & & & \\
\hline Ва & 56 & & & & & & & & & & & 7.00E-01 & & TRS 422 & & \\
\hline $\mathrm{Bi}$ & 83 & & & & & & & & & & & & & & & \\
\hline $\mathrm{Bk}$ & 97 & & & & & & & & & & & $4.00 \mathrm{E}+02$ & & TRS 422 & & \\
\hline $\mathrm{Br}$ & 35 & & & & & & & & & & & $1.00 \mathrm{E}+01$ & & Coughtrey et al. (1985) & S4.2 & \\
\hline $\mathrm{Br}$ & 35 & & & & & & & & & & & & & & & \\
\hline C & 6 & & & & & $1.00 \mathrm{E}+04$ & & & & & & & & & & Hosseini et al. (2008) \\
\hline C & 6 & & & & & & & & & & & $2.00 \mathrm{E}+04$ & & TRS 422 & & \\
\hline Ca & 20 & & & & & & & & & & & $5.00 \mathrm{E}+00$ & & TRS 422 & & \\
\hline Cd & 48 & & $3.80 \mathrm{E}+01$ & $3.00 \mathrm{E}+03$ & & & & & & & & $5.00 \mathrm{E}+03$ & & Coughtrey et al. (1985) & S4.2.2, T4.8 & \\
\hline $\mathrm{Cd}$ & 48 & & & & & $2.60 \mathrm{E}+04$ & & $4.90 \mathrm{E}+04$ & & & & & & & & Hosseini et al. (2008) \\
\hline $\mathrm{Cd}$ & 48 & & & & & & & & & & & $8.00 \mathrm{E}+04$ & & TRS 422 & & \\
\hline $\mathrm{Ce}$ & 58 & & & & & & & & & & & $5.00 \mathrm{E}+02$ & & Coughtrey et al. (1985) & S4.2.2, T4.5 & \\
\hline $\mathrm{Ce}$ & 58 & & & & $1.00 \mathrm{E}+02$ & & & & & & & & & Thompson et al. (1972) & 5335 & Bryan et al. (1966) \\
\hline $\mathrm{Ce}$ & 58 & & & & & $3.40 \mathrm{E}+03$ & & $5.70 \mathrm{E}+03$ & & & & & & & & Hosseini et al. (2008) \\
\hline $\mathrm{Cf}$ & 98 & & & & & & & & & & & $4.00 \mathrm{E}+02$ & & TRS 422 & & \\
\hline $\mathrm{Cl}$ & 17 & & & & & $5.60 \mathrm{E}-02$ & & & & & & & & & & Hosseini et al. (2008) \\
\hline $\mathrm{Cl}$ & 17 & & & & & & & & & & & 6.00E-02 & & TRS 422 & & \\
\hline $\mathrm{Cm}$ & 96 & & & & & & & & & & & $4.00 \mathrm{E}+02$ & & TRS 422 & & \\
\hline Co & 27 & & & & & $1.80 \mathrm{E}+03$ & & $2.90 \mathrm{E}+03$ & & & & & & & & Hosseini et al. (2008) \\
\hline Co & 27 & & & & & & & & & & & $7.00 \mathrm{E}+03$ & & TRS 422 & & \\
\hline $\mathrm{Cr}$ & 24 & & & & & & & & & & & $1.00 \mathrm{E}+02$ & & TRS 422 & & \\
\hline Cs & 55 & & $3.00 \mathrm{E}-01$ & $1.10 \mathrm{E}+04$ & & & & & & & & $2.00 \mathrm{E}+01$ & & Coughtrey et al. (1985) & S4.2.2 & \\
\hline Cs & 55 & & $2.90 \mathrm{E}+01$ & $3.00 \mathrm{E}+01$ & & & & & & & & & & Thompson et al. (1972) & 5335 & Bryan et al. (1966) \\
\hline Cs & 55 & & & & $3.50 \mathrm{E}+01$ & & & & & & & & & Thompson et al. (1972) & 9532 & Kamath et al. (1971) \\
\hline Cs & 55 & & & & $2.30 \mathrm{E}+01$ & & & & & & & & & Thompson et al. (1972) & 9533 & Feldt (1971) \\
\hline Cs & 55 & & & & $4.00 \mathrm{E}+01$ & & & & & & & & & Thompson et al. (1972) & 10061 & Templeton (1964) \\
\hline Cs & 55 & & & & & $4.10 \mathrm{E}+01$ & & $8.30 \mathrm{E}+01$ & & & & & & & & Hosseini et al. (2008) \\
\hline Cs & 55 & & & & & & & & & & & $5.00 \mathrm{E}+01$ & & TRS 422 & & \\
\hline $\mathrm{Cu}$ & 29 & & & & & & & & & & & & & & & \\
\hline Dy & 66 & & & & & & & & & & & $4.00 \mathrm{E}+03$ & & TRS 422 & & \\
\hline Er & 68 & & & & & & & & & & & & & & & \\
\hline Es & 99 & & & & & & & & & & & & & & & \\
\hline Eu & 63 & & & & & & & & & & & $4.00 \mathrm{E}+03$ & & TRS 422 & & \\
\hline $\mathrm{F}$ & 9 & & & & & & & & & & & & & & & \\
\hline $\mathrm{Fe}$ & 26 & & & & & & & & & & & $5.0 \mathrm{E}+05$ & & TRS 422 & & \\
\hline $\mathrm{Fm}$ & 100 & & & & & & & & & & & & & & & \\
\hline
\end{tabular}


Table A.7. (contd)

\begin{tabular}{|c|c|c|c|c|c|c|c|c|c|c|c|c|c|c|c|c|}
\hline Element & Atomic\# & $\mathrm{N}$ & Min & Max & AVG & Mean & Wmean & STD & GSD & GM & WGM & MED & 95\%ile & Document & Reference\# & Reference \\
\hline $\mathrm{Ga}$ & 31 & & & & & & & & & & & & & & & \\
\hline Gd & 64 & & & & & & & & & & & $4.00 \mathrm{E}+03$ & & TRS 422 & & \\
\hline $\mathrm{Ge}$ & 32 & & & & & & & & & & & & & & & \\
\hline $\mathrm{H}$ & 1 & & & & & & & & & & & $1.00 \mathrm{E}+00$ & & TRS 422 & & \\
\hline $\mathrm{Hf}$ & 72 & & & & & & & & & & & $4.00 \mathrm{E}+03$ & & TRS 422 & & \\
\hline $\mathrm{Hg}$ & 80 & & & & & & & & & & & $1.00 \mathrm{E}+04$ & & TRS 422 & & \\
\hline Ho & 67 & & & & & & & & & & & & & & & \\
\hline I & 53 & & & & & $3.50 \mathrm{E}+00$ & & & & & & & & & & Hosseini et al. (2008) \\
\hline I & 53 & & & & & & & & & & & $3.00 \mathrm{E}+00$ & & TRS 422 & & \\
\hline In & 49 & & & & & & & & & & & $1.00 \mathrm{E}+04$ & & TRS 422 & & \\
\hline Ir & 77 & & & & & & & & & & & $1.00 \mathrm{E}+02$ & & TRS 422 & & \\
\hline $\mathrm{K}$ & 19 & & & & & & & & & & & & & & & \\
\hline $\mathrm{La}$ & 57 & & & & & & & & & & & & & & & \\
\hline $\mathrm{Lu}$ & 71 & & & & & & & & & & & & & & & \\
\hline Md & 101 & & & & & & & & & & & & & & & \\
\hline $\mathrm{Mg}$ & 12 & & & & & & & & & & & & & & & \\
\hline $\mathrm{Mn}$ & 25 & & & & $1.50 \mathrm{E}-01$ & & & & & & & & & Thompson et al. (1972) & 10023 & Bryan et al. (1966) \\
\hline $\mathrm{Mn}$ & 25 & & & & & $2.30 \mathrm{E}+04$ & & $7.50 \mathrm{E}+04$ & & & & & & & & Hosseini et al. (2008) \\
\hline Mn & 25 & & & & & & & & & & & $5.00 \mathrm{E}+03$ & & TRS 422 & & \\
\hline Mo & 42 & & & & & & & & & & & & & & & \\
\hline $\mathrm{N}$ & 7 & & & & & & & & & & & & & & & \\
\hline $\mathrm{Na}$ & 11 & & & & & & & & & & & 7.00E-02 & & TRS 422 & & \\
\hline $\mathrm{Nb}$ & 41 & & & & & $1.00 \mathrm{E}+02$ & & $1.20 \mathrm{E}+02$ & & & & & & & & Hosseini et al. (2008) \\
\hline $\mathrm{Nb}$ & 41 & & & & & & & & & & & $2.00 \mathrm{E}+02$ & & TRS 422 & & \\
\hline $\mathrm{Ni}$ & 28 & & & & & $5.50 \mathrm{E}+02$ & & $6.40 \mathrm{E}+02$ & & & & & & & & Hosseini et al. (2008) \\
\hline $\mathrm{Ni}$ & 28 & & & & & & & & & & & $1.00 \mathrm{E}+03$ & & TRS 422 & & \\
\hline $\mathrm{Np}$ & 93 & & & & & & & & & & & $1.00 \mathrm{E}+02$ & & TRS 422 & & \\
\hline $\mathrm{O}$ & 8 & & & & & & & & & & & & & & & \\
\hline \multicolumn{17}{|l|}{ OBT } \\
\hline Os & 76 & & & & & & & & & & & & & & & \\
\hline $\mathrm{P}$ & 15 & & & & & $2.70 \mathrm{E}+04$ & & & & & & & & & & Hosseini et al. (2008) \\
\hline $\mathrm{Pa}$ & 91 & & & & & & & & & & & $1.00 \mathrm{E}+01$ & & TRS 422 & & \\
\hline $\mathrm{Pb}$ & 82 & & & & & $7.50 \mathrm{E}+03$ & & $2.10 \mathrm{E}+04$ & & & & & & & & Hosseini et al. (2008) \\
\hline $\mathrm{Pb}$ & 82 & & & & & & & & & & & $9.00 \mathrm{E}+04$ & & TRS 422 & & \\
\hline $\mathrm{Pd}$ & 46 & & & & & & & & & & & $3.00 \mathrm{E}+02$ & & TRS 422 & & \\
\hline $\mathrm{Pm}$ & 61 & & & & & & & & & & & $4.00 \mathrm{E}+03$ & & TRS 422 & & \\
\hline Po & 84 & & & & & $5.60 \mathrm{E}+04$ & & $6.60 \mathrm{E}+04$ & & & & & & & & Hosseini et al. (2008) \\
\hline Po & 84 & & & & & & & & & & & $2.00 \mathrm{E}+04$ & & TRS 422 & & \\
\hline Pr & 59 & & & & & & & & & & & & & & & \\
\hline $\mathrm{Pt}$ & 78 & & & & & & & & & & & & & & & \\
\hline Pu & 94 & & & & & $1.60 \mathrm{E}+02$ & & $1.40 \mathrm{E}+02$ & & & & & & & & Hosseini et al. (2008) \\
\hline $\mathrm{Pu}$ & 94 & & & & & & & & & & & $2.00 \mathrm{E}+02$ & & TRS 422 & & \\
\hline Ra & 88 & & & & & $1.10 \mathrm{E}+02$ & & $8.10 \mathrm{E}+01$ & & & & & & & & Hosseini et al. (2008) \\
\hline $\mathrm{Ra}$ & 88 & & & & & & & & & & & $1.00 \mathrm{E}+02$ & & TRS 422 & & \\
\hline $\mathrm{Rb}$ & 37 & & & & & & & & & & & & & & & \\
\hline $\mathrm{Re}$ & 75 & & & & & & & & & & & & & & & \\
\hline $\mathrm{Rh}$ & 45 & & & & & & & & & & & & & & & \\
\hline $\mathrm{Rn}$ & 86 & & & & & & & & & & & & & & & \\
\hline $\mathrm{Ru}$ & 44 & & & & $1.00 \mathrm{E}+01$ & & & & & & & & & Thompson et al. (1972) & 5335 & Bryan et al. (1966) \\
\hline
\end{tabular}


Table A.7. (contd)

\begin{tabular}{|c|c|c|c|c|c|c|c|c|c|c|c|c|c|c|c|c|}
\hline Element & Atomic\# & $\mathrm{N}$ & Min & Max & AVG & Mean & Wmean & STD & GSD & GM & WGM & MED & 95\%ile & Document & Reference\# & Reference \\
\hline $\mathrm{Ru}$ & 44 & & $2.50 \mathrm{E}+01$ & $6.00 \mathrm{E}+02$ & & & & & & & & & & Thompson et al. (1972) & 8915 & Preston et al. (1965) \\
\hline $\mathrm{Ru}$ & 44 & & & & & $3.20 \mathrm{E}+02$ & & $4.40 \mathrm{E}+02$ & & & & & & & & Hosseini et al. (2008) \\
\hline $\mathrm{Ru}$ & 44 & & & & & & & & & & & $1.00 \mathrm{E}+02$ & & TRS 422 & & \\
\hline $\mathrm{S}$ & 16 & & & & & $1.20 \mathrm{E}+00$ & & & & & & & & & & Hosseini et al. (2008) \\
\hline $\mathrm{s}$ & 16 & & & & & & & & & & & $1.00 \mathrm{E}+00$ & & TRS 422 & & \\
\hline $\mathrm{Sb}$ & 51 & & $1.00 \mathrm{E}+00$ & $3.00 \mathrm{E}+02$ & & & & & & & & “10-200” & & Coughtrey et al. (1985) & S4.2.2,T4.7 & \\
\hline $\mathrm{Sb}$ & 51 & & & & & $1.40 \mathrm{E}+03$ & & $2.60 \mathrm{E}+03$ & & & & & & & & Hosseini et al. (2008) \\
\hline Sb & 51 & & & & & & & & & & & $3.00 \mathrm{E}+02$ & & TRS 422 & & \\
\hline Sc & 21 & & & & & & & & & & & $3.00 \mathrm{E}+02$ & & TRS 422 & & \\
\hline Se & 34 & & & & & $7.10 \mathrm{E}+03$ & & $4.80 \mathrm{E}+03$ & & & & & & & & Hosseini et al. (2008) \\
\hline Se & 34 & & & & & & & & & & & $1.00 \mathrm{E}+04$ & & TRS 422 & & \\
\hline $\mathrm{Si}$ & 14 & & & & & & & & & & & & & & & \\
\hline Sn & 50 & & & & & & & & & & & $5.00 \mathrm{E}+05$ & & TRS 422 & & \\
\hline $\mathrm{Sr}$ & 38 & & & & $3.00 \mathrm{E}+01$ & & & & & & & & & Thompson et al. (1972) & 9533 & Feldt (1971) \\
\hline $\mathrm{Sr}$ & 38 & & & & & $1.20 \mathrm{E}+01$ & & $1.20 \mathrm{E}+01$ & & & & & & & & Hosseini et al. (2008) \\
\hline $\mathrm{Sr}$ & 38 & & & & & & & & & & & $5.00 \mathrm{E}+00$ & & TRS 422 & & \\
\hline Тa & 73 & & & & & & & & & & & $2.00 \mathrm{E}+03$ & & TRS 422 & & \\
\hline $\mathrm{Tb}$ & 65 & & & & & & & & & & & $4.00 \mathrm{E}+03$ & & TRS 422 & & \\
\hline Tc & 43 & & & & & $1.70 \mathrm{E}+04$ & & $2.20 \mathrm{E}+04$ & & & & & & & & Hosseini et al. (2008) \\
\hline Tc & 43 & & & & & & & & & & & $1.00 \mathrm{E}+03$ & & TRS 422 & & \\
\hline $\mathrm{Te}$ & 52 & & & & & & & & & & & $1.00 \mathrm{E}+03$ & & TRS 422 & & \\
\hline Th & 90 & & & & & & & & & & & $1.00 \mathrm{E}+03$ & & TRS 422 & & \\
\hline $\mathrm{Ti}$ & 22 & & & & & & & & & & & & & & & \\
\hline $\mathrm{Tl}$ & 81 & & & & & & & & & & & $1.00 \mathrm{E}+03$ & & TRS 422 & & \\
\hline $\mathrm{Tm}$ & 69 & & & & & & & & & & & $4.00 \mathrm{E}+03$ & & TRS 422 & & \\
\hline $\mathrm{V}$ & 23 & & & & & & & & & & & & & & & \\
\hline $\mathrm{w}$ & 74 & & & & & & & & & & & $1.00 \mathrm{E}+01$ & & TRS 422 & & \\
\hline $\mathrm{Xe}$ & 54 & & & & & & & & & & & $1.00 \mathrm{E}+00$ & & TRS 422 & & \\
\hline $\mathrm{Y}$ & 39 & & & & & & & & & & & $1.00 \mathrm{E}+03$ & & TRS 422 & & \\
\hline $\mathrm{Yb}$ & 70 & & & & & & & & & & & $4.00 \mathrm{E}+03$ & & TRS 422 & & \\
\hline $\mathrm{Zn}$ & 30 & & & & & & & & & & & $3.00 \mathrm{E}+05$ & & TRS 422 & & \\
\hline $\mathrm{Zr}$ & 40 & & & & $1.00 \mathrm{E}+02$ & & & & & & & & & Thompson et al. (1972) & 5335 & Bryan et al. (1966) \\
\hline $\mathrm{Zr}$ & 40 & & & & & $2.20 \mathrm{E}+02$ & & $4.00 \mathrm{E}+02$ & & & & & & & & Hosseini et al. (2008) \\
\hline $\mathrm{Zr}$ & 40 & & & & & & & & & & & $2.00 \mathrm{E}+02$ & & TRS 422 & & \\
\hline
\end{tabular}



Table A.8. Bioaccumulation in Wet Plants from Saltwater, $\mathrm{L} / \mathrm{kg}_{\text {wet }}$ (CLBMP)

\begin{tabular}{|c|c|c|c|c|c|c|c|c|c|c|c|c|c|c|c|c|}
\hline Element & Atomic\# & $\mathrm{N}$ & Min & Max & AVG & Mean & Wmean & STD & GSD & GM & WGM & MED & 95\% ile & Document & Reference\# & Reference \\
\hline $\mathrm{Ac}$ & 89 & & & & & & & & & & & $1.00 \mathrm{E}+03$ & & TRS-422 & & \\
\hline $\mathrm{Ag}$ & 47 & & & & & & & & & & & $5.00 \mathrm{E}+03$ & & TRS-422 & & \\
\hline $\mathrm{Al}$ & 13 & & & & & & & & & & & & & & & \\
\hline Am & 95 & & & & & & & & & & & $8.00 \mathrm{E}+03$ & & TRS-422 & & \\
\hline $\mathrm{Am}$ & 95 & & $2.00 \mathrm{E}+03$ & $9.00 \mathrm{E}+03$ & & & & & & & & $2.90 \mathrm{E}+02$ & & Coughtrey et al. (1985) & & \\
\hline $\mathrm{Ar}$ & 18 & & & & & & & & & & & & & & & \\
\hline As & 33 & & & & & & & & & & & & & & & \\
\hline At & 85 & & & & & & & & & & & & & & & \\
\hline $\mathrm{Au}$ & 79 & & & & & & & & & & & & & & & \\
\hline B & 5 & & & & & & & & & & & & & & & \\
\hline $\mathrm{Ba}$ & 56 & & & & & & & & & & & $7.00 \mathrm{E}+01$ & & TRS-422 & & \\
\hline Be & 4 & & & & & & & & & & & $2.00 \mathrm{E}+00$ & & TRS-422 & & \\
\hline $\mathrm{Bi}$ & 83 & & & & & & & & & & & & & & & \\
\hline $\mathrm{Bk}$ & 97 & & & & & & & & & & & $8.00 \mathrm{E}+03$ & & TRS-422 & & \\
\hline $\mathrm{Br}$ & 35 & & & & & & & & & & & $1.00 \mathrm{E}+01$ & & Coughtrey et al. (1985) & S4.2 & \\
\hline C & 6 & & & & & & & & & & & & & & & \\
\hline $\mathrm{Ca}$ & 20 & & & & & & & & & & & $6.00 \mathrm{E}+00$ & & TRS-422 & & \\
\hline $\mathrm{Cd}$ & 48 & & & & & & & & & & & $2.00 \mathrm{E}+04$ & & TRS-422 & & \\
\hline $\mathrm{Cd}$ & 48 & & $1.00 \mathrm{E}+01$ & $1.00 \mathrm{E}+04$ & & & & & & & & $1.00 \mathrm{E}+03$ & & Coughtrey et al. (1985) & S4.2.1, T4.6 & \\
\hline $\mathrm{Ce}$ & 58 & & $1.00 \mathrm{E}+02$ & $7.00 \mathrm{E}+04$ & & & & & & & & $5.00 \mathrm{E}+03$ & & Coughtrey et al. (1985) & T4.2, T4.5 & \\
\hline $\mathrm{Ce}$ & 58 & & & & $7.00 \mathrm{E}+02$ & & & & & & & & & Thompson et al. (1972) & 506 & Mauchline (1963) \\
\hline $\mathrm{Ce}$ & 58 & & $3.00 \mathrm{E}+02$ & $9.00 \mathrm{E}+02$ & & & & & & & & & & Thompson et al. (1972) & 5335 & Bryan et al. (1966) \\
\hline $\mathrm{Ce}$ & 58 & & & & & & & & & & & $5.00 \mathrm{E}+03$ & & TRS-422 & & \\
\hline $\mathrm{Cf}$ & 98 & & & & & & & & & & & $8.00 \mathrm{E}+03$ & & TRS-422 & & \\
\hline $\mathrm{Cl}$ & 17 & & & & & & & & & & & $5.00 \mathrm{E}-02$ & & TRS-422 & & \\
\hline $\mathrm{Cm}$ & 96 & & & & & & & & & & & $5.00 \mathrm{E}+03$ & & TRS-422 & & \\
\hline Co & 27 & & & & & & & & & & & $6.00 \mathrm{E}+03$ & & TRS-422 & & \\
\hline $\mathrm{Cr}$ & 24 & & & & & & & & & & & & & & & \\
\hline Cs & 55 & & $1.00 \mathrm{E}+00$ & $1.20 \mathrm{E}+04$ & & & & & & & & $7.00 \mathrm{E}+02$ & & Coughtrey et al. (1985) & S4.2.1 & \\
\hline Cs & 55 & & $1.00 \mathrm{E}+01$ & $1.70 \mathrm{E}+02$ & & & & & & & & & & Thompson et al. (1972) & 1799 & Agnedal (1965) \\
\hline Cs & 55 & & $1.80 \mathrm{E}+01$ & 7.40E+01 & $4.10 \mathrm{E}+01$ & & & & & & & & & Thompson et al. (1972) & 5335 & Bryan et al. (1966) \\
\hline Cs & 55 & & & & $7.00 \mathrm{E}+01$ & & & & & & & & & Thompson et al. (1972) & 10061 & Templeton (1964) \\
\hline Cs & 55 & & & & $7.00 \mathrm{E}+01$ & & & & & & & & & Thompson et al. (1972) & 506 & Mauchline (1963) \\
\hline Cs & 55 & & & & & & & & & & & $5.00 \mathrm{E}+01$ & & TRS-422 & & \\
\hline $\mathrm{Cu}$ & 29 & & & & & & & & & & & & & & & \\
\hline Dy & 66 & & & & & & & & & & & $3.00 \mathrm{E}+03$ & & TRS-422 & & \\
\hline Er & 68 & & & & & & & & & & & & & & & \\
\hline Es & 99 & & & & & & & & & & & & & & & \\
\hline Eu & 63 & & & & & & & & & & & $3.00 \mathrm{E}+03$ & & TRS-422 & & \\
\hline $\mathrm{F}$ & 9 & & & & & & & & & & & & & & & \\
\hline $\mathrm{Fe}$ & 26 & & & & $7.30 \mathrm{E}+02$ & & & & & & & & & Thompson et al. (1972) & 5583 & Polikarpov et al. (1957) \\
\hline $\mathrm{Fm}$ & 100 & & & & & & & & & & & & & & & \\
\hline $\mathrm{Fr}$ & 87 & & & & & & & & & & & & & & & \\
\hline Ga & 31 & & & & & & & & & & & & & & & \\
\hline Gd & 64 & & & & & & & & & & & $3.00 \mathrm{E}+03$ & & TRS-422 & & \\
\hline $\mathrm{Ge}$ & 32 & & & & & & & & & & & & & & & \\
\hline $\mathrm{H}$ & 1 & & & & & & & & & & & & & & & \\
\hline Не & 2 & & & & & & & & & & & & & & & \\
\hline $\mathrm{Hf}$ & 72 & & & & & & & & & & & $3.00 \mathrm{E}+03$ & & TRS-422 & & \\
\hline $\mathrm{Hg}$ & 80 & & & & & & & & & & & $2.00 \mathrm{E}+04$ & & TRS-422 & & \\
\hline
\end{tabular}


Table A.8. (contd)

\begin{tabular}{|c|c|c|c|c|c|c|c|c|c|c|c|c|c|c|c|c|}
\hline Element & Atomic\# & $\mathrm{N}$ & Min & Max & AVG & Mean & Wmean & STD & GSD & GM & WGM & MED & 95\%ile & Document & Reference\# & Reference \\
\hline Но & 67 & & & & & & & & & & & & & & & \\
\hline I & 53 & & & & $1.00 \mathrm{E}+03$ & & & & & & & & & Thompson et al. (1972) & 9532 & Kamath et al. (1971) \\
\hline I & 53 & & & & & & & & & & & $1.00 \mathrm{E}+04$ & & TRS-422 & & \\
\hline In & 49 & & & & & & & & & & & $5.00 \mathrm{E}+03$ & & TRS-422 & & \\
\hline Ir & 77 & & & & & & & & & & & $1.00 \mathrm{E}+03$ & & TRS-422 & & \\
\hline K & 19 & & & & & & & & & & & & & & & \\
\hline $\mathrm{Kr}$ & 36 & & & & & & & & & & & $1.00 \mathrm{E}+00$ & & TRS-422 & & \\
\hline $\mathrm{La}$ & 57 & & & & & & & & & & & & & & & \\
\hline $\mathrm{Li}$ & 3 & & & & & & & & & & & & & & & \\
\hline Lu & 71 & & & & & & & & & & & & & & & \\
\hline Md & 101 & & & & & & & & & & & & & & & \\
\hline Mg & 12 & & & & & & & & & & & & & & & \\
\hline $\mathrm{Mn}$ & 25 & & $5.50 \mathrm{E}+03$ & $2.50 \mathrm{E}+04$ & & & & & & & & & & Thompson et al. (1972) & 1848 & Black et al. (1952) \\
\hline $\mathrm{Mn}$ & 25 & & $6.80 \mathrm{E}+02$ & $5.50 \mathrm{E}+03$ & & & & & & & & & & Thompson et al. (1972) & 1405 & Folsom et al. (1963) \\
\hline $\mathrm{Mn}$ & & & & & & & & & & & & $6.00 \mathrm{E}+03$ & & TRS-422 & & \\
\hline Mo & 42 & & & & & & & & & & & & & & & \\
\hline $\mathrm{N}$ & 7 & & & & & & & & & & & & & & & \\
\hline $\mathrm{Na}$ & 11 & & & & & & & & & & & 5.00E-01 & & TRS-422 & & \\
\hline $\mathrm{Nb}$ & 41 & & $3.70 \mathrm{E}+01$ & $5.20 \mathrm{E}+02$ & & & & & & & & & & Thompson et al. (1972) & 5813 & Hampson (1967) \\
\hline $\mathrm{Nb}$ & 41 & & $4.50 \mathrm{E}+02$ & $1.00 \mathrm{E}+03$ & & & & & & & & & & Thompson et al. (1972) & 5335 & Bryan et al. (1966) \\
\hline $\mathrm{Nb}$ & 41 & & & & & & & & & & & $3.00 \mathrm{E}+03$ & & TRS-422 & & \\
\hline $\mathrm{Nd}$ & 60 & & & & & & & & & & & & & & & \\
\hline $\mathrm{Ne}$ & 10 & & & & & & & & & & & & & & & \\
\hline $\mathrm{Ni}$ & 28 & & & & & & & & & & & $2.00 \mathrm{E}+03$ & & TRS-422 & & \\
\hline $\mathrm{Np}$ & 93 & & & & & & & & & & & $5.00 \mathrm{E}+01$ & & TRS-422 & & \\
\hline $\mathrm{O}$ & 8 & & & & & & & & & & & & & & & \\
\hline Os & 76 & & & & & & & & & & & & & & & \\
\hline $\mathrm{P}$ & 15 & & $2.10 \mathrm{E}+02$ & $2.20 \mathrm{E}+02$ & & & & & & & & & & Thompson et al. (1972) & 2971 & Seymour et al. (1964) \\
\hline $\mathrm{P}$ & 15 & & & & $4.00 \mathrm{E}+03$ & & & & & & & & & Thompson et al. (1972) & 9136 & Shannon et al. (1970) \\
\hline $\mathrm{Pa}$ & 91 & & & & & & & & & & & $1.00 \mathrm{E}+02$ & & TRS-422 & & \\
\hline $\mathrm{Pb}$ & 82 & & & & $8.70 \mathrm{E}+02$ & & & & & & & & & Thompson et al. (1972) & 9136 & Shannon et al. (1970) \\
\hline $\mathrm{Pb}$ & 82 & & & & $1.00 \mathrm{E}+04$ & & & & & & & & & Thompson et al. (1972) & 9863 & Kauranen et al. (1971) \\
\hline $\mathrm{Pb}$ & 82 & & & & & & & & & & & $1.00 \mathrm{E}+03$ & & TRS-422 & & \\
\hline $\mathrm{Pd}$ & 46 & & & & & & & & & & & $1.00 \mathrm{E}+03$ & & TRS-422 & & \\
\hline $\mathrm{Pm}$ & 61 & & & & & & & & & & & $3.00 \mathrm{E}+03$ & & TRS-422 & & \\
\hline Po & 84 & & & & $2.30 \mathrm{E}+03$ & & & & & & & & & Thompson et al. (1972) & 5832 & Folsom (1966) \\
\hline Po & 84 & & & & & & & & & & & $1.00 \mathrm{E}+03$ & & TRS-422 & & \\
\hline $\operatorname{Pr}$ & 59 & & & & & & & & & & & & & & & \\
\hline $\mathrm{Pt}$ & 78 & & & & & & & & & & & & & & & \\
\hline $\mathrm{Pu}$ & 94 & & & & $3.00 \mathrm{E}+03$ & & & & & & & & & Thompson et al. (1972) & 9538 & Aarkrog (1971) \\
\hline $\mathrm{Pu}$ & 94 & & $2.60 \mathrm{E}+02$ & $3.50 \mathrm{E}+03$ & $8.90 \mathrm{E}+02$ & & & & & & & & & Thompson et al. (1972) & 10010 & Noshkin (1971) \\
\hline $\mathrm{Pu}$ & 94 & & $7.70 \mathrm{E}+02$ & $1.60 \mathrm{E}+03$ & & & & & & & & & & Thompson et al. (1972) & 1299 & Pillai et al. (1964) \\
\hline $\mathrm{Pu}$ & 94 & & & & & & & & & & & $4.00 \mathrm{E}+03$ & & TRS-422 & & \\
\hline $\mathrm{Ra}$ & 88 & & & & & & & & & & & $1.00 \mathrm{E}+02$ & & TRS-422 & & \\
\hline $\mathrm{Rb}$ & 37 & & & & & & & & & & & & & & & \\
\hline $\mathrm{Re}$ & 75 & & & & & & & & & & & & & & & \\
\hline $\mathrm{Rh}$ & 45 & & & & & & & & & & & & & & & \\
\hline $\mathrm{Rn}$ & 86 & & & & & & & & & & & & & & & \\
\hline $\mathrm{Ru}$ & 44 & & & & $2.50 \mathrm{E}+02$ & & & & & & & & & Thompson et al. (1972) & 506 & Mauchline (1963) \\
\hline $\mathrm{Ru}$ & 44 & & $1.50 \mathrm{E}+01$ & $2.00 \mathrm{E}+03$ & & & & & & & & & & Thompson et al. (1972) & 5335 & Bryan et al. (1966) \\
\hline $\mathrm{Ru}$ & 44 & & & & $1.20 \mathrm{E}+03$ & & & & & & & & & Thompson et al. (1972) & 6048 & Iwashima et al. (1966) \\
\hline $\mathrm{Ru}$ & 44 & & & & $7.60 \mathrm{E}+02$ & & & & & & & & & Thompson et al. (1972) & 6048 & Iwashima et al. (1966) \\
\hline
\end{tabular}


Table A.8. (contd)

\begin{tabular}{|c|c|c|c|c|c|c|c|c|c|c|c|c|c|c|c|c|}
\hline Element & Atomic\# & $\mathrm{N}$ & Min & Max & AVG & Mean & Wmean & STD & GSD & GM & WGM & MED & $\begin{array}{l}95 \% \text { ile } \\
\end{array}$ & Document & Reference\# & Reference \\
\hline $\mathrm{Ru}$ & 44 & & & & & & & & & & & $2.00 \mathrm{E}+03$ & & TRS-422 & & \\
\hline $\mathrm{s}$ & 16 & & & & & & & & & & & $3.00 \mathrm{E}+00$ & & TRS-422 & & \\
\hline $\mathrm{Sb}$ & 51 & & $1.00 \mathrm{E}+01$ & $1.00 \mathrm{E}+03$ & & & & & & & & “50-100” & & Coughtrey et al. (1985) & S4.2.3,T4.9 & \\
\hline sb & 51 & & & & & & & & & & & $2.00 \mathrm{E}+01$ & & TRS-422 & & \\
\hline Sc & 21 & & & & & & & & & & & $9.00 \mathrm{E}+04$ & & TRS-422 & & \\
\hline $\mathrm{Se}$ & 34 & & & & & & & & & & & $1.00 \mathrm{E}+03$ & & TRS-422 & & \\
\hline $\mathrm{Si}$ & 14 & & & & & & & & & & & & & & & \\
\hline Sm & 62 & & & & & & & & & & & $3.00 \mathrm{E}+03$ & & TRS-422 & & \\
\hline $\mathrm{Sn}$ & 50 & & & & & & & & & & & $2.00 \mathrm{E}+05$ & & TRS-422 & & \\
\hline $\mathrm{Sr}$ & 38 & & & & $4.00 \mathrm{E}+01$ & & & & & & & & & Thompson et al. (1972) & 506 & Mauchline (1963) \\
\hline $\mathrm{Sr}$ & 38 & & & & $2.50 \mathrm{E}+01$ & & & & & & & & & Thompson et al. (1972) & 1799 & Agnedal (1965) \\
\hline $\mathrm{Sr}$ & 38 & & $9.00 \mathrm{E}+01$ & $2.00 \mathrm{E}+01$ & & & & & & & & & & Thompson et al. (1972) & 5335 & Bryan et al. (1966) \\
\hline $\mathrm{Sr}$ & 38 & & & & $4.00 \mathrm{E}+01$ & & & & & & & & & Thompson et al. (1972) & 10061 & Templeton (1964) \\
\hline $\mathrm{Sr}$ & 38 & & & & & & & & & & & $1.00 \mathrm{E}+01$ & & TRS-422 & & \\
\hline Та & 73 & & & & & & & & & & & $3.00 \mathrm{E}+03$ & & TRS-422 & & \\
\hline $\mathrm{Tb}$ & 65 & & & & & & & & & & & $2.00 \mathrm{E}+03$ & & TRS-422 & & \\
\hline Tc & 43 & & & & & & & & & & & $3.00 \mathrm{E}+04$ & & TRS-422 & & \\
\hline Te & 52 & & & & & & & & & & & $1.00 \mathrm{E}+04$ & & TRS-422 & & \\
\hline Th & 90 & & & & & & & & & & & $2.00 \mathrm{E}+02$ & & TRS-422 & & \\
\hline $\mathrm{Ti}$ & 22 & & & & & & & & & & & & & & & \\
\hline $\mathrm{Tl}$ & 81 & & & & & & & & & & & $1.00 \mathrm{E}+03$ & & TRS-422 & & \\
\hline $\mathrm{Tm}$ & 69 & & & & & & & & & & & $3.00 \mathrm{E}+03$ & & TRS-422 & & \\
\hline $\mathrm{U}$ & 92 & & & & & & & & & & & $1.00 \mathrm{E}+02$ & & TRS-422 & & \\
\hline $\mathrm{V}$ & 23 & & & & & & & & & & & & & & & \\
\hline $\mathrm{W}$ & 74 & & & & & & & & & & & $6.00 \mathrm{E}+02$ & & TRS-422 & & \\
\hline Хе & 54 & & & & & & & & & & & $1.00 \mathrm{E}+00$ & & TRS-422 & & \\
\hline $\mathrm{Y}$ & 39 & & & & & & & & & & & $1.00 \mathrm{E}+03$ & & TRS-422 & & \\
\hline $\mathrm{Yb}$ & 70 & & & & & & & & & & & $8.00 \mathrm{E}+02$ & & TRS-422 & & \\
\hline $\mathrm{Zn}$ & 30 & & & & & & & & & & & $2.00 \mathrm{E}+03$ & & TRS-422 & & \\
\hline $\mathrm{Zr}$ & 40 & & & & $1.00 \mathrm{E}+03$ & & & & & & & & & Thompson et al. (1972) & 506 & Mauchline (1963) \\
\hline $\mathrm{Zr}$ & 40 & & $3.50 \mathrm{E}+02$ & $1.00 \mathrm{E}+03$ & & & & & & & & & & Thompson et al. (1972) & 5335 & Bryan et al. (1966) \\
\hline $\mathrm{Zr}$ & 40 & & & & & & & & & & & $3.00 \mathrm{E}+03$ & & TRS-422 & & \\
\hline
\end{tabular}



PNNL-21950, Appendix A

\section{A.2 References}

TRS_364

IAEA - International Atomic Energy Agency. 1994. Handbook of Parameter Values for the Prediction of the Radionuclide Transfer in Temperate Environments. Technical Report Series No. 364, Vienna, Austria.

[1] IAEA - International Atomic Energy Agency. 1994. Handbook of Parameter Values for the Prediction of the Radionuclide Transfer in Temperate Environments. Technical Report Series No. 364, p. 74, Vienna, Austria.

[2] Bogdevitch I, N Sanzharova, B Prister, and S Tarasiuk. 2002. "Countermeasures on Natural and Agricultural Areas after Chernobyl Accident.” In Role of GIS in Lifting the Cloud off Chernobyl, J Kolejka (Ed.), Kluwer Academic Publishers, pp. 147-158.

[3] Deville-Cavelin G, RM Alexakhin, IM Bogdevitch, BS Prister, H Biesold, LV Perepelyatnikova, NI Sanzharova, and SV Tarasiuk. "Countermeasures in Agriculture: Assessment of Efficiency." In Proceedings of the International Conference, "Fifteen Years after the Chernobyl Accident. Lessons Learned,” Kiev, Ukraine, pp. 118-128.

[4] Noordijk H and JM Quinault. 1992. "The Influence of Food Processing and Culinary Preparation on the Radionuclide Content of Foodstuffs: A Review of Available Data.” In Modelling of Resuspension, Seasonality and Losses during Food Processing, first report of the VAMP Terrestrial Working Group, IAEA-TECDOC-647, Vienna, pp. 35-59.

[5] Green N. 2001. “The Effect of Storage and Processing on Radionuclide Content of Fruit.” Journal of Environmental Radioactivity 52:281-290.

[6] Green N and BT Wilkins. 1995. Effects of Processing on Radionuclide Content of Foods: Derivation of Parameter Values for Use in Radiological Assessments. NRPB-M587, National Radiological Protection Board, Chilton.

[7] Long S, D Pollard, JL Cunningham, NP Astasheva, GA Donskaya, and EV Labetsky. 1995. “The Effects of Food Processing and Direct Decontamination Techniques on the Radionuclide Content of Foodstuffs: A Literature Review. Part 2: Meat, Fruit, Cereals and Drinks.” Journal of Radioecology 3(2):15-38.

[8] IAEA - International Atomic Energy Agency. 2003. Modelling the Transfer of Radionuclides to Fruit. IAEA-BIOMASS-5, IAEA, Vienna, p. 151.

[9] Ehrhardt J and A Weis (Eds.). 2000. RODOS: Decision Support System for Off-Site Nuclear Emergency Management in Europe. Report EUR 19144, ISBN No.92-828-9773-7, p. 259.

[11] State Hygienic Standards. 1997. Permissible Levels of ${ }^{137}$ Cs and ${ }^{90}$ Sr Content in Foodstuffs and Drinking Water. PL-97, Kyiv, Chornobyinterinform, p. 10.

[13] Radionuclides in Food-MAFF Food Surveillance Paper 43, HMSO, London (1994) 221.

[14] The Effects of Processing on Cadmium and Lead Levels in Food, FSA Final Report C01006 (2000) 62. 
[16] Watterson J and K Nicholson. 1995. Change in Radionuclide Content in Crops as a Result of Food Preparation. AEA/CS-10400, p. 24.

[26] Grueter H. 1971. "Radioactive Fission Product Cs-137 in Mushrooms in West Germany during 1963-1970.” Health Physics 20:655-656.

[52] Apostolatos G and A Hadjiantoniou. 1989. "Caesium Contamination Transfer in Wheat Products, Radioactivity Transfer during Food Processing and Culinary Preparation.” Proc. Seminar Cadarache, CEC, Luxembourg, pp. 377-400.

\section{NUREG/CR-5512}

Kennedy WE and DL Strenge. 1992. Residual Radioactive Contamination from Decommissioning. NUREG/CR-5512, Vol. 1, U.S. Nuclear Regulatory Commission, Washington, D.C., PNL-7994, Pacific Northwest Laboratory, Richland, Washington.

Poston and Klopfer. 1988. "Concentration Factors Used in the Assessment of Radiation Dose to Comsumers of Fish: A Review of 27 Radionuclides.” Health Physics 55:751-766.

Strenge DL, RA Peloquin, and G Whelan. 1986. LADTAP II - Technical Reference and User Guide. NUREG/CR-4013 (PNL-5270), U.S. Nuclear Regulatory Commission, Washington, D.C.

\section{UCRL-50564, Rev. 1}

Thompson, SE, AC Burton, DJ Quinn, and YC Ng. 1972. Concentration Factors of Chemical Elements in Edible Aquatic Organisms. UCRL-50564, Rev. 1, Lawrence Livermore Laboratory, Livermore, California.

Gaglione et al. (1964)

(140) Cigna et al. (1963)

(506) Mauchline (1963)

(1299) Pillai et al. (1964)

(1307) Fontaine (1962)

(1405) Folsom et al. (1963)

(1679) Krumholz et al. (1957)

(1682) Mauchline et al. (1964)

(1784) Harvey (1964)

(1799) Agnedal (1965) 
(1848) Black et al. (1952)

(2722) Pendleton et al. (1958)

(2723) Davis et al. (1958)

(2971) Seymour et al. (1964)

(3133) Berg et al. (1961)

(4081) Ewoll et al. (1963)

(5335) Bryan et al. (1966)

(5583) Polikarpov et al. (1957)

(5731) Perkins et al. (1967)

(5811) Ravera (1966)

(5813) Hampson (1967)

(5832) Folsom (1966)

(6012) Polikarpov (1966)

(6048) Iwashima et al. (1966)

(6335) Preston et al. (1967)

(8625) Kolehmainen et al. (1969)

(8687) Bortoli et al. (1969)

(8761) Seymour (1969)

(8845) Folsom et al. (1965)

(8915) Preston et al. (1965)

(8956) Wong et al. (1970)

(9136) Shannon et al. (1970)

(9532) Kamath et al. (1971)

(9533) Feldt (1971)

(9538) Aarkrog (1971) 
(9576) [unknown]

(9863) Kauranen et al. (1971)

(9903) Preston (1968)

(9957) Seelye (1970)

(9995) Wong et al. (1971)

(10010) Noshkin (1971)

(10023) Bryan et al. (1965)

(10050) Kalnina et al. (1969)

(10052) Bigliocca et al. (1969)

(10058) Ouchi et al. (1970)

(10059) Agnedal et al. (1958)

(10061) Templeton (1964)

(10067) Friend et al. (1965)

\section{PJ Coughtrey, Vol Six}

Coughtrey, PJ, D Jackson, and MC Thorne. 1985. Radionuclide Distribution and Transport in Terrestrial and Aquatic Ecosystems, Volume Six, A.A. Balkema, Boston.

(S4.2)

(S4.2.1)

(S4.2.2)

(S4.2.3)

(S4.2.4)

(T4.2)

(T4.5)

(T4.6)

(T4.7) 
TRS_422

IAEA - International Atomic Energy Agency. 2004. Sediment Distribution Coefficients and Concentration Factors for Biota in the Marine Environment. Technical Report Series No. 422, Vienna, Austria.

\section{TRS_472}

IAEA. 2010. Handbook of Parameter Values for the Prediction of Radionuclide Transfer in Terrestrial and Freshwater Environments. Technical Report Series No. 472, International Atomic Energy Agency, Vienna, Austria.

\section{Other References}

Fesenko S, J Fesenko, N Sanzharova, E Karpenko, and I Titov. 2011. “Radionuclide Transfer to Freshwater Biota Species: Review of Russian Language Studies.” Journal of Environmental Radioactivity 102(1):8-25.

Hosseini A, H Thørring, JE Brown, R Saxén, and E Ilus. 2008. "Transfer of Radionuclides in Aquatic Ecosystems - Default Concentration Ratios for Aquatic Biota in the Erica Tool.” Journal of Environmental Radioactivity 99(9):1408-1429.

Jha VN, RM Tripathi, NK Sethy, SK Sahoo, AK Shukla, and VD Puranik. 2010. "Bioaccumulation of ${ }^{226}$ Ra by Plants Growing in Fresh Water Ecosystem Around the Uranium Industry at Jaduguda, India.” Journal of Environmental Radioactivity 101(0):717-722.

Kennedy WE and DL Strenge. 1992. Residual Radioactive Contamination from Decommissioning. NUREG/CR-5512, Vol. 1, U.S. Nuclear Regulatory Commission, Washington, D.C., PNL-7994, Pacific Northwest Laboratory, Richland, Washington.

Metian M, M Warnau, J-L Teyssié, and P Bustamante. 2011. "Characterization of ${ }^{241} \mathrm{Am}$ and ${ }^{134} \mathrm{Cs}$ Bioaccumulation in the King Scallop Pecten maximus: Investigation via Three Exposure Pathways.” Journal of Environmental Radioactivity 102(6):543-550.

Outola I, RL Saxén, and S Heinävaara. 2009. “Transfer of ${ }^{90}$ Sr into Fish in Finnish Lakes.” Journal of Environmental Radioactivity 100(8):657-664. 
Pyle GG and FV Clulow. 1998. "Radionuclide Equilibria Between the Aquatic Environment and Fish Tissues.” Journal of Environmental Radioactivity 40(1):59-74.

Sheppard SC, JM Long, and B Sanipelli. 2010a. "Measured Elemental Transfer Factors for Boreal Hunter/Gatherer Scenarios: Fish, Game and Berries.” Journal of Environmental Radioactivity 101(11):902-909.

Sheppard SC, JM Long, and B Sanipelli. 2010b. "Plant/Soil Concentration Ratios for Paired Field and Garden Crops, with Emphasis on Iodine and the Role of Soil Adhesion.” Journal of Environmental Radioactivity 101(12):1032-1037. 


\section{Appendix B}

\section{Terrestrial Animal}




\section{Appendix B}

\section{Terrestrial Animal}

This appendix contains tables of feed to animal product transfer factors. References are indicated for each value.

Table B.1. Feed to Meat Transfer Factor, d/kget_as_fed $($ CLFMT)

Table B.2. Feed to Milk Transfer Factor, d/L (CLFMK)

Table B.3. Feed to Poultry Transfer Factor, d/ $\mathrm{kg}_{\text {wet_as_fed }}$ (CLFPL)

Table B.4. Feed to Egg Transfer Factor, d/kg wet_as_fed $_{\text {(CLFEG) }}$

In all tables if an element is listed once and there is no data for that element, no transfer factor information was currently identified, preferred, or available. 

Table B.1. Feed to Meat Transfer Factor, d/kg wet_as_fed $(C L F M T)$

\begin{tabular}{|c|c|c|c|c|c|c|c|c|c|c|c|c|c|c|c|}
\hline Element & Atomic\# & $\mathrm{N}$ & Min & Max & $\mathrm{AVG}$ & Mean & Wmean & STD & GSD & GM & WGM & MED & Document & Reference\# & Reference \\
\hline Ac & 89 & & & & & & & & & & & $4.00 \mathrm{E}-04$ & PNNL-13421 & LS85 & \\
\hline Ac & 89 & & & & & & & & & & & $2.50 \mathrm{E}-05$ & NUREG/CR-5512 & & \\
\hline $\mathrm{Ag}$ & 47 & & & & & & & & & & & $3.00 \mathrm{E}-03$ & PNNL-13421 & CEC87 & \\
\hline $\mathrm{Ag}$ & 47 & & & & & $4.80 \mathrm{E}-04$ & & & & & & & & & Howard et al. (2009) \\
\hline $\mathrm{Al}$ & 13 & & & & & & & & & & & & & & \\
\hline $\mathrm{Am}$ & 95 & & & & & & & & & & & 4.00E-05 & PNNL-13421 & Co90 & \\
\hline $\mathrm{Am}$ & 95 & & & & & & & & & & & $3.50 \mathrm{E}-06$ & NUREG/CR-5512 & & \\
\hline Am & 95 & & & & & $5.00 \mathrm{E}-04$ & & & & & & & & & Howard et al. (2009) \\
\hline $\mathrm{Am}$ & 95 & & & & & $1.10 \mathrm{E}-04$ & & & & & & & & & Howard et al. (2009) \\
\hline $\mathrm{Am}$ & 95 & 1 & & & & $5.00 \mathrm{E}-04$ & & & & & & & TRS_472 & & \\
\hline $\mathrm{Ar}$ & 18 & & & & & & & & & & & & & & \\
\hline As & 33 & & & & & & & & & & & $2.00 \mathrm{E}-03$ & PNNL-13421 & Ng68 & \\
\hline At & 85 & & & & & & & & & & & & & & \\
\hline $\mathrm{Au}$ & 79 & & & & & & & & & & & $5.00 \mathrm{E}-03$ & PNNL-13421 & NCRP86 & \\
\hline $\mathrm{Au}$ & 79 & & & & & & & & & & & $8.00 \mathrm{E}-03$ & NUREG/CR-5512 & & \\
\hline $\mathrm{Ba}$ & 56 & & & & & & & & & & & $2.00 \mathrm{E}-04$ & PNNL-13421 & Jo88 & \\
\hline $\mathrm{Ba}$ & 56 & & & & & & & & & & & $1.50 \mathrm{E}-04$ & NUREG/CR-5512 & & \\
\hline $\mathrm{Ba}$ & 56 & & & & & $1.40 \mathrm{E}-04$ & & & & & & & & & Howard et al. (2009) \\
\hline $\mathrm{Ba}$ & 56 & & & & & $1.30 \mathrm{E}-05$ & & & & & & & & & Howard et al. (2009) \\
\hline Ва & 56 & 2 & $5.00 \mathrm{E}-05$ & 2.30E-04 & & $1.40 \mathrm{E}-04$ & & & & & & & TRS_472 & & \\
\hline Be & 4 & & & & & & & & & & & $1.00 \mathrm{E}-03$ & PNNL-13421 & Ng68 & \\
\hline $\mathrm{Bi}$ & 83 & & & & & & & & & & & 4.00E-04 & PNNL-13421 & Ng68 & \\
\hline $\mathrm{Bk}$ & 97 & & & & & & & & & & & & & & \\
\hline $\mathrm{Br}$ & 35 & & & & & & & & & & & 2.50E-02 & PNNL-13421 & Ng68 & \\
\hline C & 6 & 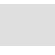 & 3.00E-01 & 6.00E-01 & & & & & & & & & & & Galeriu et al. (2007) \\
\hline $\mathrm{Ca}$ & 20 & & & & & & & & & & & $2.00 \mathrm{E}-03$ & PNNL-13421 & Ng82a & \\
\hline $\mathrm{Ca}$ & 20 & & & & & & & & & & & 7.00E-04 & NUREG/CR-5512 & & \\
\hline $\mathrm{Ca}$ & 20 & & & & & 2.00E-01 & & 3.50E-01 & & & & & & & Howard et al. (2009) \\
\hline $\mathrm{Ca}$ & 20 & 3 & $1.00 \mathrm{E}-03$ & 6.10E-01 & & & & & $3.00 \mathrm{E}+01$ & $1.30 \mathrm{E}-02$ & & & TRS_472 & & \\
\hline $\mathrm{Cd}$ & 48 & & & & & & & & & & & 4.00E-04 & PNNL-13421 & Ng82a & \\
\hline $\mathrm{Cd}$ & 48 & & & & & & & & & & & $5.50 \mathrm{E}-04$ & NUREG/CR-5512 & & \\
\hline $\mathrm{Cd}$ & 48 & & & & & $1.90 \mathrm{E}-02$ & & $2.40 \mathrm{E}-02$ & & & & & & & Howard et al. (2009) \\
\hline $\mathrm{Cd}$ & 48 & & & & & $1.20 \mathrm{E}-03$ & & & & & & & & & Howard et al. (2009) \\
\hline $\mathrm{Cd}$ & 48 & 8 & $1.50 \mathrm{E}-04$ & $6.00 \mathrm{E}-02$ & & & & & $7.80 \mathrm{E}+00$ & $5.80 \mathrm{E}-03$ & & & TRS_472 & & \\
\hline $\mathrm{Ce}$ & 58 & & & & & & & & & & & $2.00 \mathrm{E}-05$ & PNNL-13421 & CEC87 & \\
\hline $\mathrm{Ce}$ & 58 & & & & & & & & & & & 7.50E-04 & NUREG/CR-5512 & & \\
\hline $\mathrm{Ce}$ & 58 & & & & & $2.50 \mathrm{E}-04$ & & & & & & & & & Howard et al. (2009) \\
\hline $\mathrm{Cf}$ & 98 & & & & & & & & & & & 4.00E-05 & PNNL-13421 & Co90 & \\
\hline $\mathrm{Cf}$ & 98 & & & & & & & & & & & $5.00 \mathrm{E}-03$ & NUREG/CR-5512 & & \\
\hline $\mathrm{Cl}$ & 17 & & & & & & & & & & & $2.00 \mathrm{E}-02$ & PNNL-13421 & Bi89 & \\
\hline $\mathrm{Cl}$ & 17 & & & & & & & & & & & $8.00 \mathrm{E}-02$ & NUREG/CR-5512 & & \\
\hline $\mathrm{Cl}$ & 17 & & & & & $1.70 \mathrm{E}-02$ & & & & & & & & & Howard et al. (2009) \\
\hline $\mathrm{Cl}$ & 17 & 1 & & & & $1.70 \mathrm{E}-02$ & & & & & & & TRS_472 & & \\
\hline $\mathrm{Cm}$ & 96 & & & & & & & & & & & 4.00E-05 & PNNL-13421 & Co90 & \\
\hline $\mathrm{Cm}$ & 96 & & & & & & & & & & & $3.50 \mathrm{E}-06$ & NUREG/CR-5512 & & \\
\hline Co & 27 & & & & & & & & & & & $1.00 \mathrm{E}-02$ & PNNL-13421 & Ng82a & \\
\hline Co & 27 & & & & & & & & & & & $2.00 \mathrm{E}-02$ & NUREG/CR-5512 & & \\
\hline Co & 27 & & & 4.60E-04 & & & & & & & & & & & Beresford et al. (2007 \\
\hline Co & 27 & & & & & $5.20 \mathrm{E}-04$ & & 3.00E-04 & & & & & & & Howard et al. (2009) \\
\hline Co & 27 & & & & & $1.20 \mathrm{E}-02$ & & & & & & & & & Howard et al. (2009) \\
\hline
\end{tabular}


Table B.1. (contd)

\begin{tabular}{|c|c|c|c|c|c|c|c|c|c|c|c|c|c|c|c|}
\hline Element & Atomic\# & $\mathrm{N}$ & Min & Max & AVG & Mean & Wmean & STD & GSD & GM & WGM & MED & Document & Reference\# & Reference \\
\hline Co & 27 & 4 & $1.30 \mathrm{E}-04$ & $8.40 \mathrm{E}-04$ & & & & & $2.30 \mathrm{E}+00$ & $4.30 \mathrm{E}-04$ & & & TRS_472 & & \\
\hline $\mathrm{Cr}$ & 24 & & & & & & & & & & & $9.00 \mathrm{E}-03$ & PNNL-13421 & Ng82a & \\
\hline $\mathrm{Cr}$ & 24 & & & & & & & & & & & $5.50 \mathrm{E}-03$ & NUREG/CR-5512 & & \\
\hline Cs & 55 & & & & & & & & & & & $5.00 \mathrm{E}-02$ & PNNL-13421 & Co90 & \\
\hline Cs & 55 & & & & & & & & & & & $2.00 \mathrm{E}-02$ & NUREG/CR-5512 & & \\
\hline Cs & 55 & & & & & $9.30 \mathrm{E}-03$ & & $1.97 \mathrm{E}-03$ & & & & & & & Beresford et al. (2007) \\
\hline Cs & 55 & & & & & $8.30 \mathrm{E}-03$ & & $1.57 \mathrm{E}-03$ & & & & & & & Beresford et al. (2007) \\
\hline Cs & 55 & & & & & $3.00 \mathrm{E}-02$ & & 2.30E-02 & & & & & & & Howard et al. (2009) \\
\hline Cs & 55 & & & & & $4.80 \mathrm{E}-01$ & & $5.30 \mathrm{E}-01$ & & & & & & & Howard et al. (2009) \\
\hline Cs & 55 & & & & & 2.70E-01 & & 2.60E-01 & & & & & & & Howard et al. (2009) \\
\hline Cs & 55 & 58 & $4.70 \mathrm{E}-03$ & $9.60 \mathrm{E}-02$ & & & & & $2.40 \mathrm{E}+00$ & 2.20E-02 & & & TRS_472 & & \\
\hline $\mathrm{Cu}$ & 29 & & & & & & & & & & & $9.00 \mathrm{E}-03$ & PNNL-13421 & Ng82a & \\
\hline $\mathrm{Cu}$ & 29 & & & & & & & & & & & $1.00 \mathrm{E}-02$ & NUREG/CR-5512 & & \\
\hline Dy & 66 & & & & & & & & & & & 2.00E-05 & PNNL-13421 & CEC87 & \\
\hline Dy & 66 & & & & & & & & & & & $5.50 \mathrm{E}-03$ & NUREG/CR-5512 & & \\
\hline Er & 68 & & & & & & & & & & & 2.00E-05 & PNNL-13421 & CEC87 & \\
\hline Er & 68 & & & & & & & & & & & 4.00E-03 & NUREG/CR-5512 & ond & \\
\hline Es & 99 & & & & & & & & & & & & & & \\
\hline $\mathrm{Eu}$ & 63 & & & & & & & & & & & $2.00 \mathrm{E}-05$ & PNNL-13421 & CEC87 & \\
\hline $\mathrm{Eu}$ & 63 & & & & & & & & & & & $5.00 \mathrm{E}-03$ & NUREG/CR-5512 & & \\
\hline $\mathrm{F}$ & 9 & & & & & & & & & & & 1.50E-01 & PNNL-13421 & Ng68 & \\
\hline $\mathrm{Fe}$ & 26 & & & & & & & & & & & 2.00E-02 & PNNL-13421 & Ng82a & \\
\hline $\mathrm{Fe}$ & 26 & & & & & $1.50 \mathrm{E}-02$ & & $6.70 \mathrm{E}-03$ & & & & & & & Howard et al. (2009) \\
\hline $\mathrm{Fe}$ & 26 & 4 & $9.00 \mathrm{E}-03$ & $2.50 \mathrm{E}-02$ & & & & & $1.50 \mathrm{E}+00$ & $1.40 \mathrm{E}-02$ & & & TRS_472 & & \\
\hline $\mathrm{Fm}$ & 100 & & & & & & & & & & & & & & \\
\hline $\mathrm{Fr}$ & 87 & & & & & & & & & & & & & & \\
\hline $\mathrm{Ga}$ & 31 & & & & & & & & & & & $5.00 \mathrm{E}-04$ & PNNL-13421 & Ba84 & \\
\hline $\mathrm{Gd}$ & 64 & & & & & & & & & & & 2.00E-05 & PNNL-13421 & CEC87 & \\
\hline $\mathrm{Gd}$ & 64 & & & & & & & & & & & $3.50 \mathrm{E}-03$ & NUREG/CR-5512 & & \\
\hline Ge & 32 & & & & & & & & & & & & & & \\
\hline $\mathrm{H}$ & 1 & & $6.40 \mathrm{E}-01$ & $8.20 \mathrm{E}-01$ & & & & & & & & & & & Galeriu et al. (2007) \\
\hline $\mathrm{Hf}$ & 72 & & & & & & & & & & & $1.00 \mathrm{E}-03$ & PNNL-13421 & Ng68 & \\
\hline $\mathrm{Hg}$ & 80 & & & & & & & & & & & 2.50E-01 & PNNL-13421 & Ng68 & \\
\hline Ho & 67 & & & & & & & & & & & 2.00E-05 & PNNL-13421 & CEC87 & \\
\hline Но & 67 & & & & & & & & & & & 4.50E- -03 & NUREG/CR-5512 & & \\
\hline I & 53 & & & & & & & & & & & 4.00E-02 & PNNL-13421 & Bi89 & \\
\hline I & 53 & & & & & & & & & & & 7.00E-03 & NUREG/CR-5512 & & \\
\hline I & 53 & & 8.00E-04 & 2.00E-02 & & & & & & & & & & & \\
\hline I & 53 & & $8.00 \mathrm{E}-03$ & $2.00 \mathrm{E}-01$ & & & & & & & & & & & Thorne (2003) \\
\hline I & 53 & & & & & $1.20 \mathrm{E}-03$ & & $1.50 \mathrm{E}-02$ & & & & & & & Howard et al. (2009) \\
\hline I & 53 & & & & & 3.00E-02 & & & & & & & & & Howard et al. (2009) \\
\hline I & 53 & 5 & $2.00 \mathrm{E}-03$ & $3.80 \mathrm{E}-02$ & & & & & $3.20 \mathrm{E}+00$ & $6.70 \mathrm{E}-03$ & & & TRS_472 & & \\
\hline In & 49 & & & & & & & & & & & $8.00 \mathrm{E}-03$ & PNNL-13421 & Ng68 & \\
\hline Ir & 77 & & & & & & & & & & & $1.50 \mathrm{E}-03$ & PNNL-13421 & Ng68 & \\
\hline $\mathrm{K}$ & 19 & & & & & & & & & & & $2.00 \mathrm{E}-02$ & PNNL-13421 & Ng82a & \\
\hline $\mathrm{Kr}$ & 36 & & & & & & & & & & & & & & \\
\hline La & 57 & & & & & & & & & & & $2.00 \mathrm{E}-03$ & PNNL-13421 & NCRP96 & \\
\hline La & 57 & & & & & & & & & & & $3.00 \mathrm{E}-04$ & NUREG/CR-5512 & & \\
\hline La & 57 & & & & & $1.30 \mathrm{E}-04$ & & 2.00E-05 & & & & & & & Howard et al. (2009) \\
\hline $\mathrm{La}$ & 57 & 3 & $1.10 \mathrm{E}-04$ & $1.50 \mathrm{E}-04$ & & & & & $1.20 \mathrm{E}+00$ & $1.30 \mathrm{E}-04$ & & & TRS_472 & & \\
\hline $\mathrm{Lu}$ & 71 & & & & & & & & & & & & & & \\
\hline
\end{tabular}


Table B.1. (contd)

\begin{tabular}{|c|c|c|c|c|c|c|c|c|c|c|c|c|c|c|c|}
\hline Element & Atomic\# & $\mathrm{N}$ & Min & Max & AVG & Mean & Wmean & STD & GSD & GM & WGM & MED & Document & Reference\# & Reference \\
\hline $\mathrm{Mg}$ & 12 & & & & & & & & & & & $2.00 \mathrm{E}-02$ & PNNL-13421 & Ng82a & \\
\hline $\mathrm{Mg}$ & 12 & & & & & & & & & & & $5.00 \mathrm{E}-03$ & NUREG/CR-5512 & & \\
\hline Mn & 25 & & & & & & & & & & & $5.00 \mathrm{E}-04$ & PNNL-13421 & Ng82a & \\
\hline $\mathrm{Mn}$ & 25 & & & & & & & & & & & 4.00E-04 & NUREG/CR-5512 & & \\
\hline $\mathrm{Mn}$ & 25 & & & & & $6.00 \mathrm{E}-04$ & & & & & & & & & Howard et al. (2009) \\
\hline $\mathrm{Mn}$ & 25 & & & & & $9.00 \mathrm{E}-03$ & & & & & & & & & Howard et al. (2009) \\
\hline $\mathrm{Mn}$ & 25 & 2 & $6.00 \mathrm{E}-04$ & $6.00 \mathrm{E}-04$ & & $6.00 \mathrm{E}-04$ & & & & & & & TRS_472 & & \\
\hline Mo & 42 & & & & & & & & & & & $1.00 \mathrm{E}-03$ & PNNL-13421 & Jo88 & \\
\hline Mo & 42 & & & & & & & & & & & $6.00 \mathrm{E}-03$ & NUREG/CR-5512 & & \\
\hline Mo & 42 & & & & & $1.00 \mathrm{E}-03$ & & & & & & & & & Howard et al. (2009) \\
\hline Mo & 42 & 1 & & & & $1.00 \mathrm{E}-03$ & & & & & & & TRS_472 & & \\
\hline $\mathrm{N}$ & 7 & & & & & & & & & & & 7.50E-02 & PNNL-13421 & Ng68 & \\
\hline $\mathrm{Na}$ & 11 & & & & & & & & & & & 8.00E-02 & PNNL-13421 & Ng82a & \\
\hline $\mathrm{Na}$ & 11 & & & & & & & & & & & $5.50 \mathrm{E}-02$ & NUREG/CR-5512 & & \\
\hline $\mathrm{Na}$ & 11 & & & & & $1.50 \mathrm{E}-02$ & & & & & & & & & Howard et al. (2009) \\
\hline $\mathrm{Na}$ & 11 & & & & & $1.10 \mathrm{E}-01$ & & & & & & & & & Howard et al. (2009) \\
\hline $\mathrm{Na}$ & 11 & 2 & $1.00 \mathrm{E}-02$ & $2.00 \mathrm{E}-02$ & & $1.50 \mathrm{E}-02$ & & & & & & & TRS_472 & & \\
\hline $\mathrm{Nb}$ & 41 & & & & & & & & & & & $3.00 \mathrm{E}-07$ & PNNL-13421 & Jo88 & \\
\hline $\mathrm{Nb}$ & 41 & & & & & & & & & & & $2.50 \mathrm{E}-01$ & NUREG/CR-5512 & & \\
\hline $\mathrm{Nb}$ & 41 & & & $2.40 \mathrm{E}-03$ & & & & & & & & & & & Beresford et al. (2007 \\
\hline $\mathrm{Nb}$ & 41 & & & & & $2.60 \mathrm{E}-07$ & & & & & & & & & Howard et al. (2009) \\
\hline $\mathrm{Nb}$ & 41 & & & & & $6.00 \mathrm{E}-05$ & & & & & & & & & Howard et al. (2009) \\
\hline $\mathrm{Nb}$ & 41 & 1 & & & & $2.60 \mathrm{E}-07$ & & & & & & & TRS_472 & & \\
\hline $\mathrm{Nd}$ & 60 & & & & & & & & & & & 2.00E-05 & PNNL-13421 & CEC87 & \\
\hline $\mathrm{Nd}$ & 60 & & & & & & & & & & & 3.00E-04 & NUREG/CR-5512 & & \\
\hline $\mathrm{Ne}$ & 10 & & & & & & & & & & & & & & \\
\hline $\mathrm{Ni}$ & 28 & & & & & & & & & & & $5.00 \mathrm{E}-03$ & PNNL-13421 & Cr90 & \\
\hline $\mathrm{Ni}$ & 28 & & & & & & & & & & & $6.00 \mathrm{E}-03$ & NUREG/CR-5512 & & \\
\hline $\mathrm{Np}$ & 93 & & & & & & & & & & & $1.00 \mathrm{E}-03$ & PNNL-13421 & $\mathrm{Br} 79$ & \\
\hline $\mathrm{Np}$ & 93 & & & & & & & & & & & 5.50E-05 & NUREG/CR-5512 & & \\
\hline \multirow{2}{*}{\multicolumn{16}{|c|}{$\mathrm{OBT}$}} \\
\hline & & & & & & & & & & & & & & & \\
\hline Os & 76 & . & & & & & & & & & & 4.00E-01 & PNNL-13421 & Ng68 & \\
\hline $\mathrm{P}$ & 15 & & & & & & & & & & & $5.00 \mathrm{E}-02$ & PNNL-13421 & Ng82a & \\
\hline $\mathrm{P}$ & 15 & & & & & & & & & & & $5.50 \mathrm{E}-02$ & NUREG/CR-5512 & & \\
\hline $\mathrm{P}$ & 15 & & & & & $5.50 \mathrm{E}-02$ & & & & & & & & & Howard et al. (2009) \\
\hline $\mathrm{P}$ & 15 & 1 & & & & $5.50 \mathrm{E}-02$ & & & & & & & TRS_472 & & \\
\hline $\mathrm{Pa}$ & 91 & & & & & & & & & & & 4.00E-05 & PNNL-13421 & Co90 & \\
\hline $\mathrm{Pa}$ & 91 & & & & & & & & & & & $1.00 \mathrm{E}-05$ & NUREG/CR-5512 & & \\
\hline $\mathrm{Pb}$ & 82 & & & & & & & & & & & 4.00E-04 & PNNL-13421 & Ng82a & \\
\hline $\mathrm{Pb}$ & 82 & & & & & & & & & & & 3.00E-04 & NUREG/CR-5512 & & \\
\hline $\mathrm{Pb}$ & 82 & & & & & $9.30 \mathrm{E}-04$ & & $6.40 \mathrm{E}-04$ & & & & & & & Howard et al. (2009) \\
\hline $\mathrm{Pb}$ & 82 & & & & & 7.10E-03 & & & & & & & & & Howard et al. (2009) \\
\hline $\mathrm{Pb}$ & 82 & 5 & $2.00 \mathrm{E}-04$ & 1.60E-03 & & & & & $2.50 \mathrm{E}+00$ & 7.00E-04 & & & TRS_472 & & \\
\hline $\mathrm{Pd}$ & 46 & & & & & & & & & & & 4.00E-03 & PNNL-13421 & Ng68 & \\
\hline $\mathrm{Pm}$ & 61 & & & & & & & & & & & 2.00E-05 & PNNL-13421 & CEC87 & \\
\hline $\mathrm{Pm}$ & 61 & & & & & & & & & & & $5.00 \mathrm{E}-03$ & NUREG/CR-5512 & & \\
\hline Po & 84 & & & & & & & & & & & $5.00 \mathrm{E}-03$ & PNNL-13421 & Ng82a & \\
\hline Po & 84 & & & & & & & & & & & $3.00 \mathrm{E}-04$ & NUREG/CR-5512 & & \\
\hline $\operatorname{Pr}$ & 59 & & & & & & & & & & & $2.00 \mathrm{E}-05$ & PNNL-13421 & CEC87 & \\
\hline $\operatorname{Pr}$ & 59 & & & & & & & & & & & $3.00 \mathrm{E}-04$ & NUREG/CR-5512 & & \\
\hline Pt & 78 & & & & & & & & & & & & & & \\
\hline
\end{tabular}


Table B.1. (contd)

\begin{tabular}{|c|c|c|c|c|c|c|c|c|c|c|c|c|c|c|c|}
\hline Element & Atomic\# & $\mathrm{N}$ & Min & Max & $\mathrm{AVG}$ & Mean & Wmean & STD & GSD & GM & WGM & MED & Document & Reference\# & Reference \\
\hline $\mathrm{Pu}$ & 94 & & & & & & & & & & & $1.00 \mathrm{E}-05$ & PNNL-13421 & Co90 & \\
\hline $\mathrm{Pu}$ & 94 & & & & & & & & & & & $5.00 \mathrm{E}-07$ & NUREG/CR-5512 & & \\
\hline $\mathrm{Pu}$ & 94 & & & & & $4.40 \mathrm{E}-05$ & & $1.37 \mathrm{E}-05$ & & & & & & & Beresford et al. (2007) \\
\hline $\mathrm{Pu}$ & 94 & & & & & 3.90E-05 & & $1.12 \mathrm{E}-05$ & & & & & & & Beresford et al. (2007) \\
\hline $\mathrm{Pu}$ & 94 & & & & & $6.00 \mathrm{E}-05$ & & $1.30 \mathrm{E}-04$ & & & & & & & Howard et al. (2009) \\
\hline $\mathrm{Pu}$ & 94 & & & & & $5.30 \mathrm{E}-05$ & & & & & & & & & Howard et al. (2009) \\
\hline $\mathrm{Pu}$ & 94 & 5 & $8.80 \mathrm{E}-08$ & $3.00 \mathrm{E}-04$ & & & & & $2.48 \mathrm{E}+01$ & $1.10 \mathrm{E}-06$ & & & TRS_472 & & \\
\hline Ra & 88 & & & & & & & & & & & $9.00 \mathrm{E}-04$ & PNNL-13421 & MB90 & \\
\hline $\mathrm{Ra}$ & 88 & & & & & & & & & & & $2.50 \mathrm{E}-04$ & NUREG/CR-5512 & & \\
\hline $\mathrm{Ra}$ & 88 & & & & & $1.70 \mathrm{E}-03$ & & & & & & & & & Howard et al. (2009) \\
\hline $\mathrm{Ra}$ & 88 & 1 & & & & $1.70 \mathrm{E}-03$ & & & & & & & TRS_472 & & \\
\hline $\mathrm{Rb}$ & 37 & & & & & & & & & & & $1.00 \mathrm{E}-02$ & PNNL-13421 & Ng82a & \\
\hline $\mathrm{Rb}$ & 37 & & & & & & & & & & & $1.50 \mathrm{E}-02$ & NUREG/CR-5512 & & \\
\hline Re & 75 & & & & & & & & & & & $8.00 \mathrm{E}-03$ & PNNL-13421 & Ng68 & \\
\hline $\mathrm{Rh}$ & 45 & & & & & & & & & & & $2.00 \mathrm{E}-03$ & PNNL-13421 & Ng79 & \\
\hline $\mathrm{Rn}$ & 86 & & & & & & & & & & & & & & \\
\hline Ru & 44 & & & & & & & & & & & $5.00 \mathrm{E}-02$ & PNNL-13421 & Co90 & \\
\hline Ru & 44 & & & & & & & & & & & $2.00 \mathrm{E}-03$ & NUREG/CR-5512 & & \\
\hline $\mathrm{Ru}$ & 44 & & & $1.70 \mathrm{E}-04$ & & & & & & & & & & & Beresford et al. (2007) \\
\hline Ru & 44 & & & & & $3.70 \mathrm{E}-03$ & & $2.30 \mathrm{E}-03$ & & & & & & & Howard et al. (2009) \\
\hline Ru & 44 & & & & & $2.10 \mathrm{E}-03$ & & & & & & & & & Howard et al. (2009) \\
\hline Ru & 44 & 3 & $2.20 \mathrm{E}-03$ & $6.40 \mathrm{E}-03$ & & & & & $1.80 \mathrm{E}+00$ & $3.30 \mathrm{E}-03$ & & & TRS_472 & & \\
\hline $\mathrm{s}$ & 16 & & & & & & & & & & & $2.00 \mathrm{E}-01$ & PNNL-13421 & IAEA87 & \\
\hline $\mathrm{S}$ & 16 & . & & & & & & & & & & $1.00 \mathrm{E}-01$ & NUREG/CR-5512 & & \\
\hline $\mathrm{s}$ & 16 & ( & & & & $1.70 \mathrm{E}+00$ & & $4.70 \mathrm{E}-01$ & & & & & & & Howard et al. (2009) \\
\hline sb & 51 & & & & & & & & & & & $1.00 \mathrm{E}-03$ & PNNL-13421 & IAEA87 & \\
\hline Sb & 51 & & & & & $1.20 \mathrm{E}-03$ & & & & & & & & & Howard et al. (2009) \\
\hline sb & 51 & 2 & $1.10 \mathrm{E}-03$ & $1.30 \mathrm{E}-03$ & & $1.20 \mathrm{E}-03$ & & & & & & & TRS_472 & & \\
\hline Sc & 21 & & & & & & & & & & & $1.50 \mathrm{E}-02$ & PNNL-13421 & Ng68 & \\
\hline Se & 34 & & & & & & & & & & & $1.50 \mathrm{E}-02$ & PNNL-13421 & Ng68 & \\
\hline $\mathrm{Si}$ & 14 & & & & & & & & & & & $4.00 \mathrm{E}-05$ & PNNL-13421 & Ng68 & \\
\hline Sm & 62 & & & & & & & & & & & $2.00 \mathrm{E}-05$ & PNNL-13421 & CEC87 & \\
\hline Sm & 62 & & & & & & & & & & & $5.00 \mathrm{E}-03$ & NUREG/CR-5512 & & \\
\hline Sn & 50 & & & & & & & & & & & $8.00 \mathrm{E}-02$ & PNNL-13421 & Ng68 & \\
\hline $\mathrm{Sr}$ & 38 & & & & & & & & & & & $8.00 \mathrm{E}-03$ & PNNL-13421 & Co90 & \\
\hline $\mathrm{Sr}$ & 38 & & & & & & & & & & & $3.00 \mathrm{E}-04$ & NUREG/CR-5512 & & \\
\hline $\mathrm{Sr}$ & 38 & & & & & $2.10 \mathrm{E}-03$ & & $2.20 \mathrm{E}-03$ & & & & & & & Howard et al. (2009) \\
\hline $\mathrm{Sr}$ & 38 & & & & & $3.00 \mathrm{E}-03$ & & $5.10 \mathrm{E}-03$ & & & & & & & Howard et al. (2009) \\
\hline $\mathrm{Sr}$ & 38 & & & & & $1.70 \mathrm{E}-03$ & & 7.50E-04 & & & & & & & Howard et al. (2009) \\
\hline $\mathrm{Sr}$ & 38 & 35 & $2.00 \mathrm{E}-04$ & $9.20 \mathrm{E}-03$ & & & & & $2.90 \mathrm{E}+00$ & $1.30 \mathrm{E}-03$ & & & TRS_472 & & \\
\hline Та & 73 & & & & & & & & & & & $3.00 \mathrm{E}-07$ & PNNL-13421 & Jo88 & \\
\hline Та & 73 & & & & & & & & & & & $6.00 \mathrm{E}-04$ & NUREG/CR-5512 & & \\
\hline $\mathrm{Tb}$ & 65 & & & & & & & & & & & $2.00 \mathrm{E}-05$ & PNNL-13421 & CEC87 & \\
\hline $\mathrm{Tb}$ & 65 & & & & & & & & & & & $4.50 \mathrm{E}-03$ & NUREG/CR-5512 & & \\
\hline Tc & 43 & & & & & & & & & & & $1.00 \mathrm{E}-04$ & PNNL-13421 & Bi89 & \\
\hline Tc & 43 & & & & & & & & & & & $8.50 \mathrm{E}-03$ & NUREG/CR-5512 & & \\
\hline Tc & 43 & & $7.50 \mathrm{E}-05$ & $7.50 \mathrm{E}-03$ & & & & & & & & & & & Thorne (2003) \\
\hline Tc & 43 & & 7.50E-04 & 7.50E-03 & & & & & & & & & & & Thorne (2003) \\
\hline $\mathrm{Te}$ & 52 & & & & & & & & & & & $7.00 \mathrm{E}-03$ & PNNL-13421 & Jo88 & \\
\hline $\mathrm{Te}$ & 52 & & & & & & & & & & & $1.50 \mathrm{E}-02$ & NUREG/CR-5512 & & \\
\hline $\mathrm{Te}$ & 52 & & & & & $7.00 \mathrm{E}-03$ & & & & & & & & & Howard et al. (2009) \\
\hline $\mathrm{Te}$ & 52 & & & & & $2.40 \mathrm{E}-03$ & & & & & & & & & Howard et al. (2009) \\
\hline
\end{tabular}


Table B.1. (contd)

\begin{tabular}{|c|c|c|c|c|c|c|c|c|c|c|c|c|c|c|c|}
\hline Element & Atomic\# & $\mathrm{N}$ & Min & Max & $\mathrm{AVG}$ & Mean & Wmean & STD & GSD & GM & WGM & MED & Document & Reference\# & Reference \\
\hline $\mathrm{Te}$ & 52 & 1 & & & & $7.00 \mathrm{E}-03$ & & & & & & & TRS_472 & & \\
\hline Th & 90 & & & & & & & & & & & $4.00 \mathrm{E}-05$ & PNNL-13421 & Co90 & \\
\hline Th & 90 & & & & & & & & & & & $6.00 \mathrm{E}-06$ & NUREG/CR-5512 & & \\
\hline Th & 90 & & & & & 3.50E-04 & & 3.30E-04 & & & & & & & Howard et al. (2009) \\
\hline Th & 90 & 6 & $4.00 \mathrm{E}-05$ & $9.60 \mathrm{E}-04$ & & & & & $2.90 \mathrm{E}+00$ & $2.30 \mathrm{E}-04$ & & & TRS_472 & & \\
\hline $\mathrm{Ti}$ & 22 & & & & & & & & & & & & & & \\
\hline $\mathrm{Tl}$ & 81 & & & & & & & & & & & $4.00 \mathrm{E}-02$ & PNNL-13421 & Ng68 & \\
\hline $\mathrm{Tm}$ & 69 & & & & & & & & & & & & & & \\
\hline $\mathrm{U}$ & 92 & & & & & & & & & & & $3.00 \mathrm{E}-04$ & PNNL-13421 & Cr90 & \\
\hline $\mathrm{U}$ & 92 & & & & & & & & & & & $2.00 \mathrm{E}-04$ & NUREG/CR-5512 & & \\
\hline $\mathrm{U}$ & 92 & & $8.00 \mathrm{E}-01$ & $2.00 \mathrm{E}+00$ & & & & & & & & & & & Thorne (2003) \\
\hline $\mathrm{U}$ & 92 & & $9.00 \mathrm{E}-01$ & $2.20 \mathrm{E}+00$ & & & & & & & & & & & Thorne (2003) \\
\hline $\mathrm{U}$ & 92 & & & & & $4.20 \mathrm{E}-04$ & & $2.00 \mathrm{E}-04$ & & & & & & & Howard et al. (2009) \\
\hline $\mathrm{U}$ & 92 & 3 & $2.50 \mathrm{E}-04$ & $6.30 \mathrm{E}-04$ & & & & & $1.60 \mathrm{E}+00$ & $3.90 \mathrm{E}-04$ & & & TRS_472 & & \\
\hline $\mathrm{V}$ & 23 & & & & & & & & & & & & & & \\
\hline $\mathrm{W}$ & 74 & & & & & & & & & & & $4.00 \mathrm{E}-02$ & PNNL-13421 & Ng82a & \\
\hline W & 74 & & & & & & & & & & & $4.50 \mathrm{E}-02$ & NUREG/CR-5512 & & \\
\hline Xe & 54 & & & & & & & & & & & & & & Thorne (2003) \\
\hline $\mathrm{Y}$ & 39 & & & & & & & & & & & $1.00 \mathrm{E}-03$ & PNNL-13421 & Ng82a & \\
\hline $\mathrm{Y}$ & 39 & & & & & & & & & & & $3.00 \mathrm{E}-04$ & NUREG/CR-5512 & & \\
\hline Y & 39 & & & & & $5.40 \mathrm{E}-02$ & & & & & & & & & Howard et al. (2009) \\
\hline $\mathrm{Yb}$ & 70 & & & & & & & & & & & & & & \\
\hline $\mathrm{Zn}$ & 30 & & & & & & & & & & & $1.00 \mathrm{E}-01$ & PNNL-13421 & Ng82a & \\
\hline $\mathrm{Zn}$ & 30 & & & & & $2.60 \mathrm{E}-01$ & & $2.40 \mathrm{E}-01$ & & & & & & & Howard et al. (2009) \\
\hline $\mathrm{Zn}$ & 30 & & & & & $5.90 \mathrm{E}-02$ & & $4.70 \mathrm{E}-02$ & & & & & & & Howard et al. (2009) \\
\hline $\mathrm{Zn}$ & 30 & 6 & 4.00E-02 & $6.30 \mathrm{E}+00$ & & & & & $3.20 \mathrm{E}+00$ & $1.60 \mathrm{E}-01$ & & & TRS_472 & & \\
\hline $\mathrm{Zr}$ & 40 & & & & & & & & & & & $1.00 \mathrm{E}-06$ & PNNL-13421 & Jo88 & \\
\hline $\mathrm{Zr}$ & 40 & & & & & & & & & & & $5.50 \mathrm{E}-03$ & NUREG/CR-5512 & & \\
\hline $\mathrm{Zr}$ & 40 & & & & & $1.20 \mathrm{E}-06$ & & & & & & & & & Howard et al. (2009) \\
\hline $\mathrm{Zr}$ & 40 & & & & & $2.00 \mathrm{E}-05$ & & & & & & & & & Howard et al. (2009) \\
\hline $\mathrm{Zr}$ & 40 & 1 & & & & $1.20 \mathrm{E}-06$ & & & & & & & TRS_472 & & \\
\hline
\end{tabular}



Table B.2. Feed to Milk Transfer Factor, d/L (CLFMK)

\begin{tabular}{|c|c|c|c|c|c|c|c|c|c|c|c|c|c|c|c|}
\hline Element & Atomic\# & $\mathrm{N}$ & Min & Max & $\mathrm{AVG}$ & Mean & Wmean & STD & GSD & GM & MED & 95\%ile & Document & Reference\# & Reference \\
\hline Ac & 89 & & & & & & & & & & $2.00 \mathrm{E}-05$ & & PNNL-13421 & Ng77 & \\
\hline $\mathrm{Ag}$ & 47 & & & & & & & & & & $5.00 \mathrm{E}-05$ & & PNNL-13421 & CEC87 & \\
\hline $\mathrm{Ag}$ & 47 & & & & & & & & & & $2.00 \mathrm{E}-02$ & & NUREG/CR-5512 & & \\
\hline $\mathrm{Ag}$ & 47 & & & & & & & & & & $1.90 \mathrm{E}-02$ & & & & Ng et al. (1979) \\
\hline $\mathrm{Al}$ & 13 & & & & & & & & & & $2.00 \mathrm{E}-04$ & & & & Ng et al. (1979) \\
\hline $\mathrm{Am}$ & 95 & & & & & & & & & & $1.50 \mathrm{E}-06$ & & PNNL-13421 & Co90 & \\
\hline $\mathrm{Am}$ & 95 & 1 & & & & $4.20 \mathrm{E}-07$ & & & & & & & TRS_472 & & \\
\hline $\mathrm{Am}$ & 95 & & & & & & & & & & 4.00E-07 & & NUREG/CR-5512 & & \\
\hline $\mathrm{Am}$ & 95 & & & & & & & & & & $4.00 \mathrm{E}-07$ & & & & Ng et al. (1979) \\
\hline Am & 95 & & & & & 4.20E-07 & & & & & & & & & Howard et al. (2009) \\
\hline $\mathrm{Am}$ & 95 & & & & & $6.90 \mathrm{E}-06$ & & & & & & & & & Howard et al. (2009) \\
\hline $\mathrm{Ar}$ & 18 & & & & & & & & & & & & & & \\
\hline As & 33 & & & & & & & & & & $6.00 \mathrm{E}-05$ & & PNNL-13421 & Ng77 & \\
\hline As & 33 & & & & & & & & & & $6.20 \mathrm{E}-05$ & & & & Ng et al. (1979) \\
\hline At & 85 & & & & & & & & & & & & & & \\
\hline $\mathrm{Au}$ & 79 & & & & & & & & & & $5.50 \mathrm{E}-06$ & & PNNL-13421 & Ng77 & \\
\hline $\mathrm{Au}$ & 79 & & & & & & & & & & $5.30 \mathrm{E}-06$ & & & & Ng et al. (1979) \\
\hline $\mathrm{Ba}$ & 56 & & & & & & & & & & $4.00 \mathrm{E}-04$ & & PNNL-13421 & Jo88 & \\
\hline $\mathrm{Ba}$ & 56 & 15 & $3.80 \mathrm{E}-05$ & 7.30E-04 & & & & & $2.70 \mathrm{E}+00$ & $1.60 \mathrm{E}-04$ & & & TRS_472 & & \\
\hline $\mathrm{Ba}$ & 56 & & & & & & & & & & $3.50 \mathrm{E}-04$ & & NUREG/CR-5512 & & \\
\hline $\mathrm{Ba}$ & 56 & & & & & & & & & & $3.50 \mathrm{E}-04$ & & & & Ng et al. (1979) \\
\hline $\mathrm{Ba}$ & 56 & & $3.80 \mathrm{E}-05$ & 7.30E-04 & & $2.50 \mathrm{E}-04$ & & 2.40E-04 & $2.70 \mathrm{E}+00$ & $1.60 \mathrm{E}-04$ & & & & & Howard et al. (2009) \\
\hline $\mathrm{Ba}$ & 56 & & $2.10 \mathrm{E}-03$ & $1.50 \mathrm{E}-01$ & & $5.40 \mathrm{E}-02$ & & $8.70 \mathrm{E}-02$ & $9.90 \mathrm{E}+00$ & $1.10 \mathrm{E}-02$ & & & & & Howard et al. (2009) \\
\hline $\mathrm{Ba}$ & 56 & & & & & $4.10 \mathrm{E}-02$ & & & & & & & & & Howard et al. (2009) \\
\hline $\mathrm{Be}$ & 4 & & & & & & & & & & $9.00 \mathrm{E}-07$ & & PNNL-13421 & Ng77 & \\
\hline $\mathrm{Be}$ & 4 & 1 & & & & $8.30 \mathrm{E}-07$ & & & & & & & TRS_472 & & \\
\hline $\mathrm{Be}$ & 4 & & & & & & & & & & $9.00 \mathrm{E}-07$ & & & & Ng et al. (1979) \\
\hline $\mathrm{Be}$ & 4 & & & & & $8.30 \mathrm{E}-07$ & & & & & & & & & Howard et al. (2009) \\
\hline $\mathrm{Bi}$ & 83 & & & & & & & & & & $5.00 \mathrm{E}-04$ & & PNNL-13421 & Ng77 & \\
\hline $\mathrm{Bk}$ & 97 & & & & & & & & & & & & & & \\
\hline $\mathrm{Br}$ & 35 & & & & & & & & & & $2.00 \mathrm{E}-02$ & & PNNL-13421 & Ng77 & \\
\hline $\mathrm{Br}$ & 35 & & & & & & & & & & $2.00 \mathrm{E}-02$ & & & & Ng et al. (1979) \\
\hline C & 6 & & & & & & & & & & $1.50 \mathrm{E}-02$ & & & & Ng et al. (1979) \\
\hline C & 6 & & $1.30 \mathrm{E}-01$ & $2.10 \mathrm{E}-01$ & & & & & & & & & & & Galeriu et al. (2007) \\
\hline $\mathrm{Ca}$ & 20 & & & & & & & & & & $3.00 \mathrm{E}-03$ & & PNNL-13421 & СТ83 & \\
\hline $\mathrm{Ca}$ & 20 & 15 & $4.00 \mathrm{E}-03$ & 2.50E-02 & & & & & $1.70 \mathrm{E}+00$ & $1.00 \mathrm{E}-02$ & & & TRS_472 & & \\
\hline $\mathrm{Ca}$ & 20 & & & & & & & & & & $1.00 \mathrm{E}-02$ & & NUREG/CR-5512 & & \\
\hline $\mathrm{Ca}$ & 20 & & & & & & & & & & $1.10 \mathrm{E}-02$ & & & & Ng et al. (1979) \\
\hline $\mathrm{Ca}$ & 20 & & $4.00 \mathrm{E}-03$ & $2.50 \mathrm{E}-03$ & & $1.20 \mathrm{E}-02$ & & $5.90 \mathrm{E}-03$ & & & & & & & Howard et al. (2009) \\
\hline $\mathrm{Ca}$ & 20 & & $1.20 \mathrm{E}-02$ & $1.40 \mathrm{E}-01$ & & $8.30 \mathrm{E}-02$ & & 3.40E-02 & & & & & & & Howard et al. (2009) \\
\hline $\mathrm{Cd}$ & 48 & & & & & & & & & & $1.00 \mathrm{E}-03$ & & PNNL-13421 & Ng77 & \\
\hline $\mathrm{Cd}$ & 48 & 8 & $1.80 \mathrm{E}-06$ & $8.40 \mathrm{E}-03$ & & & & & $1.50 \mathrm{E}+01$ & $1.90 \mathrm{E}-04$ & & & TRS_472 & & \\
\hline $\mathrm{Cd}$ & 48 & & & & & & & & & & $1.00 \mathrm{E}-03$ & & & & Ng et al. (1979) \\
\hline $\mathrm{Cd}$ & 48 & & $1.80 \mathrm{E}-06$ & $8.40 \mathrm{E}-03$ & & $1.90 \mathrm{E}-03$ & & $3.40 \mathrm{E}-03$ & & & & & & & Howard et al. (2009) \\
\hline $\mathrm{Cd}$ & 48 & & & & & $1.60 \mathrm{E}-02$ & & & & & & & & & Howard et al. (2009) \\
\hline $\mathrm{Cd}$ & 48 & & & & & $4.90 \mathrm{E}-02$ & & & & & & & & & Howard et al. (2009) \\
\hline $\mathrm{Ce}$ & 58 & & & & & & & & & & $3.00 \mathrm{E}-05$ & & PNNL-13421 & CEC87 & \\
\hline Ce & 58 & 6 & $2.00 \mathrm{E}-06$ & $1.30 \mathrm{E}-04$ & & & & & $5.80 \mathrm{E}+00$ & 2.00E-05 & & & TRS_472 & & \\
\hline
\end{tabular}


Table B.2. (contd)

\begin{tabular}{|c|c|c|c|c|c|c|c|c|c|c|c|c|c|c|c|}
\hline Element & Atomic\# & $\mathrm{N}$ & Min & Max & AVG & Mean & Wmean & STD & GSD & GM & MED & 95\%ile & Document & Reference\# & Reference \\
\hline $\mathrm{Ce}$ & 58 & & & & & & & & & & $2.00 \mathrm{E}-05$ & & NUREG/CR-5512 & & \\
\hline $\mathrm{Ce}$ & 58 & & & & & & & & & & 2.00E-05 & & & & Ng et al. (1979) \\
\hline $\mathrm{Ce}$ & 58 & & $2.00 \mathrm{E}-06$ & $1.30 \mathrm{E}-04$ & & $4.70 \mathrm{E}-05$ & & $4.90 \mathrm{E}-05$ & & & & & & & Howard et al. (2009) \\
\hline $\mathrm{Ce}$ & 58 & & & & & 4.00E-05 & & & & & & & & & Howard et al. (2009) \\
\hline $\mathrm{Cf}$ & 98 & & & & & & & & & & $1.50 \mathrm{E}-06$ & & PNNL-13421 & Co90 & \\
\hline $\mathrm{Cf}$ & 98 & & & & & & & & & & 7.50E-07 & & NUREG/CR-5512 & & \\
\hline $\mathrm{Cl}$ & 17 & & & & & & & & & & $1.70 \mathrm{E}-02$ & & PNNL-13421 & Bi89 & \\
\hline $\mathrm{Cl}$ & 17 & & & & & & & & & & $1.50 \mathrm{E}-02$ & & NUREG/CR-5512 & & \\
\hline $\mathrm{Cl}$ & 17 & & & & & & & & & & $1.70 \mathrm{E}-02$ & & & & Ng et al. (1979) \\
\hline $\mathrm{Cm}$ & 96 & & & & & & & & & & $9.60 \mathrm{E}-05$ & & PNNL-13421 & Ng77 & \\
\hline $\mathrm{Cm}$ & 96 & & & & & & & & & & $2.00 \mathrm{E}-05$ & & NUREG/CR-5512 & & \\
\hline Co & 27 & & & & & & & & & & 3.00E-04 & & PNNL-13421 & Ba84 & \\
\hline Co & 27 & 4 & $6.00 \mathrm{E}-05$ & $3.00 \mathrm{E}-04$ & & & & & $2.00 \mathrm{E}+00$ & $1.10 \mathrm{E}-04$ & & & TRS_472 & & \\
\hline Co & 27 & & & & & & & & & & $2.00 \mathrm{E}-03$ & & NUREG/CR-5512 & & \\
\hline Co & 27 & & & & & & & & & & $1.80 \mathrm{E}-03$ & & & & Ng et al. (1979) \\
\hline Co & 27 & . & $6.00 \mathrm{E}-05$ & 3.00E-04 & & $1.30 \mathrm{E}-04$ & & $1.10 \mathrm{E}-04$ & & & & & & & Howard et al. (2009) \\
\hline Co & 27 & & & & & $5.00 \mathrm{E}-03$ & & & & & & & & & Howard et al. (2009) \\
\hline Co & 27 & & & & & $2.70 \mathrm{E}-03$ & & & & & & & & & Howard et al. (2009) \\
\hline $\mathrm{Cr}$ & 24 & & & & & & & & & & $1.00 \mathrm{E}-05$ & & PNNL-13421 & Va84 & \\
\hline $\mathrm{Cr}$ & 24 & 3 & $1.00 \mathrm{E}-05$ & 4.30E-03 & & & & & $2.60 \mathrm{E}+01$ & $4.30 \mathrm{E}-04$ & & & TRS_472 & & \\
\hline $\mathrm{Cr}$ & 24 & & & & & & & & & & $1.50 \mathrm{E}-03$ & & NUREG/CR-5512 & & \\
\hline $\mathrm{Cr}$ & 24 & & & & & & & & & & $1.60 \mathrm{E}-03$ & & & & Ng et al. (1979) \\
\hline $\mathrm{Cr}$ & 24 & & $2.90 \mathrm{E}-03$ & 2.80E-02 & & $1.50 \mathrm{E}-02$ & & & & & & & & & Howard et al. (2009) \\
\hline $\mathrm{Cr}$ & 24 & & $1.00 \mathrm{E}-05$ & 4.30E-03 & & $2.00 \mathrm{E}-03$ & & $2.10 \mathrm{E}-03$ & & & & & & & Howard et al. (2009) \\
\hline $\mathrm{Cr}$ & 24 & & & & & $2.00 \mathrm{E}-02$ & & & & & & & & & Howard et al. (2009) \\
\hline Cs & 55 & & & & & & & & & & $7.90 \mathrm{E}-03$ & & PNNL-13421 & Co90 & \\
\hline Cs & 55 & 288 & $6.00 \mathrm{E}-04$ & $6.80 \mathrm{E}-02$ & & & & & $2.00 \mathrm{E}+00$ & $4.60 \mathrm{E}-03$ & & & TRS_472 & & \\
\hline Cs & 55 & & & & & & & & & & $7.00 \mathrm{E}-03$ & & NUREG/CR-5512 & & \\
\hline Cs & 55 & & & & & & & & & $2.70 \mathrm{E}-03$ & & & & & Tsukada et al. (2003) \\
\hline Cs & 55 & & & & & & & & & & 7.10E-03 & & & & Ng et al. (1979) \\
\hline Cs & 55 & & $6.00 \mathrm{E}-04$ & $6.80 \mathrm{E}-02$ & & $6.10 \mathrm{E}-03$ & & $6.30 \mathrm{E}-03$ & & & & & & & Howard et al. (2009) \\
\hline Cs & 55 & & $7.00 \mathrm{E}-03$ & 3.30E-01 & & $1.30 \mathrm{E}-01$ & & $8.00 \mathrm{E}-02$ & & & & & & & Howard et al. (2009) \\
\hline Cs & 55 & & & & & 7.70E-02 & & & & & & & & & Howard et al. (2009) \\
\hline Cs & 55 & & $9.00 \mathrm{E}-04$ & 4.50E-03 & & & & & & & & & & & Gastbergera et al. (2001) \\
\hline Cs & 55 & & & & & $4.48 \mathrm{E}-03$ & & $3.05 \mathrm{E}-03$ & & & & & & & Panchal et al. (2011) \\
\hline $\mathrm{Cu}$ & 29 & & & & & & & & & & $2.00 \mathrm{E}-03$ & & PNNL-13421 & IAEA87 & \\
\hline $\mathrm{Cu}$ & 29 & & & & & & & & & & $1.50 \mathrm{E}-03$ & & NUREG/CR-5512 & & \\
\hline $\mathrm{Cu}$ & 29 & & & & & & & & & & $1.70 \mathrm{E}-03$ & & & & Ng et al. (1979) \\
\hline Dy & 66 & & & & & & & & & & $3.00 \mathrm{E}-05$ & & PNNL-13421 & CEC87 & \\
\hline Dy & 66 & & & & & & & & & & 2.00E-05 & & NUREG/CR-5512 & & \\
\hline Er & 68 & & & & & & & & & & 2.00E-05 & & NUREG/CR-5512 & & \\
\hline Es & 99 & & & & & & & & & & & & & & \\
\hline $\mathrm{Eu}$ & 63 & & & & & & & & & & 3.00E-05 & & PNNL-13421 & CEC87 & \\
\hline $\mathrm{Eu}$ & 63 & & & & & & & & & & $2.00 \mathrm{E}-05$ & & NUREG/CR-5512 & & \\
\hline $\mathrm{F}$ & 9 & & & & & & & & & & $1.00 \mathrm{E}-03$ & & PNNL-13421 & Ng77 & \\
\hline $\mathrm{F}$ & 9 & & & & & & & & & & $1.10 \mathrm{E}-03$ & & & & Ng et al. (1979) \\
\hline $\mathrm{Fe}$ & 26 & & & & & & & & & & $3.00 \mathrm{E}-05$ & & PNNL-13421 & Va84 & \\
\hline $\mathrm{Fe}$ & 26 & 7 & $1.00 \mathrm{E}-05$ & $9.70 \mathrm{E}-05$ & & & & & $2.00 \mathrm{E}+00$ & $3.50 \mathrm{E}-04$ & & & TRS_472 & & \\
\hline $\mathrm{Fe}$ & 26 & & & & & & & & & & 2.50E-04 & & NUREG/CR-5512 & & \\
\hline $\mathrm{Fe}$ & 26 & & & & & & & & & & 2.70E-04 & & & & Ng et al. (1979) \\
\hline $\mathrm{Fe}$ & 26 & & $1.00 \mathrm{E}-05$ & $9.70 \mathrm{E}-05$ & & $4.20 \mathrm{E}-05$ & & 2.70E-05 & & & & & & & Howard et al. (2009) \\
\hline Fm & 100 & & & & & & & & & & & & & & \\
\hline
\end{tabular}


Table B.2. (contd)

\begin{tabular}{|c|c|c|c|c|c|c|c|c|c|c|c|c|c|c|c|}
\hline Element & Atomic\# & $\mathrm{N}$ & Min & Max & AVG & Mean & Wmean & STD & GSD & GM & MED & 95\%ile & Document & Reference\# & Reference \\
\hline $\mathrm{Fr}$ & 87 & & & & & & & & & & & & & & \\
\hline $\mathrm{Ga}$ & 31 & & & & & & & & & & $5.00 \mathrm{E}-05$ & & PNNL-13421 & Ng77 & \\
\hline $\mathrm{Gd}$ & 64 & & & & & & & & & & 3.00E-05 & & PNNL-13421 & CEC87 & \\
\hline $\mathrm{Gd}$ & 64 & & & & & & & & & & 2.00E-05 & & NUREG/CR-5512 & & \\
\hline $\mathrm{Ge}$ & 32 & & & & & & & & & & & & & & \\
\hline $\mathrm{H}$ & 1 & & & & & & & & & & $1.50 \mathrm{E}-02$ & & PNNL-13421 & Va83 & \\
\hline $\mathrm{H}$ & 1 & & & & & & & & & & $1.40 \mathrm{E}-02$ & & & & Ng et al. (1979) \\
\hline $\mathrm{H}$ & 1 & & 7.60E-01 & $9.20 \mathrm{E}-01$ & & & & & & & & & & & Galeriu et al. (2007) \\
\hline $\mathrm{Hf}$ & 72 & & & & & & & & & & 5.50E- -07 & & PNNL-13421 & Jo88 & \\
\hline $\mathrm{Hf}$ & 72 & . & & & & & & & & & $5.00 \mathrm{E}-06$ & & NUREG/CR-5512 & & \\
\hline $\mathrm{Hg}$ & 80 & & & & & & & & & & 4.70E-04 & & PNNL-13421 & Ng82a & \\
\hline $\mathrm{Hg}$ & 80 & & & & & & & & & & 4.50E-04 & & NUREG/CR-5512 & & \\
\hline $\mathrm{Hg}$ & 80 & & & & & & & & & & 4.70E- -04 & & & & Ng et al. (1979) \\
\hline Ho & 67 & & & & & & & & & & 3.00E-05 & & PNNL-13421 & CEC87 & \\
\hline Ho & 67 & & & & & & & & & & $2.00 \mathrm{E}-05$ & & NUREG/CR-5512 & & \\
\hline I & 53 & & & & & & & & & & $9.00 \mathrm{E}-03$ & & PNNL-13421 & Sn94 & \\
\hline I & 53 & 104 & $4.00 \mathrm{E}-04$ & $2.50 \mathrm{E}-02$ & & & & & $2.40 \mathrm{E}+00$ & $5.40 \mathrm{E}-03$ & & & TRS_472 & & \\
\hline I & 53 & & & & & & & & & & 1.00E-02 & & NUREG/CR-5512 & & \\
\hline I & 53 & & $2.00 \mathrm{E}-03$ & $5.00 \mathrm{E}-02$ & & & & & & & & & & & Thorne (2003) \\
\hline I & 53 & & & & & & & & & & $9.90 \mathrm{E}-03$ & & & & Ng et al. (1979) \\
\hline I & 53 & & $2.00 \mathrm{E}-02$ & $1.00 \mathrm{E}+00$ & & & & & & & & & & & Thorne (2003) \\
\hline I & 53 & & 4.00E-04 & $2.50 \mathrm{E}-02$ & & $9.10 \mathrm{E}-03$ & & $7.00 \mathrm{E}-03$ & & & & & & & Howard et al. (2009) \\
\hline I & 53 & & $2.70 \mathrm{E}-02$ & $7.70 \mathrm{E}-01$ & & 3.30E-01 & & $2.30 \mathrm{E}-01$ & & & & & & & Howard et al. (2009) \\
\hline I & 53 & & & & & $3.50 \mathrm{E}-01$ & & & & & & & & & Howard et al. (2009) \\
\hline In & 49 & & & & & & & & & & $2.00 \mathrm{E}-04$ & & PNNL-13421 & NCRP86 & \\
\hline In & 49 & & & & & & & & & & $1.00 \mathrm{E}-04$ & & NUREG/CR-5512 & & \\
\hline Ir & 77 & & & & & & & & & & 2.00E-06 & & PNNL-13421 & Ng77 & \\
\hline Ir & 77 & & & & & & & & & & 2.00E-06 & & & & Ng et al. (1979) \\
\hline $\mathrm{K}$ & 19 & & & & & & & & & & $7.20 \mathrm{E}-02$ & & PNNL-13421 & Ng82a & \\
\hline $\mathrm{K}$ & 19 & & & & & & & & & & 7.00E-03 & & NUREG/CR-5512 & & \\
\hline $\mathrm{K}$ & 19 & & & & & & & & & & 7.20E-03 & & & & Ng et al. (1979) \\
\hline K & 19 & & & & & $5.30 \mathrm{E}-03$ & & $3.08 \mathrm{E}-03$ & & & & & & & Panchal et al. (2011) \\
\hline $\mathrm{Kr}$ & 36 & & & & & & & & & & & & & & \\
\hline $\mathrm{La}$ & 57 & & & & & & & & & & $2.00 \mathrm{E}-05$ & & PNNL-13421 & Ng77 & \\
\hline $\mathrm{Lu}$ & 71 & & & & & & & & & & & & & & \\
\hline Md & 101 & & & & & & & & & & & & & & \\
\hline $\mathrm{Mg}$ & 12 & & & & & & & & & & $3.90 \mathrm{E}-03$ & & PNNL-13421 & Ng82a & \\
\hline $\mathrm{Mg}$ & 12 & & & & & & & & & & 4.00E-03 & & NUREG/CR-5512 & & \\
\hline $\mathrm{Mg}$ & 12 & & & & & & & & & & $3.90 \mathrm{E}-03$ & & & & Ng et al. (1979) \\
\hline $\mathrm{Mn}$ & 25 & & & & & & & & & & $5.00 \mathrm{E}-05$ & & PNNL-13421 & Va84 & \\
\hline $\mathrm{Mn}$ & 25 & 4 & 7.00E-06 & $3.30 \mathrm{E}-04$ & & & & & $4.90 \mathrm{E}+00$ & 4.10E-05 & & & TRS_472 & & \\
\hline $\mathrm{Mn}$ & 25 & & & & & & & & & & $3.50 \mathrm{E}-04$ & & NUREG/CR-5512 & & \\
\hline $\mathrm{Mn}$ & 25 & & & & & & & & & & 3.30E-04 & & & & Ng et al. (1979) \\
\hline $\mathrm{Mn}$ & 25 & & 3.30E-04 & 7.00E-06 & & $1.00 \mathrm{E}-04$ & & $1.50 \mathrm{E}-04$ & & & & & & & Howard et al. (2009) \\
\hline $\mathrm{Mn}$ & 25 & & & & & $2.40 \mathrm{E}-03$ & & & & & & & & & Howard et al. (2009) \\
\hline Mo & 42 & & & & & & & & & & $1.70 \mathrm{E}-03$ & & PNNL-13421 & Jo88 & \\
\hline Mo & 42 & 7 & 4.30Е-04 & $5.20 \mathrm{E}-03$ & & & & & $2.30 \mathrm{E}+00$ & $1.10 \mathrm{E}-03$ & & & TRS_472 & & \\
\hline Mo & 42 & & & & & & & & & & $1.50 \mathrm{E}-03$ & & NUREG/CR-5512 & & \\
\hline Mo & 42 & & & & & & & & & & $1.40 \mathrm{E}-03$ & & & & Ng et al. (1979) \\
\hline Mo & 42 & & 4.30E-06 & $5.20 \mathrm{E}-03$ & & $1.50 \mathrm{E}-03$ & & $1.70 \mathrm{E}-03$ & & & & & & & Howard et al. (2009) \\
\hline Mo & 42 & & $5.00 \mathrm{E}-03$ & $1.10 \mathrm{E}-02$ & & 8.50E-03 & & $2.50 \mathrm{E}-03$ & & & & & & & Howard et al. (2009) \\
\hline
\end{tabular}


Table B.2. (contd)

\begin{tabular}{|c|c|c|c|c|c|c|c|c|c|c|c|c|c|c|c|}
\hline Element & Atomic\# & $\mathrm{N}$ & Min & Max & $\mathrm{AVG}$ & Mean & Wmean & STD & GSD & GM & MED & 95\%ile & Document & Reference\# & Reference \\
\hline $\mathrm{N}$ & 7 & & & & & & & & & & $2.50 \mathrm{E}-02$ & & PNNL-13421 & Ng77 & \\
\hline $\mathrm{N}$ & 7 & & & & & & & & & & 2.30E-02 & & & & Ng et al. (1979) \\
\hline $\mathrm{Na}$ & 11 & & & & & & & & & & $1.60 \mathrm{E}-02$ & & PNNL-13421 & IAEA94 & \\
\hline $\mathrm{Na}$ & 11 & 7 & $5.00 \mathrm{E}-03$ & $5.00 \mathrm{E}-03$ & & & & & $2.00 \mathrm{E}+00$ & $1.30 \mathrm{E}-02$ & & & TRS_472 & & \\
\hline $\mathrm{Na}$ & 11 & & & & & & & & & & 3.50E-02 & & NUREG/CR-5512 & & \\
\hline $\mathrm{Na}$ & 11 & & & & & & & & & & 3.50E-02 & & & & Ng et al. (1979) \\
\hline $\mathrm{Na}$ & 11 & & $5.00 \mathrm{E}-03$ & $5.00 \mathrm{E}-02$ & & $1.60 \mathrm{E}-02$ & & $1.50 \mathrm{E}-02$ & & & & & & & Howard et al. (2009) \\
\hline $\mathrm{Na}$ & 11 & & & & & $1.00 \mathrm{E}-01$ & & & & & & & & & Howard et al. (2009) \\
\hline $\mathrm{Nb}$ & 41 & & & & & & & & & & $4.10 \mathrm{E}-07$ & & PNNL-13421 & Jo88 & \\
\hline $\mathrm{Nb}$ & 41 & 1 & & & & 4.10E-07 & & & & & & & TRS_472 & & \\
\hline $\mathrm{Nb}$ & 41 & & & & & & & & & & $2.00 \mathrm{E}-02$ & & NUREG/CR-5512 & & \\
\hline $\mathrm{Nb}$ & 41 & & & & & $4.10 \mathrm{E}-07$ & & & & & & & & & Howard et al. (2009) \\
\hline $\mathrm{Nb}$ & 41 & 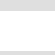 & & & & $6.40 \mathrm{E}-06$ & & & & & & & & & Howard et al. (2009) \\
\hline $\mathrm{Nd}$ & 60 & & & & & & & & & & $3.00 \mathrm{E}-05$ & & PNNL-13421 & CEC87 & \\
\hline $\mathrm{Nd}$ & 60 & & & & & & & & & & $2.00 \mathrm{E}-05$ & & NUREG/CR-5512 & & \\
\hline $\mathrm{Nd}$ & 60 & & & & & & & & & & $2.00 \mathrm{E}-05$ & & & & Ng et al. (1979) \\
\hline $\mathrm{Ne}$ & 10 & & & & & & & & & & & & & & \\
\hline $\mathrm{Ni}$ & 28 & & & & & & & & & & 1.60E-02 & & PNNL-13421 & Cr90 & \\
\hline $\mathrm{Ni}$ & 28 & 2 & $1.30 \mathrm{E}-03$ & & & $9.50 \mathrm{E}-04$ & & & $6.50 \mathrm{E}-04$ & & & & TRS_472 & & \\
\hline $\mathrm{Ni}$ & 28 & & & & & & & & & & $1.00 \mathrm{E}-03$ & & NUREG/CR-5512 & & \\
\hline $\mathrm{Ni}$ & 28 & & & & & & & & & & $1.00 \mathrm{E}-03$ & & & & Ng et al. (1979) \\
\hline $\mathrm{Ni}$ & 28 & & $6.50 \mathrm{E}-04$ & $1.30 \mathrm{E}-03$ & & $9.50 \mathrm{E}-04$ & & & & & & & & & Howard et al. (2009) \\
\hline $\mathrm{Ni}$ & 28 & & $3.20 \mathrm{E}-03$ & 1.60E-01 & & $8.30 \mathrm{E}-02$ & & & & & & & & & Howard et al. (2009) \\
\hline $\mathrm{Ni}$ & 28 & & & & & $1.00 \mathrm{E}-01$ & & & & & & & & & Howard et al. (2009) \\
\hline $\mathrm{Np}$ & 93 & & & & & & & & & & $5.00 \mathrm{E}-06$ & & PNNL-13421 & Ng82a & \\
\hline $\mathrm{O}$ & 8 & & & & & & & & & & & & & & \\
\hline \multicolumn{16}{|l|}{$\mathrm{OBT}$} \\
\hline Os & 76 & 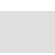 & & & & & & & & & $5.00 \mathrm{E}-03$ & & PNNL-13421 & Ng77 & \\
\hline $\mathrm{P}$ & 15 & & & & & & & & & & $1.60 \mathrm{E}-02$ & & PNNL-13421 & Ng82a & \\
\hline $\mathrm{P}$ & 15 & n/a & & & & $2.00 \mathrm{E}-02$ & & & & & & & TRS_472 & & \\
\hline $\mathrm{P}$ & 15 & & & & & & & & & & $1.50 \mathrm{E}-02$ & & NUREG/CR-5512 & & \\
\hline $\mathrm{P}$ & 15 & & & & & & & & & & $1.60 \mathrm{E}-02$ & & & & Ng et al. (1979) \\
\hline $\mathrm{Pa}$ & 91 & & & & & & & & & & 5.00E-06 & & PNNL-13421 & Ng77 & \\
\hline $\mathrm{Pb}$ & 82 & & & & & & & & & & $2.60 \mathrm{E}-04$ & & PNNL-13421 & Ng77 & \\
\hline $\mathrm{Pb}$ & 82 & 15 & $7.30 \mathrm{E}-06$ & $1.20 \mathrm{E}-03$ & & & & & $1.00 \mathrm{E}+00$ & $1.90 \mathrm{E}-04$ & & & TRS_472 & & \\
\hline $\mathrm{Pb}$ & 82 & & & & & & & & & & $2.50 \mathrm{E}-04$ & & NUREG/CR-5512 & & \\
\hline $\mathrm{Pb}$ & 82 & & & & & & & & & & 2.60E-04 & & & & Ng et al. (1979) \\
\hline $\mathrm{Pb}$ & 82 & & 7.30E-06 & $1.20 \mathrm{E}-03$ & & $3.30 \mathrm{E}-04$ & & 3.50E-04 & & & & & & & Howard et al. (2009) \\
\hline Pd & 46 & & & & & & & & & & $1.00 \mathrm{E}-02$ & & PNNL-13421 & Ng77 & \\
\hline $\mathrm{Pd}$ & 46 & & & & & $6.00 \mathrm{E}-03$ & & & & & & & & & Howard et al. (2009) \\
\hline $\mathrm{Pm}$ & 61 & & & & & & & & & & $3.00 \mathrm{E}-05$ & & PNNL-13421 & CEC87 & \\
\hline $\mathrm{Pm}$ & 61 & & & & & & & & & & $2.00 \mathrm{E}-05$ & & NUREG/CR-5512 & & \\
\hline $\mathrm{Pm}$ & 61 & & & & & 2.70E-05 & & & & & & & & & Howard et al. (2009) \\
\hline Po & 84 & & & & & & & & & & 3.40E-04 & & PNNL-13421 & Ng82a & \\
\hline Po & 84 & 4 & 8.90E-05 & $3.00 \mathrm{E}-04$ & & & & & $1.80 \mathrm{E}+00$ & $2.10 \mathrm{E}-04$ & & & TRS_472 & & \\
\hline Po & 84 & & & & & & & & & & $3.50 \mathrm{E}-04$ & & NUREG/CR-5512 & & \\
\hline Po & 84 & & 8.90E-05 & $3.00 \mathrm{E}-04$ & & $2.30 \mathrm{E}-04$ & & $9.70 \mathrm{E}-05$ & & & & & & & Howard et al. (2009) \\
\hline Po & 84 & & & & & $2.30 \mathrm{E}-03$ & & & & & & & & & Howard et al. (2009) \\
\hline Po & 84 & & & & & & & & & & 3.40E-04 & & & & Ng et al. (1979) \\
\hline $\operatorname{Pr}$ & 59 & & & & & & & & & & $3.00 \mathrm{E}-05$ & & PNNL-13421 & CEC87 & \\
\hline $\operatorname{Pr}$ & 59 & & & & & & & & & & 2.00E-05 & & NUREG/CR-5512 & & \\
\hline $\mathrm{Pt}$ & 78 & & & & & & & & & & & & & & \\
\hline
\end{tabular}


Table B.2. (contd)

\begin{tabular}{|c|c|c|c|c|c|c|c|c|c|c|c|c|c|c|c|}
\hline Element & Atomic\# & $\mathrm{N}$ & Min & Max & $\mathrm{AVG}$ & Mean & Wmean & STD & GSD & GM & MED & 95\% ile & Document & Reference\# & Reference \\
\hline $\mathrm{Pu}$ & 94 & & & & & & & & & & $1.10 \mathrm{E}-06$ & & PNNL-13421 & Co90 & \\
\hline $\mathrm{Pu}$ & 94 & $\mathrm{n} / \mathrm{a}$ & & & & $1.00 \mathrm{E}-05$ & & & & & & & TRS_472 & & \\
\hline $\mathrm{Pu}$ & 94 & & & & & & & & & & $1.00 \mathrm{E}-07$ & & NUREG/CR-5512 & & \\
\hline $\mathrm{Pu}$ & 94 & & & & & & & & & & $1.00 \mathrm{E}-07$ & & & & Ng et al. (1979) \\
\hline $\mathrm{Pu}$ & 94 & & & & & $1.00 \mathrm{E}-05$ & & & & & & & & & Howard et al. (2009) \\
\hline $\mathrm{Pu}$ & 94 & & & & & $1.00 \mathrm{E}-04$ & & & & & & & & & Howard et al. (2009) \\
\hline $\mathrm{Pu}$ & 94 & & & & & $5.80 \mathrm{E}-05$ & & $6.90 \mathrm{E}-05$ & & & & & & & Howard et al. (2007) \\
\hline $\mathrm{Ra}$ & 88 & & & & & & & & & & $1.30 \mathrm{E}-03$ & & PNNL-13421 & MB90 & \\
\hline $\mathrm{Ra}$ & 88 & 11 & $9.00 \mathrm{E}-04$ & $1.40 \mathrm{E}-03$ & & & & & $2.30 \mathrm{E}+00$ & $3.80 \mathrm{E}-04$ & & & TRS_472 & & \\
\hline $\mathrm{Ra}$ & 88 & & & & & & & & & & $4.50 \mathrm{E}-04$ & & NUREG/CR-5512 & & \\
\hline $\mathrm{Ra}$ & 88 & & & & & & & & & & 4.50E-04 & & & & Ng et al. (1979) \\
\hline $\mathrm{Ra}$ & 88 & & $9.00 \mathrm{E}-05$ & $1.40 \mathrm{E}-03$ & & $5.10 \mathrm{E}-05$ & & $3.80 \mathrm{E}-04$ & & & & & & & Howard et al. (2009) \\
\hline $\mathrm{Rb}$ & 37 & & & & & & & & & & $1.20 \mathrm{E}-02$ & & PNNL-13421 & Ng82a & \\
\hline $\mathrm{Rb}$ & 37 & & & & & & & & & & $1.00 \mathrm{E}-02$ & & NUREG/CR-5512 & & \\
\hline $\mathrm{Rb}$ & 37 & & & & & & & & & & $1.20 \mathrm{E}-02$ & & & & Ng et al. (1979) \\
\hline $\operatorname{Re}$ & 75 & & & & & & & & & & $1.50 \mathrm{E}-03$ & & PNNL-13421 & Ng77 & \\
\hline $\mathrm{Re}$ & 75 & 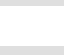 & & & & & & & & & $1.30 \mathrm{E}-03$ & & & & Ng et al. (1979) \\
\hline $\mathrm{Rh}$ & 45 & & & & & & & & & & $1.00 \mathrm{E}-02$ & & PNNL-13421 & Ng77 & \\
\hline $\mathrm{Rn}$ & 86 & 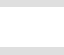 & & & & & & & & & & & & & \\
\hline $\mathrm{Ru}$ & 44 & & & & & & & & & & $3.30 \mathrm{E}-06$ & & PNNL-13421 & Co90 & \\
\hline $\mathrm{Ru}$ & 44 & 6 & 6.70E-07 & $1.40 \mathrm{E}-04$ & & & & & $8.50 \mathrm{E}+00$ & $9.40 \mathrm{E}-06$ & & & TRS_472 & & \\
\hline $\mathrm{Ru}$ & 44 & & & & & & & & & & $6.00 \mathrm{E}-07$ & & NUREG/CR-5512 & & \\
\hline $\mathrm{Ru}$ & 44 & & & & & & & & & & $6.00 \mathrm{E}-07$ & & & & Ng et al. (1979) \\
\hline $\mathrm{Ru}$ & 44 & 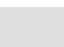 & $6.70 \mathrm{E}-07$ & $1.40 \mathrm{E}-04$ & & $3.60 \mathrm{E}-05$ & & $5.30 \mathrm{E}-05$ & & & & & & & Howard et al. (2009) \\
\hline $\mathrm{s}$ & 16 & & & & & & & & & & $1.60 \mathrm{E}-02$ & & PNNL-13421 & Ng82a & \\
\hline $\mathrm{s}$ & 16 & 1 & & & & $7.90 \mathrm{E}-03$ & & & & & & & TRS_472 & & \\
\hline $\mathrm{s}$ & 16 & & & & & & & & & & $1.50 \mathrm{E}-02$ & & NUREG/CR-5512 & & \\
\hline $\mathrm{S}$ & 16 & & & & & & & & & & $1.60 \mathrm{E}-02$ & & & & Ng et al. (1979) \\
\hline $\mathrm{s}$ & 16 & & & & & $7.90 \mathrm{E}-03$ & & & & & & & & & \\
\hline $\mathrm{s}$ & 16 & & $6.80 \mathrm{E}-02$ & $1.60 \mathrm{E}-02$ & & $4.30 \mathrm{E}-02$ & & $1.90 \mathrm{E}-02$ & & & & & & & \\
\hline $\mathrm{Sb}$ & 51 & & & & & & & & & & 2.50E-05 & & PNNL-13421 & Va82 & \\
\hline $\mathrm{Sb}$ & 51 & 3 & 2.00E-05 & $1.10 \mathrm{E}-04$ & & & & & $2.50 \mathrm{E}+00$ & $3.80 \mathrm{E}-05$ & & & TRS_472 & & \\
\hline $\mathrm{Sb}$ & 51 & & & & & & & & & & $1.00 \mathrm{E}-04$ & & NUREG/CR-5512 & & \\
\hline $\mathrm{Sb}$ & 51 & & & & & & & & & & $1.10 \mathrm{E}-04$ & & & & Ng et al. (1979) \\
\hline $\mathrm{Sb}$ & 51 & & 2.00E-05 & $1.10 \mathrm{E}-04$ & & $5.20 \mathrm{E}-05$ & $5.10 \mathrm{E}-02$ & & & & & & & & Howard et al. (2009) \\
\hline Sc & 21 & & & & & & & & & & $5.00 \mathrm{E}-06$ & & PNNL-13421 & Ng77 & \\
\hline Se & 34 & & & & & & & & & & $4.00 \mathrm{E}-03$ & & PNNL-13421 & Ng77 & \\
\hline Se & 34 & 12 & $1.50 \mathrm{E}-03$ & $1.60 \mathrm{E}-02$ & & & & & $2.10 \mathrm{E}+00$ & $4.00 \mathrm{E}-03$ & & & TRS_472 & & \\
\hline Se & 34 & & & & & & & & & & 4.00E-03 & & & & Ng et al. (1979) \\
\hline Se & 34 & & $1.50 \mathrm{E}-03$ & $1.60 \mathrm{E}-02$ & & $5.20 \mathrm{E}-03$ & & $4.50 \mathrm{E}-03$ & & & & & & & Howard et al. (2009) \\
\hline Se & 34 & & $5.90 \mathrm{E}-02$ & $7.90 \mathrm{E}-02$ & & $6.90 \mathrm{E}-02$ & & & & & & & & & Howard et al. (2009) \\
\hline $\mathrm{Si}$ & 14 & & & & & & & & & & $2.00 \mathrm{E}-05$ & & PNNL-13421 & Ng77 & \\
\hline $\mathrm{Si}$ & 14 & & & & & & & & & & 2.00E-05 & & & & Ng et al. (1979) \\
\hline Sm & 62 & & & & & & & & & & $3.00 \mathrm{E}-05$ & & PNNL-13421 & CEC87 & \\
\hline Sm & 62 & & & & & & & & & & 2.00E-05 & & NUREG/CR-5512 & & \\
\hline Sn & 50 & & & & & & & & & & $1.00 \mathrm{E}-03$ & & PNNL-13421 & NCRP86 & \\
\hline Sn & 50 & & & & & & & & & & $1.20 \mathrm{E}-03$ & & & & Ng et al. (1979) \\
\hline $\mathrm{Sr}$ & 38 & & & & & & & & & & $2.80 \mathrm{E}-03$ & & PNNL-13421 & Co90 & \\
\hline Sr & 38 & 154 & $3.40 \mathrm{E}-04$ & 4.30E-03 & & & & & $1.70 \mathrm{E}+00$ & $1.30 \mathrm{E}-03$ & & & TRS_472 & & \\
\hline $\mathrm{Sr}$ & 38 & & & & & & & & & & $1.50 \mathrm{E}-03$ & & NUREG/CR-5512 & & \\
\hline $\mathrm{Sr}$ & 38 & & & & & & & & & & $1.40 \mathrm{E}-03$ & & & & Ng et al. (1979) \\
\hline $\mathrm{Sr}$ & 38 & & $3.40 \mathrm{E}-04$ & 4.30E-03 & & $1.50 \mathrm{E}-03$ & & $8.10 \mathrm{E}-04$ & & & & & & & Howard et al. (2009) \\
\hline
\end{tabular}


Table B.2. (contd)

\begin{tabular}{|c|c|c|c|c|c|c|c|c|c|c|c|c|c|c|c|}
\hline Element & Atomic\# & $\mathrm{N}$ & Min & Max & AVG & Mean & Wmean & STD & GSD & $\overline{G M}$ & MED & 95\%ile & Document & Reference\# & Reference \\
\hline $\mathrm{Sr}$ & 38 & & $5.80 \mathrm{E}-03$ & $8.10 \mathrm{E}-02$ & & $2.10 \mathrm{E}-02$ & & $2.00 \mathrm{E}-02$ & & & & & & & Howard et al. (2009) \\
\hline $\mathrm{Sr}$ & 38 & & & & & $3.00 \mathrm{E}-02$ & & & & & & & & & Howard et al. (2009) \\
\hline $\mathrm{Sr}$ & 38 & & $5.00 \mathrm{E}-04$ & $1.20 \mathrm{E}-03$ & & & & & & & & & & & Gastbergera et al. (2001) \\
\hline $\mathrm{Ta}$ & 73 & & & & & & & & & & $4.10 \mathrm{E}-07$ & & PNNL-13421 & Jo88 & \\
\hline $\mathrm{Ta}$ & 73 & & & & & & & & & & 3.00E-06 & & NUREG/CR-5512 & & \\
\hline $\mathrm{Ta}$ & 73 & & & & & & & & & & $2.80 \mathrm{E}-06$ & & & & Ng et al. (1979) \\
\hline $\mathrm{Tb}$ & 65 & & & & & & & & & & 3.00E-05 & & PNNL-13421 & CEC87 & \\
\hline $\mathrm{Tb}$ & 65 & & & & & & & & & & $2.00 \mathrm{E}-05$ & & NUREG/CR-5512 & & \\
\hline Tc & 43 & & & & & & & & & & $1.40 \mathrm{E}-04$ & & PNNL-13421 & Jo88 & \\
\hline Tc & 43 & & & & & & & & & & $1.50 \mathrm{E}-02$ & & NUREG/CR-5512 & & \\
\hline Tc & 43 & & 7.50E-05 & $7.50 \mathrm{E}-03$ & & & & & & & & & & & Thorne (2003) \\
\hline Tc & 43 & & $7.50 \mathrm{E}-04$ & $7.50 \mathrm{E}-02$ & & & & & & & & & & & Thorne (2003) \\
\hline $\mathrm{Te}$ & 52 & 11 & 7.80E-05 & $1.00 \mathrm{E}-03$ & & & & & $2.40 \mathrm{E}+00$ & 3.40E-04 & $4.50 \mathrm{E}-04$ & & PNNL-13421 & Jo88 & \\
\hline $\mathrm{Te}$ & 52 & & & & & & & & & & $2.00 \mathrm{E}-04$ & & NUREG/CR-5512 & & \\
\hline $\mathrm{Te}$ & 52 & & & & & & & & & & $2.00 \mathrm{E}-04$ & & & & Ng et al. (1979) \\
\hline $\mathrm{Te}$ & 52 & & $7.80 \mathrm{E}-05$ & $1.00 \mathrm{E}-03$ & & $4.50 \mathrm{E}-04$ & & $2.90 \mathrm{E}-04$ & & & & & & & Howard et al. (2009) \\
\hline $\mathrm{Te}$ & 52 & & & & & 4.40E-03 & & & & & & & & & Howard et al. (2009) \\
\hline $\mathrm{Te}$ & 52 & & & & & $2.90 \mathrm{E}-03$ & & & & & & & & & Howard et al. (2009) \\
\hline Th & 90 & & & & & & & & & & 5.00E-06 & & PNNL-13421 & Ng77 & \\
\hline $\mathrm{Ti}$ & 22 & & & & & & & & & & $1.00 \mathrm{E}-02$ & & & & Ng et al. (1979) \\
\hline $\mathrm{Tl}$ & 81 & & & & & & & & & & 2.00E-03 & & PNNL-13421 & Na88 & \\
\hline $\mathrm{Tm}$ & 69 & & & & & & & & & & & & & & \\
\hline $\mathrm{U}$ & 92 & & & & & & & & & & 4.00E-04 & & PNNL-13421 & MB90 & \\
\hline $\mathrm{U}$ & 92 & 3 & $5.00 \mathrm{E}-04$ & $6.10 \mathrm{E}-03$ & & & & & $3.50 \mathrm{E}+00$ & $1.80 \mathrm{E}-03$ & & & TRS_472 & & \\
\hline $\mathrm{U}$ & 92 & & & & & & & & & & $6.00 \mathrm{E}-04$ & & NUREG/CR-5512 & & \\
\hline $\mathrm{U}$ & 92 & & $4.00 \mathrm{E}-03$ & $5.00 \mathrm{E}-02$ & & & & & & & & & & & Thorne (2003) \\
\hline $\mathrm{U}$ & 92 & & $1.00 \mathrm{E}-02$ & $1.50 \mathrm{E}-01$ & & & & & & & & & & & Thorne (2003) \\
\hline U & 92 & & & & & & & & & & $6.10 \mathrm{E}-04$ & & & & Ng et al. (1979) \\
\hline $\mathrm{U}$ & 92 & & $5.00 \mathrm{E}-04$ & $6.10 \mathrm{E}-03$ & & $2.90 \mathrm{E}-03$ & & $2.90 \mathrm{E}-03$ & & & & & & & Howard et al. (2009) \\
\hline $\mathrm{U}$ & 92 & & & & & $1.40 \mathrm{E}-03$ & & & & & & & & & Howard et al. (2009) \\
\hline $\mathrm{V}$ & 23 & & & & & & & & & & 2.00E-05 & & & & Ng et al. (1979) \\
\hline $\mathrm{w}$ & 74 & & & & & & & & & & $3.00 \mathrm{E}-04$ & & PNNL-13421 & Ng77 & \\
\hline $\mathrm{W}$ & 74 & 7 & 3.40E-05 & $6.80 \mathrm{E}-04$ & & & & & $3.10 \mathrm{E}+00$ & $1.90 \mathrm{E}-04$ & & & TRS_472 & & \\
\hline $\mathrm{w}$ & 74 & & & & & & & & & & $2.90 \mathrm{E}-04$ & & & & Ng et al. (1979) \\
\hline $\mathrm{W}$ & 74 & & $3.40 \mathrm{E}-05$ & $6.80 \mathrm{E}-04$ & & $2.90 \mathrm{E}-04$ & & $2.40 \mathrm{E}-04$ & & & & & & & Howard et al. (2009) \\
\hline Хе & 54 & & & & & & & & & & & & & & \\
\hline $\mathrm{Y}$ & 39 & & & & & & & & & & 2.00E-05 & & PNNL-13421 & Ng77 & \\
\hline $\mathrm{Yb}$ & 70 & & & & & & & & & & & & & & \\
\hline $\mathrm{Zn}$ & 30 & & & & & & & & & & $1.00 \mathrm{E}-02$ & & PNNL-13421 & Ng77 & \\
\hline $\mathrm{Zn}$ & 30 & 8 & $1.30 \mathrm{E}-04$ & $9.00 \mathrm{E}-03$ & & & & & $3.90 \mathrm{E}+00$ & $2.70 \mathrm{E}-03$ & & & TRS_472 & & \\
\hline $\mathrm{Zn}$ & 30 & & & & & & & & & & $1.00 \mathrm{E}-02$ & & & & Ng et al. (1979) \\
\hline $\mathrm{Zn}$ & 30 & & $1.30 \mathrm{E}-04$ & $9.00 \mathrm{E}-03$ & & $4.30 \mathrm{E}-03$ & & $3.00 \mathrm{E}-03$ & & & & & & & Howard et al. (2009) \\
\hline $\mathrm{Zr}$ & 40 & & & & & & & & & & $5.50 \mathrm{E}-07$ & & PNNL-13421 & Jo88 & \\
\hline $\mathrm{Zr}$ & 40 & 6 & $5.50 \mathrm{E}-07$ & $1.70 \mathrm{E}-05$ & & & & & $4.30 \mathrm{E}+00$ & $3.60 \mathrm{E}-06$ & & & TRS_472 & & \\
\hline $\mathrm{Zr}$ & 40 & & & & & & & & & & $3.00 \mathrm{E}-05$ & & NUREG/CR-5512 & & \\
\hline $\mathrm{Zr}$ & 40 & & & & & & & & & & 3.00E-05 & & & & Ng et al. (1979) \\
\hline $\mathrm{Zr}$ & 40 & & $5.50 \mathrm{E}-07$ & $1.70 \mathrm{E}-05$ & & $7.10 \mathrm{E}-06$ & & $6.90 \mathrm{E}-06$ & & & & & & & Howard et al. (2009) \\
\hline $\mathrm{Zr}$ & 40 & & & & & $5.50 \mathrm{E}-06$ & & & & & & & & & Howard et al. (2009) \\
\hline
\end{tabular}


Table B.3. Feed to Poultry Transfer Factor, d/kg wet_as_fed (CLFPL)

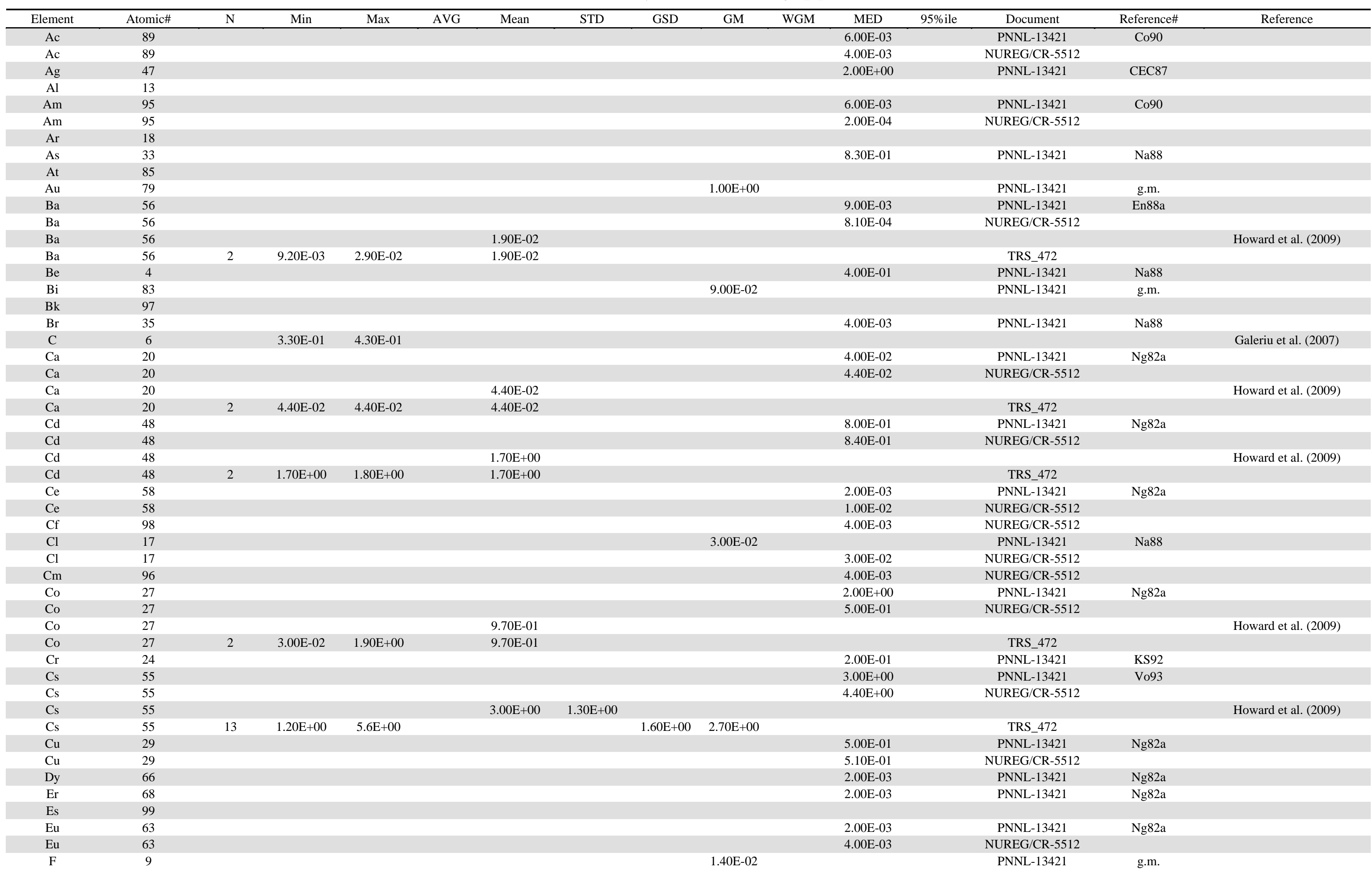


Table B.3. (contd)

\begin{tabular}{|c|c|c|c|c|c|c|c|c|c|c|c|c|c|c|c|}
\hline Element & Atomic\# & $\mathrm{N}$ & Min & Max & AVG & Mean & STD & GSD & GM & WGM & MED & 95\%ile & Document & Reference\# & Reference \\
\hline $\mathrm{Fe}$ & 26 & & & & & & & & & & $1.00 \mathrm{E}+00$ & & PNNL-13421 & Ng82a & \\
\hline $\mathrm{Fe}$ & 26 & & & & & & & & & & $1.50 \mathrm{E}+00$ & & NUREG/CR-5512 & & \\
\hline $\mathrm{Fm}$ & 100 & & & & & & & & & & & & & & \\
\hline $\mathrm{Fr}$ & 87 & & & & & & & & & & & & & & \\
\hline $\mathrm{Ga}$ & 31 & & & & & & & & & & $8.00 \mathrm{E}-01$ & & PNNL-13421 & Na88 & \\
\hline $\mathrm{Ge}$ & 32 & & & & & & & & & & & & & & \\
\hline $\mathrm{H}$ & 1 & & 7.00E-01 & 9.00E-01 & & & & & & & & & & & Galeriu et al. (2007) \\
\hline $\mathrm{Hf}$ & 72 & & & & & & & & & & $6.00 \mathrm{E}-04$ & & PNNL-13421 & En88a & \\
\hline $\mathrm{Hg}$ & 80 & & & & & & & & & & 3.00E-02 & & PNNL-13421 & Ng82a & \\
\hline $\mathrm{Hg}$ & 80 & & & & & & & & & & $1.10 \mathrm{E}-02$ & & NUREG/CR-5512 & & \\
\hline Ho & 67 & & & & & & & & & & 2.00E-03 & & PNNL-13421 & Ng82a & \\
\hline I & 53 & & & & & & & & & & $5.00 \mathrm{E}-02$ & & PNNL-13421 & Sn94 & \\
\hline I & 53 & & & & & & & & & & $1.80 \mathrm{E}-02$ & & NUREG/CR-5512 & & \\
\hline I & 53 & & 2.00E-02 & $5.00 \mathrm{E}-01$ & & & & & & & & & & & Thorne (2003) \\
\hline I & 53 & & & & & 1.00E-02 & $5.60 \mathrm{E}-03$ & & & & & & & & Howard et al. (2009 \\
\hline I & 53 & 3 & 4.00E-03 & $1.50 \mathrm{E}-02$ & & & & $2.00 \mathrm{E}+00$ & $8.70 \mathrm{E}-03$ & & & & TRS_472 & & \\
\hline In & 49 & & & & & & & & & & $8.00 \mathrm{E}-01$ & & PNNL-13421 & Na88 & \\
\hline Ir & 77 & & & & & & & & & & $2.00 \mathrm{E}+00$ & & PNNL-13421 & Ng82a & \\
\hline $\mathrm{K}$ & 19 & & & & & & & & & & 4.00E-01 & & PNNL-13421 & KS92 & \\
\hline K & 19 & & & & & & & & & & & & & & \\
\hline $\mathrm{La}$ & 57 & 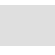 & & & & & & & & & $1.00 \mathrm{E}-01$ & & PNNL-13421 & Ng82a & \\
\hline $\mathrm{Lu}$ & 71 & & & & & & & & & & $1.00 \mathrm{~L}$ & & 1910 & & \\
\hline Md & 101 & & & & & & & & & & & & & & \\
\hline $\mathrm{Mn}$ & 25 & & & & & $1.90 \mathrm{E}-03$ & & & & & & & & & Howard et al. (2009 \\
\hline $\mathrm{Mn}$ & 25 & 2 & $1.00 \mathrm{E}-03$ & $2.80 \mathrm{E}-03$ & & $1.90 \mathrm{E}-03$ & & & & & & & TRS_472 & & \\
\hline Mo & 42 & & & & & & & & & & $1.80 \mathrm{E}-02$ & & PNNL-13421 & En88a & \\
\hline Mo & 42 & & & & & & & & & & $1.90 \mathrm{E}-01$ & & NUREG/CR-5512 & & \\
\hline Mo & 42 & & & & & $1.80 \mathrm{E}-01$ & & & & & & & & & Howard et al. (2009 \\
\hline Mo & 42 & 1 & & & & $1.80 \mathrm{E}-01$ & & & & & & & TRS_472 & & \\
\hline $\mathrm{N}$ & 7 & & & & & & & & $9.80 \mathrm{E}-02$ & & & & PNNL-13421 & g.m. & \\
\hline $\mathrm{Na}$ & 11 & & & & & & & & & & $1.00 \mathrm{E}-02$ & & PNNL-13421 & Na88 & \\
\hline $\mathrm{Na}$ & 11 & & & & & $7.00 \mathrm{E}+00$ & & & & & & & & & Howard et al. (2009 \\
\hline $\mathrm{Na}$ & 11 & 1 & & & & $7.00 \mathrm{E}+00$ & & & & & & & TRS_472 & & \\
\hline $\mathrm{Nb}$ & 41 & & & & & & & & & & $3.00 \mathrm{E}-04$ & & PNNL-13421 & En88a & \\
\hline $\mathrm{Nb}$ & 41 & & & & & & & & & & $3.10 \mathrm{E}-04$ & & NUREG/CR-5512 & & \\
\hline $\mathrm{Nb}$ & 41 & & & & & 3.00E-04 & & & & & & & & & Howard et al. (2009 \\
\hline $\mathrm{Nb}$ & 41 & 1 & & & & $3.00 \mathrm{E}-04$ & & & & & & & TRS_472 & & \\
\hline $\mathrm{Nd}$ & 60 & & & & & & & & & & 2.00E-03 & & PNNL-13421 & Ng82a & \\
\hline $\mathrm{Nd}$ & 60 & & & & & & & & & & 4.00E-03 & & NUREG/CR-5512 & & \\
\hline $\mathrm{Ne}$ & 10 & & & & & & & & & & & & & & \\
\hline $\mathrm{Ni}$ & 28 & & & & & & & & & & $1.00 \mathrm{E}-03$ & & PNNL-13421 & Na88 & \\
\hline $\mathrm{Np}$ & 93 & & & & & & & & & & $6.00 \mathrm{E}-03$ & & PNNL-13421 & Co90 & \\
\hline $\mathrm{Np}$ & 93 & & & & & & & & & & 4.00E-03 & & NUREG/CR-5512 & & \\
\hline \multirow{2}{*}{\multicolumn{16}{|c|}{ OBT }} \\
\hline & & & & & & & & & & & & & & & \\
\hline Os & 76 & & & & & & & & 8.40E-02 & & & & PNNL-13421 & g.m. & \\
\hline
\end{tabular}


Table B.3. (contd)

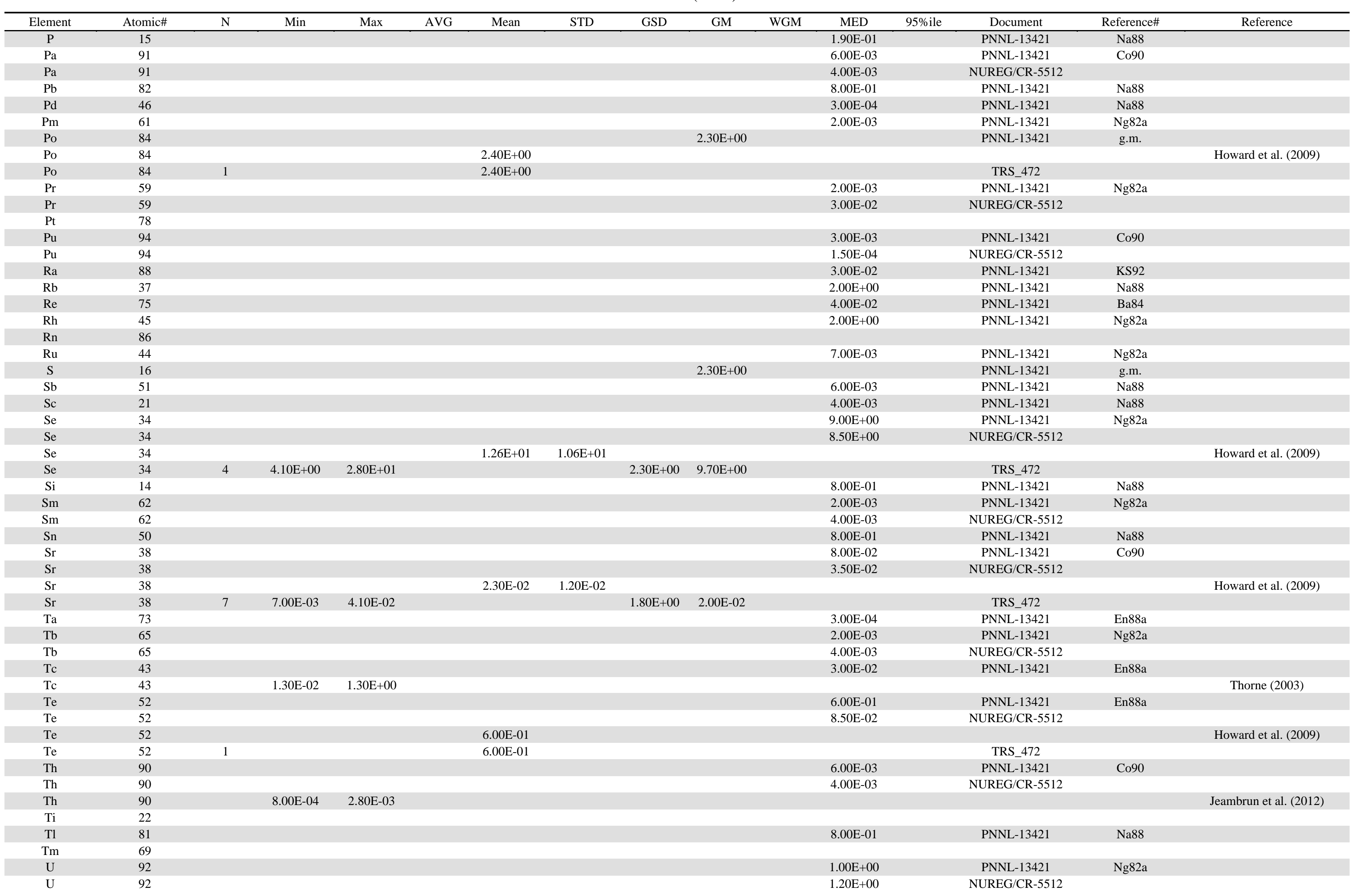


Table B.3. (contd)

\begin{tabular}{|c|c|c|c|c|c|c|c|c|c|c|c|c|c|c|c|}
\hline Element & Atomic\# & $\mathrm{N}$ & Min & Max & $\mathrm{AVG}$ & Mean & STD & GSD & GM & WGM & MED & 95\%ile & Document & Reference\# & Reference \\
\hline $\mathrm{U}$ & 92 & & $1.50 \mathrm{E}+00$ & $7.50 \mathrm{E}+00$ & & & & & & & & & & & Thorne (2003) \\
\hline $\mathrm{U}$ & 92 & & $1.80 \mathrm{E}-03$ & 7.30E-03 & & & & & & & & & & & Howard et al. (2009) \\
\hline $\mathrm{U}$ & 92 & 2 & $3.00 \mathrm{E}-01$ & $1.20 \mathrm{E}+00$ & & 7.50E-01 & & & & & & & TRS_472 & & \\
\hline $\mathrm{V}$ & 23 & & & & & & & & & & & & & & \\
\hline w & 74 & & & & & & & & & & 2.00E-01 & & PNNL-13421 & IAEA94 & \\
\hline $\mathrm{Y}$ & 39 & & & & & & & & & & $1.00 \mathrm{E}-02$ & & PNNL-13421 & Ng82a & \\
\hline $\mathrm{Yb}$ & 70 & 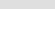 & & & & & & & & & & & & & \\
\hline $\mathrm{Zn}$ & 30 & & & & & & & & & & $7.00 \mathrm{E}+00$ & & PNNL-13421 & Ng82a & \\
\hline $\mathrm{Zn}$ & 30 & & & & & & & & & & $6.50 \mathrm{E}+00$ & & NUREG/CR-5512 & & \\
\hline $\mathrm{Zn}$ & 30 & & & & & 4.70E- 01 & $7.90 \mathrm{E}-02$ & & & & & & & & Howard et al. (2009) \\
\hline $\mathrm{Zn}$ & 30 & 3 & $3.80 \mathrm{E}-01$ & 5.30E-01 & & & & $1.20 \mathrm{E}+00$ & 4.70E-01 & & & & TRS_472 & & \\
\hline $\mathrm{Zr}$ & 40 & & & & & & & & & & $6.40 \mathrm{E}-05$ & & NUREG/CR-5512 & & \\
\hline $\mathrm{Zr}$ & 40 & & & & & $6.00 \mathrm{E}-05$ & & & & & & & & & Howard et al. (2009) \\
\hline $\mathrm{Zr}$ & 40 & 1 & & & & $6.00 \mathrm{E}-05$ & & & & & & & TRS_472 & & \\
\hline
\end{tabular}


Table B.4. Feed to Egg Transfer Factor, d/kg $\mathrm{kg}_{\text {wet as fed }}$ (CLFEG)

\begin{tabular}{|c|c|c|c|c|c|c|c|c|c|c|c|c|c|c|c|}
\hline Element & Atomic\# & $\mathrm{N}$ & Min & Max & AVG & Mean & Wmean & STD & GSD & GM & WGM & MED & Document & Reference\# & Reference \\
\hline $\mathrm{Ac}$ & 89 & & & & & & & & & & & $4.00 \mathrm{E}-03$ & PNNL-13421 & Ng82a & \\
\hline Ac & 89 & & & & & & & & & & & $2.00 \mathrm{E}-03$ & NUREG/CR-5512 & & \\
\hline $\mathrm{Ag}$ & 47 & & & & & & & & & & & $5.00 \mathrm{E}-01$ & PNNL-13421 & Ng82a & \\
\hline $\mathrm{Al}$ & 13 & & & & & & & & & & & & & & \\
\hline $\mathrm{Am}$ & 95 & & & & & & & & & & & $4.00 \mathrm{E}-03$ & PNNL-13421 & Ng82a & \\
\hline Am & 95 & & & & & & & & & & & 9.00E-05 & NUREG/CR-5512 & & \\
\hline $\mathrm{Am}$ & 95 & & & & & $3.00 \mathrm{E}-03$ & & & & & & & & & Howard et al. (2009) \\
\hline $\mathrm{Am}$ & 95 & 1 & & & & $3.00 \mathrm{E}-03$ & & & & & & & TRS_472 & & \\
\hline $\mathrm{Ar}$ & 18 & & & & & & & & & & & & & & \\
\hline As & 33 & & & & & & & & & 2.60E-01 & & & PNNL-13421 & g.m. & \\
\hline At & 85 & & & & & & & & & & & & & & \\
\hline $\mathrm{Au}$ & 79 & & & & & & & & & & & $5.00 \mathrm{E}-01$ & PNNL-13421 & Ng82a & \\
\hline $\mathrm{Ba}$ & 56 & . & & & & & & & & & & $9.00 \mathrm{E}-01$ & PNNL-13421 & En88a & \\
\hline $\mathrm{Ba}$ & 56 & & & & & & & & & & & $1.50 \mathrm{E}+00$ & NUREG/CR-5512 & & \\
\hline $\mathrm{Ba}$ & 56 & & & & & $8.70 \mathrm{E}-01$ & & & & & & & & & Howard et al. (2009) \\
\hline $\mathrm{Ba}$ & 56 & 1 & & & & $8.70 \mathrm{E}-01$ & & & & & & & TRS_472 & & \\
\hline $\mathrm{Be}$ & 4 & & & & & & & & & & & $2.00 \mathrm{E}-02$ & PNNL-13421 & Na88 & \\
\hline $\mathrm{Bi}$ & 83 & & & & & & & & & 2.60E-01 & & & PNNL-13421 & g.m. & \\
\hline $\mathrm{Bk}$ & 97 & & & & & & & & & & & & & & \\
\hline $\mathrm{Br}$ & 35 & & & & & & & & & & & $1.60 \mathrm{E}+00$ & PNNL-13421 & Na88 & \\
\hline $\mathrm{C}$ & 6 & 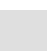 & $3.10 \mathrm{E}-01$ & $4.00 \mathrm{E}-01$ & & & & & & & & & & & Galeriu et al. (2007) \\
\hline $\mathrm{Ca}$ & 20 & & & & & & & & & & & 4.00E-01 & PNNL-13421 & Ng82a & \\
\hline $\mathrm{Ca}$ & 20 & . & & & & & & & & & & $4.40 \mathrm{E}+01$ & NUREG/CR-5512 & & \\
\hline $\mathrm{Ca}$ & 20 & & & & & 4.40E-01 & & & & & & & & & Howard et al. (2009) \\
\hline $\mathrm{Ca}$ & 20 & 1 & & & & 4.40E-01 & & & & & & & TRS_472 & & \\
\hline $\mathrm{Cd}$ & 48 & & & & & & & & & & & $1.00 \mathrm{E}-01$ & PNNL-13421 & Ng82a & \\
\hline $\mathrm{Ce}$ & 58 & & & & & & & & & & & 4.00E-05 & PNNL-13421 & CEC87 & \\
\hline $\mathrm{Ce}$ & 58 & & & & & & & & & & & 5.00E-03 & NUREG/CR-5512 & & \\
\hline $\mathrm{Ce}$ & 58 & & & & & $3.10 \mathrm{E}-03$ & & & & & & & & & Howard et al. (2009) \\
\hline $\mathrm{Ce}$ & 58 & 1 & & & & $3.10 \mathrm{E}-03$ & & & & & & & TRS_472 & & \\
\hline $\mathrm{Cf}$ & 98 & & & & & & & & & & & $4.00 \mathrm{E}-03$ & PNNL-13421 & Ng82a & \\
\hline $\mathrm{Cf}$ & 98 & & & & & & & & & & & $2.00 \mathrm{E}-03$ & NUREG/CR-5512 & & \\
\hline $\mathrm{Cl}$ & 17 & & & & & & & & & $2.70 \mathrm{E}+00$ & & & PNNL-13421 & g.m. & \\
\hline $\mathrm{Cm}$ & 96 & & & & & & & & & & & $4.00 \mathrm{E}-03$ & PNNL-13421 & Ng82a & \\
\hline $\mathrm{Cm}$ & 96 & & & & & & & & & & & $2.00 \mathrm{E}-03$ & NUREG/CR-5512 & & \\
\hline Co & 27 & & & & & & & & & & & $1.00 \mathrm{E}-01$ & PNNL-13421 & Ng82a & \\
\hline Co & 27 & & & & & $3.30 \mathrm{E}-02$ & & & & & & & & & Howard et al. (2009) \\
\hline $\mathrm{Cr}$ & 24 & & & & & & & & & & & $9.00 \mathrm{E}-01$ & PNNL-13421 & En88a & \\
\hline Cs & 55 & & & & & & & & & & & 4.00E-01 & PNNL-13421 & Co90 & \\
\hline Cs & 55 & & & & & & & & & & & 4.90E-01 & NUREG/CR-5512 & & \\
\hline Cs & 55 & & & & & $4.30 \mathrm{E}-01$ & & $1.60 \mathrm{E}-01$ & & & & & & & Howard et al. (2009) \\
\hline $\mathrm{Cu}$ & 29 & & & & & & & & & & & $5.00 \mathrm{E}-01$ & PNNL-13421 & Ng82a & \\
\hline $\mathrm{Cu}$ & 29 & & & & & & & & & & & 4.90E-01 & NUREG/CR-5512 & & \\
\hline Dy & 66 & & & & & & & & & & & $4.00 \mathrm{E}-05$ & PNNL-13421 & CEC87 & \\
\hline $\mathrm{Er}$ & 68 & & & & & & & & & & & 4.00E-05 & PNNL-13421 & CEC87 & \\
\hline Es & 99 & & & & & & & & & & & & & & \\
\hline $\mathrm{Eu}$ & 63 & & & & & & & & & & & 4.00E-05 & PNNL-13421 & CEC87 & \\
\hline $\mathrm{Eu}$ & 63 & & & & & & & & & & & $7.00 \mathrm{E}-03$ & NUREG/CR-5512 & & \\
\hline F & 9 & & & & & & & & & & & & & & \\
\hline $\mathrm{Fe}$ & 26 & & & & & & & & & & & $1.00 \mathrm{E}+00$ & PNNL-13421 & Ng82a & \\
\hline $\mathrm{Fe}$ & 26 & & & & & & & & & & & $1.30 \mathrm{E}+00$ & NUREG/CR-5512 & & \\
\hline
\end{tabular}


Table B.4. (contd)

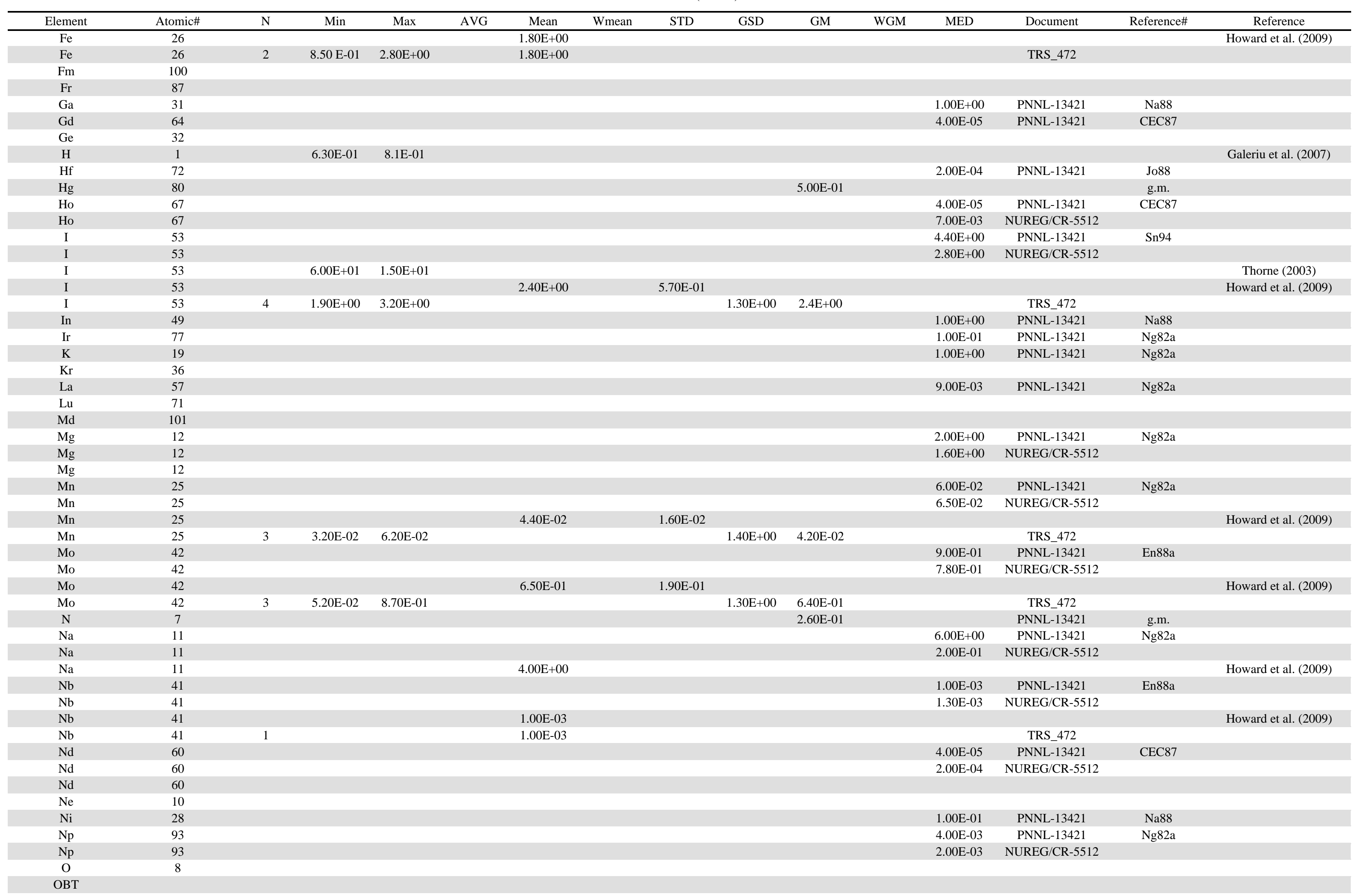


Table B.4. (contd)

\begin{tabular}{|c|c|c|c|c|c|c|c|c|c|c|c|c|c|c|c|}
\hline Element & Atomic\# & $\mathrm{N}$ & Min & Max & AVG & Mean & Wmean & STD & GSD & GM & WGM & MED & Document & Reference\# & Reference \\
\hline Os & 76 & & & & & & & & & $7.10 \mathrm{E}-02$ & & & & g.m. & \\
\hline $\mathrm{P}$ & 15 & & & & & & & & & & & $1.00 \mathrm{E}+00$ & PNNL-13421 & Na88 & \\
\hline $\mathrm{P}$ & 15 & & & & & & & & & & & $1.00 \mathrm{E}+01$ & NUREG/CR-5512 & & \\
\hline $\mathrm{P}$ & 15 & & & & & $6.40 \mathrm{E}-01$ & & & & & & & & & Howard et al. (2009) \\
\hline $\mathrm{P}$ & 15 & 1 & & & & $6.40 \mathrm{E}-01$ & & & & & & & TRS_472 & & \\
\hline Pa & 91 & & & & & & & & & & & $4.00 \mathrm{E}-03$ & PNNL-13421 & Ng82a & \\
\hline Pa & 91 & & & & & & & & & & & 2.00E-03 & NUREG/CR-5512 & & \\
\hline $\mathrm{Pb}$ & 82 & & & & & & & & & & & $1.00 \mathrm{E}+00$ & PNNL-13421 & Na88 & \\
\hline Pd & 46 & & & & & & & & & & & 4.00E-03 & PNNL-13421 & Na88 & \\
\hline $\mathrm{Pd}$ & 46 & & & & & & & & & & & & & & \\
\hline $\mathrm{Pm}$ & 61 & & & & & & & & & & & 4.00E-05 & PNNL-13421 & CEC87 & \\
\hline $\mathrm{Pm}$ & 61 & & & & & & & & & & & 2.00E-02 & NUREG/CR-5512 & & \\
\hline Po & 84 & & & & & & & & & $7.00 \mathrm{E}+00$ & & & PNNL-13421 & g.m. & \\
\hline Po & 84 & & & & & $3.10 \mathrm{E}+00$ & & & & & & & & & Howard et al. (2009) \\
\hline Po & 84 & & & & & $3.10 \mathrm{E}+00$ & & & & & & & TRS_472 & & \\
\hline $\operatorname{Pr}$ & 59 & & & & & & & & & & & $4.00 \mathrm{E}-05$ & PNNL-13421 & CEC87 & \\
\hline Pr & 59 & & & & & & & & & & & 5.00E-03 & NUREG/CR-5512 & & \\
\hline $\mathrm{Pt}$ & 78 & & & & & & & & & & & & & & \\
\hline Pu & 94 & & & & & & & & & & & $5.00 \mathrm{E}-04$ & PNNL-13421 & Ng82a & \\
\hline Pu & 94 & & & & & & & & & & & $8.00 \mathrm{E}-03$ & NUREG/CR-5512 & & \\
\hline Pu & 94 & & & & & $1.20 \mathrm{E}-03$ & & & & & & & & & Howard et al. (2009) \\
\hline Pu & 94 & 2 & $9.90 \mathrm{E}-06$ & $2.30 \mathrm{E}-03$ & & $1.20 \mathrm{E}-03$ & & & & & & & TRS_472 & & \\
\hline Ra & 88 & & & & & & & & & $3.10 \mathrm{E}-01$ & & & PNNL-13421 & g.m. & \\
\hline Ra & 88 & & & & & & & & & & & 2.00E-05 & NUREG/CR-5512 & & \\
\hline Ra & 88 & . & $1.50 \mathrm{E}-02$ & $1.10 \mathrm{E}-01$ & & & & & & & & & & & Jeambrun et al. (2012) \\
\hline $\mathrm{Rb}$ & 37 & & & & & & & & & & & $3.00 \mathrm{E}+00$ & PNNL-13421 & Na88 & \\
\hline $\mathrm{Re}$ & 75 & & & & & & & & & 4.20E-01 & & & & g.m. & \\
\hline $\mathrm{Rh}$ & 45 & & & & & & & & & & & $1.00 \mathrm{E}-01$ & PNNL-13421 & Ng82a & \\
\hline $\mathrm{Rn}$ & 86 & & & & & & & & & & & & & & \\
\hline $\mathrm{Ru}$ & 44 & & & & & & & & & & & $5.00 \mathrm{E}-03$ & PNNL-13421 & Co90 & \\
\hline $\mathrm{Ru}$ & 44 & & & & & & & & & & & $6.00 \mathrm{E}-03$ & NUREG/CR-5512 & & \\
\hline $\mathrm{Ru}$ & 44 & & & & & $4.00 \mathrm{E}-03$ & & & & & & & & & Howard et al. (2009) \\
\hline $\mathrm{Ru}$ & 44 & 1 & & & & $4.00 \mathrm{E}-03$ & & & & & & & TRS_472 & & \\
\hline $\mathrm{s}$ & 16 & & & & & & & & & $7.00 \mathrm{E}+00$ & & & PNNL-13421 & g.m. & \\
\hline Sb & 51 & & & & & & & & & & & $7.00 \mathrm{E}+00$ & PNNL-13421 & Na88 & \\
\hline Sc & 21 & & & & & & & & & 4.20E- 03 & & & PNNL-13421 & g.m. & \\
\hline Se & 34 & & & & & & & & & & & $9.00 \mathrm{E}+00$ & PNNL-13421 & $\begin{array}{l}\text { 5.m. } \\
\text { Ng82a }\end{array}$ & \\
\hline Se & 34 & & & & & & & & & & & $9.30 \mathrm{E}+00$ & NUREG/CR-5512 & & \\
\hline Se & 34 & & & & & $1.82 \mathrm{E}+01$ & & $1.04 \mathrm{E}+01$ & & & & & & & Howard et al. (2009) \\
\hline Se & 34 & 4 & $8.80 \mathrm{E}+00$ & $2.80 \mathrm{E}+01$ & & & & & $1.90 \mathrm{E}+00$ & $1.60 \mathrm{E}+01$ & & & TRS_472 & & \\
\hline $\mathrm{Si}$ & 14 & & & & & & & & & & & $1.00 \mathrm{E}+00$ & PNNL-13421 & Na88 & \\
\hline $\mathrm{Sm}$ & 62 & & & & & & & & & & & 4.00E-05 & PNNL-13421 & CEC87 & \\
\hline $\mathrm{Sm}$ & 62 & & & & & & & & & & & 7.00E-03 & NUREG/CR-5512 & & \\
\hline Sn & 50 & & & & & & & & & & & $1.00 \mathrm{E}+00$ & PNNL-13421 & Na88 & \\
\hline $\mathrm{Sr}$ & 38 & & & & & & & & & & & $2.00 \mathrm{E}-01$ & PNNL-13421 & Co90 & \\
\hline $\mathrm{Sr}$ & 38 & & & & & & & & & & & 3.00E-01 & NUREG/CR-5512 & & \\
\hline $\mathrm{Sr}$ & 38 & & & & & $8.80 \mathrm{E}-01$ & & $1.50 \mathrm{E}+00$ & & & & & & & Howard et al. (2009) \\
\hline Та & 73 & & & & & & & & & & & $1.00 \mathrm{E}-03$ & PNNL-13421 & En88a & \\
\hline $\mathrm{Tb}$ & 65 & & & & & & & & & & & 4.00E-05 & PNNL-13421 & CEC87 & \\
\hline $\mathrm{Tb}$ & 65 & & & & & & & & & & & 7.00E-03 & NUREG/CR-5512 & & \\
\hline Tc & 43 & & & & & & & & & & & $3.00 \mathrm{E}+00$ & PNNL-13421 & En88a & \\
\hline Tc & 43 & & $1.00 \mathrm{E}-01$ & $3.00 \mathrm{E}+00$ & & & & & & & & & & & Thorne (2003) \\
\hline
\end{tabular}


Table B.4. (contd)

\begin{tabular}{|c|c|c|c|c|c|c|c|c|c|c|c|c|c|c|c|}
\hline Element & Atomic\# & $\mathrm{N}$ & Min & Max & AVG & Mean & Wmean & STD & GSD & GM & WGM & MED & Document & Reference\# & Reference \\
\hline $\mathrm{Te}$ & 52 & & & & & & & & & & & $5.00 \mathrm{E}+00$ & PNNL-13421 & En88a & \\
\hline $\mathrm{Te}$ & 52 & & & & & & & & & & & $5.20 \mathrm{E}+00$ & NUREG/CR-5512 & & \\
\hline $\mathrm{Te}$ & 52 & & & & & $5.10 \mathrm{E}+00$ & & & & & & & & & Howard et al. (2009) \\
\hline $\mathrm{Te}$ & 52 & 1 & & & & $5.10 \mathrm{E}+00$ & & & & & & & TRS 442 & & \\
\hline Th & 90 & & & & & & & & & & & 4.00E-03 & PNNL-13421 & Ng82a & \\
\hline Th & 90 & & & & & & & & & & & 2.00E-03 & NUREG/CR-5512 & & \\
\hline Th & 90 & & & & & $6.00 \mathrm{E}-04$ & & 4.00E-04 & & & & & & & Jeambrun et al. (2012) \\
\hline $\mathrm{Ti}$ & 22 & & & & & & & & & & & & & & \\
\hline $\mathrm{Tl}$ & 81 & & & & & & & & & & & $1.00 \mathrm{E}+00$ & PNNL-13421 & Na88 & \\
\hline $\mathrm{Tm}$ & 69 & 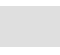 & & & & & & & & & & & & & \\
\hline $\mathrm{U}$ & 92 & & & & & & & & & & & $1.00 \mathrm{E}+00$ & PNNL-13421 & Ng82a & \\
\hline $\mathrm{U}$ & 92 & & & & & & & & & & & $9.90 \mathrm{E}-01$ & NUREG/CR-5512 & & \\
\hline $\mathrm{U}$ & 92 & 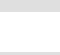 & $1.50 \mathrm{E}+00$ & $7.50 \mathrm{E}+00$ & & & & & & & & & & & Thorne (2003) \\
\hline $\mathrm{U}$ & 92 & & & & & $1.10 \mathrm{E}+00$ & & & & & & & & & Howard et al. (2009) \\
\hline $\mathrm{U}$ & 92 & & $1.80 \mathrm{E}-04$ & $1.80 \mathrm{E}-03$ & & & & & & & & & & & Jeambrun et al. (2012) \\
\hline $\mathrm{U}$ & 92 & 2 & $9.20 \mathrm{E}-01$ & $1.20 \mathrm{E}+00$ & & $1.10 \mathrm{E}+00$ & & & & & & & TRS_472 & & \\
\hline $\mathrm{V}$ & 23 & & & & & & & & & & & & & & \\
\hline $\mathrm{w}$ & 74 & & & & & & & & & & & $9.00 \mathrm{E}-01$ & PNNL-13421 & En88a & \\
\hline Хе & 54 & & & & & & & & & & & & & & \\
\hline $\mathrm{Y}$ & 39 & & & & & & & & & & & 2.00E-03 & PNNL-13421 & Ng82a & \\
\hline $\mathrm{Yb}$ & 70 & 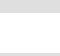 & & & & & & & & & & & & & \\
\hline $\mathrm{Zn}$ & 30 & & & & & & & & & & & $3.00 \mathrm{E}+00$ & PNNL-13421 & Ng82a & \\
\hline $\mathrm{Zn}$ & 30 & & & & & & & & & & & $2.60 \mathrm{E}+00$ & NUREG/CR-5512 & & \\
\hline $\mathrm{Zn}$ & 30 & & & & & $1.40 \mathrm{E}+00$ & & $2.90 \mathrm{E}-01$ & & & & & & & Howard et al. (2009) \\
\hline $\mathrm{Zn}$ & 30 & 4 & $1.20 \mathrm{E}+00$ & $1.90 \mathrm{E}+00$ & & & & & $1.20 \mathrm{E}+00$ & $1.40 \mathrm{E}+00$ & & & TRS_472 & & \\
\hline $\mathrm{Zr}$ & 40 & & & & & & & & & & & 2.00E-04 & PNNL-13421 & En88a & \\
\hline $\mathrm{Zr}$ & 40 & & & & & & & & & & & $1.90 \mathrm{E}-04$ & NUREG/CR-5512 & & \\
\hline $\mathrm{Zr}$ & 40 & & & & & 2.00E-04 & & & & & & & & & Howard et al. (2009) \\
\hline
\end{tabular}


PNNL-21950, Appendix B

\section{References}

PNNL-13421

Staven LH, BA Napier, K Rhoads, and DL Strenge. 2003. A Compendium of Transfer Factors for Agricultural and Animal Products. PNNL-13421, Pacific Northwest National Laboratory, Richland, Washington

(Ba84) Baes CF, RD Sharp, AL Sjoreen, and RW Shor. 1984. A Review and Analysis of Parameters for Assessing Transport of Environmentally Released Radionuclides through Agriculture. ORNL-5786, Oak Ridge National Laboratory, Oak Ridge, Tennessee.

(Bi89) Bishop GP, CJ Beetham, and YS Cuff. 1989. Review of Literature for Chlorine Technetium, Iodine and Neptunium. Nirex Radioactive Waste Disposal Safety Studies, NSS/R193, UK Nirex Ltd., Harwell, England.

(Br79) Bruland W et al. 1979. “Transfer of Organically Bound Radionuclides Through Food Chains to Man. Model-Example with Radiocobalt and Vitamin B12.” In Biological Implications of Radionuclides Released from Nuclear Industries, Vol. 2, Proceedings of a Symposium, International Atomic Energy Agency, Vienna.

(CEC87) Commission of the European Communities. 1987. Radionuclide Transfer Factors of Animal Feedingstuffs and Animal Products. CEC Doc. 7682/87, Luxembourg.

(CT83) Coughtrey PC and MC Thorne. 1983. Radionuclide Distribution and Transport in Terrestrial and Aquatic Ecosystems, A Critical Review of Data. Vols. 1-6, A.A. Balkema, Rotterdam.

(Co90) Coughtrey PJ. 1990. Radioactivity Transfer to Animal Products. EUR 12608 EN, Commission of the European Communities, Luxembourg.

(Cr90) Cramp TJ, YS Cuff, A Davis, and JE Morgan. 1990. Review of Data for Uranium, Nickel, and Cobalt. 2150-RI, Associated Nuclear Services, Ltd., Epsom, England.

(En88a) Ennis ME, Jr., GM Ward, JE Johnson, and KN Boamah. 1988. “Transfer Coefficients of Selected Radionuclides to Animal Products II. Hen Eggs and Meat.” Health Physics 54:167-170.

(IAEA87) International Atomic Energy Agency. 1987. Draft Working Document: Handbook of Parameter Values for the Prediction of Radionuclide Transfer in the Terrestrial and Freshwater Environments. IAEA, Vienna, Austria.

(IAEA94) International Atomic Energy Agency, International Union of Radioecologists. 1994. Handbook of Parameter Values for the Prediction of Radionuclide Transfer in Temperate Environments. Tech. Rep. Series No. 364, IAEA, Vienna, Austria.

(Jo88) Johnson JE, GM Ward, ME Ennis, Jr., and KN Boamah. 1988. “Transfer Coefficients of Selected Radionuclides to Animal Products, 1. Comparison of Milk and Meat from Dairy Cows and Goats.” Health Physics 54:161-166. 
PNNL-21950, Appendix B

(KS92) Kennedy WE, Jr. and DL Strenge. 1992. Residual Radioactive Contamination from Decommissioning: Technical Basis for Translating Contamination Levels to Annual Total Effective Dose Equivalent. NUREG/CR-5512 (PNL-7994), Pacific Northwest Laboratory, Richland, Washington.

(LS85) Lawson G and GM Smith. 1985. BIOS: A Model to Predict Radionuclide Transfer and Doses to Man Following Releases for Geological Repositories for Radioactive Wastes. NRPB-R169, National Radiation Protection Board, Chilton, England.

(MB90) Morgan JE and CJ Beetham. 1990. Review of Literature for Radium, Protactinium, Tin and Carbon. Nirex Radioactive Waste Disposal Safety Studies, UK Nirex LT Harwell, England.

(Na88) Napier BA, RA Peloquin, DL Strenge, and JV Ramsdell. 1988. GENII - The Hanford Environmental Radiation Dosimetry Software System. PNL-6584, Pacific Northwest Laboratory, Richland, Washington.

(NCRP86) National Council on Radiation Protection and Measurements. 1986. Screening Techniques for Determining Compliance with Environmental Standards, Releases of Radionuclides to the Atmosphere. NCRP Commentary No. 3, NCRP, Bethesda, Maryland.

(NCRP96) National Council on Ionizing Radiation and Protection. 1996. Screening Models for Releases of Radionuclides to Atmosphere, Surface Water and Ground. NCRP Report No. 123, Vol. I., NCRP, Bethesda, Maryland.

(Ng68) Ng YC, CA Burton, SE Thompson, RK Tandy, HK Kretner, and MW Pratt. 1968. "Prediction of the Maximum Dosage to Man from the Fallout of Nuclear Devices.” In Handbook for Estimating the Maximum Internal Dose from Radionuclides Released to the Biosphere. UCRL-50163, Pt. IV, Lawrence Radiation Laboratory, Livermore, California.

(Ng77) Ng YC, CS Colsher, DJ Quinn, and SE Thompson. 1977. Transfer Coefficients for the Prediction of the Dose to Man via the Forage-Cow-Milk Pathway from Radionuclides Released to the Biosphere. UCRL-51939, Lawrence Livermore National Laboratory, Livermore, California.

(Ng79) Ng YC, CS Colsher, and SE Thompson. 1979. “Transfer Coefficients for Assessing the Terrestrial Food Chains-Their Derivation and Limitations.” In Radioactivitat und Umwelt, HJ Kellermann (ed.), Vol. I, pp. 455-481. Proceedings of the 12th Annual Conference of the Fachverband fur Strahlenschutz, Nederney, West Germany, October 2-6, 1978.

(Ng82a) Ng YC, CS Colsher, and SE Thompson. 1982a. Transfer Coefficients for Assessing the Dose from Radionuclides in Meat and Eggs. NUREG/CR-2976, UCID-19464, Lawrence Livermore National Laboratory, California.

(Th94) Thiede ME, DJ Bates, EI Mart, and RW Hanf. 1994. A Guide to Environmental Monitoring Data, 1945-1972. PNWD-2226 HEDR, Battelle-Pacific Northwest Laboratories, Richland, Washington.

(Va82) Van Bruwaene R, GB Gerber, R Kirchmann, and J Colard. 1982. "Metabolism of Antimony-124 in Lactating Dairy Cows.” Health Physics 43:733-738. 
(Va83) Van Den Hoek, J, MHJ Ten Have, and GB Gerber. 1983. “The Metabolism of Tritium and Water in the Lactating Dairy Cow.” Health Physics 44:127-133.

(Va84) Van Bruwaene R, GB Gerber, R Kirchmann, J Colard, and J Van Kerkom. 1984. “Metabolism of ${ }^{51} \mathrm{Cr},{ }^{54} \mathrm{Mn},{ }^{59} \mathrm{Fe}$, and ${ }^{60} \mathrm{Co}$ in Lactating Dairy Cows.” Health Physics 46:1069-1082.

(Vo93) Voight G, H Muller, HG Paretzke, T Bauer, and G Rohrmoser. 1993. "Cs-137 Transfer after Chernobyl from Fodder into Chicken Meat and Eggs.” Health Physics 65:141-146.

\section{TRS_472}

IAEA. 2010. Handbook of Parameter Values for the Prediction of Radionuclide Transfer in Terrestrial and Freshwater Environments. Technical Report Series No. 472. International Atomic Energy Agency, Vienna, Austria

\section{Other References}

Beresford NA, BJ Howard, RW Mayes, and CS Lamb. 2007. "The Transfer of Radionuclides from Saltmarsh Vegetation to Sheep Tissues and Milk.” Journal of Environmental Radioactivity 98(1-2):36-49.

Galeriu D, A Melintescu, NA Beresford, NMJ Crout, R Peterson, and H Takeda. 2007. "Modelling ${ }^{3} \mathrm{H}$ and ${ }^{14} \mathrm{C}$ Transfer to Farm Animals and Their Products Under Steady State Conditions.” Journal of Environmental Radioactivity 98(1-2):205-217.

Gastbergera M, F Steinhäusler, MH Gerzabekb, and A Hubmera. 2001. "Fallout Strontium and Caesium Transfer from Vegetation to Cow Milk at Two Lowland and Two Alpine Pastures.” Journal of Environmental Radioactivity 54 (2) 267 \pm 273

Howard BJ, NA Beresford, CL Barnett, and S Fesenko. 2009. "Radionuclide Transfer to Animal Products: Revised Recommended Transfer Coefficient Values.” Journal of Environmental Radioactivity 100(3)263-273.

Howard BJ, NA Beresford, S Gashchak, A Arkhipov, RW Mayes, J Caborn, G Strømann, and L Wacker. 2007. “The Transfer of ${ }^{239 / 240} \mathrm{Pu}$ to Cow Milk.” Journal of Environmental Radioactivity 98(1-2):191-204.

Jeambrun M, L Pourcelot, C Mercat, B Boulet, J Loyen, X Cagnatd, and F Gauthier-Lafaye. 2012. "Study on Transfers of Uranium, Thorium and Decay Products from Grain, Water and Soil to Chicken Meat and Egg Contents.” Journal of Environmental Monitoring 14(8):2170-2180.

Ng YC, CS Colsher, and SE Thompson. 1979. "Transfer Factors for Assessing the Dose from Radionuclides in Agricultural Products.” International Symposium on Biological Implications of Radionuclides Released from Nuclear Industries, Vienna, Austria, March 26-30, 1979.

Thorne MC. 2003. "Estimation of Animal Transfer Factors for Radioactive Isotopes of Iodine, Technetium, Selenium and Uranium.” Journal of Environmental Radioactivity 70 (1-2):3-20. 
PNNL-21950, Appendix B

Tsukada H, S Hisamatsu, and J Inaba. 2003. “Transfer of ${ }^{137}$ Cs and Stable Cs in Soil-Grass-Milk Pathway in Aomori, Japan.” Journal of Radioanalytical and Nuclear Chemistry 255(3):455-458. 
PNNL-21950, Appendix C

\section{Appendix C}

\section{Terrestrial Plant}




\section{Appendix C}

\section{Terrestrial Plant}

This appendix contains tables of soil to feed or food-crop bioconcentration factors. References are indicated for each value. For elements with no data compiled, a blank row for that element is shown.

Table C.1. Bioconcentration in Leafy Vegetables from Soil, $\mathrm{kg}_{\text {dry_plant }} / \mathrm{kg}_{\text {dry_soil }}$ (CLBVLV)

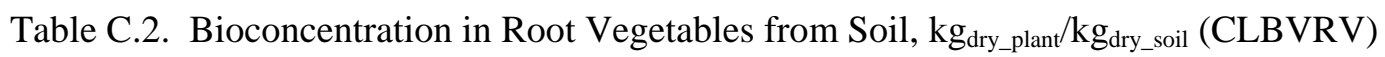

The GENII code uses the CLBVRV parameter information to indicate the bioconcentration factors for both root vegetables and other vegetables (i.e., an "other vegetable” meaning a non-leafy, non-grain, non-fruit crop). In some cases, a reference includes bioconcentration factors for a particular root vegetable and for an "other vegetable." As a result, there may be more than one bioconcentration value listed in the table from a single reference.

Table C.3. Bioconcentration in Fruit from Soil, $\mathrm{kg}_{\text {dry_plant }} / \mathrm{kg}_{\text {dry_soil }}$ (CLBVFR)

Table C.4. Bioaccumulation in Grain from Soil, $\mathrm{kg}_{\text {dry_plant }} / \mathrm{kg}_{\text {dry_soil }}$ (CLBVCL)

In all tables if an element is listed once and there is no data for that element, no bioconcentration factor information was currently identified, preferred, or available. 

Table C.1. Bioconcentration in Dry Leafy Vegetables from Soil, $\mathrm{kg}_{\text {dry_plant }} / \mathrm{kg}_{\text {dry_soil }}$ (CLBVLV)

\begin{tabular}{|c|c|c|c|c|c|c|c|c|c|c|c|c|c|c|c|c|}
\hline Element & Atomic\# & $\mathbf{N}$ & Min & Max & AVG & Mean & Wmean & STD & GSD & GM & WGM & MED & 95\% ile & Document & Reference\# & Reference \\
\hline $\mathrm{Ac}$ & 89 & & & & & & & & & & & $3.50 \mathrm{E}-03$ & & NUREG/CR-5512 & & \\
\hline Ac & 89 & & & & & & & & & & & $4.70 \mathrm{E}-04$ & & PNNL-13421 & Fr82 & \\
\hline $\mathrm{Ag}$ & 47 & & & & & & & & & & & $2.70 \mathrm{E}-04$ & & PNNL-13421 & Fr82 & \\
\hline $\mathrm{Ag}$ & 47 & 5 & 5.90E-05 & $1.30 \mathrm{E}-02$ & & & & & $3.30 \mathrm{E}+00$ & $1.80 \mathrm{E}-04$ & & & & TRS_472 & & \\
\hline $\mathrm{Ag}$ & 47 & & & & & & & & & 1.20E-01 & & & & & & Sheppard et al. (2010b) \\
\hline $\mathrm{Al}$ & 13 & & & & & & & & & & & & & & & \\
\hline Am & 95 & & & & & & & & & & & $5.80 \mathrm{E}-04$ & & NUREG/CR-5512 & & \\
\hline $\mathrm{Am}$ & 95 & & & & & & & & & & & 4.70E-04 & & PNNL-13421 & Fr82 & \\
\hline $\mathrm{Am}$ & 95 & 10 & 4.00E-05 & $1.50 \mathrm{E}-03$ & & & & & $3.30 \mathrm{E}+00$ & $2.70 \mathrm{E}-04$ & & & & TRS_472 & & \\
\hline $\mathrm{Ar}$ & 18 & & & & & & & & & & & & & & & \\
\hline As & 33 & & & & & & & & & & & $4.00 \mathrm{E}-02$ & & PNNL-13421 & Ba84 & \\
\hline As & 33 & & & & & & & & & 7.00E-03 & & & & & & Sheppard et al. (2010b) \\
\hline At & 85 & & & & & & & & & & & & & & & \\
\hline $\mathrm{Au}$ & 79 & & & & & & & & & & & $4.00 \mathrm{E}-01$ & & NUREG/CR-5512 & & \\
\hline $\mathrm{Au}$ & 79 & & & & & & & & & & & $1.00 \mathrm{E}-02$ & & PNNL-13421 & g.m & \\
\hline Ва & 56 & & & & & & & & & & & $1.50 \mathrm{E}-01$ & & PNNL-13421 & Ba84 & \\
\hline Ва & 56 & 1 & & & & $5.00 \mathrm{E}-03$ & & & & & & & & TRS_472 & & \\
\hline $\mathrm{Ba}$ & 56 & & & & & & & & & $2.80 \mathrm{E}-02$ & & & & & & Sheppard et al. (2010b) \\
\hline $\mathrm{Be}$ & 4 & & & & & & & & & & & $1.00 \mathrm{E}-02$ & & PNNL-13421 & Ba84 & \\
\hline Be & 4 & & & & & 4.20E-01 & & & & & & & & TRS_472 & & \\
\hline $\mathrm{Bi}$ & 83 & & & & & & & & & & & $3.50 \mathrm{E}-02$ & & NUREG/CR-5512 & & \\
\hline $\mathrm{Bi}$ & 83 & & & & & & & & & & & $5.00 \mathrm{E}-01$ & & PNNL-13421 & IAEA82 & \\
\hline $\mathrm{Bk}$ & 97 & & & & & & & & & & & & & & & \\
\hline $\mathrm{Br}$ & 35 & & & & & & & & & & & $1.50 \mathrm{E}+00$ & & PNNL-13421 & Fu78 & \\
\hline $\mathrm{C}$ & 6 & & & & & & & & & & & 7.00E-01 & & PNNL-13421 & & \\
\hline $\mathrm{Ca}$ & 20 & & & & & & & & & & & $3.50 \mathrm{E}+00$ & & PNNL-13421 & Ba84 & \\
\hline $\mathrm{Ca}$ & 20 & & $2.30 \mathrm{E}+00$ & $3.80 \mathrm{E}+01$ & & & & & $3.70 \mathrm{E}+00$ & $8.70 \mathrm{E}+00$ & & & & TRS_472 & & \\
\hline $\mathrm{Ca}$ & 20 & & & & & & & & & 7.90E-02 & & & & & & Sheppard et al. (2010b) \\
\hline $\mathrm{Ca}$ & 48 & & & & & & & & & & & 5.50E-01 & & PNNL-13421 & Ba84 & \\
\hline $\mathrm{Cd}$ & 48 & 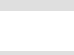 & 1.90E-01 & $5.40 \mathrm{E}+00$ & & & & & $2.20 \mathrm{E}+00$ & $2.10 \mathrm{E}+00$ & & & & TRS_472 & & \\
\hline $\mathrm{Cd}$ & 48 & & & & & & & & & 7.80E-01 & & & & & & Sheppard et al. (2010b) \\
\hline $\mathrm{Ce}$ & 58 & & & & & & & & & & & $1.00 \mathrm{E}-02$ & & NUREG/CR-5512 & & \\
\hline $\mathrm{Ce}$ & 58 & & & & & & & & & & & $2.00 \mathrm{E}-02$ & & PNNL-13421 & Ng82b & \\
\hline $\mathrm{Ce}$ & 58 & 1 & & & & $6.00 \mathrm{E}-03$ & & & & & & & & TRS_472 & & \\
\hline $\mathrm{Ce}$ & 58 & & & & & & & & & $6.20 \mathrm{E}-03$ & & & & & & Sheppard et al. (2010b) \\
\hline $\mathrm{Cf}$ & 98 & & & & & & & & & & & $1.00 \mathrm{E}-02$ & & NUREG/CR-5512 & & \\
\hline $\mathrm{Cf}$ & 98 & & & & & & & & & & & $4.70 \mathrm{E}-04$ & & PNNL-13421 & Fr82 & \\
\hline $\mathrm{Cf}$ & 98 & & & & & & & & & & & 2.50E-04 & & PNNL-13421 & Ba84 & \\
\hline $\mathrm{Cl}$ & 17 & & & & & & & & & & & $7.00 \mathrm{E}+00$ & & PNNL-13421 & Ba84 & \\
\hline $\mathrm{Cl}$ & 17 & & & & & & & & & $2.50 \mathrm{E}+01$ & & & & & & Sheppard et al. (2010b) \\
\hline $\mathrm{Cm}$ & 96 & & & & & & & & & & & 3.00E-04 & & NUREG/CR-5512 & & \\
\hline $\mathrm{Cm}$ & 96 & & & & & & & & & & & 7.70E-04 & & PNNL-13421 & Fr82 & \\
\hline $\mathrm{Cm}$ & 96 & 7 & 2.00E-04 & $8.10 \mathrm{E}-03$ & & & & & $4.50 \mathrm{E}+00$ & $1.40 \mathrm{E}-03$ & & & & TRS_472 & & \\
\hline Co & 26 & & & & & & & & & & & $8.10 \mathrm{E}-02$ & & NUREG/CR-5512 & & \\
\hline Co & 27 & & & & & & & & & & & 2.70E-01 & & PNNL-13421 & Fr89 & \\
\hline Co & 27 & 185 & $1.30 \mathrm{E}-02$ & $1.00 \mathrm{E}+00$ & & & & & $2.70 \mathrm{E}+00$ & $1.70 \mathrm{E}-01$ & & & & TRS_472 & & \\
\hline Co & 27 & & & & & & & & & $1.20 \mathrm{E}-02$ & & & & & & Sheppard et al. (2010b) \\
\hline $\mathrm{Cr}$ & 24 & & & & & & & & & & & 7.00E-03 & & PNNL-13421 & Ba84 & \\
\hline $\mathrm{Cr}$ & 24 & 1 & & & & $1.00 \mathrm{E}-03$ & & & & & & & & TRS_472 & & \\
\hline $\mathrm{Cr}$ & 24 & & & & & & & & & $1.30 \mathrm{E}-02$ & & & & & & Sheppard et al. (2010b) \\
\hline
\end{tabular}


Table C.1. (contd)

\begin{tabular}{|c|c|c|c|c|c|c|c|c|c|c|c|c|c|c|c|c|}
\hline Element & Atomic\# & $\mathbf{N}$ & Min & Max & AVG & Mean & Wmean & STD & GSD & GM & WGM & MED & 95\%ile & Document & Reference\# & Reference \\
\hline $\mathrm{Cs}$ & 55 & & & & & & & & & & & $1.30 \mathrm{E}-01$ & & NUREG/CR-5512 & & \\
\hline Cs & 55 & & & & & & & & & & & 4.60E-01 & & PNNL-13421 & Fr82 & \\
\hline Cs & 55 & 290 & $3.00 \mathrm{E}-04$ & $9.80 \mathrm{E}-01$ & & & & & $6.00 \mathrm{E}+00$ & $6.00 \mathrm{E}-02$ & & & & TRS_472 & & \\
\hline Cs & 55 & & & & & & & & & $2.20 \mathrm{E}-02$ & & & & & & Sheppard et al. (2010b) \\
\hline Cs & 55 & & & & & & & & & & & $2.30 \mathrm{E}-01$ & & & & Mollah et al. (1998) \\
\hline Cs & 55 & & & & & & & & & & & $9.10 \mathrm{E}-01$ & & & & Mollah et al. (1998) \\
\hline $\mathrm{Cu}$ & 29 & & & & & & & & & & & $4.00 \mathrm{E}-01$ & & PNNL-13421 & Ba84 & \\
\hline $\mathrm{Cu}$ & 29 & & & & & & & & & $2.70 \mathrm{E}-01$ & & & & & & Sheppard et al. (2010b) \\
\hline Dy & 66 & & & & & & & & & & & $1.00 \mathrm{E}-02$ & & NUREG/CR-5512 & & \\
\hline Dy & 66 & & & & & & & & & & & $2.00 \mathrm{E}-02$ & & PNNL-13421 & Ng82b & \\
\hline $\mathrm{Er}$ & 68 & & & & & & & & & & & $1.00 \mathrm{E}-02$ & & NUREG/CR-5512 & & \\
\hline Er & 68 & & & & & & & & & & & $2.00 \mathrm{E}-02$ & & PNNL-13421 & Ng82b & \\
\hline Es & 99 & & & & & & & & & & & & & & & \\
\hline Eu & 63 & & & & & & & & & & & $1.00 \mathrm{E}-02$ & & NUREG/CR-5512 & & \\
\hline Eu & 63 & & & & & & & & & & & $2.00 \mathrm{E}-02$ & & PNNL-13421 & Ng82b & \\
\hline $\mathrm{F}$ & 9 & & & & & & & & & & & $6.00 \mathrm{E}-02$ & & PNNL-13421 & Ba84 & \\
\hline $\mathrm{Fe}$ & 26 & & & & & & & & & & & 4.00E-03 & & NUREG/CR-5512 & & \\
\hline $\mathrm{Fe}$ & 26 & & & & & & & & & & & $5.00 \mathrm{E}-02$ & & PNNL-13421 & СТ83 & \\
\hline $\mathrm{Fe}$ & 26 & 1 & & & & $1.00 \mathrm{E}-03$ & & & & & & & & TRS_472 & & \\
\hline $\mathrm{Fm}$ & 100 & & & & & & & & & & & & & & & \\
\hline $\mathrm{Fr}$ & 87 & & & & & & & & & & & & & & & \\
\hline $\mathrm{Ga}$ & 31 & & & & & & & & & & & $4.00 \mathrm{E}-03$ & & PNNL-13421 & Ba84 & \\
\hline $\mathrm{Gd}$ & 64 & & & & & & & & & & & $1.00 \mathrm{E}-02$ & & NUREG/CR-5512 & & \\
\hline $\mathrm{Gd}$ & 64 & & & & & & & & & & & $2.00 \mathrm{E}-02$ & & PNNL-13421 & Ng82b & \\
\hline $\mathrm{Ge}$ & 32 & & & & & & & & & & & & & & & \\
\hline $\mathrm{H}$ & 1 & & & & & & & & & & & & & & & \\
\hline $\mathrm{Hf}$ & 72 & & & & & & & & & & & $3.50 \mathrm{E}-03$ & & NUREG/CR-5512 & & \\
\hline $\mathrm{Hf}$ & 72 & & & & & & & & & & & $1.00 \mathrm{E}-03$ & & PNNL-13421 & Ng82b & \\
\hline $\mathrm{Hg}$ & 80 & & & & & & & & & & & $9.00 \mathrm{E}-01$ & & NUREG/CR-5512 & & \\
\hline $\mathrm{Hg}$ & 80 & & & & & & & & & & & $8.50 \mathrm{E}-01$ & & PNNL-13421 & & \\
\hline Но & 67 & & & & & & & & & & & $1.00 \mathrm{E}-02$ & & NUREG/CR-5512 & & \\
\hline Но & 67 & & & & & & & & & & & $2.00 \mathrm{E}-02$ & & PNNL-13421 & Ng82b & \\
\hline I & 53 & & & & & & & & & & & $3.40 \mathrm{E}-03$ & & NUREG/CR-5512 & & \\
\hline I & 53 & & & & & & & & & & & $4.00 \mathrm{E}-02$ & & PNNL-13421 & Sn94 & \\
\hline I & 53 & & & & & & & & & $1.40 \mathrm{E}-02$ & & & & & & Uchida and Tagami (2011) \\
\hline In & 49 & & & & & & & & & & & 4.00E-03 & & PNNL-13421 & Fu78 & \\
\hline Ir & 77 & & & & & & & & & & & $5.50 \mathrm{E}-02$ & & PNNL-13421 & Ba84 & \\
\hline $\mathrm{K}$ & 19 & & & & & & & & & & & $1.00 \mathrm{E}+00$ & & PNNL-13421 & Ba84 & \\
\hline $\mathrm{K}$ & 19 & 2 & $1.20 \mathrm{E}+00$ & $1.30 \mathrm{E}+00$ & & $1.30 \mathrm{E}+00$ & & & & & & & & TRS_472 & & \\
\hline $\mathrm{K}$ & 19 & & $1.00 \mathrm{E}+00$ & $5.00 \mathrm{E}+00$ & & & & & & & & & & & & Al-Masri et al. (2008) \\
\hline $\mathrm{K}$ & 19 & & & & & & & & & $1.00 \mathrm{E}-01$ & & & & & & Sheppard et al. (2010b) \\
\hline $\mathrm{Kr}$ & 36 & & & & & & & & & & & & & & & \\
\hline $\mathrm{La}$ & 57 & & & & & & & & & & & $5.70 \mathrm{E}-04$ & & NUREG/CR-5512 & & \\
\hline $\mathrm{La}$ & 57 & & & & & & & & & & & $5.20 \mathrm{E}-03$ & & PNNL-13421 & Fr89 & \\
\hline $\mathrm{La}$ & 57 & 7 & $1.10 \mathrm{E}-03$ & $1.50 \mathrm{E}-02$ & & & & & $2.70 \mathrm{E}+00$ & $5.70 \mathrm{E}-03$ & & & & TRS_472 & & \\
\hline $\mathrm{La}$ & 57 & & & & & & & & & $6.80 \mathrm{E}-03$ & & & & & & Sheppard et al. (2010b) \\
\hline $\mathrm{Lu}$ & 71 & & & & & & & & & & & & & & & \\
\hline Md & 101 & & & & & & & & & & & & & & & \\
\hline $\mathrm{Mg}$ & 12 & & & & & & & & & & & $1.00 \mathrm{E}+00$ & & PNNL-13421 & Ba84 & \\
\hline $\mathrm{Mg}$ & 12 & & & & & & & & & 7.30E-01 & & & & & & Sheppard et al. (2010b) \\
\hline $\mathrm{Mn}$ & 25 & & & & & & & & & & & $5.60 \mathrm{E}-01$ & & NUREG-5512 & & \\
\hline $\mathrm{Mn}$ & 25 & & & & & & & & & & & $7.00 \mathrm{E}-01$ & & PNNL-13421 & Fr89 & \\
\hline
\end{tabular}


Table C.1. (contd)

\begin{tabular}{|c|c|c|c|c|c|c|c|c|c|c|c|c|c|c|c|c|}
\hline Element & Atomic\# & $\mathbf{N}$ & Min & Max & AVG & Mean & Wmean & STD & GSD & GM & WGM & MED & 95\%ile & Document & Reference\# & Reference \\
\hline $\mathrm{Mn}$ & 25 & 103 & $5.20 \mathrm{E}-02$ & $3.00 \mathrm{E}+00$ & & & & & $2.40 \mathrm{E}+00$ & $4.10 \mathrm{E}-01$ & & & & $\begin{array}{l}\text { TRS_472 } \\
\end{array}$ & & \\
\hline $\mathrm{Mn}$ & 25 & & & & & & & & & $1.00 \mathrm{E}-01$ & & & & & & Sheppard et al. (2010b) \\
\hline Mo & 42 & & & & & & & & & & & $2.50 \mathrm{E}-01$ & & NUREG/CR-5512 & & \\
\hline Mo & 42 & & & & & & & & & & & $8.00 \mathrm{E}-01$ & & PNNL-13421 & Ng82b & \\
\hline Mo & 42 & 1 & $2.10 \mathrm{E}-01$ & $8.00 \mathrm{E}+01$ & & $5.10 \mathrm{E}-01$ & & & & & & & & TRS_472 & & \\
\hline Mo & 42 & & & & & & & & & $3.80 \mathrm{E}+00$ & & & & & & Sheppard et al. (2010b) \\
\hline $\mathrm{N}$ & 7 & & & & & & & & & & & $3.00 \mathrm{E}+01$ & & NUREG-5512 & & \\
\hline $\mathrm{N}$ & 7 & & & & & & & & & & & $5.50 \mathrm{E}-02$ & & PNNL-13421 & Ba84 & \\
\hline $\mathrm{Na}$ & 11 & & & & & & & & & & & $7.50 \mathrm{E}-02$ & & NUREG-5512 & & \\
\hline $\mathrm{Na}$ & 11 & & & & & & & & & & & $3.00 \mathrm{E}-01$ & & PNNL-13421 & Ng82b & \\
\hline $\mathrm{Na}$ & 11 & 1 & & & & $3.00 \mathrm{E}-02$ & & & & & & & & TRS_472 & & \\
\hline $\mathrm{Na}$ & 11 & & & & & & & & & $3.20 \mathrm{E}+00$ & & & & & & Sheppard et al. (2010b) \\
\hline $\mathrm{Nb}$ & 41 & & & & & & & & & & & $2.00 \mathrm{E}-02$ & & NUREG/CR-5512 & & \\
\hline $\mathrm{Nb}$ & 41 & & & & & & & & & & & $2.50 \mathrm{E}-02$ & & PNNL-13421 & СТ83 & \\
\hline $\mathrm{Nb}$ & 41 & 2 & $8.00 \mathrm{E}-03$ & $2.50 \mathrm{E}-02$ & & $1.70 \mathrm{E}-02$ & & & & & & & & TRS_472 & & \\
\hline $\mathrm{Nb}$ & 41 & & & & & & & & & $1.70 \mathrm{E}-02$ & & & & & & Sheppard et al. (2010b) \\
\hline $\mathrm{Nd}$ & 60 & & & & & & & & & & & $1.00 \mathrm{E}-02$ & & NUREG/CR-5512 & & \\
\hline $\mathrm{Nd}$ & 60 & & & & & & & & & & & $2.00 \mathrm{E}-02$ & & PNNL-13421 & Ng82b & \\
\hline $\mathrm{Nd}$ & 60 & & & & & & & & & $4.30 \mathrm{E}-03$ & & & & & & Sheppard et al. (2010b) \\
\hline $\mathrm{Ne}$ & 10 & & & & & & & & & & & & & & & \\
\hline $\mathrm{Ni}$ & 28 & & & & & & & & & & & $2.80 \mathrm{E}-01$ & & PNNL-13421 & IUR89 & \\
\hline $\mathrm{Ni}$ & 28 & 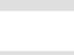 & $1.80 \mathrm{E}-02$ & $5.80 \mathrm{E}-01$ & & & & & $2.60 \mathrm{E}+00$ & $1.70 \mathrm{E}-01$ & & & & TRS_472 & & \\
\hline $\mathrm{Ni}$ & 28 & & & & & & & & & $1.80 \mathrm{E}-02$ & & & & & & Sheppard et al. (2010b) \\
\hline $\mathrm{Np}$ & 93 & 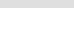 & & & & & & & & & & $1.30 \mathrm{E}-02$ & & NUREG/CR-5512 & & \\
\hline $\mathrm{Np}$ & 93 & & & & & & & & & & & $3.20 \mathrm{E}-02$ & & PNNL-13421 & Fr82 & \\
\hline $\mathrm{Np}$ & 93 & 5 & $5.00 \mathrm{E}-03$ & $8.00 \mathrm{E}-02$ & & & & & $3.00 \mathrm{E}+00$ & $2.70 \mathrm{E}-02$ & & & & TRS_472 & & \\
\hline \multirow{2}{*}{\multicolumn{17}{|c|}{ OBT }} \\
\hline & & & & & & & & & & & & & & & & \\
\hline Os & 76 & 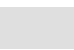 & & & & & & & & & & $1.50 \mathrm{E}-02$ & & PNNL-13421 & Ва84 & \\
\hline $\mathrm{P}$ & 15 & & & & & & & & & & & $3.50 \mathrm{E}+00$ & & PNNL-13421 & Ba84 & \\
\hline $\mathrm{P}$ & 15 & 1 & & & & $1.00 \mathrm{E}+00$ & & & & & & & & TRS_472 & & \\
\hline Рa & 91 & & & & & & & & & & & $2.50 \mathrm{E}-03$ & & NUREG/CR-5512 & & \\
\hline $\mathrm{Pa}$ & 91 & & & & & & & & & & & $4.70 \mathrm{E}-04$ & & PNNL-13421 & Fr82 & \\
\hline $\mathrm{Pb}$ & 82 & & & & & & & & & & & $5.80 \mathrm{E}-03$ & & NUREG/CR-5512 & & \\
\hline $\mathrm{Pb}$ & 82 & & & & & & & & & & & $1.00 \mathrm{E}-02$ & & PNNL-13421 & Fr89 & \\
\hline $\mathrm{Pb}$ & 82 & 31 & $3.20 \mathrm{E}-03$ & $2.50 \mathrm{E}+01$ & & & & & $1.30 \mathrm{E}+01$ & $8.00 \mathrm{E}-02$ & & & & TRS_472 & & \\
\hline $\mathrm{Pb}$ & 82 & & $2.40 \mathrm{E}-01$ & $1.43 \mathrm{E}+00$ & & & & & & & & & & & & Al-Masri et al. (2008) \\
\hline $\mathrm{Pb}$ & 82 & & & & & & & & & $4.90 \mathrm{E}-03$ & & & & & & Sheppard et al. (2010b) \\
\hline $\mathrm{Pb}$ & 82 & & $3.21 \mathrm{E}-03$ & $2.46 \mathrm{E}+01$ & & $2.11 \mathrm{E}+00$ & & $6.12 \mathrm{E}+00$ & & & & & & & & Vandenhove et al. (2009b) \\
\hline $\mathrm{Pd}$ & 46 & & & & & & & & & & & $1.50 \mathrm{E}-01$ & & PNNL-13421 & Ва84 & \\
\hline Pm & 61 & & & & & & & & & & & $1.00 \mathrm{E}-02$ & & NUREG/CR-5512 & & \\
\hline $\mathrm{Pm}$ & 61 & & & & & & & & & & & $2.00 \mathrm{E}-02$ & & PNNL-13421 & Ng82b & \\
\hline $\mathrm{Pm}$ & 61 & & $2.20 \mathrm{E}-02$ & $1.40 \mathrm{E}+00$ & & & & & $4.10 \mathrm{E}+00$ & $2.30 \mathrm{E}-01$ & & & & TRS_472 & & \\
\hline Po & 84 & & & & & & & & & & & $1.20 \mathrm{E}-03$ & & PNNL-13421 & Ho91 & \\
\hline Po & 84 & 12 & $2.50 \mathrm{E}-04$ & $5.00 \mathrm{E}-02$ & & & & & $6.90 \mathrm{E}+00$ & 7.40E-03 & & & & TRS_472 & & \\
\hline Po & 84 & & $2.80 \mathrm{E}-02$ & $2.10 \mathrm{E}-01$ & & & & & & & & & & & & Al-Masri et al. (2008) \\
\hline Po & 84 & & & & & $1.90 \mathrm{E}-02$ & $1.72 \mathrm{E}-02$ & & & & & & & & & Vandenhove et al. (2009b) \\
\hline Pr & 59 & & & & & & & & & & & $1.00 \mathrm{E}-02$ & & NUREG/CR-5512 & & \\
\hline $\operatorname{Pr}$ & 59 & & & & & & & & & & & $2.00 \mathrm{E}-02$ & & PNNL-13421 & Ng82b & \\
\hline $\operatorname{Pr}$ & 59 & 1 & & & & $2.00 \mathrm{E}-02$ & & & & & & & & TRS_472 & & \\
\hline $\operatorname{Pr}$ & 59 & & & & & & & & & $5.40 \mathrm{E}-03$ & & & & & & Sheppard et al. (2010b) \\
\hline
\end{tabular}


Table C.1. (contd)

\begin{tabular}{|c|c|c|c|c|c|c|c|c|c|c|c|c|c|c|c|c|}
\hline Element & Atomic\# & $\mathbf{N}$ & Min & Max & AVG & Mean & Wmean & STD & GSD & GM & WGM & MED & 95\%ile & Document & Reference\# & Reference \\
\hline $\mathrm{Pu}$ & 94 & & & & & & & & & & & $3.90 \mathrm{E}-04$ & & NUREG/CR-5512 & & \\
\hline $\mathrm{Pu}$ & 94 & & & & & & & & & & & $5.00 \mathrm{E}-05$ & & PNNL-13421 & Fr82 & \\
\hline Pu & 94 & 13 & $1.00 \mathrm{E}-05$ & $2.90 \mathrm{E}-04$ & & & & & $2.70 \mathrm{E}+00$ & $8.30 \mathrm{E}-05$ & & & & TRS_472 & & \\
\hline Ra & 88 & & & & & & & & & & & 7.50E-02 & & NUREG/CR-5512 & & \\
\hline Ra & 88 & & & & & & & & & & & 4.90E-02 & & PNNL-13421 & Fr89 & \\
\hline Ra & 88 & 77 & $1.80 \mathrm{E}-03$ & $1.30 \mathrm{E}+02$ & & & & & $6.70 \mathrm{E}+00$ & $9.10 \mathrm{E}-02$ & & & & TRS_472 & & \\
\hline Ra & 88 & & $2.60 \mathrm{E}-02$ & $3.80 \mathrm{E}-02$ & & & & & & & & & & & & Lauria et al. (2009) \\
\hline Ra & 88 & & $1.76 \mathrm{E}-03$ & $1.25 \mathrm{E}+02$ & & $2.58 \mathrm{E}+00$ & & $1.49 \mathrm{E}+01$ & & & & & & & & Vandenhove et al. (2009b) \\
\hline $\mathrm{Rb}$ & 37 & & & & & & & & & & & $1.50 \mathrm{E}-01$ & & NUREG/CR-5512 & & \\
\hline $\mathrm{Rb}$ & 37 & & & & & & & & & & & $9.00 \mathrm{E}-01$ & & PNNL-13421 & Ng82b & \\
\hline $\mathrm{Rb}$ & 37 & 2 & $3.40 \mathrm{E}-01$ & $9.00 \mathrm{E}-01$ & & $6.20 \mathrm{E}-01$ & & & & & & & & TRS_472 & & \\
\hline $\mathrm{Rb}$ & 37 & & & & & & & & & $6.20 \mathrm{E}-01$ & & & & & & Sheppard et al. (2010b) \\
\hline Re & 75 & & & & & & & & & & & $1.50 \mathrm{E}+00$ & & PNNL-13421 & Ba84 & \\
\hline $\mathrm{Rh}$ & 45 & & & & & & & & & & & 1.50E-01 & & PNNL-13421 & Ba84 & \\
\hline $\mathrm{Rn}$ & 86 & 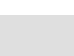 & & & & & & & & & & & & & & \\
\hline Ru & 44 & & & & & & & & & & & $5.20 \mathrm{E}-01$ & & NUREG/CR-5512 & & \\
\hline Ru & 44 & & & & & & & & & & & 4.00E-02 & & PNNL-13421 & Ng82b & \\
\hline Ru & 44 & 3 & $2.00 \mathrm{E}-02$ & $2.30 \mathrm{E}-01$ & & & & & $3.70 \mathrm{E}+00$ & $9.00 \mathrm{E}-02$ & & & & TRS_472 & & \\
\hline $\mathrm{s}$ & 16 & & & & & & & & & & & $1.50 \mathrm{E}+00$ & & PNNL-13421 & Ba84 & \\
\hline sb & 51 & & & & & & & & & & & $1.30 \mathrm{E}-04$ & & PNNL-13421 & IUR89 & \\
\hline Sb & 51 & 5 & $2.20 \mathrm{E}-05$ & $2.30 \mathrm{E}-04$ & & & & & $2.60 \mathrm{E}+00$ & $9.40 \mathrm{E}-05$ & & & & TRS_472 & & \\
\hline sb & 51 & & & & & & & & & $1.70 \mathrm{E}-02$ & & & & & & Sheppard et al. (2010b) \\
\hline Sc & 21 & & & & & & & & & & & $6.00 \mathrm{E}-03$ & & PNNL-13421 & Ba84 & \\
\hline Se & 34 & & & & & & & & & & & $2.50 \mathrm{E}-01$ & & PNNL-13421 & СТ83 & \\
\hline Se & 34 & & & & & & & & & $1.00 \mathrm{E}+00$ & & & & & & Sheppard et al. (2010b) \\
\hline $\mathrm{Si}$ & 14 & & & & & & & & & & & $3.50 \mathrm{E}-01$ & & PNNL-13421 & Ba84 & \\
\hline $\mathrm{Sm}$ & 62 & & & & & & & & & & & $1.00 \mathrm{E}-02$ & & NUREG/CR-5512 & & \\
\hline Sm & 62 & & & & & & & & & & & $2.00 \mathrm{E}-02$ & & PNNL-13421 & Ng82b & \\
\hline Sm & 62 & & & & & & & & & $4.50 \mathrm{E}-03$ & & & & & & Sheppard et al. (2010b) \\
\hline Sn & 50 & & & & & & & & & & & $3.00 \mathrm{E}-02$ & & PNNL-13421 & Fu78 & \\
\hline Sn & 50 & & & & & & & & & $1.20 \mathrm{E}-01$ & & & & & & Sheppard et al. (2010b) \\
\hline $\mathrm{Sr}$ & 38 & & & & & & & & & & & $1.60 \mathrm{E}+00$ & & NUREG/CR-5512 & & \\
\hline $\mathrm{Sr}$ & 38 & & & & & & & & & & & $3.00 \mathrm{E}+00$ & & PNNL-13421 & Fr82 & \\
\hline $\mathrm{Sr}$ & 38 & 217 & $3.90 \mathrm{E}-03$ & $7.80 \mathrm{E}+00$ & & & & & $6.00 \mathrm{E}+00$ & 7.60E-01 & & & & TRS 472 & & \\
\hline $\mathrm{Sr}$ & 38 & & & & & & & & & $1.10 \mathrm{E}+00$ & & & & & & Sheppard et al. (2010b) \\
\hline $\mathrm{Sr}$ & 38 & & & & & & & & & & & $6.90 \mathrm{E}-01$ & & & & Mollah et al. (1998) \\
\hline $\mathrm{Sr}$ & 38 & & & & & & & & & & & $5.90 \mathrm{E}-01$ & & & & Mollah et al. (1998) \\
\hline Та & 73 & & & & & & & & & & & 1.00E-02 & & NUREG/CR-5512 & & \\
\hline Та & 73 & & & & & & & & & & & $2.50 \mathrm{E}-02$ & & PNNL-13421 & СТ83 & \\
\hline $\mathrm{Tb}$ & 65 & & & & & & & & & & & $1.00 \mathrm{E}-02$ & & NUREG/CR-5512 & & \\
\hline $\mathrm{Tb}$ & 65 & & & & & & & & & & & 2.00E-02 & & PNNL-13421 & Ng82b & \\
\hline $\mathrm{Tb}$ & 65 & & & & & & & & & 7.20E-03 & & & & & & Sheppard et al. (2010b) \\
\hline Tc & 43 & & & & & & & & & & & $4.40 \mathrm{E}+01$ & & NUREG/CR-5512 & & \\
\hline Tc & 43 & & & & & & & & & & & $2.10 \mathrm{E}+01$ & & PNNL-13421 & Fr82 & \\
\hline $\mathrm{Te}$ & 52 & & & & & & & & & & & $2.50 \mathrm{E}-02$ & & NUREG/CR-5512 & & \\
\hline $\mathrm{Te}$ & 52 & & & & & & & & & & & $2.00 \mathrm{E}-02$ & & PNNL-13421 & Ba84 & \\
\hline Te & 52 & 1 & & & & $3.00 \mathrm{E}-01$ & & & & & & & & TRS_472 & & \\
\hline Th & 90 & & & & & & & & & & & $6.60 \mathrm{E}-03$ & & NUREG/CR-5512 & & \\
\hline Th & 90 & & & & & & & & & & & $1.80 \mathrm{E}-03$ & & PNNL-13421 & Fr89 & \\
\hline Th & 90 & 24 & $9.40 \mathrm{E}-05$ & 2.10E-01 & & & & & $6.00 \mathrm{E}+00$ & $1.20 \mathrm{E}-03$ & & & & TRS_472 & & \\
\hline Th & 90 & & & & & & & & & $1.36 \mathrm{E}-02$ & & & & & & Sheppard et al. (2010b) \\
\hline Th & 90 & & $9.38 \mathrm{E}-05$ & $2.11 \mathrm{E}-01$ & & $1.18 \mathrm{E}-02$ & & $4.29 \mathrm{E}-02$ & & & & & & & & Vandenhove et al. (2009b) \\
\hline
\end{tabular}


Table C.1. (contd)

\begin{tabular}{|c|c|c|c|c|c|c|c|c|c|c|c|c|c|c|c|c|}
\hline Element & Atomic\# & $\mathbf{N}$ & Min & Max & AVG & Mean & Wmean & STD & GSD & GM & WGM & MED & 95\%ile & Document & Reference\# & Reference \\
\hline $\mathrm{Ti}$ & 22 & & & & & & & & & & & & & & & \\
\hline $\mathrm{Tl}$ & 81 & & & & & & & & & & & $4.00 \mathrm{E}-03$ & & PNNL-13421 & Fu78 & \\
\hline $\mathrm{Tl}$ & 81 & & & & & & & & & $1.16 \mathrm{E}-01$ & & & & & & Sheppard et al. (2010b) \\
\hline $\mathrm{Tm}$ & 69 & & & & & & & & & & & & & & & \\
\hline $\mathrm{U}$ & 92 & & & & & & & & & & & $1.70 \mathrm{E}-02$ & & NUREG/CR-5512 & & \\
\hline $\mathrm{U}$ & 92 & & & & & & & & & & & $8.30 \mathrm{E}-03$ & & PNNL-13421 & Fr89 & \\
\hline $\mathrm{U}$ & 92 & 108 & $7.80 \mathrm{E}-05$ & $8.80 \mathrm{E}+00$ & & & & & $7.30 \mathrm{E}+00$ & $2.00 \mathrm{E}-02$ & & & & TRS_472 & & \\
\hline $\mathrm{U}$ & 92 & & 3.00E-03 & $5.70 \mathrm{E}-02$ & & & & & & & & & & & & Al-Masri et al. (2008) \\
\hline $\mathrm{U}$ & 92 & & & & & & & & & $5.20 \mathrm{E}-03$ & & & & & & Sheppard et al. (2010b) \\
\hline $\mathrm{U}$ & 92 & & $1.10 \mathrm{E}-02$ & $1.40 \mathrm{E}-02$ & & & & & & & & & & & & Lauria et al. (2009) \\
\hline $\mathrm{U}$ & 92 & & $7.84 \mathrm{E}-05$ & $8.82 \mathrm{E}+00$ & & $2.21 \mathrm{E}-01$ & & $1.14 \mathrm{E}+00$ & & & & & & & & Vandenhove et al. (2009b) \\
\hline $\mathrm{V}$ & 23 & & & & & & & & & $8.80 \mathrm{E}-03$ & & & & & & Sheppard et al. (2010b) \\
\hline $\mathrm{w}$ & 74 & & & & & & & & & & & $4.50 \mathrm{E}-02$ & & NUREG/CR-5512 & & \\
\hline $\mathrm{w}$ & 74 & & & & & & & & & & & $3.00 \mathrm{E}+00$ & & PNNL-13421 & NCRP86 & \\
\hline Xe & 54 & & & & & & & & & & & & & & & \\
\hline Y & 39 & & & & & & & & & & & $1.50 \mathrm{E}-02$ & & NUREG/CR-5512 & & \\
\hline $\mathrm{Y}$ & 39 & & & & & & & & & & & $1.00 \mathrm{E}-02$ & & PNNL-13421 & Ng82b & \\
\hline $\mathrm{Y}$ & 39 & 1 & & & & $2.00 \mathrm{E}-03$ & & & & & & & & TRS_472 & & \\
\hline $\mathrm{Y}$ & 39 & & & & & & & & & $5.50 \mathrm{E}-03$ & & & & & & Sheppard et al. (2010b) \\
\hline $\mathrm{Yb}$ & 70 & & & & & & & & & $8.00 \mathrm{E}-03$ & & & & & & Sheppard et al. (2010b) \\
\hline $\mathrm{Zn}$ & 30 & & & & & & & & & & & $1.40 \mathrm{E}+00$ & & NUREG/CR-5512 & & \\
\hline $\mathrm{Zn}$ & 30 & & & & & & & & & & & $1.30 \mathrm{E}+00$ & & PNNL-13421 & Fr89 & \\
\hline $\mathrm{Zn}$ & 30 & 112 & $1.00 \mathrm{E}-01$ & $1.70 \mathrm{E}+01$ & & & & & $2.40 \mathrm{E}+00$ & $2.40 \mathrm{E}+00$ & & & & TRS 472 & & \\
\hline $\mathrm{Zn}$ & 30 & & & & & & & & & $2.80 \mathrm{E}-01$ & & & & & & Sheppard et al. (2010b) \\
\hline $\mathrm{Zr}$ & 40 & & & & & & & & & & & $2.00 \mathrm{E}-03$ & & NUREG/CR-5512 & & \\
\hline $\mathrm{Zr}$ & 40 & & & & & & & & & & & $1.00 \mathrm{E}-03$ & & PNNL-13421 & Ng82b & \\
\hline $\mathrm{Zr}$ & 40 & 1 & & & & $4.00 \mathrm{E}-02$ & & & & & & & & TRS_472 & & \\
\hline $\mathrm{Zr}$ & 40 & & & & & & & & & 8.70E-02 & & & & & & Sheppard et al. (2010b) \\
\hline
\end{tabular}



Table C.2. Bioconcentration in Dry Root Vegetables from Soil, kgdry_plant $/ k_{\text {dry_soil }}$ (CLBVRV\&CLBVOV combined)

\begin{tabular}{|c|c|c|c|c|c|c|c|c|c|c|c|c|c|c|c|}
\hline Element & Atomic\# & $\mathrm{N}$ & Min & Max & $\mathrm{AVG}$ & Mean & Wmean & STD & GSD & GM & MED & $95 \%$ ile & Document & Reference\# & Reference \\
\hline Ac & 89 & & & & & & & & & & $3.50 \mathrm{E}-04$ & & PNNL-13421 & Fr82 & \\
\hline Ag & 47 & & & & & & & & & & $1.30 \mathrm{E}-03$ & & PNNL-13421 & Fr82 & \\
\hline $\mathrm{Ag}$ & 47 & & & & & & & & & $3.70 \mathrm{E}-02$ & & & & & Sheppard et al. (2010b) \\
\hline $\mathrm{Ag}$ & 47 & 5 & $2.50 \mathrm{E}-04$ & $2.00 \mathrm{E}-03$ & & & & & $2.30 \mathrm{E}+00$ & $6.40 \mathrm{E}-04$ & & & TRS_472 (OV) & & \\
\hline $\mathrm{Ag}$ & 47 & 6 & $5.70 \mathrm{E}-04$ & $3.90 \mathrm{E}-03$ & & & & & $2.00 \mathrm{E}+00$ & $1.30 \mathrm{E}-03$ & & & TRS_472 (RV) & & \\
\hline $\mathrm{Am}$ & 95 & & & & & & & & & & $3.50 \mathrm{E}-04$ & & PNNL-13421 & Fr82 & \\
\hline $\mathrm{Am}$ & 95 & & & & & & & & & & $4.10 \mathrm{E}-04$ & & NUREG/CR-5512 & & \\
\hline Am & 95 & & $3.00 \mathrm{E}-06$ & $1.00 \mathrm{E}-03$ & & & & & & & $1.00 \mathrm{E}-05$ & & Coughtrey et al. (1985) & S2.2, T2.1 & \\
\hline Am & 95 & 9 & $2.30 \mathrm{E}-05$ & $1.90 \mathrm{E}-03$ & & & & & $5.00 \mathrm{E}+00$ & $3.60 \mathrm{E}-04$ & & & TRS_472 (OV) & & \\
\hline $\mathrm{Am}$ & 95 & 4 & $2.00 \mathrm{E}-04$ & $1.70 \mathrm{E}-03$ & & & & & $2.40 \mathrm{E}+00$ & $6.70 \mathrm{E}-04$ & & & TRS_472 (RV) & & \\
\hline $\mathrm{Ar}$ & 18 & & & & & & & & & & & & & & \\
\hline As & 33 & & & & & & & & & & $5.00 \mathrm{E}-03$ & & NUREG/CR-5512 & & \\
\hline As & 33 & & & & & & & & & $4.00 \mathrm{E}-03$ & & & & & Sheppard et al. (2010b) \\
\hline At & 85 & & & & & & & & & & & & & & \\
\hline $\mathrm{Au}$ & 79 & & & & & & & & & & $1.80 \mathrm{E}-02$ & & PNNL-13421 & g.m. & \\
\hline $\mathrm{Au}$ & 79 & & & & & & & & & & $1.00 \mathrm{E}-01$ & & NUREG/CR-5512 & & \\
\hline $\mathrm{Ba}$ & 56 & 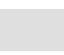 & & & & & & & & & $1.50 \mathrm{E}-02$ & & PNNL-13421 & Ba84 & \\
\hline $\mathrm{Ba}$ & 56 & & & & & & & & & 7.50E-02 & & & & & Sheppard et al. (2010b) \\
\hline $\mathrm{Ba}$ & 56 & 1 & & & & $5.00 \mathrm{E}-03$ & & & & & & & TRS_472 (OV) & & \\
\hline $\mathrm{Be}$ & 4 & & & & & & & & & & & & & & \\
\hline $\mathrm{Bi}$ & 83 & 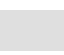 & & & & & & & & & $1.00 \mathrm{E}-05$ & & PNNL-13421 & IAEA82 & \\
\hline $\mathrm{Bi}$ & 83 & ( & & & & & & & & & $5.00 \mathrm{E}-03$ & & NUREG/CR-5512 & & \\
\hline $\mathrm{Bk}$ & 97 & 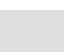 & & & & & & & & & & & & & \\
\hline $\mathrm{C}$ & 6 & & & & & & & & & & $7.00 \mathrm{E}-01$ & & PNNL-13421 & Na88 & \\
\hline $\mathrm{Ca}$ & 20 & & & & & & & & & & $3.50 \mathrm{E}-01$ & & PNNL-13421 & Ba84 & \\
\hline $\mathrm{Ca}$ & 20 & & & & & & & & & 7.10E-02 & & & & & Sheppard et al. (2010b) \\
\hline $\mathrm{Ca}$ & 20 & 6 & 53 & 75 & & & & & & $2.00 \mathrm{E}+01$ & & & TRS_472 (legume) & & \\
\hline $\mathrm{Cd}$ & 48 & & & & & & & & & & $1.50 \mathrm{E}-01$ & & PNNL-13421 & Ba84 & \\
\hline $\mathrm{Cd}$ & 48 & & & & & & & & & $2.60 \mathrm{E}-01$ & & & & & Sheppard et al. (2010b) \\
\hline $\mathrm{Cd}$ & 48 & & 7.00E-02 & $4.00 \mathrm{E}+00$ & & & & & & $8.00 \mathrm{E}-01$ & & & Coughtrey et al. (1985) & S2.1, T2.3 & \\
\hline $\mathrm{Cd}$ & 48 & 1 & $8.00 \mathrm{E}-02$ & $4.60 \mathrm{E}-01$ & & $2.70 \mathrm{E}-01$ & & & $2.70 \mathrm{E}-01$ & & & & TRS_472 (legume) & & \\
\hline $\mathrm{Cd}$ & 48 & 1 & & & & $1.50 \mathrm{E}+00$ & & & & & & & TRS_472 (tuber) & & \\
\hline $\mathrm{Ce}$ & 58 & & & & & & & & & & $2.00 \mathrm{E}-02$ & & PNNL-13421 & Ng82b & \\
\hline $\mathrm{Ce}$ & 58 & & & & & & & & & & $4.00 \mathrm{E}-03$ & & NUREG/CR-5512 & & \\
\hline $\mathrm{Ce}$ & 58 & & & & & & & & & $6.70 \mathrm{E}-04$ & & & & & Sheppard et al. (2010b) \\
\hline $\mathrm{Ce}$ & 58 & 2 & $6.00 \mathrm{E}-03$ & $2.00 \mathrm{E}-02$ & & $1.30 \mathrm{E}-02$ & & & & & & & TRS_472 (legume) & & \\
\hline $\mathrm{Ce}$ & 58 & 1 & & & & $6.00 \mathrm{E}-03$ & & & & & & & TRS_472 (RV) & & \\
\hline Cf & 98 & & & & & & & & & & $3.50 \mathrm{E}-04$ & & PNNL-13421 & Fr82 & \\
\hline $\mathrm{Cf}$ & 98 & & & & & & & & & & $1.00 \mathrm{E}-02$ & & NUREG/CR-5512 & & \\
\hline $\mathrm{Cl}$ & 17 & & & & & & & & & & $7.00 \mathrm{E}+00$ & & PNNL-13421 & Ba84 & \\
\hline $\mathrm{Cl}$ & 17 & & & & & & & & & $7.80 \mathrm{E}+00$ & & & & & Sheppard et al. (2010b) \\
\hline $\mathrm{Cm}$ & 96 & & & & & & & & & & 4.30E-04 & & PNNL-13421 & Fr82 & \\
\hline $\mathrm{Cm}$ & 96 & & & & & & & & & & $2.40 \mathrm{E}-04$ & & NUREG/CR-5512 & & \\
\hline $\mathrm{Cm}$ & 96 & 8 & $3.60 \mathrm{E}-05$ & $1.40 \mathrm{E}-03$ & & & & & $4.50 \mathrm{E}+00$ & $3.20 \mathrm{E}-04$ & & & TRS_472 (OV) & & \\
\hline $\mathrm{Cm}$ & 96 & 6 & $2.00 \mathrm{E}-04$ & $3.90 \mathrm{E}-03$ & & & & & $3.00 \mathrm{E}+00$ & $8.50 \mathrm{E}-04$ & & & TRS_472 (RV) & & \\
\hline Сo & 27 & & & & & & & & & & $6.70 \mathrm{E}-02$ & & PNNL-13421 & Fr89 & \\
\hline Сo & 27 & & & & & & & & & & $4.00 \mathrm{E}-02$ & & NUREG/CR-5512 & & \\
\hline
\end{tabular}


Table C.2. (contd)

\begin{tabular}{|c|c|c|c|c|c|c|c|c|c|c|c|c|c|c|c|}
\hline Element & Atomic\# & $\mathrm{N}$ & Min & Max & AVG & Mean & Wmean & STD & GSD & GM & MED & 95\%ile & Document & Reference\# & Reference \\
\hline Co & 27 & & & & & & & & & $4.50 \mathrm{E}-03$ & & & & & Sheppard et al. (2010b) \\
\hline Co & 27 & 7 & $5.70 \mathrm{E}-02$ & 2.30E-01 & & & & & $1.60 \mathrm{E}+00$ & $1.40 \mathrm{E}-01$ & & & TRS_472(OV) & & \\
\hline Co & 27 & 14 & 4.70E-02 & 7.20E-01 & & & & & $2.20 \mathrm{E}+00$ & 1.10E-01 & & & TRS_472 (RV) & & \\
\hline $\mathrm{Cr}$ & 24 & & & & & & & & & & $4.50 \mathrm{E}-03$ & & PNNL-13421 & Ba84 & \\
\hline $\mathrm{Cr}$ & 24 & & & & & & & & & $3.00 \mathrm{E}-03$ & & & & & Sheppard et al. (2010b) \\
\hline $\mathrm{Cr}$ & 24 & 1 & & & & $1.00 \mathrm{E}-03$ & & & & & & & TRS_472 (OV, RV) & & \\
\hline Cs & 55 & & & & & & & & & & $1.00 \mathrm{E}-01$ & & PNNL-13421 & Fr82 & \\
\hline Cs & 55 & & & & & & & & & & $4.90 \mathrm{E}-02$ & & NUREG/CR-5512 & & \\
\hline Cs & 55 & & & & & & & & & $5.00 \mathrm{E}-03$ & & & & & Sheppard et al. (2010b) \\
\hline Cs & 55 & & $5.00 \mathrm{E}-03$ & $1.20 \mathrm{E}+01$ & & & & & & & $2.50 \mathrm{E}-01$ & & Coughtrey et al. (1985) & S2.1 & \\
\hline Cs & 55 & 38 & 7.00E-04 & 7.30E-01 & & & & & $4.10 \mathrm{E}+00$ & $2.10 \mathrm{E}-02$ & & & TRS_472(OV) & & \\
\hline Cs & 55 & 81 & $1.00 \mathrm{E}-03$ & $8.80 \mathrm{E}-01$ & & & & & $3.00 \mathrm{E}+00$ & $4.20 \mathrm{E}-02$ & & & TRS_472 (RV) & & \\
\hline $\mathrm{Cu}$ & 29 & & & & & & & & & $2.00 \mathrm{E}-01$ & & & & & Sheppard et al. (2010b) \\
\hline Dy & 66 & & & & & & & & & & $2.00 \mathrm{E}-02$ & & PNNL-13421 & Ng82b & \\
\hline Dy & 66 & & & & & & & & & & 4.00E-03 & & NUREG/CR-5512 & & \\
\hline Er & 68 & & & & & & & & & & $2.00 \mathrm{E}-02$ & & PNNL-13421 & Ng82b & \\
\hline Er & 68 & & & & & & & & & & 4.00E-03 & & NUREG/CR-5512 & & \\
\hline Es & 99 & & & & & & & & & & & & & & \\
\hline Eu & 63 & & & & & & & & & & $2.00 \mathrm{E}-02$ & & PNNL-13421 & Ng82b & \\
\hline Eu & 63 & & & & & & & & & & $4.00 \mathrm{E}-03$ & & NUREG/CR-5512 & & \\
\hline $\mathrm{F}$ & 9 & & & & & & & & & & $6.00 \mathrm{E}-03$ & & PNNL-13421 & Ba84 & \\
\hline $\mathrm{Fe}$ & 26 & & & & & & & & & & $5.00 \mathrm{E}-02$ & & PNNL-13421 & СТ83 & \\
\hline $\mathrm{Fe}$ & 26 & & & & & & & & & & $1.00 \mathrm{E}-03$ & & NUREG/CR-5512 & & \\
\hline $\mathrm{Fe}$ & 26 & 3 & & & & & & & & $1.00 \mathrm{E}-03$ & & & TRS_472 (OV,RV) & & \\
\hline $\mathrm{Fm}$ & 100 & & & & & & & & & & & & & & \\
\hline $\mathrm{Ga}$ & 31 & & & & & & & & & & $4.00 \mathrm{E}-04$ & & PNNL-13421 & Ba84 & \\
\hline $\mathrm{Gd}$ & 64 & & & & & & & & & & $2.00 \mathrm{E}-02$ & & PNNL-13421 & Ng82b & \\
\hline $\mathrm{Gd}$ & 64 & & & & & & & & & & $4.00 \mathrm{E}-03$ & & NUREG/CR-5512 & & \\
\hline Ge & 32 & & & & & & & & & & & & & & \\
\hline $\mathrm{H}$ & 1 & & & & & & & & & & $1.50 \mathrm{E}-03$ & & PNNL-13421 & Ba84 & \\
\hline $\mathrm{Hf}$ & 72 & & & & & & & & & & $1.00 \mathrm{E}-03$ & & PNNL-13421 & Ng82b & \\
\hline $\mathrm{Hf}$ & 72 & & & & & & & & & & $8.50 \mathrm{E}-04$ & & NUREG/CR-5512 & & \\
\hline $\mathrm{Hg}$ & 80 & & & & & & & & & & $2.00 \mathrm{E}-01$ & & PNNL-13421 & Ba84 & \\
\hline Но & 67 & & & & & & & & & & $2.00 \mathrm{E}-02$ & & PNNL-13421 & Ng82b & \\
\hline Но & 67 & & & & & & & & & & $4.00 \mathrm{E}-03$ & & NUREG/CR-5512 & & \\
\hline Но & 67 & & & & & & & & & & & & & & \\
\hline I & 53 & & & & & & & & & & 4.00E-02 & & PNNL-13421 & Sn94 & \\
\hline I & 53 & & & & & & & & & & $5.00 \mathrm{E}-02$ & & NUREG/CR-5512 & & \\
\hline I & 53 & & & & & & & & & 7.70E-03 & & & & & Uchida and Tagami (2011) \\
\hline I & 53 & & & & & & & & & $1.40 \mathrm{E}-02$ & & & & & Uchida and Tagami (2011) \\
\hline In & 49 & & & & & & & & & & 4.00E-04 & & PNNL-13421 & Fu78 & \\
\hline In & 49 & & & & & & & & & & & & & & \\
\hline Ir & 77 & & & & & & & & & & $1.50 \mathrm{E}-02$ & & PNNL-13421 & Ba84 & \\
\hline Ir & 77 & & & & & & & & & & & & & & \\
\hline $\mathrm{K}$ & 19 & & & & & & & & & & $5.50 \mathrm{E}-01$ & & PNNL-13421 & Ba84 & \\
\hline $\mathrm{K}$ & 19 & & & & & & & & & $1.40 \mathrm{E}+00$ & & & & & Sheppard et al. (2010b) \\
\hline $\mathrm{Kr}$ & 36 & & & & & & & & & $1 .+40 \mathrm{~T}=0$ & & & & & 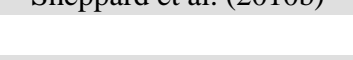 \\
\hline $\mathrm{La}$ & 57 & & & & & & & & & & $3.50 \mathrm{E}-04$ & & PNNL-13421 & Fr89 & \\
\hline $\mathrm{La}$ & 57 & & & & & & & & & & $6.40 \mathrm{E}-04$ & & NUREG/CR-5512 & & \\
\hline
\end{tabular}


Table C.2. (contd)

\begin{tabular}{|c|c|c|c|c|c|c|c|c|c|c|c|c|c|c|c|}
\hline Element & Atomic\# & $\mathrm{N}$ & Min & Max & AVG & Mean & Wmean & STD & GSD & GM & MED & 95\%ile & Document & Reference\# & Reference \\
\hline $\mathrm{La}$ & 57 & & & & & & & & & 7.50E-04 & & & & & Sheppard et al. (2010b) \\
\hline $\mathrm{La}$ & 57 & 2 & $5.90 \mathrm{E}-03$ & $6.00 \mathrm{E}-03$ & & $6.00 \mathrm{E}-03$ & & & & & & & TRS_472 (OV) & & \\
\hline La & 57 & 9 & 4.50E-04 & $6.00 \mathrm{E}-03$ & & & & & $2.70 \mathrm{E}+00$ & $1.60 \mathrm{E}-03$ & & & TRS_472 (RV) & & \\
\hline $\mathrm{Lu}$ & 71 & & & & & & & & & & & & & & \\
\hline Md & 101 & & & & & & & & & & & & & & \\
\hline $\mathrm{Mg}$ & 12 & & & & & & & & & & $5.50 \mathrm{E}-01$ & & PNNL-13421 & Ba84 & \\
\hline $\mathrm{Mg}$ & 12 & & & & & & & & & $1.40 \mathrm{E}-01$ & & & & & Sheppard et al. (2010b) \\
\hline $\mathrm{Mn}$ & 25 & & & & & & & & & & 2.00E-01 & & PNNL-13421 & Fr89 & \\
\hline $\mathrm{Mn}$ & 25 & & & & & & & & & & $1.50 \mathrm{E}-01$ & & NUREG/CR-5512 & & \\
\hline $\mathrm{Mn}$ & 25 & 3 & $1.00 \mathrm{E}-01$ & $1.50 \mathrm{E}+00$ & & & & & $4.10 \mathrm{E}+00$ & $3.10 \mathrm{E}-01$ & & & TRS_472 (OV) & & \\
\hline $\mathrm{Mn}$ & 25 & 13 & $1.50 \mathrm{E}-02$ & $3.90 \mathrm{E}+00$ & & & & & $5.50 \mathrm{E}+00$ & 4.20E- 01 & & & TRS_472 (RV) & & \\
\hline $\mathrm{Mn}$ & 25 & & & & & & & & & $1.70 \mathrm{E}-02$ & & & - & & Sheppard et al. (2010b) \\
\hline Mo & 42 & & & & & & & & & & $6.00 \mathrm{E}-02$ & & NUREG/CR-5512 & & \\
\hline Mo & 42 & & & & & & & & & 7.00E-01 & & & & & Sheppard et al. (2010b) \\
\hline Mo & 42 & 1 & & & & $5.40 \mathrm{E}+00$ & & & & & & & TRS_472 (legume) & & \\
\hline Mo & 42 & 3 & $2.30 \mathrm{E}-02$ & $4.20 \mathrm{E}-01$ & & & & & & $3.20 \mathrm{E}-01$ & & & TRS_472 (RV) & & \\
\hline $\mathrm{N}$ & 7 & & & & & & & & & & 4.20E-02 & & PNNL-13421 & Ba84 & \\
\hline $\mathrm{N}$ & 7 & & & & & & & & & & $3.00 \mathrm{E}+01$ & & NUREG/CR-5512 & & \\
\hline $\mathrm{Na}$ & 11 & & & & & & & & & & $3.00 \mathrm{E}-01$ & & PNNL-13421 & Ng82b & \\
\hline $\mathrm{Na}$ & 11 & & & & & & & & & & $5.50 \mathrm{E}-02$ & & NUREG/CR-5512 & & \\
\hline $\mathrm{Na}$ & 11 & & & & & & & & & 5.60E-01 & & & & & Sheppard et al. (2010b) \\
\hline $\mathrm{Na}$ & 11 & 1 & & & & 3.00E-02 & & & & & & & TRS_472 (OV) & & \\
\hline $\mathrm{Nb}$ & 41 & & & & & & & & & & $2.50 \mathrm{E}-02$ & & PNNL-13421 & СТ83 & \\
\hline $\mathrm{Nb}$ & 41 & 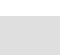 & & & & & & & & & 5.00E- 03 & & NUREG/CR-5512 & & \\
\hline $\mathrm{Nb}$ & 41 & & & & & & & & & $2.50 \mathrm{E}-03$ & & & & & Sheppard et al. (2010b) \\
\hline $\mathrm{Nb}$ & 41 & 2 & 8.00E-03 & $2.50 \mathrm{E}-02$ & & $1.70 \mathrm{E}-02$ & & & & & & & TRS_472 (RV) & & \\
\hline $\mathrm{Nd}$ & 60 & & & & & & & & & & $2.00 \mathrm{E}-02$ & & PNNL-13421 & Ng82b & \\
\hline Nd & 60 & & & & & & & & & & 4.00E-03 & & NUREG/CR-5512 & & \\
\hline $\mathrm{Nd}$ & 60 & & & & & & & & & $5.40 \mathrm{E}-04$ & & & & & Sheppard et al. (2010b) \\
\hline $\mathrm{Ne}$ & 10 & & & & & & & & & & & & & & \\
\hline $\mathrm{Ni}$ & 28 & & & & & & & & & & $6.00 \mathrm{E}-02$ & & PNNL-13421 & Ba84 & \\
\hline $\mathrm{Ni}$ & 28 & 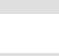 & & & & & & & & & $4.00 \mathrm{E}-02$ & & NUREG/CR-5512 & & \\
\hline $\mathrm{Ni}$ & 28 & & & & & & & & & $1.70 \mathrm{E}-02$ & & & & & Sheppard et al. (2010b) \\
\hline $\mathrm{Ni}$ & 28 & 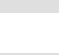 & 2.34E-02 & $2.90 \mathrm{E}-01$ & & $1.18 \mathrm{E}-01$ & & & $2.76 \mathrm{E}+00$ & 8.08E-02 & & & & & Vandenhove et al. (2009a) \\
\hline $\mathrm{Ni}$ & 28 & & $2.79 \mathrm{E}-02$ & $1.28 \mathrm{E}-01$ & & $5.38 \mathrm{E}-02$ & & & $1.73 \mathrm{E}+00$ & $4.65 \mathrm{E}-02$ & & & & & Vandenhove et al. (2009a) \\
\hline $\mathrm{Ni}$ & 28 & 27 & 7.30E-02 & $2.60 \mathrm{E}+00$ & & & & & $2.50 \mathrm{E}+00$ & 4.00E- 01 & & & TRS_472 (legume) & & \\
\hline $\mathrm{Np}$ & 93 & & & & & & & & & & $1.30 \mathrm{E}-02$ & & PNNL-13421 & Fr82 & \\
\hline $\mathrm{Np}$ & 93 & & & & & & & & & & 9.40E-03 & & NUREG/CR-5512 & & \\
\hline $\mathrm{Np}$ & 93 & 9 & $4.00 \mathrm{E}-03$ & $5.70 \mathrm{E}-02$ & & & & & $2.40 \mathrm{E}+00$ & $1.80 \mathrm{E}-02$ & & & TRS 472 (OV) & & \\
\hline $\mathrm{Np}$ & 93 & 7 & 5.00E-03 & 3.60E-02 & & & & & $2.00 \mathrm{E}+00$ & 2.20E-02 & & & TRS_472 (RV) & & \\
\hline & 8 & & & & & & & & & & & & & & \\
\hline \multicolumn{16}{|l|}{ OBT } \\
\hline Os & 76 & & & & & & & & & & $3.50 \mathrm{E}-03$ & & PNNL-13421 & Ba84 & \\
\hline $\mathrm{P}$ & 15 & & & & & & & & & & $3.50 \mathrm{E}+00$ & & PNNL-13421 & Ba84 & \\
\hline $\mathrm{P}$ & 15 & 1 & & & & $1.00 \mathrm{E}+00$ & & & & & & & TRS_472 (OV, RV) & & \\
\hline $\mathrm{Pa}$ & 91 & & & & & & & & & & 3.50E-04 & & PNNL-13421 & Fr82 & \\
\hline $\mathrm{Pa}$ & 91 & & & & & & & & & & $2.50 \mathrm{E}-04$ & & NUREG/CR-5512 & & \\
\hline $\mathrm{Pb}$ & 82 & & & & & & & & & & $6.00 \mathrm{E}-03$ & & PNNL-13421 & Fr89 & \\
\hline $\mathrm{Pb}$ & 82 & & & & & & & & & & $3.20 \mathrm{E}-03$ & & NUREG/CR-5512 & & \\
\hline $\mathrm{Pb}$ & 82 & & & & & & & & & $2.00 \mathrm{E}-03$ & & & & & Sheppard et al. (2010b) \\
\hline
\end{tabular}


Table C.2. (contd)

\begin{tabular}{|c|c|c|c|c|c|c|c|c|c|c|c|c|c|c|c|}
\hline Element & Atomic\# & $\mathrm{N}$ & Min & Max & $\mathrm{AVG}$ & Mean & Wmean & STD & GSD & GM & MED & 95\%ile & Document & Reference\# & Reference \\
\hline $\mathrm{Pb}$ & 82 & & $2.40 \mathrm{E}-04$ & $3.30 \mathrm{E}+00$ & & $4.14 \mathrm{E}-01$ & & $9.77 \mathrm{E}-01$ & & & & & & & 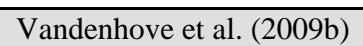 \\
\hline $\mathrm{Pb}$ & 82 & & $1.47 \mathrm{E}-03$ & $3.86 \mathrm{E}+00$ & & 7.77E-01 & & $1.72 \mathrm{E}+00$ & & & & & & & Vandenhove et al. (2009b) \\
\hline $\mathrm{Pb}$ & 82 & 5 & $1.50 \mathrm{E}-03$ & $3.90 \mathrm{E}+00$ & & & & & $2.60 \mathrm{E}+01$ & $1.50 \mathrm{E}-02$ & & & TRS_472 (OV) & & \\
\hline $\mathrm{Pb}$ & 82 & 27 & $2.40 \mathrm{E}-04$ & $3.30 \mathrm{E}+00$ & & & & & $1.60 \mathrm{E}+01$ & $1.50 \mathrm{E}-02$ & & & TRS_472 (RV) & & \\
\hline Pd & 46 & & & & & & & & & & 4.00E-02 & & PNNL-13421 & Ba84 & \\
\hline $\mathrm{Pm}$ & 61 & & & & & & & & & & $2.00 \mathrm{E}-02$ & & PNNL-13421 & Ng82b & \\
\hline $\mathrm{Pm}$ & 61 & & & & & & & & & & 4.00E-03 & & NUREG/CR-5512 & & \\
\hline $\mathrm{Pm}$ & 61 & 4 & 2.00E-02 & $1.20 \mathrm{E}+00$ & & & & & $7.40 \mathrm{E}+00$ & $1.70 \mathrm{E}-01$ & & & TRS_472 (OV) & & \\
\hline $\mathrm{Pm}$ & 61 & 5 & $3.60 \mathrm{E}-02$ & $6.00 \mathrm{E}-02$ & & & & & $1.20 \mathrm{E}+00$ & 4.20E-02 & & & TRS_472 (RV) & & \\
\hline Po & 84 & & & & & & & & & & 7.00E-03 & & PNNL-13421 & HW91 & \\
\hline Po & 84 & & & & & & & & & & $9.00 \mathrm{E}-03$ & & NUREG/CR-5512 & & \\
\hline Po & 84 & & $2.40 \mathrm{E}-04$ & $4.92 \mathrm{E}-02$ & & $1.20 \mathrm{E}-02$ & & $1.65 \mathrm{E}-02$ & & & & & & & Vandenhove et al. (2009b) \\
\hline Po & 84 & 2 & $1.60 \mathrm{E}-05$ & 3.70E-04 & & $1.90 \mathrm{E}-04$ & & & & & & & TRS_472 (OV) & & \\
\hline Po & 84 & 10 & $2.40 \mathrm{E}-04$ & $4.90 \mathrm{E}-02$ & & & & & $4.30 \mathrm{E}+00$ & $5.80 \mathrm{E}-03$ & & & TRS_472 (RV) & & \\
\hline $\operatorname{Pr}$ & 59 & & & & & & & & & & $2.00 \mathrm{E}-02$ & & PNNL-13421 & Ng82b & \\
\hline $\operatorname{Pr}$ & 59 & & & & & & & & & & 4.00E-03 & & NUREG/CR-5512 & & \\
\hline Pr & 59 & & & & & & & & & 7.50E-04 & & & & & Sheppard et al. (2010b) \\
\hline $\operatorname{Pr}$ & 59 & 1 & & & & 2.00E-02 & & & & & & & TRS_472 (RV) & & \\
\hline $\mathrm{Pt}$ & 78 & & & & & & & & & & & & & & \\
\hline $\mathrm{Pu}$ & 94 & & & & & & & & & & $1.10 \mathrm{E}-03$ & & PNNL-13421 & Fr82 & \\
\hline Pu & 94 & & & & & & & & & & $2.00 \mathrm{E}-04$ & & NUREG/CR-5512 & & \\
\hline Pu & 94 & 9 & $6.00 \mathrm{E}-06$ & $2.00 \mathrm{E}-04$ & & & & & $2.70 \mathrm{E}+00$ & $6.50 \mathrm{E}-05$ & & & TRS $472(\mathrm{OV})$ & & \\
\hline Pu & 94 & 5 & 7.00E-05 & $5.80 \mathrm{E}-03$ & & & & & $1.00 \mathrm{E}+01$ & $3.90 \mathrm{E}-04$ & & & TRS_472 (RV) & & \\
\hline Ra & 88 & & & & & & & & & & $2.00 \mathrm{E}-03$ & & PNNL-13421 & Fr89 & \\
\hline Ra & 88 & & & & & & & & & & 3.20E-03 & & NUREG/CR-5512 & & \\
\hline Ra & 88 & & $2.41 \mathrm{E}-04$ & $6.25 \mathrm{E}+00$ & & $2.62 \mathrm{E}-01$ & & $1.01 \mathrm{E}+00$ & & & & & & & Vandenhove et al. (2009b) \\
\hline Ra & 88 & 44 & 2.40E-04 & $6.30 \mathrm{E}+00$ & & & & & $8.40 \mathrm{E}+00$ & $1.70 \mathrm{E}-02$ & & & TRS 472 (OV) & & \\
\hline Ra & 88 & 60 & 2.00E-03 & $5.60 \mathrm{E}+01$ & & & & & $9.20 \mathrm{E}+00$ & 7.00E-02 & & & TRS 472 (RV) & & \\
\hline $\mathrm{Rb}$ & 37 & & & & & & & & & & $9.00 \mathrm{E}-01$ & & PNNL-13421 & Ng82b & \\
\hline $\mathrm{Rb}$ & 37 & & & & & & & & & & 7.00E-02 & & NUREG/CR-5512 & & \\
\hline $\mathrm{Rb}$ & 37 & & & & & & & & & $2.50 \mathrm{E}-01$ & & & & & Sheppard et al. (2010b) \\
\hline $\mathrm{Rb}$ & 37 & 1 & & & & $9.00 \mathrm{E}-01$ & & & & & & & TRS $472(\mathrm{RV})$ & & \\
\hline Re & 75 & & & & & & & & & & $3.50 \mathrm{E}+00$ & & PNNL-13421 & Ba84 & \\
\hline $\mathrm{Re}$ & 75 & & & & & & & & & & $3.50 \mathrm{E}-01$ & & NUREG/CR-5512 & & \\
\hline Rh & 45 & & & & & & & & & & $4.00 \mathrm{E}-02$ & & PNNL-13421 & Ba84 & \\
\hline $\mathrm{Rn}$ & 86 & & & & & & & & & & & & & & \\
\hline Ru & 44 & & & & & & & & & & $4.00 \mathrm{E}-02$ & & PNNL-13421 & Ng82b & \\
\hline Ru & 44 & & & & & & & & & & 2.00E-02 & & NUREG/CR-5512 & & \\
\hline $\mathrm{Ru}$ & 37 & 1 & & & & $2.00 \mathrm{E}-02$ & & & & & & & TRS 472 (OV) & & \\
\hline Ru & 44 & 1 & & & & $1.00 \mathrm{E}-02$ & & & & & & & TRS 472 (RV) & & \\
\hline s & 16 & & & & & & & & & & $1.50 \mathrm{E}+00$ & & PNNL-13421 & Ba84 & \\
\hline Sb & 51 & & & & & & & & & & $5.60 \mathrm{E}-04$ & & PNNL-13421 & Fr82 & \\
\hline $\mathrm{Sb}$ & 51 & & & & & & & & & 4.90E-03 & & & & & Sheppard et al. (2010b) \\
\hline Sb & 51 & & 7.00E-03 & $5.00 \mathrm{E}-02$ & & & & & & & $5.00 \mathrm{E}-02$ & & Coughtrey et al. (1985) & S2.1 & \\
\hline $\mathrm{sb}$ & 51 & 5 & $1.50 \mathrm{E}-05$ & $1.60 \mathrm{E}-03$ & & & & & $6.70 \mathrm{E}+00$ & $1.30 \mathrm{E}-04$ & & & TRS_472 (OV) & & \\
\hline $\mathrm{sb}$ & 51 & 5 & 4.00E-04 & $1.10 \mathrm{E}-03$ & & & & & $1.50 \mathrm{E}+00$ & 6.20E-04 & & & TRS_472 (RV) & & \\
\hline Sc & 21 & & & & & & & & & & $1.00 \mathrm{E}-03$ & & PNNL-13421 & Ba84 & \\
\hline Se & 34 & & & & & & & & & & 5.00E-02 & & PNNL-13421 & CT83 & \\
\hline Se & 34 & & & & & & & & & & $2.50 \mathrm{E}-02$ & & NUREG/CR-5512 & & \\
\hline Se & 34 & & & & & & & & & $6.60 \mathrm{E}-01$ & & & & & Sheppard et al. (2010b) \\
\hline
\end{tabular}


Table C.2.(contd)

\begin{tabular}{|c|c|c|c|c|c|c|c|c|c|c|c|c|c|c|c|}
\hline Element & Atomic\# & $\mathrm{N}$ & Min & Max & AVG & Mean & Wmean & STD & GSD & GM & MED & 95\%ile & $\begin{array}{l}\text { Document } \\
\end{array}$ & Reference\# & Reference \\
\hline $\mathrm{Si}$ & 14 & & & & & & & & & & $7.00 \mathrm{E}-02$ & & PNNL-13421 & Ba84 & \\
\hline Sm & 62 & & & & & & & & & & $2.00 \mathrm{E}-02$ & & PNNL-13421 & Ng82b & \\
\hline $\mathrm{Sm}$ & 62 & & & & & & & & & & 4.00E-03 & & NUREG/CR-5512 & & \\
\hline Sm & 62 & & & & & & & & & $6.40 \mathrm{E}-04$ & & & & & Sheppard et al. (2010b) \\
\hline Sn & 50 & & & & & & & & & & $6.00 \mathrm{E}-03$ & & PNNL-13421 & Fu78 & \\
\hline Sn & 50 & & & & & & & & & $1.20 \mathrm{E}+00$ & & & & & Sheppard et al. (2010b) \\
\hline $\mathrm{Sr}$ & 38 & & & & & & & & & & $5.00 \mathrm{E}-01$ & & PNNL-13421 & Fr82 & \\
\hline $\mathrm{Sr}$ & 38 & & & & & & & & & & $8.10 \mathrm{E}-01$ & & NUREG/CR-5512 & & \\
\hline $\mathrm{Sr}$ & 38 & & & & & & & & & $1.10 \mathrm{E}-01$ & & & & & Sheppard et al. (2010b) \\
\hline $\mathrm{Sr}$ & 38 & 19 & 7.10E-03 & $7.90 \mathrm{E}+00$ & & & & & $5.50 \mathrm{E}+00$ & $3.60 \mathrm{E}-01$ & & & TRS_472 (OV) & & \\
\hline $\mathrm{Sr}$ & 38 & 56 & $3.00 \mathrm{E}+02$ & $4.80 \mathrm{E}+00$ & & & & & $4.10 \mathrm{E}+00$ & 7.20E-01 & & & TRS_472 (RV) & & \\
\hline Та & 73 & & & & & & & & & & $2.50 \mathrm{E}-02$ & & PNNL-13421 & СТ83 & \\
\hline $\mathrm{Tb}$ & 65 & & & & & & & & & & 2.00E-02 & & PNNL-13421 & Ng82b & \\
\hline $\mathrm{Tb}$ & 65 & & & & & & & & & & 4.00E- 03 & & NUREG/CR-5512 & & \\
\hline $\mathrm{Tb}$ & 65 & & & & & & & & & $1.30 \mathrm{E}-03$ & & & & & Sheppard et al. (2010b) \\
\hline Tc & 43 & & & & & & & & & & $2.40 \mathrm{E}-01$ & & PNNL-13421 & Fr82 & \\
\hline Tc & 43 & & & & & & & & & & $1.10 \mathrm{E}+00$ & & NUREG/CR-5512 & & \\
\hline $\mathrm{Te}$ & 52 & & & & & & & & & & $4.00 \mathrm{E}-03$ & & PNNL-13421 & Ba84 & \\
\hline $\mathrm{Te}$ & 52 & 1 & & & & $3.00 \mathrm{E}-01$ & & & & & & & TRS_472 (OV, RV) & & \\
\hline Th & 90 & & & & & & & & & & $3.30 \mathrm{E}-04$ & & PNNL-13421 & Fr89 & \\
\hline Th & 90 & & & & & & & & & & $1.20 \mathrm{E}-04$ & & NUREG/CR-5512 & & \\
\hline Th & 90 & & & & & & & & & 4.20E-03 & & & & & Sheppard et al. (2010b) \\
\hline Th & 90 & & $8.21 \mathrm{E}-06$ & $9.50 \mathrm{E}-02$ & & $9.33 \mathrm{E}-03$ & & $2.00 \mathrm{E}-02$ & & & & & & & Vandenhove et al. (2009b) \\
\hline Th & 90 & & $6.21 \mathrm{E}-05$ & $1.62 \mathrm{E}-02$ & & 3.43E-03 & & $5.36 \mathrm{E}-03$ & & & & & & & Vandenhove et al. (2009b) \\
\hline Th & 90 & 17 & $6.20 \mathrm{E}-05$ & 1.60E-02 & & $7.80 \mathrm{E}-04$ & & & $6.80 \mathrm{E}+00$ & & & & TRS_472 (OV) & & \\
\hline $\mathrm{Ti}$ & 22 & & & & & & & & & & & & & & \\
\hline $\mathrm{Tl}$ & 81 & & & & & & & & & & $4.00 \mathrm{E}-04$ & & PNNL-13421 & Fu78 & \\
\hline $\mathrm{Tl}$ & 81 & & & & & & & & & $1.60 \mathrm{E}-02$ & & & & & Sheppard et al. (2010b) \\
\hline $\mathrm{Tm}$ & 69 & & & & & & & & & & & & & & \\
\hline $\mathrm{U}$ & 92 & & & & & & & & & & $1.20 \mathrm{E}-02$ & & PNNL-13421 & Fr89 & \\
\hline $\mathrm{U}$ & 92 & & & & & & & & & & $1.40 \mathrm{E}-02$ & & NUREG/CR-5512 & T100 & \\
\hline $\mathrm{U}$ & 92 & & & & & & & & & $3.20 \mathrm{E}-03$ & & & & & Sheppard et al. (2010b) \\
\hline $\mathrm{U}$ & 92 & & $4.91 \mathrm{E}-04$ & 2.63E-01 & & $3.61 \mathrm{E}-02$ & & $6.50 \mathrm{E}-02$ & & & & & & & Vandenhove et al. (2009b) \\
\hline $\mathrm{U}$ & 92 & & 5.23E-04 & 2.03E-01 & & $3.57 \mathrm{E}-02$ & & 5.27E-02 & & & & & & & Vandenhove et al. (2009b) \\
\hline $\mathrm{U}$ & 92 & 38 & 5.20E-04 & 2.00E-01 & & $1.50 \mathrm{E}-02$ & & & 4.20E +00 & & & & TRS_472 (OV) & & \\
\hline $\mathrm{U}$ & 92 & 46 & 4.90E-04 & 2.60E-01 & & 8.40E-03 & & & $6.20 \mathrm{E}+00$ & & & & TRS_472 (RV) & & \\
\hline $\mathrm{V}$ & 23 & & & & & & & & & $2.00 \mathrm{E}-03$ & & & & & Sheppard et al. (2010b) \\
\hline W & 74 & & & & & & & & & & $3.00 \mathrm{E}+00$ & & PNNL-13421 & NCRP86 & \\
\hline W & 74 & & & & & & & & & & $1.00 \mathrm{E}-02$ & & NUREG/CR-5512 & & \\
\hline $\mathrm{Xe}$ & 54 & & & & & & & & & & & & & & \\
\hline $\mathrm{Y}$ & 39 & & & & & & & & & & $1.00 \mathrm{E}-02$ & & PNNL-13421 & Ng82b & \\
\hline $\mathrm{Y}$ & 39 & & & & & & & & & & 6.00E-02 & & NUREG/CR-5512 & & \\
\hline $\mathrm{Y}$ & 39 & & & & & & & & & $8.00 \mathrm{E}-04$ & & & & & Sheppard et al. (2010b) \\
\hline $\mathrm{Y}$ & 39 & 1 & & & & 2.00E-03 & & & & & & & TRS_472 (OV, RV) & & \\
\hline $\mathrm{Yb}$ & 70 & & & & & & & & & $2.00 \mathrm{E}-03$ & & & & & Sheppard et al. (2010b) \\
\hline $\mathrm{Zn}$ & 30 & & & & & & & & & & 3.50E-01 & & PNNL-13421 & Fr89 & \\
\hline $\mathrm{Zn}$ & 30 & & & & & & & & & & $5.90 \mathrm{E}-01$ & & NUREG/CR-5512 & & \\
\hline $\mathrm{Zn}$ & 30 & & & & & & & & & 1.70E-01 & & & & & Sheppard et al. (2010b) \\
\hline
\end{tabular}


Table C.2. (contd)

\begin{tabular}{|c|c|c|c|c|c|c|c|c|c|c|c|c|c|c|c|}
\hline Element & Atomic\# & $\mathrm{N}$ & Min & Max & AVG & Mean & Wmean & STD & GSD & GM & MED & 95\%ile & Document & Reference\# & Reference \\
\hline $\mathrm{Zn}$ & 30 & 3 & $1.00 \mathrm{E}-01$ & $9.50 \mathrm{E}-01$ & & & & & $3.70 \mathrm{E}+00$ & $4.20 \mathrm{E}-01$ & & & TRS_472 (OV) & & \\
\hline $\mathrm{Zn}$ & 30 & 20 & $5.00 \mathrm{E}-02$ & $6.30 \mathrm{E}-01$ & & & & & $1.80 \mathrm{E}+00$ & $3.00 \mathrm{E}-01$ & & & TRS_472 (RV) & & \\
\hline $\mathrm{Zr}$ & 40 & & & & & & & & & & $1.00 \mathrm{E}-04$ & & PNNL-13421 & Ng82b & \\
\hline $\mathrm{Zr}$ & 40 & & & & & & & & & & $5.00 \mathrm{E}-04$ & & NUREG/CR-5512 & & \\
\hline $\mathrm{Zr}$ & 40 & & & & & & & & & $1.40 \mathrm{E}-02$ & & & & & Sheppard et al. (2010b) \\
\hline $\mathrm{Zr}$ & 40 & 1 & & & & 4.00E-03 & & & & & & & TRS_472 (OV, RV) & & \\
\hline
\end{tabular}


Table C.3. Bioconcentration in Fruit from Soil, $\mathrm{kg}_{\text {dry_plant }} / \mathrm{kg}_{\text {dry_soil }}$ (CLBVFR)

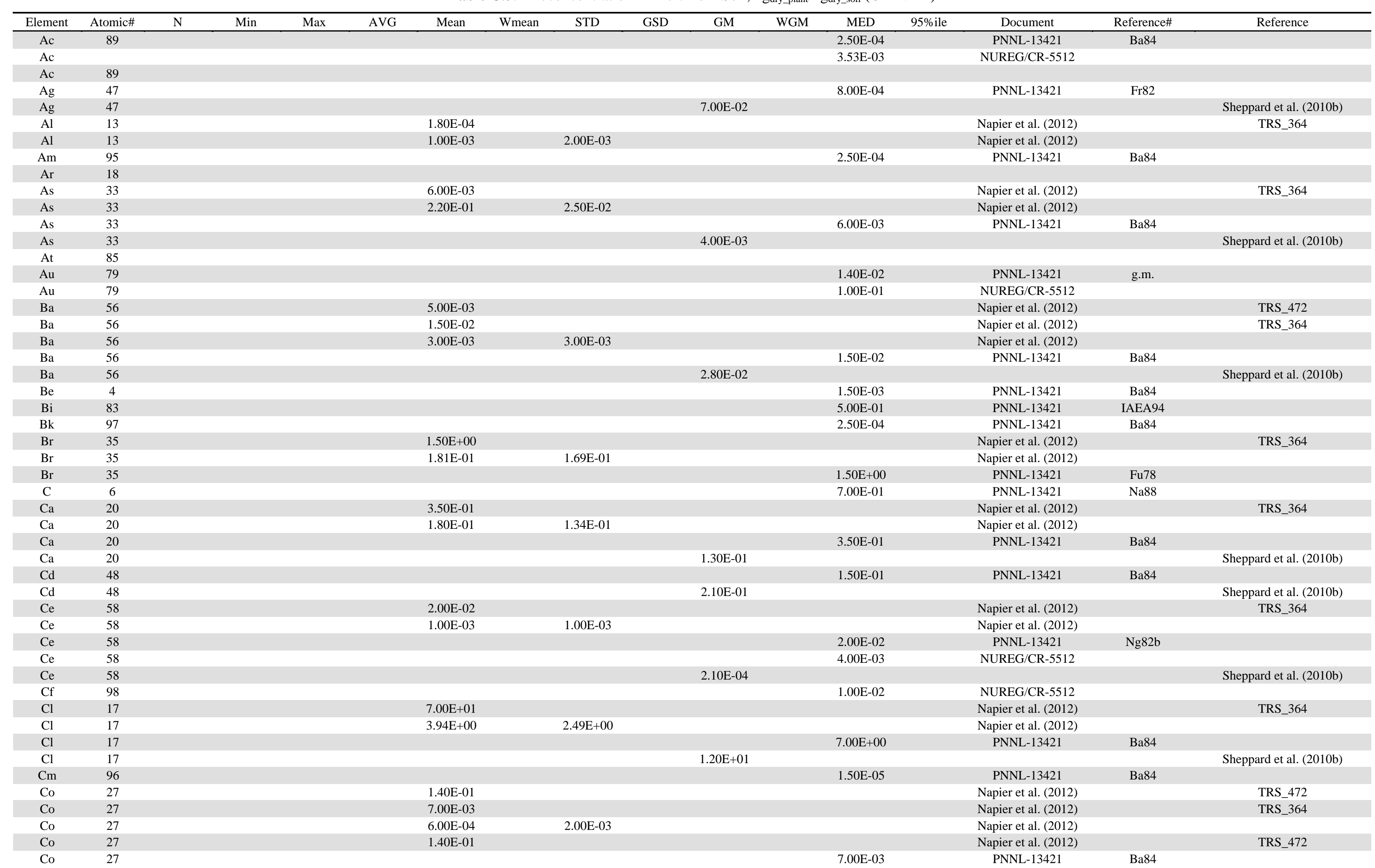


Table C.3. (contd)

\begin{tabular}{|c|c|c|c|c|c|c|c|c|c|c|c|c|c|c|c|c|}
\hline Element & Atomic\# & $\mathrm{N}$ & Min & Max & AVG & Mean & Wmean & STD & GSD & GM & WGM & MED & 95\%ile & Document & Reference\# & Reference \\
\hline Сo & 27 & & & & & & & & & $7.70 \mathrm{E}-03$ & & & & & & Sheppard et al. (2010b) \\
\hline $\mathrm{Cr}$ & 24 & & & & & $1.00 \mathrm{E}-03$ & & & & & & & & Napier et al. (2012) & & TRS_472 \\
\hline $\mathrm{Cr}$ & 24 & & & & & $4.50 \mathrm{E}-03$ & & & & & & & & Napier et al. (2012) & & TRS_364 \\
\hline $\mathrm{Cr}$ & 24 & & & & & $1.10 \mathrm{E}-02$ & & $8.00 \mathrm{E}-03$ & & & & & & Napier et al. (2012) & & \\
\hline $\mathrm{Cr}$ & 24 & & & & & & & & & & & $4.30 \mathrm{E}-03$ & & PNNL-13421 & Ва84 & \\
\hline Cs & 55 & & & & & $2.10 \mathrm{E}-02$ & & & & & & & & Napier et al. (2012) & & TRS_472 \\
\hline Cs & 55 & & & & & $2.20 \mathrm{E}-01$ & & & & & & & & Napier et al. (2012) & & TRS_364 \\
\hline Cs & 55 & & & & & $2.00 \mathrm{E}-03$ & & $1.00 \mathrm{E}-03$ & & & & & & Napier et al. (2012) & & \\
\hline Cs & 55 & & & & & & & & & & & 2.20E-01 & & PNNL-13421 & Fr89 & \\
\hline Cs & 55 & & $2.30 \mathrm{E}-03$ & $9.50 \mathrm{E}-02$ & & & & & & & & & & & & Al-Oudat et al. (2006) \\
\hline Cs & 55 & & & & & & & & & $5.40 \mathrm{E}-03$ & & & & & & Sheppard et al. (2010b) \\
\hline Cs & 55 & & $2.00 \mathrm{E}-02$ & $6.00 \mathrm{E}-02$ & & & & & & & & & & & & Velasco et al. (2012) \\
\hline $\mathrm{Cu}$ & 29 & & & & & & & & & & & $2.50 \mathrm{E}-01$ & & PNNL-13421 & Ba84 & \\
\hline $\mathrm{Cu}$ & 29 & & & & & & & & & $2.70 \mathrm{E}-01$ & & & & & & Sheppard et al. (2010b) \\
\hline Dy & 66 & & & & & & & & & & & $2.00 \mathrm{E}-02$ & & PNNL-13421 & Ng82b & \\
\hline Dy & 66 & & & & & & & & & & & 4.00E-03 & & NUREG/CR-5512 & & \\
\hline Er & 68 & & & & & & & & & & & $2.00 \mathrm{E}-02$ & & PNNL-13421 & Ng82b & \\
\hline Er & 68 & & & & & & & & & & & 4.00E-03 & & NUREG/CR-5512 & & \\
\hline Es & 99 & & & & & & & & & & & & & & & \\
\hline $\mathrm{Eu}$ & 63 & & & & & $1.00 \mathrm{E}-03$ & & $1.00 \mathrm{E}-03$ & & & & & & Napier et al. (2012) & & \\
\hline $\mathrm{Eu}$ & 63 & & & & & $2.00 \mathrm{E}-02$ & & & & & & & & Napier et al. (2012) & & TRS_364 \\
\hline $\mathrm{Eu}$ & 63 & & & & & & & & & & & $2.00 \mathrm{E}-02$ & & PNNL-13421 & Ng82b & \\
\hline $\mathrm{Eu}$ & 63 & & & & & & & & & & & $4.00 \mathrm{E}-03$ & & NUREG/CR-5512 & & \\
\hline $\mathrm{F}$ & 9 & & & & & & & & & & & $6.00 \mathrm{E}-03$ & & & Ba84 & \\
\hline Fe & 26 & & & & & $1.00 \mathrm{E}-03$ & & & & & & & & Napier et al. (2012) & & TRS_472 \\
\hline $\mathrm{Fe}$ & 26 & & & & & $5.00 \mathrm{E}-02$ & & & & & & & & Napier et al. (2012) & & TRS_364 \\
\hline $\mathrm{Fe}$ & 26 & & & & & $2.00 \mathrm{E}-03$ & & $5.00 \mathrm{E}-04$ & & & & & & Napier et al. (2012) & & \\
\hline $\mathrm{Fe}$ & 26 & & & & & & & & & & & $5.00 \mathrm{E}-02$ & & PNNL-13421 & СТ83 & \\
\hline $\mathrm{Fe}$ & 26 & & & & & & & & & & & $1.00 \mathrm{E}-03$ & & NUREG/CR-5512 & & \\
\hline $\mathrm{Fm}$ & 100 & & & & & & & & & & & & & & & \\
\hline $\mathrm{Fr}$ & 87 & & & & & & & & & & & & & & & \\
\hline $\mathrm{Ga}$ & 31 & & & & & & & & & & & $4.00 \mathrm{E}-04$ & & PNNL-13421 & Ва84 & \\
\hline $\mathrm{Gd}$ & 64 & & & & & & & & & & & $2.00 \mathrm{E}-02$ & & PNNL-13421 & Ng82b & \\
\hline $\mathrm{Gd}$ & 64 & & & & & & & & & & & $4.00 \mathrm{E}-03$ & & NUREG/CR-5512 & & \\
\hline Ge & 32 & & & & & & & & & & & & & & & \\
\hline $\mathrm{H}$ & 1 & & & & & & & & & & & & & & & \\
\hline $\mathrm{Hf}$ & 72 & & & & & $1.00 \mathrm{E}-03$ & & & & & & & & Napier et al. (2012) & & TRS 364 \\
\hline $\mathrm{Hf}$ & 72 & & & & & $1.00 \mathrm{E}-03$ & & $1.00 \mathrm{E}-03$ & & & & & & Napier et al. (2012) & & \\
\hline $\mathrm{Hf}$ & 72 & & & & & & & & & & & $1.00 \mathrm{E}-03$ & & PNNL-13421 & Ng82b & \\
\hline $\mathrm{Hf}$ & 72 & & & & & & & & & & & $8.50 \mathrm{E}-04$ & & NUREG/CR-5512 & & \\
\hline $\mathrm{Hg}$ & 80 & & & & & & & & & & & $3.70 \mathrm{E}-01$ & & PNNL-13421 & g.m. & \\
\hline $\mathrm{Hg}$ & 80 & & & & & & & & & & & $2.00 \mathrm{E}-01$ & & NUREG/CR-5512 & & \\
\hline Но & 67 & & & & & & & & & & & $2.00 \mathrm{E}-02$ & & PNNL-13421 & Ng82b & \\
\hline Но & 67 & & & & & & & & & & & $4.00 \mathrm{E}-03$ & & NUREG/CR-5512 & & \\
\hline I & 53 & & & & & & & & & & & $2.00 \mathrm{E}-02$ & & PNNL-13421 & Sn94 & \\
\hline I & 53 & & & & & & & & & & & $5.00 \mathrm{E}-02$ & & NUREG/CR-5512 & & \\
\hline In & 49 & & & & & & & & & & & $4.00 \mathrm{E}-04$ & & PNNL-13421 & Fu78 & \\
\hline
\end{tabular}


Table C.3. (contd)

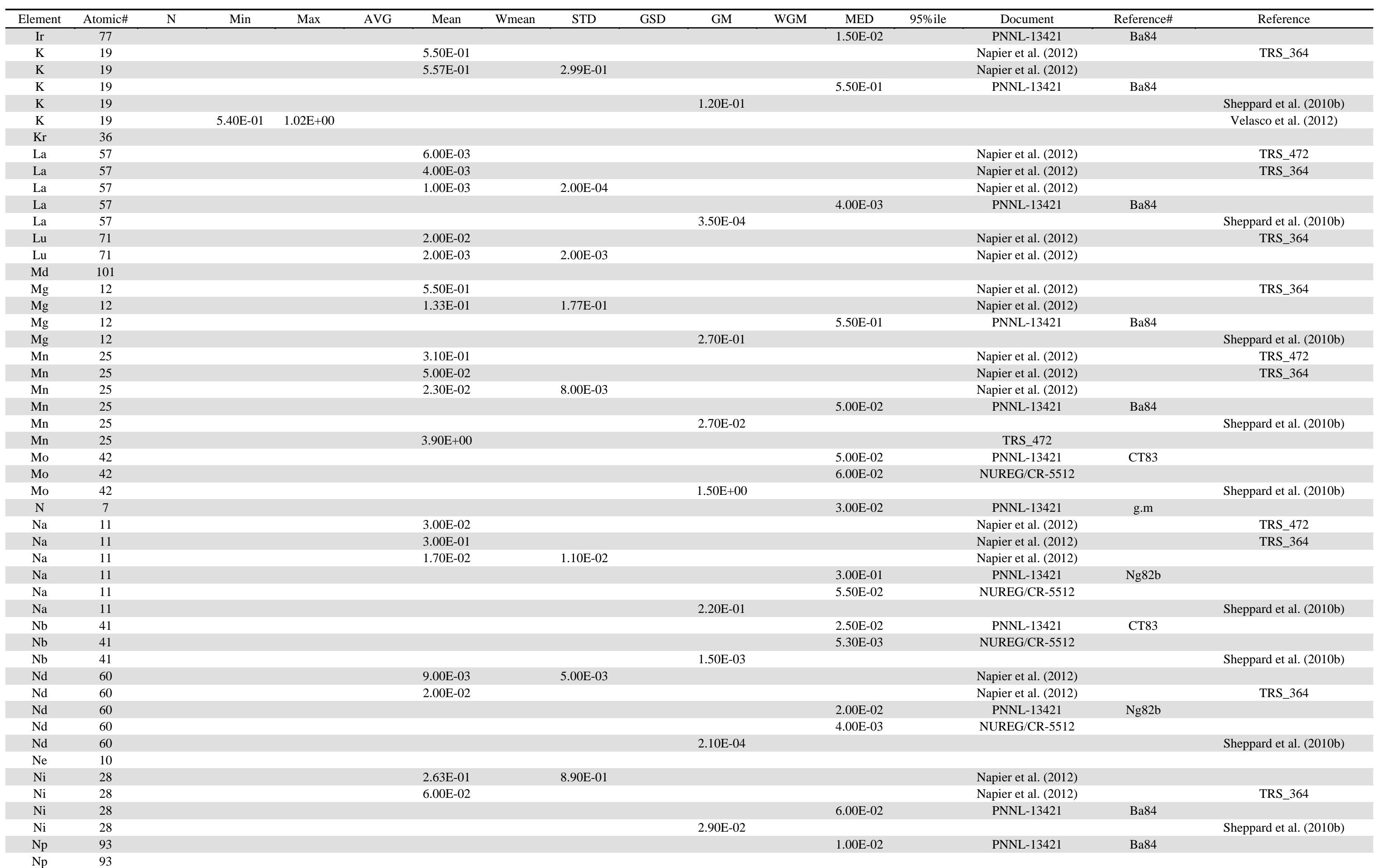


Table C.3. (contd)

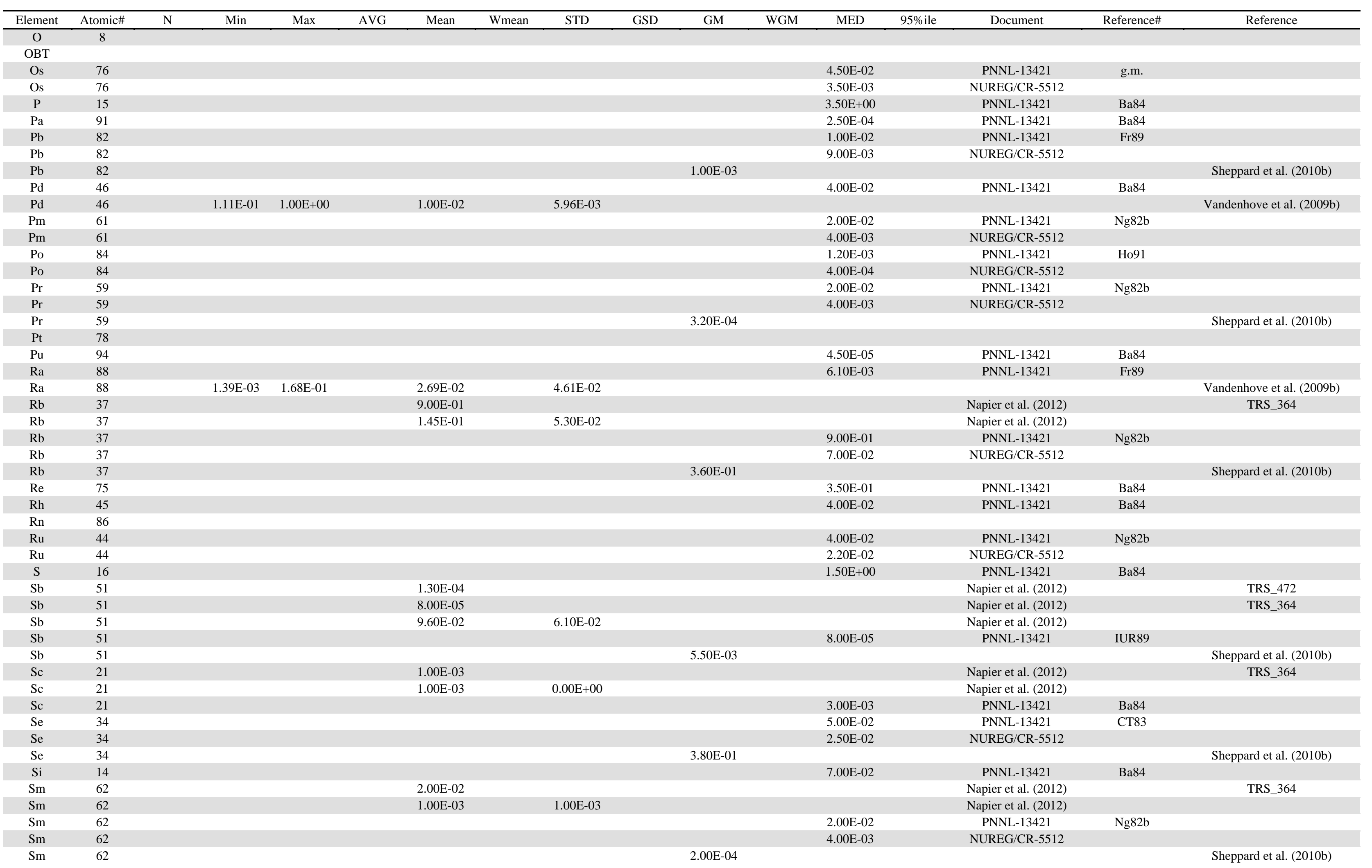


Table C.3. (contd)

\begin{tabular}{|c|c|c|c|c|c|c|c|c|c|c|c|c|c|c|c|c|}
\hline Element & Atomic\# & $\mathrm{N}$ & Min & Max & AVG & Mean & Wmean & STD & GSD & GM & WGM & MED & 95\%ile & Document & Reference\# & Reference \\
\hline Sn & 50 & & & & & & & & & & & $6.00 \mathrm{E}-03$ & & PNNL-13421 & Fu78 & \\
\hline Sn & 50 & & & & & & & & & 9.70E-01 & & & & & & Sheppard et al. (2010b) \\
\hline $\mathrm{Sr}$ & 38 & & & & & 3.60E-01 & & & & & & & & Napier et al. (2012) & & TRS_472 \\
\hline $\mathrm{Sr}$ & 38 & & & & & 2.00E-01 & & & & & & & & Napier et al. (2012) & & TRS_364 \\
\hline $\mathrm{Sr}$ & 38 & & & & & $6.80 \mathrm{E}-02$ & & 7.80E-02 & & & & & & Napier et al. (2012) & & \\
\hline $\mathrm{Sr}$ & 38 & & & & & & & & & & & $1.70 \mathrm{E}-01$ & & NUREG/CR-5512 & & \\
\hline $\mathrm{Sr}$ & 38 & & 8.00E-02 & $1.30 \mathrm{E}-01$ & & & & & & & & & & & & Al-Oudat et al. (2006) \\
\hline $\mathrm{Sr}$ & 38 & & & & & & & & & $1.80 \mathrm{E}-01$ & & & & & & Sheppard et al. (2010b) \\
\hline Та & 73 & & & & & $2.50 \mathrm{E}-02$ & & & & & & & & Napier et al. (2012) & & TRS_364 \\
\hline Та & 73 & & & & & $1.00 \mathrm{E}-03$ & & $1.00 \mathrm{E}-03$ & & & & & & Napier et al. (2012) & & \\
\hline Та & 73 & & & & & & & & & & & 2.50E-02 & & PNNL-13421 & СТ83 & \\
\hline $\mathrm{Tb}$ & 65 & & & & & $2.00 \mathrm{E}-03$ & & $2.00 \mathrm{E}-03$ & & & & & & Napier et al. (2012) & & \\
\hline $\mathrm{Tb}$ & 65 & & & & & & & & & & & $2.00 \mathrm{E}-02$ & & PNNL-13421 & Ng82b & \\
\hline $\mathrm{Tb}$ & 65 & & & & & & & & & & & $4.00 \mathrm{E}-03$ & & NUREG/CR-5512 & & \\
\hline $\mathrm{Tb}$ & 65 & & & & & & & & & $5.00 \mathrm{E}-04$ & & & & & & Sheppard et al. (2010b) \\
\hline Tc & 43 & & & & & & & & & & & $1.50 \mathrm{E}+00$ & & PNNL-13421 & Ba84 & \\
\hline Te & 52 & & & & & & & & & & & 4.00E-03 & & PNNL-13421 & Ba84 & \\
\hline Th & 90 & & & & & 7.80E-04 & & & & & & & & Napier et al. (2012) & & TRS_472 \\
\hline Th & 90 & & & & & $2.50 \mathrm{E}-04$ & & & & & & & & Napier et al. (2012) & & TRS_364 \\
\hline Th & 90 & & & & & $7.00 \mathrm{E}-04$ & & 4.00E-04 & & & & & & Napier et al. (2012) & & \\
\hline Th & 90 & & & & & & & & & & & $2.50 \mathrm{E}-04$ & & PNNL-13421 & Ba84 & \\
\hline Th & 90 & & & & & & & & & & & $8.50 \mathrm{E}-05$ & & NUREG/CR-5512 & & \\
\hline Th & 90 & & & & & & & & & $3.60 \mathrm{E}-03$ & & & & & & Sheppard et al. (2010b) \\
\hline $\mathrm{Ti}$ & 22 & & & & & & & & & & & & & & & \\
\hline $\mathrm{Tl}$ & 81 & & & & & & & & & & & 4.00E-04 & & PNNL-13421 & Ba84 & \\
\hline $\mathrm{Tl}$ & 81 & & & & & & & & & $1.20 \mathrm{E}-02$ & & & & & & Sheppard et al. (2010b) \\
\hline $\mathrm{Tm}$ & 69 & & & & & & & & & & & & & & & \\
\hline $\mathrm{U}$ & 92 & & & & & $1.50 \mathrm{E}-02$ & & & & & & & & Napier et al. (2012) & & TRS_472 \\
\hline $\mathrm{U}$ & 92 & & & & & 4.00E-03 & & & & & & & & Napier et al. (2012) & & TRS_364 \\
\hline U & 92 & & & & & $5.00 \mathrm{E}-03$ & & $6.00 \mathrm{E}-03$ & & & & & & Napier et al. (2012) & & \\
\hline $\mathrm{U}$ & 92 & & & & & & & & & & & 4.00E-03 & & PNNL-13421 & Ba84 & \\
\hline $\mathrm{U}$ & 92 & & & & & & & & & $7.00 \mathrm{E}-04$ & & & & & & Sheppard et al. (2010b) \\
\hline $\mathrm{U}$ & 92 & & $1.29 \mathrm{E}-03$ & 3.73E-01 & & $5.72 \mathrm{E}-02$ & & $1.15 \mathrm{E}-01$ & & & & & & & & Vandenhove et al. (2009b) \\
\hline $\mathrm{V}$ & 23 & & & & & $1.30 \mathrm{E}-03$ & & & & & & & & Napier et al. (2012) & & TRS_364 \\
\hline $\mathrm{V}$ & 23 & & & & & 7.00E-03 & & $1.00 \mathrm{E}-02$ & & & & & & Napier et al. (2012) & & \\
\hline $\mathrm{V}$ & 23 & & & & & & & & & & & & & & & \\
\hline $\mathrm{V}$ & 23 & & & & & & & & & 4.00E-04 & & & & & & Sheppard et al. (2010b) \\
\hline W & 74 & & & & & & & & & & & $3.00 \mathrm{E}+00$ & & PNNL-13421 & NCRP96 & \\
\hline $\mathrm{W}$ & 74 & & & & & & & & & & & $1.00 \mathrm{E}-02$ & & NUREG/CR-5512 & & \\
\hline Хе & 54 & & & & & & & & & & & & & & & \\
\hline $\mathrm{Y}$ & 39 & & & & & & & & & & & 2.00E-02 & & PNNL-13421 & Ng82b & \\
\hline $\mathrm{Y}$ & 39 & & & & & & & & & & & $6.00 \mathrm{E}-03$ & & NUREG/CR-5512 & & \\
\hline $\mathrm{Y}$ & 39 & & & & & & & & & 3.60E-04 & & & & & & Sheppard et al. (2010b) \\
\hline $\mathrm{Yb}$ & 70 & & & & & 2.00E-02 & & & & & & & & Napier et al. (2012) & & TRS_364 \\
\hline $\mathrm{Yb}$ & 70 & & & & & $5.00 \mathrm{E}-03$ & & $5.00 \mathrm{E}-03$ & & & & & & Napier et al. (2012) & & \\
\hline $\mathrm{Yb}$ & 70 & & & & & & & & & $1.00 \mathrm{E}-03$ & & & & & & Sheppard et al. (2010b) \\
\hline
\end{tabular}


Table C.3. (contd)

\begin{tabular}{|c|c|c|c|c|c|c|c|c|c|c|c|c|c|c|c|c|}
\hline Element & Atomic\# & $\mathrm{N}$ & Min & Max & AVG & Mean & Wmean & STD & GSD & GM & WGM & MED & 95\%ile & Document & Reference\# & Reference \\
\hline $\mathrm{Zn}$ & 30 & & & & & $4.20 \mathrm{E}-01$ & & & & & & & & Napier et al. (2012) & & TRS_472 \\
\hline $\mathrm{Zn}$ & 30 & & & & & $9.00 \mathrm{E}-01$ & & & & & & & & Napier et al. (2012) & & TRS_364 \\
\hline $\mathrm{Zn}$ & 30 & & & & & $1.37 \mathrm{E}-01$ & & $6.20 \mathrm{E}-02$ & & & & & & Napier et al. (2012) & & \\
\hline $\mathrm{Zn}$ & 30 & & & & & & & & & & & $9.00 \mathrm{E}-01$ & & PNNL-13421 & Ba84 & \\
\hline $\mathrm{Zn}$ & 30 & & & & & & & & & $2.30 \mathrm{E}-01$ & & & & & & Sheppard et al. (2010b) \\
\hline $\mathrm{Zr}$ & 40 & & & & & $4.00 \mathrm{E}-03$ & & 8.00E-03 & & & & & & Napier et al. (2012) & & \\
\hline $\mathrm{Zr}$ & 40 & & & & & $4.00 \mathrm{E}-03$ & & & & & & & & Napier et al. (2012) & & TRS_472 \\
\hline $\mathrm{Zr}$ & 40 & & & & & $1.00 \mathrm{E}-03$ & & & & & & & & Napier et al. (2012) & & TRS_364 \\
\hline $\mathrm{Zr}$ & 40 & & & & & & & & & & & $1.00 \mathrm{E}-03$ & & PNNL-13421 & Ng82b & \\
\hline $\mathrm{Zr}$ & 40 & & & & & & & & & & & $5.00 \mathrm{E}-04$ & & NUREG/CR-5512 & & \\
\hline $\mathrm{Zr}$ & 40 & & & & & & & & & $1.20 \mathrm{E}-02$ & & & & & & Sheppard et al. (2010b) \\
\hline
\end{tabular}


Table C.4. Bioaccumulation in Dry Grain (Feed or Food Crop) from Soil, $\mathrm{kg}_{\text {dry_plant }} / \mathrm{kg}_{\text {dry__soil }}$

\begin{tabular}{|c|c|c|c|c|c|c|c|c|c|c|c|c|c|c|c|}
\hline Element & Atomic\# & $\mathrm{N}$ & Min & Max & AVG & Mean & Wmean & STD & GSD & GM & WGM & MED & Document & Reference\# & Reference \\
\hline $\mathrm{Ac}$ & 89 & & & & & & & & & & & $3.40 \mathrm{E}-04$ & NUREG/CR-5512 & & \\
\hline Ac & 89 & & & & & & & & & & & $2.20 \mathrm{E}-05$ & PNNL-13421 & Fr82 & \\
\hline $\mathrm{Ag}$ & 47 & & & & & & & & & & & 1.00E- 01 & NUREG/CR-5512 & & \\
\hline $\mathrm{Ag}$ & 47 & & & & & & & & & & & $2.50 \mathrm{E}-01$ & PNNL-13421 & Ba84 & \\
\hline $\mathrm{Ag}$ & 47 & & & & & & & & & $3.90 \mathrm{E}-02$ & & & & & Sheppard et al. (2010b) \\
\hline $\mathrm{Al}$ & 13 & & & & & $1.80 \mathrm{E}-04$ & & & & & & & Napier et al. (2012) & & TRS_364 \\
\hline $\mathrm{Al}$ & 13 & & & & & $4.00 \mathrm{E}-03$ & & $2.00 \mathrm{E}-03$ & & & & & Napier et al. (2012) & & \\
\hline $\mathrm{Am}$ & 95 & & 3.00E-07 & $1.00 \mathrm{E}-03$ & & & & & & & & $1.00 \mathrm{E}-05$ & Coughtrey et al. (1985) & S2.1, T2.1 & \\
\hline $\mathrm{Am}$ & 95 & & & & & & & & & & & $2.20 \mathrm{E}-05$ & PNNL-13421 & Fr82 & \\
\hline $\mathrm{Am}$ & 95 & & & & & & & & & $2.30 \mathrm{E}-03$ & & & & & Uchida et al. (2009) \\
\hline Am & 95 & 83 & $7.40 \mathrm{E}+07$ & 3.40E-02 & & & & & $1.10 \mathrm{E}+01$ & 2.20E-05 & & & TRS_472 & & \\
\hline As & 33 & & & & & $6.00 \mathrm{E}-03$ & & & & & & & Napier et al. (2012) & & TRS_364 \\
\hline As & 33 & & & & & $1.90 \mathrm{E}-02$ & & $6.00 \mathrm{E}-03$ & & & & & Napier et al. (2012) & & \\
\hline As & 33 & & & & & & & & & & & $6.00 \mathrm{E}-03$ & PNNL-13421 & Ba84 & \\
\hline As & 33 & & & & & & & & & $2.70 \mathrm{E}-02$ & & & & & Sheppard et al. (2010b) \\
\hline As & 33 & & & & & & & & & 7.10E-03 & & & & & Uchida et al. (2009) \\
\hline As & 33 & & & & & & & & & $9.20 \mathrm{E}-03$ & & & & & Uchida et al. (2009) \\
\hline $\mathrm{Au}$ & 79 & & & & & & & & & & & $1.00 \mathrm{E}-01$ & NUREG/CR-5512 & & \\
\hline $\mathrm{Au}$ & 79 & & & & & & & & & & & $2.50 \mathrm{E}-01$ & PNNL-13421 & Ba84 & \\
\hline $\mathrm{Ba}$ & 56 & & & & & 1.50E-02 & & & & & & & Napier et al. (2012) & & TRS_364 \\
\hline $\mathrm{Ba}$ & 56 & & & & & $1.00 \mathrm{E}-03$ & & & & & & & Napier et al. (2012) & & TRS_472 \\
\hline $\mathrm{Ba}$ & 56 & & & & & 5.00E-03 & & $2.00 \mathrm{E}-03$ & & & & & Napier et al. (2012) & & \\
\hline Ba & 56 & & & & & & & & & & & $1.50 \mathrm{E}-02$ & PNNL-13421 & Ba84 & \\
\hline Ва & 56 & & & & & & & & & 2.60E-02 & & & & & Sheppard et al. (2010b) \\
\hline $\mathrm{Ba}$ & 56 & & & & & & & & & 3.90E-04 & & & & & Uchida et al. (2009) \\
\hline Ba & 56 & 1 & & & & $1.00 \mathrm{E}-03$ & & & & & & & TRS_472 & & \\
\hline $\mathrm{Be}$ & 4 & & & & & & & & & & & $1.50 \mathrm{E}-03$ & NUREG/CR-5512 & & \\
\hline $\mathrm{Be}$ & 4 & & & & & & & & & & & $3.00 \mathrm{E}-03$ & PNNL-13421 & g.m. & \\
\hline $\mathrm{Be}$ & 4 & & & & & & & & & $1.80 \mathrm{E}-03$ & & & & & Uchida et al. (2009) \\
\hline $\mathrm{Bi}$ & 83 & & & & & & & & & & & 5.00E- 01 & PNNL-13421 & IAEA82 & \\
\hline $\mathrm{Br}$ & 35 & & & & & $1.50 \mathrm{E}+00$ & & & & & & & Napier et al. (2012) & & TRS_364 \\
\hline $\mathrm{Br}$ & 35 & & & & & $1.06 \mathrm{E}+00$ & & $3.01 \mathrm{E}-01$ & & & & & Napier et al. (2012) & & \\
\hline $\mathrm{Br}$ & 35 & & $1.00 \mathrm{E}-02$ & $1.57 \mathrm{E}+01$ & & & & & & & & & Coughtrey et al. (1985) & S2.1 & \\
\hline $\mathrm{Br}$ & 35 & & & & & & & & & & & $1.50 \mathrm{E}+00$ & PNNL-13421 & Fu78 & \\
\hline $\mathrm{C}$ & 6 & & & & & & & & & & & 7.00E-01 & PNNL-13421 & Na88 & \\
\hline $\mathrm{Ca}$ & 20 & & & & & 3.50E-01 & & & & & & & Napier et al. (2012) & & TRS_364 \\
\hline $\mathrm{Ca}$ & 20 & & & & & $2.00 \mathrm{E}+01$ & & & & & & & Napier et al. (2012) & & TRS_472 \\
\hline $\mathrm{Ca}$ & 20 & & & & & $1.08 \mathrm{E}-01$ & & $3.10 \mathrm{E}-02$ & & & & & Napier et al. (2012) & & \\
\hline Са & 20 & & & & & & & & & & & $3.50 \mathrm{E}-01$ & PNNL-13421 & Ba84 & \\
\hline $\mathrm{Ca}$ & 20 & & & & & & & & & $4.80 \mathrm{E}-02$ & & & & & Sheppard et al. (2010b) \\
\hline Са & 20 & & & & & & & & & $4.10 \mathrm{E}-03$ & & & & & Uchida et al. (2009) \\
\hline $\mathrm{Ca}$ & 20 & & & & & & & & & $8.80 \mathrm{E}-03$ & & & & & Uchida et al. (2009) \\
\hline $\mathrm{Ca}$ & 20 & 6 & $2.30 \mathrm{E}+00$ & $3.80 \mathrm{E}+01$ & & & & & $3.70 \mathrm{E}+00$ & $8.70 \mathrm{E}+00$ & & & TRS_472 & & \\
\hline $\mathrm{Cd}$ & 48 & & & & & & & & & & & 1.50E-01 & PNNL-13421 & Ba84 & \\
\hline $\mathrm{Cd}$ & 48 & & & & & & & & & $1.20 \mathrm{E}-01$ & & & & & Sheppard et al. (2010b) \\
\hline $\mathrm{Cd}$ & 48 & & & & & & & & & $9.10 \mathrm{E}-02$ & & & & & Uchida et al. (2009) \\
\hline $\mathrm{Cd}$ & 48 & & & & & & & & & $9.40 \mathrm{E}-02$ & & & & & Uchida et al. (2009) \\
\hline $\mathrm{Cd}$ & 48 & 11 & $1.40 \mathrm{E}-01$ & 2.9 & & & & & $2.70 \mathrm{E}+00$ & $8.80 \mathrm{E}-01$ & & & TRS_472 & & \\
\hline $\mathrm{Ce}$ & 58 & & & & & 2.00E-02 & & & & & & & Napier et al. (2012) & & TRS_364 \\
\hline $\mathrm{Ce}$ & 58 & & & & & $3.00 \mathrm{E}-03$ & & & & & & & Napier et al. (2012) & & TRS_472 \\
\hline
\end{tabular}


Table C.4. (contd)

\begin{tabular}{|c|c|c|c|c|c|c|c|c|c|c|c|c|c|c|c|}
\hline Element & Atomic\# & $\mathrm{N}$ & Min & Max & AVG & Mean & Wmean & STD & GSD & GM & WGM & MED & Document & Reference\# & Reference \\
\hline $\mathrm{Ce}$ & 58 & & & & & $2.00 \mathrm{E}-03$ & & & & & & & Napier et al. (2012) & & \\
\hline $\mathrm{Ce}$ & 58 & & & & & & & & & & & $4.00 \mathrm{E}-03$ & NUREG/CR-5512 & & \\
\hline $\mathrm{Ce}$ & 58 & & & & & & & & & & & $2.00 \mathrm{E}-02$ & PNNL-13421 & Ng82b & \\
\hline $\mathrm{Ce}$ & 58 & & & & & & & & & $1.60 \mathrm{E}-04$ & & & & & Sheppard et al. (2010b) \\
\hline $\mathrm{Ce}$ & 58 & & & & & & & & & $3.30 \mathrm{E}-05$ & & & & & Uchida et al. (2009) \\
\hline $\mathrm{Ce}$ & 58 & 20 & $2.40 \mathrm{E}-04$ & $2.00 \mathrm{E}-02$ & & & & & $3.70 \mathrm{E}+00$ & $3.10 \mathrm{E}-03$ & & & TRS_472 & & \\
\hline Cf & 98 & & & & & & & & & & & $1.00 \mathrm{E}-02$ & NUREG/CR-5512 & & \\
\hline $\mathrm{Cf}$ & 98 & & & & & & & & & & & $2.20 \mathrm{E}-05$ & PNNL-13421 & Fr82 & \\
\hline $\mathrm{Cl}$ & 17 & & & & & $7.00 \mathrm{E}+01$ & & & & & & & Napier et al. (2012) & & TRS_364 \\
\hline $\mathrm{Cl}$ & 17 & & & & & $1.16 \mathrm{E}+01$ & & $2.61 E+03$ & & & & & Napier et al. (2012) & & \\
\hline $\mathrm{Cl}$ & 17 & & & & & & & & & & & $7.00 \mathrm{E}+00$ & PNNL-13421 & Ba84 & \\
\hline $\mathrm{Cl}$ & 17 & & & & & & & & & $3.20 \mathrm{E}+00$ & & & & & Sheppard et al. (2010b) \\
\hline $\mathrm{Cm}$ & 96 & & & & & & & & & & & $2.10 \mathrm{E}-05$ & PNNL-13421 & Fr82 & \\
\hline $\mathrm{Cm}$ & 96 & 67 & $1.40 \mathrm{E}-06$ & $2.00 \mathrm{E}-04$ & & & & & $3.30 \mathrm{E}+00$ & 2.30E-05 & & & TRS_472 & & \\
\hline Co & 27 & & & & & $3.70 \mathrm{E}-03$ & & & & & & & Napier et al. (2012) & & TRS_364 \\
\hline Co & 27 & & & & & $8.50 \mathrm{E}-03$ & & & & & & & Napier et al. (2012) & & TRS 472 \\
\hline Co & 27 & & & & & $2.20 \mathrm{E}-02$ & & $2.00 \mathrm{E}-03$ & & & & & Napier et al. (2012) & & \\
\hline Co & 27 & & & & & & & & & & & $3.70 \mathrm{E}-03$ & PNNL-13421 & Fr89 & \\
\hline Co & 27 & & & & & & & & & $1.60 \mathrm{E}-03$ & & & & & Sheppard et al. (2010b) \\
\hline Co & 27 & & & & & & & & & $4.60 \mathrm{E}-04$ & & & & & Uchida et al. (2009) \\
\hline Co & 27 & & & & & & & & & $9.30 \mathrm{E}-04$ & & & & & Uchida et al. (2009) \\
\hline Co & 27 & 61 & $4.00 \mathrm{E}-04$ & $7.20 \mathrm{E}-01$ & & & & & $5.50 \mathrm{E}+00$ & $8.50 \mathrm{E}-03$ & & & TRS_472 & & \\
\hline $\mathrm{Cr}$ & 24 & & & & & $4.50 \mathrm{E}-03$ & & & & & & & Napier et al. (2012) & & TRS_364 \\
\hline $\mathrm{Cr}$ & 24 & & & & & $2.00 \mathrm{E}-04$ & & & & & & & Napier et al. (2012) & & TRS 472 \\
\hline $\mathrm{Cr}$ & 24 & & & & & $2.50 \mathrm{E}-02$ & & $6.00 \mathrm{E}-03$ & & & & & Napier et al. (2012) & & \\
\hline $\mathrm{Cr}$ & 24 & & & & & & & & & & & $4.50 \mathrm{E}-03$ & PNNL-13421 & Ba84 & \\
\hline $\mathrm{Cr}$ & 24 & & & & & & & & & $4.00 \mathrm{E}-03$ & & & & & Sheppard et al. (2010b) \\
\hline $\mathrm{Cr}$ & 24 & & & & & & & & & $1.50 \mathrm{E}-03$ & & & & & Uchida et al. (2009) \\
\hline $\mathrm{Cr}$ & 24 & & & & & & & & & $2.00 \mathrm{E}-03$ & & & & & Uchida et al. (2009) \\
\hline $\mathrm{Cr}$ & 24 & 1 & & & & $2.00 \mathrm{E}-04$ & & & & & & & TRS_472 & & \\
\hline Cs & 55 & & & & & 2.60E-01 & & & & & & & Napier et al. (2012) & & TRS_364 \\
\hline Cs & 55 & & & & & $2.90 \mathrm{E}-02$ & & & & & & & Napier et al. (2012) & & TRS 472 \\
\hline Cs & 55 & & & & & 3.00E-03 & & $1.00 \mathrm{E}-03$ & & & & & Napier et al. (2012) & & \\
\hline Cs & 55 & & $3.00 \mathrm{E}-03$ & $1.00 \mathrm{E}+00$ & & & & & & & & $5.00 \mathrm{E}-02$ & Coughtrey et al. (1985) & S2.1 & \\
\hline Cs & 55 & & & & & & & & & & & $2.60 \mathrm{E}-01$ & PNNL-13421 & Fr82 & \\
\hline Cs & 55 & & & & & & & & & & & 0.28 & & & Mollah et al. (1998) \\
\hline Cs & 55 & & & & & & & & & $2.80 \mathrm{E}-03$ & & & & & Sheppard et al. (2010b) \\
\hline Cs & 55 & & & & & & & & & $\begin{array}{l}2.0 \mathrm{v}-0 \mathrm{0}-04 \\
6.00 \mathrm{E}-04\end{array}$ & & & & & Uchida et al. (2009) \\
\hline Cs & 55 & & & & & & & & & $9.40 \mathrm{E}-04$ & & & & & Uchida et al. (2009) \\
\hline Cs & 55 & 470 & $2.00 \mathrm{E}-04$ & $9.00 \mathrm{E}-01$ & & & & & $4.00 \mathrm{E}+00$ & $\begin{array}{l}2.400 \mathrm{E}-02 \\
2.92\end{array}$ & & & TRS_472 & & \\
\hline $\mathrm{Cu}$ & 29 & & & & & & & & & & & $2.50 \mathrm{E}-01$ & PNNL-13421 & Ba84 & \\
\hline $\mathrm{Cu}$ & 29 & & & & & & & & & $2.00 \mathrm{E}-01$ & & & & & Sheppard et al. (2010b) \\
\hline $\mathrm{Cu}$ & 29 & & & & & & & & & $8.80 \mathrm{E}-02$ & & & & & Uchida et al. (2009) \\
\hline $\mathrm{Cu}$ & 29 & & & & & & & & & $\begin{array}{l}0.0 \mathrm{E}-0 \mathrm{U} \\
1.00 \mathrm{E}-01\end{array}$ & & & & & Uchida et al. (2009) \\
\hline Dy & 66 & & & & & & & & & & & $4.00 \mathrm{E}-03$ & NUREG/CR-5512 & & \\
\hline Dy & 66 & & & & & & & & & & & $2.00 \mathrm{E}-02$ & PNNL-13421 & Ng82b & \\
\hline Er & 68 & & & & & & & & & & & $4.00 \mathrm{E}-03$ & NUREG/CR-5512 & & \\
\hline Er & 68 & & & & & & & & & & & $2.00 \mathrm{E}-02$ & PNNL-13421 & Ng82b & \\
\hline $\mathrm{Eu}$ & 63 & & & & & 2.00E-02 & & & & & & & Napier et al. (2012) & & TRS_364 \\
\hline Eu & 63 & & & & & $2.00 \mathrm{E}-03$ & & $1.00 \mathrm{E}-03$ & & & & & Napier et al. (2012) & & \\
\hline
\end{tabular}


Table C.4. (contd)

\begin{tabular}{|c|c|c|c|c|c|c|c|c|c|c|c|c|c|c|c|}
\hline Element & Atomic\# & $\mathrm{N}$ & Min & Max & AVG & Mean & Wmean & STD & GSD & GM & WGM & MED & Document & Reference\# & Reference \\
\hline Eu & 63 & & & & & & & & & & & $4.00 \mathrm{E}-03$ & NUREG/CR-5512 & & \\
\hline $\mathrm{Eu}$ & 63 & & & & & & & & & & & $2.00 \mathrm{E}-02$ & PNNL-13421 & Ng82b & \\
\hline Еu & 63 & & & & & & & & & $3.10 \mathrm{E}-04$ & & & & & Uchida et al. (2009) \\
\hline Eu & 63 & & & & & & & & & $2.60 \mathrm{E}-04$ & & & & & Uchida et al. (2009) \\
\hline $\mathrm{F}$ & 9 & & & & & & & & & & & $6.00 \mathrm{E}-03$ & NUREG/CR-5512 & & \\
\hline $\mathrm{Fe}$ & 26 & & & & & $5.00 \mathrm{E}-02$ & & & & & & & Napier et al. (2012) & & TRS_364 \\
\hline $\mathrm{Fe}$ & 26 & & & & & $2.00 \mathrm{E}-04$ & & & & & & & Napier et al. (2012) & & TRS_472 \\
\hline $\mathrm{Fe}$ & 26 & & & & & $4.00 \mathrm{E}-03$ & & & & & & & Napier et al. (2012) & & \\
\hline $\mathrm{Fe}$ & 26 & & & & & & & & & & & $1.00 \mathrm{E}-03$ & NUREG/CR-5512 & & \\
\hline $\mathrm{Fe}$ & 26 & & & & & & & & & & & $5.00 \mathrm{E}-02$ & PNNL-13421 & СT83 & \\
\hline $\mathrm{Fe}$ & 26 & & & & & & & & & $9.80 \mathrm{E}-05$ & & & & & Uchida et al. (2009) \\
\hline $\mathrm{Fe}$ & 26 & & & & & & & & & $2.90 \mathrm{E}-04$ & & & & & Uchida et al. (2009) \\
\hline Fe & 26 & 1 & & & & $2.20 \mathrm{E}-04$ & & & & & & & TRS_472 & & \\
\hline Ga & 31 & & & & & & & & & & & $4.00 \mathrm{E}-04$ & PNNL-13421 & Ba84 & \\
\hline $\mathrm{Gd}$ & 64 & & & & & & & & & & & $4.00 \mathrm{E}-03$ & NUREG/CR-5512 & & \\
\hline Gd & 64 & & & & & & & & & & & $2.00 \mathrm{E}-02$ & PNNL-13421 & Ng82b & \\
\hline $\mathrm{Hf}$ & 72 & & & & & $1.00 \mathrm{E}-03$ & & & & & & & Napier et al. (2012) & & TRS_364 \\
\hline $\mathrm{Hf}$ & 72 & & & & & $2.00 \mathrm{E}-03$ & & $1.00 \mathrm{E}-03$ & & & & & Napier et al. (2012) & & \\
\hline $\mathrm{Hf}$ & 72 & & & & & & & & & & & $8.50 \mathrm{E}-04$ & NUREG/CR-5512 & & \\
\hline $\mathrm{Hf}$ & 72 & & & & & & & & & & & $3.00 \mathrm{E}-03$ & PNNL-13421 & Ng82b & \\
\hline $\mathrm{Hg}$ & 80 & & & & & & & & & & & $2.00 \mathrm{E}-01$ & NUREG/CR-5512 & & \\
\hline $\mathrm{Hg}$ & 80 & & & & & & & & & & & $4.90 \mathrm{E}-01$ & PNNL-13421 & g.m. & \\
\hline Ho & 67 & & & & & & & & & & & $4.00 \mathrm{E}-03$ & NUREG/CR-5512 & & \\
\hline Но & 67 & & & & & & & & & & & $2.00 \mathrm{E}-02$ & PNNL-13421 & Ng82b & \\
\hline Но & 67 & & & & & & & & & $4.00 \mathrm{E}-04$ & & & & & Uchida et al. (2009) \\
\hline Ho & 67 & & & & & & & & & $1.80 \mathrm{E}-04$ & & & & & Uchida et al. (2009) \\
\hline I & 53 & & & & & & & & & & & $5.00 \mathrm{E}-02$ & NUREG/CR-5512 & & \\
\hline I & 53 & & & & & & & & & & & 4.00E-02 & PNNL-13421 & Sn94 & \\
\hline I & 53 & & & & & & & & & $2.00 \mathrm{E}-03$ & & & & & Uchida et al. (2009) \\
\hline I & 53 & & & & & & & & & $5.00 \mathrm{E}-03$ & & & & & Uchida et al. (2009) \\
\hline I & 53 & & & & & & & & & $4.90 \mathrm{E}-03$ & & & & & Uchida and Tagami (2011) \\
\hline In & 49 & & & & & & & & & & & $4.00 \mathrm{E}-04$ & PNNL-13421 & Fu78 & \\
\hline Ir & 77 & & & & & & & & & & & $1.50 \mathrm{E}-02$ & PNNL-13421 & Ba84 & \\
\hline $\mathrm{K}$ & 19 & & & & & $5.50 \mathrm{E}-01$ & & & & & & & Napier et al. (2012) & & TRS_364 \\
\hline $\mathrm{K}$ & 19 & & & & & 7.40E-01 & & & & & & & Napier et al. (2012) & & TRS_472 \\
\hline $\mathrm{K}$ & 19 & & & & & 2.78E-01 & & 7.10E-02 & & & & & Napier et al. (2012) & & \\
\hline $\mathrm{K}$ & 19 & & & & & & & & & & & $5.50 \mathrm{E}+00$ & PNNL-13421 & Ва84 & \\
\hline $\mathrm{K}$ & 19 & & & & & & & & & $1.80 \mathrm{E}+00$ & & & & & Sheppard et al. (2010b) \\
\hline $\mathrm{K}$ & 19 & & & & & & & & & $6.20 \mathrm{E}-02$ & & & & & Uchida et al. (2009) \\
\hline $\mathrm{K}$ & 19 & & & & & & & & & $2.20 \mathrm{E}-01$ & & & & & Uchida et al. (2009) \\
\hline $\mathrm{K}$ & 19 & 2 & 7.30E-01 & 7.40E-01 & & 7.40E-01 & & & & & & & TRS_472 & & \\
\hline $\mathrm{La}$ & 57 & & & & & $4.00 \mathrm{E}-03$ & & & & & & & Napier et al. (2012) & & TRS_364 \\
\hline $\mathrm{La}$ & 57 & & & & & $2.00 \mathrm{E}-05$ & & & & & & & Napier et al. (2012) & & TRS_472 \\
\hline $\mathrm{La}$ & 57 & & & & & $2.00 \mathrm{E}-03$ & & & & & & & Napier et al. (2012) & & \\
\hline $\mathrm{La}$ & 57 & & & & & & & & & & & $4.00 \mathrm{E}-03$ & PNNL-13421 & Ва84 & \\
\hline $\mathrm{La}$ & 57 & & & & & & & & & $2.10 \mathrm{E}-04$ & & & & & Sheppard et al. (2010b) \\
\hline $\mathrm{La}$ & 57 & & & & & & & & & $3.50 \mathrm{E}-05$ & & & & & Uchida et al. (2009) \\
\hline La & 57 & & & & & & & & & $4.90 \mathrm{E}-05$ & & & & & Uchida et al. (2009) \\
\hline $\mathrm{La}$ & 57 & 1 & & & & 2.00E-05 & & & & & & & TRS_472 & & \\
\hline $\mathrm{Lu}$ & 71 & & & & & $2.00 \mathrm{E}-02$ & & & & & & & Napier et al. (2012) & & TRS_364 \\
\hline $\mathrm{Lu}$ & 71 & & & & & $4.00 \mathrm{E}-04$ & & $1.00 \mathrm{E}-03$ & & & & & Napier et al. (2012) & & \\
\hline
\end{tabular}


Table C.4. (contd)

\begin{tabular}{|c|c|c|c|c|c|c|c|c|c|c|c|c|c|c|c|}
\hline Element & Atomic\# & $\mathrm{N}$ & Min & Max & AVG & Mean & Wmean & STD & GSD & GM & WGM & MED & Document & Reference\# & Reference \\
\hline $\mathrm{Mg}$ & 12 & & & & & $5.50 \mathrm{E}-01$ & & & & & & & Napier et al. (2012) & & $\begin{array}{l}\text { TRS_364 } \\
\end{array}$ \\
\hline $\mathrm{Mg}$ & 12 & & & & & 2.31E-01 & & $1.13 \mathrm{E}-01$ & & & & & Napier et al. (2012) & & \\
\hline $\mathrm{Mg}$ & 12 & & & & & & & & & & & $5.50 \mathrm{E}-01$ & PNNL-13421 & Ba84 & \\
\hline $\mathrm{Mg}$ & 12 & & & & & & & & & $1.90 \mathrm{E}-01$ & & & & & Sheppard et al. (2010b) \\
\hline Mn & 25 & & & & & 3.00E-01 & & & & & & & Napier et al. (2012) & & TRS_364 \\
\hline $\mathrm{Mn}$ & 25 & & & & & $2.80 \mathrm{E}-01$ & & & & & & & Napier et al. (2012) & & TRS_472 \\
\hline $\mathrm{Mn}$ & 25 & & & & & 1.49E-01 & & $1.90 \mathrm{E}-02$ & & & & & Napier et al. (2012) & & \\
\hline $\mathrm{Mn}$ & 25 & & & & & & & & & & & 2.90E-01 & NUREG/CR-5512 & & \\
\hline $\mathrm{Mn}$ & 25 & & & & & & & & & & & $3.00 \mathrm{E}-01$ & PNNL-13421 & IAEA94 & \\
\hline $\mathrm{Mn}$ & 25 & & & & & & & & & $4.50 \mathrm{E}-02$ & & & & & Sheppard et al. (2010b) \\
\hline $\mathrm{Mn}$ & 25 & & & & & & & & & $1.50 \mathrm{E}-02$ & & & & & Uchida et al. (2009) \\
\hline Mn & 25 & & & & & & & & & 4.60E-02 & & & & & Uchida et al. (2009) \\
\hline $\mathrm{Mn}$ & 25 & 78 & $1.40 \mathrm{E}-02$ & $2.70 \mathrm{E}+00$ & & & & & $3.30 \mathrm{E}+00$ & 2.80E-01 & & & TRS_472 & & \\
\hline Mo & 42 & & & & & & & & & & & $6.00 \mathrm{E}-02$ & NUREG/CR-5512 & & \\
\hline Mo & 42 & & & & & & & & & & & $8.00 \mathrm{E}-01$ & PNNL-13421 & Ng82b & \\
\hline Mo & 42 & & & & & & & & & $1.00 \mathrm{E}+00$ & & & & & Sheppard et al. (2010b) \\
\hline Mo & 42 & & & & & & & & & 7.40E-01 & & & & & Uchida et al. (2009) \\
\hline Mo & 42 & & & & & & & & & $7.50 \mathrm{E}-01$ & & & & & Uchida et al. (2009) \\
\hline Mo & 42 & 1 & & & & $8.00 \mathrm{E}-01$ & & & & & & & TRS_472 & & \\
\hline $\mathrm{N}$ & 7 & & & & & & & & & & & $3.00 \mathrm{E}+01$ & NUREG/CR-5512 & & \\
\hline $\mathrm{N}$ & 7 & & & & & & & & & & & 1.30E-01 & PNNL-13421 & g.m. & \\
\hline $\mathrm{Na}$ & 11 & & & & & 3.00E-01 & & & & & & & Napier et al. (2012) & & TRS_364 \\
\hline Na & 11 & & & & & $1.00 \mathrm{E}-02$ & & & & & & & Napier et al. (2012) & & TRS_472 \\
\hline $\mathrm{Na}$ & 11 & & & & & $4.40 \mathrm{E}-02$ & & $1.00 \mathrm{E}-02$ & & & & & Napier et al. (2012) & & \\
\hline Na & 11 & & & & & & & & & & & $5.50 \mathrm{E}-02$ & NUREG/CR-5512 & & \\
\hline $\mathrm{Na}$ & 11 & & & & & & & & & & & $3.00 \mathrm{E}-01$ & PNNL-13421 & Ng82b & \\
\hline $\mathrm{Na}$ & 11 & & & & & & & & & $4.00 \mathrm{E}-02$ & & & & & Sheppard et al. (2010b) \\
\hline $\mathrm{Na}$ & 11 & & & & & & & & & $6.90 \mathrm{E}-04$ & & & & & Uchida et al. (2009) \\
\hline Na & 11 & & & & & & & & & $9.80 \mathrm{E}-04$ & & & & & Uchida et al. (2009) \\
\hline $\mathrm{Na}$ & 11 & 1 & & & & $1.00 \mathrm{E}-02$ & & & & & & & TRS_472 & & \\
\hline $\mathrm{Nb}$ & 41 & & & & & & & & & & & $2.50 \mathrm{E}-02$ & PNNL-13421 & СТ83 & \\
\hline $\mathrm{Nb}$ & 41 & & & & & & & & & $1.50 \mathrm{E}-03$ & & & & & Sheppard et al. (2010b) \\
\hline $\mathrm{Nb}$ & 41 & 2 & 2.00E-03 & $2.50 \mathrm{E}-02$ & & $1.40 \mathrm{E}-02$ & & & & & & & TRS_472 & & \\
\hline $\mathrm{Nd}$ & 60 & & & & & $2.00 \mathrm{E}-02$ & & & & & & & Napier et al. (2012) & & TRS_364 \\
\hline $\mathrm{Nd}$ & 60 & & & & & 1.20E-02 & & $3.00 \mathrm{E}-03$ & & & & & Napier et al. (2012) & & \\
\hline $\mathrm{Nd}$ & 60 & & & & & & & & & & & $4.00 \mathrm{E}-03$ & NUREG/CR-5512 & & \\
\hline $\mathrm{Nd}$ & 60 & & & & & & & & & & & 2.00E-02 & PNNL-13421 & Ng82b & \\
\hline $\mathrm{Nd}$ & 60 & & & & & & & & & $1.00 \mathrm{E}-04$ & & & & & Sheppard et al. (2010b) \\
\hline $\mathrm{Nd}$ & 60 & & & & & & & & & 3.00E-05 & & & & & Uchida et al. (2009) \\
\hline $\mathrm{Nd}$ & 60 & & & & & & & & & $5.00 \mathrm{E}-05$ & & & & & Uchida et al. (2009) \\
\hline $\mathrm{Ni}$ & 28 & & & & & 3.00E-02 & & & & & & & Napier et al. (2012) & & TRS_364 \\
\hline $\mathrm{Ni}$ & 28 & & & & & $2.70 \mathrm{E}-02$ & & & & & & & Napier et al. (2012) & & TRS_472 \\
\hline $\mathrm{Ni}$ & 28 & & & & & 3.09E-01 & & $5.02 \mathrm{E}-01$ & & & & & Napier et al. (2012) & & \\
\hline $\mathrm{Ni}$ & 28 & & & & & & & & & & & $3.00 \mathrm{E}-02$ & PNNL-13421 & Fr89 & \\
\hline $\mathrm{Ni}$ & 28 & & & & & & & & & $1.70 \mathrm{E}-02$ & & & & & Sheppard et al. (2010b) \\
\hline $\mathrm{Ni}$ & 28 & & 0.0031 & 0.75 & & 0.0881 & & & 3.49 & 4.12E-02 & & & & & Vandenhove et al. (2009a) \\
\hline $\mathrm{Ni}$ & 28 & & & & & & & & & $1.40 \mathrm{E}-02$ & & & & & Uchida et al. (2009) \\
\hline $\mathrm{Ni}$ & 28 & & & & & & & & & 1.30E-02 & & & & & Uchida et al. (2009) \\
\hline $\mathrm{Ni}$ & 28 & 44 & $3.10 \mathrm{E}-03$ & $1.70 \mathrm{E}-01$ & & & & & $2.70 \mathrm{E}+00$ & 2.70E-02 & & & TRS_472 & & \\
\hline $\mathrm{Np}$ & 93 & & & & & & & & & & & $2.70 \mathrm{E}-03$ & PNNL-13421 & Fr82 & \\
\hline $\mathrm{Np}$ & 93 & 85 & $2.30 \mathrm{E}-05$ & $7.10 \mathrm{E}-02$ & & & & & $5.00 \mathrm{E}+00$ & $2.90 \mathrm{E}-03$ & & & TRS_472 & & \\
\hline
\end{tabular}


Table C.4. (contd)

\begin{tabular}{|c|c|c|c|c|c|c|c|c|c|c|c|c|c|c|c|}
\hline Element & Atomic\# & $\mathrm{N}$ & Min & Max & AVG & Mean & Wmean & STD & GSD & GM & WGM & MED & Document & Reference\# & Reference \\
\hline $\mathrm{O}$ & 8 & & & & & & & & & & & $6.00 \mathrm{E}-03$ & PNNL-13421 & Ba84 & \\
\hline Os & 76 & & & & & & & & & & & $3.50 \mathrm{E}-03$ & PNNL-13421 & Ba84 & \\
\hline $\mathrm{P}$ & 15 & & & & & & & & & & & $3.50 \mathrm{E}+00$ & PNNL-13421 & Ba84 & \\
\hline $\mathrm{P}$ & 15 & 1 & & & & $2.00 \mathrm{E}-01$ & & & & & & & TRS_472 & & \\
\hline $\mathrm{Pa}$ & 91 & & & & & & & & & & & $2.50 \mathrm{E}-04$ & NUREG/CR-5512 & & \\
\hline $\mathrm{Pa}$ & 91 & & & & & & & & & & & 2.20E-05 & PNNL-13421 & Fr82 & \\
\hline $\mathrm{Pb}$ & 82 & & & & & & & & & & & 4.70E- -03 & PNNL-13421 & Fr89 & \\
\hline $\mathrm{Pb}$ & 82 & & & & & & & & & $2.50 \mathrm{E}-04$ & & & & & Uchida et al. (2009) \\
\hline $\mathrm{Pb}$ & 82 & & & & & & & & & 3.30E-05 & & & & & Uchida et al. (2009) \\
\hline $\mathrm{Pb}$ & 82 & & $5.2 \mathrm{E}-04$ & 4.80E-02 & & $1.13 \mathrm{E}-02$ & & $1.41 \mathrm{E}-02$ & & & & & & & Vandenhove et al. (2009b) \\
\hline $\mathrm{Pb}$ & 82 & 9 & 1.90E-03 & $4.80 \mathrm{E}-02$ & & & & & $3.60 \mathrm{E}+00$ & $1.10 \mathrm{E}-02$ & & & TRS_472 & & \\
\hline Pd & 46 & & & & & & & & & & & $4.00 \mathrm{E}-02$ & PNNL-13421 & Ba84 & \\
\hline Pd & 46 & & & & & & & & & $2.20 \mathrm{E}-03$ & & & & & Sheppard et al. (2010b) \\
\hline $\mathrm{Pm}$ & 61 & & & & & & & & & & & 4.00E-03 & NUREG/CR-5512 & & \\
\hline $\mathrm{Pm}$ & 61 & & & & & & & & & & & $2.00 \mathrm{E}-02$ & PNNL-13421 & Ng82b & \\
\hline $\mathrm{Pm}$ & 61 & 17 & $1.70 \mathrm{E}-03$ & 2.40E-01 & & & & & $6.00 \mathrm{E}+00$ & $1.40 \mathrm{E}-02$ & & & TRS_472 & & \\
\hline Po & 84 & & & & & & & & & & & 4.00E-04 & NUREG/CR-5512 & & \\
\hline Po & 84 & & & & & & & & & & & $2.30 \mathrm{E}-03$ & PNNL-13421 & Ho91 & \\
\hline Po & 84 & & 0.000018 & 0.0168 & & 0.00356 & & 0.00743 & & & & & & & Vandenhove et al. (2009b) \\
\hline Po & 84 & 2 & $2.20 \mathrm{E}-04$ & 2.60E-04 & & $2.40 \mathrm{E}-04$ & & & & & & & TRS_472 & & \\
\hline $\mathrm{Pr}$ & 59 & & & & & & & & & & & $2.00 \mathrm{E}-02$ & PNNL-13421 & Ng82b & \\
\hline Pr & 59 & & & & & & & & & $2.30 \mathrm{E}-04$ & & & & & Sheppard et al. (2010b) \\
\hline $\operatorname{Pr}$ & 59 & & & & & & & & & $1.10 \mathrm{E}-04$ & & & & & Uchida et al. (2009) \\
\hline $\operatorname{Pr}$ & 59 & & & & & & & & & $6.40 \mathrm{E}-05$ & & & & & Uchida et al. (2009) \\
\hline $\operatorname{Pr}$ & 59 & 1 & & & & 2.00E-02 & & & & & & & TRS_472 & & \\
\hline Pu & 94 & & & & & & & & & & & $2.60 \mathrm{E}-05$ & NUREG/CR-5512 & & \\
\hline Pu & 94 & & & & & & & & & & & $8.60 \mathrm{E}-05$ & PNNL-13421 & Fr82 & \\
\hline Pu & 94 & & & & & & & & & $1.30 \mathrm{E}-03$ & & & & & Uchida et al. (2009) \\
\hline Pu & 94 & 105 & 2.00E-07 & 1.10E-03 & & & & & $6.70 \mathrm{E}+00$ & $9.50 \mathrm{E}-06$ & & & TRS_472 & & \\
\hline Ra & 88 & & & & & & & & & & & $1.20 \mathrm{E}-03$ & PNNL-13421 & Fr89 & \\
\hline Ra & 88 & & & & & & & & & 4.70E-04 & & & & & Uchida et al. (2009) \\
\hline Ra & 88 & & & & & & & & & $9.10 \mathrm{E}-04$ & & & & & Uchida et al. (2009) \\
\hline $\mathrm{Ra}$ & 88 & & 0.00008 & 0.666 & & 0.0641 & & 0.131 & & & & & & & Vandenhove et al. (2009b) \\
\hline Ra & 88 & 24 & 8.00E-05 & $6.70 \mathrm{E}-01$ & & & & & $1.20 \mathrm{E}+01$ & $1.70 \mathrm{E}-02$ & & & TRS_472 & & \\
\hline $\mathrm{Rb}$ & 37 & & & & & $9.00 \mathrm{E}-01$ & & & & & & & Napier et al. (2012) & & TRS_364 \\
\hline $\mathrm{Rb}$ & 37 & & & & & $9.00 \mathrm{E}-01$ & & & & & & & Napier et al. (2012) & & TRS_472 \\
\hline $\mathrm{Rb}$ & 37 & & & & & $5.20 \mathrm{E}-02$ & & $9.00 \mathrm{E}-03$ & & & & & Napier et al. (2012) & & \\
\hline $\mathrm{Rb}$ & 37 & & & & & & & & & & & 7.00E-02 & NUREG/CR-5512 & & \\
\hline $\mathrm{Rb}$ & 37 & & & & & & & & & & & $9.00 \mathrm{E}-01$ & PNNL-13421 & Ng82b & \\
\hline $\mathrm{Rb}$ & 37 & & & & & & & & & 1.60E-01 & & & & & Sheppard et al. (2010b) \\
\hline $\mathrm{Rb}$ & 37 & & & & & & & & & $5.60 \mathrm{E}-02$ & & & & & Uchida et al. (2009) \\
\hline $\mathrm{Rb}$ & 37 & & & & & & & & & $1.20 \mathrm{E}-01$ & & & & & Uchida et al. (2009) \\
\hline $\mathrm{Rb}$ & 37 & 1 & & & & $9.00 \mathrm{E}-01$ & & & & & & & TRS_472 & & \\
\hline $\mathrm{Re}$ & 75 & & & & & & & & & & & $3.50 \mathrm{E}-01$ & PNNL-13421 & Ba84 & \\
\hline $\mathrm{Rh}$ & 45 & & & & & & & & & & & 4.00E-02 & PNNL-13421 & Ba84 & \\
\hline Ru & 44 & & & & & & & & & & & $5.00 \mathrm{E}-03$ & PNNL-13421 & Fr89 & \\
\hline $\mathrm{Ru}$ & 44 & 12 & $6.00 \mathrm{E}-04$ & $1.00 \mathrm{E}+02$ & & & & & $2.60 \mathrm{E}+00$ & $3.00 \mathrm{E}-03$ & & & TRS_472 & & \\
\hline $\mathrm{s}$ & 16 & & & & & & & & & & & $1.50 \mathrm{E}+00$ & PNNL-13421 & Ba84 & \\
\hline sb & 51 & & & & & 3.00E-02 & & & & & & & Napier et al. (2012) & & TRS_364 \\
\hline Sb & 51 & & & & & $1.80 \mathrm{E}-03$ & & & & & & & Napier et al. (2012) & & TRS_472 \\
\hline $\mathrm{Sb}$ & 51 & & & & & 4.30E-02 & & $1.40 \mathrm{E}-02$ & & & & & Napier et al. (2012) & & \\
\hline Sb & 51 & & & & & & & & & & & $3.00 \mathrm{E}-02$ & NUREG/CR-5512 & & \\
\hline
\end{tabular}


Table C.4. (contd)

\begin{tabular}{|c|c|c|c|c|c|c|c|c|c|c|c|c|c|c|c|}
\hline Element & Atomic\# & $\mathrm{N}$ & Min & Max & AVG & Mean & Wmean & STD & GSD & GM & WGM & MED & Document & Reference\# & Reference \\
\hline $\mathrm{Sb}$ & 51 & & $7.00 \mathrm{E}-03$ & $1.10 \mathrm{E}-01$ & & & & & & & & $5.00 \mathrm{E}-02$ & Coughtrey et al. (1985) & S2.1 & \\
\hline $\mathrm{Sb}$ & 51 & & & & & & & & & & & 3.00E-02 & PNNL-13421 & Fu78 & \\
\hline $\mathrm{Sb}$ & 51 & & & & & & & & & 3.30E-03 & & & & & Sheppard et al. (2010b) \\
\hline sb & 51 & 24 & 3.00E-04 & $9.00 \mathrm{E}-03$ & & & & & $2.70 \mathrm{E}+00$ & $1.80 \mathrm{E}-03$ & & & TRS_472 & & \\
\hline Sc & 21 & & & & & $1.00 \mathrm{E}-03$ & & & & & & & Napier et al. (2012) & & TRS_364 \\
\hline Sc & 21 & & & & & $3.00 \mathrm{E}-03$ & & & & & & & Napier et al. (2012) & & \\
\hline Sc & 21 & & & & & & & & & & & $1.00 \mathrm{E}-03$ & PNNL-13421 & Ba84 & \\
\hline Se & 34 & & & & & & & & & & & $2.50 \mathrm{E}-02$ & NUREG/CR-5512 & & \\
\hline Se & 34 & & & & & & & & & & & 2.50E-01 & PNNL-13421 & СТ83 & \\
\hline Se & 34 & & & & & & & & & $1.60 \mathrm{E}+00$ & & & & & Sheppard et al. (2010b) \\
\hline Se & 34 & & & & & & & & & $5.40 \mathrm{E}-02$ & & & & & Uchida et al. (2009) \\
\hline Se & 34 & & & & & & & & & $6.70 \mathrm{E}-02$ & & & & & Uchida et al. (2009) \\
\hline $\mathrm{Si}$ & 14 & & & & & & & & & & & 7.00E-02 & PNNL-13421 & Ba84 & \\
\hline $\mathrm{Sm}$ & 62 & & & & & $2.00 \mathrm{E}-02$ & & & & & & & Napier et al. (2012) & & TRS_364 \\
\hline Sm & 62 & & & & & $2.00 \mathrm{E}-03$ & & & & & & & Napier et al. (2012) & & \\
\hline $\mathrm{Sm}$ & 62 & & & & & & & & & & & $4.00 \mathrm{E}-03$ & NUREG/CR-5512 & & \\
\hline Sm & 62 & & & & & & & & & & & $2.00 \mathrm{E}-02$ & PNNL-13421 & Ng82b & \\
\hline $\mathrm{Sm}$ & 62 & & & & & & & & & $1.10 \mathrm{E}-04$ & & & & & Sheppard et al. (2010b) \\
\hline Sn & 50 & & & & & & & & & & & $6.00 \mathrm{E}-03$ & PNNL-13421 & Fu78 & \\
\hline Sn & 50 & & & & & & & & & $1.40 \mathrm{E}-01$ & & & & & Sheppard et al. (2010b) \\
\hline Sn & 50 & & & & & & & & & $5.60 \mathrm{E}-03$ & & & & & Uchida et al. (2009) \\
\hline Sn & 50 & & & & & & & & & $6.40 \mathrm{E}-03$ & & & & & Uchida et al. (2009) \\
\hline $\mathrm{Sr}$ & 38 & & & & & $2.10 \mathrm{E}-01$ & & & & & & & Napier et al. (2012) & & TRS_364 \\
\hline $\mathrm{Sr}$ & 38 & & & & & $1.10 \mathrm{E}-01$ & & & & & & & Napier et al. (2012) & & TRS_472 \\
\hline $\mathrm{Sr}$ & 38 & & & & & $4.90 \mathrm{E}-02$ & & $2.10 \mathrm{E}-02$ & & & & & Napier et al. (2012) & & \\
\hline $\mathrm{Sr}$ & 38 & & & & & & & & & & & $1.30 \mathrm{E}-01$ & NUREG/CR-5512 & & \\
\hline $\mathrm{Sr}$ & 38 & & & & & & & & & & & 2.10E-01 & PNNL-13421 & Fr82 & \\
\hline $\mathrm{Sr}$ & 38 & & & & & & & & & 7.00E-02 & & & & & Sheppard et al. (2010b) \\
\hline $\mathrm{Sr}$ & 38 & & & & & & & & & & & 0.82 & & & Mollah et al. (1998) \\
\hline $\mathrm{Sr}$ & 38 & & & & & & & & & $1.00 \mathrm{E}-03$ & & & & & Uchida et al. (2009) \\
\hline $\mathrm{Sr}$ & 38 & & & & & & & & & $3.00 \mathrm{E}-03$ & & & & & Uchida et al. (2009) \\
\hline $\mathrm{Sr}$ & 38 & 282 & $3.60 \mathrm{E}-03$ & $1.00 \mathrm{E}+00$ & & & & & $2.70 \mathrm{E}+00$ & 1.10E-01 & & & TRS_472 & & \\
\hline Та & 73 & & & & & $2.50 \mathrm{E}-02$ & & & & & & & Napier et al. (2012) & & TRS_364 \\
\hline $\mathrm{Ta}$ & 65 & & & & & 2.00E-02 & & & & & & & Napier et al. (2012) & & TRS_364 \\
\hline Та & 73 & & & & & $2.00 \mathrm{E}-03$ & & $1.00 \mathrm{E}-03$ & & & & & Napier et al. (2012) & & \\
\hline Та & 73 & & & & & & & & & & & $2.50 \mathrm{E}-03$ & NUREG/CR-5512 & & \\
\hline Та & 73 & & & & & & & & & & & $2.50 \mathrm{E}-02$ & PNNL-13421 & СТ83 & \\
\hline $\mathrm{Tb}$ & 65 & & & & & $3.00 \mathrm{E}-03$ & & $1.00 \mathrm{E}-03$ & & & & & Napier et al. (2012) & & \\
\hline $\mathrm{Tb}$ & 65 & & & & & & & & & & & $4.00 \mathrm{E}-03$ & NUREG/CR-5512 & & \\
\hline $\mathrm{Tb}$ & 65 & & & & & & & & & & & $2.00 \mathrm{E}-02$ & PNNL-13421 & Ng82b & \\
\hline $\mathrm{Tb}$ & 65 & & & & & & & & & $4.00 \mathrm{E}-04$ & & & & & Sheppard et al. (2010b) \\
\hline Tc & 43 & & & & & & & & & & & 7.30E-01 & PNNL-13421 & Fr89 & \\
\hline Tc & 43 & & & & & & & & & $2.00 \mathrm{E}-04$ & & & & & Uchida et al. (2009) \\
\hline $\mathrm{Te}$ & 52 & & & & & & & & & & & 4.00E-03 & PNNL-13421 & Ba84 & \\
\hline Te & 52 & 1 & & & & $1.00 \mathrm{E}+01$ & & & & & & & TRS_472 & & \\
\hline Th & 90 & & & & & 3.40E-05 & & & & & & & Napier et al. (2012) & & TRS_364 \\
\hline Th & 90 & & & & & $2.10 \mathrm{E}-03$ & & & & & & & Napier et al. (2012) & & TRS_472 \\
\hline Th & 90 & & & & & $2.00 \mathrm{E}-03$ & & & & & & & Napier et al. (2012) & & \\
\hline Th & 90 & & & & & & & & & & & 3.40E-05 & PNNL-13421 & IUR89 & \\
\hline Th & 90 & & & & & & & & & $9.00 \mathrm{E}-03$ & & & & & Sheppard et al. (2010b) \\
\hline Th & 90 & & & & & & & & & 1.70E-04 & & & & & Uchida et al. (2009) \\
\hline Th & 90 & & & & & & & & & $1.40 \mathrm{E}-04$ & & & & & Uchida et al. (2009) \\
\hline
\end{tabular}


Table C.4. (contd)

\begin{tabular}{|c|c|c|c|c|c|c|c|c|c|c|c|c|c|c|c|}
\hline Element & Atomic\# & $\mathrm{N}$ & Min & Max & $\mathrm{AVG}$ & Mean & Wmean & STD & GSD & GM & WGM & MED & Document & Reference\# & Reference \\
\hline Th & 90 & & $1.24 \mathrm{E}-06$ & $2.24 \mathrm{E}-02$ & & $2.81 \mathrm{E}-03$ & & $4.20 \mathrm{E}-03$ & & & & & & & Vandenhove et al. (2009b) \\
\hline Th & 90 & 36 & $1.60 \mathrm{E}-04$ & $2.20 \mathrm{E}-02$ & & & & & $3.40 \mathrm{E}+00$ & $2.10 \mathrm{E}-03$ & & & TRS_472 & & \\
\hline $\mathrm{Ti}$ & 22 & & & & & $5.40 \mathrm{E}-05$ & & & & & & & Napier et al. (2012) & & TRS_364 \\
\hline $\mathrm{Ti}$ & 22 & & & & & $0.00 \mathrm{E}+00$ & & & & & & & Napier et al. (2012) & & \\
\hline $\mathrm{Tl}$ & 81 & & & & & & & & & & & $4.00 \mathrm{E}-04$ & PNNL-13421 & Ba84 & \\
\hline $\mathrm{Tl}$ & 81 & & & & & & & & & $5.50 \mathrm{E}-02$ & & & & & Sheppard et al. (2010b) \\
\hline $\mathrm{U}$ & 92 & & & & & $1.30 \mathrm{E}-03$ & & & & & & & Napier et al. (2012) & & TRS_364 \\
\hline $\mathrm{U}$ & 92 & & & & & $6.20 \mathrm{E}-03$ & & & & & & & Napier et al. (2012) & & TRS_472 \\
\hline U & 92 & & & & & $1.00 \mathrm{E}-02$ & & $5.00 \mathrm{E}-03$ & & & & & Napier et al. (2012) & & \\
\hline U & 92 & & & & & & & & & & & $1.30 \mathrm{E}-03$ & PNNL-13421 & Bi91 & \\
\hline $\mathrm{U}$ & 92 & & & & & & & & & $1.10 \mathrm{E}-03$ & & & & & Sheppard et al. (2010b) \\
\hline $\mathrm{U}$ & 92 & & & & & & & & & $1.90 \mathrm{E}-04$ & & & & & Uchida et al. (2009) \\
\hline U & 92 & & & & & & & & & $2.80 \mathrm{E}-04$ & & & & & Uchida et al. (2009) \\
\hline U & 92 & & 1.60E-04 & 9.64E-01 & & 5.37E-02 & & 1.67E-01 & & & & & & & Vandenhove et al. (2009b) \\
\hline U & 92 & 59 & $1.60 \mathrm{E}-04$ & $8.20 \mathrm{E}-01$ & & & & & $7.70 \mathrm{E}+00$ & $6.20 \mathrm{E}-03$ & & & TRS_472 & & \\
\hline $\mathrm{V}$ & 23 & & & & & $1.30 \mathrm{E}-03$ & & & & & & & Napier et al. (2012) & & TRS_364 \\
\hline $\mathrm{V}$ & 23 & & & & & $1.00 \mathrm{E}-02$ & & 5.00E-03 & & & & & Napier et al. (2012) & & \\
\hline $\mathrm{V}$ & 23 & & & & & & & & & $1.30 \mathrm{E}-03$ & & & & & Sheppard et al. (2010b) \\
\hline W & 74 & & & & & & & & & & & $1.00 \mathrm{E}-02$ & NUREG/CR-5512 & & \\
\hline $\mathrm{W}$ & 74 & & & & & & & & & & & $3.00 \mathrm{E}+00$ & PNNL-13421 & NCRP96 & \\
\hline $\mathrm{Y}$ & 39 & & & & & & & & & & & $1.00 \mathrm{E}-02$ & PNNL-13421 & Ng82b & \\
\hline $\mathrm{Y}$ & 39 & & & & & & & & & $2.10 \mathrm{E}-04$ & & & & & Sheppard et al. (2010b) \\
\hline $\mathrm{Y}$ & 39 & 5 & & & & & & & & $5.00 \mathrm{E}-04$ & & & TRS_472 & & \\
\hline $\mathrm{Yb}$ & 70 & & & & & $2.00 \mathrm{E}-02$ & & & & & & & Napier et al. (2012) & & TRS_364 \\
\hline $\mathrm{Yb}$ & 70 & & & & & $1.10 \mathrm{E}-02$ & & $4.00 \mathrm{E}-03$ & & & & & Napier et al. (2012) & & \\
\hline $\mathrm{Yb}$ & 70 & & & & & & & & & 8.00E-04 & & & & & Sheppard et al. (2010b) \\
\hline $\mathrm{Zn}$ & 30 & & & & & $1.60 \mathrm{E}+00$ & & & & & & & Napier et al. (2012) & & TRS_364 \\
\hline $\mathrm{Zn}$ & 30 & & & & & $1.80 \mathrm{E}+00$ & & & & & & & Napier et al. (2012) & & TRS_472 \\
\hline $\mathrm{Zn}$ & 30 & & & & & $2.11 \mathrm{E}-01$ & & 3.80E-02 & & & & & Napier et al. (2012) & & \\
\hline $\mathrm{Zn}$ & 30 & & & & & & & & & & & $1.30 \mathrm{E}+00$ & NUREG/CR-5512 & & \\
\hline $\mathrm{Zn}$ & 30 & & & & & & & & & & & $1.60 \mathrm{E}+00$ & PNNL-13421 & Fr89 & \\
\hline $\mathrm{Zn}$ & 30 & & & & & & & & & 3.70E-01 & & & & & Sheppard et al. (2010b) \\
\hline $\mathrm{Zn}$ & 30 & & & & & & & & & $2.00 \mathrm{E}-01$ & & & & & Uchida et al. (2009) \\
\hline $\mathrm{Zn}$ & 30 & & & & & & & & & $2.40 \mathrm{E}-01$ & & & & & Uchida et al. (2009) \\
\hline $\mathrm{Zn}$ & 30 & 86 & $2.00 \mathrm{E}-02$ & $1.40 \mathrm{E}+01$ & & & & & $2.70 \mathrm{E}+00$ & $1.80 \mathrm{E}+00$ & & & TRS_472 & & \\
\hline $\mathrm{Zr}$ & 40 & & & & & $1.00 \mathrm{E}-03$ & & & & & & & Napier et al. (2012) & & TRS_364 \\
\hline $\mathrm{Zr}$ & 40 & 1 & & & & $1.00 \mathrm{E}-03$ & & & & & & & Napier et al. (2012) & & TRS_472 \\
\hline $\mathrm{Zr}$ & 40 & & & & & $5.00 \mathrm{E}-03$ & & $4.00 \mathrm{E}-03$ & & & & & Napier et al. (2012) & & \\
\hline $\mathrm{Zr}$ & 40 & & & & & & & & & & & $5.00 \mathrm{E}-04$ & NUREG/CR-5512 & & \\
\hline $\mathrm{Zr}$ & 40 & & & & & & & & & & & $1.00 \mathrm{E}-03$ & PNNL-13421 & Ng82b & \\
\hline $\mathrm{Zr}$ & 40 & & & & & & & & & 7.00E-03 & & & & & Sheppard et al. (2010b) \\
\hline
\end{tabular}



PNNL-21950, Appendix C

\section{References}

PNNL-13421

Staven LH, BA Napier, K Rhoads, and DL Strenge. 2003. A Compendium of Transfer Factors for Agricultural and Animal Products. PNNL-13421, Pacific Northwest National Laboratory, Richland, Washington.

(Ba84) Baes CF, RD Sharp, AL Sjoreen, and RW Shor. 1984. A Review and Analysis of Parameters for Assessing Transport of Environmentally Released Radionuclides through Agriculture. ORNL-5786, Oak Ridge National Laboratory, Oak Ridge, Tennessee.

(Bi91) Bilo M. 1991. Untersuchugen zum Transfer des durch den Reaktorunfall von Tschernobyl abgelagerten Radiocasiums vom Boden in die Pflanze. Thesis, Personal Communication with ZN Tubingen.

(CT83) Coughtrey PC and MC Thorne. 1983. Radionuclide Distribution and Transport in Terrestrial and Aquatic Ecosystems, A Critical Review of Data. Vols. 1-6, A.A. Balkema, Rotterdam.

(Fr82) Frissel MJ. 1992. An Update of the Recommended Soil-to-Plant Transfer Factors. Eighth Report of the IUR Working Group on Soil-to-Plant Transfer Factors, IUR, Balen, Belgium.

(Fr89) Frissel MJ and KE van Bergeijk. 1989. Mean Transfer Values Derived by Simple Statistical Regression Analysis. Sixth Report of IUR Working Group on Soil-to-Plant Transfer Factors, RIVM, Bilthoven, Netherlands.

(Fu78) Furr AK, TF Parkinson, CL Heffron, JT Reid, WM Haschek, WH Gutenmann, CA Backe, LE St. John, Jr., and DJ Lisk. 1979. "Elemental Content of Tissues and Excreta of Lambs, Goats and Kids Fed White Sweet Clover Growing on Fly Ash.” Journal of Agricultural and Food Chemistry 26(4):847-851.

(Ho91) Holzer F and K Wichterey. 1991. Ermittlung von Transferfaktoren fur den Ubergang naturlicher Radionuklide vom Boden in die Pflanzen im Uranerzbergbaugebiet. Jahresbericht des Bundesamtes fur Strahlenschutz, 146.

(HW91) [assume a typo of Ho91, above. No further information in PNNL-13421]

(IAEA82) IAEA - International Atomic Energy Agency. 1982. Generic Models and Parameters for Assessing the Environmental Transfer of Radionuclides from Routine Releases, Exposures of Critical Groups. Safety Series No. 57, IAEA, Vienna, Austria.

(IUR89) International Union of Radioecologists. 1989. Sixth Report of the Working Group on Soil-toPlant Transfer Factors. RIVM, Bilthoven, The Netherlands.

(Na88) Napier BA, RA Peloquin, DL Strenge, and JV Ramsdell. 1988. GENII - The Hanford Environmental Radiation Dosimetry Software System. PNL-6584, Pacific Northwest Laboratory, Richland, Washington. 
(NCRP86) National Council on Radiation Protection and Measurements. 1986. Screening Techniques for Determining Compliance with Environmental Standards, Releases of Radionuclides to the Atmosphere. NCRP Commentary No. 3, NCRP, Bethesda, Maryland.

(NCRP96) National Council on Ionizing Radiation and Protection. 1996. Screening Models for Releases of Radionuclides to Atmosphere, Surface Water and Ground. NCRP Report No. 123, Vol. I., NCRP, Bethesda, Maryland.

(Ng82b) Ng YC, CS Colsher, and SE Thompson. 1982b. Soil-to-Plant Concentration Factors for Radiological Assessments. NUREG/CR-2975, UCID19463, Lawrence Livermore National Laboratory, Livermore, California.

(Sn94) Snyder SF, WT Farris, BA Napier, TA Ikenberry, and RO Gilbert. 1994. Parameters Used in the Environmental Pathways and Radiological Dose Modules (DESCARTES, CIDER and CRD Codes) of the Hanford Environmental Dose Reconstruction Integrated Codes (HEDRIC). PNWD-2033 HEDR, Rev. 1, Pacific Northwest Laboratories, Richland, Washington.

\section{Coughtrey et al. (1985)}

Coughtrey, PJ, D Jackson, and MC Thorne. 1985. Radionuclide Distribution and Transport in Terrestrial and Aquatic Ecosystems, Volume Six, A.A. Balkema, Boston.

\section{$\mathrm{S} 2.1$}

S2.2

T2.1

$\mathrm{T} 2.3$

\section{Napier et al. 2012}

Napier BA, RJ Fellows, and LD Minc. 2012. DRAFT. Transfer Factors for Contaminant Uptake by Tree Fruits. Pacific Northwest National Laboratory, Richland, Washington

TRS_364 - International Atomic Energy Agency, International Union of Radioecologists. 1994. Handbook of Parameter Values for the Prediction of Radionuclide Transfer in Temperate Environments. Tech. Rep. Series No. 364, IAEA, Vienna, Austria

TRS_472 - IAEA. 2010. Handbook of Parameter Values for the Prediction of Radionuclide Transfer in Terrestrial and Freshwater Environments. Technical Report Series No. 472, International Atomic Energy Agency, Vienna, Austria

\section{TRS_472}

OV = specifically, an “other vegetable” value, but Gv2 combines other and root vegetables in modeling. RV = specifically, a "root vegetable” value, but Gv2 combines other and root vegetables in modeling. 
TRS_472 - IAEA. 2010. Handbook of Parameter Values for the Prediction of Radionuclide Transfer in Terrestrial and Freshwater Environments. Technical Report Series No. 472, International Atomic Energy Agency, Vienna, Austria

\section{Other References}

Al-Masri MS, B Al-Akel, A Nashawani, Y Amin, KH Khalifa, and F Al-Ain. 2008. "Transfer of ${ }^{40} \mathrm{~K}$, ${ }^{238} \mathrm{U},{ }^{210} \mathrm{~Pb}$, and ${ }^{210} \mathrm{Po}$ from Soil to Plant in Various Locations in South of Syria.” Journal of Environmental Radioactivity 99(2):322-331.

Al-Oudat M, AF Asfary, H Mukhalallti, A Al-Hamwi, and S. Kanakri. 2006. "Transfer Factors of ${ }^{137} \mathrm{Cs}$ and ${ }^{90}$ Sr from Soil to Trees in Arid Regions.” Journal of Environmental Radioactivity 90(1):78-88.

Coughtrey PJ, D Jackson, and MC Thorne. 1985. Radionuclide Distribution and Transport in Terrestrial and Aquatic Ecosystems. Volume Six, A.A. Balkema, Boston.

Kennedy WE, Jr. and DL Strenge. 1992. Residual Radioactive Contamination from Decommissioning: Technical Basis for Translating Contamination Levels to Annual Total Effective Dose Equivalent. NUREG/CR-5512 (PNL-7994), Pacific Northwest Laboratory, Richland, Washington.

Lauria DC, FCA Ribeiro, CC Conti, and FA Loureiro. 2009. "Radium and Uranium Levels in Vegetables Grown Using Different Farming Management Systems.” Journal of Environmental Radioactivity 100(2):176-183.

Napier BA, RJ Fellows, and LD Minc. 2012. DRAFT. Transfer Factors for Contaminant Uptake by Tree Fruits. Pacific Northwest National Laboratory, Richland, Washington.

Mollah AS, A Begum, and SM Ullah. 1998. "Determination of Soil-to-Plant Transfer Factors of ${ }^{137} \mathrm{Cs}$ and ${ }^{90} \mathrm{Sr}$ in the Tropical Environment of Bangladesh.” Radiation and Environmental Biophysics 37(2):125-128.

Sheppard SC, JM Long, and B Sanipelli. 2010a. "Measured Elemental Transfer Factors for Boreal Hunter/Gatherer Scenarios: Fish, Game and Berries.” Journal of Environmental Radioactivity 101(11):902-909.

Uchida S and K Tagami. 2011. "Iodine Transfer from Agricultural Soils to Edible Part of Crops.” Proceedings in Radiochemistry Acta 1:279-283.

Uchida S, K Tagami, ZR Shang, and YH Choi. 2009. "Uptake of Radionuclides and Stable Elements from Paddy Soil to Rice: A Review.” Journal of Environmental Radioactivity 100(9):739-745.

Vandenhove H, G Olyslaegers, N Sanzharova, O Shubina, E Reed, Z Shang, and H Velasco. 2009. "Proposal for New Best Estimates of the Soil-to-Plant Transfer Factor of U, Th, Ra, Pb and Po.” Journal of Environmental Radioactivity 100(9):721-732. 
Vandenhove H, M Van Hees, G Olyslaegers, and M Vidal. 2009. "Proposal for New Best Estimates for the Soil Solid-Liquid Distribution Coefficient and Soil-to-Plant Transfer of Nickel.” Journal of Environmental Radioactivity 100(4):342-347.

Velasco H, AS Cid, RM Anjos, CB Zamboni, M Rizzotto, DL Valladares, and J Juri Ayub. 2012. "Variability of ${ }^{137} \mathrm{Cs}$ and ${ }^{40} \mathrm{~K}$ Soil-to-Fruit Transfer Factor in Tropical Lemon Trees during the Fruit Development Period.” Journal of Environmental Radioactivity 104:64-70. 


\section{Appendix D}

\section{Food Categories}




\section{Appendix D}

\section{Food Categories}

An extensive list of crop groups is listed in 40 CFR 180, Tolerances and Exemptions for Pesticide Chemical Residues in Food, Subpart B - Procedural Regulations (180.41 Crop group tables). This list was used simply because of its broad coverage of various crop types in an established manner. By no means does its use imply that the Gv2 code is useable for environmental modeling of pesticides.

The crop listing tabulated here indicates the crop group, subgroups, and examples, then indicates the Gv2 food type that would best model the members of the group. In some cases, Gv2 models do not adequately model the crop group.

There are several crops intentionally not listed; they are shown at the end of the table. 
Table D.1. [caption]

\begin{tabular}{|c|c|c|c|}
\hline $\begin{array}{l}\text { Crop } \\
\text { Group- } \\
\text { Subgroup }\end{array}$ & Name & Examples Included in This Group & $\begin{array}{l}\text { Corresponding GENIIv2 Food or Feed } \\
\text { Category }\end{array}$ \\
\hline 1 & Root and tuber vegetables & & \\
\hline $1 \mathrm{~A}$ & Root vegetable subgroup & $\begin{array}{l}\text { Carrot, radish, sugar beet, garden beet, edible burdock, } \\
\text { ginseng, horseradish, parsnip, rutabaga, turnip. }\end{array}$ & Root/other vegetables \\
\hline $1 \mathrm{~B}$ & $\begin{array}{l}\text { Root vegetables (except sugar beet) } \\
\text { subgroup }\end{array}$ & See 1-1A, but exclude sugar beet. & Root/other vegetables \\
\hline $1 \mathrm{C}$ & Tuberous and corm vegetables subgroup & $\begin{array}{l}\text { Potato, Jerusalem artichoke, cassava, ginger, sweet } \\
\text { potato, yam. }\end{array}$ & Root/other vegetables \\
\hline $1 \mathrm{D}$ & $\begin{array}{l}\text { Tuberous and corm vegetables (except } \\
\text { potato) subgroup }\end{array}$ & See 1-1C, but exclude potato. & Root/other vegetables \\
\hline 2 & Leaves of Root and tuber vegetables (food or $\mathrm{f}$ & eed crops) & \\
\hline 2 & $<$ no subgroups $>$ & $\begin{array}{l}\text { Sugar or garden beet leaves, carrot leaves, turnip leaves, } \\
\text { radish leaves. }\end{array}$ & Leafy vegetables \\
\hline 3 & Bulb vegetables (Allium spp) & & \\
\hline $3-07 \mathrm{~A}$ & Onion, bulb, subgroup & Garlic bulb, onion bulb, pearl onion bulb, shallot bulb. & Root/other vegetables \\
\hline 3-07B & Onion, green, subgroup & Chive leaves, leek, green onions, shallot leaves. & $\begin{array}{l}\text { Leafy vegetables for air pathways; } \\
\text { root/other vegetables for water } \\
\text { pathways }\end{array}$ \\
\hline 4 & Leafy vegetables (except Brassica vegetables) & & \\
\hline $4 \mathrm{~A}$ & Leafy greens subgroup & $\begin{array}{l}\text { Head lettuce, leaf lettuce, spinach, garden cress, } \\
\text { dandelion, dock, endive, parsley, radicchio, New } \\
\text { Zealand spinach, vine spinach. }\end{array}$ & Leafy vegetables \\
\hline $4 \mathrm{~B}$ & Leafy petioles subgroup & Celery, rhubarb, Swiss chard. & Leafy vegetables \\
\hline 5 & Brassica (cole) leafy vegetables & & \\
\hline $5 \mathrm{~A}$ & Head and stem brassica group & $\begin{array}{l}\text { Broccoli, cauliflower, cabbage, Chinese mustard, } \\
\text { kohlrabi, Brussels sprouts. }\end{array}$ & Leafy vegetables \\
\hline $5 B$ & Leafy brassica greens subgroup & $\begin{array}{l}\text { Broccoli raab, bok choy, collards, kale, mustard greens, } \\
\text { rape greens. }\end{array}$ & Leafy vegetables \\
\hline
\end{tabular}


Table D.1. (contd)

\begin{tabular}{|c|c|c|c|}
\hline $\begin{array}{l}\text { Crop } \\
\text { Group- } \\
\text { Subgroup }\end{array}$ & Name & Examples Included in This Group & $\begin{array}{l}\text { Corresponding GENIIv2 Food or Feec } \\
\text { Category }\end{array}$ \\
\hline 6 & Legume vegetables (succulent or dried) & & \\
\hline $6 \mathrm{~A}$ & Edible-podded legume vegetables subgroup & $\begin{array}{l}\text { Snap bean, wax bean, yardlong bean, edible-pod pea, } \\
\text { snow pea, sugar-snap pea, immature seed of soybean. }\end{array}$ & Root/other vegetables \\
\hline $6 \mathrm{~B}$ & Succulent shelled pea and bean subgroup & $\begin{array}{l}\text { Lima bean, fresh broad bean, fresh black-eyed pea, } \\
\text { freah green pea. }\end{array}$ & Root/other vegetables \\
\hline $6 \mathrm{C}$ & $\begin{array}{l}\text { Dried shelled pea (except soybean) } \\
\text { subgroup }\end{array}$ & $\begin{array}{l}\text { Dried field bean, dried kidney bean, dried navy bean, } \\
\text { dried pinto bean, dried black-eyed pea, mung bean, } \\
\text { chickpea, lentil. }\end{array}$ & Root/other vegetables \\
\hline 7 & Foliage of legume vegetables & & \\
\hline $7 \mathrm{~A}$ & $\begin{array}{l}\text { Foliage of legume vegetables (except } \\
\text { soybeans) subgroup }\end{array}$ & $\begin{array}{l}\text { ANIMAL FEED ONLY - plant parts of any legume } \\
\text { vegetable (except soybeans) included in Group } 6 \text { that } \\
\text { will be used as animal feed. }\end{array}$ & $\begin{array}{l}\text { Meat animal forage, milk animal } \\
\text { forage, meat animal feed, milk animal } \\
\text { feed. }\end{array}$ \\
\hline 8 & Fruiting vegetables (except cucurbits) & & \\
\hline $8-10 \mathrm{~A}$ & Tomato subgroup & $\begin{array}{l}\text { Standard sized tomato, bush tomato, garden } \\
\text { huckleberry, sunberry, tomatillo, tree tomato. }\end{array}$ & Root/other vegetables \\
\hline $8-10 \mathrm{~B}$ & Pepper/eggplant subgroup & African eggplant, bell pepper, nonbell pepper. & Root/other vegetables \\
\hline $8-10 \mathrm{C}$ & Nonbell pepper/eggplant subgroup & Okra. & Root/other vegetables \\
\hline 9 & Cucurbit vegetables & & \\
\hline $9 \mathrm{~A}$ & Melon subgroup & $\begin{array}{l}\text { Cantaloupe, citron melon, muskmelon, watermelon, } \\
\text { honeydew melon. }\end{array}$ & Root/other vegetables \\
\hline 9B & Squash/cucumber subgroup & $\begin{array}{l}\text { Cucumber, gherkin, hubbard squash, zucchini, } \\
\text { pumpkin, summer squash, winter squash, chayote fruit. }\end{array}$ & Root/other vegetables \\
\hline 10 & Citrus fruits (citrus spp., Fortunella spp.) & & \\
\hline $10-10 \mathrm{~A}$ & Orange subgroup & $\begin{array}{l}\text { Sweet orange, sour orange, mandarin orange, tangerine, } \\
\text { tangelo. }\end{array}$ & Fruit \\
\hline $10-10 \mathrm{~B}$ & Lemon/lime subgroup & Lime, lemon, kumquat. & Fruit \\
\hline $10-10 \mathrm{C}$ & Grapefruit subgroup & Grapefruit, pummelo. & Fruit \\
\hline 11 & Pome fruits & & \\
\hline $11-10$ & Pome fruits & Apple, crabapple, pear, quince. & Fruit \\
\hline
\end{tabular}


Table D.1. (contd)

\begin{tabular}{|c|c|c|c|}
\hline $\begin{array}{l}\text { Crop } \\
\text { Group- } \\
\text { Subgroup }\end{array}$ & Name & Examples Included in This Group & $\begin{array}{l}\text { Corresponding GENIIv2 Food or Feed } \\
\text { Category }\end{array}$ \\
\hline 12 & Stone fruits & & \\
\hline 12 & $<$ no subgroups $>$ & $\begin{array}{l}\text { Apricot, sweet cherry, tart cherry, nectarine, peach, } \\
\text { plum, fresh prune. }\end{array}$ & Fruit \\
\hline 13 & Berries & & \\
\hline $13 \mathrm{~A}$ & Caneberry subgroup & Blackberry, loganberry, raspberry. & Root/other vegetables \\
\hline 13B & Bushberry subgroup & Blueberry, currant, elderberry, gooseberry, huckleberry. & Root/other vegetables \\
\hline $13-07$ & Berry and small fruits & & \\
\hline 13-07A & Caneberry subgroup & Blackberry, loganberry, raspberry. & Root/other vegetables \\
\hline 13-07B & Bushberry subgroup & $\begin{array}{l}\text { Blueberry, currant, elderberry, gooseberry, huckleberry, } \\
\text { edible honeysuckle, lignonberry. }\end{array}$ & Root/other vegetables \\
\hline 13-07C & Large shrub/tree berry subgroup & Bayberry, chokecherry, mulberry & Root/other vegetables OR Fruit \\
\hline 13-07D & Small fruit vine climbing subgroup & Grape, fuzzy kiwifruit. & Root/other vegetables OR Fruit \\
\hline 13-07E & $\begin{array}{l}\text { Small fruit vine climbing subgroup (except } \\
\text { grape) }\end{array}$ & See 13-07D, but exclude grape. & Root/other vegetables OR Fruit \\
\hline 13-07F & $\begin{array}{l}\text { Small fruit vine climbing subgroup (except } \\
\text { kiwifruit) }\end{array}$ & See 13-07D, but exclude kiwifruit. & Root/other vegetables OR Fruit \\
\hline 13-07G & Low growing berry subgroup & Strawberry, cranberry, lingonberry. & Root/other vegetables \\
\hline 13-07H & $\begin{array}{l}\text { Low growing berry subgroup (except } \\
\text { strawberry) }\end{array}$ & See 13-07G, but exclude strawberry. & Root/other vegetables \\
\hline 14 & Tree nuts & & \\
\hline 14 & $<$ no subgroups $>$ & $\begin{array}{l}\text { almond, beech nut, brazil nut, cashew, chestnut, } \\
\text { chinquapin, filber (hazelnut), hickory nut, macadamia } \\
\text { (bush) nut, pecan, walnut }\end{array}$ & $\begin{array}{l}<\text { not adequately addressed with } \\
\text { Gv2.10> }\end{array}$ \\
\hline 15 & Cereal grains & & \\
\hline 15 & $<$ no subgroups $>$ & $\begin{array}{l}\text { barley, buckwheat, corn, millet, oats, popcorn, rice, rye, } \\
\text { sorghum, triticale, wheat, wild rice. }\end{array}$ & $\begin{array}{l}\text { Grain, milk animal feed, meat animal } \\
\text { feed, poultry feed, egg feed. }\end{array}$ \\
\hline 16 & Forage, fodder, and straw of cereal grains & & \\
\hline 16 & $<$ no subgroups $>$ & $\begin{array}{l}\text { ANIMAL FEED ONLY - non-grain portion of } \\
\text { group } 15 .\end{array}$ & $\begin{array}{l}\text { Meat animal forage, Milk animal } \\
\text { forage, Meat animal feed, Milk animal } \\
\text { feed. }\end{array}$ \\
\hline
\end{tabular}


Table D.1. (contd)

\begin{tabular}{|c|c|c|c|}
\hline $\begin{array}{l}\text { Crop } \\
\text { Group- } \\
\text { Subgroup }\end{array}$ & Name & Examples Included in This Group & $\begin{array}{l}\text { Corresponding GENIIv2 Food or Feed } \\
\text { Category }\end{array}$ \\
\hline 17 & \multicolumn{3}{|l|}{ Grass forage, fodder, and hay } \\
\hline 18 & $<$ no subgroups $>$ & $\begin{array}{l}\text { ANIMAL FEED ONLY - alfalfa, clover, kudzu, trefoil, } \\
\text { vetch. }\end{array}$ & $\begin{array}{l}\text { Milk animal feed, milk animal forage, } \\
\text { meat animal feed, meat animal forage, } \\
\text { poultry feed, egg feed. }\end{array}$ \\
\hline 19 & \multicolumn{3}{|l|}{ Herbs and spices } \\
\hline 19A & Herb subgroup & $\begin{array}{l}\text { basil, camomile, catnip, chive, dillweed, lavender, } \\
\text { lemongrass, bay, rosemary, parsley, thyme, sage. }\end{array}$ & Leafy vegetables \\
\hline 19B & Spice subgroup & $\begin{array}{l}\text { Allspice, anise seed, caper buds, cumin, mustard seed, } \\
\text { nutmeg, saffron, vanilla. }\end{array}$ & $\begin{array}{l}\text { Leafy vegetables OR root/other } \\
\text { vegetables. }\end{array}$ \\
\hline 20B & Sunflower subgroup & $\begin{array}{l}\text { Castor oil plant, euphorbia, rose hip, safflower, } \\
\text { sunflower, tea oil plant. }\end{array}$ & $\begin{array}{l}<\text { not adequately addressed with } \\
\text { Gv2.10> }\end{array}$ \\
\hline 20C & Cottonseed subgroup & Cottonseed varieties. & $\begin{array}{l}<\text { not adequately addressed with } \\
\text { Gv2.10> }\end{array}$ \\
\hline 21 & \multicolumn{3}{|l|}{ Edible fungi } \\
\hline 21 & $<$ no subgroups $>$ & $\begin{array}{l}\text { White button mushroom, oyster mushroom, shiitake } \\
\text { mushroom, truffle, morel. }\end{array}$ & $\begin{array}{l}<\text { not adequately addressed with } \\
\text { Gv2.10> }\end{array}$ \\
\hline \multicolumn{4}{|c|}{ INTENTIONALLY NOT LISTED in 40 CFR 180} \\
\hline NA & NA & Asparagus, hops & Leafy vegetable \\
\hline NA & NA & Avocado, fig, mango, papaya, pawpaw, persimmon & Fruit \\
\hline NA & NA & Globe artichoke & Root/other vegetable \\
\hline
\end{tabular}


Table D.1. (contd)

\begin{tabular}{|c|c|c|c|}
\hline $\begin{array}{c}\text { Crop } \\
\text { Group- } \\
\text { Subgroup }\end{array}$ & Name & Examples Included in This Group & $\begin{array}{l}\text { Corresponding GENIIv2 Food or Feed } \\
\text { Category }\end{array}$ \\
\hline NA & NA & Banana & $\begin{array}{l}<\text { not adequately addressed with } \\
\text { Gv2.10> }\end{array}$ \\
\hline NA & NA & Peanut, pineapple & $\begin{array}{l}\text { Possibly root/other, but careful review } \\
\text { of modeling should be done. }\end{array}$ \\
\hline NA & NA & Water chestnut, watercress & aquatic plant \\
\hline
\end{tabular}


PNNL-21950, Appendix D

\section{References}

40 CFR Part 180, Subpart B - Procedural Regulations. Section 180.41 Crop Group Tables. 


\section{Appendix E}

\section{Rainfall Rate}




\section{Appendix E}

\section{Rainfall Rate}

The National Climatic Data Center (NCDC) provides access to climatological normals based on 30-years (1981-2010) of data for thousands of stations throughout the United States. ${ }^{1}$ Climatological values include normal annual precipitation amount and the normal number of days with precipitation greater than a trace (i.e., 0.01 inches or greater). The average daily rainfall rate can be estimated by dividing the normal precipitation amount by the normal number of precipitation days. Data are provided in this appendix for numerous locations throughout the United States and some of its territories. More locations are available at the NCDC website. The procedure to acquire the data is summarized in Section 7.1.2 (RAIN Rainfall Rate) of the main text.

\footnotetext{
${ }^{1}$ See http://www1.ncdc.noaa.gov/pub/data/normals/1981-2010/station-inventories/prcp-inventory.txt.
} 
PNNL-21950, Appendix E

Table E.1. Rainfall Rate

\begin{tabular}{|c|c|c|c|c|}
\hline Name ${ }^{(a)}$ & State & $\begin{array}{c}\text { Average } \\
\text { d/yr with } \\
\text { Precipitation } \\
>0.254 \mathrm{~mm}\end{array}$ & $\begin{array}{l}\text { Normal } \\
\text { Precipitation } \\
(\mathrm{mm} / \mathrm{yr})\end{array}$ & $\underset{(\mathrm{mm} / \mathrm{d})^{(\mathrm{b})}}{\mathrm{RAIN}}$ \\
\hline DILLINGHAM FAA AP & $\mathrm{AK}$ & 141 & 643.1 & 4.6 \\
\hline PUNTILLA & $\mathrm{AK}$ & 96 & 369.8 & 3.9 \\
\hline ANNETTE ISLAND AP & AK & 231 & 2581.4 & 11.2 \\
\hline JUNEAU INTL AP & $\mathrm{AK}$ & 230 & 1581.7 & 6.9 \\
\hline GUSTAVUS & AK & 224 & 1508 & 6.7 \\
\hline KETCHIKAN INTL AP & $\mathrm{AK}$ & 234 & 3587.8 & 15.3 \\
\hline PETERSBURG 1 & AK & 233 & 2774.4 & 11.9 \\
\hline PALMER MUNI AP & AK & 87 & 302 & 3.5 \\
\hline SITKA AIRPORT & $\mathrm{AK}$ & 236 & 2205 & 9.4 \\
\hline SKAGWAY AP & $\mathrm{AK}$ & 154 & 687.1 & 4.5 \\
\hline YAKUTAT STATE AP & $\mathrm{AK}$ & 240 & 3940 & 16.4 \\
\hline KODIAK AP & AK & 203 & 1981.2 & 9.8 \\
\hline KING SALMON & $\mathrm{AK}$ & 156 & 495 & 3.2 \\
\hline ILIAMNA AP & AK & 125 & 633.2 & 5.1 \\
\hline HOMER AP & $\mathrm{AK}$ & 151 & 618.2 & 4.1 \\
\hline COLD BAY AP & AK & 249 & 1058.4 & 4.2 \\
\hline ADAK & AK & 266 & 1389.4 & 5.2 \\
\hline ST PAUL ISLAND AP & $\mathrm{AK}$ & 203 & 601.2 & 3.0 \\
\hline ELMENDORF AFB & AK & 112 & 399.5 & 3.6 \\
\hline CORDOVA M K SMITH AP & $\mathrm{AK}$ & 220 & 2296.7 & 10.4 \\
\hline FAIRBANKS INTL AP & AK & 109 & 274.6 & 2.5 \\
\hline NORTHWAY AP & AK & 92 & 266.4 & 2.9 \\
\hline BIG DELTA AP & AK & 97 & 295.1 & 3.0 \\
\hline GULKANA AP & AK & 101 & 286 & 2.8 \\
\hline SEWARD AP & $\mathrm{AK}$ & 172 & 1862.3 & 10.9 \\
\hline VALDEZ WSO & AK & 200 & 1753.4 & 8.8 \\
\hline ANCHORAGE INTL AP & $\mathrm{AK}$ & 115 & 421.1 & 3.7 \\
\hline UMIAT & AK & 40 & 122.2 & 3.1 \\
\hline MCGRATH AP & $\mathrm{AK}$ & 142 & 457.2 & 3.2 \\
\hline MINCHUMINA & AK & 99 & 353.3 & 3.6 \\
\hline KENAI MUNI AP & AK & 124 & 462.5 & 3.7 \\
\hline TALKEETNA AP & $\mathrm{AK}$ & 143 & 710.4 & 5.0 \\
\hline TANANA CALHOUN MEM AP & AK & 101 & 294.4 & 2.9 \\
\hline BETTLES AP & $\mathrm{AK}$ & 125 & 378.5 & 3.0 \\
\hline BETHEL AP & AK & 146 & 470.9 & 3.2 \\
\hline KOTZEBUE RALPH WEIN AP & $\mathrm{AK}$ & 113 & 279.4 & 2.5 \\
\hline NOME MUNI AP & AK & 133 & 427 & 3.2 \\
\hline WALES & AK & 90 & 303.8 & 3.4 \\
\hline BARROW POST ROGERS AP & AK & 88 & 115.1 & 1.3 \\
\hline SHEMYA USAF BASE & AK & 239 & 809.5 & 3.4 \\
\hline HUNTSVILLE INTL AP & $\mathrm{AL}$ & 117 & 1380.2 & 11.8 \\
\hline CENTREVILLE 6 SW & $\mathrm{AL}$ & 113 & 1453.6 & 12.9 \\
\hline BIRMINGHAM AP & $\mathrm{AL}$ & 117 & 1364.5 & 11.7 \\
\hline
\end{tabular}


PNNL-21950, Appendix E

Table E.1. (contd)

\begin{tabular}{|c|c|c|c|c|}
\hline Name $^{(a)}$ & State & $\begin{array}{c}\text { Average } \\
\mathrm{d} / \mathrm{yr} \text { with } \\
\text { Precipitation } \\
>0.254 \mathrm{~mm}\end{array}$ & $\begin{array}{l}\text { Normal } \\
\text { Precipitation } \\
(\mathrm{mm} / \mathrm{yr})\end{array}$ & $\underset{(\mathrm{mm} / \mathrm{d})^{(\mathrm{b})}}{\mathrm{RAIN}}$ \\
\hline MOBILE & AL & 115 & 1680.2 & 14.6 \\
\hline MONTGOMERY AP & $\mathrm{AL}$ & 104 & 1348 & 12.9 \\
\hline ALABASTER SHELBY CO AP & $\mathrm{AL}$ & 124 & 1388.9 & 11.2 \\
\hline LITTLE ROCK & $\mathrm{AR}$ & 107 & 1270.8 & 11.8 \\
\hline FT SMITH RGNL AP & AR & 98 & 1154.7 & 11.8 \\
\hline HARRISON BOONE CO AP & AR & 116 & 1121.2 & 9.7 \\
\hline PAGE & $\mathrm{AZ}$ & 48 & 173.5 & 3.6 \\
\hline FLAGSTAFF PULLIAM AP & $\mathrm{AZ}$ & 88 & 555.2 & 6.3 \\
\hline TUCSON INTL AP & $\mathrm{AZ}$ & 53 & 294.4 & 5.6 \\
\hline PHOENIX SKY HARBOR INTL AP & $\mathrm{AZ}$ & 37 & 204 & 5.6 \\
\hline WINSLOW MUNI AP & $\mathrm{AZ}$ & 53 & 178.1 & 3.4 \\
\hline YUMA WSO AP & $\mathrm{AZ}$ & 18 & 83.8 & 4.7 \\
\hline DOUGLAS BISBEE INL AP & AZ & 64 & 332.7 & 5.2 \\
\hline LEMOORE REEVES NAS & CA & 46 & 200.9 & 4.4 \\
\hline LONG BEACH DAUGHERTY FLD & CA & 35 & 311.4 & 8.8 \\
\hline BURBANK GLENDALE PASADENA AP & CA & 37 & 439.7 & 12.0 \\
\hline BAKERSFIELD AP & CA & 39 & 164.3 & 4.2 \\
\hline BISHOP AP & CA & 28 & 131.6 & 4.8 \\
\hline LOS ANGELES INTL AP & CA & 36 & 325.6 & 9.1 \\
\hline SANDBERG & CA & 38 & 313.2 & 8.3 \\
\hline SAN DIEGO LINDBERGH FLD & CA & 42 & 262.6 & 6.3 \\
\hline SANTA BARBARA MUNI AP & CA & 37 & 451.1 & 12.1 \\
\hline SACRAMENTO EXECUTIVE AP & CA & 60 & 470.4 & 7.8 \\
\hline SALINAS MUNICIPAL AP & CA & 57 & 325.9 & 5.8 \\
\hline SAN FRANCISCO INTL AP & CA & 68 & 524.5 & 7.7 \\
\hline STOCKTON METRO AP & CA & 57 & 357.1 & 6.3 \\
\hline ALAMEDA NAS & CA & 66 & 527.8 & 7.9 \\
\hline MOFFETT FEDERAL AIRFIELD & CA & 64 & 372.9 & 5.8 \\
\hline CONCORD BUCHANAN FLD & CA & 59 & 418.1 & 7.1 \\
\hline SANTA MARIA PUBLIC AP & CA & 48 & 354.3 & 7.4 \\
\hline EUREKA WFO WOODLEY ISLAND & CA & 128 & 1024.4 & 8.0 \\
\hline RED BLUFF MUNI AP & CA & 73 & 622 & 8.5 \\
\hline REDDING MUNI AP & CA & 82 & 879.3 & 10.7 \\
\hline EL TORO MCAS & CA & 43 & 365.3 & 8.6 \\
\hline SAN DIEGO MIRAMAR NAS & CA & 43 & 283.5 & 6.5 \\
\hline POINT MUGU NF & CA & 35 & 324.9 & 9.3 \\
\hline TUSTIN MCAF & CA & 38 & 304.5 & 8.0 \\
\hline FRESNO YOSEMITE INTL AP & CA & 48 & 292.1 & 6.1 \\
\hline HAYWARD AIR TERMINAL & CA & 65 & 458.2 & 7.1 \\
\hline DENVER INTL AP & $\mathrm{CO}$ & 80 & 379 & 4.8 \\
\hline ALAMOSA SAN LUIS AP & $\mathrm{CO}$ & 67 & 185.7 & 2.8 \\
\hline DENVER-STAPLETON & $\mathrm{CO}$ & 87 & 395.7 & 4.6 \\
\hline GRAND JUNCTION WALKER FLD & $\mathrm{CO}$ & 74 & 239.3 & 3.2 \\
\hline LIMON WSMO & $\mathrm{CO}$ & 83 & 395.2 & 4.7 \\
\hline
\end{tabular}


PNNL-21950, Appendix E

Table E.1. (contd)

\begin{tabular}{|c|c|c|c|c|}
\hline Name $^{(a)}$ & State & $\begin{array}{c}\text { Average } \\
\mathrm{d} / \mathrm{yr} \text { with } \\
\text { Precipitation } \\
>0.254 \mathrm{~mm} \\
\end{array}$ & $\begin{array}{c}\text { Normal } \\
\text { Precipitation } \\
\text { (mm/yr) }\end{array}$ & $\begin{array}{c}\text { RAIN } \\
(\mathrm{mm} / \mathrm{d})^{(\mathrm{b})}\end{array}$ \\
\hline COLORADO SPRINGS MUNI AP & $\mathrm{CO}$ & 92 & 420.1 & 4.6 \\
\hline PUEBLO MEM AP & $\mathrm{CO}$ & 72 & 319.3 & 4.4 \\
\hline HARTFORD BRADLEY INTL AP & CT & 130 & 1164.6 & 9.0 \\
\hline BRIDGEPORT SIKORSKY MEM AP & $\mathrm{CT}$ & 122 & 1085.6 & 8.9 \\
\hline WILMINGTON NEW CASTLE CO AP & $\mathrm{DE}$ & 118 & 1094.2 & 9.3 \\
\hline ORLANDO INTL AP & FL & 117 & 1288.5 & 11.0 \\
\hline GAINESVILLE RGNL AP & FL & 114 & 1204.2 & 10.5 \\
\hline APALACHICOLA AP & FL & 93 & 1465.6 & 15.8 \\
\hline DAYTONA BEACH INTL AP & FL & 115 & 1260.3 & 11.0 \\
\hline KEY WEST INTL AP & FL & 106 & 1011.7 & 9.5 \\
\hline MELBOURNE INTL AP & FL & 116 & 1320.8 & 11.4 \\
\hline MIAMI INTL AP & FL & 135 & 1572.3 & 11.6 \\
\hline TAMPA INTL AP & FL & 105 & 1176 & 11.2 \\
\hline VERO BEACH INTL AP & FL & 123 & 1317.5 & 10.7 \\
\hline WEST PALM BEACH INTL AP & FL & 136 & 1583.2 & 11.6 \\
\hline FT LAUDERDALE HOLLYWOOD AP & FL & 122 & 1579.4 & 12.9 \\
\hline JACKSONVILLE & FL & 114 & 1330.7 & 11.7 \\
\hline PENSACOLA RGNL AP & FL & 109 & 1657.9 & 15.3 \\
\hline TALLAHASSEE & FL & 111 & 1504.4 & 13.5 \\
\hline FT STEWART & GA & 99 & 1259.1 & 12.7 \\
\hline MACON MIDDLE GA RGNL AP & GA & 106 & 1160.3 & 11.0 \\
\hline AUGUSTA BUSH FLD AP & GA & 105 & 1106.7 & 10.6 \\
\hline SAVANNAH INTL AP & GA & 108 & 1218.2 & 11.3 \\
\hline WAYCROSS WSMO & GA & 108 & 1188.5 & 11.0 \\
\hline ALMA BACON CO AP & GA & 106 & 1184.9 & 11.1 \\
\hline ATHENS BEN EPPS AP & GA & 110 & 1176.8 & 10.7 \\
\hline ATLANTA HARTSFIELD INTL AP & GA & 113 & 1262.6 & 11.1 \\
\hline GUAM INTL AP & GU & 272 & 2480.1 & 9.1 \\
\hline HAINA 214 & HI & 171 & 1854.2 & 10.9 \\
\hline KULA BRANCH STN 324.5 & $\mathrm{HI}$ & 77 & 590.6 & 7.6 \\
\hline UPOLU POINT USCG 159.2 & $\mathrm{HI}$ & 180 & 835.7 & 4.6 \\
\hline WAIMANALO EXP F 795.1 & $\mathrm{HI}$ & 184 & 1099.1 & 6.0 \\
\hline HILO INTL AP & HI & 272 & 3218.7 & 11.8 \\
\hline BARKING SANDS & $\mathrm{HI}$ & 67 & 500.1 & 7.5 \\
\hline BARBERS POINT NAS & $\mathrm{HI}$ & 69 & 406.9 & 5.9 \\
\hline KAHULUI AP & $\mathrm{HI}$ & 95 & 452.9 & 4.8 \\
\hline KANEOHE BAY MCAS & $\mathrm{HI}$ & 176 & 815.1 & 4.6 \\
\hline HONOLULU INTL AP & $\mathrm{HI}$ & 89 & 434.3 & 4.9 \\
\hline MOLOKAI AP & HI & 99 & 626.9 & 6.4 \\
\hline LIHUE WSO AP 1020.1 & $\mathrm{HI}$ & 195 & 941.1 & 4.8 \\
\hline SPENCER $1 \mathrm{~N}$ & IA & 102 & 749.6 & 7.3 \\
\hline BURLINGTON MUNI AP & IA & 114 & 977.4 & 8.6 \\
\hline DES MOINES INTL AP & IA & 113 & 914.7 & 8.1 \\
\hline SIOUX CITY GATEWAY AP & IA & 101 & 704.6 & 7.0 \\
\hline
\end{tabular}


PNNL-21950, Appendix E

Table E.1. (contd)

\begin{tabular}{|c|c|c|c|c|}
\hline Name $^{(a)}$ & State & $\begin{array}{c}\text { Average } \\
\mathrm{d} / \mathrm{yr} \text { with } \\
\text { Precipitation } \\
>0.254 \mathrm{~mm} \\
\end{array}$ & $\begin{array}{c}\text { Normal } \\
\text { Precipitation } \\
\text { (mm/yr) }\end{array}$ & $\begin{array}{c}\text { RAIN } \\
(\mathrm{mm} / \mathrm{d})^{(\mathrm{b})}\end{array}$ \\
\hline CEDAR RAPIDS MUNI AP & IA & 111 & 879.1 & 7.9 \\
\hline DUBUQUE RGNL AP & IA & 120 & 921.8 & 7.7 \\
\hline WATERLOO MUNI AP & IA & 112 & 877.8 & 7.8 \\
\hline BOISE AIR TERMINAL & ID & 88 & 297.9 & 3.4 \\
\hline LEWISTON NEZ PERCE CO AP & ID & 103 & 312.7 & 3.0 \\
\hline POCATELLO RGNL AP & ID & 97 & 308.1 & 3.2 \\
\hline SALEM & IL & 110 & 1114.6 & 10.2 \\
\hline CHICAGO MIDWAY AP & IL & 123 & 992.9 & 8.1 \\
\hline PEORIA GTR PEORIA AP & IL & 117 & 926.8 & 8.0 \\
\hline MOLINE QUAD CITY INTL AP & IL & 117 & 964.2 & 8.2 \\
\hline SPRINGFIELD CAPITAL AP & $\mathrm{IL}$ & 113 & 950.7 & 8.4 \\
\hline QUINCY RGNL AP & $\mathrm{IL}$ & 111 & 948.2 & 8.5 \\
\hline ROCKFORD GTR ROCKFORD AP & IL & 119 & 920.5 & 7.7 \\
\hline CHICAGO OHARE INTL AP & $\mathrm{IL}$ & 124 & 937 & 7.6 \\
\hline FT WAYNE INTL AP & IN & 134 & 973.8 & 7.3 \\
\hline SOUTH BEND MICHIANA RGNL AP & IN & 145 & 965.2 & 6.6 \\
\hline EVANSVILLE REGIONAL AP & IN & 116 & 1150.9 & 10.0 \\
\hline INDIANAPOLIS & IN & 129 & 1078 & 8.3 \\
\hline WICHITA & KS & 88 & 829.1 & 9.4 \\
\hline MEDICINE LODGE & KS & 80 & 689.4 & 8.7 \\
\hline CONCORDIA MUNI AP & KS & 90 & 708.4 & 7.8 \\
\hline DODGE CITY & KS & 77 & 548.6 & 7.1 \\
\hline TOPEKA MUNI AP & KS & 100 & 926.1 & 9.3 \\
\hline GOODLAND & KS & 81 & 499.4 & 6.2 \\
\hline PADUCAH & KY & 110 & 1246.6 & 11.3 \\
\hline LONDON CORBIN AP & KY & 134 & 1197.4 & 8.9 \\
\hline JACKSON & KY & 144 & 1227.8 & 8.5 \\
\hline CINCINNATI NORTHERN KY AP & KY & 132 & 1080 & 8.2 \\
\hline LEXINGTON BLUEGRASS AP & KY & 130 & 1147.3 & 8.8 \\
\hline LOUISVILLE INTL AP & KY & 123 & 1140.7 & 9.3 \\
\hline SLIDELL AP & LA & 116 & 1555.8 & 13.4 \\
\hline LAKE CHARLES & LA & 109 & 1460.2 & 13.5 \\
\hline BOOTHVILLE ASOS & LA & 121 & 1508.3 & 12.5 \\
\hline NEW ORLEANS INTL AP & LA & 115 & 1591.6 & 13.9 \\
\hline SHREVEPORT & LA & 101 & 1305.8 & 12.9 \\
\hline СНАТНАМ & MA & 125 & 1194.8 & 9.5 \\
\hline BOSTON LOGAN INTL AP & MA & 126 & 1111.8 & 8.8 \\
\hline BLUE HILL & MA & 136 & 1356.9 & 10.0 \\
\hline WORCESTER RGNL AP & MA & 139 & 1221 & 8.8 \\
\hline PATUXENT RIVER NAS & MD & 116 & 1184.9 & 10.3 \\
\hline SALISBURY WICOMICO RGNL AP & MD & 117 & 1160.3 & 9.9 \\
\hline BALTIMORE WASH INTL AP & MD & 116 & 1063.8 & 9.2 \\
\hline EASTPORT & ME & 141 & 1163.8 & 8.3 \\
\hline GRAY & ME & 139 & 1275.6 & 9.2 \\
\hline
\end{tabular}


PNNL-21950, Appendix E

Table E.1. (contd)

\begin{tabular}{|c|c|c|c|c|}
\hline Name $^{(a)}$ & State & $\begin{array}{c}\text { Average } \\
\mathrm{d} / \mathrm{yr} \text { with } \\
\text { Precipitation } \\
>0.254 \mathrm{~mm} \\
\end{array}$ & $\begin{array}{c}\text { Normal } \\
\text { Precipitation } \\
\text { (mm/yr) }\end{array}$ & $\begin{array}{c}\text { RAIN } \\
(\mathrm{mm} / \mathrm{d})^{(\mathrm{b})}\end{array}$ \\
\hline BANGOR INTL AP & $\mathrm{ME}$ & 133 & 1065 & 8.0 \\
\hline CARIBOU MUNI AP & ME & 160 & 977.6 & 6.1 \\
\hline BRUNSWICK NAS & ME & 131 & 1297.9 & 9.9 \\
\hline PORTLAND INTL JETPORT & $\mathrm{ME}$ & 131 & 1200.2 & 9.2 \\
\hline GAYLORD 9SSW & MI & 172 & 923.8 & 5.4 \\
\hline WHITEFISH POINT & MI & 175 & 819.2 & 4.7 \\
\hline ESCANABA & MI & 118 & 728.2 & 6.2 \\
\hline FLINT BISHOP INTL AP & MI & 136 & 796.8 & 5.9 \\
\hline LANSING CAPITAL CITY AP & MI & 137 & 807 & 5.9 \\
\hline MARQUETTE & MI & 150 & 739.9 & 4.9 \\
\hline MUSKEGON CO AP & MI & 141 & 850.6 & 6.0 \\
\hline SAULT STE MARIE SANDERSON FLD & MI & 163 & 836.9 & 5.1 \\
\hline HANCOCK HOUGHTON CO AP & MI & 152 & 705.9 & 4.6 \\
\hline HOUGHTON LK ROSCOMMON AP & MI & 139 & 730.3 & 5.3 \\
\hline DETROIT METRO AP & MI & 135 & 850.1 & 6.3 \\
\hline ALPENA CO RGNL AP & MI & 146 & 713.7 & 4.9 \\
\hline MARQUETTE & MI & 164 & 906.3 & 5.5 \\
\hline GRAND RAPIDS & MI & 145 & 972.1 & 6.7 \\
\hline DULUTH & MN & 131 & 786.4 & 6.0 \\
\hline INTL FALLS INTL AP & $\mathrm{MN}$ & 128 & 615.2 & 4.8 \\
\hline MINNEAPOLIS/ST PAUL AP & MN & 117 & 777.5 & 6.7 \\
\hline ROCHESTER INTL AP & $\mathrm{MN}$ & 121 & 838.7 & 7.0 \\
\hline ST CLOUD RGNL AP & MN & 109 & 704.3 & 6.5 \\
\hline MONETT 4SW & MO & 100 & 1164.6 & 11.6 \\
\hline SPICKARD $7 \mathrm{~W}$ & MO & 103 & 988.3 & 9.6 \\
\hline WEST PLAINS & MO & 102 & 1199.4 & 11.8 \\
\hline CAPE GIRARDEAU MUNI AP & MO & 114 & 1189 & 10.5 \\
\hline COLUMBIA RGNL AP & MO & 112 & 1082.5 & 9.6 \\
\hline KANSAS CITY INTL AP & MO & 105 & 987 & 9.4 \\
\hline ST JOSEPH ROSECRANS AP & MO & 108 & 904.2 & 8.3 \\
\hline ST LOUIS LAMBERT INTL AP & MO & 113 & 1040.4 & 9.2 \\
\hline SPRINGFIELD & MO & 111 & 1157.5 & 10.4 \\
\hline VICHY ROLLA NATIONAL AP & MO & 111 & 1107.7 & 9.9 \\
\hline JACKSON INTL AP & MS & 109 & 1375.2 & 12.7 \\
\hline MERIDIAN KEY FLD & MS & 111 & 1426.5 & 12.8 \\
\hline GREENWOOD LEFLORE AP & MS & 102 & 1315 & 12.9 \\
\hline TUPELO RGNL AP & MS & 110 & 1397.3 & 12.7 \\
\hline KALISPELL GLACIER AP & MT & 132 & 431.5 & 3.3 \\
\hline BILLINGS LOGAN INTL AP & MT & 97 & 347 & 3.6 \\
\hline MILES CITY F WILEY FLD & MT & 84 & 316 & 3.8 \\
\hline BUTTE BERT MOONEY AP & MT & 108 & 324.4 & 3.0 \\
\hline CUT BANK MUNI AP & MT & 78 & 275.6 & 3.5 \\
\hline DILLON AP & MT & 80 & 266.7 & 3.4 \\
\hline GREAT FALLS INTL AP & MT & 100 & 374.7 & 3.8 \\
\hline
\end{tabular}


PNNL-21950, Appendix E

Table E.1. (contd)

\begin{tabular}{|c|c|c|c|c|}
\hline Name $^{(a)}$ & State & $\begin{array}{c}\text { Average } \\
\mathrm{d} / \mathrm{yr} \text { with } \\
\text { Precipitation } \\
>0.254 \mathrm{~mm}\end{array}$ & $\begin{array}{l}\text { Normal } \\
\text { Precipitation } \\
(\mathrm{mm} / \mathrm{yr})\end{array}$ & $\underset{(\mathrm{mm} / \mathrm{d})^{(\mathrm{b})}}{\mathrm{RAIN}}$ \\
\hline HELENA RGNL AP & MT & 92 & 285 & 3.1 \\
\hline MISSOULA INTL AP & MT & 123 & 358.9 & 2.9 \\
\hline GLASGOW & MT & 91 & 296.2 & 3.3 \\
\hline HAVRE CITY CO AP & MT & 87 & 284.5 & 3.3 \\
\hline HICKORY FAA AP & NC & 113 & 1175 & 10.4 \\
\hline ASHEVILLE RGNL AP & NC & 128 & 1157.5 & 9.0 \\
\hline POPE AFB & NC & 113 & 1158 & 10.3 \\
\hline RALEIGH DURHAM INTL AP & NC & 114 & 1100.8 & 9.6 \\
\hline PIEDMONT TRIAD INTL AP & NC & 111 & 1072.6 & 9.7 \\
\hline WILMINGTON INTL AP & NC & 135 & 1463.3 & 10.9 \\
\hline CHERRY POINT MCAS & NC & 121 & 1425.7 & 11.8 \\
\hline ELIZABETH CITY CGAS & NC & 125 & 1183.1 & 9.5 \\
\hline CHARLOTTE DOUGLAS AP & NC & 110 & 1057.4 & 9.6 \\
\hline CAPE HATTERAS AP & NC & 120 & 1474.2 & 12.2 \\
\hline FARGO HECTOR INTL AP & ND & 106 & 573.5 & 5.4 \\
\hline GRAND FORKS INTL AP & ND & 103 & 528.6 & 5.1 \\
\hline BISMARCK & ND & 98 & 453.4 & 4.6 \\
\hline THEODORE ROOSEVELT AP & ND & 87 & 400.6 & 4.6 \\
\hline WILLISTON SLOULIN INTL AP & ND & 97 & 365 & 3.8 \\
\hline GRAND ISLAND AP & NE & 89 & 677.2 & 7.6 \\
\hline LINCOLN MUNI AP & $\mathrm{NE}$ & 95 & 735.3 & 7.7 \\
\hline NORFOLK KARL STEFAN AP & $\mathrm{NE}$ & 96 & 696.2 & 7.2 \\
\hline OMAHA EPPLEY AIRFIELD & $\mathrm{NE}$ & 101 & 777.7 & 7.7 \\
\hline NORTH PLATTE RGNL AP & $\mathrm{NE}$ & 87 & 513.8 & 5.9 \\
\hline SCOTTSBLUFF HEILIG AP & NE & 87 & 401.1 & 4.6 \\
\hline SIDNEY MUNI AP & $\mathrm{NE}$ & 92 & 470.9 & 5.1 \\
\hline VALENTINE MILLER FLD & $\mathrm{NE}$ & 88 & 508.5 & 5.8 \\
\hline OMAHA \#1 & $\mathrm{NE}$ & 98 & 792.7 & 8.1 \\
\hline CONCORD MUNI AP & $\mathrm{NH}$ & 131 & 1031.5 & 7.9 \\
\hline MT WASHINGTON & NH & 210 & 2460.5 & 11.7 \\
\hline NEWARK INTL AP & NJ & 122 & 1174.8 & 9.6 \\
\hline ATLANTIC CITY INTL AP & NJ & 115 & 1060.5 & 9.2 \\
\hline ROSWELL IND AIR PK & NM & 55 & 327.9 & 6.0 \\
\hline ALBUQUERQUE INTL AP & NM & 61 & 240 & 3.9 \\
\hline CLAYTON MUNI AIR PK & NM & 68 & 401.1 & 5.9 \\
\hline MERCURY DESERT ROCK AP & NV & 32 & 150.6 & 4.7 \\
\hline EUREKA AIRPORT & NV & 80 & 228.6 & 2.8 \\
\hline ELY YELLAND FLD AP & NV & 75 & 247.9 & 3.3 \\
\hline LAS VEGAS MCCARRAN AP & NV & 27 & 106.4 & 4.0 \\
\hline RENO TAHOE INTL AP & NV & 52 & 188 & 3.6 \\
\hline WINNEMUCCA MUNI AP & $\mathrm{NV}$ & 71 & 210.3 & 3.0 \\
\hline BINGHAMTON & NY & 159 & 998.2 & 6.3 \\
\hline ISLIP LI MACARTHUR AP & NY & 119 & 1174.5 & 9.9 \\
\hline NEW YORK LAGUARDIA AP & NY & 118 & 1136.1 & 9.6 \\
\hline
\end{tabular}


PNNL-21950, Appendix E

Table E.1. (contd)

\begin{tabular}{|c|c|c|c|c|}
\hline Name $^{(a)}$ & State & $\begin{array}{c}\text { Average } \\
\text { d/yr with } \\
\text { Precipitation } \\
>0.254 \mathrm{~mm} \\
\end{array}$ & $\begin{array}{c}\text { Normal } \\
\text { Precipitation } \\
\text { (mm/yr) }\end{array}$ & $\begin{array}{c}\text { RAIN } \\
(\mathrm{mm} / \mathrm{d})^{(\mathrm{b})}\end{array}$ \\
\hline BUFFALO & NY & 167 & 1028.2 & 6.2 \\
\hline ALBANY AP & NY & 138 & 999.5 & 7.3 \\
\hline GLENS FALLS AP & NY & 132 & 992.1 & 7.5 \\
\hline ROCHESTER GTR INTL AP & NY & 167 & 870.5 & 5.2 \\
\hline SYRACUSE HANCOCK INTL AP & NY & 174 & 977.1 & 5.6 \\
\hline NEW YORK CNTRL PK TWR & NY & 122 & 1268.5 & 10.4 \\
\hline NEW YORK JFK INTL AP & NY & 119 & 1086.4 & 9.1 \\
\hline CLEVELAND & $\mathrm{OH}$ & 155 & 994.2 & 6.4 \\
\hline COLUMBUS PORT COLUMBUS INTL AP & $\mathrm{OH}$ & 139 & 998.5 & 7.2 \\
\hline YOUNGSTOWN RGNL AP & $\mathrm{OH}$ & 160 & 988.3 & 6.2 \\
\hline MANSFIELD LAHM MUNI AP & $\mathrm{OH}$ & 150 & 1122.4 & 7.5 \\
\hline AKRON CANTON RGNL AP & $\mathrm{OH}$ & 158 & 1005.8 & 6.4 \\
\hline DAYTON INTL AP & $\mathrm{OH}$ & 136 & 1042.9 & 7.7 \\
\hline TOLEDO EXPRESS AP & $\mathrm{OH}$ & 132 & 869.7 & 6.6 \\
\hline OKLAHOMA CITY WILL ROGERS AP & $\mathrm{OK}$ & 84 & 927.6 & 11.0 \\
\hline TULSA INTL AP & OK & 93 & 1039.6 & 11.2 \\
\hline PONCA CITY MUNI AP & OK & 84 & 884.9 & 10.5 \\
\hline GAGE AP & OK & 66 & 558 & 8.4 \\
\hline PENDLETON & OR & 98 & 321.6 & 3.3 \\
\hline EUGENE MAHLON SWEET AP & OR & 144 & 1170.9 & 8.2 \\
\hline MEDFORD ROGUE VLY AP & OR & 103 & 466.1 & 4.5 \\
\hline PORTLAND INTL AP & OR & 155 & 915.2 & 5.9 \\
\hline REDMOND ROBERTS FLD & OR & 80 & 225.6 & 2.8 \\
\hline SALEM MCNARY FLD & OR & 144 & 1007.6 & 7.0 \\
\hline BURNS MUNI AP & OR & 94 & 277.4 & 3.0 \\
\hline ASTORIA RGNL AP & OR & 191 & 1708.4 & 8.9 \\
\hline PHILADELPHIA INTL AP & PA & 118 & 1054.9 & 8.9 \\
\hline MIDDLETOWN HARRISBURG INTL AP & PA & 122 & 1034.8 & 8.5 \\
\hline ALLENTOWN INTL AP & PA & 127 & 1151.9 & 9.1 \\
\hline HARRISBURG CPTL CY AP & PA & 127 & 1039.4 & 8.2 \\
\hline WILKES-BARRE INTL AP & PA & 139 & 971.8 & 7.0 \\
\hline WILLIAMSPORT & $\mathrm{PA}$ & 133 & 1048.5 & 7.9 \\
\hline ERIE INTL AP & PA & 166 & 1070.9 & 6.4 \\
\hline PITTSBURGH INTL AP & PA & 151 & 970 & 6.4 \\
\hline SAN JUAN L M MARIN AP & PR & 199 & 1431.3 & 7.2 \\
\hline PROVIDENCE T F GREEN AP & RI & 125 & 1198.4 & 9.6 \\
\hline GREER & SC & 114 & 1198.6 & 10.5 \\
\hline CHARLESTON INTL AP & SC & 113 & 1296.2 & 11.5 \\
\hline COLUMBIA & SC & 107 & 1132.6 & 10.6 \\
\hline ANDERSON CO AP & SC & 111 & 1122.9 & 10.1 \\
\hline PICKSTOWN & SD & 85 & 613.7 & 7.2 \\
\hline ABERDEEN & SD & 94 & 551.7 & 5.8 \\
\hline HURON RGNL AP & SD & 93 & 581.7 & 6.3 \\
\hline SIOUX FALLS & SD & 105 & 670.1 & 6.4 \\
\hline
\end{tabular}


PNNL-21950, Appendix E

Table E.1. (contd)

\begin{tabular}{|c|c|c|c|c|}
\hline Name $^{(a)}$ & State & $\begin{array}{c}\text { Average } \\
\text { d/yr with } \\
\text { Precipitation } \\
>0.254 \mathrm{~mm}\end{array}$ & $\begin{array}{l}\text { Normal } \\
\text { Precipitation } \\
(\mathrm{mm} / \mathrm{yr})\end{array}$ & $\underset{(\mathrm{mm} / \mathrm{d})^{(\mathrm{b})}}{\mathrm{RAIN}}$ \\
\hline PIERRE RGNL AP & SD & 88 & 506.2 & 5.8 \\
\hline RAPID CITY RGNL AP & SD & 94 & 413.8 & 4.4 \\
\hline CROSSVILLE MEM AP & $\mathrm{TN}$ & 137 & 1399.3 & 10.2 \\
\hline BRISTOL TRI CITY AP & $\mathrm{TN}$ & 134 & 1041.7 & 7.8 \\
\hline CHATTANOOGA LOVELL AP & $\mathrm{TN}$ & 120 & 1333 & 11.1 \\
\hline KNOXVILLE MCGHEE TYSON AP & $\mathrm{TN}$ & 125 & 1215.6 & 9.7 \\
\hline MEMPHIS INTL AP & $\mathrm{TN}$ & 108 & 1363.5 & 12.7 \\
\hline NASHVILLE INTL AP & $\mathrm{TN}$ & 119 & 1200.2 & 10.1 \\
\hline OAK RIDGE ASOS & $\mathrm{TN}$ & 125 & 1293.1 & 10.4 \\
\hline FT WORTH WSFO & $\mathrm{TX}$ & 81 & 960.1 & 11.9 \\
\hline COLLEGE STN & $\mathrm{TX}$ & 91 & 1017.5 & 11.2 \\
\hline DALLAS FT WORTH AP & $\mathrm{TX}$ & 80 & 918 & 11.5 \\
\hline LONGVIEW WSMO & $\mathrm{TX}$ & 92 & 1258.1 & 13.7 \\
\hline STEPHENVILLE & $\mathrm{TX}$ & 77 & 801.1 & 10.4 \\
\hline VICTORIA RGNL AP & $\mathrm{TX}$ & 94 & 1047 & 11.1 \\
\hline PORT ARTHUR SE TX AP & $\mathrm{TX}$ & 105 & 1535.9 & 14.6 \\
\hline HOUSTON HOBBY AP & $\mathrm{TX}$ & 104 & 1388.1 & 13.4 \\
\hline BROWNSVILLE & $\mathrm{TX}$ & 74 & 697 & 9.4 \\
\hline SAN ANTONIO INTL AP & $\mathrm{TX}$ & 83 & 819.7 & 9.9 \\
\hline CORPUS CHRISTI & $\mathrm{TX}$ & 77 & 806.7 & 10.5 \\
\hline HOUSTON INTERCONT AP & $\mathrm{TX}$ & 104 & 1264.2 & 12.2 \\
\hline AUSTIN BERGSTROM AP & $\mathrm{TX}$ & 82 & 816.6 & 9.9 \\
\hline AUSTIN-CAMP MABRY & $\mathrm{TX}$ & 88 & 869.7 & 9.9 \\
\hline WACO RGNL AP & $\mathrm{TX}$ & 82 & 881.1 & 10.8 \\
\hline DALLAS LOVE FLD & $\mathrm{TX}$ & 81 & 954.3 & 11.8 \\
\hline FT WORTH MEACHAM FLD & $\mathrm{TX}$ & 80 & 916.7 & 11.5 \\
\hline ABILENE RGNL AP & $\mathrm{TX}$ & 69 & 630.4 & 9.1 \\
\hline WICHITA FALLS MUNI AP & $\mathrm{TX}$ & 74 & 734.1 & 9.9 \\
\hline DEL RIO INTL AP & $\mathrm{TX}$ & 61 & 495.8 & 8.1 \\
\hline CHILDRESS MUNI AP & $\mathrm{TX}$ & 69 & 607.6 & 8.8 \\
\hline MIDLAND ODESSA & $\mathrm{TX}$ & 52 & 370.8 & 7.2 \\
\hline SAN ANGELO & $\mathrm{TX}$ & 62 & 539.8 & 8.7 \\
\hline LUBBOCK & $\mathrm{TX}$ & 66 & 485.6 & 7.3 \\
\hline EL PASO INTL AP & $\mathrm{TX}$ & 53 & 246.6 & 4.6 \\
\hline AMARILLO & $\mathrm{TX}$ & 72 & 517.1 & 7.2 \\
\hline DALHART MUNI AP & $\mathrm{TX}$ & 64 & 446.8 & 7.0 \\
\hline ROOSEVELT RADIO & UT & 61 & 189.2 & 3.1 \\
\hline DELTA & UT & 66 & 218.7 & 3.3 \\
\hline MILFORD MUNI AP & UT & 68 & 265.4 & 3.9 \\
\hline SALT LAKE CITY INTL AP & UT & 96 & 408.9 & 4.3 \\
\hline WENDOVER AP AWOS & UT & 38 & 102.9 & 2.7 \\
\hline LANGLEY AFB & VA & 109 & 1186.2 & 10.9 \\
\hline LYNCHBURG RGNL AP & VA & 116 & 1055.9 & 9.1 \\
\hline NORFOLK INTL AP & VA & 117 & 1181.9 & 10.1 \\
\hline
\end{tabular}


PNNL-21950, Appendix E

Table E.1. (contd)

\begin{tabular}{|c|c|c|c|c|}
\hline Name $^{(a)}$ & State & $\begin{array}{l}\text { Average } \\
\text { d/yr with } \\
\text { Precipitation } \\
>0.254 \mathrm{~mm} \\
\end{array}$ & $\begin{array}{c}\text { Normal } \\
\text { Precipitation } \\
\text { (mm/yr) }\end{array}$ & $\begin{array}{c}\text { RAIN }_{(\mathrm{mm} / \mathrm{d})^{(\mathrm{b})}} \\
\end{array}$ \\
\hline RICHMOND INTL AP & VA & 114 & 1107.4 & 9.7 \\
\hline ROANOKE RGNL AP & VA & 116 & 1047.8 & 9.0 \\
\hline WASHINGTON REAGAN AP & VA & 114 & 1009.4 & 8.8 \\
\hline WASHINGTON DC DULLES AP & VA & 119 & 1055.1 & 8.9 \\
\hline WALLOPS ISLAND FLIGHT FAC & VA & 118 & 1037.3 & 8.8 \\
\hline CHRISTIANSTED AP & VI & 174 & 982.2 & 5.6 \\
\hline CHARLOTTE AMALIE AP & VI & 168 & 999.2 & 5.9 \\
\hline NEWPORT & VT & 175 & 1050.3 & 6.0 \\
\hline BURLINGTON INTL AP & VT & 156 & 935.2 & 6.0 \\
\hline SAINT JOHNSBURY & VT & 164 & 1003.3 & 6.1 \\
\hline EPHRATA MUNI AP & WA & 69 & 199.4 & 2.9 \\
\hline SPOKANE INTL AP & WA & 112 & 420.6 & 3.7 \\
\hline OLYMPIA AP & WA & 163 & 1270 & 7.8 \\
\hline SEATTLE TACOMA INTL AP & WA & 152 & 952.2 & 6.3 \\
\hline YAKIMA AIR TERMINAL & WA & 72 & 209.8 & 2.9 \\
\hline WHIDBEY ISLAND NAS & WA & 145 & 515.4 & 3.6 \\
\hline QUILLAYUTE STATE AP & WA & 206 & 2528.3 & 12.3 \\
\hline PARK FALLS DNR HQ & WI & 124 & 813.3 & 6.6 \\
\hline MADISON DANE RGNL AP & WI & 125 & 875.8 & 7.0 \\
\hline MILWAUKEE MITCHELL AP & WI & 127 & 882.9 & 7.0 \\
\hline WAUSAU DWTN AP & WI & 129 & 823.2 & 6.4 \\
\hline GREEN BAY & WI & 123 & 749.8 & 6.1 \\
\hline LA CROSSE MUNI AP & WI & 119 & 839.7 & 7.1 \\
\hline PARKERSBURG WOOD CO AP & WV & 141 & 1046.2 & 7.4 \\
\hline HUNTINGTON TRI STATE AP & WV & 139 & 1081.8 & 7.8 \\
\hline BECKLEY RALEIGH CO AP & WV & 157 & 1046.2 & 6.7 \\
\hline ELKINS RANDOLPH CO AP & WV & 175 & 1166.6 & 6.7 \\
\hline CHARLESTON YEAGER AP & WV & 151 & 1118.4 & 7.4 \\
\hline CHEYENNE & WY & 104 & 404.9 & 3.9 \\
\hline LANDER HUNT FLD AP & WY & 71 & 321.6 & 4.5 \\
\hline ROCK SPRINGS AP & WY & 78 & 217.4 & 2.8 \\
\hline SHERIDAN CO AP & WY & 101 & 359.7 & 3.6 \\
\hline RAWLINS MUNI AP & WY & 85 & 235 & 2.8 \\
\hline CASPER NATRONA CO AP & WY & 95 & 318 & 3.4 \\
\hline BIG PINEY MARBLETON AP & WY & 55 & 164.6 & 3.0 \\
\hline \multicolumn{5}{|c|}{$\begin{array}{l}\text { (a) AP = airport; CO = County, RGNL = regional, INTL = international, AFB = Air Force Base, FAC = Facility, MUN = } \\
\text { municipal; FLD = field. } \\
\text { Data are based on 30-year averages (1981-2010) from the National Climatic Data Center (e.g., } \\
\text { http://www.ncdc.noaa.gov/oa/climate/research/cag3/cag3.html). }\end{array}$} \\
\hline
\end{tabular}




\section{References}

Support documentation: A. Arguez, I Durre, S Applequist, RS Vose, MF Squires, X Yin, RR Heim, Jr, and TW Owen. 2012. NOAA’s 1981-2010 U.S. Climate Normals, An Overview. Bulletin of the American Meteorological Society, November 2012, pp. 1687-1697.

Data last accessed at: http:/www1.ncdc.noaa.gov/pub/data/normals/1981-2010/stationinventories/prcp-inventory.txt. 


\section{Appendix F}

\section{Growing Periods}




\section{Appendix F}

\section{Growing Periods}

The growing period for each human food crop type defines the deposition period for irrigation and atmospheric deposition to plants. For a given location, the user should review available data sources relevant to their region. To the extent possible, the growing period should reflect the growing period of the edible portion of the crop. Values for each crop type may be determined from a number of available data sources (e.g., frost-free days, days to maturity, planting-to-harvest dates).

The growing periods provided in this Appendix are based on planting and harvesting dates from USDA Agricultural Census data. USDA (2010) provided data on the planting and harvesting dates for a number of crops and by state. The data reflect 2008 or 2009 data. In reviewing this appendix information for parameter determinations, be cognizant of whether the crop is harvested multiple times over the growing season (e.g., melons, tomatoes, berries) or harvested at the end of its growth cycle (e.g., wheat, potatoes, corn). For those harvested multiple times, a best estimate growing period closer to the minimum Appendix value would be more appropriate. In contrast, end-of-season crops are better approximated by average values.

The USDA (2010) information was used for appendix data. For all the following calculations, corrections were included to account for harvesting in the calendar year after the planting calendar year.

Minimum and Maximum growing period for each state is calculated by determining the state-specific overall minimum and overall maximum of the following four calculations:

a. "Usual1" = $\left(\right.$ Usual Harvest Date $\left.{ }_{\text {begin }}\right)-\left(\right.$ Usual Plant Date $\left._{\text {begin }}\right)$

b. "MostActive1" = $\left(\right.$ Most Active Harvest Date $\left.{ }_{\text {start }}\right)-($ Most Active Planting Date start $)$

c. "MostActive2" = (Most Active Harvest Date $\left.{ }_{\text {end }}\right)$ - $\left(\right.$ Most Active Planting Date $\left._{\text {end }}\right)$

d. "Usual2" = (Usual Harvest Date $\left.e_{\text {end }}\right)$ - (Usual Plant Date $\left.e_{\text {end }}\right)$

The Average (most active) growing period for each state is determined as follows: average of "MostActive1" and "MostActive2".

For alfalfa hay, the Most Active (harvest and planting) data are not provided. Many state-specific Average (most active) cells are indicated as ND for alfalfa hay.

The growing period for "Summary-all US" was calculated as a simple average of state-specific values.

For Other Hay growing periods the corn-for-grain planting dates were assumed, because hay plant dates were not reported. Assumed start of growing season reflected in the corn-for-grain values. AK planting date is assumed to be the maximum date of all other states.

Table F.1. Corn for Grain Growing Periods by State

Table F.2. Corn for Silage Growing Periods by State 
Table F.3. Wheat Growing Periods by State

Table F.4. Hay Growing Periods by State

Table F.5. Alfalfa Hay Growing Periods by Reporting Region

Table F.6. Other Hay Growing Period by Reporting Region

Table F.7. Leafy Vegetables Growing Periods

Table F.8. Root/Other Vegetables Growing Periods

Table F.9. Fruits Modeled as Root/Other Vegetables Growing Periods 
PNNL-21950, Appendix F

Table F.1. Corn for Grain Growing Periods by State

\begin{tabular}{|c|c|c|c|c|}
\hline & $\begin{array}{c}2009 \text { Harvested } \\
\text { Area }\end{array}$ & \multicolumn{3}{|c|}{2009 Growing Period Estimates } \\
\hline & Corn for Grain & Corn for Grain & Corn for Grain & Corn for Grain \\
\hline & 2009 Data & $\begin{array}{c}2009 \\
\text { Minimum }\end{array}$ & $\begin{array}{c}2009 \\
\text { Maximum }\end{array}$ & $\begin{array}{l}2009 \text { Average } \\
\text { (most active) }\end{array}$ \\
\hline & acre (thousands) & $\mathrm{d}$ & $\mathrm{d}$ & $\mathrm{d}$ \\
\hline Alabama & 250 & 139 & 150 & 143.5 \\
\hline Arizona & 20 & 170 & 183 & 176.5 \\
\hline Arkansas & 410 & 143 & 150 & 147 \\
\hline California & 160 & 123 & 183 & 153 \\
\hline Colorado & 990 & 162 & 177 & 170 \\
\hline Delaware & 163 & 143 & 161 & 147.5 \\
\hline Florida & 37 & 136 & 149 & 138.5 \\
\hline Georgia & 370 & 145 & 156 & 150.5 \\
\hline Idaho & 80 & 161 & 168 & 168 \\
\hline Illinois & 11,800 & 153 & 168 & 160.5 \\
\hline Indiana & 5,460 & 148 & 168 & 157.5 \\
\hline Iowa & 13,400 & 155 & 179 & 169 \\
\hline Kansas & 3,860 & 148 & 169 & 155.5 \\
\hline Kentucky & 1,150 & 148 & 155 & 150.5 \\
\hline Louisiana & 610 & 140 & 150 & 146.5 \\
\hline Maryland & 425 & 142 & 163 & 150 \\
\hline Michigan & 2,090 & 137 & 187 & 172 \\
\hline Minnesota & 7,150 & 158 & 178 & 169 \\
\hline Mississippi & 695 & 147 & 156 & 150.5 \\
\hline Missouri & 2,920 & 148 & 193 & 155 \\
\hline Montana & 26 & 161 & 189 & 181.5 \\
\hline Nebraska & 8,850 & 152 & 183 & 169.5 \\
\hline Nevada & ND & ND & ND & ND \\
\hline New Jersey & 70 & 153 & 165 & 163.5 \\
\hline New Mexico & 50 & 163 & 184 & 168.5 \\
\hline New York & 595 & 154 & 170 & 158.5 \\
\hline North Carolina & 800 & 146 & 170 & 160.5 \\
\hline North Dakota & 1,740 & 155 & 185 & 167 \\
\hline Ohio & 3,140 & 162 & 185 & 175 \\
\hline Oklahoma & 320 & 149 & 156 & 151.5 \\
\hline Oregon & 32 & 168 & 199 & 173 \\
\hline Pennsylvania & 920 & 148 & 179 & 168.5 \\
\hline South Carolina & 320 & 137 & 158 & 155.5 \\
\hline South Dakota & 4,680 & 151 & 176 & 165 \\
\hline Tennessee & 590 & 146 & 158 & 151 \\
\hline Texas & 1,960 & 139 & 175 & 151.5 \\
\hline
\end{tabular}


PNNL-21950, Appendix F

Table F.1. (contd)

\begin{tabular}{lcccc}
\hline & $\begin{array}{c}\text { 2009 Harvested } \\
\text { Area }\end{array}$ & \multicolumn{3}{c}{ 2009 Growing Period Estimates } \\
\hline & Corn for Grain & Corn for Grain & Corn for Grain & Corn for Grain \\
\hline & 2009 Data & Minimum & Maximum & $\begin{array}{c}\text { 2009 Average } \\
\text { (most active) }\end{array}$ \\
\hline acre (thousands) & $\mathrm{d}$ & $\mathrm{d}$ & $\mathrm{d}$ \\
\hline Utah & 17 & 163 & 188 & 163 \\
Virginia & 330 & 148 & 164 & 154.5 \\
Washington & 105 & 168 & 179 & 173.5 \\
West Virginia & 30 & 148 & 168 & 160 \\
Wisconsin & 2,930 & 159 & 177 & 169.5 \\
Wyoming & 45 & 164 & 187 & 177.5 \\
Summary - all U.S. & 79,590 & 154 & 168 & 161 \\
\hline Summary - all U.S. data: Harvested Acres (total). Minimum: lowest minimum. & Maximum: \\
highest maximum. Average: & average of state specific values. & & \\
ND = No data available. & \multicolumn{5}{l}{} \\
\hline
\end{tabular}


PNNL-21950, Appendix F

Table F.2. Corn for Silage Growing Periods by State

\begin{tabular}{|c|c|c|c|c|}
\hline & \multirow{2}{*}{$\begin{array}{c}2009 \text { Harvested } \\
\text { Area } \\
\text { Corn for Silage }\end{array}$} & \multicolumn{3}{|c|}{2009 Growing Period Estimates } \\
\hline & & Corn for Silage & Corn for Silage & Corn for Silage \\
\hline & 2009 Data & 2009 Minimum & 2009 Maximum & $\begin{array}{l}2009 \text { Average } \\
\text { (most active) }\end{array}$ \\
\hline & $\begin{array}{c}\text { acre } \\
\text { (thousands) }\end{array}$ & $\mathrm{d}$ & $\mathrm{d}$ & $\mathrm{d}$ \\
\hline Arizona & 30 & 170 & 183 & 176.5 \\
\hline Colorado & 85 & 130 & 133 & 131.5 \\
\hline Delaware & 5 & 117 & 125 & 121 \\
\hline Florida & 30 & 87 & 102 & 91.5 \\
\hline Idaho & 215 & 133 & 140 & 133 \\
\hline Indiana & 110 & 96 & 117 & 106 \\
\hline Iowa & 220 & 94 & 125 & 123.5 \\
\hline Kentucky & 60 & 107 & 122 & 114 \\
\hline Maryland & 40 & 126 & 134 & 133 \\
\hline Michigan & 220 & 127 & 136 & 132.5 \\
\hline Minnesota & 380 & 127 & 139 & 135 \\
\hline Mississippi & 10 & 117 & 131 & 120.5 \\
\hline Montana & 45 & 122 & 128 & 125.5 \\
\hline "New England" & 161 & 123 & 129 & 127 \\
\hline New Jersey & 9 & 132 & 142 & 132.5 \\
\hline New Mexico & 78 & 139 & 165 & 143.5 \\
\hline New York & 470 & 110 & 138 & 119.5 \\
\hline North Carolina & 55 & 122 & 153 & 140 \\
\hline North Dakota & 170 & 123 & 132 & 128.5 \\
\hline Ohio & 170 & 126 & 137 & 132 \\
\hline Oregon & 28 & 97 & 119 & 105 \\
\hline Pennsylvania & 420 & 122 & 138 & 130.5 \\
\hline South Dakota & 250 & 120 & 131 & 127.5 \\
\hline Tennessee & 50 & 113 & 128 & 122.5 \\
\hline Texas & 140 & 61 & 139 & 95 \\
\hline Utah & 47 & 127 & 138 & 135.5 \\
\hline Virginia & 135 & 129 & 131 & 130.5 \\
\hline Washington & 65 & 127 & 148 & 140 \\
\hline West Virginia & 16 & 102 & 127 & 115 \\
\hline Wisconsin & 850 & 134 & 139 & 136.5 \\
\hline Wyoming & 32 & 127 & 133 & 131.5 \\
\hline Overall average days & 4596 & 119 & 135 & 127 \\
\hline
\end{tabular}


Table F.3. Wheat Growing Periods by State

\begin{tabular}{|c|c|c|c|c|c|c|c|c|c|c|c|c|}
\hline & \multicolumn{3}{|c|}{2009 Harvested Area } & \multicolumn{9}{|c|}{2009 Growing Period Estimates } \\
\hline & $\begin{array}{l}\text { Durum } \\
\text { Wheat }\end{array}$ & $\begin{array}{l}\text { Spring } \\
\text { Wheat }\end{array}$ & $\begin{array}{l}\text { Winter } \\
\text { Wheat }\end{array}$ & $\begin{array}{l}\text { Durum } \\
\text { Wheat }\end{array}$ & $\begin{array}{l}\text { Durum } \\
\text { Wheat }\end{array}$ & $\begin{array}{l}\text { Durum } \\
\text { Wheat }\end{array}$ & $\begin{array}{l}\text { Spring } \\
\text { Wheat }\end{array}$ & $\begin{array}{l}\text { Spring } \\
\text { Wheat }\end{array}$ & $\begin{array}{l}\text { Spring } \\
\text { Wheat }\end{array}$ & $\begin{array}{l}\text { Winter } \\
\text { Wheat }\end{array}$ & $\begin{array}{l}\text { Winter } \\
\text { Wheat }\end{array}$ & $\begin{array}{l}\text { Winter } \\
\text { Wheat }\end{array}$ \\
\hline & 2009 Data & 2009 Data & 2009 Data & $\begin{array}{c}2009 \\
\text { Minimum }\end{array}$ & $\begin{array}{c}2009 \\
\text { Maximum }\end{array}$ & $\begin{array}{c}2009 \\
\text { Average } \\
\text { (most } \\
\text { active) }\end{array}$ & $\begin{array}{c}2009 \\
\text { Minimum }\end{array}$ & $\begin{array}{c}2009 \\
\text { Maximum }\end{array}$ & $\begin{array}{c}2009 \\
\text { Average } \\
\text { (most } \\
\text { active) }\end{array}$ & $\begin{array}{c}2009 \\
\text { Minimum }\end{array}$ & $\begin{array}{c}2009 \\
\text { Maximum }\end{array}$ & $\begin{array}{c}2009 \\
\text { Average } \\
\text { (most } \\
\text { active) }\end{array}$ \\
\hline & $\begin{array}{c}\text { acre } \\
\text { (thousands) }\end{array}$ & $\begin{array}{c}\text { acre } \\
\text { (thousands) }\end{array}$ & $\begin{array}{c}\text { acre } \\
\text { (thousands) }\end{array}$ & d & $\mathrm{d}$ & d & d & d & d & $\mathrm{d}$ & d & d \\
\hline Alabama & 0 & 0 & 180 & 0 & 0 & 0 & 0 & 0 & 0 & 201 & 216 & 216 \\
\hline Arizona & 124 & 0 & 5 & 151 & 186 & 173.5 & 0 & 0 & 0 & 151 & 186 & 173.5 \\
\hline Arkansas & 0 & 0 & 390 & 0 & 0 & 0 & 0 & 0 & 0 & 213 & 241 & 226.5 \\
\hline California & 170 & 0 & 315 & 182 & 237 & 214.5 & 0 & 0 & 0 & 165 & 237 & 205 \\
\hline Colorado & 0 & 29 & 2450 & 0 & 0 & 0 & 116 & 129 & 120 & 291 & 299 & 293 \\
\hline Delaware & 0 & 0 & 67 & 0 & 0 & 0 & 0 & 0 & 0 & 238 & 257 & 246 \\
\hline Florida & 0 & 0 & 14 & 0 & 0 & 0 & 0 & 0 & 0 & 167 & 198 & 174 \\
\hline Georgia & 0 & 0 & 250 & 0 & 0 & 0 & 0 & 0 & 0 & 185 & 224 & 196.5 \\
\hline Idaho & 20 & 530 & 700 & 119 & 129 & 122 & 126 & 136 & 128 & 315 & 318 & 316 \\
\hline Illinois & 0 & 0 & 820 & 0 & 0 & 0 & 0 & 0 & 0 & 252 & 260 & 256 \\
\hline Indiana & 0 & 0 & 450 & 0 & 0 & 0 & 0 & 0 & 0 & 256 & 267 & 261.5 \\
\hline Iowa & 0 & 0 & 22 & 0 & 0 & 0 & 0 & 0 & 0 & 280 & 291 & 281.5 \\
\hline Kansas & 0 & 0 & 8800 & 0 & 0 & 0 & 0 & 0 & 0 & 256 & 278 & 268 \\
\hline Kentucky & 0 & 0 & 390 & 0 & 0 & 0 & 0 & 0 & 0 & 231 & 256 & 243 \\
\hline Louisiana & 0 & 0 & 175 & 0 & 0 & 0 & 0 & 0 & 0 & 188 & 210 & 197 \\
\hline Maryland & 0 & 0 & 195 & 0 & 0 & 0 & 0 & 0 & 0 & 243 & 260 & 251 \\
\hline Michigan & 0 & 0 & 560 & 0 & 0 & 0 & 0 & 0 & 0 & 277 & 296 & 286 \\
\hline Minnesota & 0 & 1550 & 45 & 0 & 0 & 0 & 104 & 113 & 106.5 & 329 & 350 & 336.5 \\
\hline Mississippi & 0 & 0 & 165 & 0 & 0 & 0 & 0 & 0 & 0 & 213 & 244 & 225 \\
\hline Missouri & 0 & 0 & 730 & 0 & 0 & 0 & 0 & 0 & 0 & 233 & 260 & 248.5 \\
\hline Montana & 535 & 2350 & 2420 & 108 & 113 & 111.5 & 115 & 118 & 116 & 305 & 319 & 313 \\
\hline Nebraska & 0 & 0 & 1600 & 0 & 0 & 0 & 0 & 0 & 0 & 288 & 298 & 294.5 \\
\hline Nevada & 0 & 2 & 11 & 0 & 0 & 0 & 102 & 108 & 105 & 308 & 314 & 311 \\
\hline New Jersey & 0 & 0 & 29 & 0 & 0 & 0 & 0 & 0 & 0 & 256 & 269 & 266 \\
\hline New Mexico & 0 & 0 & 140 & 0 & 0 & 0 & 0 & 0 & 0 & 273 & 294 & 286 \\
\hline
\end{tabular}


Table F.3. (contd)

\begin{tabular}{|c|c|c|c|c|c|c|c|c|c|c|c|c|}
\hline & \multicolumn{3}{|c|}{2009 Harvested Area } & \multicolumn{9}{|c|}{2009 Growing Period Estimates } \\
\hline & $\begin{array}{l}\text { Durum } \\
\text { Wheat }\end{array}$ & $\begin{array}{l}\text { Spring } \\
\text { Wheat }\end{array}$ & $\begin{array}{l}\text { Winter } \\
\text { Wheat }\end{array}$ & $\begin{array}{l}\text { Durum } \\
\text { Wheat }\end{array}$ & $\begin{array}{l}\text { Durum } \\
\text { Wheat }\end{array}$ & $\begin{array}{l}\text { Durum } \\
\text { Wheat }\end{array}$ & $\begin{array}{l}\text { Spring } \\
\text { Wheat }\end{array}$ & $\begin{array}{l}\text { Spring } \\
\text { Wheat }\end{array}$ & $\begin{array}{l}\text { Spring } \\
\text { Wheat }\end{array}$ & $\begin{array}{l}\text { Winter } \\
\text { Wheat }\end{array}$ & $\begin{array}{l}\text { Winter } \\
\text { Wheat }\end{array}$ & $\begin{array}{l}\text { Winter } \\
\text { Wheat }\end{array}$ \\
\hline & 2009 Data & 2009 Data & 2009 Data & $\begin{array}{c}2009 \\
\text { Minimum }\end{array}$ & $\begin{array}{c}2009 \\
\text { Maximum }\end{array}$ & $\begin{array}{c}2009 \\
\text { Average } \\
\text { (most } \\
\text { active) }\end{array}$ & $\begin{array}{c}2009 \\
\text { Minimum }\end{array}$ & $\begin{array}{c}2009 \\
\text { Maximum }\end{array}$ & $\begin{array}{c}2009 \\
\text { Average } \\
\text { (most } \\
\text { active) }\end{array}$ & $\begin{array}{c}2009 \\
\text { Minimum }\end{array}$ & $\begin{array}{c}2009 \\
\text { Maximum }\end{array}$ & $\begin{array}{c}2009 \\
\text { Average } \\
\text { (most } \\
\text { active) }\end{array}$ \\
\hline & $\begin{array}{c}\text { acre } \\
\text { (thousands) }\end{array}$ & $\begin{array}{c}\text { acre } \\
\text { (thousands) }\end{array}$ & $\begin{array}{c}\text { acre } \\
\text { (thousands) }\end{array}$ & d & d & d & d & $\mathrm{d}$ & d & $\mathrm{d}$ & $\mathrm{d}$ & $\mathrm{d}$ \\
\hline New York & 0 & 0 & 105 & 0 & 0 & 0 & 0 & 0 & 0 & 286 & 306 & 305.5 \\
\hline North Carolina & 0 & 0 & 600 & 0 & 0 & 0 & 0 & 0 & 0 & 217 & 252 & 230 \\
\hline North Dakota & 1570 & 6300 & 545 & 105 & 119 & 110 & 106 & 114 & 108.5 & 307 & 313 & 310 \\
\hline Ohio & 0 & 0 & 980 & 0 & 0 & 0 & 0 & 0 & 0 & 256 & 275 & 269 \\
\hline Oklahoma & 0 & 0 & 3500 & 0 & 0 & 0 & 0 & 0 & 0 & 239 & 271 & 256 \\
\hline Oregon & 0 & 127 & 750 & 0 & 0 & 0 & 120 & 141 & 130.5 & 265 & 298 & 279 \\
\hline Pennsylvania & 0 & 0 & 175 & 0 & 0 & 0 & 0 & 0 & 0 & 284 & 309 & 293 \\
\hline South Carolina & 0 & 0 & 150 & 0 & 0 & 0 & 0 & 0 & 0 & 182 & 243 & 199.5 \\
\hline South Dakota & 9 & 1470 & 1530 & 0 & 0 & 0 & 100 & 111 & 105 & 301 & 310 & 306 \\
\hline Tennessee & 0 & 0 & 340 & 0 & 0 & 0 & 0 & 0 & 0 & 217 & 253 & 232.5 \\
\hline Texas & 0 & 0 & 2450 & 0 & 0 & 0 & 0 & 0 & 0 & 231 & 263 & 251.5 \\
\hline Utah & 0 & 12 & 135 & 0 & 0 & 0 & 117 & 127 & 126.5 & 288 & 334 & 321.5 \\
\hline Virginia & 0 & 0 & 210 & 0 & 0 & 0 & 0 & 0 & 0 & 223 & 257 & 237.5 \\
\hline Washington & 0 & 585 & 1640 & 0 & 0 & 0 & 118 & 132 & 125.5 & 314 & 327 & 320.5 \\
\hline West Virginia & 0 & 0 & 5 & 0 & 0 & 0 & 0 & 0 & 0 & 262 & 283 & 272.5 \\
\hline Wisconsin & 0 & 0 & 315 & 0 & 0 & 0 & 0 & 0 & 0 & 289 & 308 & 297 \\
\hline Wyoming & 0 & 0 & 132 & 0 & 0 & 0 & 0 & 0 & 0 & 321 & 329 & 325 \\
\hline $\begin{array}{l}\text { Summary - all } \\
\text { U.S. }\end{array}$ & 2428 & 12955 & 34485 & 131 & 146 & 139 & 116 & 119 & 117 & 254 & 274 & 264 \\
\hline
\end{tabular}

Summary - all U.S. data: Harvested Acres (total). Minimum: lowest minimum. Maximum: highest maximum. Average: average of state specific values. 
Table F.4. Hay Growing Periods by State

\begin{tabular}{|c|c|c|c|c|c|c|c|c|}
\hline & \multicolumn{2}{|c|}{2009 Harvested Area } & \multicolumn{6}{|c|}{2009 Growing Period Estimates } \\
\hline \multirow{3}{*}{$\begin{array}{l}2.5 \text { alfalfa harvests } \\
\text { assumed, except CA, } \\
\text { AZ where } 3.5 \text { harvests } \\
\text { assumed }\end{array}$} & Alfalfa Hay & Other Hay & Alfalfa Hay & Alfalfa Hay & Alfalfa Hay & Other Hay & Other Hay & Other Hay \\
\hline & 2009 Data & 2009 Data & $\begin{array}{c}2009 \\
\text { Minimum }\end{array}$ & $\begin{array}{c}2009 \\
\text { Maximum }\end{array}$ & $\begin{array}{l}2009 \text { Average } \\
\text { (most active) }\end{array}$ & $\begin{array}{c}2009 \\
\text { Minimum }\end{array}$ & $\begin{array}{c}2009 \\
\text { Maximum }\end{array}$ & $\begin{array}{l}2009 \text { Average } \\
\text { (most active) }\end{array}$ \\
\hline & $\begin{array}{c}\text { acre } \\
\text { (thousands) }\end{array}$ & $\begin{array}{c}\text { acre } \\
\text { (thousands) }\end{array}$ & $\mathrm{d}$ & $\mathrm{d}$ & $\mathrm{d}$ & d & $\mathrm{d}$ & $\mathrm{d}$ \\
\hline Alabama & ND & 800 & ND & ND & ND & 22 & 60 & 43 \\
\hline Alaska & ND & 20 & ND & ND & ND & 20 & 45 & 29 \\
\hline Arizona & 280 & 30 & 21 & 52 & ND & 21 & 52 & ND \\
\hline Arkansas & 15 & 1400 & 14 & 52 & 34.4 & 14 & 52 & 34 \\
\hline California & 980 & 540 & 25 & 37 & $\mathrm{ND}$ & 22 & 61 & ND \\
\hline Colorado & 850 & 750 & 13 & 66 & 34.2 & 25 & 44 & 30 \\
\hline Delaware & 5 & 12 & 12 & 70 & ND & 11 & 66 & ND \\
\hline Florida & ND & 300 & ND & ND & ND & 28 & 80 & 44 \\
\hline Georgia & ND & 700 & ND & ND & ND & 25 & 72 & ND \\
\hline Idaho & 1140 & 370 & 12 & 53 & ND & 16 & 37 & ND \\
\hline Illinois & 340 & 270 & 10 & 43 & 27.2 & 11 & 17 & 13 \\
\hline Indiana & 300 & 320 & 10 & 41 & 0.0 & 10 & 41 & ND \\
\hline Iowa & 920 & 300 & 14 & 50 & 31.0 & 19 & 40 & 28 \\
\hline Kansas & 850 & 1700 & 14 & 64 & 37.6 & 19 & 45 & 31 \\
\hline Kentucky & 220 & 2300 & 12 & 40 & 26.2 & 16 & 50 & 30 \\
\hline Louisiana & ND & 380 & ND & ND & ND & 14 & 60 & ND \\
\hline Maryland & 40 & 170 & 9 & 70 & ND & 8 & 66 & ND \\
\hline Michigan & 700 & 290 & 16 & 52 & 30.4 & 16 & 52 & 39 \\
\hline Minnesota & 1300 & 750 & 13 & 47 & ND & 18 & 37 & ND \\
\hline Mississippi & ND & 700 & ND & ND & ND & 10 & 58 & ND \\
\hline Missouri & 280 & 3600 & 17 & 32 & 31.4 & 18 & 30 & 24 \\
\hline Montana & 1700 & 800 & 21 & 44 & ND & 22 & 50 & ND \\
\hline Nebraska & 950 & 1750 & 12 & 59 & ND & 19 & 49 & ND \\
\hline
\end{tabular}


Table F.4. (contd)

\begin{tabular}{|c|c|c|c|c|c|c|c|c|}
\hline & \multicolumn{2}{|c|}{2009 Harvested Area } & \multicolumn{6}{|c|}{2009 Growing Period Estimates } \\
\hline & Alfalfa Hay & Other Hay & Alfalfa Hay & Alfalfa Hay & Alfalfa Hay & Other Hay & Other Hay & Other Hay \\
\hline \multirow{2}{*}{$\begin{array}{l}2.5 \text { alfalfa harvests } \\
\text { assumed, except CA, } \\
\text { AZ where } 3.5 \text { harvests } \\
\text { assumed }\end{array}$} & 2009 Data & 2009 Data & $\begin{array}{c}2009 \\
\text { Minimum }\end{array}$ & $\begin{array}{c}2009 \\
\text { Maximum }\end{array}$ & $\begin{array}{l}2009 \text { Average } \\
\text { (most active) }\end{array}$ & $\begin{array}{c}2009 \\
\text { Minimum }\end{array}$ & $\begin{array}{c}2009 \\
\text { Maximum }\end{array}$ & $\begin{array}{l}2009 \text { Average } \\
\text { (most active) }\end{array}$ \\
\hline & $\begin{array}{c}\text { acre } \\
\text { (thousands) }\end{array}$ & $\begin{array}{c}\text { acre } \\
\text { (thousands) }\end{array}$ & $\mathrm{d}$ & $\mathrm{d}$ & $\mathrm{d}$ & $\mathrm{d}$ & $\mathrm{d}$ & $\mathrm{d}$ \\
\hline Nevada & 280 & 210 & 28 & 54 & ND & 39 & 42 & ND \\
\hline New England & 65 & 481 & 14 & 53 & ND & 14 & 53 & ND \\
\hline New Jersey & 25 & 85 & 12 & 56 & ND & 10 & 49 & ND \\
\hline New Mexico & 240 & 80 & 6 & 61 & ND & 6 & 61 & ND \\
\hline New York & 350 & 1010 & 14 & 45 & ND & 14 & 47 & ND \\
\hline North Carolina & 7 & 840 & 12 & 74 & ND & 12 & 74 & ND \\
\hline North Dakota & 1780 & 1180 & 18 & 38 & ND & 22 & 36 & ND \\
\hline Ohio & 380 & 660 & 12 & 47 & 28.2 & 13 & 54 & 33 \\
\hline Oklahoma & 320 & 2900 & 12 & 60 & ND & 12 & 62 & ND \\
\hline Oregon & 400 & 630 & 16 & 39 & ND & 22 & 49 & ND \\
\hline Pennsylvania & 500 & 1050 & 6 & 61 & ND & 12 & 41 & ND \\
\hline South Carolina & ND & 350 & ND & ND & ND & 26 & 73 & ND \\
\hline South Dakota & 2500 & 1300 & 13 & 43 & 28.4 & 18 & 32 & 26 \\
\hline Tennessee & 15 & 1900 & 12 & 52 & ND & 12 & 52 & ND \\
\hline Texas & 120 & 4500 & 18 & 50 & ND & 24 & 54 & ND \\
\hline Utah & 530 & 160 & 19 & 57 & ND & 32 & 34 & ND \\
\hline Virginia & 90 & 1090 & 10 & 58 & ND & 20 & 50 & ND \\
\hline Washington & 490 & 320 & 14 & 49 & 30.0 & 14 & 42 & 27 \\
\hline West Virginia & 25 & 600 & 12 & 41 & ND & 12 & 57 & ND \\
\hline Wisconsin & 1550 & 370 & 10 & 51 & ND & 12 & 41 & ND \\
\hline Wyoming & 690 & 580 & 17 & 48 & ND & 22 & 38 & ND \\
\hline Summary - all U.S. & 21,227 & 38,548 & 14 & 52 & 37.7 & 18 & 49 & 49 \\
\hline
\end{tabular}


PNNL-21950, Appendix F

Table F.5. Alfalfa Hay Growing Periods by Reporting Region

\begin{tabular}{ccccc}
\hline & \multicolumn{2}{c}{$\begin{array}{c}\text { 2009 Harvested } \\
\text { Area }\end{array}$} & \multicolumn{2}{c}{ 2009 Growing Period Estimates } \\
\hline & Alfalfa Hay & Alfalfa Hay & Alfalfa Hay & Alfalfa Hay \\
\cline { 2 - 5 } 2.5 alfalfa harvests assumed, & & & & 2009 Average \\
except CA, AZ where & 2009 Data & 2009 Minimum & 2009 Maximum & (most active) \\
\cline { 2 - 5 } 3.5 harvests assumed & acres (thousands) & $\mathrm{d}$ & $\mathrm{d}$ & $\mathrm{d}$ \\
\hline Ag Census Reporting Region (see Figure 7.2) & & 53.2 & 32.9 \\
\hline 1 & 3420 & 11.7 & 62.6 & n/a \\
2 & 167 & 11.0 & 51.0 & 32.9 \\
3 (except FL) & 975 & 13.6 & 49.7 & 31.3 \\
4 & 9630 & 17.9 & 46.9 & 30 \\
5 & 2030 & 14.3 & 44.4 & N/A \\
6 & 1260 & 23.0 & N/A & N/A \\
\hline
\end{tabular}

Table F.6. Other Hay Growing Period by Reporting Region

\begin{tabular}{|c|c|c|c|c|}
\hline \multirow{3}{*}{$\begin{array}{l}2.5 \text { alfalfa harvests assumed, } \\
\text { except CA, AZ where } \\
3.5 \text { harvests assumed }\end{array}$} & Other Hay & Other Hay & Other Hay & Other Hay \\
\hline & 2009 Data & 2009 Minimum & 2009 Maximum & $\begin{array}{l}2009 \text { Average } \\
\text { (most active) }\end{array}$ \\
\hline & acres (thousands) & & & \\
\hline \multicolumn{5}{|c|}{ Ag Census Reporting Region (see Figure 7.2) } \\
\hline 1 & 7,056 & 13.8 & 47.4 & 32.0 \\
\hline 2 & 4,562 & 17.1 & 64.7 & 43.2 \\
\hline 3 & 13,860 & 15.8 & 57.2 & 34.3 \\
\hline 4 & 5,730 & 24.8 & 39.2 & 27.8 \\
\hline 5 & 1,340 & 18.3 & 43.3 & 28.0 \\
\hline 6 & 570 & 21.9 & 56.4 & N/A \\
\hline 7 & N/A & N/A & N/A & N/A \\
\hline
\end{tabular}


PNNL-21950, Appendix F

Table F.7. Leafy Vegetables Growing Periods

\begin{tabular}{|c|c|c|c|c|c|}
\hline \multirow[b]{3}{*}{ Leafy Vegetables } & & 2008 & $\begin{array}{l}\text { GRWPA, } \\
\text { GRWP }\end{array}$ & $\begin{array}{l}\text { GRWPA, } \\
\text { GRWP }\end{array}$ & $\begin{array}{c}\text { GRWPA } \\
\text { GRWP }\end{array}$ \\
\hline & & $\begin{array}{c}\text { Harvested } \\
\text { Area }\end{array}$ & $\begin{array}{c}2009 \\
\text { Minimum }\end{array}$ & $\begin{array}{c}2009 \\
\text { Maximum }\end{array}$ & $\begin{array}{c}2009 \\
\text { Average }\end{array}$ \\
\hline & & ac & $\mathrm{d}$ & $\mathrm{d}$ & $\mathrm{d}$ \\
\hline Head Lettuce, winter & Arizona & 38,310 & 61 & 90 & 76 \\
\hline Head Lettuce, winter & California & 200,470 & 91 & 136 & 114 \\
\hline Romaine Lettuce, winter & Arizona & see above & 61 & 90 & 76 \\
\hline Romaine Lettuce, winter & \multirow[t]{2}{*}{ California } & \multirow[t]{2}{*}{ see above } & 77 & 136 & 107 \\
\hline AVERAGE, Lettuce, winter & & & 73 & 113 & 93 \\
\hline Head Lettuce, spring & California & see above & 91 & 122 & 106 \\
\hline Head Lettuce, spring & New Jersey & 918 & 56 & 92 & 72 \\
\hline Romaine Lettuce, spring & \multirow[t]{2}{*}{ California } & \multirow[t]{2}{*}{ see above } & 91 & 122 & 106 \\
\hline AVERAGE, Lettuce, spring & & & 79 & 112 & 95 \\
\hline Head Lettuce, summer & California & see above & 61 & 92 & 76 \\
\hline Head Lettuce, summer & Colorado & 4,471 & 75 & 97 & 83 \\
\hline Romaine Lettuce, summer & \multirow[t]{2}{*}{ California } & \multirow[t]{2}{*}{ see above } & 61 & 92 & 76 \\
\hline AVERAGE, Lettuce, summer & & & 66 & 94 & 78 \\
\hline Head lettuce fall & California & see above & 60.5 & 61 & 61 \\
\hline Head lettuce fall & New Jersey & see above & 73 & 112 & 90 \\
\hline Romaine Lettuce, summer & \multirow[t]{2}{*}{ California } & \multirow[t]{9}{*}{ see above } & 60.5 & 61 & 61 \\
\hline AVERAGE, Lettuce, fall & & & 65 & 78 & 71 \\
\hline Celery, winter & California & & 101 & 121.5 & 115 \\
\hline Celery, spring & California & & 106 & 121 & 116 \\
\hline Celery, summer & California & & 91.5 & 111 & 98 \\
\hline Celery, summer & Michigan & & 71 & 107 & 89 \\
\hline Celery, fall & \multirow[t]{3}{*}{ California } & & 92 & 137.5 & 122 \\
\hline AVERAGE, Celery & & & 92 & 120 & 108 \\
\hline AVERAGE, Leafy Vegetables & & & 75 & 103 & 89 \\
\hline
\end{tabular}


Table F.8. Root/Other Vegetables Growing Periods

\begin{tabular}{|c|c|c|c|c|c|}
\hline \multirow[b]{3}{*}{ Other and Root Vegetables } & & \multirow{2}{*}{$\begin{array}{c}2008 \\
\text { Harvested } \\
\text { Area } \\
\end{array}$} & $\begin{array}{c}\text { GRWPA, } \\
\text { GRWP }\end{array}$ & $\begin{array}{c}\text { GRWPA, } \\
\text { GRWP }\end{array}$ & $\begin{array}{c}\text { GRWPA, } \\
\text { GRWP }\end{array}$ \\
\hline & & & $\begin{array}{c}2009 \\
\text { Minimum }\end{array}$ & $\begin{array}{c}2009 \\
\text { Maximum }\end{array}$ & $\begin{array}{c}2009 \\
\text { Average }\end{array}$ \\
\hline & & ac & $\mathrm{d}$ & $\mathrm{d}$ & $\mathrm{d}$ \\
\hline Onions fresh, spring & Arizona & ND & 212 & 212 & 212 \\
\hline Onions fresh, spring & California & ND & 89.5 & 152 & 116 \\
\hline Onions fresh, spring & Georgia & ND & 124 & 158 & 146 \\
\hline Onions fresh, spring & \multirow[t]{2}{*}{ Texas } & \multirow[t]{2}{*}{ ND } & 113.5 & 152 & 139 \\
\hline AVERAGE, Onion fresh, spring & & & 135 & 168 & 153 \\
\hline Onions fresh, summer & California & ND & 152.5 & 184 & 173 \\
\hline Onions fresh, summer & Nevada & ND & 153 & 170 & 161 \\
\hline Onions fresh, summer & New Mexico & ND & 128 & 182.5 & 150 \\
\hline Onions fresh, summer & Texas & ND & 151 & 155 & 153 \\
\hline Onions fresh, summer & \multirow[t]{2}{*}{ Washington } & \multirow[t]{2}{*}{ ND } & 149 & 205.5 & 177 \\
\hline AVERAGE, Onion fresh, summer & & & 147 & 179 & 163 \\
\hline Onions for storage, summer & California & ND & 213 & 365 & 274 \\
\hline Onions for storage, summer & Colorado & ND & 127 & 174 & 148 \\
\hline Onions for storage, summer & Idaho & ND & 153 & 193 & 178 \\
\hline Onions for storage, summer & Michigan & ND & 110 & 153 & 135 \\
\hline Onions for storage, summer & New York & ND & 135.5 & 204 & 163 \\
\hline Onions for storage, summer & Ohio & ND & 134 & 153 & 141 \\
\hline Onions for storage, summer & Oregon & ND & 148 & 173 & 161 \\
\hline Onions for storage, summer & Utah & ND & 162 & 203 & 183 \\
\hline Onions for storage, summer & Washington & ND & 129 & 209 & 169 \\
\hline Onions for storage, summer & \multirow[t]{2}{*}{ Wisconsin } & \multirow[t]{2}{*}{ ND } & 87 & 133 & 113 \\
\hline AVERAGE, Onion for storage & & & 140 & 196 & 166 \\
\hline Carrots fresh, winter & Arizona & ND & 137 & 387 & 284 \\
\hline Carrots fresh, winter & California & ND & 123 & 152 & 138 \\
\hline Carrots fresh, winter & Georgia & ND & 136 & 182.5 & 167 \\
\hline Carrots fresh, winter & \multirow[t]{2}{*}{ Texas } & \multirow[t]{2}{*}{ ND } & 91 & 120 & 106 \\
\hline AVERAGE, Carrots, fresh winter & & & 122 & 210 & 173 \\
\hline Carrots fresh, spring & California & ND & 136 & 211 & 179 \\
\hline Carrots fresh, spring & \multirow[t]{2}{*}{ Texas } & \multirow[t]{2}{*}{ ND } & 120 & 212 & 161 \\
\hline AVERAGE, Carrots, fresh spring & & & 128 & 212 & 170 \\
\hline Carrots, summer & California & ND & 122 & 151 & 134 \\
\hline Carrots, summer & Colorado & ND & 108 & 123 & 114 \\
\hline Carrots, summer & Michigan & ND & 86 & 138 & 115 \\
\hline Carrots, summer & \multirow[t]{2}{*}{ Washington } & \multirow[t]{2}{*}{ ND } & 106 & 153 & 125 \\
\hline AVERAGE, Carrots, summer & & & 106 & 141 & 122 \\
\hline
\end{tabular}


PNNL-21950, Appendix F

Table F.8. (contd)

\begin{tabular}{|c|c|c|c|c|c|}
\hline \multirow[b]{3}{*}{ Other and Root Vegetables } & & \multirow{2}{*}{$\begin{array}{c}2008 \\
\text { Harvested } \\
\text { Area }\end{array}$} & $\begin{array}{l}\text { GRWPA, } \\
\text { GRWP }\end{array}$ & $\begin{array}{l}\text { GRWPA, } \\
\text { GRWP }\end{array}$ & $\begin{array}{l}\text { GRWPA, } \\
\text { GRWP }\end{array}$ \\
\hline & & & $\begin{array}{c}2009 \\
\text { Minimum }\end{array}$ & $\begin{array}{c}2009 \\
\text { Maximum }\end{array}$ & $\begin{array}{c}2009 \\
\text { Average }\end{array}$ \\
\hline & & ac & $\mathrm{d}$ & $\mathrm{d}$ & $\mathrm{d}$ \\
\hline Carrots fresh, fall & California & ND & 122 & 153 & 138 \\
\hline Carrots fresh, fall & \multirow[t]{2}{*}{ Texas } & \multirow[t]{2}{*}{ ND } & 92 & 153 & 123 \\
\hline AVERAGE, Carrots, fresh fall & & & 107 & 153 & 130 \\
\hline Tomatoes fresh, winter & Florida & 37,617 & 60 & 90 & 75 \\
\hline Tomatoes fresh, spring & California & see below & 106 & 122 & 112 \\
\hline Tomatoes fresh, spring & Florida & see above & 99 & 121 & 109 \\
\hline Tomatoes fresh, spring & \multirow[t]{2}{*}{ Texas } & \multirow[t]{2}{*}{256} & 121 & 150 & 140 \\
\hline $\begin{array}{l}\text { AVERAGE, Tomatoes, winter, } \\
\text { spring }\end{array}$ & & & 96 & 121 & 109 \\
\hline Tomatoes fresh, summer & Alabama & 1,145 & 106 & 122 & 114 \\
\hline Tomatoes fresh, summer & Arkansas & 2,349 & 61 & 143 & 93 \\
\hline Tomatoes fresh, summer & California & 234,239 & 93 & 122 & 107 \\
\hline Tomatoes fresh, summer & Georgia & 2,496 & 75 & 107 & 89 \\
\hline Tomatoes fresh, summer & Indiana & 3,979 & 61 & 122 & 93 \\
\hline Tomatoes fresh, summer & Michigan & 6,952 & 80 & 138 & 106 \\
\hline Tomatoes fresh, summer & New Jersey & 1,997 & 82 & 148 & 112 \\
\hline Tomatoes fresh, summer & New York & 492 & 65 & 122 & 95 \\
\hline Tomatoes fresh, summer & North Carolina & 852 & 61 & 132 & 97 \\
\hline Tomatoes fresh, summer & Ohio & 3,034 & 50 & 77 & 59 \\
\hline Tomatoes fresh, summer & Pennsylvania & 1,918 & 62 & 138 & 101 \\
\hline Tomatoes fresh, summer & South Carolina & 2,837 & 76 & 92 & 87 \\
\hline Tomatoes fresh, summer & Tennessee & 1,896 & 70 & 97.5 & 86 \\
\hline Tomatoes fresh, summer & \multirow[t]{2}{*}{ Virginia } & \multirow[t]{2}{*}{886} & 82 & 122 & 96 \\
\hline AVERAGE, Tomatoes, summer & & & 73 & 120 & 95 \\
\hline Tomatoes fresh, fall & California & see above & 91 & 152 & 112 \\
\hline Tomatoes fresh, fall & Florida & see above & 61 & 77 & 68 \\
\hline Tomatoes fresh, fall & Georgia & see above & 78 & 107 & 92 \\
\hline Tomatoes fresh, fall & \multirow[t]{2}{*}{ Ohio } & \multirow[t]{2}{*}{ see above } & 61 & 114 & 87 \\
\hline AVERAGE, Tomatoes, fall & & & 73 & 113 & 90 \\
\hline Potatoes, fall & Colorado & 55.2 & 127 & 139 & 136 \\
\hline Potatoes, fall & Idaho & 319 & 131 & 147 & 147 \\
\hline Potatoes, fall & Michigan & 43.5 & 99 & 142 & 124 \\
\hline Potatoes, fall & Minnesota & 45 & 117 & 135 & 131 \\
\hline Potatoes, fall & Montana & 9.7 & 128 & 140 & 133 \\
\hline Potatoes, fall & Maine & 55.5 & 120 & 130 & 129 \\
\hline Potatoes, fall & Massachusetts & 3.4 & 98 & 145 & 125 \\
\hline Potatoes, fall & Rhode Island & 0.4 & 98 & 131 & 120 \\
\hline Potatoes, fall & New Mexico & 6.4 & 134 & 163 & 156 \\
\hline
\end{tabular}


PNNL-21950, Appendix F

Table F.8. (contd)

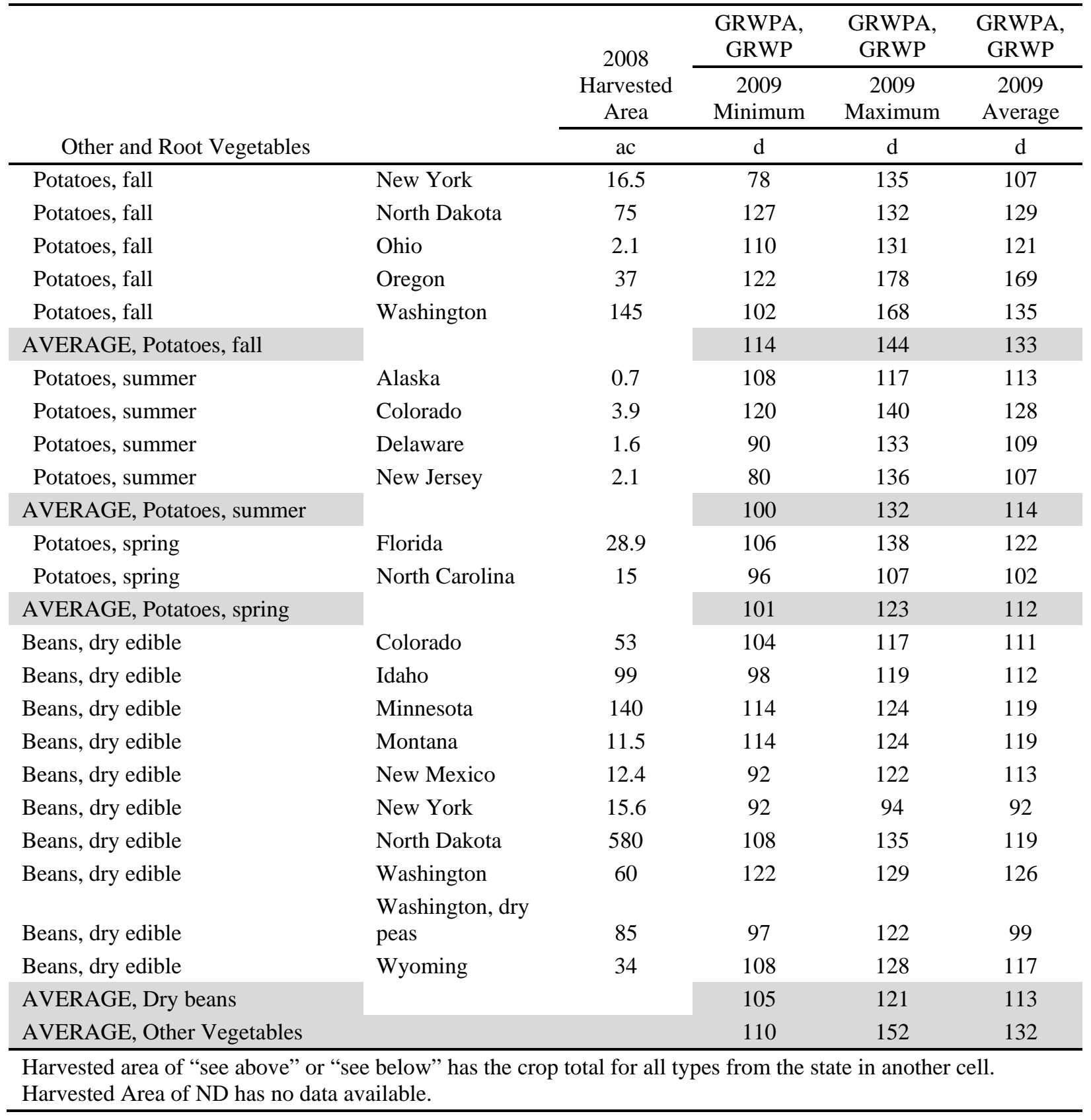


Table F.9. Fruits Modeled as Root/Other Vegetables Growing Periods

\begin{tabular}{|c|c|c|c|c|c|}
\hline \multirow[b]{2}{*}{$\begin{array}{c}\text { Other and Root Vegetables (melon } \\
\text { and strawberries) }\end{array}$} & & \multirow{2}{*}{$\begin{array}{c}2008 \\
\text { Harvested } \\
\text { Area }\end{array}$} & \multirow{2}{*}{$\begin{array}{c}\text { GRWPA, } \\
\text { GRWP } \\
2009 \\
\text { Minimum }\end{array}$} & \multirow{2}{*}{$\begin{array}{c}\text { GRWPA, } \\
\text { GRWP } \\
2009 \\
\text { Maximum }\end{array}$} & \multirow{2}{*}{$\begin{array}{c}\text { GRWPA } \\
\text { GRWP } \\
2009 \\
\text { Average }\end{array}$} \\
\hline & & & & & \\
\hline Cantaloupes fresh spring & Arizona & ND & 76 & 90 & 83 \\
\hline Cantaloupes fresh spring & California & ND & 89.5 & 135 & 112 \\
\hline Cantaloupes fresh spring & Georgia & ND & 75 & 107 & 89 \\
\hline Cantaloupes fresh spring & \multirow[t]{2}{*}{ Texas } & \multirow[t]{2}{*}{ ND } & 61 & 122 & 92 \\
\hline AVERAGE, Cantaloupe, spring & & & 75 & 114 & 94 \\
\hline Cantaloupes fresh summer & California & ND & 93 & 121 & 104 \\
\hline Cantaloupes fresh summer & Colorado & ND & 101 & 138 & 117 \\
\hline Cantaloupes fresh summer & Indiana & ND & 66 & 92 & 79 \\
\hline Cantaloupes fresh summer & Maryland & ND & 105 & 138 & 122 \\
\hline Cantaloupes fresh summer & Pennsylvania & ND & 71 & 92 & 81 \\
\hline Cantaloupes fresh summer & South Carolina & ND & 77 & 92 & 82 \\
\hline Cantaloupes fresh summer & \multirow[t]{2}{*}{ Texas } & \multirow[t]{2}{*}{ ND } & 61 & 107 & 87 \\
\hline AVERAGE, Cantaloupe, summer & & & 82 & 111 & 96 \\
\hline Cantaloupes fresh fall & Arizona & ND & 91 & 92 & 91 \\
\hline Cantaloupes fresh fall & \multirow[t]{2}{*}{ California } & \multirow[t]{2}{*}{ ND } & 91 & 106 & 96 \\
\hline AVERAGE, Cantaloupe, fall & & & 91 & 99 & 94 \\
\hline Strawberries winter & Florida & 10,184 & 66 & 166 & 118 \\
\hline Strawberries spring & California & 74,362 & 114 & 183 & 146 \\
\hline Strawberries spring & New York & 13,012 & 38 & 51 & 45 \\
\hline Strawberries spring & North Carolina & 105 & 181 & 221 & 203 \\
\hline Strawberries spring & Ohio & 468 & 36 & 40 & 38 \\
\hline Strawberries spring & \multirow[t]{2}{*}{ Oregon } & \multirow[t]{2}{*}{17,275} & 51 & 62 & 55 \\
\hline $\begin{array}{l}\text { AVERAGE, Strawberries, winter, } \\
\text { spring }\end{array}$ & & & 81 & 121 & 101 \\
\hline Strawberries summer & California & see above & 91 & 183 & 139 \\
\hline Strawberries summer & Michigan & 17,714 & 60.5 & 61 & 61 \\
\hline Strawberries summer & Pennsylvania & 826 & 56 & 70 & 64 \\
\hline Strawberries summer & Washington & N/A & 61 & 66 & 64 \\
\hline Strawberries summer & \multirow[t]{3}{*}{ Wisconsin } & \multirow[t]{3}{*}{123} & 44 & 46 & 45 \\
\hline AVERAGE, Strawberries, summer & & & 63 & 85 & 75 \\
\hline AVERAGE, “Fruit” Vegetables & & & 77 & 108 & 92 \\
\hline
\end{tabular}


PNNL-21950, Appendix F

\section{References}

USDA. 2010. Field Crops, Usual Planting and Harvesting Dates. Handbook Number 628, USDA National Agricultural Statistics Service, Washington, D.C. Last accessed at:

http://usda.mannlib.cornell.edu/MannUsda/viewDocumentInfo.do?documentID=1251 (documentID varies according to which table accessed) 


\section{Appendix G}

\section{Biomass and Yield}




\section{Appendix G}

\section{Biomass and Yield Tables}

The data presented in this Appendix provide detailed values for biomass and yield values for various food and feed categories. The detailed values can be evaluated to approximate values appropriate to the location of interest. It is unlikely that one feed or food is consumed over the year, so generalizations are necessary. The best approximation of the annual value would be entered in the Exposure module.

The Gv2 standing biomass is the total above-ground plant mass (wet weight) used to estimate interception fractions for wet and dry deposition.

The Gv2 yield of each crop type gives the total annual production of edible crop mass (wet weight) per unit area of farmland. The yield is used to calculate the harvest removal losses from the soil. Radionuclide removal due to harvesting is modeled when opted on the Agriculture/General tab. This parameter only impacts scenarios that evaluate exposures which occur more than one-year after the release.

\begin{tabular}{clccc}
\hline & & \multicolumn{3}{c}{ Feed or Forage Consumed } \\
\cline { 3 - 5 } Type & Animal Product & Grain & Hay & Grass \\
\hline Feed & Meat & $\mathrm{X}$ & & \\
& Poultry & $\mathrm{X}$ & & \\
& Milk & & $\mathrm{X}$ & \\
\multirow{5}{*}{ Forage } & Egg & $\mathrm{X}$ & & \\
& Meat & & $\mathrm{X}$ & \\
& Milk & & & $\mathrm{X}$ \\
\hline
\end{tabular}

Table G.1. Leafy Vegetable Biomass

Table G.2. Root/Other Vegetable Biomass

Table G.3. Fruit Biomass

Table G.4. Evergreen Tree Biomass

Table G.5. [caption] 
Table G.1. Leafy Vegetable Biomass

\begin{tabular}{|c|c|c|c|c|c|c|c|c|c|c|c|c|}
\hline Gv2_module & Gv2_ID & Gv2_Units & Gv2_Description & $\begin{array}{l}\text { Food } \\
\text { Type }\end{array}$ & Reference & Units Cited & Comment & $\begin{array}{c}\text { Data } \\
\text { Reported }\end{array}$ & $\begin{array}{l}\text { Average } \\
\text { Estimate }\end{array}$ & Minimum & Maximum & Units \\
\hline EXPOSURE & BIOMAS & kg_wet $/ \mathrm{m}^{2}$ & $\begin{array}{l}\text { Biomass for } \\
\text { Leafy Vegetables }\end{array}$ & 1.Leafy & $\begin{array}{l}\text { From: } \\
\text { http://usda.mannlib.cornell.edu/ } \\
\text { MannUsda/viewDocumentInfo. } \\
\text { do?documentID=1212. Last } \\
\text { accessed 7/18/12. Table 006, } \\
\text { Table 005. }\end{array}$ & $\begin{array}{l}1000 \mathrm{cwt} \text {, ac } \\
\text { (calculated to } \\
\text { lb/ac) }\end{array}$ & $\begin{array}{l}\text { Head lettuce } \\
\text { (U.S. average, } \\
\text { 1998-2010) }\end{array}$ & $\begin{array}{l}\text { Assumed } \\
\text { biomass } \\
\text { equals } \\
\text { half the } \\
\text { yield }\end{array}$ & 2.04 & 1.85 & 2.13 & kg_wet $/ \mathrm{m}^{2}$ \\
\hline EXPOSURE & BIOMAS & kg_wet $/ \mathrm{m}^{3}$ & $\begin{array}{l}\text { Biomass for } \\
\text { Leafy Vegetables }\end{array}$ & 1.Leafy & $\begin{array}{l}\text { From: } \\
\text { http://usda.mannlib.cornell.edu/ } \\
\text { MannUsda/viewDocumentInfo. } \\
\text { do?documentID=1212. Last } \\
\text { accessed 7/18/12. Table 006, } \\
\text { Table 005. }\end{array}$ & $\begin{array}{l}1000 \mathrm{cwt} \text {, ac } \\
\text { (calculated to } \\
\text { lb/ac) }\end{array}$ & $\begin{array}{l}\text { All lettuce, } \\
\text { cabbage, } \\
\text { broccoli, } \\
\text { spinach } \\
\text { (U.S. average, } \\
\text { 1998-2010) }\end{array}$ & $\begin{array}{l}\text { Assumed } \\
\text { biomass } \\
\text { equals } \\
\text { yield }\end{array}$ & 2.67 & 1.35 & 4.25 & kg_wet $/ \mathrm{m}^{2}$ \\
\hline EXPOSURE & BIOMAS & kg_wet $/ \mathrm{m}^{4}$ & $\begin{array}{l}\text { Biomass for } \\
\text { Leafy Vegetables }\end{array}$ & 1.Leafy & $\begin{array}{l}\text { From: } \\
\text { http://usda.mannlib.cornell.edu/ } \\
\text { MannUsda/viewDocumentInfo. } \\
\text { do?documentID=1212. Last } \\
\text { accessed 7/18/12. Table 006, } \\
\text { Table 005. }\end{array}$ & $\begin{array}{l}1000 \mathrm{cwt}, \mathrm{ac} \\
\text { (calculated to } \\
\text { lb/ac) }\end{array}$ & $\begin{array}{l}\text { Spinach } \\
\text { (U.S. average, } \\
\text { 1998-2010) }\end{array}$ & $\begin{array}{l}\text { Assumed } \\
\text { biomass } \\
\text { equals } \\
\text { yield }\end{array}$ & 1.71 & 1.42 & 2.09 & kg_wet $/ \mathrm{m}^{2}$ \\
\hline
\end{tabular}


Table G.2. Root/Other Vegetable Biomass

\begin{tabular}{|c|c|c|c|c|c|c|c|c|c|c|c|c|}
\hline Gv2_module & Gv2_ID & Gv2_Units & Gv2_Description & $\begin{array}{l}\text { Food } \\
\text { Type }\end{array}$ & Reference & Units Cited & Comment & $\begin{array}{c}\text { Data } \\
\text { Reported }\end{array}$ & $\begin{array}{l}\text { Average } \\
\text { Estimate }\end{array}$ & Minimum & Maximum & Units \\
\hline EXPOSURE & BIOMAS & kg_wet $/ \mathrm{m}^{2}$ & $\begin{array}{l}\text { Biomass for } \\
\text { root/other } \\
\text { vegetables }\end{array}$ & 2.other & $\begin{array}{l}\text { From: } \\
\text { http://usda.mannlib.cornell.edu } \\
\text { /MannUsda/viewDocumentInf } \\
\text { o.do?documentID=1212. Last } \\
\text { accessed 7/18/12. Table 006, } \\
\text { Table 005. }\end{array}$ & $\begin{array}{l}1000 \text { cwt, ac } \\
\text { (calculated } \\
\text { to lb/ac) }\end{array}$ & 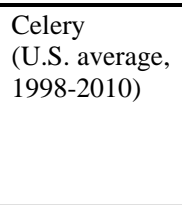 & $\begin{array}{l}\text { Assumed } \\
\text { biomass } \\
\text { equals } \\
\text { half the } \\
\text { yield }\end{array}$ & 3.90 & 3.71 & 3.99 & kg_wet $/ \mathrm{m}^{2}$ \\
\hline EXPOSURE & BIOMAS & kg_wet $/ \mathrm{m}^{2}$ & $\begin{array}{l}\text { Biomass for } \\
\text { root/other } \\
\text { vegetables }\end{array}$ & 2.other & $\begin{array}{l}\text { From: } \\
\text { http://usda.mannlib.cornell.edu } \\
\text { /MannUsda/viewDocumentInf } \\
\text { o.do?documentID=1212. Last } \\
\text { accessed 7/18/12. Table 006, } \\
\text { Table 005. }\end{array}$ & $\begin{array}{l}1000 \mathrm{cwt} \text {, ac } \\
\text { (calculated } \\
\text { to lb/ac) }\end{array}$ & $\begin{array}{l}\text { Onion, all } \\
\text { (U.S. average, } \\
\text { 1998-2010) }\end{array}$ & $\begin{array}{l}\text { Assumed } \\
\text { biomass } \\
\text { equals } \\
\text { half the } \\
\text { yield }\end{array}$ & 2.55 & 2.21 & 2.81 & kg_wet $/ \mathrm{m}^{2}$ \\
\hline EXPOSURE & BIOMAS & kg_wet $/ \mathrm{m}^{2}$ & $\begin{array}{l}\text { Biomass for } \\
\text { root/other } \\
\text { vegetables }\end{array}$ & 2.other & $\begin{array}{l}\text { From: } \\
\text { http://usda.mannlib.cornell.edu } \\
\text { /MannUsda/viewDocumentInf } \\
\text { o.do?documentID=1377. Last } \\
\text { accessed 7/17/12 SFS; } \\
\text { Table D-8. Strawberries }\end{array}$ & $\begin{array}{l}1000 \text { cwt; ac } \\
\text { (calculated } \\
\text { to lb/ac) }\end{array}$ & $\begin{array}{l}\text { Strawberries } \\
\text { (U.S. average, } \\
\text { 1998-2010) }\end{array}$ & $\begin{array}{l}\text { Assumed } \\
\text { biomass } \\
\text { equals } \\
\text { half the } \\
\text { yield }\end{array}$ & 2.42 & 2.03 & 2.81 & kg_wet $/ \mathrm{m}^{2}$ \\
\hline EXPOSURE & BIOMAS & kg_wet $/ \mathrm{m}^{2}$ & $\begin{array}{l}\text { Biomass for } \\
\text { root/other } \\
\text { vegetables }\end{array}$ & 2.other & $\begin{array}{l}\text { From: } \\
\text { http://usda.mannlib.cornell.edu } \\
\text { /MannUsda/viewDocumentInf } \\
\text { o.do?documentID=1235. Last } \\
\text { accessed 7/17/12 SFS. Table } 1\end{array}$ & $\begin{array}{l}1000 \mathrm{cwt} \text {; } \\
\text { harvested } \\
1000 \mathrm{ac} \\
\text { (calculated } \\
\text { to lb/ac) }\end{array}$ & $\begin{array}{l}\text { Fall potatoes } \\
\text { (U.S. average, } \\
\text { 1998-2006). } \\
\text { Assumed leaf } \\
\text { mass } \\
\text { negligible } \\
\text { compared to } \\
\text { potato mass. }\end{array}$ & $\begin{array}{l}\text { Assumed } \\
\text { biomass } \\
\text { equals } \\
\text { half the } \\
\text { yield }\end{array}$ & 2.15 & 2.00 & 2.28 & kg_wet $/ \mathrm{m}^{2}$ \\
\hline EXPOSURE & BIOMAS & kg_wet/m² & $\begin{array}{l}\text { Biomass for } \\
\text { root/other } \\
\text { vegetables }\end{array}$ & 2.other & $\begin{array}{l}\text { From: } \\
\text { http://usda.mannlib.cornell.edu } \\
\text { /MannUsda/viewDocumentInf } \\
\text { o.do?documentID=1235. Last } \\
\text { accessed 7/17/12 SFS. Table } 1\end{array}$ & $\begin{array}{l}\text { calculated } \\
\text { wtd average } \\
\text { of winter, } \\
\text { spring, } \\
\text { summer, fall } \\
\text { potatoes; } \\
\text { wtd by } \\
\text { acreage }\end{array}$ & $\begin{array}{l}\text { Total potatoes } \\
\text { (U.S. average, } \\
\text { 1998-2006). } \\
\text { Assumed leaf } \\
\text { mass } \\
\text { negligible } \\
\text { compared to } \\
\text { potato mass. }\end{array}$ & $\begin{array}{l}\text { Assumed } \\
\text { biomass } \\
\text { equals } \\
\text { half the } \\
\text { yield }\end{array}$ & 2.06 & 1.91 & 2.20 & kg_wet $/ \mathrm{m}^{2}$ \\
\hline
\end{tabular}


Table G.2. (contd)

\begin{tabular}{|c|c|c|c|c|c|c|c|c|c|c|c|c|}
\hline Gv2_module & Gv2_ID & Gv2_Units & Gv2_Description & $\begin{array}{l}\text { Food } \\
\text { Type }\end{array}$ & Reference & Units Cited & Comment & $\begin{array}{c}\text { Data } \\
\text { Reported }\end{array}$ & $\begin{array}{l}\text { Average } \\
\text { Estimate }\end{array}$ & Minimum & Maximum & Units \\
\hline EXPOSURE & BIOMAS & kg_wet $/ \mathrm{m}^{2}$ & $\begin{array}{l}\text { Biomass for } \\
\text { root/other } \\
\text { vegetables }\end{array}$ & 2.other & $\begin{array}{l}\text { From: } \\
\text { http://usda.mannlib.cornell.edu } \\
\text { /MannUsda/viewDocumentInf } \\
\text { o.do?documentID=1235. Last } \\
\text { accessed 7/17/12 SFS. Table } 1\end{array}$ & $\begin{array}{l}1000 \mathrm{cwt} \text {; } \\
\text { harvested } \\
1000 \mathrm{ac} \\
\text { (calculated } \\
\text { to lb/ac) }\end{array}$ & $\begin{array}{l}\text { Summer } \\
\text { potatoes } \\
\text { (U.S. average, } \\
\text { 1998-2006). } \\
\text { Assumed leaf } \\
\text { mass } \\
\text { negligible } \\
\text { compared to } \\
\text { potato mass. }\end{array}$ & $\begin{array}{l}\text { Assumed } \\
\text { biomass } \\
\text { equals } \\
\text { half the } \\
\text { yield }\end{array}$ & 1.77 & 1.57 & 1.92 & kg_wet $/ \mathrm{m}^{2}$ \\
\hline EXPOSURE & BIOMAS & kg_wet $/ \mathrm{m}^{2}$ & $\begin{array}{l}\text { Biomass for } \\
\text { root/other } \\
\text { vegetables }\end{array}$ & 2.other & $\begin{array}{l}\text { From: } \\
\text { http://usda.mannlib.cornell.edu } \\
\text { /MannUsda/viewDocumentInf } \\
\text { o.do?documentID=1212. Last } \\
\text { accessed 7/18/12. Table 006, } \\
\text { Table 005. }\end{array}$ & $\begin{array}{l}1000 \mathrm{cwt} \text {, ac } \\
\text { (calculated } \\
\text { to lb/ac) }\end{array}$ & $\begin{array}{l}\text { Carrots } \\
\text { (U.S. average, } \\
\text { 1998-2010) }\end{array}$ & $\begin{array}{l}\text { Assumed } \\
\text { biomass } \\
\text { equals } \\
\text { half the } \\
\text { yield }\end{array}$ & 1.75 & 1.61 & 1.88 & kg_wet $/ \mathrm{m}^{2}$ \\
\hline EXPOSURE & BIOMAS & kg_wet/m² & $\begin{array}{l}\text { Biomass for } \\
\text { root/other } \\
\text { vegetables }\end{array}$ & 2.other & $\begin{array}{l}\text { From: } \\
\text { http://usda.mannlib.cornell.edu } \\
\text { /MannUsda/viewDocumentInf } \\
\text { o.do?documentID=1212. Last } \\
\text { accessed 7/18/12. Table 006, } \\
\text { Table 005. }\end{array}$ & $\begin{array}{l}1000 \mathrm{cwt} \text {, ac } \\
\text { (calculated } \\
\text { to lb/ac) }\end{array}$ & $\begin{array}{l}\text { Tomatoes } \\
\text { (U.S. average, } \\
\text { 1998-2010) }\end{array}$ & $\begin{array}{l}\text { Assumed } \\
\text { biomass } \\
\text { equals } \\
\text { half the } \\
\text { yield }\end{array}$ & 1.66 & 1.51 & 1.75 & kg_wet $/ \mathrm{m}^{2}$ \\
\hline EXPOSURE & BIOMAS & kg_wet/m² & $\begin{array}{l}\text { Biomass for } \\
\text { root/other } \\
\text { vegetables }\end{array}$ & 2.other & $\begin{array}{l}\text { From: } \\
\text { http://usda.mannlib.cornell.edu } \\
\text { /MannUsda/viewDocumentInf } \\
\text { o.do?documentID=1212. Last } \\
\text { accessed 7/18/12. Table 006, } \\
\text { Table 005. }\end{array}$ & $\begin{array}{l}1000 \mathrm{cwt} \text {, ac } \\
\text { (calculated } \\
\text { to lb/ac) }\end{array}$ & $\begin{array}{l}\text { Bell peppers } \\
\text { (U.S. average, } \\
\text { 1998-2010) }\end{array}$ & $\begin{array}{l}\text { Assumed } \\
\text { biomass } \\
\text { equals } \\
\text { half the } \\
\text { yield }\end{array}$ & 1.64 & 1.44 & 1.84 & kg_wet $/ \mathrm{m}^{2}$ \\
\hline EXPOSURE & BIOMAS & kg_wet $/ \mathrm{m}^{2}$ & $\begin{array}{l}\text { Biomass for } \\
\text { root/other } \\
\text { vegetables }\end{array}$ & 2.other & $\begin{array}{l}\text { From: } \\
\text { http://usda.mannlib.cornell.edu } \\
\text { /MannUsda/viewDocumentInf } \\
\text { o.do?documentID=1235. Last } \\
\text { accessed 7/17/12 SFS. Table } 1\end{array}$ & $\begin{array}{l}1000 \mathrm{cwt} \text {; } \\
\text { harvested } \\
1000 \mathrm{ac} \\
\text { (calculated } \\
\text { to lb/ac) }\end{array}$ & $\begin{array}{l}\text { Spring } \\
\text { potatoes } \\
\text { (U.S. average, } \\
\text { 1998-2006). } \\
\text { Assumed leaf } \\
\text { mass } \\
\text { negligible } \\
\text { compared to } \\
\text { potato mass. }\end{array}$ & $\begin{array}{l}\text { Assumed } \\
\text { biomass } \\
\text { equals } \\
\text { half the } \\
\text { yield }\end{array}$ & 1.59 & 1.31 & 1.76 & kg_wet $/ \mathrm{m}^{2}$ \\
\hline EXPOSURE & BIOMAS & kg_wet/m² & $\begin{array}{l}\text { Biomass for } \\
\text { root/other } \\
\text { vegetables }\end{array}$ & 2.other & $\begin{array}{l}\text { From: } \\
\text { http://usda.mannlib.cornell.edu } \\
\text { /MannUsda/viewDocumentInf } \\
\text { o.do?documentID=1212. Last } \\
\text { accessed 7/18/12. Table 006, } \\
\text { Table 005. }\end{array}$ & $\begin{array}{l}1000 \mathrm{cwt} \text {, ac } \\
\text { (calculated } \\
\text { to lb/ac) }\end{array}$ & $\begin{array}{l}\text { Watermelon } \\
\text { (U.S. average, } \\
\text { 1998-2010) }\end{array}$ & $\begin{array}{l}\text { Assumed } \\
\text { biomass } \\
\text { equals } \\
\text { half the } \\
\text { yield }\end{array}$ & 1.52 & 1.23 & 1.79 & kg_wet $/ \mathrm{m}^{2}$ \\
\hline
\end{tabular}


Table G.2. (contd)

\begin{tabular}{|c|c|c|c|c|c|c|c|c|c|c|c|c|}
\hline Gv2_module & Gv2_ID & Gv2_Units & Gv2_Description & $\begin{array}{l}\text { Food } \\
\text { Type }\end{array}$ & Reference & Units Cited & Comment & $\begin{array}{c}\text { Data } \\
\text { Reported }\end{array}$ & $\begin{array}{l}\text { Average } \\
\text { Estimate }\end{array}$ & Minimum & Maximum & Units \\
\hline EXPOSURE & BIOMAS & kg_wet $/ \mathrm{m}^{3}$ & $\begin{array}{l}\text { Biomass for } \\
\text { root/other } \\
\text { vegetables }\end{array}$ & 2.other & $\begin{array}{l}\text { From: } \\
\text { http://usda.mannlib.cornell.edu } \\
\text { /MannUsda/viewDocumentInf } \\
\text { o.do?documentID=1235. Last } \\
\text { accessed 7/17/12 SFS. Table } 1\end{array}$ & $\begin{array}{l}1000 \mathrm{cwt} ; \\
\text { harvested } \\
1000 \mathrm{ac} \\
\text { (calculated } \\
\text { to lb/ac) }\end{array}$ & $\begin{array}{l}\text { Winter } \\
\text { potatoes } \\
\text { (U.S. average, } \\
\text { 1998-2006). } \\
\text { Assumed leaf } \\
\text { mass } \\
\text { negligible } \\
\text { compared to } \\
\text { potato mass. }\end{array}$ & $\begin{array}{l}\text { Assumed } \\
\text { biomass } \\
\text { equals } \\
\text { half the } \\
\text { yield }\end{array}$ & 1.45 & 1.12 & 1.65 & kg_wet $/ \mathrm{m}^{2}$ \\
\hline EXPOSURE & BIOMAS & kg_wet $/ \mathrm{m}^{4}$ & $\begin{array}{l}\text { Biomass for } \\
\text { root/other } \\
\text { vegetables }\end{array}$ & 2.other & $\begin{array}{l}\text { From: } \\
\text { http://usda.mannlib.cornell.edu } \\
\text { /MannUsda/viewDocumentInf } \\
\text { o.do?documentID=1212. Last } \\
\text { accessed 7/18/12 sfs. } \\
\text { Table } 003 \text {. }\end{array}$ & $\begin{array}{l}1 \mathrm{E} 6 \mathrm{cwt} / \\
1000 \mathrm{ac} \\
\text { (calculated } \\
\text { to lb/ac) }\end{array}$ & $\begin{array}{l}\text { All melons, } \\
\text { (U.S. average, } \\
\text { 1998-2010) }\end{array}$ & $\begin{array}{l}\text { Assumed } \\
\text { biomass } \\
\text { equals } \\
\text { half the } \\
\text { yield }\end{array}$ & 1.44 & 1.21 & 1.65 & kg_wet $/ \mathrm{m}^{2}$ \\
\hline EXPOSURE & BIOMAS & kg_wet $/ \mathrm{m}^{5}$ & $\begin{array}{l}\text { Biomass for } \\
\text { root/other } \\
\text { vegetables }\end{array}$ & 2.other & $\begin{array}{l}\text { From: } \\
\text { http://usda.mannlib.cornell.edu } \\
\text { /MannUsda/viewDocumentInf } \\
\text { o.do?documentID=1212. Last } \\
\text { accessed 7/18/12. Table 006, } \\
\text { Table 005. }\end{array}$ & $\begin{array}{l}1000 \mathrm{cwt} \text {, ac } \\
\text { (calculated } \\
\text { to lb/ac) }\end{array}$ & $\begin{array}{l}\text { Cantaloupe } \\
\text { (U.S. average, } \\
\text { 1998-2010) }\end{array}$ & $\begin{array}{l}\text { Assumed } \\
\text { biomass } \\
\text { equals } \\
\text { half the } \\
\text { yield }\end{array}$ & 1.38 & 1.17 & 1.55 & kg_wet $/ \mathrm{m}^{2}$ \\
\hline EXPOSURE & BIOMAS & kg_wet $/ \mathrm{m}^{6}$ & $\begin{array}{l}\text { Biomass for } \\
\text { root/other } \\
\text { vegetables }\end{array}$ & 2.other & $\begin{array}{l}\text { From: } \\
\text { http://usda.mannlib.cornell.edu } \\
\text { /MannUsda/viewDocumentInf } \\
\text { o.do?documentID=1212. Last } \\
\text { accessed 7/18/12. Table 006, } \\
\text { Table 005. }\end{array}$ & $\begin{array}{l}1000 \mathrm{cwt} \text {, ac } \\
\text { (calculated } \\
\text { to lb/ac) }\end{array}$ & $\begin{array}{l}\text { Pumpkins } \\
\text { (U.S. average, } \\
\text { 1998-2010) }\end{array}$ & $\begin{array}{l}\text { Assumed } \\
\text { biomass } \\
\text { equals } \\
\text { half the } \\
\text { yield }\end{array}$ & 1.29 & 1.16 & 1.41 & kg_wet $/ \mathrm{m}^{2}$ \\
\hline EXPOSURE & BIOMAS & kg_wet $/ \mathrm{m}^{7}$ & $\begin{array}{l}\text { Biomass for } \\
\text { root/other } \\
\text { vegetables }\end{array}$ & 2.other & $\begin{array}{l}\text { From: } \\
\text { http://usda.mannlib.cornell.edu } \\
\text { /MannUsda/viewDocumentInf } \\
\text { o.do?documentID=1212. Last } \\
\text { accessed 7/18/12. Table 006, } \\
\text { Table 005. }\end{array}$ & $\begin{array}{l}1000 \mathrm{cwt} \text {, ac } \\
\text { (calculated } \\
\text { to lb/ac) }\end{array}$ & $\begin{array}{l}\text { Honeydew } \\
\text { (U.S. average, } \\
\text { 1998-2010) }\end{array}$ & $\begin{array}{l}\text { Assumed } \\
\text { biomass } \\
\text { equals } \\
\text { half the } \\
\text { yield }\end{array}$ & 1.21 & 1.05 & 1.35 & kg_wet $/ \mathrm{m}^{2}$ \\
\hline EXPOSURE & BIOMAS & kg_wet $/ \mathrm{m}^{8}$ & $\begin{array}{l}\text { Biomass for } \\
\text { root/other } \\
\text { vegetables }\end{array}$ & 2.other & $\begin{array}{l}\text { From: } \\
\text { http://usda.mannlib.cornell.edu } \\
\text { /MannUsda/viewDocumentInf } \\
\text { o.do?documentID=1212. Last } \\
\text { accessed 7/18/12. Table 006, } \\
\text { Table 005. }\end{array}$ & $\begin{array}{l}1000 \mathrm{cwt} \text {, ac } \\
\text { (calculated } \\
\text { to lb/ac) }\end{array}$ & $\begin{array}{l}\text { Cucumbers } \\
\text { (U.S. average, } \\
\text { 1998-2010) }\end{array}$ & $\begin{array}{l}\text { Assumed } \\
\text { biomass } \\
\text { equals } \\
\text { half the } \\
\text { yield }\end{array}$ & 1.07 & 0.97 & 1.17 & kg_wet $/ \mathrm{m}^{2}$ \\
\hline
\end{tabular}


Table G.2. (contd)

\begin{tabular}{|c|c|c|c|c|c|c|c|c|c|c|c|c|}
\hline Gv2_module & Gv2_ID & Gv2_Units & Gv2_Description & $\begin{array}{l}\text { Food } \\
\text { Type }\end{array}$ & Reference & Units Cited & Comment & $\begin{array}{c}\text { Data } \\
\text { Reported }\end{array}$ & $\begin{array}{l}\text { Average } \\
\text { Estimate }\end{array}$ & Minimum & Maximum & Units \\
\hline EXPOSURE & BIOMAS & kg_wet $/ \mathrm{m}^{9}$ & $\begin{array}{l}\text { Biomass for } \\
\text { root/other } \\
\text { vegetables }\end{array}$ & 2.other & $\begin{array}{l}\text { From: } \\
\text { http://usda.mannlib.cornell.edu } \\
\text { /MannUsda/viewDocumentInf } \\
\text { o.do?documentID=1212. Last } \\
\text { accessed 7/18/12. Table 006, } \\
\text { Table 005. }\end{array}$ & $\begin{array}{l}1000 \mathrm{cwt}, \mathrm{ac} \\
\text { (calculated } \\
\text { to lb/ac) }\end{array}$ & $\begin{array}{l}\text { Broccoli } \\
\text { (U.S. average, } \\
\text { 1998-2010) }\end{array}$ & $\begin{array}{l}\text { Assumed } \\
\text { biomass } \\
\text { equals } \\
\text { yield }\end{array}$ & 1.55 & 1.35 & 1.73 & kg_wet $/ \mathrm{m}^{2}$ \\
\hline EXPOSURE & BIOMAS & kg_wet/m ${ }^{10}$ & $\begin{array}{l}\text { Biomass for } \\
\text { root/other } \\
\text { vegetables }\end{array}$ & 2.other & $\begin{array}{l}\text { From: } \\
\text { http://usda.mannlib.cornell.edu } \\
\text { /MannUsda/viewDocumentInf } \\
\text { o.do?documentID=1212. Last } \\
\text { accessed 7/18/12. Table 006, } \\
\text { Table } 005 \text {. }\end{array}$ & $\begin{array}{l}1000 \mathrm{cwt} \text {, ac } \\
\text { (calculated } \\
\text { to lb/ac) }\end{array}$ & $\begin{array}{l}\text { Snap beans } \\
\text { (U.S. average, } \\
\text { 1998-2010) }\end{array}$ & $\begin{array}{l}\text { Assumed } \\
\text { biomass } \\
\text { equals } \\
\text { yield }\end{array}$ & 0.69 & 0.62 & 0.76 & kg_wet $/ \mathrm{m}^{2}$ \\
\hline EXPOSURE & BIOMAS & kg_wet $/ \mathrm{m}^{11}$ & $\begin{array}{l}\text { Biomass for } \\
\text { root/other } \\
\text { vegetables }\end{array}$ & 2.other & $\begin{array}{l}\text { From: } \\
\text { http://usda.mannlib.cornell.edu } \\
\text { /MannUsda/viewDocumentInf } \\
\text { o.do?documentID=1377. Last } \\
\text { accessed 7/18/12. sfs. } \\
\text { Table D-2. }\end{array}$ & $\mathrm{lb} / \mathrm{ac}$ & $\begin{array}{l}\text { Blueberries } \\
\text { (AL, AR, CA, } \\
\text { FL, GA, IN, } \\
\text { MI, MS, NJ, } \\
\text { NY, NC, OR, } \\
\text { WA averages, } \\
\text { mostly } \\
\text { 1998-2010) }\end{array}$ & $\begin{array}{l}\text { Assumed } \\
\text { biomass } \\
\text { equals } \\
\text { yield }\end{array}$ & 0.49 & 0.32 & 0.64 & kg_wet $/ \mathrm{m}^{2}$ \\
\hline EXPOSURE & BIOMAS & kg_wet $/ \mathrm{m}^{12}$ & $\begin{array}{l}\text { Biomass for } \\
\text { root/other } \\
\text { vegetables }\end{array}$ & 2.other & $\begin{array}{l}\text { From: } \\
\text { http://usda.mannlib.cornell.edu } \\
\text { /MannUsda/viewDocumentInf } \\
\text { o.do?documentID=1212. Last } \\
\text { accessed 7/18/12. Table 006, } \\
\text { Table 005. }\end{array}$ & $\begin{array}{l}1000 \mathrm{cwt} \text {, ac } \\
\text { (calculated } \\
\text { to lb/ac) }\end{array}$ & $\begin{array}{l}\text { Asparagus } \\
\text { (U.S. average, } \\
\text { 1998-2010) }\end{array}$ & $\begin{array}{l}\text { Assumed } \\
\text { biomass } \\
\text { equals } \\
\text { yield }\end{array}$ & 0.24 & 0.19 & 0.28 & kg_wet $/ \mathrm{m}^{2}$ \\
\hline
\end{tabular}


Table G.3. Fruit Biomass

\begin{tabular}{|c|c|c|c|c|c|c|c|c|c|c|}
\hline Gv2_module & Gv2_ID & Gv2_Units & Gv2_Description & $\begin{array}{l}\text { Food } \\
\text { Type }\end{array}$ & Reference & Units Cited & Comment & Data Reported & $\begin{array}{l}\text { Average } \\
\text { Estimate }\end{array}$ & Units \\
\hline EXPOSURE & BIOMAS & kg_wet/m² & $\begin{array}{l}\text { Biomass for Fruit } \\
\text { trees }\end{array}$ & 3.fruit & Jenkins et al. (2004) & $<$ calculation> & $\begin{array}{l}\text { Tree foliage biomass }-15 \mathrm{~cm} \\
\text { diameter tree. Average of Aspen, soft } \\
\text { maple, mixed hardwood values. }\end{array}$ & $\begin{array}{l}\text { Results from } \\
\text { calculations } \\
\text { of foliage } \\
\text { mass }\end{array}$ & 2.19 & kg_wet $/ \mathrm{m}^{2}$ \\
\hline EXPOSURE & BIOMAS & kg_wet $/ \mathrm{m}^{2}$ & $\begin{array}{l}\text { Biomass for Fruit } \\
\text { trees }\end{array}$ & 3.fruit & Jenkins et al. (2004) & $<$ calculation> & $\begin{array}{l}\text { Tree foliage biomass }-20 \mathrm{~cm} \\
\text { diameter tree. Average of Aspen, soft } \\
\text { maple, mixed hardwood values. }\end{array}$ & $\begin{array}{l}\text { Results from } \\
\text { calculations } \\
\text { of foliage } \\
\text { mass }\end{array}$ & 2.80 & kg_wet $/ \mathrm{m}^{2}$ \\
\hline EXPOSURE & BIOMAS & kg_wet/m² & $\begin{array}{l}\text { Biomass for Fruit } \\
\text { trees }\end{array}$ & 3.fruit & Jenkins et al. (2004) & <calculation> & $\begin{array}{l}\text { Tree foliage biomass }-25 \mathrm{~cm} \\
\text { diameter tree. Average of Aspen, soft } \\
\text { maple, mixed hardwood values. }\end{array}$ & $\begin{array}{l}\text { Results from } \\
\text { calculations } \\
\text { of foliage } \\
\text { mass }\end{array}$ & 3.33 & kg_wet $/ \mathrm{m}^{2}$ \\
\hline EXPOSURE & BIOMAS & kg_wet/m² & $\begin{array}{l}\text { Biomass for Fruit } \\
\text { trees }\end{array}$ & 3.fruit & Jenkins et al. (2004) & $<$ calculation> & $\begin{array}{l}\text { Tree foliage biomass }-30 \mathrm{~cm} \\
\text { diameter tree. Average of Aspen, soft } \\
\text { maple, mixed hardwood values. }\end{array}$ & $\begin{array}{l}\text { Results from } \\
\text { calculations } \\
\text { of foliage } \\
\text { mass }\end{array}$ & 3.80 & kg_wet $/ \mathrm{m}^{2}$ \\
\hline
\end{tabular}

$\sqrt{9}$

\begin{tabular}{|c|c|c|c|c|c|c|c|c|c|c|}
\hline Gv2_module & Gv2_ID & Gv2_Units & Gv2_Description & $\begin{array}{l}\text { Food } \\
\text { Type }\end{array}$ & Reference & Units Cited & Comment & Data Reported & $\begin{array}{l}\text { Average } \\
\text { Estimate }\end{array}$ & Units \\
\hline EXPOSURE & BIOMAS & kg_wet $/ \mathrm{m}^{2}$ & $\begin{array}{l}\text { Biomass for Fruit } \\
\text { trees }\end{array}$ & 3.fruit & Jenkins et al. (2004) & $<$ calculation> & $\begin{array}{l}\text { Model forested areas as fruit-tree } \\
\text { covered area. Douglas fir values. } \\
15-30 \mathrm{~cm} \text { diameter trees. }\end{array}$ & $\begin{array}{l}\text { Results from } \\
\text { calculations } \\
\text { of foliage } \\
\text { mass }\end{array}$ & 5.30 & kg_wet $/ \mathrm{m}^{2}$ \\
\hline EXPOSURE & BIOMAS & kg_wet $/ \mathrm{m}^{2}$ & $\begin{array}{l}\text { Biomass for Fruit } \\
\text { trees }\end{array}$ & 3.fruit & Jenkins et al. (2004) & $<$ calculation $>$ & $\begin{array}{l}\text { Model forested areas as fruit-tree } \\
\text { covered area. True Fir and Spruce. } \\
15-30 \mathrm{~cm} \text { diameter trees. }\end{array}$ & $\begin{array}{l}\text { Results from } \\
\text { calculations } \\
\text { of foliage } \\
\text { mass }\end{array}$ & 4.40 & kg_wet $/ \mathrm{m}^{2}$ \\
\hline EXPOSURE & BIOMAS & kg_wet $/ \mathrm{m}^{2}$ & $\begin{array}{l}\text { Biomass for Fruit } \\
\text { trees }\end{array}$ & 3.fruit & Jenkins et al. (2004) & $<$ calculation> & $\begin{array}{l}\text { Model forested areas as fruit-tree } \\
\text { covered area. Pine. } 15-30 \mathrm{~cm} \\
\text { diameter trees. }\end{array}$ & $\begin{array}{l}\text { Results from } \\
\text { calculations } \\
\text { of foliage } \\
\text { mass }\end{array}$ & 3.80 & kg_wet $/ \mathrm{m}^{2}$ \\
\hline
\end{tabular}

Table G.4. Evergreen Tree Biomass 
Table G.5. [contd]

\begin{tabular}{|c|c|c|c|c|c|c|c|c|c|c|c|c|c|}
\hline Gv2_module & Gv2_ID & Gv2_Units & Gv2_Description & $\begin{array}{l}\text { Food } \\
\text { Type }\end{array}$ & $\begin{array}{l}\text { Data Cited } \\
\text { Crop }\end{array}$ & Reference & Units Cited & Comment & $\begin{array}{c}\text { Data } \\
\text { Reported }\end{array}$ & Average & $\begin{array}{l}\text { Min } \\
\text { Value }\end{array}$ & $\begin{array}{l}\text { Max } \\
\text { Value }\end{array}$ & Units \\
\hline EXPOSURE & YELD & kg_wet $/ \mathrm{m}^{2}$ & Food crop yield & 1.leafy & $\begin{array}{l}\text { Head lettuce } \\
\text { (U.S. average, } \\
\text { 1998-2010) }\end{array}$ & $\begin{array}{l}\text { From: } \\
\text { http://usda.mannlib.cornell.ed } \\
\text { u/MannUsda/viewDocumentIn } \\
\text { fo.do?documentID=1212. } \\
\text { Last accessed 7/18/12. } \\
\text { Table 006, Table 005. }\end{array}$ & $\begin{array}{l}1000 \mathrm{cwt} \text {, ac } \\
\text { (calculated } \\
\text { to lb/ac) }\end{array}$ & & & 4.08 & 3.7 & 4.25 & kg_wet $/ \mathrm{m}^{2}$ \\
\hline EXPOSURE & YELD & kg_wet $/ \mathrm{m}^{2}$ & Food crop yield & 1.leafy & $\begin{array}{l}\text { All lettuce, } \\
\text { cabbage, } \\
\text { broccoli, } \\
\text { spinach } \\
\text { (U.S. average, } \\
\text { 1998-2010) }\end{array}$ & $\begin{array}{l}\text { From: } \\
\text { http://usda.mannlib.cornell.ed } \\
\text { u/MannUsda/viewDocumentIn } \\
\text { fo.do?documentID=1212. } \\
\text { Last accessed 7/18/12. } \\
\text { Table 006, Table 005. }\end{array}$ & $\begin{array}{l}1000 \mathrm{cwt} \text {, ac } \\
\text { (calculated } \\
\text { to lb/ac) }\end{array}$ & & & 2.67 & 1.35 & 4.25 & kg_wet $/ \mathrm{m}^{2}$ \\
\hline EXPOSURE & YELD & kg_wet $/ \mathrm{m}^{2}$ & Food crop yield & 1.leafy & $\begin{array}{l}\text { Spinach } \\
\text { (U.S. average, } \\
\text { 1998-2010) }\end{array}$ & $\begin{array}{l}\text { From: } \\
\text { http://usda.mannlib.cornell.ed } \\
\text { u/MannUsda/viewDocumentIn } \\
\text { fo.do?documentID=1212. } \\
\text { Last accessed 7/18/12. } \\
\text { Table 006, Table 005. }\end{array}$ & $\begin{array}{l}1000 \mathrm{cwt} \text {, ac } \\
\text { (calculated } \\
\text { to lb/ac) }\end{array}$ & & & 1.71 & 1.42 & 2.09 & kg_wet $/ \mathrm{m}^{2}$ \\
\hline EXPOSURE & YELD & kg_wet $/ \mathrm{m}^{2}$ & Food crop yield & 2.other & $\begin{array}{l}\text { Celery } \\
\text { (U.S. average, } \\
\text { 1998-2010) }\end{array}$ & $\begin{array}{l}\text { From: } \\
\text { http://usda.mannlib.cornell.ed } \\
\text { u/MannUsda/viewDocumentIn } \\
\text { fo.do?documentID=1212. } \\
\text { Last accessed 7/18/12. } \\
\text { Table 006, Table 005. }\end{array}$ & $\begin{array}{l}1000 \mathrm{cwt} \text {, ac } \\
\text { (calculated } \\
\text { to } \mathrm{lb} / \mathrm{ac} \text { ) }\end{array}$ & & & 7.79 & 7.42 & 7.98 & kg_wet $/ \mathrm{m}^{2}$ \\
\hline EXPOSURE & YELD & kg_as_harvested/m² & Food crop yield & 2.other & $\begin{array}{l}\text { Onion, all } \\
\text { (U.S. average, } \\
\text { 1998-2010) }\end{array}$ & $\begin{array}{l}\text { From: } \\
\text { http://usda.mannlib.cornell.ed } \\
\text { u/MannUsda/viewDocumentIn } \\
\text { fo.do?documentID=1212. } \\
\text { Last accessed 7/18/12. } \\
\text { Table 006, Table 005. }\end{array}$ & $\begin{array}{l}1000 \mathrm{cwt} \text {, ac } \\
\text { (calculated } \\
\text { to lb/ac) }\end{array}$ & & & 5.1 & 4.41 & 5.61 & kg_wet $/ \mathrm{m}^{2}$ \\
\hline EXPOSURE & YELD & kg_fruit_wet $/ \mathrm{m}^{2}$ & Food crop yield & 2.other & $\begin{array}{l}\text { strawberries } \\
\text { (U.S. average, } \\
\text { 1998-2010) }\end{array}$ & $\begin{array}{l}\text { From: } \\
\text { http://usda.mannlib.cornell.ed } \\
\text { u/MannUsda/viewDocumentIn } \\
\text { fo.do?documentID=1377. } \\
\text { Last accessed 7/17/12 SFS; } \\
\text { Table D-8. Strawberries. }\end{array}$ & $\begin{array}{l}1000 \mathrm{cwt} \text {, ac } \\
\text { (calculated } \\
\text { to lb/ac) }\end{array}$ & & $\begin{array}{l}\text { annual, } \\
\text { U.S. totals, } \\
\text { ac, } \\
1000 \mathrm{cwt}\end{array}$ & 4.83 & 4.05 & 5.61 & kg_wet $/ \mathrm{m}^{2}$ \\
\hline EXPOSURE & YELD & kg_wet $/ \mathrm{m}^{2}$ & Food crop yield & 2.other & $\begin{array}{l}\text { fall potatoes } \\
\text { (U.S. average, } \\
\text { 1998-2006) }\end{array}$ & $\begin{array}{l}\text { From: } \\
\text { http://usda.mannlib.cornell.ed } \\
\text { u/MannUsda/viewDocumentIn } \\
\text { fo.do?documentID=1235. } \\
\text { Last accessed 7/17/12 SFS. } \\
\text { Table 1. }\end{array}$ & $\begin{array}{l}1000 \mathrm{cwt} \\
\text { harvested } \\
1000 \mathrm{ac} \\
\text { (calculated } \\
\text { to lb/ac) }\end{array}$ & $\begin{array}{l}\text { Assumed } \\
\text { leaf mass } \\
\text { negligible } \\
\text { compared } \\
\text { to potato } \\
\text { mass. }\end{array}$ & $\begin{array}{l}\text { annual, } \\
\text { U.S. totals, } \\
\text { ac, } \\
1000 \text { cwt. } \\
68-88 \% \\
\text { are fall } \\
\text { potatoes }\end{array}$ & 4.29 & 4 & 4.55 & kg_wet $/ \mathrm{m}^{2}$ \\
\hline
\end{tabular}


Table G.5. (contd)

\begin{tabular}{|c|c|c|c|c|c|c|c|c|c|c|c|c|c|}
\hline Gv2_module & Gv2_ID & Gv2_Units & Gv2_Description & $\begin{array}{l}\text { Food } \\
\text { Type }\end{array}$ & $\begin{array}{l}\text { Data Cited } \\
\text { Crop }\end{array}$ & Reference & Units Cited & Comment & $\begin{array}{c}\text { Data } \\
\text { Reported }\end{array}$ & Average & $\begin{array}{l}\text { Min } \\
\text { Value }\end{array}$ & $\begin{array}{c}\text { Max } \\
\text { Value }\end{array}$ & Units \\
\hline EXPOSURE & YELD & kg_wet $/ \mathrm{m}^{2}$ & Food crop yield & 2.other & $\begin{array}{l}\text { total potatoes } \\
\text { (U.S. average, } \\
\text { 1998-2006) }\end{array}$ & $\begin{array}{l}\text { From: } \\
\text { http://usda.mannlib.cornell.ed } \\
\text { u/MannUsda/viewDocumentIn } \\
\text { fo.do?documentID=1235. } \\
\text { Last accessed 7/17/12 SFS. } \\
\text { Table } 1 .\end{array}$ & $\begin{array}{l}\text { calculated } \\
\text { wtd average } \\
\text { of winter, } \\
\text { spring, } \\
\text { summer, fall } \\
\text { potatoes; } \\
\text { wtd by } \\
\text { acreage }\end{array}$ & $\begin{array}{l}\text { Assumed } \\
\text { leaf mass } \\
\text { negligible } \\
\text { compared } \\
\text { to potato } \\
\text { mass. }\end{array}$ & $\begin{array}{l}\text { annual, } \\
\text { U.S. totals, } \\
\text { ac, } \\
1000 \mathrm{cwt}, \\
\text { all } \\
\text { potatoes }\end{array}$ & 4.12 & 3.81 & 4.39 & kg_wet $/ \mathrm{m}^{2}$ \\
\hline EXPOSURE & YELD & kg_wet $/ \mathrm{m}^{2}$ & Food crop yield & 2.other & $\begin{array}{l}\text { summer } \\
\text { potatoes } \\
\text { (U.S. average, } \\
\text { 1998-2006) }\end{array}$ & $\begin{array}{l}\text { From: } \\
\text { http://usda.mannlib.cornell.ed } \\
\text { u/MannUsda/viewDocumentIn } \\
\text { fo.do?documentID=1235. } \\
\text { Last accessed 7/17/12 SFS. } \\
\text { Table } 1 .\end{array}$ & $\begin{array}{l}1000 \text { cwt; } \\
\text { harvested } \\
1000 \mathrm{ac} \\
\text { (calculated } \\
\text { to lb/ac) }\end{array}$ & $\begin{array}{l}\text { Assumed } \\
\text { leaf mass } \\
\text { negligible } \\
\text { compared } \\
\text { to potato } \\
\text { mass. }\end{array}$ & $\begin{array}{l}\text { annual, } \\
\text { U.S. totals, } \\
\text { ac, } \\
1000 \mathrm{cwt}\end{array}$ & 3.53 & 3.13 & 3.83 & kg_wet $/ \mathrm{m}^{2}$ \\
\hline EXPOSURE & YELD & kg_wet $/ \mathrm{m}^{2}$ & Food crop yield & 2.other & $\begin{array}{l}\text { Carrots } \\
\text { (U.S. average, } \\
\text { 1998-2010) }\end{array}$ & $\begin{array}{l}\text { From: } \\
\text { http://usda.mannlib.cornell.ed } \\
\text { u/MannUsda/viewDocumentIn } \\
\text { fo.do?documentID=1212. } \\
\text { Last accessed 7/18/12. } \\
\text { Table 006, Table 005. }\end{array}$ & $\begin{array}{l}1000 \mathrm{cwt} \text {, ac } \\
\text { (calculated } \\
\text { to lb/ac) }\end{array}$ & & & 3.5 & 3.21 & 3.75 & kg_wet $/ \mathrm{m}^{2}$ \\
\hline EXPOSURE & YELD & kg_fruit_wet $/ \mathrm{m}^{2}$ & Food crop yield & 2.other & $\begin{array}{l}\text { Tomatoes } \\
\text { (U.S. average, } \\
\text { 1998-2010) }\end{array}$ & $\begin{array}{l}\text { From: } \\
\text { http://usda.mannlib.cornell.ed } \\
\text { u/MannUsda/viewDocumentIn } \\
\text { fo.do?documentID=1212. } \\
\text { Last accessed 7/18/12. } \\
\text { Table 006, Table 005. }\end{array}$ & $\begin{array}{l}1000 \text { cwt, ac } \\
\text { (calculated } \\
\text { to lb/ac) }\end{array}$ & & & 3.31 & 3.01 & 3.49 & kg_wet $/ \mathrm{m}^{2}$ \\
\hline EXPOSURE & YELD & kg_fruit_wet $/ \mathrm{m}^{2}$ & Food crop yield & 2.other & $\begin{array}{l}\text { Bell peppers } \\
\text { (U.S. average, } \\
\text { 1998-2010) }\end{array}$ & $\begin{array}{l}\text { From: } \\
\text { http://usda.mannlib.cornell.ed } \\
\text { u/MannUsda/viewDocumentIn } \\
\text { fo.do?documentID=1212. } \\
\text { Last accessed 7/18/12. } \\
\text { Table 006, Table 005. }\end{array}$ & $\begin{array}{l}1000 \mathrm{cwt} \text {, ac } \\
\text { (calculated } \\
\text { to lb/ac) }\end{array}$ & & & 3.28 & 2.88 & 3.68 & kg_wet $/ \mathrm{m}^{2}$ \\
\hline EXPOSURE & YELD & kg_wet $/ \mathrm{m}^{2}$ & Food crop yield & 2.other & $\begin{array}{l}\text { spring potatoes } \\
\text { (U.S. average, } \\
\text { 1998-2006) }\end{array}$ & $\begin{array}{l}\text { From: } \\
\text { http://usda.mannlib.cornell.ed } \\
\text { u/MannUsda/viewDocumentIn } \\
\text { fo.do?documentID=1235. } \\
\text { Last accessed 7/17/12 SFS. } \\
\text { Table } 1 \text {. }\end{array}$ & $\begin{array}{l}1000 \mathrm{cwt} \text {; } \\
\text { harvested } \\
1000 \mathrm{ac} \\
\text { (calculated } \\
\text { to lb/ac) }\end{array}$ & $\begin{array}{l}\text { Assumed } \\
\text { leaf mass } \\
\text { negligible } \\
\text { compared } \\
\text { to potato } \\
\text { mass. }\end{array}$ & $\begin{array}{l}\text { annual, } \\
\text { U.S. totals, } \\
\text { ac, } \\
1000 \text { cwt }\end{array}$ & 3.17 & 2.62 & 3.52 & kg_wet $/ \mathrm{m}^{2}$ \\
\hline EXPOSURE & YELD & kg_fruit_wet $/ \mathrm{m}^{2}$ & Food crop yield & 2.other & $\begin{array}{l}\text { Watermelon } \\
\text { (U.S. average, } \\
\text { 1998-2010) }\end{array}$ & $\begin{array}{l}\text { From: } \\
\text { http://usda.mannlib.cornell.ed } \\
\text { u/MannUsda/viewDocumentIn } \\
\text { fo.do?documentID=1212. } \\
\text { Last accessed 7/18/12. } \\
\text { Table 006, Table 005. }\end{array}$ & $\begin{array}{l}1000 \mathrm{cwt} \text {, ac } \\
\text { (calculated } \\
\text { to lb/ac) }\end{array}$ & & & 3.03 & 2.45 & 3.57 & kg_wet $/ \mathrm{m}^{2}$ \\
\hline
\end{tabular}


Table G.5. (contd)

\begin{tabular}{|c|c|c|c|c|c|c|c|c|c|c|c|c|c|}
\hline Gv2_module & Gv2_ID & Gv2_Units & Gv2_Description & $\begin{array}{l}\text { Food } \\
\text { Type }\end{array}$ & $\begin{array}{l}\text { Data Cited } \\
\text { Crop }\end{array}$ & Reference & Units Cited & Comment & $\begin{array}{c}\text { Data } \\
\text { Reported }\end{array}$ & Average & $\begin{array}{c}\text { Min } \\
\text { Value }\end{array}$ & $\begin{array}{l}\text { Max } \\
\text { Value }\end{array}$ & Units \\
\hline EXPOSURE & YELD & kg_wet $/ \mathrm{m}^{2}$ & Food crop yield & 2.other & $\begin{array}{l}\text { winter potatoes } \\
\text { (U.S. average, } \\
\text { 1998-2006) }\end{array}$ & $\begin{array}{l}\text { From: } \\
\text { http://usda.mannlib.cornell.ed } \\
\text { u/MannUsda/viewDocumentIn } \\
\text { fo.do?documentID=1235. } \\
\text { Last accessed 7/17/12 SFS. } \\
\text { Table 1. }\end{array}$ & $\begin{array}{l}1000 \mathrm{cwt} ; \\
\text { harvested } \\
1000 \mathrm{ac} \\
\text { (calculated } \\
\text { to lb/ac) }\end{array}$ & $\begin{array}{l}\text { Assumed } \\
\text { leaf mass } \\
\text { negligible } \\
\text { compared } \\
\text { to potato } \\
\text { mass. }\end{array}$ & $\begin{array}{l}\text { annual, } \\
\text { U.S. totals, } \\
\text { ac, } \\
1000 \text { cwt }\end{array}$ & 2.9 & 2.23 & 3.29 & kg_wet $/ \mathrm{m}^{2}$ \\
\hline EXPOSURE & YELD & kg_fruit_wet $/ \mathrm{m}^{2}$ & Food crop yield & 2.other & $\begin{array}{l}\text { All melons, } \\
\text { (U.S. average, } \\
\text { 1998-2010) }\end{array}$ & $\begin{array}{l}\text { From: } \\
\text { http://usda.mannlib.cornell.ed } \\
\text { u/MannUsda/viewDocumentIn } \\
\text { fo.do?documentID=1212. } \\
\text { Last accessed 7/18/12 sfs. } \\
\text { Table 003. }\end{array}$ & $\begin{array}{l}1 \mathrm{E} 6 \mathrm{cwt} / \\
1000 \mathrm{ac} \\
\text { (calculated } \\
\text { to lb/ac) }\end{array}$ & & $\begin{array}{l}\text { annual } \\
\text { melons, } \\
1 \mathrm{e} 6 \mathrm{cwt} \\
1000 \mathrm{ac}\end{array}$ & 2.88 & 2.41 & 3.29 & kg_wet $/ \mathrm{m}^{2}$ \\
\hline EXPOSURE & YELD & kg_fruit_wet $/ \mathrm{m}^{2}$ & Food crop yield & 2.other & $\begin{array}{l}\text { Cantaloupe } \\
\text { (U.S. average, } \\
\text { 1998-2010) }\end{array}$ & $\begin{array}{l}\text { From: } \\
\text { http://usda.mannlib.cornell.ed } \\
\text { u/MannUsda/viewDocumentIn } \\
\text { fo.do?documentID=1212. } \\
\text { Last accessed 7/18/12. } \\
\text { Table 006, Table 005. }\end{array}$ & $\begin{array}{l}1000 \mathrm{cwt} \text {, ac } \\
\text { (calculated } \\
\text { to lb/ac) }\end{array}$ & & & 2.75 & 2.34 & 3.1 & kg_wet $/ \mathrm{m}^{2}$ \\
\hline EXPOSURE & YELD & kg_fruit_wet $/ \mathrm{m}^{2}$ & Food crop yield & 2.other & $\begin{array}{l}\text { Pumpkins } \\
\text { (U.S. average, } \\
\text { 1998-2010) }\end{array}$ & $\begin{array}{l}\text { From: } \\
\text { http://usda.mannlib.cornell.ed } \\
\text { u/MannUsda/viewDocumentIn } \\
\text { fo.do?documentID=1212. } \\
\text { Last accessed 7/18/12. } \\
\text { Table 006, Table 005. }\end{array}$ & $\begin{array}{l}1000 \mathrm{cwt} \text {, ac } \\
\text { (calculated } \\
\text { to lb/ac) }\end{array}$ & & & 2.57 & 2.31 & 2.82 & kg_wet $/ \mathrm{m}^{2}$ \\
\hline EXPOSURE & YELD & kg_fruit_wet $/ \mathrm{m}^{2}$ & Food crop yield & 2.other & $\begin{array}{l}\text { Honeydew } \\
\text { (U.S. average, } \\
\text { 1998-2010) }\end{array}$ & $\begin{array}{l}\text { From: } \\
\text { http://usda.mannlib.cornell.ed } \\
\text { u/MannUsda/viewDocumentIn } \\
\text { fo.do?documentID=1212. } \\
\text { Last accessed 7/18/12. } \\
\text { Table 006, Table 005. }\end{array}$ & $\begin{array}{l}1000 \mathrm{cwt} \text {, ac } \\
\text { (calculated } \\
\text { to lb/ac) }\end{array}$ & & & 2.42 & 2.1 & 2.7 & kg_wet $/ \mathrm{m}^{2}$ \\
\hline EXPOSURE & YELD & kg_fruit_wet $/ \mathrm{m}^{2}$ & Food crop yield & 2.other & $\begin{array}{l}\text { Cucumbers } \\
\text { (U.S. average, } \\
\text { 1998-2010) }\end{array}$ & $\begin{array}{l}\text { From: } \\
\text { http://usda.mannlib.cornell.ed } \\
\text { u/MannUsda/viewDocumentIn } \\
\text { fo.do?documentID=1212. } \\
\text { Last accessed 7/18/12. } \\
\text { Table 006, Table 005. }\end{array}$ & $\begin{array}{l}1000 \mathrm{cwt} \text {, ac } \\
\text { (calculated } \\
\text { to lb/ac) }\end{array}$ & & & 2.14 & 1.93 & 2.34 & kg_wet $/ \mathrm{m}^{2}$ \\
\hline EXPOSURE & YELD & kg_wet $/ \mathrm{m}^{2}$ & Food crop yield & 2.other & $\begin{array}{l}\text { Broccoli } \\
\text { (U.S. average, } \\
\text { 1998-2010) }\end{array}$ & $\begin{array}{l}\text { From: } \\
\text { http://usda.mannlib.cornell.ed } \\
\text { u/MannUsda/viewDocumentIn } \\
\text { fo.do?documentID=1212. } \\
\text { Last accessed 7/18/12. } \\
\text { Table 006, Table 005. }\end{array}$ & $\begin{array}{l}1000 \mathrm{cwt} \text {, ac } \\
\text { (calculated } \\
\text { to lb/ac) }\end{array}$ & & $\begin{array}{l}1000 \mathrm{cwt} \text {, } \\
\text { ac. }\end{array}$ & 1.55 & 1.35 & 1.73 & kg_wet $/ \mathrm{m}^{2}$ \\
\hline
\end{tabular}


Table G.5. (contd)

\begin{tabular}{|c|c|c|c|c|c|c|c|c|c|c|c|c|c|}
\hline Gv2_module & Gv2_ID & Gv2_Units & Gv2_Description & $\begin{array}{l}\text { Food } \\
\text { Type }\end{array}$ & $\begin{array}{l}\text { Data Cited } \\
\text { Crop }\end{array}$ & Reference & Units Cited & Comment & $\begin{array}{c}\text { Data } \\
\text { Reported }\end{array}$ & Average & $\begin{array}{l}\text { Min } \\
\text { Value }\end{array}$ & $\begin{array}{c}\text { Max } \\
\text { Value }\end{array}$ & Units \\
\hline EXPOSURE & YELD & kg_fruit_wet $/ \mathrm{m}^{2}$ & Food crop yield & 2.other & $\begin{array}{l}\text { Snap beans } \\
\text { (U.S. average, } \\
\text { 1998-2010) }\end{array}$ & $\begin{array}{l}\text { From: } \\
\text { http://usda.mannlib.cornell.ed } \\
\text { u/MannUsda/viewDocumentIn } \\
\text { fo.do?documentID=1212. } \\
\text { Last accessed 7/18/12. } \\
\text { Table 006, Table 005. }\end{array}$ & $\begin{array}{l}1000 \text { cwt, ac } \\
\text { (calculated } \\
\text { to lb/ac) }\end{array}$ & & & 0.689 & 0.624 & 0.756 & kg_wet $/ \mathrm{m}^{2}$ \\
\hline EXPOSURE & YELD & kg_fruit_wet $/ \mathrm{m}^{2}$ & Food crop yield & 2.other & $\begin{array}{l}\text { blueberries } \\
\text { (AL, AR, CA, } \\
\text { FL, GA, IN, } \\
\text { MI, MS, NJ, } \\
\text { NY, NC, OR, } \\
\text { WA, averages, } \\
\text { mostly } \\
\text { 1998-2010) }\end{array}$ & $\begin{array}{l}\text { From: } \\
\text { http://usda.mannlib.cornell.ed } \\
\text { u/MannUsda/viewDocumentIn } \\
\text { fo.do?documentID=1377. } \\
\text { Last accessed 7/18/12. sfs. } \\
\text { Table D-2. }\end{array}$ & $\mathrm{lb} / \mathrm{ac}$ & & $\begin{array}{l}\text { annual, } \\
\text { select } \\
\text { states } \\
\text { reporting, } \\
\text { lb/ac, } \\
\text { average } \\
\text { average, } \\
\text { avg min, } \\
\text { avg max }\end{array}$ & 0.489 & 0.323 & 0.637 & kg_wet $/ \mathrm{m}^{2}$ \\
\hline EXPOSURE & YELD & kg_wet $/ \mathrm{m}^{2}$ & Food crop yield & 2.other & $\begin{array}{l}\text { Asparagus } \\
\text { (U.S. average, } \\
\text { 1998-2010) }\end{array}$ & $\begin{array}{l}\text { From: } \\
\text { http://usda.mannlib.cornell.ed } \\
\text { u/MannUsda/viewDocumentIn } \\
\text { fo.do?documentID=1212. } \\
\text { Last accessed 7/18/12. } \\
\text { Table 006, Table 005. }\end{array}$ & $\begin{array}{l}1000 \text { cwt, ac } \\
\text { (calculated } \\
\text { to lb/ac) }\end{array}$ & & $\begin{array}{l}1000 \mathrm{cwt}, \\
\text { ac }\end{array}$ & 0.24 & 0.19 & 0.278 & kg_wet $/ \mathrm{m}^{2}$ \\
\hline EXPOSURE & YELD & kg_fruit_wet $/ \mathrm{m}^{2}$ & Food crop yield & 3. fruit & $\begin{array}{l}\text { peaches } \\
\text { (U.S. average, } \\
\text { 1998-2010) }\end{array}$ & $\begin{array}{l}\text { From: } \\
\text { http://usda.mannlib.cornell.ed } \\
\text { u/MannUsda/viewDocumentIn } \\
\text { fo.do?documentID=1377. } \\
\text { Last accessed 7/18/12 sfs. } \\
\text { Table B-24, Table A-4. }\end{array}$ & $\begin{array}{l}1 \mathrm{E} 6 \text { pounds; } \\
1000 \text { ac } \\
\text { (calculated } \\
\text { to lb/ac) }\end{array}$ & & $\begin{array}{l}\text { annual, } \\
\text { U.S. totals, } \\
\text { ac, 1E6 lbs }\end{array}$ & 1.94 & 1.68 & 2.19 & kg_wet $/ \mathrm{m}^{2}$ \\
\hline EXPOSURE & YELD & kg_fruit_wet $/ \mathrm{m}^{2}$ & Food crop yield & 3.fruit & $\begin{array}{l}\text { FL oranges - } \\
\text { assumed all } \\
\text { juice } \\
\text { (1998-2011 } \\
\text { harvest year) }\end{array}$ & $\begin{array}{l}\text { From: } \\
\text { http://usda.mannlib.cornell.ed } \\
\text { u/MannUsda/viewDocumentIn } \\
\text { fo.do?documentID=1377. } \\
\text { Last accessed 7/18/12 SFS. } \\
\text { Table C-19, Table C-21. }\end{array}$ & $\begin{array}{l}\text { short tons/ac } \\
\text { (FL) }\end{array}$ & $\begin{array}{l}\text { Juice } \\
\text { oranges - } \\
\text { Florida } \\
\text { only }\end{array}$ & $\begin{array}{l}\text { annual } \\
\text { oranges, } \\
\text { FL, ton/ac }\end{array}$ & 3.47 & 2.73 & 4.32 & kg_wet $/ \mathrm{m}^{2}$ \\
\hline EXPOSURE & YELD & kg_fruit_wet/m² & Food crop yield & 3.fruit & $\begin{array}{l}\text { apples } \\
\text { (U.S. average, } \\
\text { 1998-2010) }\end{array}$ & $\begin{array}{l}\text { From: } \\
\text { http://usda.mannlib.cornell.ed } \\
\text { u/MannUsda/viewDocumentIn } \\
\text { fo.do?documentID=1377. } \\
\text { Last accessed 7/17/12 SFS; } \\
\text { Table B-4 (Apples) and } \\
\text { Table A-4 (Bearing acreage). }\end{array}$ & $\begin{array}{l}1 \mathrm{E} 6 \text { pounds; } \\
1000 \text { ac } \\
\text { (calculated } \\
\text { to lb/ac) }\end{array}$ & & $\begin{array}{l}\text { annual, } \\
\text { U.S. totals, } \\
\text { ac, 1E6 lbs }\end{array}$ & 2.78 & 2.38 & 3.05 & kg_wet $/ \mathrm{m}^{2}$ \\
\hline
\end{tabular}


Table G.5. (contd)

\begin{tabular}{|c|c|c|c|c|c|c|c|c|c|c|c|c|c|}
\hline Gv2_module & Gv2_ID & Gv2_Units & Gv2_Description & $\begin{array}{l}\text { Food } \\
\text { Type }\end{array}$ & $\begin{array}{l}\text { Data Cited } \\
\text { Crop }\end{array}$ & Reference & Units Cited & Comment & $\begin{array}{c}\text { Data } \\
\text { Reported }\end{array}$ & Average & $\begin{array}{l}\text { Min } \\
\text { Value }\end{array}$ & $\begin{array}{c}\text { Max } \\
\text { Value }\end{array}$ & Units \\
\hline EXPOSURE & YELD & kg_fruit_wet $/ \mathrm{m}^{2}$ & Food crop yield & 3.fruit & $\begin{array}{l}\text { CA, AZ, TX } \\
\text { oranges - } \\
\text { assumed all } \\
\text { fresh } \\
\text { consumption } \\
\text { (1998-2010 } \\
\text { harvest year) }\end{array}$ & $\begin{array}{l}\text { From: } \\
\text { http://usda.mannlib.cornell.ed } \\
\text { u/MannUsda/viewDocumentIn } \\
\text { fo.do?documentID=1377. } \\
\text { Last accessed 7/18/12 SFS. } \\
\text { Table C-19, Table C-21. }\end{array}$ & $\begin{array}{l}\text { short tons/ac } \\
\text { (CA, TX, } \\
\text { AZ) }\end{array}$ & $\begin{array}{l}\text { Fresh } \\
\text { oranges - } \\
\text { CA, TX, } \\
\text { AZ only }\end{array}$ & $\begin{array}{l}\text { annual } \\
\text { oranges, } \\
\text { CA TX, } \\
\text { AZ, ton/ac }\end{array}$ & 1.84 & 1.45 & 2.59 & kg_wet $/ \mathrm{m}^{2}$ \\
\hline EXPOSURE & YELD & kg_fruit_wet $/ \mathrm{m}^{2}$ & Food crop yield & 3.fruit & $\begin{array}{l}\text { grapes } \\
\text { (U.S. average, } \\
\text { 1998-2009) }\end{array}$ & $\begin{array}{l}\text { From: } \\
\text { http://usda.mannlib.cornell.ed } \\
\text { u/MannUsda/viewDocumentIn } \\
\text { fo.do?documentID=1377. } \\
\text { Last accessed 7/18/12 SFS. } \\
\text { Table B-14, Table A-4. }\end{array}$ & $\begin{array}{l}1000 \text { short } \\
\text { tons; } \\
1000 \text { ac } \\
\text { (calculated } \\
\text { to lb/ac) }\end{array}$ & & $\begin{array}{l}\text { annual, } \\
\text { U.S. totals, } \\
\text { ac, } \\
1000 \text { short } \\
\text { tons. }\end{array}$ & 1.65 & 1.49 & 1.87 & kg_wet $/ \mathrm{m}^{2}$ \\
\hline EXPOSURE & YELD & kg_wet $/ \mathrm{m}^{2}$ & Food crop yield & 3.fruit & $\begin{array}{l}\text { bananas, } \\
\text { Hawaii } \\
\text { (1998 2010) }\end{array}$ & $\begin{array}{l}\text { From: } \\
\text { http://usda.mannlib.cornell.ed } \\
\text { u/MannUsda/viewDocumentIn } \\
\text { fo.do?documentID=1377. } \\
\text { Last accessed 7/17/12 SFS. } \\
\text { Table B-10. }\end{array}$ & & & $\begin{array}{l}\text { annual, } \\
\text { Hawaii, } \\
\text { ac, } \\
1000 \text { lbs }\end{array}$ & 0.0016 & 0.0012 & 0.0024 & kg_wet $/ \mathrm{m}^{2}$ \\
\hline EXPOSURE & YELD & kg_wet $/ \mathrm{m}^{2}$ & Food crop yield & 4.grain & $\begin{array}{l}\text { Sweet corn } \\
\text { (U.S. average, } \\
\text { 1998-2010) }\end{array}$ & $\begin{array}{l}\text { From: } \\
\text { http://usda.mannlib.cornell.ed } \\
\text { u/MannUsda/viewDocumentIn } \\
\text { fo.do?documentID=1212. } \\
\text { Last accessed 7/18/12. } \\
\text { Table 006, Table 005. }\end{array}$ & $\begin{array}{l}1000 \text { cwt, ac } \\
\text { (calculated } \\
\text { to lb/ac) }\end{array}$ & $\begin{array}{l}\text { Assumed } \\
\text { most of } \\
\text { stalk, } \\
\text { leaves also } \\
\text { harvested. } \\
\text { Assumed } \\
\text { stalk and } \\
\text { leaf mass } \\
\text { equals } \\
\text { edible corn } \\
\text { mass. }\end{array}$ & & 2.58 & 2.42 & 2.78 & kg_wet $/ \mathrm{m}^{2}$ \\
\hline
\end{tabular}




\section{References}

Jenkins JC, DC Chojnacky, LS Heath, and RA Birdsey. 2004. Comprehensive Database of Diameterbased Biomass Regressions for North American Tree Species. General Technical Report NE-319, Newtown Square, Pennsylvania.

USDA. 2010. Field Crops, Usual Planting and Harvesting Dates, Handbook Number 628, USDA National Agricultural Statistics Service, Washington, D.C. Last accessed at:

http://usda.mannlib.cornell.edu/MannUsda/viewDocumentInfo.do?documentID=1251 (documentID varies according to which table accessed). 


\section{Appendix $\mathrm{H}$}

\section{Dry-to-Wet Ratios}




\section{Appendix $\mathrm{H}$}

\section{Dry-to-Wet Ratios}

The dry-to-wet ratio for each food crop is used to convert between dry and wet weight bases. These data are also helpful when information found in open literature must be converted to the appropriate dryor wet-bases for model input. Aquatic plant concentrations are modeled solely from bioconcentration from water with no sediment-to-plant uptake, as done with terrestrial crop models. Therefore, no dry-towet ratios for aquatic foods are provided.

Table H.1. Meat Animal Feed

Table H.2. Poultry Feed

Table H.3. Milk Feed

Table H.4. Egg Layer Feed

Table H.5. Meat Animal Forage

Table H.6. Milk Forage

Table H.7. Leafy Vegetables

Table H.8. Other Vegetables

Table H.9. Fruit

Table H.10. Grains 
Table H.1. Meat Animal Feed

\begin{tabular}{|c|c|c|c|c|c|c|c|c|c|c|c|}
\hline Gv2_module & Gv2_ID & Gv2_Units $^{(a)}$ & Gv2_Description & Feed Type & Reference & $\begin{array}{l}\text { Values } \\
\text { Cited }\end{array}$ & Units Cited & Comment & $\begin{array}{c}\text { Data } \\
\text { Reported }\end{array}$ & $\begin{array}{l}\text { Value in } \\
\text { Gv2 } \\
\text { Units }\end{array}$ & Units $^{(a)}$ \\
\hline EXPOSURE & DRYFA2 & kg_dry/kg_as-fed & $\begin{array}{l}\text { Dry-to-wet conversion } \\
\text { factor for animal feed. }\end{array}$ & 1.meat animal feed & $\begin{array}{l}\text { Ensminger et al. } \\
\text { (1990, Table V-1) }\end{array}$ & 0.09 & $\begin{array}{l}\% \text { converted to } \\
\text { fraction }\end{array}$ & fresh turnip root & Datum & 0.09 & dry/wet \\
\hline EXPOSURE & DRYFA2 & kg_dry/kg_as-fed & $\begin{array}{l}\text { Dry-to-wet conversion } \\
\text { factor for animal feed. }\end{array}$ & 1.meat animal feed & IAEA (2009, Table 2) & 22 & $\begin{array}{l}\text { percent dry } \\
\text { matter, as fed }\end{array}$ & sugar beet & Datum & 0.22 & dry/wet \\
\hline EXPOSURE & DRYFA2 & kg_dry/kg_as-fed & $\begin{array}{l}\text { Dry-to-wet conversion } \\
\text { factor for animal feed. }\end{array}$ & 1.meat animal feed & IAEA (2009, Table 2) & 26 & $\begin{array}{l}\text { percent dry } \\
\text { matter, as fed }\end{array}$ & $\begin{array}{l}\text { alfalfa, vegetative } \\
\text { mass }\end{array}$ & Datum & 0.26 & dry/wet \\
\hline EXPOSURE & DRYFA2 & kg_dry/kg_as-fed & $\begin{array}{l}\text { Dry-to-wet conversion } \\
\text { factor for animal feed. }\end{array}$ & 1.meat animal feed & IAEA (2009, Table 3) & 0.26 & $\begin{array}{l}\text { dry matter } \\
\text { fraction, as fed }{ }^{(b)}\end{array}$ & grass silage & Datum & 0.26 & dry/wet \\
\hline EXPOSURE & DRYFA2 & kg_dry/kg_as-fed & $\begin{array}{l}\text { Dry-to-wet conversion } \\
\text { factor for animal feed. }\end{array}$ & 1.meat animal feed & IAEA (2009, Table 3) & 0.34 & $\begin{array}{l}\text { dry matter } \\
\text { fraction, as fed }{ }^{(b)}\end{array}$ & $\begin{array}{l}\text { lucerne silage (aka } \\
\text { alfalfa silage) }\end{array}$ & Datum & 0.34 & dry/wet \\
\hline EXPOSURE & DRYFA2 & kg_dry/kg_as-fed & $\begin{array}{l}\text { Dry-to-wet conversion } \\
\text { factor for animal feed. }\end{array}$ & 1.meat animal feed & $\begin{array}{l}\text { Ensminger et al. } \\
\text { (1990, Table V-1) }\end{array}$ & 0.5 & $\begin{array}{l}\% \text { converted to } \\
\text { fraction }\end{array}$ & fresh acorn & Datum & 0.5 & dry/wet \\
\hline EXPOSURE & DRYFA2 & kg_dry/kg_wet & $\begin{array}{l}\text { Dry-to-wet conversion } \\
\text { factor for food crops. }\end{array}$ & 1.meat animal feed & IAEA (2009, Table 2) & 85.2 & percent dry matter & maize (corn), grain & Datum & 0.85 & dry/wet \\
\hline EXPOSURE & DRYFA2 & kg_dry/kg_as-fed & $\begin{array}{l}\text { Dry-to-wet conversion } \\
\text { factor for animal feed. }\end{array}$ & 1.meat animal feed & IAEA (2009, Table 3) & 0.86 & $\begin{array}{l}\text { dry matter } \\
\text { fraction, as fed }{ }^{(b)}\end{array}$ & grass hay & Datum & 0.86 & dry/wet \\
\hline EXPOSURE & DRYFA2 & kg_dry/kg_as-fed & $\begin{array}{l}\text { Dry-to-wet conversion } \\
\text { factor for animal feed. }\end{array}$ & 1.meat animal feed & IAEA (2009, Table 3) & 0.86 & $\begin{array}{l}\text { dry matter } \\
\text { fraction, as fed }{ }^{(b)}\end{array}$ & $\begin{array}{l}\text { lucerne hay (aka } \\
\text { alfalfa hay) }\end{array}$ & Datum & 0.86 & dry/wet \\
\hline EXPOSURE & DRYFA2 & kg_dry/kg_wet & $\begin{array}{l}\text { Dry-to-wet conversion } \\
\text { factor for food crops. }\end{array}$ & 1.meat animal feed & IAEA (2009, Table 2) & 86.7 & percent dry matter & oats, grain & Datum & 0.87 & dry/wet \\
\hline EXPOSURE & DRYFA2 & kg_dry/kg_wet & $\begin{array}{l}\text { Dry-to-wet conversion } \\
\text { factor for food crops. }\end{array}$ & 1.meat animal feed & IAEA (2009, Table 2) & 87 & percent dry matter & winter rye, grain & Datum & 0.87 & dry/wet \\
\hline EXPOSURE & DRYFA2 & kg_dry/kg_wet & $\begin{array}{l}\text { Dry-to-wet conversion } \\
\text { factor for food crops. }\end{array}$ & 1.meat animal feed & IAEA (2009, Table 2) & 87 & percent dry matter & barley, grain & Datum & 0.87 & dry/wet \\
\hline EXPOSURE & DRYFA2 & kg_dry/kg_wet & $\begin{array}{l}\text { Dry-to-wet conversion } \\
\text { factor for food crops. }\end{array}$ & 1.meat animal feed & IAEA (2009, Table 2) & 87 & percent dry matter & sorghum, grain & Datum & 0.87 & dry/wet \\
\hline EXPOSURE & DRYFA2 & kg_dry/kg_as-fed & $\begin{array}{l}\text { Dry-to-wet conversion } \\
\text { factor for animal feed. }\end{array}$ & 1.meat animal feed & $\begin{array}{l}\text { Ensminger et al. } \\
\text { (1990, Table V-1) }\end{array}$ & 0.87 & $\begin{array}{l}\% \text { converted to } \\
\text { fraction }\end{array}$ & rye grain & Datum & 0.87 & dry/wet \\
\hline EXPOSURE & DRYFA2 & kg_dry/kg_as-fed & $\begin{array}{l}\text { Dry-to-wet conversion } \\
\text { factor for animal feed. }\end{array}$ & 1.meat animal feed & IAEA (2009, Table 3) & 0.88 & $\begin{array}{l}\text { dry matter } \\
\text { fraction, as fed }\end{array}$ & "concentrate feed" & Datum & 0.88 & dry/wet \\
\hline EXPOSURE & DRYFA2 & kg_dry/kg_wet & $\begin{array}{l}\text { Dry-to-wet conversion } \\
\text { factor for food crops. }\end{array}$ & 1.meat animal feed & IAEA (2009, Table 2) & 88 & percent dry matter & wheat, grain & Datum & 0.88 & dry/wet \\
\hline EXPOSURE & DRYFA2 & kg_dry/kg_as-fed & $\begin{array}{l}\text { Dry-to-wet conversion } \\
\text { factor for animal feed. }\end{array}$ & 1.meat animal feed & $\begin{array}{l}\text { Ensminger et al. } \\
\text { (1990, Table V-1) }\end{array}$ & 0.88 & $\begin{array}{l}\% \text { converted to } \\
\text { fraction }\end{array}$ & barley grain & Datum & 0.88 & dry/wet \\
\hline EXPOSURE & DRYFA2 & kg_dry/kg_as-fed & $\begin{array}{l}\text { Dry-to-wet conversion } \\
\text { factor for animal feed. }\end{array}$ & 1.meat animal feed & $\begin{array}{l}\text { Ensminger et al. } \\
\text { (1990, Table V-1) }\end{array}$ & 0.88 & $\begin{array}{l}\% \text { converted to } \\
\text { fraction }\end{array}$ & corn grain & Datum & 0.88 & dry/wet \\
\hline
\end{tabular}


Table H.1. (contd)

\begin{tabular}{|c|c|c|c|c|c|c|c|c|c|c|c|}
\hline Gv2_module & Gv2_ID & Gv2_Units $^{(a)}$ & Gv2_Description & Feed Type & Reference & $\begin{array}{l}\text { Values } \\
\text { Cited }\end{array}$ & Units Cited & Comment & $\begin{array}{c}\text { Data } \\
\text { Reported }\end{array}$ & $\begin{array}{l}\text { Value in } \\
\text { Gv2 } \\
\text { Units }\end{array}$ & Units $^{(\mathrm{a})}$ \\
\hline EXPOSURE & DRYFA2 & kg_dry/kg_as-fed & $\begin{array}{l}\text { Dry-to-wet conversion } \\
\text { factor for animal feed. }\end{array}$ & 1.meat animal feed & $\begin{array}{l}\text { Ensminger et al. } \\
\text { (1990, Table V-1) }\end{array}$ & 0.88 & $\begin{array}{l}\% \text { converted to } \\
\text { fraction }\end{array}$ & durum wheat & Datum & 0.88 & dry/wet \\
\hline EXPOSURE & DRYFA2 & kg_dry/kg_as-fed & $\begin{array}{l}\text { Dry-to-wet conversion } \\
\text { factor for animal feed. }\end{array}$ & 1.meat animal feed & $\begin{array}{l}\text { Ensminger et al. } \\
\text { (1990, Table V-1) }\end{array}$ & 0.88 & $\begin{array}{l}\% \text { converted to } \\
\text { fraction }\end{array}$ & lentil grain & Datum & 0.88 & dry/wet \\
\hline EXPOSURE & DRYFA2 & kg_dry/kg_as-fed & $\begin{array}{l}\text { Dry-to-wet conversion } \\
\text { factor for animal feed. }\end{array}$ & 1.meat animal feed & $\begin{array}{l}\text { Ensminger et al. } \\
\text { (1990, Table V-1) }\end{array}$ & 0.88 & $\begin{array}{l}\% \text { converted to } \\
\text { fraction }\end{array}$ & millet & Datum & 0.88 & dry/wet \\
\hline EXPOSURE & DRYFA2 & kg_dry/kg_as-fed & $\begin{array}{l}\text { Dry-to-wet conversion } \\
\text { factor for animal feed. }\end{array}$ & 1.meat animal feed & IAEA (2009, Table 2) & 88.6 & percent dry matter & soybean seeds & Datum & 0.89 & dry/wet \\
\hline EXPOSURE & DRYFA2 & kg_dry/kg_as-fed & $\begin{array}{l}\text { Dry-to-wet conversion } \\
\text { factor for animal feed. }\end{array}$ & 1.meat animal feed & $\begin{array}{l}\text { Ensminger et al. } \\
\text { (1990, Table V-1) }\end{array}$ & 0.89 & $\begin{array}{l}\% \text { converted to } \\
\text { fraction }\end{array}$ & dry kidney bean & Datum & 0.89 & dry/wet \\
\hline EXPOSURE & DRYFA2 & kg_dry/kg_as-fed & $\begin{array}{l}\text { Dry-to-wet conversion } \\
\text { factor for animal feed. }\end{array}$ & 1.meat animal feed & $\begin{array}{l}\text { Ensminger et al. } \\
\text { (1990, Table V-1) }\end{array}$ & 0.89 & $\begin{array}{l}\% \text { converted to } \\
\text { fraction }\end{array}$ & oat grain & Datum & 0.89 & dry/wet \\
\hline EXPOSURE & DRYFA2 & kg_dry/kg_as-fed & $\begin{array}{l}\text { Dry-to-wet conversion } \\
\text { factor for animal feed. }\end{array}$ & 1.meat animal feed & $\begin{array}{l}\text { Ensminger et al. } \\
\text { (1990, Table V-1) }\end{array}$ & 0.89 & $\begin{array}{l}\% \text { converted to } \\
\text { fraction }\end{array}$ & rice grain & Datum & 0.89 & dry/wet \\
\hline EXPOSURE & DRYFA2 & kg_dry/kg_as-fed & $\begin{array}{l}\text { Dry-to-wet conversion } \\
\text { factor for animal feed. }\end{array}$ & 1.meat animal feed & $\begin{array}{l}\text { Ensminger et al. } \\
\text { (1990, Table V-1) }\end{array}$ & 0.89 & $\begin{array}{l}\% \text { converted to } \\
\text { fraction }\end{array}$ & $\begin{array}{l}\text { sun-cured alfalfa- } \\
\text { orchardgrass hay }\end{array}$ & Datum & 0.89 & dry/wet \\
\hline EXPOSURE & DRYFA2 & kg_dry/kg_as-fed & $\begin{array}{l}\text { Dry-to-wet conversion } \\
\text { factor for animal feed. }\end{array}$ & 1.meat animal feed & $\begin{array}{l}\text { Ensminger et al. } \\
\text { (1990, Table V-1) }\end{array}$ & 0.89 & $\begin{array}{l}\% \text { converted to } \\
\text { fraction }\end{array}$ & wheat grain & Datum & 0.89 & dry/wet \\
\hline EXPOSURE & DRYFA2 & kg_dry/kg_as-fed & $\begin{array}{l}\text { Dry-to-wet conversion } \\
\text { factor for animal feed. }\end{array}$ & 1.meat animal feed & $\begin{array}{l}\text { Ensminger et al. } \\
\text { (1990, Table V-1) }\end{array}$ & 0.9 & $\begin{array}{l}\% \text { converted to } \\
\text { fraction }\end{array}$ & sorghum grain & Datum & 0.9 & dry/wet \\
\hline EXPOSURE & DRYFA2 & kg_dry/kg_as-fed & $\begin{array}{l}\text { Dry-to-wet conversion } \\
\text { factor for animal feed. }\end{array}$ & 1.meat animal feed & $\begin{array}{l}\text { Ensminger et al. } \\
\text { (1990, Table V-1) }\end{array}$ & 0.9 & $\begin{array}{l}\% \text { converted to } \\
\text { fraction }\end{array}$ & soybean mill feed & Datum & 0.9 & dry/wet \\
\hline EXPOSURE & DRYFA2 & kg_dry/kg_as-fed & $\begin{array}{l}\text { Dry-to-wet conversion } \\
\text { factor for animal feed. }\end{array}$ & 1.meat animal feed & $\begin{array}{l}\text { Ensminger et al. } \\
\text { (1990, Table V-1) }\end{array}$ & 0.91 & $\begin{array}{l}\% \text { converted to } \\
\text { fraction }\end{array}$ & $\begin{array}{l}\text { sun-cured alfalfa } \\
\text { grass hay }\end{array}$ & Datum & 0.91 & dry/wet \\
\hline EXPOSURE & DRYFA2 & kg_dry/kg_as-fed & $\begin{array}{l}\text { Dry-to-wet conversion } \\
\text { factor for animal feed. }\end{array}$ & 1.meat animal feed & $\begin{array}{l}\text { Ensminger et al. } \\
\text { (1990, Table V-1) }\end{array}$ & 0.92 & $\begin{array}{l}\% \text { converted to } \\
\text { fraction }\end{array}$ & soybean seed & Datum & 0.92 & dry/wet \\
\hline EXPOSURE & DRYFA2 & kg_dry/kg_as-fed & $\begin{array}{l}\text { Dry-to-wet conversion } \\
\text { factor for animal feed. }\end{array}$ & 1.meat animal feed & $\begin{array}{l}\text { Ensminger et al. } \\
\text { (1990, Table V-1) }\end{array}$ & 0.93 & $\begin{array}{l}\% \text { converted to } \\
\text { fraction }\end{array}$ & $\begin{array}{l}\text { sun-cured alfalfa } \\
\text { timothy grass hay }\end{array}$ & Datum & 0.93 & dry/wet \\
\hline EXPOSURE & DRYFA2 & kg_dry/kg_as-fed & $\begin{array}{l}\text { Dry-to-wet conversion } \\
\text { factor for animal feed. }\end{array}$ & 1.meat animal feed & $\begin{array}{l}\text { Ensminger et al. } \\
\text { (1990, Table V-1) }\end{array}$ & 90 & $\begin{array}{l}\% \text { converted to } \\
\text { fraction }\end{array}$ & dry pinto bean & Datum & 90 & dry/wet \\
\hline
\end{tabular}


Table H.2. Poultry Feed

\begin{tabular}{|c|c|c|c|c|c|c|c|c|c|c|c|}
\hline Gv2_module & Gv2_ID & Gv2_Units $^{(a)}$ & Gv2_Description & Feed Type & Reference & $\begin{array}{l}\text { Values } \\
\text { Cited }\end{array}$ & Units Cited & Comment & $\begin{array}{c}\text { Data } \\
\text { Reported }\end{array}$ & $\begin{array}{l}\text { Value in } \\
\text { Gv2 } \\
\text { Units }\end{array}$ & Units $^{(a)}$ \\
\hline EXPOSURE & DRYFA2 & kg_dry/kg_as-fed & $\begin{array}{l}\text { Dry-to-wet conversion } \\
\text { factor for animal feed. }\end{array}$ & 2.poultry feed & IAEA (2009, Table 3) & 0.34 & $\begin{array}{l}\text { dry matter } \\
\text { fraction, as fed }\end{array}$ & $\begin{array}{l}\text { lucerne silage (aka } \\
\text { alfalfa silage) }\end{array}$ & Datum & 0.34 & dry/wet \\
\hline EXPOSURE & DRYFA2 & kg_dry/kg_wet & $\begin{array}{l}\text { Dry-to-wet conversion } \\
\text { factor for food crops. }\end{array}$ & 2.poultry feed & IAEA (2009, Table 2) & 85.2 & percent dry matter & maize (corn), grain & Datum & 0.85 & dry/wet \\
\hline EXPOSURE & DRYFA2 & kg_dry/kg_as-fed & $\begin{array}{l}\text { Dry-to-wet conversion } \\
\text { factor for animal feed. }\end{array}$ & 2.poultry feed & $\begin{array}{l}\text { Ensminger et al. (1990, } \\
\text { Table V-1) }\end{array}$ & $0.85-0.90$ & $\begin{array}{l}\% \text { converted to } \\
\text { fraction }\end{array}$ & dried corn & $\begin{array}{l}\text { mean of } \\
\text { range }\end{array}$ & 0.88 & dry/wet \\
\hline
\end{tabular}

Table H.3. Milk Feed

\begin{tabular}{|c|c|c|c|c|c|c|c|c|c|c|c|}
\hline Gv2_module & Gv2_ID & Gv2_Units ${ }^{(a)}$ & Gv2_Description & Feed Type & Reference & $\begin{array}{l}\text { Values } \\
\text { Cited }\end{array}$ & Units Cited & Comment & $\begin{array}{c}\text { Data } \\
\text { Reported }\end{array}$ & $\begin{array}{c}\text { Value in } \\
\text { Gv2 } \\
\text { Units }\end{array}$ & Units $^{(\mathrm{a})}$ \\
\hline EXPOSURE & DRYFA2 & kg_dry/kg_as-fed & $\begin{array}{l}\text { Dry-to-wet conversion } \\
\text { factor for animal feed. }\end{array}$ & 3. milk feed & see meat animal feed & & & & Datum & & dry/wet \\
\hline
\end{tabular}

Table H.4. Egg Layer Feed

\begin{tabular}{|c|c|c|c|c|c|c|c|c|c|c|c|}
\hline Gv2_module & Gv2_ID & Gv2_Units ${ }^{(a)}$ & Gv2_Description & Feed Type & Reference & $\begin{array}{l}\text { Values } \\
\text { Cited }\end{array}$ & Units Cited & Comment & $\begin{array}{c}\text { Data } \\
\text { Reported }\end{array}$ & $\begin{array}{c}\text { Value in } \\
\text { Gv2 } \\
\text { Units }\end{array}$ & Units $^{(\mathrm{a})}$ \\
\hline EXPOSURE & DRYFA2 & kg_dry/kg_wet & $\begin{array}{l}\text { Dry-to-wet conversion } \\
\text { factor for food crops. }\end{array}$ & 4.egg layer feed & IAEA (2009, Table 2) & 85.2 & percent dry matter & maize (corn), grain & Datum & 0.85 & dry/wet \\
\hline EXPOSURE & DRYFA2 & kg_dry/kg_as-fed & $\begin{array}{l}\text { Dry-to-wet conversion } \\
\text { factor for animal feed. }\end{array}$ & 4.egg layer feed & $\begin{array}{l}\text { Ensminger et al. (1990, } \\
\text { Table V-1) }\end{array}$ & $0.85-0.90$ & $\begin{array}{l}\% \text { converted to } \\
\text { fraction }\end{array}$ & dried corn & Datum & 0.875 & dry/wet \\
\hline
\end{tabular}


Table H.5. Meat Animal Forage

\begin{tabular}{|c|c|c|c|c|c|c|c|c|c|c|c|}
\hline Gv2_module & Gv2_ID & Gv2_Units ${ }^{(a)}$ & Gv2_Description & Feed Type & Reference & $\begin{array}{l}\text { Values } \\
\text { Cited }\end{array}$ & Units Cited & Comment & $\begin{array}{c}\text { Data } \\
\text { Reported }\end{array}$ & $\begin{array}{c}\text { Value in } \\
\text { Gv2 } \\
\text { Units }\end{array}$ & Units $^{(a)}$ \\
\hline EXPOSURE & DRYFA2 & kg_dry/kg_as-fed & $\begin{array}{l}\text { Dry-to-wet conversion } \\
\text { factor for animal feed. }\end{array}$ & $\begin{array}{l}\text { 5.meat animal } \\
\text { forage }\end{array}$ & IAEA (2009, Table 3) & 0.25 & $\begin{array}{l}\text { dry matter } \\
\text { fraction, as fed }{ }^{(b)}\end{array}$ & corn silage & Datum & 0.25 & dry/wet \\
\hline EXPOSURE & DRYFA2 & kg_dry/kg_as-fed & $\begin{array}{l}\text { Dry-to-wet conversion } \\
\text { factor for animal feed. }\end{array}$ & $\begin{array}{l}\text { 5.meat animal } \\
\text { forage }\end{array}$ & $\begin{array}{l}\text { Ensminger et al. (1990, } \\
\text { Table V-1) }\end{array}$ & $0.13-0.38$ & $\begin{array}{l}\% \text { converted to } \\
\text { fraction }\end{array}$ & silage- sweet potato & $\begin{array}{l}\text { mean of } \\
\text { range }\end{array}$ & 0.26 & dry/wet \\
\hline EXPOSURE & DRYFA2 & kg_dry/kg_as-fed & $\begin{array}{l}\text { Dry-to-wet conversion } \\
\text { factor for animal feed. }\end{array}$ & $\begin{array}{l}\text { 5.meat animal } \\
\text { forage }\end{array}$ & $\begin{array}{l}\text { Ensminger et al. (1990, } \\
\text { Table V-1) }\end{array}$ & $0.22-0.30$ & $\begin{array}{l}\% \text { converted to } \\
\text { fraction }\end{array}$ & silage- sugarcane & $\begin{array}{l}\text { mean of } \\
\text { range }\end{array}$ & 0.26 & dry/wet \\
\hline EXPOSURE & DRYFA2 & kg_dry/kg_as-fed & $\begin{array}{l}\text { Dry-to-wet conversion } \\
\text { factor for animal feed. }\end{array}$ & $\begin{array}{l}\text { 5.meat animal } \\
\text { forage }\end{array}$ & $\begin{array}{l}\text { Ensminger et al. (1990, } \\
\text { Table V-1) }\end{array}$ & 0.26 & $\begin{array}{l}\% \text { converted to } \\
\text { fraction }\end{array}$ & silage- sunflower & Datum & 0.26 & dry/wet \\
\hline EXPOSURE & DRYFA2 & kg_dry/kg_as-fed & $\begin{array}{l}\text { Dry-to-wet conversion } \\
\text { factor for animal feed. }\end{array}$ & $\begin{array}{l}\text { 5.meat animal } \\
\text { forage }\end{array}$ & $\begin{array}{l}\text { Ensminger et al. (1990, } \\
\text { Table V-1) }\end{array}$ & 0.27 & $\begin{array}{l}\% \text { converted to } \\
\text { fraction }\end{array}$ & $\begin{array}{l}\text { silage- corn- } \\
\text { soybean }\end{array}$ & Datum & 0.27 & dry/wet \\
\hline EXPOSURE & DRYFA2 & kg_dry/kg_as-fed & $\begin{array}{l}\text { Dry-to-wet conversion } \\
\text { factor for animal feed. }\end{array}$ & $\begin{array}{l}\text { 5.meat animal } \\
\text { forage }\end{array}$ & $\begin{array}{l}\text { Ensminger et al. (1990, } \\
\text { Table V-1) }\end{array}$ & 0.27 & $\begin{array}{l}\% \text { converted to } \\
\text { fraction }\end{array}$ & silage- oats-vetch & Datum & 0.27 & dry/wet \\
\hline EXPOSURE & DRYFA2 & kg_dry/kg_as-fed & $\begin{array}{l}\text { Dry-to-wet conversion } \\
\text { factor for animal feed. }\end{array}$ & $\begin{array}{l}\text { 5.meat animal } \\
\text { forage }\end{array}$ & $\begin{array}{l}\text { Ensminger et al. (1990, } \\
\text { Table V-1) }\end{array}$ & 0.3 & $\begin{array}{l}\% \text { converted to } \\
\text { fraction }\end{array}$ & silage- clover-grass & Datum & 0.3 & dry/wet \\
\hline EXPOSURE & DRYFA2 & kg_dry/kg_as-fed & $\begin{array}{l}\text { Dry-to-wet conversion } \\
\text { factor for animal feed. }\end{array}$ & $\begin{array}{l}\text { 5.meat animal } \\
\text { forage }\end{array}$ & $\begin{array}{l}\text { Ensminger et al. (1990, } \\
\text { Table V-1) }\end{array}$ & $0.26-0.35$ & $\begin{array}{l}\% \text { converted to } \\
\text { fraction }\end{array}$ & silage- grass legume & $\begin{array}{l}\text { mean of } \\
\text { range }\end{array}$ & 0.31 & dry/wet \\
\hline EXPOSURE & DRYFA2 & kg_dry/kg_as-fed & $\begin{array}{l}\text { Dry-to-wet conversion } \\
\text { factor for animal feed. }\end{array}$ & $\begin{array}{l}\text { 5.meat animal } \\
\text { forage }\end{array}$ & $\begin{array}{l}\text { Ensminger et al. (1990, } \\
\text { Table V-1) }\end{array}$ & 0.31 & $\begin{array}{l}\% \text { converted to } \\
\text { fraction }\end{array}$ & $\begin{array}{l}\text { silage- sweet corn } \\
\text { (canning residue) }\end{array}$ & Datum & 0.31 & dry/wet \\
\hline EXPOSURE & DRYFA2 & kg_dry/kg_as-fed & $\begin{array}{l}\text { Dry-to-wet conversion } \\
\text { factor for animal feed. }\end{array}$ & $\begin{array}{l}\text { 5.meat animal } \\
\text { forage }\end{array}$ & $\begin{array}{l}\text { Ensminger et al. (1990, } \\
\text { Table V-1) }\end{array}$ & 0.33 & $\begin{array}{l}\% \text { converted to } \\
\text { fraction }\end{array}$ & $\begin{array}{l}\text { silage- meadow } \\
\text { fescue }\end{array}$ & Datum & 0.33 & dry/wet \\
\hline EXPOSURE & DRYFA2 & kg_dry/kg_as-fed & $\begin{array}{l}\text { Dry-to-wet conversion } \\
\text { factor for animal feed. }\end{array}$ & $\begin{array}{l}\text { 5.meat animal } \\
\text { forage }\end{array}$ & $\begin{array}{l}\text { Ensminger et al. (1990, } \\
\text { Table V-1) }\end{array}$ & 0.34 & $\begin{array}{l}\% \text { converted to } \\
\text { fraction }\end{array}$ & $\begin{array}{l}\text { silage- corn- } \\
\text { sorghum }\end{array}$ & Datum & 0.34 & dry/wet \\
\hline EXPOSURE & DRYFA2 & kg_dry/kg_as-fed & $\begin{array}{l}\text { Dry-to-wet conversion } \\
\text { factor for animal feed. }\end{array}$ & $\begin{array}{l}\text { 5.meat animal } \\
\text { forage }\end{array}$ & $\begin{array}{l}\text { Ensminger et al. (1990, } \\
\text { Table V-1) }\end{array}$ & 0.35 & $\begin{array}{l}\% \text { converted to } \\
\text { fraction }\end{array}$ & silage- oats & Datum & 0.35 & dry/wet \\
\hline EXPOSURE & DRYFA2 & kg_dry/kg_as-fed & $\begin{array}{l}\text { Dry-to-wet conversion } \\
\text { factor for animal feed. }\end{array}$ & $\begin{array}{l}\text { 5.meat animal } \\
\text { forage }\end{array}$ & $\begin{array}{l}\text { Ensminger et al. (1990, } \\
\text { Table V-1) }\end{array}$ & $0.33-0.43$ & $\begin{array}{l}\% \text { converted to } \\
\text { fraction }\end{array}$ & silage- timothy & $\begin{array}{l}\text { mean of } \\
\text { range }\end{array}$ & 0.38 & dry/wet \\
\hline EXPOSURE & DRYFA2 & kg_dry/kg_as-fed & $\begin{array}{l}\text { Dry-to-wet conversion } \\
\text { factor for animal feed. }\end{array}$ & $\begin{array}{l}\text { 5.meat animal } \\
\text { forage }\end{array}$ & $\begin{array}{l}\text { Ensminger et al. (1990, } \\
\text { Table V-1) }\end{array}$ & $0.25-0.54$ & $\begin{array}{l}\% \text { converted to } \\
\text { fraction }\end{array}$ & silage-corn & $\begin{array}{l}\text { mean of } \\
\text { range }\end{array}$ & 0.40 & dry/wet \\
\hline EXPOSURE & DRYFA2 & kg_dry/kg_as-fed & $\begin{array}{l}\text { Dry-to-wet conversion } \\
\text { factor for animal feed. }\end{array}$ & $\begin{array}{l}\text { 5.meat animal } \\
\text { forage }\end{array}$ & $\begin{array}{l}\text { Ensminger et al. (1990, } \\
\text { Table V-1) }\end{array}$ & $0.26-0.56$ & $\begin{array}{l}\% \text { converted to } \\
\text { fraction }\end{array}$ & $\begin{array}{l}\text { silage- alfalfa } \\
\text { bromegrass }\end{array}$ & $\begin{array}{l}\text { mean of } \\
\text { range }\end{array}$ & 0.41 & dry/wet \\
\hline EXPOSURE & DRYFA2 & kg_dry/kg_as-fed & $\begin{array}{l}\text { Dry-to-wet conversion } \\
\text { factor for animal feed. }\end{array}$ & $\begin{array}{l}\text { 5.meat animal } \\
\text { forage }\end{array}$ & $\begin{array}{l}\text { Ensminger et al. (1990, } \\
\text { Table V-1) }\end{array}$ & $0.27-0.57$ & $\begin{array}{l}\% \text { converted to } \\
\text { fraction }\end{array}$ & silage- alfalfa & $\begin{array}{l}\text { mean of } \\
\text { range }\end{array}$ & 0.42 & dry/wet \\
\hline EXPOSURE & DRYFA2 & kg_dry/kg_as-fed & $\begin{array}{l}\text { Dry-to-wet conversion } \\
\text { factor for animal feed. }\end{array}$ & $\begin{array}{l}\text { 5.meat animal } \\
\text { forage }\end{array}$ & $\begin{array}{l}\text { Ensminger et al. (1990, } \\
\text { Table V-1) }\end{array}$ & $0.28-0.57$ & $\begin{array}{l}\% \text { converted to } \\
\text { fraction }\end{array}$ & $\begin{array}{l}\text { silage- alfalfa } \\
\text { orchardgrass }\end{array}$ & $\begin{array}{l}\text { mean of } \\
\text { range }\end{array}$ & 0.43 & dry/wet \\
\hline EXPOSURE & DRYFA2 & kg_dry/kg_as-fed & $\begin{array}{l}\text { Dry-to-wet conversion } \\
\text { factor for animal feed. }\end{array}$ & $\begin{array}{l}\text { 5.meat animal } \\
\text { forage }\end{array}$ & $\begin{array}{l}\text { Ensminger et al. (1990, } \\
\text { Table V-1) }\end{array}$ & $0.24-0.62$ & $\begin{array}{l}\% \text { converted to } \\
\text { fraction }\end{array}$ & silage- sorghum & $\begin{array}{l}\text { mean of } \\
\text { range }\end{array}$ & 0.43 & dry/wet \\
\hline
\end{tabular}


Table H.5. (contd)

\begin{tabular}{|c|c|c|c|c|c|c|c|c|c|c|c|}
\hline Gv2_module & Gv2_ID & Gv2_Units ${ }^{(a)}$ & Gv2_Description & Feed Type & Reference & $\begin{array}{l}\text { Values } \\
\text { Cited }\end{array}$ & Units Cited & Comment & $\begin{array}{c}\text { Data } \\
\text { Reported }\end{array}$ & $\begin{array}{l}\text { Value in } \\
\text { Gv2 } \\
\text { Units }\end{array}$ & Units $^{(a)}$ \\
\hline EXPOSURE & DRYFA2 & kg_dry/kg_as-fed & $\begin{array}{l}\text { Dry-to-wet conversion } \\
\text { factor for animal feed. }\end{array}$ & $\begin{array}{l}\text { 5.meat animal } \\
\text { forage }\end{array}$ & $\begin{array}{l}\text { Ensminger et al. (1990, } \\
\text { Table V-1) }\end{array}$ & 0.43 & $\begin{array}{l}\% \text { converted to } \\
\text { fraction }\end{array}$ & $\begin{array}{l}\text { silage-corn ear with } \\
\text { husk }\end{array}$ & Datum & 0.43 & dry/wet \\
\hline EXPOSURE & DRYFA2 & kg_dry/kg_as-fed & $\begin{array}{l}\text { Dry-to-wet conversion } \\
\text { factor for animal feed. }\end{array}$ & $\begin{array}{l}\text { 5.meat animal } \\
\text { forage }\end{array}$ & $\begin{array}{l}\text { Ensminger et al. (1990, } \\
\text { Table V-1) }\end{array}$ & 0.7 & $\begin{array}{l}\% \text { converted to } \\
\text { fraction }\end{array}$ & silage-corn ear & Datum & 0.7 & dry/wet \\
\hline EXPOSURE & DRYFA2 & kg_dry/kg_as-fed & $\begin{array}{l}\text { Dry-to-wet conversion } \\
\text { factor for animal feed. }\end{array}$ & $\begin{array}{l}\text { 5.meat animal } \\
\text { forage }\end{array}$ & $\begin{array}{l}\text { Ensminger et al. (1990, } \\
\text { Table V-1) }\end{array}$ & 0.78 & $\begin{array}{l}\% \text { converted to } \\
\text { fraction }\end{array}$ & silage-corn husk & Datum & 0.78 & dry/wet \\
\hline EXPOSURE & DRYFA2 & kg_dry/kg_as-fed & $\begin{array}{l}\text { Dry-to-wet conversion } \\
\text { factor for animal feed. }\end{array}$ & $\begin{array}{l}\text { 5.meat animal } \\
\text { forage }\end{array}$ & $\begin{array}{l}\text { Ensminger et al. (1990, } \\
\text { Table V-1) }\end{array}$ & 0.82 & $\begin{array}{l}\% \text { converted to } \\
\text { fraction }\end{array}$ & $\begin{array}{l}\text { sun-cured corn } \\
\text { fodder with ears, } \\
\text { husks, mature }\end{array}$ & Datum & 0.82 & dry/wet \\
\hline EXPOSURE & DRYFA2 & kg_dry/kg_as-fed & $\begin{array}{l}\text { Dry-to-wet conversion } \\
\text { factor for animal feed. }\end{array}$ & $\begin{array}{l}\text { 5.meat animal } \\
\text { forage }\end{array}$ & $\begin{array}{l}\text { Ensminger et al. (1990, } \\
\text { Table V-1) }\end{array}$ & 0.85 & $\begin{array}{l}\% \text { converted to } \\
\text { fraction }\end{array}$ & $\begin{array}{l}\text { sun-cured stover } \\
\text { without ears or } \\
\text { husks }\end{array}$ & Datum & 0.85 & dry/wet \\
\hline EXPOSURE & DRYFA2 & kg_dry/kg_as-fed & $\begin{array}{l}\text { Dry-to-wet conversion } \\
\text { factor for animal feed. }\end{array}$ & $\begin{array}{l}\text { 5.meat animal } \\
\text { forage }\end{array}$ & $\begin{array}{l}\text { Ensminger et al. (1990, } \\
\text { Table V-1) }\end{array}$ & 0.88 & $\begin{array}{l}\% \text { converted to } \\
\text { fraction }\end{array}$ & $\begin{array}{l}\text { sun-cured barley } \\
\text { hay }\end{array}$ & Datum & 0.88 & dry/wet \\
\hline EXPOSURE & DRYFA2 & kg_dry/kg_as-fed & $\begin{array}{l}\text { Dry-to-wet conversion } \\
\text { factor for animal feed. }\end{array}$ & $\begin{array}{l}\text { 5.meat animal } \\
\text { forage }\end{array}$ & $\begin{array}{l}\text { Ensminger et al. (1990, } \\
\text { Table V-1) }\end{array}$ & 0.88 & $\begin{array}{l}\% \text { converted to } \\
\text { fraction }\end{array}$ & $\begin{array}{l}\text { sun-cured clover } \\
\text { hay }\end{array}$ & Datum & 0.88 & dry/wet \\
\hline EXPOSURE & DRYFA2 & kg_dry/kg_as-fed & $\begin{array}{l}\text { Dry-to-wet conversion } \\
\text { factor for animal feed. }\end{array}$ & $\begin{array}{l}\text { 5.meat animal } \\
\text { forage }\end{array}$ & $\begin{array}{l}\text { Ensminger et al. (1990, } \\
\text { Table V-1) }\end{array}$ & 0.88 & $\begin{array}{l}\% \text { converted to } \\
\text { fraction }\end{array}$ & $\begin{array}{l}\text { sun-cured red clover } \\
\text { grass }\end{array}$ & Datum & 0.88 & dry/wet \\
\hline EXPOSURE & DRYFA2 & kg_dry $/ \mathrm{kg} \_a s-f e d$ & $\begin{array}{l}\text { Dry-to-wet conversion } \\
\text { factor for animal feed. }\end{array}$ & $\begin{array}{l}\text { 5.meat animal } \\
\text { forage }\end{array}$ & $\begin{array}{l}\text { Ensminger et al. (1990, } \\
\text { Table V-1) }\end{array}$ & 0.88 & $\begin{array}{l}\% \text { converted to } \\
\text { fraction }\end{array}$ & $\begin{array}{l}\text { sun-cured sorghum } \\
\text { fodder }\end{array}$ & Datum & 0.88 & dry/wet \\
\hline EXPOSURE & DRYFA2 & kg_dry/kg_as-fed & $\begin{array}{l}\text { Dry-to-wet conversion } \\
\text { factor for animal feed. }\end{array}$ & $\begin{array}{l}\text { 5.meat animal } \\
\text { forage }\end{array}$ & $\begin{array}{l}\text { Ensminger et al. (1990, } \\
\text { Table V-1) }\end{array}$ & 0.89 & $\begin{array}{l}\% \text { converted to } \\
\text { fraction }\end{array}$ & $\begin{array}{l}\text { sun-cured Kentucky } \\
\text { bluegrass }\end{array}$ & Datum & 0.89 & dry/wet \\
\hline EXPOSURE & DRYFA2 & kg_dry/kg_as-fed & $\begin{array}{l}\text { Dry-to-wet conversion } \\
\text { factor for animal feed. }\end{array}$ & $\begin{array}{l}\text { 5.meat animal } \\
\text { forage }\end{array}$ & $\begin{array}{l}\text { Ensminger et al. (1990, } \\
\text { Table V-1) }\end{array}$ & 0.89 & $\begin{array}{l}\% \text { converted to } \\
\text { fraction }\end{array}$ & sun-cured wheat hay & Datum & 0.89 & dry/wet \\
\hline EXPOSURE & DRYFA2 & kg_dry/kg_as-fed & $\begin{array}{l}\text { Dry-to-wet conversion } \\
\text { factor for animal feed. }\end{array}$ & $\begin{array}{l}\text { 5.meat animal } \\
\text { forage }\end{array}$ & $\begin{array}{l}\text { Ensminger et al. (1990, } \\
\text { Table V-1) }\end{array}$ & 0.9 & $\begin{array}{l}\% \text { converted to } \\
\text { fraction }\end{array}$ & $\begin{array}{l}\text { sun-cured bahia } \\
\text { grass }\end{array}$ & Datum & 0.9 & dry/wet \\
\hline EXPOSURE & DRYFA2 & kg_dry/kg_as-fed & $\begin{array}{l}\text { Dry-to-wet conversion } \\
\text { factor for animal feed. }\end{array}$ & $\begin{array}{l}\text { 5.meat animal } \\
\text { forage }\end{array}$ & $\begin{array}{l}\text { Ensminger et al. (1990, } \\
\text { Table V-1) }\end{array}$ & 0.9 & $\begin{array}{l}\% \text { converted to } \\
\text { fraction }\end{array}$ & $\begin{array}{l}\text { sun-cured sorghum } \\
\text { fodder with heads }\end{array}$ & Datum & 0.90 & dry/wet \\
\hline EXPOSURE & DRYFA2 & kg_dry/kg_as-fed & $\begin{array}{l}\text { Dry-to-wet conversion } \\
\text { factor for animal feed. }\end{array}$ & $\begin{array}{l}\text { 5.meat animal } \\
\text { forage }\end{array}$ & $\begin{array}{l}\text { Ensminger et al. (1990, } \\
\text { Table V-1) }\end{array}$ & 0.91 & $\begin{array}{l}\% \text { converted to } \\
\text { fraction }\end{array}$ & $\begin{array}{l}\text { sun-cured Bermuda } \\
\text { grass hay }\end{array}$ & Datum & 0.91 & dry/wet \\
\hline EXPOSURE & DRYFA2 & kg_dry/kg_as-fed & $\begin{array}{l}\text { Dry-to-wet conversion } \\
\text { factor for animal feed. }\end{array}$ & $\begin{array}{l}\text { 5.meat animal } \\
\text { forage }\end{array}$ & $\begin{array}{l}\text { Ensminger et al. (1990, } \\
\text { Table V-1) }\end{array}$ & 0.91 & $\begin{array}{l}\% \text { converted to } \\
\text { fraction }\end{array}$ & sun-cured oats hay & Datum & 0.91 & dry/wet \\
\hline EXPOSURE & DRYFA2 & kg_dry/kg_as-fed & $\begin{array}{l}\text { Dry-to-wet conversion } \\
\text { factor for animal feed. }\end{array}$ & $\begin{array}{l}\text { 5.meat animal } \\
\text { forage }\end{array}$ & $\begin{array}{l}\text { Ensminger et al. (1990, } \\
\text { Table V-1) }\end{array}$ & 0.92 & $\begin{array}{l}\% \text { converted to } \\
\text { fraction }\end{array}$ & $\begin{array}{l}\text { immature } \\
\text { dehydrated cereals }\end{array}$ & Datum & 0.92 & dry/wet \\
\hline
\end{tabular}

(a) Gv2 units for feed types indicate kg_dry/kg_wet, where kg_wet is the as-fed mass. This can differ from the as-harvested mass of the feed crop.

(b) Corrects error in reported units, which were \%. 
Table H.6. Milk Forage

\begin{tabular}{|c|c|c|c|c|c|c|c|c|c|c|c|}
\hline Gv2_module & Gv2_ID & Gv2_Units ${ }^{(a)}$ & Gv2_Description & Feed Type & Reference & $\begin{array}{l}\text { Values } \\
\text { Cited }\end{array}$ & Units Cited & Comment & $\begin{array}{c}\text { Data } \\
\text { Reported }\end{array}$ & $\begin{array}{l}\text { Value in } \\
\text { Gv2 } \\
\text { Units }\end{array}$ & Units $^{(a)}$ \\
\hline EXPOSURE & DRYFA2 & kg_dry/kg_as-fed & $\begin{array}{l}\text { Dry-to-wet conversion } \\
\text { factor for animal feed. }\end{array}$ & 6. milk forage & $\begin{array}{l}\text { Ensminger et al. (1990, } \\
\text { Table V-1) }\end{array}$ & 0.13 & $\begin{array}{l}\% \text { converted to } \\
\text { fraction }\end{array}$ & fresh turnip & Datum & 0.13 & dry/wet \\
\hline EXPOSURE & DRYFA2 & kg_dry/kg_as-fed & $\begin{array}{l}\text { Dry-to-wet conversion } \\
\text { factor for animal feed. }\end{array}$ & 6. milk forage & $\begin{array}{l}\text { Ensminger et al. (1990, } \\
\text { Table V-1) }\end{array}$ & 0.14 & $\begin{array}{l}\% \text { converted to } \\
\text { fraction }\end{array}$ & $\begin{array}{l}\text { fresh dandelion } \\
\text { leaves }\end{array}$ & Datum & 0.14 & dry/wet \\
\hline EXPOSURE & DRYFA2 & kg_dry/kg_as-fed & $\begin{array}{l}\text { Dry-to-wet conversion } \\
\text { factor for animal feed. }\end{array}$ & 6. milk forage & $\begin{array}{l}\text { Ensminger et al. (1990, } \\
\text { Table V-1) }\end{array}$ & $0.15-0.17$ & $\begin{array}{l}\% \text { converted to } \\
\text { fraction }\end{array}$ & fresh sunflower & $\begin{array}{l}\text { mean of } \\
\text { range }\end{array}$ & 0.16 & dry/wet \\
\hline EXPOSURE & DRYFA2 & kg_dry/kg_as-fed & $\begin{array}{l}\text { Dry-to-wet conversion } \\
\text { factor for animal feed. }\end{array}$ & 6. milk forage & $\begin{array}{l}\text { Ensminger et al. (1990, } \\
\text { Table V-1) }\end{array}$ & 0.17 & $\begin{array}{l}\% \text { converted to } \\
\text { fraction }\end{array}$ & fresh rape & Datum & 0.17 & dry/wet \\
\hline EXPOSURE & DRYFA2 & kg_dry/kg_as-fed & $\begin{array}{l}\text { Dry-to-wet conversion } \\
\text { factor for animal feed. }\end{array}$ & 6. milk forage & $\begin{array}{l}\text { Ensminger et al. (1990, } \\
\text { Table V-1) }\end{array}$ & 0.17 & $\begin{array}{l}\% \text { converted to } \\
\text { fraction }\end{array}$ & $\begin{array}{l}\text { fresh sugar beet } \\
\text { with tops, crowns }\end{array}$ & Datum & 0.17 & dry/wet \\
\hline EXPOSURE & DRYFA2 & kg_dry/kg_as-fed & $\begin{array}{l}\text { Dry-to-wet conversion } \\
\text { factor for animal feed. }\end{array}$ & 6. milk forage & $\begin{array}{l}\text { Ensminger et al. (1990, } \\
\text { Table V-1) }\end{array}$ & 0.18 & $\begin{array}{l}\% \text { converted to } \\
\text { fraction }\end{array}$ & $\begin{array}{l}\text { fresh corn- } \\
\text { sunflower }\end{array}$ & Datum & 0.18 & dry/wet \\
\hline EXPOSURE & DRYFA2 & kg_dry/kg_as-fed & $\begin{array}{l}\text { Dry-to-wet conversion } \\
\text { factor for animal feed. }\end{array}$ & 6. milk forage & IAEA (2009, Table 2) & 20 & $\begin{array}{l}\text { percent dry } \\
\text { matter, as fed }\end{array}$ & $\begin{array}{l}\text { sudan grass, } \\
\text { vegetative mass }\end{array}$ & Datum & 0.20 & dry/wet \\
\hline EXPOSURE & DRYFA2 & kg_dry/kg_as-fed & $\begin{array}{l}\text { Dry-to-wet conversion } \\
\text { factor for animal feed. }\end{array}$ & 6. milk forage & IAEA (2009, Table 2) & 20 & $\begin{array}{l}\text { percent dry } \\
\text { matter, as fed }\end{array}$ & $\begin{array}{l}\text { annual ryegrass, } \\
\text { vegetative mass }\end{array}$ & Datum & 0.20 & dry/wet \\
\hline EXPOSURE & DRYFA2 & kg_dry/kg_as-fed & $\begin{array}{l}\text { Dry-to-wet conversion } \\
\text { factor for animal feed. }\end{array}$ & 6. milk forage & IAEA (2009, Table 2) & 20 & $\begin{array}{l}\text { percent dry } \\
\text { matter, as fed }\end{array}$ & $\begin{array}{l}\text { meadow fescue, } \\
\text { vegetative mass }\end{array}$ & Datum & 0.20 & dry/wet \\
\hline EXPOSURE & DRYFA2 & kg_dry/kg_as-fed & $\begin{array}{l}\text { Dry-to-wet conversion } \\
\text { factor for animal feed. }\end{array}$ & 6. milk forage & IAEA (2009, Table 3) & 0.20 & $\begin{array}{l}\text { dry matter } \\
\text { fraction, as fed }{ }^{(\mathrm{b})}\end{array}$ & pasture & Datum & 0.20 & dry/wet \\
\hline EXPOSURE & DRYFA2 & kg_dry/kg_as-fed & $\begin{array}{l}\text { Dry-to-wet conversion } \\
\text { factor for animal feed. }\end{array}$ & 6. milk forage & IAEA (2009, Table 2) & 20.8 & $\begin{array}{l}\text { percent dry } \\
\text { matter, as fed }\end{array}$ & $\begin{array}{l}\text { fussion brome grass, } \\
\text { vegetative mass }\end{array}$ & Datum & 0.21 & dry/wet \\
\hline EXPOSURE & DRYFA2 & kg_dry/kg_as-fed & $\begin{array}{l}\text { Dry-to-wet conversion } \\
\text { factor for animal feed. }\end{array}$ & 6. milk forage & $\begin{array}{l}\text { Ensminger et al. (1990, } \\
\text { Table V-1) }\end{array}$ & 0.21 & $\begin{array}{l}\% \text { converted to } \\
\text { fraction }\end{array}$ & fresh barley & Datum & 0.21 & dry/wet \\
\hline EXPOSURE & DRYFA2 & kg_dry/kg_as-fed & $\begin{array}{l}\text { Dry-to-wet conversion } \\
\text { factor for animal feed. }\end{array}$ & 6. milk forage & IAEA (2009, Table 2) & 22 & $\begin{array}{l}\text { percent dry } \\
\text { matter, as fed }\end{array}$ & $\begin{array}{l}\text { red clover, } \\
\text { vegetative mass }\end{array}$ & Datum & 0.22 & dry/wet \\
\hline EXPOSURE & DRYFA2 & kg_dry/kg_as-fed & $\begin{array}{l}\text { Dry-to-wet conversion } \\
\text { factor for animal feed. }\end{array}$ & 6. milk forage & IAEA (2009, Table 2) & 22 & $\begin{array}{l}\text { percent dry } \\
\text { matter, as fed }\end{array}$ & $\begin{array}{l}\text { meadow grass, } \\
\text { vegetative mass }\end{array}$ & Datum & 0.22 & dry/wet \\
\hline EXPOSURE & DRYFA2 & kg_dry/kg_as-fed & $\begin{array}{l}\text { Dry-to-wet conversion } \\
\text { factor for animal feed. }\end{array}$ & 6. milk forage & $\begin{array}{l}\text { Ensminger et al. (1990, } \\
\text { Table V-1) }\end{array}$ & 0.22 & $\begin{array}{l}\% \text { converted to } \\
\text { fraction }\end{array}$ & fresh alfalfa-timothy & Datum & 0.22 & dry/wet \\
\hline EXPOSURE & DRYFA2 & kg_dry/kg_as-fed & $\begin{array}{l}\text { Dry-to-wet conversion } \\
\text { factor for animal feed. }\end{array}$ & 6. milk forage & $\begin{array}{l}\text { Ensminger et al. (1990, } \\
\text { Table V-1) }\end{array}$ & $0.18-0.26$ & $\begin{array}{l}\% \text { converted to } \\
\text { fraction }\end{array}$ & fresh clover & $\begin{array}{l}\text { mean of } \\
\text { range }\end{array}$ & 0.22 & dry/wet \\
\hline EXPOSURE & DRYFA2 & kg_dry/kg_as-fed & $\begin{array}{l}\text { Dry-to-wet conversion } \\
\text { factor for animal feed. }\end{array}$ & 6. milk forage & $\begin{array}{l}\text { Ensminger et al. (1990, } \\
\text { Table V-1) }\end{array}$ & 0.22 & $\begin{array}{l}\% \text { converted to } \\
\text { fraction }\end{array}$ & fresh wheat & Datum & 0.22 & dry/wet \\
\hline EXPOSURE & DRYFA2 & kg_dry/kg_as-fed & $\begin{array}{l}\text { Dry-to-wet conversion } \\
\text { factor for animal feed. }\end{array}$ & 6. milk forage & IAEA (2009, Table 2) & 22.6 & $\begin{array}{l}\text { percent dry } \\
\text { matter, as fed }\end{array}$ & $\begin{array}{l}\text { winter rye, } \\
\text { vegetative mass, no } \\
\text { grain }\end{array}$ & Datum & 0.23 & dry/wet \\
\hline
\end{tabular}


Table H.6. (contd)

\begin{tabular}{|c|c|c|c|c|c|c|c|c|c|c|c|}
\hline Gv2_module & Gv2_ID & Gv2_Units ${ }^{(a)}$ & Gv2_Description & Feed Type & Reference & $\begin{array}{l}\text { Values } \\
\text { Cited }\end{array}$ & Units Cited & Comment & $\begin{array}{c}\text { Data } \\
\text { Reported }\end{array}$ & $\begin{array}{c}\text { Value in } \\
\text { Gv2 } \\
\text { Units }\end{array}$ & Units $^{(a)}$ \\
\hline EXPOSURE & DRYFA2 & kg_dry/kg_as-fed & $\begin{array}{l}\text { Dry-to-wet conversion } \\
\text { factor for animal feed. }\end{array}$ & 6. milk forage & $\begin{array}{l}\text { Ensminger et al. (1990, } \\
\text { Table V-1) }\end{array}$ & $0.2-0.26$ & $\begin{array}{l}\% \text { converted to } \\
\text { fraction }\end{array}$ & fresh alfalfa & $\begin{array}{l}\text { mean of } \\
\text { range }\end{array}$ & 0.23 & dry/wet \\
\hline EXPOSURE & DRYFA2 & kg_dry/kg_as-fed & $\begin{array}{l}\text { Dry-to-wet conversion } \\
\text { factor for animal feed. }\end{array}$ & 6. milk forage & $\begin{array}{l}\text { Ensminger et al. (1990, } \\
\text { Table V-1) }\end{array}$ & 0.23 & $\begin{array}{l}\% \text { converted to } \\
\text { fraction }\end{array}$ & fresh soybean & Datum & 0.23 & dry/wet \\
\hline EXPOSURE & DRYFA2 & kg_dry/kg_as-fed & $\begin{array}{l}\text { Dry-to-wet conversion } \\
\text { factor for animal feed. }\end{array}$ & 6. milk forage & $\begin{array}{l}\text { Ensminger et al. (1990, } \\
\text { Table V-1) }\end{array}$ & 0.24 & $\begin{array}{l}\% \text { converted to } \\
\text { fraction }\end{array}$ & fresh grass & Datum & 0.24 & dry/wet \\
\hline EXPOSURE & DRYFA2 & kg_dry/kg_as-fed & $\begin{array}{l}\text { Dry-to-wet conversion } \\
\text { factor for animal feed. }\end{array}$ & 6. milk forage & IAEA (2009, Table 2) & 25 & $\begin{array}{l}\text { percent dry } \\
\text { matter, as fed }\end{array}$ & $\begin{array}{l}\text { sorghum, vegetative } \\
\text { mass }\end{array}$ & Datum & 0.25 & dry/wet \\
\hline EXPOSURE & DRYFA2 & kg_dry/kg_as-fed & $\begin{array}{l}\text { Dry-to-wet conversion } \\
\text { factor for animal feed. }\end{array}$ & 6. milk forage & IAEA (2009, Table 2) & 25.5 & $\begin{array}{l}\text { percent dry } \\
\text { matter, as fed }\end{array}$ & $\begin{array}{l}\text { timothy grass, } \\
\text { vegetative mass }\end{array}$ & Datum & 0.26 & dry/wet \\
\hline EXPOSURE & DRYFA2 & kg_dry/kg_as-fed & $\begin{array}{l}\text { Dry-to-wet conversion } \\
\text { factor for animal feed. }\end{array}$ & 6. milk forage & $\begin{array}{l}\text { Ensminger et al. (1990, } \\
\text { Table V-1) }\end{array}$ & $0.22-0.3$ & $\begin{array}{l}\% \text { converted to } \\
\text { fraction }\end{array}$ & fresh tall oatgrasses & $\begin{array}{l}\text { mean of } \\
\text { range }\end{array}$ & 0.26 & dry/wet \\
\hline EXPOSURE & DRYFA2 & kg_dry/kg_as-fed & $\begin{array}{l}\text { Dry-to-wet conversion } \\
\text { factor for animal feed. }\end{array}$ & 6. milk forage & $\begin{array}{l}\text { Ensminger et al. (1990, } \\
\text { Table V-1) }\end{array}$ & 0.27 & $\begin{array}{l}\% \text { converted to } \\
\text { fraction }\end{array}$ & fresh ragweed & Datum & 0.27 & dry/wet \\
\hline EXPOSURE & DRYFA2 & kg_dry/kg_as-fed & $\begin{array}{l}\text { Dry-to-wet conversion } \\
\text { factor for animal feed. }\end{array}$ & 6. milk forage & $\begin{array}{l}\text { Ensminger et al. (1990, } \\
\text { Table V-1) }\end{array}$ & $0.2-0.35$ & $\begin{array}{l}\% \text { converted to } \\
\text { fraction }\end{array}$ & fresh sorghum & $\begin{array}{l}\text { mean of } \\
\text { range }\end{array}$ & 0.28 & dry/wet \\
\hline EXPOSURE & DRYFA2 & kg_dry/kg_as-fed & $\begin{array}{l}\text { Dry-to-wet conversion } \\
\text { factor for animal feed. }\end{array}$ & 6. milk forage & IAEA (2009, Table 2) & 27.6 & $\begin{array}{l}\text { percent dry } \\
\text { matter, as fed }\end{array}$ & $\begin{array}{l}\text { oats, vegetative } \\
\text { mass, no grain }\end{array}$ & Datum & 0.28 & dry/wet \\
\hline EXPOSURE & DRYFA2 & kg_dry/kg_as-fed & $\begin{array}{l}\text { Dry-to-wet conversion } \\
\text { factor for animal feed. }\end{array}$ & 6. milk forage & $\begin{array}{l}\text { Ensminger et al. (1990, } \\
\text { Table V-1) }\end{array}$ & 0.28 & $\begin{array}{l}\% \text { converted to } \\
\text { fraction }\end{array}$ & $\begin{array}{l}\text { fresh meadow } \\
\text { fescue }\end{array}$ & Datum & 0.28 & dry/wet \\
\hline EXPOSURE & DRYFA2 & kg_dry/kg_as-fed & $\begin{array}{l}\text { Dry-to-wet conversion } \\
\text { factor for animal feed. }\end{array}$ & 6. milk forage & $\begin{array}{l}\text { Ensminger et al. (1990, } \\
\text { Table V-1) }\end{array}$ & 0.28 & $\begin{array}{l}\% \text { converted to } \\
\text { fraction }\end{array}$ & $\begin{array}{l}\text { fresh meadow } \\
\text { foxtail }\end{array}$ & Datum & 0.28 & dry/wet \\
\hline EXPOSURE & DRYFA2 & kg_dry/kg_as-fed & $\begin{array}{l}\text { Dry-to-wet conversion } \\
\text { factor for animal feed. }\end{array}$ & 6. milk forage & $\begin{array}{l}\text { Ensminger et al. (1990, } \\
\text { Table V-1) }\end{array}$ & 0.28 & $\begin{array}{l}\% \text { converted to } \\
\text { fraction }\end{array}$ & fresh sugarcane & Datum & 0.28 & dry/wet \\
\hline EXPOSURE & DRYFA2 & kg_dry/kg_as-fed & $\begin{array}{l}\text { Dry-to-wet conversion } \\
\text { factor for animal feed. }\end{array}$ & 6. milk forage & $\begin{array}{l}\text { Ensminger et al. (1990, } \\
\text { Table V-1) }\end{array}$ & 0.28 & $\begin{array}{l}\% \text { converted to } \\
\text { fraction }\end{array}$ & fresh tall fescue & Datum & 0.28 & dry/wet \\
\hline EXPOSURE & DRYFA2 & kg_dry/kg_as-fed & $\begin{array}{l}\text { Dry-to-wet conversion } \\
\text { factor for animal feed. }\end{array}$ & 6. milk forage & $\begin{array}{l}\text { Ensminger et al. (1990, } \\
\text { Table V-1) }\end{array}$ & 0.28 & $\begin{array}{l}\% \text { converted to } \\
\text { fraction }\end{array}$ & fresh timothy & Datum & 0.28 & dry/wet \\
\hline EXPOSURE & DRYFA2 & kg_dry/kg_as-fed & $\begin{array}{l}\text { Dry-to-wet conversion } \\
\text { factor for animal feed. }\end{array}$ & 6. milk forage & $\begin{array}{l}\text { Ensminger et al. (1990, } \\
\text { Table V-1) }\end{array}$ & 0.29 & $\begin{array}{l}\% \text { converted to } \\
\text { fraction }\end{array}$ & fresh barley grass & Datum & 0.29 & dry/wet \\
\hline EXPOSURE & DRYFA2 & kg_dry/kg_as-fed & $\begin{array}{l}\text { Dry-to-wet conversion } \\
\text { factor for animal feed. }\end{array}$ & 6. milk forage & $\begin{array}{l}\text { Ensminger et al. (1990, } \\
\text { Table V-1) }\end{array}$ & 0.3 & $\begin{array}{l}\% \text { converted to } \\
\text { fraction }\end{array}$ & fresh bahiagrass & Datum & 0.30 & dry/wet \\
\hline EXPOSURE & DRYFA2 & kg_dry/kg_as-fed & $\begin{array}{l}\text { Dry-to-wet conversion } \\
\text { factor for animal feed. }\end{array}$ & 6. milk forage & $\begin{array}{l}\text { Ensminger et al. (1990, } \\
\text { Table V-1) }\end{array}$ & $0.22-0.38$ & $\begin{array}{l}\% \text { converted to } \\
\text { fraction }\end{array}$ & fresh corn plants & Datum & 0.30 & dry/wet \\
\hline EXPOSURE & DRYFA2 & kg_dry/kg_as-fed & $\begin{array}{l}\text { Dry-to-wet conversion } \\
\text { factor for animal feed. }\end{array}$ & 6. milk forage & IAEA (2009, Table 2) & 34 & $\begin{array}{l}\text { percent dry } \\
\text { matter, as fed }\end{array}$ & $\begin{array}{l}\text { slender wheatgrass, } \\
\text { vegetative mass }\end{array}$ & Datum & 0.34 & dry/wet \\
\hline
\end{tabular}


Table H.6. (contd)

\begin{tabular}{|c|c|c|c|c|c|c|c|c|c|c|c|}
\hline Gv2_module & Gv2_ID & Gv2_Units ${ }^{(a)}$ & Gv2_Description & Feed Type & Reference & $\begin{array}{l}\text { Values } \\
\text { Cited }\end{array}$ & Units Cited & Comment & $\begin{array}{c}\text { Data } \\
\text { Reported }\end{array}$ & $\begin{array}{l}\text { Value in } \\
\text { Gv2 } \\
\text { Units }\end{array}$ & Units $^{(a)}$ \\
\hline EXPOSURE & DRYFA2 & kg_dry/kg_as-fed & $\begin{array}{l}\text { Dry-to-wet conversion } \\
\text { factor for animal feed. }\end{array}$ & 6. milk forage & $\begin{array}{l}\text { Ensminger et al. (1990, } \\
\text { Table V-1) }\end{array}$ & $0.31-0.42$ & $\begin{array}{l}\% \text { converted to } \\
\text { fraction }\end{array}$ & fresh bluegrass & $\begin{array}{l}\text { mean of } \\
\text { range }\end{array}$ & 0.37 & dry/wet \\
\hline EXPOSURE & DRYFA2 & kg_dry/kg_as-fed & $\begin{array}{l}\text { Dry-to-wet conversion } \\
\text { factor for animal feed. }\end{array}$ & 6. milk forage & $\begin{array}{l}\text { Ensminger et al. (1990, } \\
\text { Table V-1) }\end{array}$ & 0.38 & $\begin{array}{l}\% \text { converted to } \\
\text { fraction }\end{array}$ & fresh rabbitbrush & Datum & 0.38 & dry/wet \\
\hline EXPOSURE & DRYFA2 & kg_dry/kg_as-fed & $\begin{array}{l}\text { Dry-to-wet conversion } \\
\text { factor for animal feed. }\end{array}$ & 6. milk forage & $\begin{array}{l}\text { Ensminger et al. (1990, } \\
\text { Table V-1) }\end{array}$ & $0.22-0.55$ & $\begin{array}{l}\% \text { converted to } \\
\text { fraction }\end{array}$ & fresh cheatgrass & $\begin{array}{l}\text { mean of } \\
\text { range }\end{array}$ & 0.39 & dry/wet \\
\hline EXPOSURE & DRYFA2 & kg_dry/kg_as-fed & $\begin{array}{l}\text { Dry-to-wet conversion } \\
\text { factor for animal feed. }\end{array}$ & 6. milk forage & $\begin{array}{l}\text { Ensminger et al. (1990, } \\
\text { Table V-1) }\end{array}$ & $0.23-0.34$ & $\begin{array}{l}\text { \% converted to } \\
\text { fraction }\end{array}$ & fresh orchard grass & $\begin{array}{l}\text { mean of } \\
\text { range }\end{array}$ & 0.39 & dry/wet \\
\hline EXPOSURE & DRYFA2 & kg_dry/kg_as-fed & $\begin{array}{l}\text { Dry-to-wet conversion } \\
\text { factor for animal feed. }\end{array}$ & 6. milk forage & $\begin{array}{l}\text { Ensminger et al. (1990, } \\
\text { Table V-1) }\end{array}$ & 0.48 & $\begin{array}{l}\% \text { converted to } \\
\text { fraction }\end{array}$ & $\begin{array}{l}\text { fresh Indian } \\
\text { ricegrass }\end{array}$ & Datum & 0.48 & dry/wet \\
\hline EXPOSURE & DRYFA2 & kg_dry/kg_as-fed & $\begin{array}{l}\text { Dry-to-wet conversion } \\
\text { factor for animal feed. }\end{array}$ & 6. milk forage & $\begin{array}{l}\text { Ensminger et al. (1990, } \\
\text { Table V-1) }\end{array}$ & $0.28-0.75$ & $\begin{array}{l}\% \text { converted to } \\
\text { fraction }\end{array}$ & $\begin{array}{l}\text { fresh crested } \\
\text { wheatgrass }\end{array}$ & $\begin{array}{l}\text { mean of } \\
\text { range }\end{array}$ & 0.52 & dry/wet \\
\hline EXPOSURE & DRYFA2 & kg_dry/kg_as-fed & $\begin{array}{l}\text { Dry-to-wet conversion } \\
\text { factor for animal feed. }\end{array}$ & 6. milk forage & $\begin{array}{l}\text { Ensminger et al. (1990, } \\
\text { Table V-1) }\end{array}$ & $0.28-0.75$ & $\begin{array}{l}\% \text { converted to } \\
\text { fraction }\end{array}$ & fresh wheatgrass & $\begin{array}{l}\text { mean of } \\
\text { range }\end{array}$ & 0.52 & dry/wet \\
\hline
\end{tabular}


Table H.7. Leafy Vegetables

\begin{tabular}{|c|c|c|c|c|c|c|c|c|c|c|c|}
\hline Gv2_module & Gv2_ID & Gv2_Units & Gv2_Description & Food type $\mathrm{a}^{(\mathrm{a})}$ & Reference $^{(\mathrm{b})}$ & $\begin{array}{l}\text { Values } \\
\text { Cited }\end{array}$ & Units Cited & Comment & $\begin{array}{c}\text { Data } \\
\text { Reported }\end{array}$ & $\begin{array}{l}\text { Value in } \\
\text { Gv2 } \\
\text { units }\end{array}$ & Units \\
\hline Exposure & DRYFAC & kg_dry/kg_wet & $\begin{array}{l}\text { Dry-to-wet } \\
\text { conversion factor } \\
\text { for food crops. }\end{array}$ & 1. Leafy veg & $\begin{array}{l}\text { USDA (2011, Nutrient } \\
\text { Database, Release 24) }\end{array}$ & 95.64 & g water/100 g veg & head lettuce & Datum & 0.05 & dry/wet \\
\hline Exposure & DRYFAC & kg_dry/kg_wet & $\begin{array}{l}\text { Dry-to-wet } \\
\text { conversion factor } \\
\text { for food crops. }\end{array}$ & 1. Leafy veg & $\begin{array}{l}\text { USDA (2011, Nutrient } \\
\text { Database, Release 24) }\end{array}$ & 95.64 & g water $/ 100 \mathrm{~g}$ veg & leaf lettuce & Datum & 0.05 & dry/wet \\
\hline Exposure & DRYFAC & kg_dry/kg_wet & $\begin{array}{l}\text { Dry-to-wet } \\
\text { conversion factor } \\
\text { for food crops. }\end{array}$ & 1. Leafy veg & $\begin{array}{l}\text { Gebhardt and Thomas } \\
\text { (2002, H\&G } \\
\text { Bulletin 72) }\end{array}$ & 96 & percent water & $\begin{array}{l}\text { butterhead lettuce. } \\
\text { Value limited by } \\
\text { GENII limit of } 0.05 \text {. }\end{array}$ & Datum & 0.05 & dry/wet \\
\hline Exposure & DRYFAC & kg_dry/kg_wet & $\begin{array}{l}\text { Dry-to-wet } \\
\text { conversion factor } \\
\text { for food crops. }\end{array}$ & 1. Leafy veg & $\begin{array}{l}\text { Gebhardt and Thomas } \\
\text { (2002, H\&G } \\
\text { Bulletin 72) }\end{array}$ & 96 & percent water & $\begin{array}{l}\text { crisphead (iceberg) } \\
\text { lettuce. }\end{array}$ & Datum & 0.05 & dry/wet \\
\hline Exposure & DRYFAC & kg_dry/kg_wet & $\begin{array}{l}\text { Dry-to-wet } \\
\text { conversion factor } \\
\text { for food crops. }\end{array}$ & 1. Leafy veg & $\begin{array}{l}\text { Gebhardt and Thomas } \\
\text { (2002, H\&G } \\
\text { Bulletin 72) }\end{array}$ & 95 & percent water & romaine lettuce & Datum & 0.05 & dry/wet \\
\hline Exposure & DRYFAC & kg_dry/kg_wet & $\begin{array}{l}\text { Dry-to-wet } \\
\text { conversion factor } \\
\text { for food crops. }\end{array}$ & 1. Leafy veg & $\begin{array}{l}\text { Gebhardt and Thomas } \\
\text { (2002, H\&G } \\
\text { Bulletin 72) }\end{array}$ & 94 & percent water & mustard greens & Datum & 0.06 & dry/wet \\
\hline Exposure & DRYFAC & kg_dry/kg_wet & $\begin{array}{l}\text { Dry-to-wet } \\
\text { conversion factor } \\
\text { for food crops. }\end{array}$ & 1. Leafy veg & IAEA (2009, Table 2) & 8 & percent dry matter & lettuce & Datum & 0.08 & dry/wet \\
\hline Exposure & DRYFAC & kg_dry/kg_wet & $\begin{array}{l}\text { Dry-to-wet } \\
\text { conversion factor } \\
\text { for food crops. }\end{array}$ & 1. Leafy veg & IAEA (2009, Table 2) & 8 & percent dry matter & spinach & Datum & 0.08 & dry/wet \\
\hline Exposure & DRYFAC & kg_dry/kg_wet & $\begin{array}{l}\text { Dry-to-wet } \\
\text { conversion factor } \\
\text { for food crops. }\end{array}$ & 1. Leafy veg & $\begin{array}{l}\text { Gebhart and Thomas } \\
\text { (2002, H\&G } \\
\text { Bulletin 72) }\end{array}$ & 92 & percent water & cabbage & Datum & 0.08 & dry/wet \\
\hline Exposure & DRYFAC & kg_dry/kg_wet & $\begin{array}{l}\text { Dry-to-wet } \\
\text { conversion factor } \\
\text { for food crops. }\end{array}$ & 1. Leafy veg & $\begin{array}{l}\text { USDA (2011, Nutrient } \\
\text { Database, Release 24) }\end{array}$ & 90.55 & $\mathrm{~g}$ water/100 g veg & collard greens & Datum & 0.09 & dry/wet \\
\hline Exposure & DRYFAC & kg_dry/kg_wet & $\begin{array}{l}\text { Dry-to-wet } \\
\text { conversion factor } \\
\text { for food crops. }\end{array}$ & 1. Leafy veg & $\begin{array}{l}\text { USDA (2011, Nutrient } \\
\text { Database, Release 24) }\end{array}$ & 91.4 & $\mathrm{~g}$ water/100 g veg & fresh spinach & Datum & 0.09 & dry/wet \\
\hline Exposure & DRYFAC & kg_dry/kg_wet & $\begin{array}{l}\text { Dry-to-wet } \\
\text { conversion factor } \\
\text { for food crops. }\end{array}$ & 1. Leafy veg & IAEA (2009, Table 2) & 12 & percent dry matter & cabbage & Datum & 0.12 & dry/wet \\
\hline
\end{tabular}


Table H.7. (contd)

\begin{tabular}{|c|c|c|c|c|c|c|c|c|c|c|c|}
\hline Gv2_module & Gv2_ID & Gv2_Units & Gv2_Description & Food type $\mathrm{e}^{(\mathrm{a})}$ & Reference $^{(b)}$ & $\begin{array}{l}\text { Values } \\
\text { Cited }\end{array}$ & Units Cited & Comment & $\begin{array}{c}\text { Data } \\
\text { Reported }\end{array}$ & $\begin{array}{l}\text { Value in } \\
\text { Gv2 } \\
\text { units }\end{array}$ & Units \\
\hline Exposure & DRYFAC & kg_dry/kg_wet & $\begin{array}{l}\text { Dry-to-wet } \\
\text { conversion factor } \\
\text { for food crops. }\end{array}$ & 1. Leafy veg/high SA & IAEA (2009, Table 2) & 6 & percent dry matter & celery & Datum & 0.06 & dry/wet \\
\hline Exposure & DRYFAC & kg_dry/kg_wet & $\begin{array}{l}\text { Dry-to-wet } \\
\text { conversion factor } \\
\text { for food crops. }\end{array}$ & 1. Leafy veg/high SA & $\begin{array}{l}\text { Gebhardt and Thomas } \\
\text { (2002, H\&G } \\
\text { Bulletin 72) }\end{array}$ & 91 & percent water & broccoli & Datum & 0.09 & dry/wet \\
\hline $\begin{array}{ll}\text { (a) } & \text { "Leafy } \\
& \text { Gv2. "C } \\
& \text { material } \\
\text { (b) Standar }\end{array}$ & $\begin{array}{l}\text { Vegetables" } \\
\text { Dther vegetab } \\
\text { s that can be } \\
\text { d deviation o }\end{array}$ & $\begin{array}{l}\text { food types furthe } \\
\text { les” food types } f \\
\text { used for feed or } \\
\text { n water fraction }\end{array}$ & $\begin{array}{l}\text { r qualified by /high S } \\
\text { urther qualified as /rc } \\
\text { forage by terrestrial a } \\
\text { provided in USDA (2 }\end{array}$ & $\begin{array}{l}\text { A to indicate edible crop } \\
\text { ot, /berry, /melon-cuke, } \\
\text { nimals. } \\
\text { O11) database. }\end{array}$ & $\begin{array}{l}\text { s with high surface area- } \\
\text { /bean FW (fresh-weight }\end{array}$ & $\begin{array}{l}\text { olume ra } \\
\text { n), or /be }\end{array}$ & $\begin{array}{l}\text { ios, which can be ad } \\
\text { an DW (dried bean) }\end{array}$ & $\begin{array}{l}\text { equately represen } \\
\text { o provide further }\end{array}$ & $\begin{array}{l}\text { he leafy veg } \\
\text { Feed/Forag }\end{array}$ & $\begin{array}{l}\text { able mo } \\
\text { are leafy }\end{array}$ & of \\
\hline
\end{tabular}


Table H.8. Other Vegetables

\begin{tabular}{|c|c|c|c|c|c|c|c|c|c|c|c|}
\hline Gv2_module & Gv2_ID & Gv2_Units & Gv2_Description & Food type $^{(a)}$ & Reference $^{(\mathrm{b})}$ & $\begin{array}{l}\text { Values } \\
\text { Cited }\end{array}$ & Units Cited & Comment & $\begin{array}{c}\text { Data } \\
\text { Reported }\end{array}$ & $\begin{array}{l}\text { Value in } \\
\text { Gv2 } \\
\text { units }\end{array}$ & Units \\
\hline Exposure & DRYFAC & kg_dry/kg_wet & $\begin{array}{l}\text { Dry-to-wet } \\
\text { conversion factor } \\
\text { for food crops. }\end{array}$ & 2. Other veg & $\begin{array}{l}\text { Gebhardt and Thomas } \\
\text { (2002, H\&G } \\
\text { Bulletin 72) }\end{array}$ & 94 & percent water & tomato & Datum & 0.06 & dry/wet \\
\hline Exposure & DRYFAC & kg_dry/kg_wet & $\begin{array}{l}\text { Dry-to-wet } \\
\text { conversion factor } \\
\text { for food crops. }\end{array}$ & 2. Other veg & IAEA (2009, Table 2) & 6 & percent dry matter & tomato & Datum & 0.06 & dry/wet \\
\hline Exposure & DRYFAC & kg_dry/kg_wet & $\begin{array}{l}\text { Dry-to-wet } \\
\text { conversion factor } \\
\text { for food crops. }\end{array}$ & 2. Other veg & $\begin{array}{l}\text { USDA (2011, Nutrient } \\
\text { Database, Release 24) }\end{array}$ & 92.54 & g water/100 g veg & mushroom & Datum & 0.08 & dry/wet \\
\hline Exposure & DRYFAC & kg_dry/kg_wet & $\begin{array}{l}\text { Dry-to-wet } \\
\text { conversion factor } \\
\text { for food crops. }\end{array}$ & 2. Other veg & $\begin{array}{l}\text { Gebhardt and Thomas } \\
\text { (2002, H\&G } \\
\text { Bulletin 72) }\end{array}$ & 92 & percent water & raw mushroom & Datum & 0.08 & dry/wet \\
\hline Exposure & DRYFAC & kg_dry/kg_wet & $\begin{array}{l}\text { Dry-to-wet } \\
\text { conversion factor } \\
\text { for food crops. }\end{array}$ & 2. Other veg & $\begin{array}{l}\text { Gebhardt and Thomas } \\
\text { (2002, H\&G } \\
\text { Bulletin 72) }\end{array}$ & 92 & percent water & sweet pepper & Datum & 0.08 & dry/wet \\
\hline Exposure & DRYFAC & kg_dry/kg_wet & $\begin{array}{l}\text { Dry-to-wet } \\
\text { conversion factor } \\
\text { for food crops. }\end{array}$ & 2. Other veg & $\begin{array}{l}\text { Gebhardt and Thomas } \\
\text { (2002, H\&G } \\
\text { Bulletin 72) }\end{array}$ & 92 & percent water & tomatillo & Datum & 0.08 & dry/wet \\
\hline Exposure & DRYFAC & kg_dry/kg_wet & $\begin{array}{l}\text { Dry-to-wet } \\
\text { conversion factor } \\
\text { for food crops. }\end{array}$ & 2. Other veg & $\begin{array}{l}\text { USDA (2011, Nutrient } \\
\text { Database, Release 24) }\end{array}$ & 90.95 & g water/100 g veg & strawberries, raw & Datum & 0.09 & dry/wet \\
\hline Exposure & DRYFAC & kg_dry/kg_wet & $\begin{array}{l}\text { Dry-to-wet } \\
\text { conversion factor } \\
\text { for food crops. }\end{array}$ & 2. Other veg & $\begin{array}{l}\text { USDA (2011, Nutrient } \\
\text { Database, Release 24) }\end{array}$ & 90.32 & g water/100 g veg & fresh snap bean & Datum & 0.10 & dry/wet \\
\hline Exposure & DRYFAC & kg_dry/kg_wet & $\begin{array}{l}\text { Dry-to-wet } \\
\text { conversion factor } \\
\text { for food crops. }\end{array}$ & 2. Other veg & $\begin{array}{l}\text { Gebhardt and Thomas } \\
\text { (2002, H\&G } \\
\text { Bulletin 72) }\end{array}$ & 89 & percent water & peas, edible pod & Datum & 0.11 & dry/wet \\
\hline Exposure & DRYFAC & kg_dry/kg_wet & $\begin{array}{l}\text { Dry-to-wet } \\
\text { conversion factor } \\
\text { for food crops. }\end{array}$ & 2. Other veg & $\begin{array}{l}\text { Gebhardt and Thomas } \\
\text { (2002, H\&G } \\
\text { Bulletin 72) }\end{array}$ & 89 & percent water & snap beans & Datum & 0.11 & dry/wet \\
\hline Exposure & DRYFAC & kg_dry/kg_wet & $\begin{array}{l}\text { Dry-to-wet } \\
\text { conversion factor } \\
\text { for food crops. }\end{array}$ & 2. Other veg & IAEA (2009, Table 2) & 11 & percent dry matter & cauliflower & Datum & 0.11 & dry/wet \\
\hline Exposure & DRYFAC & kg_dry/kg_wet & $\begin{array}{l}\text { Dry-to-wet } \\
\text { conversion factor } \\
\text { for food crops. }\end{array}$ & 2. Other veg & $\begin{array}{l}\text { Gebhardt and Thomas } \\
\text { (2002, H\&G } \\
\text { Bulletin 72) }\end{array}$ & 87 & percent water & pineapple & Datum & 0.13 & dry/wet \\
\hline Exposure & DRYFAC & kg_dry/kg_wet & $\begin{array}{l}\text { Dry-to-wet } \\
\text { conversion factor } \\
\text { for food crops. }\end{array}$ & 2. Other veg & $\begin{array}{l}\text { USDA (2011, Nutrient } \\
\text { Database, Release 24) }\end{array}$ & 76.05 & g water/100 g veg & $\begin{array}{l}\text { fresh sweet corn. See } \\
\text { grain, for dehydrated } \\
\text { grain corn. }\end{array}$ & Datum & 0.24 & dry/wet \\
\hline
\end{tabular}


Table H.8. (contd)

\begin{tabular}{|c|c|c|c|c|c|c|c|c|c|c|c|}
\hline Gv2_module & Gv2_ID & Gv2_Units & Gv2_Description & Food type $^{(\mathrm{a})}$ & Reference $^{(\mathrm{b})}$ & $\begin{array}{l}\text { Values } \\
\text { Cited }\end{array}$ & Units Cited & Comment & $\begin{array}{c}\text { Data } \\
\text { Reported }\end{array}$ & $\begin{array}{l}\text { Value in } \\
\text { Gv2 } \\
\text { units }\end{array}$ & Units \\
\hline Exposure & DRYFAC & kg_dry/kg_wet & $\begin{array}{l}\text { Dry-to-wet } \\
\text { conversion factor } \\
\text { for food crops. }\end{array}$ & 2. Other veg & $\begin{array}{l}\text { Gebhardt and Thomas } \\
\text { (2002, H\&G } \\
\text { Bulletin 72) }\end{array}$ & 75 & percent water & black-eyed peas & Datum & 0.25 & dry/wet \\
\hline Exposure & DRYFAC & kg_dry/kg_wet & $\begin{array}{l}\text { Dry-to-wet } \\
\text { conversion factor } \\
\text { for food crops. }\end{array}$ & 2. Other veg & $\begin{array}{l}\text { Gebhardt and Thomas } \\
\text { (2002, H\&G } \\
\text { Bulletin 72) }\end{array}$ & 70 & percent water & $\begin{array}{l}\text { fresh raw sweet corn } \\
\text { kernels }\end{array}$ & Datum & 0.30 & dry/wet \\
\hline Exposure & DRYFAC & kg_dry/kg_wet & $\begin{array}{l}\text { Dry-to-wet } \\
\text { conversion factor } \\
\text { for food crops. }\end{array}$ & 2. Other veg & $\begin{array}{l}\text { Gebhardt and Thomas } \\
\text { (2002, H\&G } \\
\text { Bulletin 72) }\end{array}$ & 69 & percent water & soybean, green cooked & Datum & 0.31 & dry/wet \\
\hline Exposure & DRYFAC & kg_dry/kg_wet & $\begin{array}{l}\text { Dry-to-wet } \\
\text { conversion factor } \\
\text { for food crops. }\end{array}$ & $\begin{array}{l}\text { 2. Other veg/bean } \\
\text { DW }\end{array}$ & IAEA (2009, Table 2) & 83 & percent dry matter & garden pea, seeds & Datum & 0.83 & dry/wet \\
\hline Exposure & DRYFAC & kg_dry/kg_wet & $\begin{array}{l}\text { Dry-to-wet } \\
\text { conversion factor } \\
\text { for food crops. }\end{array}$ & $\begin{array}{l}\text { 2. Other veg/bean } \\
\text { DW }\end{array}$ & IAEA (2009, Table 2) & 85.1 & percent dry matter & field pea, seeds & Datum & 0.85 & dry/wet \\
\hline Exposure & DRYFAC & kg_dry/kg_wet & $\begin{array}{l}\text { Dry-to-wet } \\
\text { conversion factor } \\
\text { for food crops. }\end{array}$ & $\begin{array}{l}\text { 2. Other veg/bean } \\
\text { DW }\end{array}$ & IAEA (2009, Table 2) & 88 & percent dry matter & broadbean seeds & Datum & 0.88 & dry/wet \\
\hline Exposure & DRYFAC & kg_dry/kg_wet & $\begin{array}{l}\text { Dry-to-wet } \\
\text { conversion factor } \\
\text { for food crops. }\end{array}$ & $\begin{array}{l}\text { 2. Other veg/bean } \\
\text { DW }\end{array}$ & IAEA (2009, Table 2) & 88.6 & percent dry matter & soybean seeds & Datum & 0.89 & dry/wet \\
\hline Exposure & DRYFAC & kg_dry/kg_wet & $\begin{array}{l}\text { Dry-to-wet } \\
\text { conversion factor } \\
\text { for food crops. }\end{array}$ & 2. Other veg/berry & IAEA (2009, Table 4) & 10.8 & $\begin{array}{l}\text { percent dry } \\
\text { matter, mean }\end{array}$ & cranberry & Datum & 0.11 & dry/wet \\
\hline Exposure & DRYFAC & kg_dry/kg_wet & $\begin{array}{l}\text { Dry-to-wet } \\
\text { conversion factor } \\
\text { for food crops. }\end{array}$ & 2. Other veg/berry & $\begin{array}{l}\text { Gebhardt and Thomas } \\
\text { (2002, H\&G } \\
\text { Bulletin 72) }\end{array}$ & 87 & percent water & raspberries & Datum & 0.13 & dry/wet \\
\hline Exposure & DRYFAC & kg_dry/kg_wet & $\begin{array}{l}\text { Dry-to-wet } \\
\text { conversion factor } \\
\text { for food crops. }\end{array}$ & 2. Other veg/berry & IAEA (2009, Table 4) & 13.2 & $\begin{array}{l}\text { percent dry } \\
\text { matter, mean }\end{array}$ & blueberry & Datum & 0.13 & dry/wet \\
\hline Exposure & DRYFAC & kg_dry/kg_wet & $\begin{array}{l}\text { Dry-to-wet } \\
\text { conversion factor } \\
\text { for food crops. }\end{array}$ & 2. Other veg/berry & $\begin{array}{l}\text { Gebhardt and Thomas } \\
\text { (2002, H\&G } \\
\text { Bulletin 72) }\end{array}$ & 86 & percent water & blackberries & Datum & 0.14 & dry/wet \\
\hline Exposure & DRYFAC & kg_dry/kg_wet & $\begin{array}{l}\text { Dry-to-wet } \\
\text { conversion factor } \\
\text { for food crops. }\end{array}$ & 2. Other veg/berry & IAEA (2009, Table 4) & 15.4 & $\begin{array}{l}\text { percent dry } \\
\text { matter, mean }\end{array}$ & wild strawberry & Datum & 0.15 & dry/wet \\
\hline Exposure & DRYFAC & kg_dry/kg_wet & $\begin{array}{l}\text { Dry-to-wet } \\
\text { conversion factor } \\
\text { for food crops. }\end{array}$ & 2. Other veg/berry & IAEA (2009, Table 2) & 16 & percent dry matter & raspberry & Datum & 0.16 & dry/wet \\
\hline
\end{tabular}


Table H.8. (contd)

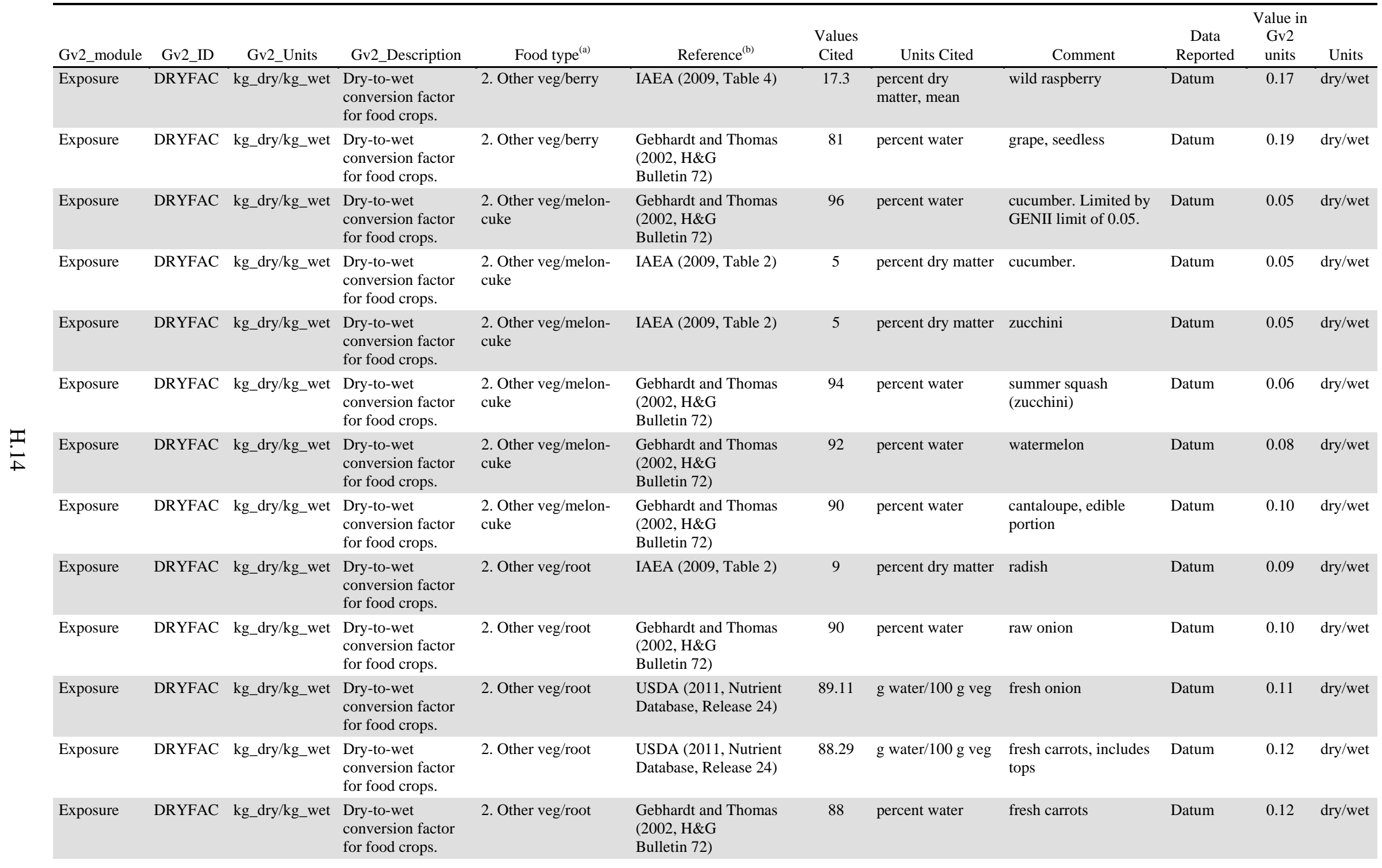


Table H.8. (contd)

\begin{tabular}{|c|c|c|c|c|c|c|c|c|c|c|c|}
\hline Gv2_module & Gv2_ID & Gv2_Units & Gv2_Description & Food type $^{(a)}$ & Reference $^{(b)}$ & $\begin{array}{l}\text { Values } \\
\text { Cited }\end{array}$ & Units Cited & Comment & $\begin{array}{c}\text { Data } \\
\text { Reported }\end{array}$ & $\begin{array}{l}\text { Value in } \\
\text { Gv2 } \\
\text { units }\end{array}$ & Units \\
\hline Exposure & DRYFAC & kg_dry/kg_wet & $\begin{array}{l}\text { Dry-to-wet } \\
\text { conversion factor } \\
\text { for food crops. }\end{array}$ & 2. Other veg/root & IAEA (2009, Table 2) & 14 & percent dry matter & carrot & Datum & 0.14 & dry/wet \\
\hline Exposure & DRYFAC & kg_dry/kg_wet & $\begin{array}{l}\text { Dry-to-wet } \\
\text { conversion factor } \\
\text { for food crops. }\end{array}$ & 2. Other veg/root & $\begin{array}{l}\text { USDA (2011, Nutrient } \\
\text { Database, Release 24) }\end{array}$ & 78.58 & g water $/ 100 \mathrm{~g}$ veg & fresh potato & Datum & 0.21 & dry/wet \\
\hline Exposure & DRYFAC & kg_dry/kg_wet & $\begin{array}{l}\text { Dry-to-wet } \\
\text { conversion factor } \\
\text { for food crops. }\end{array}$ & 2. Other veg/root & IAEA (2009, Table 2) & 21 & percent dry matter & potato & Datum & 0.21 & dry/wet \\
\hline Exposure & DRYFAC & kg_dry/kg_wet & $\begin{array}{l}\text { Dry-to-wet } \\
\text { conversion factor } \\
\text { for food crops. }\end{array}$ & 2. Other veg/root & $\begin{array}{l}\text { USDA (2011, Nutrient } \\
\text { Database, Release 24) }\end{array}$ & 77.28 & g water $/ 100 \mathrm{~g}$ veg & fresh sweet potato & Datum & 0.23 & dry/wet \\
\hline Exposure & DRYFAC & kg_dry/kg_wet & $\begin{array}{l}\text { Dry-to-wet } \\
\text { conversion factor } \\
\text { for food crops. }\end{array}$ & 2. Other veg/root & $\begin{array}{l}\text { Derived from Gebhardt } \\
\text { and Thomas (2002, } \\
\text { H\&G Bulletin 72) }\end{array}$ & 67 & percent water & $\begin{array}{l}\text { raw potato (potato } \\
\text { skin, peeled potato) }\end{array}$ & Datum & 0.33 & dry/wet \\
\hline \multicolumn{12}{|c|}{$\begin{array}{l}\text { (a) "Leafy Vegetables" food types further qualified by /high SA to indicate edible crops with high surface area-to-volume ratios, which can be adequately represented by the leafy vegetable model of } \\
\text { Gv2. "Other vegetables" food types further qualified as /root, /berry, /melon-cuke, /bean FW (fresh-weight bean), or /bean DW (dried bean) to provide further details. Feed/Forage are leafy or grain } \\
\text { materials that can be used for feed or forage by terrestrial animals. } \\
\text { (b) Standard deviation on water fraction provided in USDA (2011) database. }\end{array}$} \\
\hline
\end{tabular}


Table H.9. Fruit

\begin{tabular}{|c|c|c|c|c|c|c|c|c|c|c|c|}
\hline Gv2_module & Gv2_ID & Gv2_Units & Gv2_Description & Food type $^{(a)}$ & Reference $^{(\mathrm{b})}$ & $\begin{array}{l}\text { Values } \\
\text { Cited }\end{array}$ & Units Cited & Comment & $\begin{array}{c}\text { Data } \\
\text { Reported }\end{array}$ & $\begin{array}{l}\text { Value in } \\
\text { Gv2 } \\
\text { units }\end{array}$ & Units \\
\hline Exposure & DRYFAC & kg_dry/kg_wet & $\begin{array}{l}\text { Dry-to-wet } \\
\text { conversion factor } \\
\text { for food crops. }\end{array}$ & 3. Fruit & $\begin{array}{l}\text { Gebhardt and Thomas } \\
\text { (2002, H\&G } \\
\text { Bulletin 72) }\end{array}$ & 89 & percent water & lemon, no peel & Datum & 0.11 & dry/wet \\
\hline Exposure & DRYFAC & kg_dry/kg_wet & $\begin{array}{l}\text { Dry-to-wet } \\
\text { conversion factor } \\
\text { for food crops. }\end{array}$ & 3. Fruit & $\begin{array}{l}\text { Gebhardt and Thomas } \\
\text { (2002, H\&G } \\
\text { Bulletin 72) }\end{array}$ & 88 & percent water & Asian pear & Datum & 0.12 & dry/wet \\
\hline Exposure & DRYFAC & kg_dry/kg_wet & $\begin{array}{l}\text { Dry-to-wet } \\
\text { conversion factor } \\
\text { for food crops. }\end{array}$ & 3. Fruit & $\begin{array}{l}\text { Gebhardt and Thomas } \\
\text { (2002, H\&G } \\
\text { Bulletin 72) }\end{array}$ & 88 & percent water & peach, no pit & Datum & 0.12 & dry/wet \\
\hline Exposure & DRYFAC & kg_dry/kg_wet & $\begin{array}{l}\text { Dry-to-wet } \\
\text { conversion factor } \\
\text { for food crops. }\end{array}$ & 3. Fruit & $\begin{array}{l}\text { Gebhardt and Thomas } \\
\text { (2002, H\&G } \\
\text { Bulletin 72) }\end{array}$ & 87 & percent water & orange, no peel or seed & Datum & 0.13 & dry/wet \\
\hline Exposure & DRYFAC & kg_dry/kg_wet & $\begin{array}{l}\text { Dry-to-wet } \\
\text { conversion factor } \\
\text { for food crops. }\end{array}$ & 3. Fruit & $\begin{array}{l}\text { USDA (2011, Nutrient } \\
\text { Database, Release 24) }\end{array}$ & 86.35 & g water/100 g veg & apricots, raw & Datum & 0.14 & dry/wet \\
\hline Exposure & DRYFAC & kg_dry/kg_wet & $\begin{array}{l}\text { Dry-to-wet } \\
\text { conversion factor } \\
\text { for food crops. }\end{array}$ & 3. Fruit & $\begin{array}{l}\text { USDA (2011, Nutrient } \\
\text { Database, Release 24) }\end{array}$ & 85.56 & g water/100 g veg & apple, raw with skin & Datum & 0.15 & dry/wet \\
\hline Exposure & DRYFAC & kg_dry/kg_wet & $\begin{array}{l}\text { Dry-to-wet } \\
\text { conversion factor } \\
\text { for food crops. }\end{array}$ & 3. Fruit & $\begin{array}{l}\text { Gebhardt and Thomas } \\
\text { (2002, H\&G } \\
\text { Bulletin 72) }\end{array}$ & 85 & percent water & plum & Datum & 0.15 & dry/wet \\
\hline Exposure & DRYFAC & kg_dry/kg_wet & $\begin{array}{l}\text { Dry-to-wet } \\
\text { conversion factor } \\
\text { for food crops. }\end{array}$ & 3. Fruit & $\begin{array}{l}\text { USDA (2011, Nutrient } \\
\text { Database, Release 24) }\end{array}$ & 83.71 & g water/100 g veg & pears, raw & Datum & 0.16 & dry/wet \\
\hline Exposure & DRYFAC & kg_dry/kg_wet & $\begin{array}{l}\text { Dry-to-wet } \\
\text { conversion factor } \\
\text { for food crops. }\end{array}$ & 3. Fruit & $\begin{array}{l}\text { Gebhardt and Thomas } \\
\text { (2002, H\&G } \\
\text { Bulletin 72) }\end{array}$ & 84 & percent water & apple, raw unpeeled & Datum & 0.16 & dry/wet \\
\hline Exposure & DRYFAC & kg_dry/kg_wet & $\begin{array}{l}\text { Dry-to-wet } \\
\text { conversion factor } \\
\text { for food crops. }\end{array}$ & 3. Fruit & $\begin{array}{l}\text { Gebhardt and Thomas } \\
\text { (2002, H\&G } \\
\text { Bulletin 72) }\end{array}$ & 84 & percent water & pear, cored & Datum & 0.16 & dry/wet \\
\hline Exposure & DRYFAC & kg_dry/kg_wet & $\begin{array}{l}\text { Dry-to-wet } \\
\text { conversion factor } \\
\text { for food crops. }\end{array}$ & 3. Fruit & $\begin{array}{l}\text { Gebhardt and Thomas } \\
\text { (2002, H\&G } \\
\text { Bulletin 72) }\end{array}$ & 83 & percent water & kiwi fruit, no skin & Datum & 0.17 & dry/wet \\
\hline Exposure & DRYFAC & kg_dry/kg_wet & $\begin{array}{l}\text { Dry-to-wet } \\
\text { conversion factor } \\
\text { for food crops. }\end{array}$ & 3. Fruit & $\begin{array}{l}\text { Gebhardt and Thomas } \\
\text { (2002, H\&G } \\
\text { Bulletin 72) }\end{array}$ & 82 & percent water & mango, no skin or seed & Datum & 0.18 & dry/wet \\
\hline Exposure & DRYFAC & kg_dry/kg_wet & $\begin{array}{l}\text { Dry-to-wet } \\
\text { conversion factor } \\
\text { for food crops. }\end{array}$ & 3. Fruit & $\begin{array}{l}\text { Gebhardt and Thomas } \\
\text { (2002, H\&G } \\
\text { Bulletin 72) }\end{array}$ & 81 & percent water & $\begin{array}{l}\text { sweet cherries, no pits } \\
\text { or stems }\end{array}$ & Datum & 0.19 & dry/wet \\
\hline
\end{tabular}


Table H.9. (contd)

\begin{tabular}{|c|c|c|c|c|c|c|c|c|c|c|c|}
\hline Gv2_module & Gv2_ID & Gv2_Units & Gv2_Description & Food type $\mathrm{e}^{(\mathrm{a})}$ & Reference $^{(\mathrm{b})}$ & $\begin{array}{l}\text { Values } \\
\text { Cited }\end{array}$ & Units Cited & Comment & \multicolumn{3}{|c|}{$\begin{array}{l}\text { Value in } \\
\text { Gv2 }\end{array}$} \\
\hline Exposure & & kg_dry/kg_wet & $\begin{array}{l}\text { Dry-to-wet } \\
\text { conversion factor } \\
\text { for food crops. }\end{array}$ & 3. Fruit & $\begin{array}{l}\text { USDA (2011, Nutrient } \\
\text { Database, Release 24) }\end{array}$ & 78.81 & g water/100 g veg & avocado, raw (FLA) & Datum & 0.21 & dry/wet \\
\hline Exposure & DRYFAC & kg_dry/kg_wet & $\begin{array}{l}\text { Dry-to-wet } \\
\text { conversion factor } \\
\text { for food crops. }\end{array}$ & 3. Fruit & $\begin{array}{l}\text { Gebhardt and Thomas } \\
\text { (2002, H\&G } \\
\text { Bulletin 72) }\end{array}$ & 74 & percent water & banana & Datum & 0.26 & dry/wet \\
\hline Exposure & DRYFAC & kg_dry/kg_wet & $\begin{array}{l}\text { Dry-to-wet } \\
\text { conversion factor } \\
\text { for food crops. }\end{array}$ & 3. Fruit & $\begin{array}{l}\text { USDA (2011, Nutrient } \\
\text { Database, Release 24) }\end{array}$ & 72.33 & g water/100 g veg & avocado, raw (CA) & Datum & 0.28 & dry/wet \\
\hline Exposure & DRYFAC & kg_dry/kg_wet & $\begin{array}{l}\text { Dry-to-wet } \\
\text { conversion factor } \\
\text { for food crops. }\end{array}$ & 3. Fruit & $\begin{array}{l}\text { Gebhardt and Thomas } \\
\text { (2002, H\&G } \\
\text { Bulletin 72) }\end{array}$ & 15 & percent water & raisins, as eaten & Datum & 0.85 & dry/wet \\
\hline Exposure & DRYFAC & kg_dry/kg_wet & $\begin{array}{l}\text { Dry-to-wet } \\
\text { conversion factor } \\
\text { for food crops. }\end{array}$ & 3. Fruit/nut & $\begin{array}{l}\text { Gebhardt and Thomas } \\
\text { (2002, H\&G } \\
\text { Bulletin 72) }\end{array}$ & 5 & percent water & $\begin{array}{l}\text { Tree growth, other veg } \\
\text { - almond (edible nut) }\end{array}$ & Datum & 0.95 & dry/wet \\
\hline Exposure & DRYFAC & kg_dry/kg_wet & $\begin{array}{l}\text { Dry-to-wet } \\
\text { conversion factor } \\
\text { for food crops. }\end{array}$ & 3. Fruit/nut & $\begin{array}{l}\text { Gebhardt and Thomas } \\
\text { (2002, H\&G } \\
\text { Bulletin 72) }\end{array}$ & 5 & percent water & $\begin{array}{l}\text { Tree growth, other veg } \\
\text { - hazelnut (edible nut) }\end{array}$ & Datum & 0.95 & dry/wet \\
\hline Exposure & DRYFAC & kg_dry/kg_wet & $\begin{array}{l}\text { Dry-to-wet } \\
\text { conversion factor } \\
\text { for food crops. }\end{array}$ & 3. Fruit/nut & $\begin{array}{l}\text { Gebhardt and Thomas } \\
\text { (2002, H\&G } \\
\text { Bulletin 72) }\end{array}$ & 4 & percent water & $\begin{array}{l}\text { Tree growth, other veg } \\
\text { - walnut (edible nut). } \\
\text { Limited by Gv2 max } \\
\text { of } 0.95 \text {. }\end{array}$ & Datum & 0.95 & dry/wet \\
\hline $\begin{array}{ll}\text { (a) "Leafy I } \\
\text { Gv2. "O } \\
\text { material } \\
\text { (b) Standard }\end{array}$ & $\begin{array}{l}\text { Vegetables" } \\
\text { ther vegetal } \\
\text { s that can be } \\
\text { deviation o }\end{array}$ & used for feed or & $\begin{array}{l}\text { qualified by /high } \\
\text { urther qualified as / } 1 \\
\text { forage by terrestrial }\end{array}$ & $\begin{array}{l}\text { to indicate edible } \\
\text { t, /berry, /melon-c } \\
\text { imals. } \\
\text { 11) database. }\end{array}$ & $\begin{array}{l}\text { with high surface area-t } \\
\text { bean FW (fresh-weight }\end{array}$ & $\begin{array}{l}\text { yolume ra } \\
\text { n), or /bea }\end{array}$ & $\begin{array}{l}\text { ios, which can be ac } \\
\text { in DW (dried bean) }\end{array}$ & $\begin{array}{l}\text { equately represented by th } \\
\text { o provide further details. }\end{array}$ & $\begin{array}{l}\text { he leafy veg } \\
\text { Feed/Forag }\end{array}$ & $\begin{array}{l}\text { ble mo } \\
\text { re leafy }\end{array}$ & of \\
\hline
\end{tabular}


Table H.10. Grains

\begin{tabular}{|c|c|c|c|c|c|c|c|c|c|c|c|}
\hline Gv2_module & Gv2_ID & Gv2_Units & Gv2_Description & Food type $^{(a)}$ & Reference $^{(\mathrm{b})}$ & $\begin{array}{l}\text { Values } \\
\text { Cited }\end{array}$ & Units Cited & Comment & $\begin{array}{c}\text { Data } \\
\text { Reported }\end{array}$ & $\begin{array}{l}\text { Value in } \\
\text { Gv2 } \\
\text { units }\end{array}$ & Units \\
\hline Exposure & DRYFAC & kg_dry/kg_wet & $\begin{array}{l}\text { Dry-to-wet } \\
\text { conversion factor } \\
\text { for food crops. }\end{array}$ & 4. Grain & IAEA (2009, Table 2) & 85.2 & percent dry matter & maize (corn), grain & Datum & 0.85 & dry/wet \\
\hline Exposure & DRYFAC & kg_dry/kg_wet & $\begin{array}{l}\text { Dry-to-wet } \\
\text { conversion factor } \\
\text { for food crops. }\end{array}$ & 4. Grain & IAEA (2009, Table 2) & 86.7 & percent dry matter & oats, grain & Datum & 0.87 & dry/wet \\
\hline Exposure & DRYFAC & kg_dry/kg_wet & $\begin{array}{l}\text { Dry-to-wet } \\
\text { conversion factor } \\
\text { for food crops. }\end{array}$ & 4. Grain & IAEA (2009, Table 2) & 87 & percent dry matter & winter rye, grain & Datum & 0.87 & dry/wet \\
\hline Exposure & DRYFAC & kg_dry/kg_wet & $\begin{array}{l}\text { Dry-to-wet } \\
\text { conversion factor } \\
\text { for food crops. }\end{array}$ & 4. Grain & IAEA (2009, Table 2) & 87 & percent dry matter & barley, grain & Datum & 0.87 & dry/wet \\
\hline Exposure & DRYFAC & kg_dry/kg_wet & $\begin{array}{l}\text { Dry-to-wet } \\
\text { conversion factor } \\
\text { for food crops. }\end{array}$ & 4. Grain & IAEA (2009, Table 2) & 87 & percent dry matter & sorghum, grain & Datum & 0.87 & dry/wet \\
\hline Exposure & DRYFAC & kg_dry/kg_wet & $\begin{array}{l}\text { Dry-to-wet } \\
\text { conversion factor } \\
\text { for food crops. }\end{array}$ & 4. Grain & $\begin{array}{l}\text { Gebhardt and Thomas } \\
\text { (2002, H\&G } \\
\text { Bulletin 72) }\end{array}$ & 12 & percent water & rice, white raw & Datum & 0.88 & dry/wet \\
\hline Exposure & DRYFAC & kg_dry/kg_wet & $\begin{array}{l}\text { Dry-to-wet } \\
\text { conversion factor } \\
\text { for food crops. }\end{array}$ & 4. Grain & IAEA (2009, Table 2) & 88 & percent dry matter & wheat, grain & Datum & 0.88 & dry/wet \\
\hline Exposure & DRYFAC & kg_dry/kg_wet & $\begin{array}{l}\text { Dry-to-wet } \\
\text { conversion factor } \\
\text { for food crops. }\end{array}$ & 4. Grain & $\begin{array}{l}\text { Gebhardt and Thomas } \\
\text { (2002, H\&G } \\
\text { Bulletin 72) }\end{array}$ & 10 & percent water & cornmeal, whole grain & Datum & 0.90 & dry/wet \\
\hline Exposure & DRYFAC & kg_dry/kg_wet & $\begin{array}{l}\text { Dry-to-wet } \\
\text { conversion factor } \\
\text { for food crops. }\end{array}$ & 4. Grain & $\begin{array}{l}\text { Gebhardt and Thomas } \\
\text { (2002, H\&G } \\
\text { Bulletin 72) }\end{array}$ & 9 & percent water & couscous, uncooked & Datum & 0.91 & dry/wet \\
\hline Exposure & DRYFAC & kg_dry/kg_wet & $\begin{array}{l}\text { Dry-to-wet } \\
\text { conversion factor } \\
\text { for food crops. }\end{array}$ & 4. Grain & $\begin{array}{l}\text { Gebhardt and Thomas } \\
\text { (2002, H\&G } \\
\text { Bulletin 72) }\end{array}$ & 9 & percent water & $\begin{array}{l}\text { bulgur, uncooked } \\
\text { (wheat) }\end{array}$ & Datum & 0.91 & dry/wet \\
\hline Exposure & DRYFAC & kg_dry/kg_wet & $\begin{array}{l}\text { Dry-to-wet } \\
\text { conversion factor } \\
\text { for food crops. }\end{array}$ & 4.Grain & $\begin{array}{l}\text { Gebhardt and Thomas } \\
\text { (2002, H\&G } \\
\text { Bulletin 72) }\end{array}$ & 10 & percent water & barley, uncooked & Datum & 0.90 & dry/wet \\
\hline $\begin{array}{ll}\text { (a) "Leafy V } \\
\text { Gv2. "O } \\
\text { materials } \\
\text { (b) Standard }\end{array}$ & $\begin{array}{l}\text { Vegetables" } \\
\text { Dther vegetal } \\
\text { s that can be } \\
\text { d deviation o }\end{array}$ & $\begin{array}{l}\text { used for feed or } \\
\text { n water fraction }\end{array}$ & $\begin{array}{l}\text { qualified by /high S } \\
\text { urther qualified as /r } \\
\text { forage by terrestrial } \\
\text { orovided in USDA (2 }\end{array}$ & $\begin{array}{l}\text { to indicate edible } \\
\text { t, /berry, /melon-c } \\
\text { imals. } \\
\text { 11) database. }\end{array}$ & $\begin{array}{l}\text { with high surface area- } \\
\text { bean FW (fresh-weight }\end{array}$ & $\begin{array}{l}\text { olume ra } \\
\text { n), or /be }\end{array}$ & $\begin{array}{l}\text { ios, which can be ac } \\
\text { in DW (dried bean) }\end{array}$ & $\begin{array}{l}\text { lequately represented by } \\
\text { to provide further details }\end{array}$ & $\begin{array}{l}\text { he leafy veg } \\
\text { Feed/Forag }\end{array}$ & $\begin{array}{l}\text { ble mo } \\
\text { re leafy }\end{array}$ & $\begin{array}{l}\text { el of } \\
\text { or grain }\end{array}$ \\
\hline
\end{tabular}


PNNL-21950, Appendix H

\section{References}

Ensminger ME, JE Oldfield, and WW Heinemann. 1990. Feeds and Nutrition, second edition. Ensminger Publishing Company, Clovis, California.

Gebhardt SE and RG Thomas. 2002. Nutritive Value of Foods, Home and Garden Bulletin Number 72, USDA, Agricultural Research Service, Nutrient Data Laboratory, Beltsville, Maryland.

IAEA. 2009. "Quantification of Radionuclide Transfer in Terrestrial and Freshwater Environments for Radiological Assessments.” S Fenko, N Sanzharova, M Vidal, H Vandenhoeve, Y Thiry, E Reed, BJ Howard, G Prohl, G Zibold, B Varga, and A Rantavaara. Radioecological Definitions, Soil, Plant Classifications and Reference Ecological Data for Radiological Assessments. IAEA-TECDOC-1616, pp. 7-26, International Atomic Energy Agency, Vienna.

USDA. 2011. Composition of Foods, Raw, Processed, Prepared, USDA National Nutrient Database for Standard Reference, Release 24. USDA, Agricultural Research Service, Beltsville, Maryland. Last accessed at: http://www.nal.usda.gov/fnic/foodcomp/search/. 
PNNL-21950, Appendix I

\section{Appendix I}

\section{Holdup Times}




\section{Appendix I}

\section{Holdup Times}

This appendix lists hold-up times found from a single USDA reference and other reasonable

anecdotal values from internet sources. Apply values as they seem reasonable for the behaviors, climate, and practices in the region of interest for the indicated food types. 
Table I.1. Holdup Times

\begin{tabular}{|c|c|c|c|c|c|c|c|c|c|c|c|c|c|c|}
\hline $\begin{array}{l}\mathrm{Gv} 2_{-} \\
\text {module }\end{array}$ & Gv2_ID & $\begin{array}{l}\text { Gv2 } \\
\text { Units }\end{array}$ & Gv2_Description & Food Type & Qualifier & Reference & $\begin{array}{l}\text { Values } \\
\text { Cited }\end{array}$ & $\begin{array}{l}\text { Units } \\
\text { Cited }\end{array}$ & Comment & $\begin{array}{c}\text { Data } \\
\text { Reported }\end{array}$ & $\begin{array}{l}\text { Recom- } \\
\text { mended }\end{array}$ & $\begin{array}{l}\text { Min } \\
\text { Value }\end{array}$ & $\begin{array}{l}\text { Max } \\
\text { Value }\end{array}$ & Units \\
\hline Exposure & HLDUP & d & $\begin{array}{l}\text { Intake delay } \\
\text { harvest\&consumption }\end{array}$ & $\begin{array}{l}\text { 1.leafy } \\
\text { vegetable }\end{array}$ & Fresh, refrigerated & $\begin{array}{l}\text { (Internet } \\
\text { review) }\end{array}$ & & & e.g., lettuce, spinach & range & 4 & 1 & 7 & d \\
\hline Exposure & HLDUP & d & $\begin{array}{l}\text { Intake delay } \\
\text { harvest\&consumption }\end{array}$ & $\begin{array}{l}\text { 1.leafy } \\
\text { vegetable }\end{array}$ & Frozen & $\begin{array}{l}\text { (Internet } \\
\text { review) }\end{array}$ & & & e.g., spinach, collards & range & 180 & 30 & 360 & d \\
\hline Exposure & HLDUP & d & $\begin{array}{l}\text { Intake delay } \\
\text { harvest\&consumption }\end{array}$ & $\begin{array}{l}\text { 1.leafy } \\
\text { vegetable }\end{array}$ & $\begin{array}{l}\text { Canned - low acid } \\
\text { food }\end{array}$ & $\begin{array}{l}\text { USDA FSIS } \\
(2012)\end{array}$ & $2-5$ & $\mathrm{yr}$ & $\begin{array}{l}\text { e.g., spinach (not } \\
\text { sauerkraut/cabbage) }\end{array}$ & datum & 365 & & & d \\
\hline Exposure & HLDUP & d & $\begin{array}{l}\text { Intake delay } \\
\text { harvest\&consumption }\end{array}$ & $\begin{array}{l}\text { 1.leafy } \\
\text { vegetable }\end{array}$ & $\begin{array}{l}\text { Canned - high acid } \\
\text { food }\end{array}$ & $\begin{array}{l}\text { USDA FSIS } \\
(2012)\end{array}$ & 1.5 & $\mathrm{yr}$ & e.g., sauerkruat & datum & 240 & & & d \\
\hline Exposure & HLDUP & d & $\begin{array}{l}\text { Intake delay } \\
\text { harvest\&consumption }\end{array}$ & $\begin{array}{l}\text { 2.other } \\
\text { vegetable }\end{array}$ & Frozen & $\begin{array}{l}\text { (Internet } \\
\text { review) }\end{array}$ & & & $\begin{array}{l}\text { e.g., asparagus, carrots, } \\
\text { peas, lima beans, broccoli, } \\
\text { green beans, mushrooms }\end{array}$ & range & 180 & 30 & 360 & d \\
\hline Exposure & HLDUP & d & $\begin{array}{l}\text { Intake delay } \\
\text { harvest\&consumption }\end{array}$ & $\begin{array}{l}\text { 2.other } \\
\text { vegetable }\end{array}$ & Frozen & $\begin{array}{l}\text { (Internet } \\
\text { review) }\end{array}$ & & & $\begin{array}{l}\text { e.g., bell peppers, } \\
\text { tomatoes }\end{array}$ & range & 90 & 15 & 240 & d \\
\hline Exposure & HLDUP & d & $\begin{array}{l}\text { Intake delay } \\
\text { harvest\&consumption }\end{array}$ & $\begin{array}{l}\text { 2.other } \\
\text { vegetable }\end{array}$ & $\begin{array}{l}\text { Canned - low acid } \\
\text { food }\end{array}$ & $\begin{array}{l}\text { USDA FSIS } \\
\text { (2012) }\end{array}$ & $2-5$ & $\mathrm{yr}$ & $\begin{array}{l}\text { e.g., carrot, canned beans } \\
\text { (not tomato) }\end{array}$ & datum & 365 & & & d \\
\hline Exposure & HLDUP & d & $\begin{array}{l}\text { Intake delay } \\
\text { harvest\&consumption }\end{array}$ & $\begin{array}{l}\text { 2.other } \\
\text { vegetable }\end{array}$ & $\begin{array}{l}\text { Canned - high acid } \\
\text { food }\end{array}$ & $\begin{array}{l}\text { USDA FSIS } \\
(2012)\end{array}$ & 1.5 & yr & e.g., tomato, pickled beets & datum & 240 & & & d \\
\hline Exposure & HLDUP & d & $\begin{array}{l}\text { Intake delay } \\
\text { harvest\&consumption }\end{array}$ & $\begin{array}{l}\text { 2.other } \\
\text { vegetable }\end{array}$ & $\begin{array}{l}\text { commercially } \\
\text { packaged nuts }\end{array}$ & $\begin{array}{l}\text { USDA FSIS } \\
(2012)\end{array}$ & $6-12$ & mo & Nuts & range & 180 & 180 & 360 & d \\
\hline Exposure & HLDUP & d & $\begin{array}{l}\text { Intake delay } \\
\text { harvest\&consumption }\end{array}$ & 3.fruit & $\begin{array}{l}\text { Canned - low acid } \\
\text { food }\end{array}$ & $\begin{array}{l}\text { USDA FSIS } \\
\text { (2012) }\end{array}$ & $2-5$ & $\mathrm{yr}$ & $\begin{array}{l}\text { e.g., pears (not pineapple, } \\
\text { citrus) }\end{array}$ & datum & 365 & & & d \\
\hline Exposure & HLDUP & d & $\begin{array}{l}\text { Intake delay } \\
\text { harvest\&consumption }\end{array}$ & 3.fruit & $\begin{array}{l}\text { Canned - high acid } \\
\text { food }\end{array}$ & $\begin{array}{l}\text { USDA FSIS } \\
(2012)\end{array}$ & 1.5 & $\mathrm{yr}$ & e.g., pineapple, grapefruit & datum & 240 & & & d \\
\hline Exposure & HLDUP & d & $\begin{array}{l}\text { Intake delay } \\
\text { harvest\&consumption }\end{array}$ & 3.fruit & $\begin{array}{l}\text { fresh, refrigerated or } \\
\text { room temp }\end{array}$ & $\begin{array}{l}\text { USDA FSIS } \\
(2012)\end{array}$ & $0.4-3$ & wk & Varies by type & range & 12 & 3 & 21 & d \\
\hline Exposure & HLDUP & d & $\begin{array}{l}\text { Intake delay } \\
\text { harvest\&consumption }\end{array}$ & 3.fruit & dried & $\begin{array}{l}\text { USDA FSIS } \\
(2012)\end{array}$ & $1-6$ & mo & $\begin{array}{l}\text { Minimum is room } \\
\text { temperature storage, } \\
\text { maximum is refrigerated } \\
\text { storage }\end{array}$ & range & 90 & 30 & 180 & d \\
\hline Exposure & HLDUP & d & $\begin{array}{l}\text { Intake delay } \\
\text { harvest\&consumption }\end{array}$ & 3.fruit & jams, jellies & $\begin{array}{l}\text { USDA FSIS } \\
(2012)\end{array}$ & $6-12$ & mo & $\begin{array}{l}\text { Unopened room } \\
\text { temperature or refrigerator } \\
\text { storage }\end{array}$ & range & 180 & 180 & 365 & d \\
\hline Exposure & HLDUPA & d & $\begin{array}{l}\text { Intake delay } \\
\text { harvest\&consumption }\end{array}$ & 1.meat & $\begin{array}{l}\text { FROZEN } 0^{\circ} \mathrm{F} . \\
\text { Quality degrades } \\
\text { after cited time. }\end{array}$ & $\begin{array}{l}\text { USDA FSIS } \\
\text { (2012) }\end{array}$ & $1-2$ & mo & $\begin{array}{l}\text { Bacon, sausage, ham, hot } \\
\text { dogs, lunchmeats }\end{array}$ & range & 45 & 30 & 60 & d \\
\hline Exposure & HLDUPA & d & $\begin{array}{l}\text { Intake delay } \\
\text { harvest\&consumption }\end{array}$ & 1.meat & $\begin{array}{l}\text { FROZEN } 0^{\circ} \mathrm{F} \text {. } \\
\text { Quality degrades } \\
\text { after cited time. }\end{array}$ & $\begin{array}{l}\text { USDA FSIS } \\
(2012)\end{array}$ & $4-12$ & mo & $\begin{array}{l}\text { Uncooked, not ground } \\
\text { meat }\end{array}$ & range & 180 & 120 & 365 & d \\
\hline
\end{tabular}


Table I.1. (contd)

\begin{tabular}{|c|c|c|c|c|c|c|c|c|c|c|c|c|c|c|}
\hline $\begin{array}{l}\mathrm{Gv}_{2} \\
\text { module }\end{array}$ & Gv2_ID & $\begin{array}{l}\text { Gv2 } \\
\text { Units }\end{array}$ & Gv2_Description & Food Type & Qualifier & Reference & $\begin{array}{l}\text { Values } \\
\text { Cited }\end{array}$ & $\begin{array}{l}\text { Units } \\
\text { Cited }\end{array}$ & Comment & $\begin{array}{c}\text { Data } \\
\text { Reported }\end{array}$ & $\begin{array}{l}\text { Recom- } \\
\text { mended }\end{array}$ & $\begin{array}{l}\text { Min } \\
\text { Value }\end{array}$ & $\begin{array}{l}\text { Max } \\
\text { Value }\end{array}$ & Units \\
\hline Exposure & HLDUPA & $\mathrm{d}$ & $\begin{array}{l}\text { Intake delay } \\
\text { harvest\&consumption }\end{array}$ & 1.meat & $\begin{array}{l}\text { FROZEN } 0^{\circ} \mathrm{F} \text {. } \\
\text { Quality degrades } \\
\text { after cited time. }\end{array}$ & $\begin{array}{l}\text { USDA FSIS } \\
\text { (2012) }\end{array}$ & $3-4$ & mo & Uncooked ground meat & range & 90 & 90 & 120 & $\mathrm{~d}$ \\
\hline Exposure & HLDUPA & d & $\begin{array}{l}\text { Intake delay } \\
\text { harvest\&consumption }\end{array}$ & 1.meat & $\begin{array}{l}\text { FROZEN } 0^{\circ} \mathrm{F} \text {. } \\
\text { Quality degrades } \\
\text { after cited time. }\end{array}$ & $\begin{array}{l}\text { USDA FSIS } \\
(2012)\end{array}$ & $2-3$ & mo & Soups and stews & range & 75 & 60 & 90 & d \\
\hline Exposure & HLDUPA & d & $\begin{array}{l}\text { Intake delay } \\
\text { harvest\&consumption }\end{array}$ & 1.meat & $\begin{array}{l}\text { FROZEN } 0^{\circ} \mathrm{F} \text {. } \\
\text { Quality degrades } \\
\text { after cited time. }\end{array}$ & $\begin{array}{l}\text { USDA FSIS } \\
(2012)\end{array}$ & $8-12$ & mo & Uncooked wild game & range & 240 & 240 & 365 & d \\
\hline Exposure & HLDUPA & d & $\begin{array}{l}\text { Intake delay } \\
\text { harvest\&consumption }\end{array}$ & 1.meat & $\begin{array}{l}\text { Canned - low acid } \\
\text { food }\end{array}$ & $\begin{array}{l}\text { USDA FSIS } \\
(2012)\end{array}$ & $2-5$ & $\mathrm{yr}$ & e.g., canned ham. & datum & 365 & & & d \\
\hline Exposure & HLDUPA & d & $\begin{array}{l}\text { Intake delay } \\
\text { harvest\&consumption }\end{array}$ & 1.meat & fresh, refrigerated & $\begin{array}{l}\text { USDA FSIS } \\
(2012)\end{array}$ & $3-5$ & d & $\begin{array}{l}\text { Refrigerated storage after } \\
\text { purchase. Assumed } \\
10-14 \mathrm{~d} \text { for processing and } \\
\text { transport. }\end{array}$ & range & 14 & 13 & 19 & d \\
\hline Exposure & HLDUPA & d & $\begin{array}{l}\text { Intake delay } \\
\text { harvest\&consumption }\end{array}$ & 1.meat & fresh, refrigerated & $\begin{array}{l}\text { USDA FSIS } \\
(2012)\end{array}$ & $1-2$ & d & $\begin{array}{l}\text { Ground meat, stew meat. } \\
\text { Assumed } 10-14 \mathrm{~d} \text { for } \\
\text { processing and transport. }\end{array}$ & range & 14 & 11 & 16 & d \\
\hline Exposure & HLDUPA & d & $\begin{array}{l}\text { Intake delay } \\
\text { harvest\&consumption }\end{array}$ & 1.meat & fresh, refrigerated & $\begin{array}{l}\text { USDA FSIS } \\
(2012)\end{array}$ & $5-7$ & d & $\begin{array}{l}\text { Cured ham. Assumed } \\
10-14 \mathrm{~d} \text { for processing and } \\
\text { transport. }\end{array}$ & range & 18 & 15 & 21 & d \\
\hline Exposure & HLDUPA & d & $\begin{array}{l}\text { Intake delay } \\
\text { harvest\&consumption }\end{array}$ & 1.meat & fresh, refrigerated & $\begin{array}{l}\text { USDA FSIS } \\
(2012)\end{array}$ & $1-2$ & d & $\begin{array}{l}\text { Variety meats (tongue, } \\
\text { kidney, heart, liver). } \\
\text { Assumed 10-14 d for } \\
\text { processing and transport. }\end{array}$ & range & 15 & 11 & 16 & d \\
\hline Exposure & HLDUPA & d & $\begin{array}{l}\text { Intake delay } \\
\text { harvest\&consumption }\end{array}$ & 1.meat & fresh, refrigerated & $\begin{array}{l}\text { USDA FSIS } \\
\text { (2012) }\end{array}$ & 2 & wk & $\begin{array}{l}\text { Hot dogs and luncheon } \\
\text { meats, unopened package. } \\
\text { Assumed 5-14 d for } \\
\text { processing. }\end{array}$ & range & 20 & 19 & 28 & d \\
\hline Exposure & HLDUPA & d & $\begin{array}{l}\text { Intake delay } \\
\text { harvest\&consumption }\end{array}$ & 2.dairy & refrigerated & $\begin{array}{l}\text { USDA FSIS } \\
(2012)\end{array}$ & $0.5-6$ & mo & $\begin{array}{l}\text { Cheese, processed or hard. } \\
\text { Minimum is sliced. }\end{array}$ & range & 75 & 14 & 180 & d \\
\hline Exposure & HLDUPA & d & $\begin{array}{l}\text { Intake delay } \\
\text { harvest\&consumption }\end{array}$ & 3.poultry & $\begin{array}{l}\text { FROZEN } 0^{\circ} \mathrm{F} \text {. } \\
\text { Quality degrades } \\
\text { after cited time. }\end{array}$ & $\begin{array}{l}\text { USDA FSIS } \\
(2012)\end{array}$ & $9-12$ & mo & Uncooked poultry & range & 270 & 270 & 365 & d \\
\hline Exposure & HLDUPA & d & $\begin{array}{l}\text { Intake delay } \\
\text { harvest\&consumption }\end{array}$ & 3.poultry & $\begin{array}{l}\text { FROZEN } 0^{\circ} \mathrm{F} \text {. } \\
\text { Quality degrades } \\
\text { after cited time. }\end{array}$ & $\begin{array}{l}\text { USDA FSIS } \\
(2012)\end{array}$ & 4 & mo & Cooked poultry & datum & 120 & & 120 & d \\
\hline Exposure & HLDUPA & d & $\begin{array}{l}\text { Intake delay } \\
\text { harvest\&consumption }\end{array}$ & 3.poultry & $\begin{array}{l}\text { Canned - low acid } \\
\text { food }\end{array}$ & $\begin{array}{l}\text { USDA FSIS } \\
(2012)\end{array}$ & $2-5$ & $\mathrm{yr}$ & e.g., canned chicken & datum & 365 & & & d \\
\hline
\end{tabular}


Table I.1. (contd)

\begin{tabular}{|c|c|c|c|c|c|c|c|c|c|c|c|c|c|c|}
\hline $\begin{array}{l}\text { Gv2_- } \\
\text { module }\end{array}$ & Gv2_ID & $\begin{array}{l}\text { Gv2 } \\
\text { Units }\end{array}$ & Gv2_Description & Food Type & Qualifier & Reference & $\begin{array}{l}\text { Values } \\
\text { Cited }\end{array}$ & $\begin{array}{l}\text { Units } \\
\text { Cited }\end{array}$ & Comment & $\begin{array}{c}\text { Data } \\
\text { Reported }\end{array}$ & $\begin{array}{l}\text { Recom- } \\
\text { mended }\end{array}$ & $\begin{array}{l}\text { Min } \\
\text { Value }\end{array}$ & $\begin{array}{l}\text { Max } \\
\text { Value }\end{array}$ & Units \\
\hline Exposure & HLDUPA & d & $\begin{array}{l}\text { Intake delay } \\
\text { harvest\&consumption }\end{array}$ & 3.poultry & fresh, refrigerated & $\begin{array}{l}\text { USDA FSIS } \\
(2012)\end{array}$ & $1-2$ & d & $\begin{array}{l}\text { Refrigerated storage after } \\
\text { purchase. Assumed 5-6 d } \\
\text { for processing and } \\
\text { transport. }\end{array}$ & range & 7 & 6 & 8 & d \\
\hline Exposure & HLDUPA & d & $\begin{array}{l}\text { Intake delay } \\
\text { harvest\&consumption }\end{array}$ & 3.poultry & fresh, refrigerated & $\begin{array}{l}\text { USDA FSIS } \\
(2012)\end{array}$ & $1-2$ & d & $\begin{array}{l}\text { Game birds. Assumed } 1 \mathrm{~d} \\
\text { for transport. }\end{array}$ & range & 2 & 2 & 3 & d \\
\hline Exposure & HLDUPA & d & $\begin{array}{l}\text { Intake delay } \\
\text { harvest\&consumption }\end{array}$ & 3.poultry & $\begin{array}{l}\text { commerically } \\
\text { smoked (cooked), } \\
\text { refrigerated }\end{array}$ & $\begin{array}{l}\text { USDA FSIS } \\
(2012)\end{array}$ & 7 & d & Turkey & datum & 7 & & 7 & d \\
\hline Exposure & HLDUPA & d & $\begin{array}{l}\text { Intake delay } \\
\text { harvest\&consumption }\end{array}$ & 3.poultry & $\begin{array}{l}\text { commerically } \\
\text { smoked (cooked), } \\
\text { frozen }\end{array}$ & $\begin{array}{l}\text { USDA FSIS } \\
(2012)\end{array}$ & 6 & mo & Turkey & datum & 100 & & 180 & d \\
\hline Exposure & HLDUPA & d & $\begin{array}{l}\text { Intake delay } \\
\text { harvest\&consumption }\end{array}$ & 4.egg & $\begin{array}{l}\text { FROZEN } 0^{\circ} \mathrm{F} \text {. } \\
\text { Quality degrades } \\
\text { after cited time. }\end{array}$ & $\begin{array}{l}\text { USDA FSIS } \\
(2012)\end{array}$ & 1 & $\mathrm{yr}$ & Egg whites & datum & 180 & & 365 & d \\
\hline Exposure & HLDUPA & d & $\begin{array}{l}\text { Intake delay } \\
\text { harvest\&consumption }\end{array}$ & 4.egg & fresh, refrigerated & $\begin{array}{l}\text { USDA FSIS } \\
(2012)\end{array}$ & $3-5$ & wk & $\begin{array}{l}\text { Maximum refrigerated } \\
\text { storage after purchase. } \\
\text { Assumed 2-3 d for } \\
\text { processing and transport. }\end{array}$ & range & 23 & 14 & 38 & d \\
\hline Exposure & HLDUP2 & d & $\begin{array}{l}\text { Intake delay } \\
\text { harvest\&consumption }\end{array}$ & 1.fish & fresh, refrigerated & $\begin{array}{l}\text { USDA FSIS } \\
(2012)\end{array}$ & $1-2$ & d & $\begin{array}{l}\text { Fresh fish and shellfish. } \\
\text { Assumed 1-3 d for } \\
\text { transport. }\end{array}$ & range & 3 & 2 & 5 & d \\
\hline Exposure & HLDUP2 & d & $\begin{array}{l}\text { Intake delay } \\
\text { harvest\&consumption }\end{array}$ & 1.fish & $\begin{array}{l}\text { commercially } \\
\text { smoked, vacuum } \\
\text { package }\end{array}$ & $\begin{array}{l}\text { USDA FSIS } \\
\text { (2012); } \\
\text { Internet } \\
\text { review }\end{array}$ & 1 & $\mathrm{yr}$ & $\begin{array}{l}\text { Salmon. Shelf-stable } \\
\text { vacuum package. }\end{array}$ & datum & 180 & 60 & 365 & d \\
\hline Exposure & HLDUP2 & d & $\begin{array}{l}\text { Intake delay } \\
\text { harvest\&consumption }\end{array}$ & 1.fish & $\begin{array}{l}\text { home smoked, not } \\
\text { vacuum packed }\end{array}$ & $\begin{array}{l}\text { Iinternet } \\
\text { review) }\end{array}$ & & & & & 5 & 1 & 14 & d \\
\hline Exposure & HLDUP2 & d & $\begin{array}{l}\text { Intake delay } \\
\text { harvest\&consumption }\end{array}$ & 1.fish & frozen & $\begin{array}{l}\text { USDA FSIS } \\
(2012)\end{array}$ & 1 & $\mathrm{yr}$ & Frozen seafood entrees & datum & 180 & & 365 & d \\
\hline Exposure & HLDUP2 & d & $\begin{array}{l}\text { Intake delay } \\
\text { harvest\&consumption }\end{array}$ & 1.fish & frozen & $\begin{array}{l}\text { (Internet } \\
\text { review) }\end{array}$ & & & Frozen lean fish (e.g., cod) & & 90 & 30 & 180 & d \\
\hline Exposure & HLDUP2 & d & $\begin{array}{l}\text { Intake delay } \\
\text { harvest\&consumption }\end{array}$ & 1.fish & frozen & $\begin{array}{l}\text { (Internet } \\
\text { review) }\end{array}$ & & & $\begin{array}{l}\text { Frozen fatty fish (e.g., } \\
\text { salmon) }\end{array}$ & & 60 & 30 & 90 & d \\
\hline Exposure & HLDUP2 & d & $\begin{array}{l}\text { Intake delay } \\
\text { harvest\&consumption }\end{array}$ & 1.fish & frozen & $\begin{array}{l}\text { (Internet } \\
\text { review) }\end{array}$ & & & Commercial fishsticks & & 270 & 60 & 365 & d \\
\hline Exposure & HLDUP2 & d & $\begin{array}{l}\text { Intake delay } \\
\text { harvest\&consumption }\end{array}$ & 2.mollusk & fresh, refrigerated & $\begin{array}{l}\text { USDA FSIS } \\
(2012)\end{array}$ & $1-2$ & d & $\begin{array}{l}\text { Fresh fish and shellfish. } \\
\text { Assumed 1-3 d for } \\
\text { transport. }\end{array}$ & range & 3 & 2 & 5 & d \\
\hline
\end{tabular}


Table I.1. (contd)

\begin{tabular}{|c|c|c|c|c|c|c|c|c|c|c|c|c|c|c|}
\hline $\begin{array}{l}\mathrm{Gv}_{2} \\
\text { module }\end{array}$ & Gv2_ID & $\begin{array}{l}\text { Gv2 } \\
\text { Units }\end{array}$ & Gv2_Description & Food Type & Qualifier & Reference & $\begin{array}{l}\text { Values } \\
\text { Cited }\end{array}$ & $\begin{array}{l}\text { Units } \\
\text { Cited }\end{array}$ & Comment & $\begin{array}{c}\text { Data } \\
\text { Reported }\end{array}$ & $\begin{array}{l}\text { Recom- } \\
\text { mended }\end{array}$ & $\begin{array}{c}\text { Min } \\
\text { Value }\end{array}$ & $\begin{array}{l}\text { Max } \\
\text { Value }\end{array}$ & Units \\
\hline Exposure & HLDUP2 & $\mathrm{d}$ & $\begin{array}{l}\text { Intake delay } \\
\text { harvest\&consumption }\end{array}$ & 3.crustacean & fresh, refrigerated & $\begin{array}{l}\text { USDA FSIS } \\
(2012)\end{array}$ & $1-2$ & $\mathrm{~d}$ & $\begin{array}{l}\text { Fresh fish and shellfish. } \\
\text { Assumed 1-3 d for } \\
\text { transport. }\end{array}$ & range & 3 & 2 & 5 & $\mathrm{~d}$ \\
\hline Exposure & HLDUP2 & d & $\begin{array}{l}\text { Intake delay } \\
\text { harvest\&consumption }\end{array}$ & 3.crustacean & cooked then frozen & $\begin{array}{l}\text { (Internet } \\
\text { review) }\end{array}$ & & & shellfish & & 90 & 30 & 120 & d \\
\hline Exposure & HLDUP2 & d & $\begin{array}{l}\text { Intake delay } \\
\text { harvest\&consumption }\end{array}$ & 3.crustacean & cooked then frozen & $\begin{array}{l}\text { (Internet } \\
\text { review) }\end{array}$ & & & crab & & 30 & 24 & 60 & d \\
\hline Exposure & HLDUP2 & d & $\begin{array}{l}\text { Intake delay } \\
\text { harvest\&consumption }\end{array}$ & 3.crustacean & $\begin{array}{l}\text { live lobster, } \\
\text { refrigerated }\end{array}$ & $\begin{array}{l}\text { USDA FSIS } \\
(2012)\end{array}$ & $1-2$ & d & $\begin{array}{l}\text { Lobster. Assumed } 1 \mathrm{~d} \text { for } \\
\text { transport. }\end{array}$ & range & 2 & 2 & 3 & d \\
\hline
\end{tabular}

ज्ञ 


\section{References}

USDA FSIS. 2012. Food Safety and Inspection Service [online] Fact Sheets (multiple). USDA Food Safety and Inspection Service, Washington, D.C. Last accessed at:

http://www.fsis.usda.gov/Fact_Sheets/. 


\section{Appendix J}

\section{Distribution Assumptions}


PNNL-21950, Appendix J

\section{Appendix J}

\section{Distribution Assumptions}

Parameters used in an environmental model are simplified to individual values. Such discrete parameter definition results in discrete final results. Models can calculate an uncertainty in the final result by defining the range of values appropriate to a parameter and the distribution of values within that range (i.e., probability density function (PDF)). Software, hardware, and research advancements have enabled more sophistication in the parameter definitions. The PDF and minimum and maximum limits (i.e., the range) for parameters can be defined in more complex models. In addition, parameter correlations may be possible with some software. Care must be taken that assumptions regarding parameter distributions, limits, and correlations do not unrealistically characterize the parameters. Sometime such unrealistic results are not known until results are reviewed in detail.

A full discussion regarding parameter uncertainty is not developed here. Uncertainty is inherent to virtually all environmental parameters. The user can refer to the scientific literature for additional information.

The main text of this document indicates ranges and discrete values for the Gv2 parameters. This section reviews distributions generally appropriate to the Gv2 parameter. The term "generally appropriate" is used because geographic application and scenario scope among users is quite broad. The user will ultimately need to decide how best to characterize the parameter distribution and range for their scenario. Possible results from non-discrete parameter definitions vary. Use of broad range(s) can result in such a wide uncertainty that the result is meaningless (e.g., dose ranges from far less than background to a lethal dose).

It is recommended that the next step from discrete parameter values be toward a narrow parameter range definition. The parameters with the strongest technical strength could be defined to the extent supported by research. Then it is suggested that the user experiment between broadening the range and varying the distribution type. The data to support decisions may not be available to the user, so caution is advised for range and distribution assumptions not supported by available information. Given the potential number of parameters for which ranges and distributions can be assigned, this task could be daunting.

Gv2 has a defined set of possible PDFs available in the Sensitivity/Uncertainty Multimedia Modeling module (Napier 2012, Section 5). Table J.1, taken directly from the Gv2 SDD (Napier et al. 2012, Section 4.2.3) indicates distribution names and parameter requirements for that distribution. In order to include parameter distributions in Gv2 scenarios, the user must incorporate the Sensitivity module (see Figure J.1. Gv2 example cases 17, 18, and 19 can all be reviewed, as these include uncertainty.

The generally appropriate parameter distributions are indicated in Table J.2. The first two columns indicate the module and parameter ID, followed by the distribution recommendation. The parameter description and units follow. The next columns, Explicit and Implicit dependency, provide an indication of other parameters or model considerations that the user-defined distribution depends upon. This is important, for example, if the user wants to apply a certain distribution to the intake rate of one age group, 
but not another, then separate evaluations must be done. Sometimes the separate evaluations could be done within one scenario; sometimes it is better to organize the separate evaluation in a separate scenario. Implicit dependencies are good to keep in mind, but generally are considered "givens" for the model. The final columns in Table J.2 provide additional commentary.

The parameter distributions indicated in Table J.2 were determined, to the extent possible from the resources used to assign the discrete values, as described in the main text sections (e.g., the majority of the receptor intake module parameter distributions were determined from review of the EPA Exposure Factors Handbook (EPA 2011)). For parameters with limited data, the simplest distribution (uniform) was typically assigned. For strongly linked parameters such as irrigation rate and irrigation time, the decision was made to keep the time component fixed and suggest a distribution only for the rate. Retaining this practice is up to the user.

As a final note, the user is cautioned to proceed with parameter distribution and limit definitions cautiously and to review results with respect to input values. Full understanding of the assumptions used to generate an impact estimate should be understood prior to finalizing or accepting code output.

Additional information and issues related to determining parameter distributions for environmental modeling include Kamboj et al (2000); Chapter 2 of EPA (2011) which discusses variability and uncertainty; and NCRP Report 76 (NCRP 1984) which discuss environmental parameter uncertainty. ICRP Report 101(ICRP 2006), Annex B, also reviews the topic of probabilistic dose estimation.

Table J.1. PDFs Implemented in Gv2

\begin{tabular}{|c|c|c|}
\hline Index & Distribution & Parameterization Requirements \\
\hline 0 & Constant & Single value \\
\hline 1 & Uniform & Lower limit and upper limit \\
\hline 2 & Loguniform (base e) & Lower limit and upper limit are in data (not log) units \\
\hline 3 & Normal & Mean and standard deviation \\
\hline 4 & Lognormal (base e) & $\begin{array}{l}\text { Mean and standard deviation of the underlying normal } \\
\text { distribution }\end{array}$ \\
\hline 5 & Triangular & Lower limit, mode, and upper limit \\
\hline 6 & User-supplied & $\begin{array}{l}\text { Table of } n \text { pairs of values }\left(\mathrm{x}_{\mathrm{i}}, \mathrm{p}_{\mathrm{i}}\right) \text {. The } \mathrm{x}_{\mathrm{i}} \text { must be unique } \\
\text { and ordered in increasing value. The } \mathrm{p}_{\mathrm{i}} \text { must be unique } \\
\text { and ordered in increasing value with } \mathrm{p}_{1}=0.0 \text { and } \mathrm{p}_{\mathrm{n}}=1.0\end{array}$ \\
\hline
\end{tabular}




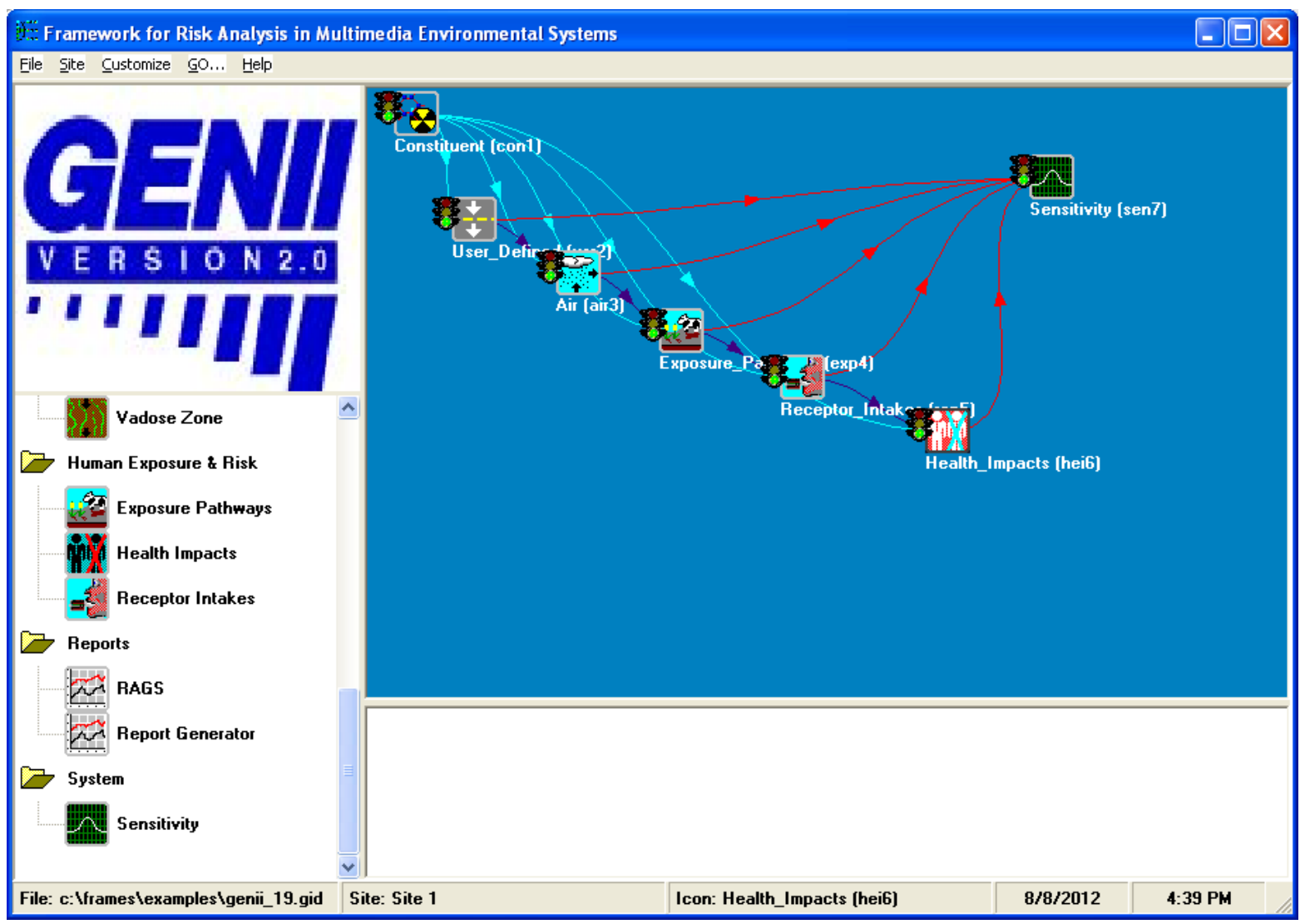

Figure J.1. Gv2.10, Example 19, Indicates Use of Sensitivity Module

\section{References}

EPA. 2011. Exposure Factors Handbook: 2011 Edition. EPA/600/R-090/052F, U.S. Environmental Protection Agency, Office of Research and Development, Washington, D.C.

ICRP. 2006. Assessing Dose of the Representative Person for the Purpose of Radiation Protection of the Public and the Optimisation of Radiological Protection: Broadening the Process. ICRP (International Commission on Radiological Protection) Publication 101, Ann. ICRP 36(3).

ICRP. 2009. Environmental Protection: Transfer Parameters for Reference Animals and Plants. ICRP (International Commission on Radiological Protection) Publication 114, Ann. ICRP 39(6).

Kamboj S, D LePoire, E Gnanapragasam, BM Biwer, J Cheng, J Arnish, C Yu, and SY Chen. 2000. Probabilistic Dose Analysis Using Parameter Distributions Developed for RESRAD and RESRADBUILD Codes. NUREG/CR-6676, U.S. Nuclear Regulatory Commission, Washington, D.C.

Napier BA. 2012. GENII Version 2 User's Guide. PNNL-14583, Rev 4, Pacific Northwest National Laboratory, Richland, Washington.

Napier BA, DL Strenge, JV Ramdell, Jr., PW Eslinger, and C Fosmire. 2012. GENII Version 2, Software Design Document. PNNL-14584, Rev 4, Pacific Northwest National Laboratory, Richland, Washington. 
NCRP. 1984. Radiological Assessment: Predicting the Transport, Bioaccumulation, and Uptake by Man of Radionuclides Released to the Environment. National Council on Radiation Protection and Measurements Report No. 76, Bethesda, Maryland.

Pettoussi N, P Jacob, M Zankl, and K Saito. 1991. "Organ Doses for Foetuses, Babies, Children and Adults from Environmental Gamma Rays.” Radiation Protection Dosimetry 37(1):31-41.

Snyder SF, WT Farris, BA Napier, TA Ikenberry, and RO Gilbert. 1994. Parameters Used in the Environmental and Radiological Dose Modules (DESCARTES, CIDER, and CRD Codes) of the Hanford Environmental Dose Reconstruction Integrated Codes (HEDRIC). PNWD-2023 HEDR, Rev. 1, Pacific Northwest Laboratory, Richland, Washington. 
Table J.2. Guideline for Chronic Scenario Parameter Distributions in Gv2

\begin{tabular}{|c|c|c|c|c|c|c|c|c|}
\hline Gv2 Module & GENIIv2 ID & $\begin{array}{c}\text { Chronic Scenario } \\
\text { Distribution - } \\
\text { Assumption } \\
\end{array}$ & GENII Description & Units & Explicit Dependency & Implicit Dependency & $\begin{array}{l}\text { Additional Information } \\
\text { Regarding Parameter } \\
\text { Distribution }\end{array}$ & Comment \\
\hline Constituent & CLWPF & $<$ none suggested $>$ & Water purification factor & unitless & chemical & Type of treatment plant & & \\
\hline Constituent & CLDFAx & lognormal & Inhalation dose factor & $\mathrm{rem} / \mathrm{pCi}$ & nuclide, age group & $\begin{array}{l}\text { ICRP 60/70 modeling and } \\
\text { ICRP } 66 \text { lung model }\end{array}$ & Snyder et al. (1994) & \\
\hline Constituent & CLRDFGx & lognormal & Ingestion dose factor & rem $/ \mathrm{pCi}_{\text {ingested }}$ & nuclide, age group & ICRP 60/70 modeling & Snyder et al. (1994) & \\
\hline Constituent & CLDEX & lognormal & $\begin{array}{l}\text { External dose factor, air } \\
\text { immersion }\end{array}$ & $\begin{array}{l}\mathrm{mrem} / \mathrm{hr} \text { per } \\
\mathrm{pCi} / \mathrm{m}^{3} \text { air }\end{array}$ & nuclide & ICRP 60 modeling & Snyder et al. (1994) & $\begin{array}{l}\text { Adult factors used for all, see Pettousi et al } \\
\text { (1991) for discussion of age-dependence. }\end{array}$ \\
\hline Constituent & CLDIMR & lognormal & $\begin{array}{l}\text { External dose factor, water } \\
\text { immersion }\end{array}$ & $\begin{array}{l}\mathrm{mrem} / \mathrm{hr} \text { per } \\
\mathrm{pCi} / \mathrm{m}^{3} \text { water }\end{array}$ & nuclide & ICRP 60 modeling & Snyder et al. (1994) & $\begin{array}{l}\text { Adult factors used for all, see Pettousi et al } \\
\text { (1991) for discussion of age-dependence. }\end{array}$ \\
\hline Constituent & CLDSH15 & uniform & $\begin{array}{l}\text { External dose factor, ground } \\
\text { contamination to } 15 \mathrm{~cm}\end{array}$ & $\begin{array}{c}\mathrm{mrem} / \mathrm{hr} \mathrm{per} \\
\mathrm{pCi} / \mathrm{m}^{3} \text { soil to a soil } \\
\text { depth of } 15 \mathrm{~cm}\end{array}$ & nuclide & ICRP 60 modeling & Snyder et al. (1994) & $\begin{array}{l}\text { Adult factors used for all, see Pettousi et al } \\
\text { (1991) for discussion of age-dependence. }\end{array}$ \\
\hline Constituent & CLBFx, CLBMx & lognormal & $\begin{array}{l}\text { Bioaccumulation factor for } \\
\text { Aquatic Animals } \\
\text { (F=freshwater, } \mathrm{M}=\text { =marine) }\end{array}$ & $\mathrm{L} / \mathrm{kg}_{\text {wet }}$ & $\begin{array}{l}\text { nuclide, freshwater vs. } \\
\text { marine; aquatic animal type }\end{array}$ & & $\begin{array}{l}\text { ICRP (2009); Kamboj et al. } \\
\text { (2000) }\end{array}$ & $\begin{array}{l}\text { Chemical-specific information varies widely } \\
\text { in quality and quantity. }\end{array}$ \\
\hline Constituent & CLBFP, CLBMP & lognormal & $\begin{array}{l}\text { Bioaccumulation factor for } \\
\text { Aquatic Plants ( } F=\text { freshwater, } \\
\text { M=marine) }\end{array}$ & $\mathrm{L} / \mathrm{kg}_{\text {wet }}$ & $\begin{array}{l}\text { nuclide, freshwater vs. } \\
\text { marine }\end{array}$ & & ICRP (2009) & $\begin{array}{l}\text { Chemical-specific information varies widely } \\
\text { in quality and quantity. }\end{array}$ \\
\hline Constituent & CLFMT & lognormal & Feed to meat transfer factor & $\mathrm{d} / \mathrm{kg}_{\text {wet }}$ & nuclide & Average type of feed consumed & $\begin{array}{l}\text { ICRP (2009); Kamboj et al. } \\
\text { (2000) }\end{array}$ & $\begin{array}{l}\text { Chemical-specific information varies widely } \\
\text { in quality and quantity. }\end{array}$ \\
\hline Constituent & CLFMK & lognormal & Feed to milk transfer factor & $d / L$ & nuclide & Average type of feed consumed & $\begin{array}{l}\text { ICRP (2009); Kamboj et al. } \\
\text { (2000) }\end{array}$ & $\begin{array}{l}\text { Chemical-specific information varies widely } \\
\text { in quality and quantity. }\end{array}$ \\
\hline Constituent & CLFPL and CLFEG & lognormal & $\begin{array}{l}\text { Feed to poultry; and feed to egg } \\
\text { transfer factors }\end{array}$ & $\mathrm{d} / \mathrm{kg}_{\text {wet }}$ & nuclide, poultry or egg & Average type of feed consumed & ICRP (2009) & \\
\hline Constituent & CLBVx & lognormal & $\begin{array}{l}\text { Bioconcentration to edible crop } \\
\text { portion from soil }\end{array}$ & kgdry_plant $/ \mathrm{kg}_{\text {dry__oil }}$ & nuclide, food type & $\begin{array}{l}\text { Value assigned to a food type } \\
\text { (e.g., root vegetables) applies to } \\
\text { all crops in that category (e.g., } \\
\text { potato, tomato) }\end{array}$ & $\begin{array}{l}\text { ICRP (2009); Kamboj et al. } \\
\text { (2000) }\end{array}$ & \\
\hline Constituent & CLBVAx & lognormal & $\begin{array}{l}\text { Bioconcentration to edible feed } \\
\text { crop portion from soil }\end{array}$ & 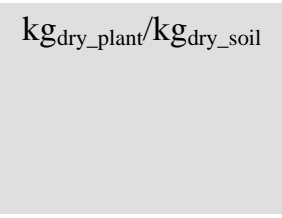 & nuclide, feed type & $\begin{array}{l}\text { Value assigned to a feed type } \\
\text { (e.g., meat feed) corresponds to } \\
\text { the average value applicable to } \\
\text { all feed crops in that category } \\
\text { (e.g., corn silage, grass hay) }\end{array}$ & $\begin{array}{l}\text { ICRP (2009); Kamboj et al. } \\
\text { (2000) }\end{array}$ & \\
\hline $\begin{array}{l}\text { Air, Chronic } \\
\text { Plume model }\end{array}$ & ARMINRISESPD & $<$ none suggested $>$ & $\begin{array}{l}\text { Minimum air speed during } \\
\text { plume rise }\end{array}$ & $\mathrm{m} / \mathrm{s}$ & & & & \\
\hline $\begin{array}{l}\text { Air, Chronic } \\
\text { Plume model }\end{array}$ & ARMINSIGYSHIFT & $<$ none suggested $>$ & $\begin{array}{l}\text { Sigma shift to semi-infinite } \\
\text { cloud shine model }\end{array}$ & $\mathrm{m}$ & & & & \\
\hline $\begin{array}{l}\text { Air, Chronic } \\
\text { Plume model }\end{array}$ & ARTRANSRESIST & $<$ none suggested $>$ & $\begin{array}{l}\text { Transfer resistance for iodine } \\
\text { and particles }\end{array}$ & $\mathrm{s} / \mathrm{m}$ & & & & \\
\hline $\begin{array}{l}\text { Air, Chronic } \\
\text { Plume model }\end{array}$ & ARMINWIND & constant & $\begin{array}{l}\text { Maximum wind speed for } \\
\text { "calm" }\end{array}$ & $\mathrm{m} / \mathrm{s}$ & & & & Determined by instrumentation. \\
\hline $\begin{array}{l}\text { Exposure, } \\
\text { Chronic }\end{array}$ & ABSHUM & normal & Absolute humidity & $\mathrm{kg} / \mathrm{m}^{3}$ & $\begin{array}{l}\text { spatial, growing season } \\
\text { average value }\end{array}$ & $\begin{array}{l}\text { Specific activity equivalence } \\
\text { model }\end{array}$ & & Relevant to tritium only \\
\hline
\end{tabular}


Table J.2. (contd)

\begin{tabular}{|c|c|c|c|c|c|c|c|c|}
\hline Gv2 Module & GENIIv2 ID & $\begin{array}{l}\text { Chronic Scenario } \\
\text { Distribution - } \\
\text { Recommended } \\
\text { Assumption }\end{array}$ & GENII Description & Units & Explicit Dependency & Implicit Dependency & $\begin{array}{c}\text { Additional Information } \\
\text { Regarding Parameter } \\
\text { Distribution }\end{array}$ & Comment \\
\hline $\begin{array}{l}\text { Exposure, } \\
\text { Chronic }\end{array}$ & RF1 & constant & Root fraction & unitless & & $\begin{array}{l}\text { Relatively uniform distribution } \\
\text { of nuclide in top } 15 \mathrm{~cm} \text { of soil. }\end{array}$ & & $\begin{array}{l}\text { Assumption is that all roots are in top } 15 \mathrm{~cm} \\
\text { of soil; and that soil contamination is } \\
\text { distributed relatively uniformly in the top } \\
15 \mathrm{~cm} \text { of soil. }\end{array}$ \\
\hline $\begin{array}{l}\text { Exposure, } \\
\text { Chronic }\end{array}$ & RAIN & normal & Rainfall rate & $\mathrm{mm} / \mathrm{d}$ & spatial & & & $\begin{array}{l}\text { Suggest that dry years use the same rate. The } \\
\text { meteorology file will indicate the number of } \\
\text { days it rains and thereby adjust for drier years. }\end{array}$ \\
\hline $\begin{array}{l}\text { Exposure, } \\
\text { Chronic }\end{array}$ & RIRRR, IRTIMR & normal, constant & $\begin{array}{l}\text { Residential land irrigation rate } \\
\text { and irrigation time }\end{array}$ & in/yr and mo/yr & spatial & & & $\begin{array}{l}\text { Dependent parameters - recommend varying } \\
\text { one; leave other constant. }\end{array}$ \\
\hline $\begin{array}{l}\text { Exposure, } \\
\text { Chronic }\end{array}$ & HOLDDW & uniform & $\begin{array}{l}\text { Delay time for water } \\
\text { distribution }\end{array}$ & d & $\begin{array}{l}\text { typically, distance from } \\
\text { treatment plant }\end{array}$ & & & \\
\hline $\begin{array}{l}\text { Exposure, } \\
\text { Chronic }\end{array}$ & SEDDN & uniform & Shoreline sediment density & $\mathrm{kg} / \mathrm{m}^{2}$ & spatial & $\begin{array}{l}\text { Density average across shoreline } \\
\text { location of interest. }\end{array}$ & & \\
\hline $\begin{array}{l}\text { Exposure, } \\
\text { Chronic }\end{array}$ & $\begin{array}{l}\text { DWFACA, } \\
\text { DWATER }\end{array}$ & constant, normal & $\begin{array}{l}\text { Contaminated fraction and } \\
\text { animal drinking water rate }\end{array}$ & fraction and $\mathrm{L} / \mathrm{d}$ & animal product type & $\begin{array}{l}\text { Type of meat animal (e.g., cow, } \\
\text { pig, sheep) }\end{array}$ & & $\begin{array}{l}\text { Dependent parameters - strongly recommend } \\
\text { varying intake rate and leaving DWFACA } \\
\text { fixed. }\end{array}$ \\
\hline $\begin{array}{l}\text { Exposure, } \\
\text { Chronic }\end{array}$ & RIRR, IRTIMT & normal, constant & $\begin{array}{l}\text { Food crop irrigation rate and } \\
\text { irrigation time }\end{array}$ & in/yr and mo/yr & spatial & $\begin{array}{l}\text { Value assigned to a food type } \\
\text { (e.g., root vegetables) applies to } \\
\text { all crops in that category (e.g., } \\
\text { potato, tomato) }\end{array}$ & & $\begin{array}{l}\text { Dependent parameters - recommend varying } \\
\text { one; leave other constant. }\end{array}$ \\
\hline $\begin{array}{l}\text { Exposure, } \\
\text { Chronic }\end{array}$ & RIRRA, IRTIMA & normal, constant & $\begin{array}{l}\text { Feed and forage crop irrigation } \\
\text { rate and irrigation time }\end{array}$ & in/yr and mo/yr & spatial, weather, feed type & & & $\begin{array}{l}\text { Dependent parameters -- recommend varying } \\
\text { one; leave other constant. }\end{array}$ \\
\hline $\begin{array}{l}\text { Exposure, } \\
\text { Chronic }\end{array}$ & SLDN, SURCM & uniform, constant & $\begin{array}{l}\text { Surface soil areal density and } \\
\text { surface soil thickness }\end{array}$ & $\mathrm{kg} / \mathrm{m}^{2}$ and $\mathrm{cm}$ & spatial & $\begin{array}{l}\text { Density average across receptor } \\
\text { location }\end{array}$ & Snyder et al. (1994) & $\begin{array}{l}\text { Dependent parameters - strongly recommend } \\
\text { fixing SURCM at } 15 \mathrm{~cm} \text { and varying SLDNN. } \\
\text { The SLDN of this module and the Health } \\
\text { Impacts module should be consistently } \\
\text { parameterized, with difference resulting only } \\
\text { from different units. }\end{array}$ \\
\hline $\begin{array}{l}\text { Exposure, } \\
\text { Chronic }\end{array}$ & XMLF & lognormal & $\begin{array}{l}\text { Mass loading factor for } \\
\text { resuspension }\end{array}$ & $\mathrm{g} / \mathrm{m}^{3}$ & & & & \\
\hline $\begin{array}{l}\text { Exposure, } \\
\text { Chronic }\end{array}$ & LEAFRS & $<$ none suggested $>$ & $\begin{array}{l}\text { Soil to plant resuspension } \\
\text { factor }\end{array}$ & $\mathrm{m}^{-1}$ & & $\begin{array}{l}\text { Parameter applied to all feed, } \\
\text { forage, and food crops. }\end{array}$ & & \\
\hline $\begin{array}{l}\text { Exposure, } \\
\text { Chronic }\end{array}$ & DPVRES & constant & Soil to plant deposition velocity & $\mathrm{m} / \mathrm{s}$ & & $\begin{array}{l}\text { Parameter applied to all feed, } \\
\text { forage, and food crops. }\end{array}$ & & Fixed value recommended. \\
\hline $\begin{array}{l}\text { Exposure, } \\
\text { Chronic }\end{array}$ & WTIM & normal & Plant weathering constant & d & & $\begin{array}{l}\text { Parameter applied to all feed, } \\
\text { forage, and food crops. }\end{array}$ & & $\begin{array}{l}\text { Recommend that spatial variability not } \\
\text { considered for this parameter }\end{array}$ \\
\hline $\begin{array}{l}\text { Exposure, } \\
\text { Chronic }\end{array}$ & $\begin{array}{l}\text { BIOMA2, GRWPA, } \\
\text { YELDA, TRANSA }\end{array}$ & $\begin{array}{l}\text { normal, uniform, } \\
\text { normal, } \\
\text { loguniform }\end{array}$ & $\begin{array}{l}\text { Animal feed and forage crop } \\
\text { field characteristics: biomass; } \\
\text { growing period; yield; and } \\
\text { translocation factor }\end{array}$ & $\begin{array}{l}\mathrm{kg}_{\text {wel }} / \mathrm{d} ; \mathrm{d} ; \mathrm{kg}_{\text {wet as- }} \\
\text { feed } / \mathrm{m}^{2} ; \text { unitless }\end{array}$ & spatial, weather, feed type & & & $\begin{array}{l}\text { Dependent parameters - recommend not } \\
\text { varying all; leave some as constants. }\end{array}$ \\
\hline $\begin{array}{l}\text { Exposure, } \\
\text { Chronic }\end{array}$ & $\begin{array}{l}\text { CONSUM, } \\
\text { STORTM, DIETFR }\end{array}$ & $\begin{array}{l}\text { normal, uniform, } \\
\text { constant }\end{array}$ & $\begin{array}{l}\text { Animal feed and forage intake } \\
\text { parameters: consumption, } \\
\text { storage time, dieffr }\end{array}$ & $\mathrm{kg} / \mathrm{d}$; d; unitless & spatial, feed or forage type & & & $\begin{array}{l}\text { Dependent parameters - recommend not } \\
\text { varying all; leave some as constants. }\end{array}$ \\
\hline
\end{tabular}


Table J.2. (contd)

\begin{tabular}{|c|c|c|c|c|c|c|c|c|}
\hline Gv2 Module & GENIIv2 ID & $\begin{array}{l}\text { Chronic Scenario } \\
\text { Distribution - } \\
\text { Recommended } \\
\text { Assumption } \\
\end{array}$ & GENII Description & Units & Explicit Dependency & Implicit Dependency & $\begin{array}{c}\text { Additional Information } \\
\text { Regarding Parameter } \\
\text { Distribution }\end{array}$ & Comment \\
\hline $\begin{array}{l}\text { Exposure, } \\
\text { Chronic }\end{array}$ & DRYFA2 & uniform & $\begin{array}{l}\text { Dry to wet ratio for animal } \\
\text { feeds }\end{array}$ & unitless & feed crop type & $\begin{array}{l}\text { Value assigned to a feed type } \\
\text { (e.g., meat feed) corresponds to } \\
\text { the average value applicable to } \\
\text { all feed crops in that category } \\
\text { (e.g., corn silage, grass hay) }\end{array}$ & & \\
\hline $\begin{array}{l}\text { Exposure, } \\
\text { Chronic }\end{array}$ & SLCONA & uniform & Soil intake rate of animals & $\mathrm{kg} / \mathrm{d}$ & $\begin{array}{l}\text { farming practice (e.g., barn, } \\
\text { feedlot, pasture) }\end{array}$ & & & \\
\hline $\begin{array}{l}\text { Exposure, } \\
\text { Chronic }\end{array}$ & $\begin{array}{l}\text { BIOMAS, GRWP, } \\
\text { YELD, TRANS }\end{array}$ & $\begin{array}{l}\text { normal, uniform, } \\
\text { normal, } \\
\text { loguniform }\end{array}$ & $\begin{array}{l}\text { Human food crop field } \\
\text { characteristics: biomass; } \\
\text { growing period; yield; and } \\
\text { translocation factor }\end{array}$ & $\begin{array}{c}\mathrm{kg}_{\text {wee }} / \mathrm{d} ; \mathrm{d} ; \\
\mathrm{kg}_{\text {weel }} / \mathrm{m}^{2} ; \text { unitless }\end{array}$ & $\begin{array}{l}\text { spatial, weather, food crop } \\
\text { type }\end{array}$ & & & $\begin{array}{l}\text { Dependent parameters - recommend not } \\
\text { varying all; leave some as constants. }\end{array}$ \\
\hline $\begin{array}{l}\text { Exposure, } \\
\text { Chronic }\end{array}$ & DRYFAC & uniform & $\begin{array}{l}\text { Dry to wet ratio for food crops } \\
\text { consumed by humans }\end{array}$ & & food crop type & $\begin{array}{l}\text { Value assigned to a food type } \\
\text { (e.g., root vegetables) applies to } \\
\text { all crops in that category (e.g., } \\
\text { potato, tomato) }\end{array}$ & & \\
\hline $\begin{array}{l}\text { Exposure, } \\
\text { Chronic }\end{array}$ & $\begin{array}{l}\text { HLDUP, HLDUPA, } \\
\text { HLDUP2 }\end{array}$ & uniform & $\begin{array}{l}\text { Intake delays between harvest } \\
\text { and consumption of food: food } \\
\text { crops, animal products, and } \\
\text { aquatic foods. }\end{array}$ & d & $\begin{array}{l}\mathrm{MEI} / \mathrm{POP} \text {, processing and } \\
\text { storage conditions }\end{array}$ & & & $\begin{array}{l}\text { Unless it is known that only canned or frozen } \\
\text { foods are consumed, it is generally assumed } \\
\text { that food is consumed fresh with the shelflife } \\
\text { determined based on refrigeration. }\end{array}$ \\
\hline Receptor Intake & $<$ virtually all $>$ & -- & -- & -- & -- & -- & -- & $\begin{array}{l}\text { Almost all Receptor intake parameters are } \\
\text { dependent on whether the Maximally exposed } \\
\text { individual (MEI) or population (POP) is under } \\
\text { consideration. In addition, the age of the MEI } \\
\text { or POP group also results in variable } \\
\text { parameter values. }\end{array}$ \\
\hline Receptor Intake & UEXAIR, TEXAIR & & $\begin{array}{l}\text { External dose from air } \\
\text { parameters. Daily hours and } \\
\text { annual days the receptor is } \\
\text { exposed to the contaminated } \\
\text { plume at one location. }\end{array}$ & $\mathrm{hr} ; \mathrm{d}$ & age, MEI/POP & & EPA (2011) & $\begin{array}{l}\text { Recommend that TEXAIR; FRINH, FTOUT, } \\
\text { FRINHR, and FRINDR (see below) are } \\
\text { consistently parameterized. }\end{array}$ \\
\hline Receptor Intake & $\begin{array}{l}\text { UEXGRD, } \\
\text { TEXGRD; FTIN } \\
\text { and FTOUT; SHIN } \\
\text { and SHOUT }\end{array}$ & $<$ none suggested $>$ & $\begin{array}{l}\text { External dose from soil } \\
\text { parameters. Daily hours and } \\
\text { annual days the receptor is } \\
\text { exposed to the contaminated } \\
\text { ground at one location, with } \\
\text { consideration of indoor and } \\
\text { outdoor fraction to further } \\
\text { qualify shielding assumptions } \\
\text { when indoors and outdoors. }\end{array}$ & $\begin{array}{l}\text { hr; d; unitless, } \\
\text { unitless; unitless, } \\
\text { unitless }\end{array}$ & age, MEI/POP & & EPA (2011) & $\begin{array}{l}\text { Dependent parameters - recommend not } \\
\text { varying all; leave some as constants. } \\
\text { Recommend that FTIN and FTOUT; } \\
\text { TEXAIR (see above); FRINH, TTOUT, and } \\
\text { FRINHR and FRINDR (see below) are } \\
\text { consistently parameterized. }\end{array}$ \\
\hline Receptor Intake & $\begin{array}{l}\text { EVSWIM, } \\
\text { TESWIM, TSWIM }\end{array}$ & $\begin{array}{l}\text { lognormal, } \\
\text { normal, constant }\end{array}$ & $\begin{array}{l}\text { Surface water dose parameters. } \\
\text { Daily swimming events; hours } \\
\text { per event; and annual days } \\
\text { swimming occurs. }\end{array}$ & events/d; hr; d & age, MEI/POP & & EPA (2011) & $\begin{array}{l}\text { Dependent parameters - recommend not } \\
\text { varying all; leave some as constants. }\end{array}$ \\
\hline
\end{tabular}


Table J.2. (contd)

\begin{tabular}{|c|c|c|c|c|c|c|c|c|}
\hline Gv2 Module & GENIIv2 ID & $\begin{array}{l}\text { Chronic Scenario } \\
\text { Distribution - } \\
\text { Recommended } \\
\text { Assumption }\end{array}$ & GENII Description & Units & Explicit Dependency & Implicit Dependency & $\begin{array}{l}\text { Additional Information } \\
\text { Regarding Parameter } \\
\text { Distribution }\end{array}$ & Comment \\
\hline Receptor Intake & USWIM & normal & $\begin{array}{l}\text { Ingestion rate of surface water } \\
\text { during swimming }\end{array}$ & $\mathrm{L} / \mathrm{hr}$ & age, MEI/POP & & EPA (2011) & \\
\hline Receptor Intake & $\begin{array}{c}\text { EVBOAT, } \\
\text { TEBOAT, TBOAT }\end{array}$ & $\begin{array}{l}\text { normal, constant, } \\
\text { constant }\end{array}$ & $\begin{array}{l}\text { Surface water dose parameters. } \\
\text { Daily boating events; hours per } \\
\text { event; and annual days boating } \\
\text { occurs. }\end{array}$ & events/d; hr; d & age, MEI/POP & & EPA (2011) & \\
\hline Receptor Intake & SFBOAT & $<$ constant $>$ & $\begin{array}{l}\text { Shielding factor representative } \\
\text { of surface water to receptor } \\
\text { distance on boat. }\end{array}$ & unitless & type of boat & & EPA (2011) & \\
\hline Receptor Intake & UCRP, TCRP & $\begin{array}{l}\text { lognormal, } \\
\text { constant }\end{array}$ & $\begin{array}{l}\text { Food crop ingestion } \\
\text { parameters. Daily intake rate } \\
\text { and annual intake days. }\end{array}$ & $\mathrm{kg} / \mathrm{d} ; \mathrm{d} / \mathrm{yr}$ & $\begin{array}{l}\text { age, MEI/POP, food crop } \\
\text { type }\end{array}$ & & EPA (2011) & $\begin{array}{l}\text { Dependent parameters - strongly recommend } \\
\text { varying intake rate and leaving TCRP fixed at } \\
365 \mathrm{~d} / \mathrm{yr} \text {. }\end{array}$ \\
\hline Receptor Intake & UANM, TANM & $\begin{array}{l}\text { lognormal, } \\
\text { constant }\end{array}$ & $\begin{array}{l}\text { Animal product ingestion } \\
\text { parameters. Daily intake rate } \\
\text { and annual intake days. }\end{array}$ & $\mathrm{kg} / \mathrm{d} ; \mathrm{d} / \mathrm{yr}$ & $\begin{array}{l}\text { age, MEI/POP, animal } \\
\text { product type }\end{array}$ & & EPA (2011) & $\begin{array}{l}\text { Dependent parameters - strongly recommend } \\
\text { varying intake rate and leaving TANM fixed } \\
\text { at } 365 \mathrm{~d} / \mathrm{yr} \text {. }\end{array}$ \\
\hline Receptor Intake & UAQU, TAQU & $\begin{array}{l}\text { lognormal, } \\
\text { constant }\end{array}$ & $\begin{array}{l}\text { Aquatic food ingestion } \\
\text { parameters. Daily intake rate } \\
\text { and annual intake days. }\end{array}$ & $\mathrm{kg} / \mathrm{d} ; \mathrm{d} / \mathrm{yr}$ & $\begin{array}{l}\text { age, MEI/POP, aquatic food } \\
\text { type }\end{array}$ & & EPA (2011) & $\begin{array}{l}\text { Dependent parameters - strongly recommend } \\
\text { varying intake rate and leaving TAQU fixed } \\
\text { at } 365 \mathrm{~d} / \mathrm{yr} \text {. }\end{array}$ \\
\hline Receptor Intake & UDW, TDW & normal, constant & $\begin{array}{l}\text { Drinking water ingestion } \\
\text { parameters. Daily intake rate } \\
\text { and annual intake days. }\end{array}$ & $\mathrm{L} / \mathrm{d} ; \mathrm{d} / \mathrm{yr}$ & $\begin{array}{l}\text { age, MEI/POP; } \\
\text { lactating/pregnant status for } \\
\text { females }\end{array}$ & & & $\begin{array}{l}\text { Dependent parameters - strongly recommend } \\
\text { varying intake rate and leaving TDW fixed at } \\
365 \mathrm{~d} / \mathrm{yr} \text {. }\end{array}$ \\
\hline Receptor Intake & $\begin{array}{l}\text { EVSHWR, } \\
\text { TESHWR, TSHWR }\end{array}$ & $\begin{array}{l}\text { constant, normal, } \\
\text { constant }\end{array}$ & $\begin{array}{l}\text { Incidental shower water } \\
\text { ingestion parameters. Daily } \\
\text { shower events; hours per event; } \\
\text { annual shower days. }\end{array}$ & events/d; hr; d & age, $\mathrm{MEI} / \mathrm{POP}$ & & EPA (2011) & $\begin{array}{l}\text { Dependent parameters - strongly recommend } \\
\text { leaving TSHWR fixed at } 365 \mathrm{~d} / \mathrm{yr} \text {. }\end{array}$ \\
\hline Receptor Intake & USHIN & normal & $\begin{array}{l}\text { Incidental shower water } \\
\text { consumption rate. }\end{array}$ & $\mathrm{L} / \mathrm{hr}$ & age, MEI/POP & & EPA (2011) & \\
\hline Receptor Intake & USOIL, TSOIL & normal, constant & $\begin{array}{l}\text { Incidental soil ingestion } \\
\text { parameters. Daily intake rate } \\
\text { and annual soil contact days }\end{array}$ & $\mathrm{mg} / \mathrm{d} ; \mathrm{d}$ & age, MEI/POP & & EPA (2011) & $\begin{array}{l}\text { Dependent parameters - strongly recommend } \\
\text { leaving TSOIL fixed at } 365 \mathrm{~d} / \mathrm{yr} \text {. }\end{array}$ \\
\hline Receptor Intake & $\begin{array}{l}\text { UINH, TINH, } \\
\text { FRINH }\end{array}$ & $\begin{array}{l}\text { lognormal, } \\
\text { constant, constant }\end{array}$ & $\begin{array}{l}\text { Plume air inhalation } \\
\text { parameters. Daily intake rate; } \\
\text { annual days of exposure; } \\
\text { fraction of time outdoors. }\end{array}$ & $\mathrm{m}^{3} / \mathrm{d} ; \mathrm{d} / \mathrm{yr}$; unitless & age, MEI/POP & & NCRP (1984) & $\begin{array}{l}\text { Dependent parameters - strongly recommend } \\
\text { leaving TINH fixed at } 365 \mathrm{~d} / \mathrm{yr} \text {. Recommend } \\
\text { that FRINH; FTOUT (see above); and } \\
\text { FRINHR and FRINDR (see below) are } \\
\text { consistently parameterized. }\end{array}$ \\
\hline Receptor Intake & $\begin{array}{l}\text { UINHR, TINHR, } \\
\text { FRINHR }\end{array}$ & $\begin{array}{l}\text { lognormal, } \\
\text { constant, constant }\end{array}$ & $\begin{array}{l}\text { Resuspended soil inhalation } \\
\text { parameters. Daily intake rate; } \\
\text { annual days of exposure; } \\
\text { fraction of time outdoors. }\end{array}$ & $\mathrm{m}^{3} / \mathrm{d}$; d/yr; unitless & age, MEI/POP & & NCRP (1984) & $\begin{array}{l}\text { Dependent parameters - strongly recommend } \\
\text { leaving TINHR fixed at } 365 \mathrm{~d} / \mathrm{yr} \text {. } \\
\text { Recommend that FRINHR; FRINH and } \\
\text { FTOUT (see above); and FRINDR (see } \\
\text { below) are consistently parameterized. }\end{array}$ \\
\hline
\end{tabular}


Table J.2.(contd)

\begin{tabular}{|c|c|c|c|c|c|c|c|c|}
\hline Gv2 Module & GENIIv2 ID & $\begin{array}{c}\text { Chronic Scenario } \\
\text { Distribution - } \\
\text { Recommended } \\
\text { Assumption }\end{array}$ & GENII Description & Units & Explicit Dependency & Implicit Dependency & $\begin{array}{l}\text { Additional Information } \\
\text { Regarding Parameter } \\
\text { Distribution }\end{array}$ & Comment \\
\hline Receptor Intake & $\begin{array}{l}\text { UINDRH, TINDRH, } \\
\text { FRINDR }\end{array}$ & $\begin{array}{l}\text { lognormal, } \\
\text { constant, constant }\end{array}$ & $\begin{array}{l}\text { Indoor air inhalation } \\
\text { parameters. Daily intake rate; } \\
\text { annual days of exposure; } \\
\text { fraction of time indoors. }\end{array}$ & $\mathrm{m}^{3} / \mathrm{d}$; d/yr; unitless & age, MEI/POP & & NCRP (1984) & $\begin{array}{l}\text { Dependent parameters - strongly recommend } \\
\text { leaving TINDRH fixed at } 365 \text { d/yr. } \\
\text { Recommend that FRINDR; FRINH, FTOUT, } \\
\text { and FRINHR (see above) are consistently } \\
\text { parameterized. }\end{array}$ \\
\hline Health Impacts & SOILT, SLDN & constant, uniform & $\begin{array}{l}\text { Soil thickness and density (to } \\
15 \mathrm{~cm} \text { ). }\end{array}$ & $\mathrm{m} ; \mathrm{kg} / \mathrm{m}^{3}$ & spatial & $\begin{array}{l}\text { Density average across receptor } \\
\text { location }\end{array}$ & Snyder et al. (1994) & $\begin{array}{l}\text { Dependent parameters - strongly recommend } \\
\text { leaving SOILT at } 0.15 \mathrm{~m} \text {. The SLDN of this } \\
\text { module and the Exposure module should be } \\
\text { consistently parameterized, with difference } \\
\text { resulting only from different units. }\end{array}$ \\
\hline
\end{tabular}




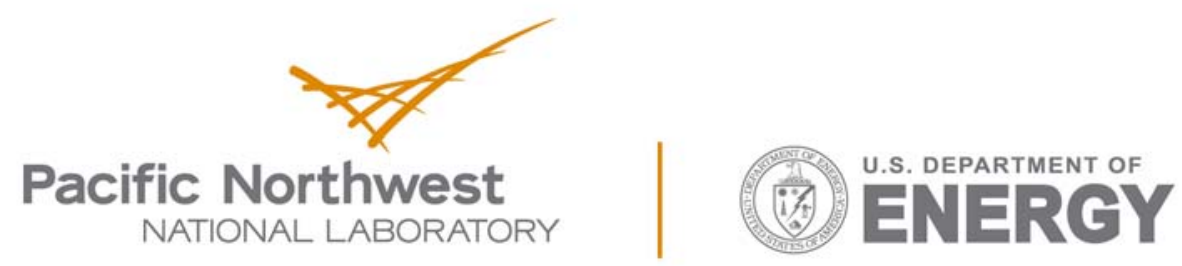

Proudly Operated by Battelle Since 1965

902 Battelle Boulevard

P.O. Box 999

Richland, WA 99352

1-888-375-PNNL (7665)

www.pnnl.gov 